\author{
Rereners \\ औis \\ $0.8 \pi$
}

September 1994

Prepared by the

U.S Department of Energy

Albuquerque, New Mexico

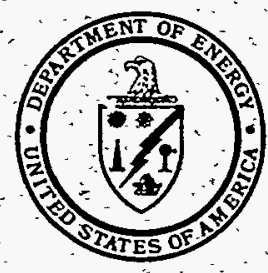




\section{INTENDED FOR PUBLIC RELEASE}

This report has been reproduced from the best available copy. Available in paper copy and microfiche.

Number of pages in this report: 661

DOE and DOE contractors can obtain copies of this report from:

Office of Scientific and Technical Information

P:0. Box. 62

Oak Ridge; TN 37831

(615) $576-8401$

This report is publicly available from:

National Techinicaí Informâtion Service

Department of Commerce

5285 Port Royal Road

Springfield, VA 22161

(703) $487-4650$ 


\section{DISCLAIMER}

This report was prepared as an account of work sponsored by an agency of the United States Government. Neither the United States Government nor any agency thereof, nor any of their employees, make any warranty, express or implied, or assumes any legal liability or responsibility for the accuracy, completeness, or usefulness of any information, apparatus, product, or process disclosed, or represents that its use would not infringe privately owned rights. Reference herein to any specific commercial product, process, or service by trade name, trademark, manufacturer, or otherwise does not necessarily constitute or imply its endorsement, recommendation, or favoring by the United States Government or any agency thereof. The views and opinions of authors expressed herein do not necessarily state or reflect those of the United States Government or any agency thereof. 


\section{DISCLAIMER}

Portions of this document may be illegible in electronic image products. Images are produced from the best available original document. 


\title{
SITE OBSERVATIONAL WORK PLAN FOR THE UMTRA PROJECT SITE \\ AT SPOOK, WYOMING
}

September 1994

\author{
Prepared for \\ U.S. Department of Energy \\ UMTRA Project Office \\ Albuquerque, New Mexico \\ Prepared by \\ Jacobs Engineering Group Inc. \\ Albuquerque, New Mexico
}




\section{EXECUTIVE SUMMARY}

The requirements for ground water compliance for UMTRA Project sites, including the Spook, Wyoming, processing site, are found in the Uranium Mill Tailings Radiation Control Act (UMTRCA) of 1978 (42 USC $\$ 7901$ et seq.) and Subparts B and Subpart C of U.S. Environmental Protection Agency's (EPA) "Health and Environmental Protection Standards for Uranium and Thorium Mill Tailings" codified in 40 CFR Part 192 (1994), and the associated proposed 1987 standards published in 52 FR 36000 (1987).

Ground water in the uppermost aquifer beneath and downgradient from the Spook site was contaminated by uranium-processing activities that occurred from 1962 until 1966. Contamination from the tailings pile and the acid pond (including uranium; selenium, nitrate, and radium-226 and -228) leached into the subsurface by infiltration and migrated into ground water, forming a plume extending approximately 2500 feet $(\mathrm{ft})(760$ meters [m]) downgradient from the site.

The surface remedial action at the Spook site under Subpart A of 40 CFR Part 192 consisted of stabilizing the uranium mill tailings and other residual radioactive materials in a disposal cell in the bottom of the Spook open-pit mine and covering the cell with overburden material. The ground water protection strategy was supplemental standards based on Class III ground water in the uppermost aquifer because of widespread ambient contamination.

The proposed ground water protection strategy to comply with Subpart B of 40 CFR Part 192 at the Spook site is to perform no remediation. This strategy is based on ground water in the uppermost aquifer (also the zone of contamination) being classified as Class III, which allows the application of supplemental standards. Class III ground water beneath the Spook site is neither a current nor a potential source of drinking water because of widespread, ambient contamination that cannot be cleaned up using treatment methods reasonably employed in public water supply systems (40 CFR $\$ 192.11(\mathrm{e})$ ). Ground water at the Spook site contains widespread, ambient uranium and selenium contamination resulting from naturally-occurring conditions (natural uranium mineralization associated with an alteration front) and from the effects of broadscale human activity not related to uranium-milling operations (uranium exploration and mining activities). There is no apparent risk to human health and the environment because there are no known exposure pathways for ground water from the uppermost aquifer. No one is using or is projected to use the ground water from this aquifer for any purpose and there is no discharge of ground water from the uppermost aquifer to deeper aquifers used for domestic and agricultural purposes or to the surface or to surface water. 


\section{TABLE OF CONTENTS}

Section

Page

1.0 INTRODUCTION .......................... 1-1

1.1 Purpose and content ........................ 1-1

1.2 Ground water compliance strategy .................. 1-1

1.3 Relationship to other ground water documents $\ldots \ldots \ldots \ldots \ldots \ldots \ldots$

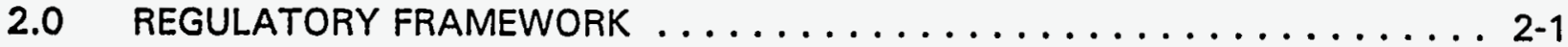

2.1 Uranium mill tailings radiation control act $\ldots \ldots \ldots \ldots \ldots \ldots \ldots \ldots$. . . . . . . . . .

2.1.1 EPA ground water standards ................. 2-1

2.1.2 Cooperative agreements and state requirements ....... 2-4

2.2 National environmental policy act . . . . . . . . . . . . . . . 2-4

3.0 SUMMARY OF SITE CONDITIONS $\ldots \ldots \ldots \ldots \ldots \ldots \ldots \ldots \ldots \ldots \ldots \ldots$

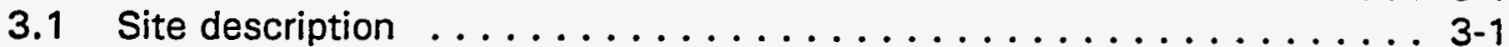

3.1 .1 Location ....................... 3-1

3.1.2 History of operations and surface remediation ........ 3-1

3.1.3 Land and water use ...................... 3-4

3.1 .4 Sources of existing data $\ldots \ldots \ldots \ldots \ldots \ldots \ldots \ldots . \ldots \ldots$

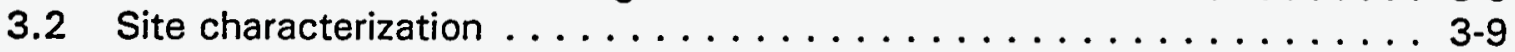

3.2.1 Hydrogeology . . . . . . . . . . . . . . . . . . 3-9

3.2.2 Geochemistry and background ground water quality ..... 3-15

3.3 Conceptual site model . . . . . . . . . . . . . . . . . 3-19

3.3.1 Contaminant Sources . . . . . . . . . . . . . . . 3-22

3.3.2 Extent and magnitude of contamination . . . . . . . . 3-22

3.3.3 Potential release mechanisms ................ 3-26

3.3.4 Contaminant fate and transport mechanisms . . . . . . . . 3-26

3.3.5 Background risk evaluation ................ 3-27

3.3.6 Treatability issues . . . . . . . . . . . . . . . 3-28

3.4 Data deficiencies . . . . . . . . . . . . . . . . 3-30

4.0 GROUND WATER COMPLIANCE STRATEGY SELECTION $\ldots \ldots \ldots \ldots . \ldots . . .4$

4.1 Decision framework process $\ldots \ldots \ldots \ldots \ldots \ldots \ldots \ldots \ldots \ldots . \ldots \ldots$

4.2 Conclusion .......................... 4-3

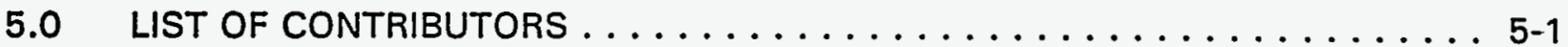

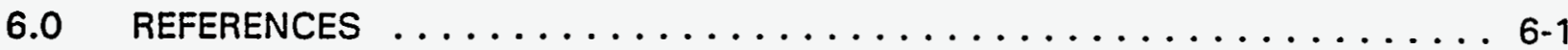

APPENDIX A - MONITOR WELL LITHOLOGIC LOGS

APPENDIX B - MONITOR WELL COMPLETION RECORDS

APPENDIX C - GROUND WATER ELEVATIONS

APPENDIX D - GROUND WATER QUALITY DATA BY PARAMETER

APPENDIX E - AQUIFER PUMPING TEST CALCULATION SET

APPENDIX F - RISK ANALYSIS 


\section{LIST OF FIGURES}

Figure

Page

3.1 Location of the Spook, Wyoming, site . . . . . . . . . . . . . . . 3-2

3.2 Monitor well location map, Spook, Wyoming, site . . . . . . . . . . . . 3-6

3.3 Cross section A-A', Spook, Wyoming, site .................. 3-10

3.4 Cross section B-B', Spook, Wyoming, site . . . . . . . . . . . . 3-11

3.5 Potentiometric surface map of the upper sandstone unit, Spook, Wyoming, site ................................... 3-13

3.6 Potentiometric surface map of the lower sandstone unit, Spook, Wyoming,

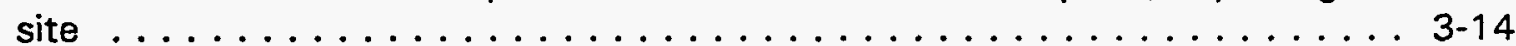

3.7 Alteration front in the vicinity of the Spook, Wyoming, site . . . . . . . . . 3-16

3.8 Extent of contamination in upper sandstone unit based on distribution of ammonium, acid $\mathrm{pH}$, and nitrate at the Spook, Wyoming, site . . . . . . . 3-21

3.9 Conceptual model for the Spook, Wyoming, site . . . . . . . . . . . . . 3-23

4.1 Ground water compliance strategy selection framework . . . . . . . . . 4-2

F.1 Extent of contamination in upper sandstone unit based on distribution of ammonium, acid $\mathrm{pH}$, and nitrate at the Spook, Wyoming, site . . . . . . . F -5 


\section{LIST OF TABLES}

Table

Page

2.1 MCLs for inorganic constituents for ground water protection for UMTRA Project sites .......................... 2-3

3.1 Monitor well information at the Spook, Wyoming, site . . . . . . . . . . . 3-7

3.2 Ground water quality sampling history at the Spook, Wyoming, site . . . . . . 3-8

3.3 Summary of regional ground water quality in altered and unaltered sandstone of the Wasatch Formation, Converse County, Wyoming . . . . . . 3-18

3.4 Summary of ground water quality in the upper sandstone unit at the Spook,

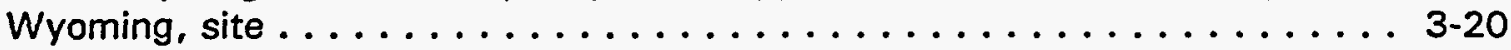

3.5 Summary of ground water quality in the lower sandstone unit at the Spook, Wyoming, site . . . . . . . . . . . . . . . . . . 3-25

F.1 Background, agricultural irrigation water, and livestock drinking water contaminant concentration comparison at the Spook, Wyoming, site . . . . . F-2 
LIST OF ACRONYMS AND ABBREVIATIONS

\section{Acronym Definition}

ac

acre

$A C L$

BAT

alternate concentration limit

$\mathrm{cm}$

best available treatment

$\mathrm{cm} / \mathrm{s}$

centimeter

EA

centimeters per second

EPA

$\mathrm{ft}$

environmental assessment

gpm

U.S. Environmental Protection Agency

feet

ha

gallons per minute

$\mathrm{km}$

hectare

kilometer

L/S

$m$

$m^{3}$

liters per second

meter

cubic meters

$\mathrm{MCL}$

$\mathrm{mg} / \mathrm{L}$

mi

maximum concentration limit

milligrams per liter

mile

NEPA

NRC

National Environmental Policy Act

U.S. Nuclear Regulatory Commission

PEIS

pCi/L

RAP

Programmatic Environmental Impact Statement

picocuries per liter

Remedial Action Plan

RME

RRM

Rocky Mountain Energy

SOWP

TAGR

residual radioactive materials

site observational work plan

Technical Approach to Groundwater Restoration

UMTRA

Uranium Mill Tailing Remedial Action

UMTRCA Uranium Mill Tailings Radiation Control Act

$\mathrm{yd}^{3}$

cubic yards 


\subsection{INTRODUCTION}

The Spook, Wyoming, site observational work plan (SOWP) proposes site-specific activities to achieve compliance with Subpart B of 40 CFR Part 192 (1994) of the proposed U. S. Environmental Protection Agency (EPA) ground water protection standards (52 FR 36000 (1987)) at this Uranium Mill Tailing Remedial Action (UMTRA) Project site.

\subsection{PURPOSE AND CONTENT}

The purpose of the SOWP is to recommend the site-specific ground water compliance strategy at the Spook UMTRA Project processing site. This draft SOWP (Revision 0) presents a comprehensive summary of existing site characterization data, a conceptual site model of the nature and extent of ground water contamination, exposure pathways, and potential impact to human health and the environment. Section 2.0 describes the requirements for meeting ground water standards at UMTRA Project sites. Section 3.0 defines past and current conditions, describes potential environmental and human health risks, and provides site-specific data that support the proposed ground water compliance strategy. Section 4.0 provides the justification for selecting the proposed ground water compliance strategy.

\subsection{GROUND WATER COMPLIANCE STRATEGY}

Ground water in the uppermost aquifer beneath and downgradient from the Spook site was contaminated by uranium processing activities that occurred from 1962 until 1966. Contamination from the tailings pile and the acid pond (including uranium, selenium, nitrate, and radium-226 and -228) was released into the subsurface by infiltration and migrated into ground water, forming a plume extending approximately 2500 feet $(\mathrm{ft})$ (760 meters [m]) downgradient from the site.

The proposed ground water compliance strategy, in accordance with Subpart B of 40 CFR Part 192 (1994), is to perform no remediation at the Spook site. This strategy is based on ground water in the uppermost aquifer (also the zone of contamination) being classified as Class III, which allows the application of supplemental standards. Class III ground water beneath the Spook site is not a current or potential source of drinking water because of widespread, ambient contamination that cannot be cleaned up using treatment methods reasonably employed in public water supply systems (40 CFR \$192.11(e)). Ground water in the uppermost aquifer contains widespread, ambient uranium and selenium contamination resulting from naturally-occurring conditions (natural uranium mineralization associated with an alteration front) and from the effects of broadscale human activity not related to uranium milling operations (uranium exploration and mining activities). The potential for impacts to human health and the environment related to ground water contamination associated with the Spook site is minimal because 1) ground water in the area has been affected by naturally-occurring uranium mineralization and human activities related to 
regional uranium exploration and mining, 2) ground water in the uppermost aquifer is not locally used for any purposes, 3 ) the area is remote and has a low population density, 4) adequate ground water resources are available from deeper sandstone units, some under artesian conditions, and 5) there is no evidence of discharge of contaminated ground water from the uppermost aquifer to deeper aquifers used for domestic and agricultural purposes or to the surface or to surface water.

\subsection{RELATIONSHIP TO OTHER GROUND WATER DOCUMENTS}

Programmatic documents that provide guidance for the SOWP include the DOE UMTRA Groundwater Program Plan (DOE, 1992a), the "Programmatic Environmental Impact Statement for the Uranium Mill Tailings Remedial Action Ground Water Project" (PEIS) (DOE, 1994) and the Technical Approach to Groundwater Restoration (TAGR) (DOE, 1993a). The UMTRA Groundwater Program Plan states the objectives for the UMTRA Ground Water Project and provides an overall technical plan and management approach for conducting the program. The PEIS provides a programmatic decision-making framework for conducting the UMTRA Ground Water Project; assesses the potential programmatic impacts of conducting the Project; provides a method for determining the site-specific ground water compliance strategies; and provides data and information that can be used to prepare site-specific National Environmental Policy Act (NEPA) (42 USC $\$ 4321$ et seq.) documentation more efficiently. The TAGR provides general technical guidance concerning how the Ground Water Project will be conducted.

The environmental impacts from the proposed compliance strategy presented in this SOWP will be assessed in appropriate site-specific NEPA documentation. The UMTRA Surface Project Remedial Action Plan and Site Conceptual Design for Stabilization of the Inactive Uranium Mill Tailings Site at Spook, Wyoming (RAP) (DOE, 1990) will be modified and submitted to the U.S. Nuclear Regulatory Commission (NRC) for concurrence on the UMTRA Project's proposed compliance strategy. 


\subsection{REGULATORY FRAMEWORK}

The requirements for selecting a ground water compliance strategy for the Spook, Wyoming, processing site are described in this section. These requirements are found in the Uranium Mill Tailings Radiation Control Act (UMTRCA) of 1978 (42 USC $\S 7901$ et seq.) and Subpart B and of EPA's "Health and Environmental Protection Standards for Uranium and Thorium Mill Tailings" (40 CFR Part 192 (1994)).

\section{$2.1 \quad$ URANIUM MILL TAILINGS RADIATION CONTROL ACT}

The U.S. Congress passed the UMTRCA in 1978 in response to public concerns about the potential health hazards from exposure to uranium mill tailings over long periods of time. The UMTRCA authorized the DOE to stabilize, dispose of, and control uranium mill tailings and other residual radioactive materials (RRM) at uranium mill processing sites. The UMTRCA designates 24 inactive processing sites to undergo remediation; directs the EPA to promulgate standards; mandates the DOE to perform remedial action in accordance with standards prescribed by the EPA; directs the NRC to concur in the remedial actions; directs the NRC to license the disposal sites for long-term care; and directs the DOE to enter into cooperative agreements with the affected states and Indian tribes.

In 1988, Congress amended UMTRCA and authorized the DOE to extend without limitation the time needed to complete ground water restoration activities at the processing sites. Section 108 of the UMTRCA requires that the DOE comply with the proposed EPA standards (40 CFR Part 192 (1994)) in the absence of final standards.

\subsubsection{EPA ground water standards}

The UMTRCA requires the EPA to promulgate standards for protecting public health, safety, and the environment from radiological hazards and nonradiological hazards associated with processing of uranium and the resulting RRM. UMTRCA defines RRM as waste in the form of tailings resulting from the processing of ores for the extraction of uranium, and other wastes (which the Secretary of the Department of Energy determines to be radioactive) at a processing site that relate to such processing, including any residual stock of unprocessed ores or low-grade materials. The 1994 draft final rule for "Groundwater Standards for Remedial Actions at Inactive Uranium Processing Sites" (EPA, 1994) includes any residual stock of unprocessed ores or low grade materials, such as sludges and captured contaminated water from the processing site.

On January 5, 1983, the EPA published standards (40 CFR Part 192) for the disposal and cleanup of RRM. The EPA published proposed changes to the ground water standards in the Federal Register on September 24, 1987 (52 FR 36000). The standards address two ground water contamination scenarios: 
Subpart $A$ addresses future ground water contamination that may occur from RRM disposal, and Subpart $B$ addresses the cleanup of contamination of ground water that occurred at the processing sites before disposal of the RRM. Protection of the ground water from future contamination at the disposal sites is required by Subpart $A$ and has been addressed in the UMTRA Surface Project. The UMTRA Ground Water Project addresses the ground water contamination that occurred at the processing sites and is regulated by Subparts $B$ and $C$ of the proposed standards.

\section{Subpart B}

Subpart B, "Standards for Cleanup of Land and Buildings Contaminated with Residual Radioactive Materials from Inactive Uranium Processing Sites," requires that remedial action at processing sites be conducted to ensure that the amounts of RRM in ground water meet any one of three ground water standards:

- Background levels: concentrations of constituents in nearby ground water that were not affected by processing activities (52 FR 36007, 40 CFR $\S 192.12(\mathrm{c}))$.

- Maximum concentration limits (MCL): the EPA's maximum limits for concentrations of certain hazardous constituents in ground water, as proposed for the UMTRA Project. The MCLs for inorganic constituents that apply to UMTRA Project sites are provided in Table 2.1 (52 FR 36007, 40 CFR §192.12(c)).

- Alternate concentration limits (ACL): alternate limits for hazardous constituents that do not pose a substantial present or potential hazard to human health or the environment as long as the limits are not exceeded (52 FR 36007, 40 CFR \$192.12(c)(2)).

Subpart B also provides for active ground water remediation or for selecting natural flushing as a means to meet the proposed standards. Active ground water remediation would require the application of engineered ground water remediation methods such as gradient manipulation, ground water extraction and treatment, and in situ ground water treatment to achieve compliance with the proposed standards. Natural flushing means allowing natural ground water processes to reduce the contamination in ground water, and these processes must allow the standards to be met within 100 years. Institutional controls that protect human health (measures that restrict access to contamination and satisfy beneficial uses of ground water) and an adequate monitoring program must be established and maintained during the period of natural flushing. Ground water also must not currently be, or be projected to become, a source of drinking water during the period of natural flushing (52 FR 36007, 40 CFR $\S 192.12(\mathrm{c})(4))$. 
Table 2.1 MCLs for inorganic constituents for ground water protection for UMTRA Project sites ${ }^{a}$

\begin{tabular}{|c|c|}
\hline Constituent & Maximum concentration ${ }^{b}$ \\
\hline Arsenic & 0.05 \\
\hline Barium & 1.0 \\
\hline Cadmium & 0.01 \\
\hline Chromium & 0.05 \\
\hline Lead & 0.05 \\
\hline Mercury & 0.002 \\
\hline Molybdenum & 0.1 \\
\hline Nitrate (as N) & $10.0^{c}$ \\
\hline Selenium & 0.01 \\
\hline Silver & 0.05 \\
\hline Combined radium-226 and radium-228 & $5 \mathrm{pCi} / \mathrm{L}$ \\
\hline Combined uranium-234 and uranium-238 & $30 \mathrm{pCi} / \mathrm{L}^{d}$ \\
\hline $\begin{array}{l}\text { Gross alpha-particle activity } \\
\text { (excluding radon and uranium) }\end{array}$ & $15 \mathrm{pCi} / \mathrm{L}$ \\
\hline $\begin{array}{l}\mathrm{a}_{52} \mathrm{FR} 36007 \text { (1987). } \\
\mathrm{b}_{\text {Milligrams per liter unless otherwise note }} \\
c_{\text {Equivalent to } 44 \text { milligrams per liter (mg/l }} \\
d_{\text {Equivalent to } 0.044 \text { ma/L. }}\end{array}$ & nitrate expressed as $\mathrm{NO}_{3}$. \\
\hline
\end{tabular}




\section{Subpart C}

Subpart C, "Implementation," includes guidance for implementing methods and procedures to provide reasonable assurance that the provisions of Subpart $B$ are satisfied. Subpart $C$ requires that the conditions of Subpart $B$ be met on a sitespecific basis, using information gathered from site characterization and monitoring. A remedial action plan must be presented, stating the schedule and steps necessary to complete the cleanup of ground water at the sites to meet the conditions of Subpart B (52 FR 36008, 40 CFR \$192.20(b)(4)).

Subpart $C$ provides guidance for implementing methods and procedures to provide reasonable assurance that the provisions of Subpart $B$ are satisfied (52 FR 36008, 40 CFR §192.21(g)).

\subsubsection{Cooperative agreements and state requirements}

The UMTRCA requires that remedial action be accomplished with the full participation and concurrence of the affected states and Indian tribes on whose lands the uranium mill tailings are located or disposed. The UMTRCA also directed the DOE to enter into cooperative agreements with these states and Indian tribes. No Indian tribes are affected by the activities at the Spook site. A cooperative agreement for the UMTRA Surface Project with the state of Wyoming was executed February 16, 1984.

The Wyoming state regulations for ground water are in Chapter VIII of the state of Wyoming water quality standards (State of Wyoming, 1993) and are presented as points of reference that support the Class III EPA ground water definition. Ground water found closely associated with commercial deposits of minerals, as found at the Spook site, may be considered Class $V$ ground water and is not suitable for domestic, agricultural, and livestock uses, fish and aquatic life, or industrial purposes (Section $4(d)(8)(b)$ ). In addition, Class VI ground water may be unusable or unsuitable for use because it is so contaminated that it would be economically or technologically impractical to make the water usable (Section $4(d)(9)(b)$ ). In classifying ground water, the state takes into account the technical practicality and economic reasonableness of treating ambient water quality to meet suitability standards for use (Section 5(a)). The state's water quality MCLs for domestic use (Section $4(d)(1)$ ) are similar to those proposed by EPA MCLs with the exception of uranium. The state established a MCL of $5.0 \mathrm{mg} / \mathrm{L}$; the EPA established a MCL for uranium of $0.044 \mathrm{mg} / \mathrm{L}$.

\subsection{NATIONAL ENVIRONMENTAL POLICY ACT}

Implementation of the UMTRCA represents a major federal action subject to the requirements of NEPA. The Council on Environmental Quality's regulations that implement the NEPA are codified in 40 CFR Parts 1500-1508 (1994) and require that each federal agency develop its own implementing procedures $(40$ CFR \$1507.3). The DOE NEPA regulations are contained in "National 
Environmental Policy Act; Implementing Procedures" (10 CFR Part 1021 (1994)). DOE guidance is provided in Recommendations for the Preparation of Environmental Assessments and Environmental Impact Statements (DOE, $1993 b)$.

Pursuant to the NEPA, the DOE drafted a PEIS in 1994 that analyzed potential impacts of implementing programmatic alternatives for conducting the Ground Water Project. The preferred alternative for conducting the Ground Water Project will be selected by the DOE and published in a record of decision. All subsequent actions on the UMTRA Ground Water Project, including preparation of site-specific NEPA documentation, must comply with the record of decision. 


\subsection{SUMMARY OF SITE CONDITIONS}

This section describes the location and history of the Spook site, including local geography, past uranium mining and milling activities at and in the vicinity of the site, and the surface remedial action and associated ground water protection strategy. Site characterization data, and the conceptual site model are also presented.

\subsection{SITE DESCRIPTION}

\subsubsection{Location}

The Spook processing and disposal site is in northern Converse County, Wyoming, approximately 48 miles (mi) $(77$ kilometers [km]) northeast of Casper (Figure 3.1). The area is sparsely populated, with the nearest town, Glenrock, $31 \mathrm{mi}(50 \mathrm{~km})$ south of the site. The climate is semiarid and cool with moderate humidity. The average annual precipitation (measured in the Casper area) is 11 inches (28 centimeters [cm]) with 60 percent of the precipitation falling during the spring and summer months (DOE, 1989). The ground is covered principally with short-grass prairie vegetation.

The Spook site is situated in the southern end of the Powder River Basin, a deep structural basin that lies along the east flank of the Middle Rocky Mountains. Sedimentary rocks in the area generally dip less than 2 degrees to the northeast. The gently rolling topography in the vicinity of the site has an elevation of approximately $5100 \mathrm{ft}(1555 \mathrm{~m})$. Drainage in the area is to the east and southeast toward the Dry Fork of the Cheyenne River, an intermittent stream approximately $1 \mathrm{mi}(1.6 \mathrm{~km})$ south of the site. Surface drainage is controlled to the north by the Cheyenne River Divide.

The southern part of the Powder River Basin contains significant uranium and coal deposits. The site is underlain by uranium mineralization associated with an alteration front in sandstone in the Tertiary Wasatch Formation. Uranium has been mined from open-pit mines (including the Spook pit) along the alteration front in the area. The Bear Creek uranium mill site (an UMTRCA Title II site) is approximately $2 \mathrm{mi}(3 \mathrm{~km})$ north of the Spook site.

\subsubsection{History of operations and surface remediation}

Through the 1960s, much of the uranium ore mined in the United States was processed by private companies under contract to the federal government for use in national defense research and weapons development. The Spook site was formerly mined for this purpose by the Wyoming Mining and Milling Company and Western Nuclear, Inc.

The Spook open-pit mine was first excavated in 1962 and operated as a producing mine until 1966. A total of 42,600 tons $(38,647$ tonnes) of uranium ore were extracted. Initially uranium ore from the Spook mine was processed at 


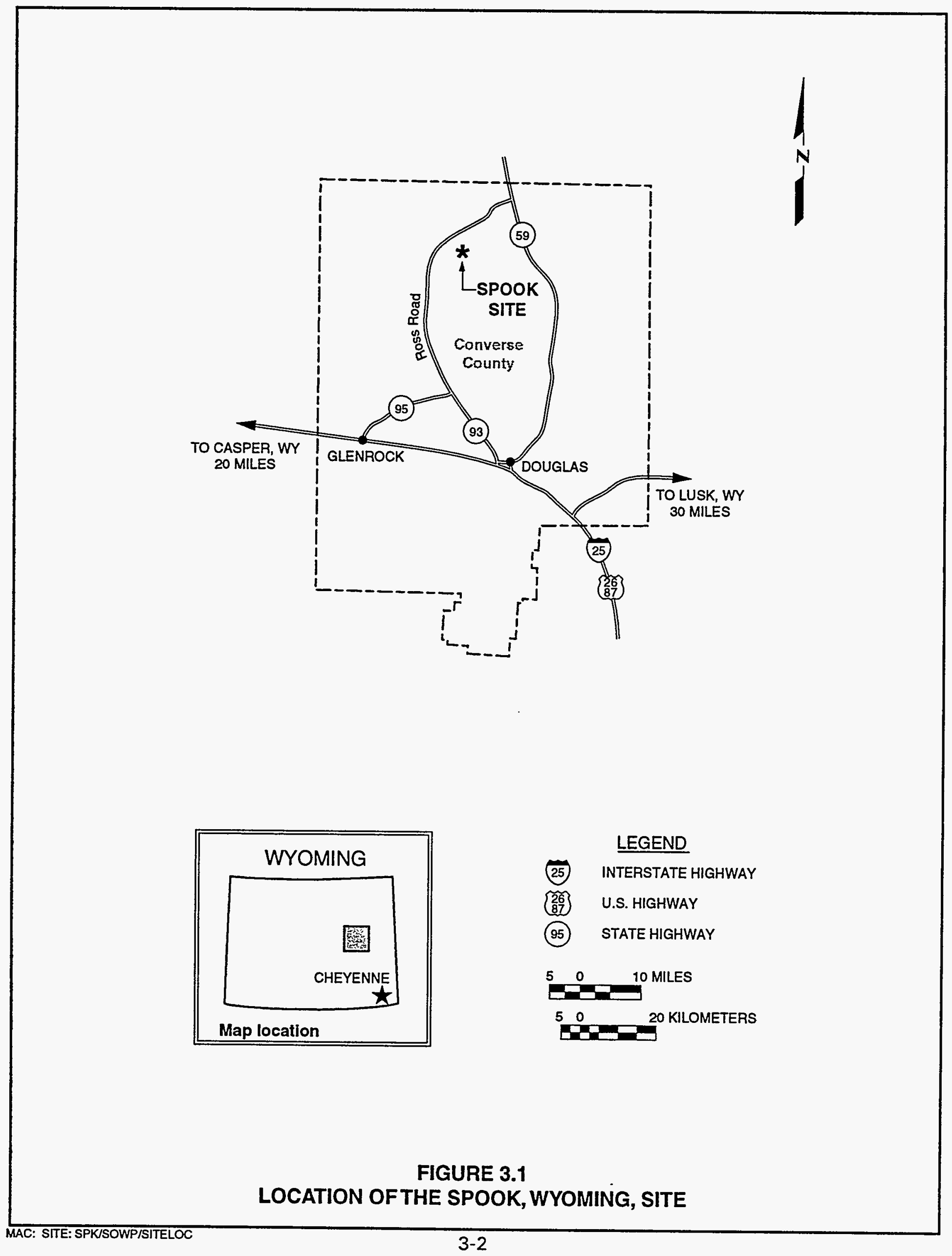


the uranium mill at Jeffrey City, Wyoming, $165 \mathrm{mi}(265 \mathrm{~km})$ west of the site. As lower-grade ore was mined, it became more economical to process the ore on the site, so a uranium upgrading plant was established at the Spook site.

The uranium milling process at the Spook upgrader consisted of an acid-curing process used in conjunction with percolation heap leaching. In this process, crushed sandstone containing the uranium was agglomerated into small pellets containing ore, water, and sulfuric acid. These pellets were loaded into large vats and allowed to cure (given time for the interstitial sulfuric acid to react with the ore the pellets). After curing, an acidified solution was circulated through the pellet bed within the vats and then drained. This pregnant, uranium-bearing solution was processed by ion exchange. The pelletized ore was then washed, and this wash solution was used to leach uranium from the next batch of pellets. The barren, washed pellets were disposed of in the open-pit mine. The uranium-bearing solution was passed through ion exchange columns containing an ammonium-bearing exchange resin. Uranyl sulfate complexes in the acid uranium-bearing solution were exchanged for ammonium, separating the uranium from other metals in the pregnant solution. The uranium was then removed from the exchanger (eluted) by passing another solution through the exchange column that released the uranium from exchange sites. There are several possible elutants, including 1) sulfuric acid solution or 2) acidified solutions of ammonium chloride or sodium nitrate. The latter elutant was probably used at the Spook upgrader, since this would explain the elevated concentrations of nitrate in ground water. After elution, the resins were regenerated using a solution of nitric acid followed by solutions of sodium and ammonium hydroxide. The eluted uranium was precipitated from solution as an oxide by neutralization with anhydrous ammonia. Some liquid was removed from this precipitate, leaving a thick slurry that was trucked to the uranium mill at Jeffrey City. The residue sands (tailings) were placed on the surface at the mill site or receded into the open-pit mine. The solutions used in the process were disposed of on the tailings pile and in the acid pond $1500 \mathrm{ft}(460 \mathrm{~m})$ south of the mill site (Merritt, 1971; Mashbir 1964).

As a result of this operation, process reagents in the contaminated ground water at the site include sulfate, ammonium, sodium, nitrate, and, possibly, chloride. In addition, leaching of the ore would have solubilized several metals and nonmetals, including aluminum, iron, calcium, magnesium, potassium, manganese, selenium, molybdenum, chrome, nickel, zinc, and the thorium progeny of uranium decay, including thorium-230, radium-226, and lead-210.

Water used for ore processing came from two wells near the mill site completed to 350 and $700 \mathrm{ft}(107$ and $213 \mathrm{~m})$ respectively. The volume of water used in the processing activities is not known, but the wells were capable of yielding 60 and 150 gallons per minute (gpm) (4 to 9.5 liters per second [L/s]) respectively, based on well permit information from the Wyoming State Engineer's Office.

Surface remediation at the Spook processing site under Subpart A of 40 CFR Part 192 (1994) consisted of stabilizing the RRM in a disposal cell in the bottom 
of the Spook open-pit mine. Surface remedial action was completed in November 1989. An estimated 269,600 cubic yards ( $\left.y d^{3}\right)(206,200$ cubic meters $\left[\mathrm{m}^{3}\right]$ ) of uranium mill tailings and other RRM were consolidated and stabilized in the disposal cell. The disposal cell includes a basal clay layer and is covered with a fine-grained infiltration and radon barrier. These features were designed to minimize infiltration, seepage, and migration of leachate from the bottom of the cell. The state of Wyoming then backfilled the Spook open-pit mine with overburden material. The surface was restored and revegetated. The top of the disposal cell is approximately $35 \mathrm{ft}(11 \mathrm{~m})$ below the existing land surface.

The surface remedial action ground water protection strategy at the Spook site, under Subpart A of 40 CFR Part 192, is application of supplemental standards based on Class III ground water in the uppermost aquifer because of widespread ambient contamination. The Class III designation is based on ground water in the uppermost aquifer not being currently or potentially a source of drinking water because it contains widespread, ambient uranium and selenium contamination resulting from 1) naturally-occurring conditions (natural uranium mineralization associated with an alteration front), and 2) the effects of broadscale human activity not related to uranium milling operations (uranium exploration and mining activities) such that it cannot be effectively cleaned up for drinking or other beneficial purposes using treatment methods reasonably employed in public water supply systems 52 FR 36007.

The state of Wyoming, as agent for the United States of America, acquired title to a 13.5-acre (ac) (5.5-hectare [ha]) surface area over the disposal cell. The NRC developed regulations for issuing a general license for the long-term care of Title I disposal sites (10 CFR part 40 (1994)). Under these regulations, the general license becomes effective when the NRC concurs with the DOE's certification that the surface remedial action at the site is complete, including the ground water protection strategy. In September 1993, the general license for Subpart A was issued for the Spook site.

\subsubsection{Land and water use}

The land surrounding the site is used predominantly for sheep and cattle ranching. Some hay production takes place along the Dry Fork of the Cheyenne River. Prior to 1982, uranium mines were also in production throughout the . area.

Good quality ground water from confined aquifers in the Tertiary Wasatch and Fort Union Formations is used for domestic and stock watering purposes in the area. Ground water from the unconfined mineralized sandstone (uppermost aquifer) beneath the Spook site is not used because it is of marginal quality (because of mineralization and related mining and milling activities) and the sustained yield of ground water from the aquifer is less than yields from underlying confined aquifers. 


\subsubsection{Sources of existing data}

Development and evaluation of the conceptual site model for the Spook site are based on a review of DOE site characterization data generated for the surface remediation phase of the UMTRA Project and reported in the RAP (DOE, 1990). Site characterization data include reconnaissance information, U.S. Geological Survey topographic maps, aerial photography, core and corehole logs, lithologic logs and well completion records from drilling and monitor well installation, data from water quality sampling, and aquifer testing data. Monitor well and water quality data are available in the UMTRA Project Software Program for Environmental Analysis and Reporting data base (DOE, 1992b).

DOE installed 35 monitor wells (SPK-01-910 through -944) in hydrogeologic units beneath the Spook site between December 1986 and August 1988 (Figure 3.2 and Table 3.1). Several monitor wells were decommissioned to facilitate remedial activities (SPK-01-910, $-911,-928,-929$ and -940 ) or because of well construction problems (SPK-01-926). Monitor wells were sampled for ground water quality from February 1987 through December 1988 (Table 3.2). The number of sampling rounds per well varies from two to six, depending on when the monitor wells were installed.

Water quality data through August 1988 were included in the RAP (DOE, 1990). A water sampling round was completed in December 1988 to verify data from the August 1988 sampling round, particularly for the new monitor wells installed during August 1988. These data fulfilled the requirements for site characterization in the RAP and allowed development of a ground water compliance strategy and concurrence from the NRC on the surface remedial action at the Spook site. With supplemental standards, based on Class III ground water, as the compliance strategy, the NRC, DOE, and state agreed that it would not be necessary to continue ground water monitoring for compliance purposes. Therefore ground water sampling was discontinued after December 1988 (DOE, 1993c).

Water levels were measured in the monitor wells during these sampling rounds, as well as during July 1994. Three domestic wells (SPK-01-800, -803, and -903 ) completed in deeper aquifers in the vicinity were also sampled for ground water quality. Details of well construction are not known. Water levels were not taken from these three wells, because two flowed at the surface and one had a windmill to provide water.

Relevant site characterization data are attached as Appendices $A$ through $E$ to this report. These data are monitor well lithologic logs (Appendix $A$ ); monitor well completion records (Appendix B); ground water elevations (Appendix C); ground water quality data by parameter (Appendix D); and a calculation set for aquifer pumping tests (Appendix E). Industry and government reports and 


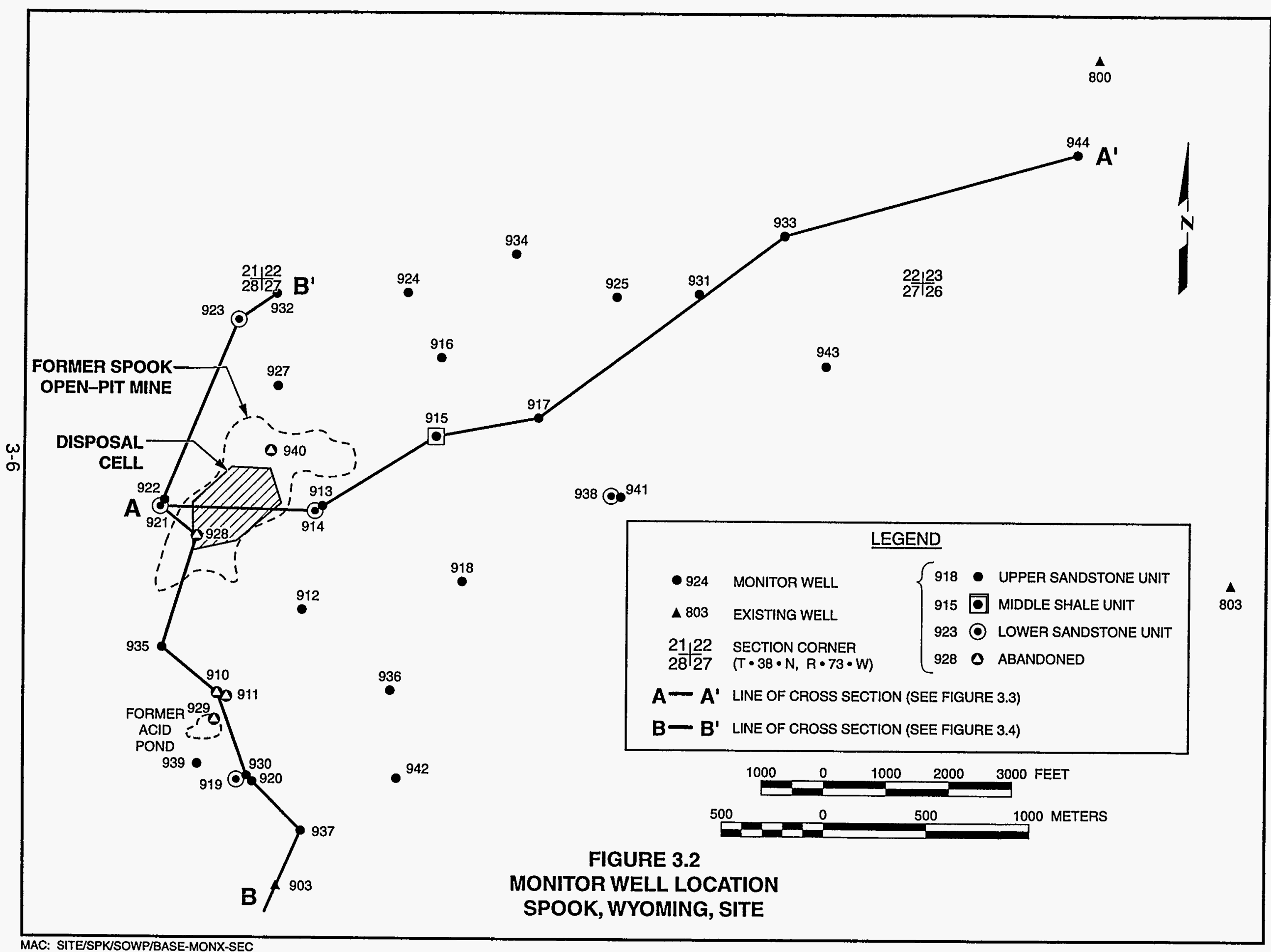


TABLE 3.1 MONITOR HELL INFORMATION AT THE SPOOK, WYONING SITE

SITE: SPKOI SPOOK

REPORT DATE: $09 / 22 / 94$

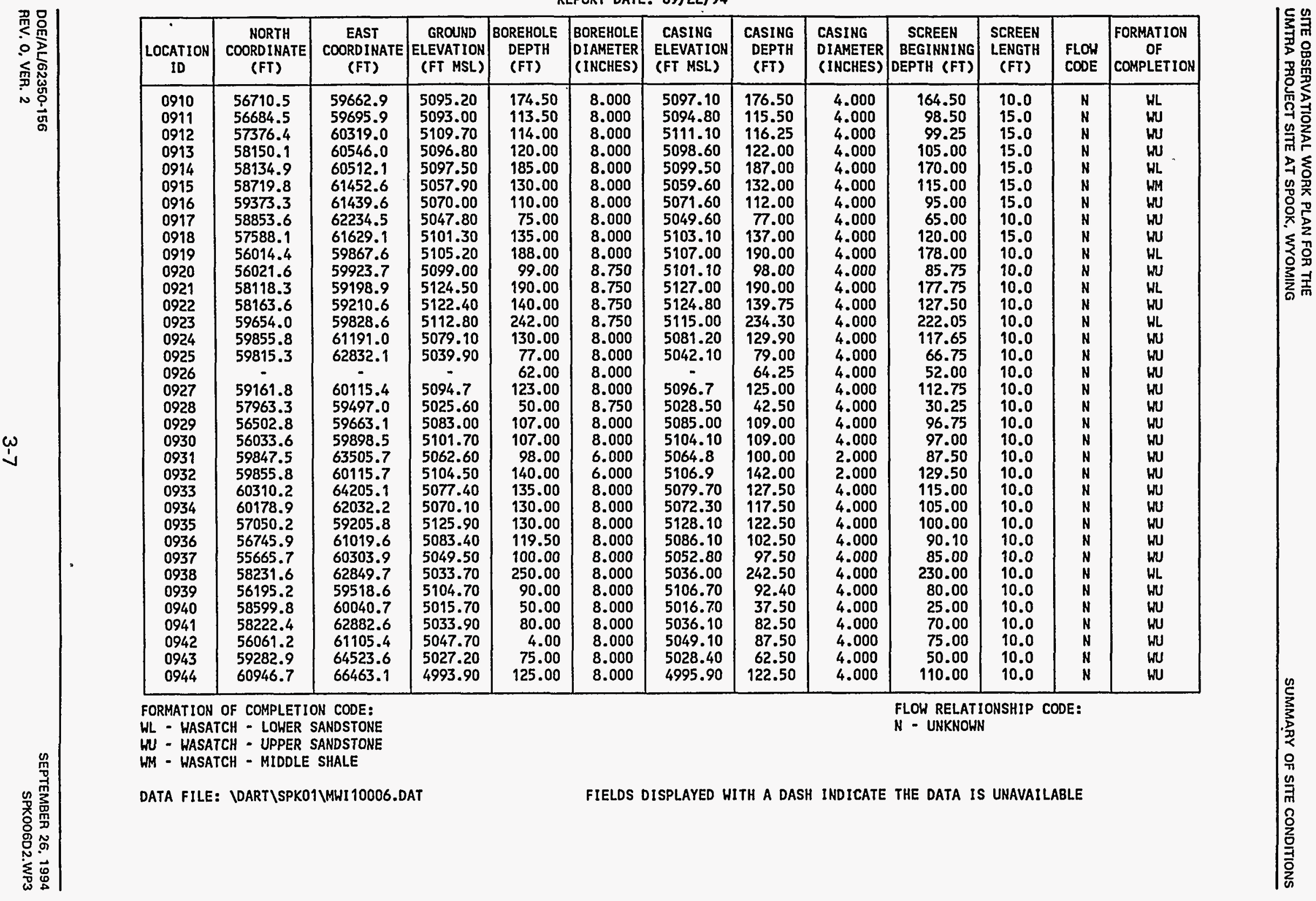




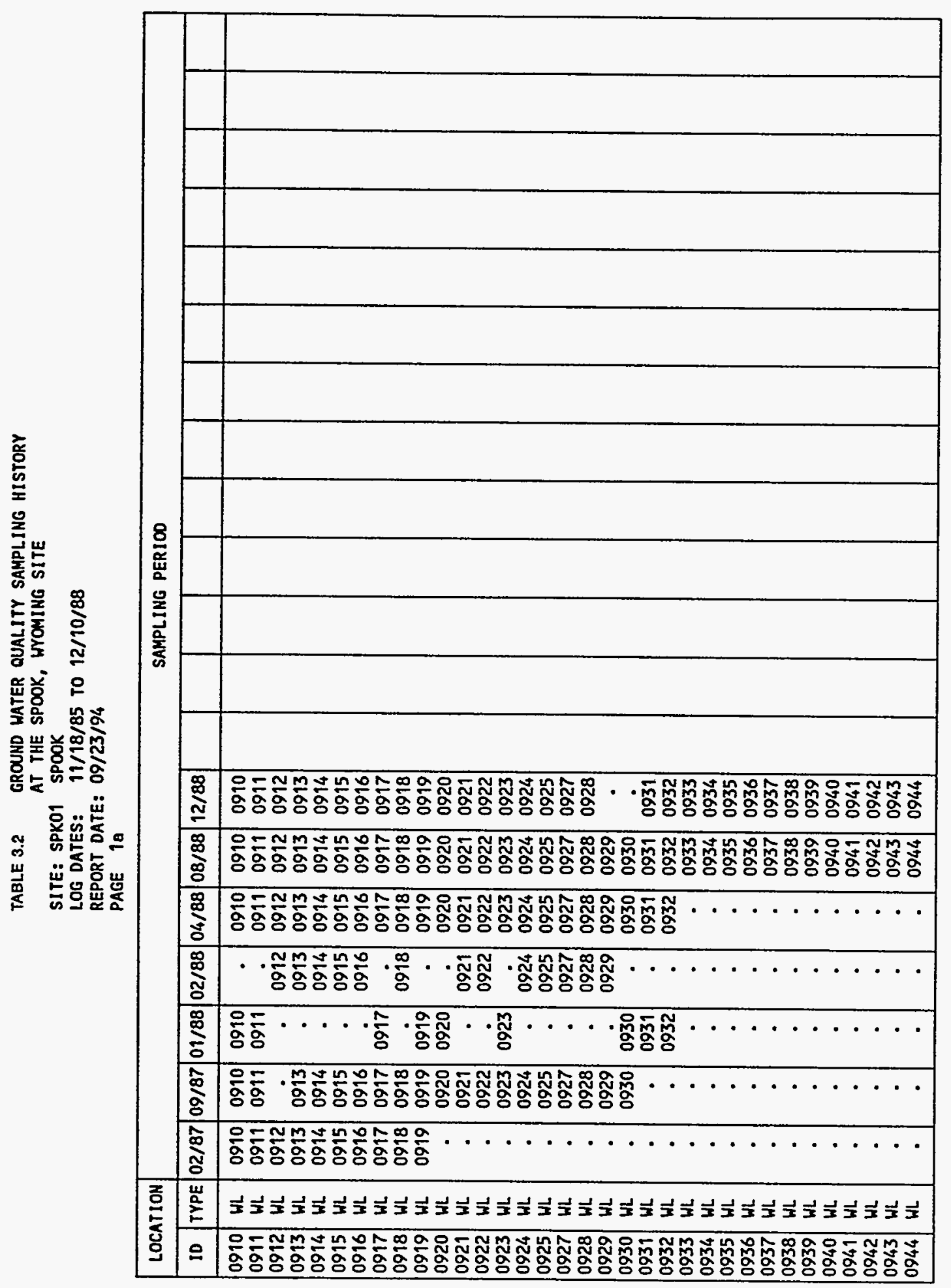


information from the literature were also used in the investigation and are listed in the reference section.

\subsection{SITE CHARACTERIZATION}

\subsubsection{Hydrogeology}

Continental fluvial sediments of the Tertiary Wasatch Formation, in excess of $300 \mathrm{ft}(90 \mathrm{~m})$ thick, underlie the Spook site and dip slightly to the northeast. The Wasatch Formation consists of lenticular fine-grained to conglomeratic arkosic sandstone, siltstone, claystone, carbonaceous shale, and coal. The Wasatch Formation is divided into three distinct hydrogeologic units: the upper sandstone unit, the middle shale unit, and the lower sandstone unit. The upper sandstone unit is considered the uppermost aquifer for demonstrating compliance with Subpart B of the proposed EPA ground water protection standards (52 FR 36000 (1987)).

The upper sandstone unit consists of moderately cemented, fine- to coarsegrained sandstone containing silty shale lenses. It ranges in thickness from 70 to $140 \mathrm{ft}(21$ to $43 \mathrm{~m})$. The middle shale unit is composed of well-consolidated silty shale. It ranges in thickness from $40 \mathrm{ft}(12 \mathrm{~m})$ in the vicinity of the site to greater than $180 \mathrm{ft}(55 \mathrm{~m})$ east of the site. The lower sandstone unit consists of moderately cemented, fine- to coarse-grained sandstone, with the top of the unit in excess of $160 \mathrm{ft}(49 \mathrm{~m})$ below the surface near the site (Figures 3.2, 3.3, and 3.4).

Ground water beneath the Spook site occurs in the upper and lower sandstone units of the Wasatch Formation under unconfined and confined conditions, respectively. Depths to the water table in the upper sandstone unit range from 40 to $120 \mathrm{ft}(12$ to $37 \mathrm{~m})$ below the ground surface. The observed saturated thickness of the upper sandstone unit is approximately $20 \mathrm{ft}(6 \mathrm{~m})$ in the vicinity of the site. The upper and lower sandstone units are separated by an aquitard consisting of a thick, laterally extensive silty-shale unit (middle shale unit) with no apparent hydraulic interconnection between the two units.

Ground water in the upper sandstone unit in the vicinity of the site is recharged by infiltration of precipitation and seepage from intermittent streams. Recharge also resulted from processing solutions used in milling during the 1960 s. Ground water in the upper sandstone unit discharges by ground water underflow. There is no evidence of discharge of ground water from the upper sandstone unit to the land surface or surface water in the vicinity of the site (based on field observations and conversations with local ranchers). Inflow and outflow of ground water in the lower sandstone unit is by ground water underflow. Seasonal fluctuations of the potentiometric surfaces of the upper and lower sandstone units are generally less than $1 \mathrm{ft}(0.3 \mathrm{~m})$. Comparison of ground water elevations measured during 1987-1988 with elevations measured during July 1994 indicates relatively stable ground water conditions in the upper and lower sandstone units in the vicinity of the Spook site. 


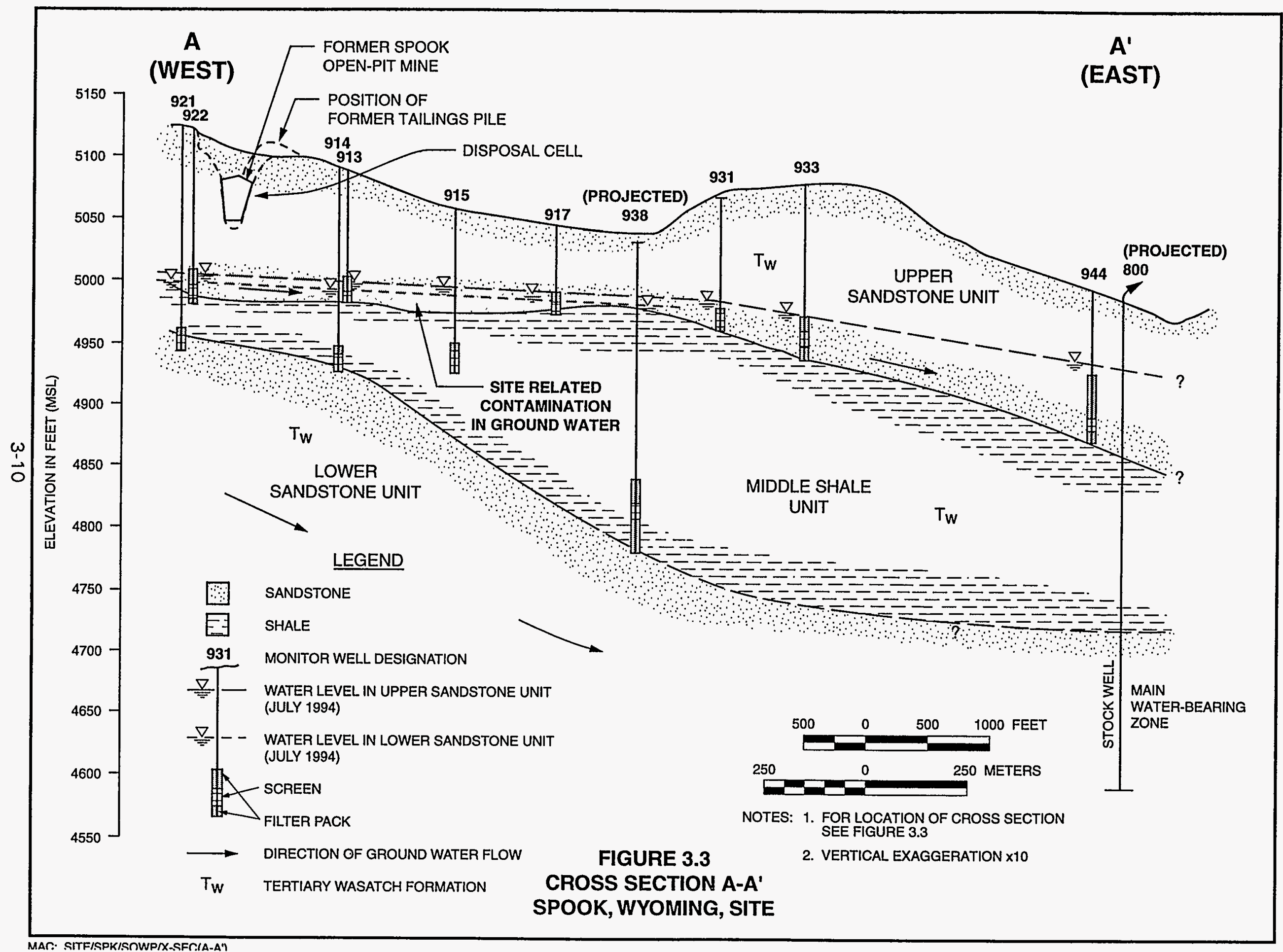




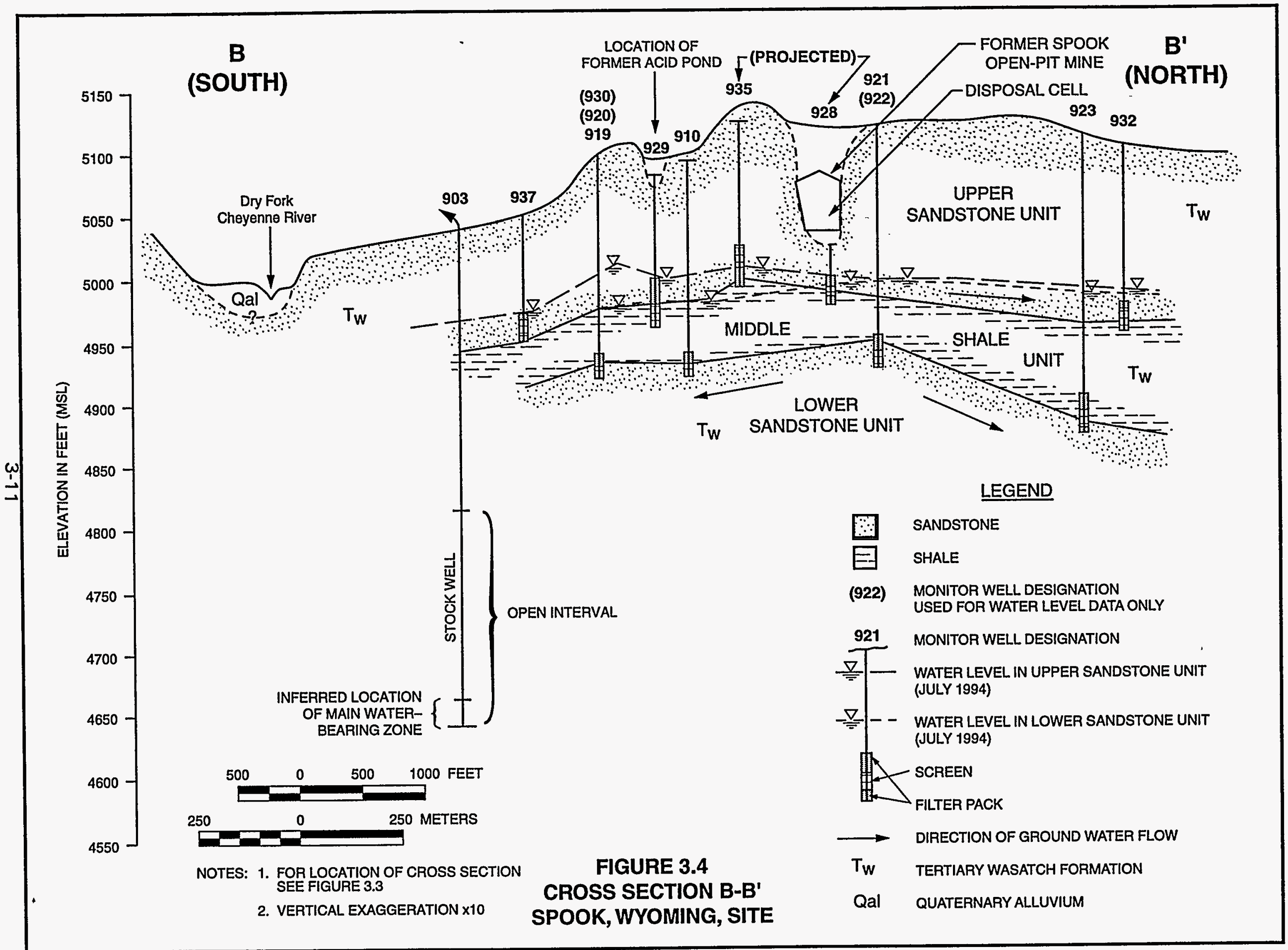


The predominant ground water flow direction in the upper sandstone unit is to the northeast, under a hydraulic gradient averaging 0.005 (Figure 3.5). The gradient becomes steeper northeast and southeast of the site. The configuration of the ground water table in the upper sandstone unit is controlled by the elevation of the top of the middle shale unit as well as land surface topography and recharge. The ground water flow direction in the lower sandstone unit is generally to the southeast under a hydraulic gradient averaging 0.01 (Figure 3.6).

Aquifer pumping tests were conducted in monitor wells SPK-01-913 and -928 to measure hydraulic parameters of the upper sandstone unit. The tests were run for 13.1 and 16.8 hours with an average discharge rate of 3.3 and $4.2 \mathrm{gpm}$ $(0.2 \mathrm{~L} / \mathrm{s}$ and $0.26 \mathrm{~L} / \mathrm{s})$, respectively. The two tests indicated an average transmissivity of $225 \mathrm{ft}^{2} /$ day $\left(2.42 \mathrm{~cm}^{2} / \mathrm{s}\right)$ in the upper sandstone unit. The average hydraulic conductivity of the upper sandstone unit, determined by dividing the transmissivity by the average saturated thickness $(14.5 \mathrm{ft},[4.4 \mathrm{~m}])$, was $15 \mathrm{ft} /$ day $\left(5.3 \times 10^{-3} \mathrm{~cm} / \mathrm{s}\right)$. The average linear ground water velocity in the upper sandstone unit is $0.38 \mathrm{ft} /$ day $\left(1.34 \times 10^{-4} \mathrm{~cm} / \mathrm{s}\right)$, based on the above hydraulic conductivity, a hydraulic gradient of 0.005 , and an estimated effective porosity of 0.20 . The aquifer pumping test calculations are presented in Appendix E.

Although the average discharge rate (yield) of ground water from the uppermost aquifer was sustained at approximately $4 \mathrm{gpm}(0.25 \mathrm{~L} / \mathrm{s})$ during the aquifer pumping tests, steady-state conditions were not reached. Drawdown of approximately 4 to $5 \mathrm{ft}(1$ to $2 \mathrm{~m}$ ) was observed in the monitor wells from an average saturated thickness of $14.5 \mathrm{ft}(4.4 \mathrm{~m})$. Thus, it is not anticipated that this aquifer would be able to sustain long-term pumping to provide an adequate supply of water for domestic purposes.

Based on the average linear ground water velocity in the upper sandstone unit, it is estimated that the contaminants in ground water could have migrated approximately $2500 \mathrm{ft}(760 \mathrm{~m})$ downgradient from the site since the period of uranium processing operations during the 1960s. This estimate is based on limited data and could change, depending on the spatial variability of aquifer parameters and the temporal variation in recharge to the system during and after uranium-processing activities. The maximum influx of fluids (and contaminants) into the ground water system would have occurred during the time uraniumprocessing activities were in progress. Fluid from the tailings (which were deposited adjacent to and into the Spook open-pit mine) would infiltrate from the bottom of the pit into ground water at a depth of approximately $20 \mathrm{ft}(6 \mathrm{~m})$ below the surface. After milling operations ceased, the principal driving force for contaminant migration decreased and steady-state conditions were reached in the aquifer over time. After completion of remedial action at the Spook site, the open-pit mine was filled in with overburden materials, causing the vertical distance to the water table to increase from $20 \mathrm{ft}(6 \mathrm{~m})$ to approximately $100 \mathrm{ft}$ $(30 \mathrm{~m})$, reducing the ability of fluids to migrate into the ground water system. 


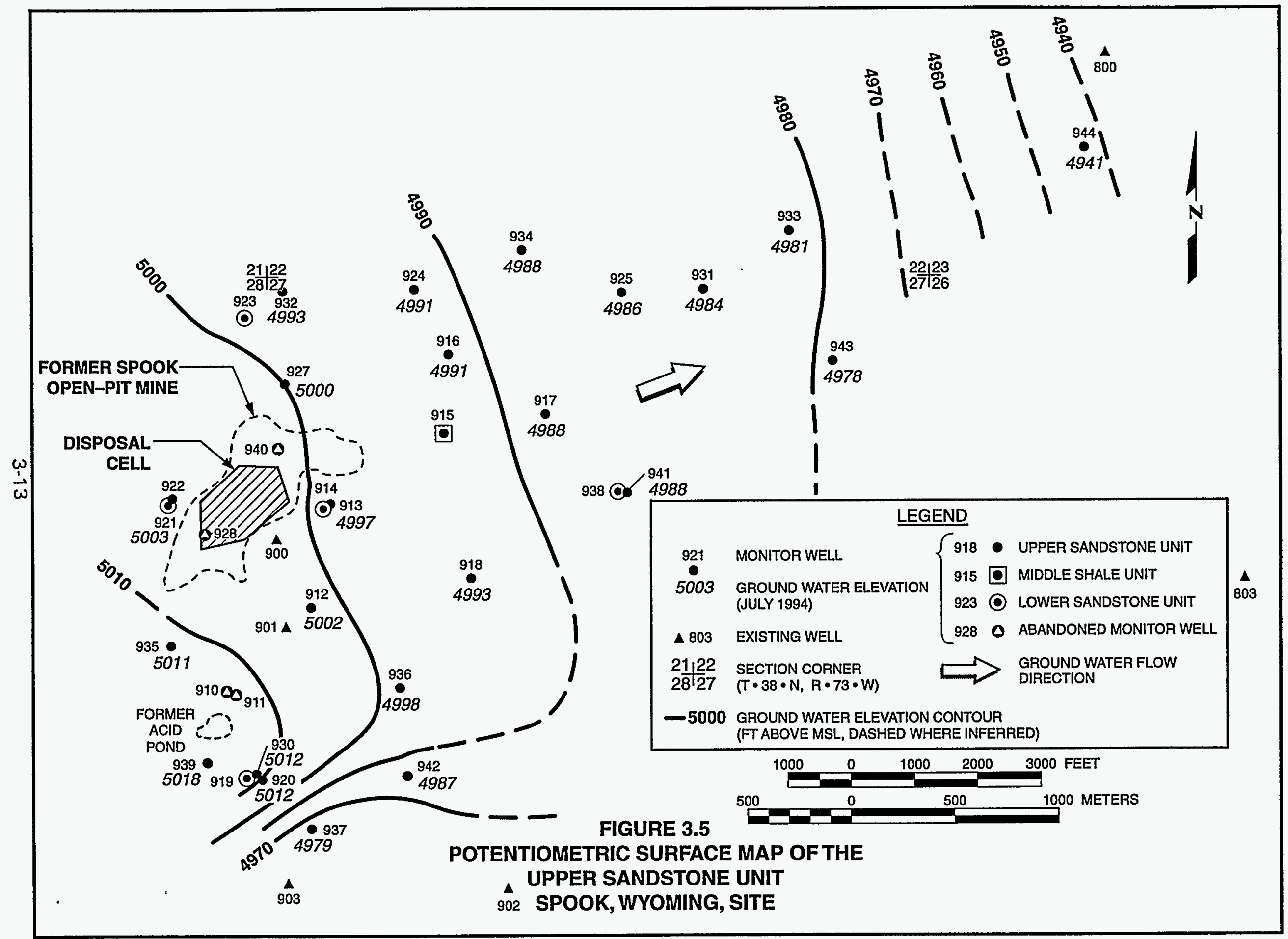




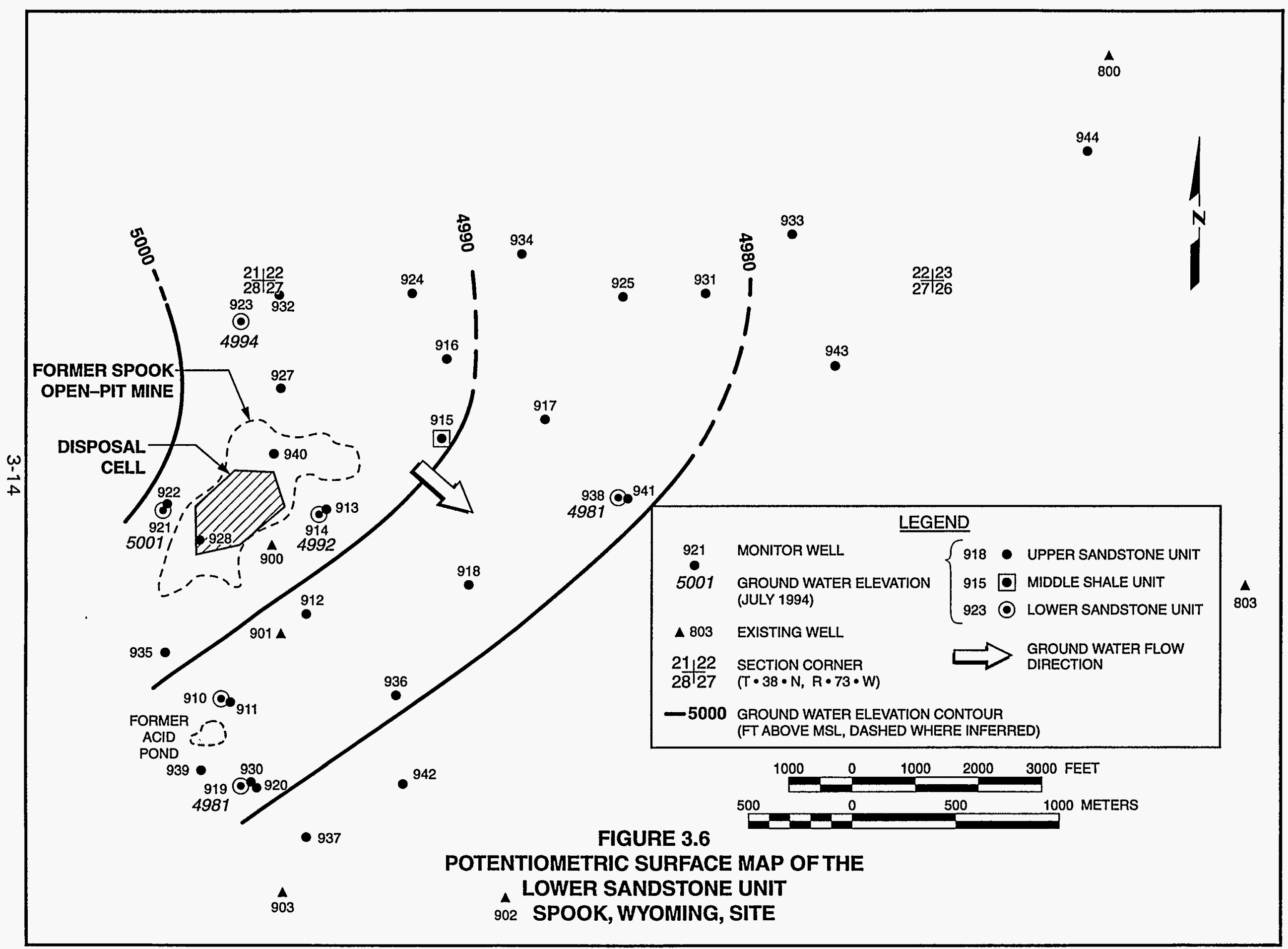




\subsubsection{Geochemistry and background ground water quality}

The Spook uranium mill site is underlain by uranium mineralization associated with an alteration front in the upper sandstone unit. Uranium ore deposits characteristically occur at irregular intervals along the alteration front, as evidenced by the Bear Creek open-pit mine north of the Spook and Kerr McGee open-pit mines south of the site. The primary regional mineralization episode probably occurred during the Oligocene through early Miocene Ages as oxidizing ground water moved from the south through sandstone aquifers in the Wasatch Formation and through overlying formations, dissolving disseminated minerals that contained trace amounts of uranium, molybdenum, and selenium (Galloway, 1979). The metal-bearing ground water migrated toward the west, north, and east, until changes in physical and geochemical conditions caused precipitation of uranium, selenium, and molybdenum along the edges of the alteration front. Figure 3.7 shows the resulting pattern of mineralization, with the alteration front separating the unaltered sandstones (west, north, and east of the front) from the altered sandstones in the interior of the front. The Spook site lies on that front, at the boundary between altered and unaltered sandstones.

There is generally a geochemical gradient within uranium-mineralizing alteration fronts, with vanadium and selenium precipitates being concentrated on the altered (interior) side of the front, uranium concentrated in the middle, and molybdenum concentrated on the unaltered (exterior) side. In the Powder River basin, selenium precipitates have been identified as being ferroselite in the interior of the front, with some elemental selenium occurring on the reduced side (Granger and Warren, 1974).

At present, the ground water flow direction in the upper sandstone unit beneath the Spook site is from the southwest to northeast, moving from the unaltered sandstones, which contain no uranium mineralization, through the uraniumbearing alteration front, and into the oxidized, altered sandstones. As the ground water encounters uranium- and selenium-bearing minerals, it dissolves those minerals. Thus, as naturally-occurring ground water moves from the unaltered zones into the area of altered sandstones, ground water quality can be expected to degrade as naturally-occurring uranium and selenium are dissolved into the ground water. Evidence that this natural degradation of ground water quality has occurred in the vicinity of the alteration front is provided in Figure 3.7, which shows that uranium and selenium concentrations in natural ground waters are higher within the area of altered sandstones.

Ground water beneath the Spook site is located within the altered, oxidized interior of the alteration front. Ground water from the west side of this front (farther upgradient) would encounter conditions in unaltered, reduced sandstones, which are not representative of conditions beneath and downgradient from the Spook site. 


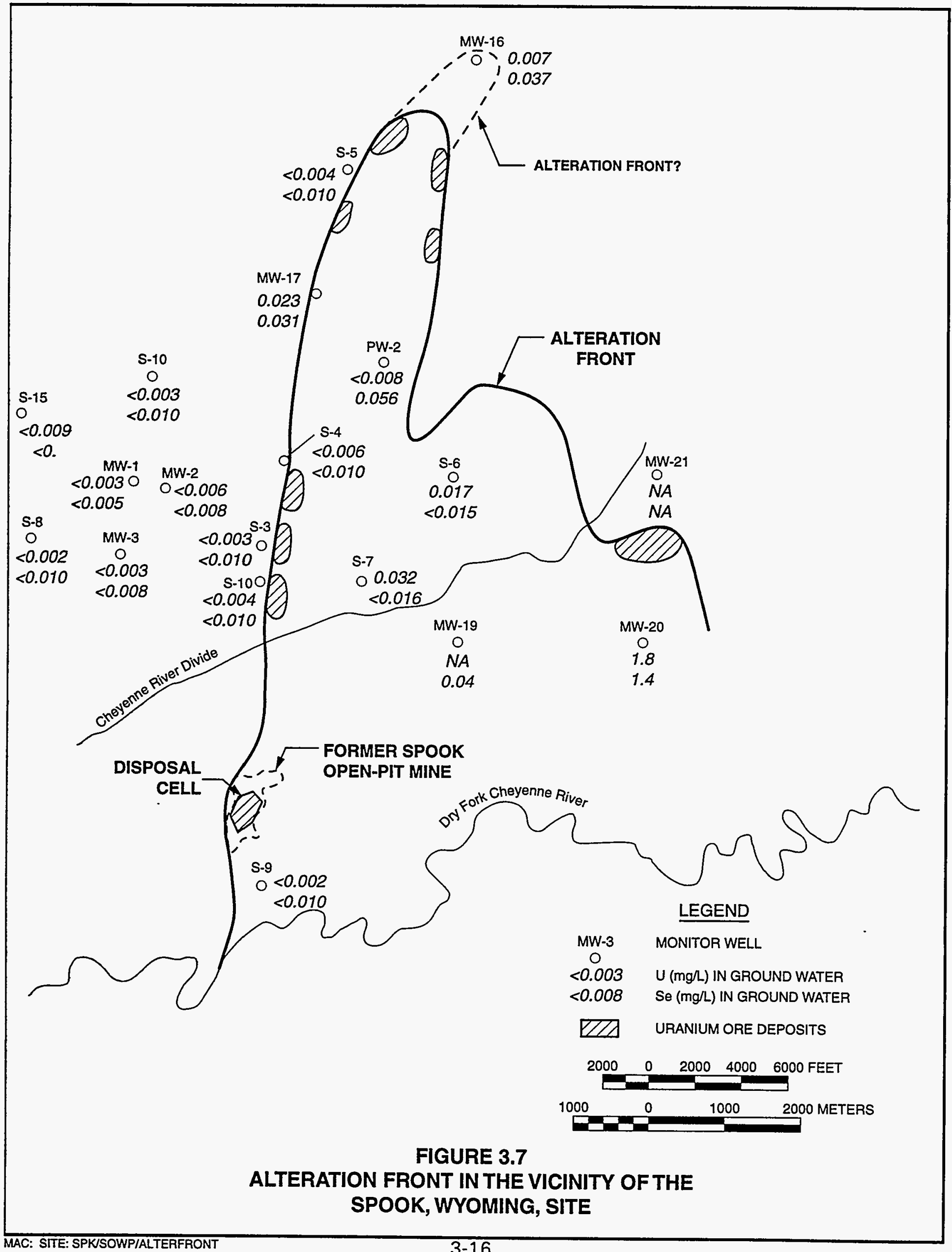


Because of the influence of the alteration front geochemical gradient on ground water chemistry at the site, background ground water quality must be assessed using available information on regional background conditions and information from downgradient monitor wells that have not been impacted by processing operations. Background ground water quality is the quality of ground water expected in the vicinity of the Spook site if contamination had not occurred as a result of uranium-processing activities.

Regional background ground water quality data from the sandstone in the Wasatch Formation were collected by Rocky Mountain Energy (RME) from 22 domestic wells and monitor wells during the period between 1974 and 1977 (RME, 1983). The wells were completed in both the altered and unaltered portions of the Wasatch Formation, as determined by their location relative to the alteration front (Figure 3.7). The data were collected as part of a mining permit application, and ground water was sampled prior to mining and milling activities. Thus, the data represent natural, ambient ground water quality. These data are available from the state of Wyoming (RME, 1983). The average concentration of selected constituents and the depth for each well are summarized in Table 3.3, and the complete set of data are provided in Appendix D.

Regionally, in unaltered sandstones, uranium concentrations are generally less than $0.005 \mathrm{mg} / \mathrm{L}$ and selenium concentrations are generally less than $0.01 \mathrm{mg} / \mathrm{L}$, reflecting the absence of mineralization within the unaltered sandstones (Table 3.3). Other metals associated with mineralization (molybdenum, and chrome) are also not present at levels above detection. Nitrate concentrations range up to $8.8 \mathrm{mg} / \mathrm{L}$; ammonium is generally less than $0.3 \mathrm{mg} / \mathrm{L}$; and the $\mathrm{pH}$ is slightly alkaline.

In contrast, regional data for the altered sandstones demonstrate that most ground water within the altered sandstones has elevated concentrations of uranium and selenium compared to ground water in the unaltered sandstones. Of the eight wells located within the altered sandstone, six have selenium concentrations in excess of the UMTRA Project proposed $\mathrm{MCL}$ for selenium $(0.01 \mathrm{mg} / \mathrm{L})$. Uranium exceeds the proposed $\mathrm{MCL}$ for uranium $(0.044 \mathrm{mg} / \mathrm{L})$ in one well. Molybdenum and chromium occur at levels that are close to or below detection levels. Ammonium is generally below detection; nitrate ranges from less than 0.2 to $38 \mathrm{mg} / \mathrm{L}$ (averaging $7.7 \mathrm{mg} / \mathrm{L}$ ); and $\mathrm{pH}$ is slightly alkaline (Table 3.3).

The regional data demonstrate that ground water from the altered sandstones has uranium and selenium concentrations greater than those in the unaltered sandstones and that the altered sandstones may contain ground water with ambient uranium and selenium concentrations in excess of the proposed MCLs for UMTRA Project sites. 
Table 3.3 Summary of regional ground water quality in altered and unaltered sandstone of the Wasatch Formation, Converse County, Wyoming

\begin{tabular}{|c|c|c|c|c|c|c|c|c|c|}
\hline Well Id & $\begin{array}{c}\text { Depth } \\
\text { (ft) }\end{array}$ & $\begin{array}{c}\mathrm{Se} \\
\mathrm{mg} / \mathrm{L}\end{array}$ & $\begin{array}{c}\mathrm{U} \\
\mathrm{mg} / \mathrm{L}\end{array}$ & $\begin{array}{c}\mathrm{Cr} \\
\mathrm{mg} / \mathrm{L}\end{array}$ & $\begin{array}{c}\text { Mo } \\
\mathrm{mg} / \mathrm{L}\end{array}$ & $\begin{array}{c}\mathrm{NO}_{3} \\
\text { as } \\
\mathrm{mg} / \mathrm{L} \\
\mathrm{NO3}\end{array}$ & $\begin{array}{l}\mathrm{SO}_{4} \\
\mathrm{mg} / \mathrm{L}\end{array}$ & $\begin{array}{c}\mathrm{NH}_{4} \\
\text { as } \\
\mathrm{N} \\
\mathrm{mg} / \mathrm{L}\end{array}$ & $\mathrm{pH}$ \\
\hline \multicolumn{10}{|c|}{ UNALTERED SANDSTONES ${ }^{a}$} \\
\hline $\begin{array}{l}\text { Site } 1 \\
\text { Site } 2 \\
\text { Site } 3 \\
\text { Site } 4 \\
\text { Site } 5 \\
\text { Site } 8 \\
\text { Site } 9 \\
\text { Site } 10 \\
\text { Site } 15 \\
\text { Site } 16 \\
M W-1 \text { b } \\
M W-2^{b} \\
M W-3^{b} \\
M W-21^{b}\end{array}$ & $\begin{array}{r}360 \\
325 \\
270 \\
260 \\
80 \\
515 \\
410 \\
430 \\
126 \\
240 \\
200 \\
273 \\
290 \\
203\end{array}$ & $\begin{array}{r}<0.010 \\
<0.010 \\
<0.010 \\
<0.010 \\
<0.010 \\
<0.010 \\
<0.010 \\
<0.010 \\
<0.012 \\
0.010 \\
<0.005 \\
<0.008 \\
<0.008 \\
\text { na }\end{array}$ & $\begin{array}{r}<0.004 \\
<0.013 \\
<0.003 \\
<0.006 \\
<0.004 \\
<0.002 \\
<0.002 \\
<0.003 \\
<0.009 \\
<0.007 \\
<0.003 \\
0.006 \\
<0.003 \\
\text { na }\end{array}$ & $\begin{array}{r}<0.006 \\
<0.004 \\
<0.006 \\
<0.006 \\
<0.008 \\
<0.007 \\
<0.006 \\
<0.009 \\
<0.006 \\
0.004 \\
<0.004 \\
<0.006 \\
0.003 \\
\text { na }\end{array}$ & $\begin{array}{r}<0.020 \\
<0.009 \\
<0.010 \\
<0.030 \\
<0.006 \\
<0.020 \\
<0.009 \\
<0.008 \\
<0.009 \\
0.004 \\
<0.010 \\
<0.020 \\
<0.005 \\
\text { na }\end{array}$ & $\begin{array}{l}3.3 \\
1.7 \\
3.9 \\
4.2 \\
6.8 \\
2.5 \\
2.8 \\
2.8 \\
3.9 \\
8.8 \\
0.6 \\
5.8 \\
5.6 \\
0.4\end{array}$ & $\begin{array}{r}423 \\
369 \\
167 \\
171 \\
770 \\
26 \\
78 \\
90 \\
1392 \\
384 \\
577 \\
352 \\
562 \\
274\end{array}$ & $\begin{array}{r}<0.5 \\
0.4 \\
0.5 \\
0.5 \\
<0.1 \\
<0.2 \\
0.3 \\
<0.2 \\
<0.1 \\
1.0 \\
<0.1 \\
<0.1 \\
<0.2 \\
<0.1\end{array}$ & $\begin{array}{l}7.4 \\
7.3 \\
7.6 \\
7.5 \\
7.3 \\
8.0 \\
7.6 \\
8.2 \\
7.1 \\
8.3 \\
7.5 \\
7.2 \\
7.3 \\
7.1\end{array}$ \\
\hline AVERAGE & 284 & $<0.009$ & $<0.005$ & $<0.005$ & $<0.011$ & 3.8 & 403 & 0.31 & 7.5 \\
\hline \multicolumn{10}{|c|}{ ALTERED SANDSTONES } \\
\hline $\begin{array}{l}\text { Site } 6 \\
\text { Site } 7 \\
M W-20^{b} \\
M W-18^{b} \\
M W-19^{b} \\
M W-17^{b} \\
P W-2^{b} \\
M W-16^{b}\end{array}$ & $\begin{array}{l}265 \\
322 \\
197 \\
271 \\
237 \\
176 \\
222 \\
240\end{array}$ & $\begin{array}{r}<0.015 \\
<0.016 \\
1.400 \\
0.065 \\
0.040 \\
0.031 \\
0.056 \\
0.037\end{array}$ & $\begin{array}{r}0.017 \\
0.032 \\
1.800 \\
0.036 \\
\text { na } \\
0.023 \\
<0.008 \\
0.007\end{array}$ & $\begin{array}{r}<0.004 \\
<0.006 \\
<0.02 \\
<0.02 \\
0.05 \\
<0.02 \\
0.02 \\
0.05\end{array}$ & $\begin{array}{r}<0.006 \\
<0.010 \\
<0.050 \\
<0.050 \\
0.050 \\
<0.050 \\
0.050 \\
0.100\end{array}$ & $\begin{array}{r}38.0 \\
3.6 \\
17.6 \\
1.0 \\
<0.2 \\
0.4 \\
0.6 \\
0.3\end{array}$ & $\begin{array}{l}128 \\
551 \\
955 \\
445 \\
221 \\
888 \\
435 \\
766\end{array}$ & $\begin{array}{r}<0.1 \\
<0.1 \\
<0.1 \\
<0.1 \\
<0.1 \\
<0.1 \\
0.3 \\
<0.1\end{array}$ & $\begin{array}{l}7.2 \\
7.5 \\
7.8 \\
7.6 \\
7.4 \\
7.7 \\
7.7 \\
7.7\end{array}$ \\
\hline AVERAGE & 241 & 0.208 & 0.240 & $<0.024$ & $<0.046$ & 7.7 & 549 & $<0.13$ & 7.6 \\
\hline
\end{tabular}


Background ground water quality at the Spook processing site

Six monitor wells (SPK-01-918, -925, -931, -933, -943, and -944) are located downgradient but beyond the area of contamination as defined above. That is, these samples had no ammonium, a neutral to slightly alkaline $\mathrm{pH}$, and nitrate concentrations less than $13 \mathrm{mg} / \mathrm{L}$. Data from these wells were also checked against other positive indicators of contamination, such as chloride, sodium, and sulfate. One monitor well at the edge of the identified area of ground water contamination, monitor well SPK-01-918, had a chloride concentration of $26 \mathrm{mg} / \mathrm{L}$ that may indicate contamination. However, the other five samples all had low chloride (less than 1 to $5.3 \mathrm{mg} / \mathrm{L}$ ) and low sulfate (61 to $393 \mathrm{mg} / \mathrm{L}$ ). The plume extends approximately $2500 \mathrm{ft}(760 \mathrm{~m})$ downgradient from the former tailings pile and acid pond. One well (SPK-01-925) lies close to the edge of the plume (within about 200 feet $[61 \mathrm{~m}]$ ) and was discounted as background simply because of its proximity to the plume.

The four remaining monitor wells in the upper sandstone (SPK-01-931, -933, -943 , and -944) display no trends in nitrate, chloride, sulfate, or sodium as a function of distance from the core of contamination, and $\mathrm{pH}$ and major element chemistry are within the range of the regional background. These four wells are located 2900 to $6700 \mathrm{ft}(880$ to $2000 \mathrm{~m})$ downgradient from the Spook site and 700 to $4200 \mathrm{ft}(213$ to $1300 \mathrm{~m})$ beyond the edge of the plume.

Data from these four monitor wells determined to be beyond the influence of contamination were used to determine the quality of background ground water in the altered upper sandstone at the Spook site. The ground water quality data for these four wells are summarized in Table 3.4; locations are shown in Figure 3.8.

As with the regional background data, data from these background monitor wells on the site demonstrate the natural occurrence of uranium and selenium, in excess of MCLs for UMTRA Project sites, in ground water within the altered sandstones of the Wasatch Formation. Selenium concentrations (up to $1.1 \mathrm{mg} / \mathrm{L}$ ) are within the observed range of regional background for altered sandstones (up to $1.4 \mathrm{mg} / \mathrm{L}$ ), but uranium concentrations (0.89 to $2.9 \mathrm{mg} / \mathrm{L}$ ) are slightly greater than observed regional range (up to $1.8 \mathrm{mg} / \mathrm{L}$ ). Overall, the comparison to regional background ground water quality data demonstrates that background ground water from altered sandstones near the site is typical of regional background; together, both sets of data demonstrate that the concentrations of selenium in background ground water locally exceeds the MCLs for UMTRA Project sites for selenium and uranium. In addition, chromium exceeds the MCL in background ground water within the upper sandstone at the Spook site (Table 3.4).

\subsection{CONCEPTUAL SITE MODEL}

A conceptual model of the Spook site has been developed to provide a basis for assessing the interaction of site-related contaminants with the environmental 


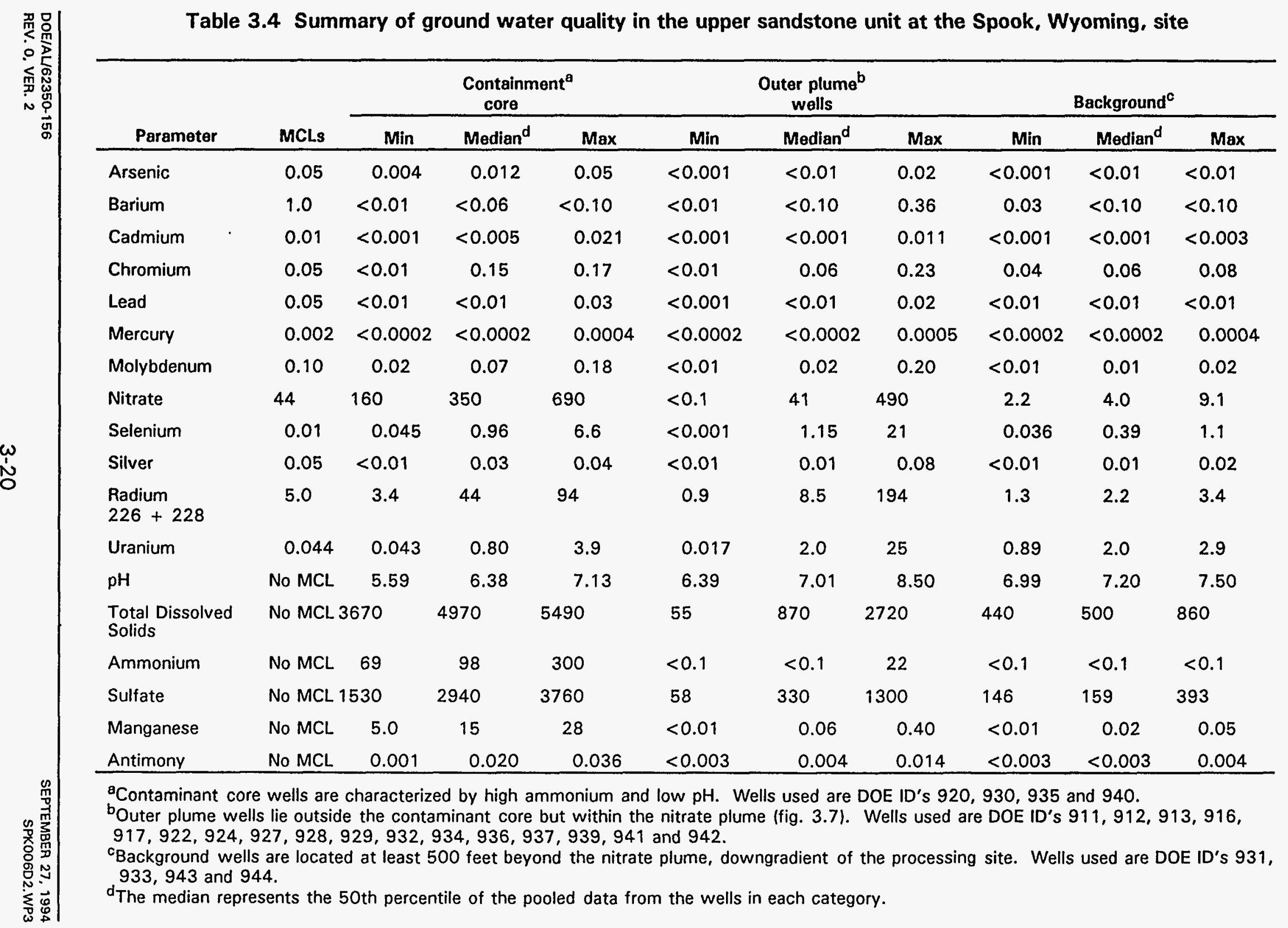




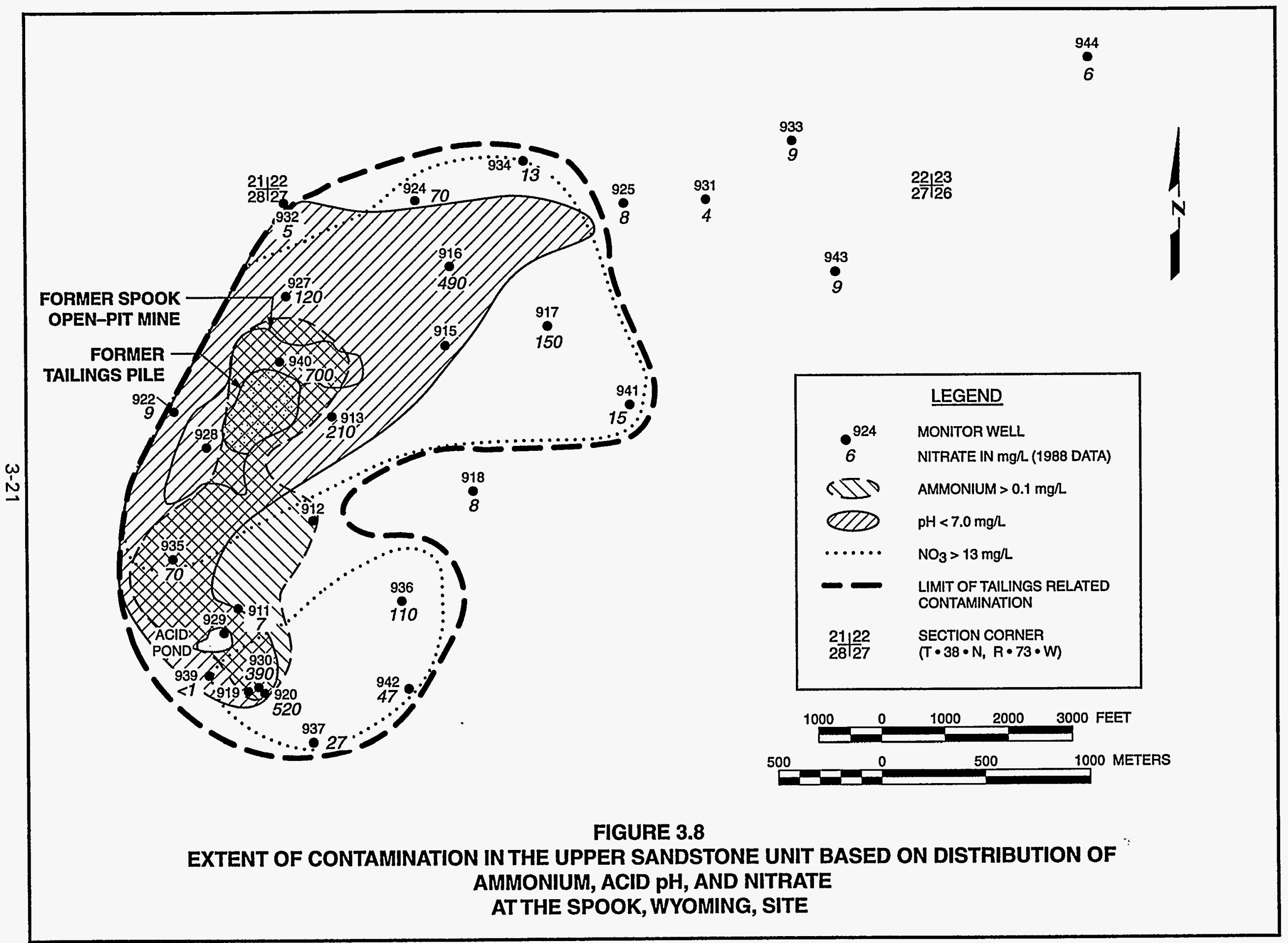


setting, contaminant release mechanisms, contaminant fate and transport, and potential risks to human health and the environment. The conceptual model is used to identify additional data needs and is the foundation upon which an appropriate ground water compliance strategy is determined. Figure 3.9 depicts the conceptual site model for the Spook site.

\subsubsection{Contaminant sources}

Ground water in the uppermost aquifer (upper sandstone unit of the Tertiary Wasatch Formation) beneath and downgradient of the site has been contaminated by uranium-processing activities that occurred from 1962 until 1966. Site-related contaminants in ground water have been detected approximately $2500 \mathrm{ft}(760 \mathrm{~m})$ downgradient and include uranium, selenium, chromium, molybdenum, and radium-266 and -228. The primary sources of site-related contamination in ground water in the upper sandstone unit include chemical and radiological constituents derived from tailings and ore piles, the acid pond, and windblown tailings subjected to leaching during precipitation infiltration. The original site-related sources of shallow ground water contamination in the upper sandstone aquifer are no longer active, having been stabilized within the Spook open-pit mine.

Naturally-occurring alteration front mineralization within the saturated zone also constitutes a primary source of contamination for ground water in the upper sandstone unit.

\subsubsection{Extent and magnitude of contamination}

Process reagents that could reasonably be anticipated in the contaminated ground water at the site include sulfate, ammonium, sodium, nitrate, and possibly chloride. Comparison of the regional background ground water quality (Table 3.3) with samples from DOE monitor wells in the upper sandstone unit (Table 3.4) indicates that all of these anticipated process reagent contaminants are present at levels significantly greater than background levels. Of these, nitrate is the most useful for identifying the extent of contamination.

Ammonium and $\mathrm{pH}$ are buffered by reactions with the solid rock matrix and only serve to identify the area most intensely affected by contaminants. In contrast, nitrate is a chemically conservative constituent (generally not affected by reactions with aquifer matrix) and delineates areas that have received lesser . amounts of contaminated solutions because of dilution and chemical reactions. Nitrate is used in respiration by bacteria (denitrification); however, denitrification will not occur in the presence of dissolved oxygen (Rowell, 1981). Dissolved oxygen is present in ground waters in the region (RME, 1978) and on the site (DOE, 1990) in measurable amounts (averaging about $1.6 \mathrm{mg} / \mathrm{L}$ in the upper sandstone on the site). Thus, nitrate is probably not appreciably affected by denitrification in the upper sandstone on the site.

Together, ammonium and $\mathrm{pH}$ define a core of contamination in the upper sandstone centered on the sources of contamination, including the tailings pile, 


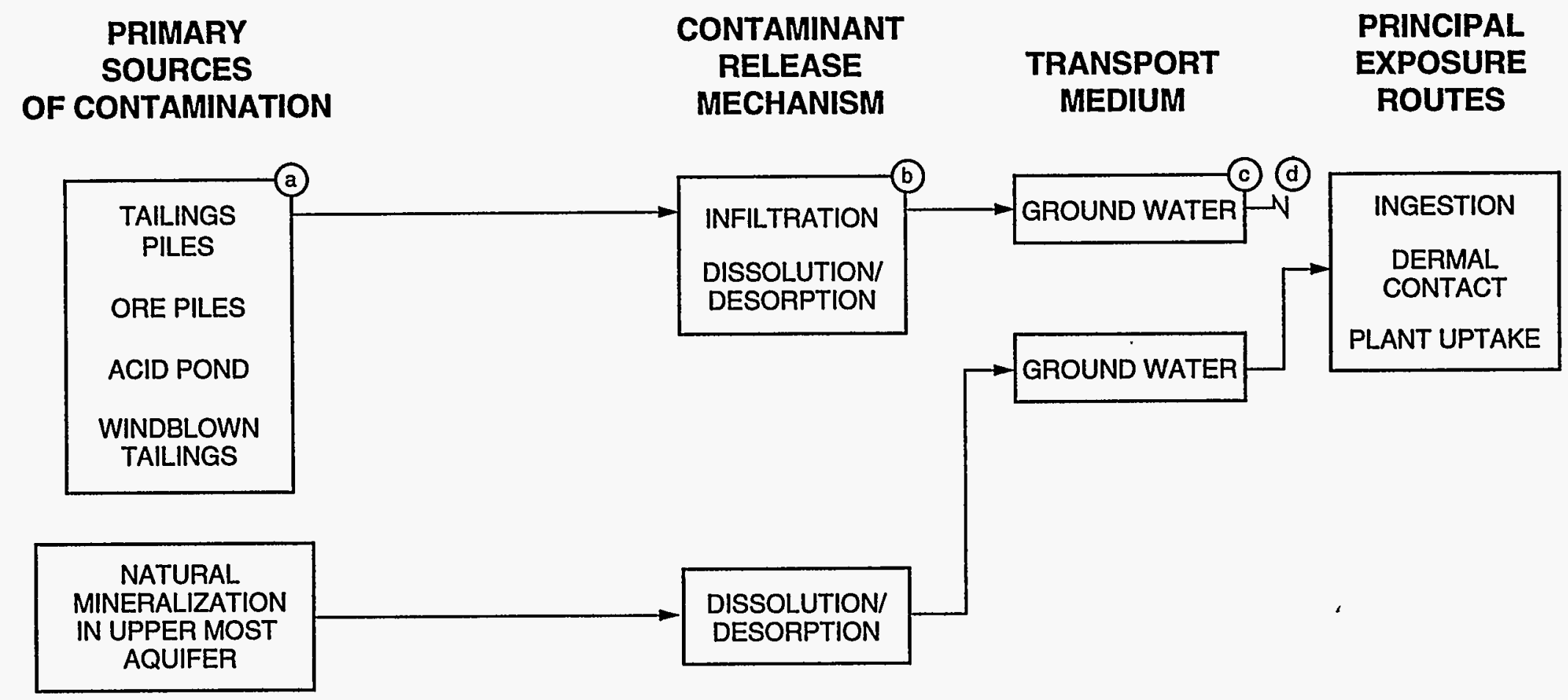

\section{LEGEND}

a SURFACE CONTAMINATION STABILIZED IN EXISTING MINE PIT (DOE 1990).

b EXPOSURE PATHWAYS NOT EVALUATED IN FAVOR OF ANALYSIS OF BACKGROUND CONDITIONS.

c RELEASE MECHANISM ACTIVE PRIOR TO SURFACE STABILIZATION

d INCOMPLETE PATHWAY

FIGURE 3.9

CONCEPTUAL MODEL FOR THE

SPOOK, WYOMING, SITE 
acid pond, and areas between (Table 3.4 and Figure 3.8). In this core area, ammonium generally is present at levels above those found in the regional background (less than $0.1 \mathrm{mg} / \mathrm{L}$ ) and/or the $\mathrm{pH}$ is less than 7.0 , lower than that observed in regional background ( $\mathrm{pH}$ greater than 7.0). In addition, nitrate and sulfate are present in the contaminated ground water at relatively high concentrations in the core of contamination (up to $690 \mathrm{mg} / \mathrm{L}$ nitrate and $3760 \mathrm{mg} / \mathrm{L}$ sulfate) (Table 3.4 ).

Nitrate concentration in regional background ground water ranges from less than 0.2 to $38 \mathrm{mg} / \mathrm{L}$, with an average of $5.2 \mathrm{mg} / \mathrm{L}$ and a standard deviation of 8.3 $\mathrm{mg} / \mathrm{L}$. Nitrate values at the site in excess of the regional mean plus one standard deviation $(13 \mathrm{mg} / \mathrm{L}$ ) delineate the contaminated area that includes the core of contamination and an outer zone of contamination with elevated nitrate, but little or no ammonium and generally neutral to slightly alkaline pH (Table 3.4 and Figure 3.8). The nitrate delineates two plumes: one extending northeast of the tailings pile, and a second extending east of the acid pond. The trends of these plumes are consistent with the hydrologic gradient, and the extent of the plumes is consistent with the ground water flow velocity computed from hydrologic tests. Not enough date exists to determine long-term time-dependent trends in the concentrations within the core and outer portions of the plumes.

The depth of contamination is restricted vertically to the upper sandstone unit, which is underlain by a persistent shale aquitard. Ground water in the lower sandstone unit is not contaminated as a result of milling operations at the Spook site, and concentrations of all constituents are below proposed MCLs for UMTRA Project sites (Table 3.5; Appendix D). Elevated concentrations of nitrate and uranium detected in monitor well SPK-01-910 during the initial round of sampling in February 1987 (a few weeks after completion of the well) were possibly the result of contamination introduced into the ground water during drilling and installation of the well. Five sampling rounds from September 1987 to December 1988 displayed a significant decrease in nitrate (from 60 to less than $10 \mathrm{mg} / \mathrm{L}$ ) and uranium (from 0.08 to $0.01 \mathrm{mg} / \mathrm{L}$ ), demonstrating that mixing and dilution with natural ground water are returning the ground water near this well to its natural state.

Data from the DOE background monitor wells in the upper sandstone unit were compared to the water quality data from wells located within the core and outer portions of the contaminant plume associated with the tailings pile and acid pond. The comparison of background to plume data indicate that, within the upper sandstone unit, several constituents in the plume are at concentrations above MCLs for UMTRA Project sites, including chromium, molybdenum, nitrate, selenium, uranium, and combined radium-226 and -228 . In addition, a few samples within the plume exceeded the MCL for cadmium; however, most contaminated ground water contained less than $0.001 \mathrm{mg} / \mathrm{L}$ cadmium.

About half of the contaminants in the plume that exceed UMTRA Project proposed MCLs are present at levels 10 times or less that of background. These include chromium (about 2 times background), molybdenum (about 10 
Table 3.5 Ground water quality in the lower sandstone unit at the Spook, Wyoming, site

\begin{tabular}{|c|c|c|c|c|c|c|}
\hline Parameter & MW-910 & MW-914 & MW-919 & $M W-921$ & MW-923 & MW-938 \\
\hline Alkalinity ${ }^{1}$ & 135 & 111 & 165 & 214 & 145 & 213 \\
\hline Ammonium & 0.5 & $<0.1$ & $<0.1$ & $<0.1$ & $<0.1$ & $<0.1$ \\
\hline Arsenic & $<0.01$ & $<0.01$ & $<0.01$ & $<0.01$ & $<0.01$ & $<0.01$ \\
\hline Cadmium & $<0.001$ & $<0.001$ & $<0.001$ & $<0.001$ & $<0.001$ & $<0.001$ \\
\hline Calcium & 50 & 34 & 57 & 104 & 50 & 10 \\
\hline Chloride & $<1.0$ & $<1.0$ & $<1.0$ & 2.1 & $<1.0$ & $<1.0$ \\
\hline Chromium & 0.05 & 0.01 & 0.02 & 0.04 & 0.02 & $<0.01$ \\
\hline Gross alpha 4 & 12 & 11 & 5 & 0 & 4 & 2 \\
\hline Gross beta ${ }^{4}$ & 7 & 4 & 7 & 17 & 6 & 4 \\
\hline Iron & 0.04 & $<0.03$ & 0.05 & 0.68 & 0.07 & $<0.03$ \\
\hline Lead & $<0.01$ & $<0.01$ & $<0.01$ & $<0.01$ & $<0.01$ & $<0.01$ \\
\hline Magnesium & 9 & 5 & 10 & 22 & 9 & 2 \\
\hline Manganese & 0.04 & 0.02 & 0.03 & 0.07 & 0.04 & $<0.01$ \\
\hline Nitrate $^{2}$ & 8 & 7 & 1 & 5 & 6 & 6 \\
\hline $\mathrm{pH}$ & 8.0 & 8.2 & 7.6 & 7.3 & 7.7 & 8.6 \\
\hline Potassium & 5 & 4 & 5 & 7 & 6 & 4 \\
\hline Radium-2264 & 0.7 & 0.3 & 1.3 & 0.6 & 0.8 & 0.3 \\
\hline Radium-2284 & 0.9 & 0.8 & 1.7 & 1.8 & 0.6 & 0.0 \\
\hline Selenium & 0.006 & 0.005 & 0.006 & $<0.005$ & $<0.005$ & $<0.005$ \\
\hline Silver & $<0.01$ & $<0.01$ & $<0.01$ & $<0.01$ & $<0.01$ & $<0.01$ \\
\hline Sodium & 77 & 56 & 58 & 36 & 98 & 80 \\
\hline Sulfate & 200 & 110 & 160 & 210 & 210 & 2 \\
\hline TDS $^{3}$ & 470 & 340 & 60 & 560 & 510 & 260 \\
\hline Uranium & 0.0101 & 0.0012 & 0.0015 & 0.0006 & 0.0011 & 0.0004 \\
\hline Vanadium & $<0.01$ & $<0.01$ & $<0.01$ & 0.02 & $<0.01$ & $<0.01$ \\
\hline
\end{tabular}

1. Alkalinity as $\mathrm{mg} / \mathrm{L} \mathrm{CaCO}$.

2. Nitrate expressed as $\mathrm{NO}_{3}$.

3. TDS - total dissolved solids.

Data are for filtered samples collected December 10, 1988. All data expressed as milligrams per liter $(\mathrm{mg} / \mathrm{L}$ ) unless noted as picocuries per liter ( $\mathrm{pCi} / \mathrm{L})$. 
times background), and uranium (about the same as background). Selenium is also generally present at concentrations near that of background (within 3 times that of background), though locally (in monitor well SPK-01-916), selenium exceeds background by a factor of about 20 . Nitrate in the area of the plume is considerably above background levels (up to about 90 times background) but decreases steadily in the outer portions of the plume to near background levels. Combined radium-226 and radium-228 is present locally at concentrations up to 60 times background.

\subsubsection{Potential release mechanisms}

Based on available information regarding the uranium milling process, characterization of tailings solutions, and the distribution of contaminants in ground water, a reasonable model of the potential contaminant release mechanisms has been developed.

Acid solutions used in the uranium milling process at the Spook site were disposed of on the tailings pile and in the acid pond. Consequently, these solutions percolated into the vadose zone, where they were neutralized by reactions with carbonate minerals. However, several constituents remained in neutralized solutions, including uranium, selenium, and nitrate. Seepage of the process solutions from the tailings appears to have effectively drained process solutions from the tailings, such that, prior to surface remediation, the tailings were no longer saturated (DOE, 1990). Seepage from the acid pond resulted in the complete drainage of acid solutions from the pond prior to surface remediation. Thus the primary release of contaminants is thought to have been seepage of process solutions into the vadose zone prior to surface remediation. Potential secondary release mechanisms would include leaching of contaminants from tailings and subsoils immediately beneath the tailings and acid pond by infiltration of precipitation. Typically, leaching mechanisms include dissolution, desorption and cation exchange.

Surface remediation at the site has resulted in stabilization of site wastes in a disposal cell which incorporates features designed to provide long term hydrologic isolation. As a result, the primary and secondary release mechanisms as discussed above have been mitigated.

\subsubsection{Contaminant fate and transport mechanisms}

A geochemical reaction effective in decreasing or removing contaminants from the process solutions was neutralization of acid process solutions by carbonate minerals within soils immediately beneath the tailings pile and acid pond. This neutralization occurred within $12 \mathrm{ft}(3.7 \mathrm{~m})$ of the contact between the tailings and the Wasatch Formation (Shepard and Brown, 1983); thus, the $\mathrm{pH}$ of the contaminant plume beneath the site is neutral to slightly acid (Table 3.2). Geochemical modeling of contaminated ground water within the plume area was performed using the computer code PHREEQE (Parkhurst et al., 1980). This modeling indicates that most of the ground water is now equilibrated with 
calcite (a calcium carbonate mineral responsible for neutralizing the acid process solutions). Neutralization is generally effective in decreasing total dissolved solids, sulfate, aluminum, iron, radium, and cation trace metal concentrations in contaminant solutions produced by uranium processing.

A second effective process for decreasing contaminant concentrations in the ground water is dilution. The decrease in contaminant concentrations in the outer portions of the plume, relative to the core of contamination, demonstrates that dilution has occurred at the Spook site (Table 3.2). This dilution is caused by the movement of ground water from southwest to northeast across the site. Mixing of the contaminated waters with natural waters downgradient of the site has resulted in five-fold or greater decreases in total dissolved solids, sulfate, and nitrate. Further dilution will occur in conjunction with contaminated ground water movement toward the northeast.

Dilution of the plume has little or no effect on the concentrations of uranium, molybdenum, selenium, and chromium because these constituents are present in background ground water at concentrations that are similar to, or even greater than, those in the plume. For example, most uranium concentrations in background ground water are the same as or greater than those in the core of the plume (Table 3.2), reflecting the fact that the principal source of uranium in both plume and background ground water is dissolution of uranium ores within the Wasatch Formation.

As with uranium and selenium, radium concentrations in the plume may also result from dissolution of the uranium ore deposit in the alteration front. The extent of radium-226 and radium-228 migration into the Wasatch Formation beneath the tailings pile was generally less than $3 \mathrm{ft}(1 \mathrm{~m})$, due to coprecipitation with sulfates formed in response to neutralization reactions (FBDU, 1981). Considering the general effectiveness of this process, it is likely that locally high concentrations of radium in the plume (up to $194 \mathrm{pCi} / \mathrm{L}$ combined radium-226 and -228 in monitoring well SPK-01-924) are related to local dissolution of ore in the alteration front. Radium is adsorbed onto clay minerals, iron oxyhydroxides, and manganese oxyhydroxides (Ames et al., 1983). These solids are present within the altered upper sandstone on the site (DOE, 1989) and thus adsorption may limit the transport of radium from local sources.

\subsubsection{Background risk evaluation}

A baseline risk assessment of site-related contamination was not performed for the Spook site because of the lack of exposure pathways for the uppermost aquifer and because of the naturally contaminated nature of background ground water from that aquifer.

The geochemical study presented above has demonstrated that widespread, ambient contamination exists in background ground water. To demonstrate the contaminated nature of the ambient background ground water from the 
uppermost aquifer, a screening level risk analysis was performed using data from the four background wells within the alteration front, but beyond the farthest extent of tailings-related contamination (SPK-01-931, -933, -943, and -944). The risk analysis is provided in Appendix $F$. The risk analysis determined that site conditions support the proposed compliance strategy of performing no remediation.

\section{Potential exposure pathways}

There are no known current exposure pathways for ground water from the uppermost aquifer because no one is using the water and there is no ground water discharge to the surface. Water supply wells in the vicinity of the Spook site, some of which are under artesian (flowing) conditions, readily obtain good quality ground water for domestic purposes and stock watering from aquifers below the uppermost sandstone unit, protected from the effects of processing activities at the site.

The Spook site is in a remote area of Wyoming, well removed from any large population centers. The nearest residence is $1.4 \mathrm{mi}(2.3 \mathrm{~km})$ southwest of the Spook site. An estimated 10 residents live within a $3-\mathrm{mi}(5-\mathrm{km})$ radius of the site. Conversations with the Converse County Planning Commissioner and the local ranchers indicated that the large ranches in the vicinity of the Spook site have been owned and operated by several generations of the same families currently in residence, and it appears that this trend likely will continue into the future (DOE, 1990). Thus, the current population density is very low and is likely to remain very low in the foreseeable future.

Conditions in the site area support the conclusion that there currently are no complete exposure pathways to ground water from the uppermost aquifer. Because there are no current or foreseeable future exposure pathways to ground water in the uppermost aquifer, this ground water does not present a risk to human health or the environment.

\subsubsection{Treatability issues}

The proposed EPA UMTRA Project site ground water cleanup standard for selenium is $0.01 \mathrm{mg} / \mathrm{L}$ (52 FR 36000 (1987)). Chapter VIII (4, (d)) of the Wyoming regulation states the ground water MCLs for domestic, agricultural, . and livestock uses are $0.01,0.02$, and $0.05 \mathrm{mg} / \mathrm{L}$ respectively (State of Wyoming, 1993). Background ground water selenium concentrations at the Spook site are well above both the state ground water MCLs and proposed EPA ground water cleanup standards. The maximum background selenium concentration observed was $1.1 \mathrm{mg} / \mathrm{L}$ (Table 3.4). In addition, the background ground water concentrations of uranium exceed both the state and EPA limits described above (Table 3.4).

Selenium may be removed by several treatment methods. Treatment methods that are considered as best available treatment (BAT) methods by the EPA are 
electrodialysis, lime softening, activated alumina fluoride (alumina), and ion exchange. Although not listed as a BAT, reverse osmosis and distillation will also remove selenium along with other dissolved metals and salts. Since the water must also be treated for uranium removal, the process design for water treatment becomes more complicated.

The elevated levels of selenium ( $1.1 \mathrm{mg} / \mathrm{L}$ maximum), uranium $(2.9 \mathrm{mg} / \mathrm{L}$ maximum), calcium (179 $\mathrm{mg} / \mathrm{L}$ maximum) and sulfate ( $393 \mathrm{mg} / \mathrm{L}$ maximum) make treatment of this water more akin to an industrial waste water treatment process than a public water supply treatment process. The need to achieve at least a 99 percent removal of selenium and uranium (to meet MCLs of $0.01 \mathrm{mg} / \mathrm{L}$ for selenium and $30 \mathrm{pCi} / \mathrm{L}$, or $0.044 \mathrm{mg} / \mathrm{L}$ for uranium) emphasizes the difficulty.

A treatment system to meet the EPA drinking water MCLs for these two elements could consist of a pretreatment system such as lime softening and filtration to remove calcium (which can cause scale formation), followed by either reverse osmosis or electrodialysis to remove of the selenium and uranium as well as all other metals and salts. Because of the need to achieve 99 percent reduction of uranium and selenium, the treated water would be very low in total dissolved solids, which would require the addition of some salts to prevent the water from being excessively corrosive in water system distribution lines.

Alternately, pretreatment with a lime-softening unit could be used, followed by neutralization, filtration, and polishing with specific ion exchange resin units for selenium and uranium removal. The lime-softening pretreatment could remove 30 to 50 percent of the selenium and up to 90 percent of the uranium. Before polishing the water with the ion exchange units, ozone or chlorine would probably need to be added, as the selenium in the ground water is probably present as selenite $\left(\mathrm{SeO}_{3}{ }^{-2}\right)$. Selenium removal with ion exchange resins is preferential to the removal of selenate $\left(\mathrm{SeO}_{4}{ }^{-4}\right)$, as opposed to selenite. The same requirement for conversion of selenite to selenate exists with the use of alumina for the removal of selenium (Montgomery, 1985).

In addition to the complexity of the treatment system, the treatment processes used to remove the selenium and uranium may generate hazardous or mixed wastes in the sludges and brines generated during treatment. If these wastes meet hazardous waste criteria, proper disposal of these residues will be very costly.

Consistently achieving 99-percent-plus removal rates for selenium and uranium would be technically difficult. In addition to the high capital and operating costs for these systems, well-trained operating, maintenance, and laboratory personnel will be required at such a facility.

None of these treatment processes are used on a widespread basis within Wyoming or within EPA Region VIII for public water supply treatment (Jones, 1994). 
The extensive contamination of the aquifer caused by natural mineralization and the inability to employ reasonable treatment methods qualify the aquifer in the upper sandstone unit as Class III ground water. The concentrations of selenium and uranium found in background ground water cannot be cleaned up by using treatment methods reasonably employed in public water supply systems.

\section{$3.4 \quad$ DATA DEFICIENCIES}

No data deficiencies have been identified, based on evaluation of existing data and the conceptual site model. Therefore, no additional site characterization data are required to support the selection of the compliance strategy for Subpart B of 40 CFR Part 192 at the Spook site.

The lack of recent ground water quality data is not considered a significant data deficiency. Since completion of surface remediation activities in November 1989 , the source of ground water contamination has been minimized and the amount of infiltration through the contaminated materials has been significantly reduced. Existing site-related contamination in ground water would be diluted over time by ground water flow beneath the site. The extent of the contaminated area may increase with time, but concentrations of potentially hazardous constituents should decrease because of dilution. Based on existing characterization data and regional ground water quality (as previously discussed), any changes in ground water conditions beneath and downgradient from the Spook site are not expected to have any significant impact on human health and the environment. 


\subsection{GROUND WATER COMPLIANCE STRATEGY SELECTION}

This section describes how requirements for meeting standards at UMTRA Project sites presented in Section 2.0 are fulfilled through the ground water compliance strategy selection framework (Figure 4.1). The site-specific data presented in Section 3.0 are used to answer the questions posed in the framework. This framework was developed in the UMTRA Ground Water Project PEIS (DOE, 1994). The three compliance strategies that could result from the use of the framework are as follows:

- No remediation: The strategy of no remediation means that compliance with the proposed standards would be met without altering the ground water or cleaning it up in any way. This could be applied at sites that have no contamination above MCLs or background levels or at sites that have contamination above MCLs or background levels but qualify for supplemental standards or ACLs.

- Natural flushing: This strategy allows the natural ground water movement and geochemical processes to reduce the constituent concentrations to levels within regulatory limits in a given time period. This could be applied at sites where ground water compliance would be achieved with the application of natural flushing within 100 years, where effective monitoring and institutional controls could be maintained, and where the ground water is not currently, and is not projected to be, a drinking water source.

- Active ground water remediation: This strategy would require the application of engineered ground water remediation methods such as gradient manipulation, ground water extraction and treatment, or in situ ground water treatment to achieve compliance with the proposed standards.

\subsection{DECISION FRAMEWORK PROCESS}

Figure 4.1 shows the ground water compliance strategy selection framework used to determine the strategy for the Spook site.

- Assessment of existing data: The first step in selecting the compliance strategy was to assess whether the existing data adequately characterized the hydrogeologic and ground water contamination conditions, or the collection of additional data was necessary. Section 3.0 provides a conceptual site model that includes the hydrogeologic setting, nature and extent of contamination, and contaminant fate and transport. Evaluation of the conceptual site model indicates that adequate hydrogeological and ground water contamination characterization has been completed. Ground water in the uppermost aquifer beneath and downgradient from the Spook site has been contaminated as a result of uranium processing activities that occurred from 1962 until 1966. Site-related contaminants in ground water have been detected approximately $2500 \mathrm{ft}(760 \mathrm{~m})$ downgradient, and include uranium, selenium, and nitrate. 
CHARACTERIZE PLUME AND

HYDROLOGICAL CONDITIONS USING

EXISTING DATA AND NEW DATA AS

REQUIRED.

\section{1 \\ IS GROUND WATER CONTAMINATION PRESENT IN EXCESS OF MAXIMUM CONCENTRATION LIMITS OR BACKGROUND?}

\section{YES}

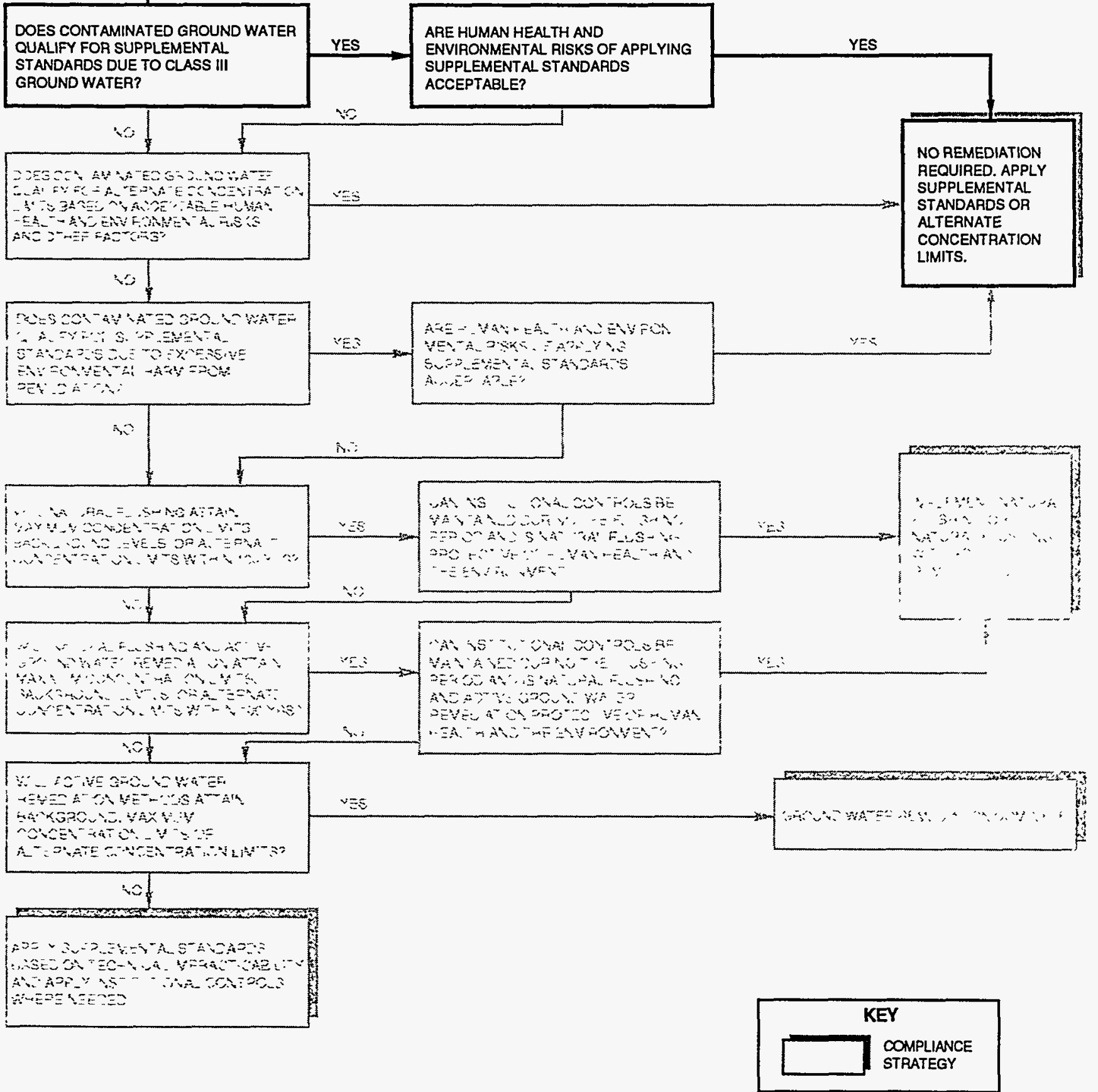

FIGURE 4.1

GROUND WATER COMPLIANCE STRATEGY SELECTION FRAMEWORK SPOOK, WYOMING, SITE 
- Comparison with MCLs or background concentrations: The second step was to determine whether ground water contamination exceeds MCLs or background ground water quality. Section 3.0 summarizes the results of analytical data that show that site-related ground water contamination is present in excess of the MCLs for chromium, molybdenum, nitrate, selenium, uranium, and combined radium-226 and -228 .

- Applicability of supplemental standards: The third step was to determine whether the contaminated ground water qualified for supplemental standards because of the classification of ground water as Class III. The conceptual site model describes the regional background ground water quality and the ground water quality of the uranium mineralization belt in the area of the Spook site. Section 3.2.2 shows that widespread, ambient contamination by uranium and selenium occurs in background ground water in the vicinity of the site. The widespread, ambient contamination and technical impracticability of treating the ground water meet the requirements for Class III designation under the surface remedial action ground water protection strategy. Section 3.3.6 discusses the treatability issues.

- Risk acceptability: The fourth step was to determine whether human health and environmental risks that result from applying supplemental standards were acceptable. The risk evaluation for the Spook site provided in Section 3.3.5 determines that ground water from the upper sandstone unit in the vicinity of the Spook site is not used as a source of drinking water or for any other beneficial purpose because of poor water quality resulting from naturally-occurring contamination and marginal sustained yield. The risk evaluation concludes that the ground water is not a present risk to human health or the environment because 1) the poor quality of the natural water from the upper aquifer is well-known; 2) good quality water is available from the lower confined aquifers, and 3 ) there are no current or foreseeable future exposure pathways to ground water in the uppermost aquifer.

- Compliance strategy: The fifth and final step in the framework leads to the compliance strategy to perform no remediation. This is based on ground water in the uppermost aquifer (also the zone of contamination) being classified as Class III, which allows the application of supplemental standards based on Class III classification. Class III ground water includes ground water that is neither a current nor a potential source of drinking water because of widespread, ambient contamination that cannot be cleaned up using treatment methods reasonably employed in public water supply systems (52 FR 36007) (40 CFR \$192.11(e)).

\subsection{CONCLUSION}

Based on the decision framework process, it has been determined that ground water in the uppermost aquifer was contaminated by uranium processing activities at the Spook UMTRA Project site. Contaminants from site source 
areas were released into the environment by infiltration and migrated into the ground water system in the uppermost aquifer, forming a plume measuring approximately $2500 \mathrm{ft}(762 \mathrm{~m})$ downgradient from the site. It has also been determined that widespread ambient contamination exists in ground water in the uppermost aquifer because of naturally-occurring conditions (natural uranium mineralization associated with an alteration front) and from the effects of broadscale human activity not related to uranium milling operations (uranium exploration and mining activities). Ground water from the uppermost aquifer is not used as a source of drinking water and cannot be effectively cleaned up. Therefore, ground water contamination resulting from uranium processing activities at the Spook site is in an aquifer already contaminated and not usable as a drinking water source. There is not apparent risk to human health and the environment because there are no known exposure pathways for contaminated ground water from the uppermost aquifer to lower aquifers or the surface. No one is using the ground water from this aquifer for any purpose and there is no discharge of ground water from the uppermost aquifer to the surface or to surface water.

The recommended compliance strategy for the Spook site is to perform no remediation based on the justification that ground water in the uppermost aquifer is Class III, and supplemental standards will be applied.

The next step in documenting compliance with Subpart B at the Spook site will include preparation of an environmental assessment (EA). Then, Section E4 of the RAP (DOE, 1990) will be modified to include the information in the SOWP and provide justification for the selection. The RAP will be modified and submitted to the NRC for concurrence of the proposed compliance strategy for Subpart B of 40 CFR Part 192 (1994). 


\subsection{LIST OF CONTRIBUTORS}

The following individuals contributed to the preparation of this report.

\begin{tabular}{ll}
\hline Name & Contribution \\
\hline M. Gawthrop-Cooper & Document coordination \\
A. Holm, J. Gibb, M. Leaf, & Peer reviewers \\
K. Smith, R. Sarr & \\
P. Briggs, R. Bowen & Senior reviewers \\
T. Jackson & Geochemistry \\
K. Smith & Statistics \\
R. Heydenburg, J. Carman & Hydrogeology \\
L. Sanchez & Text processing \\
B. Harvey & Graphic design \\
R. Woodward & Technical editing \\
\hline
\end{tabular}




\subsection{REFERENCES}

Adler, H. H., 1974. "Concepts of Uranium-Ore Formation in Reducing Environments in Sandstone and Other Sediments," in Formation of Uranium Ore Deposits, International Atomic Energy Agency, Vienna, Austria

Ames et al. (L. L. Ames, J. E. McGarrah, B. A. Walker, and P. F. Salter), 1983. "Uranium and Radium Sorption on Amorphous Ferric Oxyhydroxide," in Chemical Geology, Vol. 40, pp. 135-148.

Buturla, F. J., and M. E. Schwenk, 1976. "The Bear Creek Uranium Project," in Geology and Energy Resources of the Powder River, Wyoming Geological Association, 28th Annual Field Conference Guidebook, pp. 231-234.

Curry, D. L., 1976. "Evaluation of Uranium Resources in the Powder River Basin, Wyoming," in Geology and Energy Resources of the Powder River, Wyoming Geological Association, 28th Annual Field Conference Guidebook, pp. 235-242.

Dahl, A. R., and J. L. Hagmaier, 1974. "Genesis and Characteristics of the Southern Powder River Basin Uranium Deposits, Wyoming, USA," in Formation of Uranium Ore Deposits, International Atomic Energy Agency, Vienna, Austria.

DOE (U.S. Department of Energy), 1994. "Programmatic Environmental Impact Statement for the Uranium Mill Tailings Remedial Action Ground Water Project," draft, DOE/EIS-0198, Rev. 3, April 1994, prepared by the U.S. Department of Energy, UMTRA Project Office, Albuquerque Operations Office, Albuquerque, New Mexico.

DOE (U.S. Department of Energy), 1993a. Technical Approach to Groundwater Restoration, DOE/AL/62350-26F, Rev 1, July 1993, U.S. Department of Energy, UMTRA Project Office, Albuquerque Operations Office, Albuquerque, New Mexico.

DOE (U.S. Department of Energy), 1993b. Recommendations for the Preparation of Environmental Assessments and Environmental Impact Statements, Office of NEPA Oversight, U.S. Department of Energy, Washington, D.C.

DOE (U.S. Department of Energy), 1993c. Long-Term Surveillance Plan for the Spook, Wyoming, Disposal Site, final, UMTRA-DOE/AL-350215.0000, prepared by the U.S. Department of Energy, UMTRA Project Office, Albuquerque Operations Office, Albuquerque, New Mexico.

DOE (U.S. Department of Energy), 1992a. UMTRA Groundwater Program Plan, May 1992, prepared by the U.S. Department of Energy, UMTRA Project Office, Albuquerque Operations Office, Albuquerque, New Mexico. 
DOE (U.S. Department of Energy), 1992b. Software Program for Environmental Analysis and Reporting (SPEAR) Program System, U.S. Department of Energy, UMTRA Project Office, Albuquerque Operations Office, Albuquerque, New Mexico.

DOE (U.S. Department of Energy), 1990. Remedial Action Plan and Site Conceptual Design for Stabilization of the Inactive Uranium Mill Tailings Site at Spook, Wyoming, UMTRA-DOE/AL-050515.0000, April 1990, prepared by the U.S. Department of Energy, UMTRA Project Office, Albuquerque Operations Office, Albuquerque, New Mexico.

DOE (U.S. Department of Energy), 1989. Environmental Assessment of Remedial Action at the Spook Uranium Mill Tailings Site, Spook, Wyoming, DOE/EA-0345, April 1989, prepared by the U.S. Department of Energy, UMTRA Project Office, Albuquerque Operations Office, Albuquerque, New Mexico.

Domenico, P. A., and Robbins, G. A., 1985. "A New Method of Contaminant Plume Analysis," in Ground Water, Vol. 23, pp. 476-485.

EPA (U.S. Environmental Protection Agency), 1994. Draft Final Rule, Groundwater Standards for Remedial Actions at Inactive Uranium Processing Sites, UMTRA Project Document Control Center file location number 0.13.5.1, no date, prepared by the U.S. Environmental Protection Agency, Washington, D.C.

EPA (U.S. Environmental Protection Agency), 1989. Risk Assessment Guidance for Superfund, Volume I, Human Health Evaluation Manual, Part A, Interim Final, EPA/540/1-89/002, Office of Solid Waste, Waste Management Division, Washington, D.C.

EPA (U.S. Environmental Protection Agency), 1972. Quality Criteria for Water 1972, A Report of the Committee on Water Quality Criteria, prepared by the National Academy of Sciences--National Academy of Engineering. Environmental Studies Board, Washington.

FBDU (Ford, Bacon, \& Davis Utah Inc.), 1981. Engineering Assessment of Inactive Uranium Mill Tailings, Spook Site, Converse County, Wyoming, DOE/UMT-0119, FBDU 360-15, UC-70, prepared by FBDU, Salt Lake City, Utah, for the U.S. Department of Energy, UMTRA Project Office, Albuquerque Operations Office, Albuquerque, New Mexico.

Galloway, W. E., 1979. "Early Tertiary-Wyoming Intermontane Basins," in Depositional and Ground-Water Flow Systems in the Exploration for Uranium, Texas Bureau of Economic Geology Research Colloquium.

Granger, H. C., and C. G. Warren, 1974. "Zoning in the Altered Tongue Associated with Roll-Type Uranium Deposits, " in Formation of Uranium Ore Deposits, International Atomic Energy Agency, Vienna, Austria. 
Harris et al. (R. E. Harris, E. D. Hausel, and J.E. Meyer), 1985. "Metallic and Industrial Minerals Map of Wyoming," Map Series 14 (MS-14), Geological Survey of Wyoming.

Harshman, E. N., and S. S. Adams, 1981. "Geology and Recognition Criteria for Roll-Type Uranium Deposits in Continental Sandstones," prepared for the U.S. Department of Energy and Bendix Field Engineering Corporation, GJBX-1 (81).

Hodson et al., (W. G. Hodson, R. H. Pearl, and S. A. Druse), 1973. "Water Resources of the Powder River Basin and Adjacent Areas, Northeastern Wyoming," USGS Hydrologic Investigation Atlas HA-465, including "Geologic Map and Generalized Structure Contours."

Hydro-Engineering, 1987. Report of Investigation for Abandoned Mine Land Program 15-3, Spook Site, prepared by Hydro-Engineering, Casper, Wyoming, for the state of Wyoming, Department of Environmental Quality.

Jones, Betty, 1994. Personal Communication between B. Jones, USEPA Region 8 , and George Hartman, TAC UMTRA Project, Albuquerque, New Mexico, UPDCC File No. 15.1.11.1.

Mashbir, D. S., 1964. "Heap Leaching of Low Grade Uranium Ore, " in Mining Congress Journal, December 1964.

Merritt, R. C., 1971. The Extractive Metallurgy of Uranium, Colorado School of Mines Research Institute, prepared under contract with U.S. Atomic Energy Commission, Johnson Publishing Company, Boulder, Colorado.

Montgomery (James M. Montgomery, Consulting Engineers, Inc.), 1985. Water Treatment--Principles and Designs, John Wiley \& Sons, New York, New York.

MSRD (Mountain States Research and Development), 1982. "Economic Evaluation of Inactive Uranium Mill Tailings, Spook Site, Converse County, Wyoming," UMTRA-DOE/ALO-179, prepared by MSRD, Tucson, Arizona, for Sandia National Laboratories, Albuquerque, New Mexico.

Parkhurst et al. (D. L. Parkhurst, D. C. Thorstenson, and L. N. Plummer), 1980. PHREEQEA Computer Program for Geochemical Calculations, U.S. Geological Survey, Water-Resources Investigations 80-96, 209 pages.

RME (Rocky Mountain Energy), 1983. Environmental Report for Bear Creek Project, Permit No. 399.

Rowell, D. L., 1981. "Oxidation and Reduction," In The Chemistry of Soil Processes, Greenland, D. J., and Hayes, H.B., eds., John Wiley \& Sons, New York, pp. 401-462. 
Shepard, T.A., and S. Brown, 1983. "Interpretation of Contaminant Migration Conditions at 12 UMTRA Project Inactive Uranium Tailings Sites," prepared for the U.S. Department of Energy, UMTRA Project Office, Albuquerque Operations Office, Albuquerque, New Mexico.

State of Wyoming, 1993, Water Quality Standards, Wyoming Department of Environmental Quality, Water Quality Division.

\section{CODE OF FEDERAL REGULATIONS}

10 CFR Part 40. "Domestic Licensing of Source Material," U.S. Nuclear Regulatory Commission (1994).

10 CFR Part 1021, "National Environmental Policy Act; Implementing Procedures," U.S. Department of Energy (1994).

40 CFR Part 192, "Health and Environmental Protection Standards for Uranium and Thorium Mill Tailings," U.S. Environmental Protection Agency (1994).

40 CFR Part 300, "National Oil and Hazardous Substance Pollution Contingency Plan," U.S. Environmental Protection Agency (1994).

40 CFR Part 1500, "Regulation for Implementing the Procedural Provision of the National Environmental Policy Act," Council on Environmental Quality (1994).

\section{FEDERAL REGISTER}

52 FR 36000, "EPA's Proposed Standards for Remedial Action at Inactive Uranium Processing Sites with Groundwater Contamination," U.S. Environmental Protection Agency, September 24, 1987.

\section{UNITED STATES CODE}

42 USC $\$ 4321$ et seq.. National Environmental Policy Act, January 1, 1970.

42 USC $\$ 7901$ et seq., Uranium Mill Tailings Radiation Control Act, November 8, 1978. 
APPENDIX A

MONITOR WELL LITHOLOGIC LOGS 



\section{BOREHOLE LOG (ROCK)}

LOCATION MAP:

SITE ID: SPK- OI LOCATION ID: 900910 SITE COORDINATES (ft.):

N

GROUND ELEVATION (ft. MSL): 5089

DRILLING METHOD: Rataicy - Aot

DRILLING CONTR:: Bayles Bros,

DATE STARTED:

DATE COMPLETED: $11-24-86$

See p. 1

FIELD REP.:

$11-26-86$

L. Phife

GROUNDWATER LEVELS

\begin{tabular}{|c|c|c|}
\hline \multicolumn{3}{|c|}{ GROUNDWATER LEVEL } \\
\hline DATE & TIME & DEPTH (ft.) \\
\hline & & \\
\hline & & \\
\hline & & \\
\hline
\end{tabular}

\section{LOCATION DESCRIPTION}

SITE CONDITION

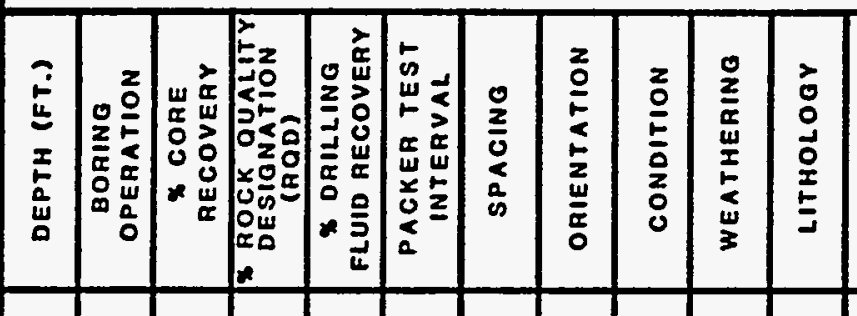
1t. grey

Sthale, some frue sand, fissle, high plastiz clays, interbedded coal, tgrey

COMMENTS: 


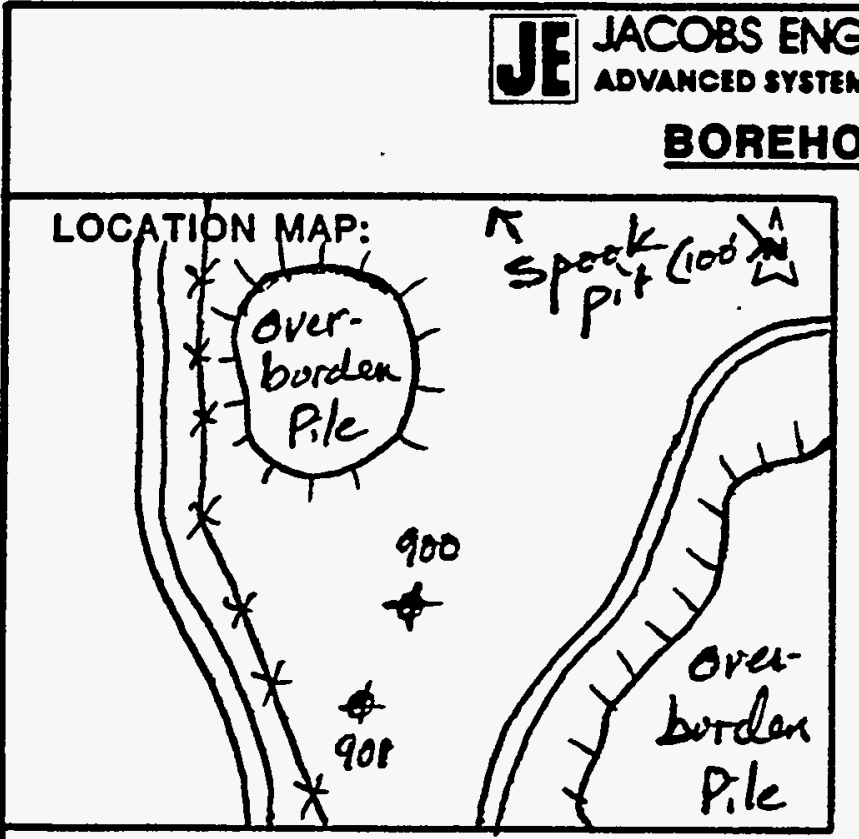

INEERING GROUP INC.

IMS DIVISION, ALBUEUERQUE OPERATIONS

BOREHOLE LOG (ROCK)

Page $\leq$ of $\underline{3}$

SITE ID: SRK QI LOCATION ID: FOT QII SITE COORDINATES (ft.):

N

GROUND ELEVATION (ft. MSL): $\quad 5090$

DRILLING METHOD: Rotury - Ar

DRILLING CONTR.: Berytes Bros.

DATE STARTED: $1 / 1-23-86$

DATE COMPLETED: $11-24-84$

FIELD REP.:

L.Phyte

\begin{tabular}{|c|c|c|}
\hline \multicolumn{3}{|c|}{ GROUNDWATER LEVELS } \\
\hline DATE & TIME & DEPTH (ft.) \\
\hline II-23-8\% & $3: 30$ & $\approx 23^{\prime}$ \\
\hline & & \\
\hline & & \\
\hline
\end{tabular}
LOCATION DESCRIPTION Tuat inside tailings area sauth of ab pite
SITE CONDITION

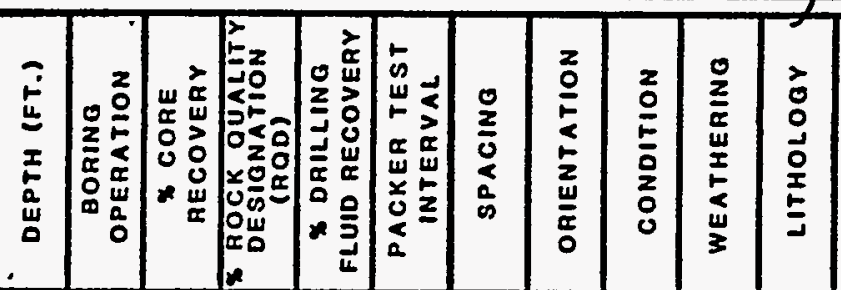

ROCK TYPE \& REMARKS

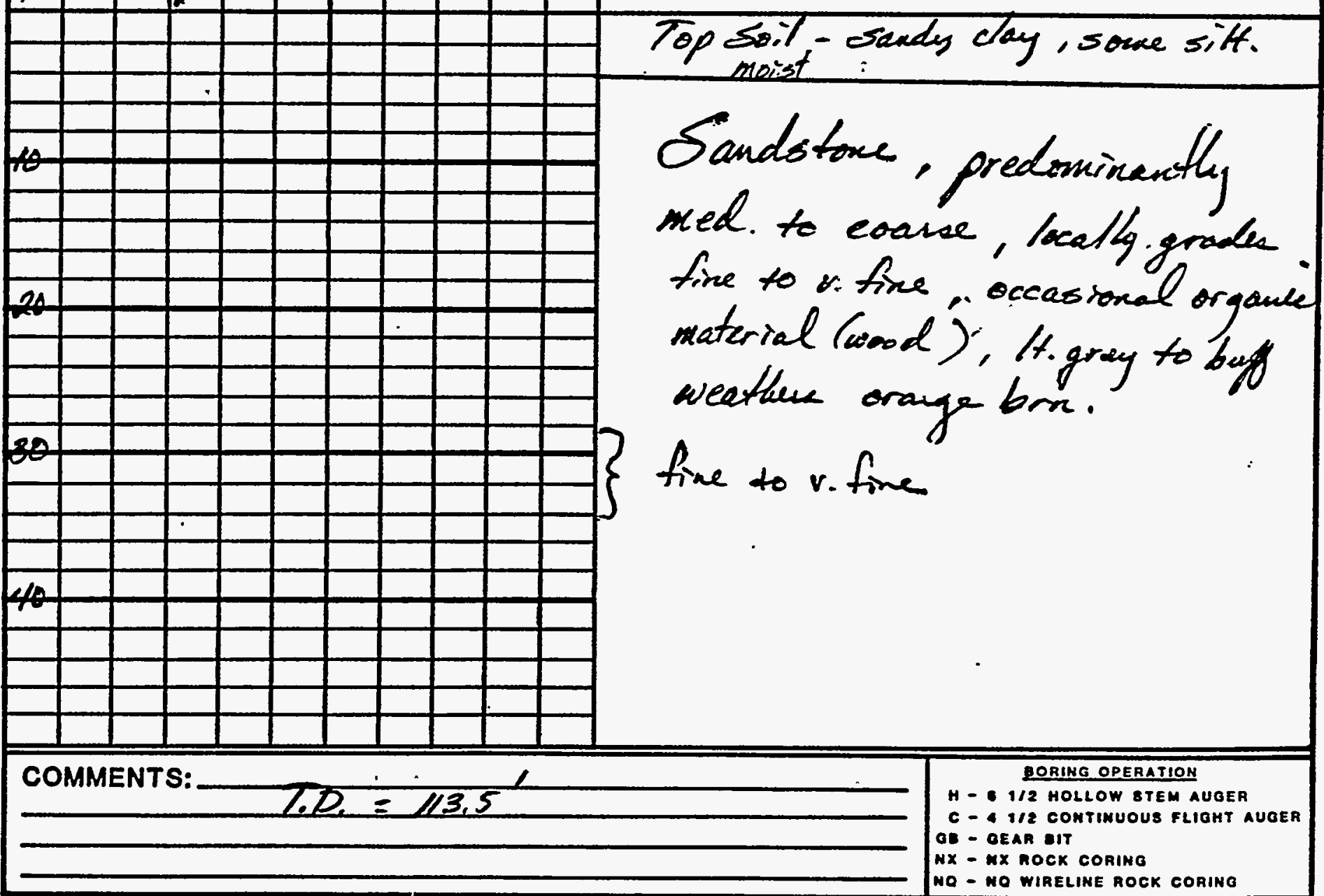


JE IACOBS ENGINEERING GROUP IINC.

BOREHOLE LOG (ROCK) Page $\underline{Z}$ of $\underline{3}$

LOCATION MAP:

Se pg.
SITE ID: SPK_ $6 /$ LOCATION ID: LOS 911

SITE COORDINATES (ft.):

N.

GROUND ELEVATION (ft. MSL): 5090

DRILLING METHOD:

DRILLING CONTR.:

DATE STARTED:

DATE COMPLETED:

FIELD REP.:

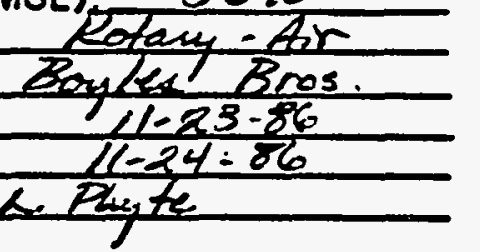

GROUNDWATER LEVELS

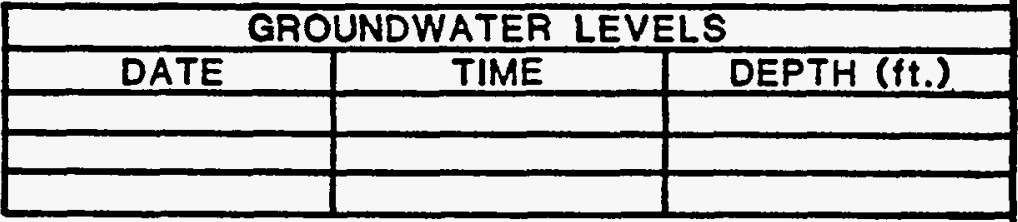

\section{LOCATION DESCRIPTION} SITE CONDITION

\begin{tabular}{|c|c|c|c|c|c|c|c|c|c|}
\hline 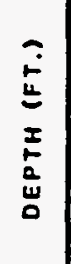 & 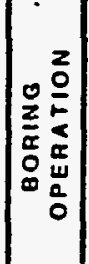 & 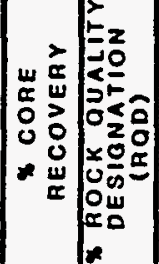 & 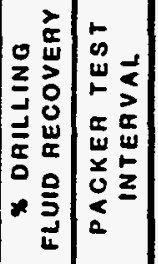 & $\begin{array}{l}0 \\
0 \\
z \\
0 \\
\vdots \\
\vdots \\
\infty\end{array}$ & $\begin{array}{l}z \\
\frac{0}{2} \\
\frac{5}{z} \\
\frac{w}{\tilde{z}} \\
0\end{array}$ & $\begin{array}{l}z \\
0 \\
0 \\
0 \\
0 \\
0 \\
0\end{array}$ & 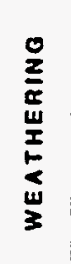 & 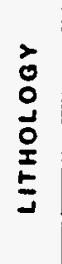 & ROCK TYPE \& REMARKS \\
\hline
\end{tabular}

100

60

(a)

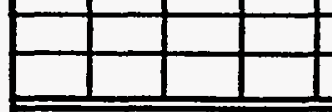

\begin{tabular}{ll|l}
\hline & & \\
\hline & &
\end{tabular}

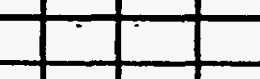

$+$

$+$

$+1$

$+1$

$+1$

70

\begin{tabular}{llll|l|l|l}
\hline & & & & \\
\hline & & & & \\
\hline & & & &
\end{tabular}

COMMENTS:

color change to arange bre.

$\log$

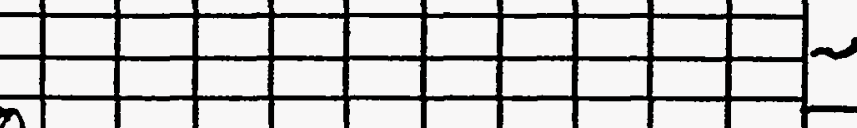

Shale, savely, fissle, It. guey

occasional coal othringen

Sandstore, fine to med. grained, greyish blue, domp to wet 
LOCATION MAP:

Se pg, $\stackrel{\hat{\omega}}{\omega}$

SITE ID: SPK-Q/LOCATION ID: fot GII SITE COORDINATES (ft.):

$N$ GROUND ELEVATION ( $f t . M S L$ ): DRILLING METHOD:

DRILLING CONTR.:

DATE STARTED:

DATE COMPLETED:

FIELD REP.:

\begin{tabular}{|c|c|c|}
\hline \multicolumn{3}{|c|}{ GROUNDWATER LEVELS } \\
\hline DATE & TIME & DEPTH (ft.) \\
\hline & & \\
\hline & & \\
\hline & & \\
\hline
\end{tabular}

\section{LOCATION DESCRIPTION}

SITE CONDITION

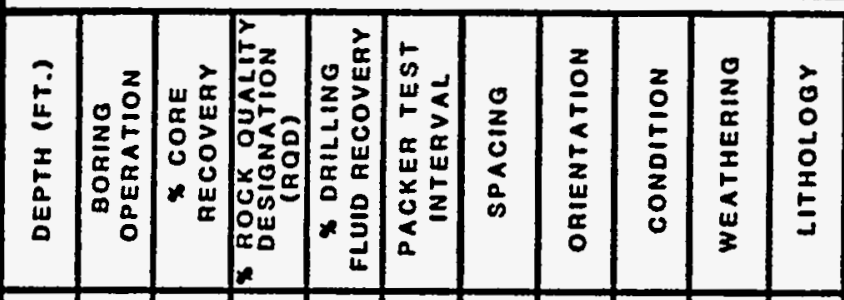

Shale, saudy, duy, grayish blue. coel stringers

$$
\text { T.D. } 1 / 3.5^{\prime}
$$


JACOBS ENGINEERING GROUP INC.

ADVANCED SYSTEMS DIVISION, ALLUEUERQUE OPERATIONS

BOREHOLE LOG (ROCK)

Page 2 of 3

LOCATION MAP:

$\stackrel{\wedge}{\omega}$

SITE ID: SPK- 6 I LOCATION ID: TOZ 912

SITE COORDINATES (ft.):

N.

GROUND ELEVATION (ft. MSL): $5 / 00$

DRILLING METHOD: Catein- Aic/MLEt

$\operatorname{sen}^{\prime}{ }^{\prime}$

DRILLING CONTR.:

DATE STARTED:

DATE COMPLETED:

FIELD REP.:
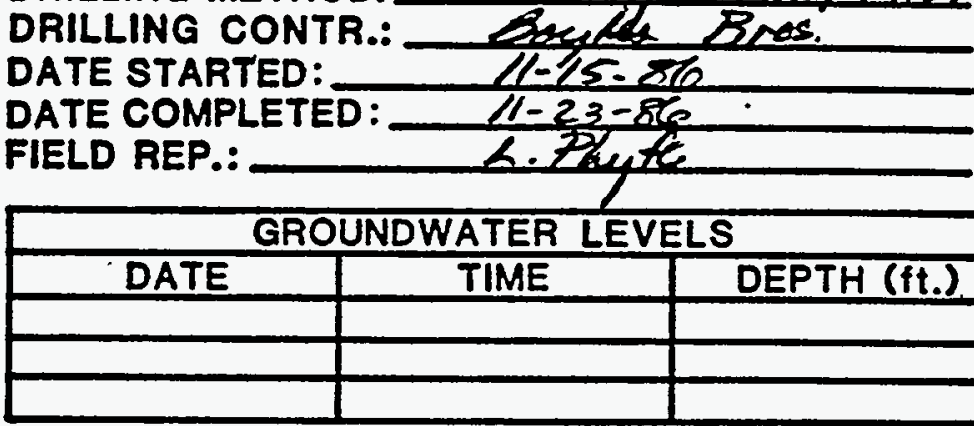

\section{LOCATION DESCRIPTION}

SITE CONDITION

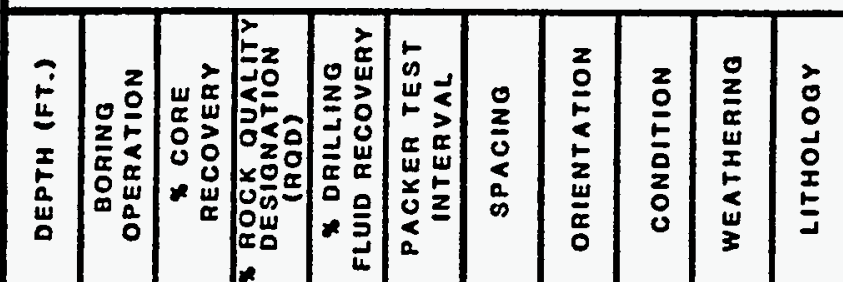

ROCK TYPE \& REMARKS

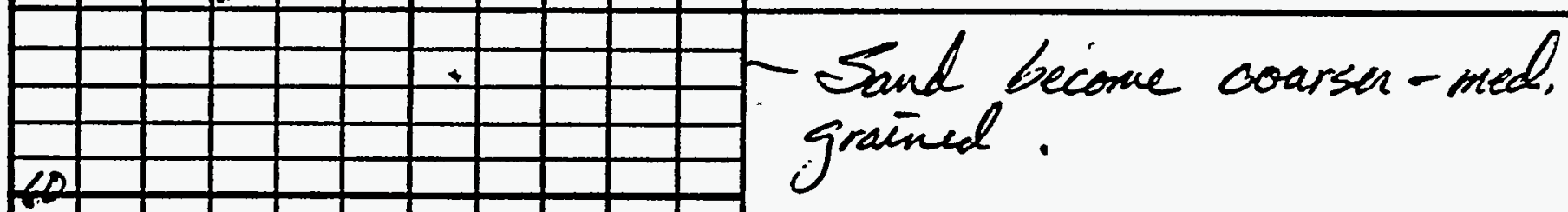


1]. IACOBS ENGINEERING GROUP INC.

ADVANCED SYSTEMS DIVISION, ALLUQUERQUE OPERATIONS

BOREHOLE LOG (ROCK) Page $\underline{3}$ of $\underline{3}$

LOCATION MAP:

$\stackrel{\omega}{\omega}$

SITE ID: SPK- $\$ /$ LOCATION ID: 462912

SITE COORDINATES (ft.):

N

GROUND ELEVATION (ft. MSL): SICO

DRILLING METHOD:

See pg 1

DRILLING CONTR.:

DATE STARTED:

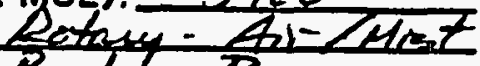

DATE COMPLETED:

FIELD REP.:

$111-15-86$

\begin{tabular}{|c|c|c|}
\hline \multicolumn{3}{|c|}{ GROUNDWATER LEVELS } \\
\hline DATE & TIME & DEPTH (ft.) \\
\hline IE-22-g, & li3o & 110 \\
\hline & & \\
\hline & & \\
\hline
\end{tabular}

LOCATION DESCRIPTION -

SITE CONDITION

so

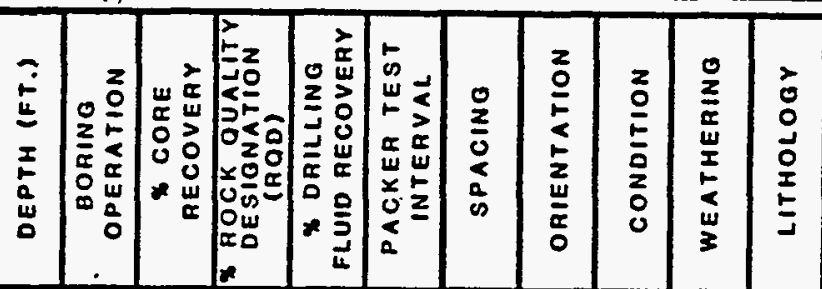

ROCK TYPE REMARKS

Sandistine, nied to cause, sonce skule, mast. - coal stringes

\begin{tabular}{|l|l|l|l|l|l|l|l|l|l|l|}
\hline & & & & & & & & & & \\
\hline
\end{tabular}

grey te black, wot

$$
\text { T.D. } 1 / 5^{\circ}
$$


1]. JACOBS ENGINEERING GROUP INC.

BOREHOLE LOG (ROCK) Page 1 of 3

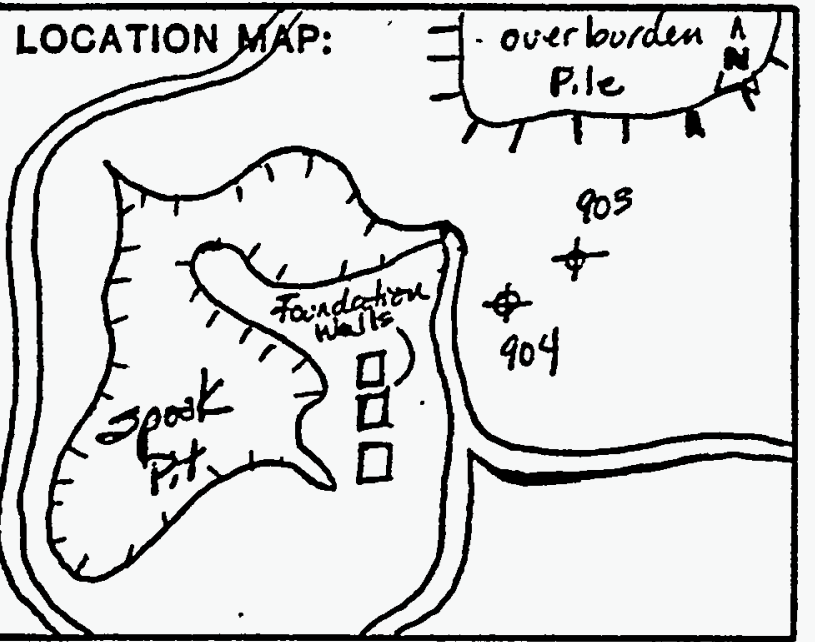

SITE ID: SPK- \&1 LOCATION ID: RYS 913

SITE COORDINATES (ft.):

$N$

GROUND ELEVATION (ft. MSL): 5090

DRILLING METHOD: Rotan-Air /Mist

DRILLING CONTR.: Boyles Beos,

DATE STARTED:

DATE COMPLETED:

FIELD REP.: $12-13-86$

\begin{tabular}{|c|c|c|}
\hline \multicolumn{3}{|c|}{ GROUNDWATER LEVELS } \\
\hline DATE & TIME & DEPTH (ft.) \\
\hline $12-13-86$ & 5,00 & 100 \\
\hline & & \\
\hline & & \\
\hline
\end{tabular}

LOCATION DESCRIPTION Ix cunter of mitlarea $\approx 60^{\prime}$ from spook pit SITE CONDITION Tairly flat, sparsly vegitated, showy t uindblows.

\begin{tabular}{|c|c|c|c|c|c|c|c|}
\hline 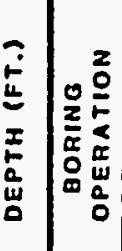 & 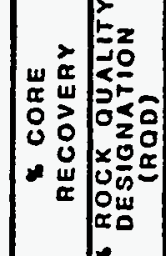 & 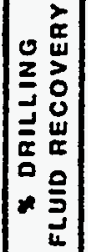 & 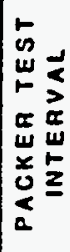 & $\begin{array}{l}0 \\
\frac{2}{0} \\
0 \\
0 \\
0\end{array}$ & 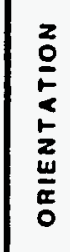 & $\begin{array}{l}5 \\
0 \\
0 \\
0\end{array}$ & 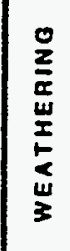 \\
\hline
\end{tabular}

ROCK TYPE \& REMARKS

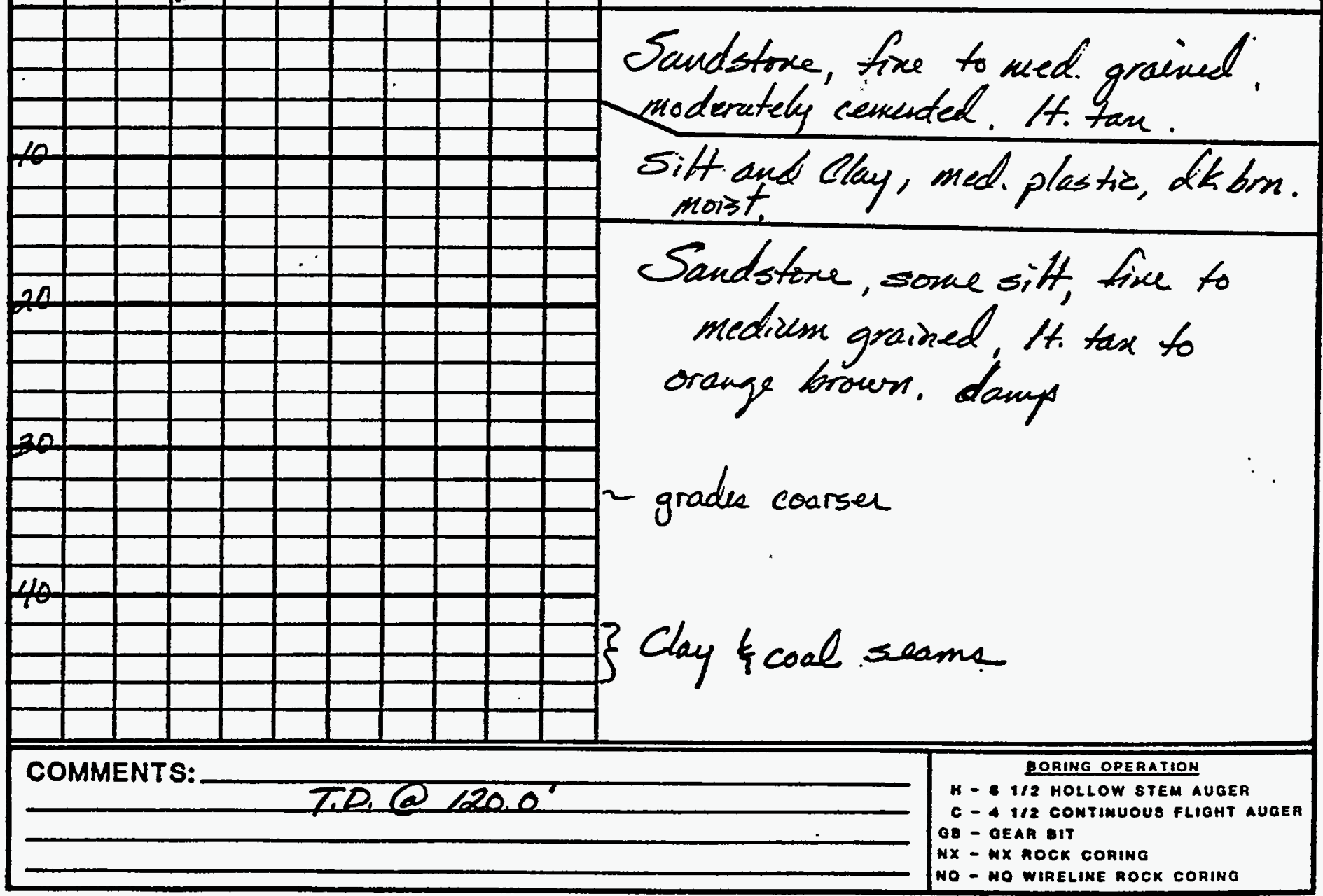

JEG-AL-ENG-2A (4/85) 


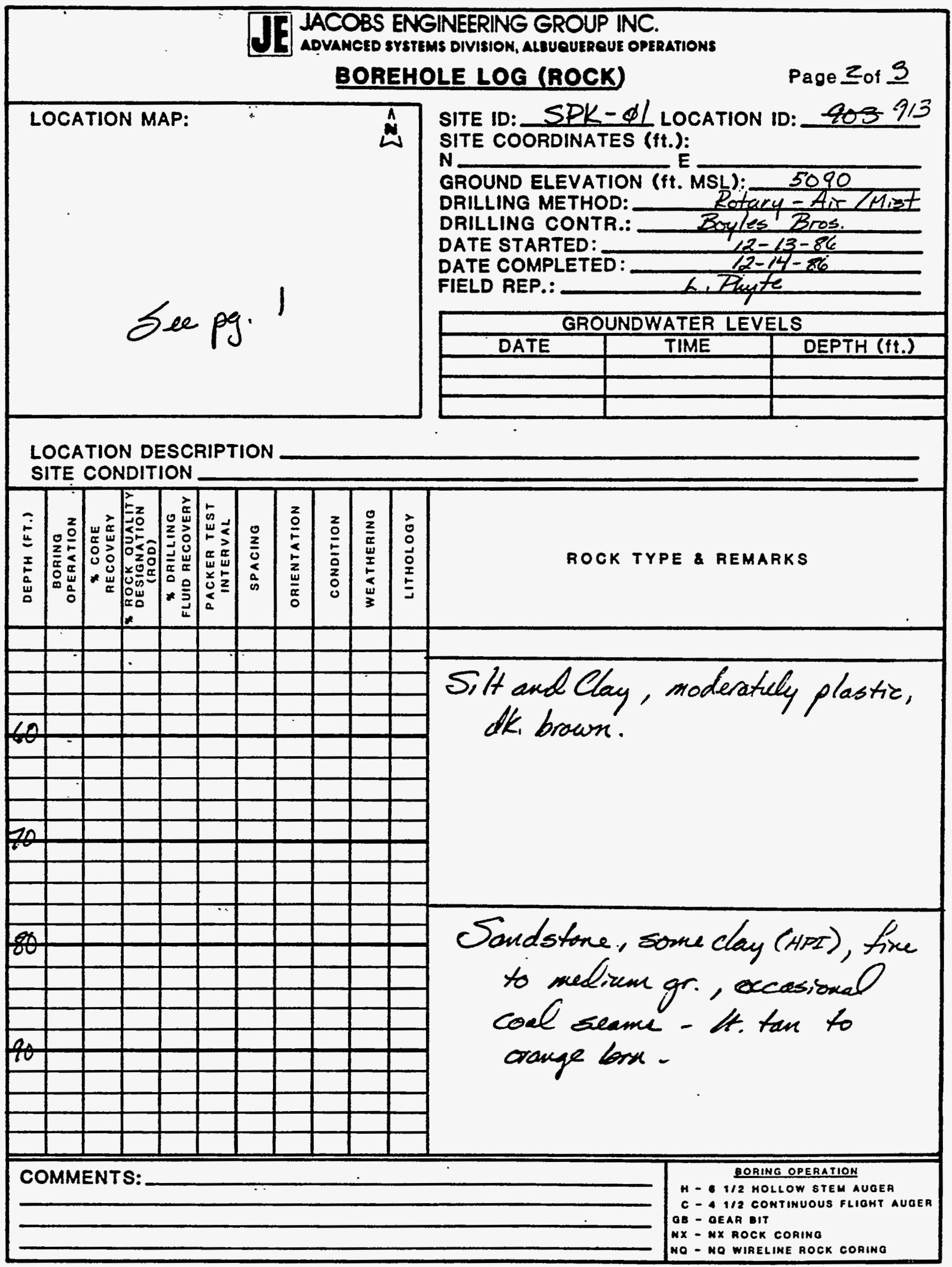

JEG-AL-ENG-2A (4/85) 
IACOBS ENGINEERING GROUP INC.

ADVAKEED SYSTEMS DIVISION, ALDUQUERQUE OPERATIONS

BOREHOLE LOG (ROCK)

Page $\underline{3}$ of 3

LOCATION MAP:

$\stackrel{\omega}{\omega}$

See $p g, 1$
SITE ID: SPK- $6 /$ LOCATION ID: RQS 913

SITE COORDINATES (ft.):

N

GROUND ELEVATION (ft. MSL): 5090

DRILLING METHOD:_- Poteny-Air /Mist

DRILLING CONTR.: _ Boyles Bros,

DATE STARTED:

DATE COMPLETED:

FIELD REP.:

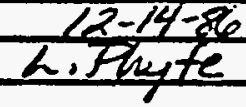

\begin{tabular}{|c|c|c|}
\hline \multicolumn{3}{|c|}{ GROUNDWATER LEVELS } \\
\hline DATE & TIME & DEPTH (ft.) \\
\hline $12-13-86$ & $5: 00$ & $100^{\prime}$ \\
\hline & & \\
\hline & & \\
\hline
\end{tabular}

\section{LOCATION DESCRIPTION}

SITE CONDITION

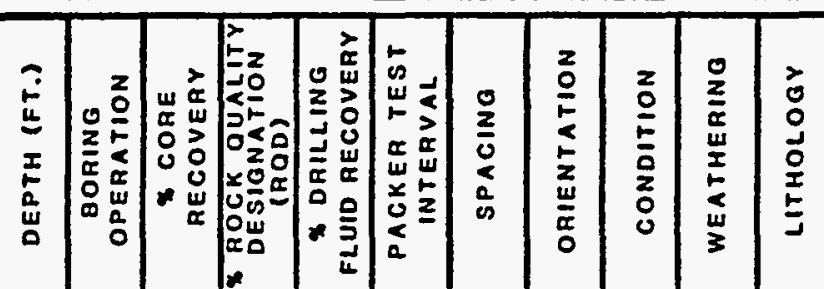

ROCK TYPE REMARKS

Shale, sandy, fissle, with \begin{tabular}{|l|l|l|l|l|l|l|l|l|l|l|}
\hline 120 & & & & & & & & & & \\
\hline & & & & & & & & & & \\
\hline & & & & & & & & & & \\
\hline & & & & & & & & & & \\
\hline
\end{tabular}

$$
+1
$$

\begin{tabular}{l|l|l|}
\hline & 4 & \\
\hline
\end{tabular}
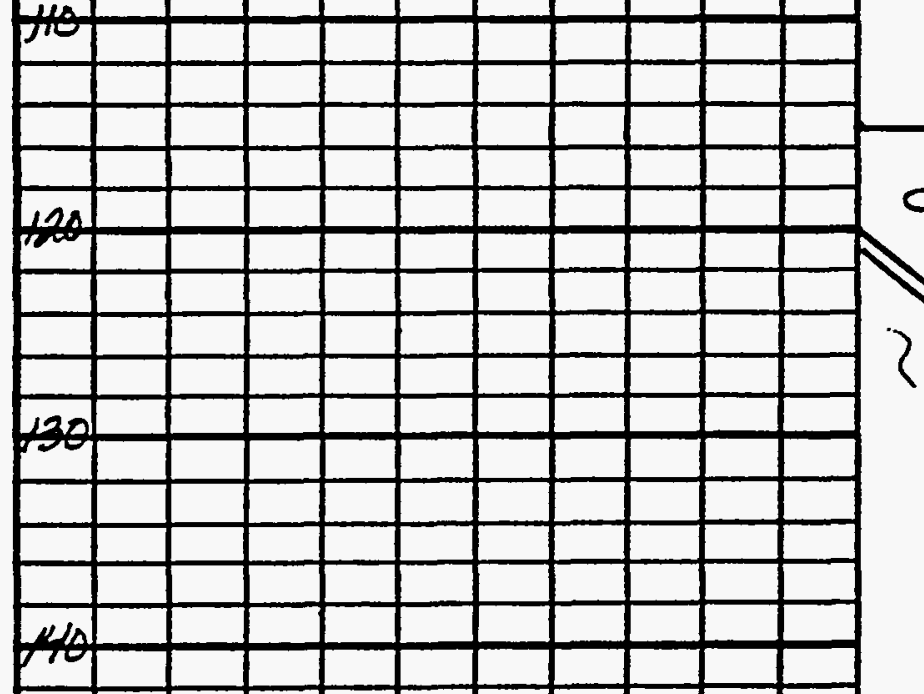

interbed

?

T.J.Q120.0'

COMMENTS: 
IACOBS ENGINEERING GROUP INC.

ADVANCED SYSTEMS DIVIBIOK, aLBUQUERQUE OPERATIONS

BOREHOLE LOG (ROCK)

Page $f$ of 4

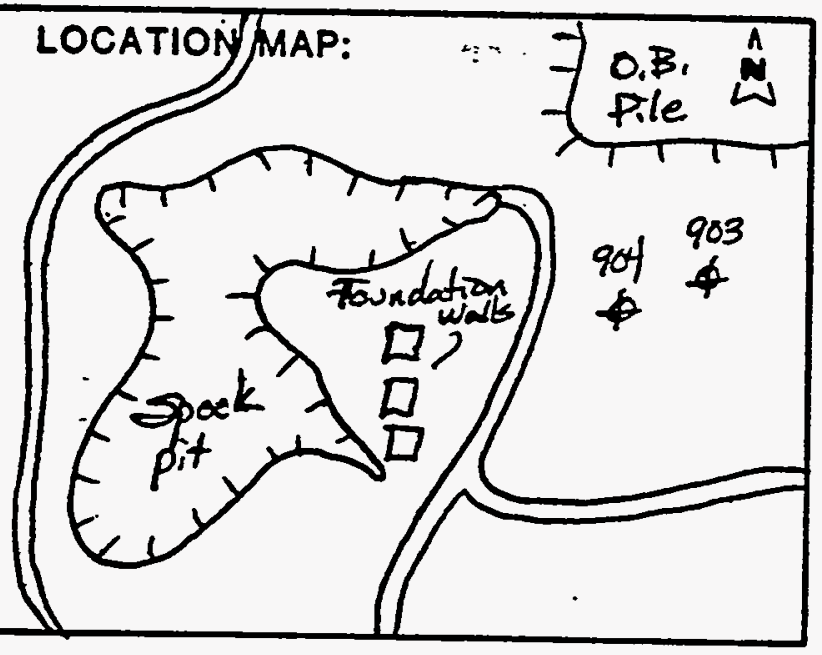

SITE ID:SPK- QI LOCATION ID: 914

SITE COORDINATES ( $f t$.):

N

GROUND ELEVATION ( $\mathrm{ft}$. MSL):

5090

DRILLING METHOD: Rotary-Air lMist

DRILLING CONTR.:

DATE STARTED:

Beyles Bros

DATE COMPLETED:

FIELD REP.:

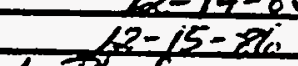

\begin{tabular}{|c|c|c|}
\hline \multicolumn{3}{|c|}{ GROUNDWATER LEVELS } \\
\hline DATE & TIME & DEPTH (ft.) \\
\hline $12-14-86$ & $3: 30$ & $1 / 4^{\prime}$ \\
\hline
\end{tabular}

LOCATION DESCRIPTION. Ta center of mill area east of foundation wealts SITE CONDITION Farly flat, wind bluwn snowiy, sparsly vegitated

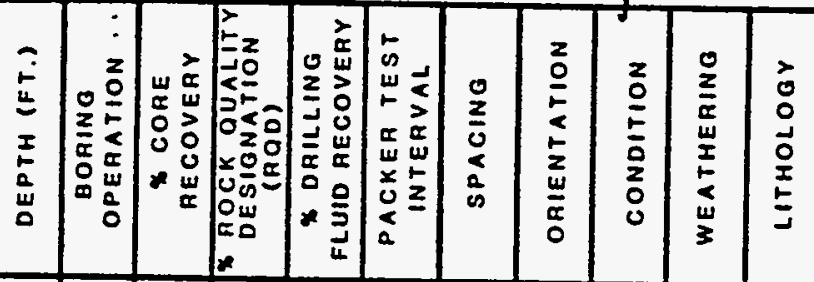

ROCK TYPE \& REMARKS

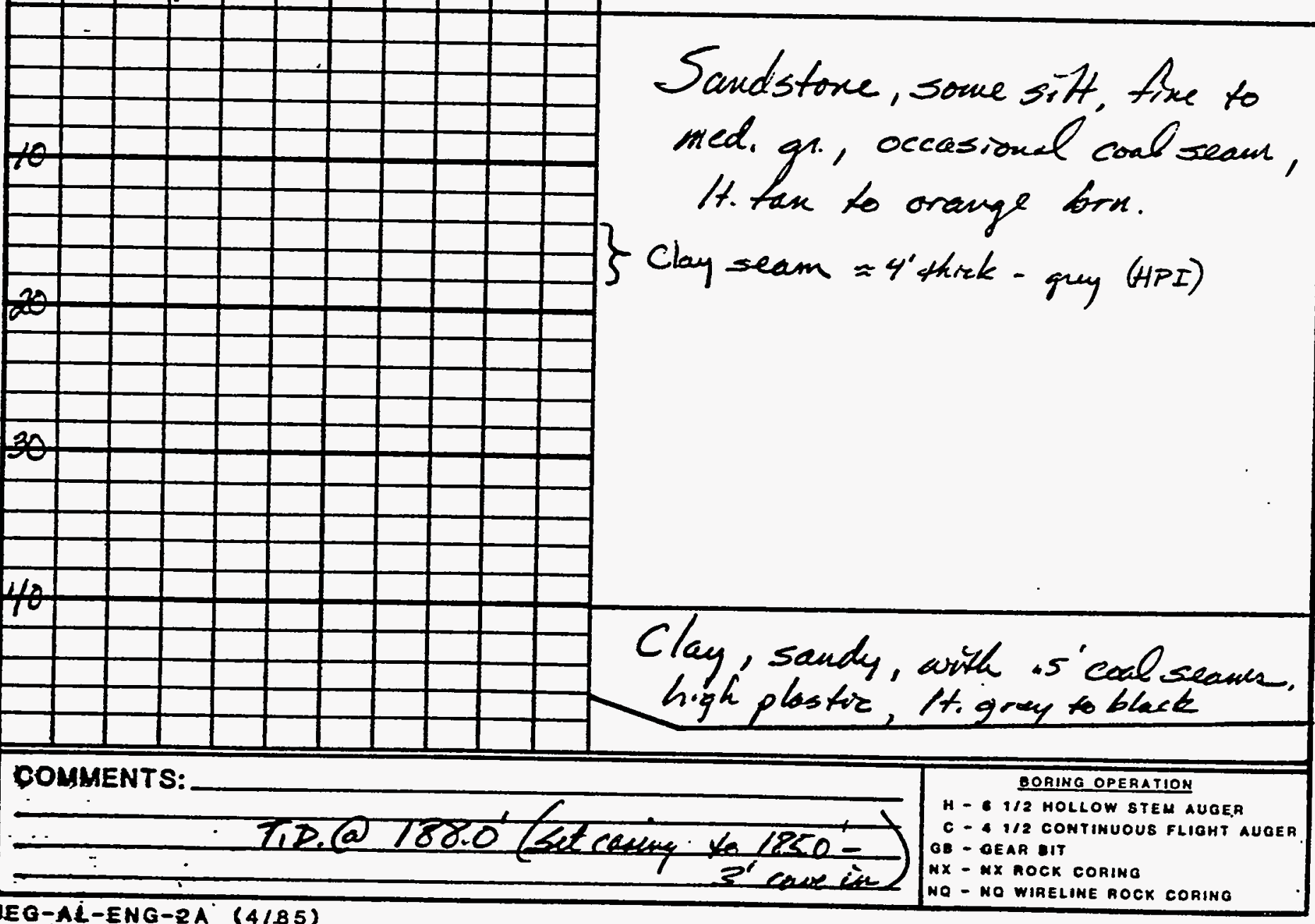




\section{BOREHOLE LOG (ROCK)

LOCATION MAP:

SITE ID: SPK- $\phi \mid$

LOCATION ID: Gof 914

SITE COORDINATES (ft.):

N GROUND ELEVATION (ft. M

DRILLING METHOD:

MSL): 5090

DRILLING CONTR.:

DATE STARTED:

DATE COMPLETED:

FIELD REP.:

\begin{tabular}{|c|c|c|}
\hline \multicolumn{3}{|c|}{ GROUNDWATER LEVELS } \\
\hline DATE & TIME & DEPTH (ft.) \\
\hline & & \\
\hline & & \\
\hline & & \\
\hline
\end{tabular}

\section{LOCATION DESCRIPTION SITE CONDITION}

\begin{tabular}{|c|c|c|c|c|c|c|c|c|c|}
\hline 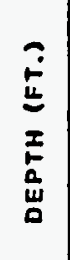 & 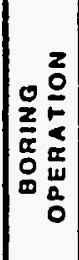 & 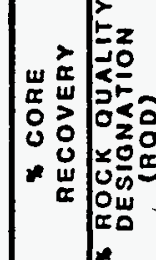 & 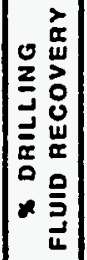 & 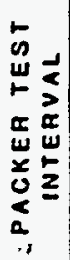 & $\begin{array}{l}0 \\
\frac{2}{0} \\
0 \\
0 \\
0\end{array}$ & $\begin{array}{l}\frac{2}{0} \\
\frac{a}{2} \\
\frac{z}{z} \\
\frac{w}{\alpha} \\
0\end{array}$ & $\begin{array}{l}2 \\
0 \\
\frac{0}{0} \\
0 \\
0 \\
0\end{array}$ & 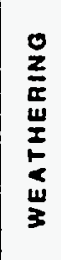 & 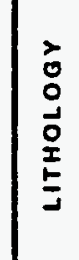 \\
\hline
\end{tabular}

Silty Clay, some coane sand, med. plastir, hrown.

\begin{tabular}{lllll|l|l|l|l|l|l|}
\hline 70 & & & $\cdot$ & & & & & & & \\
\hline & & & & & & & & & & \\
\hline
\end{tabular}

Eundstare, med. to coose gr., sonel silt, brown 


\section{BOREHOLE LOG (ROCK) Page 3 of 4}

LOCATION MAP:

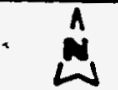

Sue pg. 1
SITE ID: SPK- $\$ 1$ LOCATION ID: 904 914

SITE COORDINATES ( $f t$. ):

$N$ $E$

GROUND ELEVATION (ft. MSL): 5090 DRILLING METHOD: DRILLING CONTR.: Rotany-Ar lHist

DATE STARTED:

DATE COMPLETED:

FIELD REP.: Boyles Bros.

$2-14-86$ $42-15-86$

\begin{tabular}{|c|c|c|}
\hline \multicolumn{3}{|c|}{ GROUNDWATER LEVELS } \\
\hline DATE & TIME & DEPTH (ft.) \\
\hline $12-14-86$ & $3: 30$ & $\mathbb{1 4}^{\prime}$ \\
\hline & & \\
\hline & & \\
\hline
\end{tabular}

\section{LOCATION DESCRIPTION}

SITE CONDITION

$100^{\circ}$

\begin{tabular}{|c|c|c|c|c|c|c|c|c|}
\hline & & & & & & & & \\
\hline 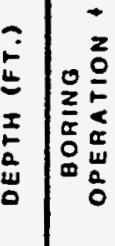 & 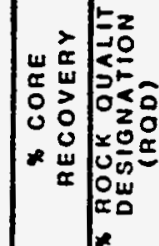 & 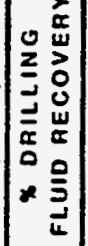 & 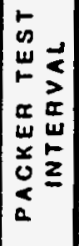 & $\begin{array}{l}0 \\
\frac{2}{0} \\
\vdots \\
\vdots \\
\text { si }\end{array}$ & $\begin{array}{l}\frac{5}{2} \\
\frac{1}{2} \\
z \\
\frac{u}{z} \\
0\end{array}$ & $\begin{array}{l}z \\
0 \\
0 \\
0 \\
0 \\
0 \\
0\end{array}$ & 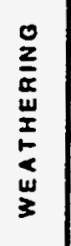 & \\
\hline
\end{tabular}


LOCATION MAP:

Seepg. 1
SITE ID SPK - $\phi 1$

$\hat{\omega}$

\section{LOCATION DESCRIPTION}

SITE CONDITION

SITE COORDINATES (ft.):

$\mathrm{N}$

LOCATION ID: 204 914

EATION (ft. MSL

GROUND ELEVATION (ft. MSL): 5090

DRILLING METHOD:

Rotary-Aor/Mat

DRILLING CONTR.:

Bogles Bros.

DATE STARTED:

DATE COMPLETED:

FIELD REP.:

\begin{tabular}{|c|c|c|}
\hline \multicolumn{3}{|c|}{ GROUNDWATER LEVELS } \\
\hline DATE & TIME & DEPTH (ft.) \\
\hline & & \\
\hline & & \\
\hline & & \\
\hline
\end{tabular}

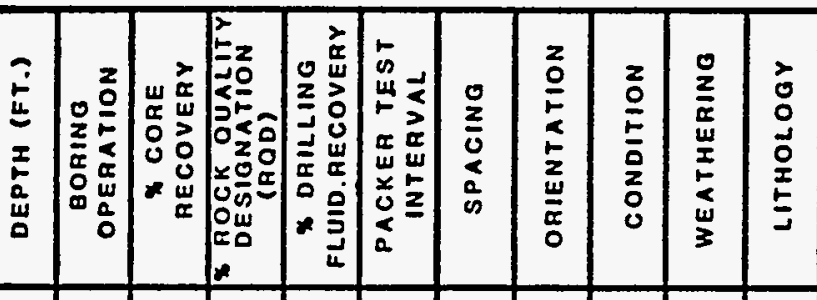
to.

Shale aud Cool, tissle, soneday, ti gray to black. Siltstonc, some shale, Higreyish blue of silt, H.greysh blue.

T.D.Q188.0' 


\section{JE JACOBS ENGINEERING GROUP INC:}

BOREHOLE LOG (ROCK)

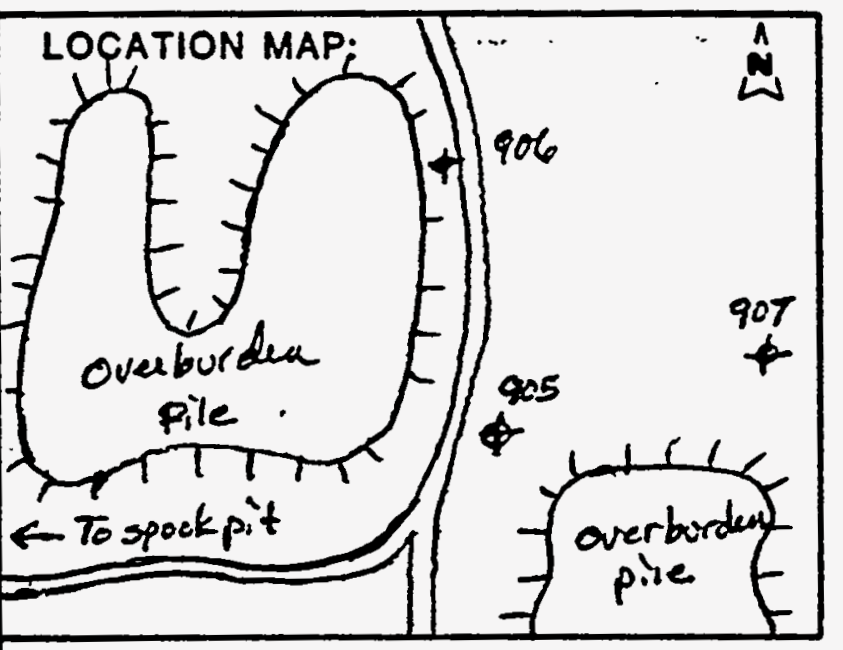

SITE ID: SPK- $\$$ I LOCATION ID: SITE COORDINATES ( $f t$.):

$N$

GROUND ELEVATION ( 4 . MSL): -5074

DRILLING METHOD: Betacy - Air LMLt

DRILLING CONTR.:

DATE STARTED:

DATE COMPLETED:

FIELD REP.:

Eogles Euses. $\frac{12-6-86}{10}$

$12-10-86$ L. He Plunte

\begin{tabular}{|c|c|c|}
\hline \multicolumn{3}{|c|}{ GROUNDWATER LEVELS } \\
\hline DATE & TIME & DEPTH (f1.) \\
\hline & & \\
\hline $12-9-86$ & 123 PM & 124.0 \\
\hline & & \\
\hline
\end{tabular}

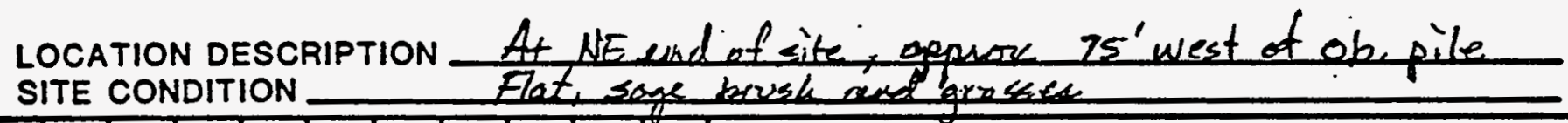

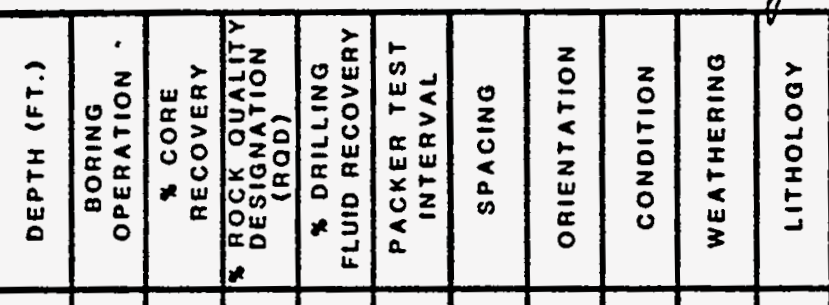

ROCK TYPE \& REMARKS

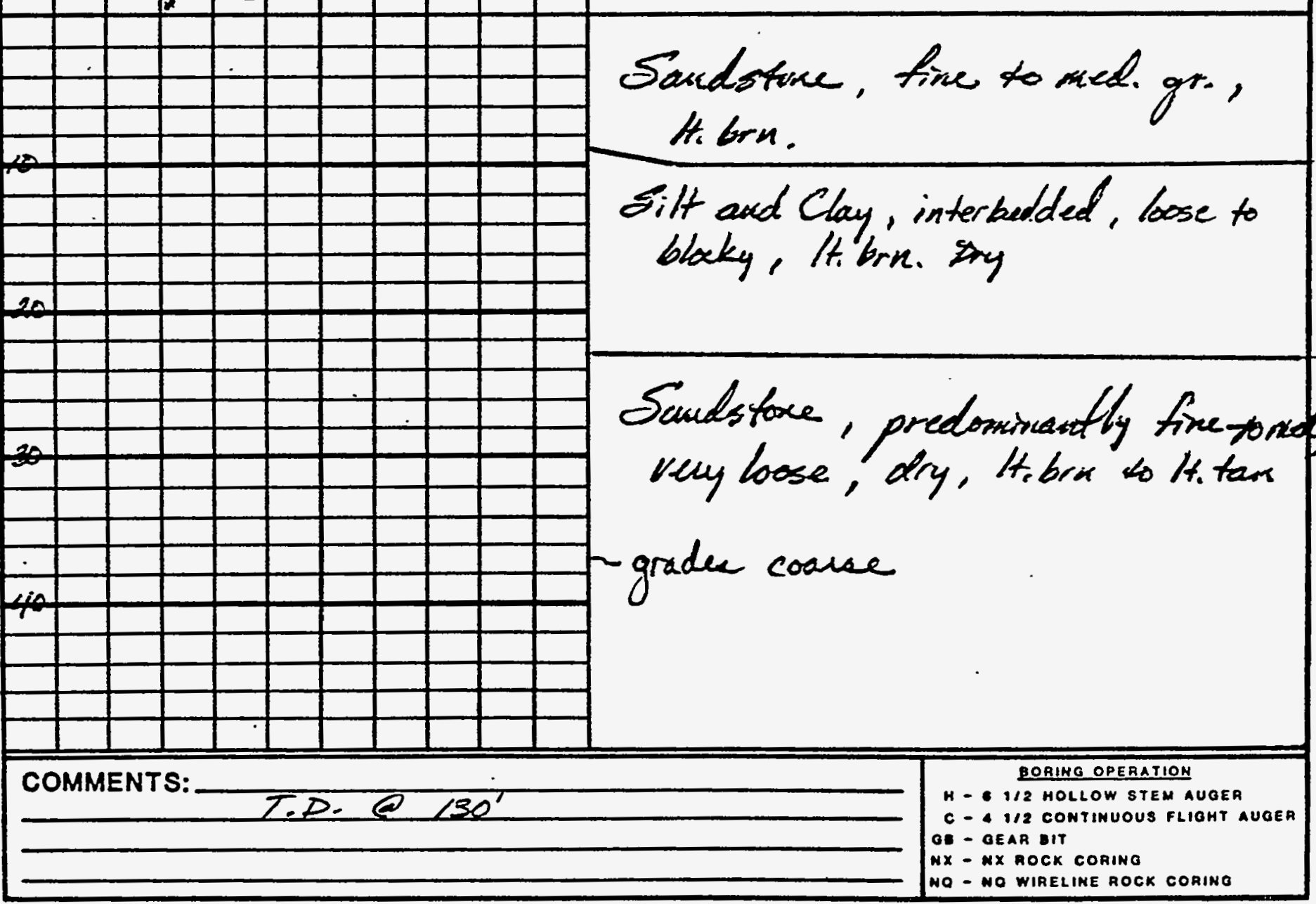




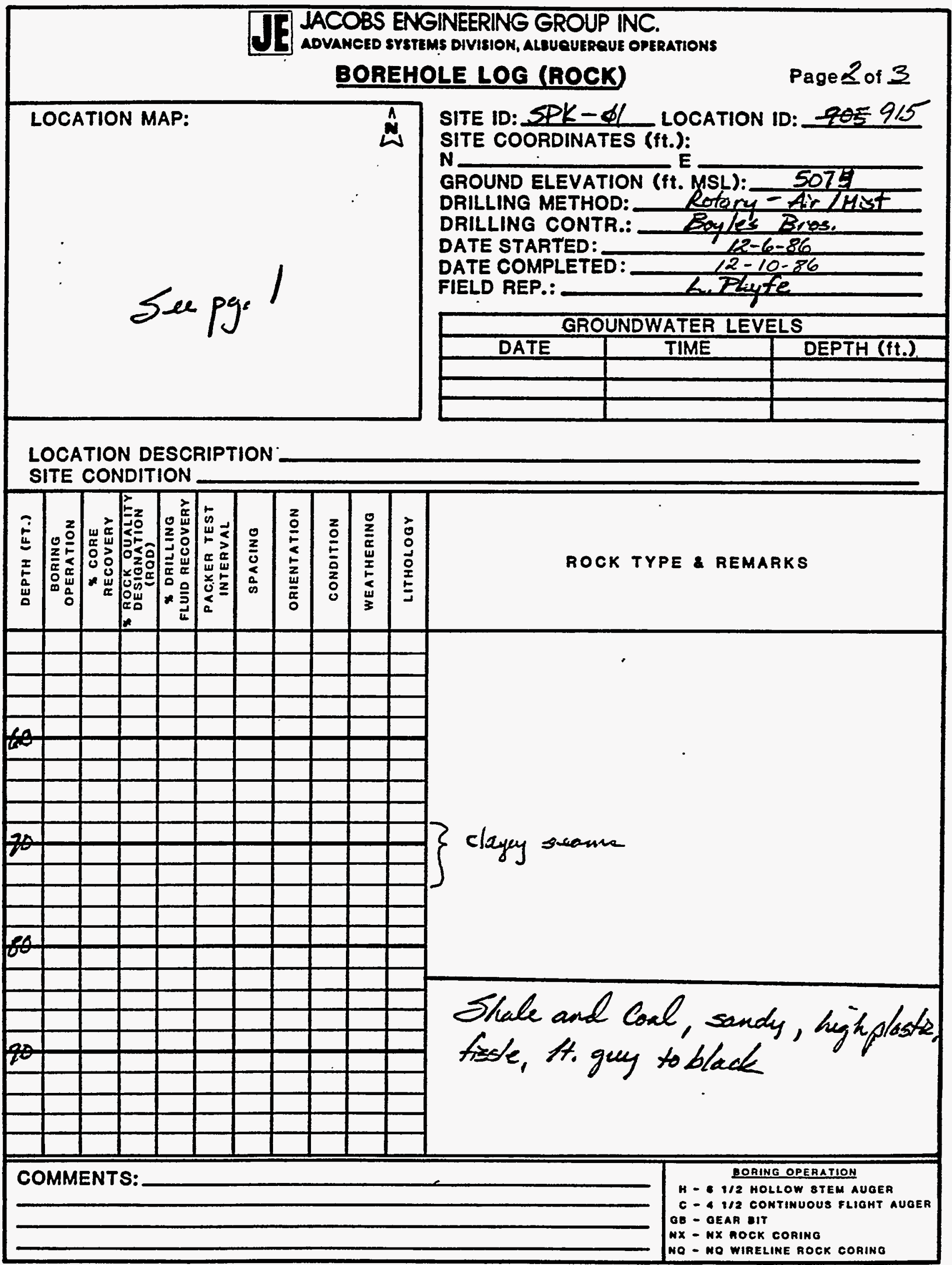




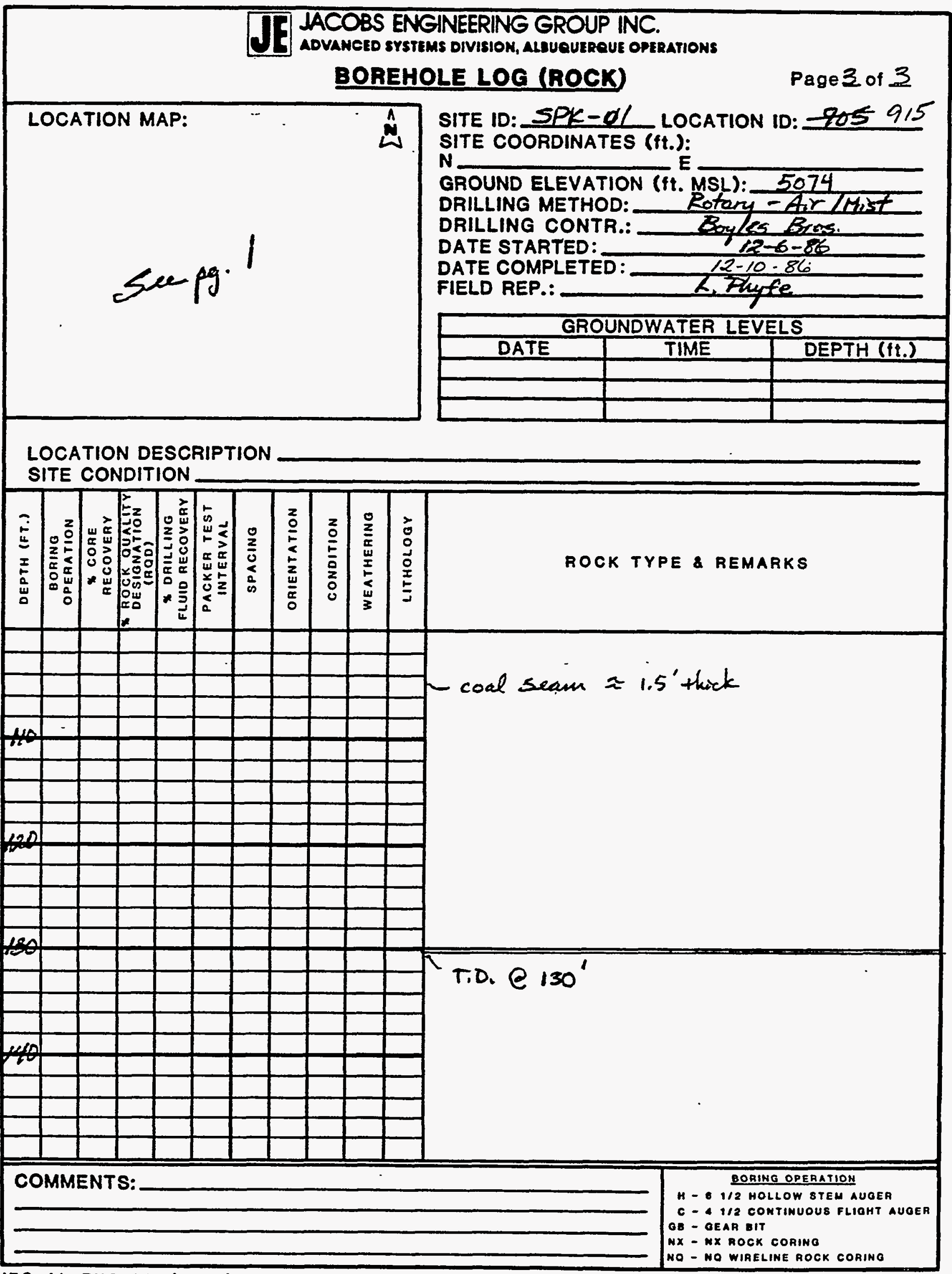

JEG-AL-ENG-2A (4/85) 


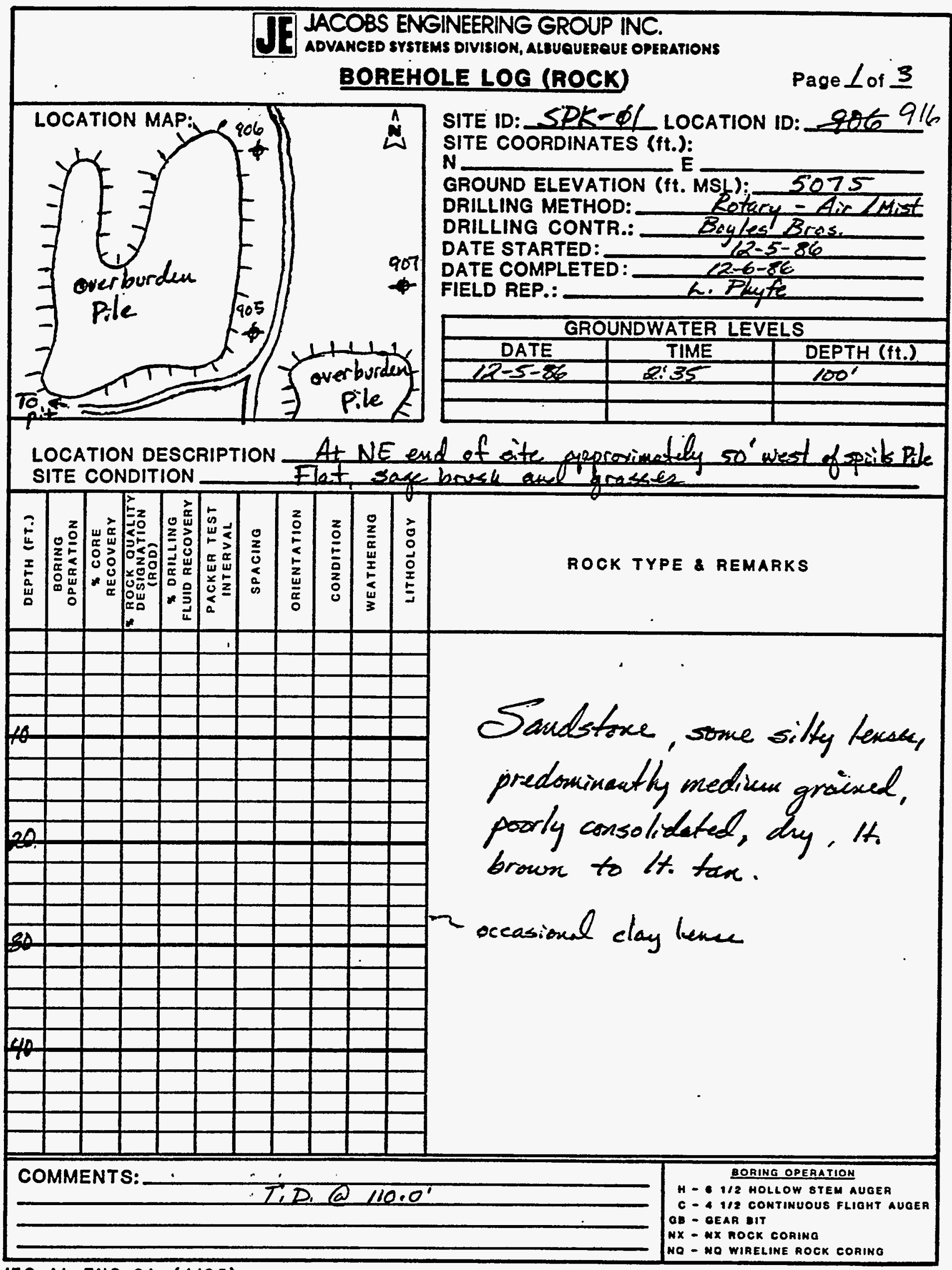

JEG-AL-ENG-2A (4/85) 


\section{BOREHOLE LOG (ROCK) \\ Page 2 of 3}

LOCATION MAP:

See pg. 1
出

SITE ID: SPK- 1 LOCATION ID: FOt 916 SITE COORDINATES (ft.):

$N$ DRILLING METHOD: DRILLING CONTR.:

DATE STARTED:

DATE COMPLETED :

FIELD REP.:

\section{E}

MSL): 5075 Rotary - Air TMant Boykes Bues

$12-6-8$ K. M. Phyte

\begin{tabular}{|c|c|c|}
\hline \multicolumn{3}{|c|}{ GROUNDWATER LEVELS } \\
\hline DATE & TIME & DEPTH (ft.) \\
\hline & & \\
\hline & & \\
\hline & & \\
\hline
\end{tabular}

LOCATION DESCRIPTION SITE CONDITION

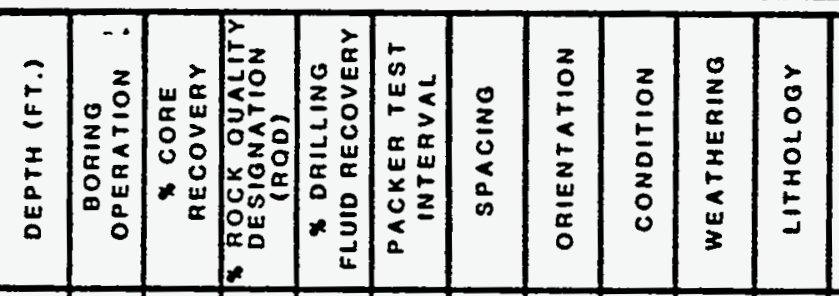

Sardstone, med. to coane. - $\downarrow$ It. $\tan$ to orange bon.

$$
\cdot
$$




\section{.15 IACOBS ENGINEERING GROUP INC. \\ ADVAMCED SYSTEMS DIVISION, ALIUQUERQUE OPERATIONS}

\section{BOREHOLE LOG (ROCK)}

Page 3 of $\underline{3}$

LOCATION MAP:

Seep. 1

$\stackrel{\wedge}{\omega}$

SITE ID: SPK- $\phi /$ LOCATION ID: 916 SITE COORDINATES (ft.):

$N$ GROUND ELEVATION (ft, MSL):

DRILLING METHOD:

DRILLING CONTR.:

DATE STARTED:

DATE COMPLETED:

FIELD REP.:

\section{Petary = fic leist}

Eayter Bres.

$12-5-86$

$: \frac{12-6-86}{617}$
L. Plupte

\begin{tabular}{|c|c|c|}
\hline \multicolumn{2}{|c|}{ GROUNDWATER LEVELS } \\
\hline DATE & TIME & DEPTH (ft.) \\
\hline $12-5-86$ & D. 95 & 100 \\
\hline & & \\
\hline & & \\
\hline
\end{tabular}

\section{LOCATION DESCRIPTION}

SITE CONDITION

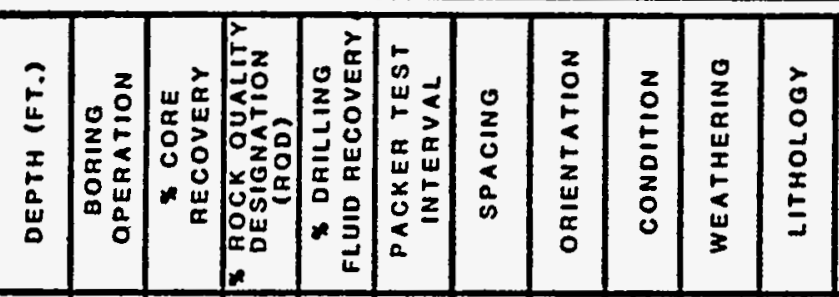

\section{D2.}




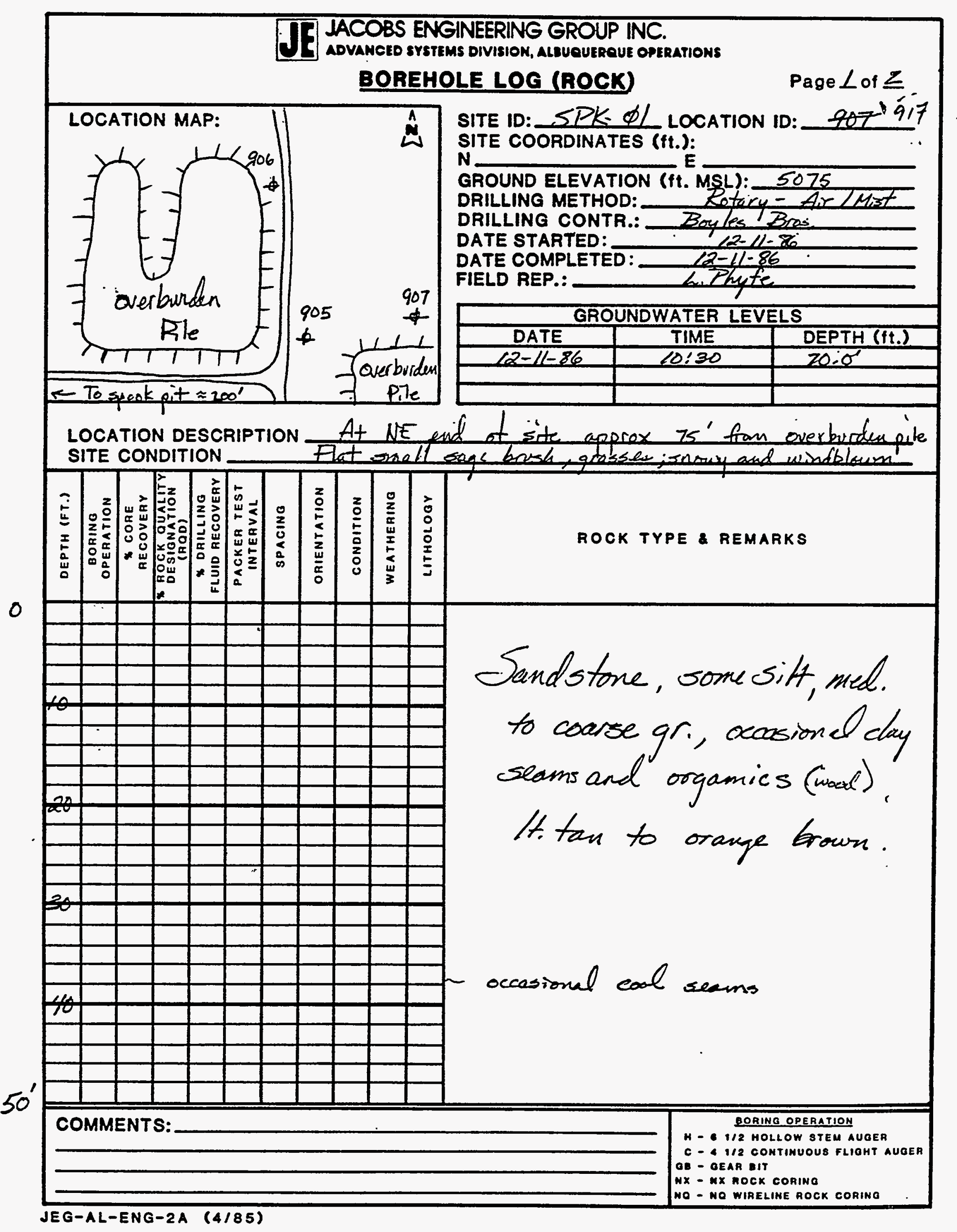


LOCATION MAP:

SITE ID:SP/S- $\$$ / LOCATION ID: FO 917 SITE COORDINATES ( $f$ t.):

N

GROUND ELEVATION (ft. MSL): 5075

DRILLING METHOD:

See pl

DRILLING CONTR.:

DATE STARTED:

DATE COMPLETED:

FIELD REP.: $<$. Phyfe

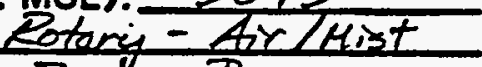

Boyles Bros. $2-11-86$

\begin{tabular}{|c|c|c|}
\hline \multicolumn{3}{|c|}{ GROUNDWATER LEVELS } \\
\hline DATE & TIME & DEPTH (ft.) \\
\hline & & \\
\hline & & \\
\hline & & \\
\hline
\end{tabular}

\section{LOCATION DESCRIPTION}

SITE CONDITION

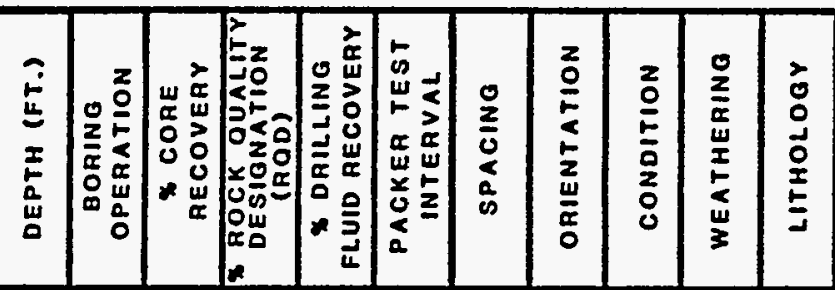

- color change to orange brown 


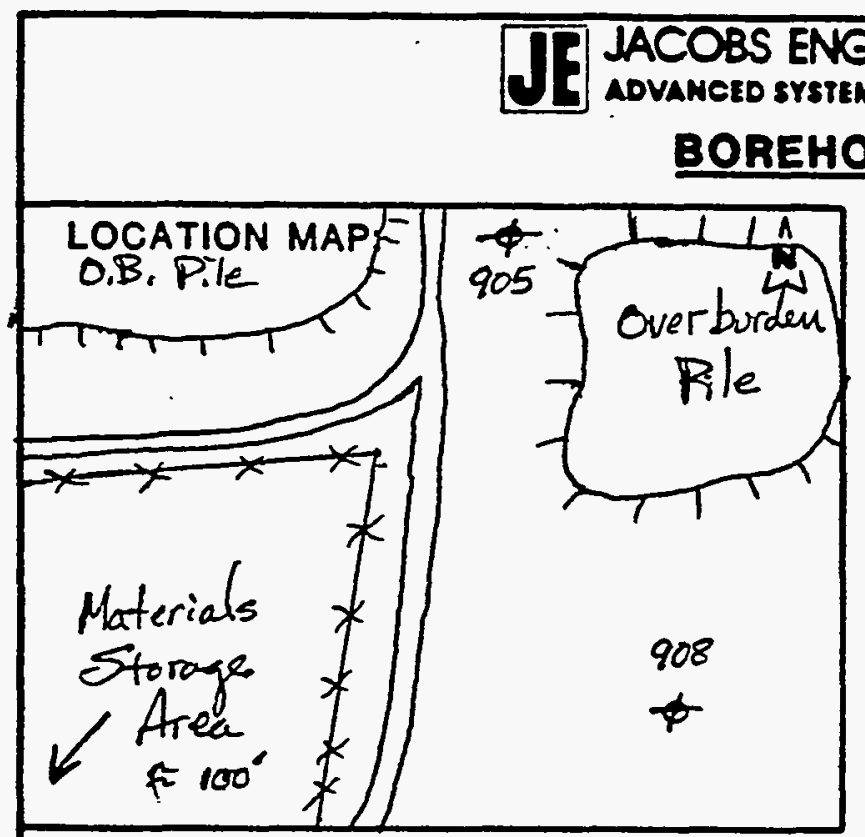

Page $L$ of 3

SITE ID: SPK- QI LOCATION ID: SITE COORDINATES (ft.):

$N$

GROUND ELEVATION ( $\mathrm{ft}$. MSL): 5100

DRILLING METHOD:

DRILLING CONTR.:

DATE STARTED:

DATE COMPLETED:

FIELD REP.:

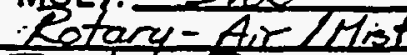

Rotary-Ave

$12-41-86$ h. Phife

\begin{tabular}{|c|c|c|}
\hline \multicolumn{3}{|c|}{ GROUNDWATER LEVELS } \\
\hline DATE & TIME & DEPTH $(\mathrm{ft.})$ \\
\hline $12-12.86$ & $10: 30$ & $125.0^{\prime}$ \\
\hline & & \\
\hline & & \\
\hline
\end{tabular}

LOCATION DESCRIPTION Approximately $350^{\circ}$ east of material storage area. SITE CONDITION

ROCK TYPE \& REMARKS

0

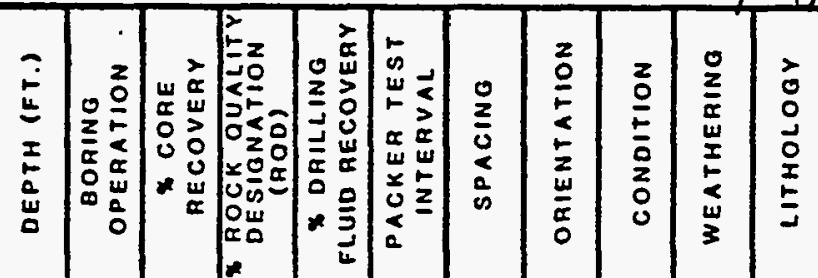

Sandstorce, silty, fine to meds grained, loose. It.tan. dry

Siltstone, sandy, blocky, Itigray

\begin{tabular}{lllllllllllll}
\hline & & & & & & & & & & & & \\
\hline
\end{tabular}

b COMMENTS:

Sandistore. fine to vifine, loose H. tan, damp 


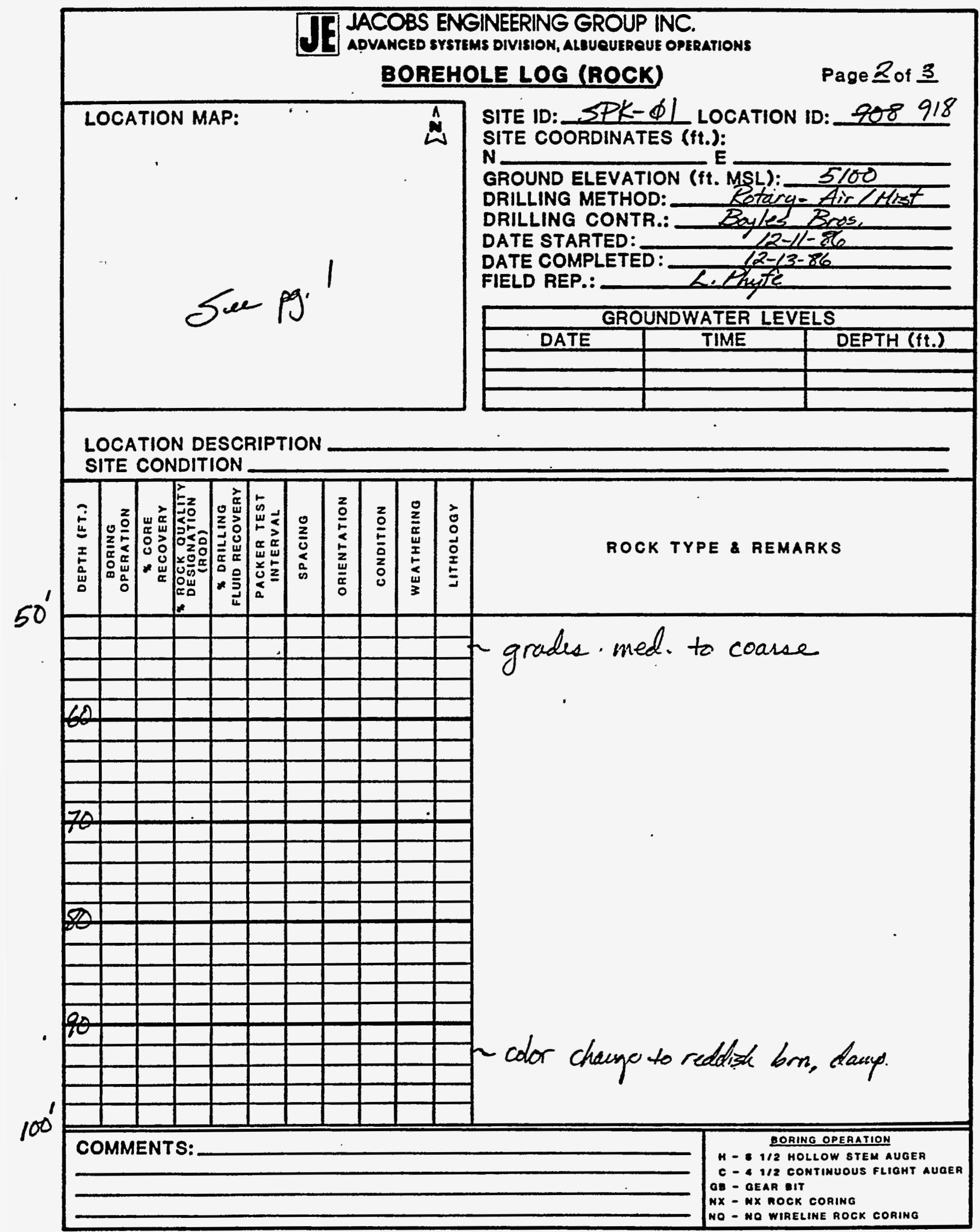

JEG-AL-ENG-2A (4/85) 


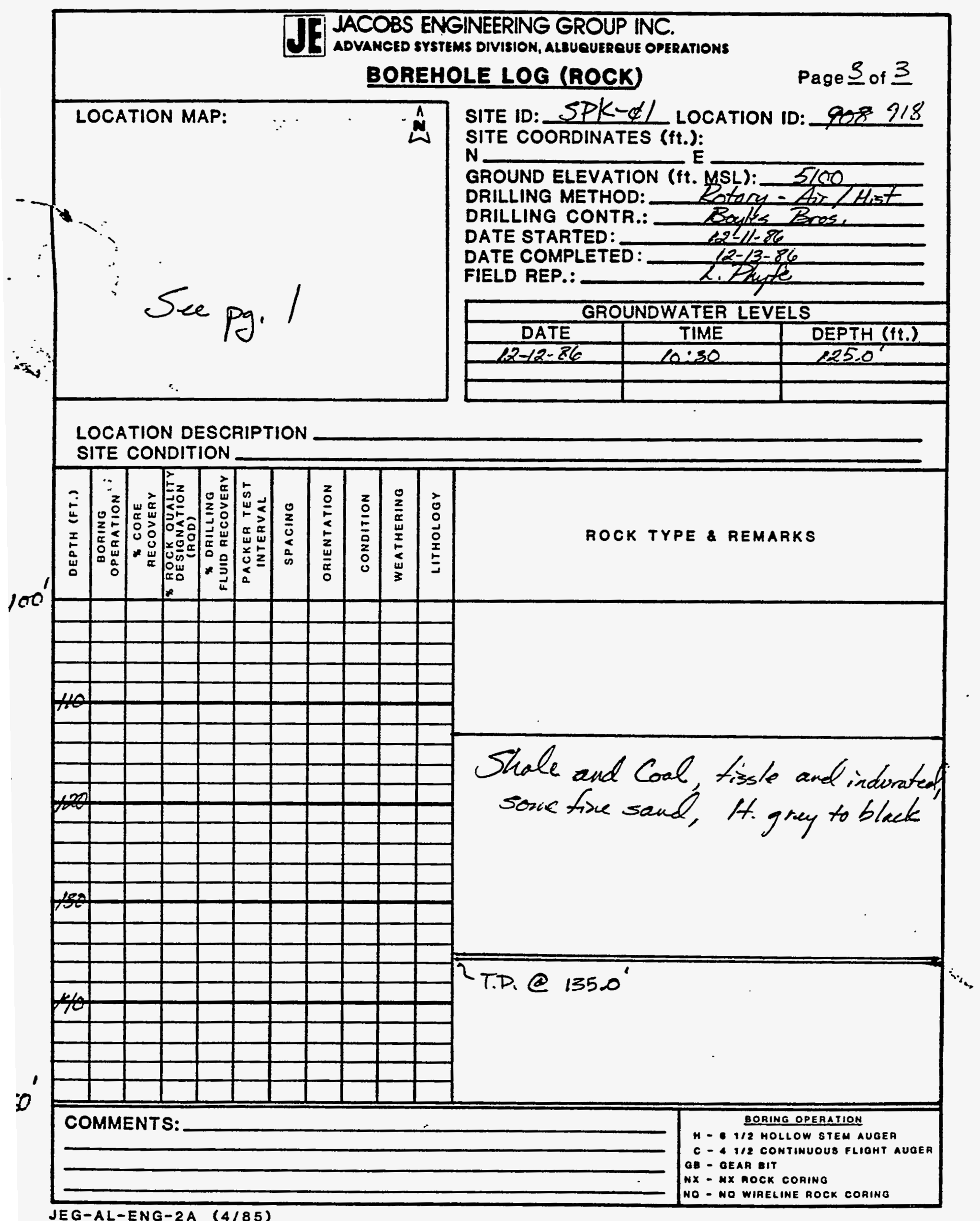




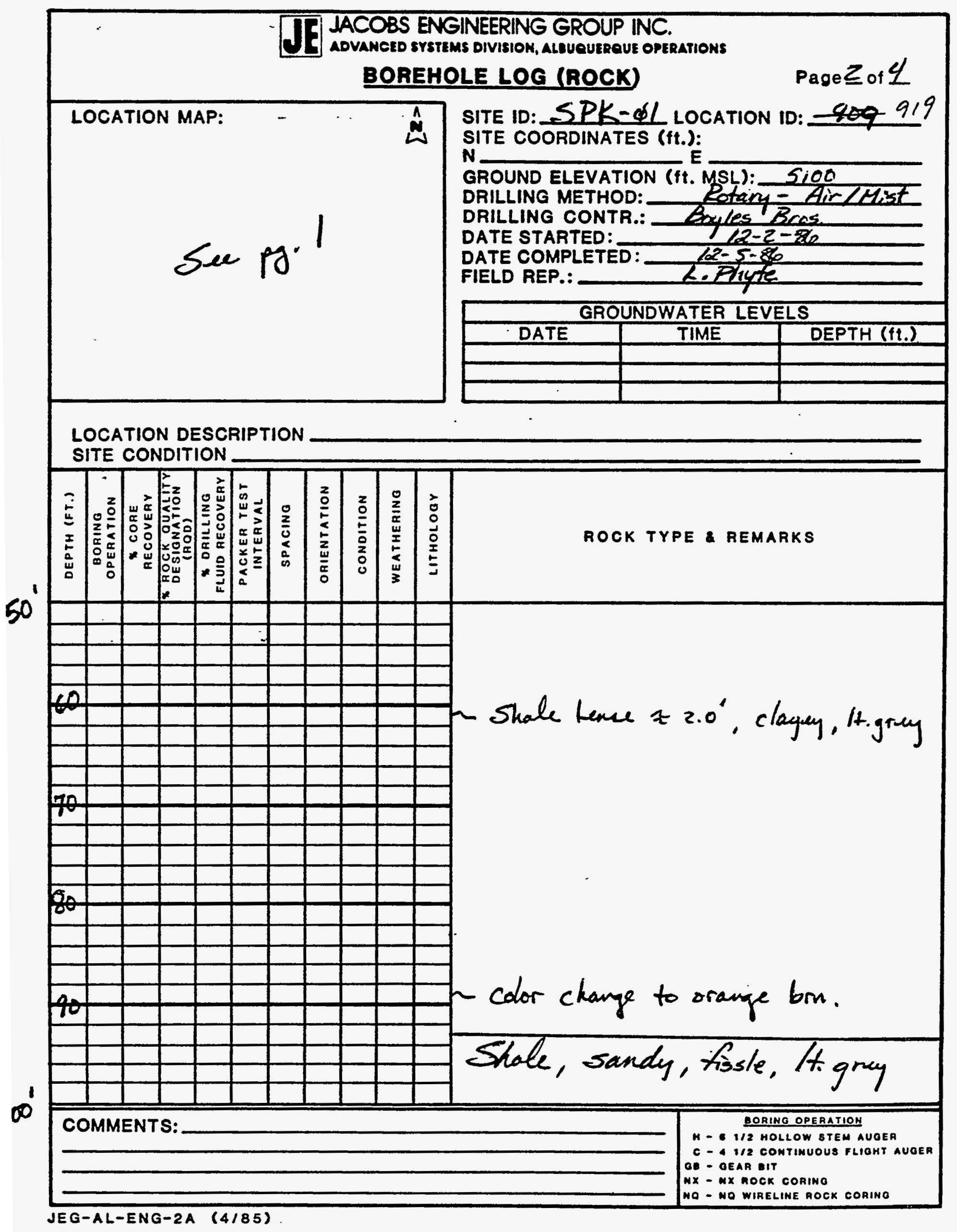




\section{BOREHOLE LOG (ROCK) \\ Page 3 of 4}

LOCATION MAP:

SITE ID: SPK- 61 LOCATION ID: FO9 919

SITE COORDINATES (ft.):

N

GROUND ELEVATION ( $\mathrm{ft} . \mathrm{MSL}$ ): 5100

DRILLING METHOD: Retary- Air / Hist

DRILLING CONTR.: Boykes Bros.

DATE STARTED: $12-2-86$

See pg. 1

DATE COMPLETED:

FIELD REP.:

$12-5-86$

\begin{tabular}{|c|c|c|}
\hline \multicolumn{3}{|c|}{ GROUNDWATER LEVELS } \\
\hline DATE & TIME & DEPTH (ft.) \\
\hline & & \\
\hline & & \\
\hline & & \\
\hline
\end{tabular}

LOCATION DESCRIPTION

SITE CONDITION

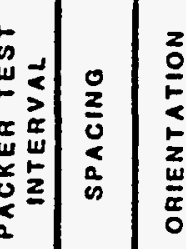

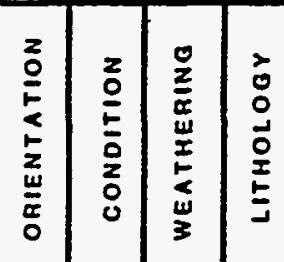

$\sim$ occasiond coal seames

Siltstore, trace fine sand, some shaluy interbed's, H. gray.

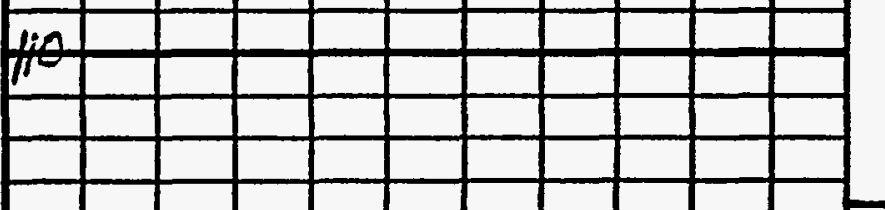

Saxdstore, sketry, fire to r. time, Higrey to bleck, moisto Shale, ence silt, tissle, H.gry to black. duy

Silfstore, sorre shale. Higray to black, moist. 


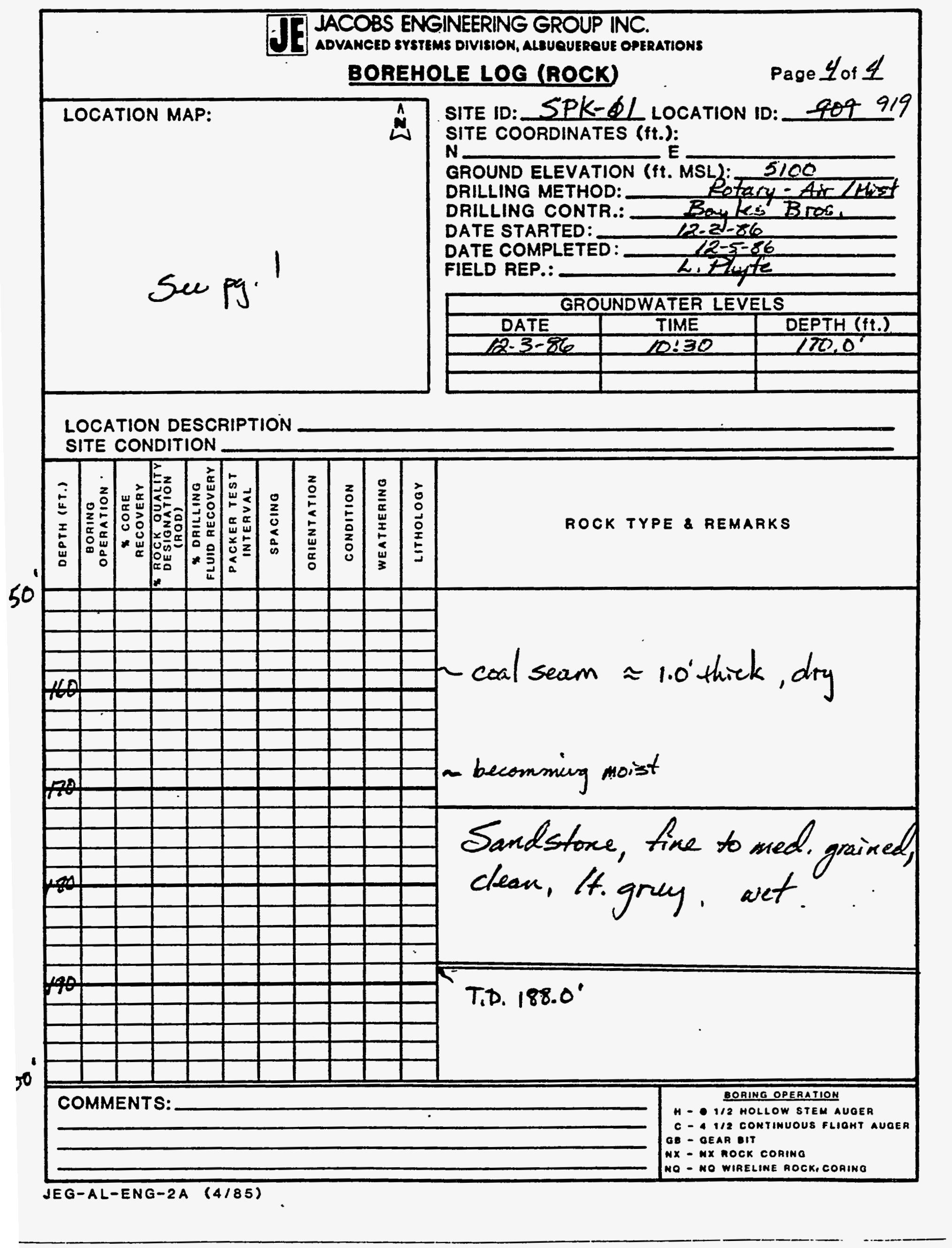




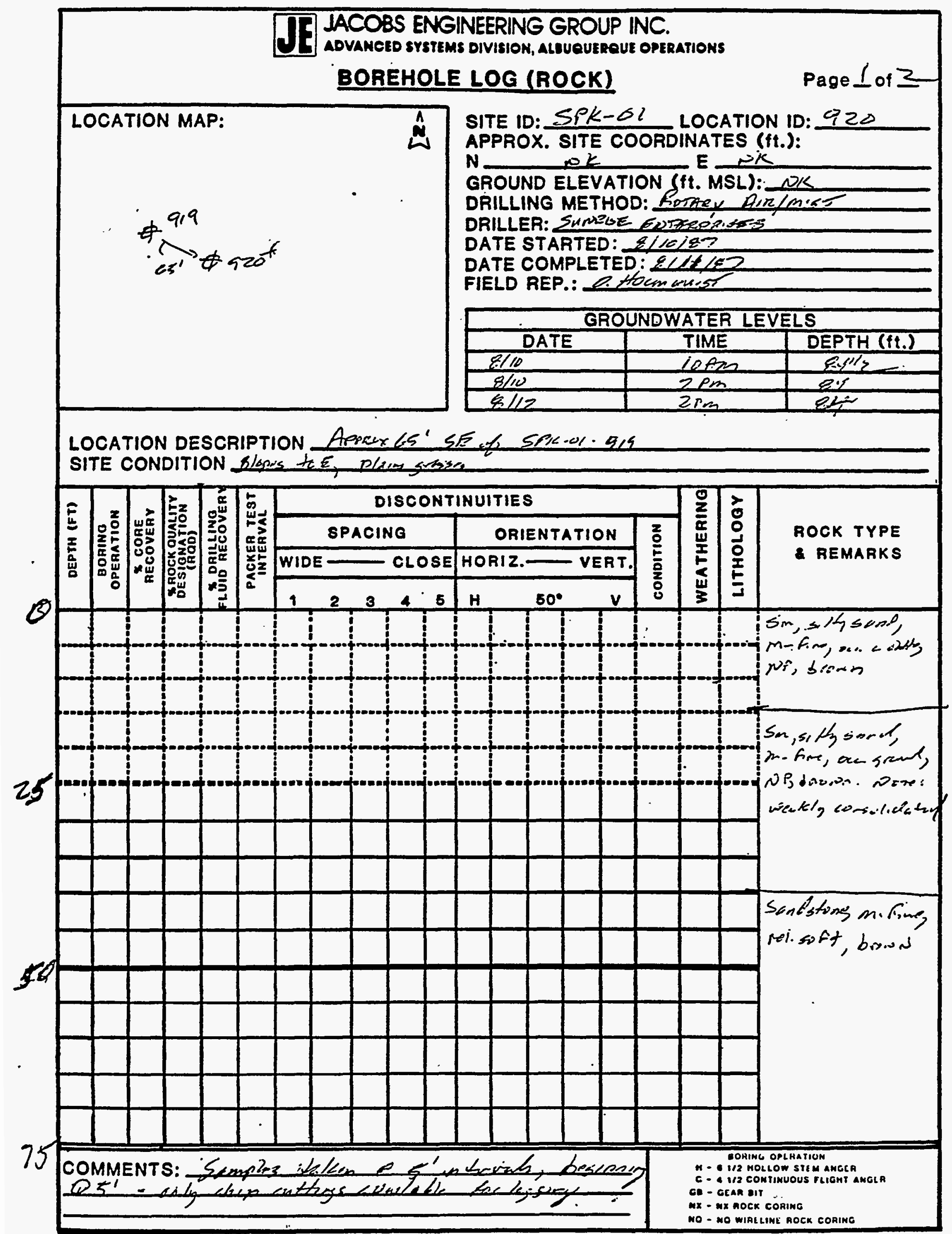




\section{LACOBS ENGINEERING GROUP INC.}

BOREHOLE LOG (ROCK)

Page 2 of 2

LOCATION MAP:

$\stackrel{\Lambda}{\omega}$

SITE ID: SPEOOI

LOCATION ID: 9Z0

APPROX. SITE COORDINATES ( $\mathrm{ft}$.):

$N$ a K K E iK

GROUND ELEVATION (ft. MSL): NK

DRILLING METHOD: Aire/mist Izy

DRILLER: Suprese Eaterpouss

DATE STARTED: 8 Licis?

SFE BSlot 2

DATE COMPLETED: $\& U \angle B>$

FIELD REP.: \& theim and

\begin{tabular}{|c|c|c|}
\hline \multicolumn{3}{|c|}{ GROUNDWATER LEVELS } \\
\hline DATE & TIME & DEPTH (ft.) \\
\hline & & \\
\hline & & \\
\hline & & \\
\hline
\end{tabular}

LOCATION DESCRIPTION

SITE CONDITION

\begin{tabular}{|c|c|c|c|c|c|c|c|c|c|c|c|}
\hline \multirow{4}{*}{ 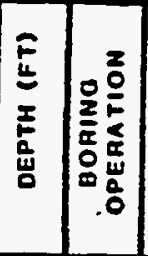 } & \multirow{4}{*}{\multicolumn{2}{|c|}{ 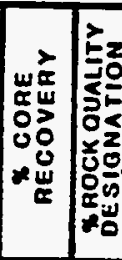 }} & \multirow{4}{*}{ 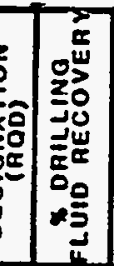 } & \multirow{4}{*}{ 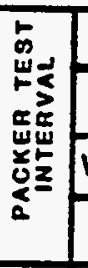 } & \multicolumn{5}{|c|}{ DISCONTINUITIES } & \multirow{4}{*}{ 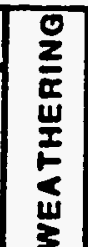 } & \multirow{4}{*}{ ב⿱ } \\
\hline & & & & & \multicolumn{2}{|r|}{ SPACING } & \multirow{2}{*}{\multicolumn{2}{|c|}{$\begin{array}{l}\text { ORIENTATION } \\
\text { HORIZ. VERT. }\end{array}$}} & \multirow{3}{*}{$\begin{array}{l}z \\
0 \\
0 \\
0 \\
0 \\
0 \\
0\end{array}$} & & \\
\hline & & & & & & $D E=$ CLOSE & & & & & \\
\hline & & & & & 1 & 34 & $50^{\circ}$ & v & & & \\
\hline
\end{tabular}

ROCK TYPE

REMARKS

メ

(1)

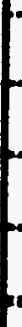

1 $+\infty$ it :

(1)

Shale boopn clestictysoft Sandstone, M-fing bixpon

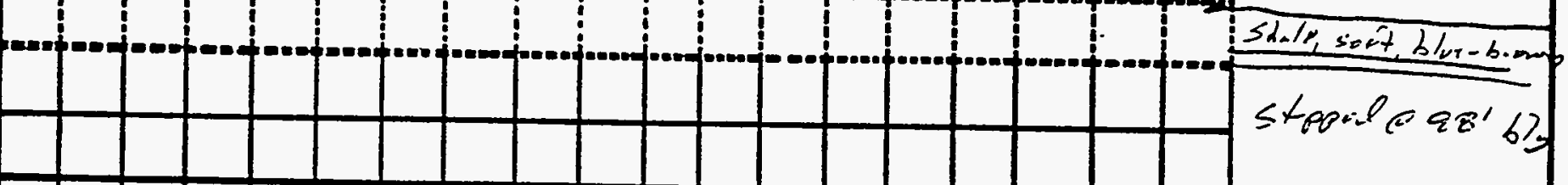

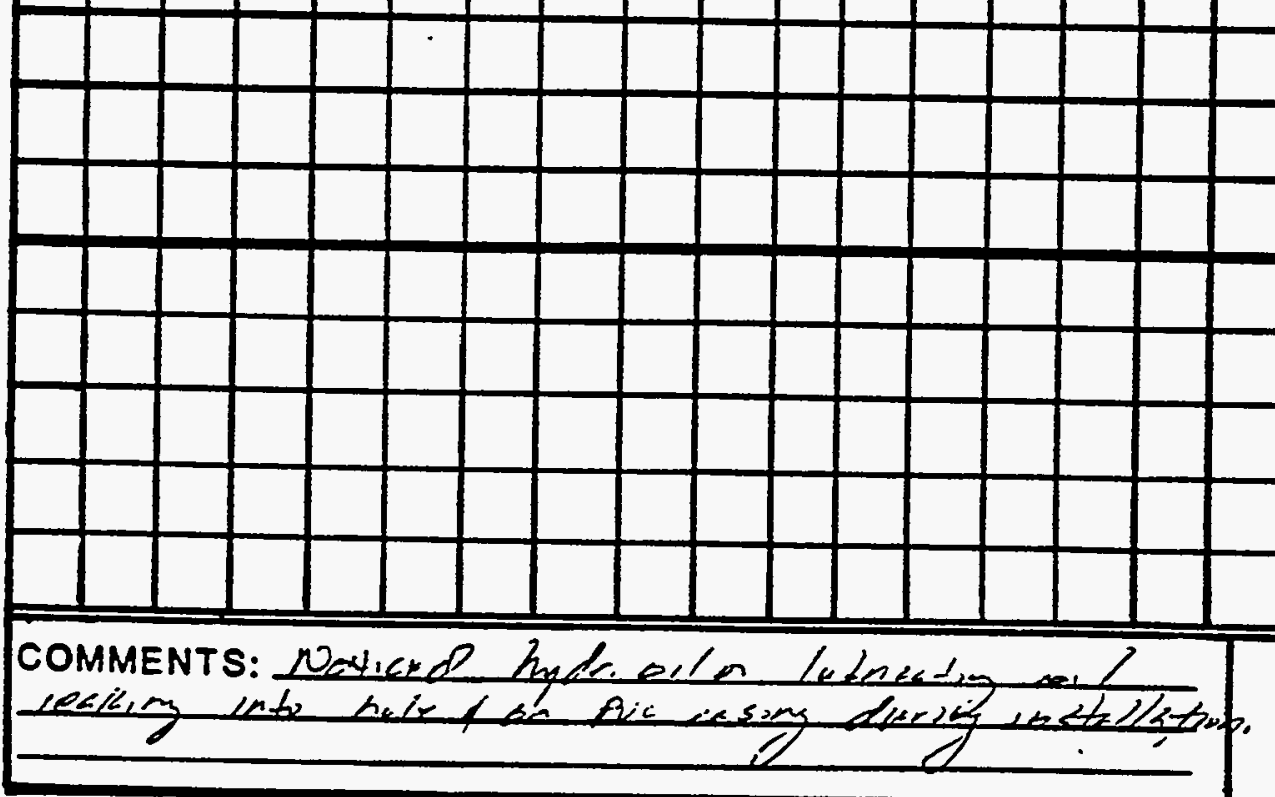




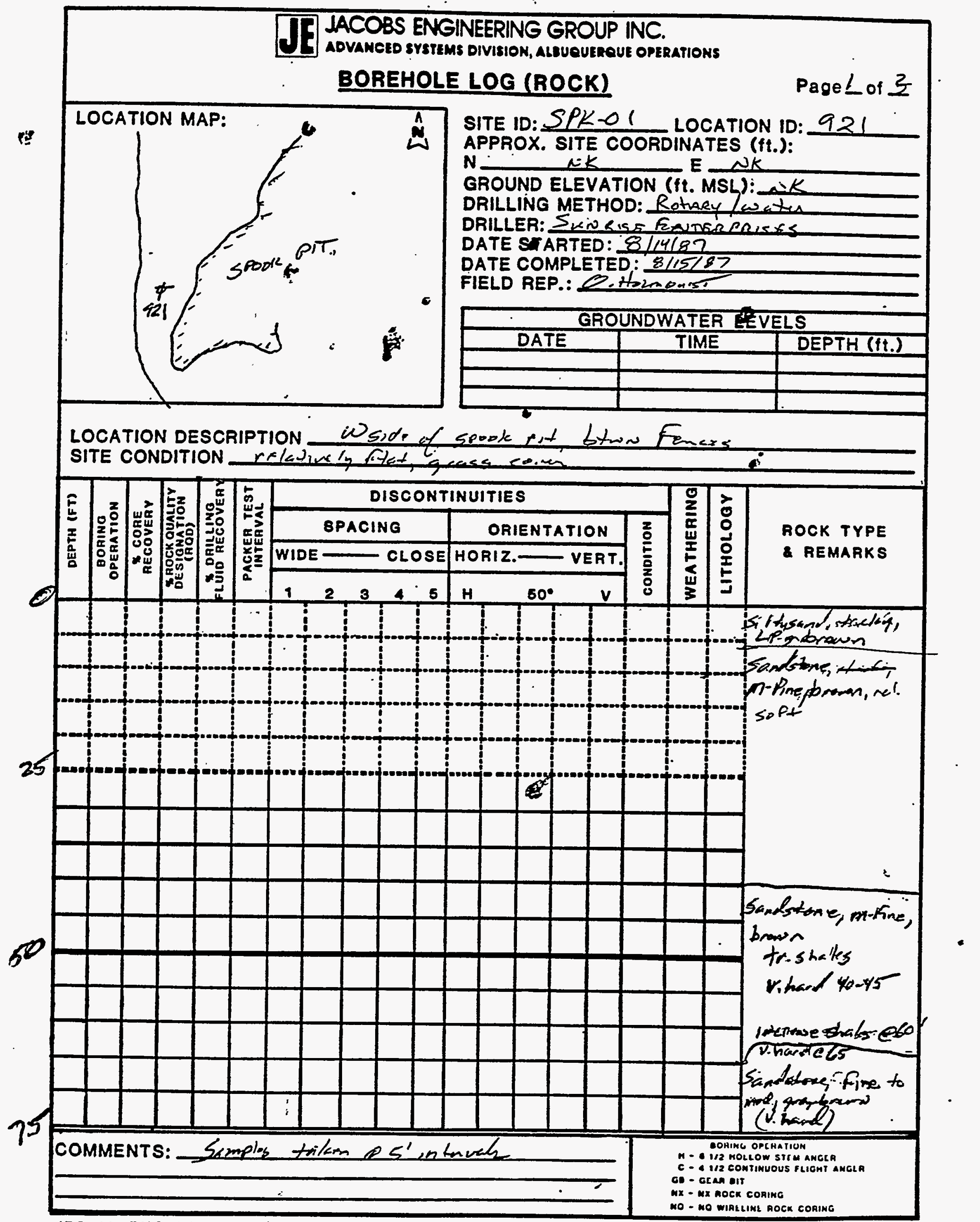


IE HACOBS ENGINEERING GROUP INC.

ADVAMEED SYETEMS DIVISION, ALUUUERQUE ORERATIONS

BOREHOLE LOG (ROCK)

Page? of 3

LOCATION MAP:

SITE ID: SPKDI LOCATION ID: 921 APPROX. SITE COORDINATES ( $f t$.):

N. E

GROUND ELEVATION (\$1. MSL):

DRILLING METHOD: RoTARY/WTAER

SFF bi d o 3

DRILLER: SUNRISE Fonterencshs

DATE STARTED: $5 / 14 / 87$

DATE COMPLETED: $8 / 15 / 87$

FIELD REP.: OH Herm 6 wsts

\begin{tabular}{|c|c|c|}
\hline \multicolumn{3}{|c|}{ GROUNDWATER LEVELS } \\
\hline DATE & TIME & DEPTH (fi.) \\
\hline & & \\
\hline & & \\
\hline & & \\
\hline
\end{tabular}

LOCATION DESCRIPTION

SITE CONDITION

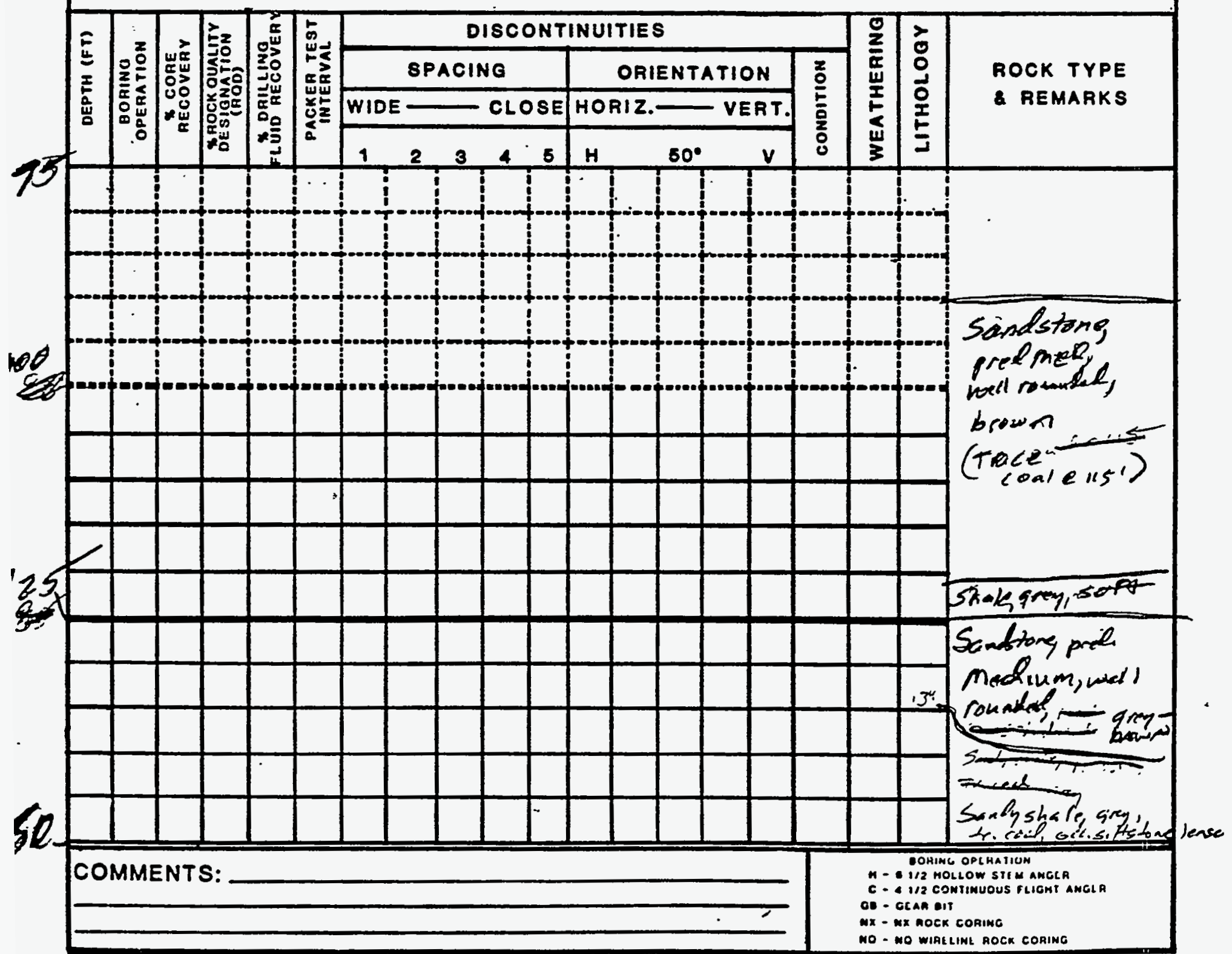




\section{BOREHOLE LOG (ROCK)}

Page 2 of 3

LOCATION MAP:

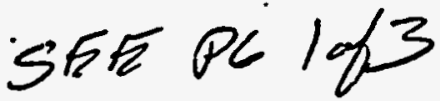

\section{$\stackrel{\Lambda}{\omega}$}

SITE ID: SPK-DI

APPROX. SITE COORDINATES ( $f$.):

N

GROUND ELEVATION (ft. MSL):

DRILLING METHOD: RoTOR.2/ IwADEA

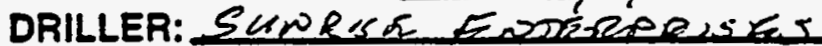

DATE STARTED: $\& / 14187$

DATE COMPLETED: $9 / 1</ 87$

FIELD REP.: D. Halmen.st

\begin{tabular}{|l|c|c|}
\hline \multicolumn{3}{|c|}{ GROUNDWATER LEVELS } \\
\hline DATE & TIME & DEPTH (ft.) \\
\hline & & \\
\hline & & \\
\hline
\end{tabular}

LOCATION DESCRIPTION

SITE CONDITION

\begin{tabular}{|c|c|c|c|c|c|c|c|c|c|c|c|}
\hline \multirow{4}{*}{$\begin{array}{l}\text { E } \\
\underline{u} \\
z \\
\frac{z}{5} \\
\stackrel{4}{0}\end{array}$} & \multirow{4}{*}{ 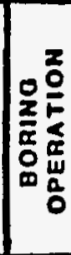 } & \multirow{4}{*}{ 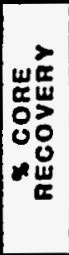 } & \multirow{4}{*}{ 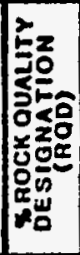 } & \multirow{4}{*}{ } & \multirow{4}{*}{ 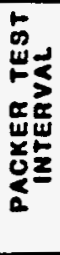 } & \multicolumn{3}{|c|}{ DISCONTINUITIES } & \multirow{4}{*}{ 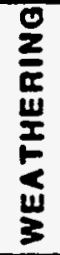 } & \multirow{4}{*}{$\begin{array}{l}x \\
0 \\
0 \\
0 \\
0 \\
0\end{array}$} & \multirow{4}{*}{$\begin{array}{l}\text { ROCK TYPE } \\
\text { \& REMARKS }\end{array}$} \\
\hline & & & & & & SPACING & ORIENTATION & 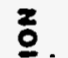 & & & \\
\hline & & & & & & WIDE _ CLOSE & HORIZ. $\longrightarrow$ VERT. & $\frac{5}{8}$ & & & \\
\hline & & & & & & $4^{\cdot} 5$ & $50^{\circ}$ & 8 & & & \\
\hline
\end{tabular}

$+$

!

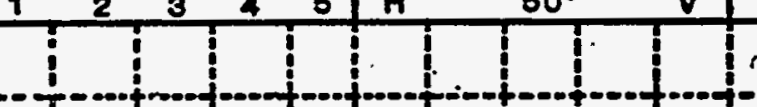

iscrelstane, m-Five,

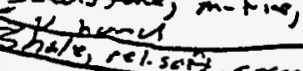

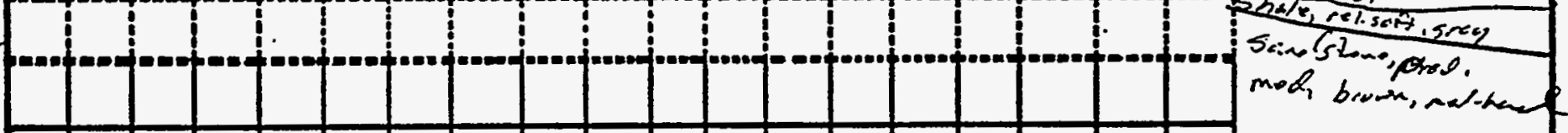

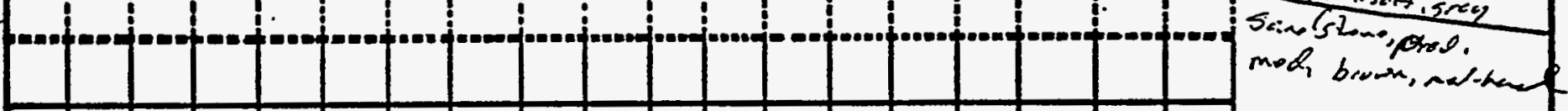

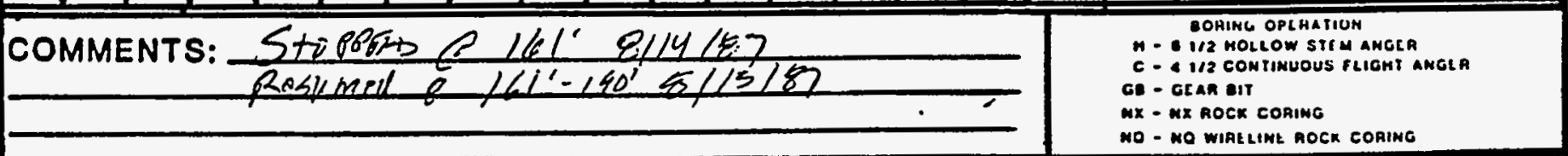




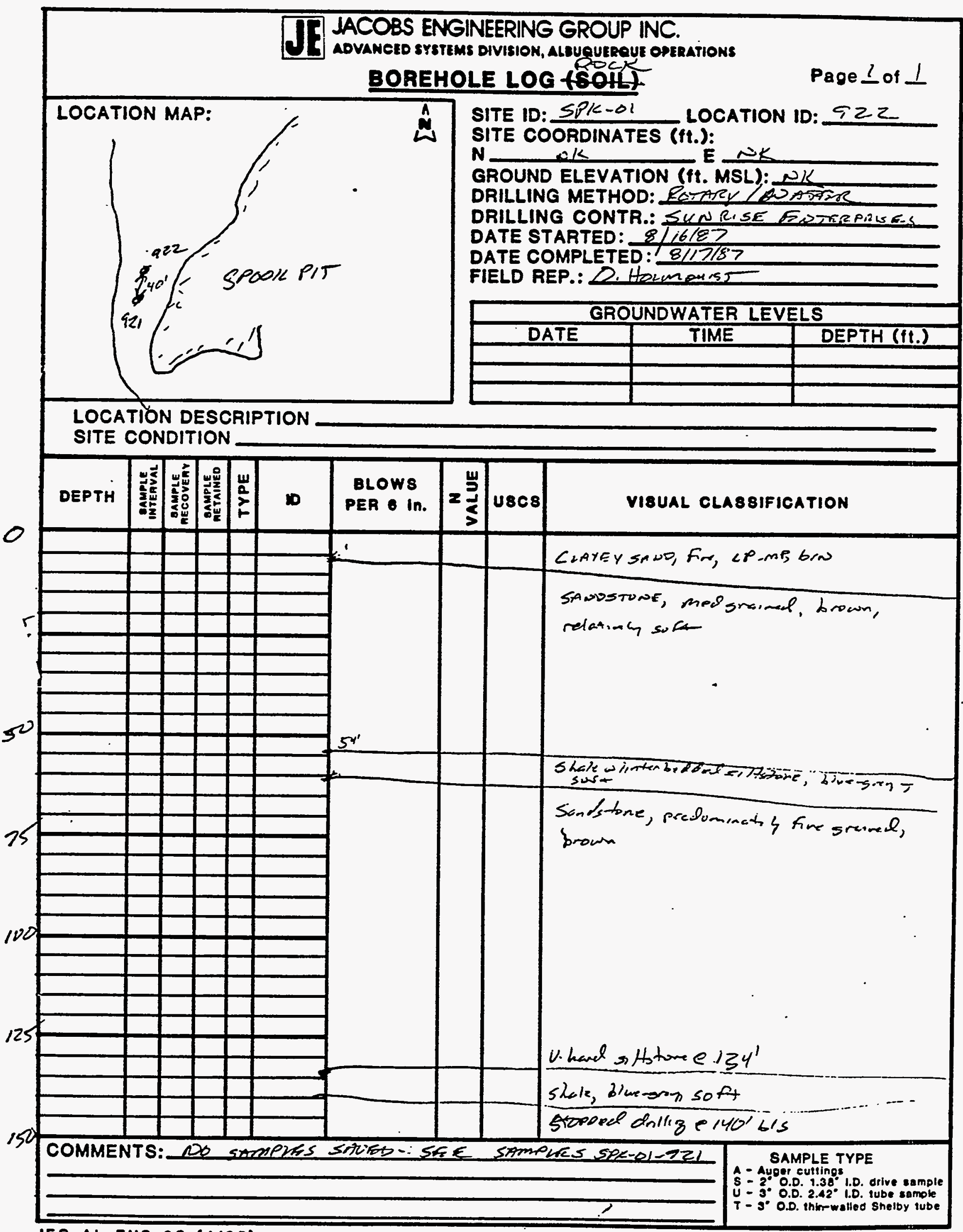




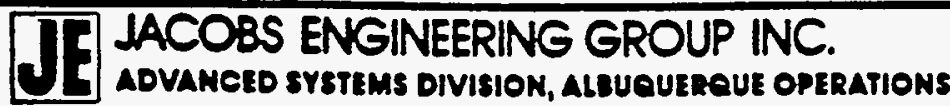

BOREHOLE LOG (ROCK)

LOCATION MAP:

$$
\text { SAR } 81064
$$

SITE ID: SPK V L LOCATION ID: 923 APPROX. SITE COORDINATES ( $f$ t.):

N GROUND ELEVATION (ft. MSL):

DRILLING METHOD: Rotany Y in porar DRILLER: SUNRLSE ENTERPLSTS DATE STARTED: $\& / L E / 2 ?$ DATE COMPLETED;

FIELD REP.: S. themenest

\begin{tabular}{|c|c|c|}
\hline \multicolumn{3}{|c|}{ GROUNDWATER LEVELS } \\
\hline DATE & TIME & DEPTH ( $f$. $)$ \\
\hline & & \\
\hline & & \\
\hline & & \\
\hline
\end{tabular}

LOCATION DESCRIPTION SITE CONDITION

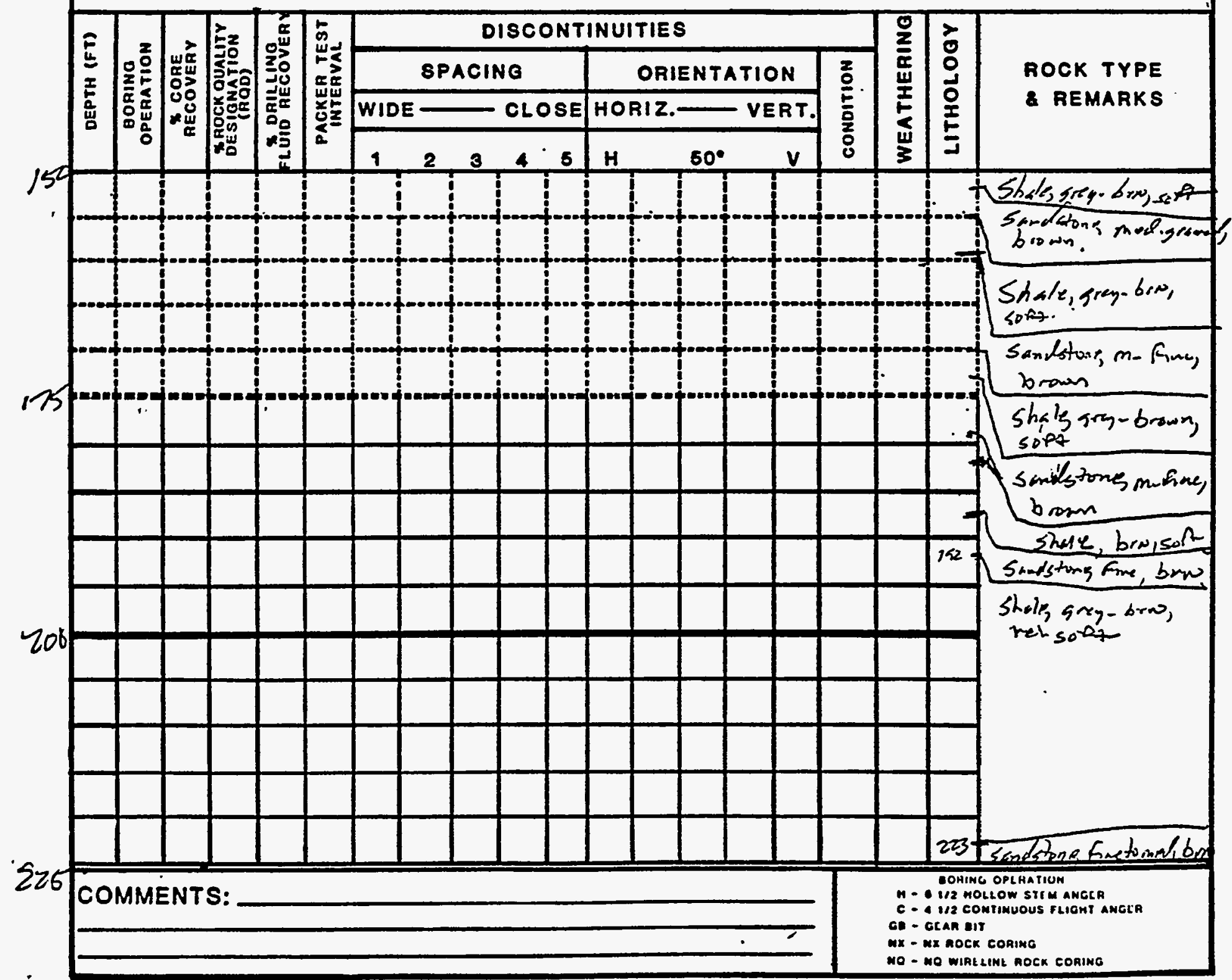


LOCATION MAP:

$\stackrel{\wedge}{\omega}$

SITE ID:SPK-O I

APP

$N$

GROUND ELEVATION (ft. MSL):

DRILLING METHOD: Rrtur wous

DRILLER: SunR,SE EarkeRRIS Es

DATE STARTED: \&IBLE

DATE COMPLETED

FIELD REP.: D.thlesereat

\begin{tabular}{|c|c|c|}
\hline \multicolumn{3}{|c|}{ GROUNOWATER LEVELS } \\
\hline DATE & TIME & DEPTH (ft.) \\
\hline & & \\
\hline & & \\
\hline & & \\
\hline
\end{tabular}

LOCATION DESCRIPTION

SITE CONDITION

\begin{tabular}{|c|c|c|c|c|c|c|c|c|c|c|c|c|c|}
\hline \multirow{4}{*}{ 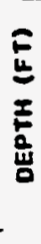 } & \multirow{4}{*}{ 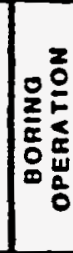 } & \multirow{4}{*}{ 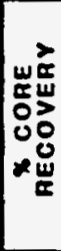 } & \multirow{4}{*}{ 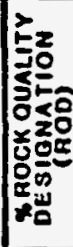 } & \multirow{4}{*}{ o } & \multirow{4}{*}{ 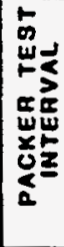 } & \multicolumn{6}{|c|}{ DISCONTINUITIES } & \multirow{4}{*}{ 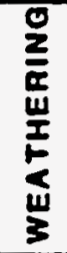 } & \multirow{4}{*}{$\begin{array}{l}2 \\
0 \\
0 \\
0 \\
0 \\
\underline{2} \\
\end{array}$} \\
\hline & & & & & & & & & ORIENTA & & $z$ & & \\
\hline & & & & & & WIDE - & 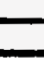 & CLOSE & HORIZ. & RT. & $\frac{1}{2}$ & & \\
\hline & & & & & & 1 & 3 & 4 & $50^{\circ}$ & $v$ & v & & \\
\hline
\end{tabular}

ROCK TYPE

- REMARKS i

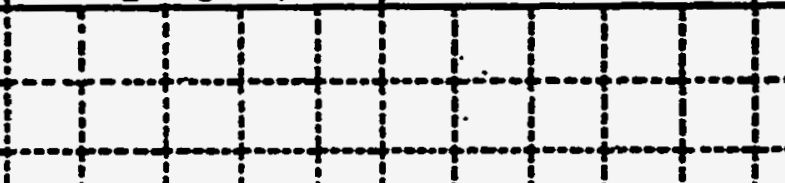
Nori:7re.conl in.enthings 1 mod pit Shale, sarely

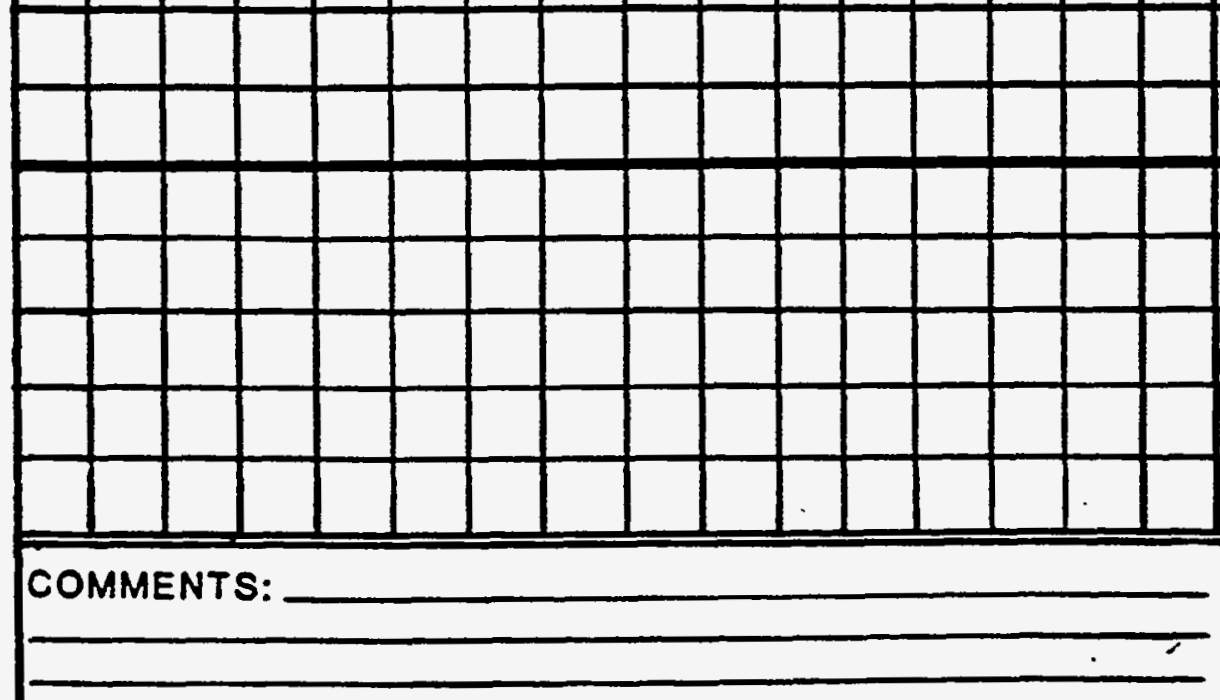


LOCATION MAP:

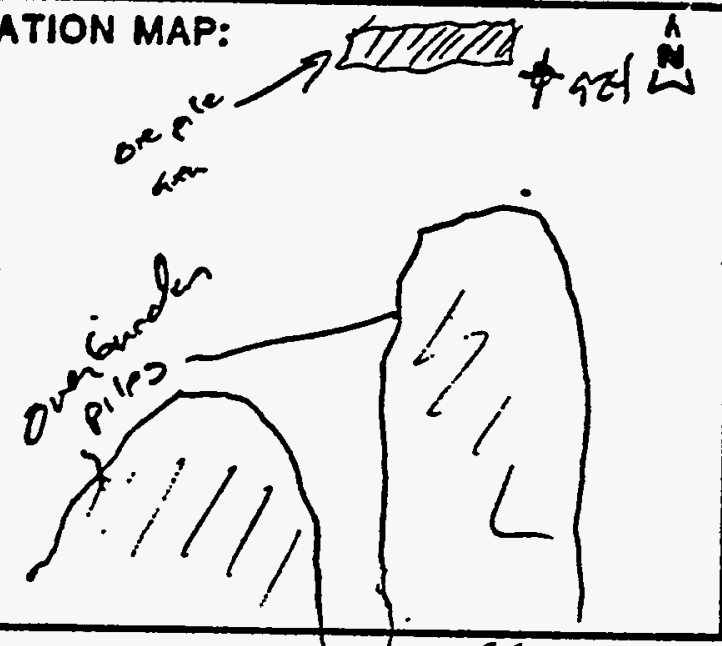

SITE ID: SPK-OI LOCATION ID: 524

SITE COORDINATES (ft.):

$N$ NK

GROUND ELEVATION (ft. MSL): 5080 (3thate)

DRILLING METHOD: RoRaY I WATKR

DRILLING CONTR.: SLONRISE EVRERPR.SE?

DATE STARTED: 82.9 \%

DATE COMPLETED: $2 / 38 / 28$

FIELD REP.: \& fio (manez)

LOCATION DESCRIPTION Sé cormen of ore pile aree

SITE CONDITION rlal, GEASSY

\begin{tabular}{|c|c|c|c|c|c|}
\hline PTH & 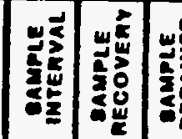 & 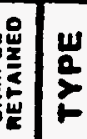 & $\boldsymbol{D}$ & $\begin{array}{l}\text { BLOW8 } \\
\text { PER } 6 \text { in. }\end{array}$ & \\
\hline
\end{tabular}

VIBUAL CLAB8IFICATION

clapesead, LP-mp, bod

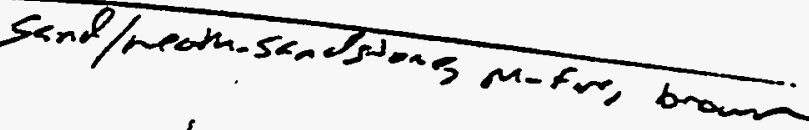

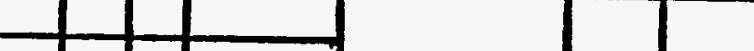

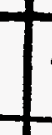




\section{IACOBS ENGINEERING GROUP INC.
ADVAHCED SYSTEMS DIVISION, ALQUQUERQUE OPERATIONS}

BOREHOLE LOG (ROCK)

LOCATION MAP:

$q_{25} \stackrel{A}{\omega}$

SITE ID: SPK-OI

$N$ QJK

GROUND ELEVATION (ft. MSL): KA

DRILLING METHOD: ReTARV ivaDERT

DRILLER: SUNRISE FANTÉ ERLSES

DATE STARTED: $\& / z y / \varepsilon 7$

DATE COMPLETED:

FIELD REP.: O. Heimenis

\begin{tabular}{|c|c|c|}
\hline \multicolumn{3}{|c|}{ GROUNDWATER LEVELS } \\
\hline DATE & TIME & DEPTH (ft.) \\
\hline & & \\
\hline & & \\
\hline & & \\
\hline
\end{tabular}

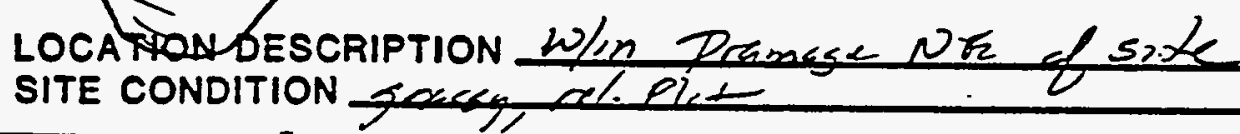

\begin{tabular}{|c|c|c|c|c|c|c|c|c|c|c|c|c|c|}
\hline & & & & & $0_{0}^{2}$ & 点 & & DISCONI & INUITIES & & & & \\
\hline$\Sigma$ & 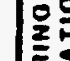 & & 然 & 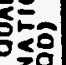 & 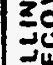 & 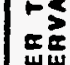 & & SPACING & ORIENTA & & & & נ? \\
\hline 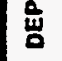 & 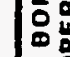 & 年 & 总 & 促 & $\bar{\sigma}_{\varepsilon}$ & 蕞 & & IDE $\longrightarrow$ CLOSE & HORIZ. & ERT. & & & 足 \\
\hline & & & & & & & & 2 & $80^{\circ}$ & $v$ & & & $\Xi$ \\
\hline
\end{tabular}

ROCK TYPE

R REMARKS

:

:

25

(1:

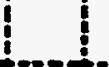

$+1$

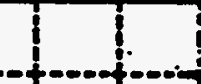

is, hysant, the clan

Lf, bre

sanbshiskm

50

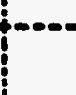

(1)

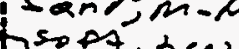

Sandy shale, solis

brown, occ.

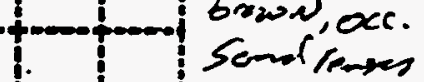

75

-

.

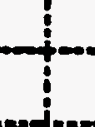

-...

+at

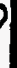

$+$

1

[

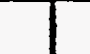

\begin{tabular}{|l|l|l|l|}
\hline & & & \\
\hline & & & \\
\hline & & & \\
\hline & & & \\
\hline
\end{tabular}

$+$

$+$

$+$

$+$

$+1$

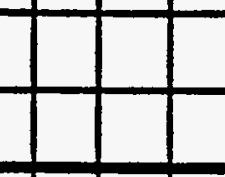

\begin{tabular}{ll|l|l|l|l|l|}
\hline & & & & & & \\
\hline & & & & \\
\hline
\end{tabular}

Sandstros, m- Race, sofis brom

Sandotera Ainct aned. mand hand, redibram

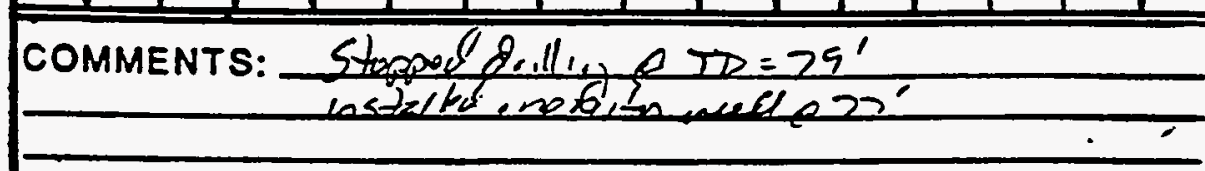




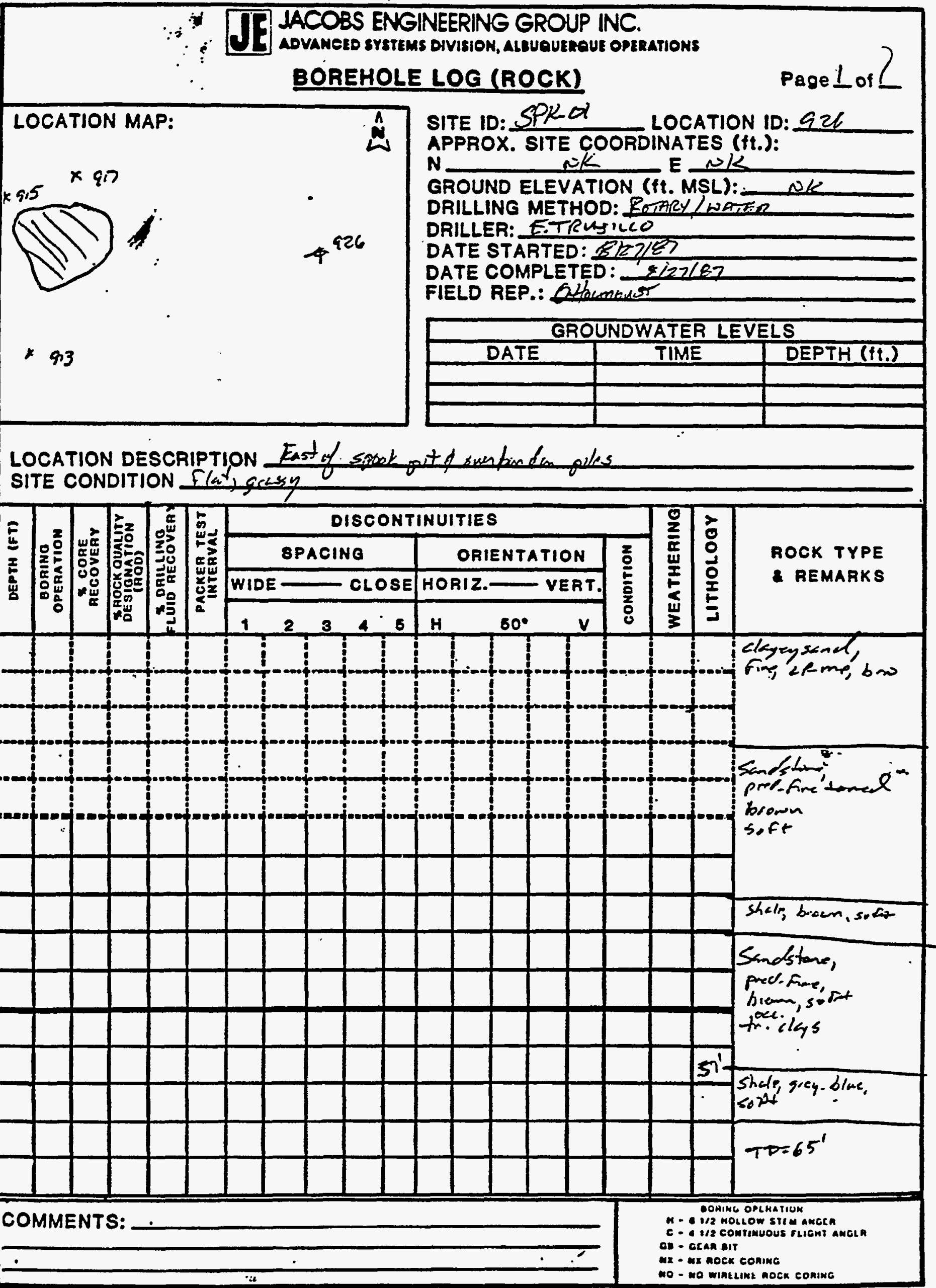




\section{LACOBS ENGINEERING GROUP INC.}

BOREHOLE LOG (SOIL)

Page $\leq$ of 2

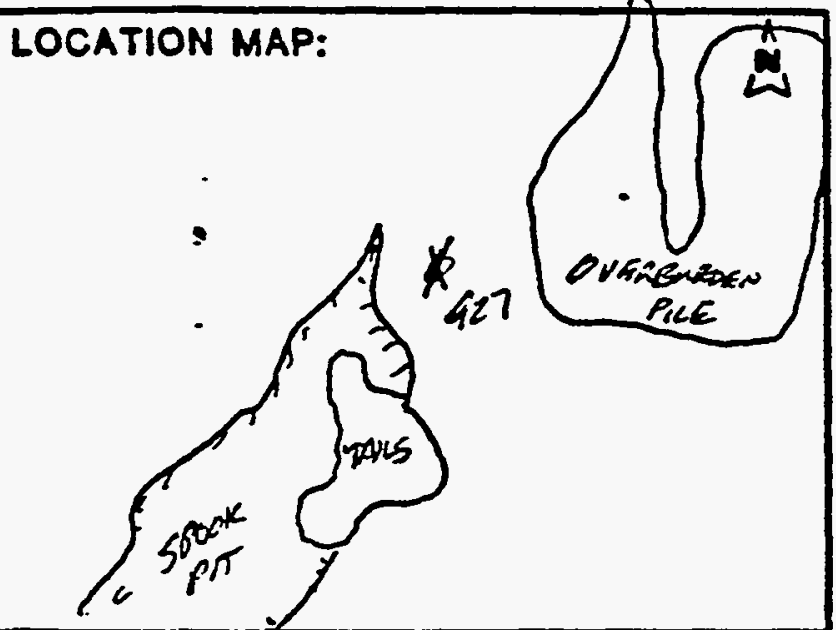

SITE ID: SPस-OI

LOCATION ID: 92

SITE COORDINATES (ft.):

N.

GROUND ELEVATION (ft. MSL):

DRILLING METHOD: Ponary / waman

DRILLING CONTR.: Janolse Enterpostss

DATE STARTED: B/2T/E2

DATE COMPLETED:

FIELD REP.: D Shemesist

\begin{tabular}{|c|c|c|}
\hline \multicolumn{3}{|c|}{ GROUNDWATER LEVELS } \\
\hline DATE & TIME & DEPTH (ft.) \\
\hline & & \\
\hline & & \\
\hline & & \\
\hline
\end{tabular}

LOCATION DESCRIPTION

SITE CONOITION 


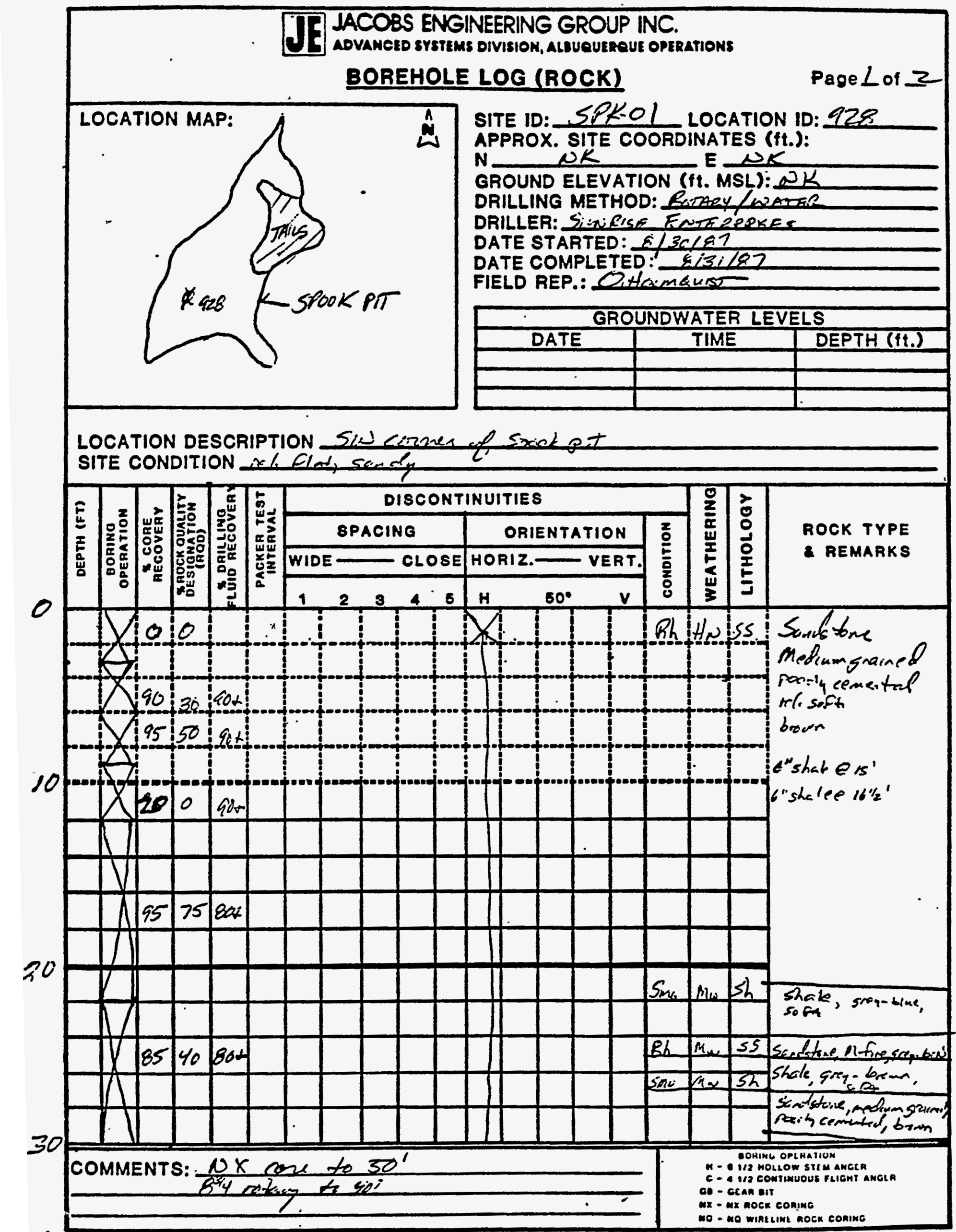




\section{JE LACOBS ENGINEERING GROUP INC.}

BOREHOLE LOG (ROCK)

Page zof 2

LOCATION MAP:

$\operatorname{sic}^{2}$ of 2 of
SITE ID: SAK-OI LOCATION ID: QZS APPROX. SITE COORDINATES ( $f$.):

$N$ NK $E \frac{\Omega L}{2 K}$

GROUND ELEVATION (ft. MSL): NK

DRILLING METHOD: $\ell_{L}=R Y$ /WA

DRILLER: Sinieice Fueperises

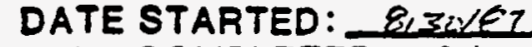

DATE COMPLETED: $8 / 3 / / 27$

FIELD REP.: Dtarmon'sT

LOCATION DESCRIPTION

SITE CONDITION

:0

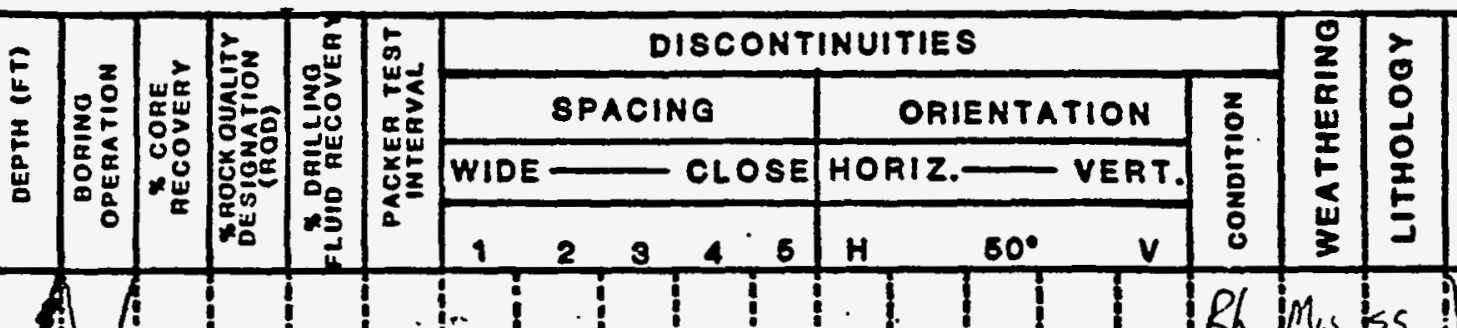

$23: 30: 892$

\begin{tabular}{|c|c|c|}
\hline \multicolumn{3}{|c|}{ GROUNDWATER LEVELS } \\
\hline DATE & TIME & DEPTH (ft.) \\
\hline & & \\
\hline & & \\
\hline & & \\
\hline
\end{tabular}

0

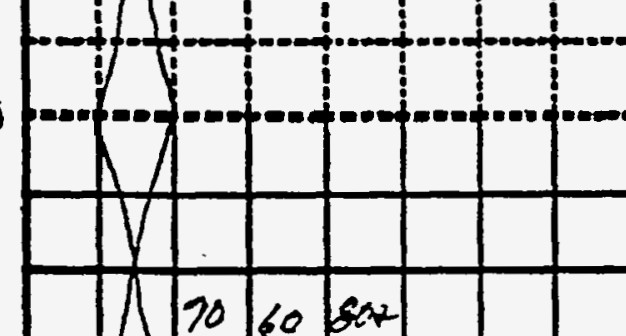

1

so sar

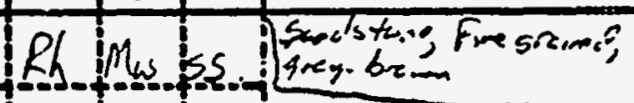
$-1-\infty+\infty+\infty$

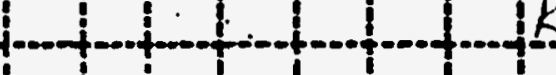

Sandstine, mefismind $-+\cdots+$

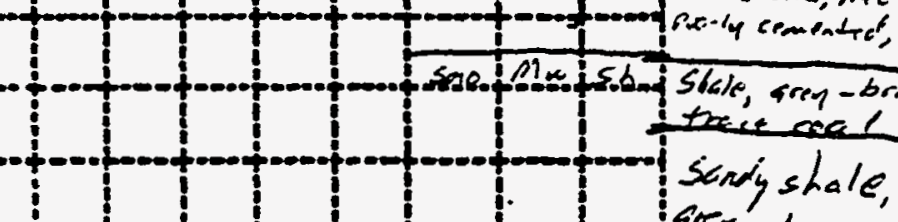
dondy shale, 1 1 $+1$

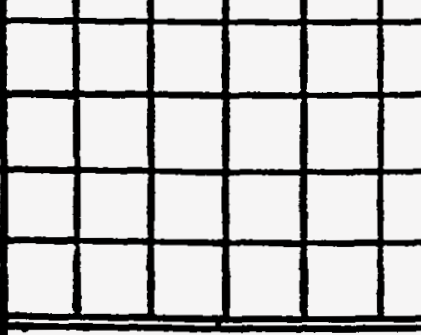
COMMENTS: 


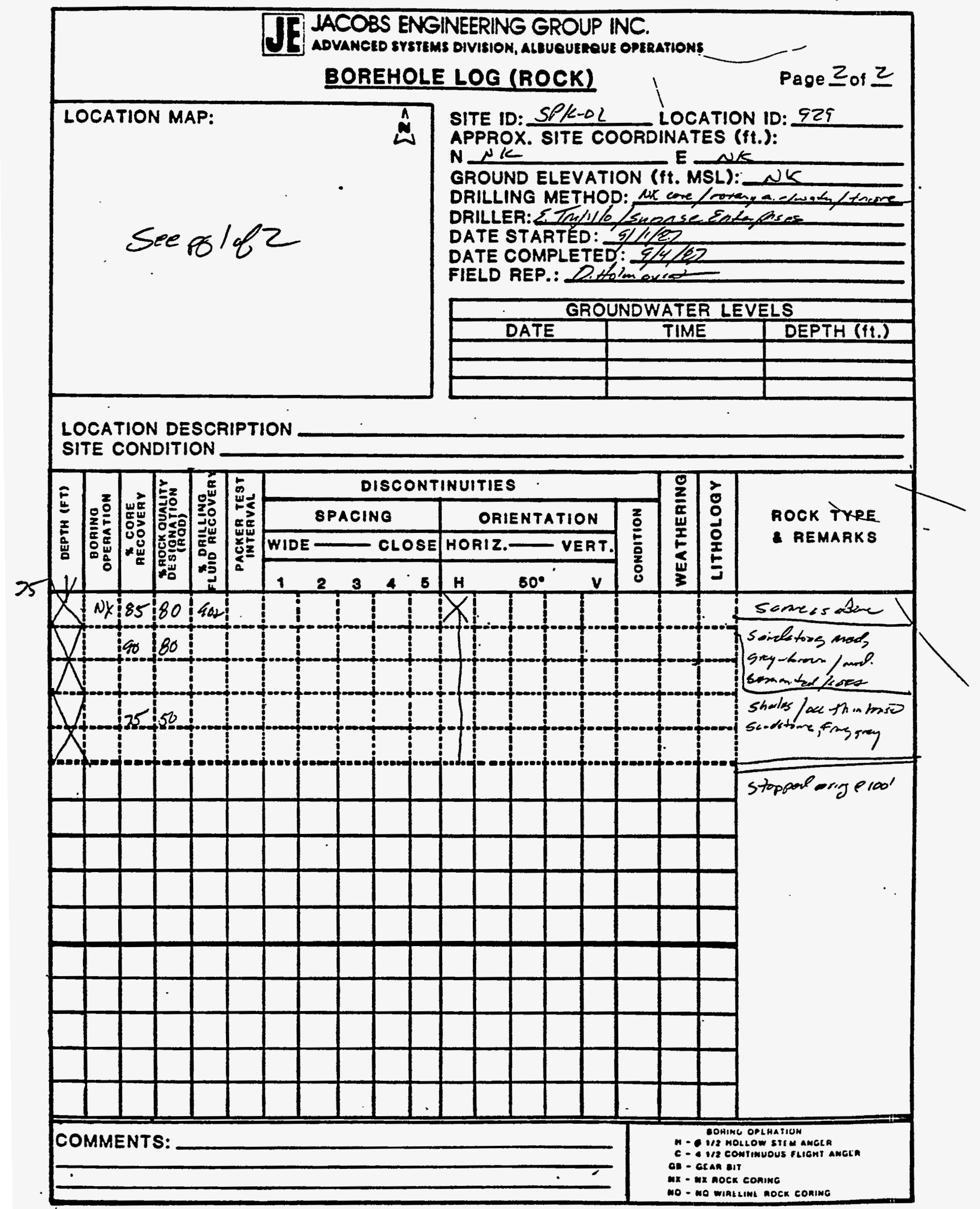




\section{LOCATION MAP:}

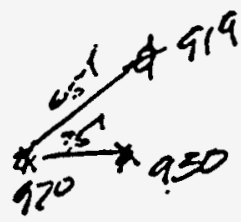

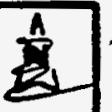

$b^{2}$
SITE ID: SPK - 01 SITE COORDINATES (It.)

N GROUND ELE ELVATION (ft. MSL): NK DRILLING METHOD: Tricone Retart fuesten DRILLING CONTR.: Smanse Enterours

DATE STARTED: $9 / 13 / 87$

DATE COMPLETED: 4/10/e7

FIELD REP.: D.thlnweres

\begin{tabular}{|c|c|c|}
\hline \multicolumn{3}{|c|}{ GROUNDWATER LEVELS } \\
\hline DATE & TIME & DEPTH (ft.) \\
\hline & & \\
\hline & & \\
\hline & & \\
\hline
\end{tabular}

LOCATION DESCRIPTION $35^{\circ} \mathrm{NE}$ of 920

SITE CONDITION Smuer 428

$+$

sitty sond, pred medpum to fince; siay-brown 


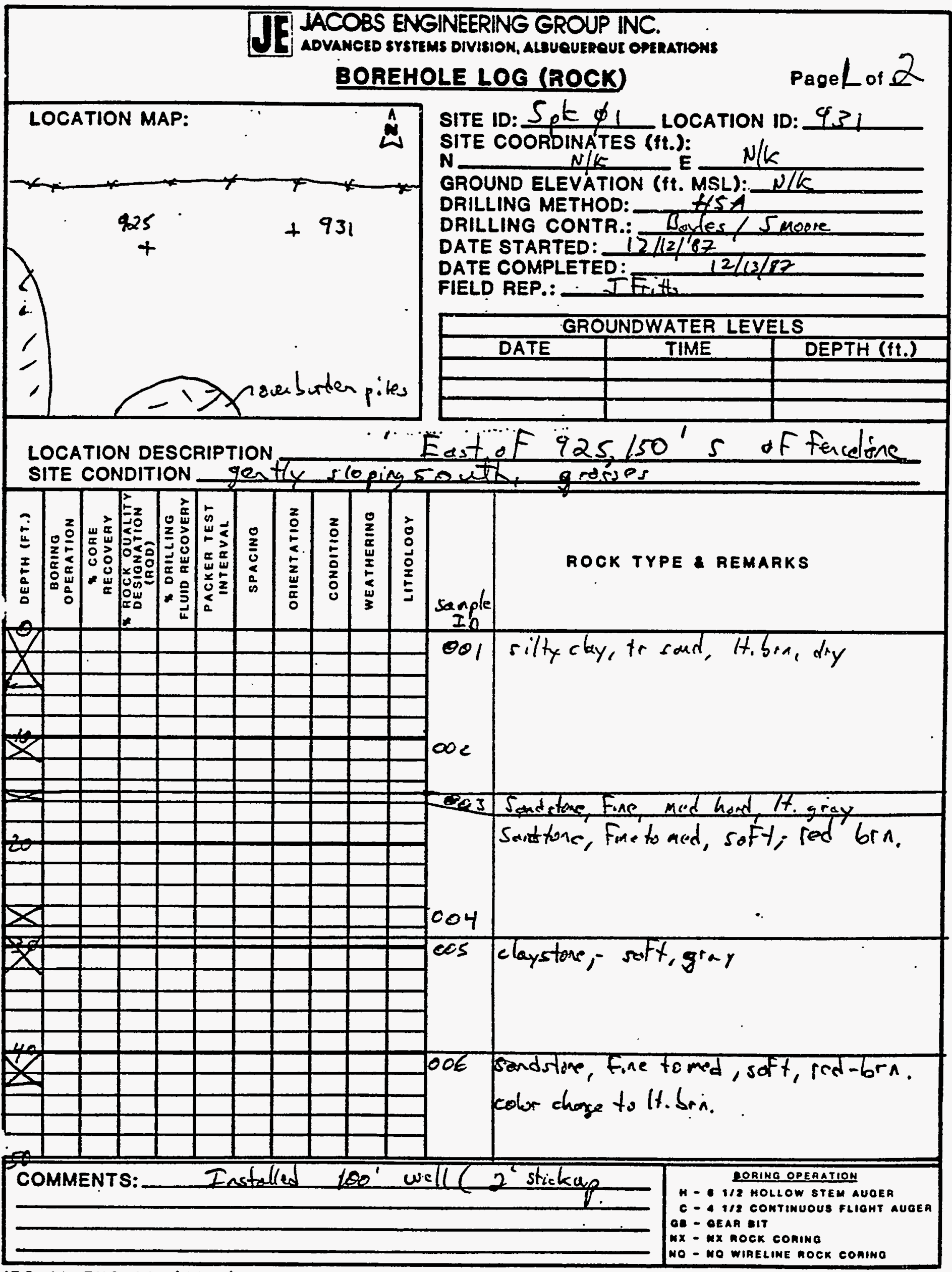




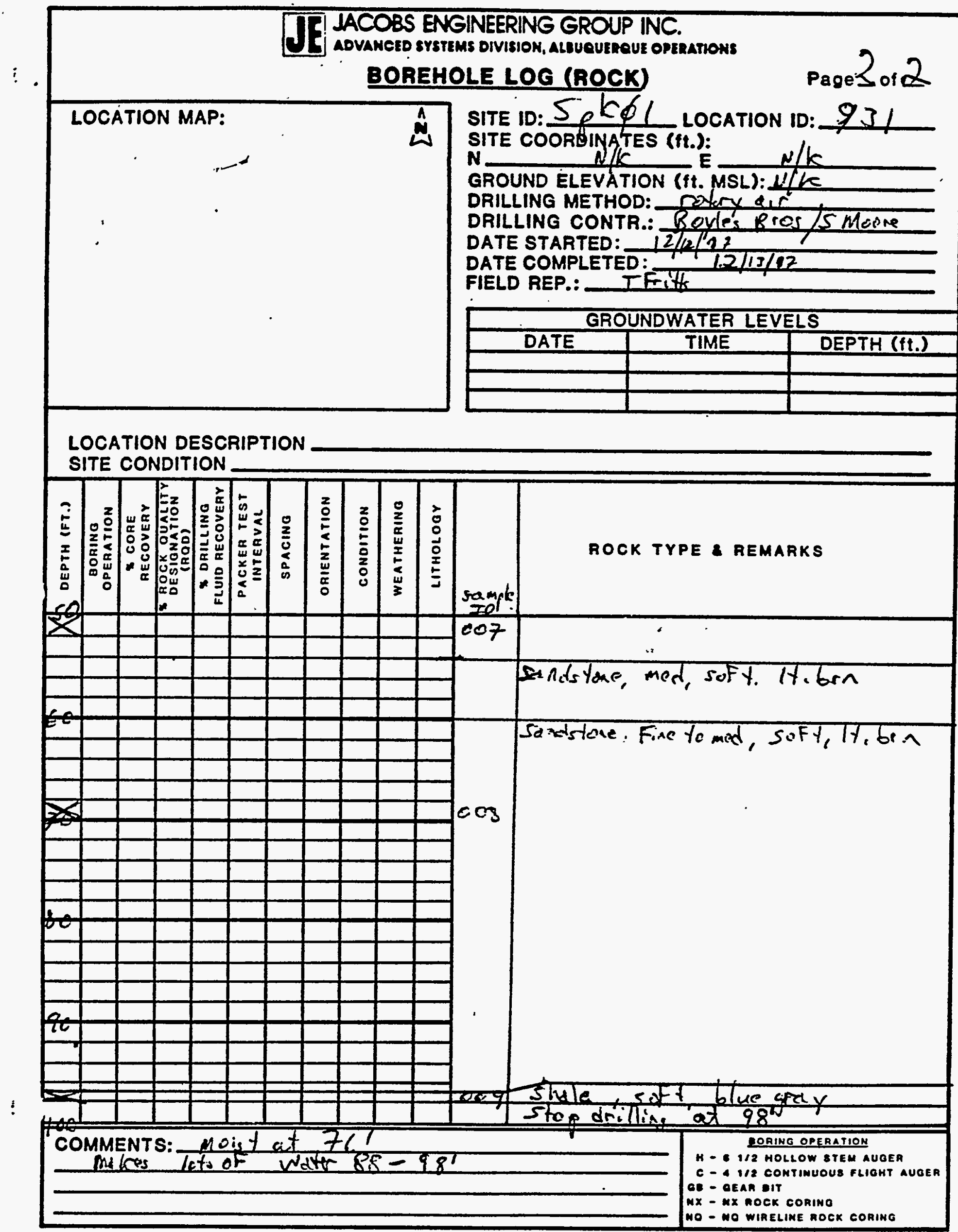




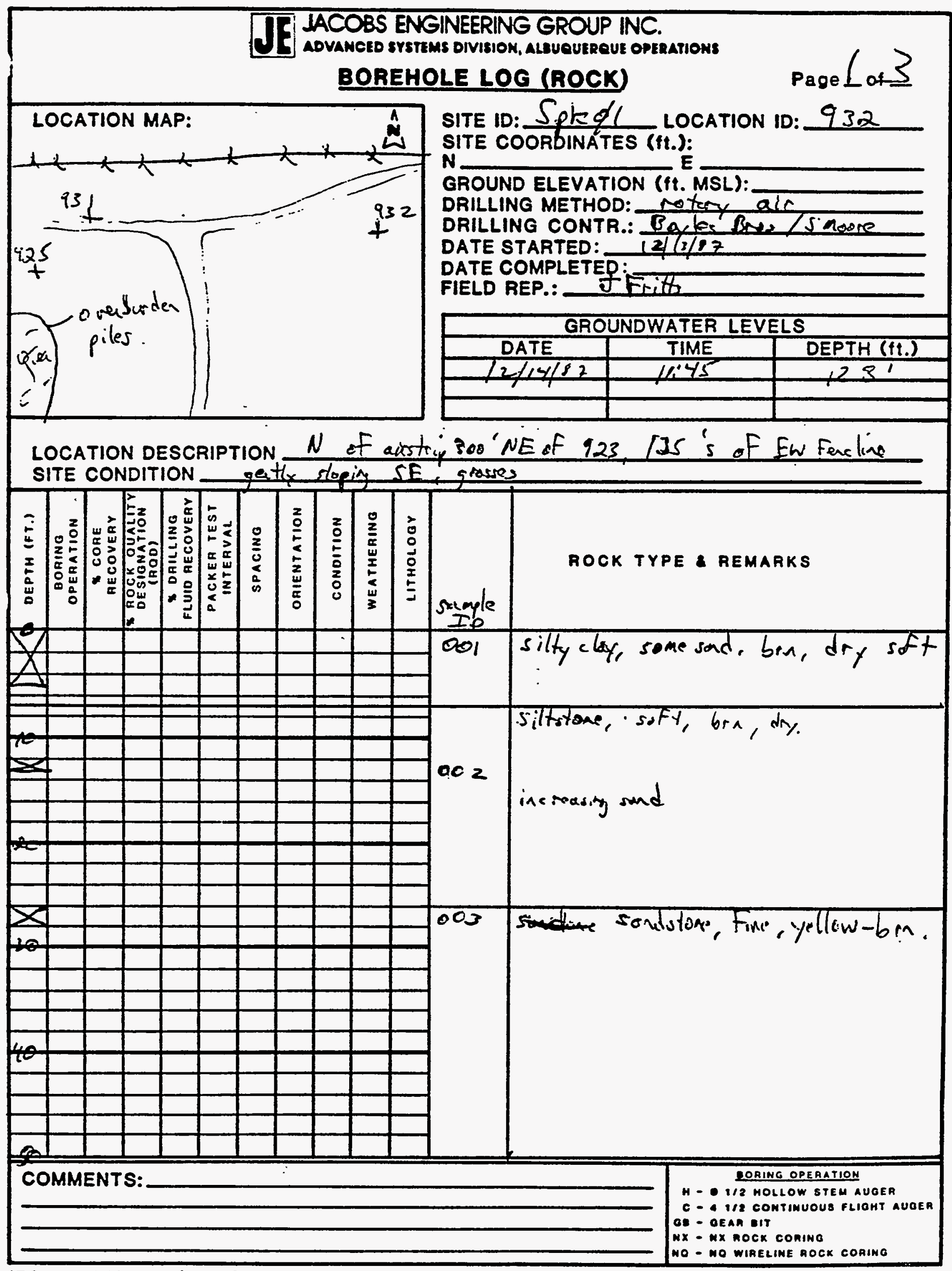




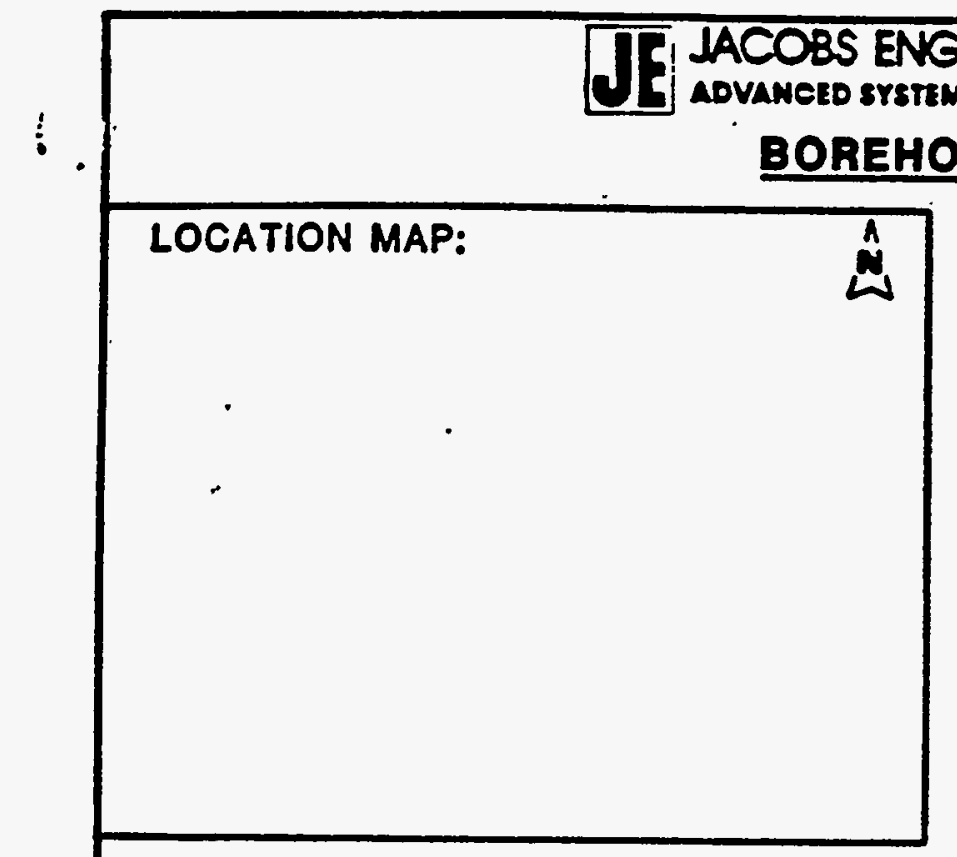

GINEERING GROUP INC.

AMCED SYSTEMS DIVISION, aLLUQUERQUE OPERATLOHS

BOREHOLE LOG (ROCK)

Page 2 of 3

LOCATION MAP:

SITE ID: $\frac{\text { Sple } \alpha}{2}$ LOCATION ID: $9 \times 2$

SITE COORDINATES (ft.):

$N$

GROUND ELEVATION ( $f t . M S L$ ):

DRILLING METHOD: retery dic

DRILLING CONTR:: PoLles / S Aadre

DATE STARTED: $\frac{1}{12 / 43 / 37}$

DATE COMPLETED:

FIELD REP.: T E. TIS

\begin{tabular}{|c|c|c|}
\hline \multicolumn{3}{|c|}{ GROUNDWATER LEVELS } \\
\hline DATE & TIME : & DEPTH (ft.) \\
\hline & $\ddots$ & \\
\hline & & \\
\hline & & \\
\hline
\end{tabular}

\section{LOCATION DESCRIPTION}

SITE CONDITION

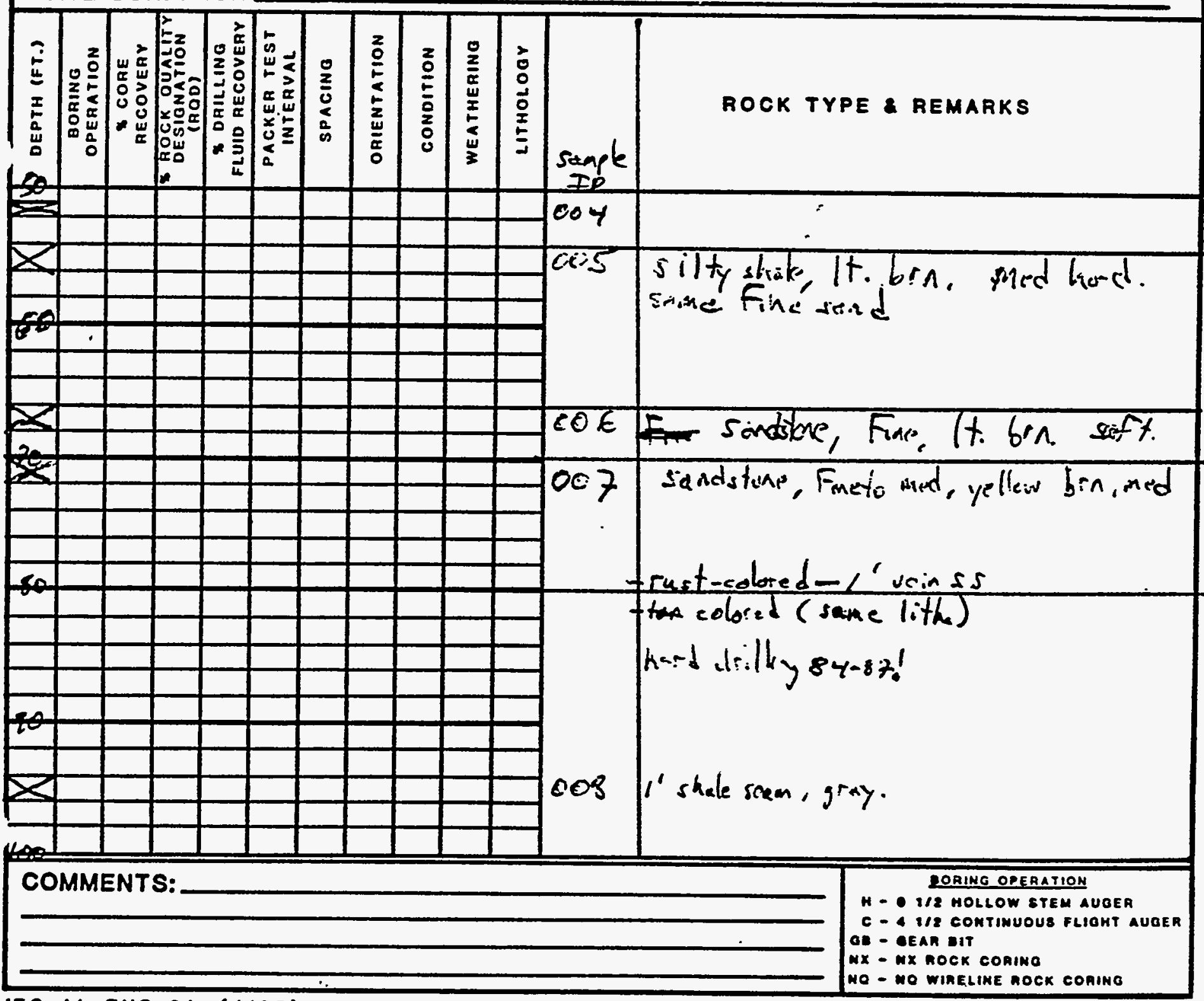


LOCATION MAP:
SITE ID: SpK \&!

SITE COORDINATES (ft.):

N.

GROUND ELEVATION (ft. MSL):

DRILLING METHOD:

DRILLING CONTR.:

DATE STARTED:

DATE COMPLETED:

FIELD REP.:

\begin{tabular}{|c|c|c|}
\hline \multicolumn{3}{|c|}{ GROUNDWATER LEVELS } \\
\hline DATE & TIME & DEPTH (ft.) \\
\hline & & \\
\hline & & \\
\hline & & \\
\hline
\end{tabular}

\section{LOCATION DESCRIPTION}

SITE CONDITION

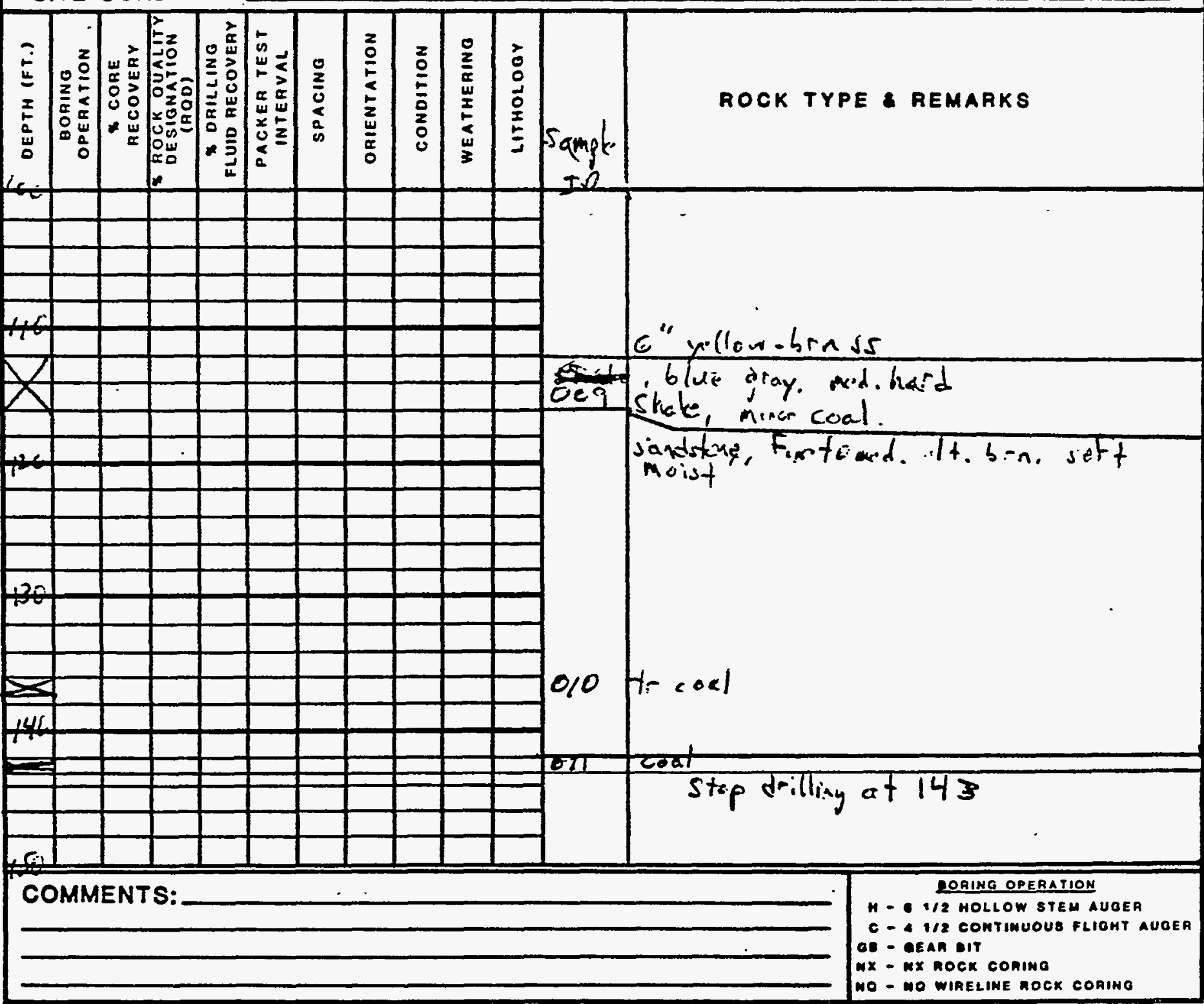




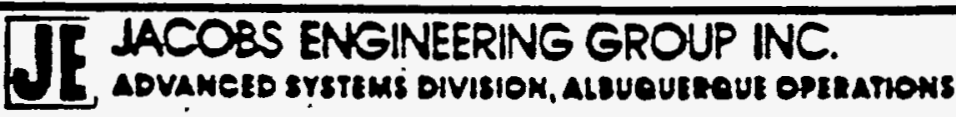

BOREHOLE LOG (SOIL)

Pagelof 5

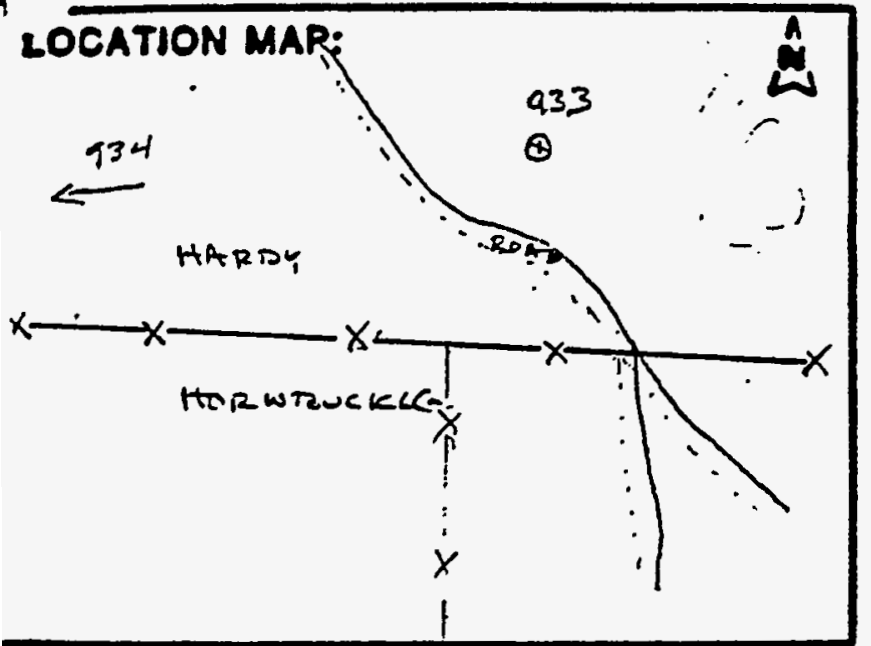

GITE ID: SPK- 15

LOCATION ID:

433

SITE COORDINATES (fI.):

N.

GROUND ELEVATION (ft. MSL):

DRILLING METHOD: ROTARY AIR

DRILLING CONTR.: BARNItART

DATE STARTED: $7-28-88$

DATE COMPLETED:

FIELD REP.: - O. METZLE R

\begin{tabular}{|c|c|c|}
\hline \multicolumn{3}{|c|}{ GROUNDWATER LEVELS } \\
\hline DATE & TIME & DEPTH (I1.) \\
\hline & & \\
\hline & & \\
\hline & & \\
\hline
\end{tabular}

LOCATION DESCRIPTION ON HAI2 4 LANP.75' wist if smull rize

SITE CONDITION moO. flet same

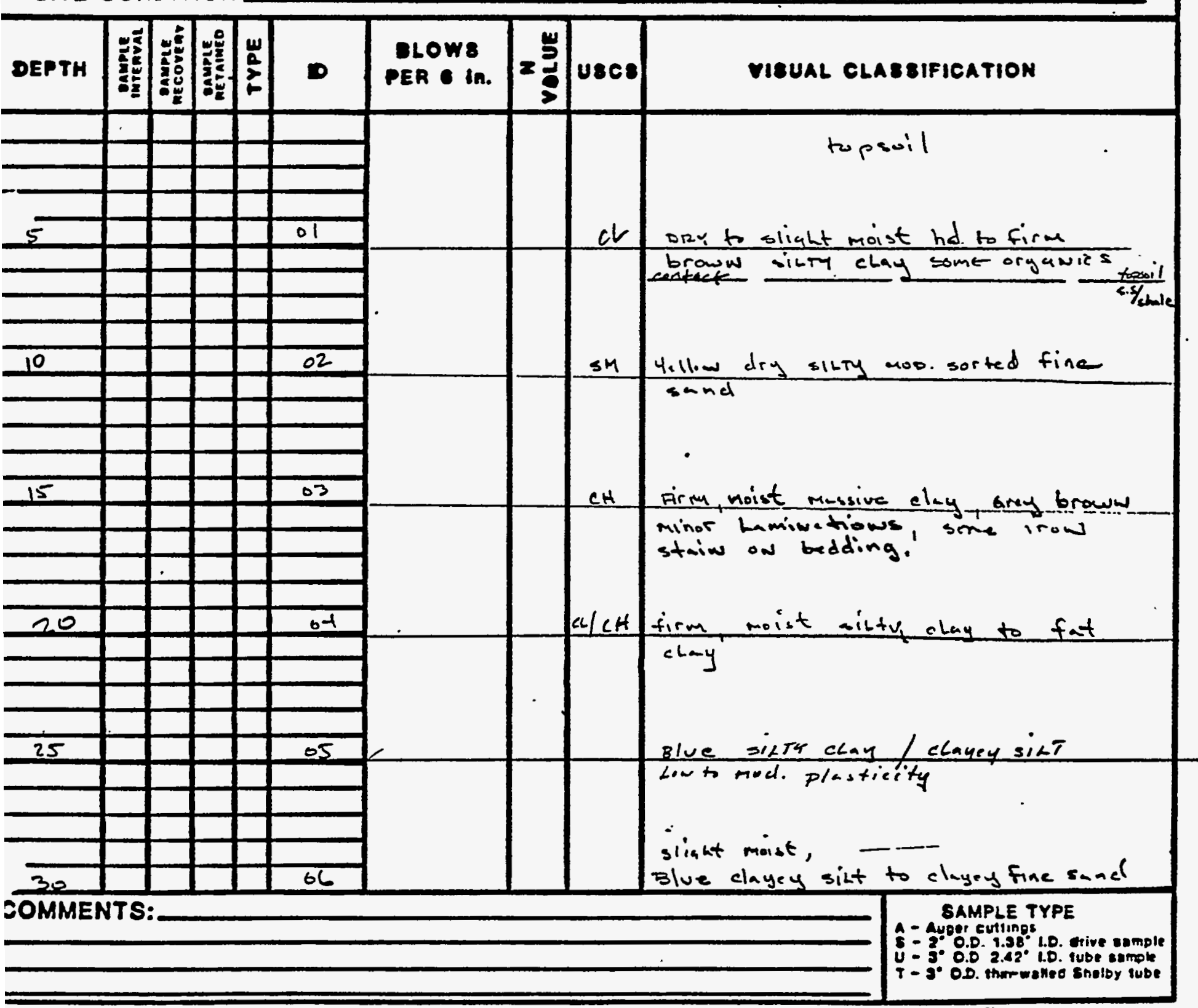




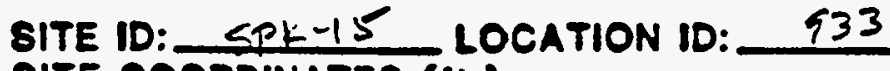

SITE COORDINATES (ft.):

N.

GROUND ELEVATION (It. MSL):

DRILLING METHOD:

DRILLING CONTR.:

DATE STARTED:

DATE COMPLETED:

FIELD REP.:

\begin{tabular}{|c|c|c|}
\hline \multicolumn{3}{|c|}{ GROUNDWATER LEVELS } \\
\hline DATE & TIME & DEPTH (ft.) \\
\hline & & \\
\hline & & \\
\hline & & \\
\hline
\end{tabular}

LOCATION DESCRIPTION

SITE CONDITION.

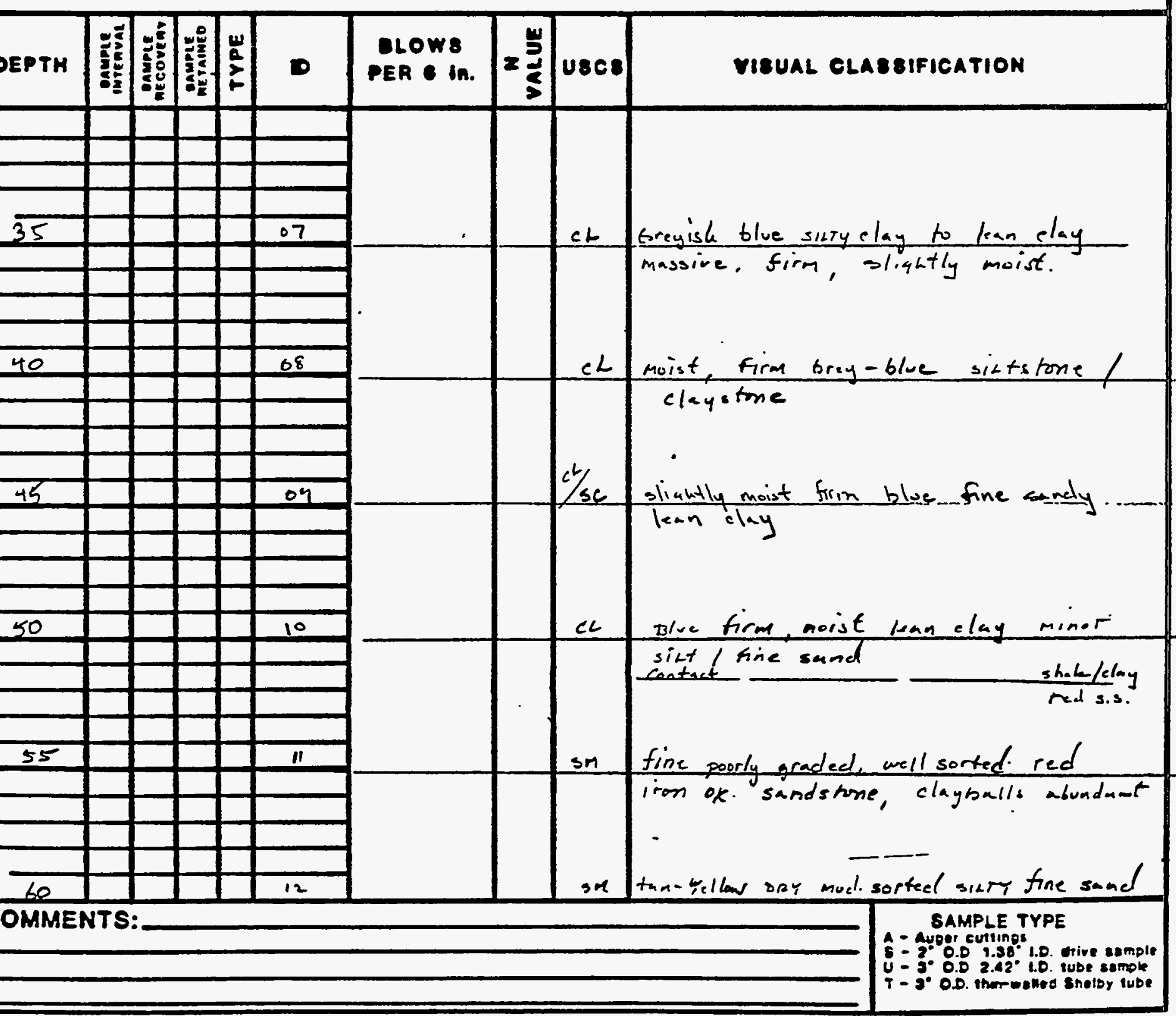



If LACOBS ENGINEERING GROUP INC.

ADVAMCED EYSTEMS DIVIBIOK, ALLUQULREUI OPLATIONS

BOREHOLE LOG (SOIL)

Paoe 4 or 15

LOCATION MAP:

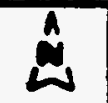

SITE ID: SPK-1S

SITE COORDINATES (II.):

N.

GROUND ELEVATION (ft. MSL):

DRILLING METHOD:

DRILLING CONTR.:

DATE STARTED:

DATE COMPLETED:

FIELD REP.:

\begin{tabular}{|c|c|c|}
\hline \multicolumn{3}{|c|}{ GROUNDWATER LEVELS } \\
\hline DATE & TIME & DEPTH (ff.) \\
\hline & & \\
\hline & & \\
\hline & & \\
\hline
\end{tabular}

LOCATION DESCRIPTION

SITE CONDITION

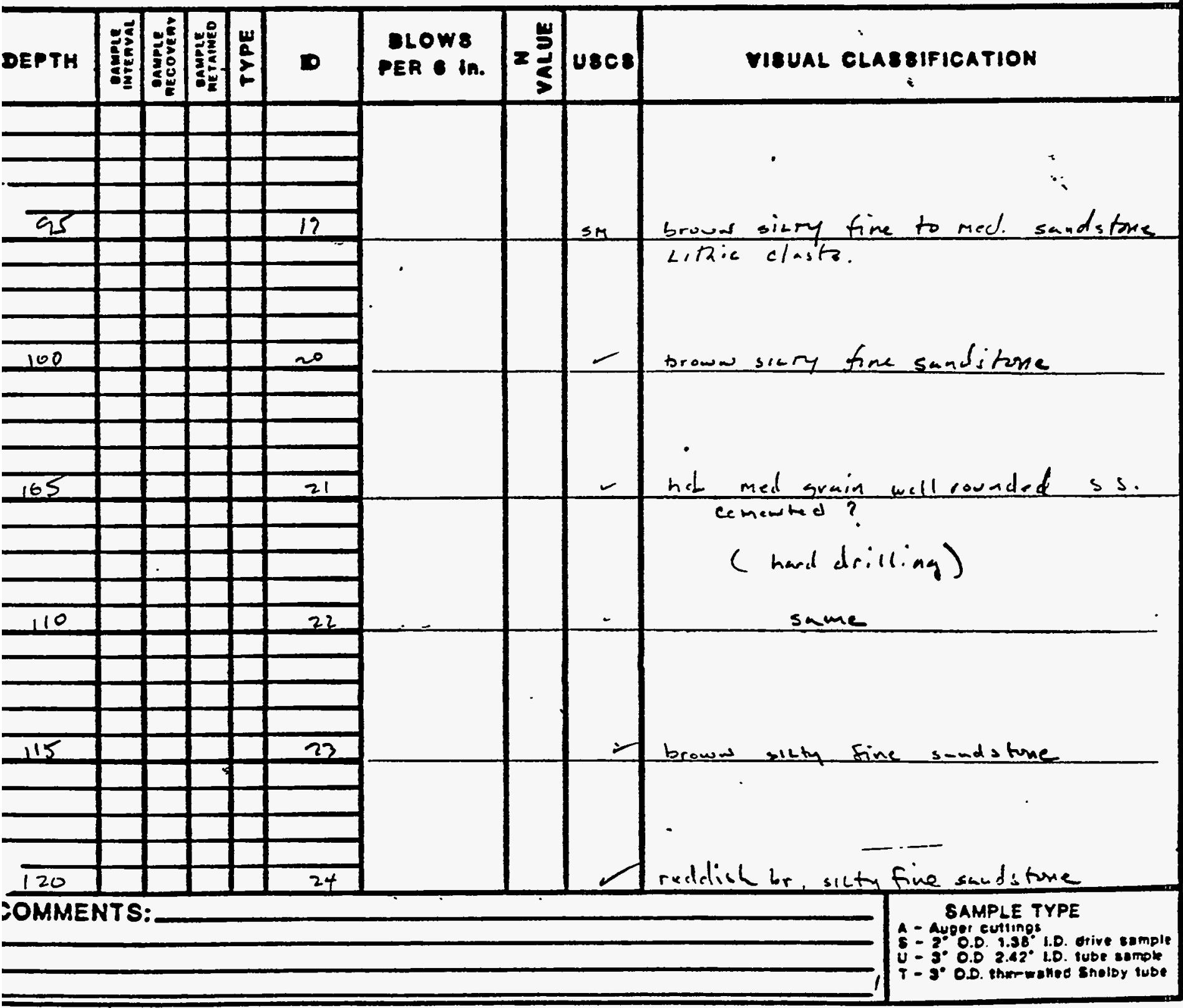


J1 IACOBS ENGINEERING GROUP INC.

ADVANCED EYBTEMS DIVIBION, ALBUQUERQUR OPREATONS

BOREHOLE LOG (SOIL)

Pave 3 of 5

LOCATION MAP:

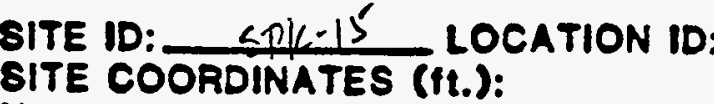

$\omega$

SITE COORDINATES (it.):

N.

GROUND ELEVATION (ft. MSL):

DRILLING METHOD:

DRILLING CONTR.:

DATE STARTED:

DATE COMPLETED:

FIELD REP.:

\begin{tabular}{|c|c|c|}
\hline \multicolumn{3}{|c|}{ GROUNDWATER LEVELS } \\
\hline DATE & TIME & DEPTH (ff.) \\
\hline & & \\
\hline & & \\
\hline & & \\
\hline
\end{tabular}

LOCATION DESCRIPTION

SITE CONDITION

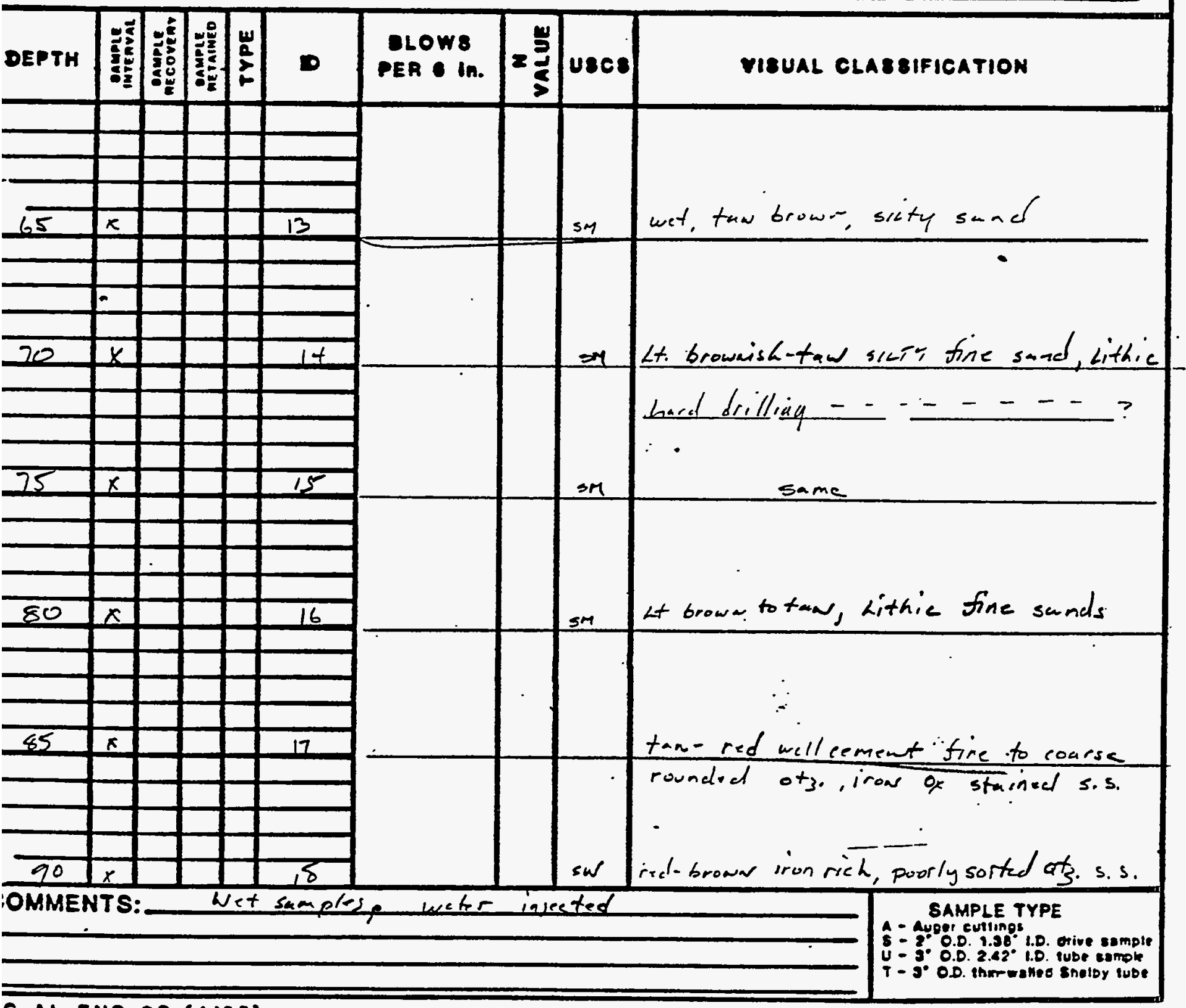


SITE ID: SPK-15

LOCATION ID: 934

SITE COORDINATES (ft.):

N.

GROUND ELEVATION (11. MSL):

DRILLING METHOD:

DRILLING CONTR.:

DATE STARTED:

DATE COMPLETED:

FIELD REP.:

\begin{tabular}{|c|c|c|}
\hline \multicolumn{3}{|c|}{ GROUNDWATER LEVELS } \\
\hline DATE & TIME & DEPTH (ft.) \\
\hline & & \\
\hline & & \\
\hline & & \\
\hline
\end{tabular}

LOCATION DESCRIPTION

SITE CONDITION

\begin{tabular}{|c|c|c|c|c|c|c|c|c|}
\hline DEPTH & 告 & 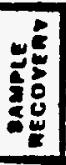 & \begin{tabular}{|l|l}
0 \\
0
\end{tabular} & D & $\begin{array}{l}\text { OLOW8 } \\
\text { PER } P \text { in. }\end{array}$ & 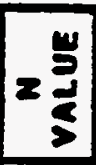 & usces & VISUAL CLABSIFICATION \\
\hline & & & & & \multirow[t]{3}{*}{ - } & & & \multirow{3}{*}{$\begin{array}{l}\text { no } \operatorname{san} x<6 \\
\text { sume }\end{array}$} \\
\hline & & & & & & & & \\
\hline 95 & & & & & & & & \\
\hline & & & & & & & \multirow[b]{3}{*}{ sin } & \multirow[b]{3}{*}{ veir eenrent. redelish br med. srain ats sis } \\
\hline & & & & & & & & \\
\hline 100 & $x$ & & & 19 & & & & \\
\hline \multirow{2}{*}{ 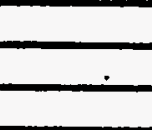 } & & & & & & & \multirow[b]{3}{*}{ sin. } & \multirow{3}{*}{ wet grey trown viodirately sorted } \\
\hline & & & & & & & & \\
\hline \multirow[t]{2}{*}{105} & $\bar{x}$ & & & 20 & & & & \\
\hline & & & & & & & & \multirow{3}{*}{$\begin{array}{c}\text { Lithle sandstone "waele } / \\
\text { sume. mucleret ly sonted ot }\end{array}$} \\
\hline$\because$ & & & & & & & \multirow{2}{*}{$\sin$} & \\
\hline \multirow{2}{*}{110} & $x$ & & & 21 & & & & \\
\hline & & & & & & & & $\longrightarrow$ \\
\hline & & & & & & & & . \\
\hline 115 & $x$ & & & 22 & & & sm & same \\
\hline 117 & $x$ & & & 23 & & & -6 & Dy. ahorolet br. 10uly saindy shake \\
\hline & & & & & & & & - - note samplis / \\
\hline 120 & $x$ & & & 24 & & & $\Delta$ & Blue- oray eclaystone coming take : \\
\hline & & & & & & & & 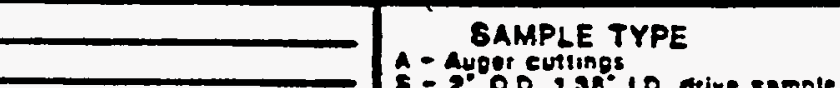 \\
\hline
\end{tabular}


LACOBS ENGINEERING GROUP INC.

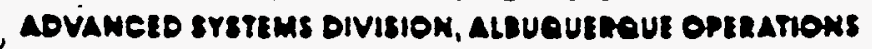

BOREHOLE LOG (SOIL)

Page 5 of 5

LOCATION MAP:

$\because \quad \stackrel{\omega}{\omega}$

SITE ID: SOK-12

LOCATION ID:

934

SITE COORDINATES (ft.):

N $E$

GROUND ELEVATION (ft. MSL):

DRILLING METHOD:

DRILLING CONTR.:

DATE STARTED:

DATE COMPLETED:

FIELD REP.:

\begin{tabular}{|c|c|c|}
\hline \multicolumn{3}{|c|}{ GROUNDWATER LEVELS } \\
\hline DATE & TIME & DEPTH ( 1.$)$ \\
\hline & & \\
\hline & & \\
\hline & & \\
\hline
\end{tabular}

LOCATION DESCRIPTION

SITE CONDITION

\begin{tabular}{|c|c|c|c|c|c|c|c|c|c|}
\hline DEPTH & 罗 & 资 & 资 & $\frac{\omega}{2}$ & D & $\begin{array}{l}\text { DLOW8 } \\
\text { PER In. }\end{array}$ & $=\frac{m}{3}$ & usces & VIBUAL CLABEIFICATION \\
\hline
\end{tabular}

\section{$+2$}

\begin{tabular}{|c|c|c|c|c|}
\hline & & & & \\
\hline 125 & $x$ & & & 25 \\
\hline & & & & \\
\hline & & & & \\
\hline 130 & $c$ & & & 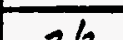 \\
\hline & & & & \\
\hline & & & & \\
\hline & & & & \\
\hline & & & & \\
\hline & & & & \\
\hline & & & & \\
\hline & & . & & \\
\hline & 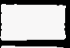 & & & \\
\hline & 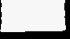 & & & \\
\hline & - & & & \\
\hline & 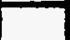 & & & \\
\hline & - & & & \\
\hline & & & & \\
\hline & & & & \\
\hline & & & & \\
\hline & - & & & \\
\hline & & & & \\
\hline
\end{tabular}

e4 elf Rlue ary firm, moist masive now Inminatud claystone sore ahole?

$$
\begin{aligned}
& \text { T.D. } 130^{\prime} \\
& 9^{10} \mathrm{~nm}
\end{aligned}
$$


IACOBS ENGINEERING GROUP NNC.

BOREHOLE LOQ (SOIL)

SITE ID:

LOCATION ID: 935

SITE COORDINATES (ft.):

$N$ E

GROUND ELEVATION (ft. MSL):

DRILLING METHOD:

DRILLING CONTR.:

DATE STARTED:

DATE COMPLETED:

FIELD REP.:

\begin{tabular}{|c|c|c|}
\hline \multicolumn{3}{|c|}{ GROUNDWATER LEVELS } \\
\hline DATE & TIME & DEPTH (fI.) \\
\hline & & \\
\hline & & \\
\hline & & \\
\hline
\end{tabular}

LOCATION DESCRIPTION

SITE CONDITION SPARSE GRASS AND SAGERRUSH

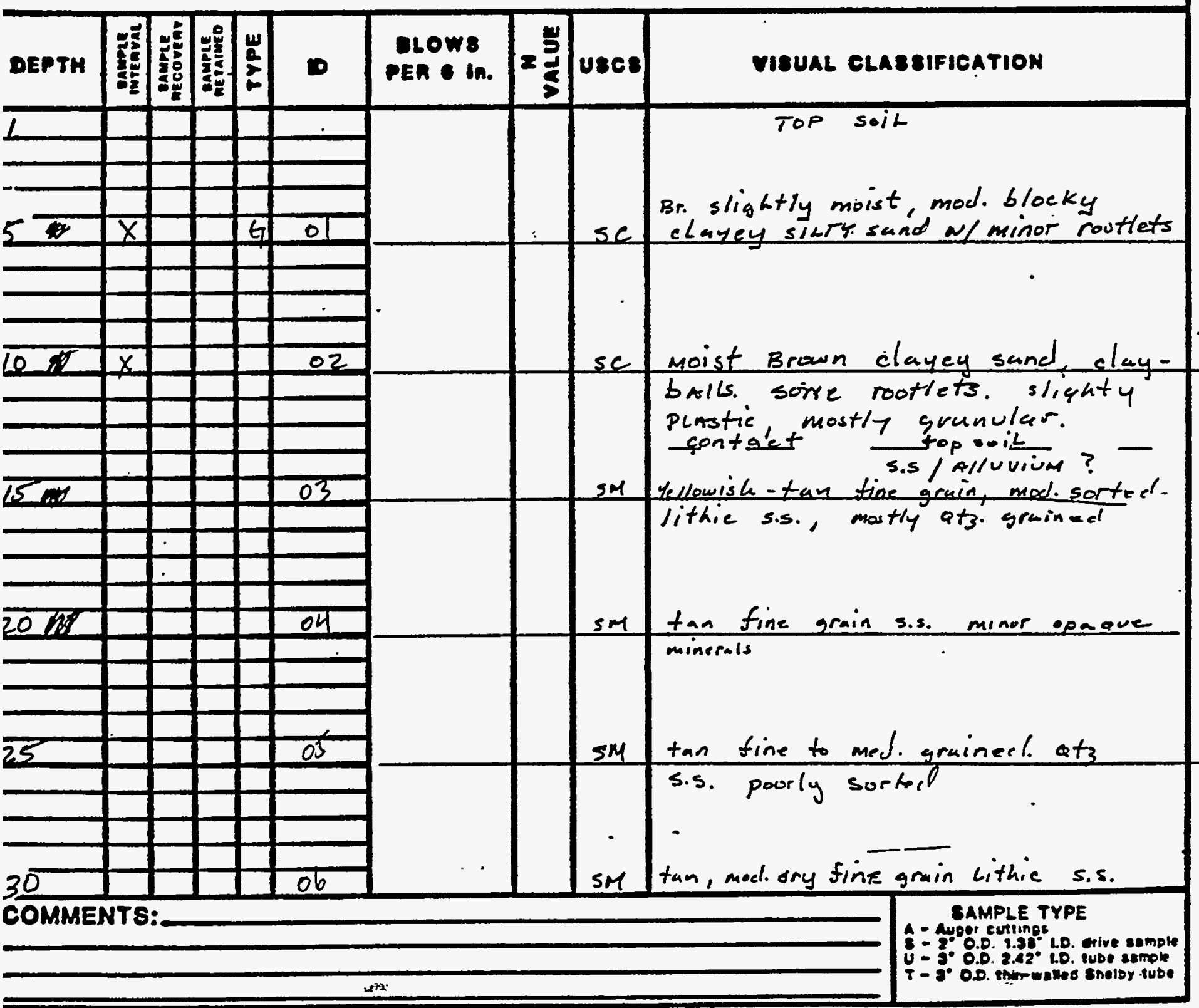


IIT IACOBS ENGINEERING GROUP NC.

aDVAneto srstims DIVIsion, alsueutmaut omtranons

BOREHOLE LOG (SOIL)

LOCATION MAP:

ڤิ

a

SITE ID:

SITE COORDINATES (ft.):

$N$

GROUND ELEVATION (ft. MSL):

DRILLING METHOD:

DRILLING CONTR.:

DATE STARTED:

DATE COMPLETED:

FIELD REP.:

\begin{tabular}{|c|c|c|}
\hline \multicolumn{3}{|c|}{ GROUNDWATER LEVELS } \\
\hline DATE & TIME & DEPTH (11.) \\
\hline & & \\
\hline & & \\
\hline & & \\
\hline
\end{tabular}

LOCATION DESCRIPTION

SITE CONDITION

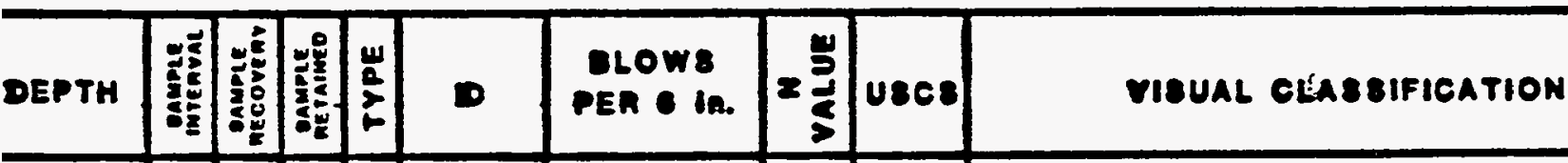

\begin{tabular}{l|l|l|l|l|l|l|}
\hline \\
\hline
\end{tabular}

-

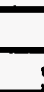

$$
5
$$

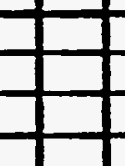

12

$+19$

1

$\longrightarrow$

$$
\underline{s}
$$

$+\frac{1}{x}$

$+09$

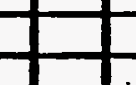

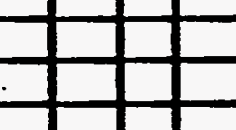

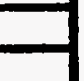

10

\begin{tabular}{|l|l|}
\hline & 10 \\
\hline & \\
\hline
\end{tabular}

\begin{tabular}{llllll} 
& & & & & \\
\hline & & & & & \\
& & & &
\end{tabular}


IACOBS ENGINEERING GROUP INC.

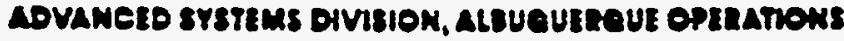

BOREHOLE LOG (SOIL)

Page 3 or 5

LOCATION MAP:

ฝ

SITE ID:

LOCATION ID: $\quad 935$

SITE COORDINATES (ft.):

$N$

GROUND ELEVATION (ft. MSL):

DRILLING METHOD:

DRILLING CONTR.:

DATE STARTED:

DATE COMPLETED:

FIELD REP.:

\begin{tabular}{|c|c|c|}
\hline \multicolumn{3}{|c|}{ GROUNDWATER LEVELS } \\
\hline DATE & TIME & DEPTH (fi.) \\
\hline & & \\
\hline & & \\
\hline & & \\
\hline
\end{tabular}

LOCATION DESCRIPTION

SITE CONDITION

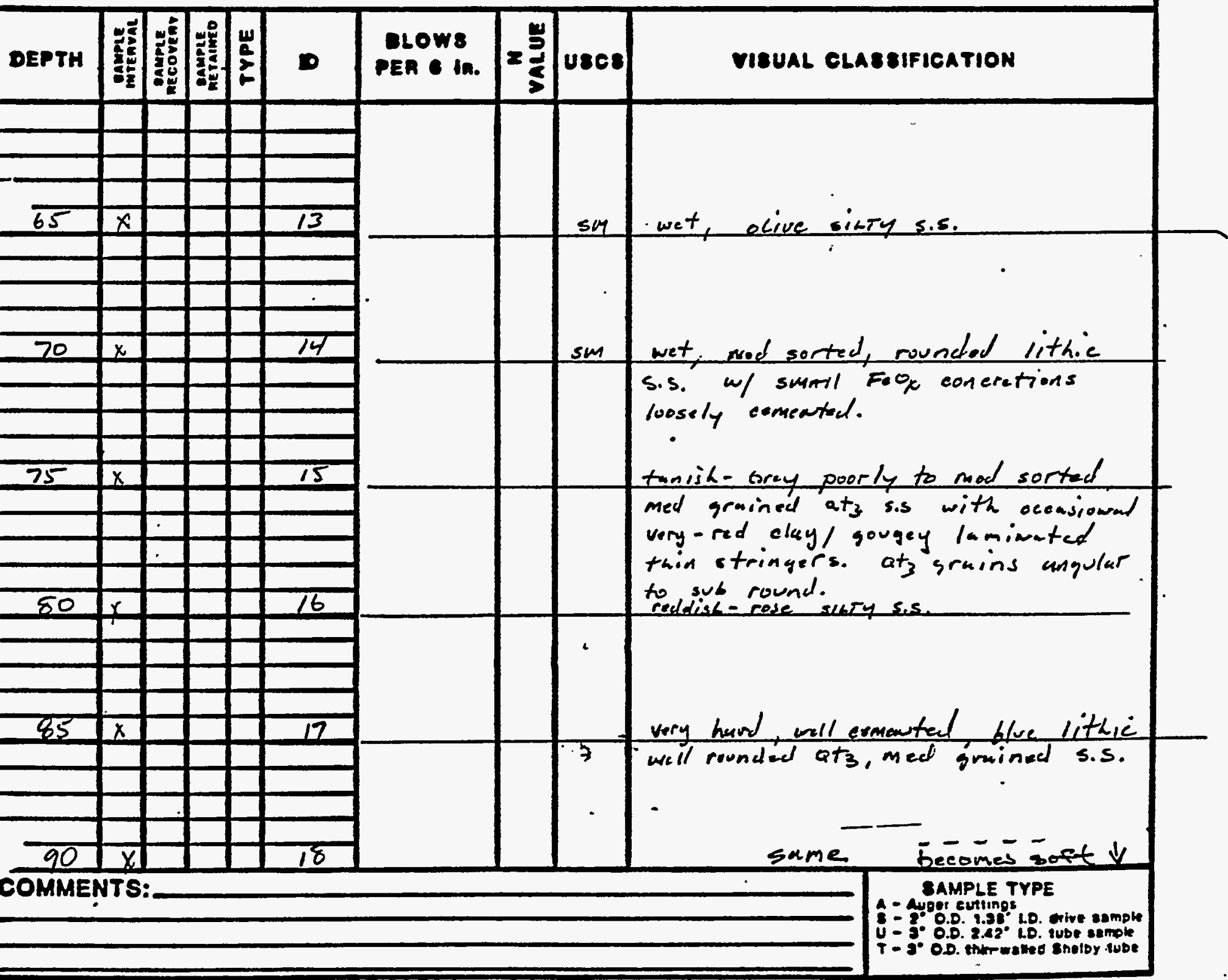




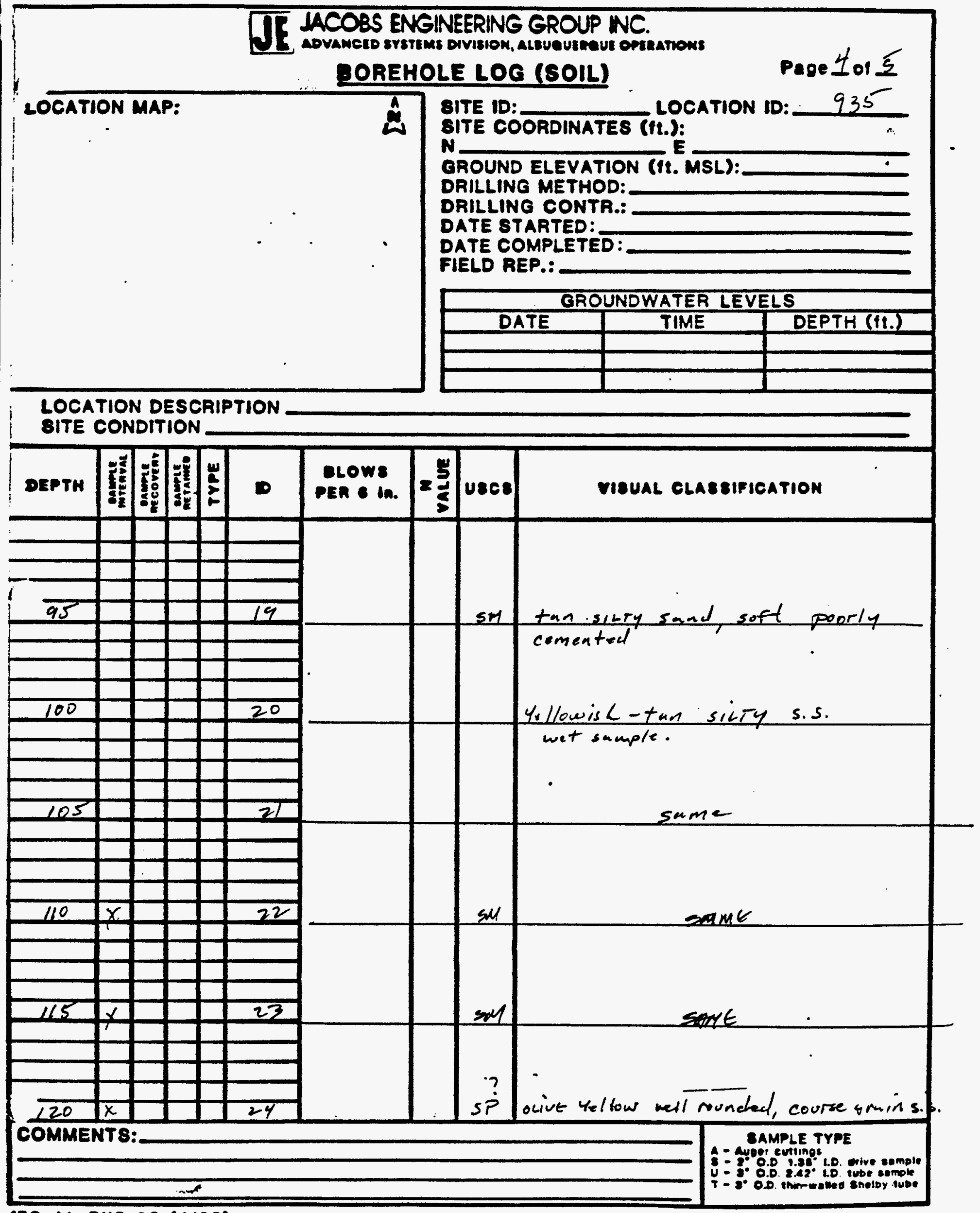




\section{BOREHOLE LOG (SOIL)}

SITE ID: SITE COORDINATES ( $t$ t.):

N LOCATION ID: $\quad 936$

GROUND ELEVATION (ft. MSL):

DRILLING METHOD:

DRILLING CONTR.:

DATE STARTED:

DATE COMPLETED:

FIELD REP.:

\begin{tabular}{|c|c|c|}
\hline \multicolumn{3}{|c|}{ GROUNDWATER LEVELS } \\
\hline DATE & TIME & DEPTH (ft.) \\
\hline & & \\
\hline & & \\
\hline & & \\
\hline
\end{tabular}

LOCATION DESCRIPTION SITE CONDITION

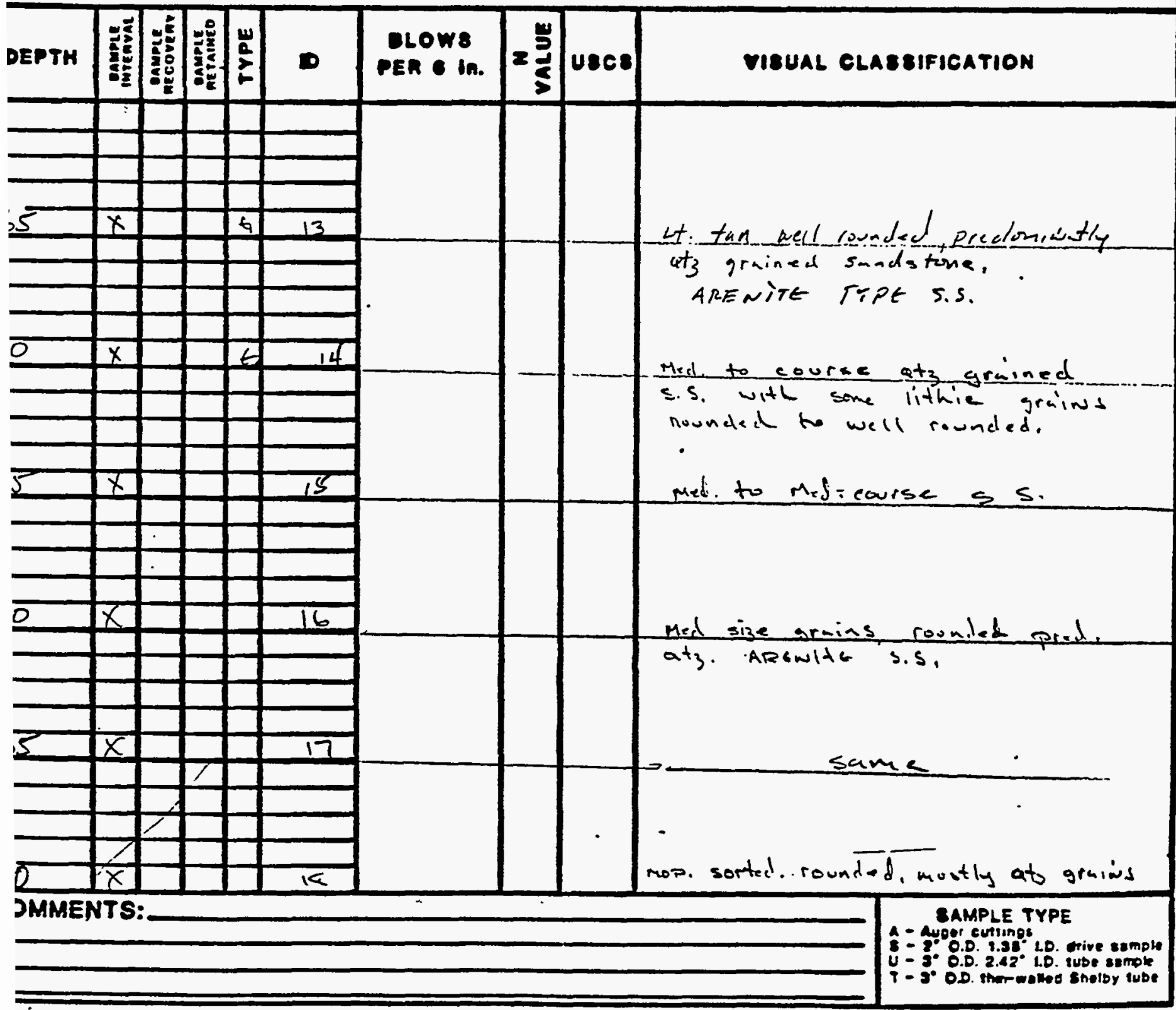




\section{LOCATION MAP:}

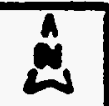

LOCATION DESCRIPTION

SITE CONDITION

BITE ID:

SITE COORDINATES (ft.):

N

GROUND ELEVATION (It. MSL):

DRILLING METHOD:

DRILLING CONTR.:

DATE STARTED:

DATE COMPLETED:

FIELD REP.:

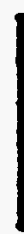

\begin{tabular}{|c|c|c|}
\hline \multicolumn{3}{|c|}{ GROUNDWATER LEVELS } \\
\hline DATE & TIME & DEPTH (fl.) \\
\hline & & \\
\hline & & \\
\hline & & \\
\hline
\end{tabular}

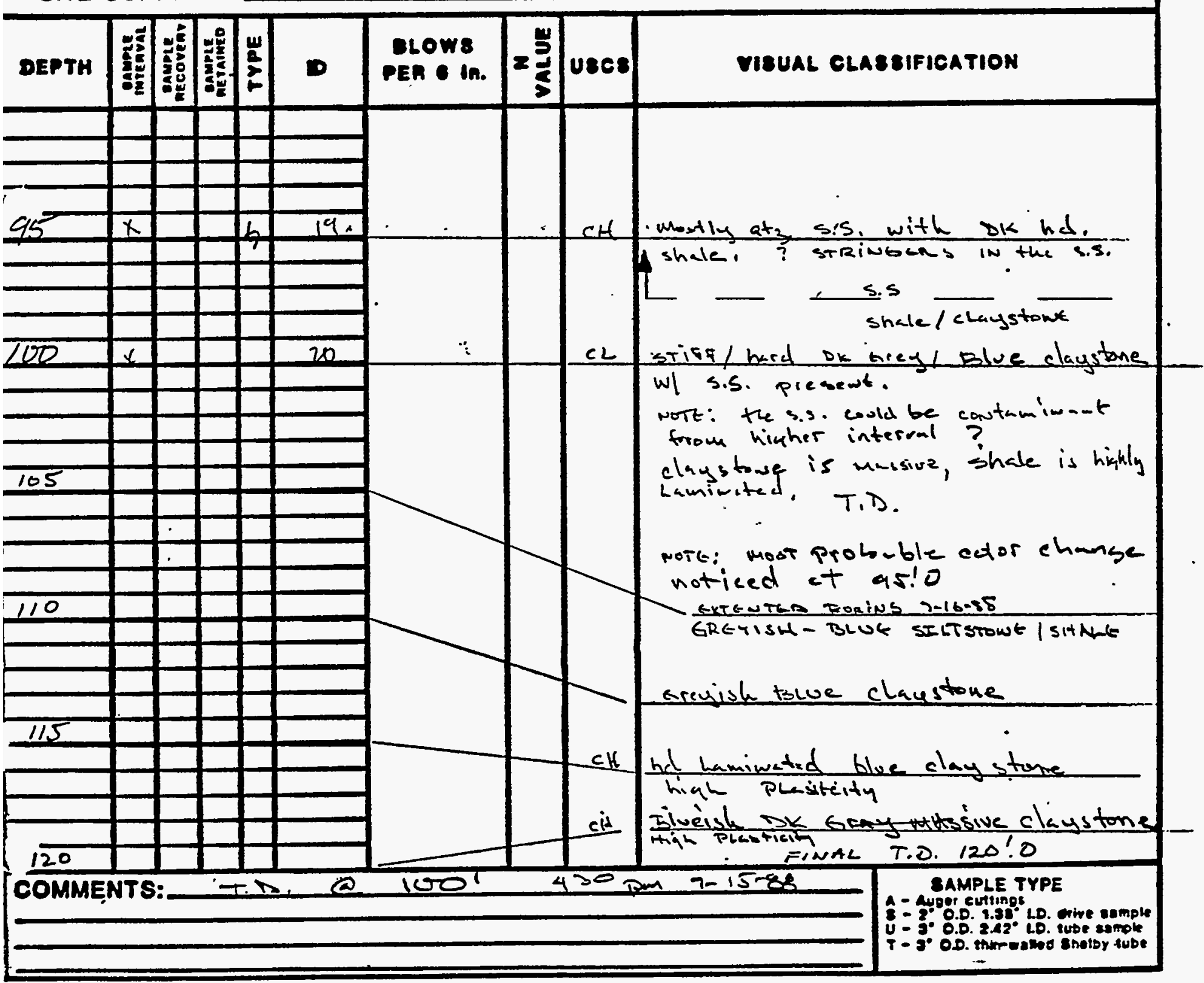


DRILLING METHOD:

DRILLING CONTR.:

DATE STARTED:

DATE COMPLETED:

FIELD REP.:

\begin{tabular}{|c|c|c|}
\hline \multicolumn{3}{|c|}{ GROUNDWATER LEVELS } \\
\hline DATE & TIME & DEPTH (II.) \\
\hline & & \\
\hline & & \\
\hline & & \\
\hline
\end{tabular}

LOCATION DESCRIPTION

SITE CONDITION

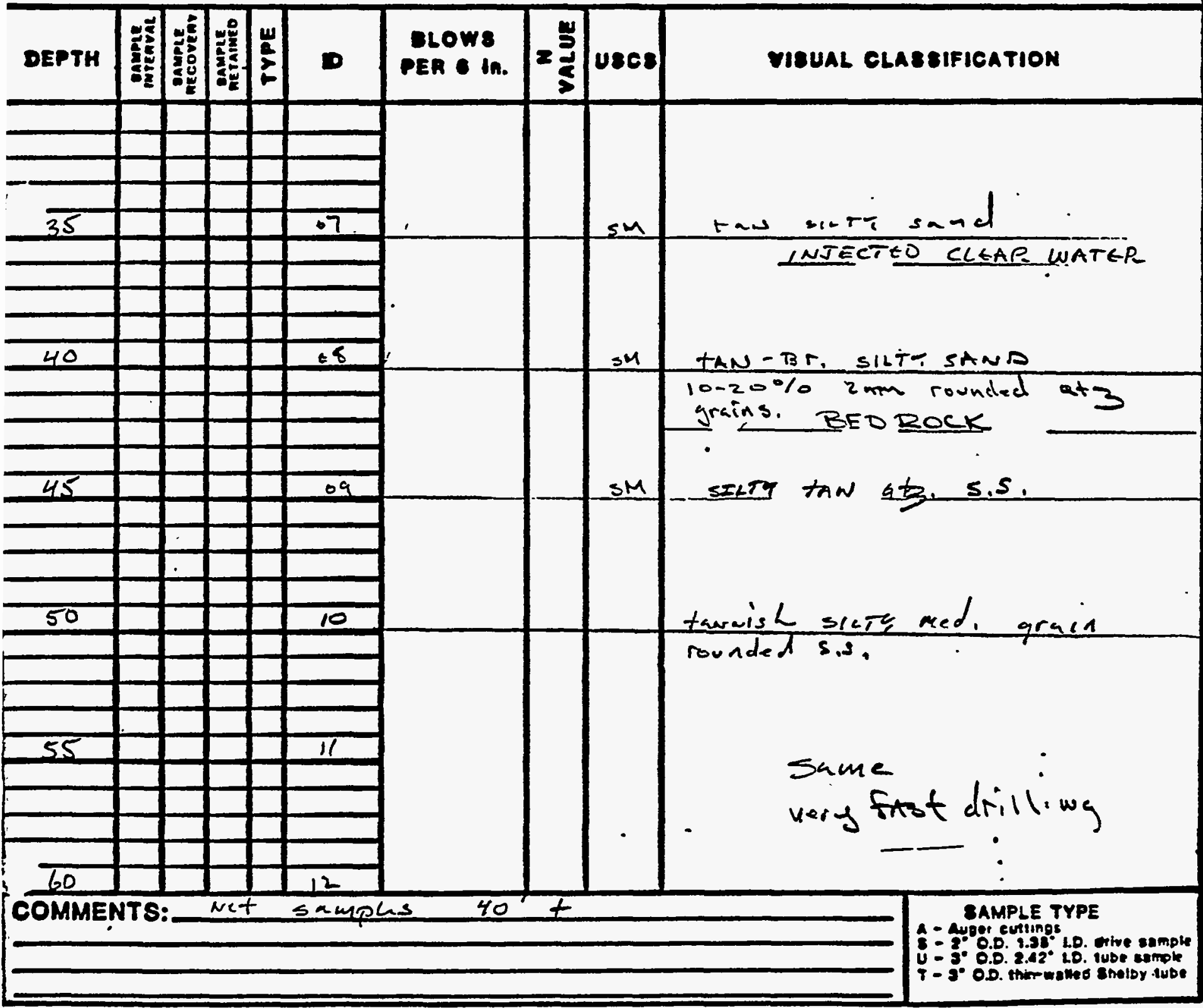


BITE DD:

$N$

SITE COORDINATES (ft.): E

GROUND ELEVATION (ft. MSL):

DRILLING METHOD:

DRILLING CONTR.:

DATE STARTED:

DATE COMPLETED:

FIELD REP.:

\begin{tabular}{|c|c|c|}
\hline \multicolumn{3}{|c|}{ GROUNDWATER LEVELS } \\
\hline DATE & TIME & DEPTH (ft.) \\
\hline & & \\
\hline & & \\
\hline & & \\
\hline
\end{tabular}

LOCATION DESCRIPTION

SITE CONDITION

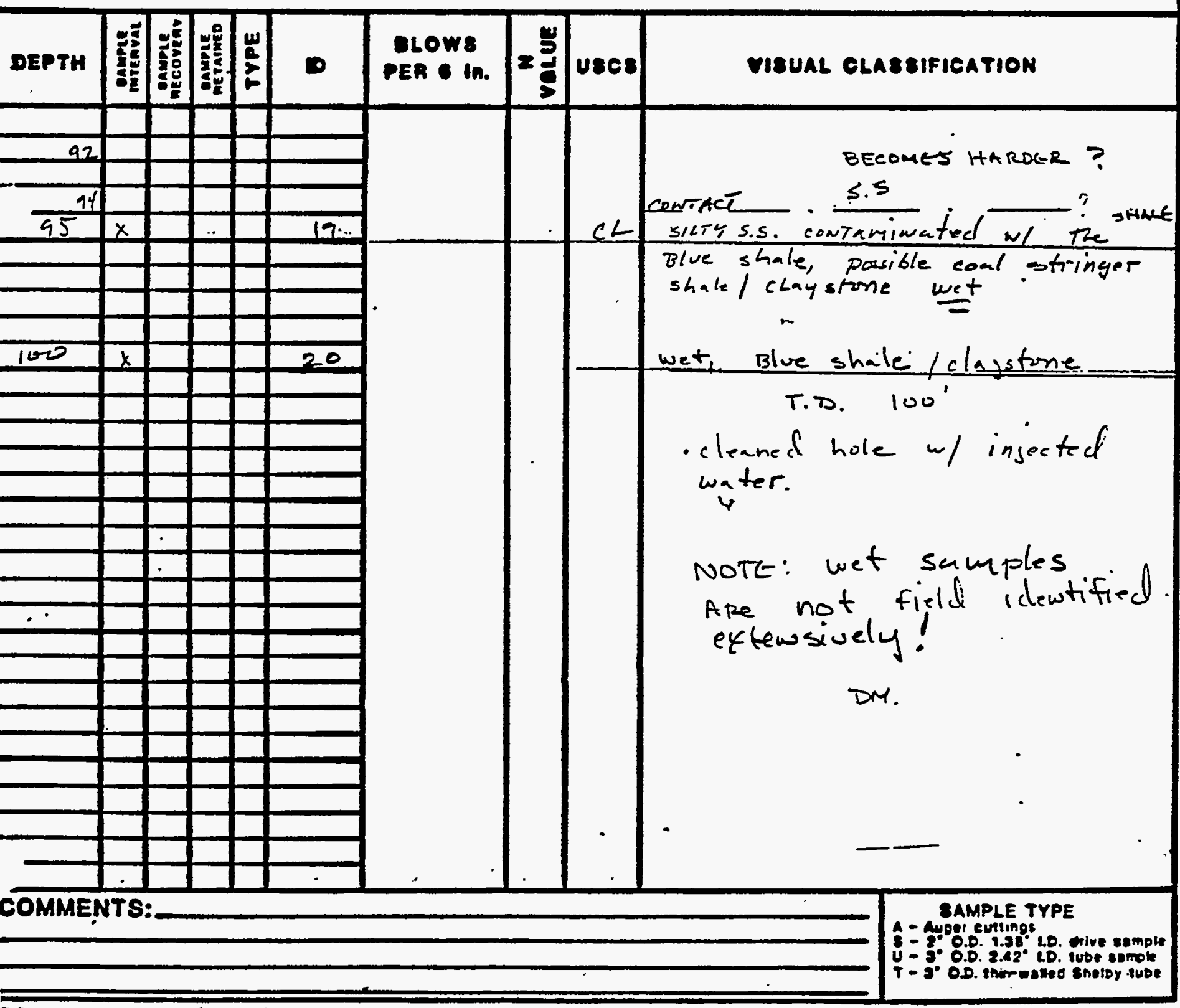


TE IACOBS ENGINEERING GROUP INC.

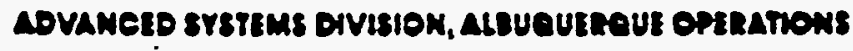

BOREHOLE LOG (SOIL)

Page 1 or 9

LOCATION MAP:

ฝ

LOCATION DESCRIPTION

BITE CONDITION

\begin{tabular}{|c|c|c|c|c|c|c|c|}
\hline DEPTH & 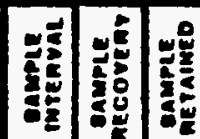 & $\frac{w}{2}$ & $\boldsymbol{D}$ & $\begin{array}{l}\text { DLOW8 } \\
\text { PEA IA. }\end{array}$ & $=\frac{m}{3}$ & usces & VISUAL CLABsIFICATION \\
\hline
\end{tabular}

95

8ITE ID:

LOCATION ID: $\quad 138$

SITE COORDINATES (ft.):

$N$

E

GROUND ELEVATION (ft. MSL):

DRILLING METHOD:

DRILLING CONTR.:

DATE STARTED:

DATE COMPLETED:

FIELD REP.:

\begin{tabular}{|c|c|c|}
\hline \multicolumn{3}{|c|}{ GROUNDWATER LEVELS } \\
\hline DATE & TIME & DEPTH (II.) \\
\hline & & \\
\hline & & \\
\hline
\end{tabular}
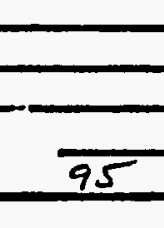

100

- 10

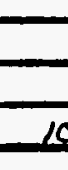

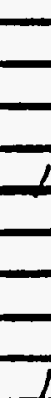 \\ $\bar{z}$}

年

$+1$

20

$\cdot$

9

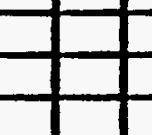

105

7

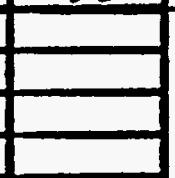

$\square$

1 120

COMMENTS:

shale wh hard black bituminous coal, concoutid fruetrons in eed

B/ue elaystone with hard Black conl chase darystorre

elonse blue-grey claystone, sub ligarte coul stringere far couting on claystone fructure surfuce.
SAMPLE TYPE

A - Auget cutings

- 20.0. 1.30 to. ctive sample

O.D. 2.2. 2.0. Iube sample

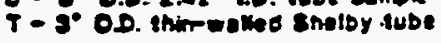


17 LCOBS ENGINEERING GROUP INC.

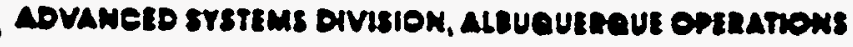

BOREHOLE LOG (SOIL)

LOCATION MAP:

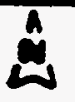

SITE ID:

SITE COORDINATES (ft.):

$N$

GROUND ELEVATION (ft. MSL):

DRILLING METHOD:

DRILLING CONTR.:

DATE STARTED:

DATE COMPLETED:

FIELD REP.:

\begin{tabular}{|c|c|c|}
\hline \multicolumn{3}{|c|}{ GROUNDWATER LEVELS } \\
\hline DATE & TIME & DEPTH (ft.) \\
\hline & & \\
\hline & & \\
\hline
\end{tabular}

LOCATION DESCRIPTION

SITE CONDITION

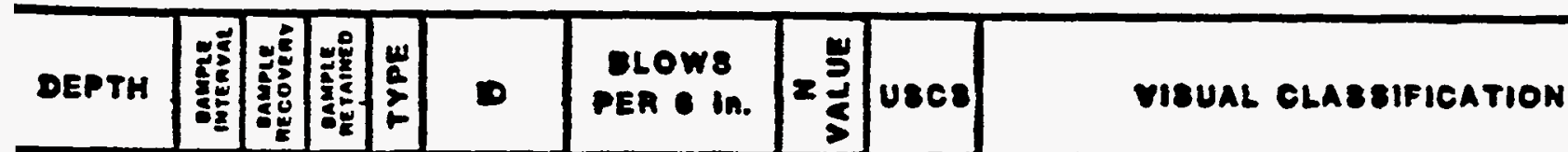

\begin{tabular}{|c|c|c|c|}
\hline & & - & \\
\hline & & - & \\
\hline 125 & $x$ & & 25 \\
\hline & & & \\
\hline & & & \\
\hline & & & \\
\hline 130 & $x$ & & 26 \\
\hline & & & \\
\hline & & & \\
\hline 135 & $x$ & & 27 \\
\hline & $\pi$ & & \\
\hline & & & \\
\hline 170 & $\infty$ & & 25 \\
\hline & & & \\
\hline & & & \\
\hline 145 & $\bar{x}$ & - & 29 \\
\hline & & & \\
\hline & & & \\
\hline 150 & & & 30 \\
\hline
\end{tabular}

SOMMENTS:

cL blue / corey shale/cluystone

mosty blece clayptone of ok euthy

shale

blue / brey daystones of occessondy

soft elay.

mestly blee / unuy claystore

claystomes, stringers 8 herd coal

SAMPLE TYPE

A - Mupoet curtungr

G- 
IT JACOBS ENGINEERING GROUP NC.

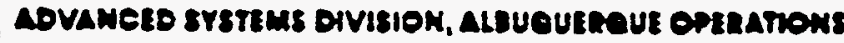

BOREHOLE LOG (SOIL)

Page 7 of 9

LOCATION MAP:

$\stackrel{\omega}{\omega}$.

SITE ID:

SITE COORDINATES (ft.):

N.

GROUND ELEVATION (ft. MSL):

DRILLING METHOD:

DRILLING CONTR.:

DATE STARTED:

DATE COMPLETED:

FIELD REP.:

\begin{tabular}{|c|c|c|}
\hline \multicolumn{3}{|c|}{ GROUNDWATER LEVELS } \\
\hline DATE & TIME & DEPTH (11.) \\
\hline & & \\
\hline & & \\
\hline & & \\
\hline
\end{tabular}

LOCATION DESCRIPTION

SITE CONDITION

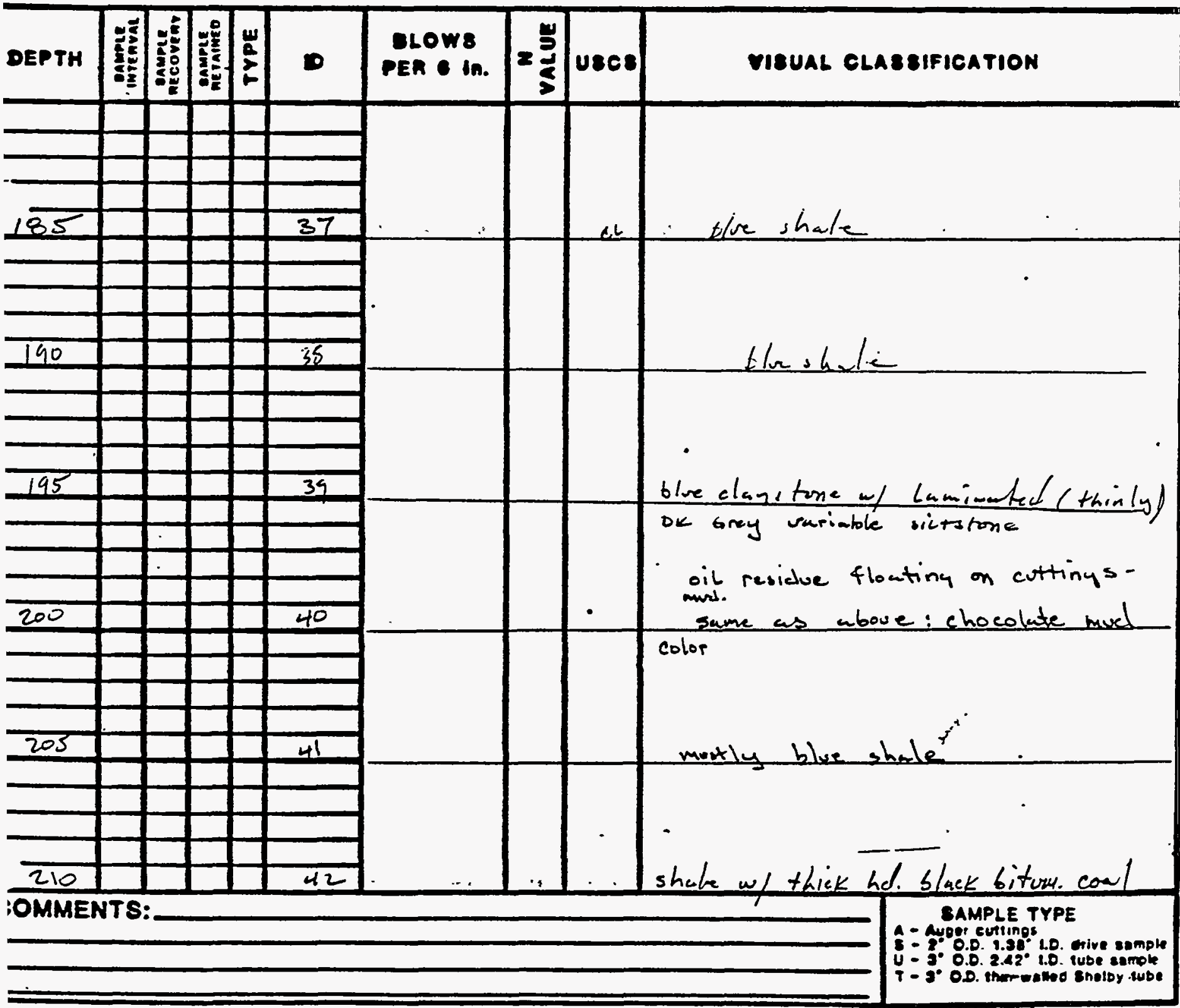

LOCATION ID: 938 


\section{TIT IACOBS ENGINEERING GROUP NC.

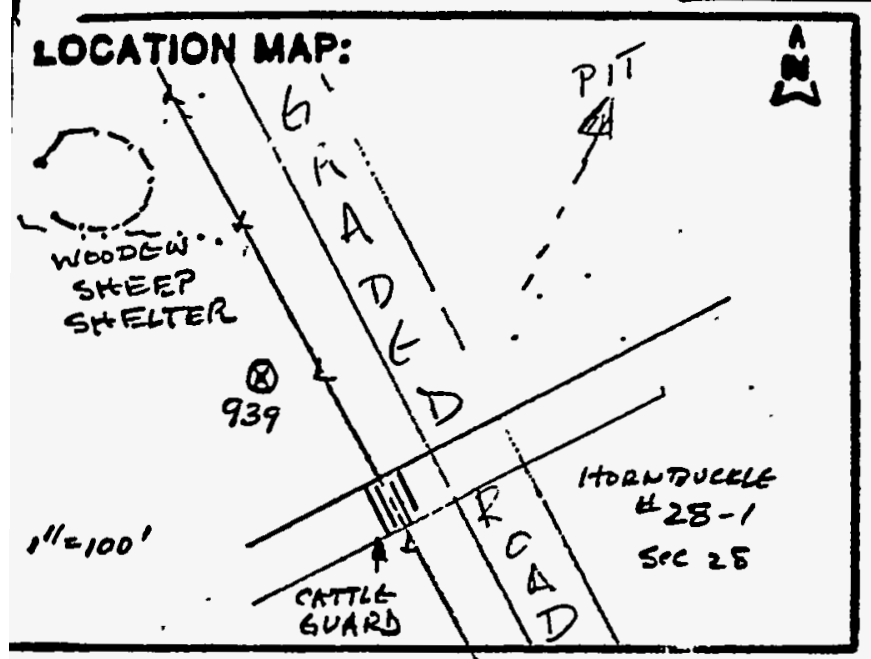

LOCATION DESCRIPTION MOREDATELT

SITE CONDITION SPARSE

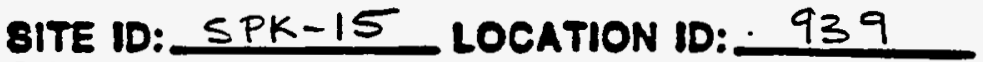
SITE COORDINATES (ft.):

N NK E NK

GROUND ELEVATION (ft. MSL): NK

DRILLING METHOD: 5 HIR ROTAR,

DRILLING CONTR: TARNILART

DATE STARTED:

$7-16-8 \delta$

DATE COMPLETED:

FIELD REP.: D. METZLCR

\begin{tabular}{|c|c|c|}
\hline \multicolumn{3}{|c|}{ GROUNDWATER LEVELS } \\
\hline DATE & TIME & DEPTH (11.) \\
\hline & & \\
\hline & & \\
\hline & & \\
\hline
\end{tabular}

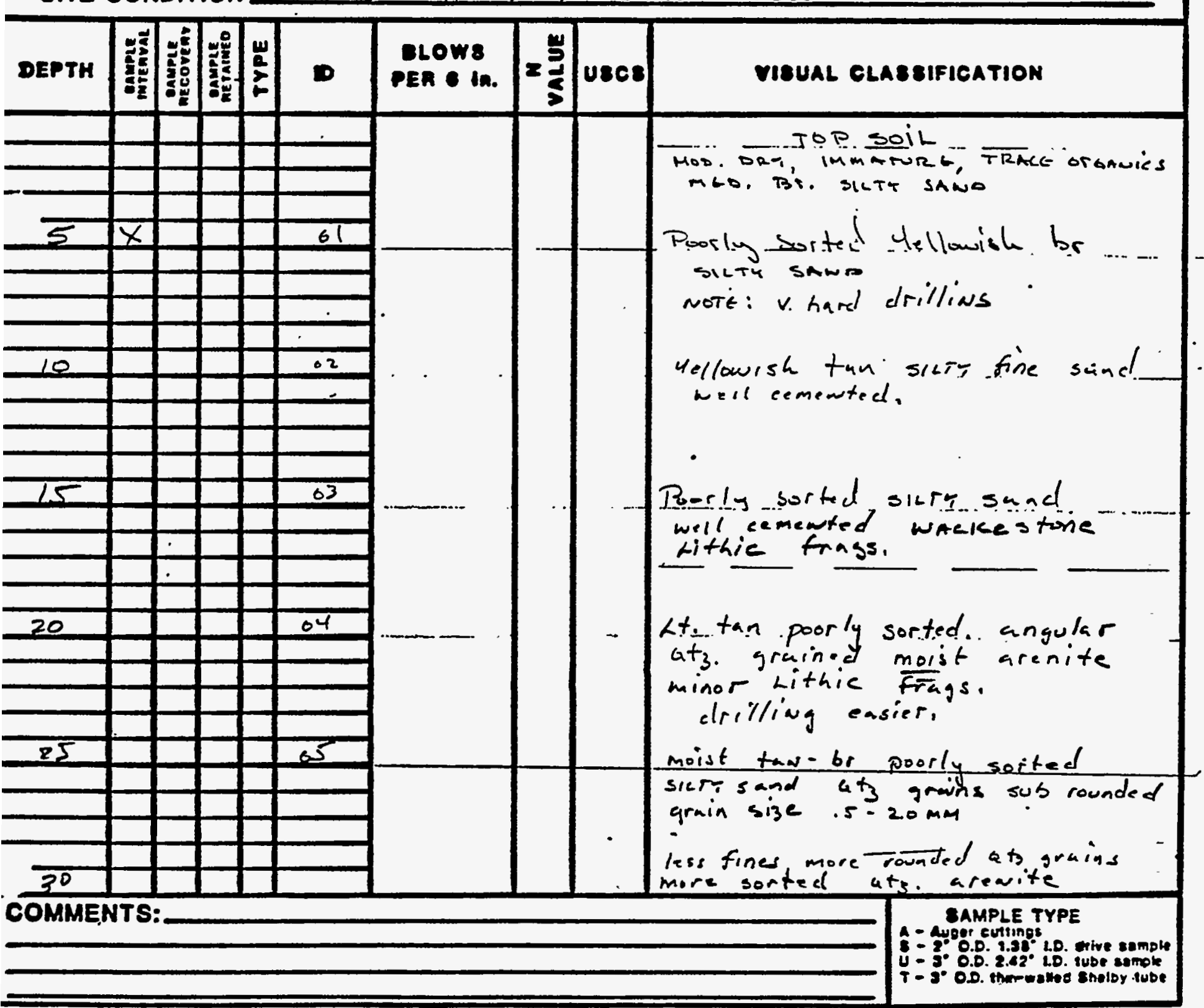


TI LACOBS ENGINEERING GROUP INC.

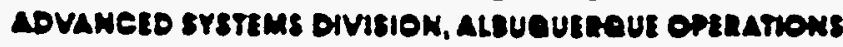

BOREHOLE LOG (SOIL)

Page 2 of 3

LOCATION MAP:

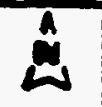

8ITE ID:

SITE COORDINATES (ft.):

N

GROUND ELEVATION (ft. MSL):

DRILLING METHOD:

DRILLING CONTR.:

DATE STARTED:

DATE COMPLETED:

FIELD REP.:

\begin{tabular}{|c|c|c|}
\hline \multicolumn{3}{|c|}{ GROUNDWATER LEVELS } \\
\hline DATE & TIME & DEPTH (f?.) \\
\hline & & \\
\hline & & \\
\hline & & \\
\hline
\end{tabular}

LOCATION DESCRIPTION

SITE CONDITION

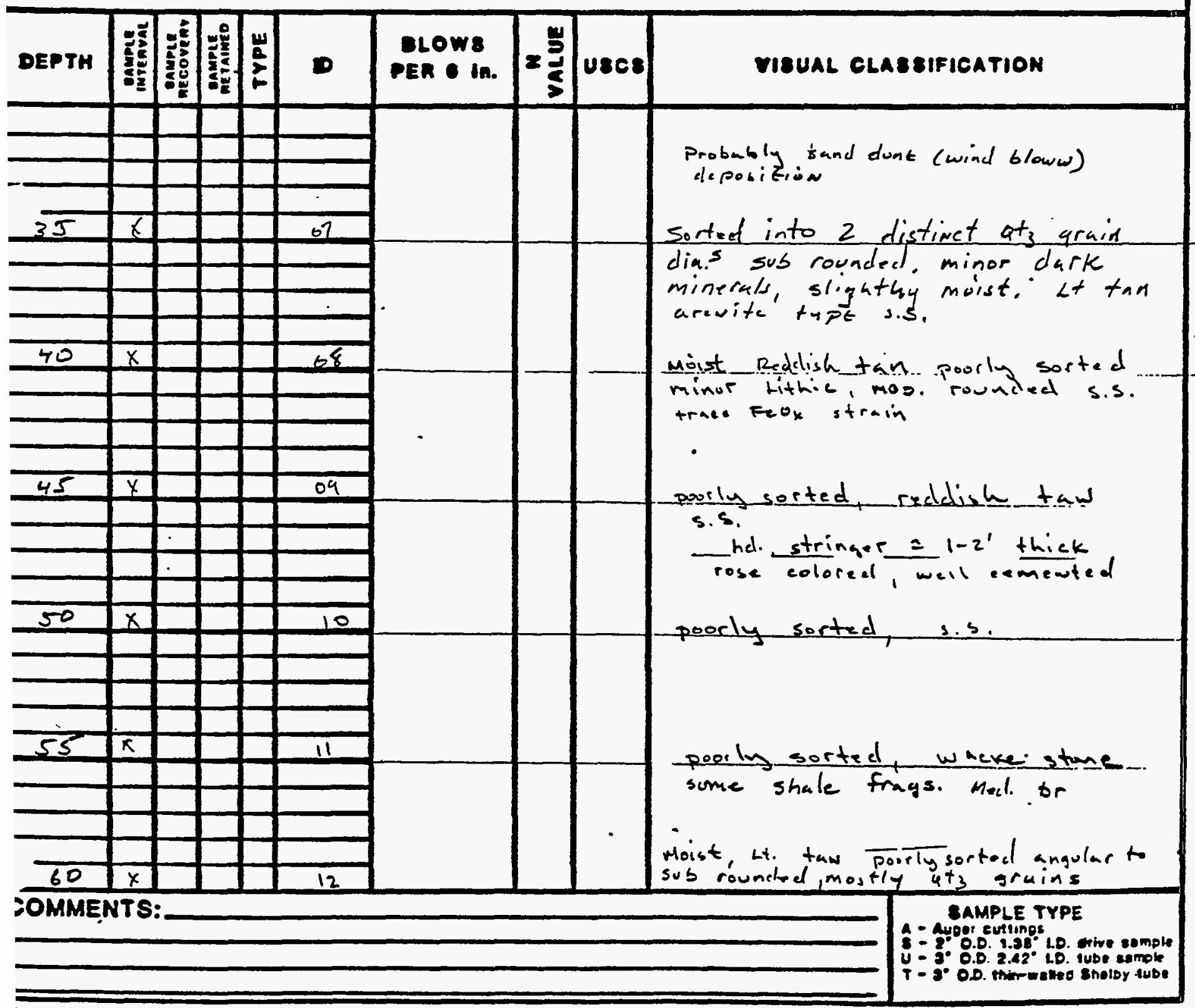


IT IACOBS ENGINEERING GROUP NC.

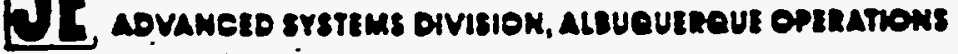

\section{BOREHOLE LOG (SOIL)}

Page 3 of 3

LOCATION MAP:
SITE ID:

SITE COORDINATES (ft.):

N GROUND ELEVATION (It. MSL):

DRILLING METHOD:

DRILLING CONTR.:

DATE STARTED:

DATE COMPLETED:

FIELD REP.:
LOCATION ID: 939 E

\begin{tabular}{|c|c|c|}
\hline \multicolumn{3}{|c|}{ GROUNDWATER LEVELS } \\
\hline DATE & TIME & DEPTH (II.) \\
\hline & & \\
\hline & & \\
\hline & & \\
\hline
\end{tabular}

LOCATION DESCRIPTION

SITE CONDITION

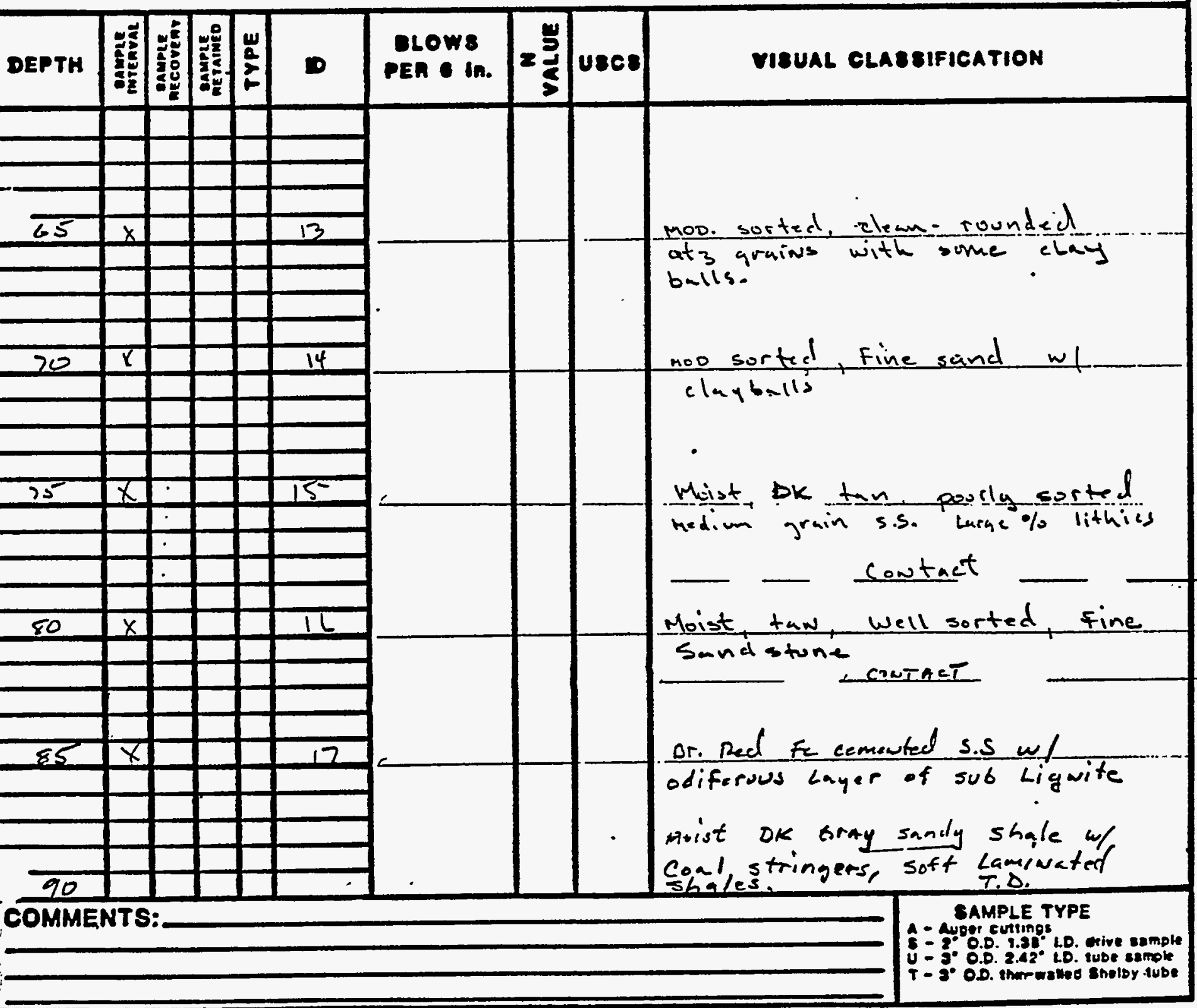




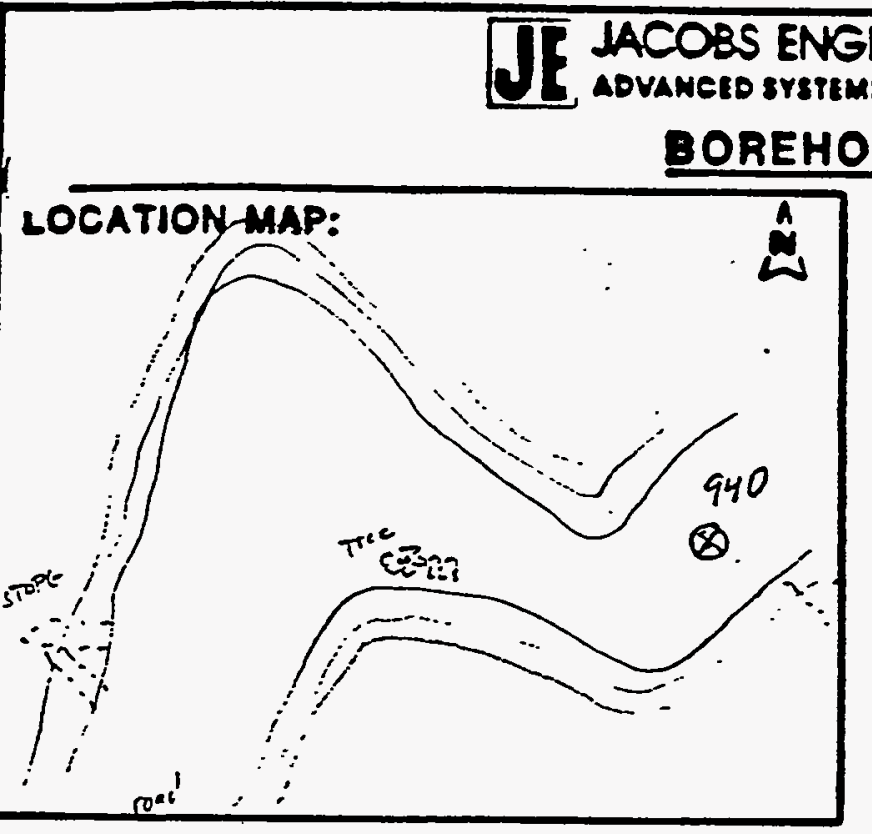

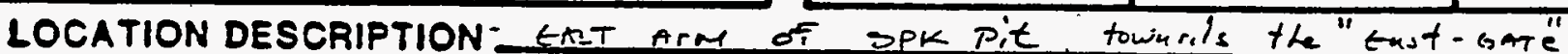
SITE CONDITION Loose sands, few weeds dey surface

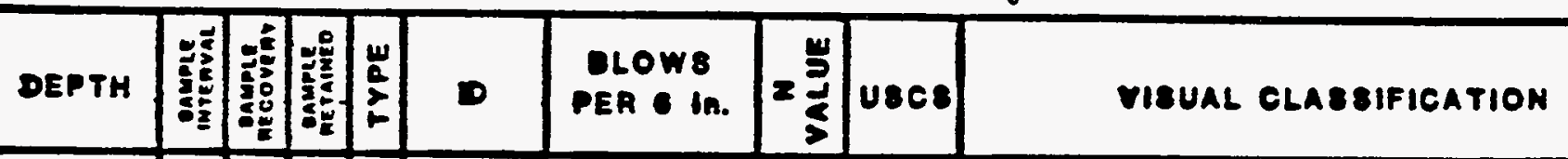

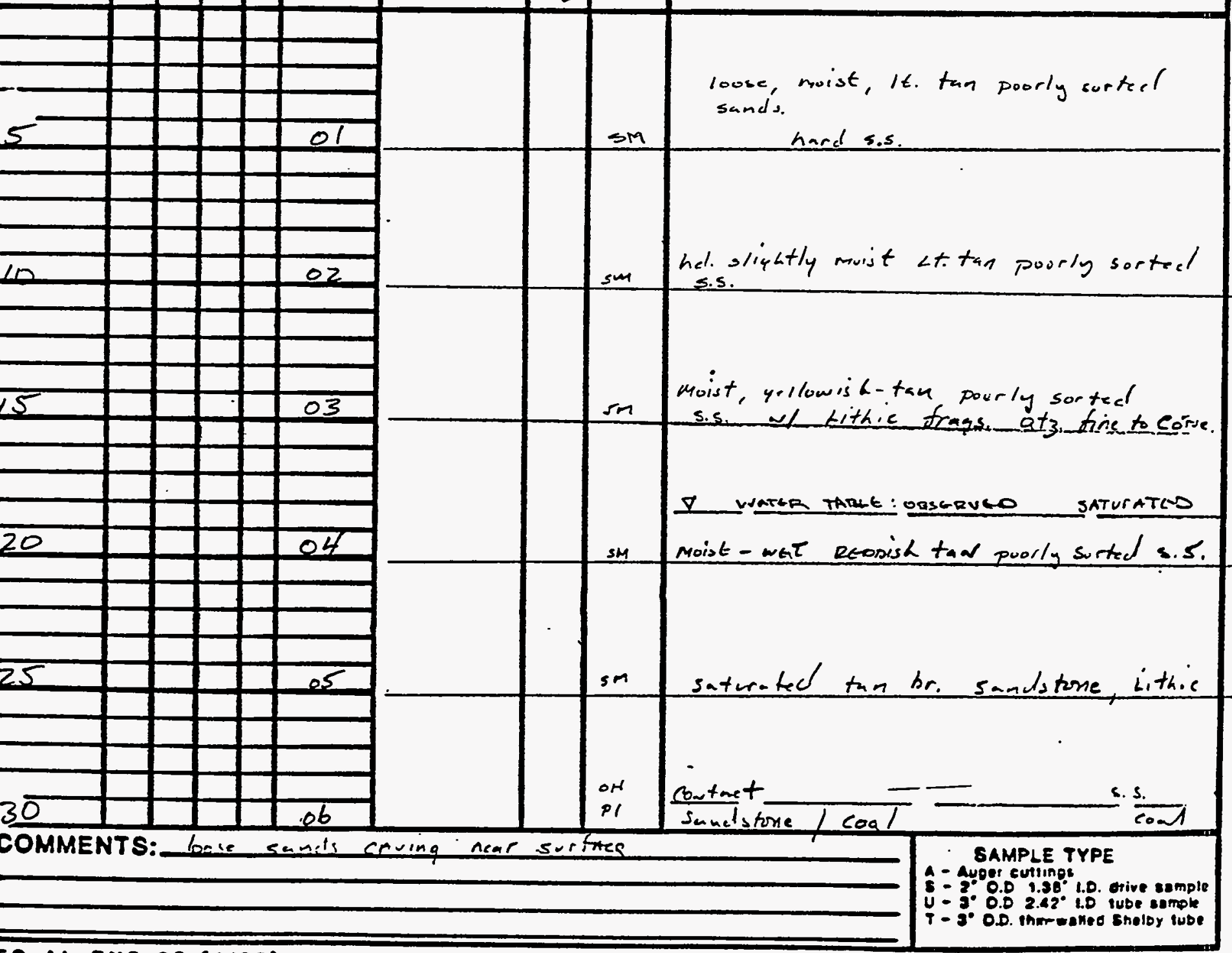




\section{TT IACOBS ENGINEERING GROUP INC

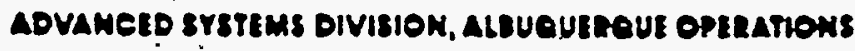

BOREHOLE LOG (SOIL)

Page 2012

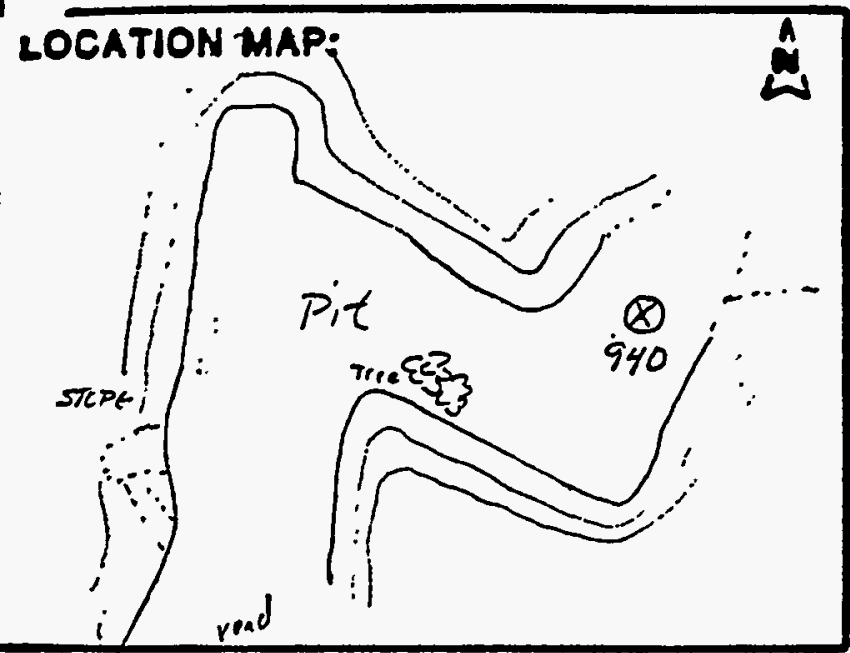

SITE ID: SPL-15

LOCATION ID: 940

SITE COORDINATES (It.):

N

GROUND ELEVATION (ft. MSL):

DRILLING METHOD:

DRILLING CONTR.:

DATE STARTED:

DATE COMPLETED:

FIELD REP.:

\begin{tabular}{|c|c|c|}
\hline \multicolumn{3}{|c|}{ GROUNDWATER LEVELS } \\
\hline DATE & TIME & DEPTH (ft.) \\
\hline & & \\
\hline & & \\
\hline & & \\
\hline
\end{tabular}

LOCATION DESCRIPTION

SITE CONDITION

\begin{tabular}{|c|c|c|c|c|c|}
\hline DEPTH & 雚 & 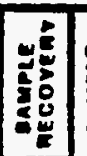 & 帒 & 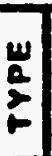 & D \\
\hline & & & & & \\
\hline & & & & & \\
\hline & & & & & \\
\hline 35 & & & & & 67 \\
\hline & & & & & \\
\hline & & & & & \\
\hline & & & & & \\
\hline 40 & & & & & 68 \\
\hline & & & & & \\
\hline & & & & & \\
\hline 45 & & & & & 09 \\
\hline & & & & & \\
\hline & & & & & \\
\hline & & & & & \\
\hline 50 & & 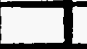 & & & 10 \\
\hline & & $E$ & & & \\
\hline & & & & & \\
\hline & & & & & \\
\hline & & & & & \\
\hline & & & & & \\
\hline & & & & & \\
\hline & & & & & \\
\hline
\end{tabular}

COMMENTS:

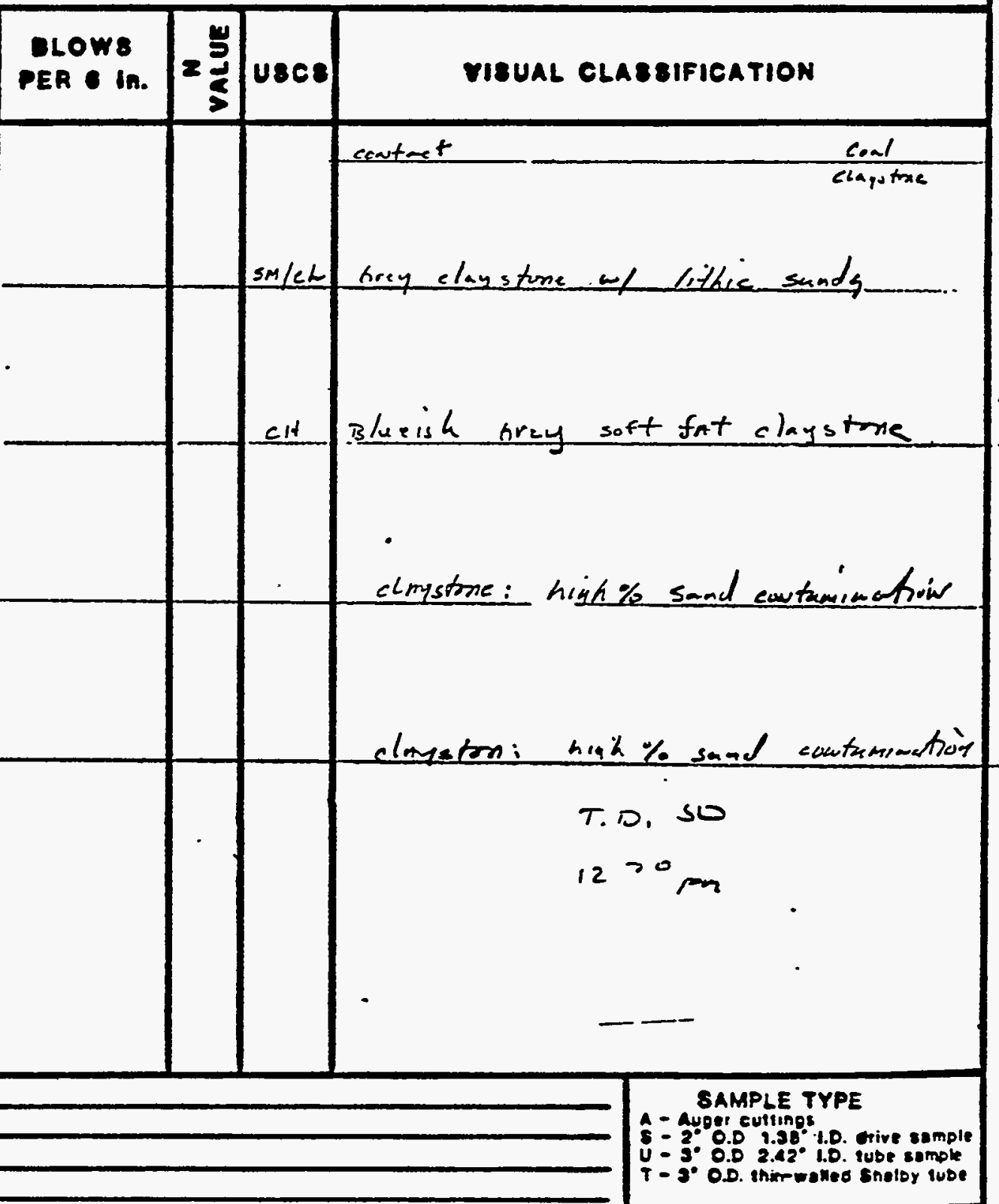




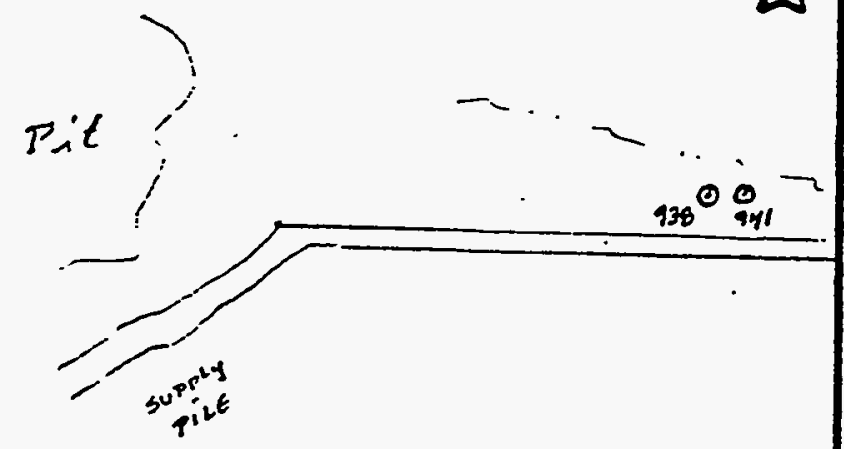

N N N

GROUND ELEVATION (ft. MSL): NK

DRILLING METHOD: RUTARY AIR

DRILLING CONTR.: BARNAART

DATE STARTED: $\frac{7-26.8 \delta}{7-26-8 \delta}$

DATE COMPLETED: $7-26-88$

FIELD REP.:

D. METZLCP.

\begin{tabular}{|c|c|c|}
\hline \multicolumn{3}{|c|}{ GROUNDWATER LEVELS } \\
\hline DATE & TIME & DEPTH (f1.) \\
\hline $2-27-88$ & 745.4 & 45.0 \\
\hline & & \\
\hline & & \\
\hline
\end{tabular}

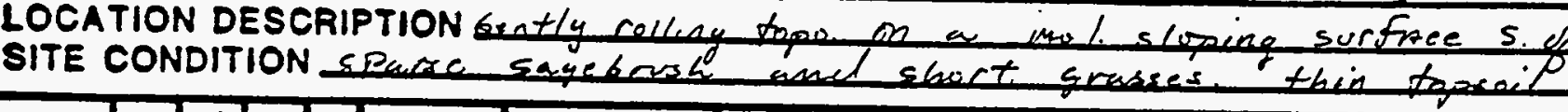

\begin{tabular}{|c|c|c|c|}
\hline DEPTH & 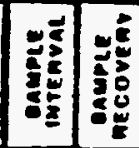 & 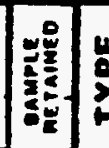 & 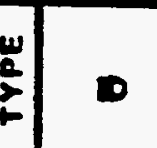 \\
\hline & & & \\
\hline & & & \\
\hline & & & \\
\hline & $x$ & 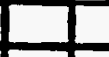 & 01 \\
\hline & 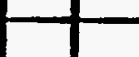 & - & \\
\hline & $L$ & & \\
\hline & $x$ & & 02 \\
\hline & 5 & & \\
\hline & $\leftarrow$ & & \\
\hline & \begin{tabular}{|l|l} 
& \\
$x$ &
\end{tabular} & & 03 \\
\hline & 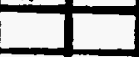 & & \\
\hline & & & \\
\hline & $y$ & & 03.5 \\
\hline & & & 04 \\
\hline & $E$ & & \\
\hline & & & \\
\hline & $x$ & & 05 \\
\hline & - & & \\
\hline & & & \\
\hline & & & \\
\hline & & 7 & 06 \\
\hline
\end{tabular}

OMMENTS:

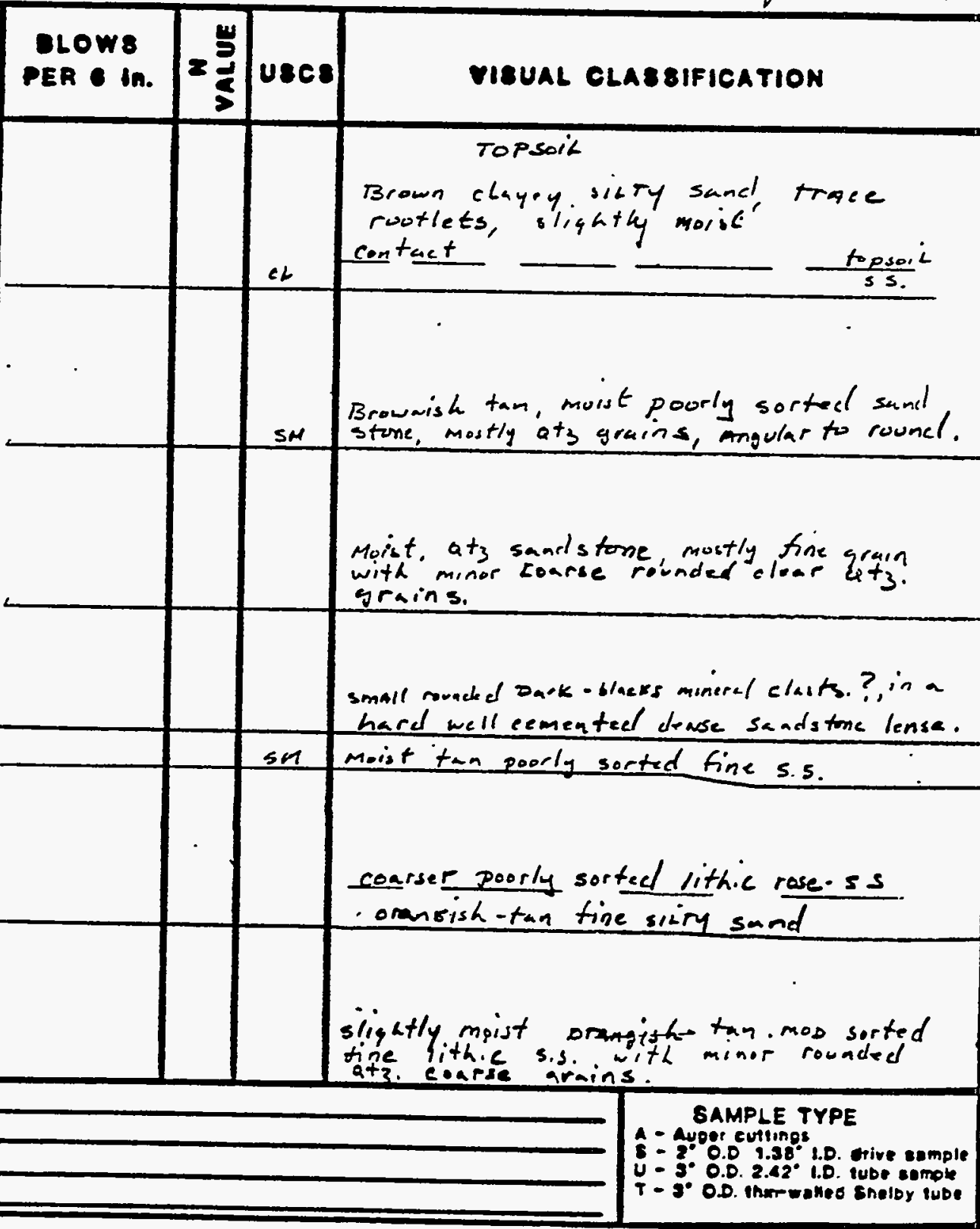


17. IACOBS ENGINEERING GROUP INC.

\section{LOCATION MAP:}

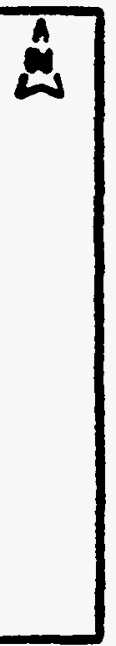

SITE ID:

SITE COORDINATES (ft.):

$N$

GROUND ELEVATION (Ft. MSL):

DRILLING METHOD:

DRILLING CONTR.:

DATE STARTED:

DATE COMPLETED:

FIELD REP.:

\begin{tabular}{|c|c|c|}
\hline \multicolumn{3}{|c|}{ GROUNDWATER LEVELS } \\
\hline DATE & TIME & DEPTH (ft.) \\
\hline & & \\
\hline & & \\
\hline & & \\
\hline
\end{tabular}

LOCATION DESCRIPTION

SITE CONDITION

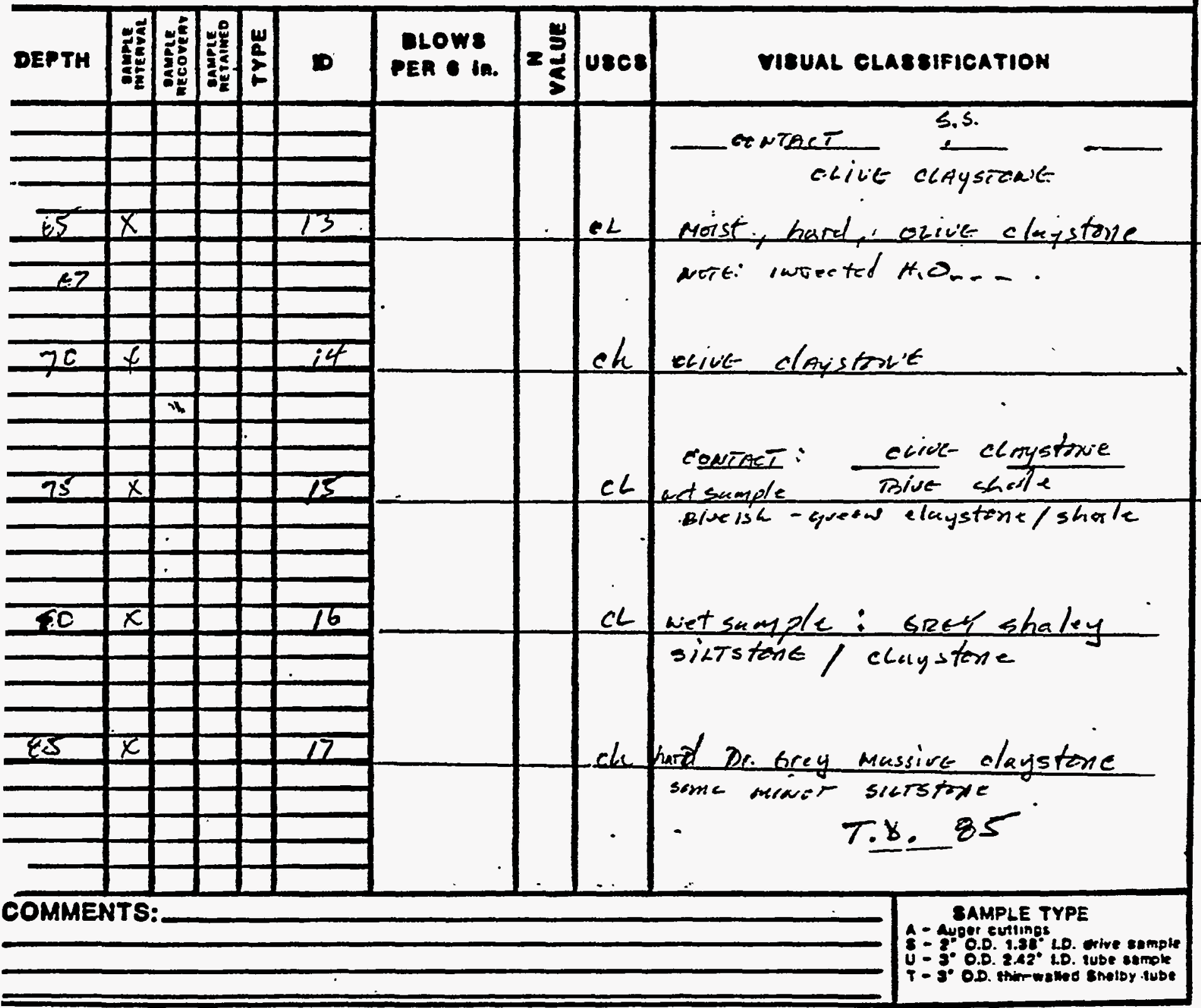




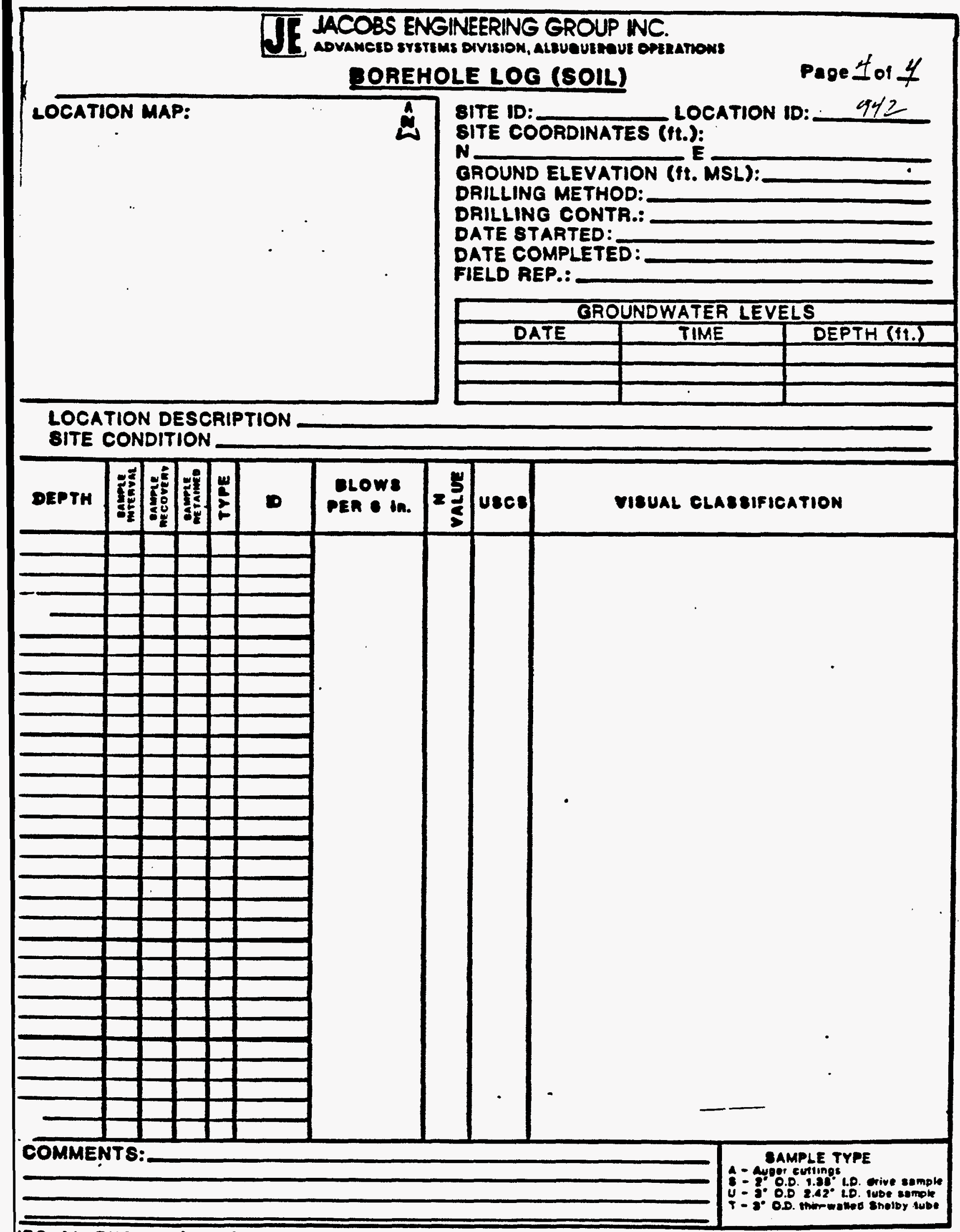


JE IACOBS ENGINEERING GROUP INC.

BOREHOLE LOG (SOIL)

LOCATION MAP:

kxrov,

a

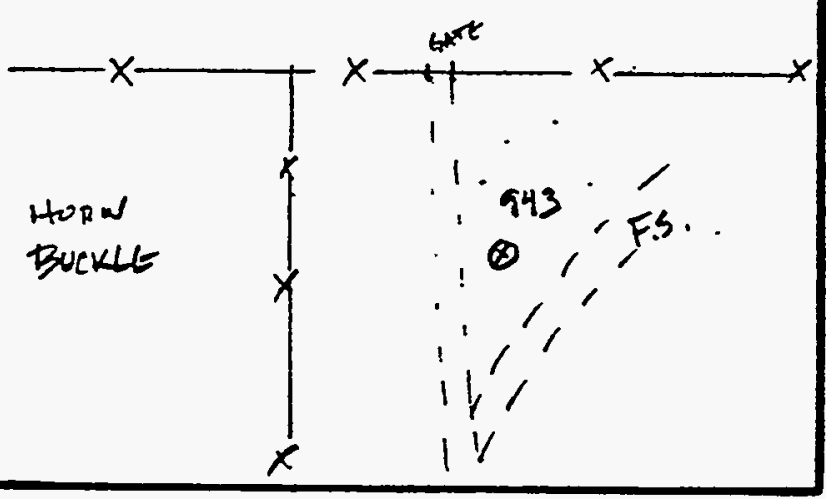

8ITE ID: SPK-1S

SITE COORDINATES (ft.):

N

GROUND ELEVATION (ft. MSL):

DRILLING METHOD:

DRILLING CONTR.:

DATE STARTED:

DATE COMPLETED:

FIELD REP.:

E

\section{LOCATION DESCRIPTION}

BITE CONDITION

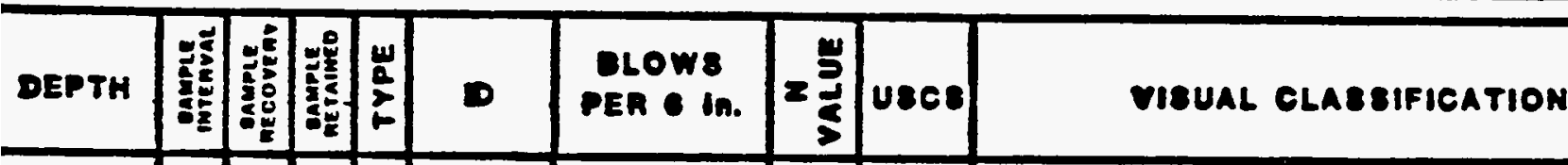

$45 \times$

\begin{tabular}{ll|l|l}
\hline$x$ & & & 07 \\
\hline
\end{tabular}

35

40

40

a

50

$x$

10

\begin{tabular}{|c|c|c|}
\hline \multicolumn{3}{|c|}{ GROUNDWATER LEVELS } \\
\hline DATE & TIME & DEPTH (II.) \\
\hline & & \\
\hline & & \\
\hline & & \\
\hline
\end{tabular}

LOCATION ID: $\quad 743$ 


\section{BOREHOLE LOG (ROCK)}

LOCATION MAP:

\section{$\hat{\omega}$}

ran out of soil boring

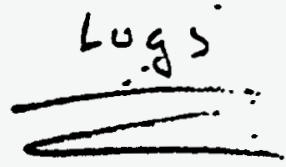

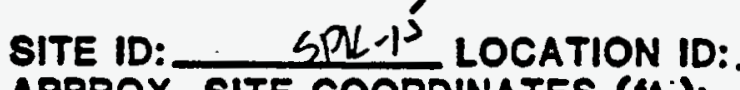

APPROX. SITE COORDINATES (ft:):

N

GROUND ELEVATION (ft. MSL):

DRILLING METHOD:

DRILLER:

DATE STARTED:

DATE COMPLETED:

FIELD REP.:

\begin{tabular}{|c|c|c|}
\hline \multicolumn{2}{|c|}{ GROUNDWATER LEVELS } \\
\hline DATE & TIME & DEPTH (ft.) \\
\hline & & \\
\hline & & \\
\hline & & \\
\hline
\end{tabular}

\section{LOCATION DESCRIPTION}

SITE CONDITION

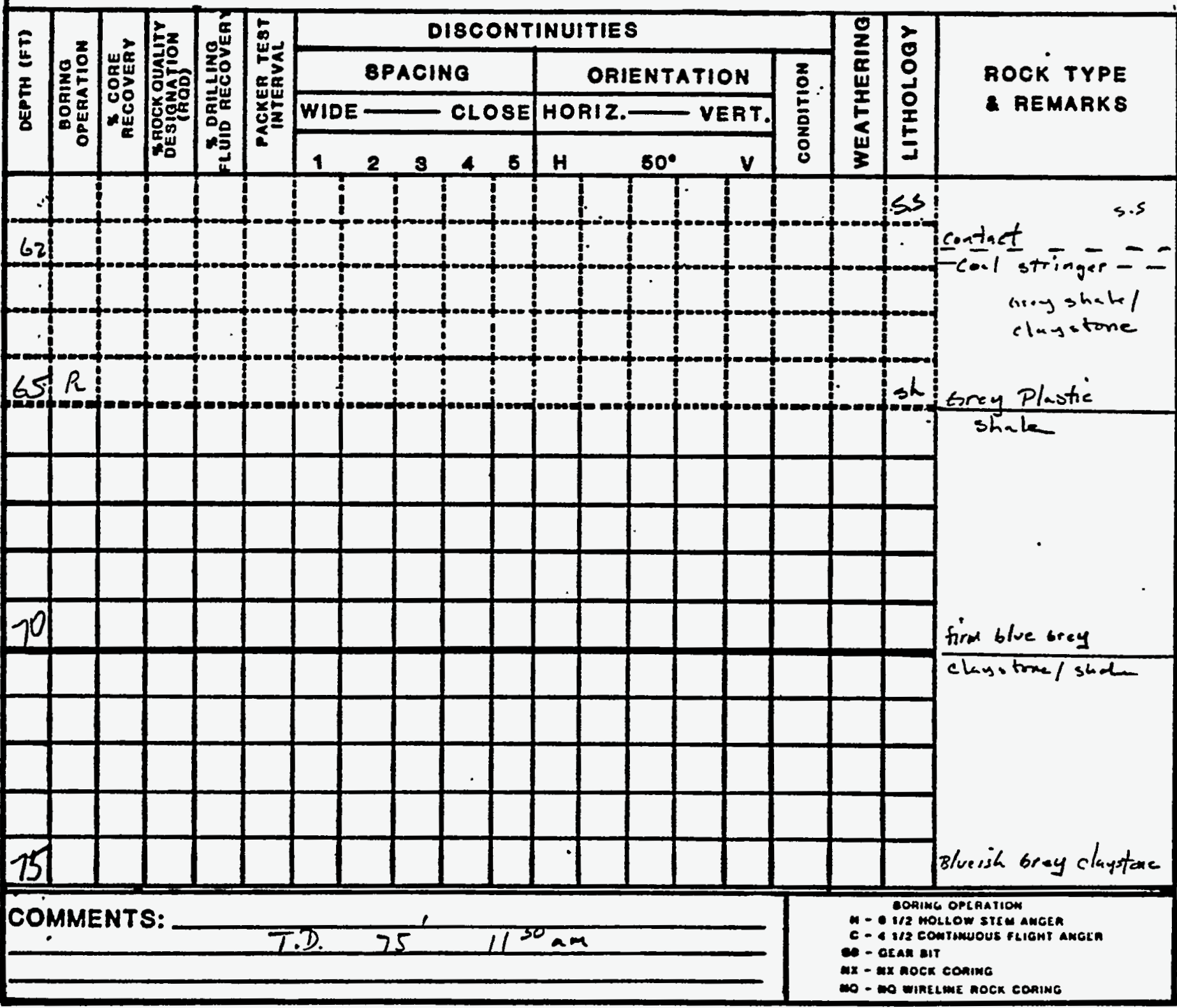


OCATION MAP:

HAROM

RANCH sumbl in 6 L
Corent

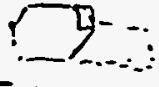<smiles>C[Al][Mg]</smiles><smiles>[CH2-]C</smiles>

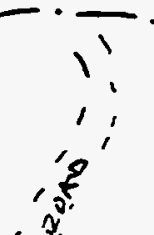

wiwenibl

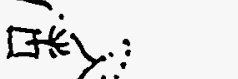

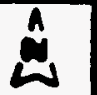

N NE NE

SITE ID: SPK-15.

LOCATION ID: $\quad 944$

SITE COORDINATES (ft.):

GROUND ELEVATION (fI. MSL): NK

DRILLING METHOD: ROTARY AIR

DRILLING CONTR.: BARNHABT

DATE STARTED: 7-29-

DATE COMPLETED:

FIELD REP.: D. METZLC-R

\begin{tabular}{|c|c|c|}
\hline \multicolumn{3}{|c|}{ GROUNDWATER LEVELS } \\
\hline DATE & TIME & DEPTH (11.) \\
\hline & & \\
\hline & & \\
\hline & & \\
\hline
\end{tabular}

LOCATION DESCRIPTION $400^{\prime}$ Suth of HaRD'S wiUTMILL

SITE CONDITION MODERATLT EAT SPARSE SAGE I SHTET GILASS

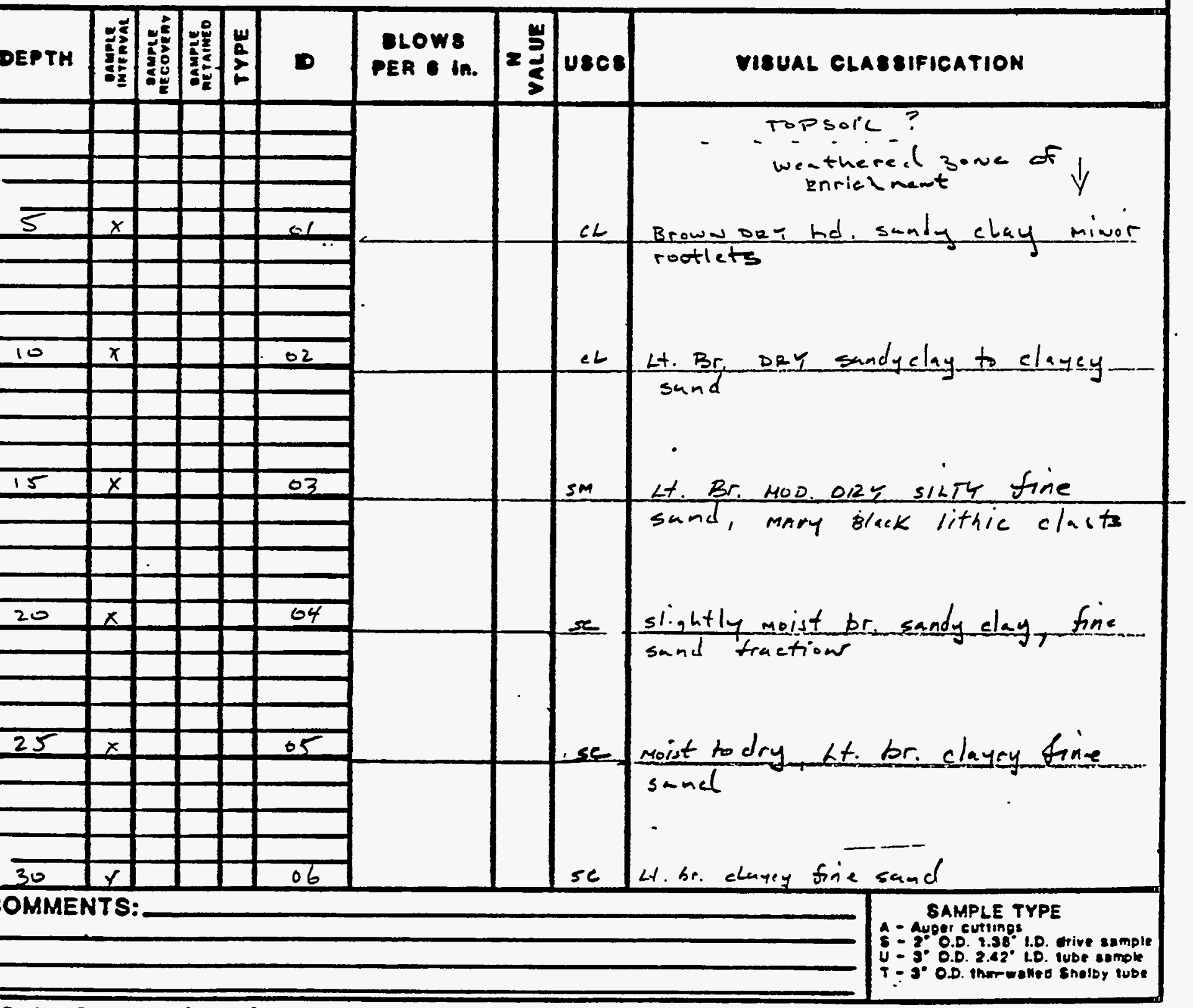


SITE ID: COK-1S LOCATION ID: 941

SITE COORDINATES (fI.):

$\mathrm{N}$ - E

GROUND ELEVATION (ft. MSL):

DRILLING METHOD:

DRILLING CONTR.:

DATE STARTED:

DATE COMPLETED:

FIELD REP.:

\begin{tabular}{|c|c|c|}
\hline \multicolumn{3}{|c|}{ GROUNDWATER LEVELS } \\
\hline DATE & TIME & DEPTH (ft.) \\
\hline & & \\
\hline & & \\
\hline & & \\
\hline
\end{tabular}

LOCATION DESCRIPTION

SITE CONDITION

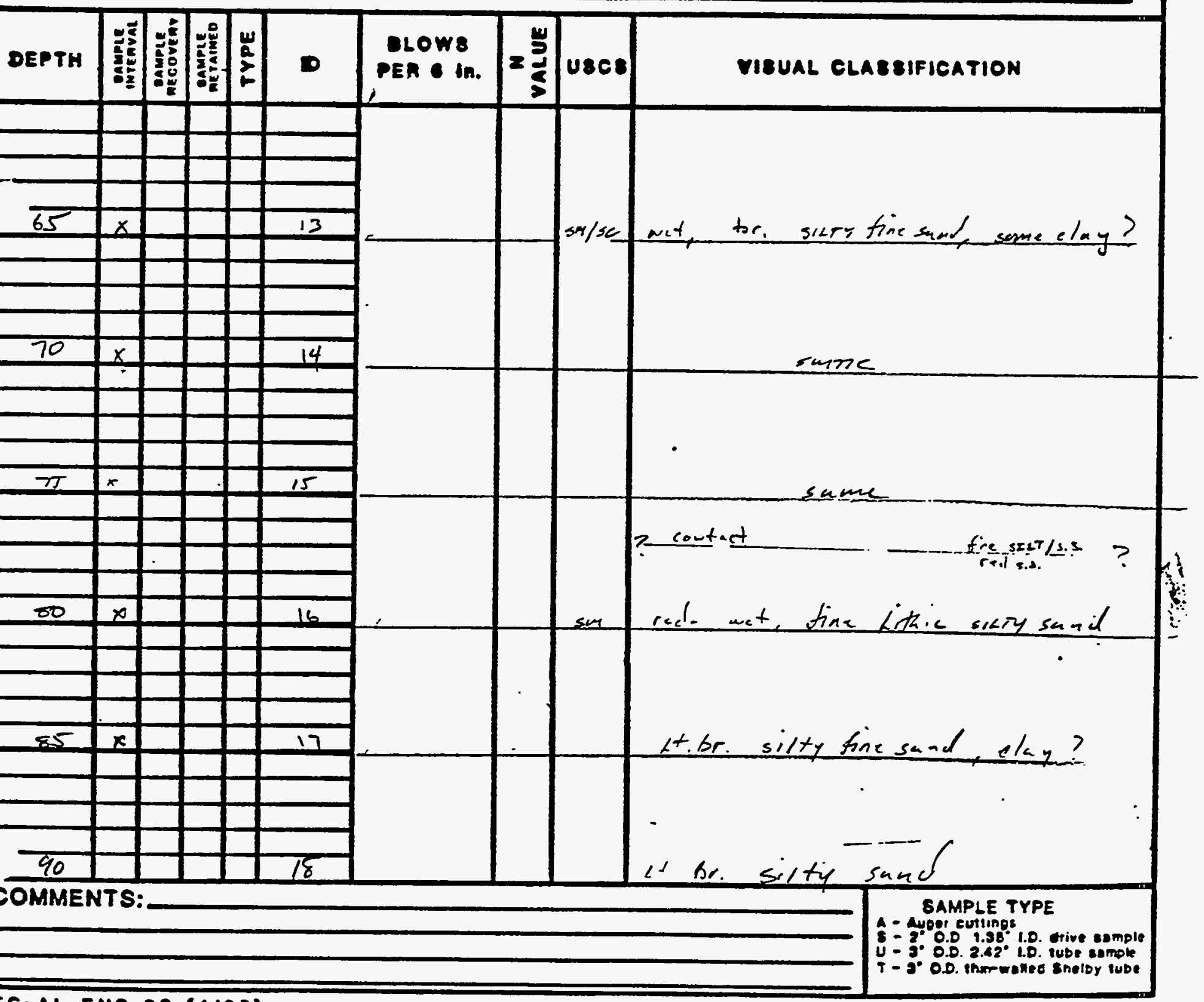


LOCATION DESCRIPTION

SITE CONDITION

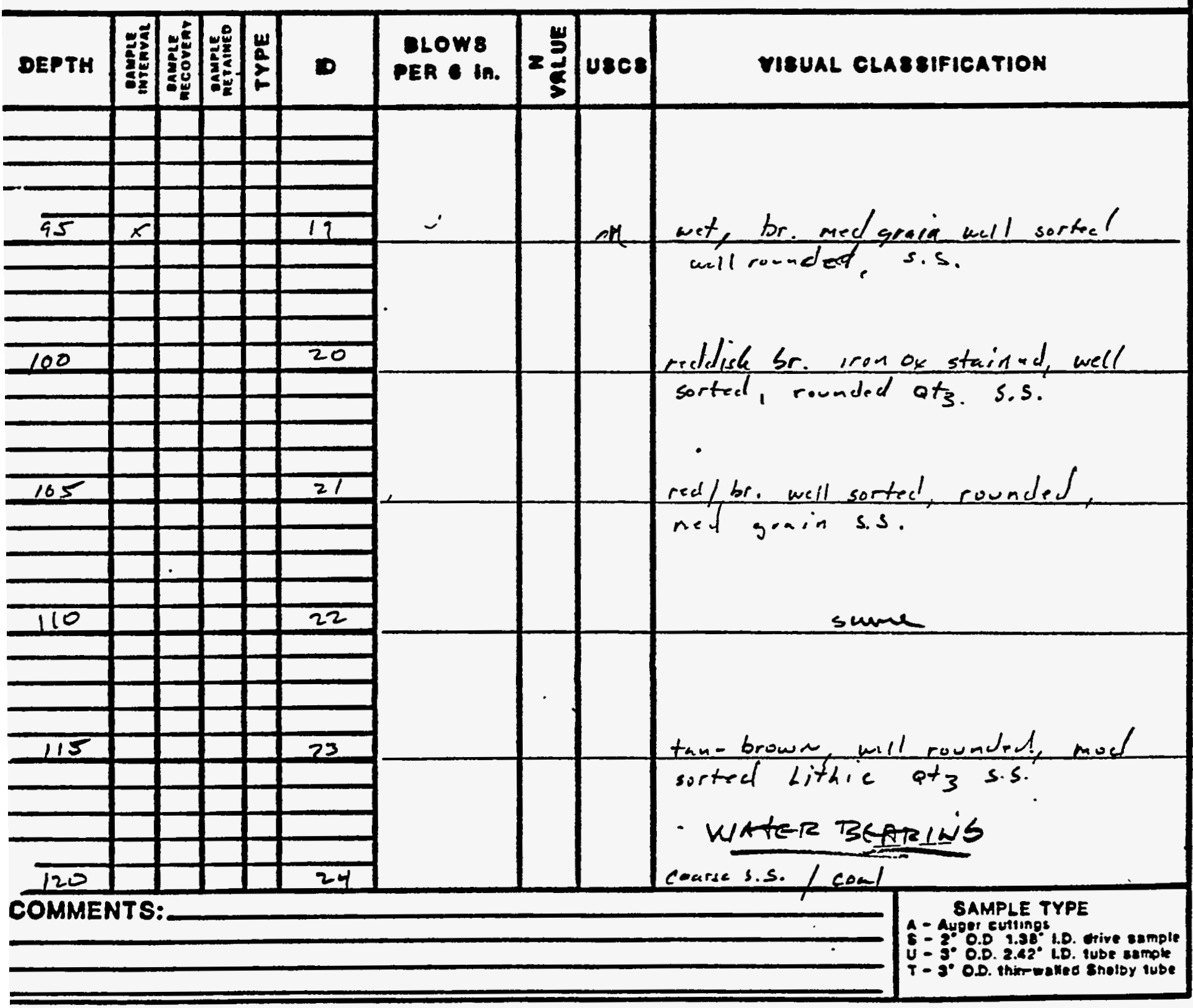

SITE ID: SPK-15 LOCATION ID: 144 SITE COOFIDINATES (ft.):

N. E

GROUND ELEVATION (ft. MSL):

DRILLING METHOD:

DRILLING CONTR.:

DATE STARTED:

DATE COMPLETED:

FIELD REP.:

*

\begin{tabular}{|c|c|c|}
\hline \multicolumn{3}{|c|}{ GROUNDWATER LEVELS } \\
\hline DATE & TIME & DEPTH (f1.) \\
\hline & & \\
\hline & & \\
\hline & & \\
\hline
\end{tabular}


7] LACOBS ENGINEERING GROUP INC.

ADVAMELD SYBTIMS DIVIBION, ALLUQUERQUE ORIRATIONS

BOREHOLE LOG (SOIL)

Page 5 of 5

LOCATION MAP:

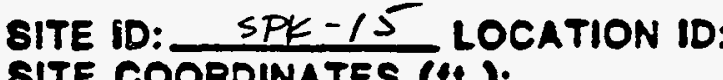

944

SITE COORDINATES (ft.):

N

E

GROUND ELEVATION (It. MSL):

DRILLING METHOD:

DRILLING CONTR.:

DATE STARTED:

DATE COMPLETED:

FIELD REP.:

\begin{tabular}{|c|c|c|}
\hline \multicolumn{3}{|c|}{ GROUNDWATER LEVELS } \\
\hline DATE & TIME & DEPTH (fi.) \\
\hline & & \\
\hline & & \\
\hline & & \\
\hline
\end{tabular}

LOCATION DESCRIPTION

SITE CONDITION

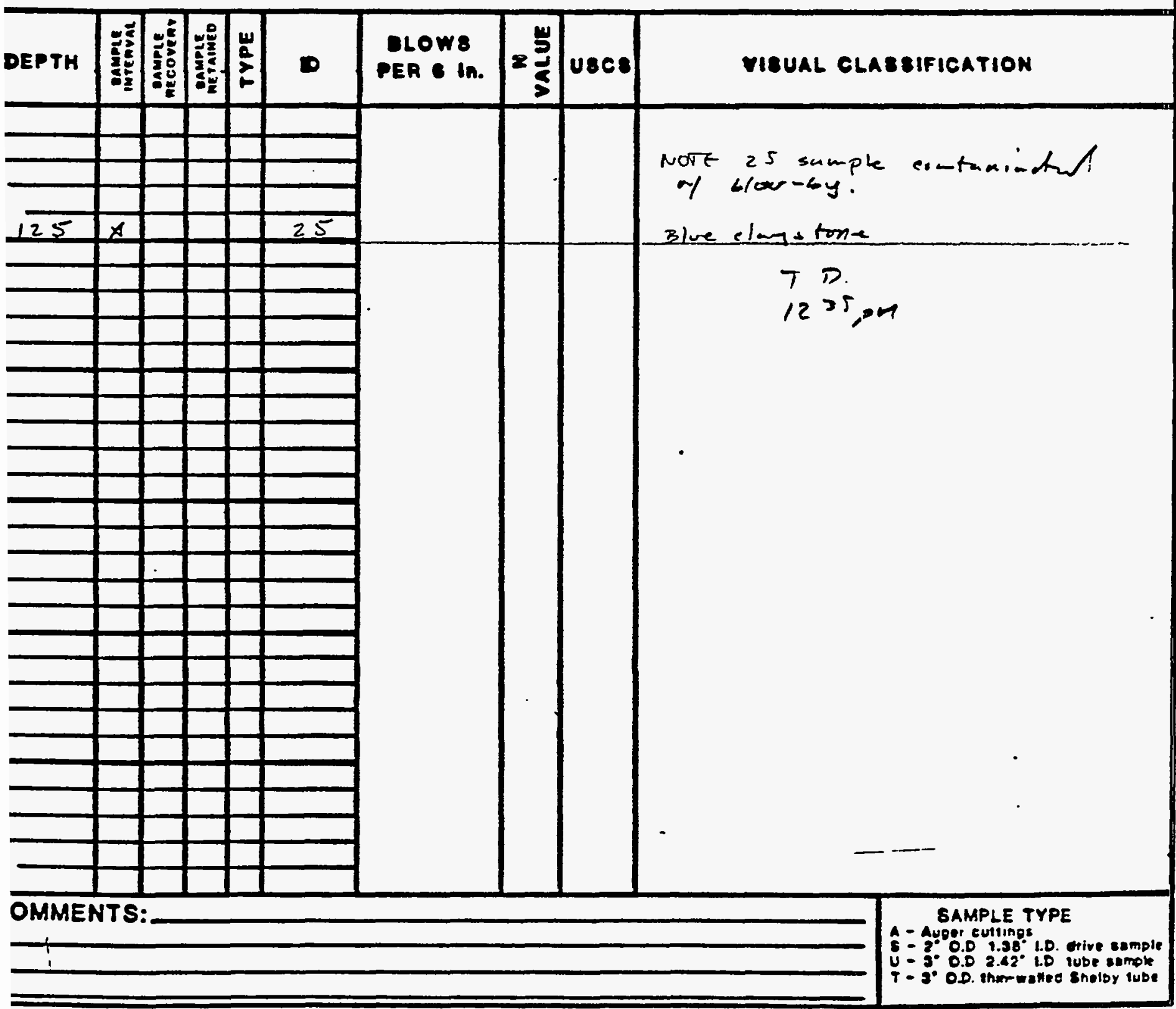


APPENDIX B

MONITOR WELL COMPLETION RECORDS 


\section{TT MCO3S ENeNEERTG EROUP NK. \\ $\because=$ \\ 3 \\ $\therefore$ YELL COMPLETION RECORD}

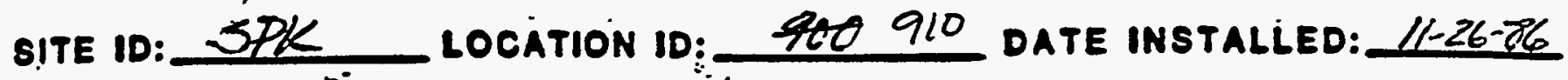
APPROX. SITE COOORDINATES:(FT.) $\mathrm{N}$ E OPEN AREA DER LINEAL FTGI (IN2/FT.)

FORMATION OF COMPLETION: FIELD REP.:

L. Plugfe

Wassatch

Sindstaxe

DRILLER: Boyles Bros.

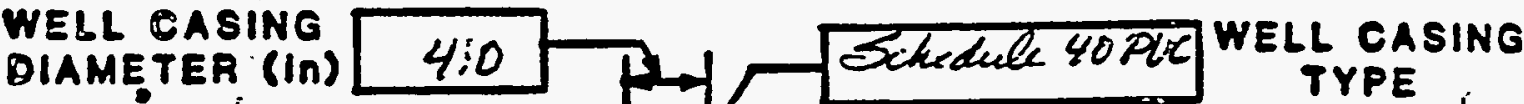

HOLE gIAMETER $(\ln ) 8.0$

GROUND SURFACE

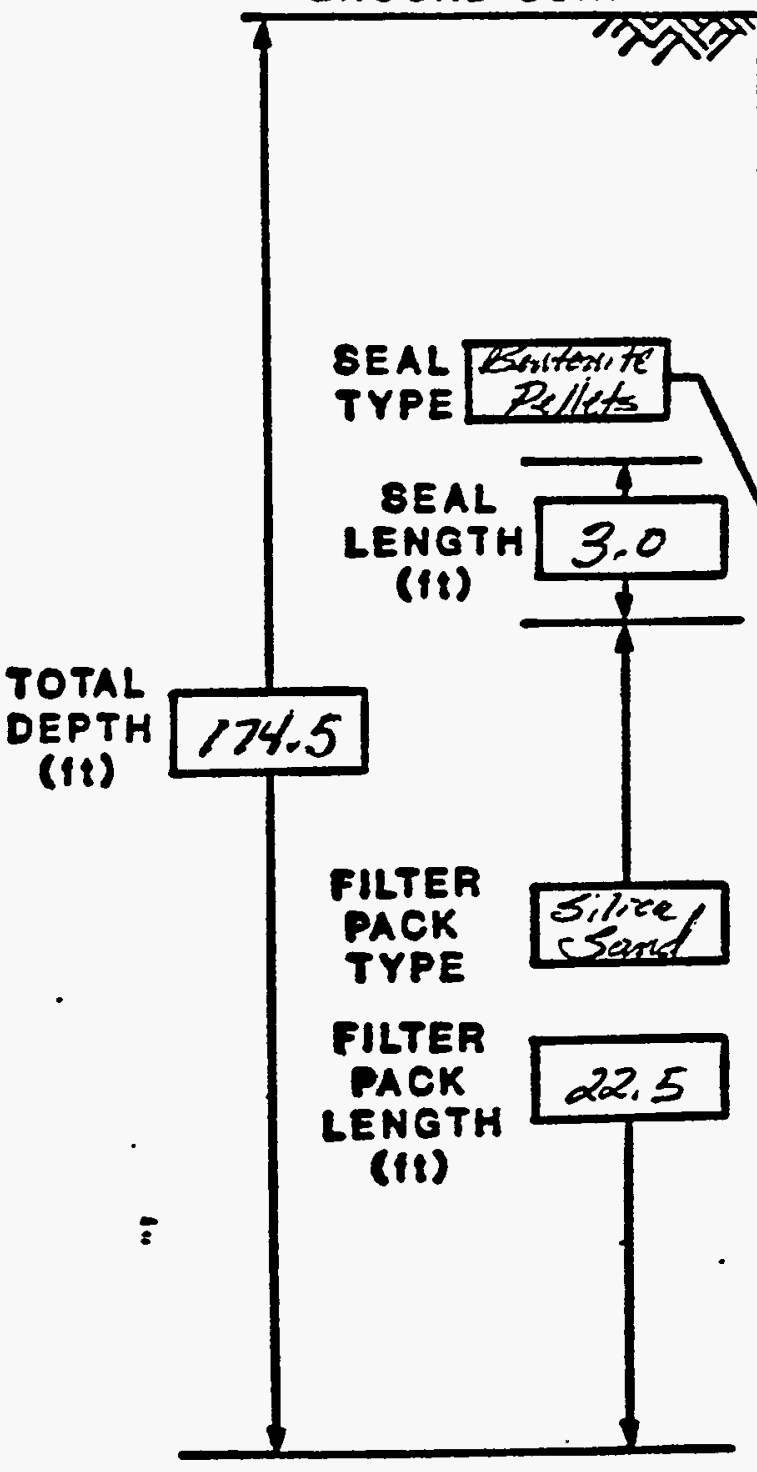

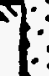

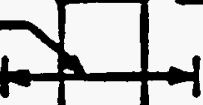

2.0 JROUND (It) $_{\text {GT. ABOVE }}^{\text {HT }}$

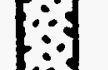

$\therefore$

BACKFILL TYPE

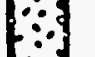

$\therefore$

Cement-Brestonide

$\therefore$

साष्र

$\because \vdots$
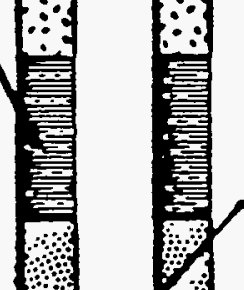

BLOT

OPENING

(in)
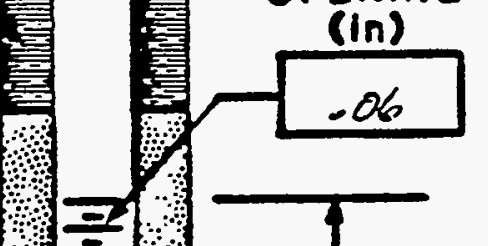

मी $=2$

OPEN OR

SLOTTED

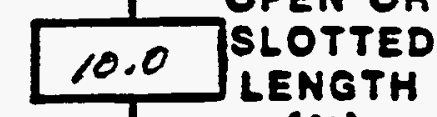

(II)

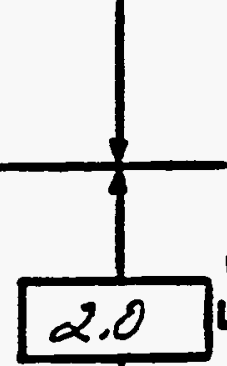

BLANK LENGTH

176.5

CASING LENGTH

(tt)

COMMENT8: 


\section{LACOBS ENGN ERTNG GROUP NC.

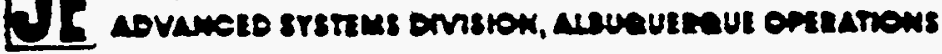 \\ BOREHOLE/WELL CONSTRUCTION LOG}

SITE ID:SFK LOCATION ID: 910 FIELD REP: L. Phafe APPROX. SITE COORDINATES (FT.): N E GROUND ELEVATION (FT. MSL): 5089 COMPLETION DATE: $11-26-86$

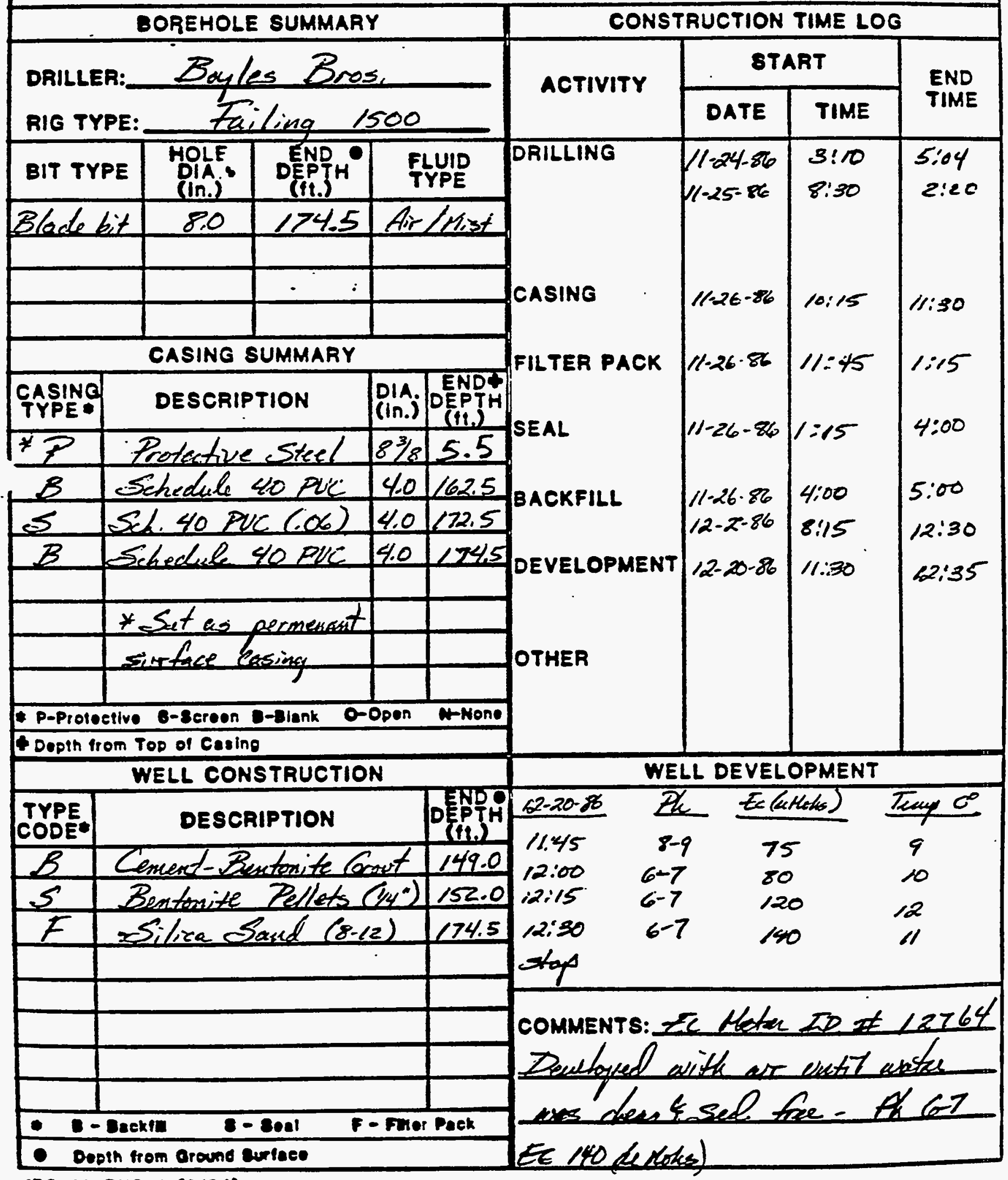




\section{IIE HAOBS ENGNEERNG CROUP NC.}

\section{. WELL COMPLETION RECORD}

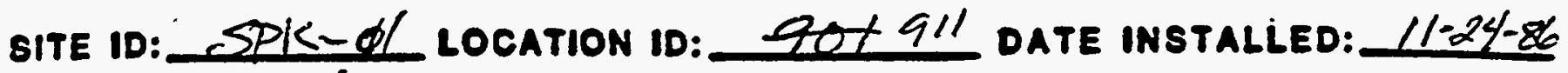
APPROX. SITE COORDINATES:(FT.) N OPEN AREA PER LINEAL FT. (IN2/FT.)

FORMATION OF COMPLETJON: Wlasatch (Sandstome)

FIELD REP.: DRILLER: E

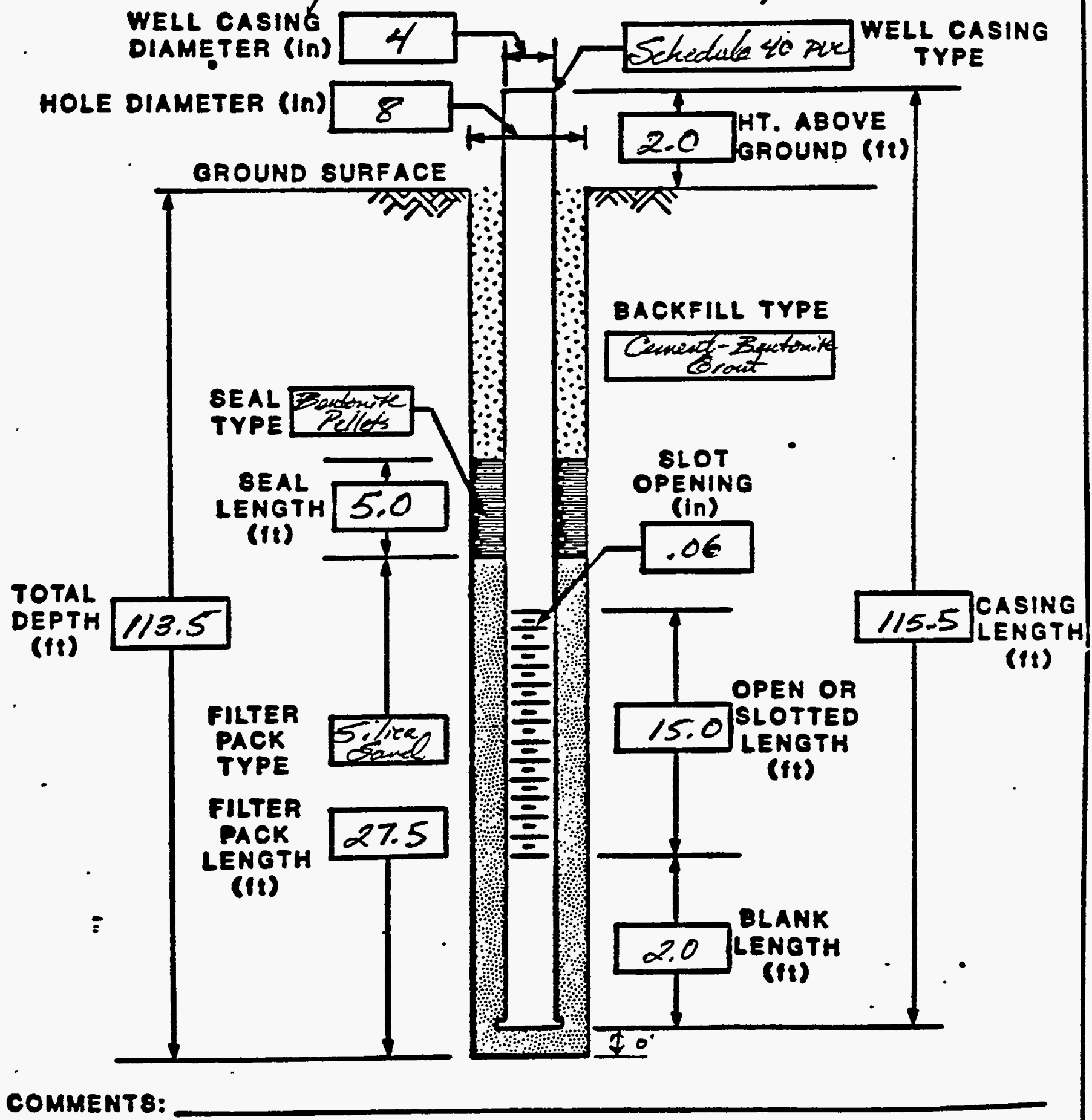

COMMENT 8: 


\section{TF JACOBS ENGN EERTV GROUP NC.

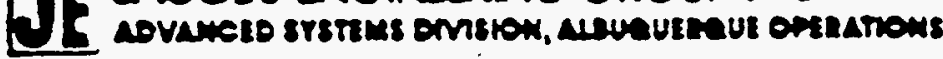 \\ BOREHOLE/WELL CONSTRUCTION LOG}

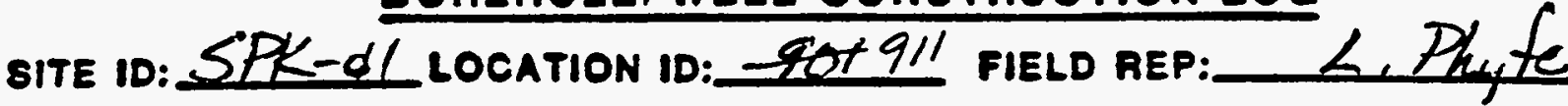
APPROX. BITE COORDINATES (FT.): $N$

OROUND ELEVATION (FT. MSL): 5090 E BOREHOLE SUMMARY

DRILLER:_Bxiles Bros.

RIG TYPE: Teiling

\begin{tabular}{|c|c|c|c|}
\hline BIT TYPE & $\begin{array}{l}\text { HOLE } \\
\text { DIA." } \\
\text { (In.) }\end{array}$ & $\begin{array}{c}\text { ENND } \\
\text { DEPTH } \\
\text { (fi.) }\end{array}$ & $\begin{array}{l}\text { FLUID } \\
\text { TYPE } \\
\end{array}$ \\
\hline Elade bit & 8 & 113.5 & Air \\
\hline & & & \\
\hline & & - & \\
\hline & & & \\
\hline
\end{tabular}

CASING SUMMARY

\begin{tabular}{|c|c|c|c|}
\hline CASING & DESCRIPTION & $\begin{array}{c}\text { DIA. } \\
\text { (IN.) }\end{array}$ & $\begin{array}{c}\text { ENPPT } \\
\text { THF. }\end{array}$ \\
\hline
\end{tabular}

* P Protective Steel $8 \% 18$ 5.0 B Sekedule 40 PLK 496.5

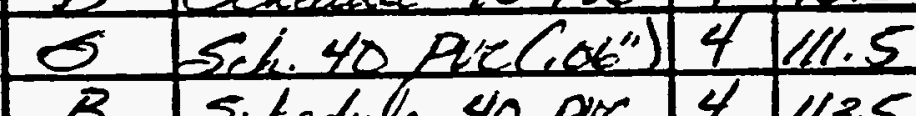

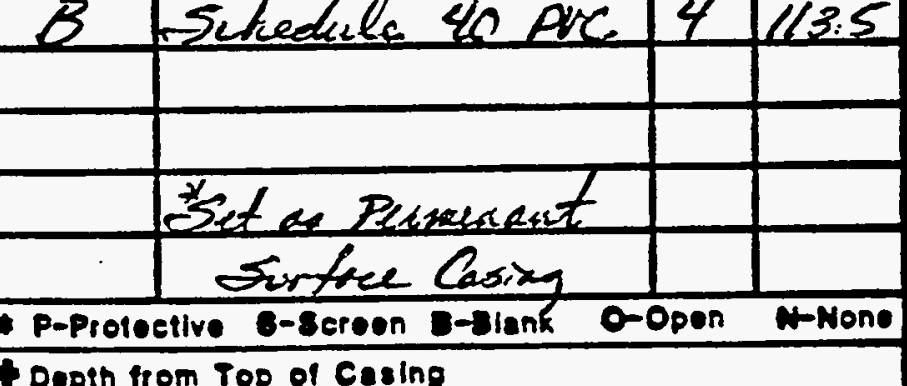
WELL CONSTRUCTION \begin{tabular}{|c|c|c|}
\hline $\begin{array}{c}\text { TYPE } \\
\text { CODE* }\end{array}$ & DESCRIPTION & DEFPPTH \\
\hline
\end{tabular}

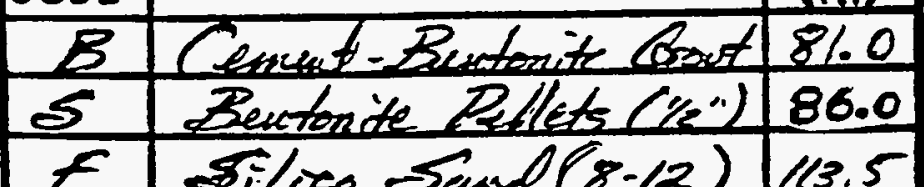
\begin{tabular}{|c|c|c|}
\hline$f$ & Siliza Seund $(8-12)$ & 13.5 \\
\hline & & \\
\hline
\end{tabular}
CASING CONSTRUCTION TIME LOG

\begin{tabular}{|c|c|c|c|}
\hline \multicolumn{4}{|c|}{ CONSTRUCTION TIME LO } \\
\hline \multirow{2}{*}{ ACTIVITY } & \multicolumn{2}{|c|}{ START } & \multirow{2}{*}{$\begin{array}{l}\text { END } \\
\text { TIME }\end{array}$} \\
\hline & DATE & TIME & \\
\hline DRILLING & $11-23-86$ & 2.04 & $3: 45$ \\
\hline CASING & $|1|-24-86$ & 10100 & 10.57 \\
\hline FILTER PACK & $1 /=24-86$ & $11: \infty$ & $12: 30$ \\
\hline SEAL & $11-24 \cdot 86$ & 12.130 & $2: 20$ \\
\hline BACKFILL & $11 / 224-86$ & 2.40 & 4,50 \\
\hline DEVELOPMENT & $\mid 12-20-88$ & $9: 30$ & $11: 00$ \\
\hline OTHER & & & \\
\hline \multicolumn{4}{|c|}{ WELL DEVELOPMENT } \\
\hline $12-20-26$ & \& & e(nots): & $\operatorname{mop}\left(e^{-}\right)$ \\
\hline $9: 45$ & $6-7$ & 80 & 9 \\
\hline $10: 15$ & $6-7$ & & 9 \\
\hline $10: 55$ & $6-7$ & & 10 \\
\hline $10: 50$ & $6-7$ & & 11 \\
\hline $\begin{array}{l}\text { COMMENTS: - } \\
\text { Deneloged }\end{array}$ & $\begin{array}{l}\text { Ee Mote } \\
\text { sith ase }\end{array}$ & $=12$ & $\frac{64}{\text { uratu }}$ \\
\hline
\end{tabular}

- Dooln from Oround curface

- Fuer Paek JEO-AL-ENO-I (B/84). 


\section{IE MCOBS ENCNEERTG GROUP INC.}

\section{WELL COMPLETION RECORD}

SITE ID: SAKE LI LOCATION ID: LLZ 912 DATE INSTALIED: $11-23.86$ APPROX. SITE COOORDINATES:(FT.) N E OPEN AREA.PER LINEAL FT. (IN2/FT.) FORMATION OF COMPLETION: FIELD REP.: Wasatels FIELD REP.

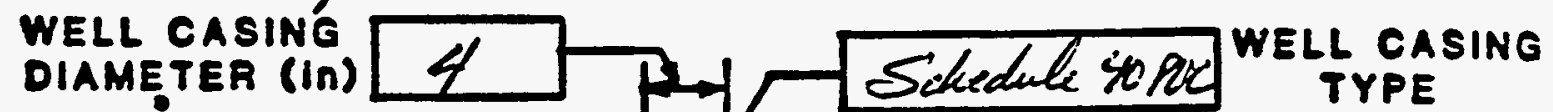
HOLE DIAMETER (in)

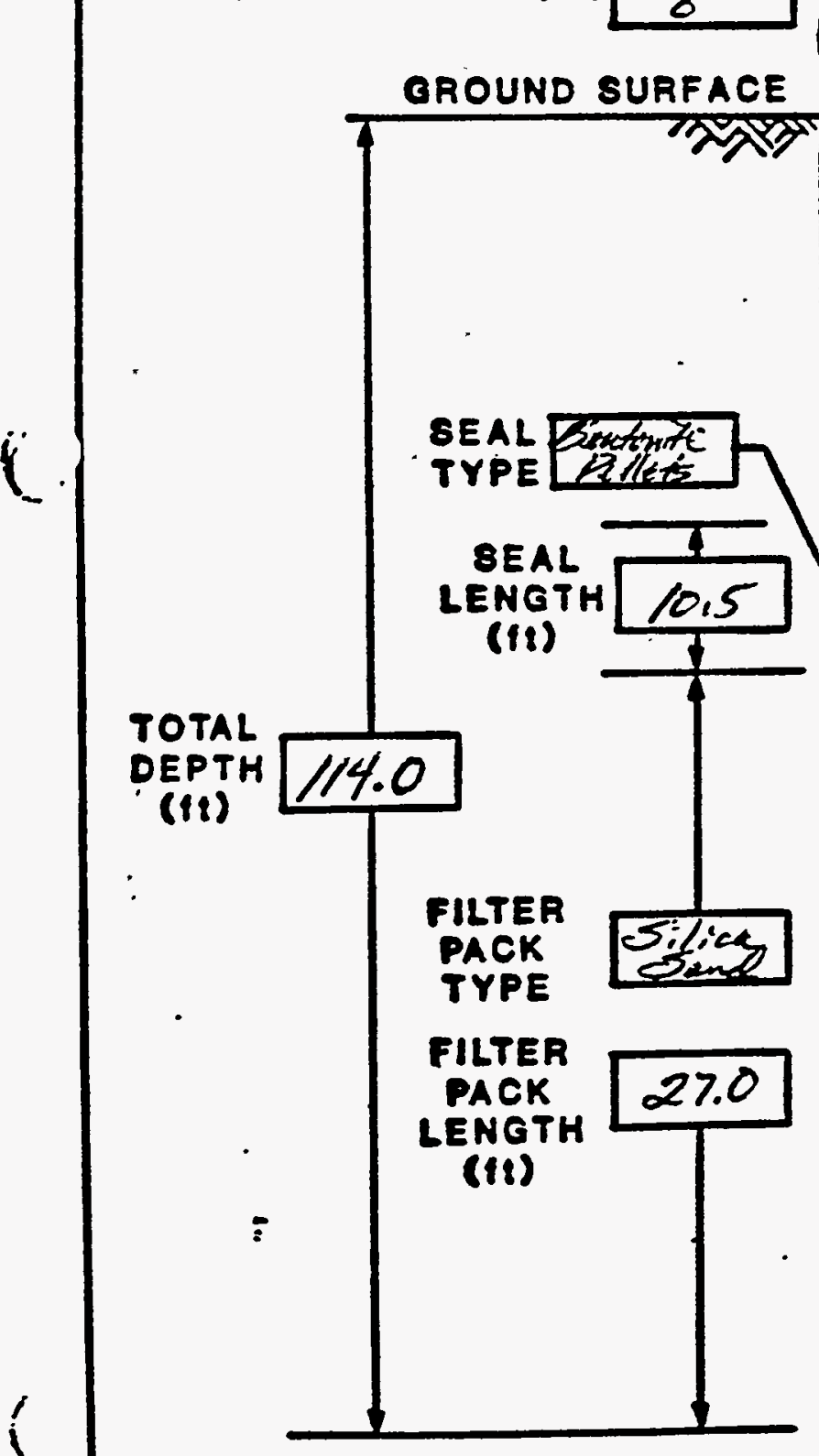

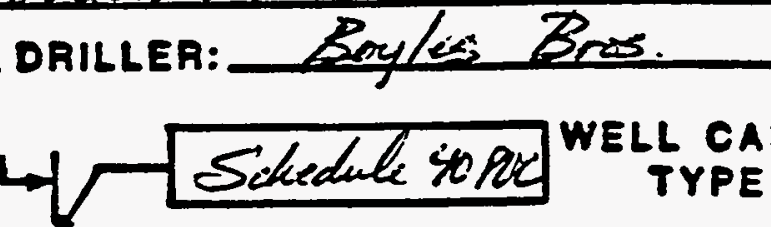<smiles>C1C2C3C4C1C1C2C3C41</smiles>

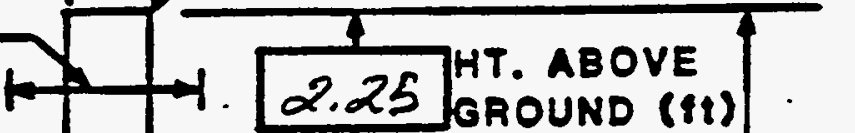

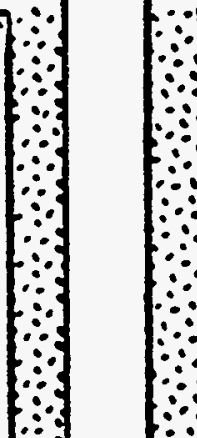

BACKFILL TYPE Cemigt-genterift
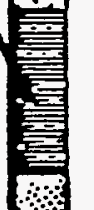

BLOT
OPENING (in)
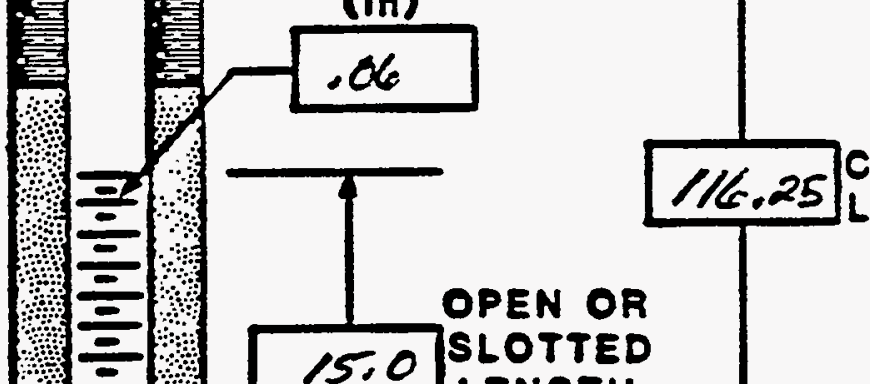

OPEN OR

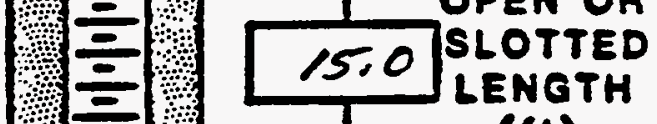

(fis)
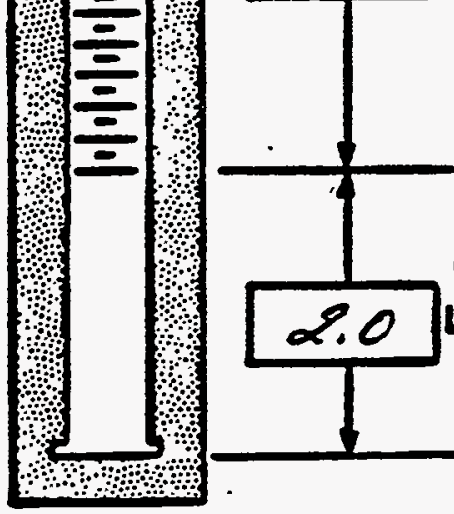

BLANK LENGTH (f1)

COMMENT 8 : 


\section{TF LACOBS ENGNEERIV GROUP NC.

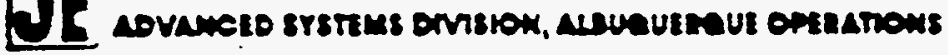 \\ BOREHOLE/WELL CONSTRUCTION LOG}

SITE ID: SEK- Q1 LOCATION ID: GEZ 912 FIELD REP: APPROX. SITE COORDINATES (FT.): $N$ E GROUND ELEVATION (FT. MSL): 5100 COMPLETION DATE: $11-23.86$

BOREHOLE SUMMARY

DRILLER: Beyles Pros.

RIG TYPE:

Fiv

\begin{tabular}{|c|c|c|c|}
\hline BIT TYPE & 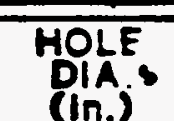 & DEN & $\begin{array}{l}\text { FUUID } \\
\text { TYPE }\end{array}$ \\
\hline Trieone & 12 & 5.5 & As \\
\hline Trues & 8 & 114.0 & qicl thist \\
\hline & & & \\
\hline
\end{tabular}

CASING SUMMARY

\begin{tabular}{|c|c|}
\hline $\begin{array}{l}\text { CASING } \\
\text { TYPE: }\end{array}$ & DESCRIPTION \\
\hline
\end{tabular}

\begin{tabular}{l|l|l|l|}
$*$ & Protective Steel & 878 & 5.5 \\
\hline
\end{tabular}

\begin{tabular}{|c|c|c|c|}
\hline$B$ & Seluedule te PIC & 4 & 97.0 \\
\hline 5 & Sols 40 pic slatid & 4 & 112.0 \\
\hline$B$ & Sebedich to Plic & 4 & 14,0 \\
\hline & & & \\
\hline & +Setas piemenant & & \\
\hline & Eurface I & & \\
\hline & . & & \\
\hline
\end{tabular}

- P-Protective S-Bcreen B-Blank O-Open W-None Sopih from Top of Casing

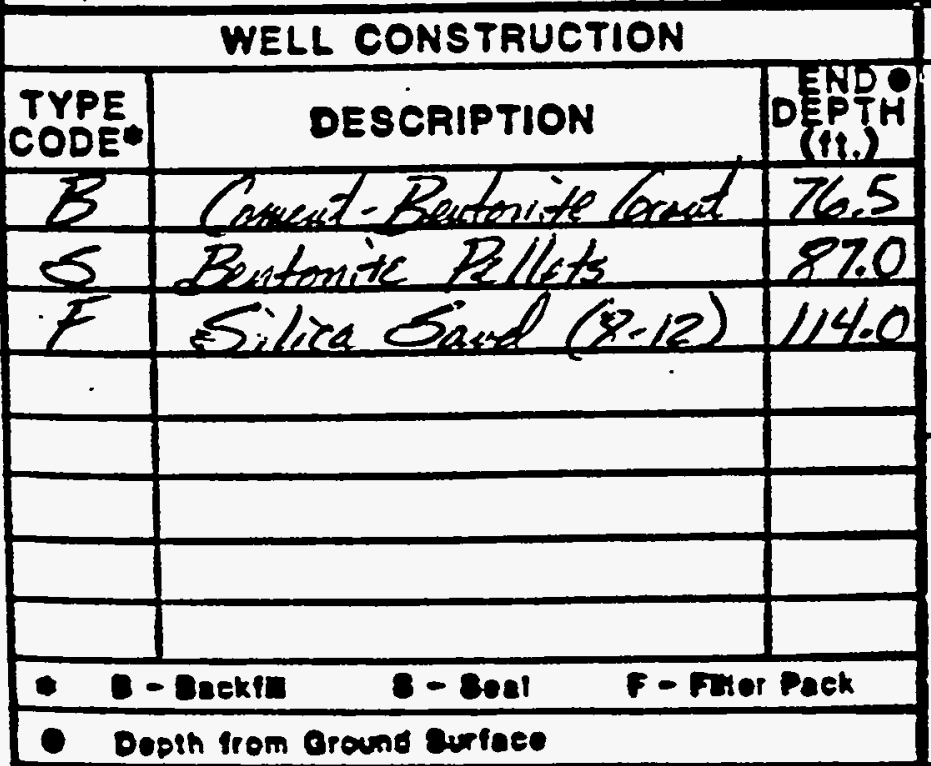

\section{CASING} CONSTRUCTION TIME LOG

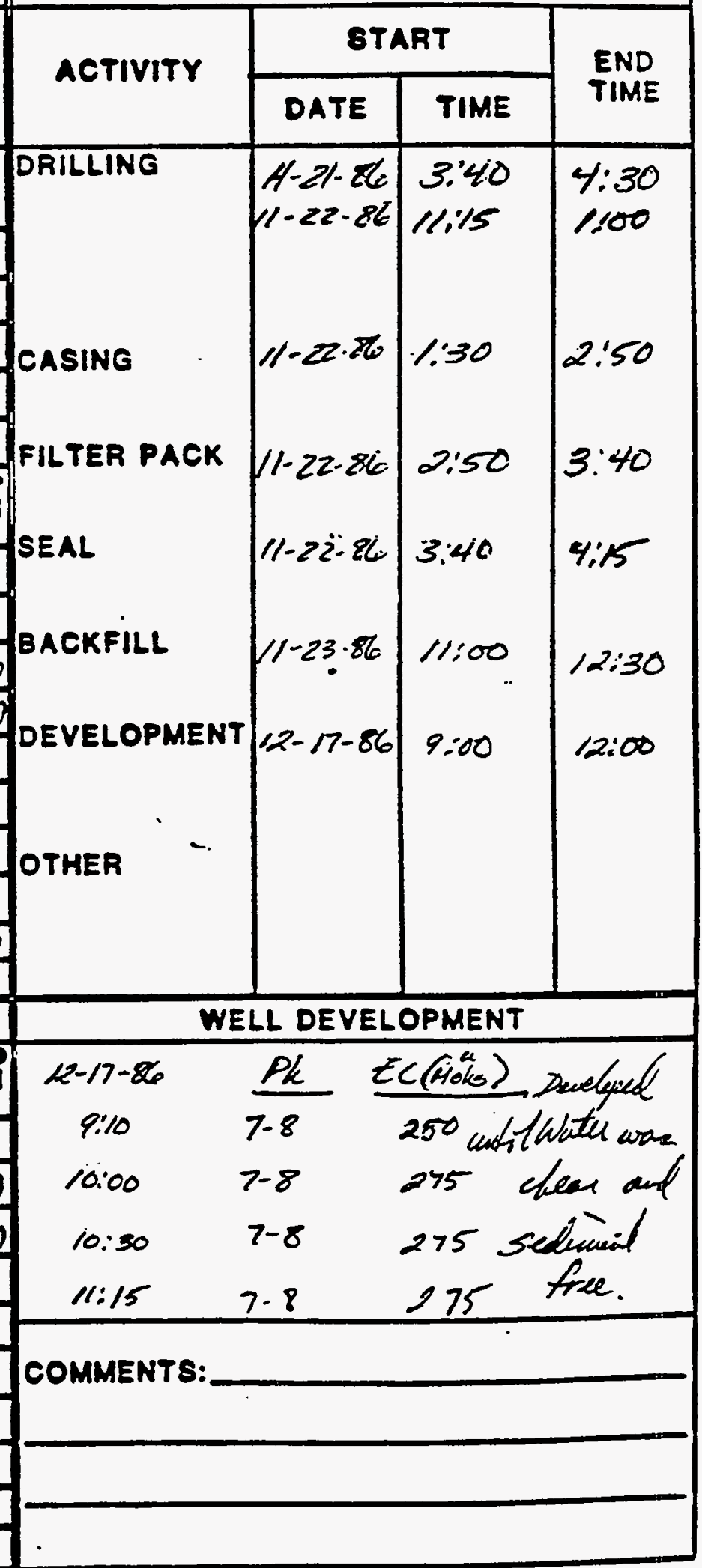




\section{TT MCOBS ENEN EBRING GROUP NC.

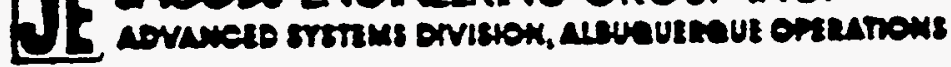

WELL COMPLETION RECORD

SITE ID: SPK- D/ LOCATION ID: 9O3 913 DATE INSTALLED: $12.14-86$ APPROX. SITE COORDINATES:(FT.) N E OPEN AREA.PER LINEAL FT. (IN2/FT.) FORMATION OF COMPLETION: FIELD REP.: K. Zlüfe Wasatch (ss/sk) WELL CASING 4.0 SiNhedule 40 PLE WELL CASING
DIAMETER (In)

HOLE DIAMETER (In) 8.0 GROUND SURFACE

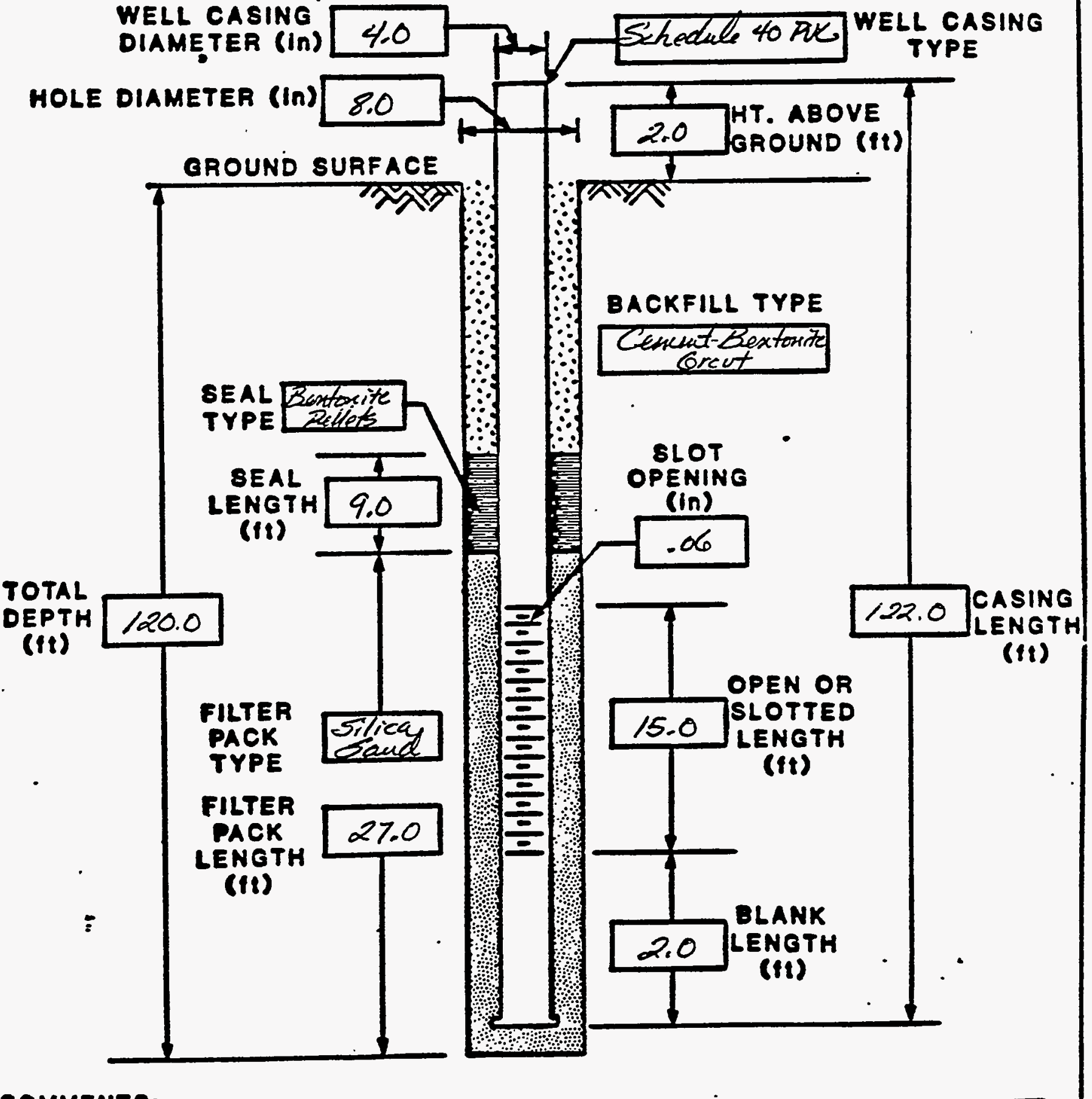
ORILLER: Boykes Bras.

COMMENT 8: 


\section{TF LCOBS BNENEERTE EROUP NC.

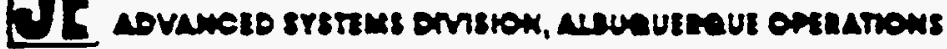 \\ BOREHOLE/WELL CONSTRUCTION LOG}

SITE ID:SPK- SL LOCATION ID: 93913 FIELD REP:

APPROX. BITE COORDINATES (FT.): $N$ E

GROUND ELEVATION (FT. MSL):

5090

COMPLETION DATE: $12-14-86$

\begin{tabular}{l} 
BOREHOLE SUMMARY \\
DRILLER:_Boyles Bros. \\
RIG TYPE: Tailing 1500 \\
\hline
\end{tabular}

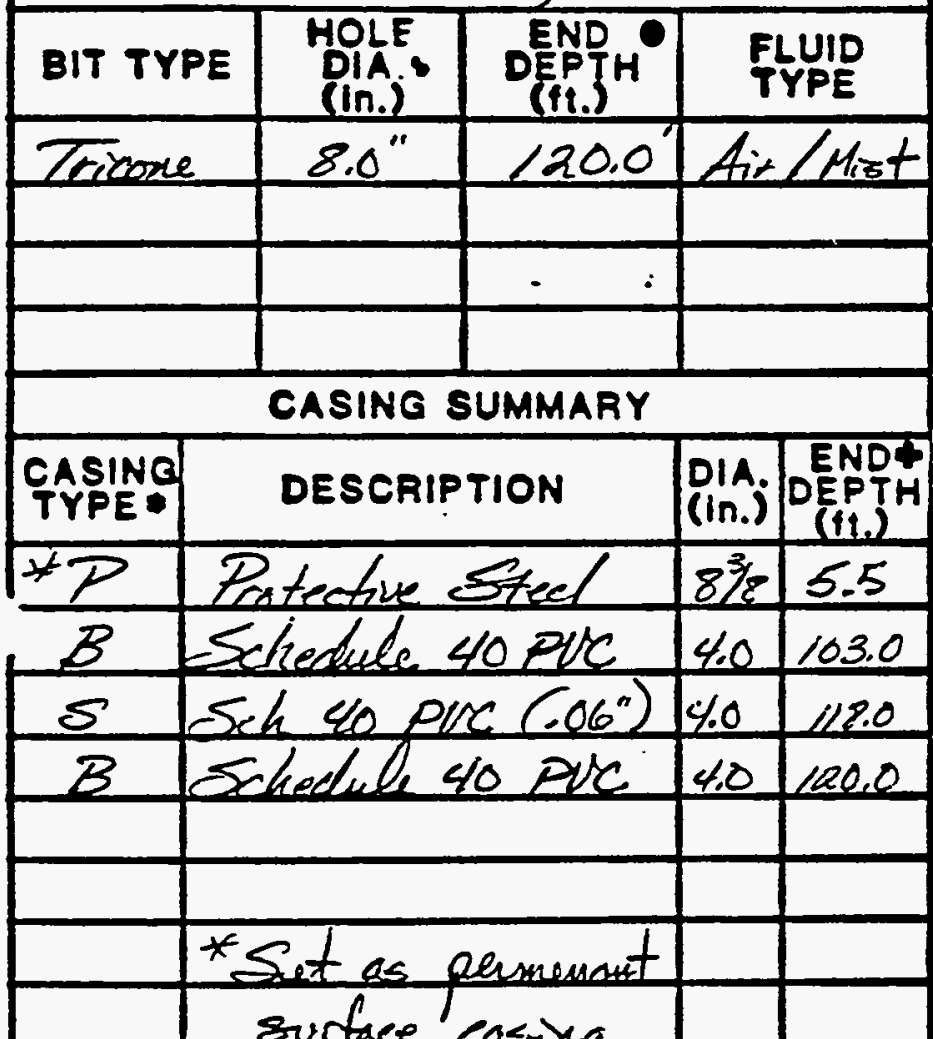
- p-Protective B-sereon D-siank O-Opon a-None - Depth from Tod of Casing

\begin{tabular}{|c|c|c|}
\hline \multicolumn{3}{|c|}{ WELL CONSTRUCTION } \\
\hline $\begin{array}{l}\text { TYPE } \\
\text { CODE }\end{array}$ & DESCRIPTION & DEF \\
\hline $\bar{B}$ & A- Bentorite Gront & 84.0 \\
\hline$\delta$ & Bestonite Pelilets ("k.) & 93.0 \\
\hline$F$ & Edo Silire Sand $8-12)$ & 120.0 \\
\hline & & \\
\hline & & \\
\hline & & \\
\hline & & \\
\hline & & \\
\hline - & $8-8001$ & Peek \\
\hline - $D_{0}$ & in from around eurfece & \\
\hline
\end{tabular}

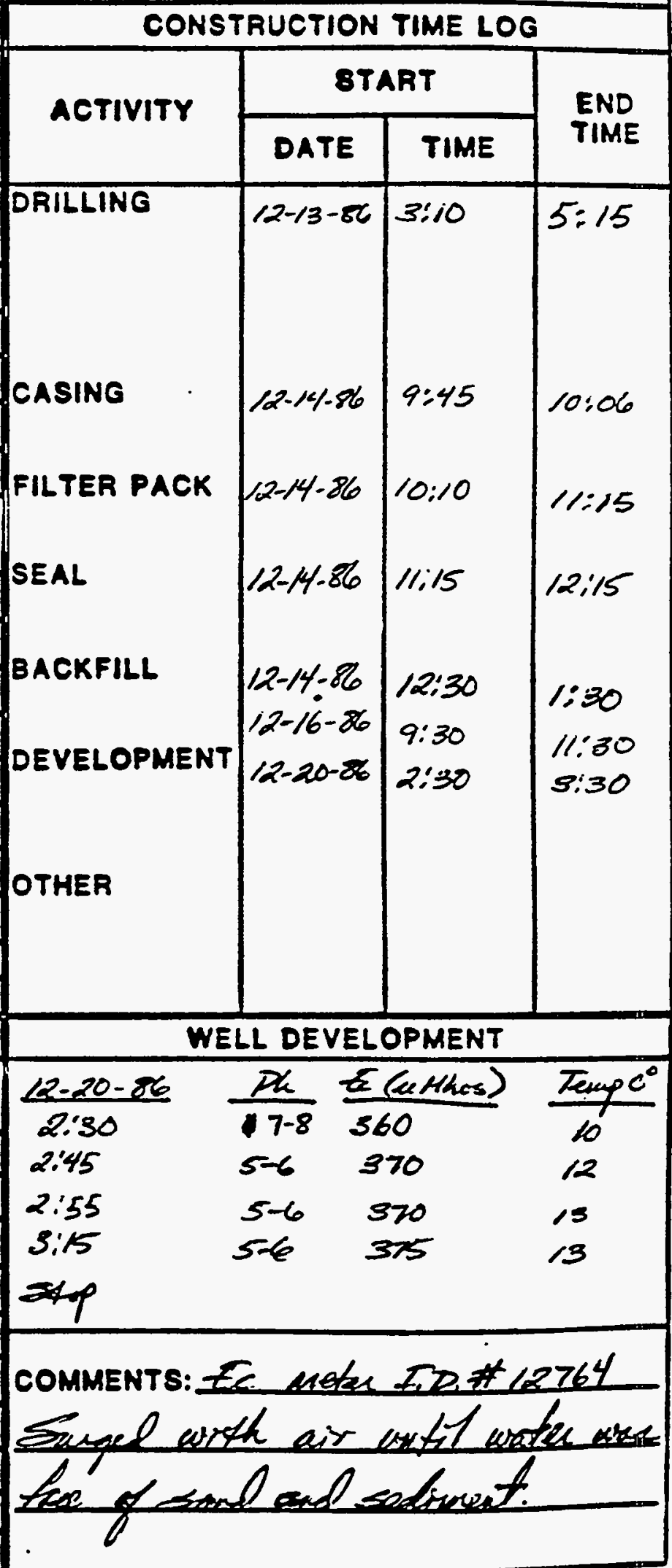




\section{WE MAOOBS BNENEERNG GROUP NC.}

\section{WELL COMPLETION RECORD}

SITE ID:SPE-D/ LOCATION ID: 904914 DATE INSTALLED: $12-15-816$ APPROX. SITE COORDINATES:(FT.) N E OPEN AREA.PER LINEAL FT. (IN2/FT.) FORMATION OF COMPLETION: FIELD REP.: Wasatel (sk/ss)

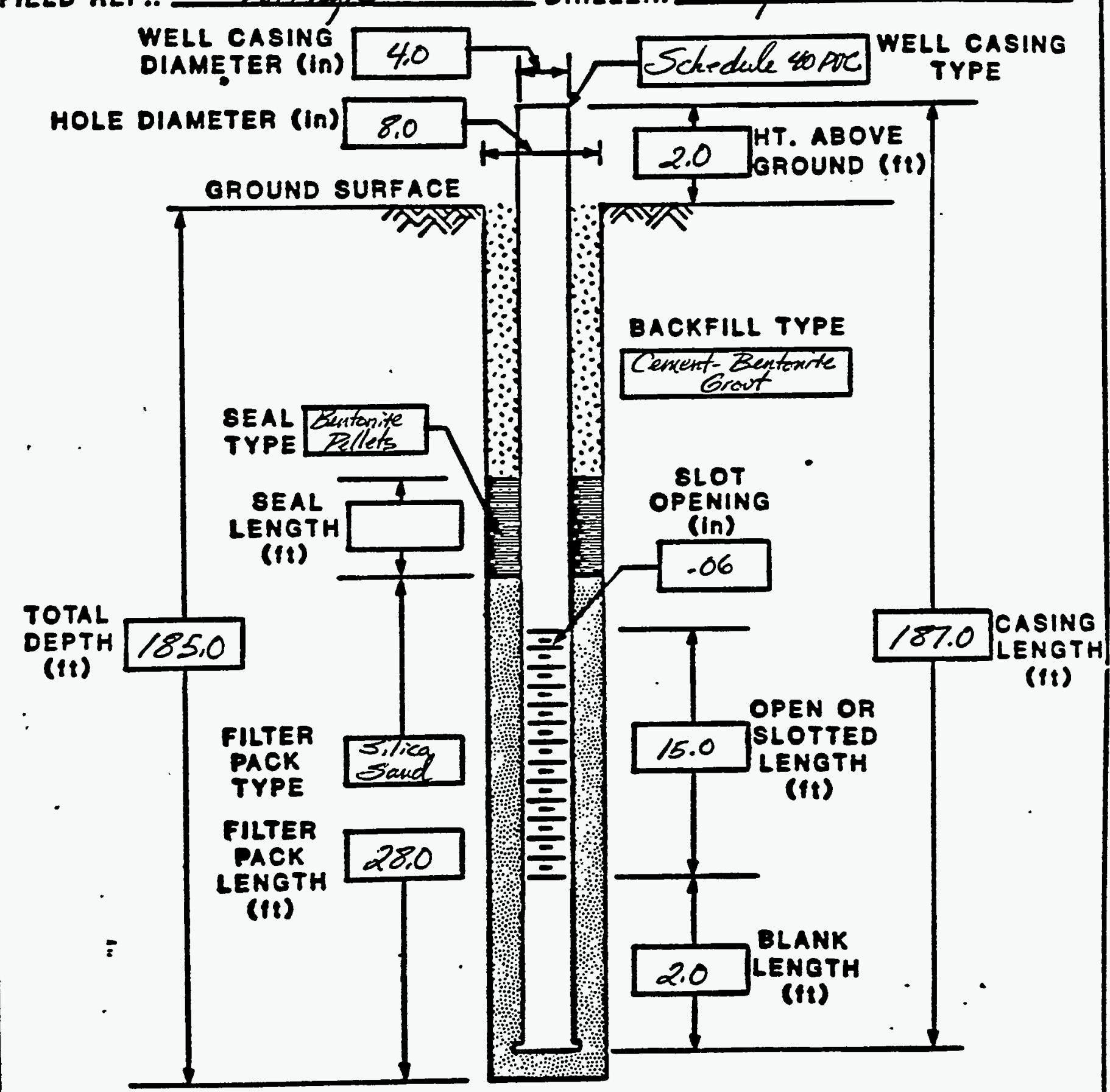

COMMENT 8: 


\section{TIE HAOOBS ENG EERTE GROUP INC.

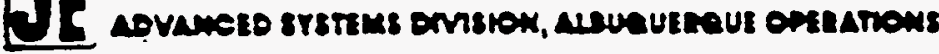 \\ BOREHOLE/WELL CONSTRUCTION LOG}

SITE ID:SRE- d/ LOCATION ID: GOH 914 FIELD REP:_L. Phyfe APPROX. SITE COORDINATES (FT.): N E GROUND ELEVATION (FT. MSL): $\quad 5090$

COMPLETION DATE: $122-15-86$

\section{BOREHOLE SUMMARY}

DriLler: Boyjes Bros.

RIG TYPE:

BIT TYPE

\begin{tabular}{|c|c|c|c|}
\hline BIT TYPE & $\begin{array}{l}\text { HOLE } \\
\text { DiA.) } \\
\text { (In.) }\end{array}$ & $\begin{array}{l}\text { END } \\
\text { DEPTH } \\
\text { (ft.) }\end{array}$ & $\begin{array}{l}\text { FLUID } \\
\text { TYPE }\end{array}$ \\
\hline Tricouce & 8.0 & 185.0 & Art lMist \\
\hline & & & \\
\hline & & - & \\
\hline & & & \\
\hline
\end{tabular}

CASING SUMMARY

\begin{tabular}{|l|l|}
\hline CASING & DESCRIPTION \\
\hline TYPE:
\end{tabular}

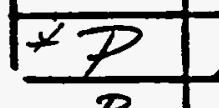

Protective

tai

\begin{tabular}{|c|c|}
\hline DIA. & $\begin{array}{c}\text { ENDO } \\
\text { (In.) }\end{array}$ \\
\hline DETH $_{(41 .)}$ \\
\hline $3 / 8$ & 5.5 \\
\hline
\end{tabular}

$B$ Schedule 40 pre $4.0 \quad 168.0$

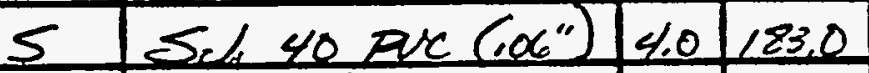

\begin{tabular}{l|l|l|l}
3 & Sehedule 40 PuC & 4.0 & 185.0
\end{tabular}

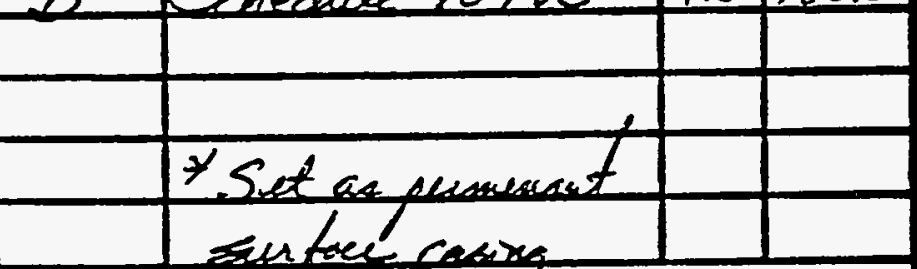

- p-Protoetive ésereon D-alimik O-Opon a-None

CDopth from TOD of Casing

WELL CONSTRUCTION

\begin{tabular}{|c|c|c|}
\hline $\begin{array}{l}\text { TYPE } \\
\text { CODE }\end{array}$ & $\therefore \quad$ DESCRIPTION & DEPTH \\
\hline 8 & Cenent-Bentonit Grout & 147.0 \\
\hline 5 & - Bestonite Rellets ("1.") & 157.0 \\
\hline$F$ & Colo. Siliea -Saud $(8-12)$ & 1850 \\
\hline & & \\
\hline & & \\
\hline & & \\
\hline & & \\
\hline & & \\
\hline - 8- & $8-80 a 1$ & Paek \\
\hline \multicolumn{3}{|c|}{ - Depin from Oround eurfaeo } \\
\hline
\end{tabular}

CASING

\begin{tabular}{|c|c|c|c|}
\hline \multicolumn{4}{|c|}{ CONSTAUCTION TIME LOG } \\
\hline \multirow{2}{*}{ ACTIVITY } & \multicolumn{2}{|c|}{ START } & \multirow{2}{*}{$\begin{array}{l}\text { END } \\
\text { TIME }\end{array}$} \\
\hline & DATE & TIME & \\
\hline DRILLING & $\mid 12-14-86$ & $1: 45$ & $5: 00$ \\
\hline CASING & $12-15-80$ & liso & $2: 40$ \\
\hline FILTER PACK & $12-15 \cdot 26$ & $2: 45$ & $3: 26$ \\
\hline SEAL & $12-15 \cdot 86$ & $3: 26$ & $4: 00$ \\
\hline BACKFILL & $12-16-86$ & $A=30$ & $2: 00$ \\
\hline DEVELOPMENT & $12-20-86$ & $4: 00$ & $5: 5$ \\
\hline OTHER & & & \\
\hline \multicolumn{4}{|c|}{ WELL DEVELOPMENT } \\
\hline $12-20-86$ & $P K$ E & (uthos) & Iuxp $C^{\circ}$ \\
\hline $4: \infty$ & 5 & 400 & 10 \\
\hline $4: 20$ & $5-6$ & 400 & 10 \\
\hline $4: 40$ & $6-7$ & $\begin{array}{l}330 \\
340\end{array}$ & 11 \\
\hline $\begin{array}{l}4: 55 \\
540\end{array}$ & $6-7$ & & / \\
\hline $\begin{array}{l}\text { COMMENTS: } \\
\text { Dueloped as }\end{array}$ & $\begin{array}{l}\text { Is mete } \\
\text { sith air }\end{array}$ & $\frac{I D}{\text { entil }}$ & $\frac{12764}{6}$ \\
\hline
\end{tabular}




\section{TT MCOBS BNENEERIVG GROUP NC.

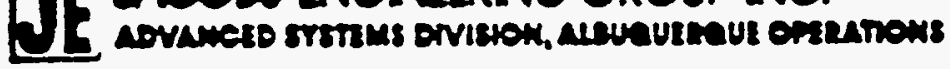 \\ WELL COMPLETION RECORD}

SITE ID: SPK-OD/ LOCATION ID: TOE 915 DATE INSTALIED: $15-10-86$ APPROX. SITE COORDINATES:(FT.) $N$ E OPEN AREA.PER LINEAL FT. $\left(I N^{2} / F T.\right)$

FORMATION OF COMPLETION:

FIELD REP.: L Pugfe

DRILLER: Bugles Bras

WELl CASING 40
DIAMETER (IN) 4.0

HOLE DIAMETER $(\ln ) 8.0$
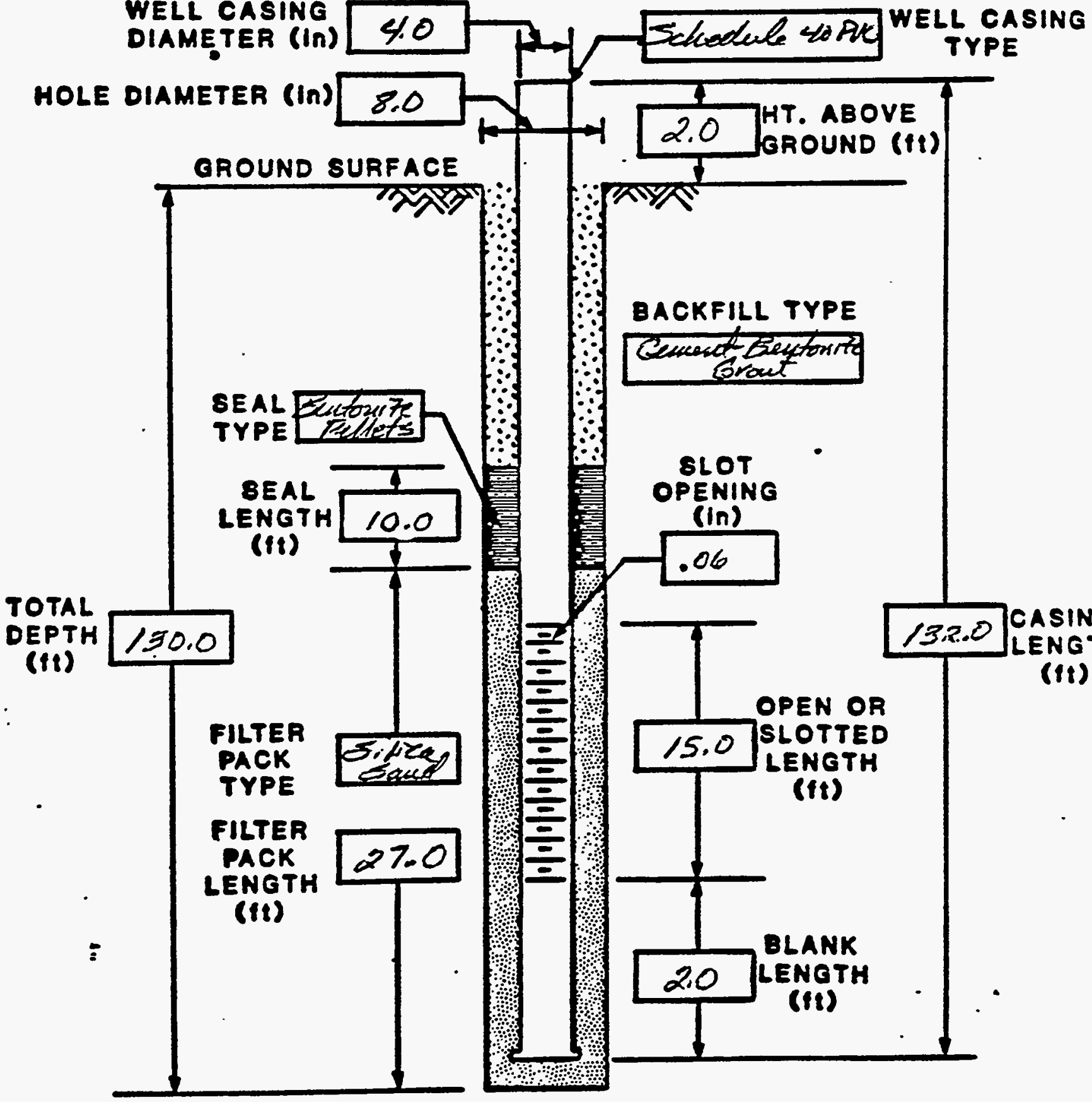

TOTAL DEPTH (1t)

COMMENT8:

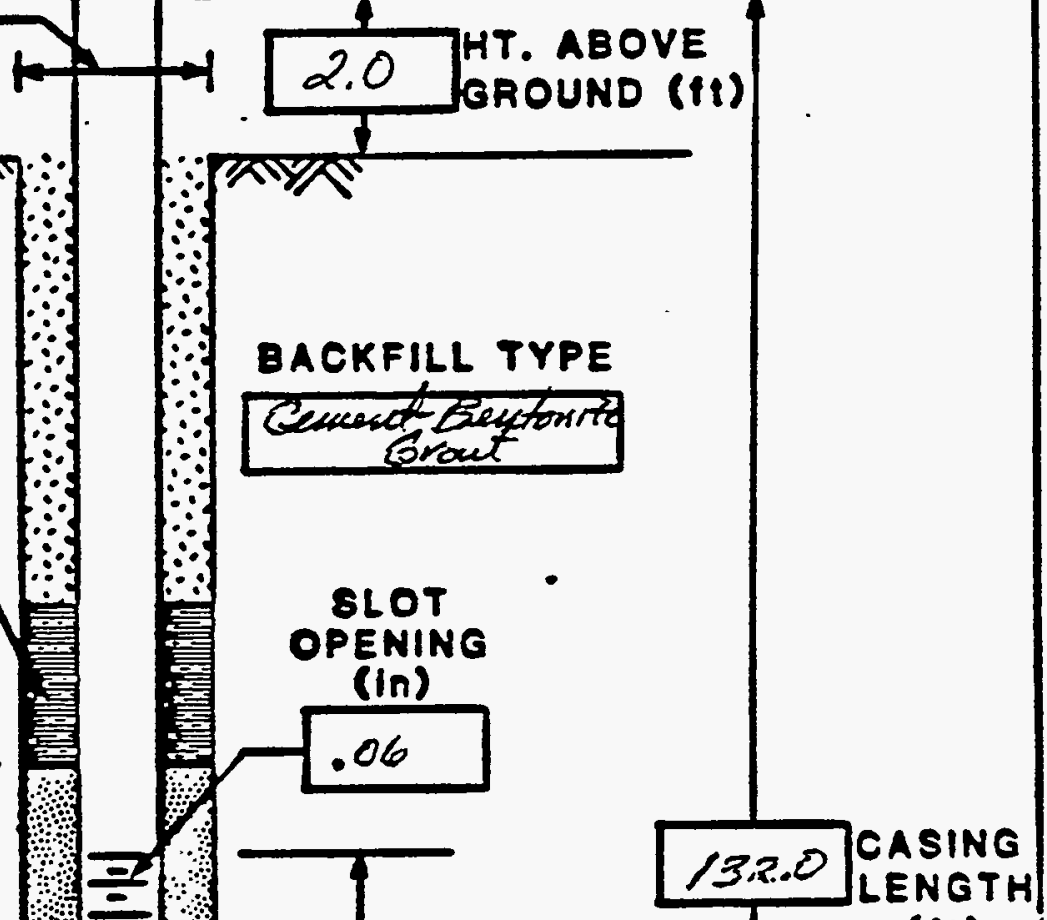

(II) 


\section{TF 14 COSS ENGI EERTG EROUP INC.

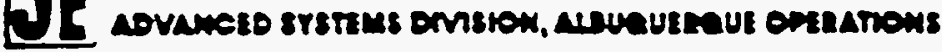 \\ BOREHOLE/WELL CONSTRUCTION LOG}

SITE ID: SFK- dL LOCATION ID: fOF 915 FIELD REP:_L Pluste APPROX. SITE COORDINATES (FT.): $N$ E GROUND ELEVATION (FT. MSL):

COMPLETION DATE: $12-10-86$ BOREHOLE SUMMARY

DRILLER: Boyles Bres. RIG TYPE: Friling 1500

\begin{tabular}{|c|c|c|c|}
\hline BIT TYPE & $\begin{array}{l}\text { HOLE } \\
\text { DiA.; } \\
\text { (In.) }\end{array}$ & $\begin{array}{l}\text { END } \\
\text { DEPFH } \\
\text { (ft.) }\end{array}$ & $\begin{array}{l}\text { FLUID } \\
\text { TYPE }\end{array}$ \\
\hline Tricione & 80 & 130.0 & $A=/ H_{1} \leq t$ \\
\hline & & & \\
\hline & & - & \\
\hline & & & \\
\hline
\end{tabular}

CASING SUMMARY

\begin{tabular}{|c|c|c|c|}
\hline $\begin{array}{l}\text { CASING } \\
\text { TYPE }\end{array}$ & DESCRIPTION & $\begin{array}{l}\text { DiA. } \\
\text { (in.) }\end{array}$ & $\begin{array}{l}\text { END' } \\
\text { DEPTH } \\
(4 t .)\end{array}$ \\
\hline$* p$ & Patective sted & $8 \frac{3}{8}$ & 5.5 \\
\hline$B$ & sckedule 40 pre & $\div 0$ & 1130 \\
\hline 5 & Sekedule 40 pre Coto & 4.0 & 128.0 \\
\hline$E$ & Shledule is pre & 4.0 & 130.0 \\
\hline & & & \\
\hline & & & \\
\hline & * Setas & & \\
\hline & ourface Casing & & \\
\hline
\end{tabular}

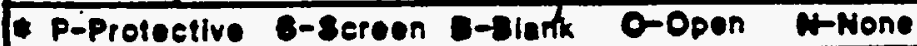
Coppth from TOD ol Casing

\begin{tabular}{|c|c|c|}
\hline \multicolumn{3}{|c|}{ WELL CONSTRUCTION } \\
\hline $\begin{array}{l}\text { TYPE } \\
\text { CODE }\end{array}$ & DESCRIPTION & De \\
\hline$B$ & Cement- Berctorite burt & 93.0 \\
\hline 5 & Bustonite Pellet ("4") & 10000 \\
\hline$F$ & Solor Silica Sind $(8-12)$ & 130.0 \\
\hline & & \\
\hline & & \\
\hline & & \\
\hline & & \\
\hline & & \\
\hline E. & $8-80.2$ & Paek \\
\hline D. & sh from oround surface & \\
\hline
\end{tabular}

JEQ-AL-ENO-I (E/QA)

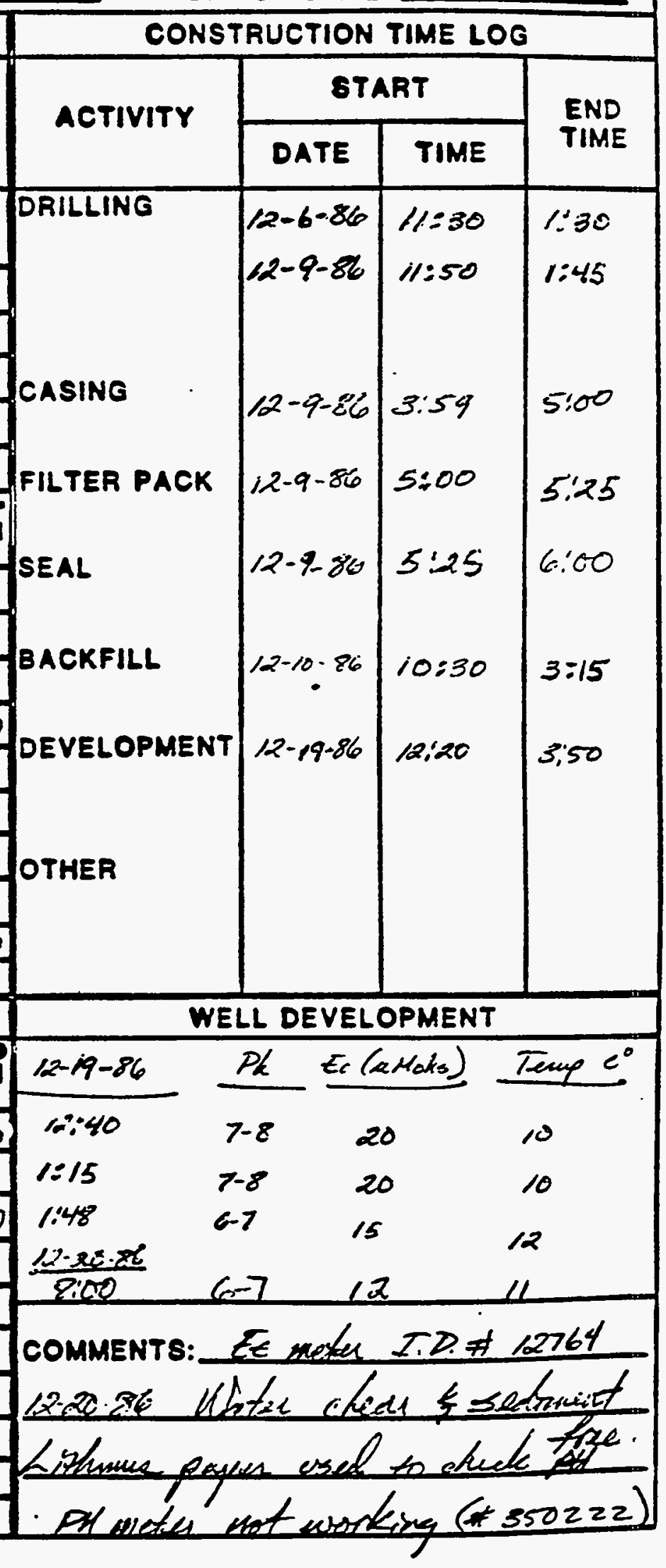




\section{TT MCOBS ENGN EERTV EROUP NC.

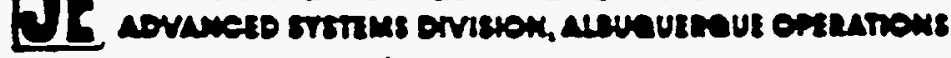

\section{WELL COMPLETION RECORD}

SITE ID: SPK- D/ LOCATION ID: TO 916 DATE INSTALLED: $12-6-86$ APPROX. SITE COORDINATES: (FT.) N OPEN AREA.PER LINEAL FT. $\left(I N^{2} / F T.\right)$ FORMATION OF COMPLETION: FIELD REP.:

Whatele $(5 s / 4 a)$

WELL CASING 4.0
DIAMETER (In) 4.0 .04

HOLE DIAMETER $(\ln ) 8.0$ DRILLER:

Boyles Bese.

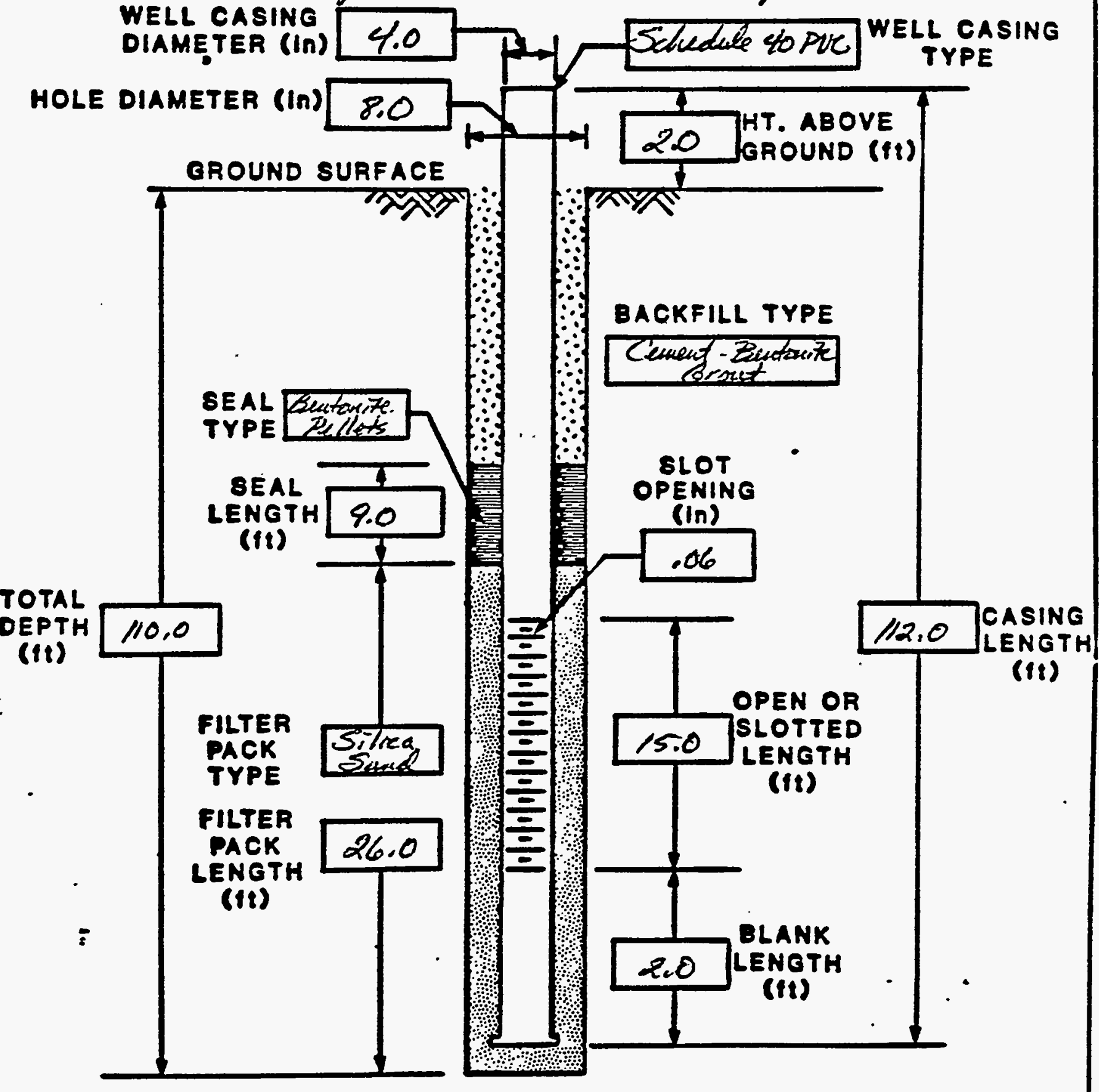

COMMENT 8: 


\section{TI IACOBS BNGN EETNG GROUP NC. \\ Je stons \\ BOREHOLE/WELL CONSTRUCTION LOG}

SITE ID: SPK- QI LOCATION ID: QOT 916 FIELD REP:

APPROX. SITE COORDINATES (FT.): N E

GROUND ELEVATION (FT. MSL): 5075

COMPLETION DATE: $12-6-\not \%$

BOREHOLE SUMMARY

DRILLER:

Boykes Bros.

RIG TYPE:

Filing 1500

OIT TYPE HOLE

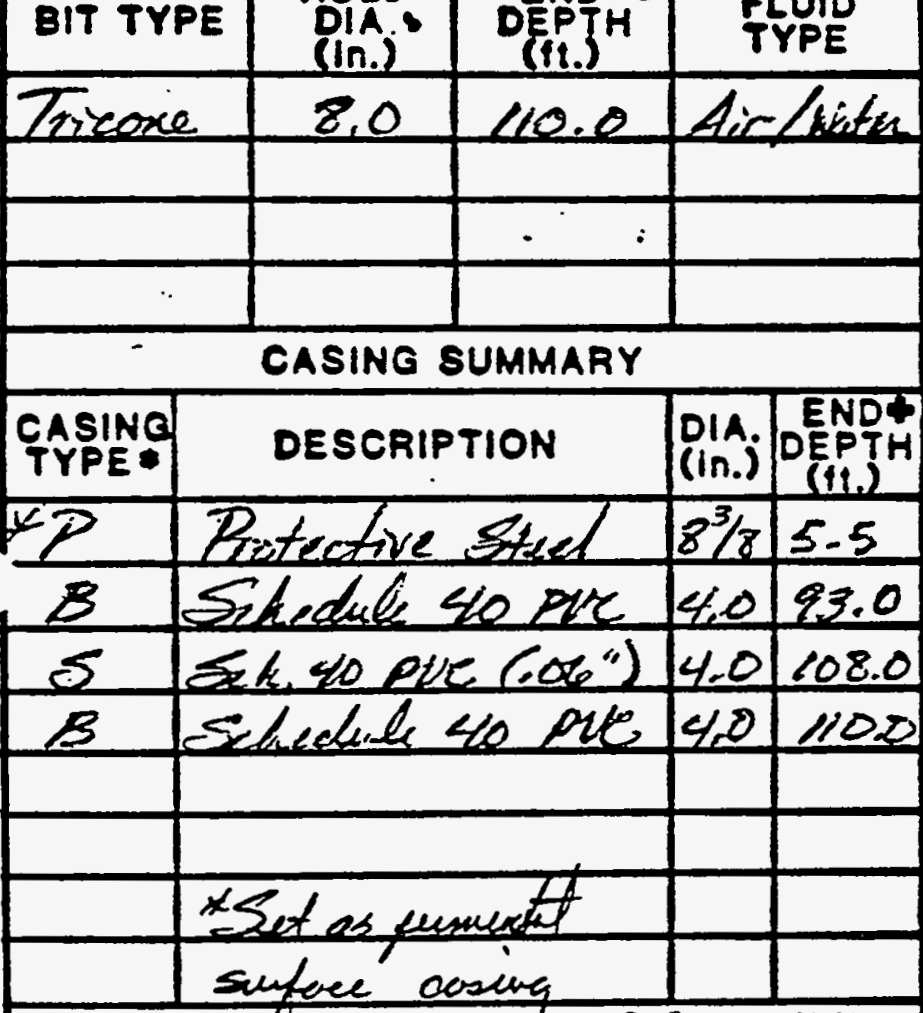

- p-protective b-seroon D-Bichik O-Opon a-None

Dopth from Top of Casing

WELL CONSTRUCTION

\begin{tabular}{|c|c|c|}
\hline $\begin{array}{l}\text { TYPE. } \\
\text { SODE }\end{array}$ & DESCRIPTION & 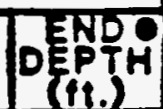 \\
\hline 3 & Prest-Butanch Arent & $75 x$ \\
\hline 5 & Bestorite Pellet (kiv) & 84.0 \\
\hline$F$ & Bdor silies Saud (t-12 & 710. \\
\hline & & \\
\hline & & \\
\hline & & \\
\hline & & \\
\hline & 800 & \\
\hline
\end{tabular}

- Dopth from arownd artace

JEO-AL-ENO-1 (aIS4).
CONSTRUCTION TIME LOG

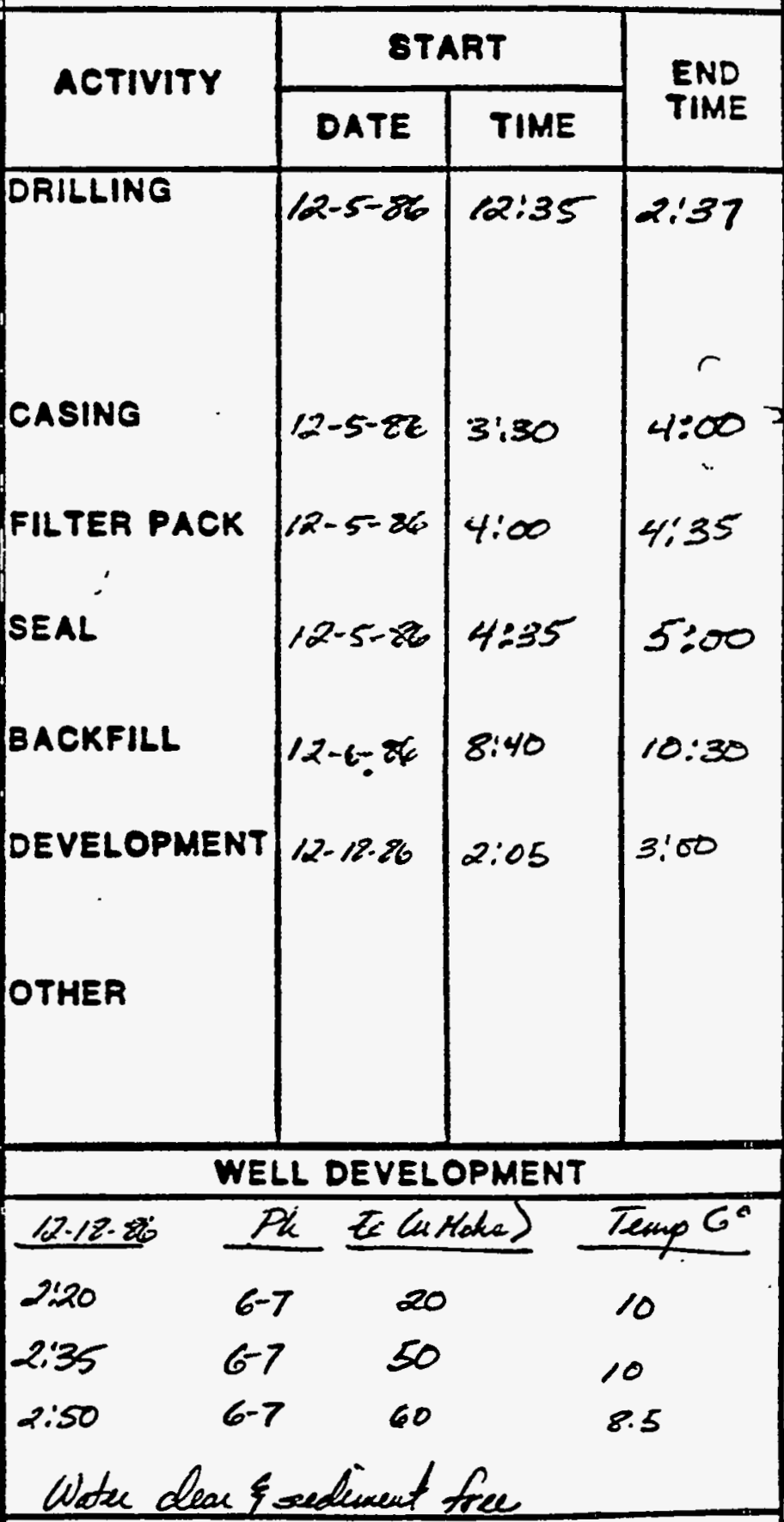

coMMENTS: $Z_{C}$ Heter IR 12764

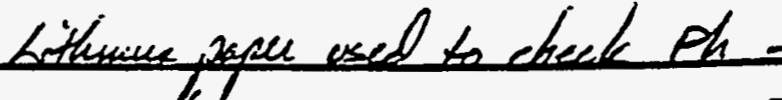
inwetes IDAt 350222 not ievorkizes 


\section{TT MCOBS EKGNEERIV GROUP NC.

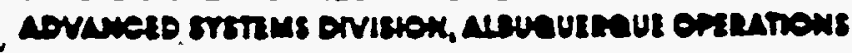

\section{WELL COMPLETION RECORD}

SITE ID: SPK- $8 /$ LOCATION ID: ROF 917 DATE INSTALIED: $22-11-86$ APPROX. SITE COORDINATES:(FT.) N OPEN. AREA.PER LINEAL FT. (IN²/FT.)

FORMATION OF COMPLETION: FIELD REP.: Wasatch (ss/sk) E

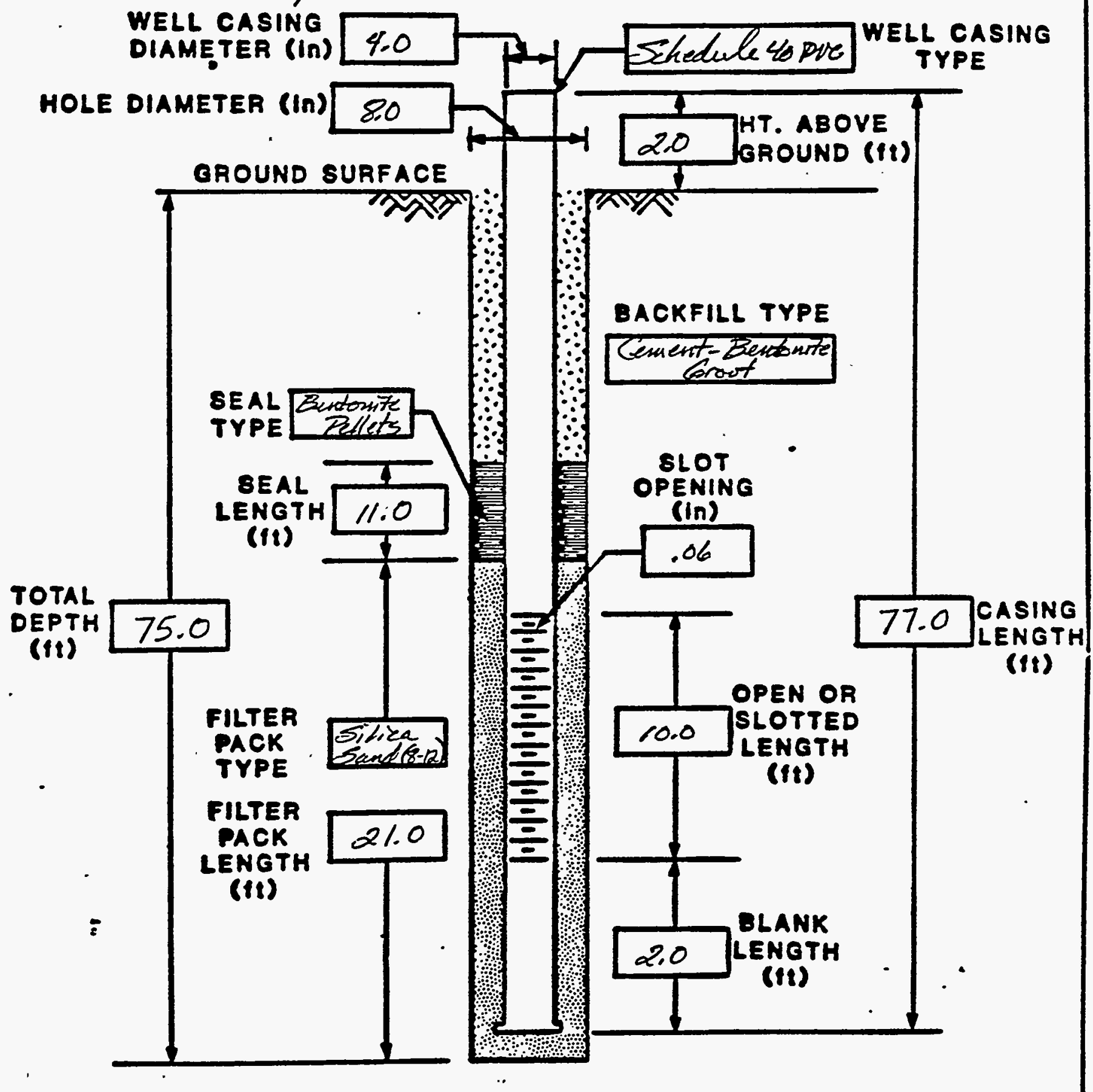

COMMENT8: 


\section{TT $A C O B S$ BNGNERTIG GROUP NC.

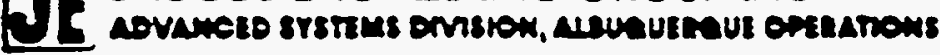 BOREHOLE/WELL CONSTRUCTION LOG}

SITE ID: SFE-Q1 LOCATION 1D: 907917 FIELD REP:_L Phufe
APPROX. 8ITE COORDINATES (FT.): N GROUND ELEVVATION (FT. MSL): 5075 COMPLETION DATE: $12 \cdot 1 /-86$

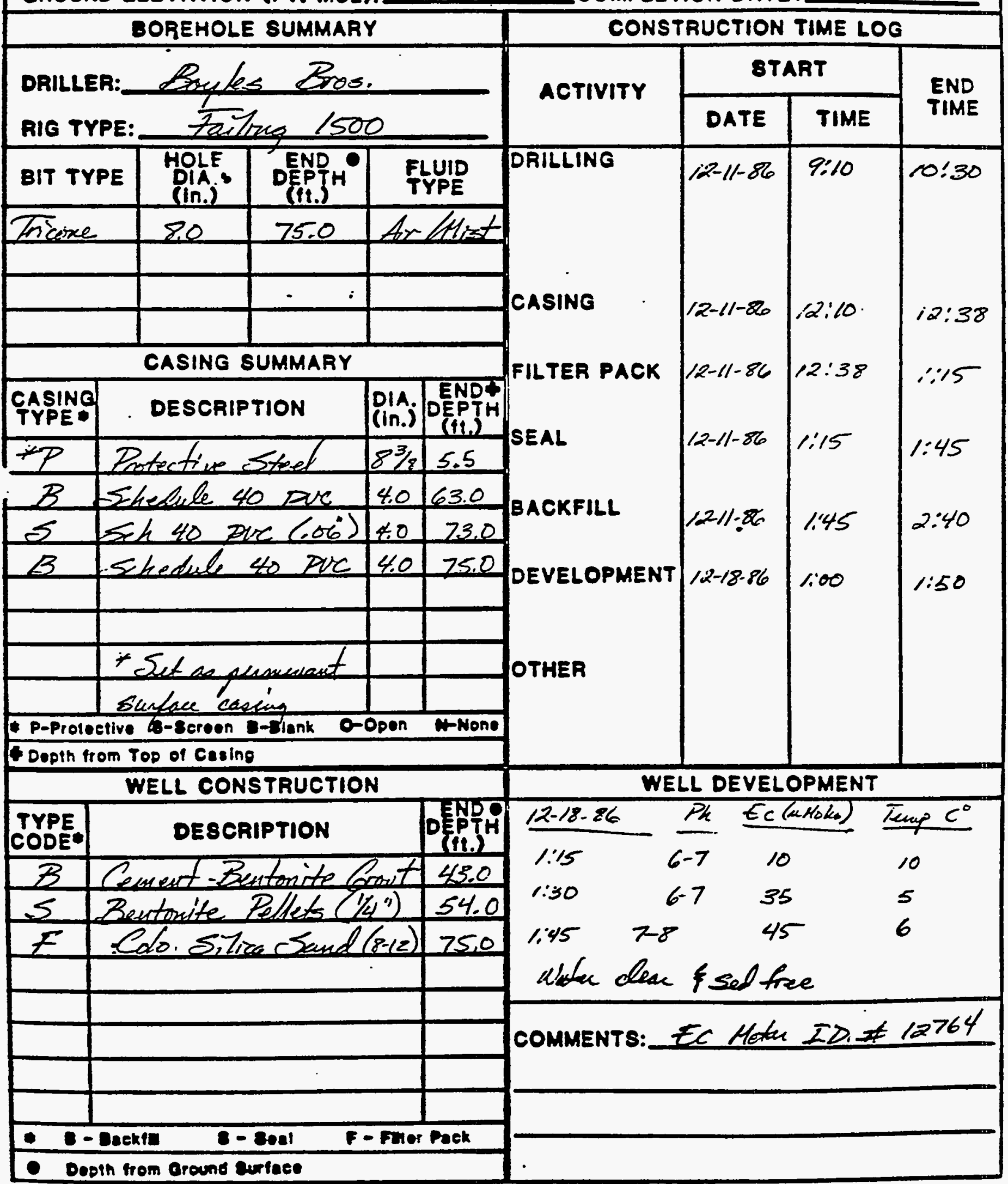




\section{TF MCOSS ENGNEBRIG GROUP NC.

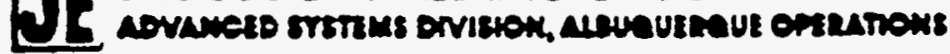

\section{WELL COMPLETION RECORD}

SITE ID: SPK- $\$ 1$ ' LOCATION ID: 988 918 DATE INSTALLED: $12-13-86$ APPROX. SITE COORDINATES:(FT.) $N$ OPEN AREA.PER LINEAL FT. (IN²/FT.)

FORMATION OF COMPLETJON:

FIELD REP.: L L, Phyfe DRILLER: Boykes Bros.

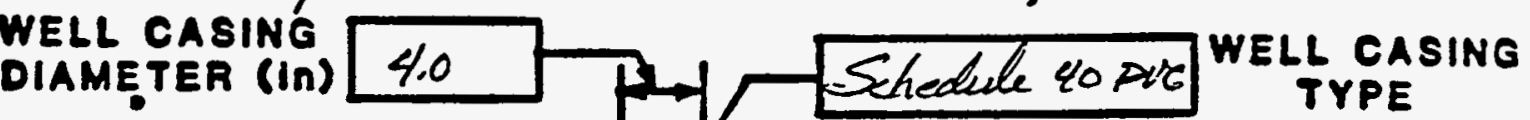

HOLE DIAMETER $(\ln ) 8.0$
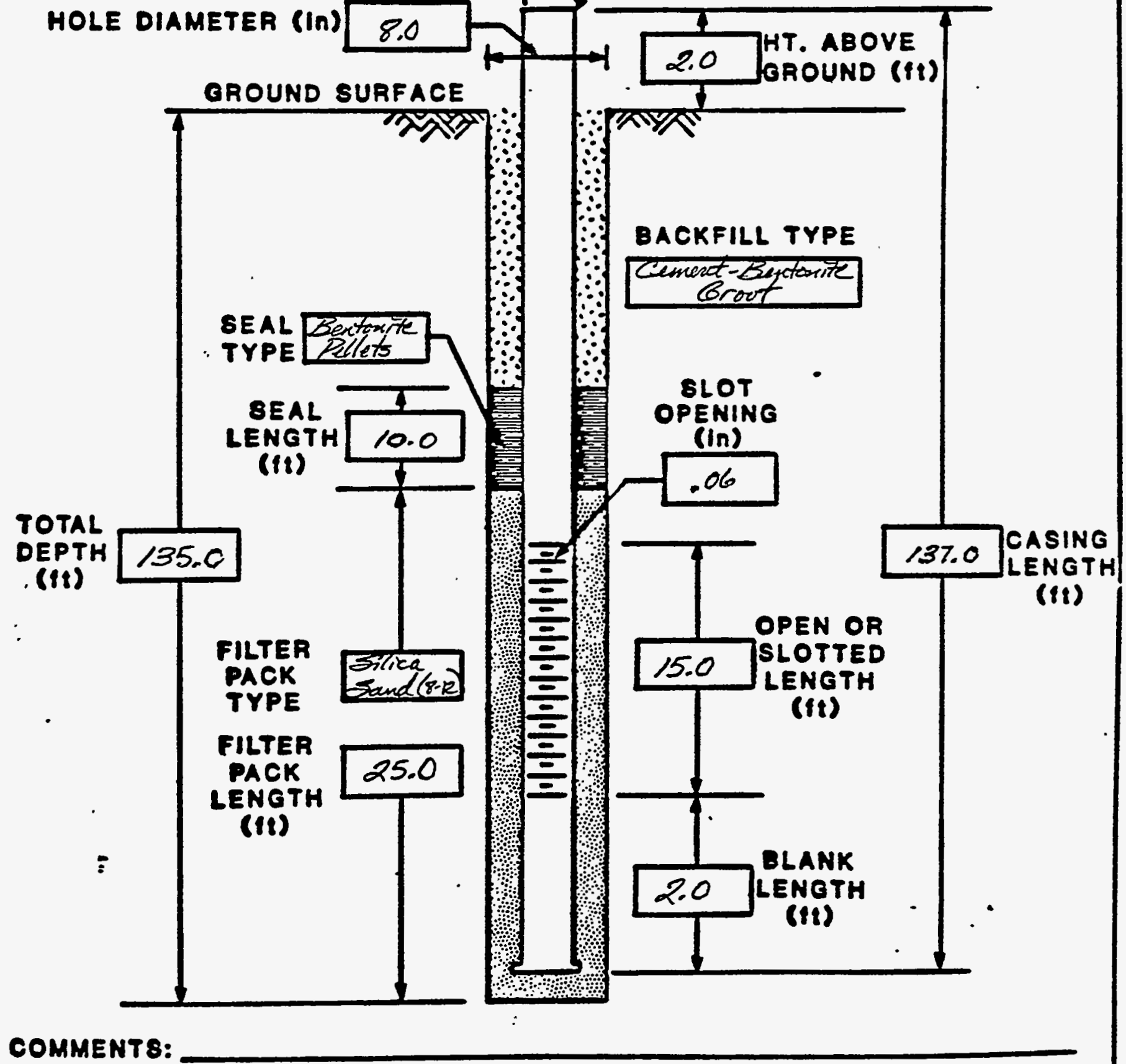

COMMENT 8: 


\section{TTE HACOBS BNGN EERTE GROUP NC. \\ Je \\ BOREHOLE/WELL CONSTRUCTION LOG}

SITE ID: SPK- $\phi$ LOCATION 1D: QO8918 FIELD REP: L Phyfe APPROX. SITE COORDINATES (FT.): N GROUND ELEVATION (FT. MSL): 5100 BOREHOLE SUMMARY

DriLler: Boyles Bros.

RIG TYPE: Failing 1500

\begin{tabular}{|c|c|c|c|}
\hline BIT TYPE & $\begin{array}{l}\text { HOLE } \\
\text { DIA.: } \\
(\operatorname{In.} .)\end{array}$ & 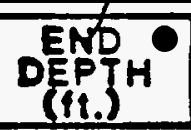 & $\begin{array}{l}\text { FLUID } \\
\text { TYPE }\end{array}$ \\
\hline Fricone & 8.0 & 135.0 & Ar $/ M_{\text {ist }}$ \\
\hline & & & \\
\hline & & & \\
\hline & & & \\
\hline
\end{tabular}

$$
\begin{aligned}
& \hline \\
& \hline x
\end{aligned}
$$

\begin{tabular}{|c|c|c|c|}
\hline $\begin{array}{l}\text { CASING } \\
\text { TYPE: }\end{array}$ & DESCRIPTION & $\begin{array}{l}\text { DiA. } \\
(\text { in. })\end{array}$ & $\begin{array}{l}\text { END' } \\
\text { DEPTH } \\
\text { (fi,.) }\end{array}$ \\
\hline$* P$ & Protective Strel & $83 / 8$ & 5.5 \\
\hline$B$ & Schedul to prr & 4.0 & 118.0 \\
\hline$s$ & Shle to pic $\left(100^{\prime \prime}\right)$ & 4,0 & 133.0 \\
\hline$B$ & Schediale 40 pir & 4,0 & $\angle 35.0$ \\
\hline & & & \\
\hline & & & \\
\hline & $*$ Set & & \\
\hline & & & \\
\hline
\end{tabular}
* P-Protoctive E-Eercen D-Blank O-Open A-None Popin irom Too of Casino

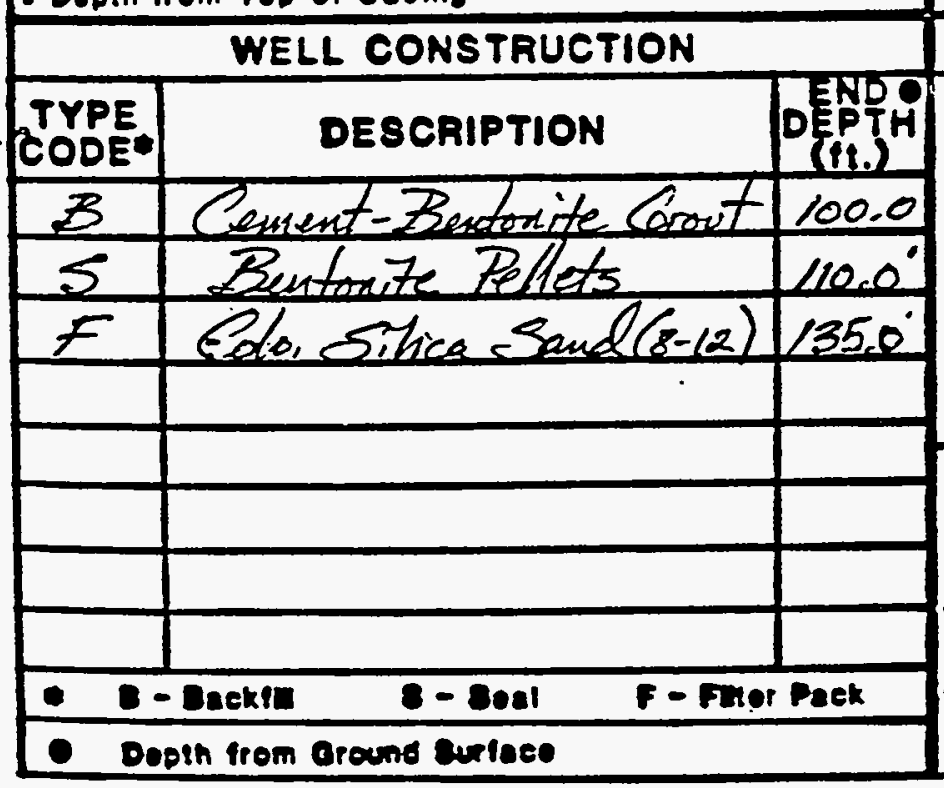

\section{COMPLETION DATE: $12-13-86$}

CONSTRUCTION TIME LOG

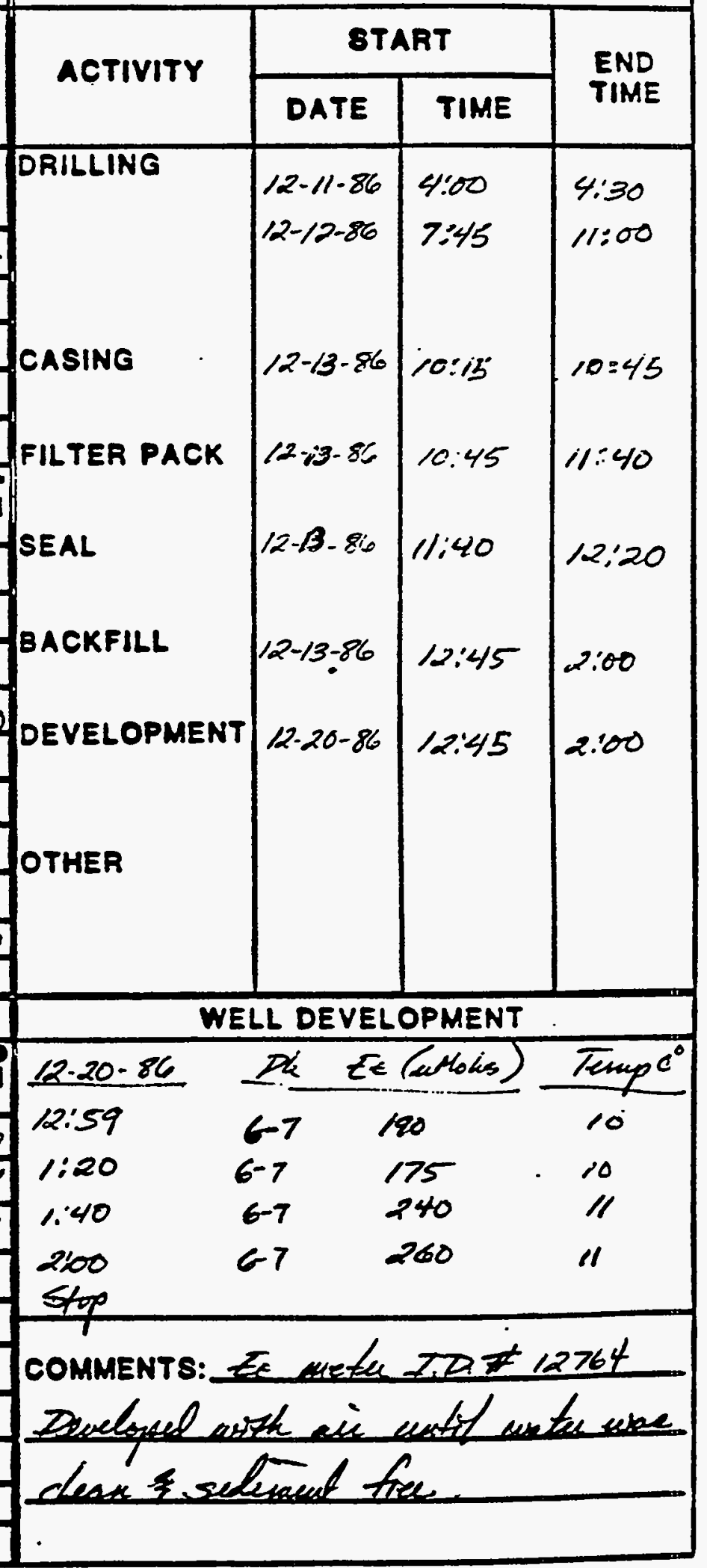




\section{TT MCOBS ENGNEERTE GROUP NC.

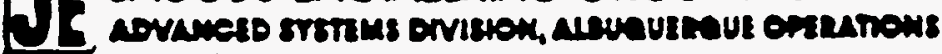

\section{: WELL COMPLETION RECORD}

SITE ID: SPK $\because$ LOCATION 10: ROF 919 DATE INSTALLED: 12-5-86 APPROX. SITE COORDINATES:(FT.) N $\boldsymbol{E}$ OPEN AREA.PER LINEAL FT. (IN2/FT.)

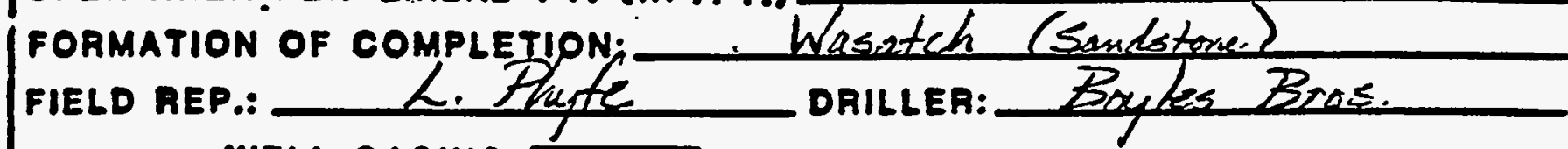
WIAMETER (In) 8.0 Seledule 40 PUT WELL CASING HOLE DIAMETER (in) 4.0 GROUND SURFACE
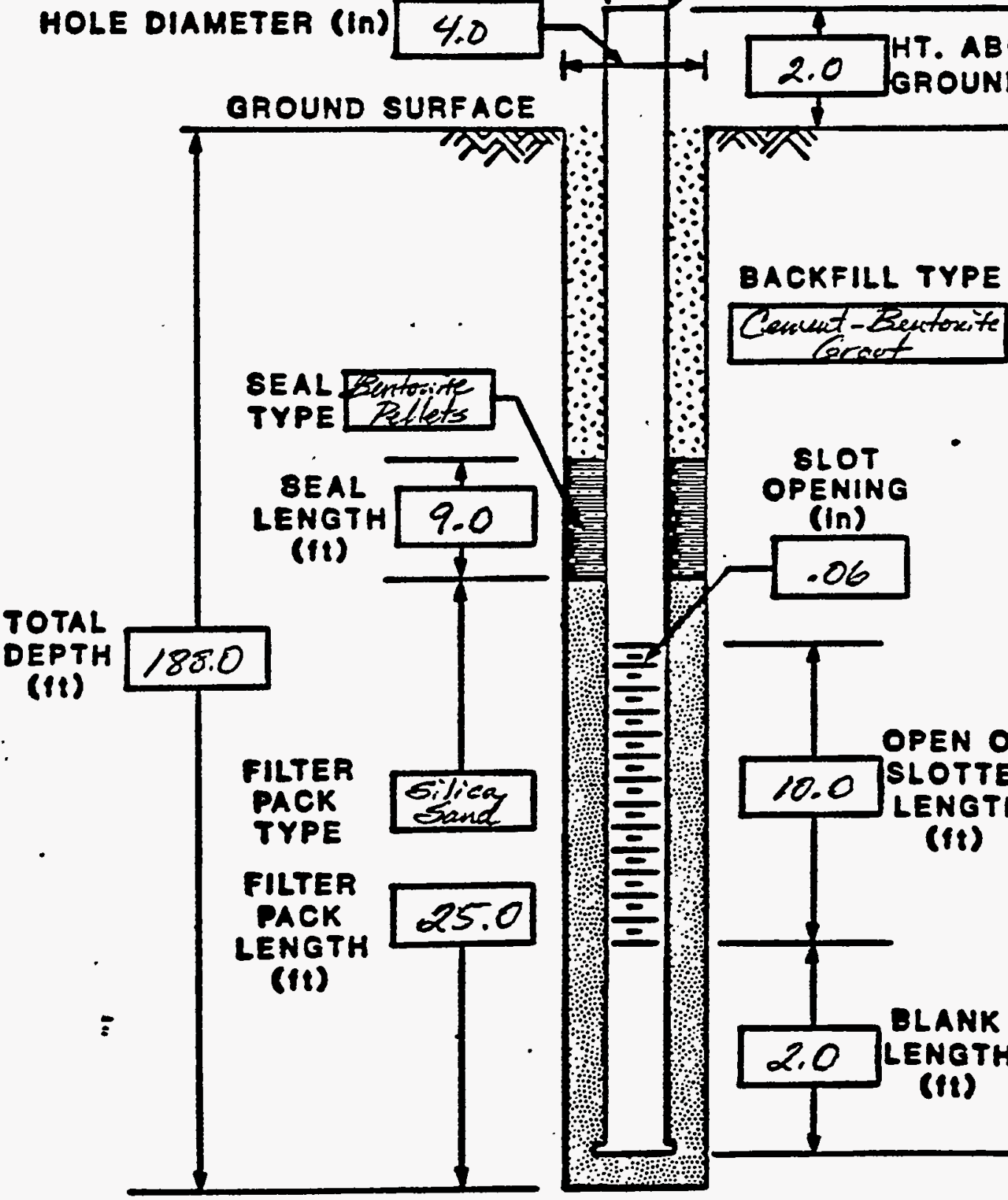

$\therefore-1$ 20 HT. ABOVE

TOTAL

DEPTH

(18)
SLOT

OPENING (In)

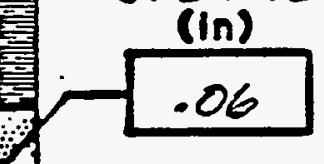




\section{WE JACOBS ENGN EERTG GROUP NC. \\ BOREHOLE/WELL CONSTRUCTION LOG}

SITE ID: SPK LOCATION ID: GE 919FIELD REP: APPROX. BITE COORDINATES (FT.): $N$ E L. Pryte OROUND ELEVATION (FT. MSL): $5 / 00$ COMPLETION DATE: $12-5-86$ BOREHOLE SUMMARY

DRILLER:

RIG TYPE: Boules Bros. Tailing 1500 BIT TYPE

\begin{tabular}{|c|c|c|c|}
\hline Blade b.7 & 8.0 & 1880 & Air /Mist \\
\hline & & & \\
\hline & &. & \\
\hline & & & \\
\hline
\end{tabular}

CASING SUMMARY

\begin{tabular}{|l|l|l|}
\hline CASING \\
TYPE
\end{tabular} \begin{tabular}{l|l|l|l|}
\hline 7 & Protective Steel & $83 / 8$ & 5.5 \\
\hline
\end{tabular} $B$ Sikedule 40 PVC 4.0174 .0 5 $B$ Stedule 40 PVC 4.0188 .0

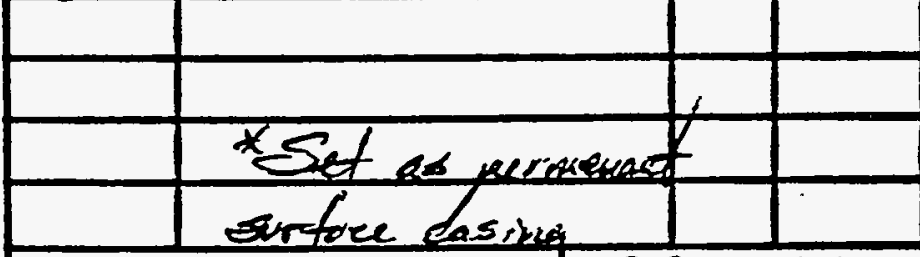
- P-Pioloctive 8-sereon s-siege O-Opon H-Nono + Dopsin from TOD ol cesing WELL CONSTRUCTION

\begin{tabular}{|c|c|c|}
\hline TYPEE & DESCRIPTION & DENPTH \\
\hline CODEA
\end{tabular}
B Cescunt-Bentancte boint 154.0 5 Buntoicte Pellots $\left(1 / \mathrm{H}^{3}\right) \quad 163.0$

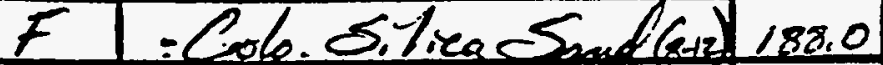

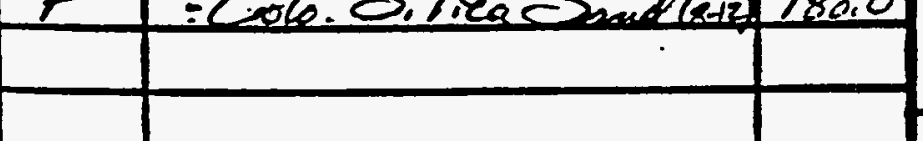

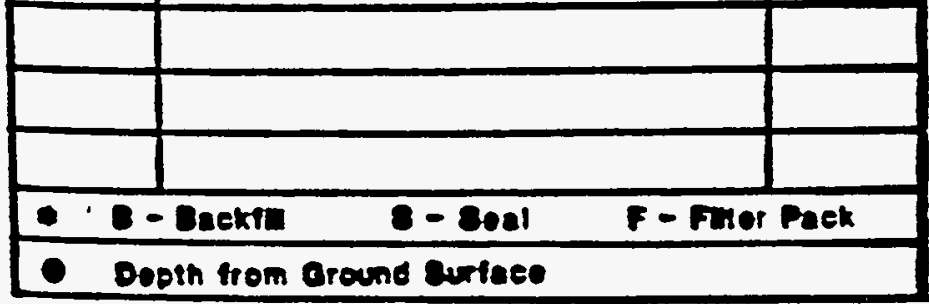

CASING FILTER PACK OTHER DEVELOPMENT

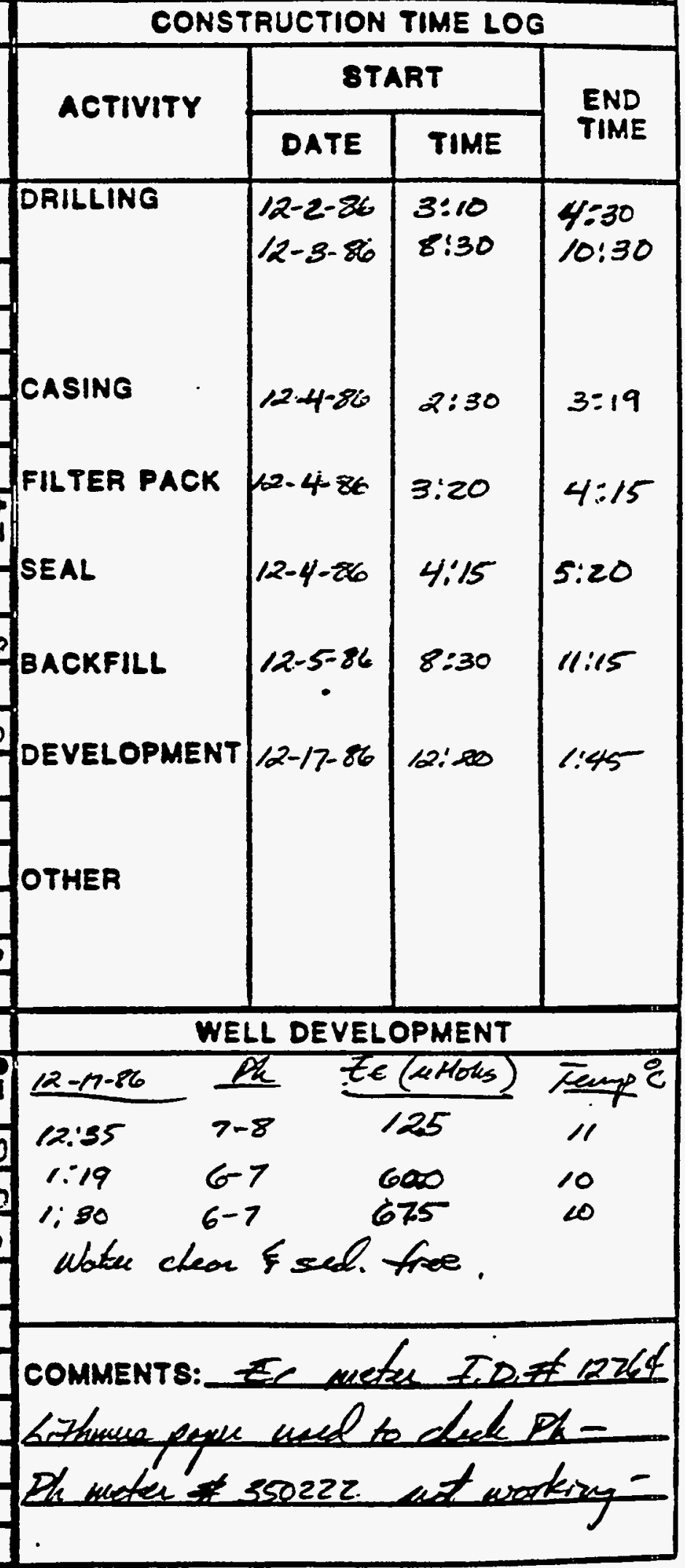




\section{TT LACOBS ENGNERING GROUP NC.

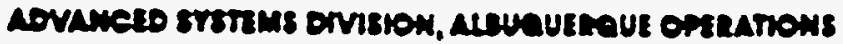

\section{WELL COMPLETION RECORD}

SITE ID: SPK-OI LOCATION ID: 920 DATE INSTALLED: $8 / 11 / 87$ *PAO 8ITE COORDINATES:(FT.) N 560.21 .6 OPEN AREA PER LINEAL FT. (IN2/FT.) .05 5 lot

FORMATION OF COMPLETION: WASATEA EM - UppE SOndNTOX

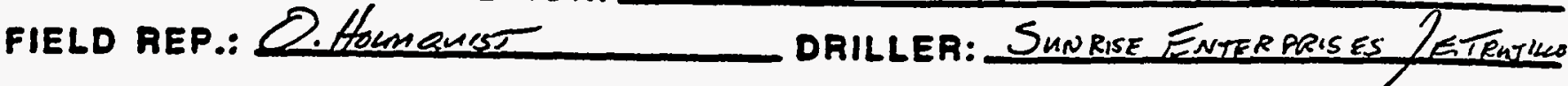

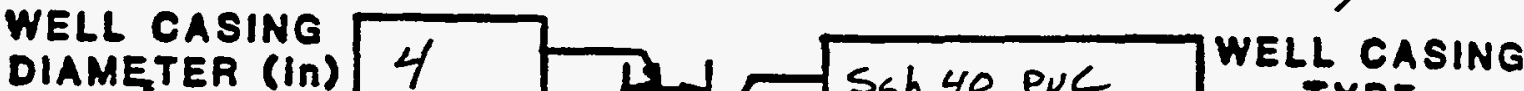
DIAMETER (In) 4 Sh 40 PUC WY TYP

HOLE DIAMETER (In) 83/4 5101.1

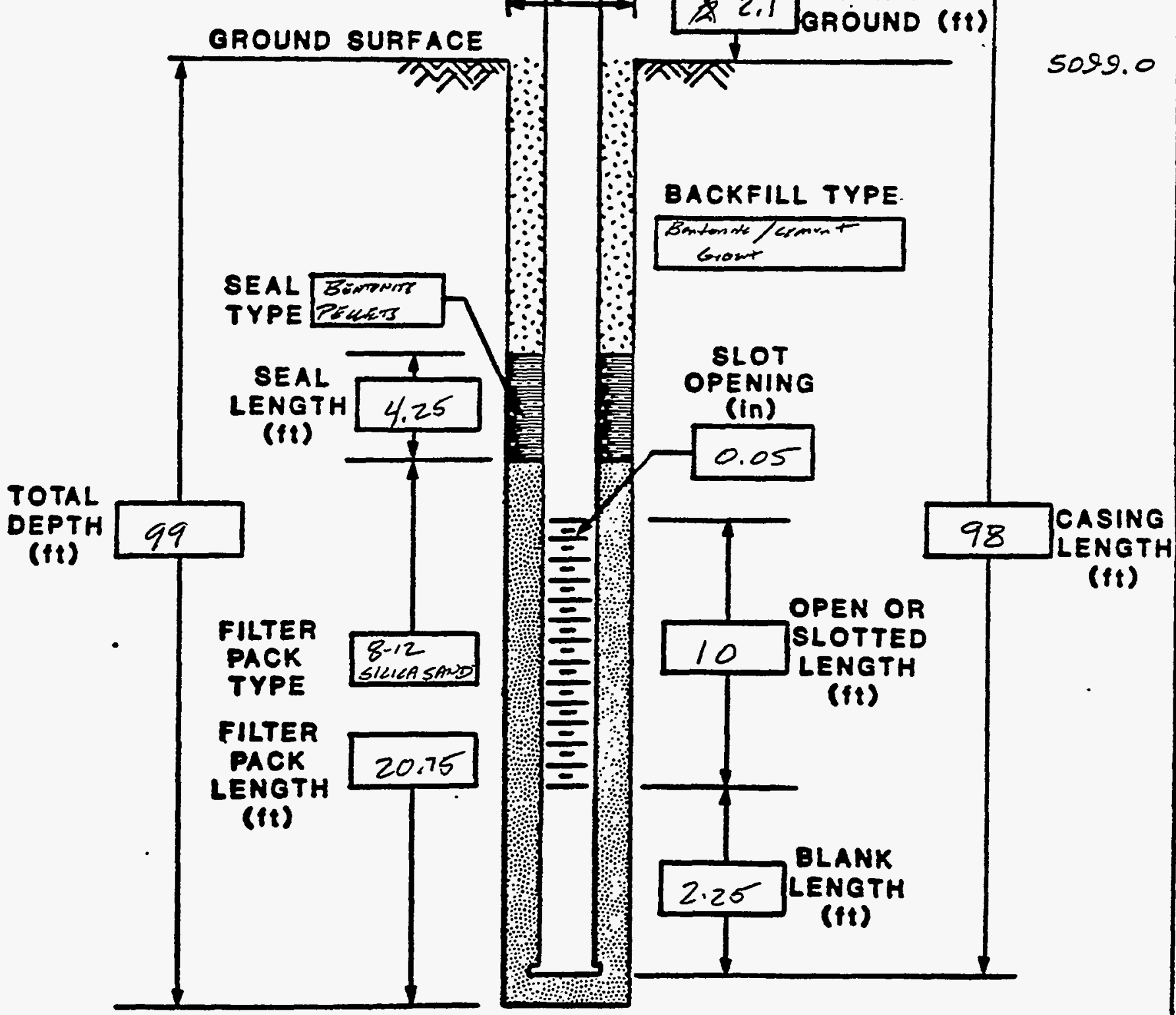

\section{COMMENTS:}




\section{TI. LACOBS ENGINEERING GROUP INC. ADVANCED SYBTEMS ONVISHON, ALUUQUERQUE OPERATONS \\ BOREHOLE/WELL CONSTRUCTION LOG}

SITE ID: SPK-01 LOCATION ID: 920 FIELD REP: 0 . Homomso APPROX. SITE COORDINATES (FT.): N_ iK N N N N BROUND ELEVATION (FT. MSL): גiK_COMPLETION DATE: $8 / 1 / 6)$

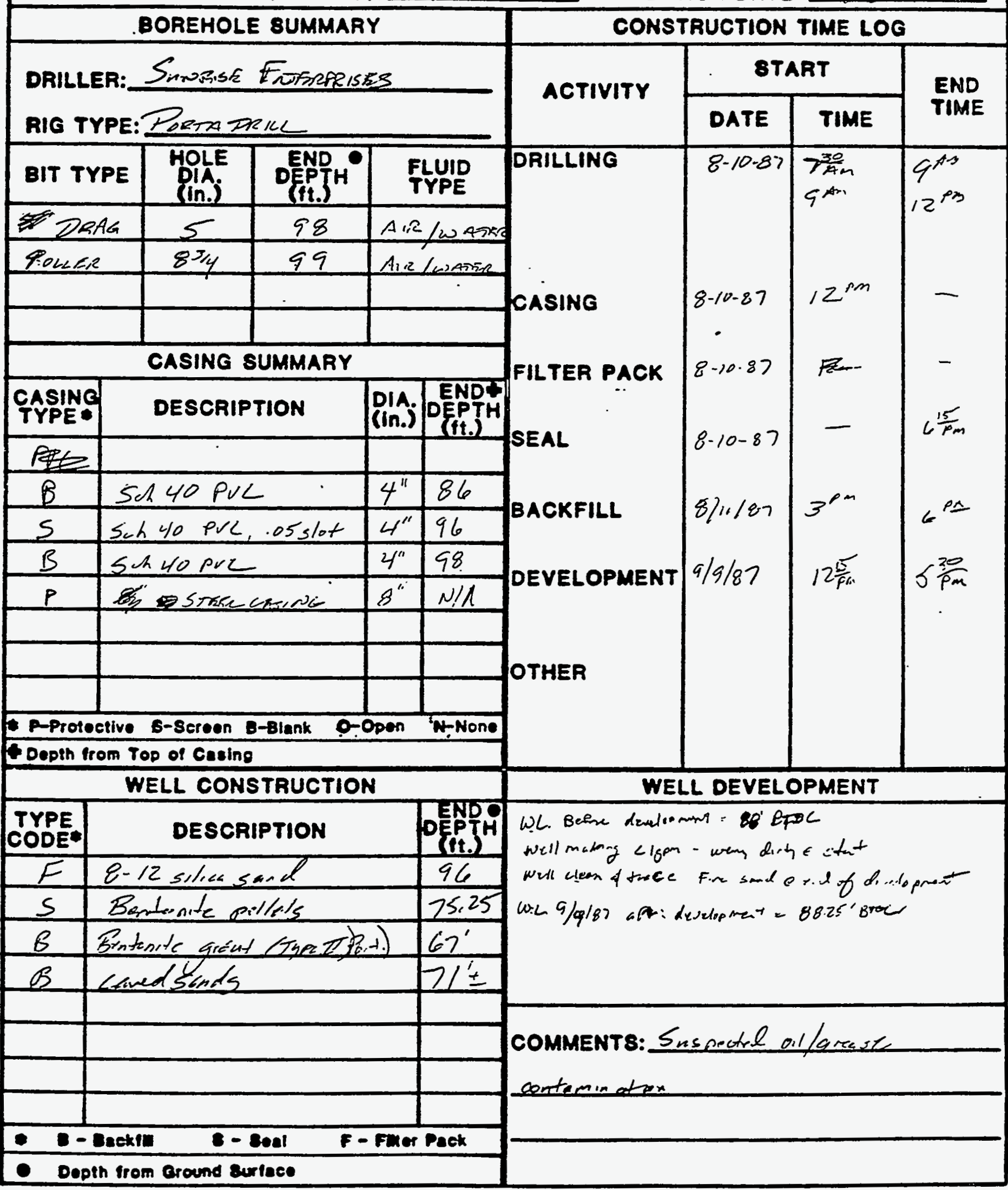




\section{DE HOCOBS ENGNEERNG GROUP NC.}

\section{WELL COMPLETION RECORD}

SITE ID: SPK-01 LOCATION ID: 921 DATE INSTALLED: 8-15-87 APITE COORDINATES:(FT.) N $58,118.3$ OPEN AREA PPER LINEAL FT. (IN2/FT.) 0.05 SlotE 592198.9

FORMATION OF COMPLETION: WASATCHF Fm - Lower Sandrtone

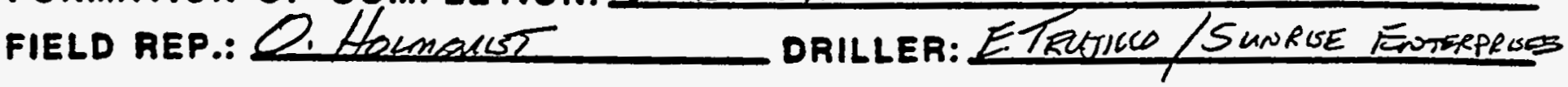
WIALLCASING 4 HOLE DIAMETER (in) B3/4 GROUND SURFACE

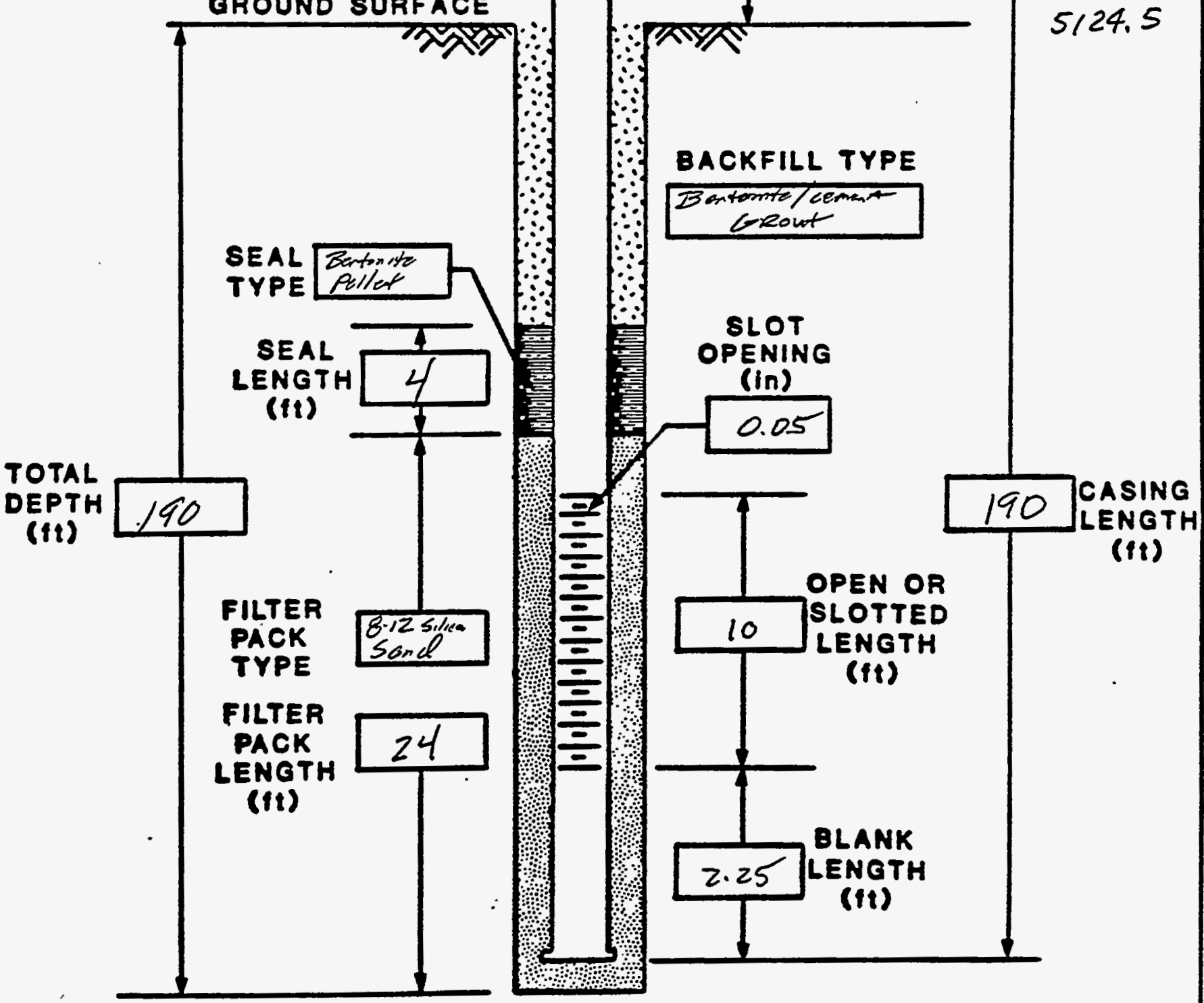

COMMENTS: 


\section{TE. HACOBS ENGNEERING GROUP NC.

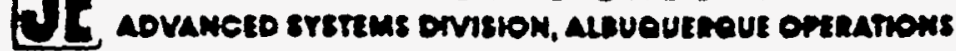 BOREHOLE/WELL CONSTRUCTION LOG}

SITE ID: SPKOO LOCATION ID: 921 FIELD REP: D. Hormonest APPROX. BITE COORDINATES (FT.): N NK E L GROUND ELEVATION (FT. MSL): DK COMPLETION DATE: 8/15/8?

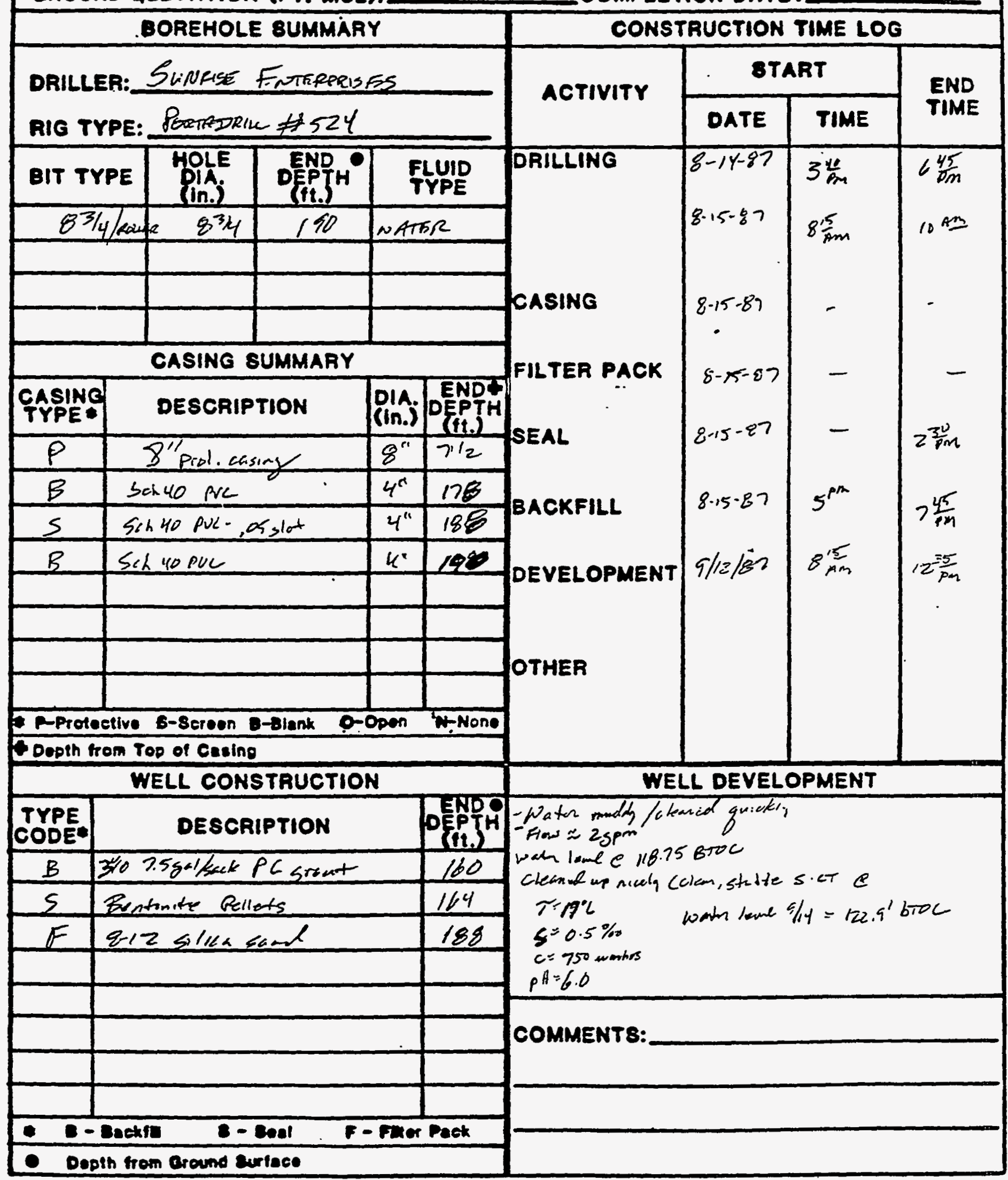




\section{TIE MCOSS ENGNEERNG CROUP NC.

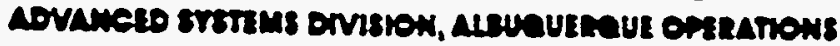

\section{WELL COMPLETION RECORD}

SITE ID: SPK-OI LOCATION ID: $9 Z 2$ APPROX. 8ITE COOORDINATES:(FT.) N $58,163.6$ DATE INSTALLED: $8 / 17 / 87$ OPEN AREA PER LINEAL FT. (IN2/FT.). .05 Slot

FORMATION OF COMPLETION: WASACH Fm - Uphen SindFtone FIELD REP.: D. Horm aust

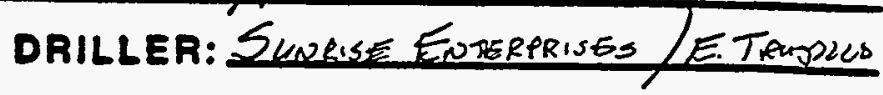

WELL CASING DIAMETER (In)

HOLE DIAMETER (in) $83 / 4$

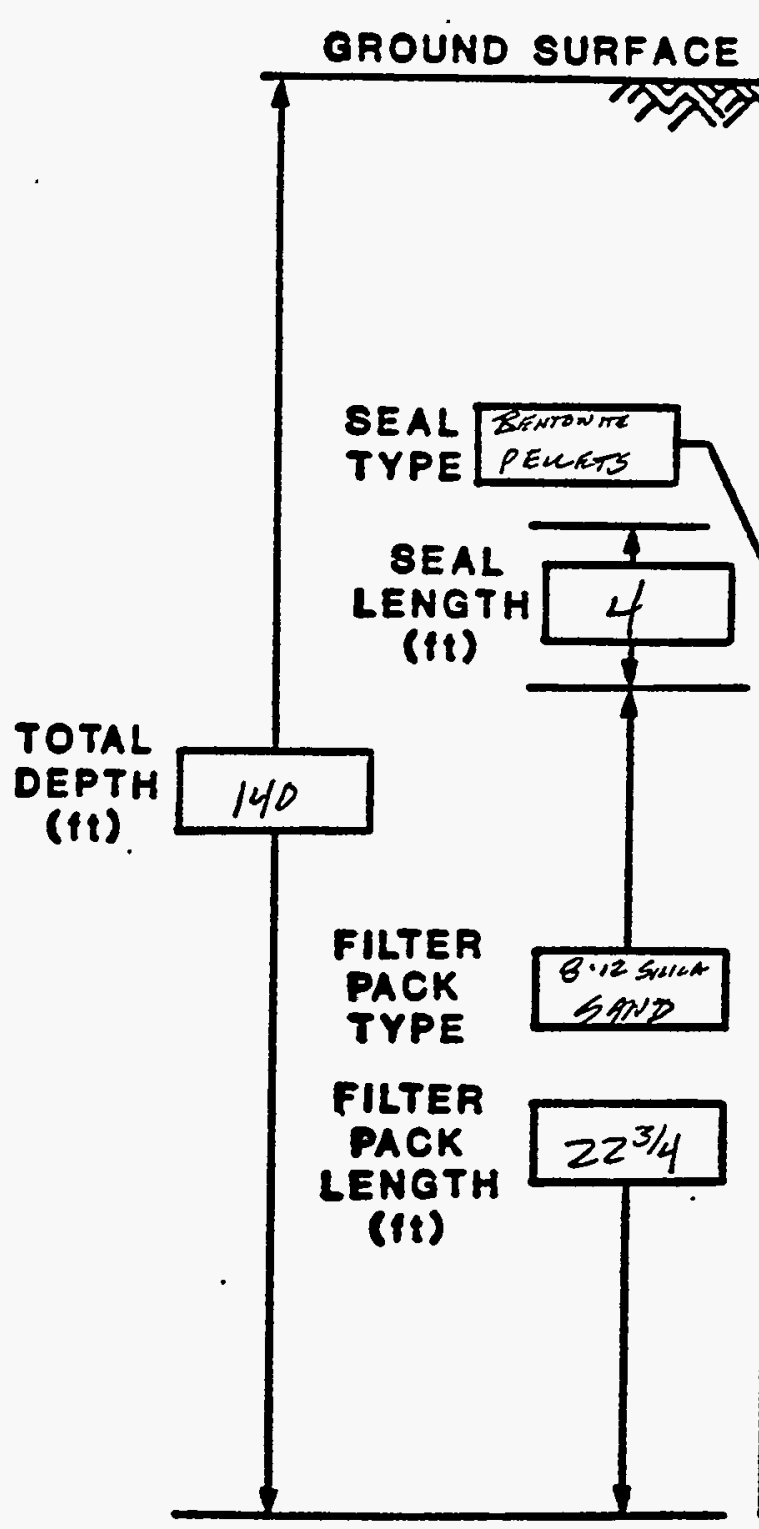

COMMENTS:
WELL CASING TYPE
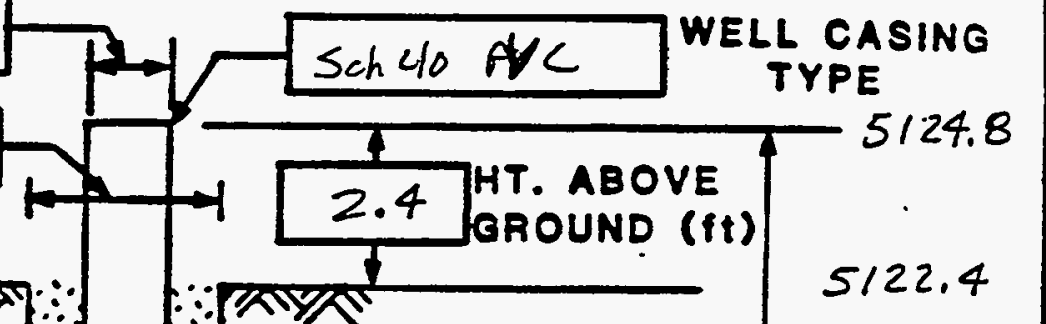

$\because$ अश्र

5122.4

BACKFILL TYPE

BEFTONITE-CFMSNT

GRowt

$\therefore$

$\because \because 1$

$\therefore$ SLOT

OPENING

(in)
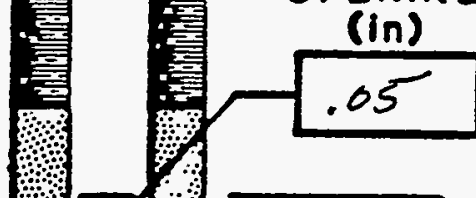

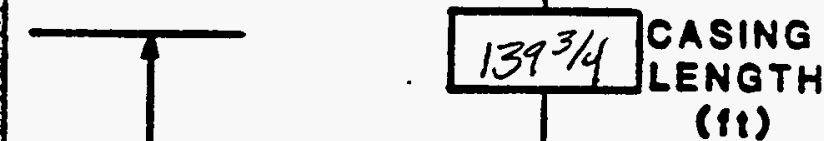

(it) 


\section{T. JACOBS ENGNEERNG GROUP NKC.

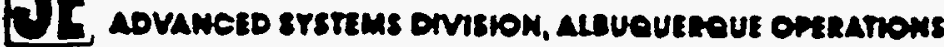 BOREHOLE/WELL CONSTRUCTION LOG}

SITE ID: SPK-OI LLCATION ID: $92 Z$ APPROX. BITE COORDINATES (FT.): N NK OROUND ELEVATION (FT. MSL): NK BOREHOLE BUMMARY

DRILLER:SUnRISE ENTERPRUES/E.Truossua

RIG TYPE: PortadeIL 524

\begin{tabular}{|c|c|c|c|}
\hline BIT TYPE & $\begin{array}{c}\text { HOLE } \\
\text { DIA. } \\
\text { (In.) }\end{array}$ & $\begin{array}{c}\text { END } \\
\text { DEPJH } \\
\text { (Ft.) }\end{array}$ & $\begin{array}{c}\text { FLUID } \\
\text { TYPE }\end{array}$ \\
\hline TRICONE GAABR & $83 \mu$ & 140 & WATER \\
\hline & & & \\
\hline & & & \\
\hline & & & \\
\hline
\end{tabular}

CASING SUMMARY

\begin{tabular}{|c|c|c|c|}
\hline $\begin{array}{l}\text { CASING } \\
\text { TYPE }\end{array}$ & DESCRIPTION & $\begin{array}{l}\text { pia. } \\
\text { (In.) }\end{array}$ & $\begin{array}{l}\text { END } \\
\text { DEPPH } \\
\text { (ft.) }\end{array}$ \\
\hline$P$ & Sch 40 StFEC CASIND & 8 & $5( \pm)$ \\
\hline$B$ & $\operatorname{Seh} 40$ PUC BLAPUL & 4 & $127 \%$ \\
\hline$\leq$ & Seh 40 PrL $.05 \leqslant 10+$ & 4 & $157 \%$ \\
\hline$B$ & Sek vo PVL sump & 4 & $139^{3 / 4}$ \\
\hline & & & \\
\hline & & & \\
\hline & & & \\
\hline & & & \\
\hline & - S-8eroen 8-Blank & pon & Nono \\
\hline
\end{tabular}
WELL CONSTRUCTION

\begin{tabular}{|c|c|c|}
\hline $\begin{array}{l}\text { TYPE } \\
\text { CODE* }\end{array}$ & DESCAIPTION & OEPPD \\
\hline$B$ & BENDDNITE/CEMGNT GEOUT & 111 \\
\hline$s$ & BENTONITE PEUAT SEAL & 115 \\
\hline$E$ & B-12 Silla sand & $B 73 / 4$ \\
\hline & & \\
\hline & & \\
\hline & & \\
\hline & & \\
\hline & & \\
\hline e- & $8-$ Soal & Poek \\
\hline \multicolumn{3}{|c|}{ - Dopin from Ground eurfece } \\
\hline
\end{tabular}

FIELD REP: O. HOLMQRIST ENK

COMPLETION DATE: $8 / 17 / 87$ CONSTRUCTION TIME LOG

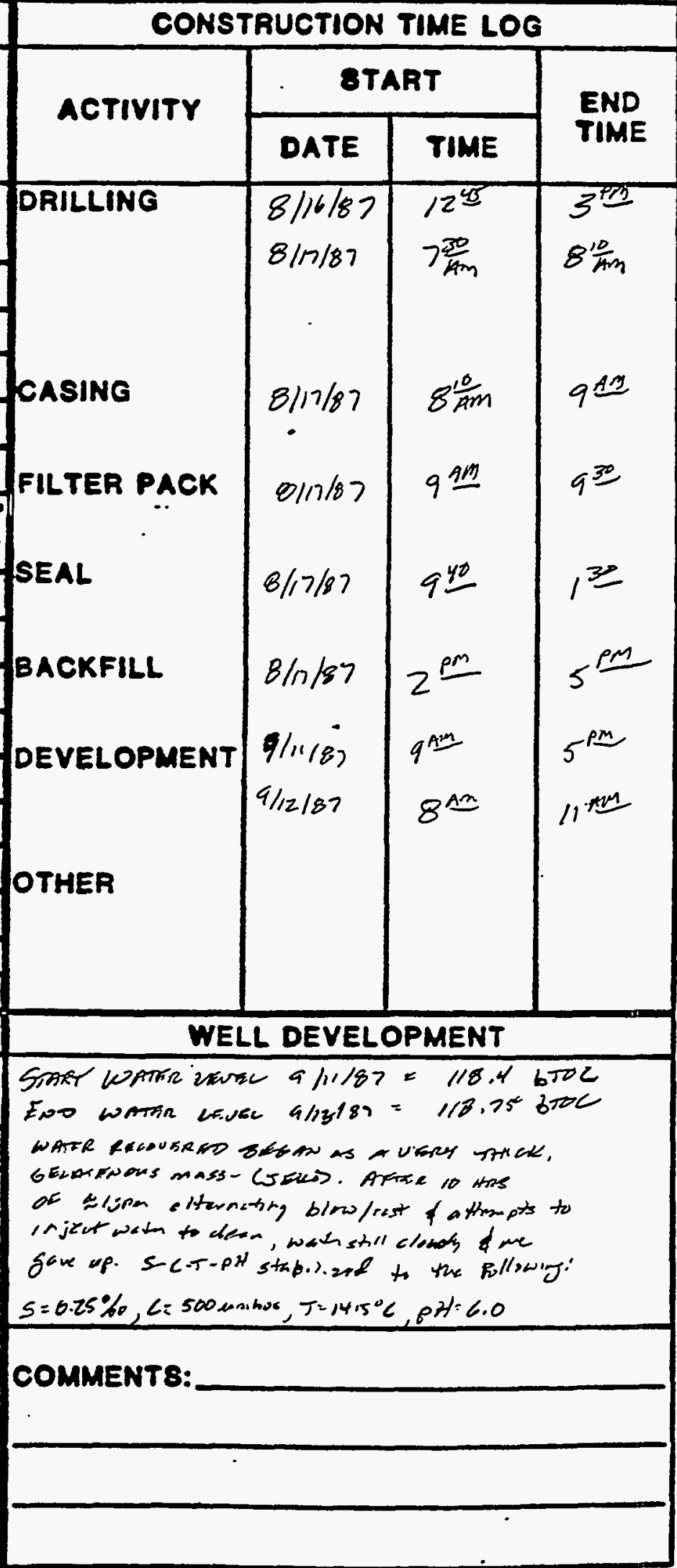




\section{TIE HOOBS ENGNEERNG GROUP NC.

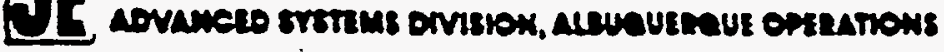

\section{* WELL COMPLETION RECORD}

SITE ID: SPK.O I LOCATION ID: 923 DATE INSTALLLD: $8 / 18 / 87$ APPROX. 8ITE COORDINATES:(FT.) N $59,654.0$ E 59.828 .6 OPEN AREA PER LINEAL FT. (IN2/FT.) .05 Stot

FORMATION OF COMPLETION: WAS ATCHA Fu - Lower Sandstone

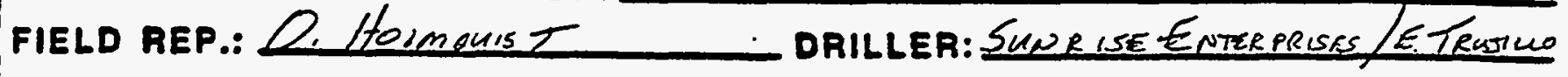

WELL CASING 4 SCh 40 PULS WELL CASING DIAMETER (in) 4 Sch 40 PUC TYPE

HOLE DIAMETER (In) 83/4 5115.0

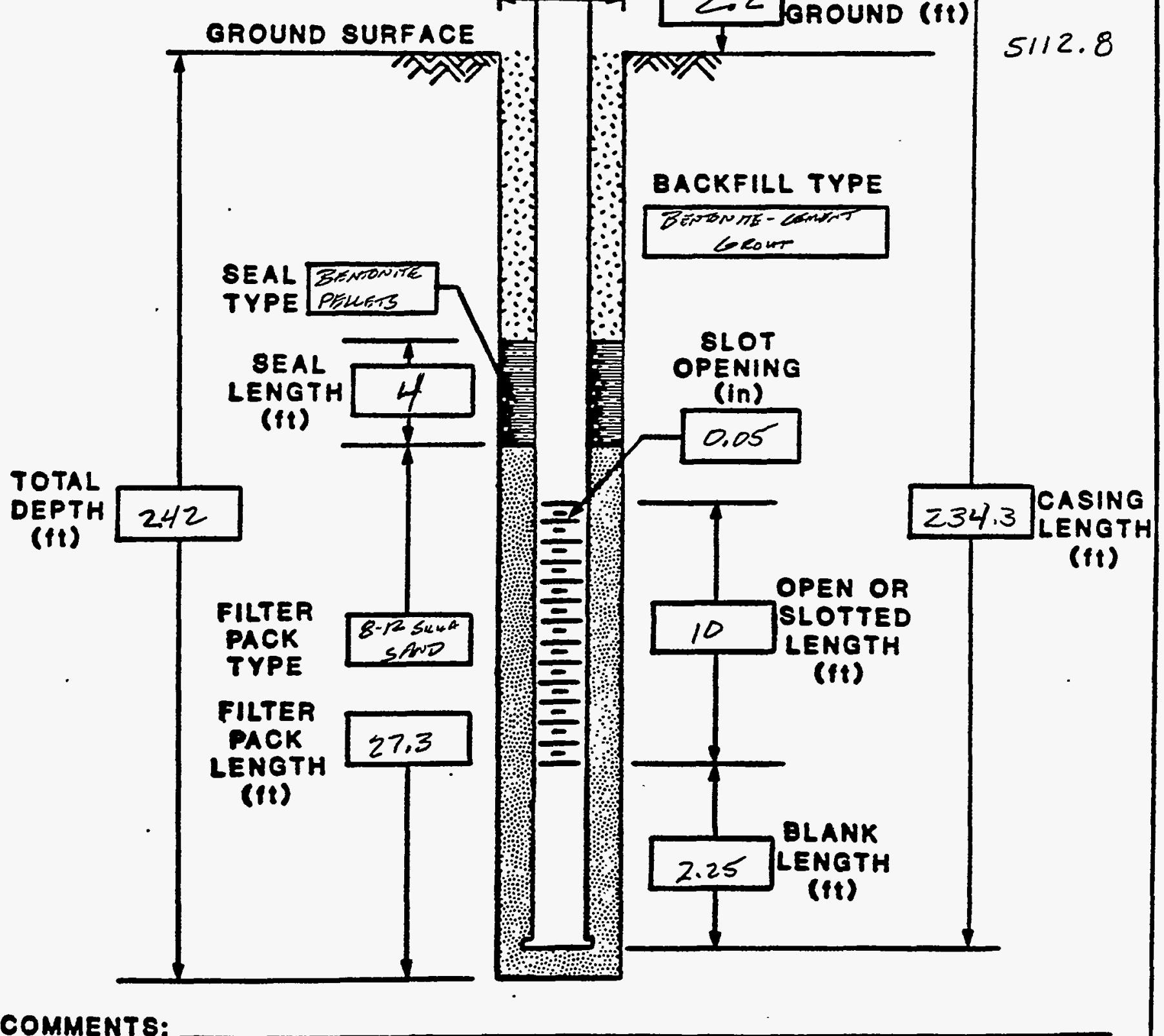

COMMENTS: 


\section{T.

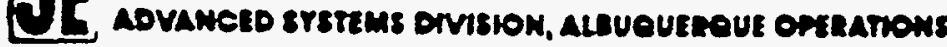 \\ BOREHOLE/WELL CONSTRUCTION LOG}

SITE ID: SPK-OI LOCATION ID: 923 FIELD REP: O. HOLM QWNTT

APPROX. BITE COORDINATES (FT.): $N$ NK

QROUND ELEVATION (FT. MSL): NK E NK

\section{BOREHOLE BUMMARY}

DRILLER:SUNRISE ENTERPRISES/E.TRUTUCO

RIG TYPE: PermpPIC 524

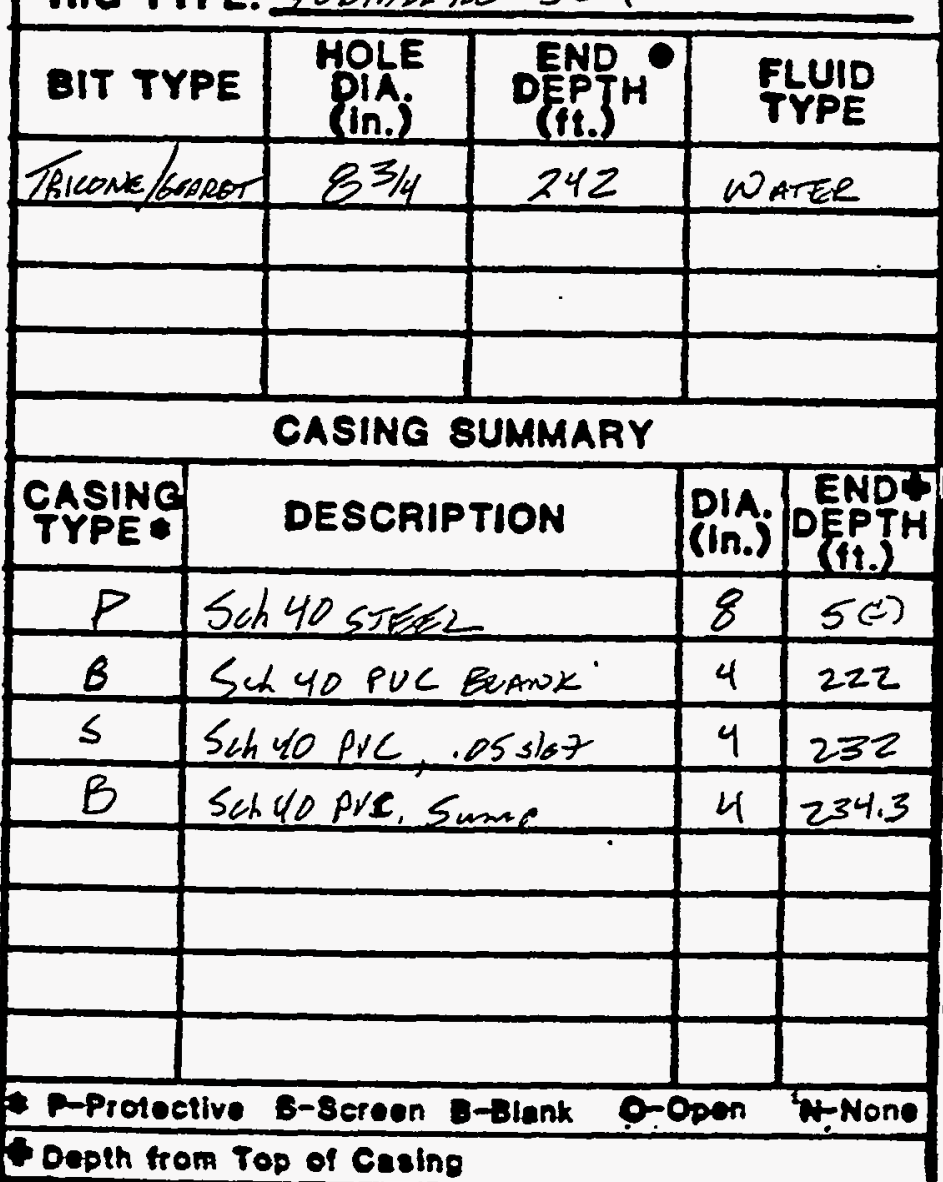

\begin{tabular}{|c|c|c|}
\hline \multicolumn{3}{|c|}{ WELL CONSTRUCTION } \\
\hline $\begin{array}{l}\text { TYPE } \\
\text { CODE }\end{array}$ & DESCRIPTION & DEPTH \\
\hline$B$ & 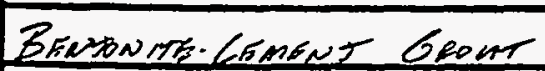 & 201 \\
\hline 5 & Bewronire Peutrs & 205 \\
\hline$E$ & 8.12 sinICA $\leq$ AND & 232.3 \\
\hline & & \\
\hline & & \\
\hline & & \\
\hline & & \\
\hline 8 & Beckfin & 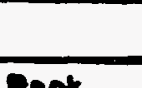 \\
\hline$-D_{0}$ & oth from Ground surface & \\
\hline
\end{tabular}

COMPLETION DATE: B/28/82

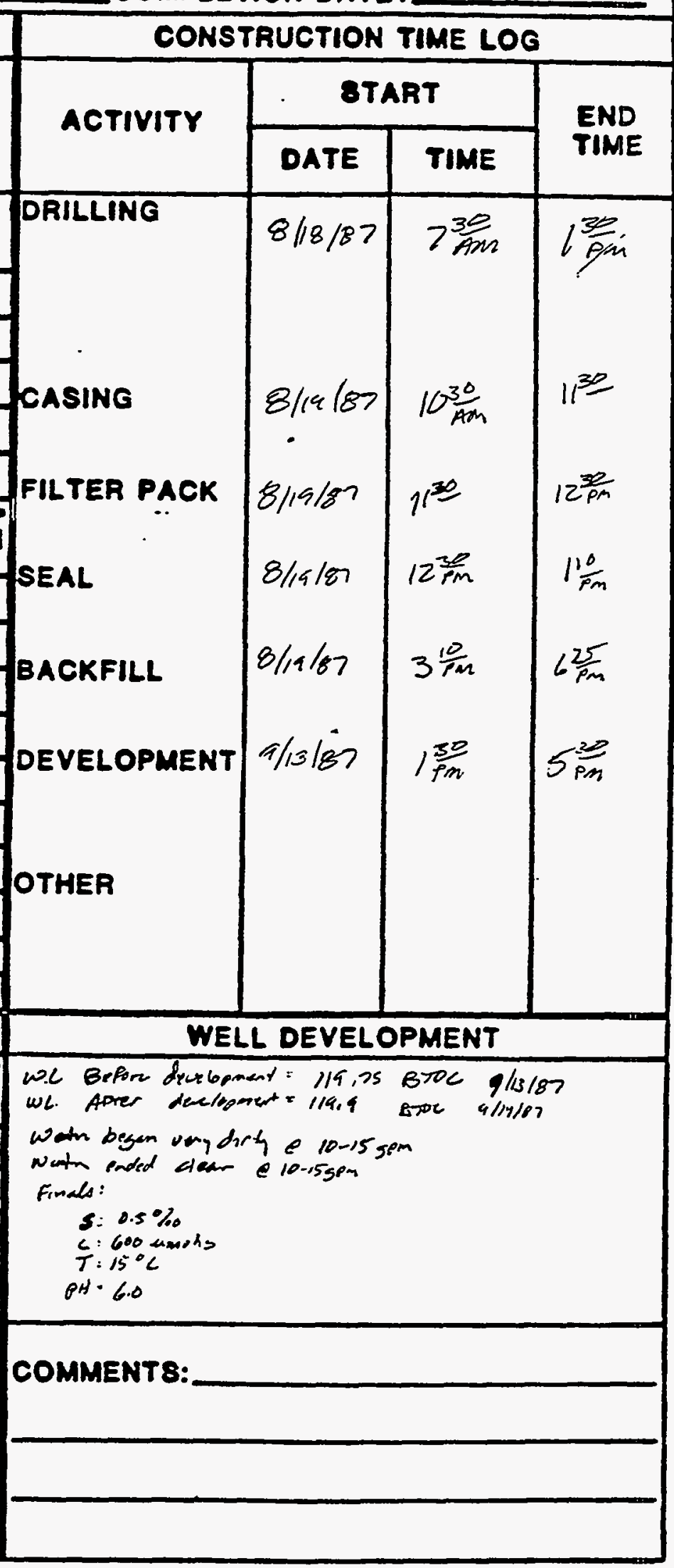




\section{TIE HOOBS ENGNERTNG GROUP NC.

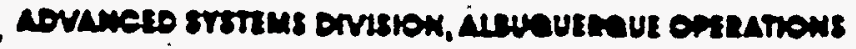

\section{WELL COMPLETION RECORD}

SITE ID: SPK-01 LOCATION ID: 924 DATE INSTALLLE: $8 / 30 / 87$ APPROX. BITE COORDINATES: (FT.) N NK

E NK

OPEN AREA PER LINEAL FT. (IN2/FT.). O5S/OT

FORMATION OF COMPLETION: W ATCH

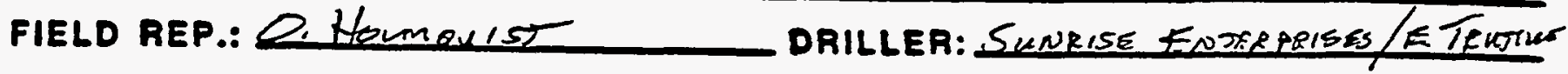

WELLCASING 4 DIAMETER (In) 4 Soh40 PUL WELL CASING

HOLE DIAMETER (In)

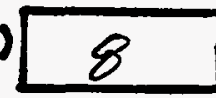

OROUND SURFACE
TOTAL DEPTH (ti)

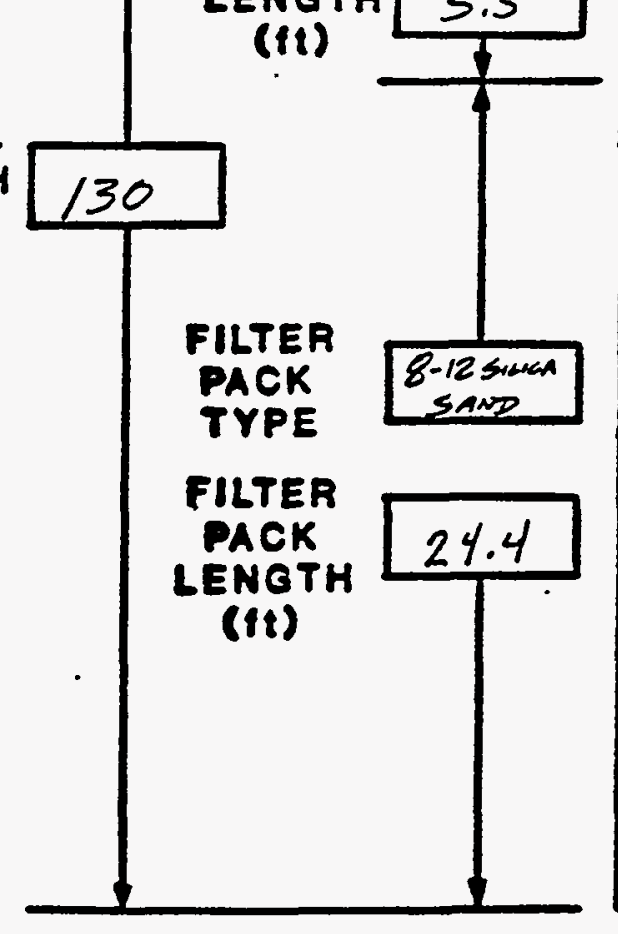

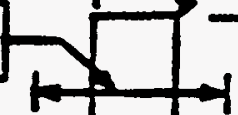

みूY

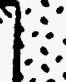

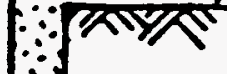

BACKFILL TYPE

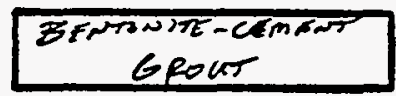

SEAL B=FODFE

TYPE PEHETS

SEAL

LENGTH

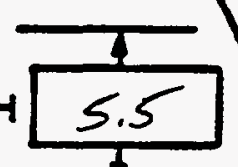

(11)
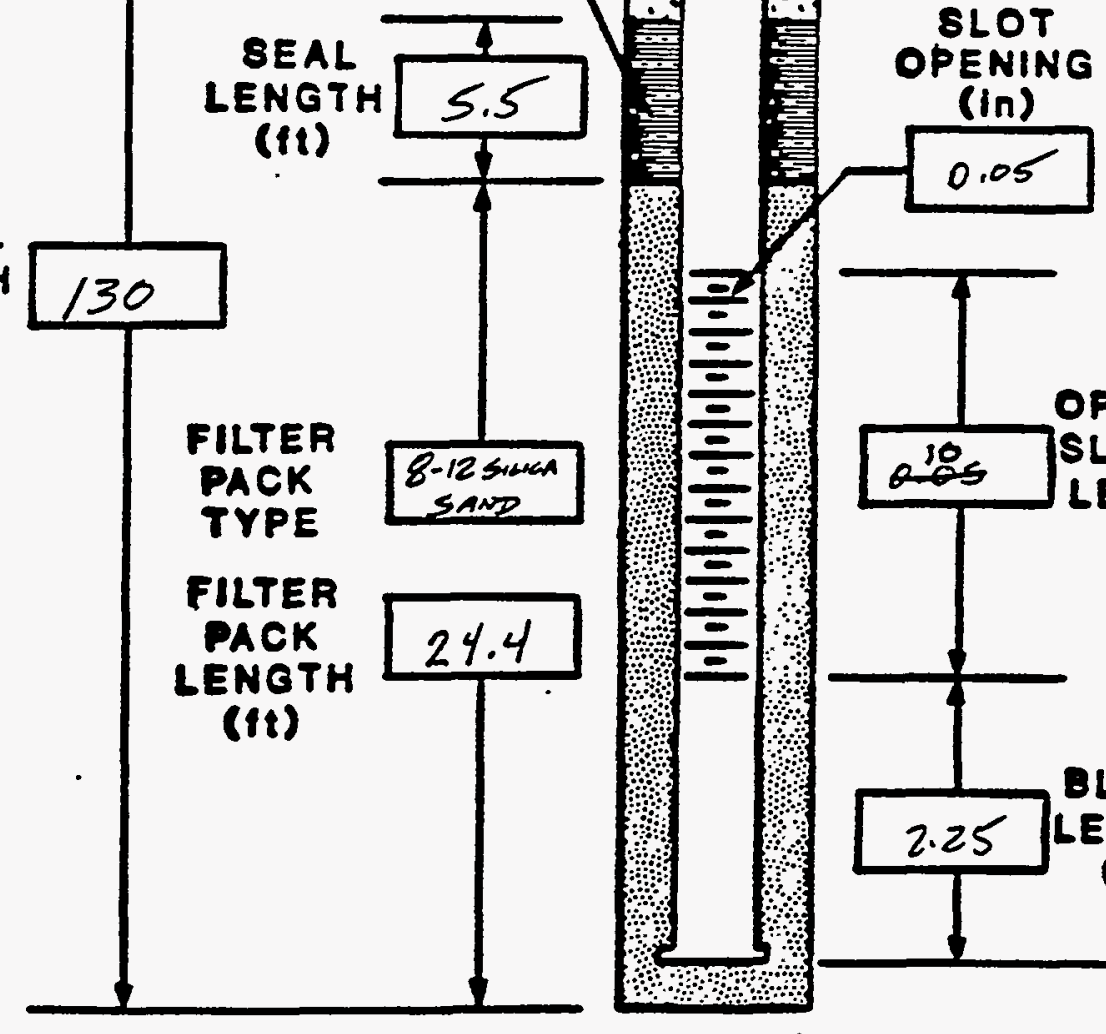

SLOT
OPENING

(in)

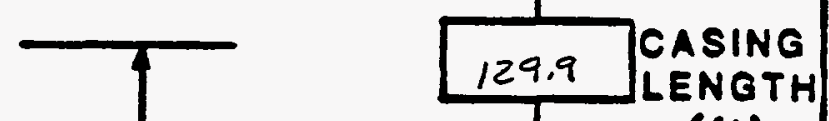

(1i)

\section{COMMENTS:}




\section{TF LACOBS ERTENEERNG GROUP NC.

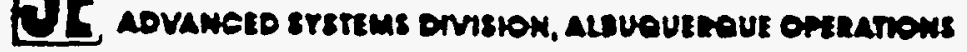 \\ BOREHOLE/WELL CONSTRUCTION LOG}

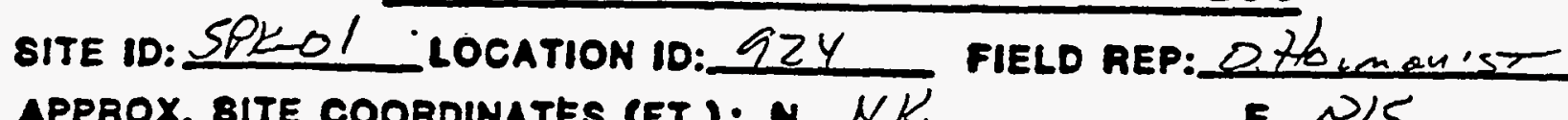

APPROX. 8ITE COORDINATES (FT.): N.NK. NL

QROUND ELEVATION (FT. MSL): NR

\section{BOREHOLE BUMMARY}

DRILLER:SKNGISE FOUTBRR PRISES

RIG TYPE: ParisieicL E524

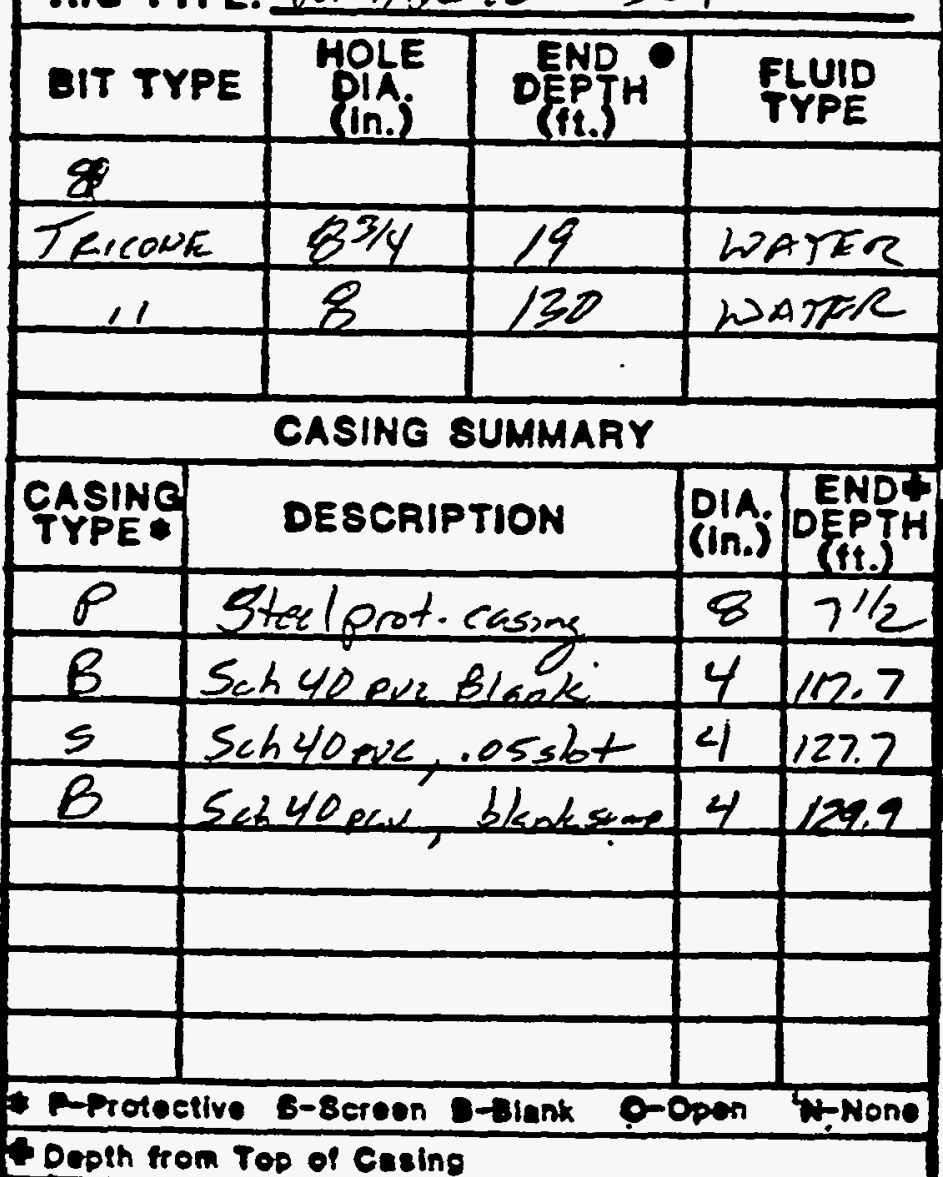

\begin{tabular}{|c|c|c|}
\hline \multicolumn{3}{|c|}{ WELL CONSTRUCTION } \\
\hline $\begin{array}{l}\text { TYPE } \\
\text { CODE }\end{array}$ & DESCRIPTION & DENPTH \\
\hline$B$ & 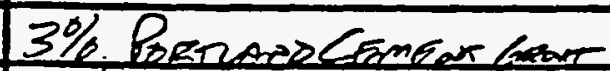 & 98.0 \\
\hline 5 & 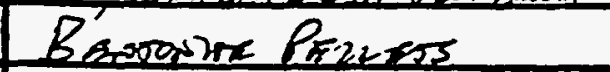 & 103.5 \\
\hline$F$ & $8-12$ SILICA SAND & 1239 \\
\hline & & $2 \% 4$ \\
\hline & & \\
\hline & & \\
\hline & & \\
\hline & & \\
\hline e. & $8-8001$ & Pack \\
\hline 은 & from Ground Qurtaes & \\
\hline
\end{tabular}

COMPLETION DATE: $8 / 50 / 57$

CONSTRUCTION TIME LOG

\begin{tabular}{|c|c|c|c|}
\hline \multicolumn{4}{|c|}{ CONSTRUCTION TIME LOG } \\
\hline \multirow{2}{*}{ ACTIVITY } & \multicolumn{2}{|c|}{ BTART } & \multirow{2}{*}{$\begin{array}{l}\text { END } \\
\text { TIME }\end{array}$} \\
\hline & DATE & TIME & \\
\hline DRILLING & $8 / 25 / 87$ & $2 \frac{Y D}{P a n}$ & 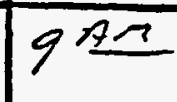 \\
\hline CASING & $3 / 50 / 87$ & $9 \frac{\sqrt{5}}{A m}$ & $9 \frac{22}{\text { An }}$ \\
\hline FILTER PACK & $8 / 30$ & $9 \frac{45}{42 n}$ & $10^{A}=$ \\
\hline SEAL & 88130 & & 1020 \\
\hline BACKFILL & $8 / 30 / 87$ & $11 \frac{30}{7 m}$ & $20,12_{p=}^{15}$ \\
\hline DEVELOPMENT & $9 / 14 / 87$ & $8 \frac{45}{A m}$ & $12 \frac{4 T}{\sin }$ \\
\hline OTHER & & & \\
\hline \multicolumn{4}{|c|}{ WELL DEVELOPMENT } \\
\hline \multicolumn{4}{|c|}{ 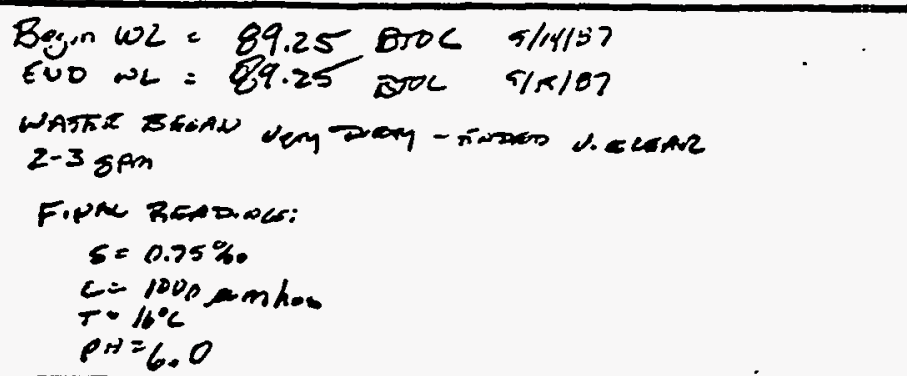 } \\
\hline \multicolumn{4}{|c|}{ COMMENT8: } \\
\hline & & & \\
\hline & & & \\
\hline
\end{tabular}




\section{TT MCOBS ENENEERING CROUP NC.

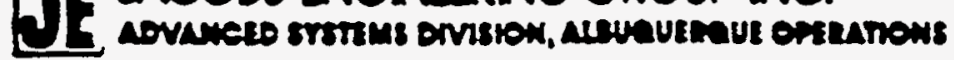

\section{WELL COMPLETION RECORD}

SITE ID: SPK-OI LOCATION ID: 925 DATE INSTALLED: 8/24/8? APPROX. BITE COOORDINATES:(FT.) N NK E NK

OPEN AREA DER LINEAL FT. (IN²/FT.) .05 Slot

(FORMATION OF COMPLETION: WASATCH

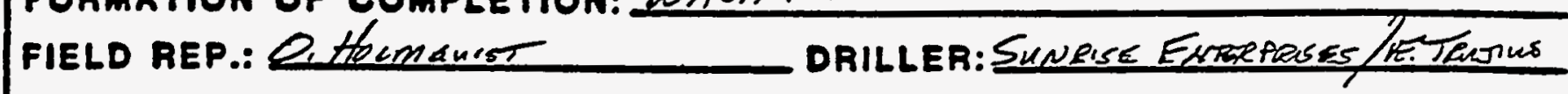

WELL CASING 4

DIAMETER (In) 4 Sal 40 fVC TYPE

HOLE DIAMETER $($ in) 8

TOTAL

DEPTH (11)

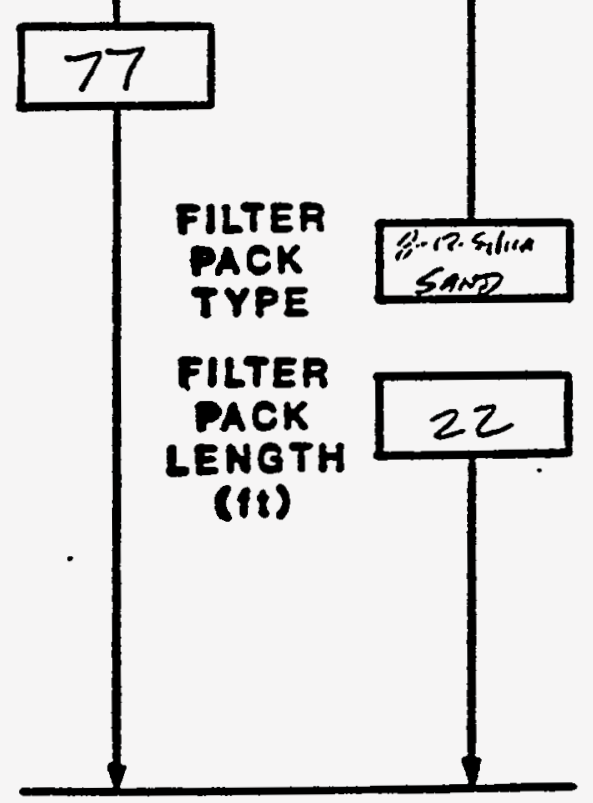

GROUND SURFACE

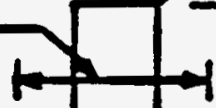

XYOTO

$\because$

SEAL FFMTNFE

TYPE PEMES

SEAL

LENGTH

(ft)

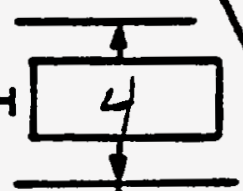

$\because$ अप्र

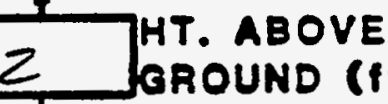

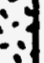

$\because$

$\because$

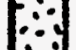

BACKFILL TYPE

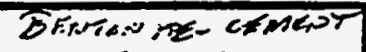

GLNT
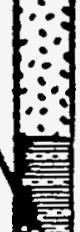

$\because \vdots 1$

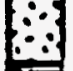

ELOT

OPENING
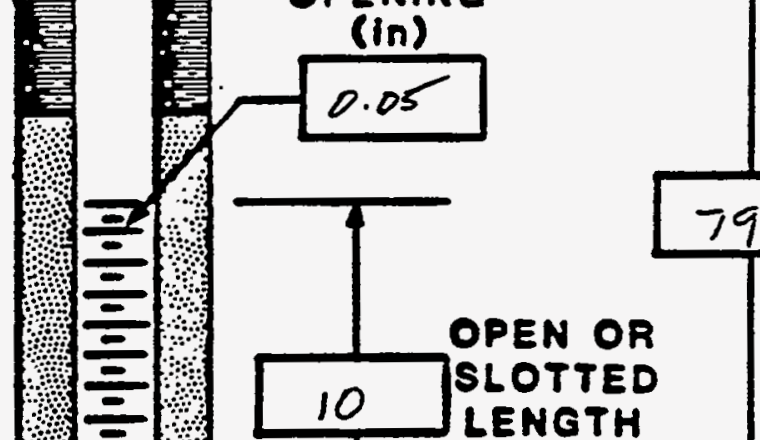

CASING LENGTH (ft)

\section{OPEN OR} SLOTTED LENGTH (ft)

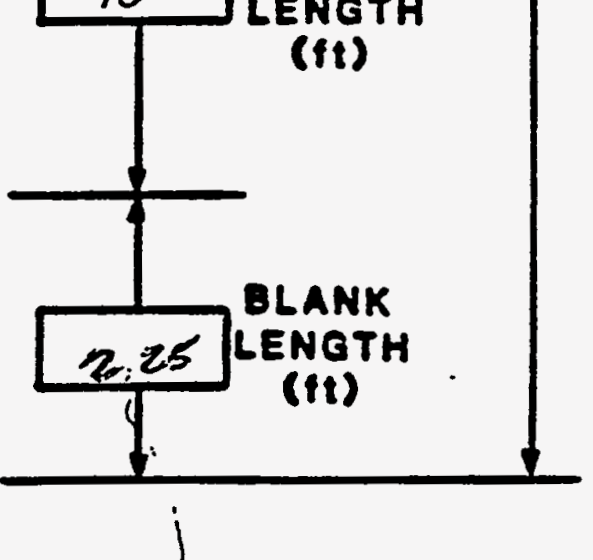

COMMENTS: 


\section{TI JACOBS ENGMEERNG GROUP NC.

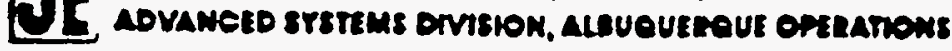 BOREHOLE/WELL CONSTRUCTION LOG}

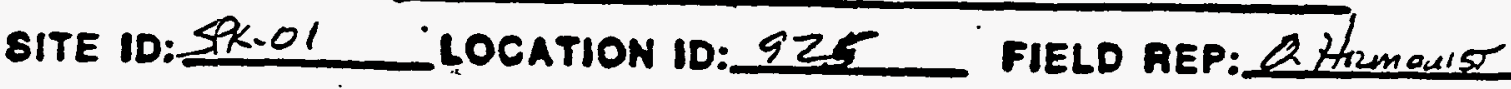

APPROX. 8ITE COORDINATES (FT.): NWK NK

GROUND ELEVATION (FT. MSL): NK COMPLETION DATE: 8/2Y /P7 BOREHOLE BUMMARY

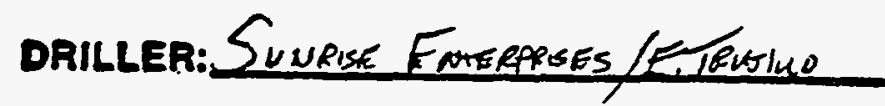

RIG TYPE: PDPRIL WSEY

\begin{tabular}{|c|c|}
\hline$\left|\begin{array}{c}\text { TYPE } \\
\text { CODE }\end{array}\right|$ & DESCRIPTION \\
\hline
\end{tabular}

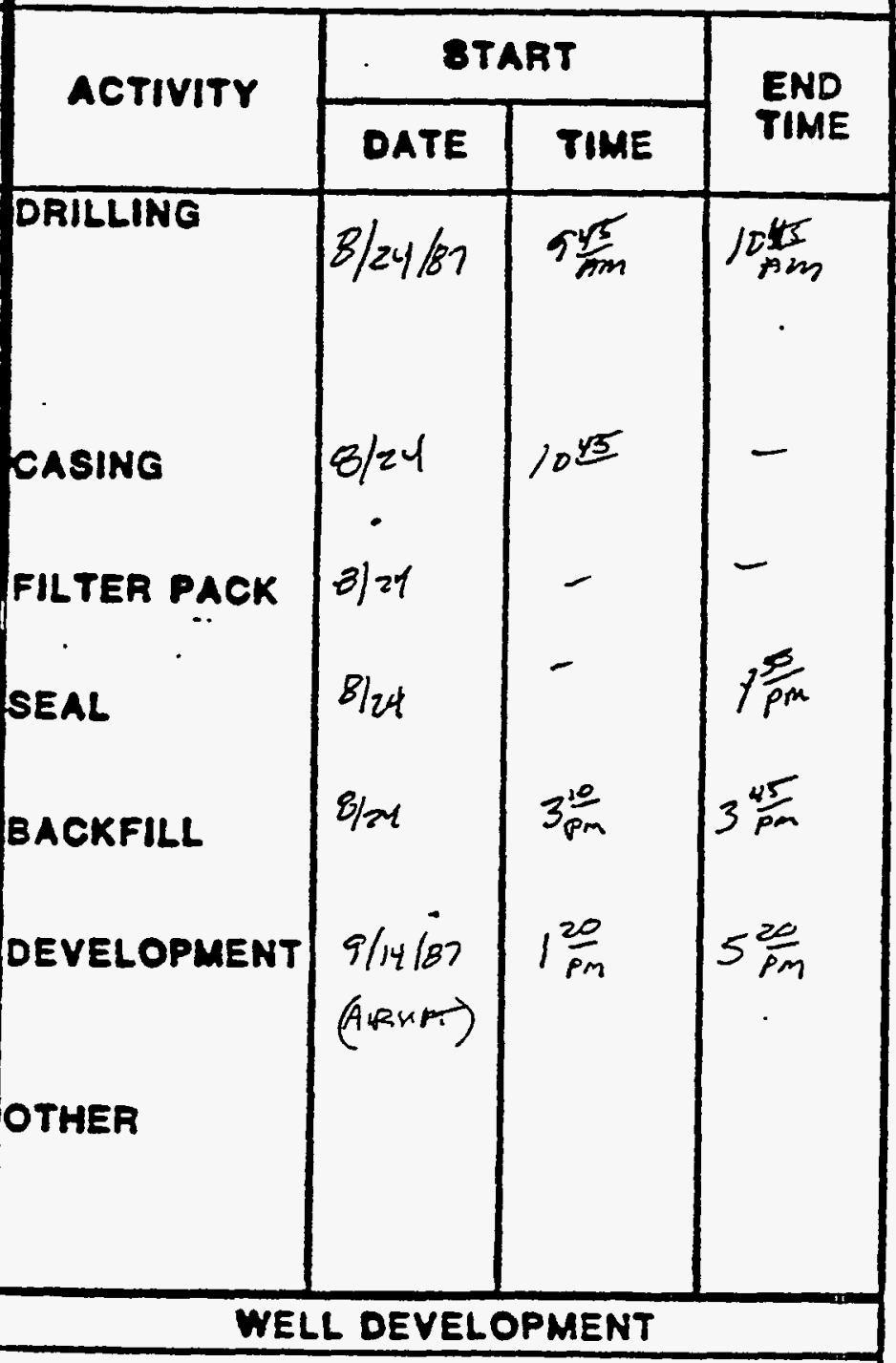

Bogin WL $=55.5$ GTOC $9 / 14 / 87$

EDD NOL $=55.6$ GNOC $5 / 45187$

wath began vidirty - ead viciean

1-2grm

PINAL RAAD,NES:

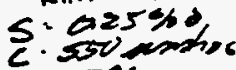

$T=15^{\circ} \mathrm{C}$

pd $x<0$

\begin{tabular}{|l|l|l|}
\hline & & \\
\hline & & \\
\hline & & \\
\hline e-Deckin 8-seal F-Fur Peck \\
\hline
\end{tabular}

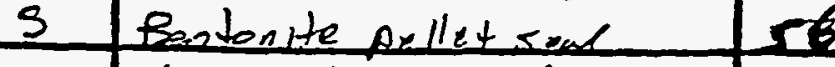

E 8 -12 silicasent 77

COMMENT8: 


\section{TI HCOBS ENENEERTE GROUP NC.

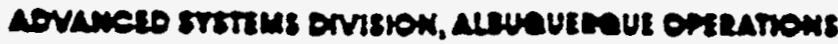

\section{WELL COMPLETION RECORD}

SITE ID:SPK-O/_LCATION ID: 926 DATE INSTALLED: $8 / 27 / 87$ APPROX. 8ITE COORDINATES:(FT.) N NK E NK OPEN AREA PER LINEAL FT. (IN'/FT.) OSS/OT

FORMATION OF COMPLETION: 12 AS PTLH

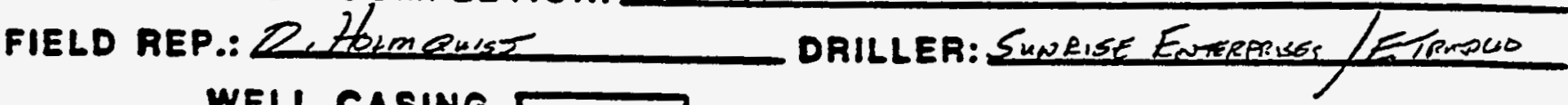
WELL CASING DIAMETER (in)

HOLE DIAMETER (in) 6
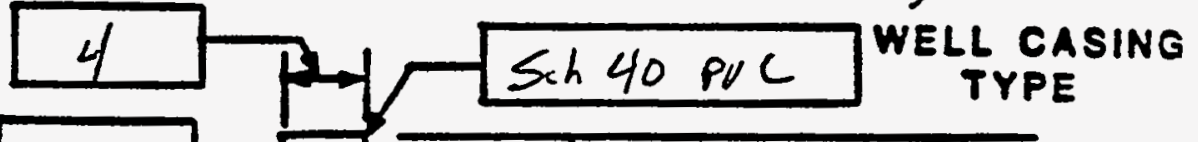

TOTAL DEPTH (ti) GROUND SURFACE
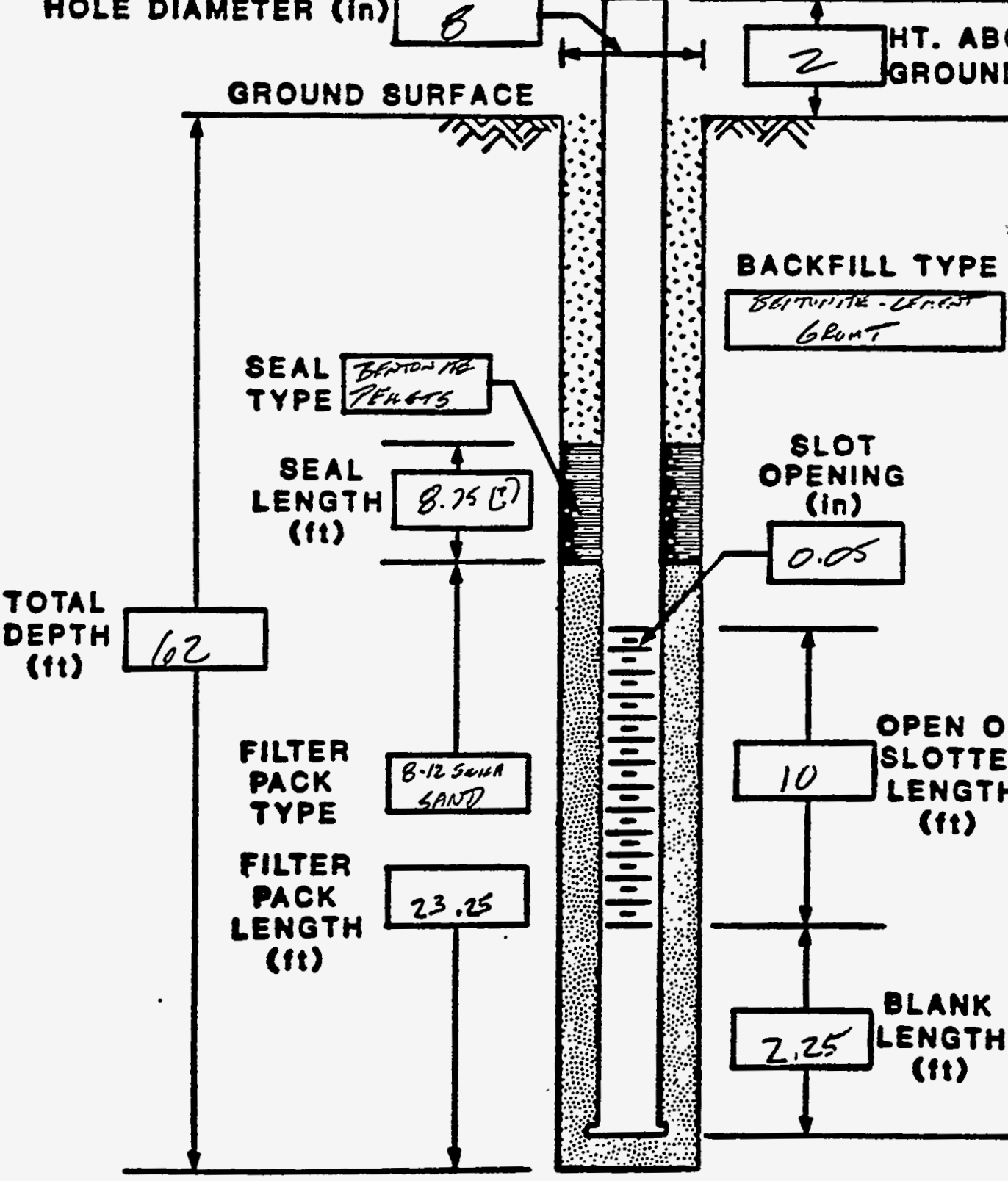

$\therefore$
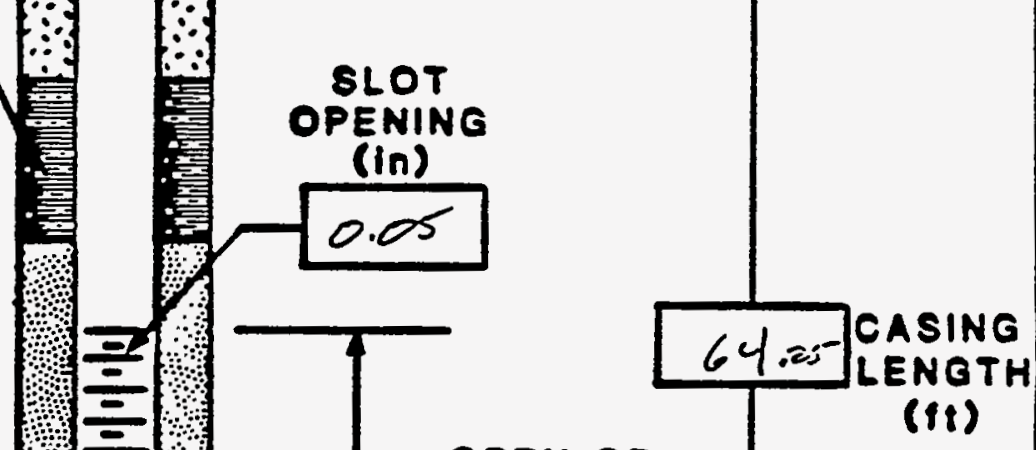

COMMENTS: 


\section{TF LACOBS ENGMEERNG GROUP WC.

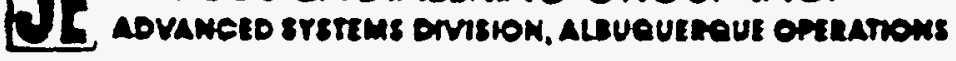 \\ BOREHOLE/WELL CONSTRUCTION LOG}

SITE ID: SPK.OI LOCATION ID: 926 FIELD REP: Q Holmenes APPROX. BITE COORDINATES (FT.): N NK BROUND ELEVATION (FT. MSL): DK E NK

BOREHOLE BUMMARY

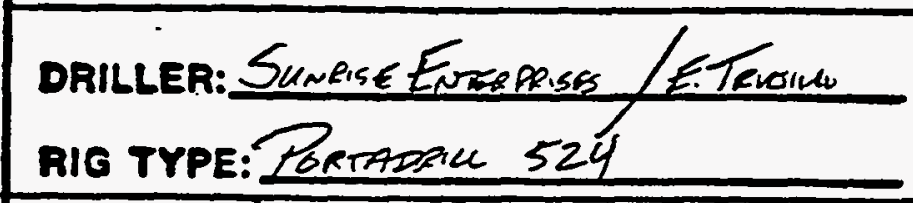

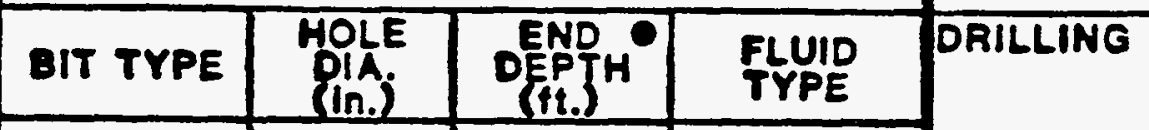

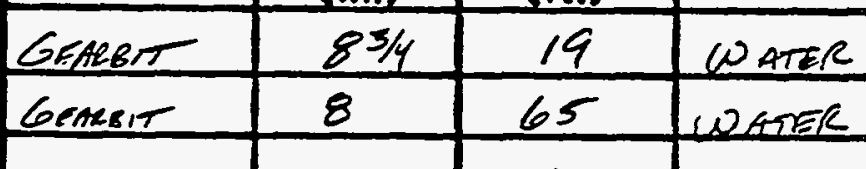

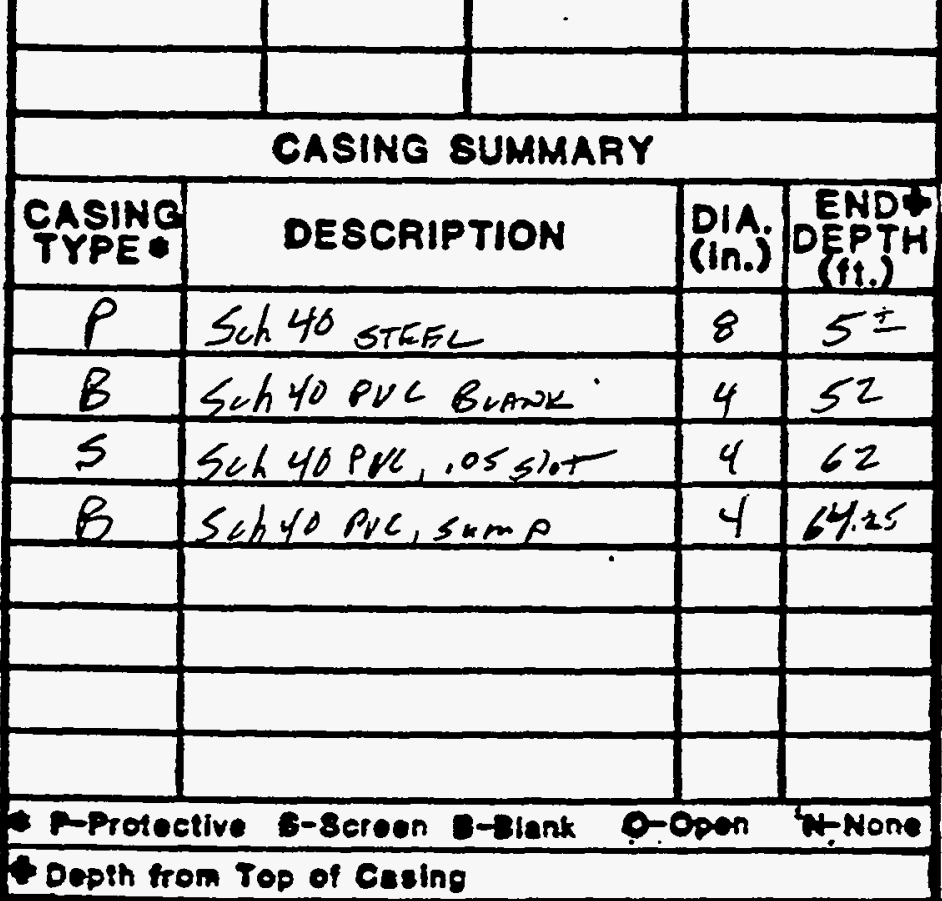

\begin{tabular}{|c|c|c|}
\hline \multicolumn{3}{|c|}{ WELL CONSTRUCTION } \\
\hline $\begin{array}{l}\text { TYPE } \\
\text { CODE }\end{array}$ & DESCRIPTION & DEPTH \\
\hline B & Benmuin-LEmRar GRont & 30 \\
\hline 5 & Bentonite Paneds & 38.75 \\
\hline$F$ & $8-12$ Salse 5 nom & 62 \\
\hline & & \\
\hline & & \\
\hline & & \\
\hline & & \\
\hline & & \\
\hline 0 & $e-80 a 1$ & Peck \\
\hline - Do & in from cround suriece & \\
\hline
\end{tabular}

COMPLETION DATE: $8(2)$ /e? CONSTRUCTION TIME LOG

\begin{tabular}{|c|c|c|c|}
\hline \multirow{2}{*}{ ACTIVITY } & \multicolumn{2}{|c|}{ BTART } & \multirow{2}{*}{$\begin{array}{l}\text { END } \\
\text { TIME }\end{array}$} \\
\hline & DAte & TIME & \\
\hline DRILLING & $8120 / 87$ & $q^{A x}$ & $1015 / 40$ \\
\hline CASING & $8 / 27 / 87$ & $10=$ & $1 / A+1$ \\
\hline FILTER PACK & 812187 & 1105 & 1125 \\
\hline SEAL & $8120 / 87$ & 115 & ij \\
\hline BACKFILL & $8 / 27 / 27$ & 135 & 145 \\
\hline DEVELOPMENT & 91,5187 & $12 \frac{25}{p m}$ & $1 \frac{30}{10}$ \\
\hline OTHER & & & \\
\hline & & & \\
\hline
\end{tabular}

WELL DEVELOPMENT

Bum wL"

Werl making very Dirhy waten \&! a large omowe to of send ( filtru pad)

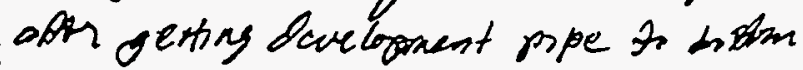
oneces could nit ses thene agam.

comments: Beteer sereen for casing-notederloped 


\section{TF MCOSS ENGNERTE CROUP NC.

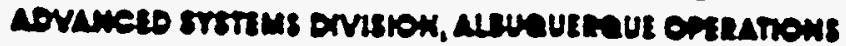

\section{WELL COMPLETION RECORD}

SITE ID: SPK. OI LOCATION ID: 927

APPROX. 8ITE COOORDINATES:(FT.) N NK

OPEN AREA PER LINEAL FT. (IN2/FT.) 0.05 SIOT

FORMATION OF COMPLETION: COASATCH

FIELD REP.: Q Ho LM OUIST

WELL CASING DIAMETER (In)

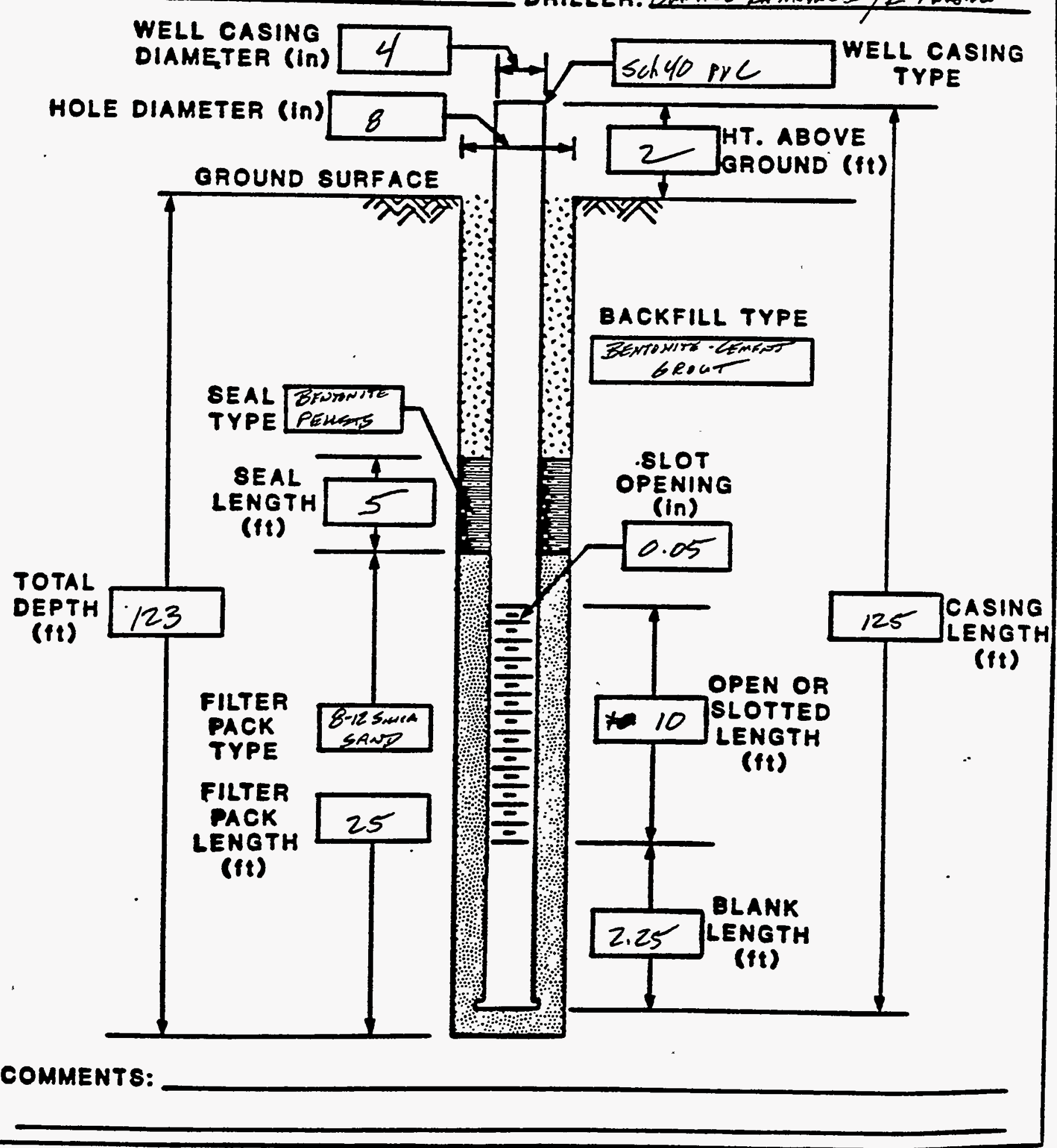

HOLE DIAMETER (in)
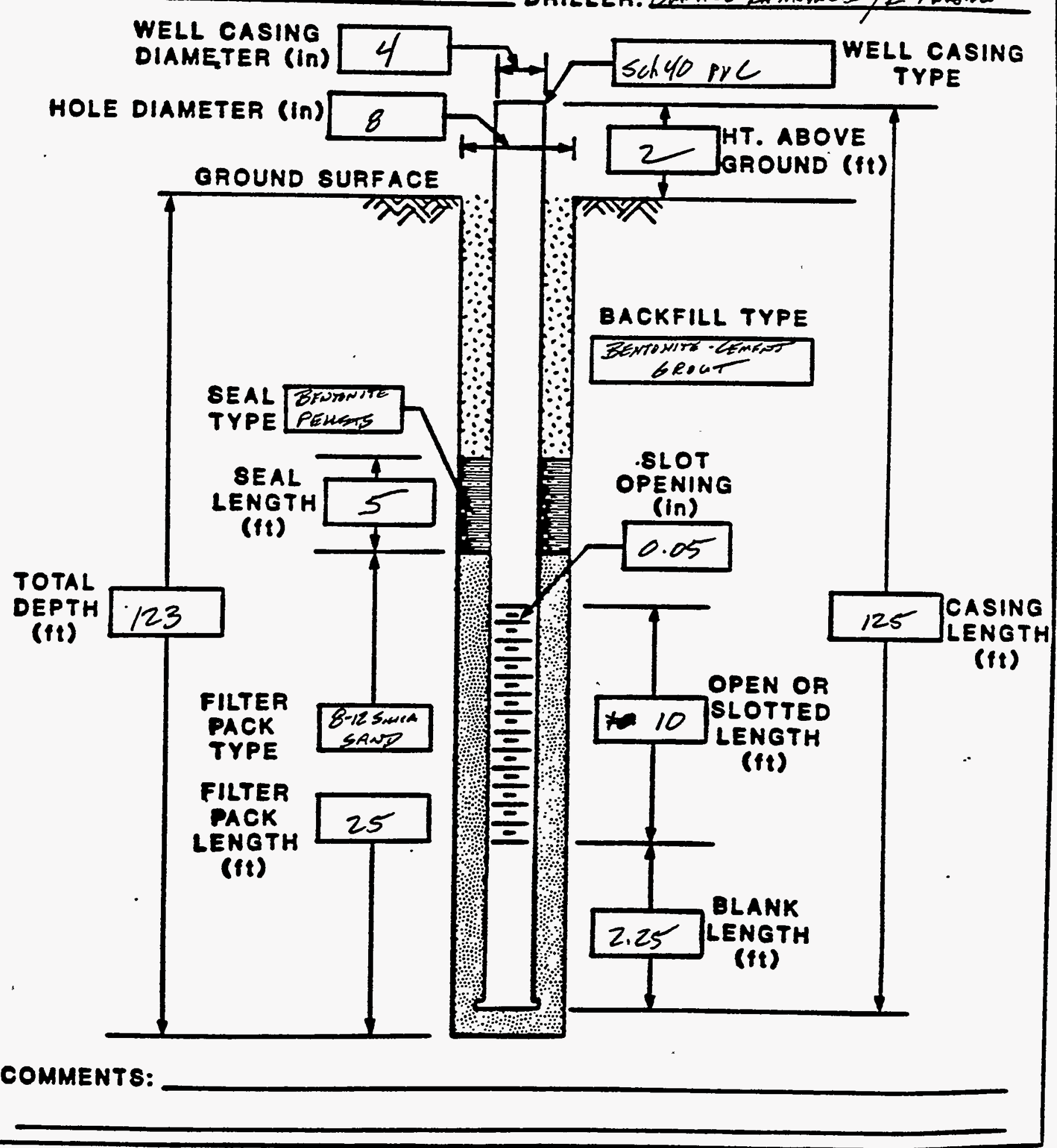

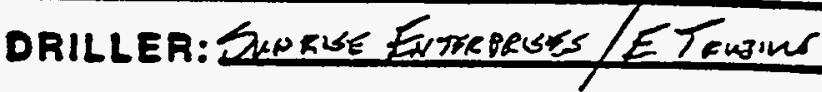
DATE INSTALLED: 8/28/87 E NIK 


\section{TI JACOBS ENGNEERING GROUP NC.

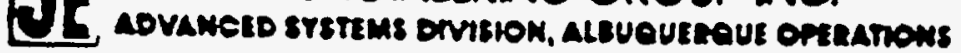 \\ BOREHOLE/WELL CONSTRUCTION LOG}

SITE ID:SPK-0) LOCATION 10: 927 FIELO REP:O H dLmQUIST APPROX. 8ITE COORDINATES (FT.): N N/K _ NSK

OROUND ELEVATION (FT. MSL): N/K_COMPLETION DATE: $8 / 28 / 87$ BOREHOLE BUMMAAY

DRILLER:SUUREE ENTERPRISES[ETEUTILO RIG TYPE: Prespons $\Rightarrow 24$

BIT TYPE HOLE

\begin{tabular}{|c|c|c|c|}
\hline & (in. & $(\text { tit. })^{n}$ & PE \\
\hline TRlas & $83 / 4$ & 20 & WATFR \\
\hline 111 & 8 & 125 & $\angle A T E R$ \\
\hline
\end{tabular}

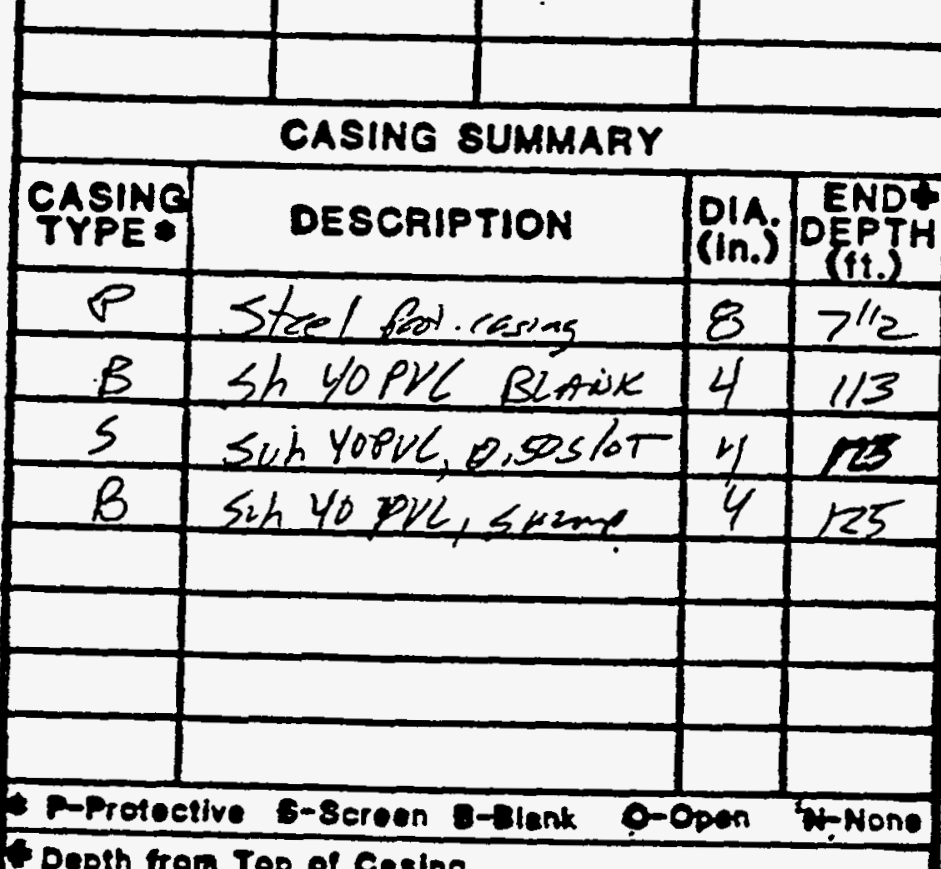

\section{WELL CONSTRUCTION}

\begin{tabular}{|c|c|c|}
\hline $\begin{array}{l}\text { TYPE } \\
\text { CODE: }\end{array}$ & DESCRIPTION & DEPTS \\
\hline $\bar{\beta}$ & $3 \%$ bertante / Poatlen $\&$ Gement scout & 95 \\
\hline$\underline{s}$ & Eentoarte Peilet seal & 98 \\
\hline$E$ & $8+12$ silica sead & 123 \\
\hline & & \\
\hline & & \\
\hline & & \\
\hline & & \\
\hline & & \\
\hline 28 & Eetin & Pack \\
\hline
\end{tabular}
casing CONSTRUCTION TIME LOG

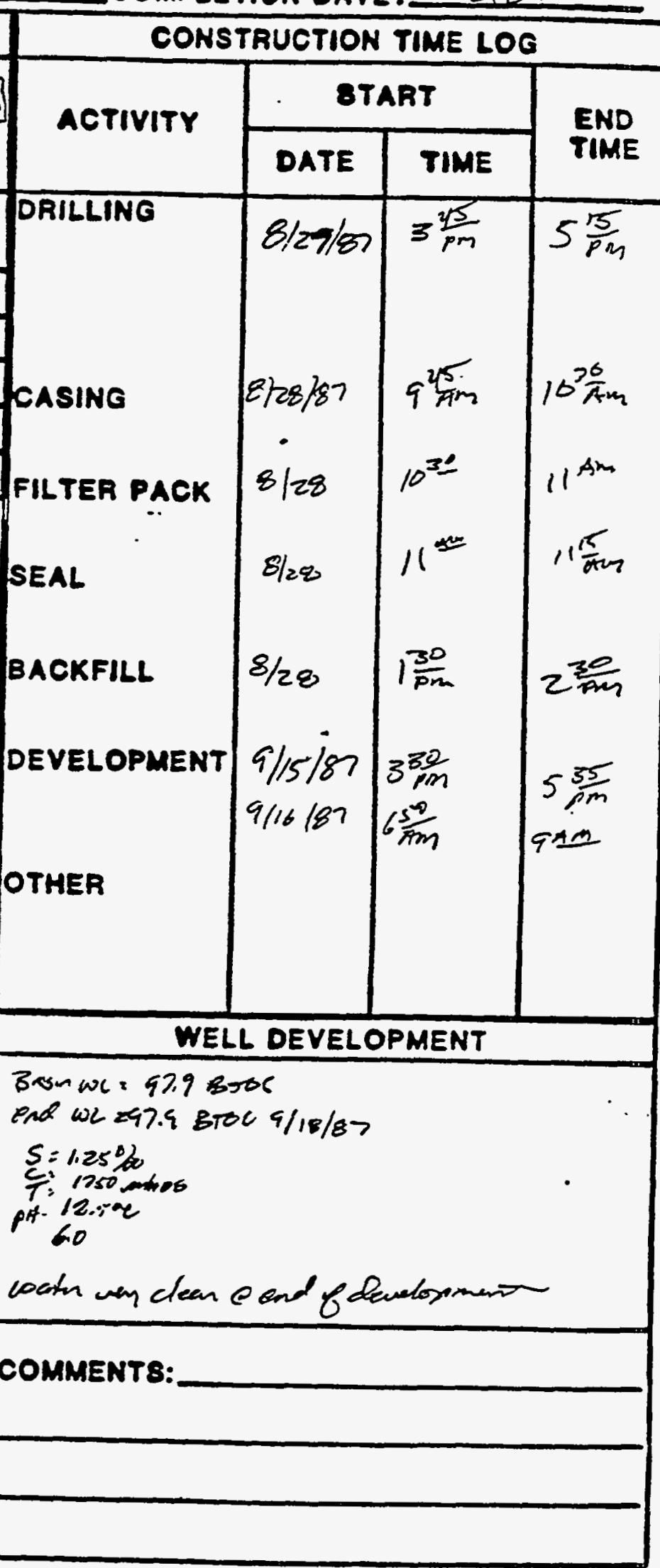




\section{TF MCOBS ENGNEERTG EROUP NK.

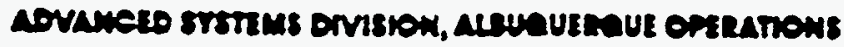

\section{WELL COMPLETION RECORD}

SITE ID:SPK-OI LOCATION ID: 928 DATE INSTALLED: 8131/87 APPROX. SITE COORDINATES:(FT.) NNK E $N$ KK

OPEN AREA PER LINEAL FT. (IN2/FT.) O.05 Slot

FORMATION OF COMPLETION: 12 ASATCH

FIELD REP.: O. HoLm Cu'sT

DRILLER:SANRISE FOTRRPISES

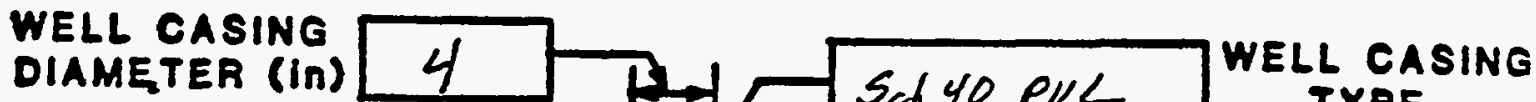

HOLE DIAMETER (In) $83 / 4$

GROUND SURFACE
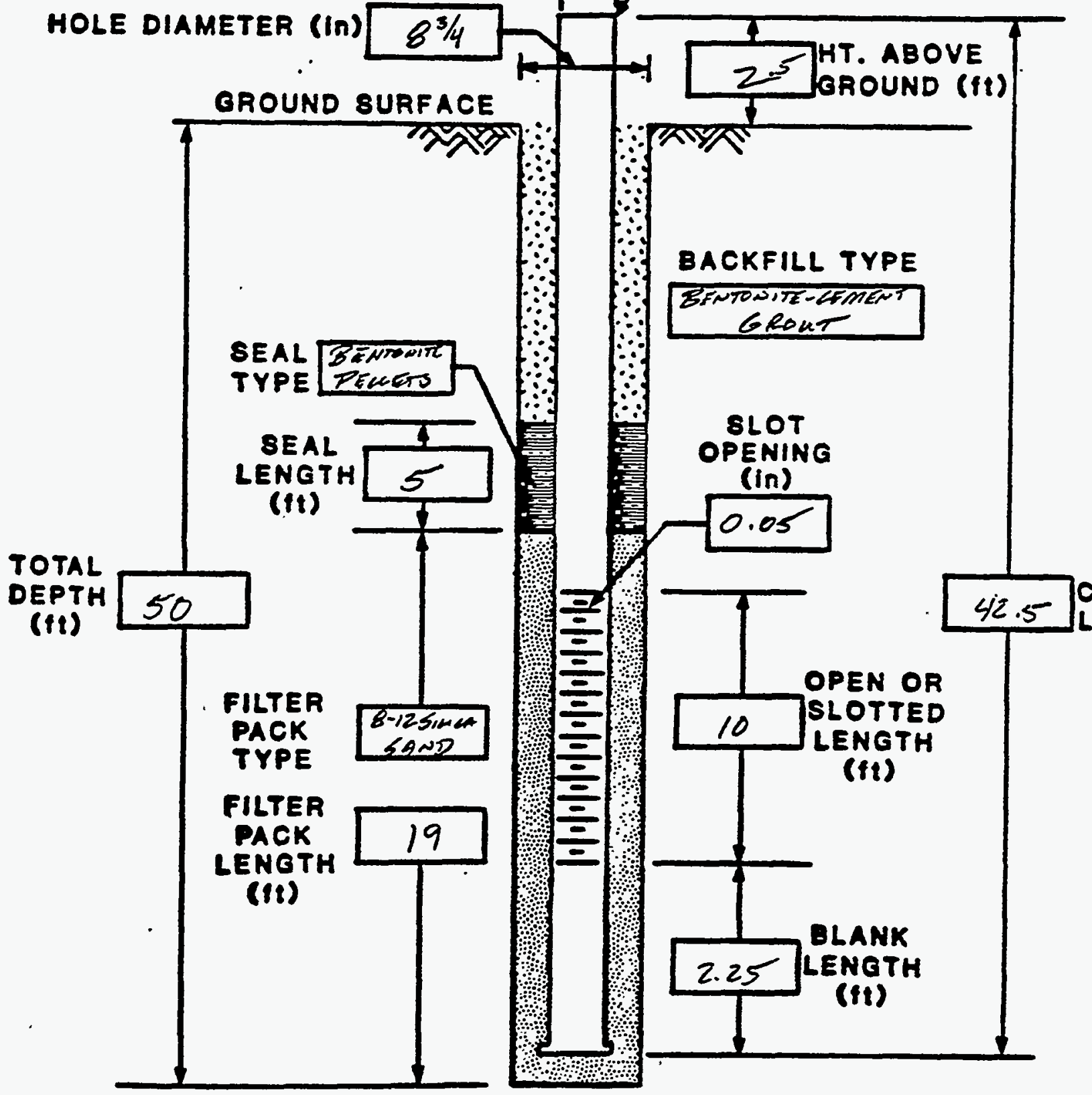

BACKFILL TYPE

EFNTONTE-EFMENT GROAT
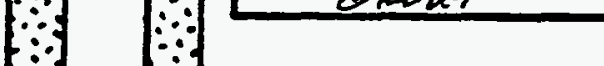

$\because$
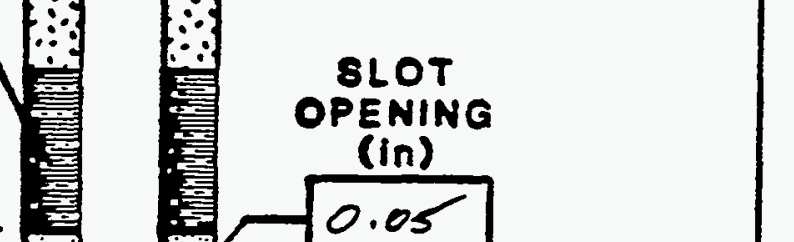

TOTAL DEPTH (11)
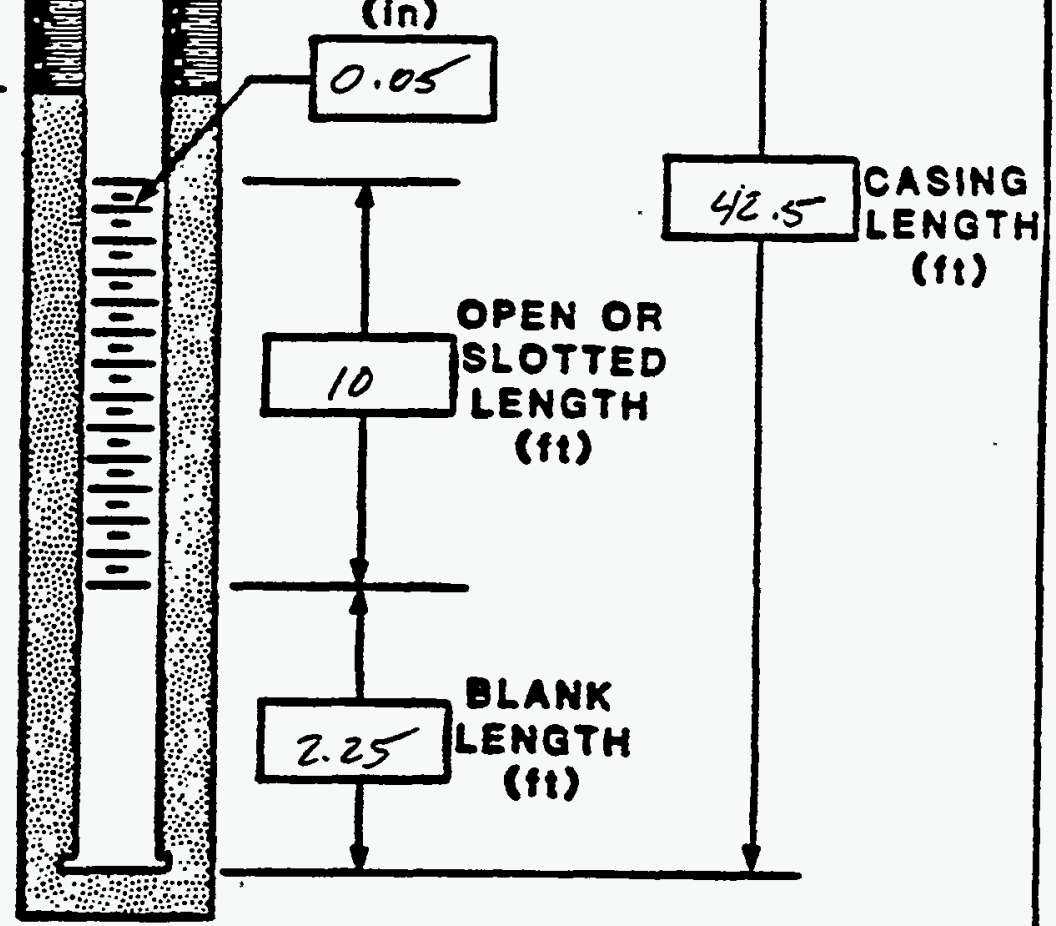

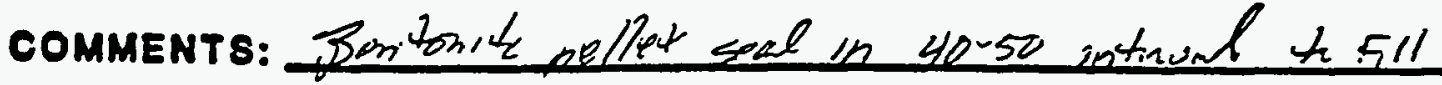

- coreshole suerstax 


\section{II $A A C O B S$ ENGNEERNG GROUP NC. De BOREHOLE/WELL CONSTRUCTION LOG}

SITE ID: SPK-OI LOCATION ID: 928 FIELD REP:O.ARManCST APPROX. BITE COORDINATES (FT.): N NK BROUND ELEVATION (FT. MSL): NIL E NK

\section{. BOREHOLE BUMMARY}

DRILLER:SUNRSE FNTEPRISES/ETRUTILU RIG TYPE: Poctasal 524

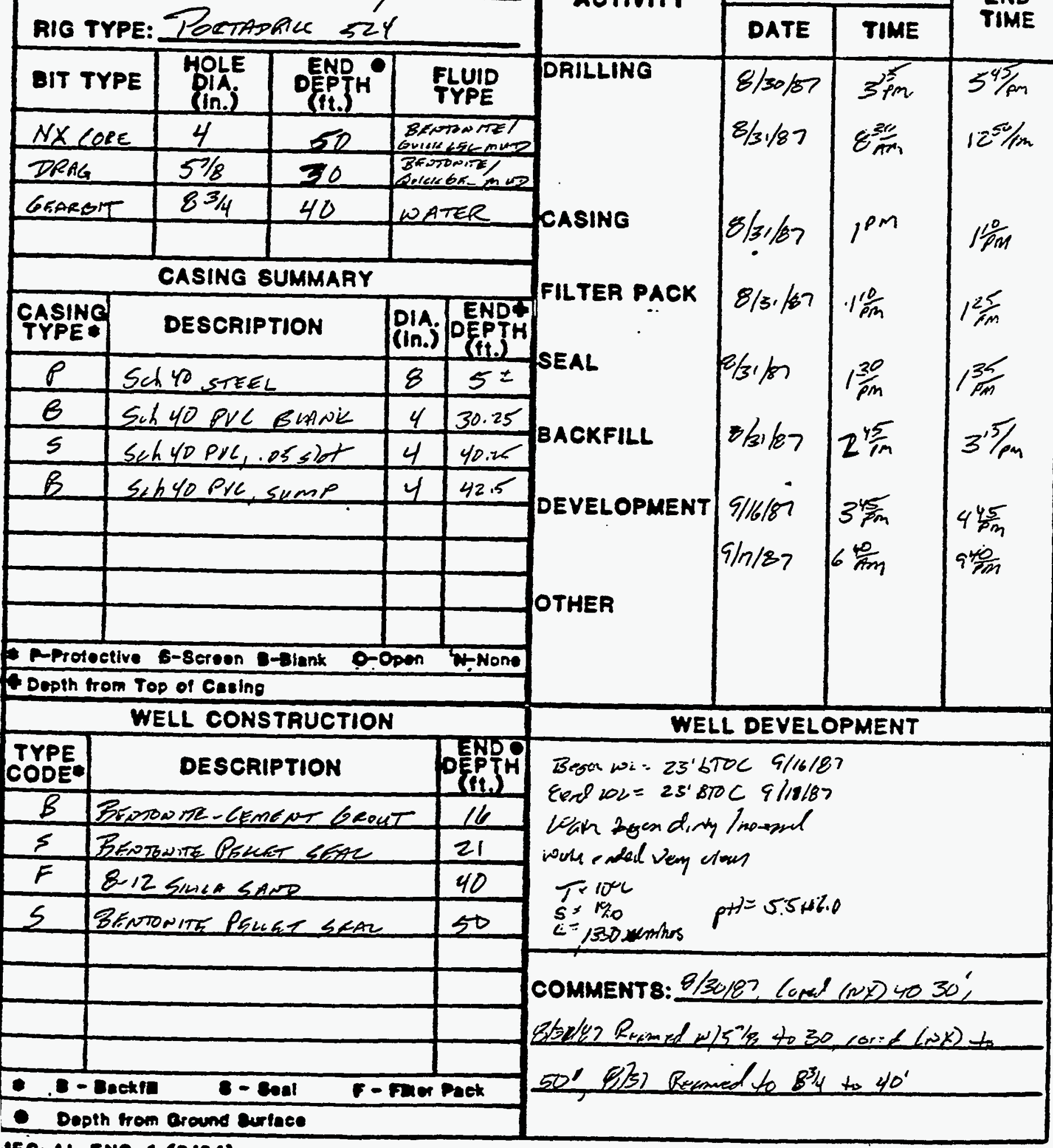




\section{TE HCOSS ENENEERTG GROUP NC.

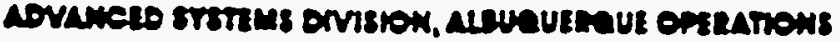

\section{WELL COMPLETION RECORD}

SITE ID: SPK-01 LO'CATION ID: 929

APPROX. 8ITE COOORDINATES:(FT.) N DK

OPEN AREA DER LINEAL FT. (IN2/FT.) 0.05 POT

FORMATION OF COMPLETION: WOASATCH

DRILLER: SUNRISE ENTERPRSES/ETRUTILA

WELL CASING DIAMETER (In)

HOLE DIAMETER (In)

\section{TOTAL} DEPTH (ti) FIELD REP.: O. Heanuarg DATE INSTALLED: 9/4/82 I E NE

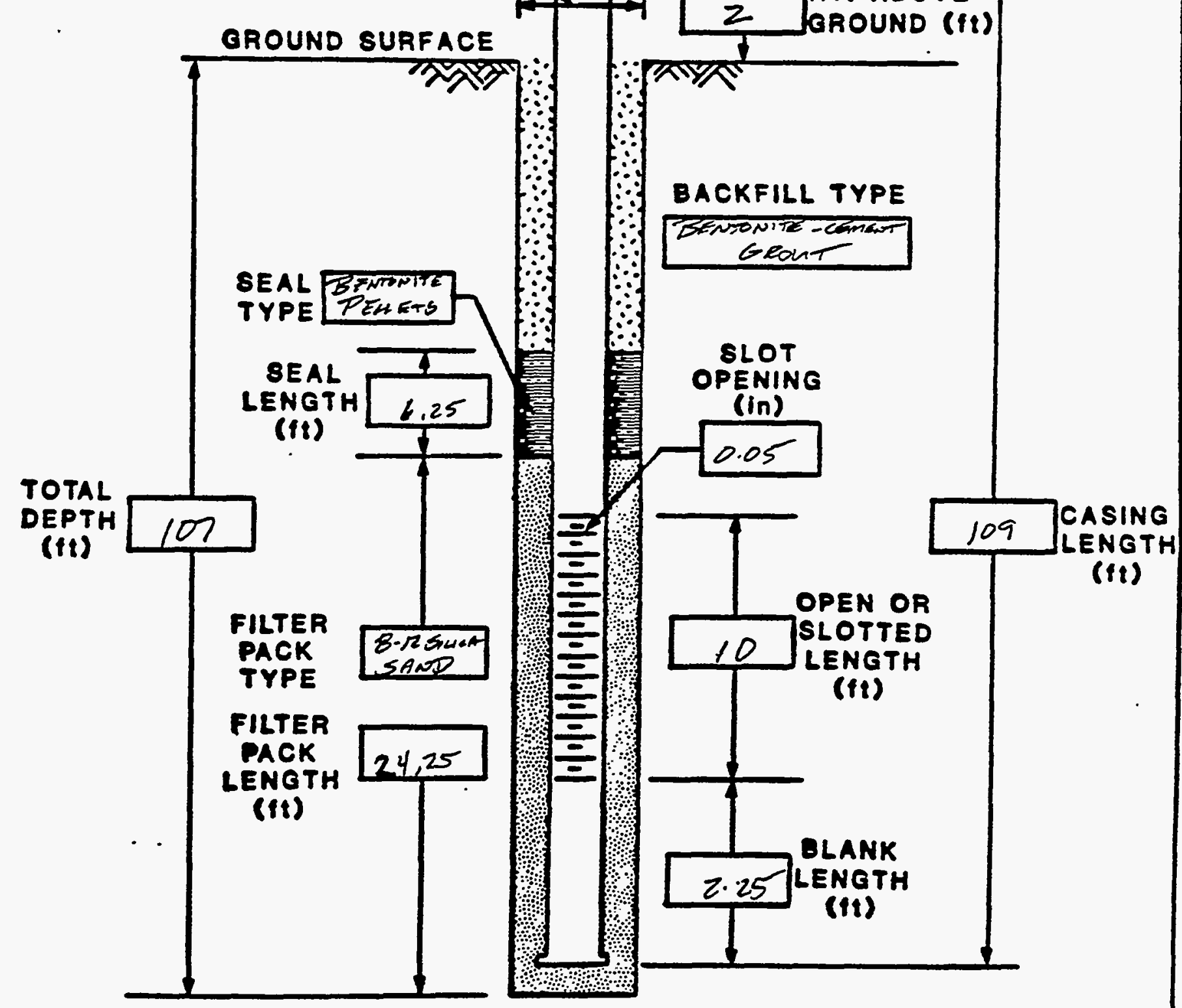

COMMENTS: 


\section{TF. LACOBS ENGNEERIV GROUP NC.

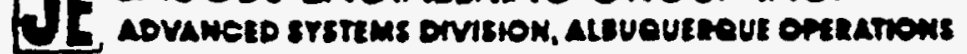 BOREHOLE/WELL CONSTRUCTION LOG}

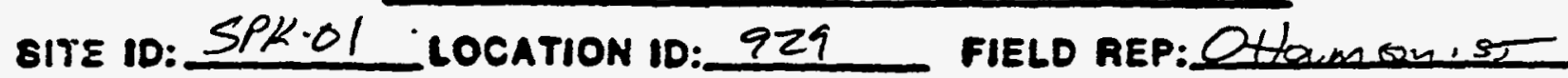
APPROX. 8ITE COORDINATES (FT.): N_NK E DK OROUND ELEVATION (FT. MSL): D2K

\section{BOREHOLE BUMMARY}

DRILLER: E.TCM, TIIO

RIo TYPE: Bothdrill 524

\begin{tabular}{|l|l|l|l|l}
\hline HOLE & END O \\
\hline
\end{tabular}

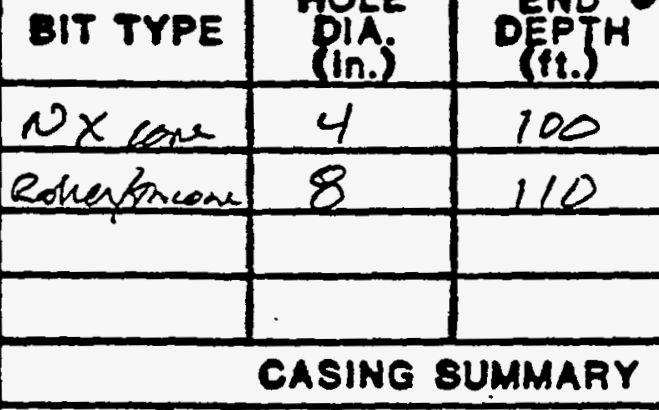

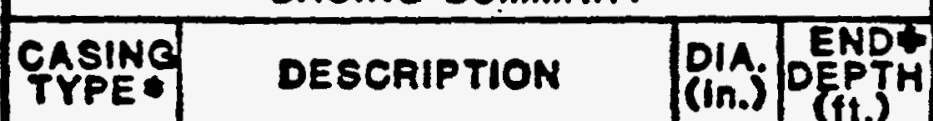

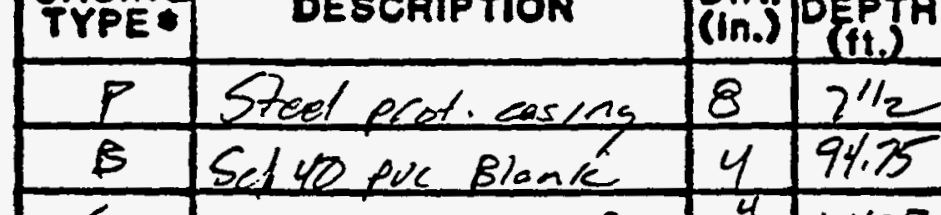

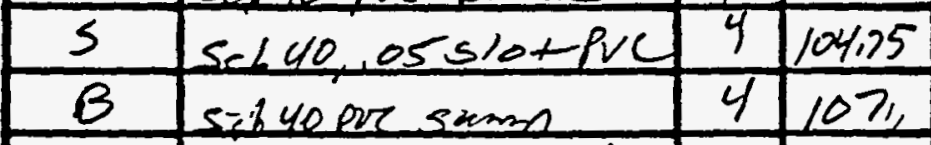

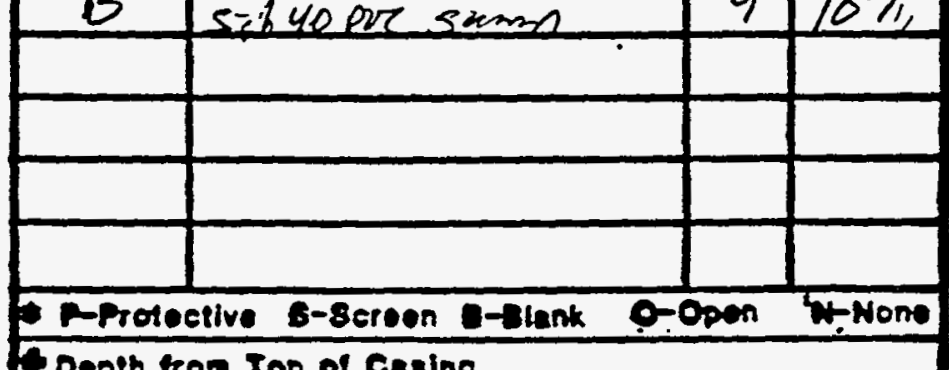

Dopth trom Top of Cesing

\begin{tabular}{|c|c|c|}
\hline \multicolumn{3}{|c|}{ WELL CONSTRUCTION } \\
\hline $\begin{array}{l}\text { TYPE } \\
\text { CODE }\end{array}$ & DESCRIPTION & DEPYH \\
\hline $\bar{B}$ & Bentonite lacout seal & 82.75 \\
\hline$s$ & Bewonite peuezo & 8275 \\
\hline$E$ & $83-12$ Salea Sand & 107 \\
\hline & & \\
\hline & & \\
\hline & & \\
\hline & & \\
\hline & & \\
\hline e. & $-2-201$ & Poet \\
\hline
\end{tabular}

- Dopth from cround eurtece

Coopth from Ground suret
COMPLETION DATE: $9 / 4 / 82$ CONSTRUCTION TIME LOG

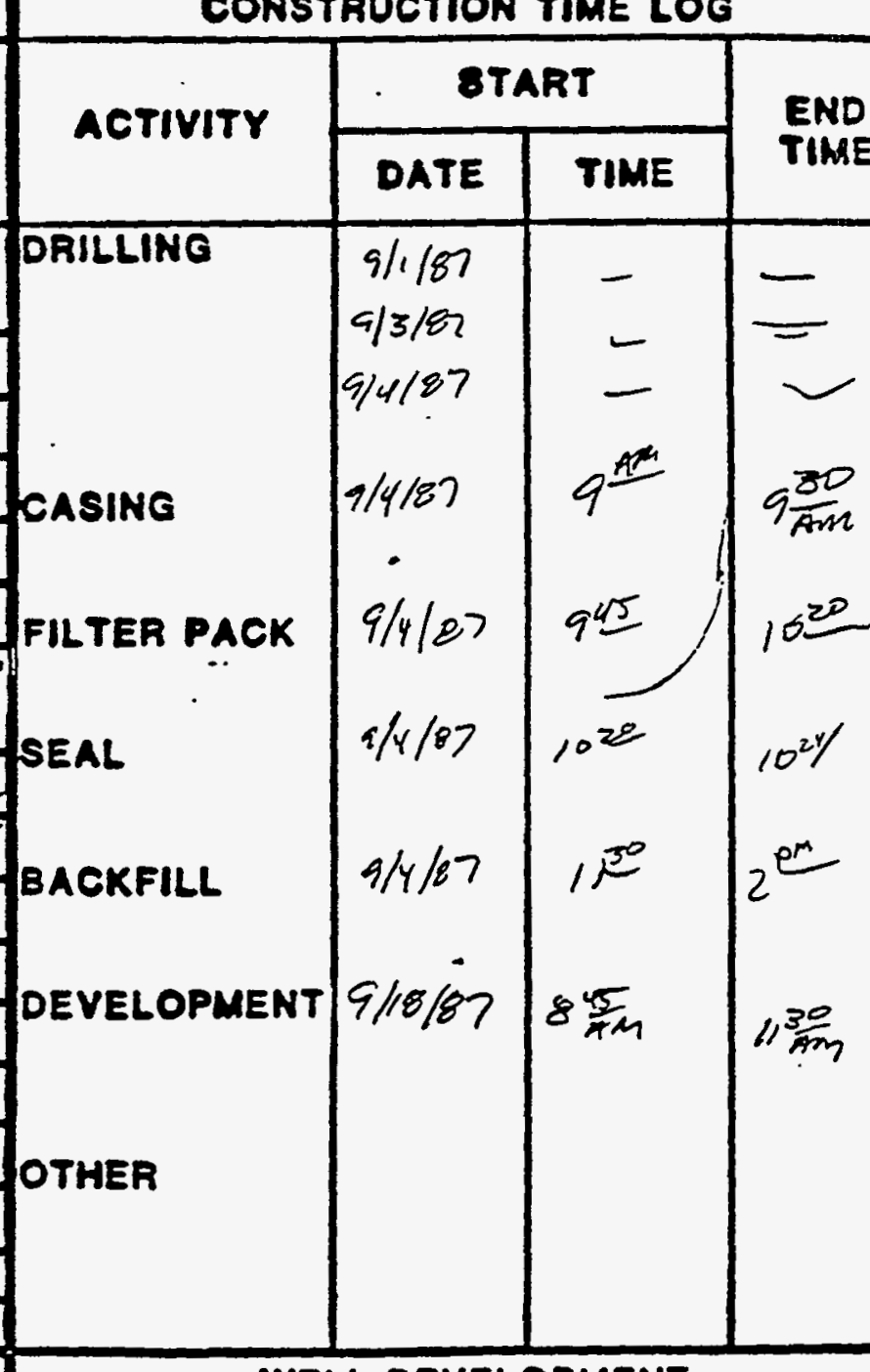

WELL DEVELOPMENT

$$
\text { Bym ma: } 79.5 \text { BTOC }
$$

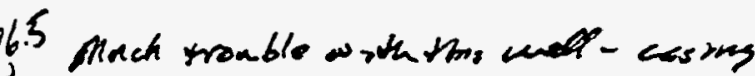

boken dritled Bkern -

dacloped $23 / 1$ hrs - ptt $\approx 8.529 .0$

vay fitthe on no wan recomy

COMMENT8: 


\section{TIF HCOSS ENENEERTG GROUP NC.

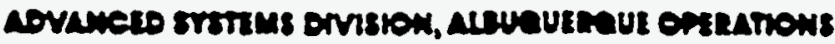

\section{WELL COMPLETION RECORD}

SITE ID: SPK.OI LOCATION ID: 930

APPROX. 8ITE COORDINATES:(FT.) N NK
DATE INSTALLED: $9 / 10 / 87$ E NK

\section{OPEN AREA PER LINEAL FT. (IN2/FT.) .05 Slot}

IFORMATION OF COMPLETION: WASATLH

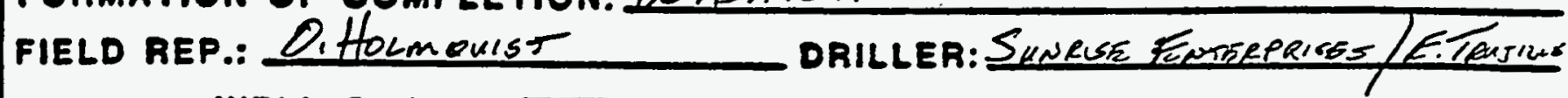
WELL CASING 4 DIAMETER (In) 4 SCh 40 PUL
WELL CASING

HOLE DIAMETER (in)

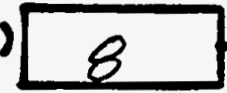

GROUND SURFACE

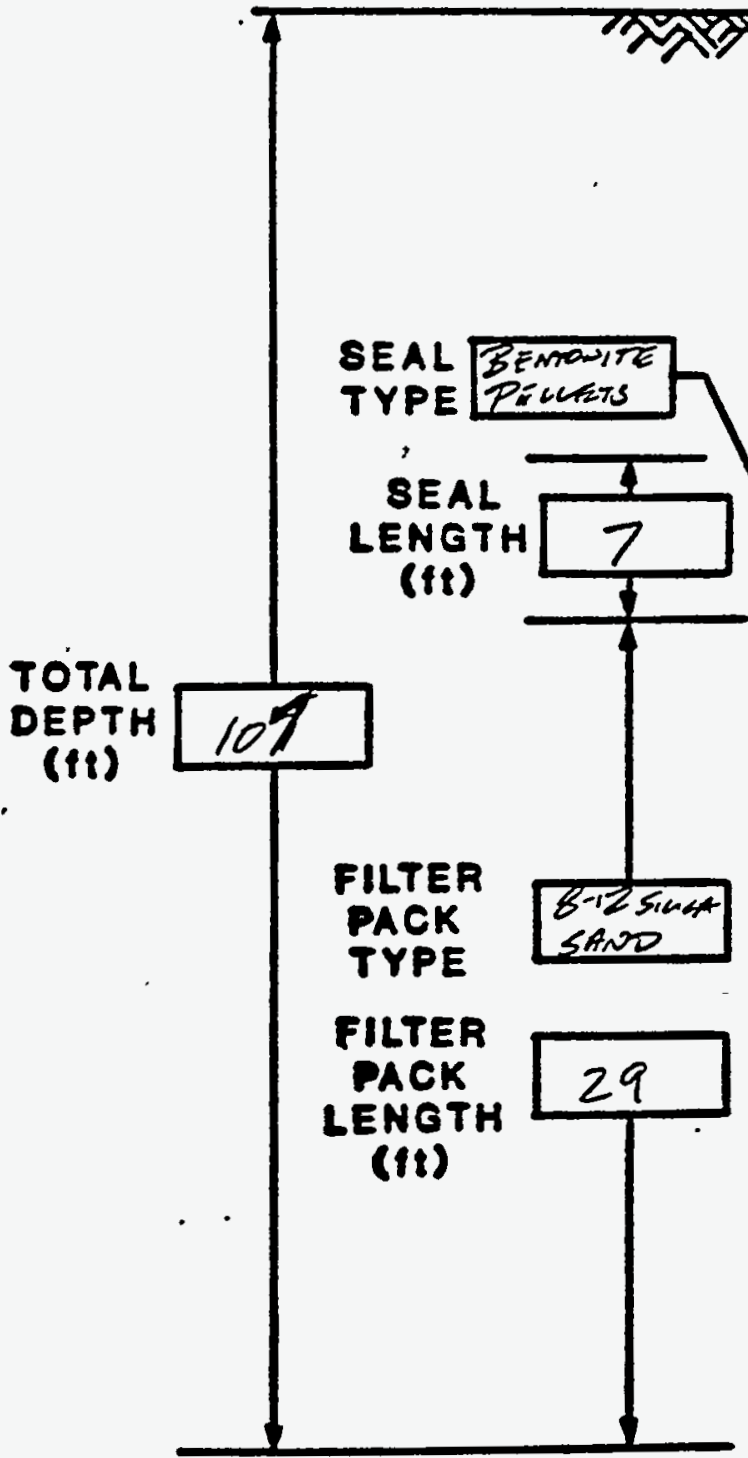

$\because$<smiles>CCC(C)(C)C(C)C</smiles>

$\because$ सיण TYPE

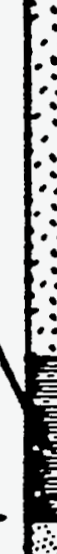

BACKFILL TYPE

BEPTODITE- CEMTAS Geont
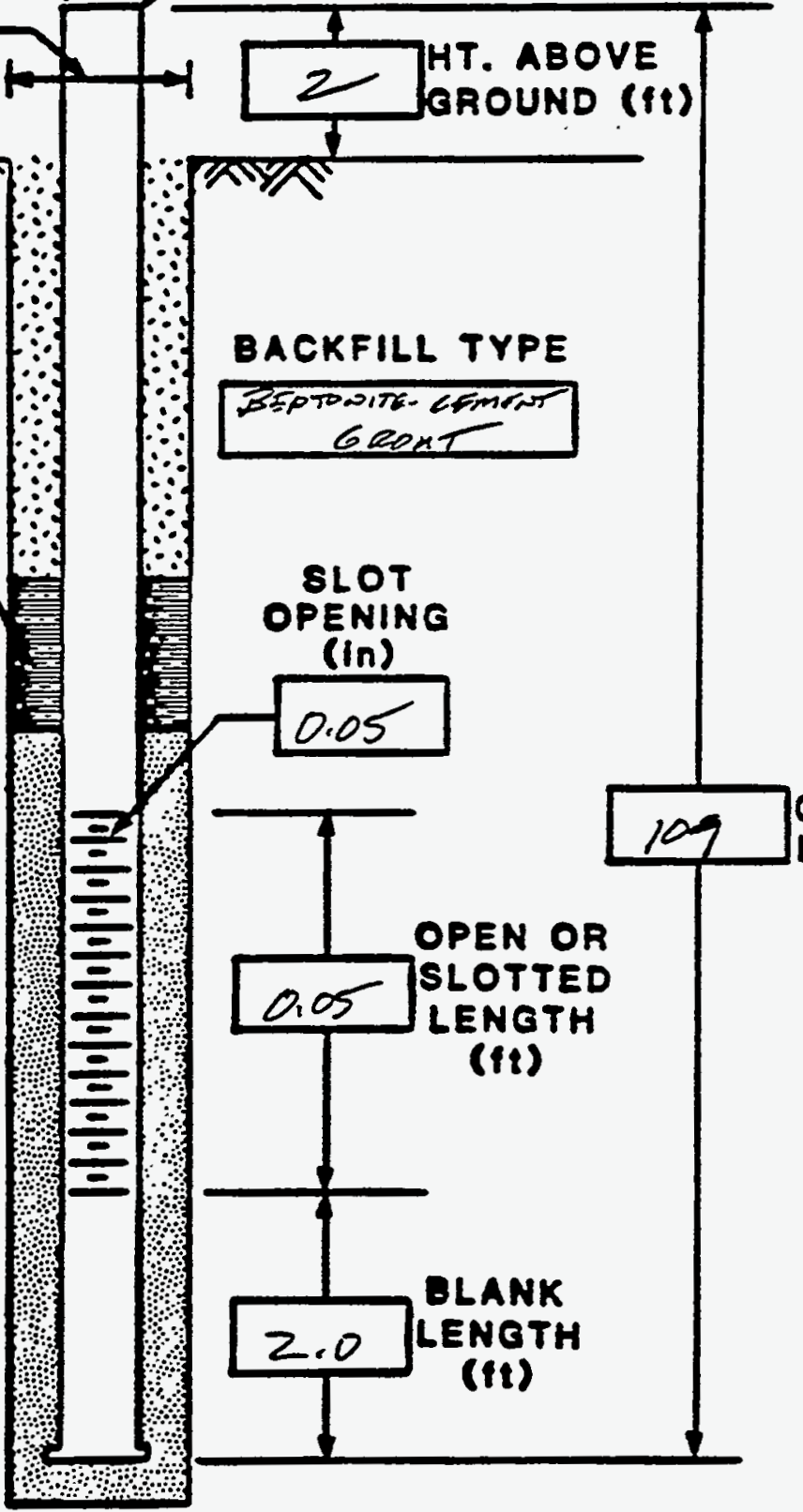

SLOT

OPENING (In)

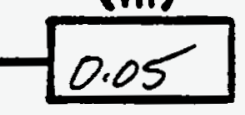

$\longrightarrow$

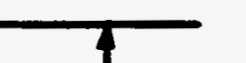

COMMENTS: 


\section{TF $2 A C O B S$ ENGNEERING GROUP NC.

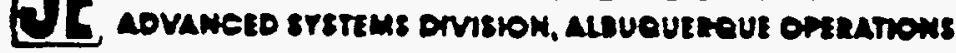 BOREHOLE/WELL CONSTAUCTION LOG}

SITE ID: SPK-OI LLOCATION 1D: 930 FIELD REP:O HoLmou'st APPROX. BITE COORDINATES (FT.): N NK OROUND ELEVATION (FT. MSL): NIL EN BOREHOLE BUMMARY DRILLER: E.TMillo /Smase Entupuses RIG TYPE: Fandall 524

\begin{tabular}{|c|c|c|c|}
\hline DIT TYPE & $\begin{array}{l}\text { HOLE } \\
\text { OiAA.) } \\
\text { in.. }\end{array}$ & OENPH & $\begin{array}{l}\text { FLUID } \\
\text { TYPE }\end{array}$ \\
\hline \multicolumn{4}{|l|}{82} \\
\hline Rollew frusine & $83 / 4$ & 19 & Watn \\
\hline Rojlostrainen & 8 & 107 & Wadn \\
\hline & & & \\
\hline
\end{tabular}

\begin{tabular}{|c|c|c|c|}
\hline \multicolumn{4}{|c|}{ CASING SUMMARY } \\
\hline $\begin{array}{l}\text { CASING } \\
\text { TYPE }\end{array}$ & DESCRIPTION & $\begin{array}{l}\text { ola. } \\
\text { (in.) }\end{array}$ & $\begin{array}{l}\text { END } \\
\text { DEPPH }\end{array}$ \\
\hline$p$ & $8^{\prime \prime}$ seh40 steed & $8^{\prime \prime}$ & 5 \\
\hline$B$ & Sch 40 puC blank & 4 & 95 \\
\hline 5 & $\operatorname{seh} 40$ PlC .05sit & 4 & 105 \\
\hline$B$ & sch yo pre sumpo & 4 & 107 \\
\hline & & & \\
\hline & & & \\
\hline & & & \\
\hline & & & \\
\hline 4 Dopth & Top of Casing & & \\
\hline
\end{tabular}

\begin{tabular}{|c|c|c|}
\hline \multicolumn{3}{|c|}{ WELL CONSTRUCTION } \\
\hline $\begin{array}{l}\text { TYPE } \\
\text { CODEA }\end{array}$ & DESCRIPTION & DEPTH \\
\hline$B$ & Endontulcomind scout & 71 \\
\hline 5 & 212 Bent, Pellots & 78 \\
\hline$\pi$ & guic oplies sand & $\tan 10^{3}$ \\
\hline & & \\
\hline & & \\
\hline & & \\
\hline & & \\
\hline & & \\
\hline 2. & $3-\cos$ & Paek \\
\hline
\end{tabular}

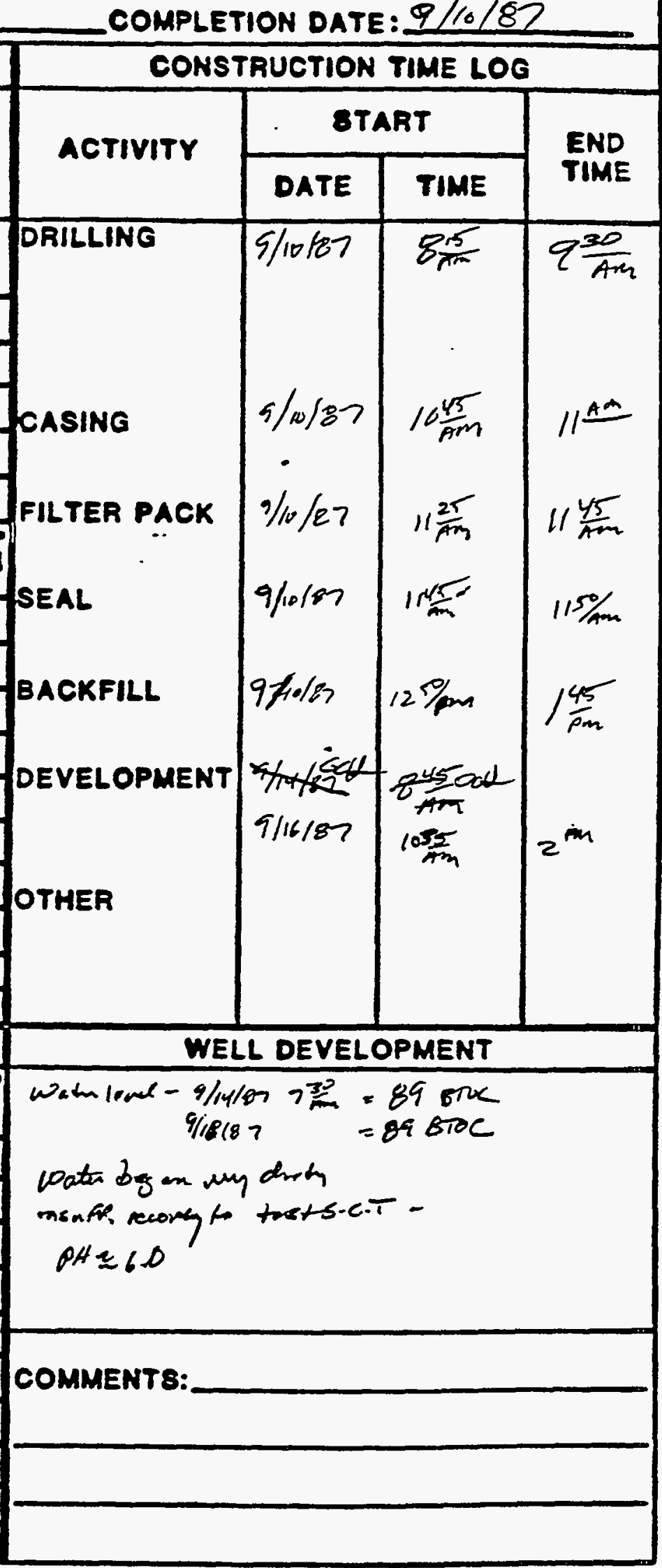




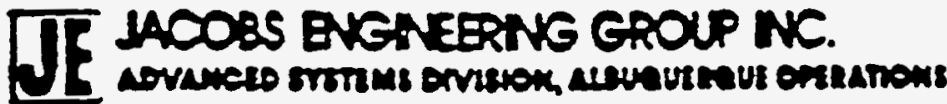

\section{WELL COMPLETION RECORD}

SITE ID: Spleqf LOCATION ID: 931 DATE INSTALLED: $12 / 13 / 87$ APPROX. 8ITE COOORDINATES:(FT.) $N$ OPEN AREA PER LINEAL FT. (IN2/FT.) w/k N/K E N/Ke FORMATION OF COMPLETION: Wasateh FIELD REP.: IFritt Driller: Boyles Bras/S mooce WELl CASING
DIAME TEA (In) $2^{\prime \prime}$ HOLE DIAMETER $(\mathrm{in}) 66^{\pi}$
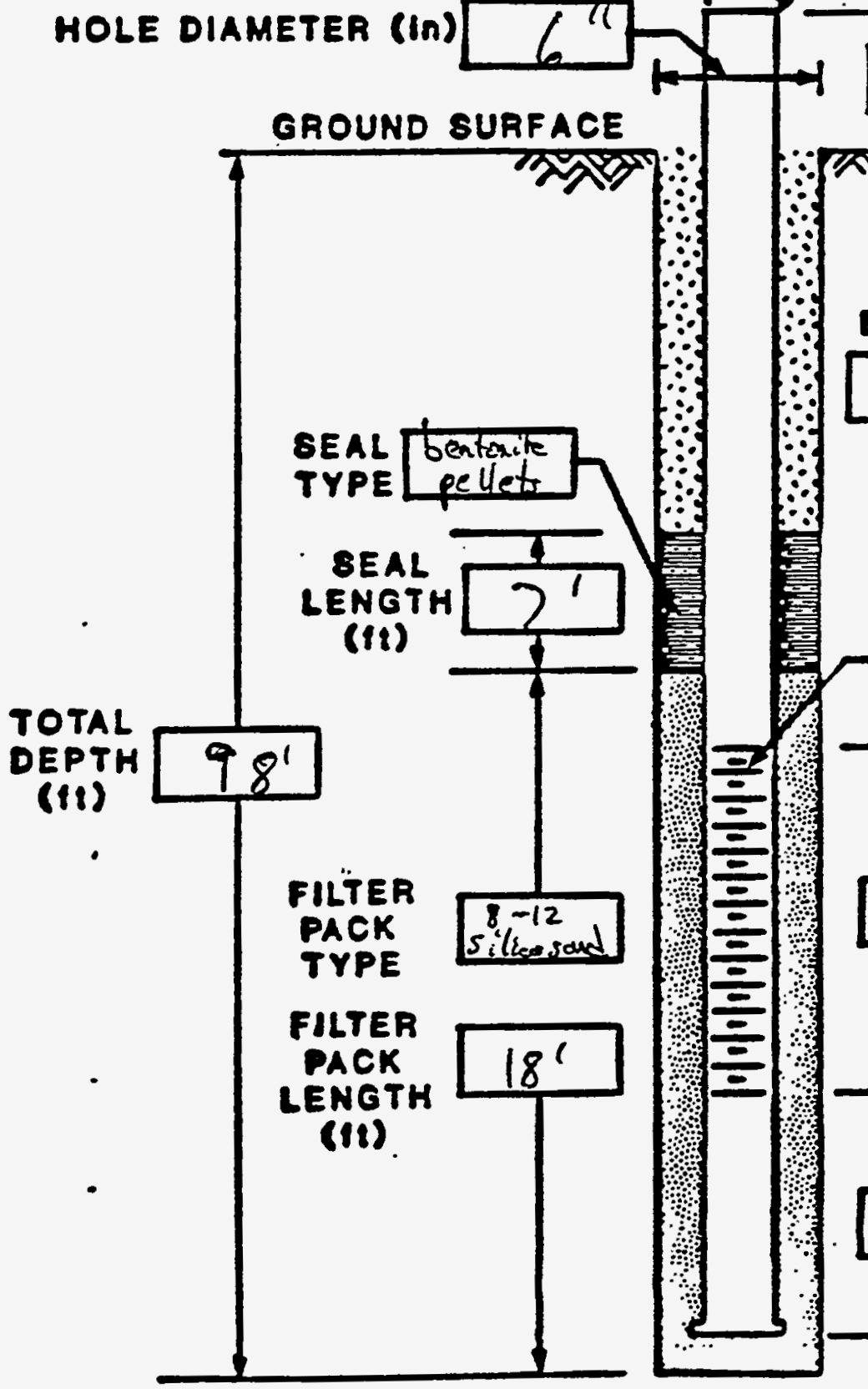

BACKFILL TYPE
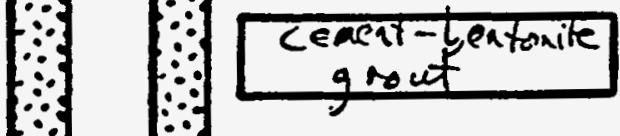
a mout a
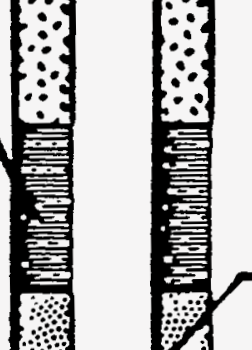

\section{BLOT}

OPENING (In)

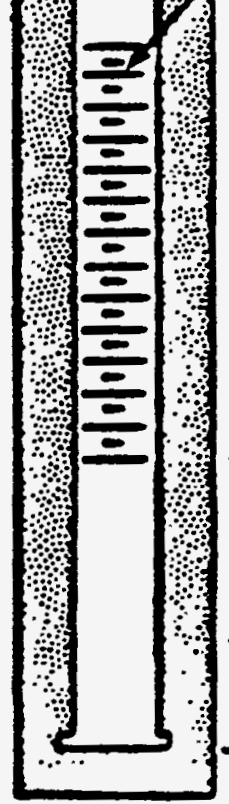
TYPE 


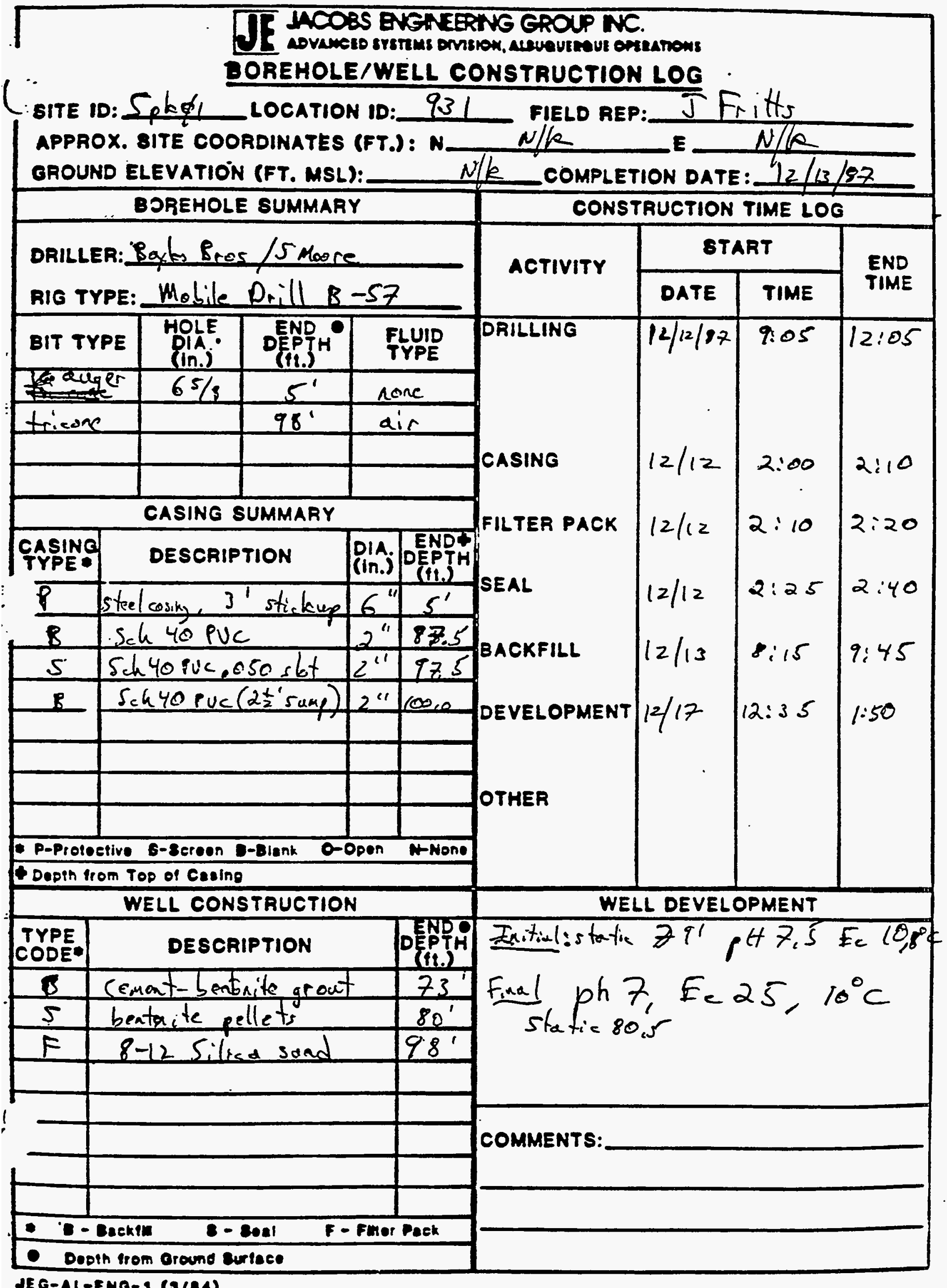




\section{TT' LACOSS ENGNEERING GROUP NC.

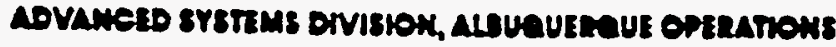

\section{WELL COMPLETION RECORD}

SITE ID: $\int p k \phi l$ LOCATION ID: $\frac{932}{91}$ DATE INSTALLED: $12 / 16 / 02$ APPROX. SITE COORDINATES:(FT.) N OPEN AREA PPER LINEAL FT. (IN²/FT.)

$N / k$ E N/K

\section{IFORMATION OF COMPLETION: Inlosoteh}

FIELD REP.: TFrits DRILLER: Bolles Bror /S Moore

WELL CASING DIAMETER (In)

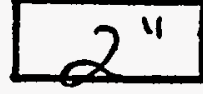

HOLE DIAMETER (in)

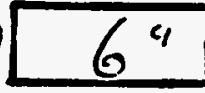

\section{GROUND SURFACE}

TOTAL

DEPTH (ft)

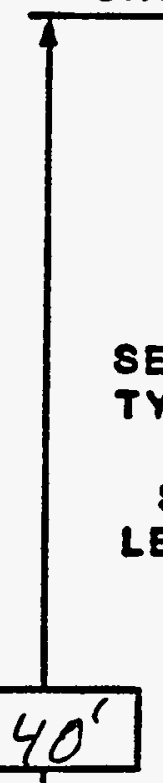

SEAL Sentorite

TYPE Lallets

SEAL LENGTH (ti)
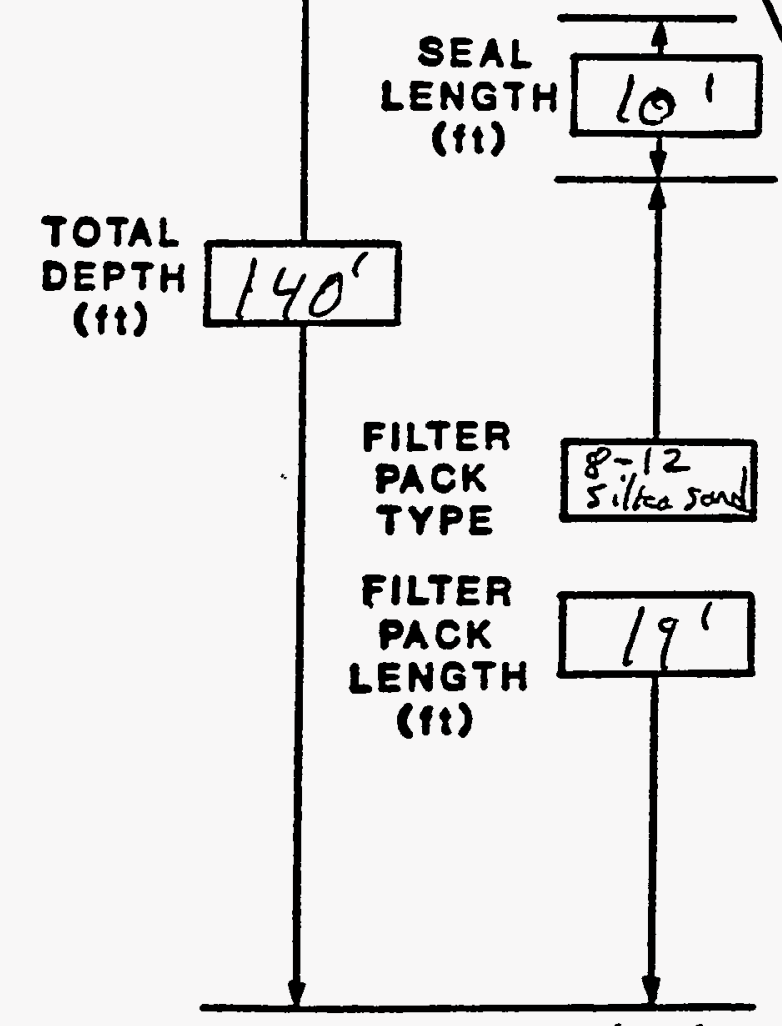

COMMENTS: drilled to

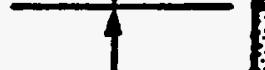
w/k

WELL CASING TYPE<smiles>[AlH2]</smiles>

BACKFILL TYPE cement-bertoute
grent

SLOT

OPENING (in)

.050
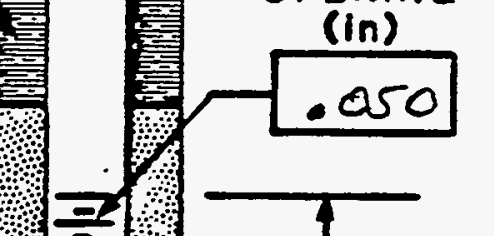

CASING

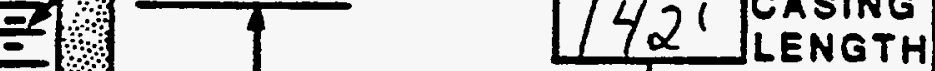
(ft)

OPEN OR

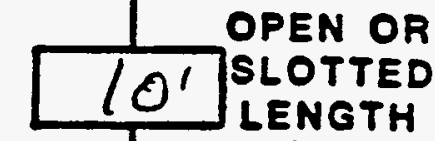

(ft)

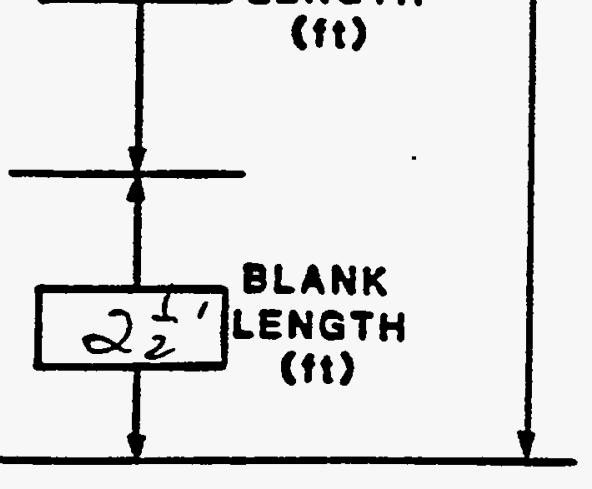

$3^{\prime}$ cave in 


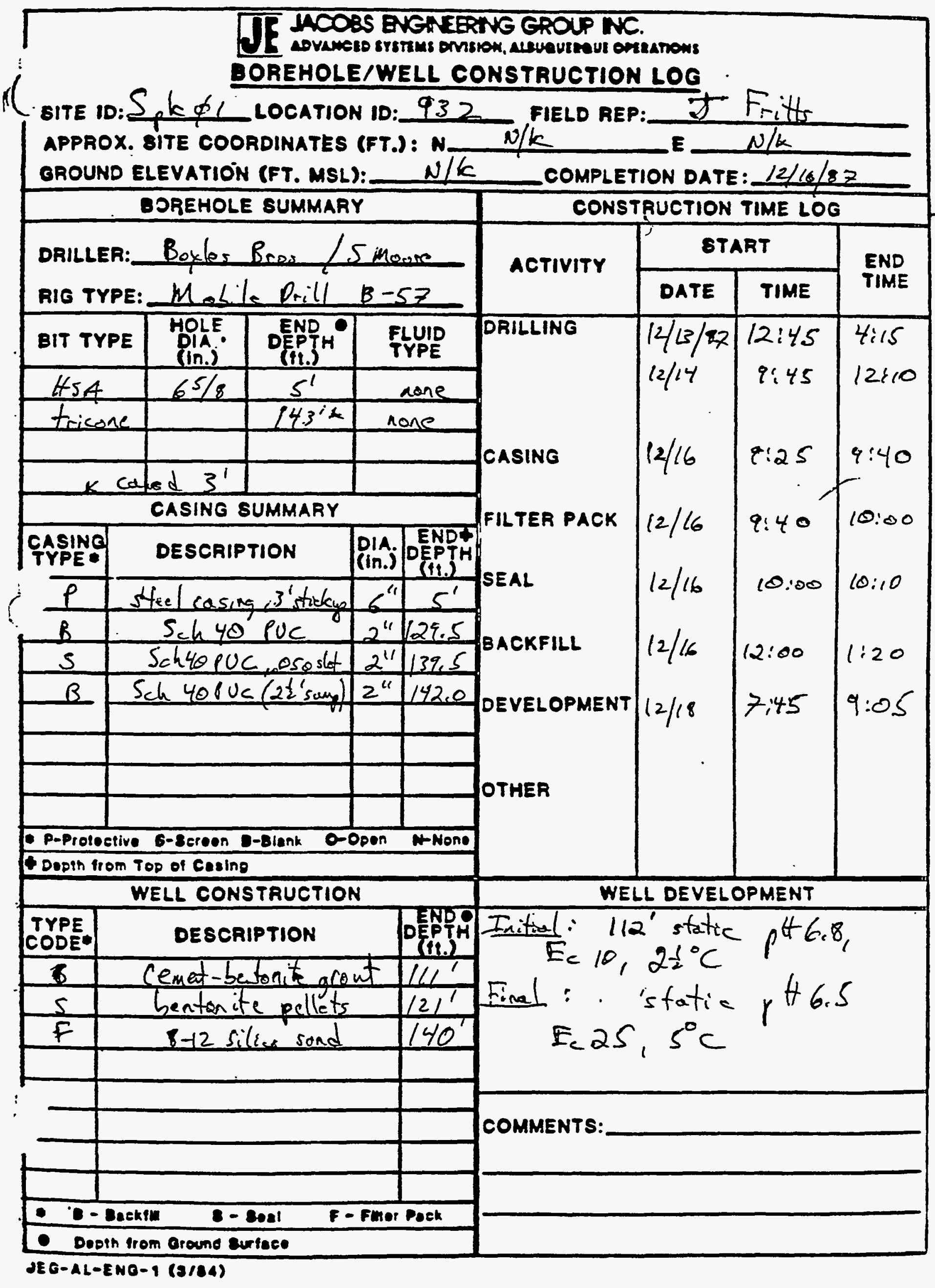




\section{WELL COMPLETION RECORD}

SITE ID: APPROX. SITE COOORDINATES:(FT.) N $\frac{N K}{\left(7^{2 / r}-4^{1}\right)^{2}}$ E OPEN AREA PER LINEAL FT. $\left(\right.$ IN $\left.^{2} / F T.\right) \frac{\left(7^{7 / r}-4^{\prime \prime}\right)^{2}}{(T / L}$ (FORMATION OF COMPLETION: Wasateh fm. FIELD REP.: DRILLER: T3arnhart

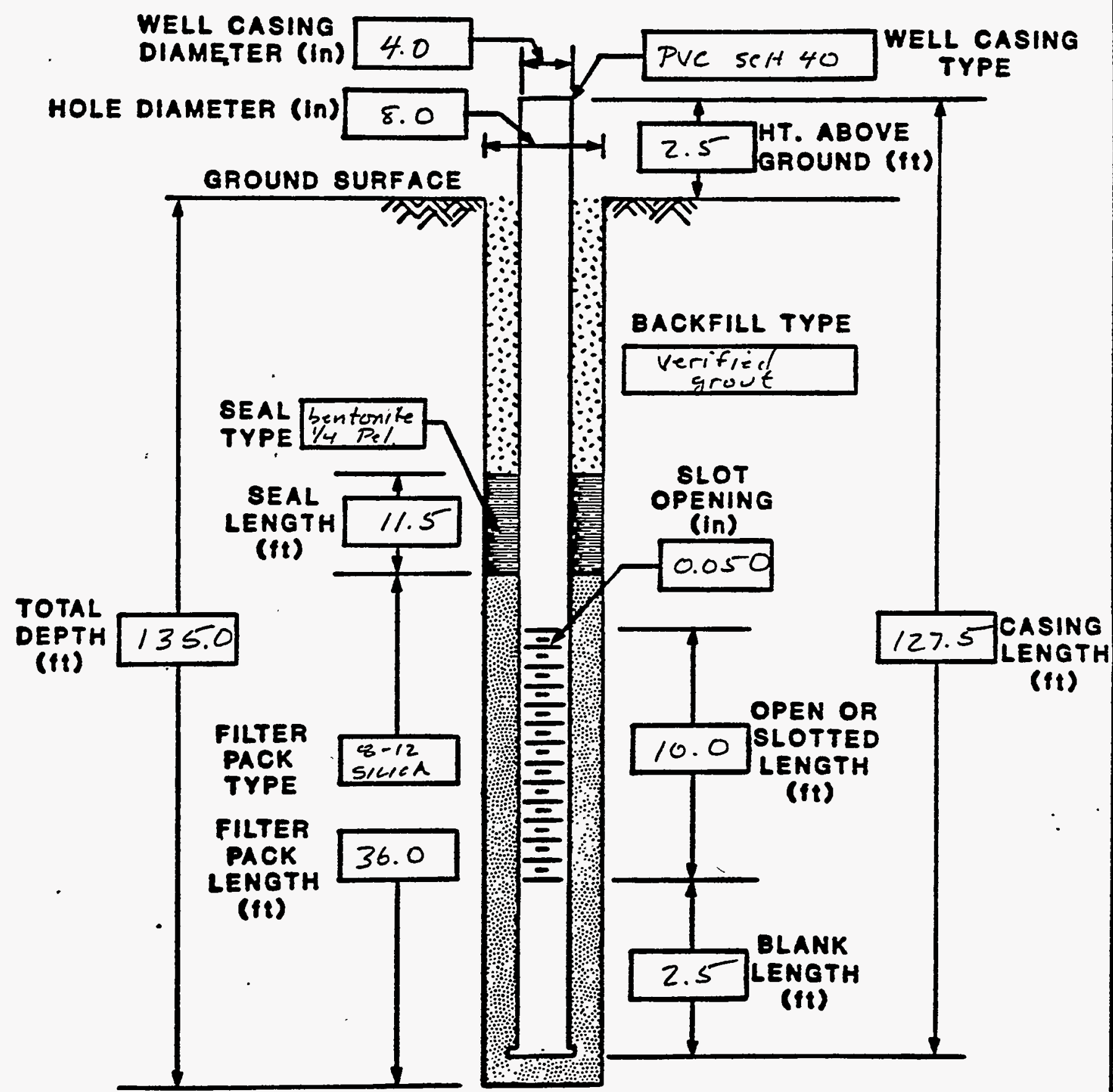

COMMENTS: 


\section{TT LACOSS ENGNEERNG GROUP NC.

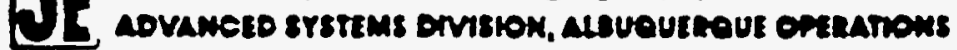 \\ BOREHOLE/WELL CONSTRUCTION LOG}

8ITE 1D: SPK-15 LOCATION ID: 933 FIELD AEP:D. METZLGR

APPROX. BITE COORDINATES (FT.): N_NK N N N

OROUND ELEVATION (FT. MSL): NK

. OOREHOLE BUMMARY

DRILLER: BARWHART

RIG TYPE:

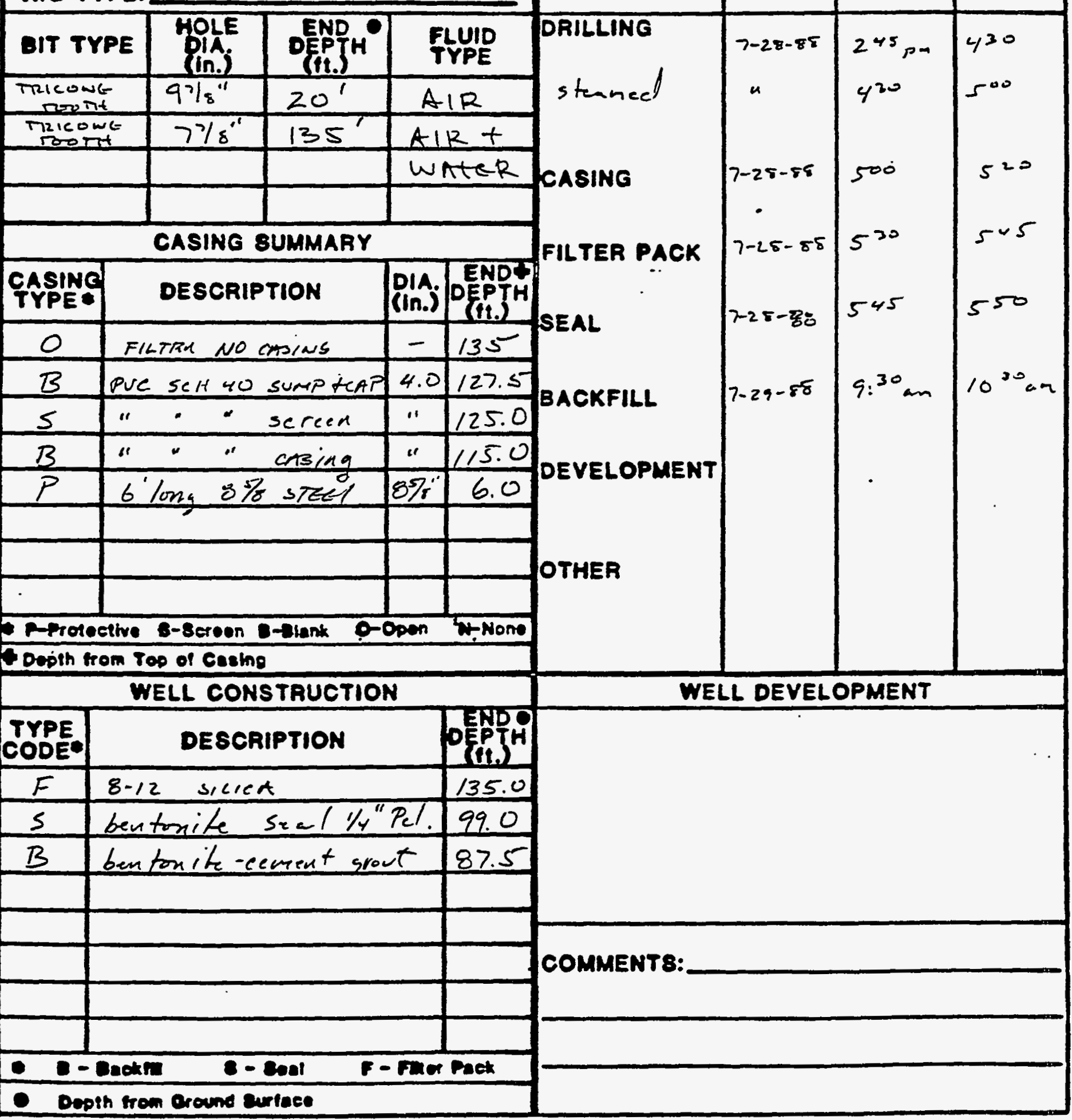




\section{WI IACOBS ENGNEERNG GROUP NC.}

\section{WELL COMPLETION RECORD}

SITE ID: $\frac{\text { SPK }-15}{5}$ LOCATION ID: 934 DATE INSTALLED: $228-88$ APPROX. SITE COOORDINATES:(FT.) N

OPEN AREA PPER LINEAL FT. (IN'/FT.) $\left(77 / 8^{\prime \prime}-4.0^{\circ}\right)^{2}$

E NK

FORMATION OF COMPLETION: WASATCK $4 M$.

FIELD REP.: D. METZLER

DRILLER: BARNIFART

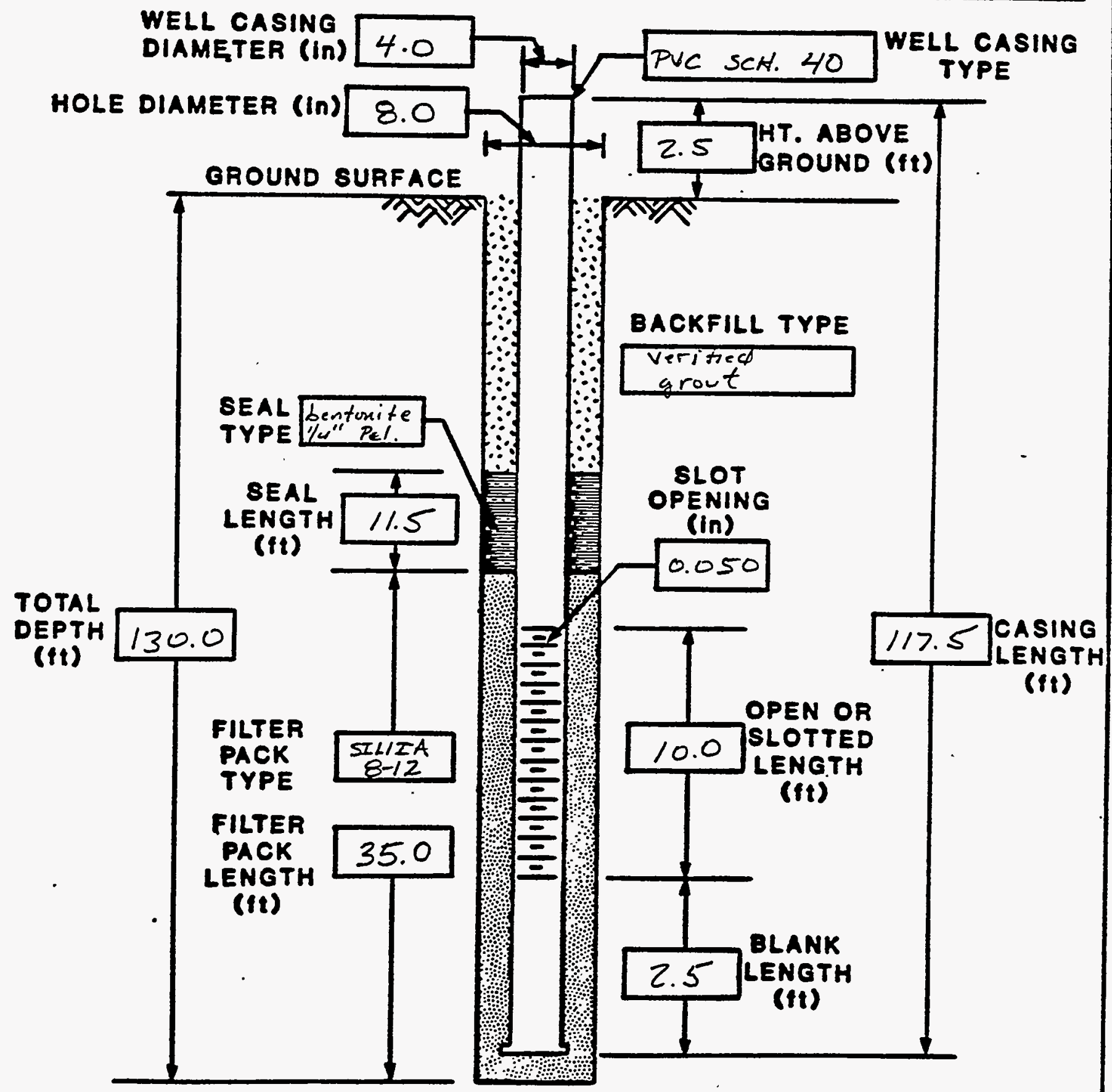

COMMENT 8: 


\section{T. LACOBS ENGNEERNG GROUP NC.

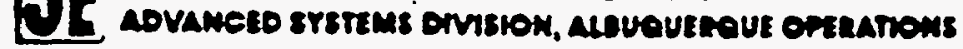 \\ BOREHOLE/WELL CONSTAUCTION LOG}

SITE ID: SPK-15 LOCATION ID: 934 FIELD REP: D. METZLEP

APPROX. BITE COORDINATES (FT.): $N$

NIK

E

NK

OROUND ELEVATION (FT. MSL):

NK

COMPLETION DATE: $7-28-88$

. OOREHOLE BUMMARY

ORILLER: BARNHART

RIG TYPE: 5 D ISOO ROTARY

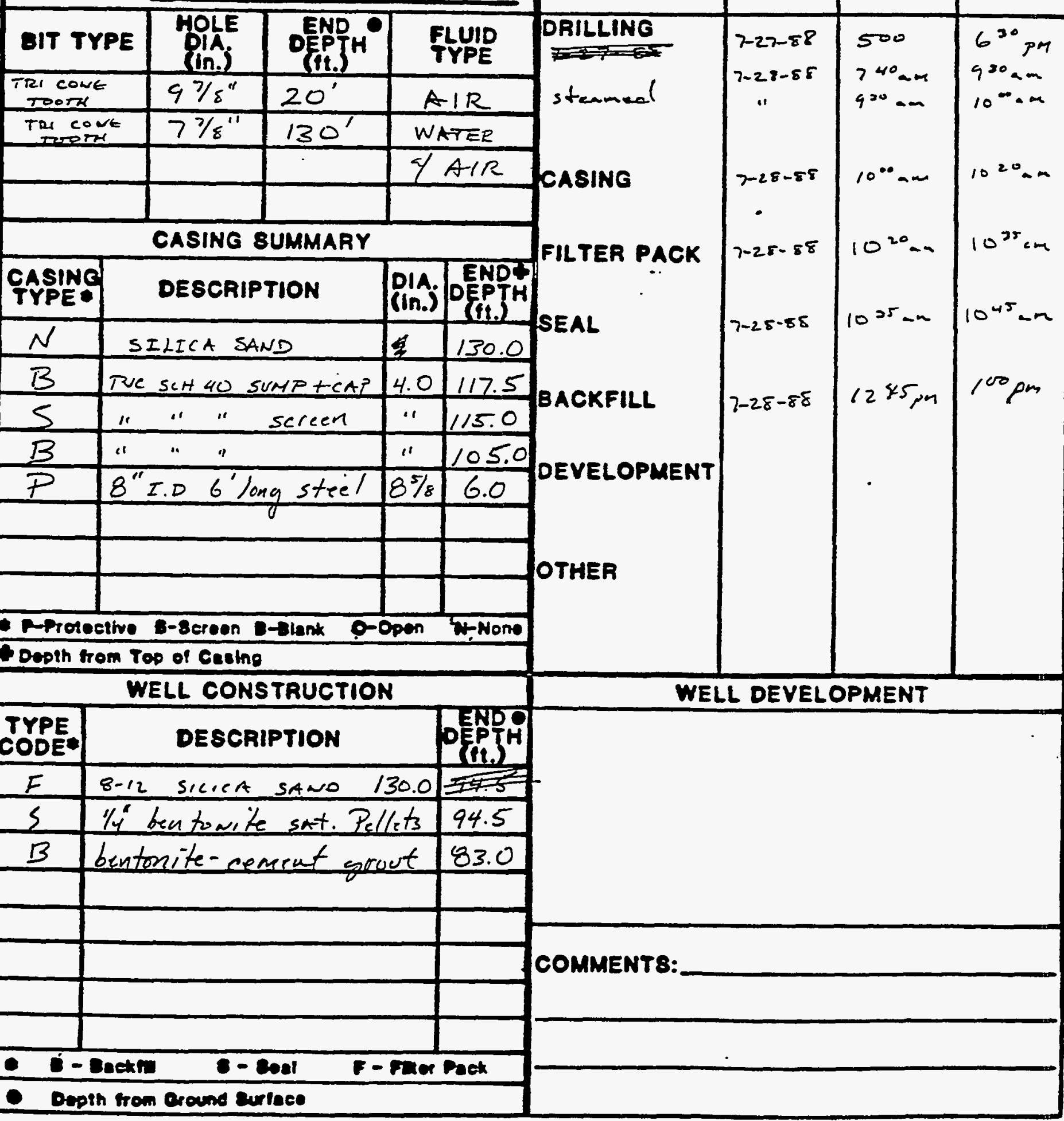




\section{WELL COMPLETION RECORD}

SITE ID: SPK -15 LOCATION ID: 935 DATE INSTALLED: $7-19-88$ APPROX. 8ITE COORDINATES:(FT.) N $\frac{N K}{\left(2^{\prime \prime}-45^{\prime \prime}\right)_{2}}$ NK NK OPEN AREA PER LINEAL FT. (IN2/FT. $\left.-(2 \%)^{\prime \prime}-40^{\prime \prime}\right)^{2} / f t$ |FORMATION OF COMPLETION: WASATCh FM.

FIELD REP.: D. MEZZLER DRILLER: BARNHART

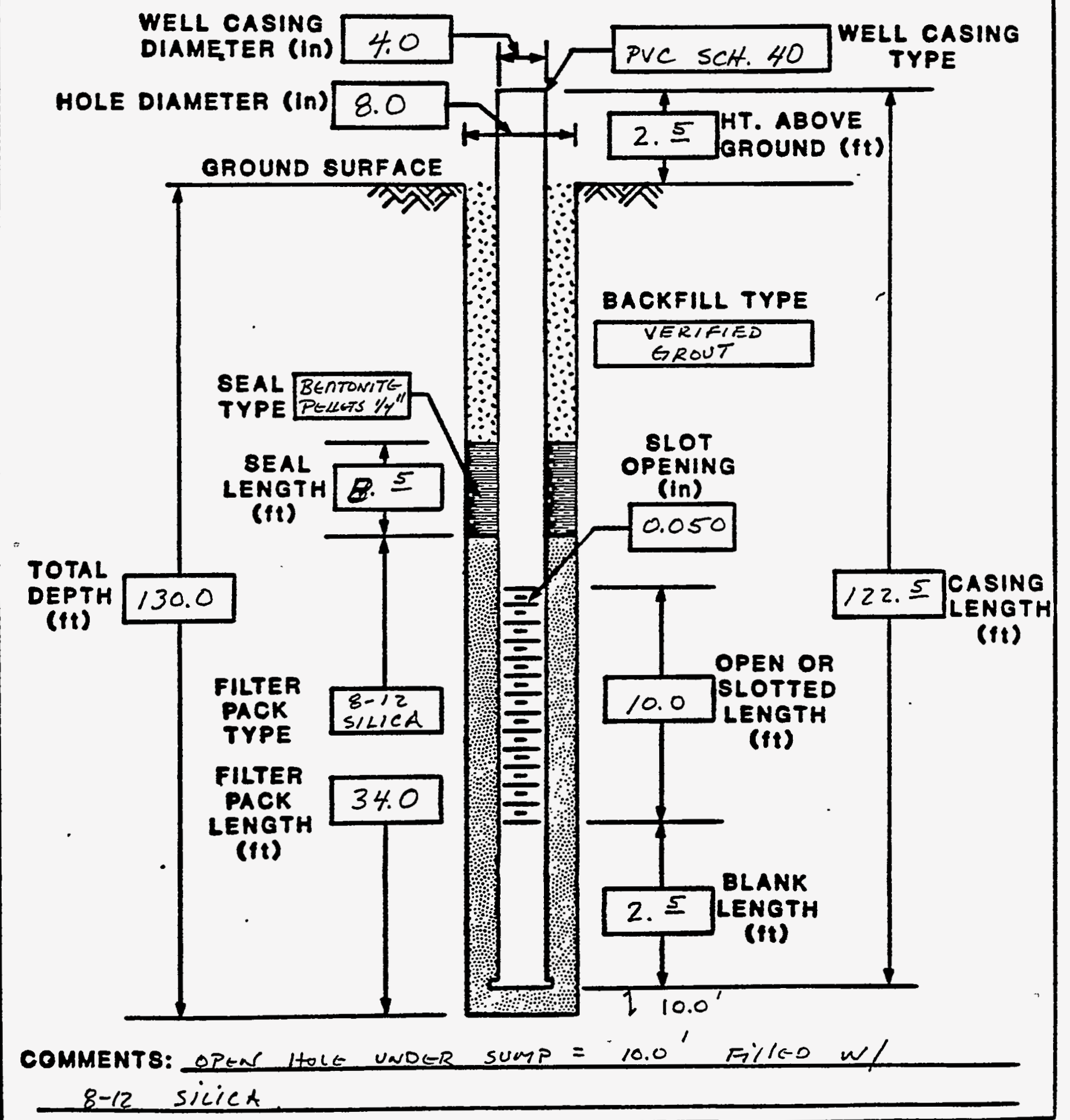




\section{T. JACOBS ENGMEERNG GROUP NK.

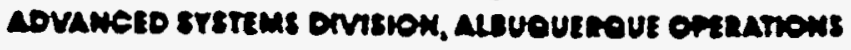 BOREHOLE/WELL CONSTRUCTION LOG}

SITE 10: SPK-15 LOCATION ID: 935 FIELD REP:D D.METZLER

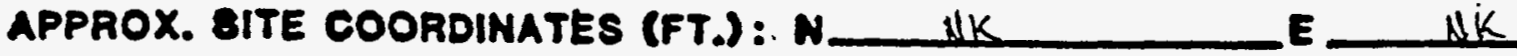

OROUND ELEVATION (FT. MSL):_ NK . DOREHOLE BUMMARY

COMPLETION DATE: $7-19-88$

\begin{tabular}{|c|}
\hline . POREHOLE SUMMARY \\
DRILLER:_BARNHART \\
RIG TYPE: G.D. IS WD ROTARY \\
\hline
\end{tabular}

\begin{tabular}{|c|c|c|c|}
\hline BIT TYPE & $\begin{array}{c}\text { HOLE } \\
\text { SIA.) }\end{array}$ & $\begin{array}{c}\text { ENP } \\
\text { DEPH }\end{array}$ & $\begin{array}{c}\text { FLUID } \\
\text { TYPE }\end{array}$ \\
\hline TRI cONE & $778 "$ & 130.0 & AlR + \\
\hline
\end{tabular}

\begin{tabular}{|l|l|l|l|}
\hline & & & WIATER \\
\hline & & & \\
\hline & & & \\
\hline & & & \\
\hline
\end{tabular}

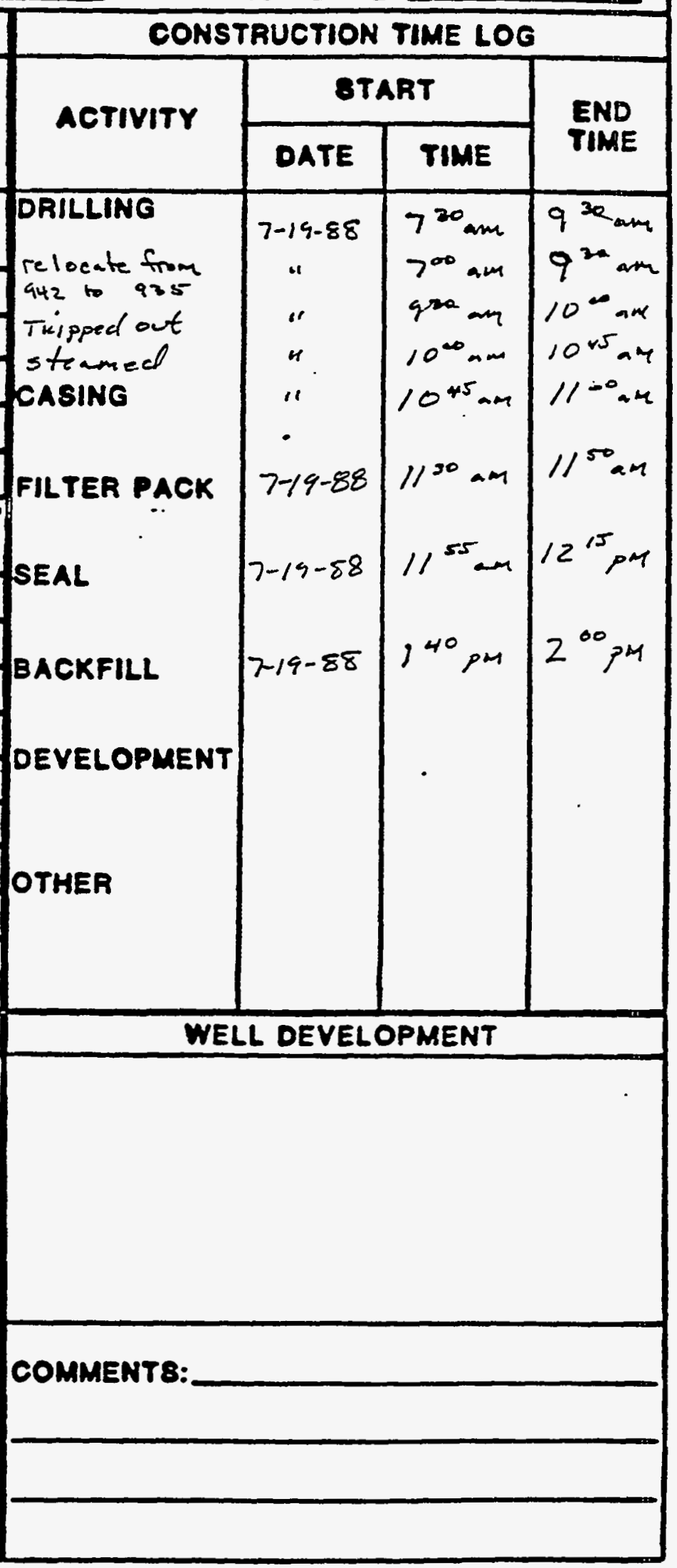

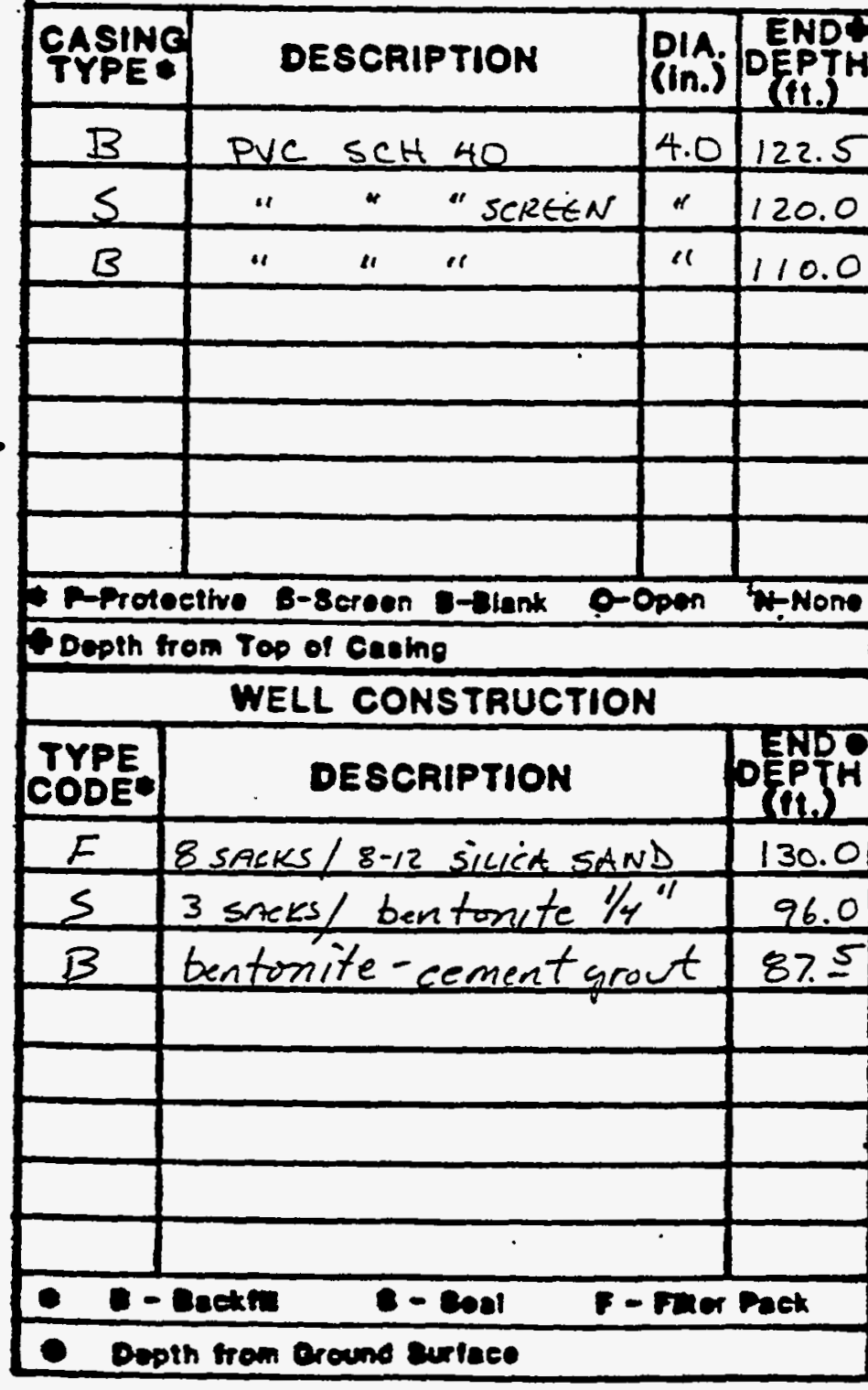




\section{TT LACOBS ENGNEERING GROUP NC.

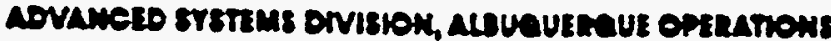

\section{WELL COMPLETION RECORD}

SITE ID: 5 SP - 15 LOCATION ID: 936 DATE INSTALLED: $7-16-88$ APPROX. BITE COORDINATES:(FT.) N $\frac{N K}{(g / 4-4.0)}$ E NK

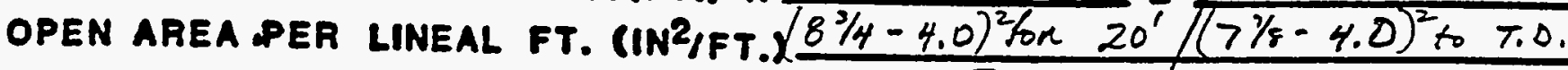
FORMATION OF COMPLETION: FIELD REP.: D. METZLER WASATCH FM.

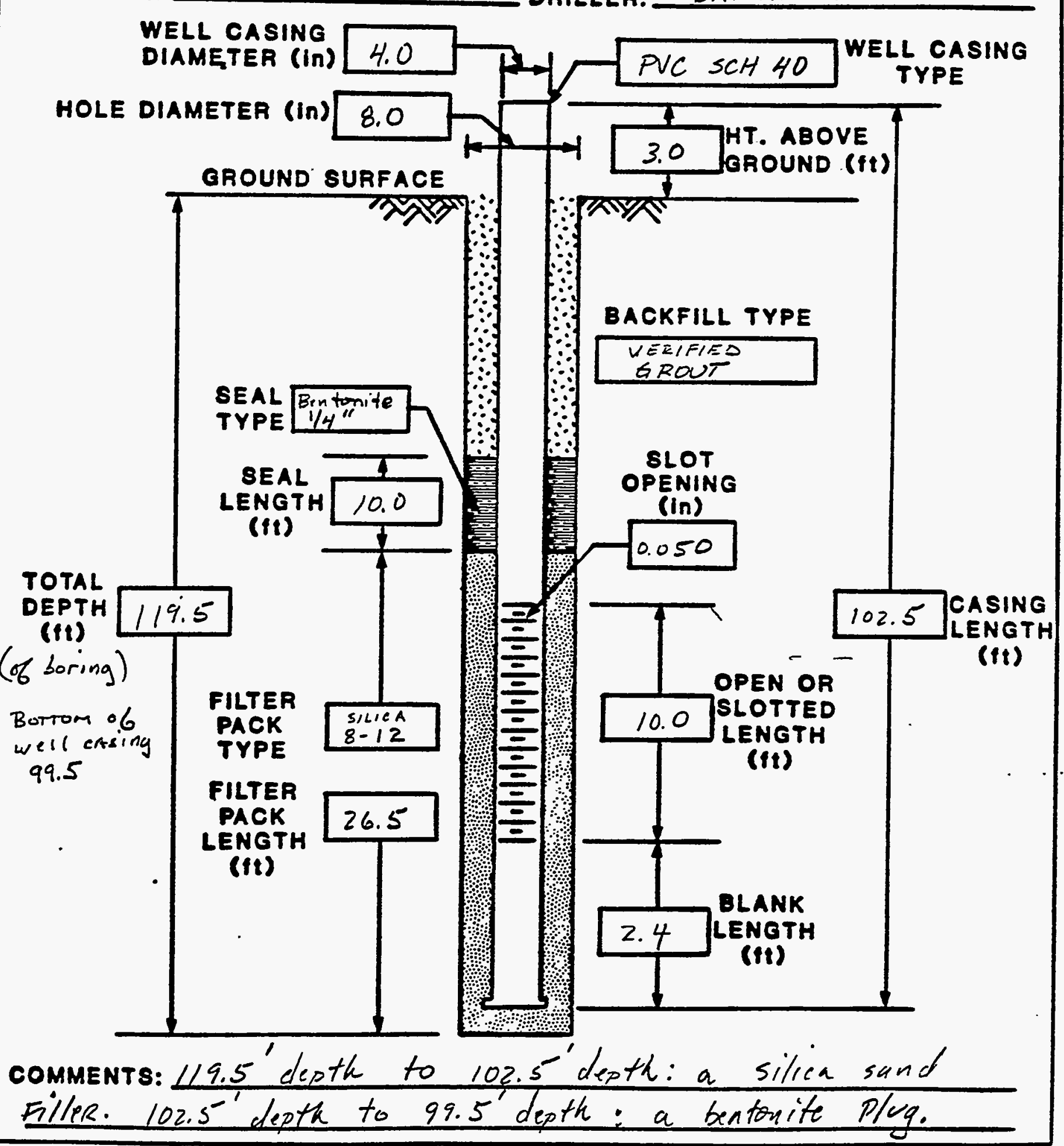
DRILLER: BARNAART

WELL CASING 


\section{TI JACOBS ENGINEERHE GROUP HNC.

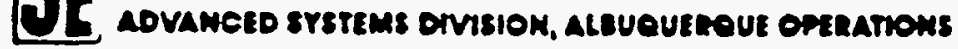 BOREHOLE/WELL CONSTRUCTION LOG}

SITE ID: SPK-15 LOCATION ID: 936 FIELD REP: D. METZLER APPROX. BITE COORDINATES (FT.): N_NK. N_E NK GROUND ELEVATION (FT. MSL): NK COMPLETION DATE: $7-16-88$

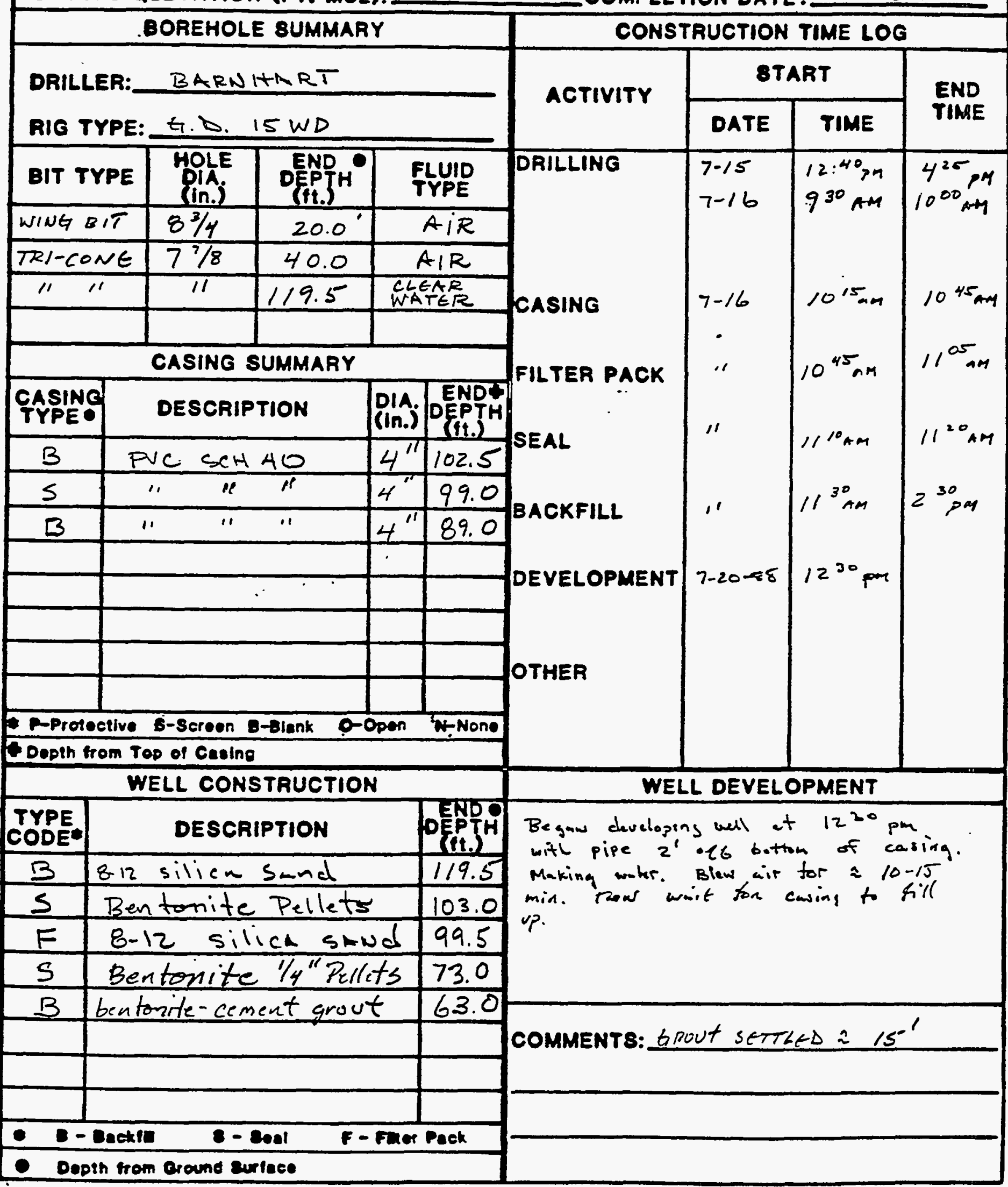




\section{TF LACOBS ANGNEERING GROUP NC.

\section{WELL COMPLETION RECORD}

SITE ID: SPK-15 LOCATION ID: 937 DATE INSTALLED: $9-18-8 \delta$ APPROX. SITE COORDINATES:(FT.) N NK OPEN AREA PER LINEAL FT. (IN2/FT.) $\left(8^{\prime \prime}-4^{\prime \prime}\right)^{2} / f^{2}$ fFORMATION OF COMPLETION: WABATCH fol. fep if plue shale FIELD REP.: D.METZLER DRILLER:

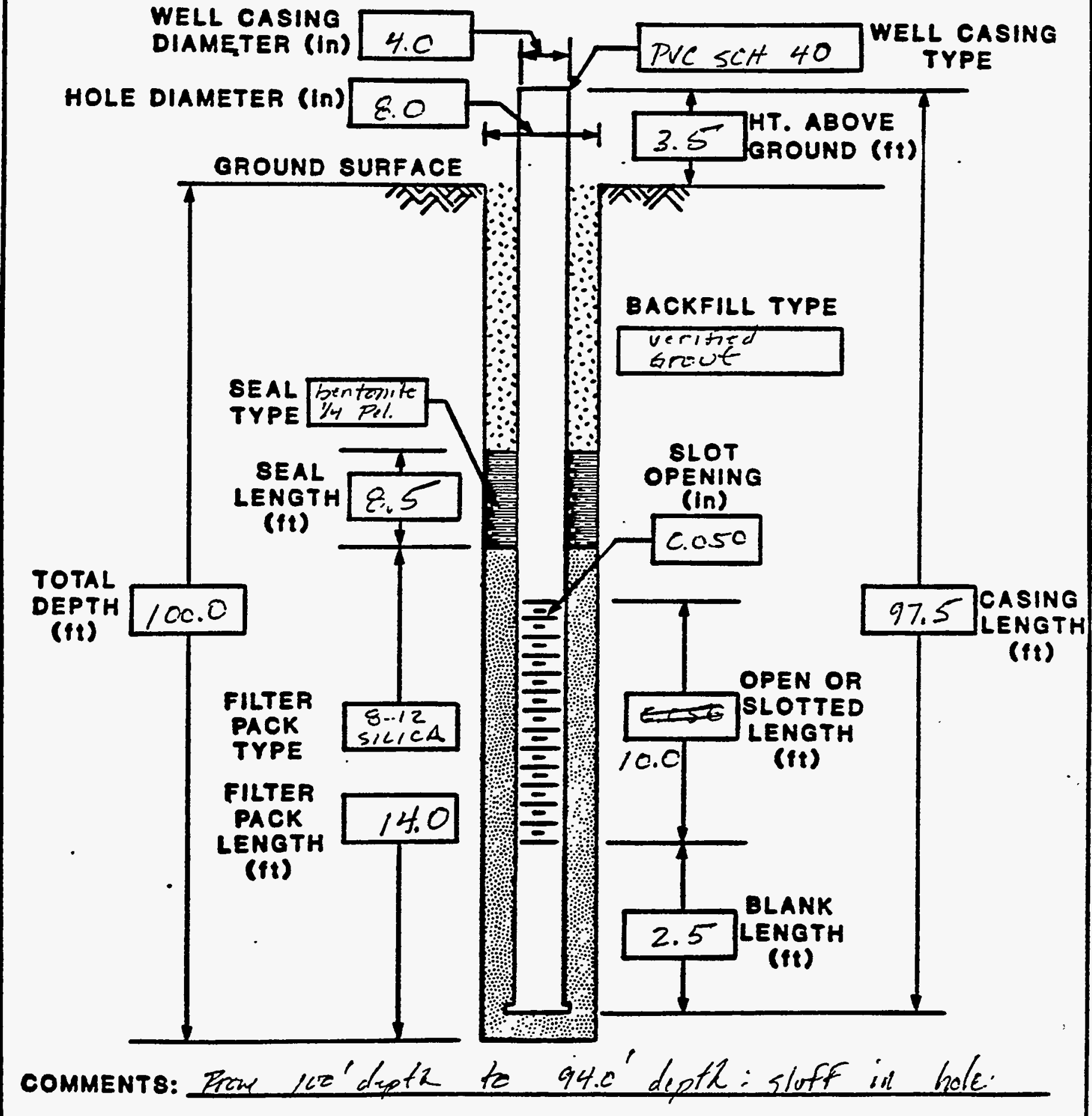




\section{TI JACOBS ENGMEERNG GROJP NKC.

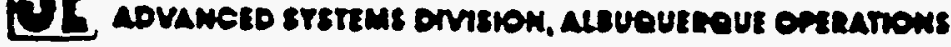 \\ BOREHOLE/WELL CONSTRUCTION LOG}

SITE ID:SPK-1.5 LOCATION ID: 937 FIELD REP:D. METZLER

APPROX. 8ITE COORDINATES (FT.): N_l'K.

BROUND ELEVATION (FT. MSL):

Q

COMPLETION DATE: $7-18-8 \varepsilon$

OOREHOLE BUMMARY

DRILLER: BARNHART

RIG TYPE: GD. 15WD ROTARY

\begin{tabular}{|c|c|c|c|}
\hline BIT TYPE & $\begin{array}{l}\text { MOLE } \\
\text { PIA. } \\
\text { In.) }\end{array}$ & 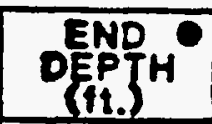 & $\begin{array}{l}\text { FLUID } \\
\text { TYPE }\end{array}$ \\
\hline 70 & $\pi$ & & \\
\hline
\end{tabular}

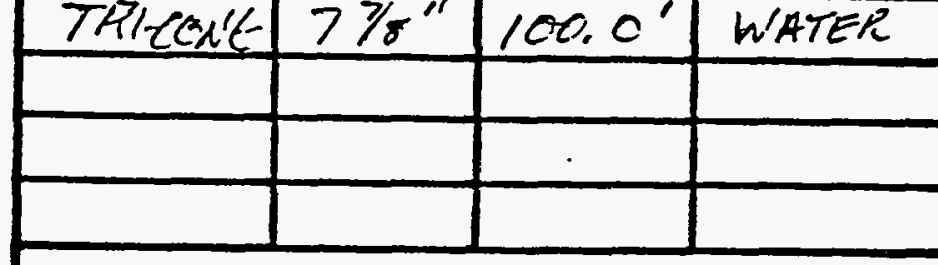

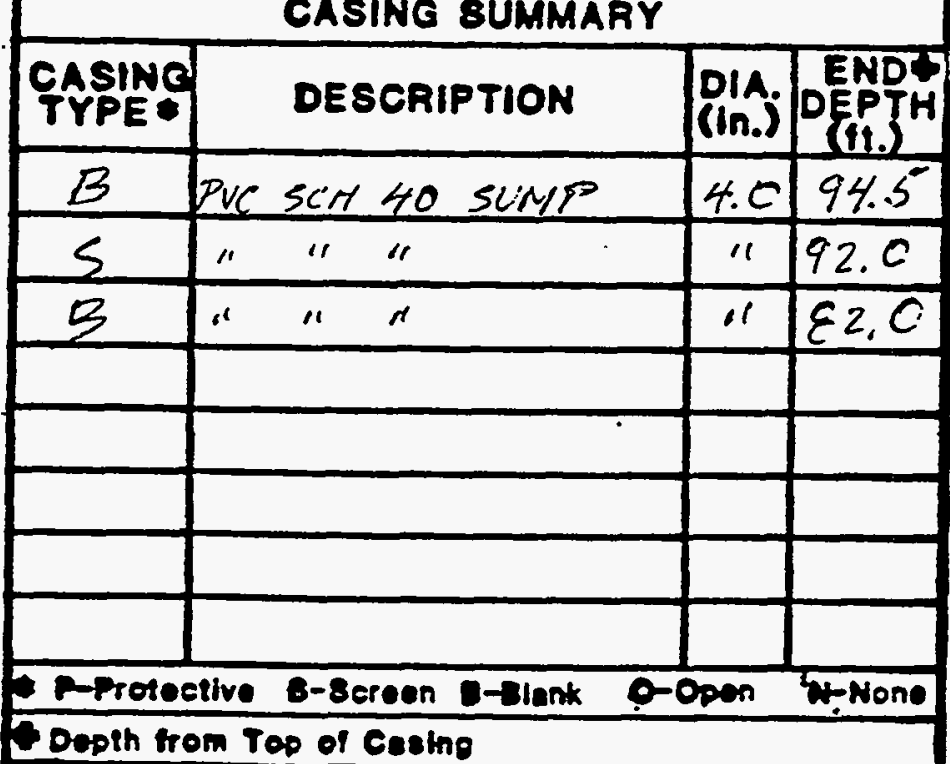

WELL CONSTAUCTION

\begin{tabular}{|c|c|c|}
\hline $\begin{array}{l}\text { TYPE } \\
\text { CODE }\end{array}$ & DESCRIPTION & o \\
\hline$B$ & BORING KIAlI SLL'FF & $1 c c .0$ \\
\hline$F$ & 12 SACKS / E-12 SiliCa S. & 94.5 \\
\hline 5 & 3 snexs beritarite $1 / 4 "$ & $\varepsilon c . c$ \\
\hline$B$ & bautomite-cenreat areut & 715 \\
\hline & & \\
\hline & & \\
\hline & & \\
\hline & & \\
\hline E- & $8-20 a 1$ & $\overline{\text { Peck }}$ \\
\hline
\end{tabular}

Depen from eround eurfece

dEO-AL-EMO-g (EIOA)

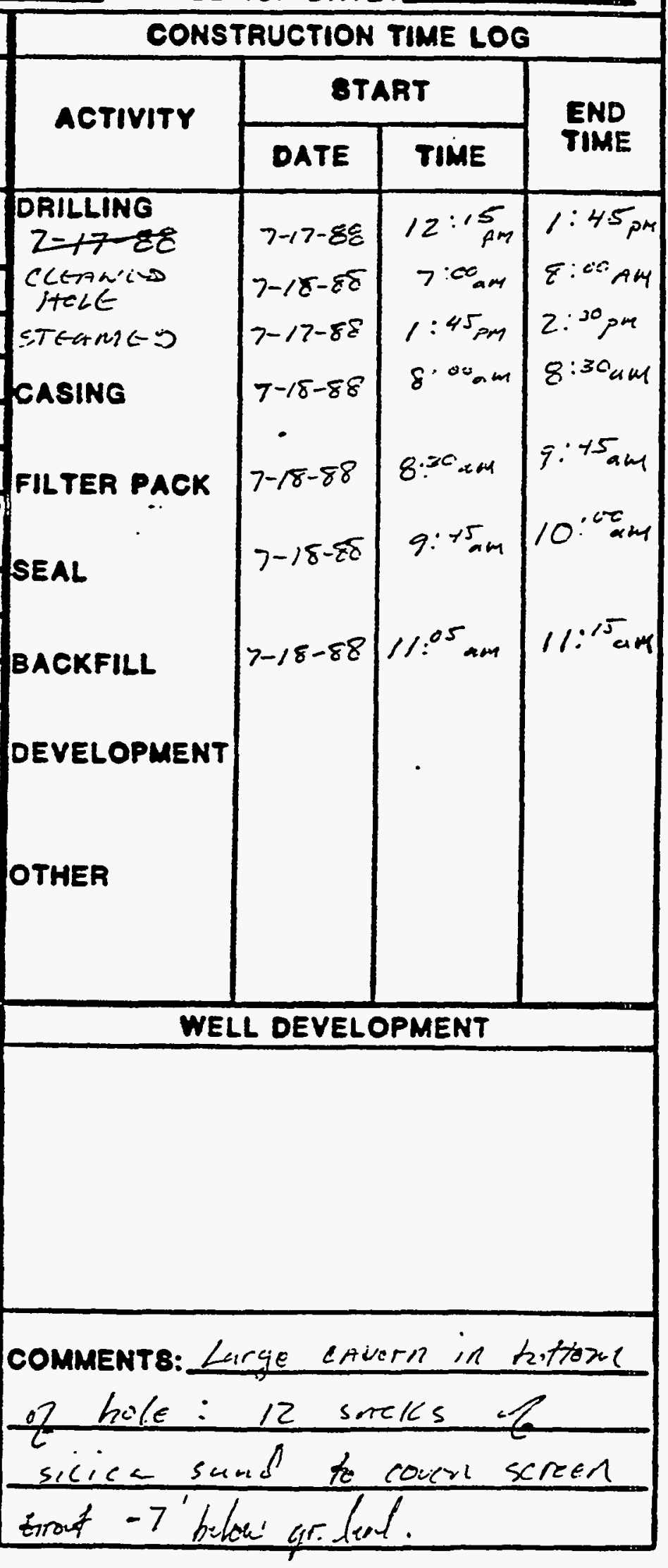


DI ACOBS ENGMEERNG GROUP NC.

\section{WELL COMPLETION RECORD}

SITE ID: SPK-15 LOCATION ID: 938 DATE INSTALLED: 7-20.88 APPROX. 8ITE COORDINATES:(FT.) N $\frac{N / K}{\left(7 \% s^{4}-4.0^{\prime \prime}\right)^{2}}$
OPEN AREA PER LINEAL FT. (IN2/FT.)

fFORMATION OF COMPLETION: WATSATCH FM: LOWET COWFINZO AQUIFER

FIELD REP.: D.METZLEP DRILLER: BARNITART WELL CASING 4.0 PIAMETER (IN)
DIAC. SCH 40 WELL CASING

HOLE DIAMETER (in) 8.0 GROUND SURFACE

TOTAL DEPTH (ft)
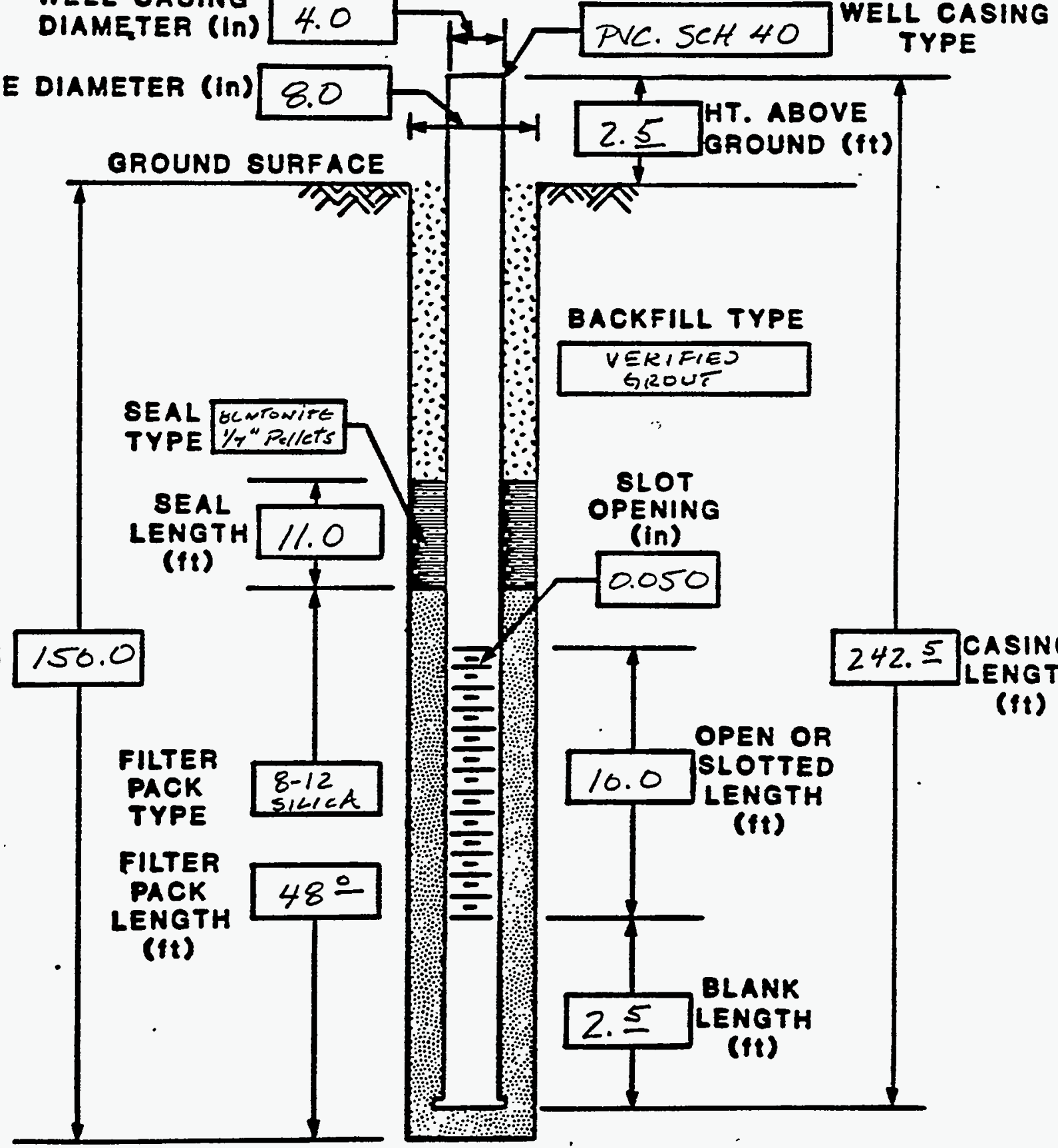

SEAL GLNTONITE TYPE $1 / 7$ "Pellets

SEAL

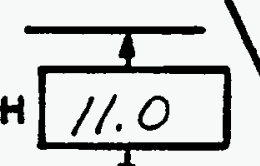
$\because$ LENGTH (fi)
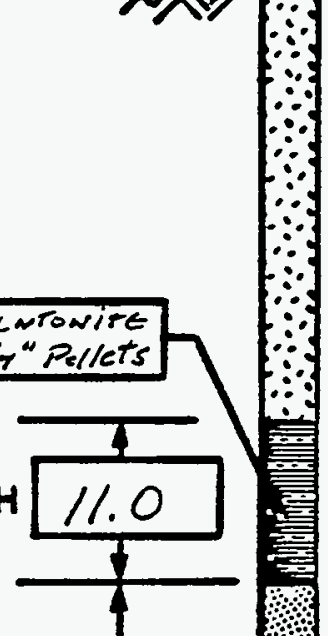
2. 5 JROUND (ft)

BACKFILL TYPE
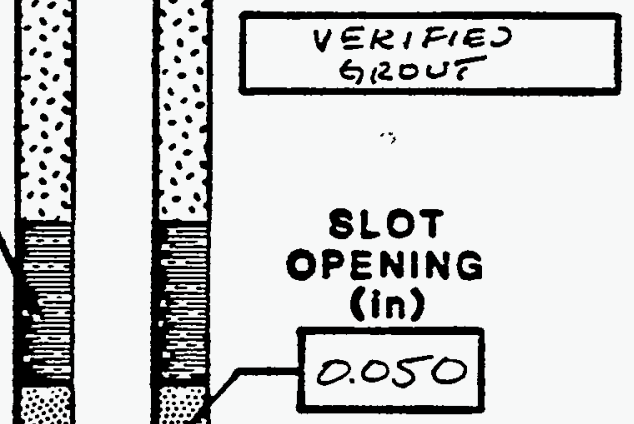
\begin{tabular}{l}
\hline VERIFIED \\
GROUT
\end{tabular}
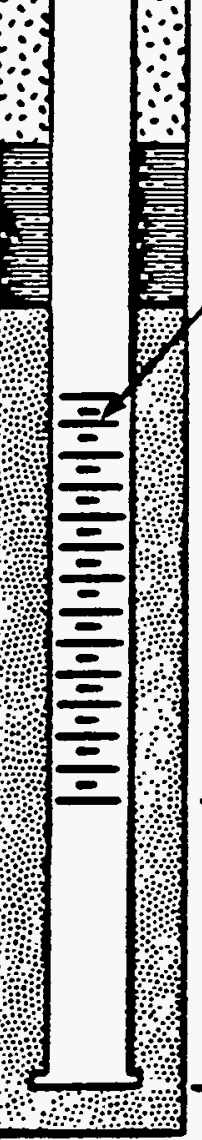

\section{SLOT}

OPENING (in) 0.050
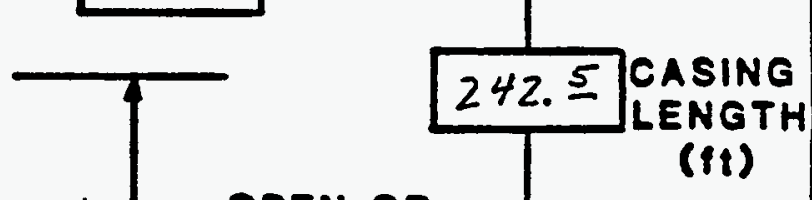

COMMENTS: 


\section{Tf 14 COBS ENGMEERNG GROUP NC.

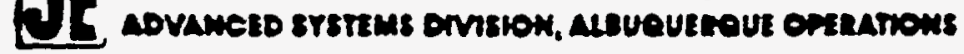 \\ BOREHOLE/WELL CONSTRUCTION LOG}

SITE ID: SPK-15 LLCATION 10: 938 FIELD REP:D METZLER

APPROX. BITE COORDINATES (FT.): N_L_NK. E_ NK

BROUND ELEVATION (FT. MSL):_UK_U_COMPLETION DATE: $7-20-8 \delta$

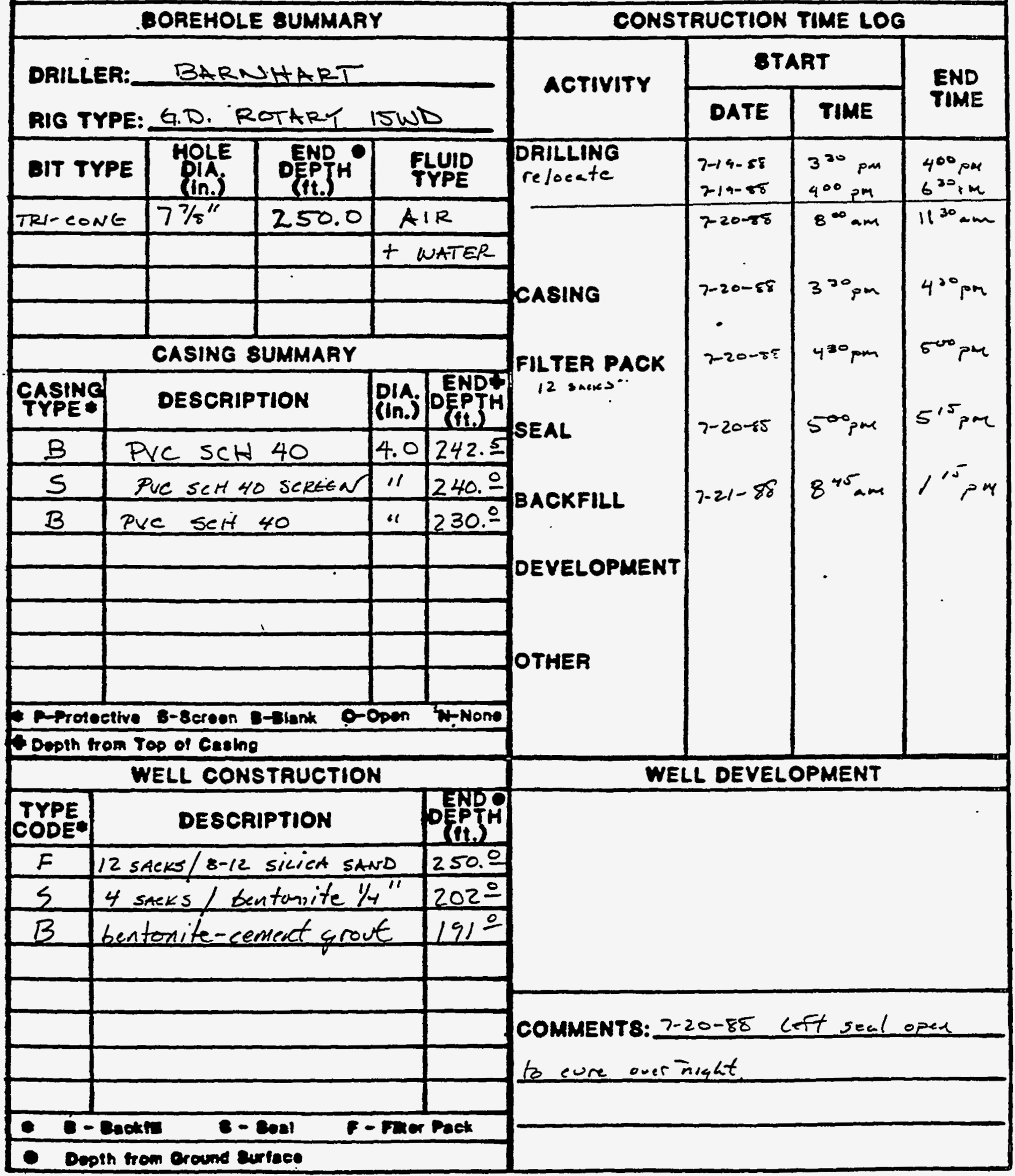




\section{WELL COMPLETION RECORD}

SITE ID: $\quad$ SPK-15 LOCATION ID: 939 DATE INSTALLED: 7-17-88

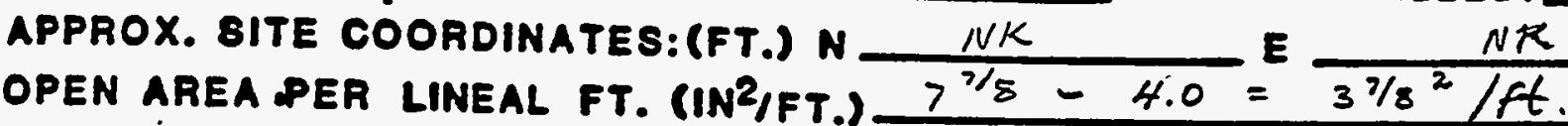

FORMATION OF COMPLETION: WASATCH FM:

FIELD REP.: D. METZLER DRILLER: BARN HART WELL CASING 4.0 PVC SCH 40 WELL CASING
DIAMETER (In)

HOLE DIAMETER (in) 8.0

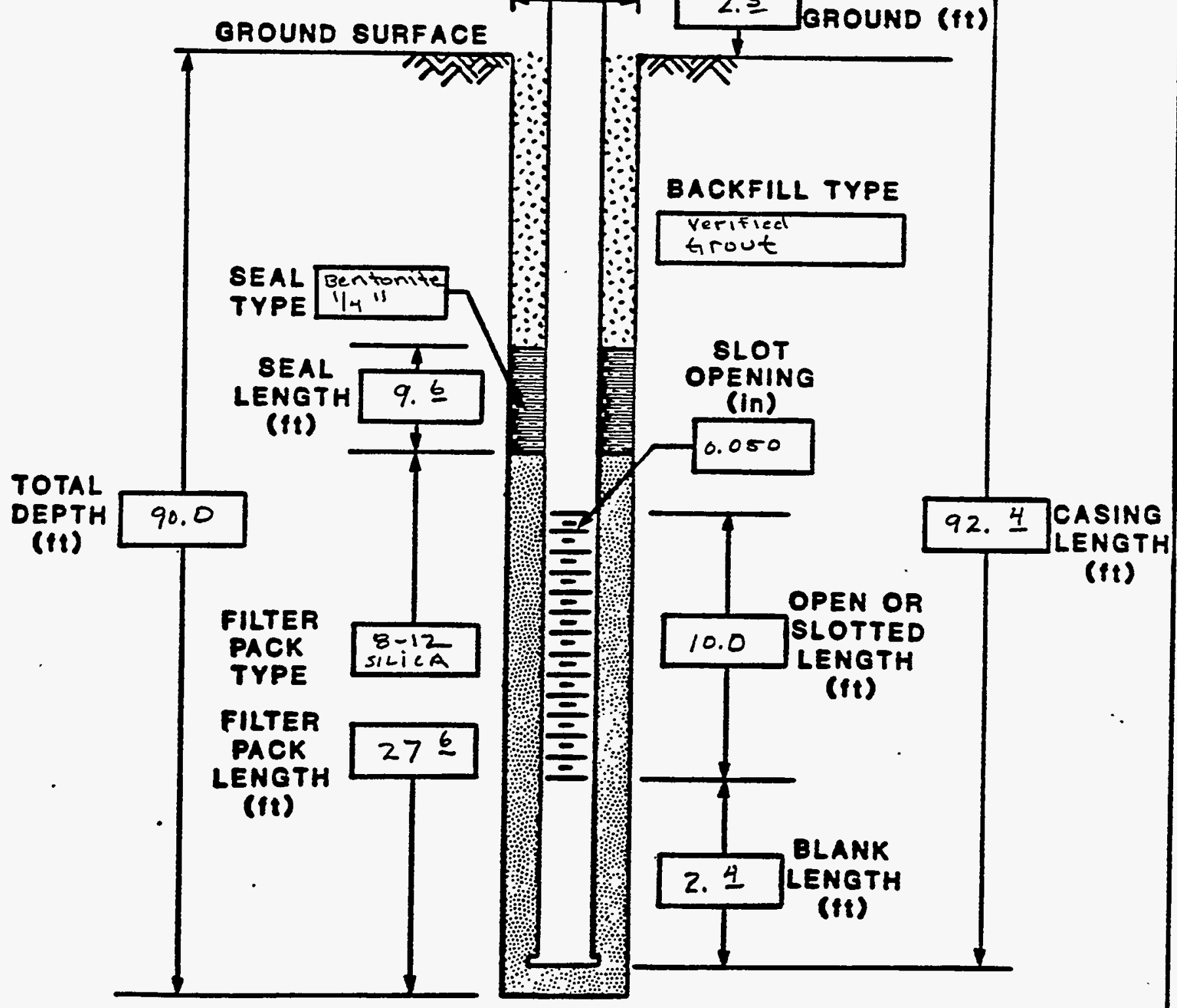

COMMENT8: observations and time recorded. Tagged w/ $100^{\prime}$ tape. 


\section{TF LACOBS ENGNEERNG GROUP NK.

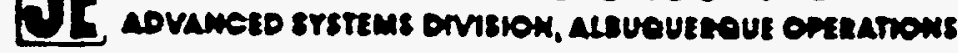 BOREHOLE/WELL CONSTRUCTION LOG}

SITE ID: SPK-16 - LOCATION ID: 939 FIELD REP: D. METZLER APPROX. BITE COORDINATES (FT.): N_NK. N_E NK

OROUND ELEVATION (FT. MSL): NK NK DOREHOLE BUMMARY

ORILLER: IBARN NART WY

RIG TYPE: $G D^{\circ}$ ISWD ROTARY

\begin{tabular}{|c|c|c|c|}
\hline DIT TYPE & $\begin{array}{l}\text { MOLE } \\
\text { giA. } \\
\text { (in.) }\end{array}$ & DEFD & $\begin{array}{l}\text { FLUID } \\
\text { TYPE }\end{array}$ \\
\hline TRI-CONE & $77 / 8^{\prime \prime}$ & 90.9 & AIR \\
\hline $711 \quad 11$ & $\pi$ & 90.0 & NATER \\
\hline * clenned & hole N/ & water & \\
\hline NOTE :DID N & set sur & Aace & 79 \\
\hline
\end{tabular}

CASING BUMMARY

\begin{tabular}{|c|c|c|c|c|}
\hline $\begin{array}{l}\text { CASING } \\
\text { TYPE: }\end{array}$ & & DESCRIPTION & $\begin{array}{l}\text { pin. } \\
\text { (in.) }\end{array}$ & $\begin{array}{l}\text { END' } \\
\text { OEPTH }\end{array}$ \\
\hline$B$ & PVC & SCH4O SUMP & 4.0 & 89.7 \\
\hline$s$ & $"$ & " " & 11 & 87.4 \\
\hline$B$ & $" 1$ & 11 & 11 & 77.4 \\
\hline & & & & \\
\hline & & & & \\
\hline & & & & \\
\hline & & & & \\
\hline & & & & \\
\hline Protoes & & B-8ereon B-8lank & pen & Nons \\
\hline
\end{tabular}

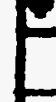

\begin{tabular}{c|}
\hline TYPE \\
CODE
\end{tabular}

WELL CONSTRUCTION

\begin{tabular}{|c|c|c|}
\hline CODE & DES & Tits \\
\hline$F$ & 8-12 silica sand & 897 \\
\hline 5 & Bentonite Pellets $1 / 4 " \mathrm{H}_{2} \mathrm{O}$ & 622 \\
\hline$B$ & bentonite-cemeat grout & $52^{5}$ \\
\hline & & \\
\hline & & \\
\hline & & \\
\hline & & \\
\hline & & \\
\hline 25 & $8-2001$ & Paot \\
\hline 00 & in from cround surlace & \\
\hline
\end{tabular}

COMPLETION DATE: $7-17-88$ CONSTRUCTION TIME LOG

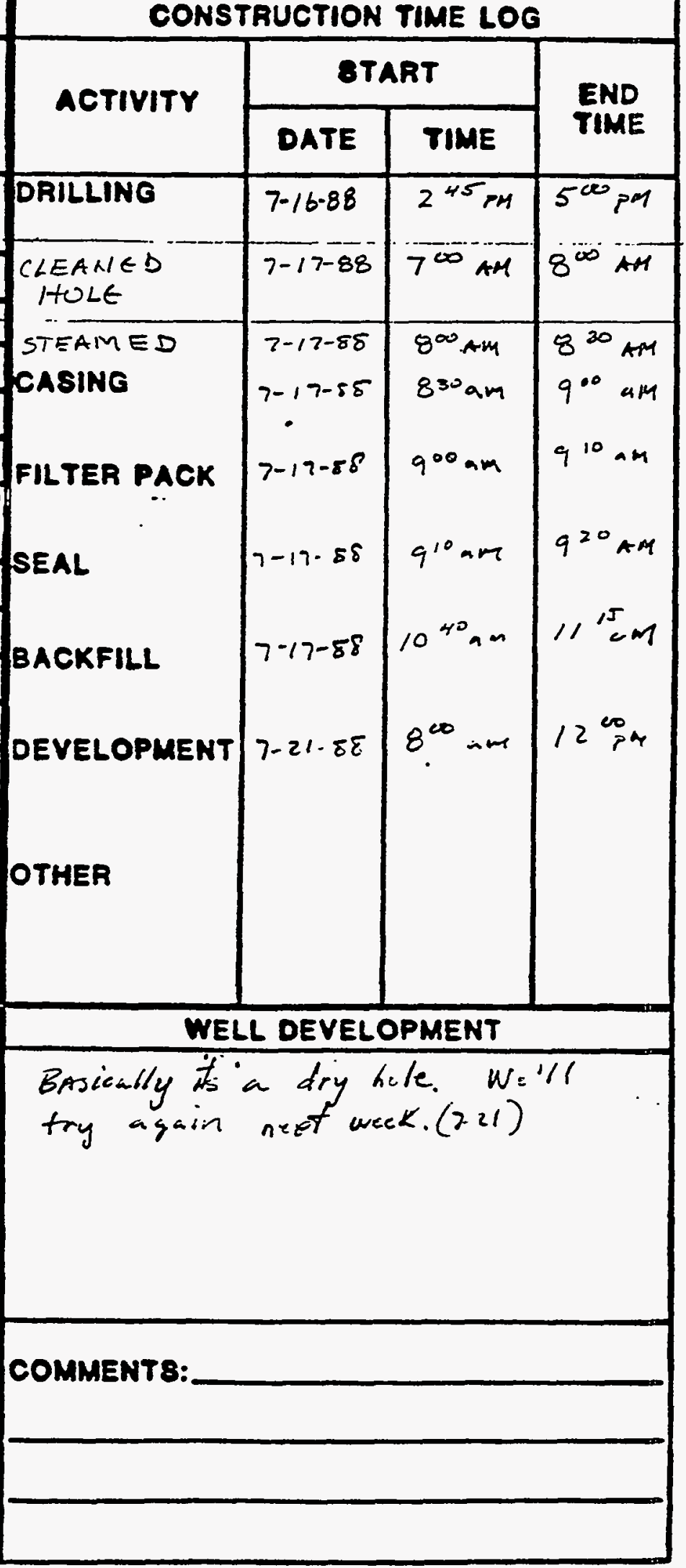




\section{WELL COMPLETION RECORD}

SITE ID: SPK-15 LOCATION ID: 940 DATE INSTALLED: 7-27-88 APPROX. SITE COORDINATES:(FT.) $N$ OPEN AREA PER LINEAL FT. (IN2/FT.) $\left(7 \% 5^{\prime \prime}-4.0^{\prime \prime}\right)^{2}$

FORMATION OF COMPLETION: ROTFOM of Pit WASAtch fM.

FIELD REP.: D. is $D$ L $2 \angle R$ DRILLER: BAEWIMART

WELL CASING 4.0
DIAMETER (IN) 4.0

HOLE DIAMETER (In) 8.0

GROUND SURFACE

TOTAL

DEPTH (11)
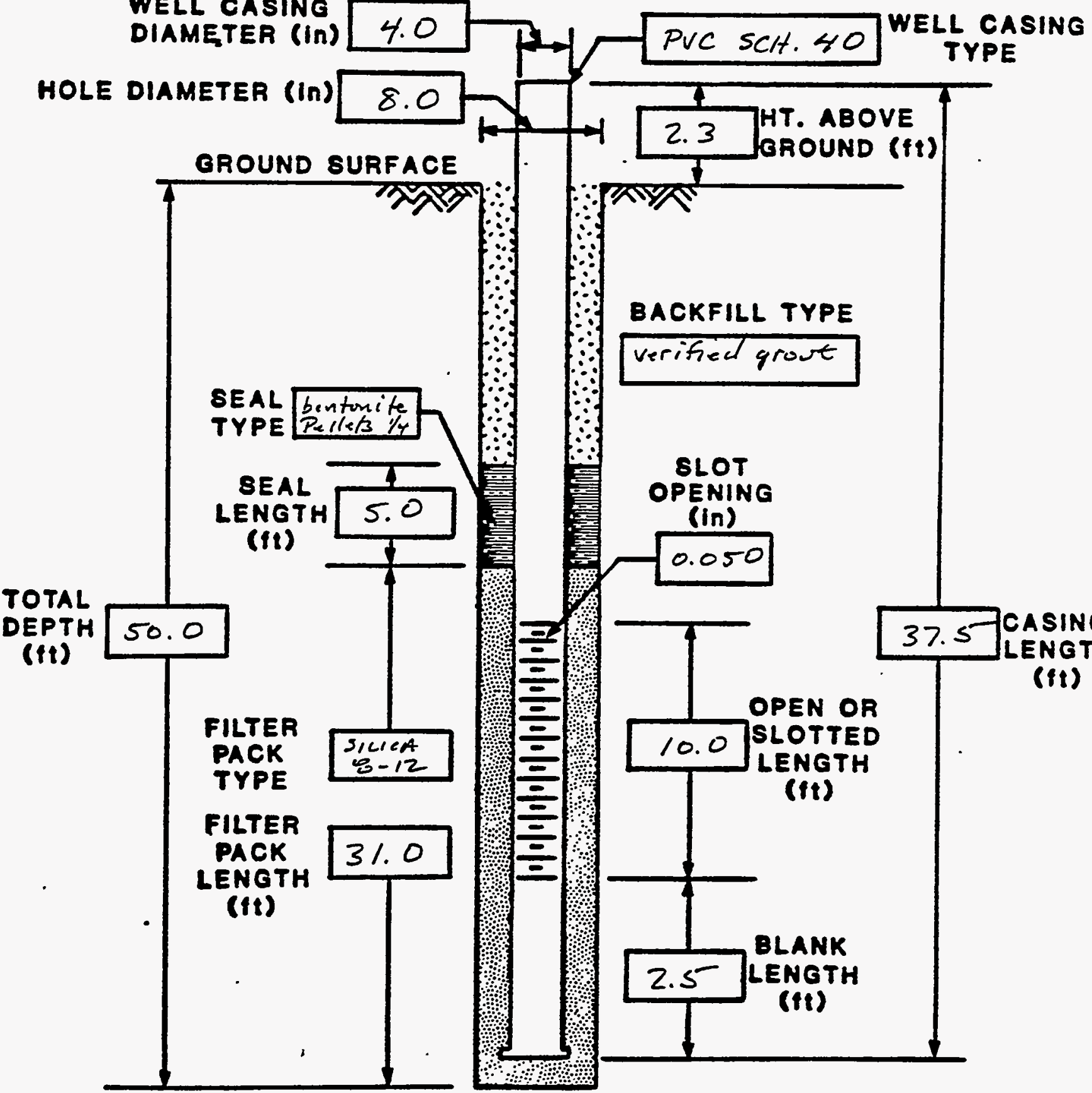
GROUND ( $f t)$
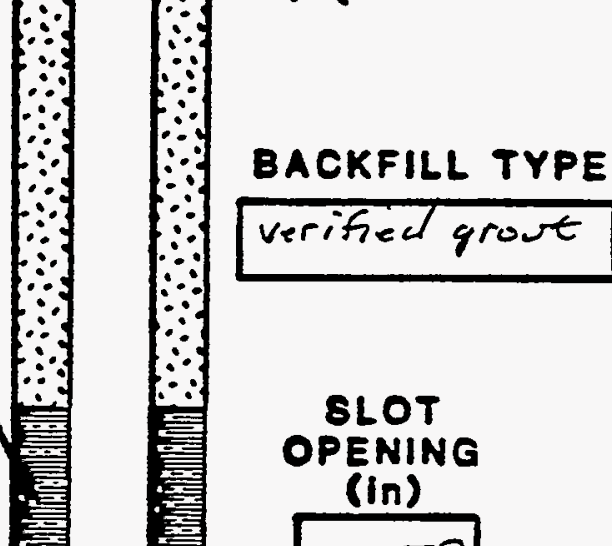

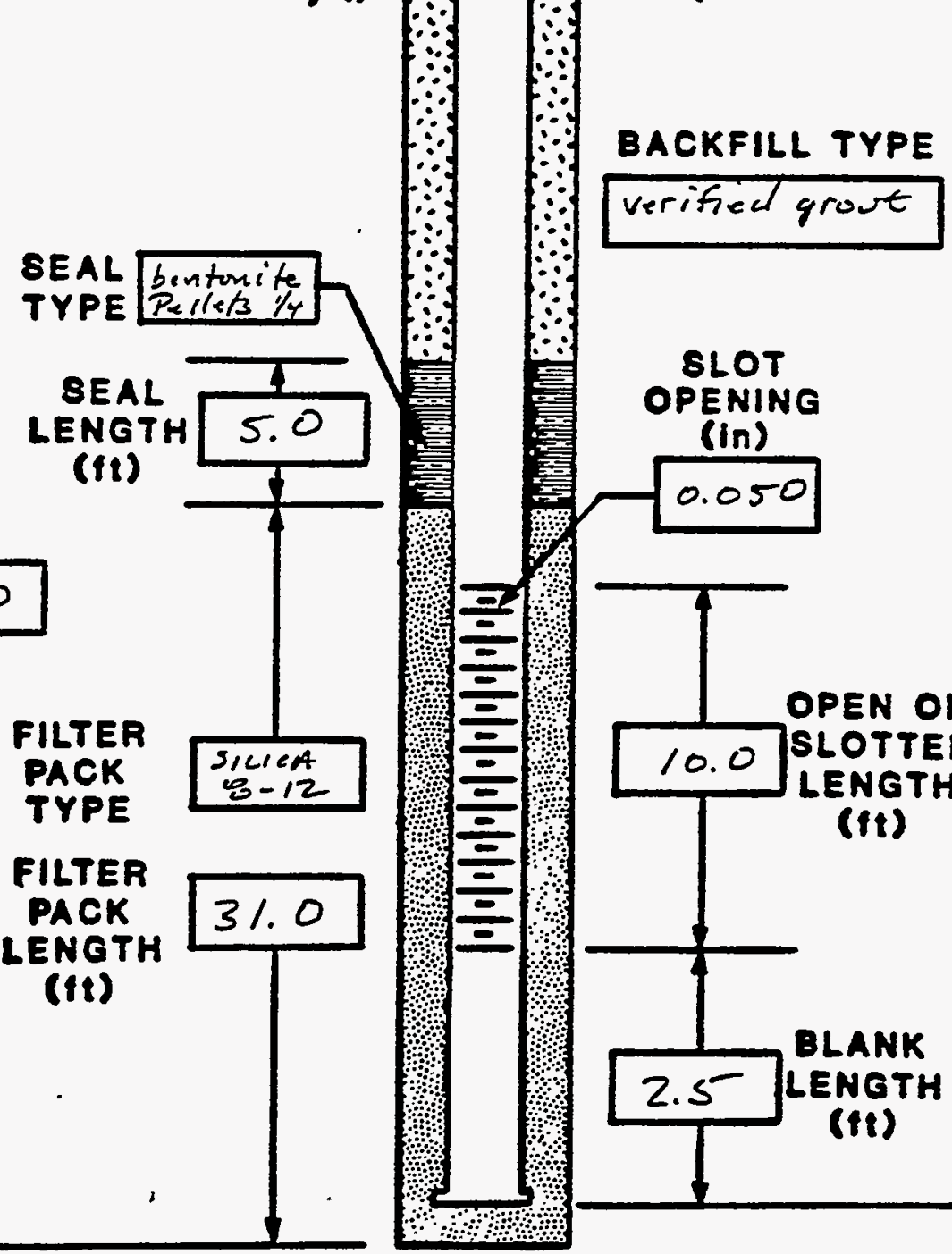

$N K$ 


\section{T. LACOBS ENGNEERHG GROUP NKC.

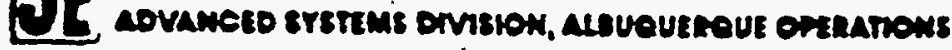 BOREHOLE/WELL CONSTRUCTION LOG}

SITE ID: SPK-15 LOCATION 10: 940 FIELD AEP:D. METZCER APPROX. SITE COORDINATES (FT.): N_N NK NK NK

OROUND ELEVATION (FT. MSL): _ NK BOREHOLE BUMMARY

DRILLER: BARN.HART

RIG TYPE: G.D: 1500 WD RETART

\begin{tabular}{|c|c|c|c|}
\hline DIT TYPE & $\begin{array}{l}\text { HoLE } \\
\text { gita.j } \\
\text { Iin.) }\end{array}$ & OFNPH & $\begin{array}{l}\text { FLUID } \\
\text { TYPE }\end{array}$ \\
\hline $\begin{array}{l}\text { TRI CONE } \\
\text { Tond BIL }\end{array}$ & $77 / 8$ & 50.0 & $A / R$ \\
\hline & & & \\
\hline & & & \\
\hline & & & \\
\hline
\end{tabular}

\section{CASINC}

TYPE:

-

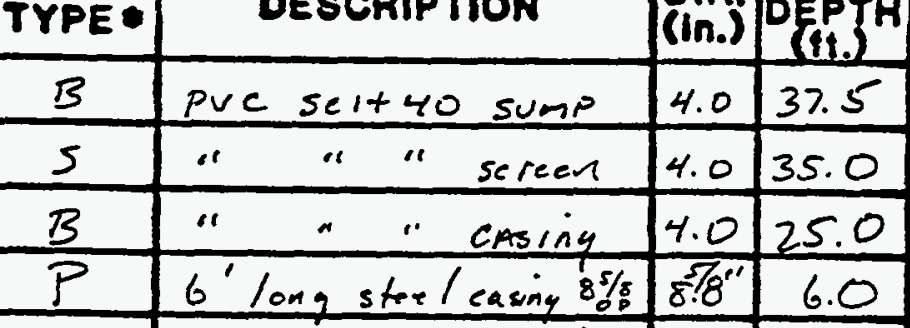

\begin{tabular}{|l|l|l|l|}
\hline & & & \\
\hline & & & \\
\hline & & & \\
\hline & & & \\
\hline Eprotective B-8croen D-8iank O-Opon & \\
\hline
\end{tabular} Depth from Top of Cesho

\begin{tabular}{|c|c|c|}
\hline \multicolumn{3}{|c|}{ WELL CONSTAUCTION } \\
\hline $\begin{array}{l}\text { TYPE } \\
\text { CODE }\end{array}$ & DESCRIPTION & OEPFH \\
\hline$E$ & $3-12$ SICICA $5 \mathrm{mD} D$ & 50.0 \\
\hline 5 & bentonite 14 "Pellets & 19.0 \\
\hline$B$ & bentonite-cement grout & 14.0 \\
\hline & & \\
\hline & & \\
\hline & & \\
\hline & & \\
\hline E- & $8-801$ & Poek \\
\hline - Dor & in from cround surface & \\
\hline
\end{tabular}

COMPLETION DATE: 7-27-8E CONSTRUCTION TIME LOG

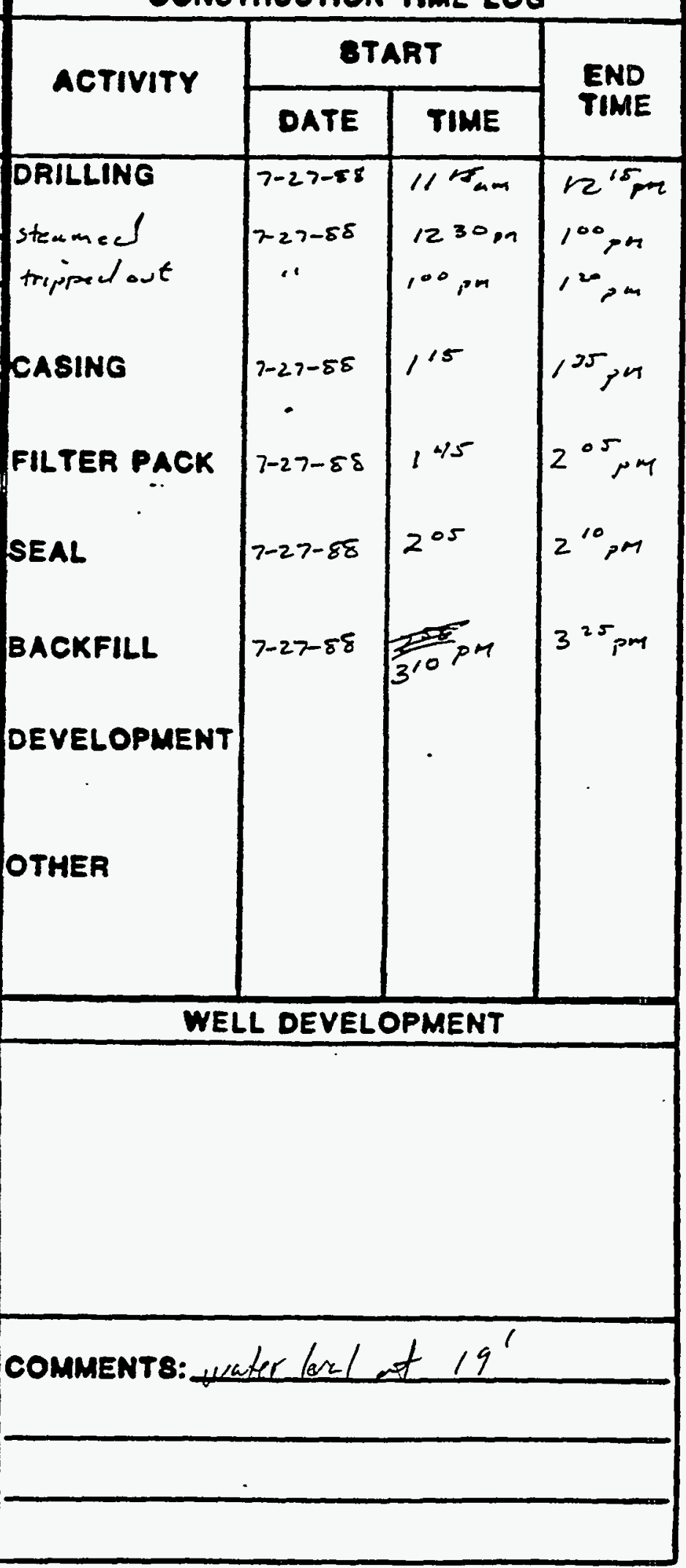




\section{TT MCOAS ANENEERNG GROUP NC.

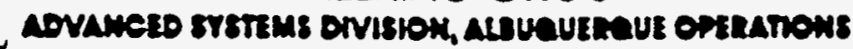

\section{WELL COMPLETION RECORD}

SITE ID: SPK-15 LOCATION ID: 941 DATE INSTALLED: 7-26-88 APPROX. SITE COOORDINATES:(FT.) N $\frac{N K}{N T^{2}}$ E OPEN AREA PER LINEAL FT. (IN2/FT.) $\left(7^{\left.7 / 8^{11}-4.0^{\prime \prime}\right)^{2}}\right.$ FORMATION OF COMPLETION: shadely uper zOne of wateh fM. FIELD REP.: D, METZLER DRILLER: TSARWLART

WELL CASING
DIAMETER (In) 4.0 SCH. 40 PVC WELL CASING
TYPE

HOLE DIAMETER $(\ln ) 8.0$ GROUND SURFACE

TOTAL DEPTH (ft)

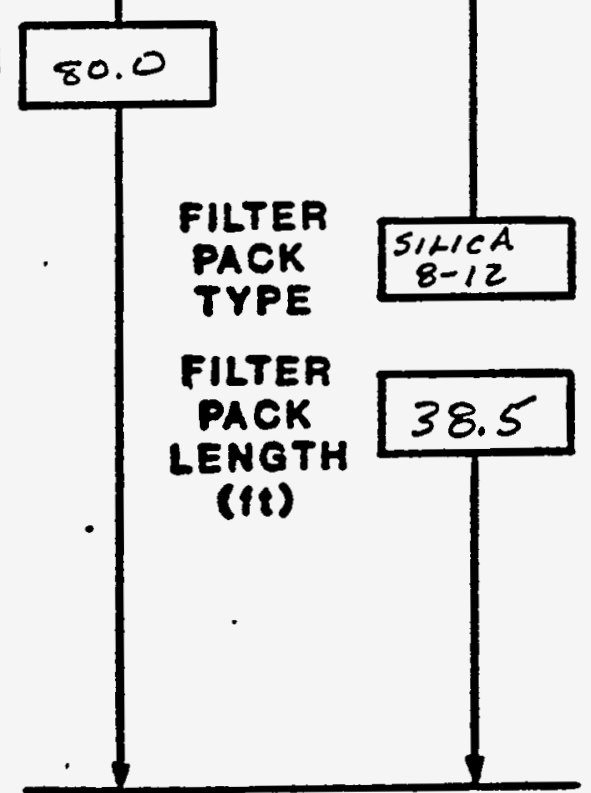

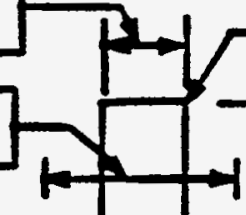

BACKFILL TYPE

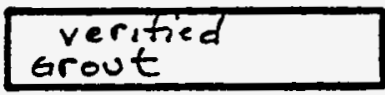

SEAL bentonite

TYPE [y "Pellets

SEAL

LENGTH

(it)
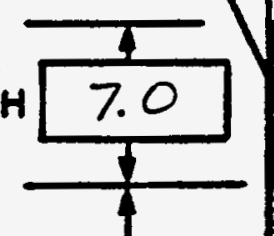

$\because$

$\because$ खण्र

2.5 HT. ABOVE GROUND (ft)

.

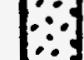

$\therefore$

$\therefore$

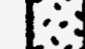

$\therefore$
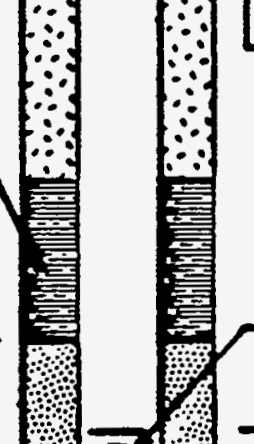

SLOT

OPENING

(in)
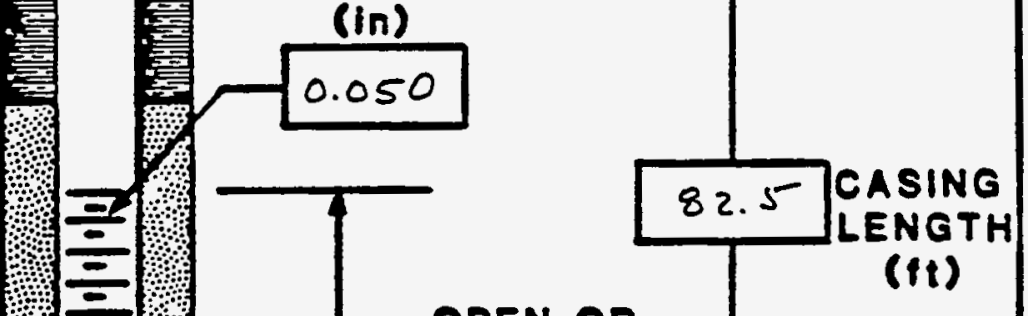

COMMENTS: water Level 7-2>-58 720 am et $45.0^{\prime}$ bentowite seal cured all rinkt 


\section{TT LACOSS ENGNKERNG GROUP NC.

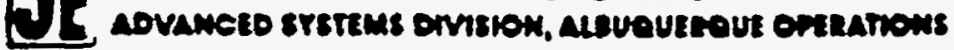 \\ BOREHOLE/WELL CONSTRUCTION LOG}

SITE 1D: SPK-15 LOCATION ID: 941 FIELD REP:D. MGTZLGR APPROX. BITE COORDINATES (FT.): N_NK N N N N

OROUND ELEVATION (FT. MSL):_ NK N BOREHOLE BUMMARY

DRILLER: T3ARNHART

RIG TYPE:

\begin{tabular}{|c|c|c|c|}
\hline BIT TYPE & $\begin{array}{l}\text { HoLe } \\
\text { giA. } \\
\text { In.j) }\end{array}$ & 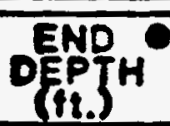 & $\begin{array}{l}\text { FLUID } \\
\text { TYPE }\end{array}$ \\
\hline TRI CONE & $97 / 8^{11}$ & $20^{\prime}$ & $A \mid R$ \\
\hline "senz"sir & $778^{\prime \prime}$ & 80 & AlR + \\
\hline & & & WATER \\
\hline & & & \\
\hline
\end{tabular}

\begin{tabular}{l} 
CASING \\
TYPE: \\
\hline
\end{tabular}

CASING SUMMARY

\begin{tabular}{|c|c|c|c|}
\hline $\begin{array}{l}\text { ASING } \\
\text { YPE: }\end{array}$ & DESCRIPTION & Din. & DEPTH \\
\hline$B$ & SeA 40 PUC & 4.0 & 80.0 \\
\hline 5 & $"$ " " screen & $"$ & 67.5 \\
\hline$B$ & " " & $"$ & 57.5 \\
\hline$P$ & $6^{\prime}$ oug 8 ${ }^{\prime \prime}$ x.0 skel & 8.0 & 6.0 \\
\hline & & & \\
\hline & & & \\
\hline & & & \\
\hline & & & \\
\hline
\end{tabular}

Sopin from Top of Cesing

$$
\text { WELL CONSTAUCTION }
$$

\begin{tabular}{|c|c|c|}
\hline $\begin{array}{l}\text { TYPE } \\
\text { CODE }\end{array}$ & DESCRIPTION & DEPP \\
\hline$F$ & siciet $8-12$ sand & 80.0 \\
\hline$\leq$ & bertanite srout 1/4"? & 41.5 \\
\hline$B$ & bentaite-cement grout & 34.5 \\
\hline & & \\
\hline & & \\
\hline & & \\
\hline & & \\
\hline & & \\
\hline E- & $2-\cos x$ & Paek \\
\hline
\end{tabular}

- Depth tron crownd Eurface

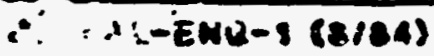

COMPLETION DATE:2-26.88/ CONSTRUCTION TIME LOG

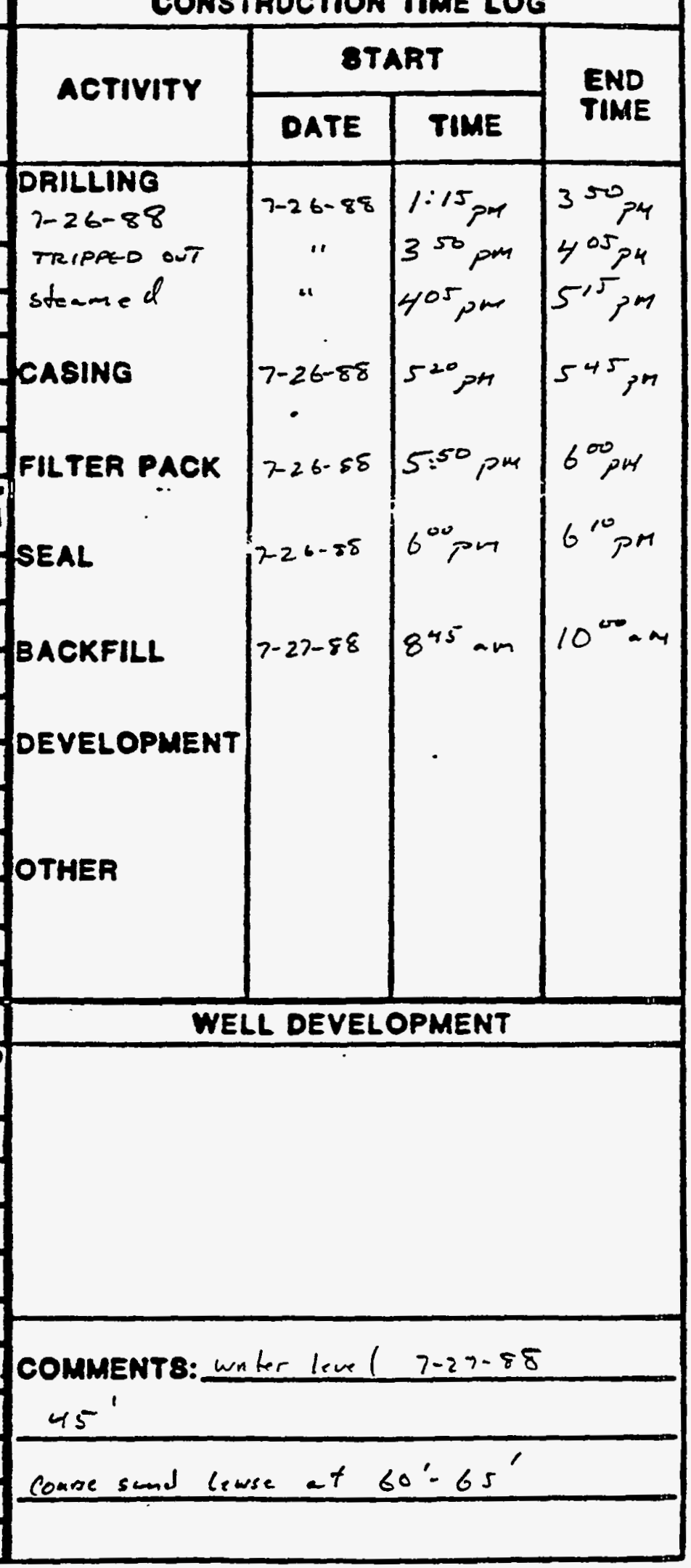




\section{WELL COMPLETION RECORD}

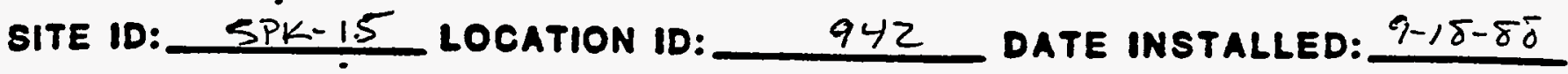
APPROX. SITE COORDINATES:(FT.) N MiK E NK OPEN AREA PPER LINEAL FT. (IN2/FT.) $\left(7^{\left.1 / 8^{\prime \prime}-4^{\prime \prime}\right)^{2}}\right.$ fA $^{2}$ FORMATION OF COMPLETION: QNASATCh SM.

FIELD REP.: T. MUETZLCR DRILLER: BARWIAARF

WELL CASING
DIAMETER (In) 4.0 WELL CASING

HOLE DIAMETER (in) 8.0 GROUND SURFACE

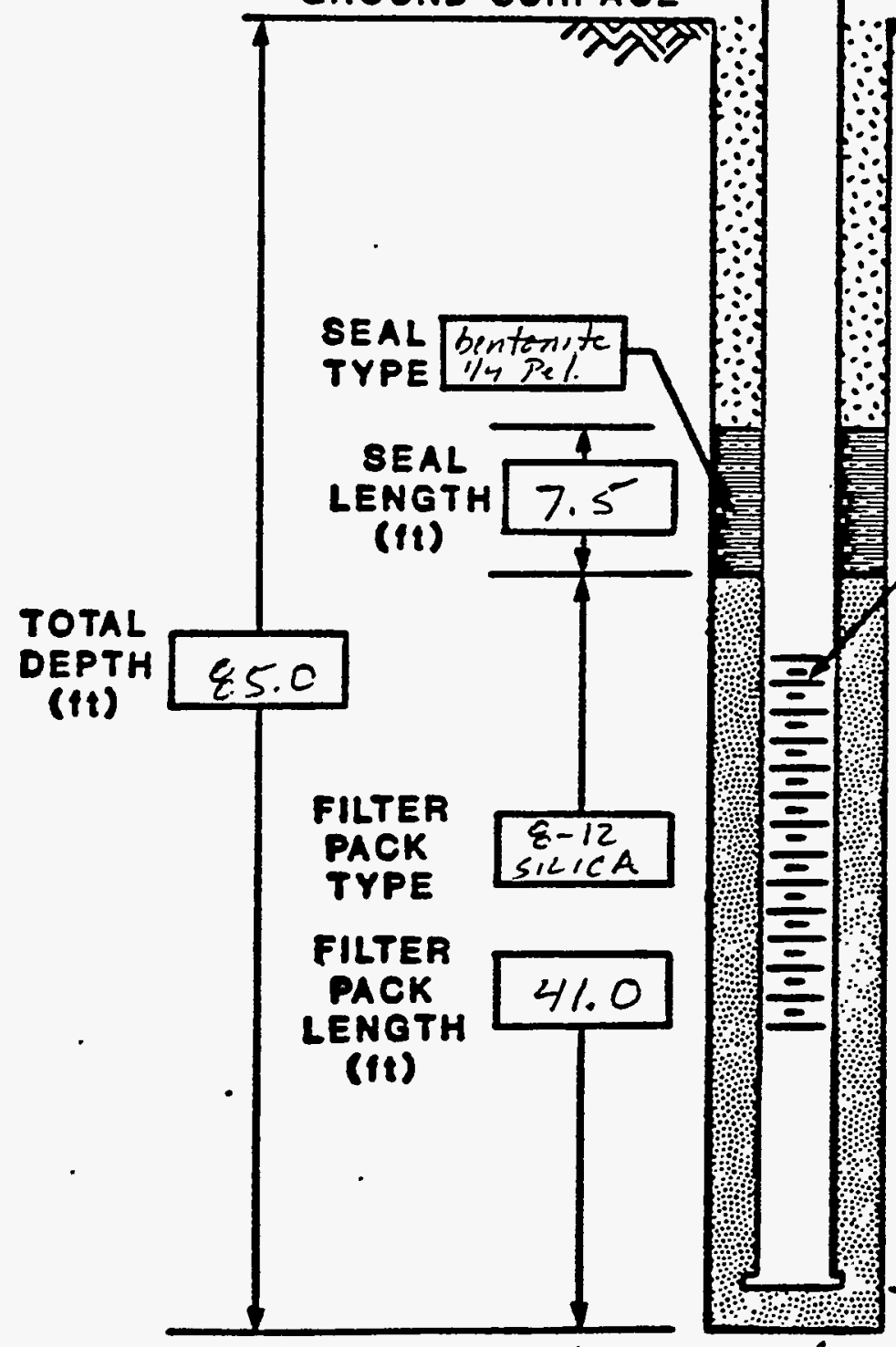

BACKFILL TYPE

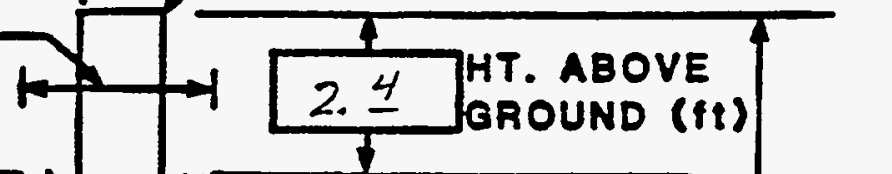
TYPE

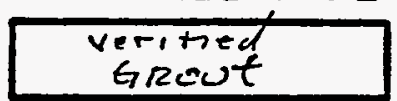

comments: $5.5 /$ shale contuc.t' at Ez.0' chepta 


\section{TE LACOBS ENGNEERNG GROUP NC.

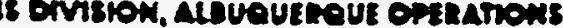 BOREHOLE/WELL CONSTRUCTION LOG}

SITE ID: SPK-15 LOCATION 1D: 942 FIELD REP: D. METZLFR APPROX. 8ITE COORDINATES (FT.): N_NK N N N

OROUND ELEVATION (FT. MSL): BOREHOLE BUMMARY

DRILLER: PBARNHART

RIG TYPE: G. D. 15 WD ROTARL

\begin{tabular}{|c|c|c|c|}
\hline BIT TYPE & $\begin{array}{l}\text { HOLE } \\
\text { PIA } \\
\text { In.j) }\end{array}$ & $\begin{array}{c}\text { END } \\
\text { OEPTH } \\
\text { (fit.) }\end{array}$ & $\begin{array}{l}\text { FLUID } \\
\text { TYPE }\end{array}$ \\
\hline$T R 1-\cos t$ & $778^{11}$ & 85.0 & $A / R+$ \\
\hline & & & scovt $\mathrm{He}$ \\
\hline & & & \\
\hline & & & \\
\hline
\end{tabular}

CASING BUMMARY

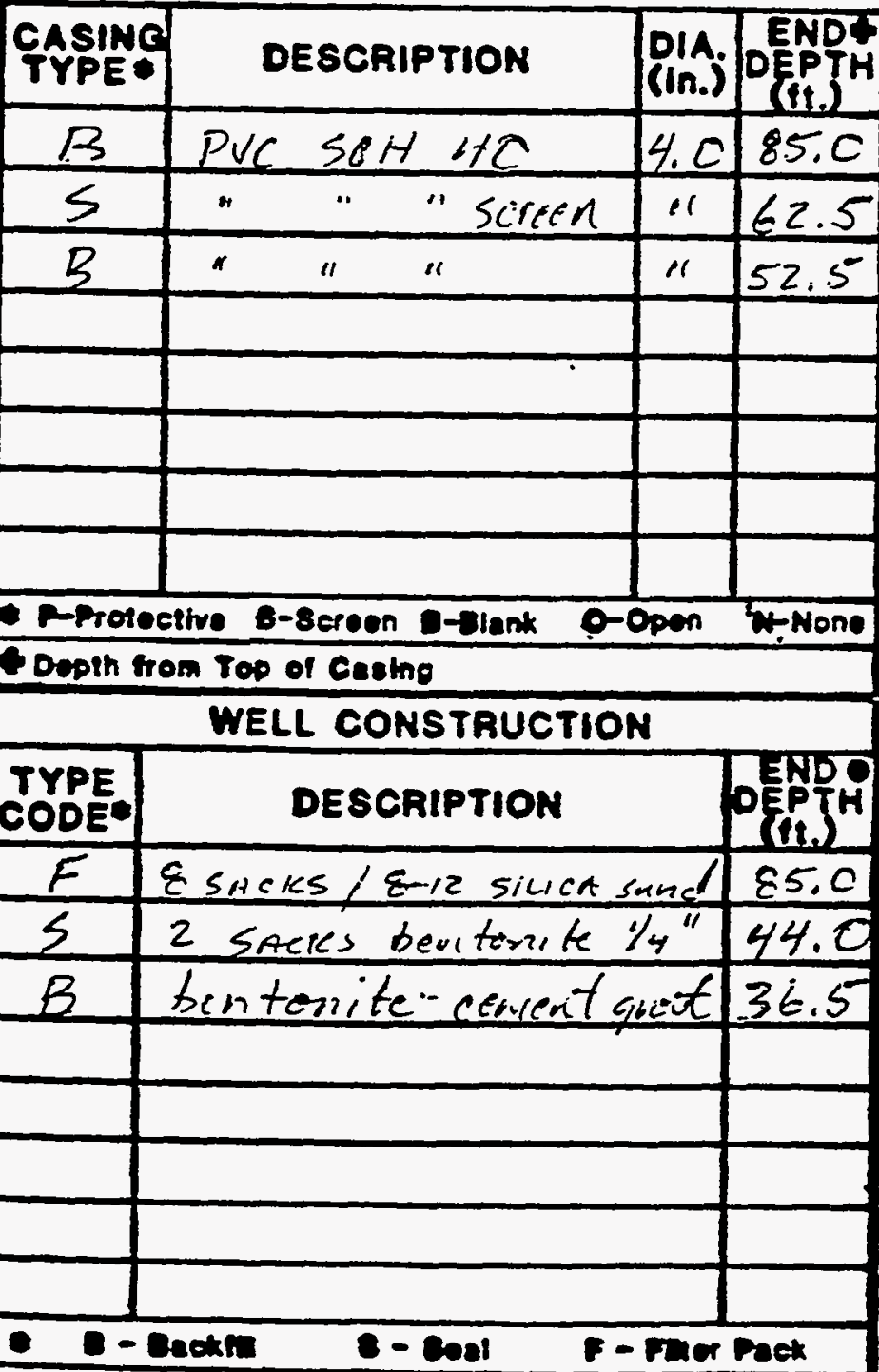

CASING

FILTER PACK

SEAL

BACKFILL

DEVELOPMENT

OTHER
COMPLETION DATE: $7-1 \delta-\bar{\delta}$

CONSTRUCTION TIME LOG

ACTIVITY

START

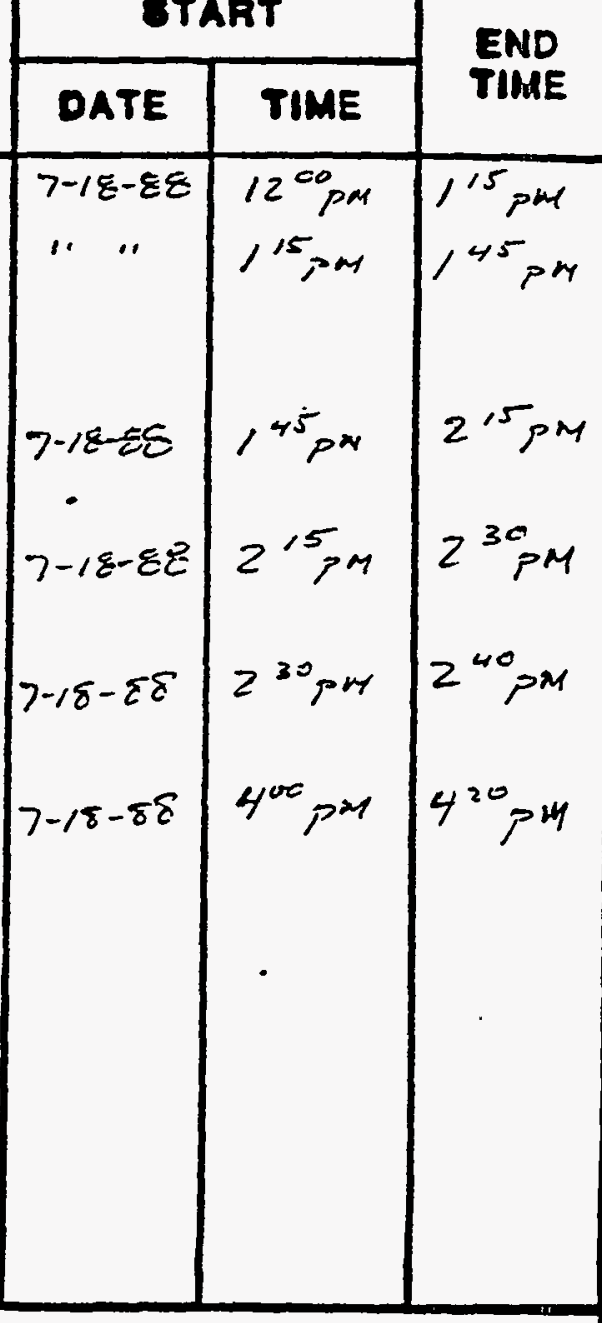

WELL DEVELOPMENT

comMEnT8: $22.5^{\prime}$ sueup in bettree 7 hale: puttinc ize screen of coutact 5.5./shale. 


\section{WELL COMPLETION RECORD}

SITE ID: $\frac{\text { SPK-15 }}{20 C A T I O N ~ I D:} 943$ DATE INSTALLED: $7-30-88$ APPROX. 8ITE COORDINATES:(FT.) N NK E OPEN AREA PER LINEAL FT. (IN2/FT.) $(77 / 5-40)^{2}$

FORMATION OF COMPLETION:

FIELD REP.: D METZLER WASATCA FM

DRILLER: BARNIAART WELL CASING
DIAMETER (IN) 4.0 PVC SCA 40 WELL CASING

HOLE DIAMETER (in) 8.0 GROUND SURFACE
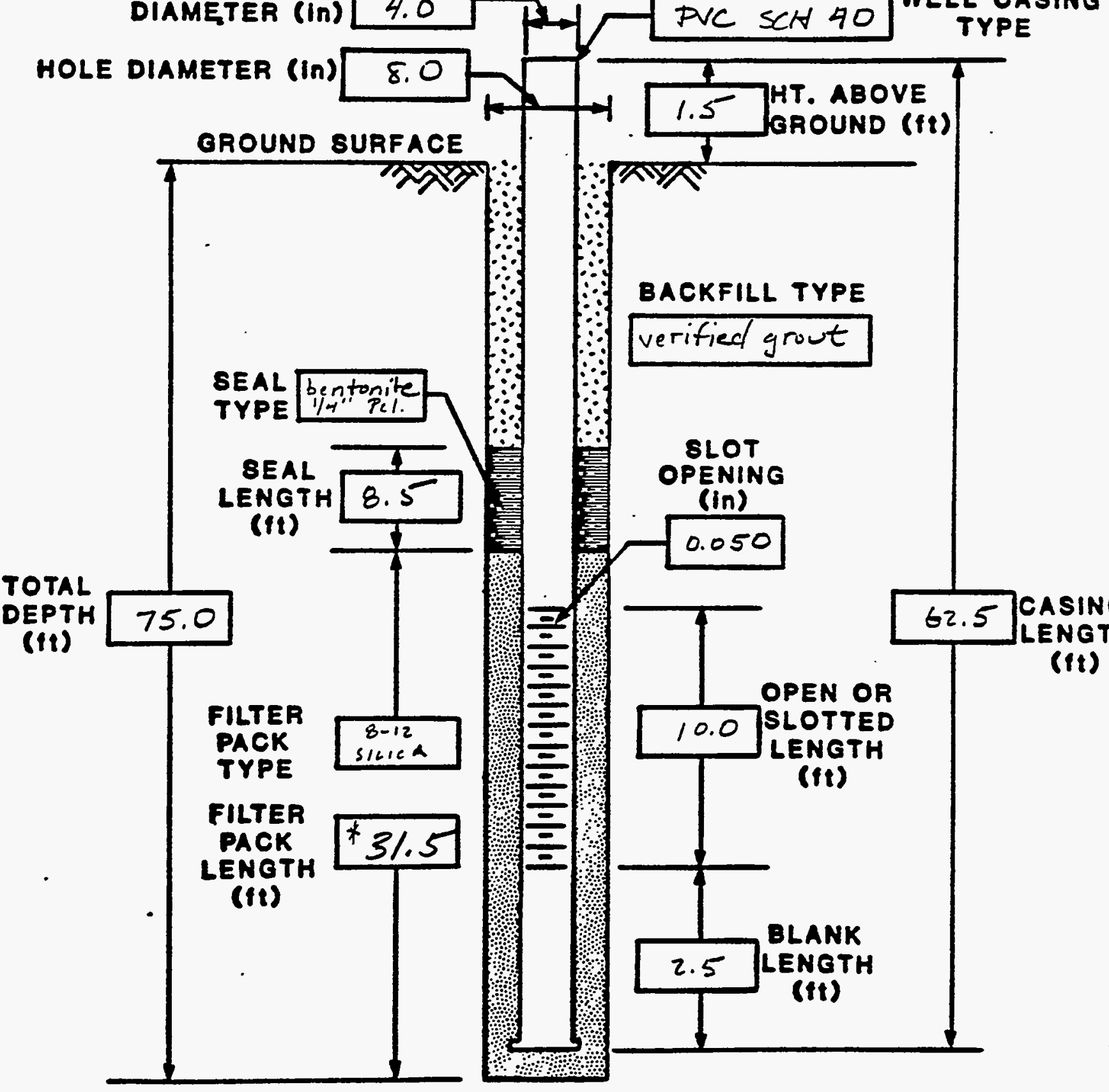

प्राy $1.5 \int_{G R O U N D}^{H T}$ ABOVE (ft)

BACKFILL TYPE verified grout

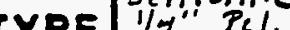
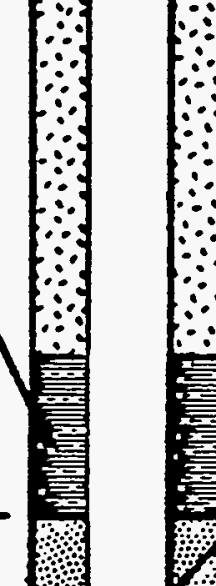

$\because$
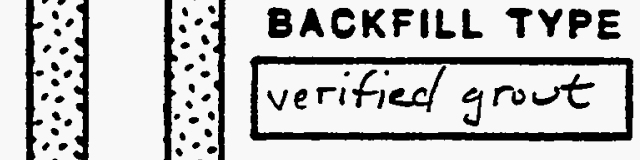

TOTAL

1

SLOT

OPENING

(in)

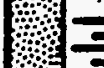

$=1$
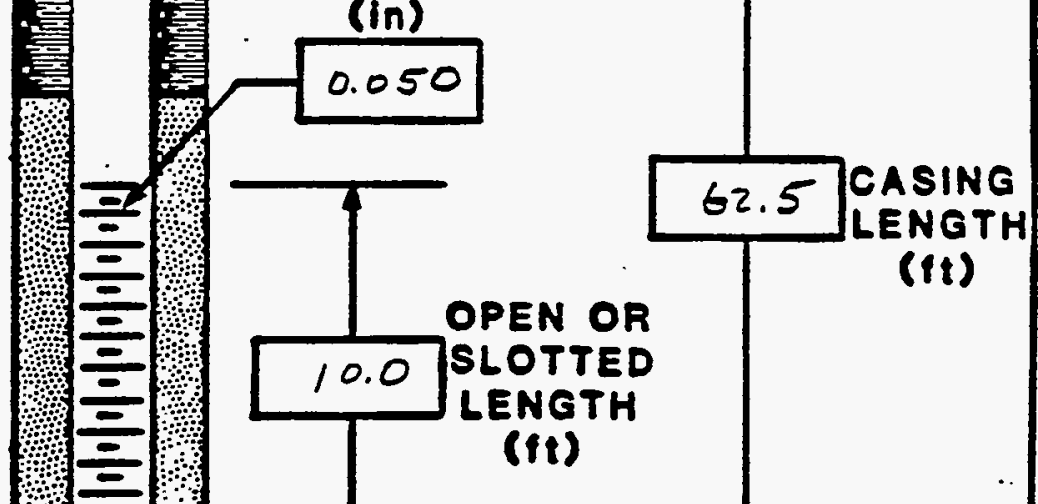

OPEN OR

(It)

comment8: $1 \%{ }^{\prime}$ of bentonite seal at $64-62 \%$ : plug 


\section{BOREHOLE/WELL CONSTRUCTION LOG}

SITE 1D: SPK-15 LOCATION 10: 943 FIELD REP: D. METZLEZ

APPROX. 8ITE COORDINATES (FT.): N_NK NK NK

BROUND ELEVATION (FT. MSL):_ NK COMPLETION DATE: $7-30-88$

BOREHOLE BUMMARY

DRILLER:

BARNIART

RIG TYPE: G.D.'1500 WO ROTARY BIT TYPE

TRI COA Toont
HOLE

(1)

$7 \% s^{\circ}$

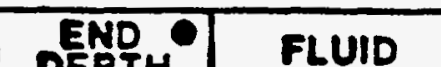

DEPTH (fit 75.0

\section{CONSTRUCTION TIME LOG}

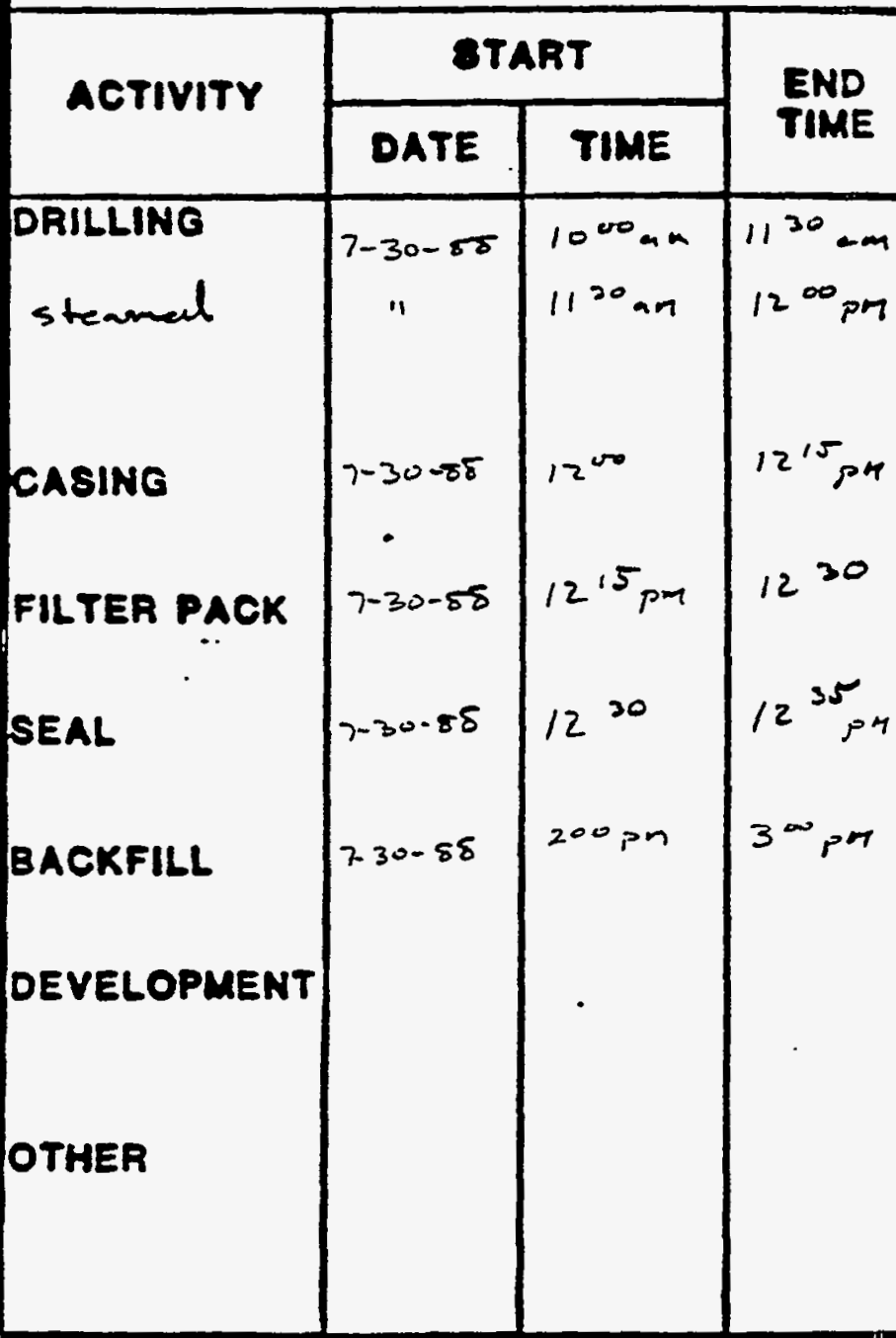

WELL DEVELOPMENT

- Protective B-sereen D-Tlank O-Open W-None Sopth trom Top of Cacing

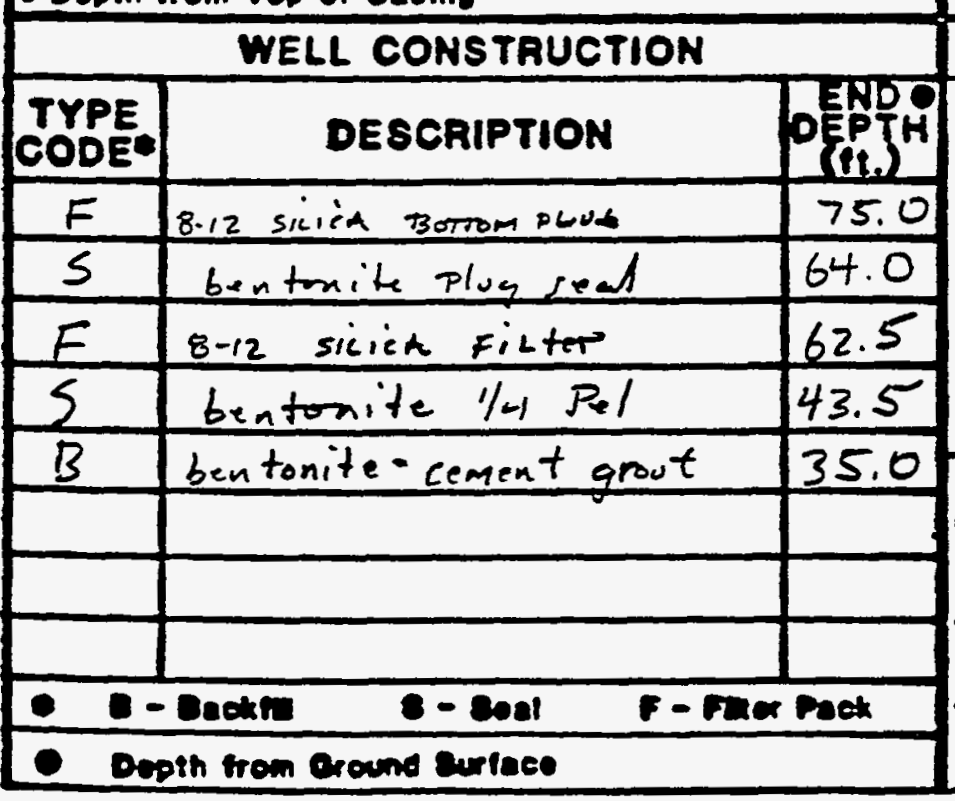

COMMENT8: Diek on site fal observation 4 


\section{WELL COMPLETION RECORD}

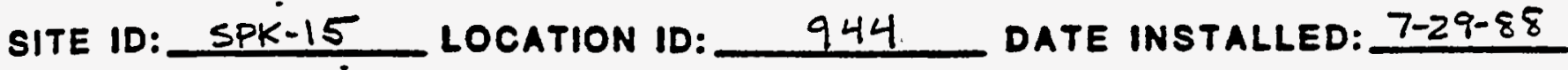
APPROX. SITE COORDINATES:(FT.) N NK E OPEN AREA PER LINEAL FT. $\left(I N^{2} / F T.\right)\left(77 / s^{\prime \prime}-4.0\right)^{2}$ (FORMATION OF COMPLETION: WATSATCh Fm.

FIELD REP.: D. METZLGR DRILLER: NK

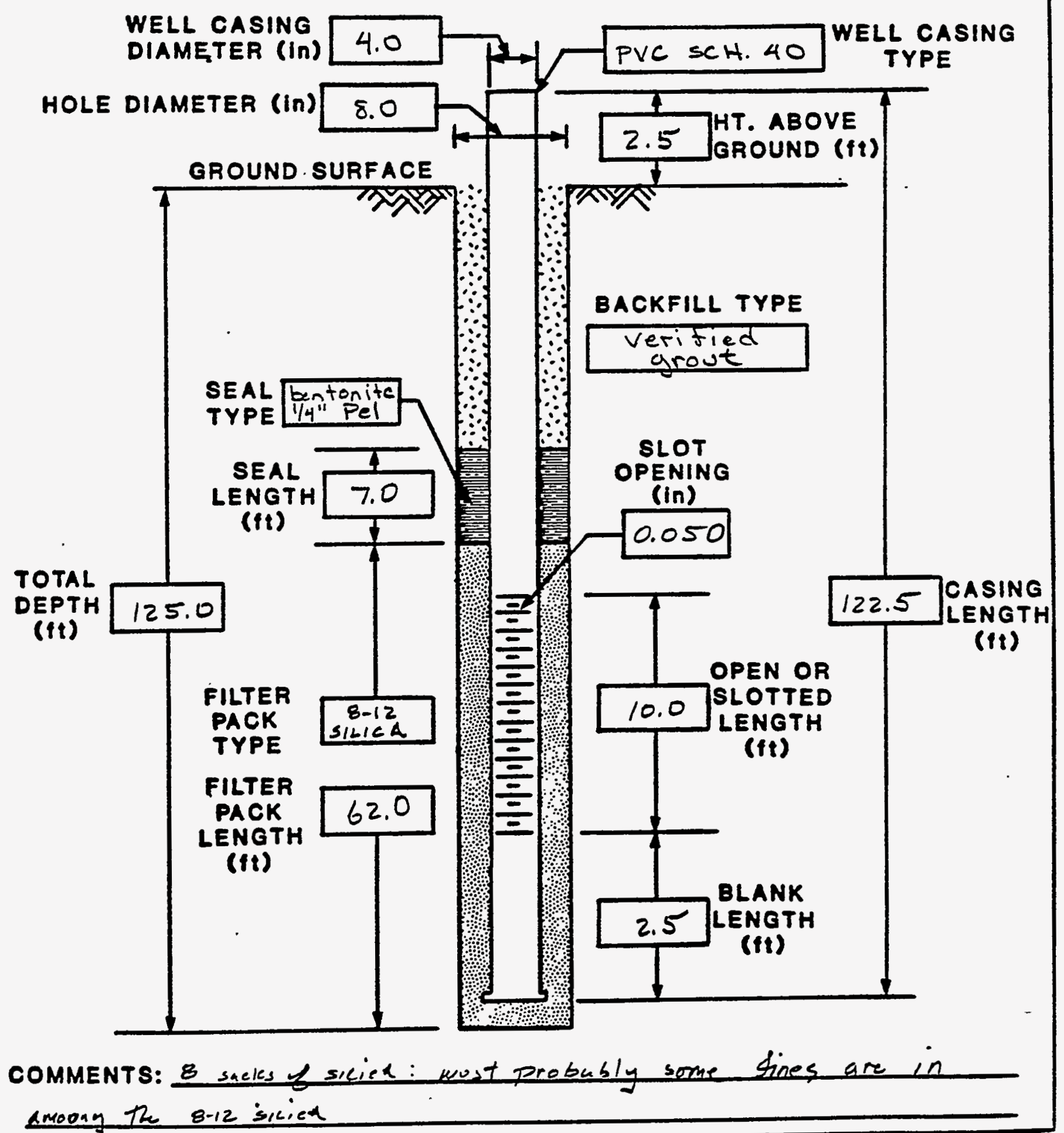




\section{TI LACOBS ENGNEERNG GROUP INC.

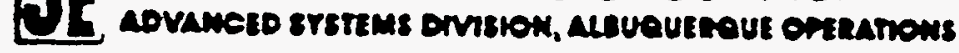 \\ BOREHOLE/WELL CONSTRUCTION LOG}

SITE ID:_SPK-15 LOCATION 10: 944 FIELD REP:_D. METZLER

APPROX, BITE COORDINATES (FT.): $N$ MK NK

OROUND ELEVATION (FT. MSL):_NK COMPLETION DATE: 7-30-88

BOREHOLE 8UMMARY

DRILLER: BARNHART

RIG TYPE: G.D.' ROTARY AIR

\begin{tabular}{|c|c|c|c|}
\hline BIT TYPE & $\begin{array}{l}\text { HOLE } \\
\text { OIA } \\
\text { in.j }\end{array}$ & DENP & $\begin{array}{l}\text { FLUID } \\
\text { TYPE }\end{array}$ \\
\hline $\begin{array}{l}\text { TRI CONE } \\
\text { T00TH }\end{array}$ & $77 / 8^{11}$ & 125.0 & $A \mid R+$ \\
\hline & & & WATERT \\
\hline & & & bentonite \\
\hline & & & \\
\hline
\end{tabular}

CASING

TYPE:

DESCRIPTION

\begin{tabular}{l|l|l|l|}
\hline$N$ & SLUFF & & 125.0 \\
\hline$B$ & PVE SCH. 40 SUMP & 4.0 & 120.0 \\
\hline
\end{tabular}

\begin{tabular}{l|l|l|l|l|}
\hline & " $" ~$ & screen & " & 117.5 \\
\hline
\end{tabular}

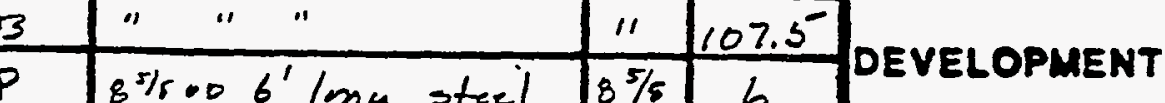

\begin{tabular}{l|l|l|l|}
$P$ & $8 \% 006^{\prime} \mathrm{lmy}$ steel & $85 / 5$ & 6 \\
\hline
\end{tabular}

CASING

hung up

FILTER PACK

SEAL

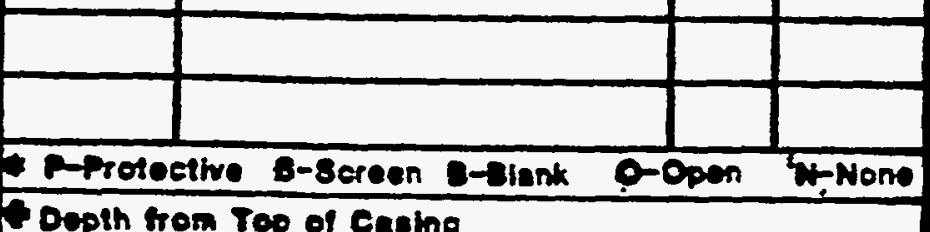

WELL CONSTRUCTION

\begin{tabular}{|c|c|c|}
\hline $\begin{array}{l}\text { TYPE } \\
\text { CODE }\end{array}$ & DESCAIPTION & DE \\
\hline$F$ & 8 sucks + s10ff & 125.0 \\
\hline$s$ & bentonite $1 / 4^{\prime \prime} \mathrm{Pel}$. & 63.0 \\
\hline 3 & beutonite-cement quot & 56.0 \\
\hline & & \\
\hline & & \\
\hline & & \\
\hline & & \\
\hline & & \\
\hline e. & $8-8021$ & Pack \\
\hline e & in trom cround Eurtece & \\
\hline
\end{tabular}

OTHER

WELL DEVELOPMENT

bentorite slurry was pumped in to mall to prevent enving. Well was developed with. air ten $=1 \mathrm{hr}$ atter samd east injtallod.

COMMENT8: EAving at $70^{\prime}$ down

fine sibts and clays. 
APPENDIX C

GROUND WATER ELEVATIONS 
STATIC GROUNDHATER LEVELS

SITE: SPKO1 SPOOK

REPORT DATE: 08/03/94

\begin{tabular}{|c|c|c|c|c|c|c|c|c|c|c|c|}
\hline $\begin{array}{c}\text { LOCATION } \\
\text { ID }\end{array}$ & $\begin{array}{c}\text { NORTH } \\
\text { COORDINATE } \\
\text { (FT) }\end{array}$ & $\begin{array}{c}\text { EAST } \\
\text { COORDINATE } \\
\text { (FT) }\end{array}$ & $\begin{array}{l}\text { FORMATION } \\
\text { OF } \\
\text { COMPLETIOH }\end{array}$ & $\begin{array}{l}\text { FLOW } \\
\text { CODE }\end{array}$ & $\begin{array}{l}\text { CASIHG } \\
\text { ELEVATION } \\
\text { (FT MSL) }\end{array}$ & $\begin{array}{l}\text { GROUND } \\
\text { ELEVATION } \\
\text { (FT MSL) }\end{array}$ & LOG DATE & $\begin{array}{l}\text { LOG } \\
\text { TIME }\end{array}$ & $\begin{array}{l}\text { DEPTH FROH } \\
\text { TOP OF } \\
\text { CASING (FT) }\end{array}$ & $\begin{array}{l}\text { DEPTH FROM } \\
\text { GROUND } \\
\text { (FT) }\end{array}$ & $\begin{array}{l}\text { GROUNDWATER } \\
\text { ELEVATION } \\
\text { (FT MSL) }\end{array}$ \\
\hline 0910 & 56710.5 & 59662.9 & WL & N & 5097.10 & 5095.20 & $\begin{array}{l}12 / 20 / 86 \\
02 / 17 / 87 \\
01 / 29 / 88 \\
04 / 19 / 88 \\
08 / 11 / 88 \\
12 / 09 / 88\end{array}$ & $\begin{array}{l}15: 30 \\
09: 35 \\
09: 30 \\
10: 10 \\
10: 30 \\
14: 41\end{array}$ & $\begin{array}{l}112.27 \\
112.48 \\
102.45 \\
111.81 \\
112.00 \\
113.70\end{array}$ & $\begin{array}{l}110.37 \\
110.58 \\
100.55 \\
109.91 \\
110.10 \\
111.80\end{array}$ & $\begin{array}{l}4984.83 \\
4984.62 \\
4994.65 \\
4985.29 \\
4985.10 \\
4983.40\end{array}$ \\
\hline 0911 & 56684.5 & 59695.9 & $W U$ & $N$ & 5094.80 & 5093.00 & $\begin{array}{l}12 / 20 / 86 \\
02 / 17 / 87 \\
01 / 28 / 88 \\
04 / 16 / 88 \\
08 / 11 / 88 \\
12 / 07 / 88\end{array}$ & $\begin{array}{l}15: 30 \\
09: 40 \\
09: 45 \\
12: 25 \\
09: 10 \\
14: 00\end{array}$ & $\begin{array}{l}85.90 \\
87.10 \\
86.76 \\
86.21 \\
86.71 \\
87.43\end{array}$ & $\begin{array}{l}84.10 \\
85.30 \\
84.96 \\
84.41 \\
84.91 \\
85.63\end{array}$ & $\begin{array}{l}5008.90 \\
5007.70 \\
5008.04 \\
5008.59 \\
5008.09 \\
5007.37\end{array}$ \\
\hline 0912 & 57376.4 & 60399.0 & WS & N & 5111.10 & 5109.70 & $\begin{array}{l}12 / 20 / 86 \\
02 / 18 / 87 \\
01 / 30 / 88 \\
04 / 21 / 88 \\
08 / 26 / 88 \\
11 / 30 / 88 \\
07 / 29 / 94\end{array}$ & $\begin{array}{l}15: 30 \\
09: 00 \\
09: 30 \\
13: 00 \\
18: 00 \\
13: 00 \\
15: 47\end{array}$ & $\begin{array}{l}107.80 \\
108.00 \\
108.42 \\
108.42 \\
108.85 \\
109.16 \\
108.62\end{array}$ & $\begin{array}{l}106.40 \\
106.60 \\
107.02 \\
107.02 \\
107.45 \\
107.76 \\
107.22\end{array}$ & $\begin{array}{l}5003.30 \\
5003.10 \\
5002.68 \\
5002.68 \\
5002.25 \\
5001.94 \\
5002.48\end{array}$ \\
\hline 0913 & 58150.1 & 60546.0 & WU & $N$ & 5098.60 & 5096.80 & $\begin{array}{l}12 / 20 / 86 \\
02 / 18 / 87 \\
01 / 30 / 88 \\
04 / 22 / 88 \\
08 / 04 / 88 \\
12 / 08 / 88 \\
07 / 29 / 94\end{array}$ & $\begin{array}{l}15: 30 \\
09: 10 \\
13: 35 \\
09: 10 \\
13: 00 \\
12: 50 \\
14: 10\end{array}$ & $\begin{array}{r}99.85 \\
100.28 \\
100.12 \\
100.15 \\
100.15 \\
100.55 \\
101.62\end{array}$ & $\begin{array}{l}98.05 \\
98.48 \\
98.32 \\
98.35 \\
98.35 \\
98.75 \\
99.82\end{array}$ & $\begin{array}{l}4998.75 \\
4998.32 \\
4998.48 \\
4998.45 \\
4998.45 \\
4998.05 \\
4996.98\end{array}$ \\
\hline 0914 & 58134.9 & 60512.1 & WL & $N$ & 5099.50 & 5097.50 & $\begin{array}{l}12 / 20 / 86 \\
02 / 18 / 87 \\
01 / 30 / 88 \\
04 / 19 / 88 \\
08 / 07 / 88 \\
12 / 09 / 88 \\
07 / 29 / 94\end{array}$ & $\begin{array}{l}15: 30 \\
01: 18 \\
15: 26 \\
15: 10 \\
09: 40 \\
12: 50 \\
14: 15\end{array}$ & $\begin{array}{c}104.15 \\
105.10 \\
105.00 \\
105.01 \\
105.00 \\
105.67 \\
107.05\end{array}$ & $\begin{array}{l}102.15 \\
103.10 \\
103.00 \\
103.01 \\
103.00 \\
103.67 \\
105.05\end{array}$ & $\begin{array}{l}4995.35 \\
4994.40 \\
4994.50 \\
4994.49 \\
4994.50 \\
4993.83 \\
4992.45\end{array}$ \\
\hline 0915 & 58719.8 & 61452.6 & WM & N & 5059.60 & 5057.90 & $\begin{array}{l}12 / 20 / 86 \\
02 / 19 / 87 \\
02 / 01 / 88\end{array}$ & $\begin{array}{l}15: 30 \\
01: 30 \\
14: 00\end{array}$ & $\begin{array}{l}68.20 \\
65.98 \\
66.69\end{array}$ & $\begin{array}{l}66.50 \\
64.28 \\
64.99\end{array}$ & $\begin{array}{l}4991.40 \\
4993.62 \\
4992.91\end{array}$ \\
\hline
\end{tabular}


STATIC GROUNDWATER LEVELS

SITE: SPKO1 SPOOK

REPORT DATE: 08/03/94

\begin{tabular}{|c|c|c|c|c|c|c|c|c|c|c|c|}
\hline $\begin{array}{c}\text { LOCATION } \\
\text { ID }\end{array}$ & $\begin{array}{c}\text { NORTH } \\
\text { COORDINATE } \\
\text { (FT) }\end{array}$ & $\begin{array}{c}\text { EAST } \\
\text { COORD INATE } \\
\text { (FT) }\end{array}$ & $\begin{array}{l}\text { FORMATION } \\
\text { OF } \\
\text { COMPLETION }\end{array}$ & $\begin{array}{l}\text { FLOW } \\
\text { CODE }\end{array}$ & $\begin{array}{l}\text { CASING } \\
\text { ELEVATIOH } \\
\text { (FT MSL) }\end{array}$ & $\begin{array}{l}\text { GROUND } \\
\text { ELEVATION } \\
\text { (FT MSL) }\end{array}$ & LOG DATE & $\begin{array}{l}\text { LOG } \\
\text { TIME }\end{array}$ & $\begin{array}{l}\text { DEPTH FROM } \\
\text { TOP OF } \\
\text { CASING (FT) }\end{array}$ & $\begin{array}{l}\text { DEPTH FROM } \\
\text { GROUND } \\
\text { (FT) }\end{array}$ & $\begin{array}{l}\text { GROUNDWATER } \\
\text { ELEVATION } \\
\text { (FT MSL) }\end{array}$ \\
\hline 0915 & 58719.8 & 61452.6 & WM & $N$ & 5059.60 & 5057.90 & $\begin{array}{l}04 / 20 / 88 \\
08 / 09 / 88 \\
12 / 08 / 88 \\
07 / 29 / 94\end{array}$ & $\begin{array}{l}12: 15 \\
12: 45 \\
09: 30 \\
13: 24\end{array}$ & $\begin{array}{l}66.13 \\
66.04 \\
66.15 \\
67.85\end{array}$ & $\begin{array}{l}64.43 \\
64.34 \\
64.45 \\
66.15\end{array}$ & $\begin{array}{l}4993.47 \\
4993.56 \\
4993.45 \\
4991.75\end{array}$ \\
\hline 0916 & 59373.3 & 61439.6 & WU & $N$ & 5071.60 & 5070.00 & $\begin{array}{l}12 / 20 / 86 \\
02 / 20 / 87 \\
02 / 02 / 88 \\
04 / 20 / 88 \\
08 / 04 / 88 \\
12 / 05 / 88 \\
07 / 29 / 94\end{array}$ & $\begin{array}{l}15: 30 \\
02: 30 \\
10: 15 \\
10: 00 \\
10: 00 \\
13: 50 \\
13: 17\end{array}$ & $\begin{array}{l}78.68 \\
80.22 \\
79.89 \\
79.86 \\
79.94 \\
80.00 \\
81.02\end{array}$ & $\begin{array}{l}77.08 \\
78.62 \\
78.29 \\
78.26 \\
78.34 \\
78.40 \\
79.42\end{array}$ & $\begin{array}{l}4992.92 \\
4991.38 \\
4991.71 \\
4991.74 \\
4991.66 \\
4991.60 \\
4990.58\end{array}$ \\
\hline 0917 & 58853.6 & 62234.5 & WU & $\mathbf{N}$ & 5049.60 & 5047.80 & $\begin{array}{l}12 / 20 / 86 \\
02 / 19 / 87 \\
01 / 28 / 88 \\
04 / 21 / 88 \\
08 / 26 / 88 \\
12 / 06 / 88 \\
07 / 29 / 94\end{array}$ & $\begin{array}{l}15: 30 \\
11: 50 \\
14: 10 \\
09: 19 \\
17: 02 \\
09: 30 \\
13: 30\end{array}$ & $\begin{array}{l}60.17 \\
60.48 \\
60.46 \\
60.43 \\
60.80 \\
60.73 \\
61.43\end{array}$ & $\begin{array}{l}58.37 \\
58.68 \\
58.66 \\
58.63 \\
59.00 \\
58.93 \\
59.63\end{array}$ & $\begin{array}{l}4989.43 \\
4989.12 \\
4989.14 \\
4989.17 \\
4988.80 \\
4988.87 \\
4988.17\end{array}$ \\
\hline 0918 & 57588.1 & 61629.1 & WU & $N$ & 5103.10 & 5101.30 & $\begin{array}{l}12 / 20 / 86 \\
02 / 20 / 87 \\
01 / 31 / 88 \\
04 / 21 / 88 \\
08 / 25 / 88 \\
12 / 03 / 88 \\
07 / 29 / 94\end{array}$ & $\begin{array}{l}15: 30 \\
10: 35 \\
09: 30 \\
10: 35 \\
15: 15 \\
10: 45 \\
14: 00\end{array}$ & $\begin{array}{l}108.90 \\
108.70 \\
109.05 \\
108.86 \\
108.92 \\
109.41 \\
110.18\end{array}$ & $\begin{array}{l}107.10 \\
106.90 \\
107.25 \\
107.06 \\
107.12 \\
107.61 \\
108.38\end{array}$ & $\begin{array}{l}4994.20 \\
4994.40 \\
4994.05 \\
4994.24 \\
4994.18 \\
4993.69 \\
4992.92\end{array}$ \\
\hline 0919 & 56014.4 & 59867.6 & WL & $\mathbf{N}$ & 5107.00 & 5105.20 & $\begin{array}{l}12 / 20 / 86 \\
02 / 17 / 87 \\
01 / 27 / 88 \\
04 / 23 / 88 \\
08 / 26 / 88 \\
12 / 09 / 88 \\
07 / 29 / 94\end{array}$ & $\begin{array}{l}15: 30 \\
03: 00 \\
15: 50 \\
09: 48 \\
10: 15 \\
09: 15 \\
15: 05\end{array}$ & $\begin{array}{l}126.00 \\
126.26 \\
126.61 \\
125.57 \\
126.10 \\
127.00 \\
126.25\end{array}$ & $\begin{array}{l}124.20 \\
124.46 \\
124.81 \\
123.77 \\
124.30 \\
125.20 \\
124.45\end{array}$ & $\begin{array}{l}4981.00 \\
4980.74 \\
4980.39 \\
4981.43 \\
4980.90 \\
4980.00 \\
4980.75\end{array}$ \\
\hline 0920 & 56021.6 & 59923.7 & WU & $\mathbf{N}$ & 5101.10 & 5099.00 & $\begin{array}{l}01 / 27 / 88 \\
04 / 23 / 88 \\
08 / 26 / 88 \\
12 / 07 / 88\end{array}$ & $\begin{array}{l}10: 30 \\
11: 50 \\
13: 00 \\
11: 30\end{array}$ & $\begin{array}{l}86.72 \\
85.38 \\
86.82 \\
87.27\end{array}$ & $\begin{array}{l}84.62 \\
83.28 \\
84.72 \\
85.17\end{array}$ & $\begin{array}{l}5014.38 \\
5015.72 \\
5014.28 \\
5013.83\end{array}$ \\
\hline
\end{tabular}

FORMATION OF COMPLETION CODE:

FLOW RELATIONSHIP CODE:

UM - HASATCH - MIDDLE SHALE

N - UNKNOWN

WU - WASATCH - UPPER SANDSTONE

WL - HASATCH - LOHER SANDSTONE 
STATIC GROUNDHATER LEVELS

SITE: SPKOI SPOOK

REPORT DATE: $08 / 03 / 94$

\begin{tabular}{|c|c|c|c|c|c|c|c|c|c|c|c|}
\hline $\begin{array}{c}\text { LOCATION } \\
\text { ID }\end{array}$ & $\begin{array}{c}\text { NORTH } \\
\text { COORDINATE } \\
\text { (FT) }\end{array}$ & $\begin{array}{c}\text { EAST } \\
\text { COORDINATE } \\
\text { (FT) }\end{array}$ & $\begin{array}{c}\text { FORMATION } \\
\text { OF } \\
\text { COMPLETION }\end{array}$ & $\begin{array}{l}\text { FLOH } \\
\text { CODE }\end{array}$ & $\begin{array}{l}\text { CASING } \\
\text { ELEVATION } \\
\text { (FT MSL) }\end{array}$ & $\begin{array}{l}\text { GROUND } \\
\text { ELEVATION } \\
\text { (FT MSL) }\end{array}$ & LOG DATE & $\begin{array}{l}\text { LOG } \\
\text { TIME }\end{array}$ & $\begin{array}{l}\text { DEPTH FROM } \\
\text { TOP OF } \\
\text { CASING (FT) }\end{array}$ & $\begin{array}{l}\text { DEPTH FROM } \\
\text { GROUND } \\
\text { (FT) }\end{array}$ & $\begin{array}{l}\text { GROUNDWATER } \\
\text { ELEVATION } \\
\text { (FT MSL) }\end{array}$ \\
\hline 0920 & 56021.6 & 59923.7 & $\mathbf{W}$ & N & 5101.10 & 5099.00 & $07 / 29 / 94$ & $14: 59$ & 88.83 & 86.73 & 5012.27 \\
\hline 0921 & 58118.3 & 59198.9 & WL. & N & 5127.00 & 5124.50 & $\begin{array}{l}02 / 03 / 88 \\
04 / 24 / 88 \\
08 / 09 / 88 \\
12 / 07 / 88 \\
07 / 29 / 94\end{array}$ & $\begin{array}{l}10: 00 \\
09: 15 \\
10: 40 \\
14: 50 \\
11: 35\end{array}$ & $\begin{array}{l}123.25 \\
122.26 \\
123.55 \\
124.38 \\
125.76\end{array}$ & $\begin{array}{l}120.75 \\
119.76 \\
121.05 \\
121.88 \\
123.26\end{array}$ & $\begin{array}{l}5003.75 \\
5004.74 \\
5003.45 \\
5002.62 \\
5001.24\end{array}$ \\
\hline 0922 & 58163.6 & 59210.6 & WU & N & 5124.80 & 5122.40 & $\begin{array}{l}02 / 02 / 88 \\
04 / 24 / 88 \\
08 / 07 / 88 \\
12 / 07 / 88 \\
07 / 29 / 94\end{array}$ & $\begin{array}{l}13: 30 \\
11: 20 \\
10: 45 \\
16: 02 \\
11: 30\end{array}$ & $\begin{array}{l}119.58 \\
119.68 \\
119.63 \\
120.46 \\
121.80\end{array}$ & $\begin{array}{l}117.18 \\
117.28 \\
117.23 \\
118.06 \\
119.40\end{array}$ & $\begin{array}{l}5005.22 \\
5005.12 \\
5005.17 \\
5004.34 \\
5003.00\end{array}$ \\
\hline 0923 & 59654.0 & 59828.6 & HL. & N & 5115.00 & 5112.80 & $\begin{array}{l}01 / 29 / 88 \\
04 / 25 / 88 \\
08 / 08 / 88 \\
12 / 09 / 88 \\
07 / 29 / 94\end{array}$ & $\begin{array}{l}12: 45 \\
09: 35 \\
14: 20 \\
10: 00 \\
11: 47\end{array}$ & $\begin{array}{l}119.96 \\
119.86 \\
119.84 \\
120.73 \\
121.32\end{array}$ & $\begin{array}{l}117.76 \\
117.66 \\
117.64 \\
118.53 \\
119.12\end{array}$ & $\begin{array}{l}4995.04 \\
4995.14 \\
4995.16 \\
4994.27 \\
4993.68\end{array}$ \\
\hline 0924 & 59855.8 & 61191.0 & WU & $N$ & 5081.20 & 5079.10 & $\begin{array}{l}01 / 03 / 88 \\
04 / 25 / 88 \\
08 / 25 / 88 \\
12 / 06 / 88 \\
07 / 29 / 94\end{array}$ & $\begin{array}{l}14: 00 \\
12: 35 \\
13: 15 \\
12: 00 \\
12: 22\end{array}$ & $\begin{array}{l}89.46 \\
89.50 \\
89.65 \\
88.29 \\
90.45\end{array}$ & $\begin{array}{l}87.36 \\
87.40 \\
87.55 \\
86.19 \\
88.35\end{array}$ & $\begin{array}{l}4991.74 \\
4991.70 \\
4991.55 \\
4992.91 \\
4990.75\end{array}$ \\
\hline 0925 & 59815.3 & 62832.1 & WU & N & 5042.10 & 5039.90 & $\begin{array}{l}02 / 02 / 88 \\
04 / 25 / 88 \\
08 / 25 / 88 \\
12 / 04 / 88 \\
07 / 29 / 94\end{array}$ & $\begin{array}{l}13: 45 \\
13: 57 \\
10: 08 \\
10: 45 \\
12: 42\end{array}$ & $\begin{array}{l}55.98 \\
55.86 \\
56.17 \\
56.05 \\
56.31\end{array}$ & $\begin{array}{l}53.78 \\
53.66 \\
53.97 \\
53.85 \\
54.11\end{array}$ & $\begin{array}{l}4986.12 \\
4986.24 \\
4985.93 \\
4986.05 \\
4985.79\end{array}$ \\
\hline 0927 & 59161.8 & 60115.4 & WU & N & 5096.7 & 5094.7 & $\begin{array}{l}01 / 03 / 88 \\
04 / 24 / 88 \\
08 / 24 / 88 \\
12 / 03 / 88 \\
07 / 29 / 94\end{array}$ & $\begin{array}{l}12: 30 \\
14: 10 \\
15: 15 \\
14: 20 \\
12: 10\end{array}$ & $\begin{array}{l}98.57 \\
98.67 \\
99.10 \\
99.10 \\
96.77\end{array}$ & $\begin{array}{l}96.57 \\
96.67 \\
97.10 \\
97.10 \\
94.77\end{array}$ & $\begin{array}{l}4998.13 \\
4998.03 \\
4997.60 \\
4997.60 \\
4999.93\end{array}$ \\
\hline 0928 & 57963.3 & 59497.0 & WU & $N$ & 5028.50 & 5025.60 & $\begin{array}{l}01 / 31 / 88 \\
04 / 22 / 88 \\
08 / 03 / 88 \\
12 / 02 / 88\end{array}$ & $\begin{array}{l}11: 00 \\
11: 28 \\
13: 15 \\
09: 00\end{array}$ & $\begin{array}{l}23.65 \\
23.76 \\
24.08 \\
24.39\end{array}$ & $\begin{array}{l}20.75 \\
20.86 \\
21.18 \\
21.49\end{array}$ & $\begin{array}{l}5004.85 \\
5004.74 \\
5004.42 \\
5004.11\end{array}$ \\
\hline
\end{tabular}

FORMATION OF COMPLETION CODE:

WU - HASATCH - UPPER SANDSTONE

FLOW RELATIONSHIP CODE:

HL - HASATCH - LOHER SANDSTONE 
STATIC GROUNDWATER LEVELS

SITE: SPKO1 SPOOK

REPORT DATE: 08/03/94

\begin{tabular}{|c|c|c|c|c|c|c|c|c|c|c|c|}
\hline $\begin{array}{c}\text { LOCATION } \\
\text { ID }\end{array}$ & $\begin{array}{c}\text { NORTH } \\
\text { COORDINATE } \\
\text { (FT) }\end{array}$ & $\begin{array}{c}\text { EAST } \\
\text { COORDINATE } \\
\text { (FT) }\end{array}$ & $\begin{array}{l}\text { FORMATION } \\
\text { OF } \\
\text { COMPLETION }\end{array}$ & $\begin{array}{l}\text { FLOW } \\
\text { CODE }\end{array}$ & $\begin{array}{l}\text { CASING } \\
\text { ELEVATION } \\
\text { (FT MSL) }\end{array}$ & $\begin{array}{l}\text { GROUND } \\
\text { ELEVATION } \\
\text { (FT MSL) }\end{array}$ & LOG DATE & $\begin{array}{l}\text { LOG } \\
\text { TIME }\end{array}$ & $\begin{array}{l}\text { DEPTH FROM } \\
\text { TOP OF } \\
\text { CASING (FT) }\end{array}$ & $\begin{array}{c}\text { DEPTH FROM } \\
\text { GROUND } \\
\text { (FT) }\end{array}$ & $\begin{array}{c}\text { GROUNDHATER } \\
\text { ELEVATION } \\
\text { (FT MSL.) }\end{array}$ \\
\hline 0929 & 56502.8 & 59663.1 & $W$ & N & 5085.00 & 5083.00 & $\begin{array}{l}01 / 30 / 88 \\
04 / 26 / 88 \\
08 / 11 / 88\end{array}$ & $\begin{array}{l}13: 15 \\
15: 10 \\
11: 10\end{array}$ & $\begin{array}{l}81.33 \\
81.25 \\
81.12\end{array}$ & $\begin{array}{l}79.33 \\
79.25 \\
79.12\end{array}$ & $\begin{array}{l}5003.67 \\
5003.75 \\
5003.88\end{array}$ \\
\hline 0930 & 56033.6 & 59898.5 & WU & $\mathbf{N}$ & 5104.10 & 5101.70 & $\begin{array}{l}01 / 27 / 88 \\
04 / 23 / 88 \\
08 / 26 / 88 \\
07 / 29 / 94\end{array}$ & $\begin{array}{l}14: 30 \\
13: 20 \\
14: 44 \\
15: 02\end{array}$ & $\begin{array}{l}89.86 \\
89.51 \\
89.86 \\
91.75\end{array}$ & $\begin{array}{l}87.46 \\
87.11 \\
87.46 \\
89.35\end{array}$ & $\begin{array}{l}5014.24 \\
5014.59 \\
5014.24 \\
5012.35\end{array}$ \\
\hline 0931 & 59847.5 & 63505.7 & WU & $N$ & 5064.8 & 5062.60 & $\begin{array}{l}01 / 26 / 88 \\
04 / 27 / 88 \\
08 / 09 / 88 \\
12 / 04 / 88 \\
07 / 29 / 94\end{array}$ & $\begin{array}{l}13: 56 \\
13: 30 \\
10: 10 \\
10: 30 \\
12: 50\end{array}$ & $\begin{array}{l}80.65 \\
80.49 \\
80.19 \\
80.62 \\
80.80\end{array}$ & $\begin{array}{l}78.45 \\
78.29 \\
77.99 \\
78.42 \\
78.60\end{array}$ & $\begin{array}{l}4984.15 \\
4984.31 \\
4984.61 \\
4984.18 \\
4984.00\end{array}$ \\
\hline 0932 & 59855.8 & 60115.7 & WU & $\mathbf{N}$ & 5106.9 & 5104.50 & $\begin{array}{l}01 / 29 / 88 \\
04 / 26 / 88 \\
08 / 08 / 88 \\
12 / 05 / 88 \\
07 / 29 / 94\end{array}$ & $\begin{array}{l}11: 10 \\
11: 00 \\
14: 35 \\
09: 15 \\
12: 00\end{array}$ & $\begin{array}{l}112.70 \\
112.50 \\
112.26 \\
112.75 \\
113.51\end{array}$ & $\begin{array}{l}110.30 \\
110.10 \\
109.86 \\
110.35 \\
111.11\end{array}$ & $\begin{array}{l}4994.20 \\
4994.40 \\
4994.64 \\
4994.15 \\
4993.39\end{array}$ \\
\hline 0933 & 60310.2 & 64205.1 & WU & $\mathbf{N}$ & 5079.70 & 5077.40 & $\begin{array}{l}08 / 09 / 88 \\
12 / 05 / 88 \\
07 / 29 / 94\end{array}$ & $\begin{array}{l}12: 50 \\
12: 45 \\
13: 00\end{array}$ & $\begin{array}{l}98.22 \\
98.38 \\
98.57\end{array}$ & $\begin{array}{l}95.92 \\
96.08 \\
96.27\end{array}$ & $\begin{array}{l}4981.48 \\
4981.32 \\
4981.13\end{array}$ \\
\hline 0934 & 60178.9 & 62032.2 & wu & $\mathbf{N}$ & 5072.30 & 5070.10 & $\begin{array}{l}08 / 10 / 88 \\
12 / 05 / 88 \\
07 / 29 / 94\end{array}$ & $\begin{array}{l}14: 31 \\
11: 00 \\
12: 33\end{array}$ & $\begin{array}{l}83.64 \\
84.01 \\
84.37\end{array}$ & $\begin{array}{l}81.44 \\
81.81 \\
82.17\end{array}$ & $\begin{array}{l}4988.66 \\
4988.29 \\
4987.93\end{array}$ \\
\hline 0935 & 57050.2 & 59205.8 & WU & $N$ & 5128.10 & 5125.90 & $\begin{array}{l}08 / 07 / 88 \\
11 / 30 / 88 \\
07 / 29 / 94\end{array}$ & $\begin{array}{l}12: 15 \\
11: 20 \\
14: 42\end{array}$ & $\begin{array}{l}115.60 \\
116.32 \\
117.15\end{array}$ & $\begin{array}{l}113.40 \\
114.12 \\
114.95\end{array}$ & $\begin{array}{l}5012.50 \\
5011.78 \\
5010.95\end{array}$ \\
\hline 0936 & 56745.9 & 61019.6 & WU & $N$ & 5086.10 & 5083.40 & $\begin{array}{l}08 / 06 / 88 \\
12 / 02 / 88 \\
07 / 29 / 94\end{array}$ & $\begin{array}{l}09: 40 \\
12: 00 \\
15: 56\end{array}$ & $\begin{array}{l}88.50 \\
88.89 \\
88.53\end{array}$ & $\begin{array}{l}85.80 \\
86.19 \\
85.83\end{array}$ & $\begin{array}{l}4997.60 \\
4997.21 \\
4997.57\end{array}$ \\
\hline 0937 & 55665.7 & 60303.9 & WU & N & 5052.80 & 5049.50 & $\begin{array}{l}08 / 06 / 88 \\
12 / 01 / 88 \\
07 / 29 / 94\end{array}$ & $\begin{array}{l}09: 45 \\
09: 00 \\
15: 25\end{array}$ & $\begin{array}{l}76.84 \\
75.29 \\
73.98\end{array}$ & $\begin{array}{l}73.54 \\
71.99 \\
70.68\end{array}$ & $\begin{array}{l}4975.96 \\
4977.51 \\
4978.82\end{array}$ \\
\hline 0938 & 58231.6 & 62849.7 & WL & N & 5036.00 & 5033.70 & $08 / 07 / 88$ & $14: 10$ & 56.15 & 53.85 & 4979.85 \\
\hline
\end{tabular}

FORMATION OF COMPLETION CODE:

WU - WASATCH - UPPER SANDSTONE

FLOW RELATIONSHIP CODE:

WL - WASATCH - LOHER SANDSTONE

N - UNKNOWH 
STATIC GROUNDHATER LEVELS

SITE: SPKO1 SPOOK

REPORT DATE: 08/03/94

\begin{tabular}{|c|c|c|c|c|c|c|c|c|c|c|c|}
\hline$\underset{\text { ID }}{\text { LOCATION }}$ & $\begin{array}{c}\text { MORTH } \\
\text { COORDINATE } \\
\text { (FT) }\end{array}$ & $\begin{array}{l}\text { EAST } \\
\text { COORDIHATE } \\
\text { (FT) }\end{array}$ & $\begin{array}{c}\text { FORMATION } \\
\text { OF } \\
\text { COMPLETION }\end{array}$ & $\begin{array}{l}\text { FLOW } \\
\text { CODE }\end{array}$ & $\begin{array}{l}\text { CASING } \\
\text { ELEVATION } \\
\text { (FT MSL) }\end{array}$ & $\begin{array}{l}\text { GROUND } \\
\text { ELEVATION } \\
\text { (FT MSL) }\end{array}$ & LOG DATE & $\begin{array}{l}\text { LOG } \\
\text { TIME }\end{array}$ & $\begin{array}{l}\text { DEPTH FROM } \\
\text { TOP OF } \\
\text { CASING (FT) }\end{array}$ & $\begin{array}{l}\text { DEPTH FROM } \\
\text { GROUND } \\
\text { (FT) }\end{array}$ & $\begin{array}{l}\text { GROUNDWATER } \\
\text { ELEVATION } \\
\text { (FT MSL) }\end{array}$ \\
\hline 0938 & 58231.6 & 62849.7 & HL & N & 5036.00 & 5033.70 & $\begin{array}{l}12 / 06 / 88 \\
07 / 29 / 94\end{array}$ & $\begin{array}{l}12: 10 \\
13: 42\end{array}$ & $\begin{array}{l}55.78 \\
54.73\end{array}$ & $\begin{array}{l}53.48 \\
52.43\end{array}$ & $\begin{array}{l}4980.22 \\
4981.27\end{array}$ \\
\hline 0939 & 56195.2 & 59518.6 & WU & N & 5106.70 & 5104.70 & $\begin{array}{l}08 / 05 / 88 \\
11 / 30 / 88 \\
07 / 29 / 94\end{array}$ & $\begin{array}{l}13: 35 \\
14: 50 \\
14: 49\end{array}$ & $\begin{array}{l}88.20 \\
88.00 \\
88.49\end{array}$ & $\begin{array}{l}86.20 \\
86.00 \\
86.49\end{array}$ & $\begin{array}{l}5018.50 \\
5018.70 \\
5018.21\end{array}$ \\
\hline 0940 & 58599.8 & 60040.7 & $W$ & N & 5016.70 & 5015.70 & $\begin{array}{l}08 / 06 / 88 \\
12 / 01 / 88\end{array}$ & $\begin{array}{l}11: 15 \\
13: 20\end{array}$ & $\begin{array}{l}16.11 \\
16.48\end{array}$ & $\begin{array}{l}15.11 \\
15.48\end{array}$ & $\begin{array}{l}5000.59 \\
5000.22\end{array}$ \\
\hline 0941 & 58222.4 & 62882.6 & WU & N & 5036.10 & 5033.90 & $\begin{array}{l}08 / 06 / 88 \\
12 / 07 / 88 \\
07 / 29 / 94\end{array}$ & $\begin{array}{l}14: 40 \\
13: 00 \\
13: 39\end{array}$ & $\begin{array}{l}47.72 \\
48.19 \\
48.39\end{array}$ & $\begin{array}{l}45.52 \\
45.99 \\
46.19\end{array}$ & $\begin{array}{l}4988.38 \\
4987.91 \\
4987.71\end{array}$ \\
\hline 0942 & 56061.2 & 61105.4 & WU & N & 5049.10 & 5047.70 & $\begin{array}{l}08 / 06 / 88 \\
12 / 01 / 88 \\
07 / 29 / 94\end{array}$ & $\begin{array}{l}13: 00 \\
11: 20 \\
15: 15\end{array}$ & $\begin{array}{l}62.10 \\
62.52 \\
61.96\end{array}$ & $\begin{array}{l}60.70 \\
61.12 \\
60.56\end{array}$ & $\begin{array}{l}4987.00 \\
4986.58 \\
4987.14\end{array}$ \\
\hline 0943 & 59282.9 & 64523.6 & WU & N & 5028.40 & 5027.20 & $\begin{array}{l}08 / 08 / 88 \\
12 / 06 / 88 \\
07 / 29 / 94\end{array}$ & $\begin{array}{l}12: 00 \\
10: 45 \\
16: 48\end{array}$ & $\begin{array}{l}49.84 \\
50.00 \\
50.30\end{array}$ & $\begin{array}{l}48.64 \\
48.80 \\
49.10\end{array}$ & $\begin{array}{l}4978.56 \\
4978.40 \\
4978.10\end{array}$ \\
\hline 0944 & 60946.7 & 66463.1 & WU & N & 4995.90 & 4993.90 & $\begin{array}{l}08 / 10 / 88 \\
12 / 05 / 88 \\
07 / 29 / 94\end{array}$ & $\begin{array}{l}10: 00 \\
14: 15 \\
17: 03\end{array}$ & $\begin{array}{l}56.01 \\
56.35 \\
55.03\end{array}$ & $\begin{array}{l}54.01 \\
54.35 \\
53.03\end{array}$ & $\begin{array}{l}4939.89 \\
4939.55 \\
4940.87\end{array}$ \\
\hline
\end{tabular}

FORMATION OF COMPLETION COOE:

WL - HASATCH - LOWER SANDSTONE

FLOW RELATIONSHIP COOE:

WU - WASATCH - UPPER SANDSTONE

N - UNKNOWN

DATA FILE: M: IDARTISPKO1 IGWL 10004.DAT 


\section{APPENDIX D}

\section{GROUND WATER QUALITY DATA BY PARAMETER}


GROUNDWATER QUALITY DATA BY PARAMETER

UPPER SANDSTONE UNIT

SITE: SPKOI SPOOK

02/17/87 TO $12 / 10 / 88$

REPORT DATE: $07 / 29 / 94$

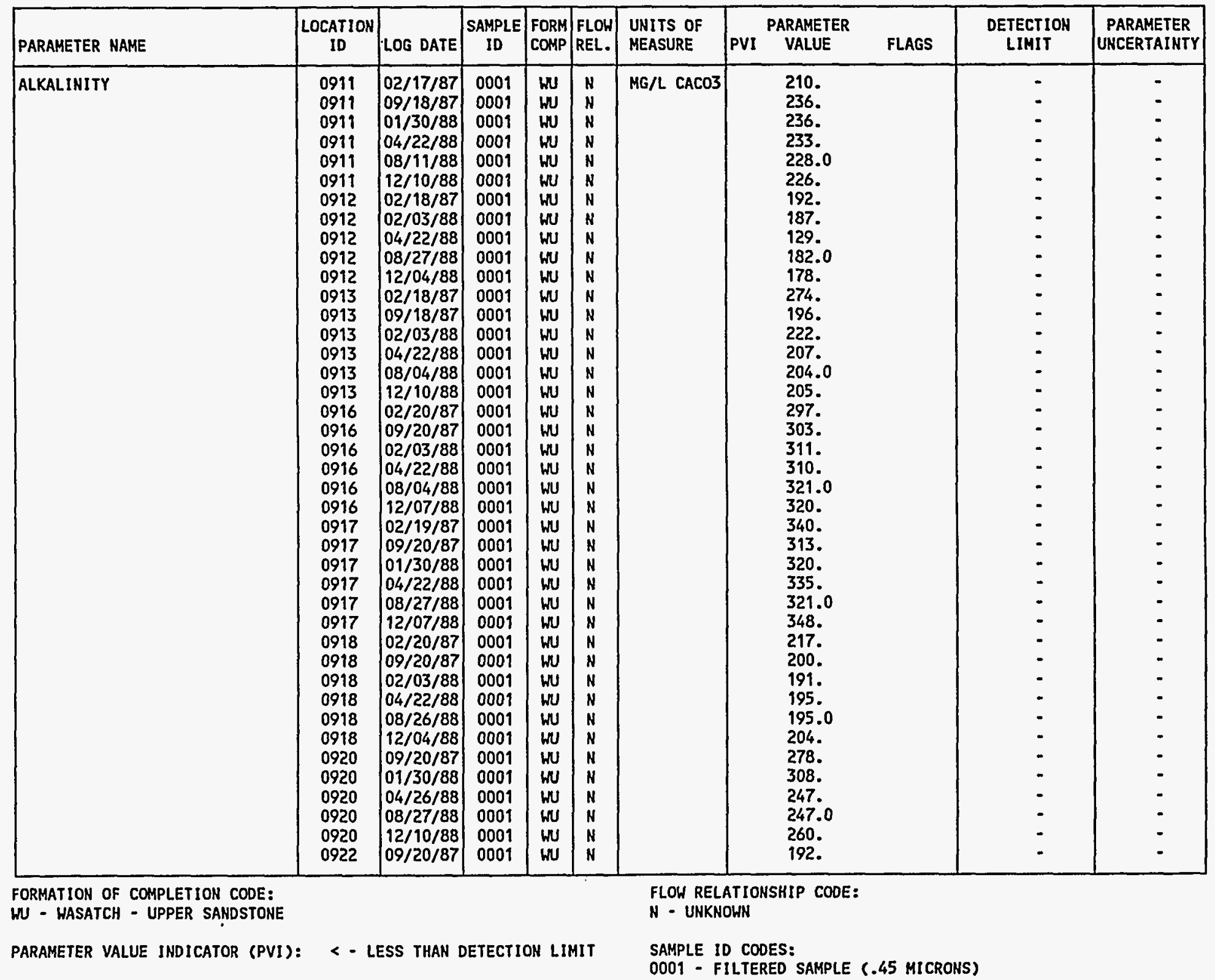


GROUNDWATER QUALITY DATA BY PARAMETER

UPPER SANDSTONE UNIT

SITE: SPK01 SP0OK

$02 / 17 / 87$ TO $12 / 10 / 88$

REPORT DATE: $07 / 29 / 94$

\begin{tabular}{|c|c|c|c|c|c|c|c|c|c|c|c|}
\hline PARAMETER NAME & $\begin{array}{c}\text { LOCATION } \\
\text { ID }\end{array}$ & LOG DATE & $\begin{array}{c}\text { SAMPLE } \\
\text { ID }\end{array}$ & $\begin{array}{l}\text { FORM } \\
\text { COMP }\end{array}$ & $\begin{array}{l}\text { FLOW } \\
\text { REL. }\end{array}$ & $\begin{array}{l}\text { UNITS OF } \\
\text { MEASURE }\end{array}$ & PVI & $\begin{array}{l}\text { PARAMETER } \\
\text { VALUE }\end{array}$ & FLAGS & $\begin{array}{l}\text { DETECTION } \\
\text { LIMIT }\end{array}$ & $\begin{array}{l}\text { PARAMETER } \\
\text { UNCERTAINTY }\end{array}$ \\
\hline ALKAL INITY & $\begin{array}{l}0922 \\
0922 \\
0922 \\
0922 \\
0924 \\
0924 \\
0924 \\
0924 \\
0924 \\
0925 \\
0925 \\
0925 \\
0925 \\
0925 \\
0927 \\
0927 \\
0927 \\
0927 \\
0927 \\
0928 \\
0928 \\
0928 \\
0928 \\
0928 \\
0929 \\
0929 \\
0929 \\
0929 \\
0930 \\
0930 \\
0930 \\
0930 \\
0931 \\
0931 \\
0931 \\
0931 \\
0932 \\
0932 \\
0932 \\
0932\end{array}$ & $\begin{array}{l}02 / 03 / 88 \\
04 / 26 / 88 \\
08 / 08 / 88 \\
12 / 10 / 88 \\
09 / 20 / 87 \\
02 / 04 / 88 \\
04 / 26 / 88 \\
08 / 26 / 88 \\
12 / 07 / 88 \\
09 / 20 / 87 \\
02 / 03 / 88 \\
04 / 26 / 88 \\
08 / 26 / 88 \\
12 / 07 / 88 \\
09 / 20 / 87 \\
02 / 04 / 88 \\
04 / 26 / 88 \\
08 / 26 / 88 \\
12 / 04 / 88 \\
09 / 20 / 87 \\
02 / 03 / 88 \\
04 / 22 / 88 \\
08 / 04 / 88 \\
12 / 04 / 88 \\
09 / 20 / 87 \\
02 / 03 / 88 \\
04 / 28 / 88 \\
08 / 11 / 88 \\
09 / 20 / 87 \\
01 / 30 / 88 \\
04 / 26 / 88 \\
08 / 27 / 88 \\
01 / 30 / 88 \\
04 / 28 / 88 \\
08 / 10 / 88 \\
12 / 04 / 88 \\
01 / 30 / 88 \\
04 / 28 / 88 \\
08 / 09 / 88 \\
12 / 07 / 88\end{array}$ & $\begin{array}{l}0001 \\
0001 \\
0001 \\
0001 \\
0001 \\
0001 \\
0001 \\
0001 \\
0001 \\
0001 \\
0001 \\
0001 \\
0001 \\
0001 \\
0001 \\
0001 \\
0001 \\
0001 \\
0001 \\
0001 \\
0001 \\
0001 \\
0001 \\
0001 \\
0001 \\
0001 \\
0001 \\
0001 \\
0001 \\
0001 \\
0001 \\
0001 \\
0001 \\
0001 \\
0001 \\
0001 \\
0001 \\
0001 \\
0001 \\
0001\end{array}$ & $\begin{array}{l}W U \\
W U \\
W U \\
W U \\
W U \\
W U \\
W U \\
W U \\
W U \\
W U \\
W U \\
W U \\
W U \\
W U \\
W U \\
W U \\
W U \\
W U \\
W U \\
W U \\
W U \\
W U \\
W U \\
W U \\
W U \\
W U \\
W U \\
W U \\
W U \\
W U \\
W U \\
W U \\
W U \\
W U \\
W U \\
W U \\
W U \\
W U \\
W U \\
W U\end{array}$ & $\begin{array}{l}N \\
N \\
N \\
N \\
N \\
N \\
N \\
N \\
N \\
N \\
N \\
N \\
N \\
N \\
N \\
N \\
N \\
N \\
N \\
N \\
N \\
N \\
N \\
N \\
N \\
N \\
N \\
N \\
N \\
N \\
N \\
N \\
N \\
N \\
N \\
N \\
N \\
N \\
N \\
N\end{array}$ & MG/L CACO3 & & $\begin{array}{l}185 . \\
162 . \\
160.0 \\
168 . \\
161 . \\
172 . \\
161 . \\
151.0 \\
166 . \\
221 . \\
233 . \\
224 . \\
212 . \\
228 . \\
138 . \\
180 . \\
166 . \\
161.0 \\
162 . \\
39 . \\
52 . \\
47 . \\
53.0 \\
51 . \\
77 . \\
228 . \\
400 . \\
242.0 \\
138 . \\
156 . \\
142 . \\
215.0 \\
255 . \\
228 . \\
223.0 \\
223 . \\
240 . \\
251 . \\
248.0 \\
241 .\end{array}$ & $\mathbf{J}$ & $\begin{array}{l}- \\
: \\
- \\
- \\
: \\
: \\
- \\
- \\
- \\
- \\
- \\
- \\
- \\
- \\
- \\
- \\
- \\
- \\
- \\
- \\
- \\
- \\
- \\
- \\
- \\
- \\
-\end{array}$ & $\begin{array}{l}- \\
- \\
- \\
- \\
- \\
- \\
- \\
- \\
- \\
- \\
- \\
- \\
- \\
- \\
- \\
- \\
- \\
- \\
- \\
- \\
- \\
- \\
- \\
- \\
- \\
- \\
- \\
- \\
- \\
- \\
- \\
-\end{array}$ \\
\hline
\end{tabular}

FORMATION OF COMPLETION CODE:

WU - WASATCH - UPPER SANDSTONE

FLOW RELATIONSHIP CODE:

PARAMETER VALUE INDICATOR (PVI): < - LESS THAN DETECTION LIMIT

SAMPLE ID CODES:

0001 - FILTERED SAMPLE (.45 MICRONS)

OTHER PARAMETER VALUE FLAGS:

$J$ - ESTIMATED VALUE 


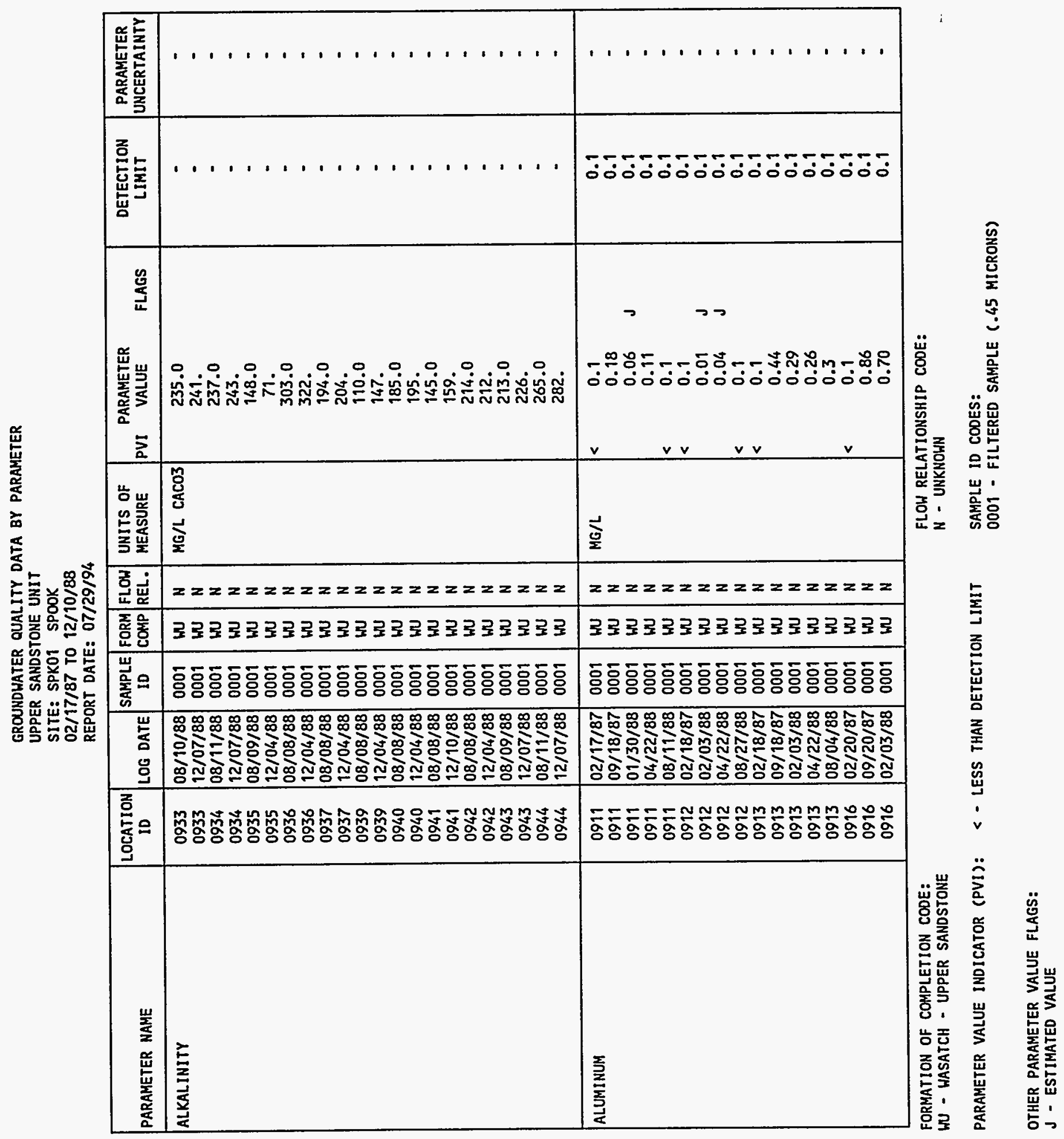




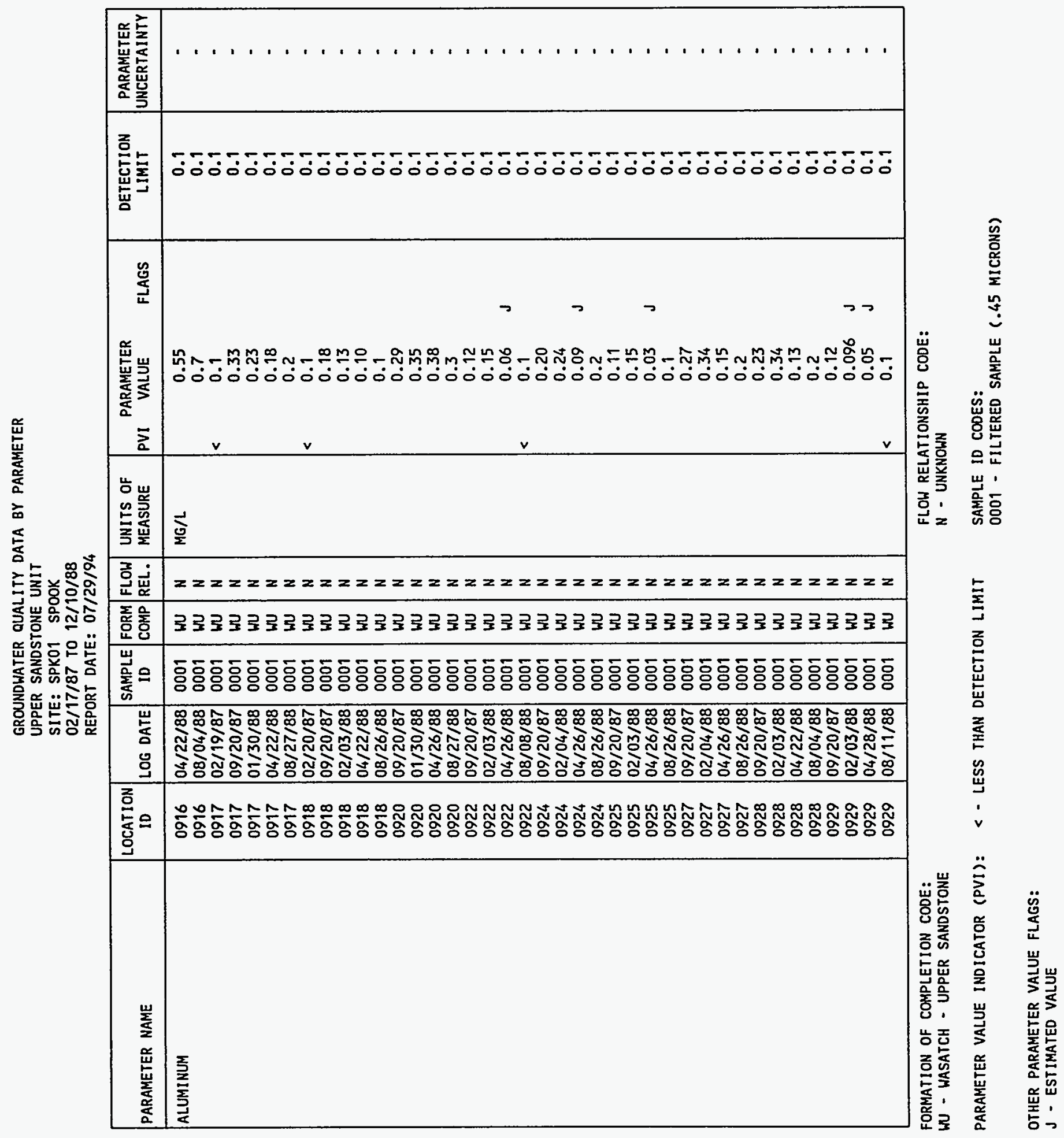




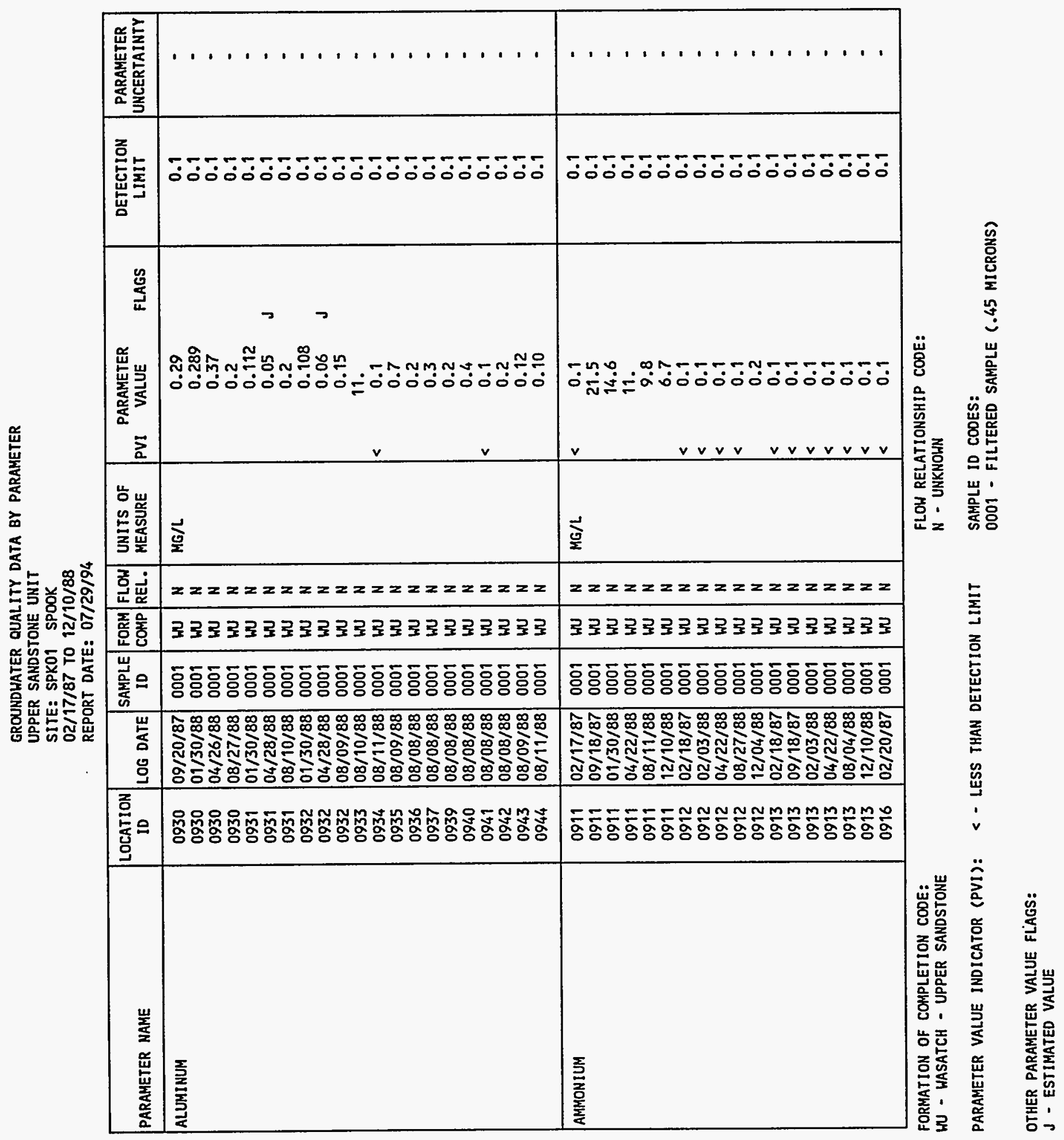




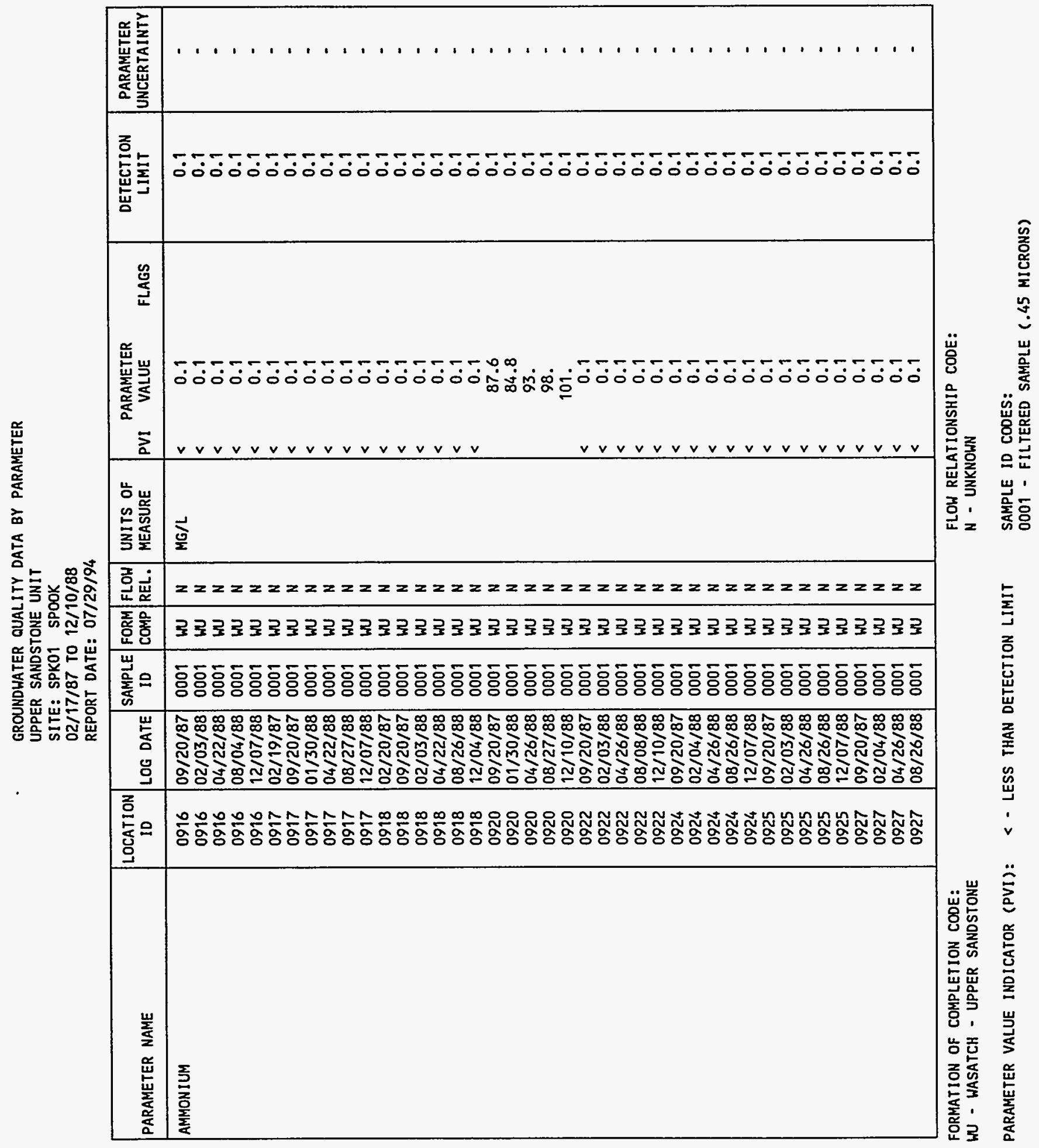




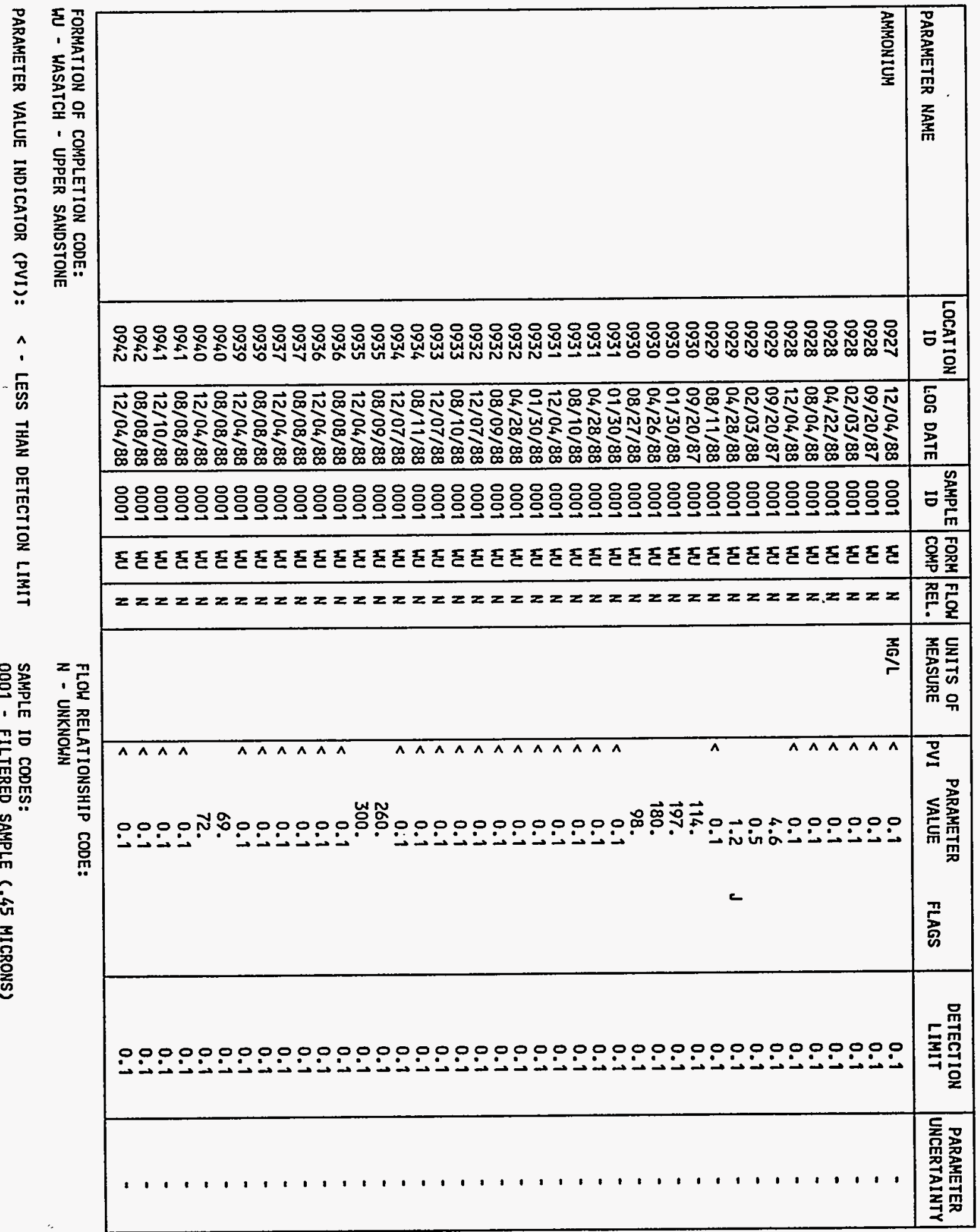




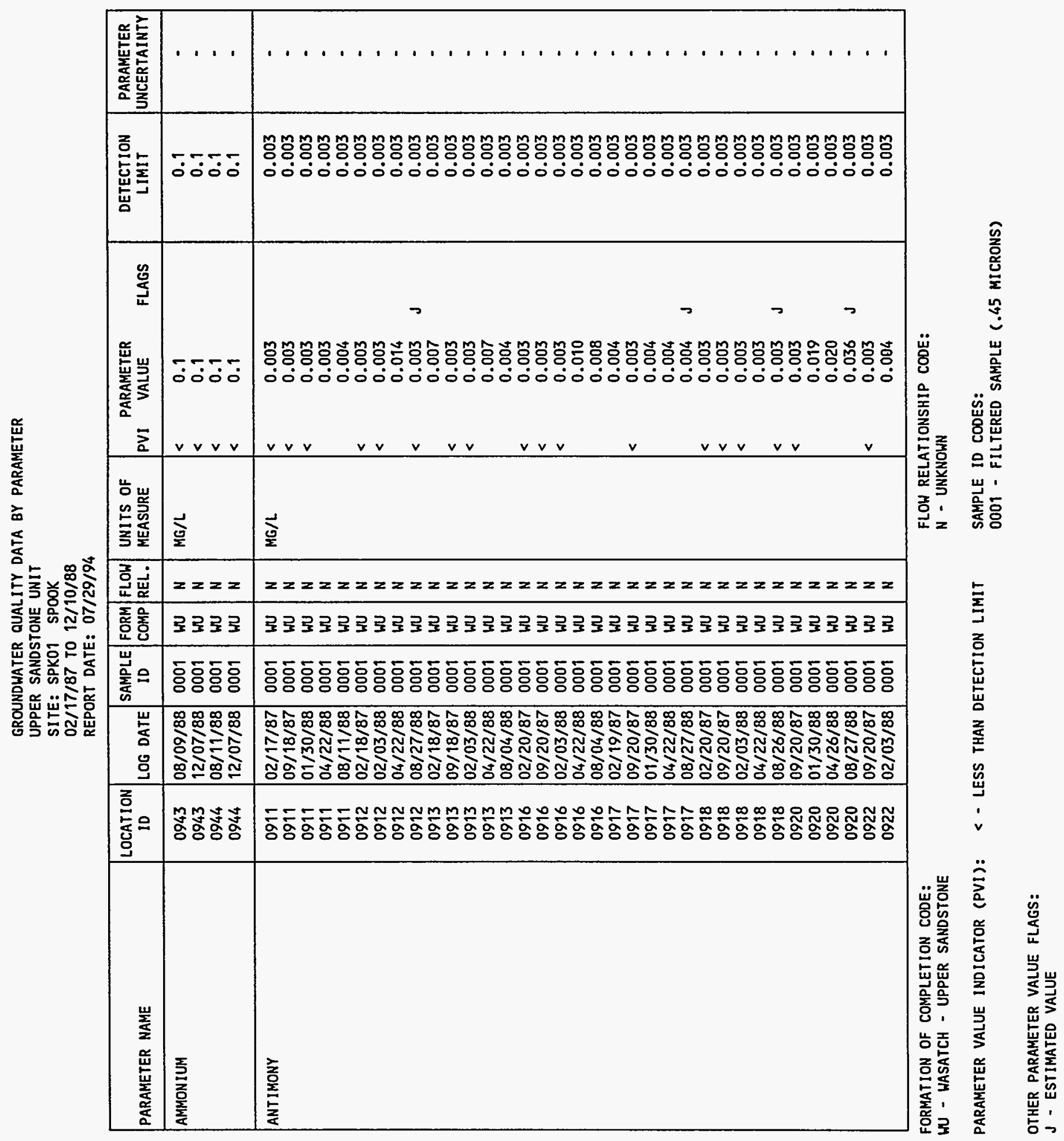


GROUNDHATER QUALITY DATA BY PARAMETER

UPPER SAMDSTOME UHIT

SITE: SPKOI SPOOK

02/17/87 TO $12 / 10 / 88$

REPORT DATE: $07 / 29 / 94$

\begin{tabular}{|c|c|c|c|c|c|c|c|c|c|c|c|}
\hline PARAMETER NAME & $\underset{\text { ID }}{\text { LOCATION }}$ & LOG DATE & $\begin{array}{c}\text { SAMPLE } \\
\text { ID }\end{array}$ & $\begin{array}{l}\text { FORM } \\
\text { COMP }\end{array}$ & $\begin{array}{l}\text { FLOW } \\
\text { REL. }\end{array}$ & $\begin{array}{l}\text { UNITS OF } \\
\text { MEASURE }\end{array}$ & PVI & $\begin{array}{l}\text { PARAMETER } \\
\text { VALUE }\end{array}$ & FLAGS & $\begin{array}{l}\text { DETECTION } \\
\text { LIMIT }\end{array}$ & $\begin{array}{l}\text { PARAMETER } \\
\text { UNCERTAINTY }\end{array}$ \\
\hline ANT IMONY & $\begin{array}{l}0922 \\
0922 \\
0924 \\
0924 \\
0924 \\
0924 \\
0925 \\
0925 \\
0925 \\
0925 \\
0927 \\
0927 \\
0927 \\
0927 \\
0928 \\
0928 \\
0928 \\
0928 \\
0929 \\
0929 \\
0929 \\
0929 \\
0930 \\
0930 \\
0930 \\
0930 \\
0931 \\
0931 \\
0931 \\
0932 \\
0932 \\
0932 \\
0933 \\
0934 \\
0935 \\
0936 \\
0937 \\
0939 \\
0940 \\
0941\end{array}$ & $\begin{array}{l}04 / 26 / 88 \\
08 / 08 / 88 \\
09 / 20 / 87 \\
02 / 04 / 88 \\
04 / 26 / 88 \\
08 / 26 / 88 \\
09 / 20 / 87 \\
02 / 03 / 88 \\
04 / 26 / 88 \\
08 / 26 / 88 \\
09 / 20 / 87 \\
02 / 04 / 88 \\
04 / 26 / 88 \\
08 / 26 / 88 \\
09 / 20 / 87 \\
02 / 03 / 88 \\
04 / 22 / 88 \\
08 / 04 / 88 \\
09 / 20 / 87 \\
02 / 03 / 88 \\
04 / 28 / 88 \\
08 / 11 / 88 \\
09 / 20 / 87 \\
01 / 30 / 88 \\
04 / 26 / 88 \\
08 / 27 / 88 \\
01 / 30 / 88 \\
04 / 28 / 88 \\
08 / 10 / 88 \\
01 / 30 / 88 \\
04 / 28 / 88 \\
08 / 09 / 88 \\
08 / 10 / 88 \\
08 / 11 / 88 \\
08 / 09 / 88 \\
08 / 08 / 88 \\
08 / 08 / 88 \\
08 / 08 / 88 \\
08 / 08 / 88 \\
08 / 08 / 88\end{array}$ & $\begin{array}{l}0001 \\
0001 \\
0001 \\
0001 \\
0001 \\
0001 \\
0001 \\
0001 \\
0001 \\
0001 \\
0001 \\
0001 \\
0001 \\
0001 \\
0001 \\
0001 \\
0001 \\
0001 \\
0001 \\
0001 \\
0001 \\
0001 \\
0001 \\
0001 \\
0001 \\
0001 \\
0001 \\
0001 \\
0001 \\
0001 \\
0001 \\
0001 \\
0001 \\
0001 \\
0001 \\
0001 \\
0001 \\
0001 \\
0001 \\
0001\end{array}$ & $\begin{array}{l}W U \\
W U \\
W U \\
W U \\
W U \\
W U \\
W U \\
W U \\
W U \\
W U \\
W U \\
W U \\
W U \\
W U \\
W U \\
W U \\
W U \\
W U \\
W U \\
W U \\
W U \\
W U \\
W U \\
W U \\
W U \\
W U \\
W U \\
W U \\
W U \\
W U \\
W U \\
W U \\
W U \\
W U \\
W U \\
W U \\
W U \\
W U \\
W U \\
W U\end{array}$ & $\begin{array}{l}N \\
N \\
N \\
N \\
N \\
N \\
N \\
N \\
N \\
N \\
N \\
N \\
N \\
N \\
N \\
N \\
N \\
N \\
N \\
N \\
N \\
N \\
N \\
N \\
N \\
N \\
N \\
N \\
N \\
N \\
N \\
N \\
N \\
N \\
N \\
N \\
N \\
N \\
N \\
N\end{array}$ & $M G / L$ & $\mid \begin{array}{l}< \\
< \\
< \\
<\end{array}$ & $\begin{array}{l}0.003 \\
0.003 \\
0.003 \\
0.008 \\
0.007 \\
0.012 \\
0.003 \\
0.003 \\
0.003 \\
0.005 \\
0.005 \\
0.012 \\
0.010 \\
0.013 \\
0.003 \\
0.012 \\
0.010 \\
0.007 \\
0.003 \\
0.004 \\
0.004 \\
0.008 \\
0.015 \\
0.019 \\
0.028 \\
0.024 \\
0.004 \\
0.003 \\
0.003 \\
0.006 \\
0.005 \\
0.003 \\
0.003 \\
0.003 \\
0.016 \\
0.003 \\
0.003 \\
0.009 \\
0.011 \\
0.003\end{array}$ & $\begin{array}{l}\mathbf{J} \\
\mathbf{J}\end{array}$ & $\begin{array}{l}0.003 \\
0.003 \\
0.003 \\
0.003 \\
0.003 \\
0.003 \\
0.003 \\
0.003 \\
0.003 \\
0.003 \\
0.003 \\
0.003 \\
0.003 \\
0.003 \\
0.003 \\
0.003 \\
0.003 \\
0.003 \\
0.003 \\
0.003 \\
0.003 \\
0.003 \\
0.003 \\
0.003 \\
0.003 \\
0.003 \\
0.003 \\
0.003 \\
0.003 \\
0.003 \\
0.003 \\
0.003 \\
0.003 \\
0.003 \\
0.003 \\
0.003 \\
0.003 \\
0.003 \\
0.003 \\
0.003\end{array}$ & $\begin{array}{l}- \\
- \\
- \\
- \\
- \\
- \\
- \\
- \\
- \\
- \\
- \\
- \\
- \\
- \\
- \\
- \\
- \\
- \\
- \\
- \\
- \\
- \\
- \\
- \\
- \\
- \\
- \\
- \\
- \\
-\end{array}$ \\
\hline
\end{tabular}

FORMATION OF COMPLETION CODE:

FLOH RELATIONSHIP COOE:

WU - WASATCH - UPPER SANDSTONE

N - UNKNOWN

PARAMETER VALUE INDICATOR (PVI): \& - LESS THAN DETECTION LIMIT

SAMPLE ID CODES:

0001 - FILTERED SAMPLE (.45 MICRONS)

OTHER PARAMETER VALUE FLAGS:

J - ESTIMATED VALUE 


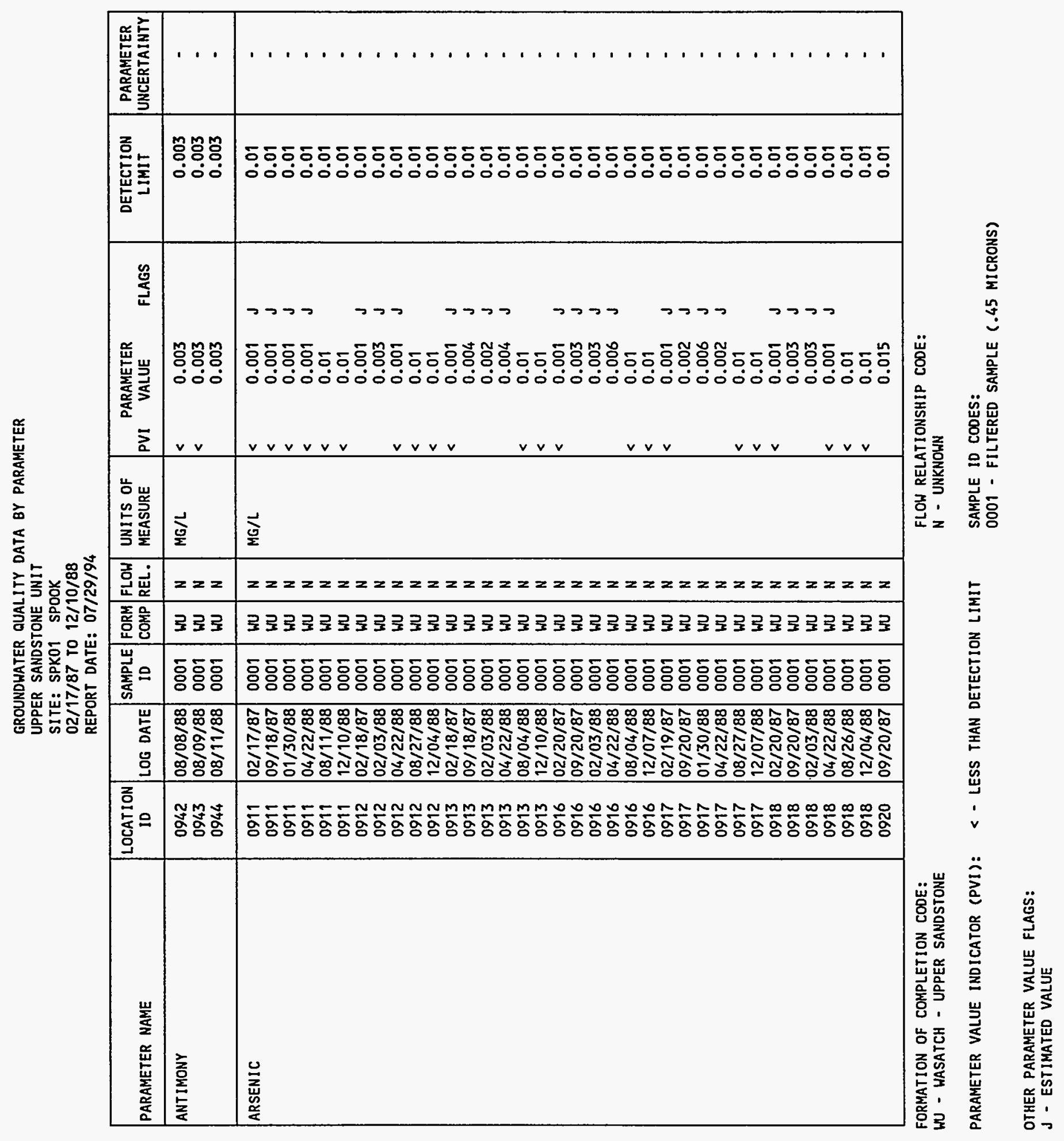


UPPER SANDSTONE UNIT

SITE: SPK01 SPOOK

02/17/87 TO $12 / 10 / 88$

\begin{tabular}{|c|c|c|c|c|c|c|c|c|c|c|c|}
\hline PARAMETER NAME & $\underset{\text { ID }}{\text { LOCATION }}$ & LOG DATE & $\begin{array}{c}\text { SAMPLE } \\
\text { ID }\end{array}$ & $\begin{array}{l}\text { FORM } \\
\text { COMP }\end{array}$ & $\begin{array}{l}\text { FLOW } \\
\text { REL. }\end{array}$ & $\begin{array}{l}\text { UNITS OF } \\
\text { MEASURE }\end{array}$ & PVI & $\begin{array}{l}\text { PARAMETER } \\
\text { VALUE }\end{array}$ & FLAGS & $\begin{array}{l}\text { DETECTION } \\
\text { LIMIT }\end{array}$ & $\begin{array}{l}\text { PARAMETER } \\
\text { UNCERTAINTY }\end{array}$ \\
\hline ARSENIC & $\begin{array}{l}0920 \\
0920 \\
0920 \\
0920 \\
0922 \\
0922 \\
0922 \\
0922 \\
0922 \\
0924 \\
0924 \\
0924 \\
0924 \\
0924 \\
0925 \\
0925 \\
0925 \\
0925 \\
0925 \\
0927 \\
0927 \\
0927 \\
0927 \\
0927 \\
0928 \\
0928 \\
0928 \\
0928 \\
0928 \\
0929 \\
0929 \\
0929 \\
0929 \\
0930 \\
0930 \\
0930 \\
0930 \\
0931 \\
0931 \\
0931\end{array}$ & $\begin{array}{l}01 / 30 / 88 \\
04 / 26 / 88 \\
08 / 27 / 88 \\
12 / 10 / 88 \\
09 / 20 / 87 \\
02 / 03 / 88 \\
04 / 26 / 88 \\
08 / 08 / 88 \\
12 / 10 / 88 \\
09 / 20 / 87 \\
02 / 04 / 88 \\
04 / 26 / 88 \\
08 / 26 / 88 \\
12 / 07 / 88 \\
09 / 20 / 87 \\
02 / 03 / 88 \\
04 / 26 / 88 \\
08 / 26 / 88 \\
12 / 07 / 88 \\
09 / 20 / 87 \\
02 / 04 / 88 \\
04 / 26 / 88 \\
08 / 26 / 88 \\
12 / 04 / 88 \\
09 / 20 / 87 \\
02 / 03 / 88 \\
04 / 22 / 88 \\
08 / 04 / 88 \\
12 / 04 / 88 \\
09 / 20 / 87 \\
02 / 03 / 88 \\
04 / 28 / 88 \\
08 / 11 / 88 \\
09 / 20 / 87 \\
01 / 30 / 88 \\
04 / 26 / 88 \\
08 / 27 / 88 \\
01 / 30 / 88 \\
04 / 28 / 88 \\
08 / 10 / 88\end{array}$ & $\begin{array}{l}0001 \\
0001 \\
0001 \\
0001 \\
0001 \\
0001 \\
0001 \\
0001 \\
0001 \\
0001 \\
0001 \\
0001 \\
0001 \\
0001 \\
0001 \\
0001 \\
0001 \\
0001 \\
0001 \\
0001 \\
0001 \\
0001 \\
0001 \\
0001 \\
0001 \\
0001 \\
0001 \\
0001 \\
0001 \\
0001 \\
0001 \\
0001 \\
0001 \\
0001 \\
0001 \\
0001 \\
0001 \\
0001 \\
0001 \\
0001\end{array}$ & $\begin{array}{l}W U \\
W U \\
W U \\
W U \\
W U \\
W U \\
W U \\
W U \\
W U \\
W U \\
W U \\
W U \\
W U \\
W U \\
W U \\
W U \\
W U \\
W U \\
W U \\
W U \\
W U \\
W U \\
W U \\
W U \\
W U \\
W U \\
W U \\
W U \\
W U \\
W U \\
W U \\
W U \\
W U \\
W U \\
W U \\
W U \\
W U \\
W U \\
W U \\
W U\end{array}$ & $\begin{array}{l}N \\
N \\
N \\
N \\
N \\
N \\
N \\
N \\
N \\
N \\
N \\
N \\
N \\
N \\
N \\
N \\
N \\
N \\
N \\
N \\
N \\
N \\
N \\
N \\
N \\
N \\
N \\
N \\
N \\
N \\
N \\
N \\
N \\
N \\
N \\
N \\
N \\
N \\
N\end{array}$ & $M G / L$ & $\begin{array}{l}< \\
< \\
< \\
< \\
< \\
< \\
< \\
< \\
< \\
< \\
< \\
< \\
< \\
< \\
< \\
< \\
< \\
< \\
< \\
< \\
< \\
< \\
< \\
< \\
<\end{array}$ & $\begin{array}{l}0.012 \\
0.004 \\
0.05 \\
0.01 \\
0.002 \\
0.01 \\
0.001 \\
0.01 \\
0.01 \\
0.004 \\
0.01 \\
0.001 \\
0.02 \\
0.01 \\
0.001 \\
0.01 \\
0.001 \\
0.01 \\
0.01 \\
0.010 \\
0.01 \\
0.001 \\
0.01 \\
0.01 \\
0.009 \\
0.01 \\
0.001 \\
0.01 \\
0.01 \\
0.006 \\
0.003 \\
0.001 \\
0.01 \\
0.012 \\
0.013 \\
0.015 \\
0.03 \\
0.001 \\
0.001 \\
0.01\end{array}$ & $\begin{array}{l}J \\
J \\
J \\
J \\
J \\
J \\
J\end{array}$ & $\begin{array}{l}0.01 \\
0.01 \\
0.01 \\
0.01 \\
0.01 \\
0.01 \\
0.01 \\
0.01 \\
0.01 \\
0.01 \\
0.01 \\
0.01 \\
0.01 \\
0.01 \\
0.01 \\
0.01 \\
0.01 \\
0.01 \\
0.01 \\
0.01 \\
0.01 \\
0.01 \\
0.01 \\
0.01 \\
0.01 \\
0.01 \\
0.01 \\
0.01 \\
0.01 \\
0.01 \\
0.01 \\
0.01 \\
0.01 \\
0.01 \\
0.01 \\
0.01 \\
0.01 \\
0.01 \\
0.01 \\
0.01\end{array}$ & $\begin{array}{l}- \\
- \\
- \\
- \\
- \\
- \\
- \\
- \\
- \\
- \\
- \\
- \\
- \\
- \\
- \\
- \\
- \\
- \\
- \\
- \\
- \\
- \\
- \\
- \\
- \\
- \\
- \\
- \\
- \\
- \\
-\end{array}$ \\
\hline
\end{tabular}

FORMATION OF COMPLETION CODE:

FLOW RELATIONSHIP CODE

WU - WASATCH - UPPER SANDSTONE

N - UNKNOWN

PARAMETER VALUE INDICATOR (PVI): < - LESS THAN DETECTION LIMIT 


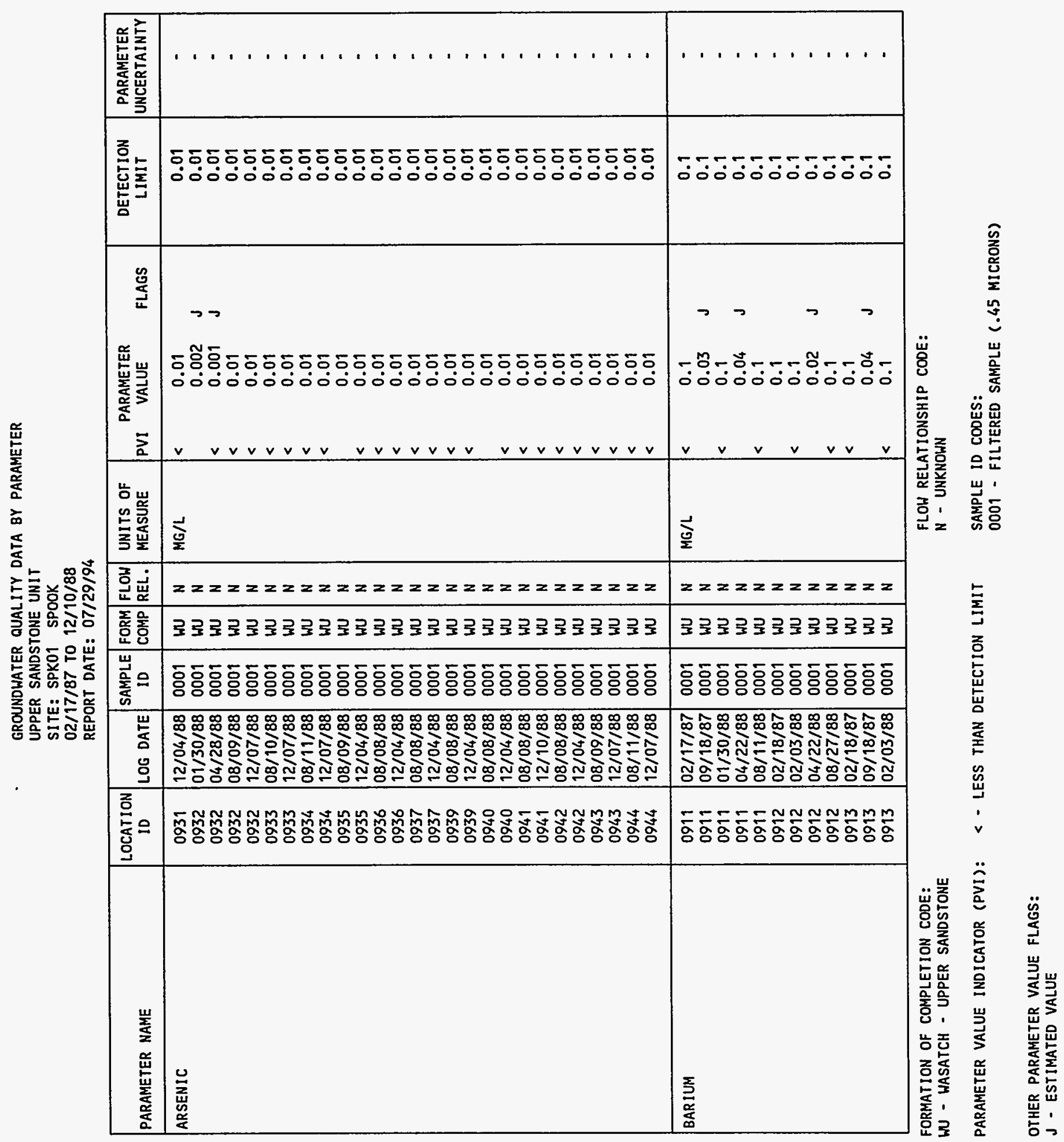




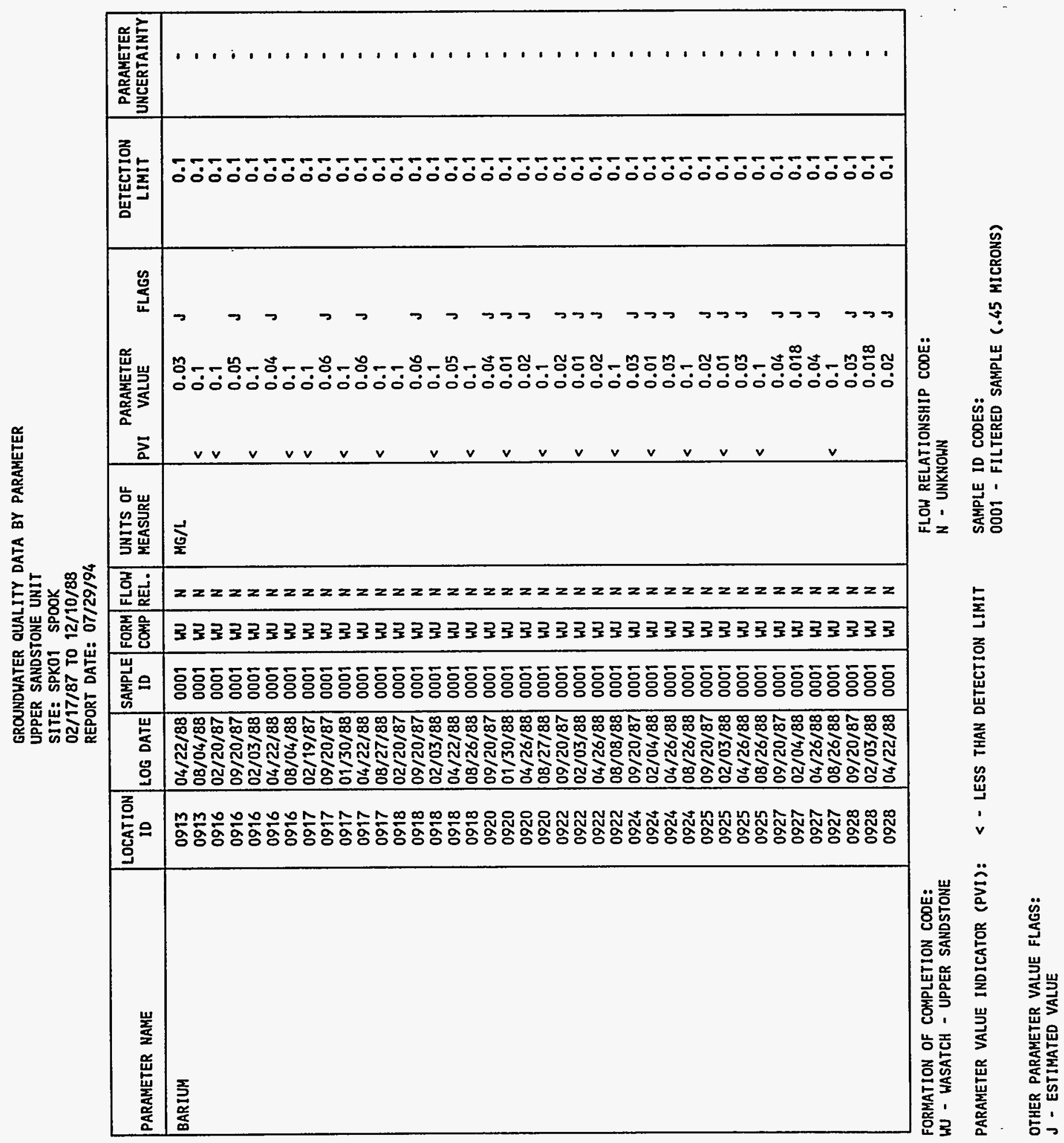




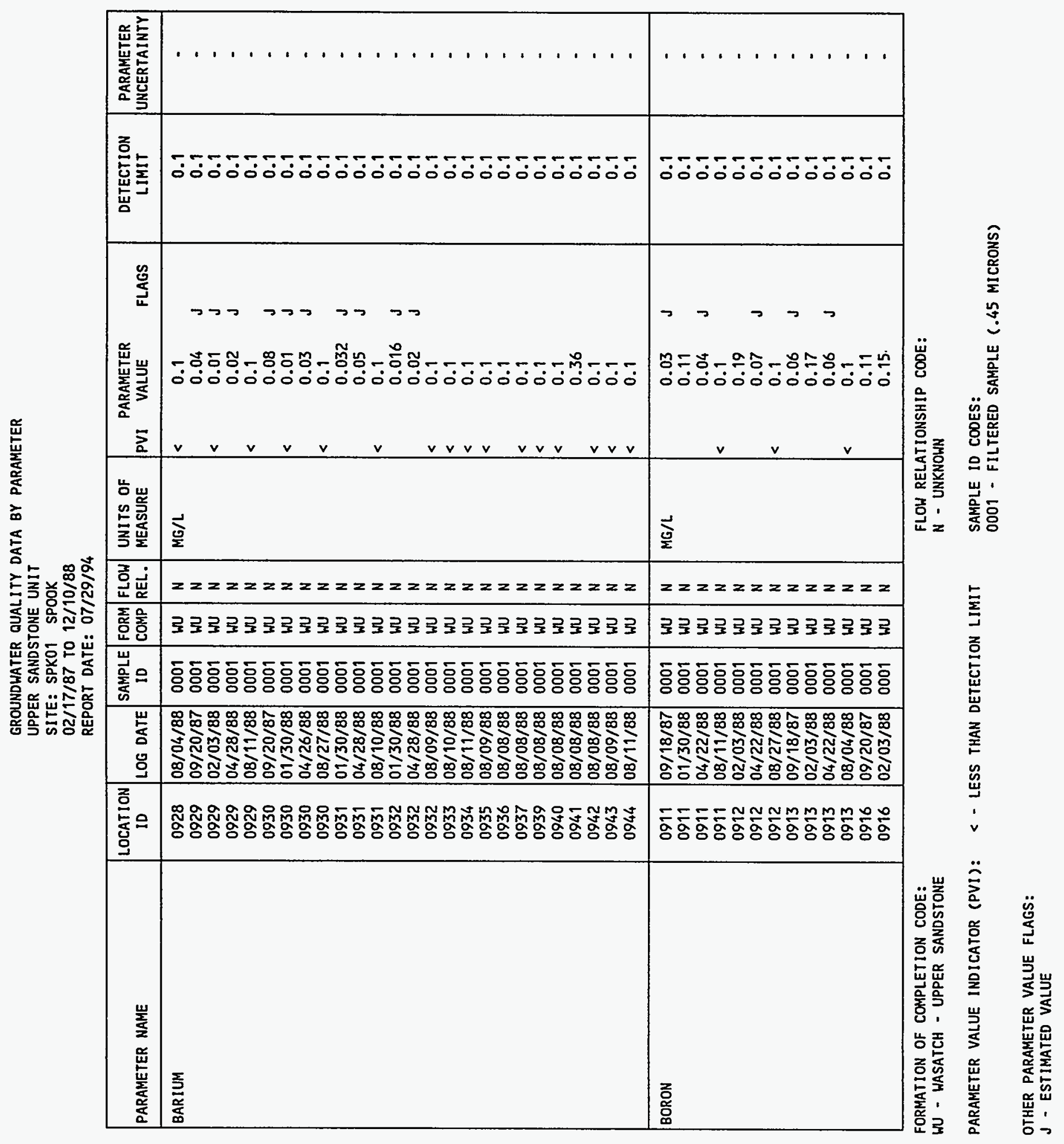




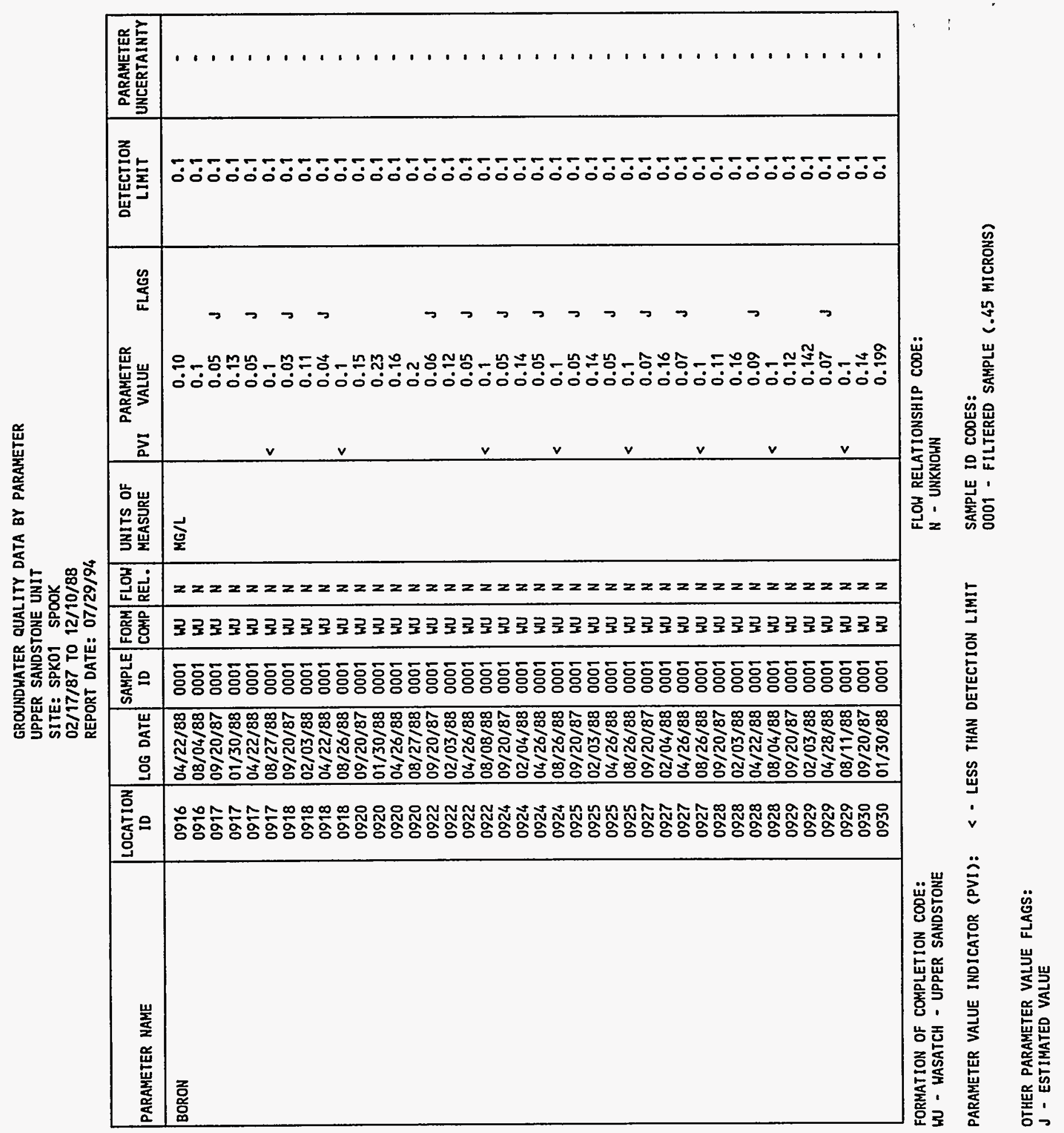




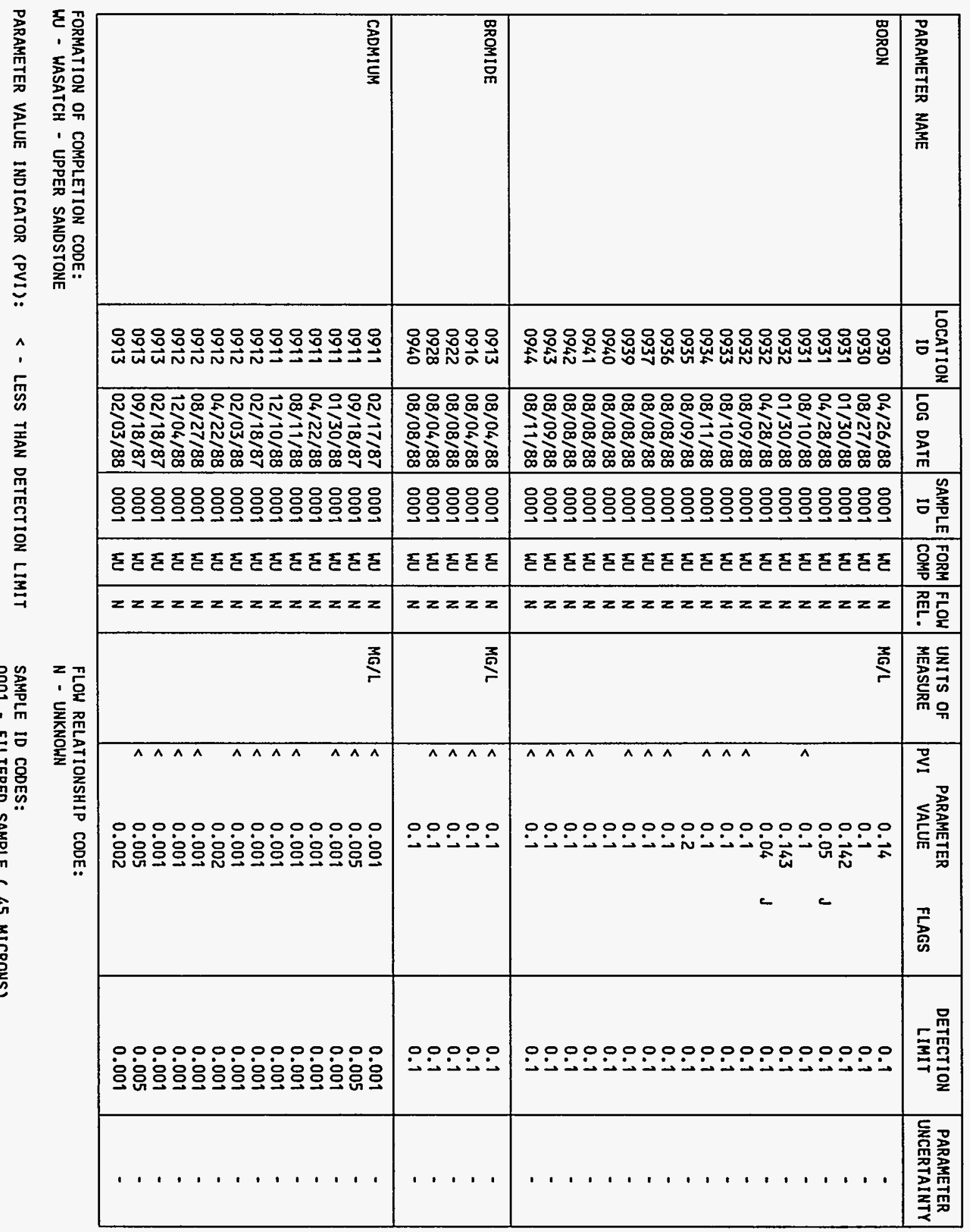

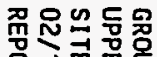




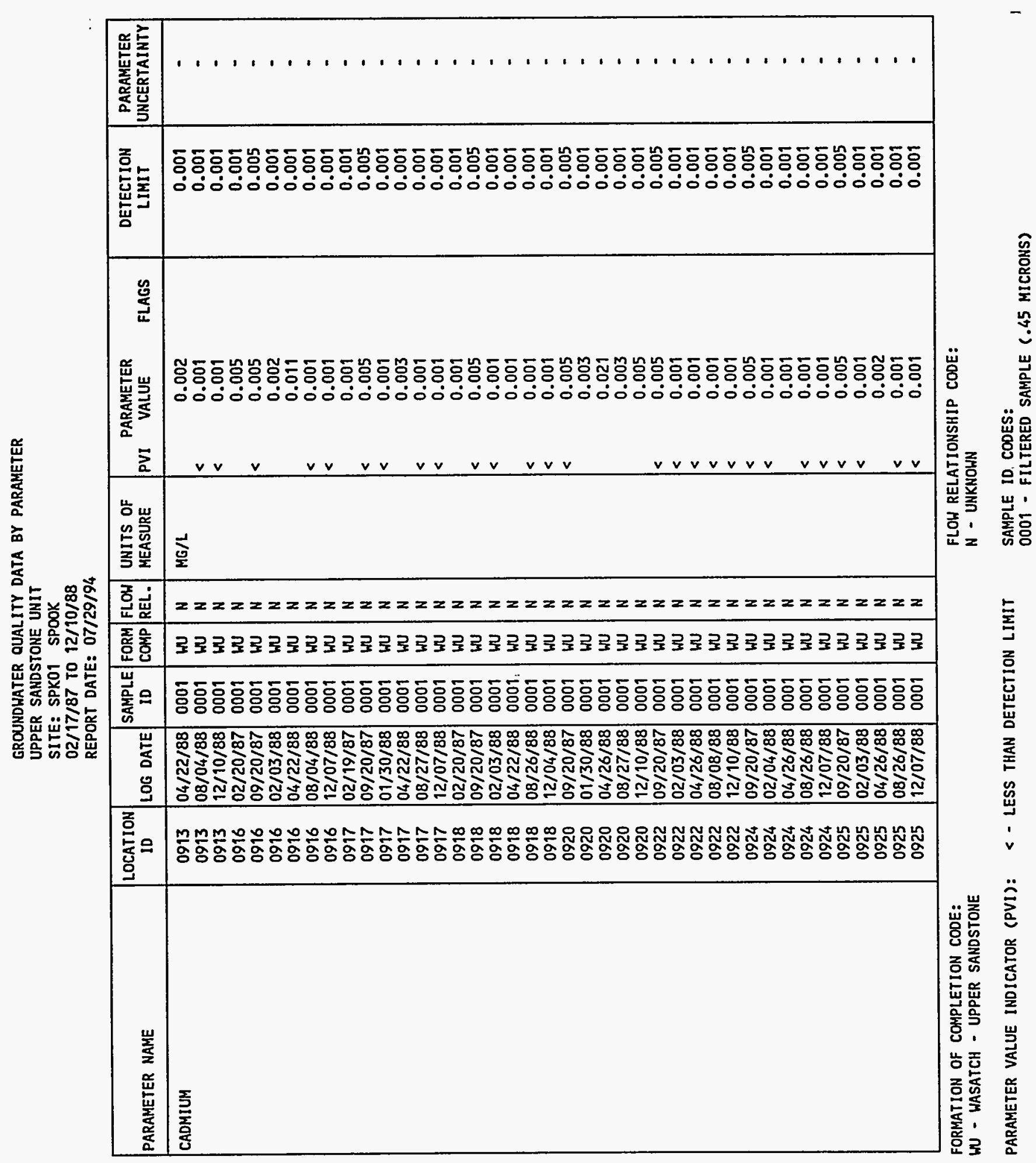




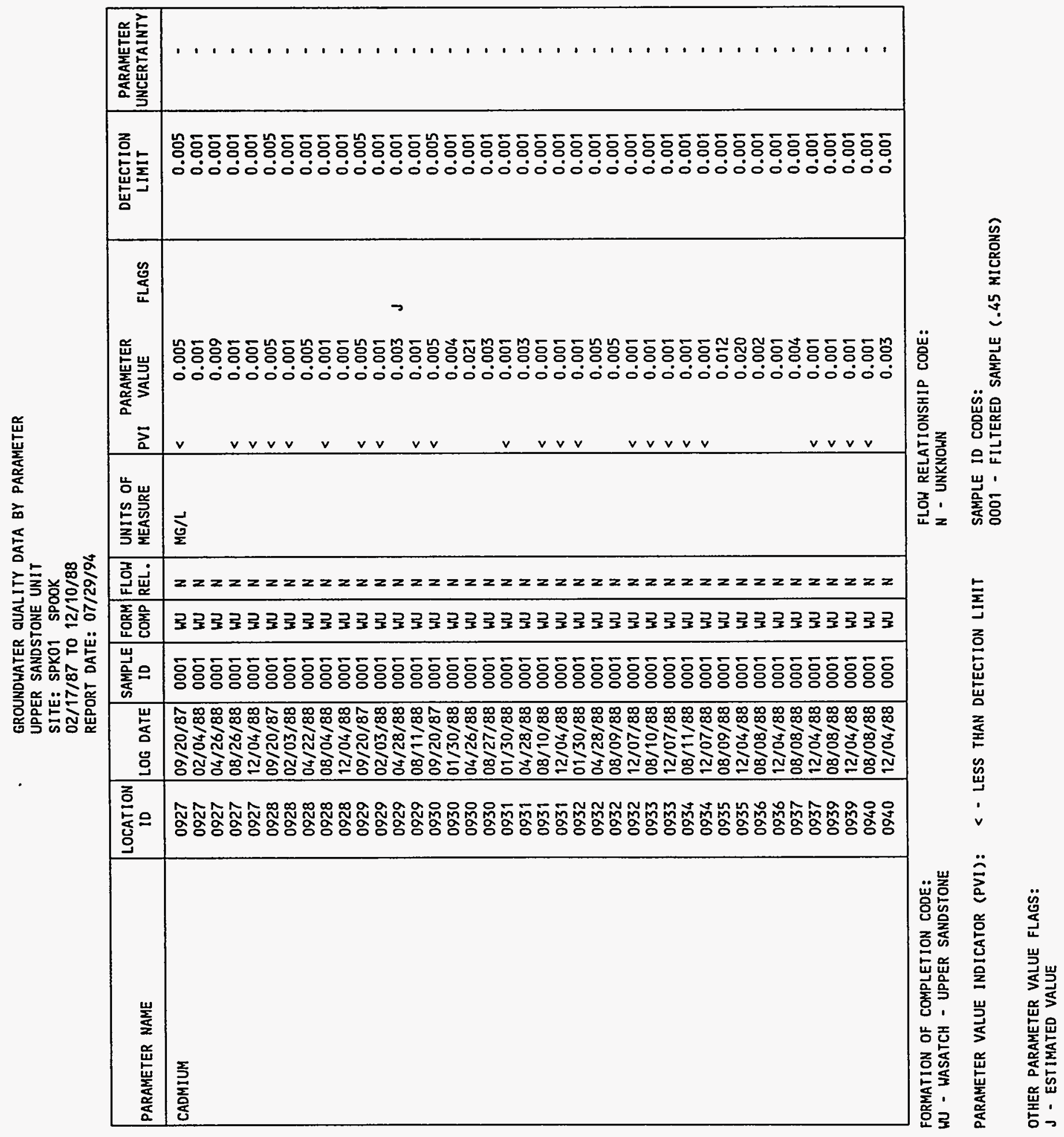




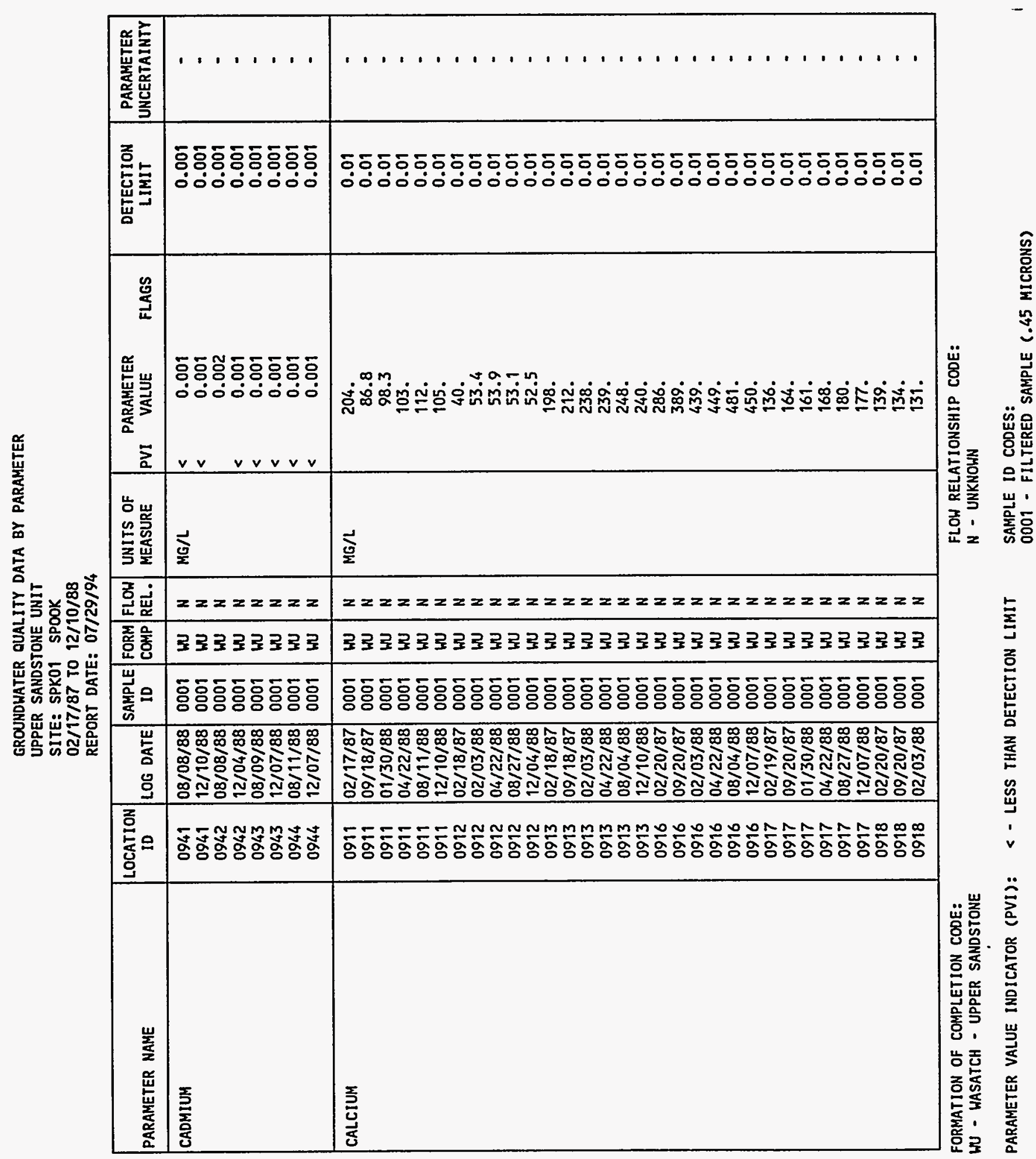


REPORT DATE: $07 / 29 / 9$

\begin{tabular}{|c|c|c|c|c|c|c|c|c|c|c|c|}
\hline PARAMETER NAME & $\underset{\text { ID }}{\text { LOCATION }}$ & LOG DATE & $\begin{array}{c}\text { SAMPLE } \\
\text { ID }\end{array}$ & $\begin{array}{l}\text { FORM } \\
\text { COMP }\end{array}$ & $\begin{array}{l}\text { FLOW } \\
\text { REL. }\end{array}$ & $\begin{array}{l}\text { UNITS OF } \\
\text { MEASURE }\end{array}$ & PVI & $\begin{array}{l}\text { PARAMETER } \\
\text { VALUE }\end{array}$ & FLAGS & $\begin{array}{l}\text { DETECTION } \\
\text { LIMIT }\end{array}$ & $\begin{array}{l}\text { PARAMETER } \\
\text { UNCERTAINTY }\end{array}$ \\
\hline CALCIUM & $\begin{array}{l}0918 \\
0918 \\
0918 \\
0920 \\
0920 \\
0920 \\
0920 \\
0920 \\
0922 \\
0922 \\
0922 \\
0922 \\
0922 \\
0924 \\
0924 \\
0924 \\
0924 \\
0924 \\
0925 \\
0925 \\
0925 \\
0925 \\
0925 \\
0927 \\
0927 \\
0927 \\
0927 \\
0927 \\
0928 \\
0928 \\
0928 \\
0928 \\
0928 \\
0929 \\
0929 \\
0929 \\
0929 \\
0930 \\
0930 \\
0930\end{array}$ & $\begin{array}{l}04 / 22 / 88 \\
08 / 26 / 88 \\
12 / 04 / 88 \\
09 / 20 / 87 \\
01 / 30 / 88 \\
04 / 26 / 88 \\
08 / 27 / 88 \\
12 / 10 / 88 \\
09 / 20 / 87 \\
02 / 03 / 88 \\
04 / 26 / 88 \\
08 / 08 / 88 \\
12 / 10 / 88 \\
09 / 20 / 87 \\
02 / 04 / 88 \\
04 / 26 / 88 \\
08 / 26 / 88 \\
12 / 07 / 88 \\
09 / 20 / 87 \\
02 / 03 / 88 \\
04 / 26 / 88 \\
08 / 26 / 88 \\
12 / 07 / 88 \\
09 / 20 / 87 \\
02 / 04 / 88 \\
04 / 26 / 88 \\
08 / 26 / 88 \\
12 / 04 / 88 \\
09 / 20 / 87 \\
02 / 03 / 88 \\
04 / 22 / 88 \\
08 / 04 / 88 \\
12 / 04 / 88 \\
09 / 20 / 87 \\
02 / 03 / 88 \\
04 / 28 / 88 \\
08 / 11 / 88 \\
09 / 20 / 87 \\
01 / 30 / 88 \\
04 / 26 / 88\end{array}$ & $\begin{array}{l}0001 \\
0001 \\
0001 \\
0001 \\
0001 \\
0001 \\
0001 \\
0001 \\
0001 \\
0001 \\
0001 \\
0001 \\
0001 \\
0001 \\
0001 \\
0001 \\
0001 \\
0001 \\
0001 \\
0001 \\
0001 \\
0001 \\
0001 \\
0001 \\
0001 \\
0001 \\
0001 \\
0001 \\
0001 \\
0001 \\
0001 \\
0001 \\
0001 \\
0001 \\
0001 \\
0001 \\
0001 \\
0001 \\
0001 \\
0001\end{array}$ & $\begin{array}{l}W U \\
W U \\
W U \\
W U \\
W U \\
W U \\
W U \\
W U \\
W U \\
W U \\
W U \\
W U \\
W U \\
W U \\
W U \\
W U \\
W U \\
W U \\
W U \\
W U \\
W U \\
W U \\
W U \\
W U \\
W U \\
W U \\
W U \\
W U \\
W U \\
W U \\
W U \\
W U \\
W U \\
W U \\
W U \\
W U \\
W U \\
W U \\
W U \\
W U \\
W U \\
W U \\
W U \\
W U \\
W U \\
W U \\
W U\end{array}$ & $\begin{array}{l}N \\
N \\
N \\
N \\
N \\
N \\
N \\
N \\
N \\
N \\
N \\
N \\
N \\
N \\
N \\
N \\
N \\
N \\
N \\
N \\
N \\
N \\
N \\
N \\
N \\
N \\
N \\
N \\
N \\
N \\
N \\
N \\
N \\
N \\
N \\
N \\
N \\
N \\
N \\
N\end{array}$ & MG/L & & $\begin{array}{l}139 . \\
144 . \\
138 . \\
564 . \\
491 . \\
534 . \\
535 . \\
503 . \\
152 . \\
111 . \\
119 . \\
120 . \\
118 . \\
246 . \\
234 . \\
244 . \\
249 . \\
245 . \\
105 . \\
104 . \\
106 . \\
107 . \\
107 . \\
448 . \\
424 . \\
459 . \\
466 . \\
443 . \\
351 . \\
311 . \\
341 . \\
340 . \\
320 . \\
184 . \\
71.7 \\
82.7 \\
86.7 \\
565 . \\
458 . \\
498 .\end{array}$ & $\mathrm{J}$ & $\begin{array}{l}0.01 \\
0.01 \\
0.01 \\
0.01 \\
0.01 \\
0.01 \\
0.01 \\
0.01 \\
0.01 \\
0.01 \\
0.01 \\
0.01 \\
0.01 \\
0.01 \\
0.01 \\
0.01 \\
0.01 \\
0.01 \\
0.01 \\
0.01 \\
0.01 \\
0.01 \\
0.01 \\
0.01 \\
0.01 \\
0.01 \\
0.01 \\
0.01 \\
0.01 \\
0.01 \\
0.01 \\
0.01 \\
0.01 \\
0.01 \\
0.01 \\
0.01 \\
0.01 \\
0.01 \\
0.01 \\
0.01\end{array}$ & $\begin{array}{l}- \\
- \\
- \\
- \\
- \\
- \\
- \\
- \\
- \\
- \\
- \\
- \\
- \\
- \\
- \\
- \\
- \\
- \\
- \\
- \\
- \\
- \\
- \\
- \\
- \\
- \\
- \\
- \\
- \\
- \\
- \\
-\end{array}$ \\
\hline
\end{tabular}

FORMATION OF COMPLETION CODE: WU - HASATCH - UPPER SANDSTONE

PARAMETER VALUE INDICATOR (PVI): < - LESS THAN DETECTION LIMIT
FLOW RELATIONSHIP CODE

N - UNKNOWN

SAMPLE ID CODES

0001 - FILTERED SAMPLE (.45 MICRONS)

OTHER PARAMETER VALUE FLAGS

$J$ - ESTIMATED VALUE 


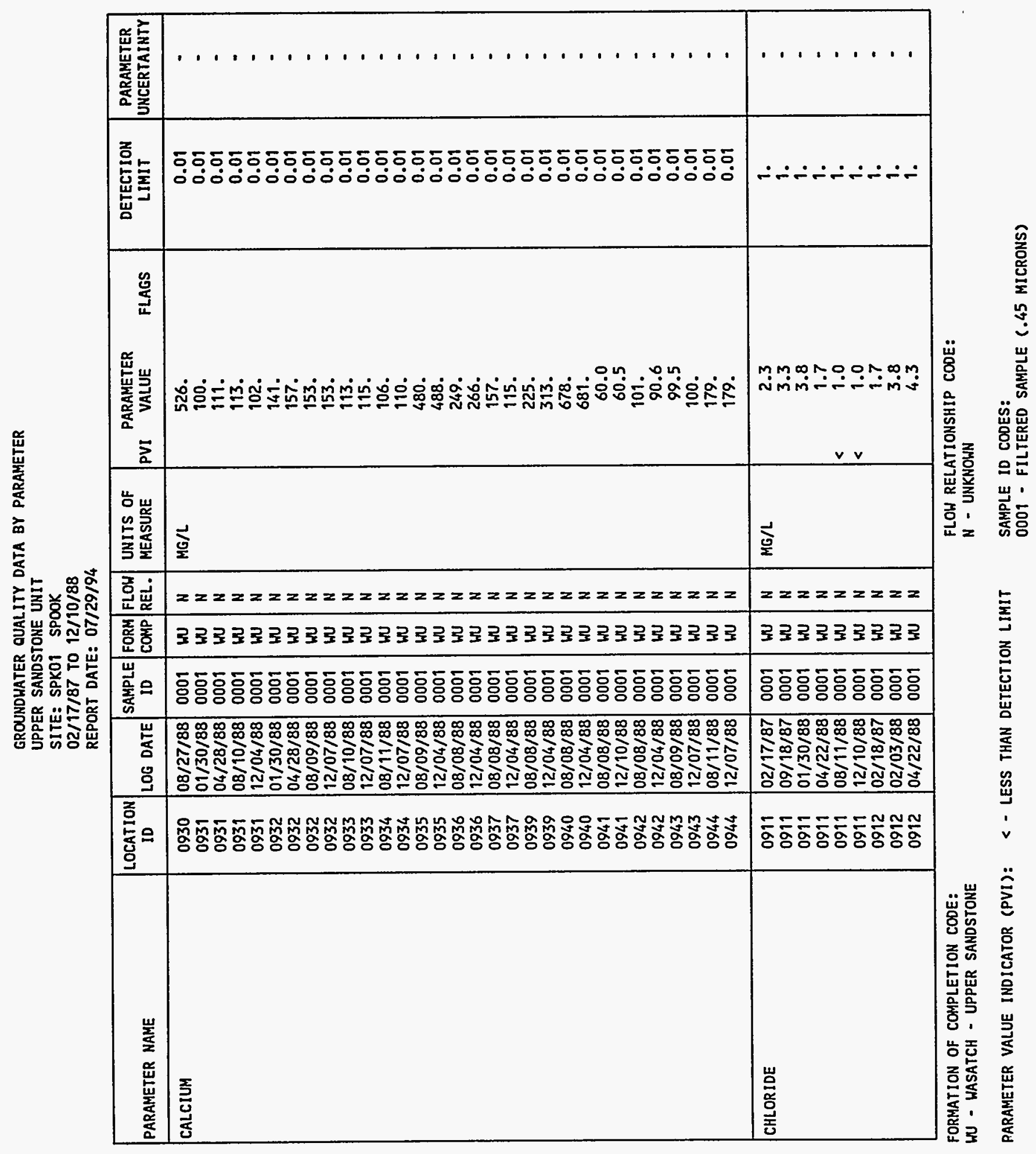




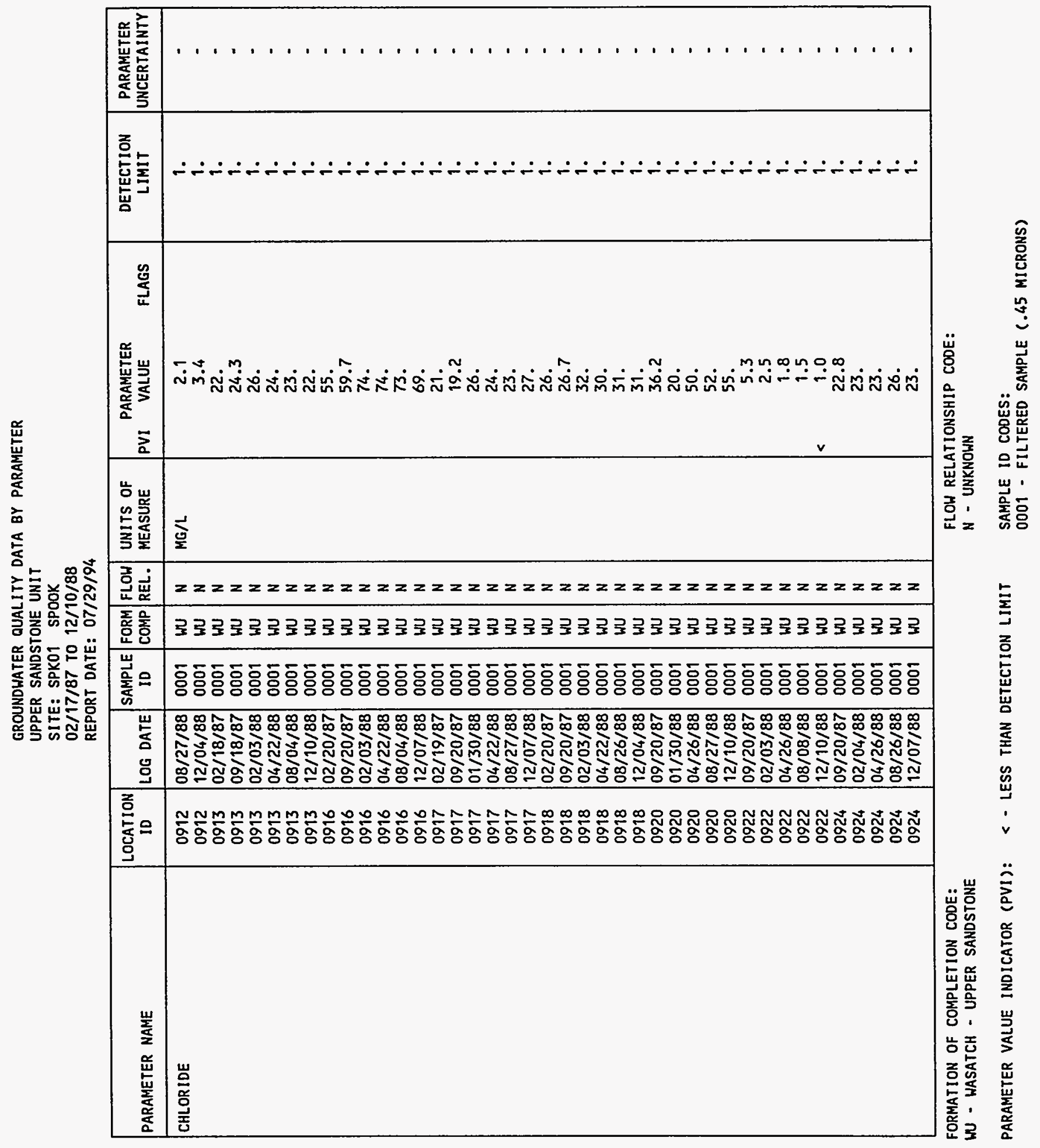




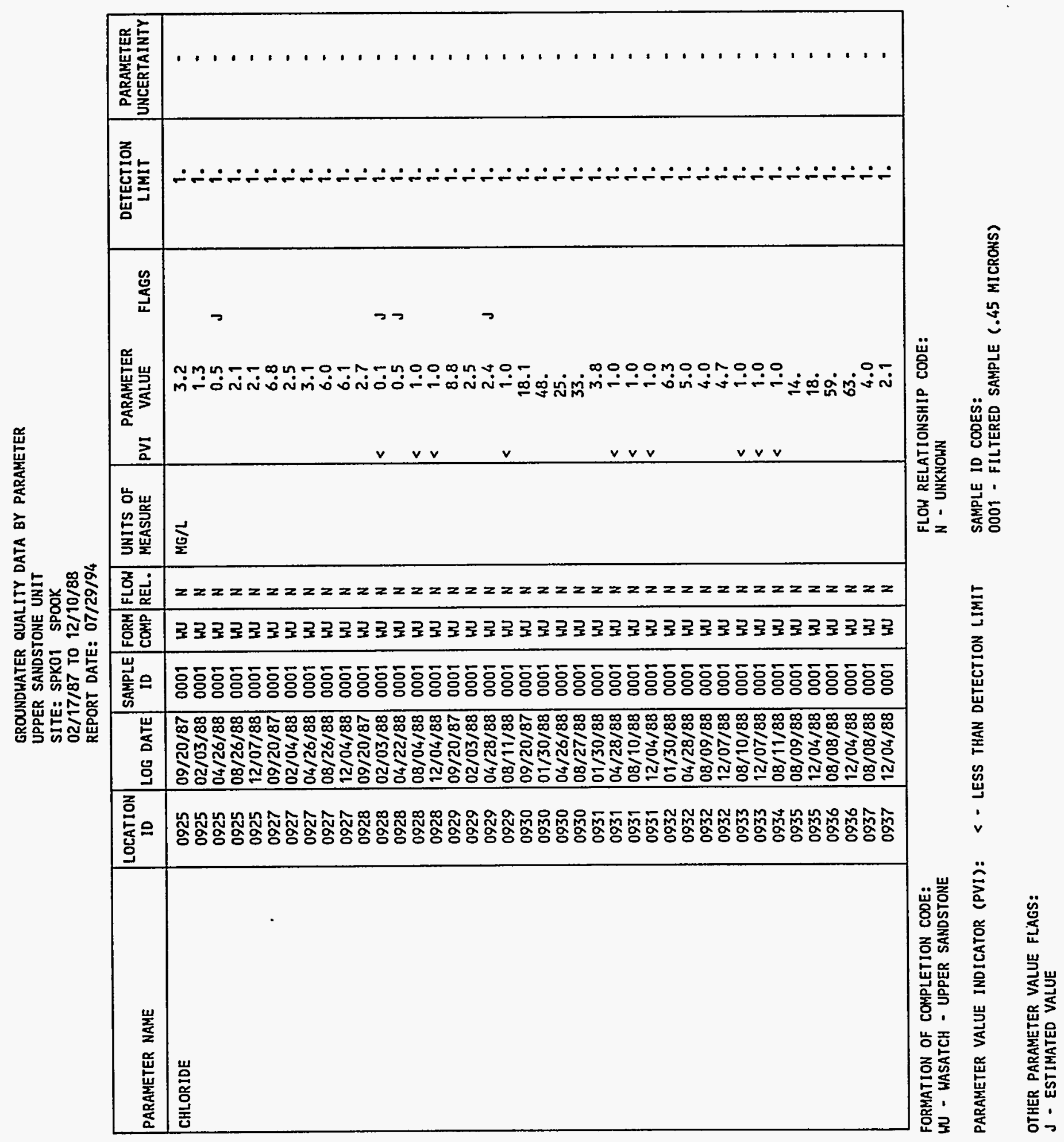




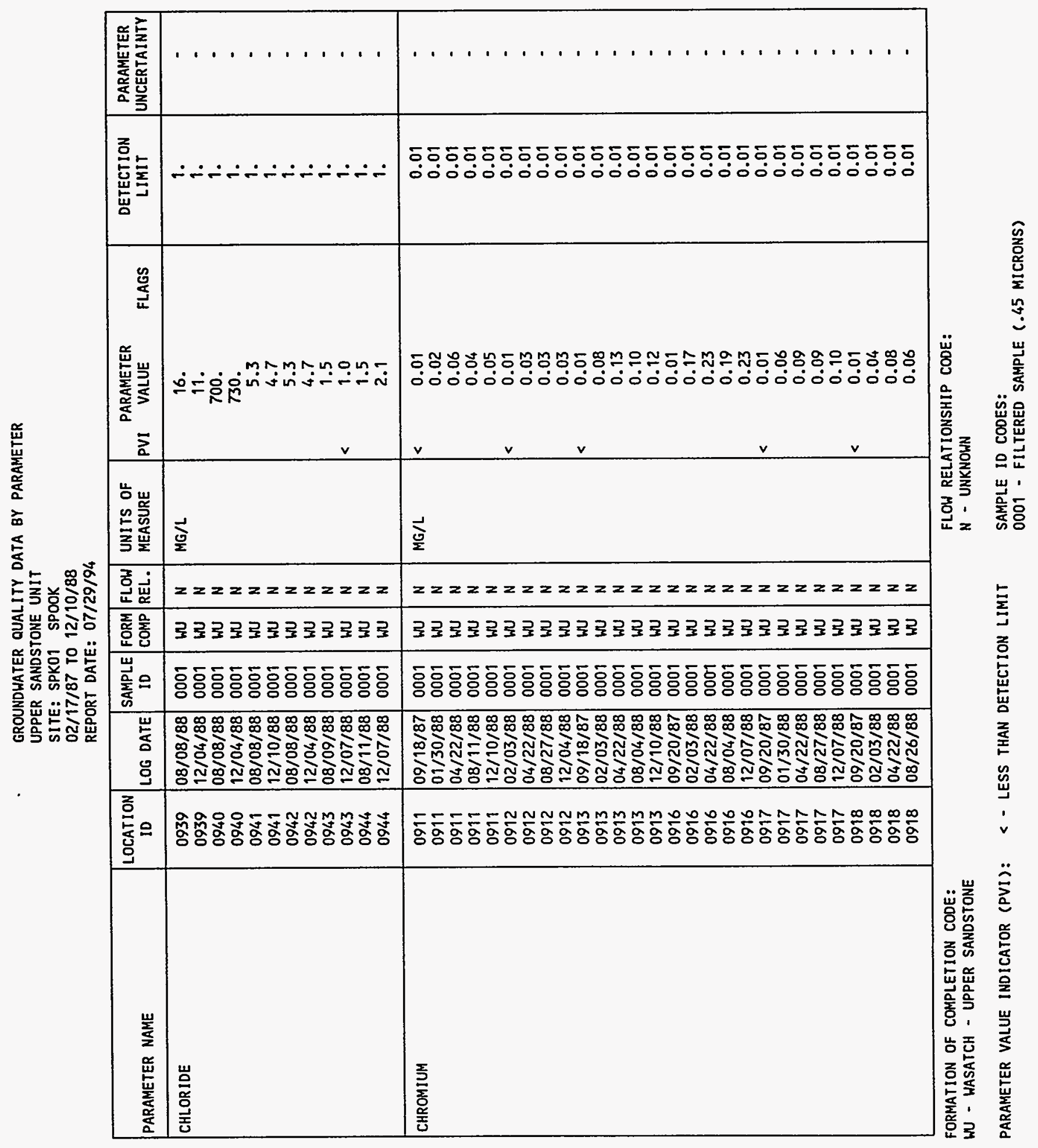




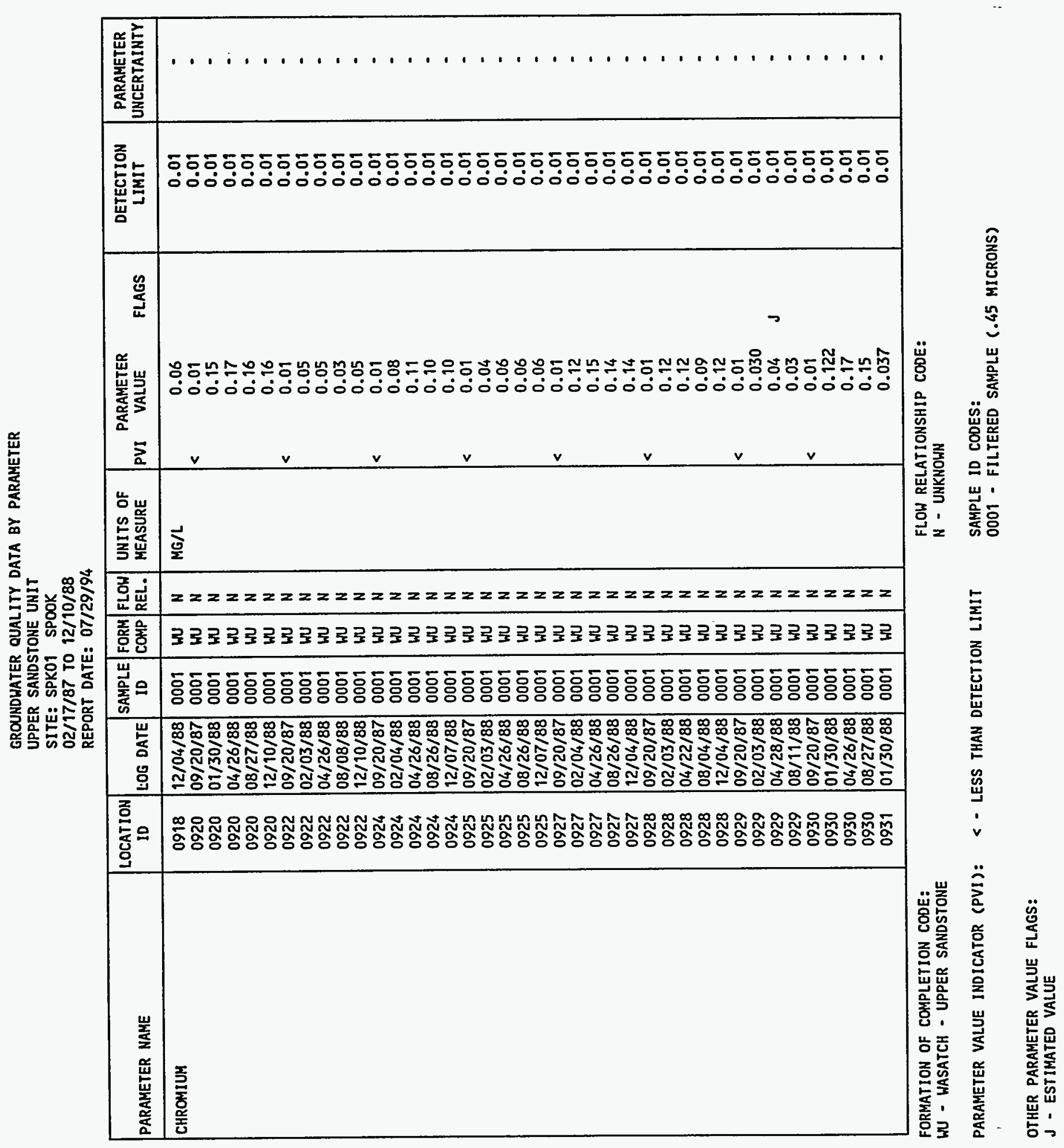




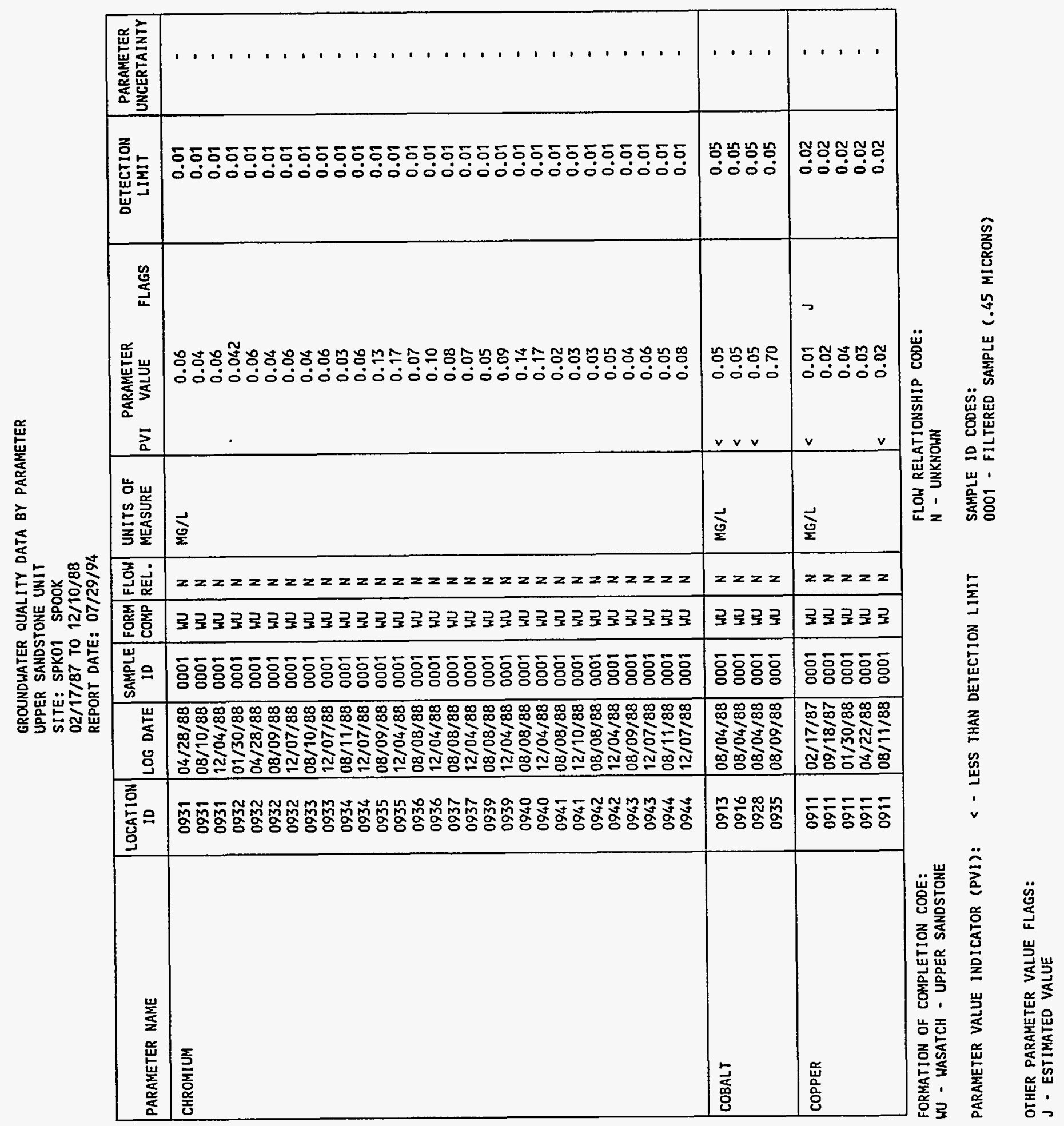




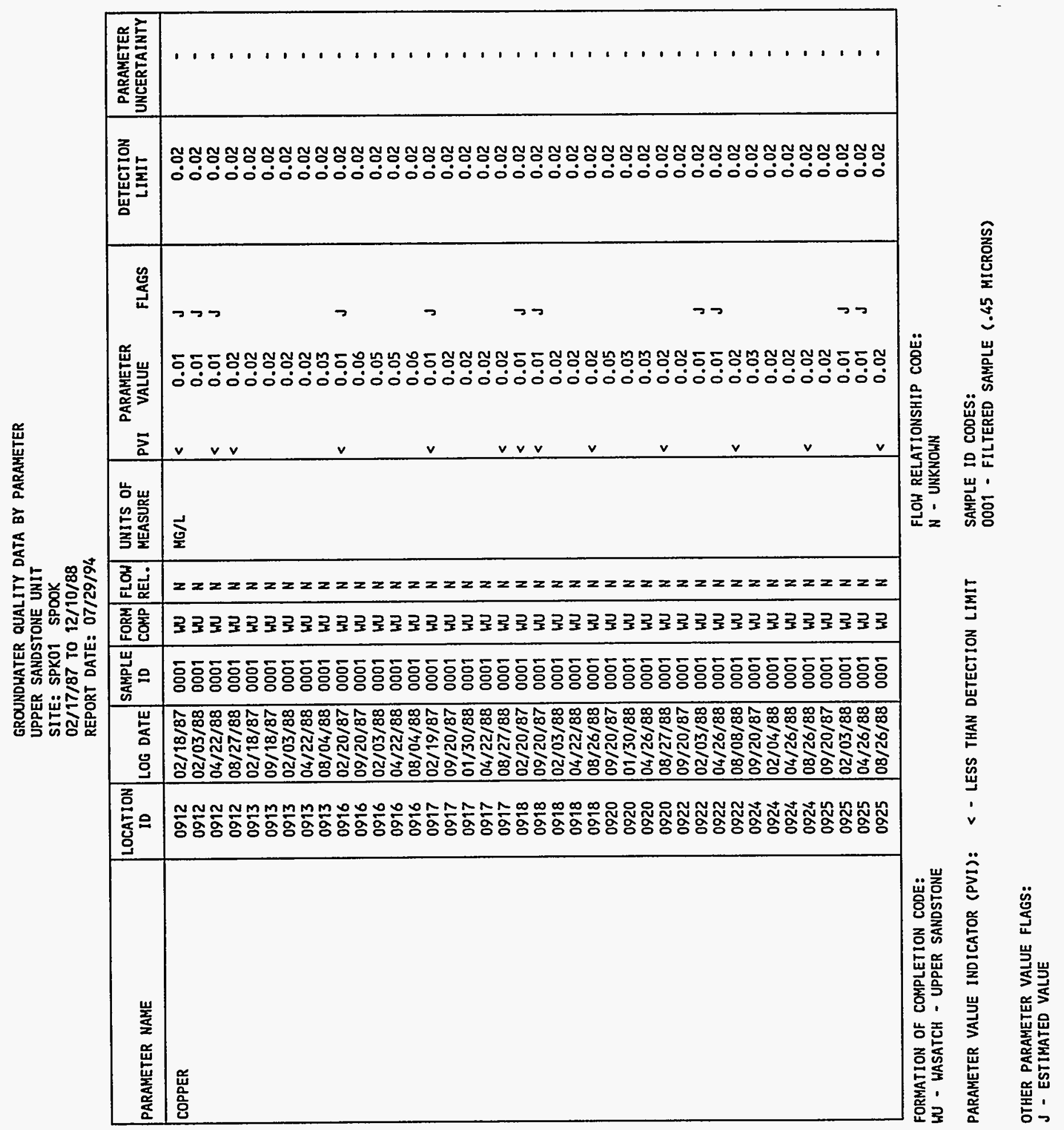




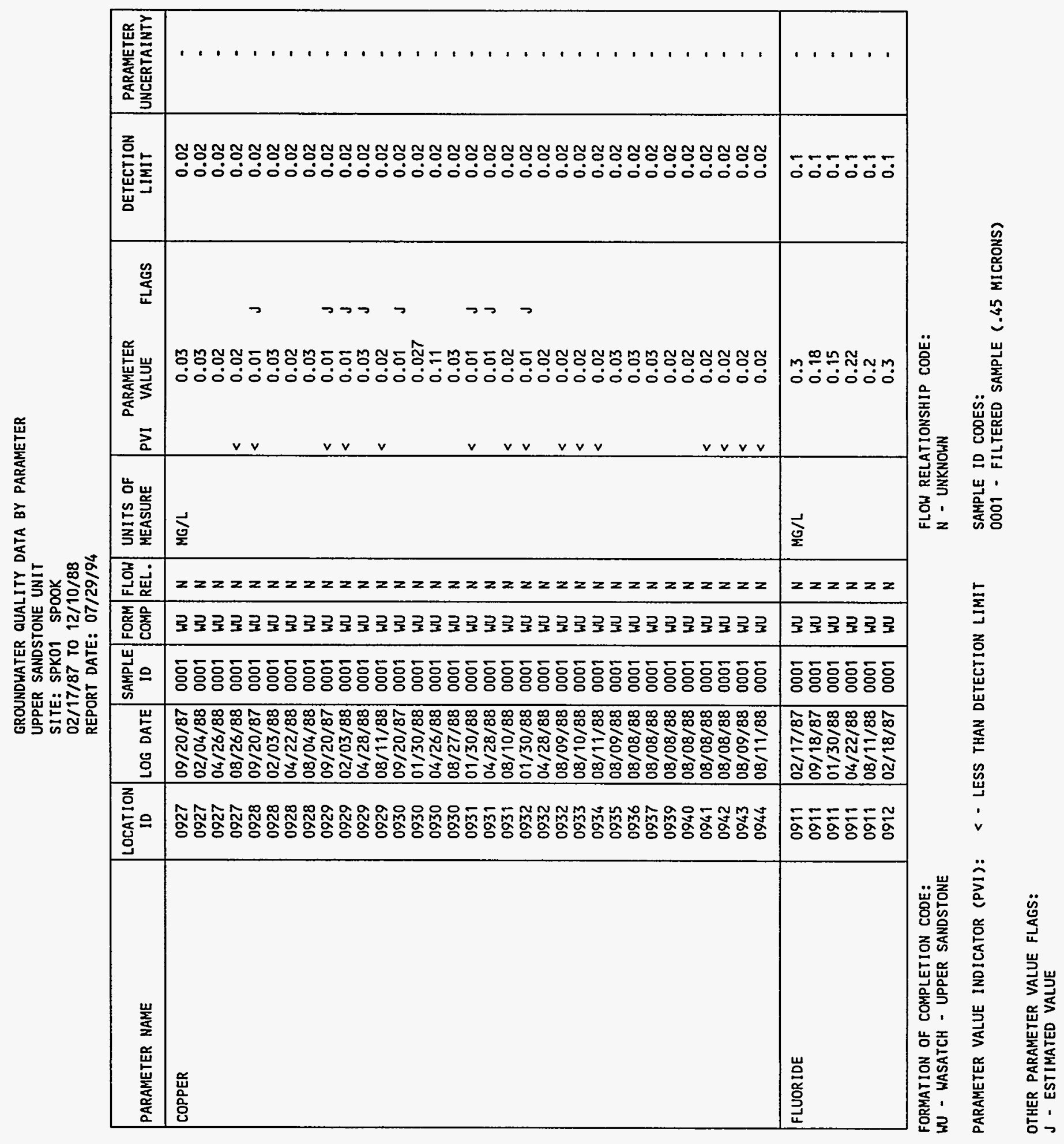


GROUNDWATER QUALITY DATA BY PARAMETER

UPPER SANDSTONE UNIT

SITE: SPKOI SPOOK

02/17/87 TO 12/10/88

REPORT DATE: $07 / 29 / 94$

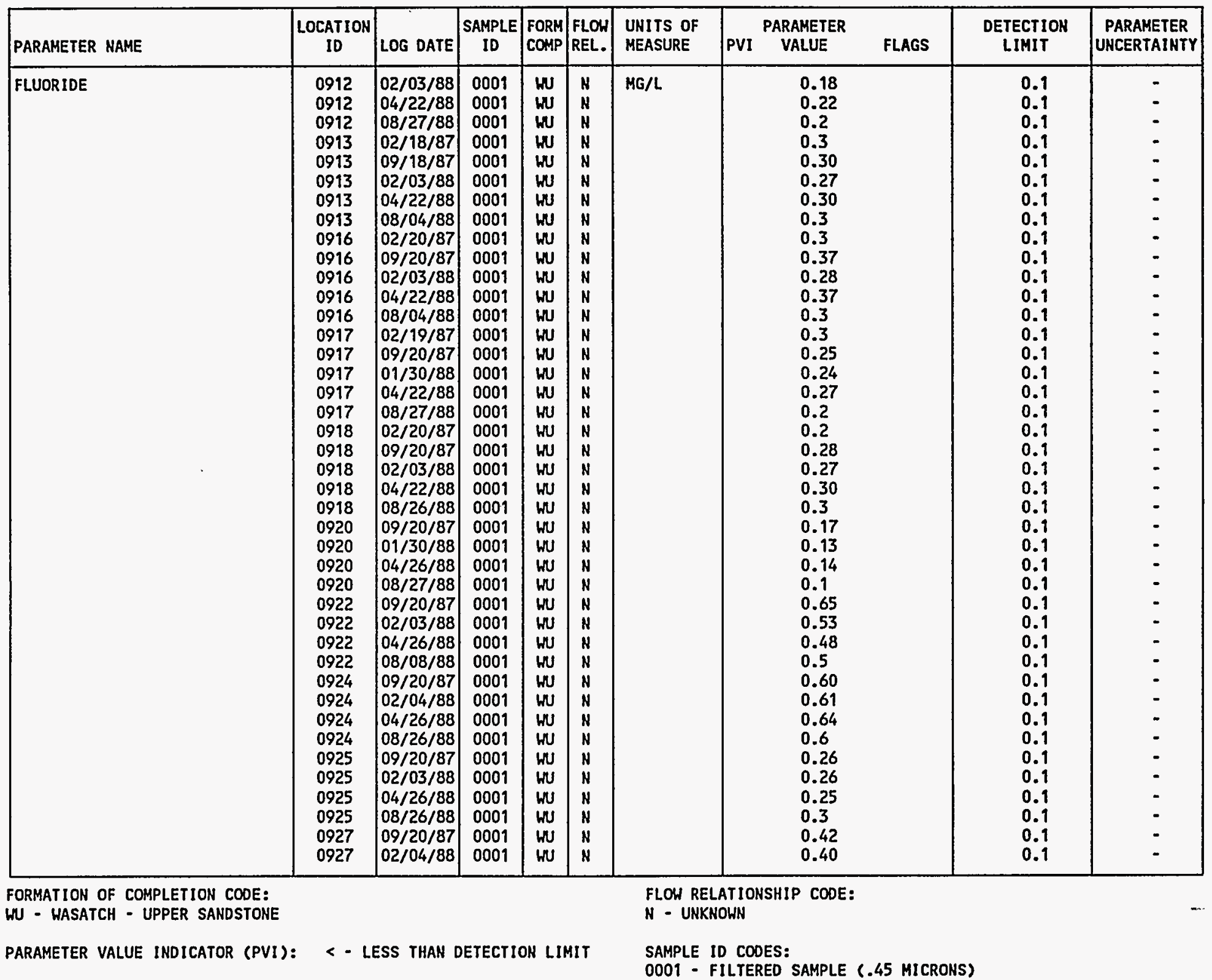


REPORT DATE: $07 / 29 / 94$

\begin{tabular}{|c|c|c|c|c|c|c|c|c|c|c|c|}
\hline PARAMETER NAME & $\underset{\text { ID }}{\text { LOCATION }}$ & LOG DATE & $\begin{array}{c}\text { SAMPLE } \\
\text { ID }\end{array}$ & $\begin{array}{l}\text { FORM } \\
\text { COMP }\end{array}$ & $\begin{array}{l}\text { FLOW } \\
\text { REL. }\end{array}$ & $\begin{array}{l}\text { UNITS OF } \\
\text { MEASURE }\end{array}$ & PVI & $\begin{array}{l}\text { PARAMETER } \\
\text { VALUE }\end{array}$ & FLAGS & $\begin{array}{l}\text { DETECTION } \\
\text { LIMIT }\end{array}$ & $\begin{array}{l}\text { PARAMETER } \\
\text { UNCERTAINTY }\end{array}$ \\
\hline FLUORIDE & $\begin{array}{l}0927 \\
0927 \\
0928 \\
0928 \\
0928 \\
0928 \\
0929 \\
0929 \\
0929 \\
0929 \\
0930 \\
0930 \\
0930 \\
0930 \\
0931 \\
0931 \\
0931 \\
0932 \\
0932 \\
0932 \\
0933 \\
0934 \\
0935 \\
0936 \\
0937 \\
0939 \\
0940 \\
0941 \\
0942 \\
0943 \\
0944\end{array}$ & $\begin{array}{l}04 / 26 / 88 \\
08 / 26 / 88 \\
09 / 20 / 87 \\
02 / 03 / 88 \\
04 / 22 / 88 \\
08 / 04 / 88 \\
09 / 20 / 87 \\
02 / 03 / 88 \\
04 / 28 / 88 \\
08 / 11 / 88 \\
09 / 20 / 87 \\
01 / 30 / 88 \\
04 / 26 / 88 \\
08 / 27 / 88 \\
01 / 30 / 88 \\
04 / 28 / 88 \\
08 / 10 / 88 \\
01 / 30 / 88 \\
04 / 28 / 88 \\
08 / 09 / 88 \\
08 / 10 / 88 \\
08 / 11 / 88 \\
08 / 09 / 88 \\
08 / 08 / 88 \\
08 / 08 / 88 \\
08 / 08 / 88 \\
08 / 08 / 88 \\
08 / 08 / 88 \\
08 / 08 / 88 \\
08 / 09 / 88 \\
08 / 11 / 88\end{array}$ & $\begin{array}{l}0001 \\
0001 \\
0001 \\
0001 \\
0001 \\
0001 \\
0001 \\
0001 \\
0001 \\
0001 \\
0001 \\
0001 \\
0001 \\
0001 \\
0001 \\
0001 \\
0001 \\
0001 \\
0001 \\
0001 \\
0001 \\
0001 \\
0001 \\
0001 \\
0001 \\
0001 \\
0001 \\
0001 \\
0001 \\
0001 \\
0001\end{array}$ & $\begin{array}{l}W U \\
W U \\
W U \\
W U \\
W U \\
W U \\
W U \\
W U \\
W U \\
W U \\
W U \\
W U \\
W U \\
W U \\
W U \\
W U \\
W U \\
W U \\
W U \\
W U \\
W U \\
W U \\
W U \\
W U \\
W U \\
W U \\
W U \\
W U \\
W U \\
W U \\
W U\end{array}$ & $\begin{array}{l}N \\
N \\
N \\
N \\
N \\
N \\
N \\
N \\
N \\
N \\
N \\
N \\
N \\
N \\
N \\
N \\
N \\
N \\
N \\
N \\
N \\
N \\
N \\
N \\
N \\
N \\
N \\
N \\
N \\
N \\
N\end{array}$ & $M G / L$ & & $\begin{array}{l}0.42 \\
0.4 \\
0.37 \\
0.40 \\
0.30 \\
0.3 \\
0.29 \\
0.18 \\
0.24 \\
0.2 \\
0.40 \\
0.13 \\
0.30 \\
0.2 \\
0.22 \\
0.23 \\
0.2 \\
0.46 \\
0.45 \\
0.5 \\
1.5 \\
0.5 \\
1.5 \\
0.2 \\
0.3 \\
0.3 \\
0.2 \\
0.1 \\
0.3 \\
0.2 \\
0.2\end{array}$ & $J$ & $\begin{array}{l}0.1 \\
0.1 \\
0.1 \\
0.1 \\
0.1 \\
0.1 \\
0.1 \\
0.1 \\
0.1 \\
0.1 \\
0.1 \\
0.1 \\
0.1 \\
0.1 \\
0.1 \\
0.1 \\
0.1 \\
0.1 \\
0.1 \\
0.1 \\
0.1 \\
0.1 \\
0.1 \\
0.1 \\
0.1 \\
0.1 \\
0.1 \\
0.1 \\
0.1 \\
0.1 \\
0.1\end{array}$ & $\begin{array}{l}- \\
- \\
- \\
- \\
- \\
- \\
- \\
- \\
- \\
- \\
- \\
- \\
- \\
- \\
- \\
- \\
- \\
- \\
- \\
- \\
- \\
- \\
-\end{array}$ \\
\hline GROSS ALPHA & $\begin{array}{l}0911 \\
0911 \\
0911 \\
0911 \\
0911 \\
0912 \\
0912 \\
0912\end{array}$ & $\begin{array}{l}09 / 18 / 87 \\
01 / 30 / 88 \\
04 / 22 / 88 \\
08 / 11 / 88 \\
12 / 10 / 88 \\
02 / 03 / 88 \\
04 / 22 / 88 \\
08 / 27 / 88\end{array}$ & $\begin{array}{l}0001 \\
0001 \\
0001 \\
0001 \\
0001 \\
0001 \\
0001 \\
0001\end{array}$ & $\begin{array}{l}W U \\
W U \\
W U \\
W U \\
W U \\
W U \\
W U \\
W U\end{array}$ & $\begin{array}{l}N \\
N \\
N \\
N \\
N \\
N \\
N \\
N\end{array}$ & $\mathrm{PCl} / \mathrm{L}$ & & $\begin{array}{c}980 . \\
970 . \\
1310 . \\
1060 . \\
1190 . \\
380 \\
480 \\
280\end{array}$ & & $\begin{array}{l}0.2 \\
0.2 \\
0.2 \\
0.2 \\
0.2 \\
0.2 \\
0.2 \\
0.2\end{array}$ & $\begin{array}{l}30 . \\
30 . \\
40 . \\
30 . \\
30 . \\
20 . \\
20 . \\
10 .\end{array}$ \\
\hline
\end{tabular}

FORMATION OF COMPLETION CODE: WU - HASATCH - UPPER SANDSTONE

PARAMETER VALUE INDICATOR (PVI): < - LESS THAN DETECTION LIMIT
FLOH RELATIONSHIP CODE:

N - UNKNOWN

SAMPLE ID CODES

0001 - FILTERED SAMPLE (.45 MICRONS)

OTHER PARAMETER VALUE FLAGS:

$J$ - ESTIMATED VALUE 
UPPER SANDSTONE UNIT

SITE: SPKO1 SPOOK

$02 / 17 / 87$ TO $12 / 10 / 88$

REPORT DATE: 07/29/94

\begin{tabular}{|c|c|c|c|c|c|c|c|c|c|c|c|}
\hline PARAMETER NAME & $\underset{10}{\text { LOCATION }}$ & LOG DATE & $\underset{10}{\text { SAMPLE }}$ & $\begin{array}{l}\text { FORM } \\
\text { COMP }\end{array}$ & $\begin{array}{l}\text { FLOW } \\
\text { REL. }\end{array}$ & $\begin{array}{l}\text { UNITS OF } \\
\text { MEASURE }\end{array}$ & PVI & $\begin{array}{l}\text { PARAMETER } \\
\text { VALUE }\end{array}$ & FLAGS & $\begin{array}{l}\text { DETECTION } \\
\text { LIMIT }\end{array}$ & $\begin{array}{c}\text { PARAMETER } \\
\text { UNCERTAINTYY }\end{array}$ \\
\hline GROSS ALPHA & $\begin{array}{l}0912 \\
0913 \\
0913 \\
0913 \\
0913 \\
0913 \\
0916 \\
0916 \\
0916 \\
0916 \\
0916 \\
0917 \\
0917 \\
0917 \\
0917 \\
0917 \\
0918 \\
0918 \\
0918 \\
0918 \\
0918 \\
0920 \\
0920 \\
0920 \\
0920 \\
0920 \\
0922 \\
0922 \\
0922 \\
0922 \\
0922 \\
0924 \\
0924 \\
0924 \\
0924 \\
0924 \\
0925 \\
0925 \\
0925 \\
0925 \\
0925\end{array}$ & $\begin{array}{l}12 / 04 / 88 \\
09 / 18 / 87 \\
02 / 03 / 88 \\
04 / 22 / 88 \\
08 / 04 / 88 \\
12 / 10 / 88 \\
09 / 20 / 87 \\
02 / 03 / 88 \\
04 / 22 / 88 \\
08 / 04 / 88 \\
12 / 07 / 88 \\
09 / 20 / 87 \\
01 / 30 / 88 \\
04 / 22 / 88 \\
08 / 27 / 88 \\
12 / 07 / 88 \\
09 / 20 / 87 \\
02 / 03 / 88 \\
04 / 22 / 88 \\
08 / 26 / 88 \\
12 / 04 / 88 \\
09 / 20 / 87 \\
01 / 30 / 88 \\
04 / 26 / 88 \\
08 / 27 / 88 \\
12 / 10 / 88 \\
09 / 20 / 87 \\
02 / 03 / 88 \\
04 / 26 / 88 \\
08 / 08 / 88 \\
12 / 10 / 88 \\
09 / 20 / 87 \\
02 / 04 / 88 \\
04 / 26 / 88 \\
08 / 26 / 88 \\
12 / 07 / 88 \\
09 / 20 / 87 \\
02 / 03 / 88 \\
04 / 26 / 88 \\
08 / 26 / 88 \\
12 / 07 / 88\end{array}$ & $\begin{array}{l}0001 \\
0001 \\
0001 \\
0001 \\
0001 \\
0001 \\
0001 \\
0001 \\
0001 \\
0001 \\
0001 \\
0001 \\
0001 \\
0001 \\
0001 \\
0001 \\
0001 \\
0001 \\
0001 \\
0001 \\
0001 \\
0001 \\
0001 \\
0001 \\
0001 \\
0001 \\
0001 \\
0001 \\
0001 \\
0001 \\
0001 \\
0001 \\
0001 \\
0001 \\
0001 \\
0001 \\
0001 \\
0001 \\
0001 \\
0001 \\
0001\end{array}$ & $\begin{array}{l}W U \\
W U \\
W U \\
W U \\
W U \\
W U \\
W U \\
W U \\
W U \\
W U \\
W U \\
W U \\
W U \\
W U \\
W U \\
W U \\
W U \\
W U \\
W U \\
W U \\
W U \\
W U \\
W U \\
W U \\
W U \\
W U \\
W U \\
W U \\
W U \\
W U \\
W U \\
W U \\
W U \\
W U \\
W U \\
W U \\
W U \\
W U \\
W U \\
W U\end{array}$ & $\begin{array}{l}N \\
N \\
N \\
N \\
N \\
N \\
N \\
N \\
N \\
N \\
N \\
N \\
N \\
N \\
N \\
N \\
N \\
N \\
N \\
N \\
N \\
N \\
N \\
N \\
N \\
N \\
N \\
N \\
N \\
N \\
N \\
N \\
N \\
N \\
N \\
N \\
N \\
N \\
N \\
N \\
N\end{array}$ & $\mathrm{PCI} / \mathrm{L}$ & & $\begin{array}{c}410 . \\
6000 . \\
4800 . \\
6000 . \\
9100 . \\
7200 . \\
12700 . \\
11800 . \\
14200 . \\
17700 . \\
11700 . \\
3400 . \\
3400 . \\
4300 . \\
2400 . \\
3100 . \\
1070 . \\
1000 . \\
1410 . \\
970 . \\
990 . \\
870 . \\
840 . \\
1300 . \\
890 . \\
750 . \\
150 . \\
100 . \\
66 . \\
72 . \\
78 . \\
2300 . \\
2000 . \\
2400 . \\
2200 . \\
2800 . \\
1220 . \\
1320 . \\
1560 . \\
1350 . \\
1190 .\end{array}$ & & $\begin{array}{l}0.2 \\
0.2 \\
0.2 \\
0.2 \\
0.2 \\
0.2 \\
0.2 \\
0.2 \\
0.2 \\
0.2 \\
0.2 \\
0.2 \\
0.2 \\
0.2 \\
0.2 \\
0.2 \\
0.2 \\
0.2 \\
0.2 \\
0.2 \\
0.2 \\
0.2 \\
0.2 \\
0.2 \\
0.2 \\
0.2 \\
0.2 \\
0.2 \\
0.2 \\
0.2 \\
0.2 \\
0.2 \\
0.2 \\
0.2 \\
0.2 \\
0.2 \\
0.2 \\
0.2 \\
0.2 \\
0.2 \\
0.2\end{array}$ & $\begin{array}{c}20 . \\
100 . \\
100 . \\
100 . \\
100 . \\
100 . \\
100 . \\
300 . \\
200 . \\
300 . \\
200 . \\
100 . \\
100 . \\
100 . \\
100 . \\
100 . \\
40 . \\
40 . \\
40 . \\
30 . \\
30 . \\
70 . \\
90 . \\
100 . \\
120 . \\
70 . \\
20 . \\
10 . \\
9.0 \\
9.0 \\
9.0 \\
100 . \\
100 . \\
100 . \\
100 . \\
100 . \\
30 . \\
30 . \\
40 . \\
30 . \\
30 .\end{array}$ \\
\hline
\end{tabular}

FORMATION OF COMPLETION CODE:

FLOW RELATIONSHIP CODE

WU - WASATCH - UPPER SANDSTONE

N - UNKNOWN

PARAMETER VALUE INDICATOR (PVI): < - LESS THAN DETECTION LIMIT

PLE ID CODES:

0001 - FILTERED SAMPLE (.45 MICRONS) 


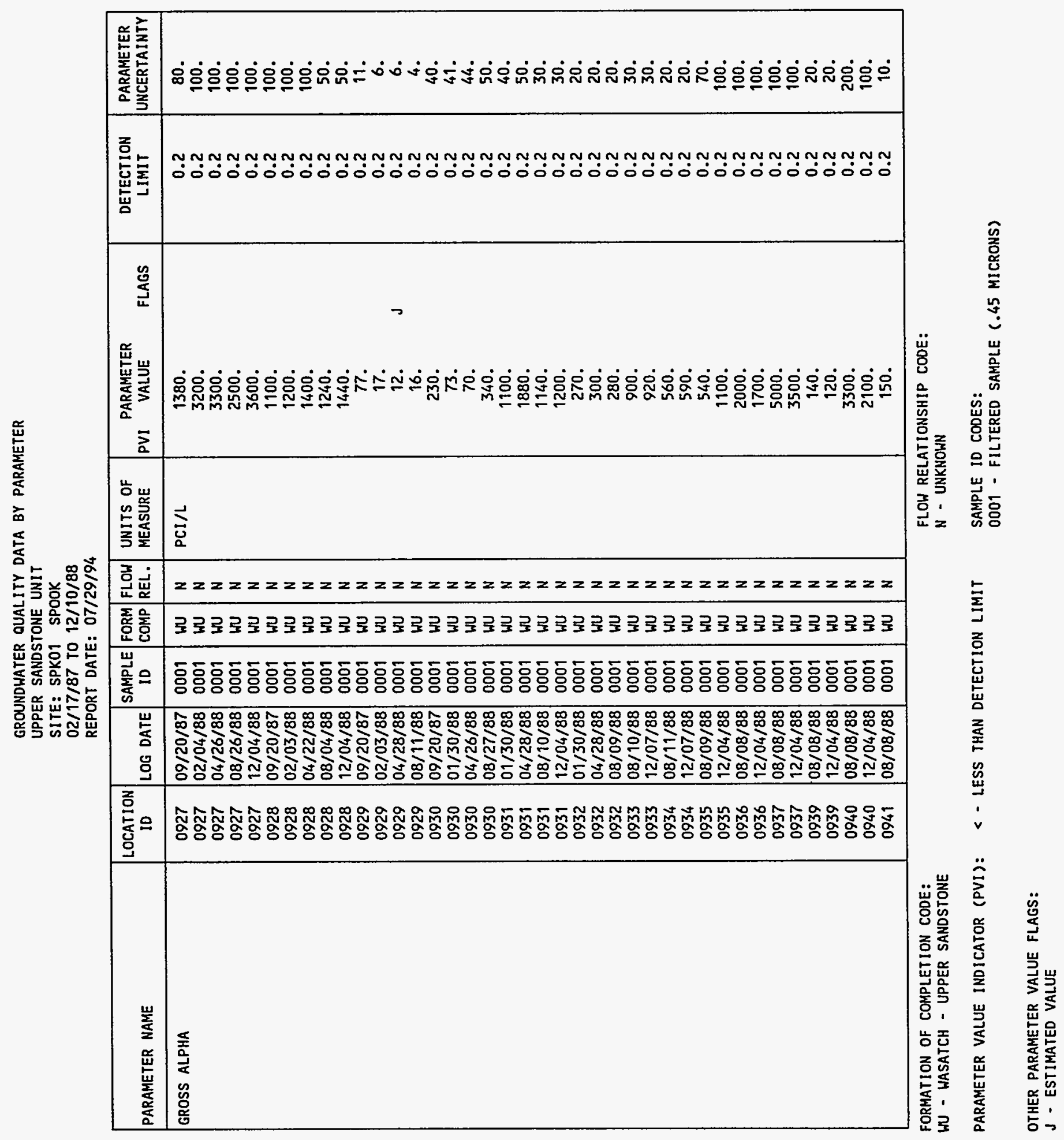


GROUNDWATER QUALITY DATA BY PARAMETER

UPPER SANDSTONE UNIT

SITE: SPKO1 SPOOK

02/17/87 TO $12 / 10 / 88$

REPORT DATE: $07 / 29 / 94$

\begin{tabular}{|c|c|c|c|c|c|c|c|c|c|c|c|}
\hline PARAMETER NAME & $\underset{\text { LOCATION }}{\text { LD }}$ & LOG DATE & $\begin{array}{c}\text { SAMPLE } \\
\text { ID }\end{array}$ & $\begin{array}{l}\text { FORM } \\
\text { COMP }\end{array}$ & $\begin{array}{l}\text { FLOW } \\
\text { REL. }\end{array}$ & $\begin{array}{l}\text { UNITS OF } \\
\text { MEASURE }\end{array}$ & PVI & $\begin{array}{l}\text { PARAMETER } \\
\text { VALUE }\end{array}$ & FLAGS & $\begin{array}{l}\text { DETECTION } \\
\text { LIMIT }\end{array}$ & $\begin{array}{l}\text { PARAMETER } \\
\text { UNCERTAINTY }\end{array}$ \\
\hline GROSS ALPHA & $\begin{array}{l}0941 \\
0942 \\
0942 \\
0943 \\
0943 \\
0944 \\
0944\end{array}$ & $\begin{array}{l}12 / 10 / 88 \\
08 / 08 / 88 \\
12 / 04 / 88 \\
08 / 09 / 88 \\
12 / 07 / 88 \\
08 / 11 / 88 \\
12 / 07 / 88\end{array}$ & $\begin{array}{l}0001 \\
0001 \\
0001 \\
0001 \\
0001 \\
0001 \\
0001\end{array}$ & $\begin{array}{l}W U \\
W U \\
W U \\
W U \\
W U \\
W U \\
W U\end{array}$ & $\begin{array}{l}N \\
N \\
N \\
N \\
N \\
N \\
N\end{array}$ & $\mathrm{PCI} / \mathrm{L}$ & & $\begin{array}{c}160 . \\
550 . \\
370 \\
1640 \\
1720 \\
590 \\
560 .\end{array}$ & & $\begin{array}{l}0.2 \\
0.2 \\
0.2 \\
0.2 \\
0.2 \\
0.2 \\
0.2\end{array}$ & $\begin{array}{l}10 . \\
20 . \\
20 . \\
40 . \\
30 . \\
30 . \\
30 .\end{array}$ \\
\hline GROSS BETA & $\begin{array}{l}0911 \\
0911 \\
0911 \\
0911 \\
0911 \\
0912 \\
0912 \\
0912 \\
0912 \\
0913 \\
0913 \\
0913 \\
0913 \\
0913 \\
0916 \\
0916 \\
0916 \\
0916 \\
0916 \\
0917 \\
0917 \\
0917 \\
0917 \\
0917 \\
0918 \\
0918 \\
0918 \\
0918 \\
0918 \\
0920 \\
0920 \\
0920 \\
0920\end{array}$ & $\begin{array}{l}09 / 18 / 87 \\
01 / 30 / 88 \\
04 / 22 / 88 \\
08 / 11 / 88 \\
12 / 10 / 88 \\
02 / 03 / 88 \\
04 / 22 / 88 \\
08 / 27 / 88 \\
12 / 04 / 88 \\
09 / 18 / 87 \\
02 / 03 / 88 \\
04 / 22 / 88 \\
08 / 04 / 88 \\
12 / 10 / 88 \\
09 / 20 / 87 \\
02 / 03 / 88 \\
04 / 22 / 88 \\
08 / 04 / 88 \\
12 / 07 / 88 \\
09 / 20 / 87 \\
01 / 30 / 88 \\
04 / 22 / 88 \\
08 / 27 / 88 \\
12 / 07 / 88 \\
09 / 20 / 87 \\
02 / 03 / 88 \\
04 / 22 / 88 \\
08 / 26 / 88 \\
12 / 04 / 88 \\
09 / 20 / 87 \\
01 / 30 / 88 \\
04 / 26 / 88 \\
08 / 27 / 88\end{array}$ & $\begin{array}{l}0001 \\
0001 \\
0001 \\
0001 \\
0001 \\
0001 \\
0001 \\
0001 \\
0001 \\
0001 \\
0001 \\
0001 \\
0001 \\
0001 \\
0001 \\
0001 \\
0001 \\
0001 \\
0001 \\
0001 \\
0001 \\
0001 \\
0001 \\
0001 \\
0001 \\
0001 \\
0001 \\
0001 \\
0001 \\
0001 \\
0001 \\
0001 \\
0001\end{array}$ & $\begin{array}{l}W U \\
W U \\
W U \\
W U \\
W U \\
W U \\
W U \\
W U \\
W U \\
W U \\
W U \\
W U \\
W U \\
W U \\
W U \\
W U \\
W U \\
W U \\
W U \\
W U \\
W U \\
W U \\
W U \\
W U \\
W U \\
W U \\
W U \\
W U \\
W U \\
W U \\
W U \\
W U\end{array}$ & $\begin{array}{l}N \\
N \\
N \\
N \\
N \\
N \\
N \\
N \\
N \\
N \\
N \\
N \\
N \\
N \\
N \\
N \\
N \\
N \\
N \\
N \\
N \\
N \\
N \\
N \\
N \\
N \\
N \\
N \\
N \\
N \\
N \\
N \\
N\end{array}$ & $\mathrm{PCI} / \mathrm{L}$ & & $\begin{array}{c}560 . \\
470 . \\
340 . \\
300 . \\
210 . \\
210 . \\
138 . \\
110 . \\
156 . \\
3600 . \\
2380 . \\
2250 . \\
2570 . \\
1120 . \\
8100 . \\
5600 . \\
5900 . \\
6000 . \\
3600 . \\
2300 . \\
1730 . \\
1440 . \\
1010 . \\
950 . \\
650 . \\
380 . \\
450 . \\
360 . \\
280 . \\
600 . \\
460 . \\
450 . \\
370 .\end{array}$ & & $\begin{array}{l}1 . \\
1 . \\
1 . \\
1 . \\
1 . \\
1 . \\
1 . \\
1 . \\
1 . \\
1 . \\
1 . \\
1 . \\
1 . \\
1 . \\
1 . \\
1 . \\
1 . \\
1 . \\
1 . \\
1 . \\
1 . \\
1 . \\
1 . \\
1 . \\
1 . \\
1 . \\
1 . \\
1 . \\
1 .\end{array}$ & $\begin{array}{c}10 . \\
10 . \\
10 . \\
10 . \\
10 . \\
10 . \\
4 . \\
4 . \\
4 . \\
100 . \\
30 . \\
30 . \\
30 . \\
20 . \\
100 . \\
100 . \\
100 . \\
100 . \\
100 . \\
10 . \\
30 . \\
20 . \\
20 . \\
20 . \\
10 . \\
10 . \\
10 . \\
10 . \\
10 . \\
30 . \\
40 . \\
30 . \\
50 .\end{array}$ \\
\hline
\end{tabular}

FORMATION OF COMPLETION CODE:

FLOW RELATIONSHIP CODE:

WU - WASATCH - UPPER SANDSTONE

N - UNKNOWN

PARAMETER VALUE INDICATOR (PVI): < - LESS THAN DETECTION LIMIT 
GROUNDWATER QUALITY DATA BY PARAMETER

UPPER SANDSTONE UNIT

SITE: SPKO1 SPOOK

$02 / 17 / 87$ TO $12 / 10 / 88$

REPORT DATE: $07 / 29 / 94$

\begin{tabular}{|c|c|c|c|c|c|c|c|c|c|c|c|}
\hline PARAMETER NAME & $\begin{array}{c}\text { LOCATION } \\
\text { ID }\end{array}$ & LOG DATE & $\begin{array}{c}\text { SAMPLE } \\
\text { ID }\end{array}$ & $\begin{array}{l}\text { FORM } \\
\text { COMP }\end{array}$ & $\begin{array}{l}\text { FLOW } \\
\text { REL. }\end{array}$ & $\begin{array}{l}\text { UNITS OF } \\
\text { MEASURE }\end{array}$ & PVI & $\begin{array}{l}\text { PARAMETER } \\
\text { VALUE }\end{array}$ & FLAGS & $\begin{array}{l}\text { DETECTION } \\
\text { LIMIT }\end{array}$ & $\begin{array}{l}\text { PARAMETER } \\
\text { UNCERTAINTY }\end{array}$ \\
\hline GROSS BETA & $\begin{array}{l}0920 \\
0922 \\
0922 \\
0922 \\
0922 \\
0922 \\
0924 \\
0924 \\
0924 \\
0924 \\
0924 \\
0925 \\
0925 \\
0925 \\
0925 \\
0925 \\
0927 \\
0927 \\
0927 \\
0927 \\
0927 \\
0928 \\
0928 \\
0928 \\
0928 \\
0928 \\
0929 \\
0929 \\
0929 \\
0929 \\
0930 \\
0930 \\
0930 \\
0930 \\
0931 \\
0931 \\
0931 \\
0931 \\
0932 \\
0932\end{array}$ & $\begin{array}{l}12 / 10 / 88 \\
09 / 20 / 87 \\
02 / 03 / 88 \\
04 / 26 / 88 \\
08 / 08 / 88 \\
12 / 10 / 88 \\
09 / 20 / 87 \\
02 / 04 / 88 \\
04 / 26 / 88 \\
08 / 26 / 88 \\
12 / 07 / 88 \\
09 / 20 / 87 \\
02 / 03 / 88 \\
04 / 26 / 88 \\
08 / 26 / 88 \\
12 / 07 / 88 \\
09 / 20 / 87 \\
02 / 04 / 88 \\
04 / 26 / 88 \\
08 / 26 / 88 \\
12 / 04 / 88 \\
09 / 20 / 87 \\
02 / 03 / 88 \\
04 / 22 / 88 \\
08 / 04 / 88 \\
12 / 04 / 88 \\
09 / 20 / 87 \\
02 / 03 / 88 \\
04 / 28 / 88 \\
08 / 11 / 88 \\
09 / 20 / 87 \\
01 / 30 / 88 \\
04 / 26 / 88 \\
08 / 27 / 88 \\
01 / 30 / 88 \\
04 / 28 / 88 \\
08 / 10 / 88 \\
12 / 04 / 88 \\
01 / 30 / 88 \\
04 / 28 / 88\end{array}$ & $\begin{array}{l}0001 \\
0001 \\
0001 \\
0001 \\
0001 \\
0001 \\
0001 \\
0001 \\
0001 \\
0001 \\
0001 \\
0001 \\
0001 \\
0001 \\
0001 \\
0001 \\
0001 \\
0001 \\
0001 \\
0001 \\
0001 \\
0001 \\
0001 \\
0001 \\
0001 \\
0001 \\
0001 \\
0001 \\
0001 \\
0001 \\
0001 \\
0001 \\
0001 \\
0001 \\
0001 \\
0001 \\
0001 \\
0001 \\
0001 \\
0001\end{array}$ & $\begin{array}{l}W U \\
W U \\
W U \\
W U \\
W U \\
W U \\
W U \\
W U \\
W U \\
W U \\
W U \\
W U \\
W U \\
W U \\
W U \\
W U \\
W U \\
W U \\
W U \\
W U \\
W U \\
W U \\
W U \\
W U \\
W U \\
W U \\
W U \\
W U \\
W U \\
W U \\
W U \\
W U \\
W U \\
W U \\
W U \\
W U \\
W U \\
W U \\
W U \\
W U\end{array}$ & $\begin{array}{l}N \\
N \\
N \\
N \\
N \\
N \\
N \\
N \\
N \\
N \\
N \\
N \\
N \\
N \\
N \\
N \\
N \\
N \\
N \\
N \\
N \\
N \\
N \\
N \\
N \\
N \\
N \\
N \\
N \\
N \\
N \\
N \\
N \\
N \\
N \\
N \\
N \\
N \\
N \\
N\end{array}$ & $\mathrm{PCl} / \mathrm{L}$ & & $\begin{array}{c}250 . \\
100 . \\
50 . \\
39 . \\
33 . \\
24 . \\
1210 . \\
850 . \\
900 . \\
920 . \\
750 . \\
900 . \\
575 . \\
520 . \\
460 . \\
390 . \\
2250 . \\
1200 . \\
1120 . \\
980 . \\
790 . \\
600 . \\
510 . \\
510 . \\
520 . \\
450 . \\
58 . \\
30 . \\
18 . \\
20 . \\
190 . \\
93 . \\
82 . \\
160 . \\
610 . \\
680 . \\
530 . \\
370 . \\
150 . \\
140 .\end{array}$ & J & $\begin{array}{l}1 . \\
1 . \\
1 . \\
1 . \\
1 . \\
1 . \\
1 . \\
1 . \\
1 . \\
1 . \\
1 . \\
1 . \\
1 . \\
1 . \\
1 . \\
1 . \\
1 . \\
1 . \\
1 . \\
1 . \\
1 . \\
1 . \\
1 . \\
1 . \\
1 . \\
1 . \\
1 . \\
1 . \\
1 . \\
1 . \\
1 . \\
1 . \\
1 . \\
1 .\end{array}$ & $\begin{array}{c}20 . \\
10 . \\
5 . \\
3 . \\
3 . \\
3 . \\
20 . \\
20 . \\
20 . \\
20 . \\
20 . \\
20 . \\
10 . \\
10 . \\
10 . \\
10 . \\
40 . \\
30 . \\
30 . \\
30 . \\
20 . \\
30 . \\
20 . \\
20 . \\
20 . \\
10 . \\
6 . \\
3 . \\
3 . \\
2 . \\
20 . \\
23 . \\
16 . \\
20 . \\
10 . \\
10 . \\
10 . \\
10 . \\
10 . \\
10 .\end{array}$ \\
\hline
\end{tabular}

FORMATION OF COMPLETION CODE:

WU - WASATCH - UPPER SANDSTONE

PARAMETER VALUE INDICATOR (PVI): < - LESS THAN DETECTION LIMIT
FLOH RELATIONSHIP CODE:

$N$ - UNKNOWN

SAMPLE ID CODES:

0001 - FILTERED SAMPLE (.45 MICRONS)

OTHER PARAMETER VALUE FLAGS

$J$ - ESTIMATED VALUE 
GROUNDHATER QUALITY DATA BY PARAMETER

UPPER SANDSTONE UNIT

SITE: SPKO1 SPOOK

$02 / 17 / 87$ TO $12 / 10 / 88$

REPORT DATE: $07 / 29 / 94$

\begin{tabular}{|c|c|c|c|c|c|c|c|c|c|c|c|}
\hline PARAMETER NAME & $\begin{array}{c}\text { LOCATION } \\
\text { ID }\end{array}$ & LOG DATE & $\begin{array}{c}\text { SAMPLE } \\
\text { ID }\end{array}$ & $\begin{array}{l}\text { FORM } \\
\text { COMP }\end{array}$ & $\begin{array}{l}\text { FLOW } \\
\text { REL. }\end{array}$ & $\begin{array}{l}\text { UNITS OF } \\
\text { MEASURE }\end{array}$ & PVI & $\begin{array}{l}\text { PARAMETER } \\
\text { VALUE }\end{array}$ & FLAGS & $\begin{array}{l}\text { DETECTION } \\
\text { LIMIT }\end{array}$ & $\begin{array}{l}\text { PARAMETER } \\
\text { UNCERTAINTY }\end{array}$ \\
\hline GROSS BETA & $\begin{array}{l}0932 \\
0932 \\
0933 \\
0933 \\
0934 \\
0934 \\
0935 \\
0935 \\
0936 \\
0936 \\
0937 \\
0937 \\
0939 \\
0939 \\
0940 \\
0940 \\
0941 \\
0941 \\
0942 \\
0942 \\
0943 \\
0943 \\
0944 \\
0944\end{array}$ & $\begin{array}{l}08 / 09 / 88 \\
12 / 07 / 88 \\
08 / 10 / 88 \\
12 / 07 / 88 \\
08 / 11 / 88 \\
12 / 07 / 88 \\
08 / 09 / 88 \\
12 / 04 / 88 \\
08 / 08 / 88 \\
12 / 04 / 88 \\
08 / 08 / 88 \\
12 / 04 / 88 \\
08 / 08 / 88 \\
12 / 04 / 88 \\
08 / 08 / 88 \\
12 / 04 / 88 \\
08 / 08 / 88 \\
12 / 10 / 88 \\
08 / 08 / 88 \\
12 / 04 / 88 \\
08 / 09 / 88 \\
12 / 07 / 88 \\
08 / 11 / 88 \\
12 / 07 / 88\end{array}$ & $\begin{array}{l}0001 \\
0001 \\
0001 \\
0001 \\
0001 \\
0001 \\
0001 \\
0001 \\
0001 \\
0001 \\
0001 \\
0001 \\
0001 \\
0001 \\
0001 \\
0001 \\
0001 \\
0001 \\
0001 \\
0001 \\
0001 \\
0001 \\
0001 \\
0001\end{array}$ & $\begin{array}{l}W U \\
W U \\
W U \\
W U \\
W U \\
W U \\
W U \\
W U \\
W U \\
W U \\
W U \\
W U \\
W U \\
W U \\
W U \\
W U \\
W U \\
W U \\
W U \\
W U \\
W U \\
W U \\
W U \\
W U\end{array}$ & $\begin{array}{l}N \\
N \\
N \\
N \\
N \\
N \\
N \\
N \\
N \\
N \\
N \\
N \\
N \\
N \\
N \\
N \\
N \\
N \\
N \\
N \\
N \\
N \\
N \\
N\end{array}$ & $\mathrm{PCI} / \mathrm{L}$ & & $\begin{array}{c}140 . \\
110 . \\
350 . \\
320 . \\
210 . \\
200 . \\
290 . \\
330 . \\
700 . \\
510 . \\
1720 . \\
860 . \\
71 . \\
49 . \\
1400 . \\
860 . \\
57 . \\
9.3 \\
190 . \\
110 . \\
640 . \\
510 . \\
220 . \\
180 .\end{array}$ & & $\begin{array}{l}1 . \\
i . \\
i . \\
i . \\
i . \\
i . \\
1 . \\
1 . \\
1 . \\
1 . \\
1 . \\
1 . \\
1 . \\
i . \\
1 . \\
i . \\
i . \\
1 .\end{array}$ & $\begin{array}{c}10 . \\
10 . \\
10 . \\
10 . \\
10 . \\
10 . \\
20 . \\
20 . \\
20 . \\
10 . \\
20 . \\
10 . \\
6 . \\
6 . \\
100 . \\
40 . \\
3 . \\
2.4 \\
10 . \\
4 . \\
10 . \\
10 . \\
10 . \\
10 .\end{array}$ \\
\hline IRON & $\begin{array}{l}0911 \\
0911 \\
0911 \\
0911 \\
0911 \\
0911 \\
0912 \\
0912 \\
0912 \\
0912 \\
0912 \\
0913 \\
0913 \\
0913 \\
0913\end{array}$ & \begin{tabular}{|l|}
$02 / 17 / 87$ \\
$09 / 18 / 87$ \\
$01 / 30 / 88$ \\
$04 / 22 / 88$ \\
$08 / 11 / 88$ \\
$12 / 10 / 88$ \\
$02 / 18 / 87$ \\
$02 / 03 / 88$ \\
$04 / 22 / 88$ \\
$08 / 27 / 88$ \\
$12 / 04 / 88$ \\
$02 / 18 / 87$ \\
$09 / 18 / 87$ \\
$02 / 03 / 88$ \\
$04 / 22 / 88$
\end{tabular} & $\begin{array}{l}0001 \\
0001 \\
0001 \\
0001 \\
0001 \\
0001 \\
0001 \\
0001 \\
0001 \\
0001 \\
0001 \\
0001 \\
0001 \\
0001 \\
0001\end{array}$ & $\begin{array}{l}W U \\
W U \\
W U \\
W U \\
W U \\
W U \\
W U \\
W U \\
W U \\
W U \\
W U \\
W U \\
W U \\
W U \\
W U\end{array}$ & $\begin{array}{l}N \\
N \\
N \\
N \\
N \\
N \\
N \\
N \\
N \\
N \\
N \\
N \\
N \\
N \\
N\end{array}$ & $\mathrm{HG} / \mathrm{L}$ & $\mid \begin{array}{l}< \\
< \\
< \\
< \\
< \\
< \\
< \\
<\end{array}$ & $\begin{array}{l}0.03 \\
0.01 \\
0.49 \\
0.04 \\
0.03 \\
0.03 \\
0.03 \\
0.16 \\
0.02 \\
0.06 \\
0.03 \\
0.03 \\
0.01 \\
1.77 \\
0.08\end{array}$ & J & $\begin{array}{l}0.03 \\
0.03 \\
0.03 \\
0.03 \\
0.03 \\
0.03 \\
0.03 \\
0.03 \\
0.03 \\
0.03 \\
0.03 \\
0.03 \\
0.03 \\
0.03 \\
0.03\end{array}$ & $\begin{array}{l}- \\
- \\
- \\
- \\
- \\
- \\
- \\
- \\
- \\
-\end{array}$ \\
\hline
\end{tabular}

FORMATION OF COMPLETION CODE:

FLOW RELATIONSHIP CODE:

HU - HASATCH - UPPER SANDSTONE

N - UNKNOWM

PARAMETER VALUE INDICATOR (PVI): < - LESS THAN DETECTION LIMIT

SAMPLE ID CODES:

0001 - FILTERED SAMPLE (.45 MICRONS)

OTHER PARAMETER VALUE FLAGS:

$J$ - ESTIMATED VALUE 


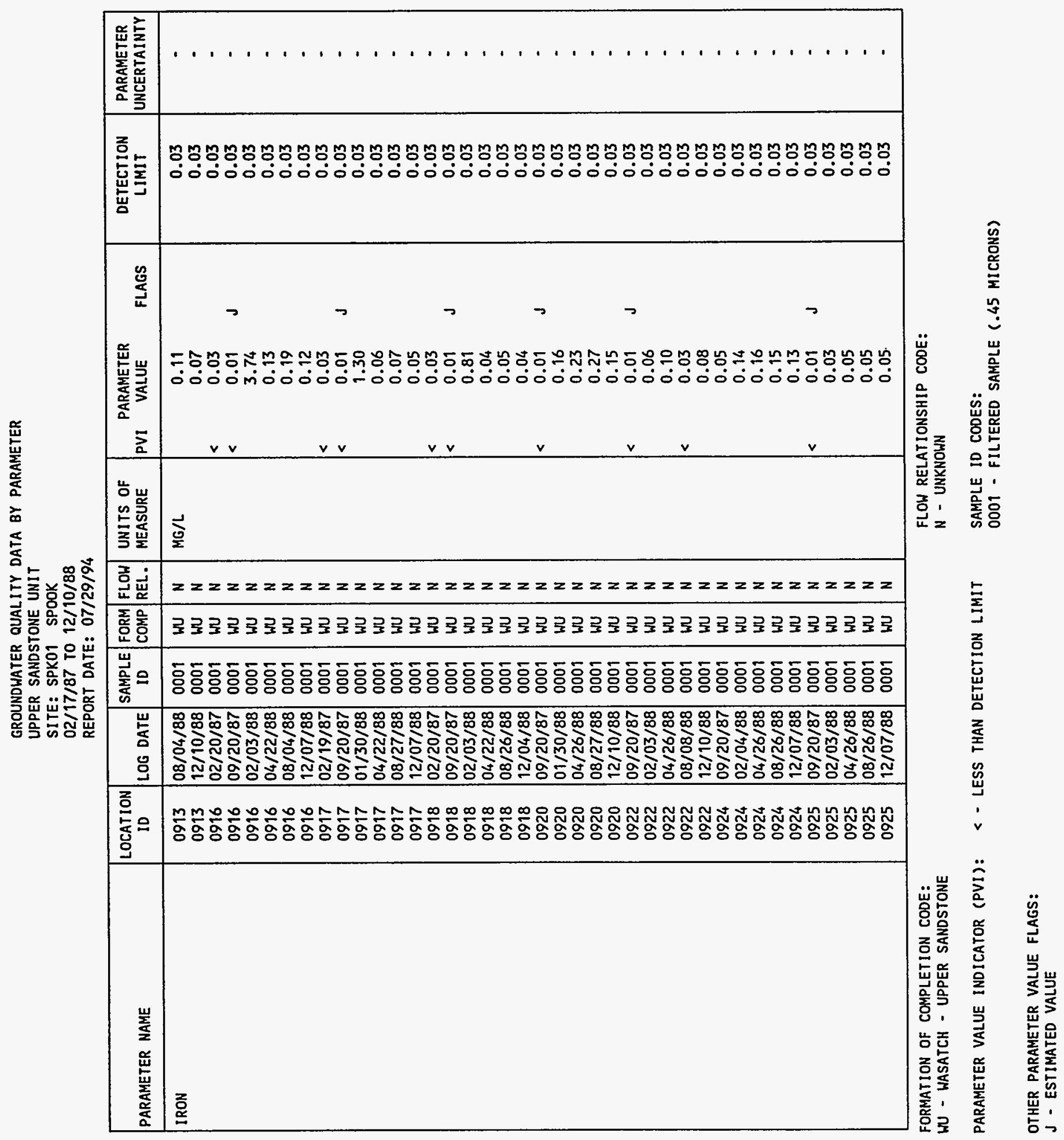




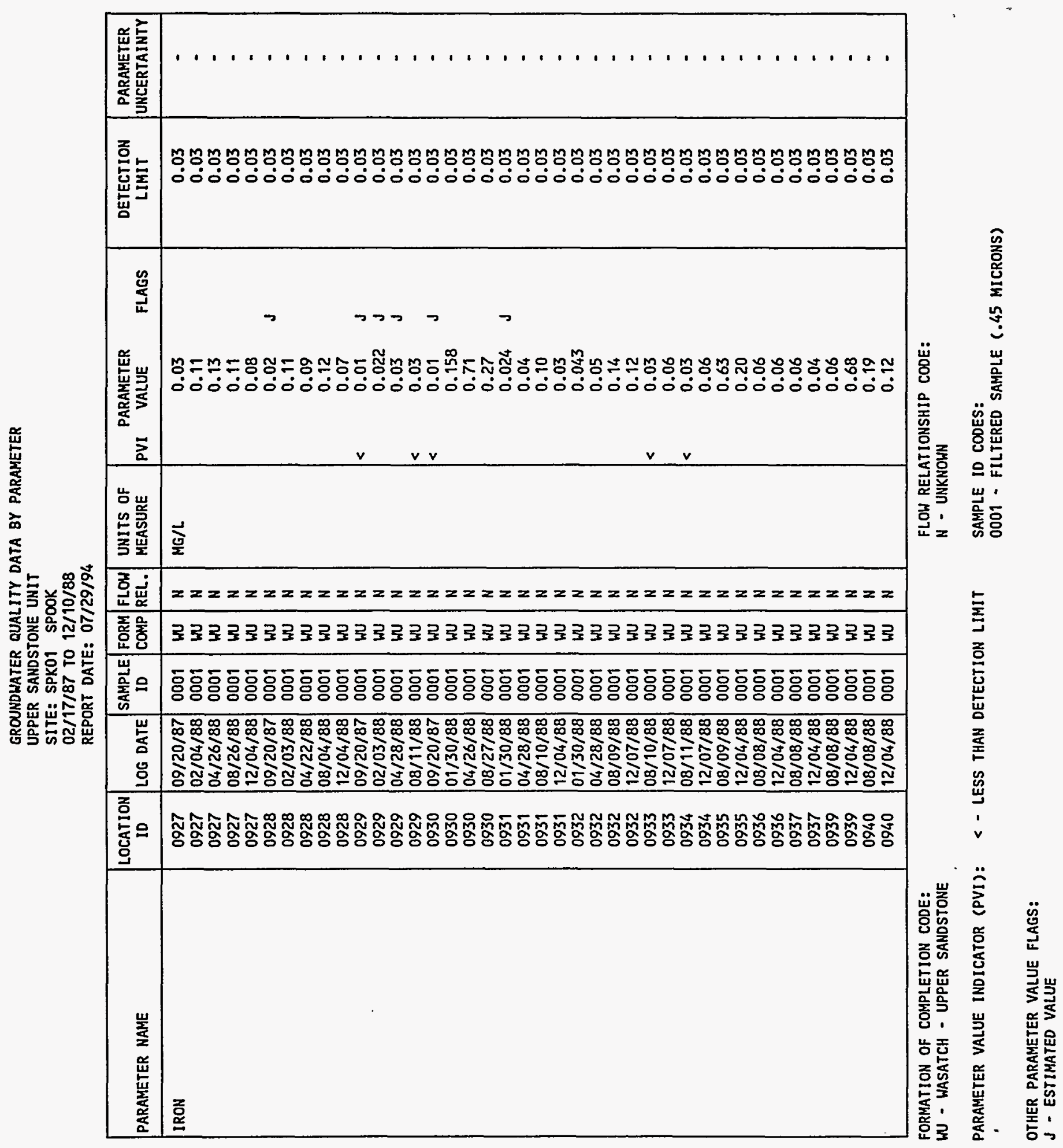




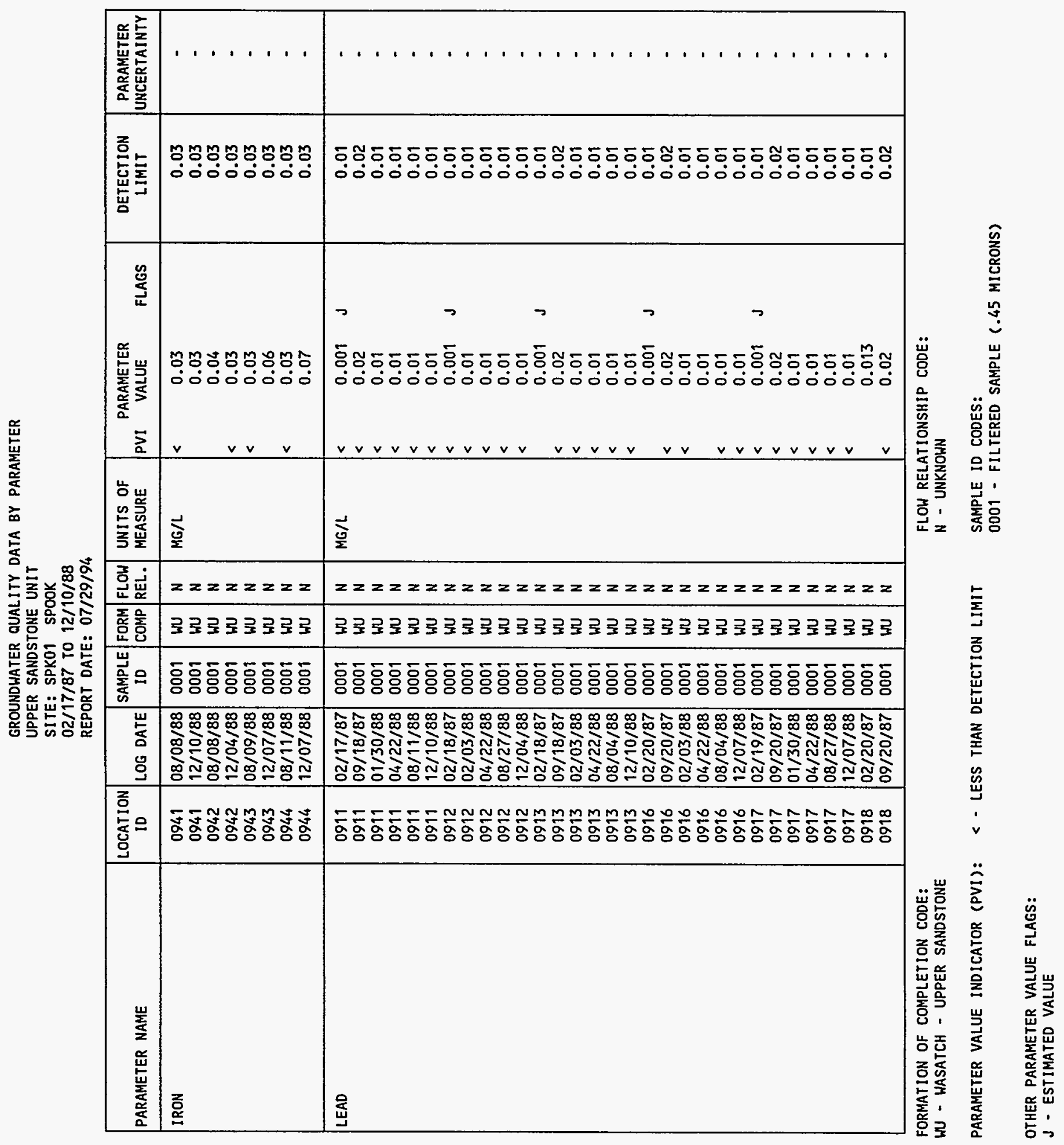




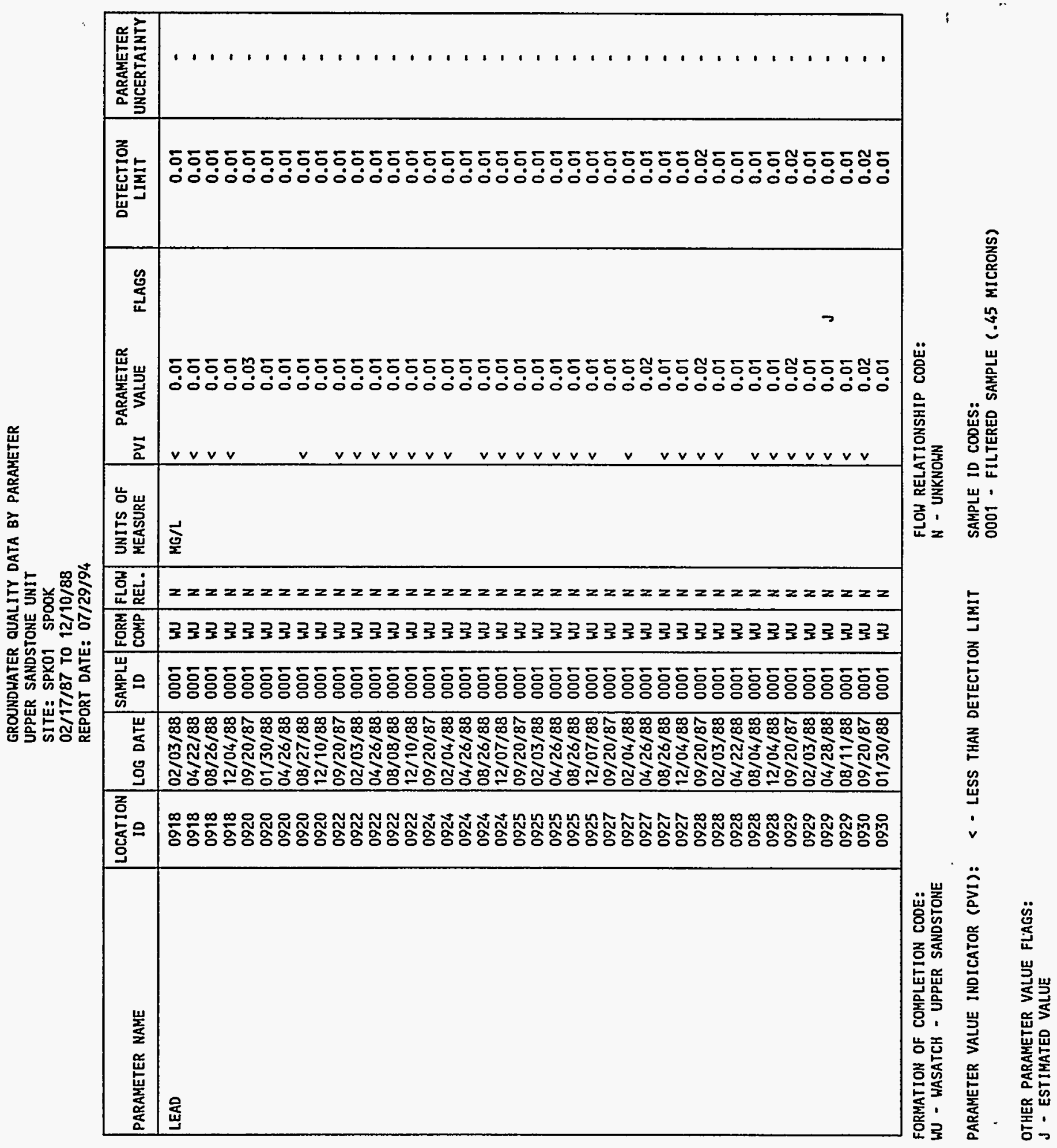




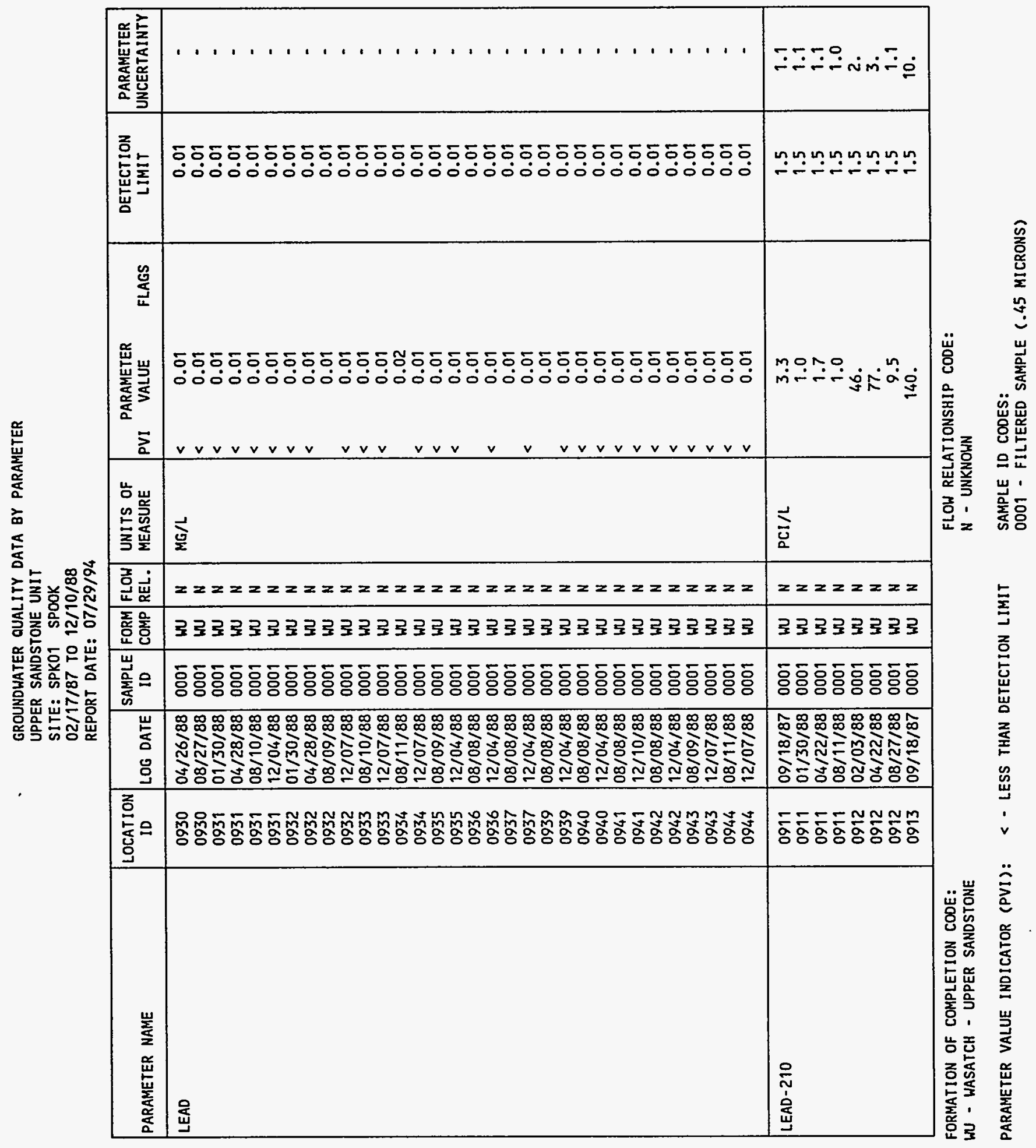


GROUNDHATER QUALITY DATA BY PARAMETER

UPPER SANDSTONE UNIT

SITE: SPKO1 SPOOK

$02 / 17 / 87$ TO $12 / 10 / 88$

REPORT DATE: $07 / 29 / 94$

\begin{tabular}{|c|c|c|c|c|c|c|c|c|c|c|c|}
\hline PARAMETER NAME & $\underset{\text { ID }}{\text { LOCATION }}$ & LOG DATE & $\begin{array}{c}\text { SAMPLE } \\
\text { ID }\end{array}$ & $\begin{array}{l}\text { FORM } \\
\text { COAPP }\end{array}$ & $\begin{array}{l}\text { FLOW } \\
\text { REL. }\end{array}$ & $\begin{array}{l}\text { UNITS OF } \\
\text { MEASURE }\end{array}$ & |PVI & $\begin{array}{l}\text { PARAMETER } \\
\text { VALUE }\end{array}$ & FLAGS & $\begin{array}{l}\text { DETECTION } \\
\text { LIMIT }\end{array}$ & $\begin{array}{l}\text { PARAMETER } \\
\text { UNCERTAINTY }\end{array}$ \\
\hline LEAD-210 & $\begin{array}{l}0913 \\
0913 \\
0913 \\
0916 \\
0916 \\
0916 \\
0916 \\
0917 \\
0917 \\
0917 \\
0917 \\
0918 \\
0918 \\
0918 \\
0918 \\
0920 \\
0920 \\
0920 \\
0920 \\
0922 \\
0922 \\
0922 \\
0922 \\
0924 \\
0924 \\
0924 \\
0924 \\
0925 \\
0925 \\
0925 \\
0925 \\
0927 \\
0927 \\
0927 \\
0927 \\
0928 \\
0928 \\
0928 \\
0928 \\
0929 \\
0929\end{array}$ & $\begin{array}{l}02 / 03 / 88 \\
04 / 22 / 88 \\
08 / 04 / 88 \\
09 / 20 / 87 \\
02 / 03 / 88 \\
04 / 22 / 88 \\
08 / 04 / 88 \\
09 / 20 / 87 \\
01 / 30 / 88 \\
04 / 22 / 88 \\
08 / 27 / 88 \\
09 / 20 / 87 \\
02 / 03 / 88 \\
04 / 22 / 88 \\
08 / 26 / 88 \\
09 / 20 / 87 \\
01 / 30 / 88 \\
04 / 26 / 88 \\
08 / 27 / 88 \\
09 / 20 / 87 \\
02 / 03 / 88 \\
04 / 26 / 88 \\
08 / 08 / 88 \\
09 / 20 / 87 \\
02 / 04 / 88 \\
04 / 26 / 88 \\
08 / 26 / 88 \\
09 / 20 / 87 \\
02 / 03 / 88 \\
04 / 26 / 88 \\
08 / 26 / 88 \\
09 / 20 / 87 \\
02 / 04 / 88 \\
04 / 26 / 88 \\
08 / 26 / 88 \\
09 / 20 / 87 \\
02 / 03 / 88 \\
04 / 22 / 88 \\
08 / 04 / 88 \\
09 / 20 / 87 \\
02 / 03 / 88\end{array}$ & $\begin{array}{l}0001 \\
0001 \\
0001 \\
0001 \\
0001 \\
0001 \\
0001 \\
0001 \\
0001 \\
0001 \\
0001 \\
0001 \\
0001 \\
0001 \\
0001 \\
0001 \\
0001 \\
0001 \\
0001 \\
0001 \\
0001 \\
0001 \\
0001 \\
0001 \\
0001 \\
0001 \\
0001 \\
0001 \\
0001 \\
0001 \\
0001 \\
0001 \\
0001 \\
0001 \\
0001 \\
0001 \\
0001 \\
0001 \\
0001 \\
0001 \\
0001\end{array}$ & $\begin{array}{l}W U \\
W U \\
W U \\
W U \\
W U \\
W U \\
W U \\
W U \\
W U \\
W U \\
W U \\
W U \\
W U \\
W U \\
W U \\
W U \\
W U \\
W U \\
W U \\
W U \\
W U \\
W U \\
W U \\
W U \\
W U \\
W U \\
W U \\
W U \\
W U \\
W U \\
W U \\
W U \\
W U \\
W U \\
W U \\
W U \\
W U \\
W U \\
W U \\
W U \\
W U\end{array}$ & $\begin{array}{l}N \\
N \\
N \\
N \\
N \\
N \\
N \\
N \\
N \\
N \\
N \\
N \\
N \\
N \\
N \\
N \\
N \\
N \\
N \\
N \\
N \\
N \\
N \\
N \\
N \\
N \\
N \\
N \\
N \\
N \\
N \\
N \\
N \\
N \\
N \\
N \\
N \\
N \\
N \\
N \\
N\end{array}$ & $\mathrm{PCI} / \mathrm{L}$ & & $\begin{array}{c}100 . \\
22 . \\
94 . \\
19 . \\
20 . \\
5.0 \\
8.1 \\
2.9 \\
0.0 \\
1.2 \\
2.1 \\
4.1 \\
0.6 \\
0.3 \\
0.9 \\
9.5 \\
15 . \\
11 . \\
14 . \\
0.9 \\
1.1 \\
0.0 \\
1.8 \\
21 . \\
18 . \\
9.9 \\
15 . \\
1.8 \\
1.3 \\
1.3 \\
0.0 \\
0.4 \\
5.7 \\
5.5 \\
3.3 \\
5.5 \\
8.8 \\
8.6 \\
7.3 \\
1.3 \\
0.4 \\
0.0\end{array}$ & & $\begin{array}{l}1.5 \\
1.5 \\
1.5 \\
1.5 \\
1.5 \\
1.5 \\
1.5 \\
1.5 \\
1.5 \\
1.5 \\
1.5 \\
1.5 \\
1.5 \\
1.5 \\
1.5 \\
1.5 \\
1.5 \\
1.5 \\
1.5 \\
1.5 \\
1.5 \\
1.5 \\
1.5 \\
1.5 \\
1.5 \\
1.5 \\
1.5 \\
1.5 \\
1.5 \\
1.5 \\
1.5 \\
1.5 \\
1.5 \\
1.5 \\
1.5 \\
1.5 \\
1.5 \\
1.5 \\
1.5 \\
1.5 \\
1.5\end{array}$ & $\begin{array}{l}3 . \\
2 . \\
4 . \\
2 . \\
2 . \\
1.2 \\
1.2 \\
1.1 \\
0.9 \\
1.0 \\
0.8 \\
1.1 \\
1.0 \\
0.8 \\
0.8 \\
1.4 \\
1 . \\
1 . \\
1 . \\
1.0 \\
0.8 \\
0.9 \\
1.1 \\
2 . \\
1 . \\
1.1 \\
1.1 \\
1.2 \\
0.8 \\
0.8 \\
0.8 \\
1.5 \\
1.0 \\
1.0 \\
1.1 \\
1.3 \\
1.1 \\
1.1 \\
1.1 \\
1.0 \\
0.8\end{array}$ \\
\hline
\end{tabular}

FORMATION OF COMPLETION CODE:

FLOW RELATIONSHIP CODE:

WU - HASATCH - UPPER SANDSTONE

N - UNKNOWN

PARAMETER VALUE INDICATOR (PVI): < - LESS THAN DETECTION LIMIT SAMPLE ID CODES:

0001 - FILTERED SAMPLE (.45 MICRONS) 
GROUNDHATER QUALITY DATA BY PARAMETER

UPPER SANDSTONE UNIT

SITE: SPKO1 SPOOK

REPORT DATE: $07 / 29 / 94$

\begin{tabular}{|c|c|c|c|c|c|c|c|c|c|c|c|}
\hline PARAMETER NAME & $\underset{10}{\text { LOCATION }}$ & LOG DATE & $\begin{array}{c}\text { SAMPLE } \\
\text { ID }\end{array}$ & $\begin{array}{l}\text { FORM } \\
\text { COMP }\end{array}$ & $\begin{array}{l}\text { FLOW } \\
\text { REL. }\end{array}$ & $\begin{array}{l}\text { UNITS OF } \\
\text { MEASURE }\end{array}$ & PVI & $\begin{array}{l}\text { PARAMETER } \\
\text { VALUE }\end{array}$ & FLAGS & $\begin{array}{l}\text { DETECTION } \\
\text { LIMIT }\end{array}$ & $\begin{array}{l}\text { PARAMETER } \\
\text { UNCERTAINTY }\end{array}$ \\
\hline LEAD-210 & $\begin{array}{l}0929 \\
0929 \\
0930 \\
0930 \\
0930 \\
0930 \\
0931 \\
0931 \\
0931 \\
0932 \\
0932 \\
0932 \\
0933 \\
0934 \\
0935 \\
0936 \\
0937 \\
0939 \\
0940 \\
0941 \\
0942 \\
0943 \\
0944\end{array}$ & $\begin{array}{l}04 / 28 / 88 \\
08 / 11 / 88 \\
09 / 20 / 87 \\
01 / 30 / 88 \\
04 / 26 / 88 \\
08 / 27 / 88 \\
01 / 30 / 88 \\
04 / 28 / 88 \\
08 / 10 / 88 \\
01 / 30 / 88 \\
04 / 28 / 88 \\
08 / 09 / 88 \\
08 / 10 / 88 \\
08 / 11 / 88 \\
08 / 09 / 88 \\
08 / 08 / 88 \\
08 / 08 / 88 \\
08 / 08 / 88 \\
08 / 08 / 88 \\
08 / 08 / 88 \\
08 / 08 / 88 \\
08 / 09 / 88 \\
08 / 11 / 88\end{array}$ & $\begin{array}{l}0001 \\
0001 \\
0001 \\
0001 \\
0001 \\
0001 \\
0001 \\
0001 \\
0001 \\
0001 \\
0001 \\
0001 \\
0001 \\
0001 \\
0001 \\
0001 \\
0001 \\
0001 \\
0001 \\
0001 \\
0001 \\
0001 \\
0001\end{array}$ & $\begin{array}{l}\text { WU } \\
W U \\
W U \\
W U \\
W U \\
W U \\
W U \\
W U \\
W U \\
W U \\
W U \\
W U \\
W U \\
W U \\
W U \\
W U \\
W U \\
W U \\
W U \\
W U \\
W U \\
W U \\
W U\end{array}$ & $\begin{array}{l}N \\
N \\
N \\
N \\
N \\
N \\
N \\
N \\
N \\
N \\
N \\
N \\
N \\
N \\
N \\
N \\
N \\
N \\
N \\
N \\
N \\
N \\
N\end{array}$ & $\mathrm{PCI} / \mathrm{L}$ & & $\begin{array}{c}0.7 \\
0.5 \\
0.9 \\
1.4 \\
2.1 \\
1.6 \\
0.9 \\
0.7 \\
13 . \\
24 . \\
21 . \\
29 . \\
2.9 \\
2.4 \\
7.2 \\
3.4 \\
5.2 \\
1.8 \\
20 . \\
0.8 \\
2.2 \\
1.9 \\
1.0\end{array}$ & $\mathbf{J}$ & $\begin{array}{l}1.5 \\
1.5 \\
1.5 \\
1.5 \\
1.5 \\
1.5 \\
1.5 \\
1.5 \\
1.5 \\
1.5 \\
1.5 \\
1.5 \\
1.5 \\
1.5 \\
1.5 \\
1.5 \\
1.5 \\
1.5 \\
1.5 \\
1.5 \\
1.5 \\
1.5 \\
1.5\end{array}$ & $\begin{array}{l}1.2 \\
0.8 \\
1.1 \\
1.0 \\
1.4 \\
0.9 \\
0.8 \\
1.1 \\
1.1 \\
1 . \\
2 . \\
2 . \\
1.6 \\
1.3 \\
1.5 \\
1.2 \\
1.2 \\
1.2 \\
2 . \\
1.0 \\
1.2 \\
1.2 \\
0.7\end{array}$ \\
\hline MAGNESIUM & $\begin{array}{l}0911 \\
0911 \\
0911 \\
0911 \\
0911 \\
0911 \\
0912 \\
0912 \\
0912 \\
0912 \\
0912 \\
0913 \\
0913 \\
0913 \\
0913 \\
0913\end{array}$ & $\begin{array}{l}02 / 17 / 87 \\
09 / 18 / 87 \\
01 / 30 / 88 \\
04 / 22 / 88 \\
08 / 11 / 88 \\
12 / 10 / 88 \\
02 / 18 / 87 \\
02 / 03 / 88 \\
04 / 22 / 88 \\
08 / 27 / 88 \\
12 / 04 / 88 \\
02 / 18 / 87 \\
09 / 18 / 87 \\
02 / 03 / 88 \\
04 / 22 / 88 \\
08 / 04 / 88\end{array}$ & $\begin{array}{l}0001 \\
0001 \\
0001 \\
0001 \\
0001 \\
0001 \\
0001 \\
0001 \\
0001 \\
0001 \\
0001 \\
0001 \\
0001 \\
0001 \\
0001 \\
0001\end{array}$ & $\begin{array}{l}W U \\
W U \\
W U \\
W U \\
W U \\
W U \\
W U \\
W U \\
W U \\
W U \\
W U \\
W U \\
W U \\
W U \\
W U \\
W U\end{array}$ & $\begin{array}{l}N \\
N \\
N \\
N \\
N \\
N \\
N \\
N \\
N \\
N \\
N \\
N \\
N \\
N \\
N \\
N\end{array}$ & MG/L & & $\begin{array}{l}48.3 \\
21.3 \\
23.4 \\
22.8 \\
22.7 \\
23.4 \\
5.25 \\
11.3 \\
10.3 \\
10.7 \\
10.7 \\
39.4 \\
44.8 \\
49.6 \\
46.3 \\
48.7\end{array}$ & & $\begin{array}{l}0.001 \\
0.001 \\
0.001 \\
0.001 \\
0.001 \\
0.001 \\
0.001 \\
0.001 \\
0.001 \\
0.001 \\
0.001 \\
0.001 \\
0.001 \\
0.001 \\
0.001 \\
0.001\end{array}$ & $\begin{array}{l}- \\
- \\
- \\
- \\
- \\
- \\
- \\
- \\
- \\
- \\
-\end{array}$ \\
\hline
\end{tabular}

FORMATION OF COMPLETION CODE:

HU - HASATCH - UPPER SANDSTONE

FLOW RELATIONSHIP CODE:

PARAMETER VALUE INDICATOR (PVI): < - LESS THAN DETECTION LIMIT

SAMPLE ID CODES:

0001 - FILTERED SAMPLE (.45 MICRONS)

OTHER PARAMETER VALUE FLAGS:

$J$ - ESTIMATED VALUE 


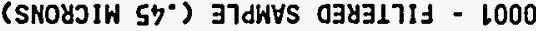
: $\$ \exists 000$ al $\exists 7 d W \forall S$

: 3000 dIHSNOIHY7Э M07:

\begin{tabular}{|c|c|c|c|c|c|c|c|c|c|c|}
\hline $\begin{array}{l}- \\
- \\
- \\
- \\
- \\
- \\
- \\
- \\
- \\
- \\
- \\
- \\
- \\
- \\
- \\
- \\
- \\
- \\
- \\
- \\
- \\
- \\
- \\
- \\
- \\
- \\
-\end{array}$ & $\begin{array}{l}100^{\circ} 0 \\
100^{\circ} 0 \\
100^{\circ} 0 \\
100^{\circ} 0 \\
100^{\circ} 0 \\
100^{\circ} 0 \\
100^{\circ} 0 \\
100^{\circ} 0 \\
100^{\circ} 0 \\
100^{\circ} 0 \\
100^{\circ} 0 \\
100^{\circ} 0 \\
100^{\circ} 0 \\
100^{\circ} 0 \\
100^{\circ} 0 \\
100^{\circ} 0 \\
100^{\circ} 0 \\
100^{\circ} 0 \\
100^{\circ} 0 \\
100^{\circ} 0 \\
100^{\circ} 0 \\
100^{\circ} 0 \\
100^{\circ} 0 \\
100^{\circ} 0 \\
100^{\circ} 0 \\
100^{\circ} 0 \\
100^{\circ} 0 \\
100^{\circ} 0 \\
100^{\circ} 0 \\
100^{\circ} 0 \\
100^{\circ} 0 \\
100^{\circ} 0 \\
100^{\circ} 0 \\
100^{\circ} 0 \\
100^{\circ} 0 \\
100^{\circ} 0 \\
100^{\circ} 0 \\
100^{\circ} 0 \\
100^{\circ} 0 \\
100^{\circ} 0 \\
100^{\circ} 0\end{array}$ & & 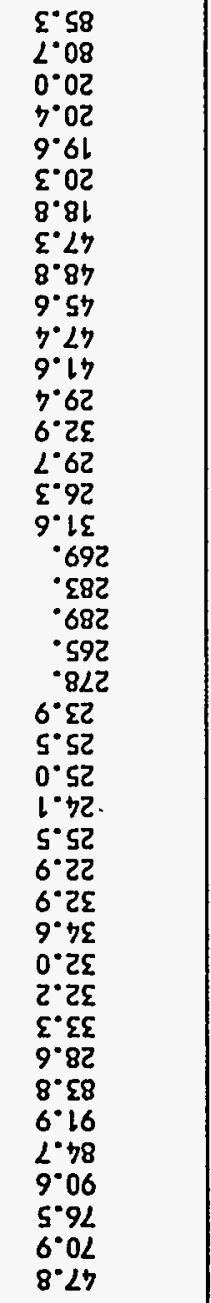 & 列 & $\begin{array}{l}N \\
N \\
N \\
N \\
N \\
N \\
N \\
N \\
N \\
N \\
N \\
N \\
N \\
N \\
N \\
N \\
N \\
N \\
N \\
N \\
N \\
N \\
N \\
N \\
N \\
N \\
N \\
N \\
N \\
N \\
N \\
N \\
N \\
N \\
N \\
N \\
N \\
N \\
N \\
N \\
N\end{array}$ & $\begin{array}{l}n M \\
n M \\
n M \\
n M \\
n M \\
n M \\
n M \\
n M \\
n M \\
n M \\
n M \\
n M \\
n M \\
n M \\
n M \\
n M \\
n M \\
n M \\
n M \\
n M \\
n M \\
n M \\
n M \\
n M \\
n M \\
n M \\
n M \\
n M \\
n M \\
n M \\
n M \\
n M \\
n M \\
n M \\
n M \\
n M \\
M M \\
n M \\
n M \\
n M \\
n M\end{array}$ & $\begin{array}{l}1000 \\
1000 \\
1000 \\
1000 \\
1000 \\
1000 \\
1000 \\
1000 \\
1000 \\
1000 \\
1000 \\
1000 \\
1000 \\
1000 \\
1000 \\
1000 \\
1000 \\
1000 \\
1000 \\
1000 \\
1000 \\
1000 \\
1000 \\
1000 \\
1000 \\
1000 \\
1000 \\
1000 \\
1000 \\
1000 \\
1000 \\
1000 \\
1000 \\
1000 \\
1000 \\
1000 \\
1000 \\
1000 \\
1000 \\
1000 \\
1000\end{array}$ & 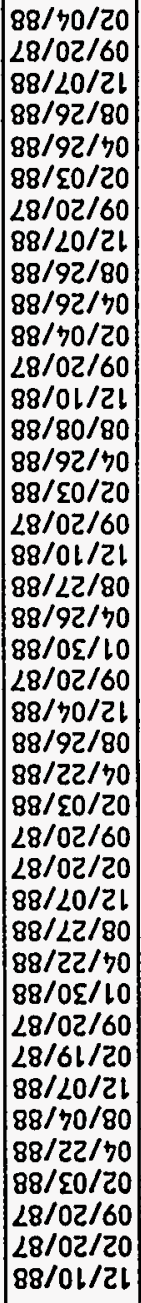 & 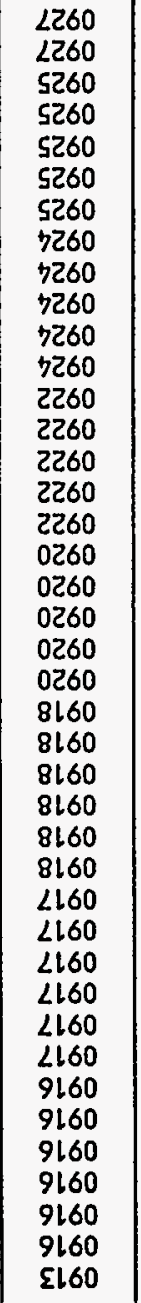 & พกISอNงษพ \\
\hline 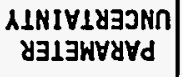 & $\begin{array}{c}\text { 1IWI7 } \\
\text { NOI1J3130 }\end{array}$ & נטาง & 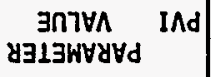 & 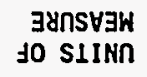 & -7 738 & $\begin{array}{l}\text { dWOS } \\
\text { WYOS }\end{array}$ & $\begin{array}{c}\text { QI } \\
\text { כ7dWUS }\end{array}$ & 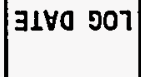 & $\begin{array}{c}\text { OI } \\
\text { NOIIษวOา }\end{array}$ & 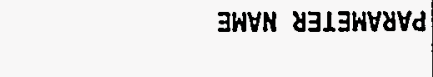 \\
\hline
\end{tabular}

$76 / 62 / 20$ : $\exists 170$ LYOdヨy

$88 / 01 / 21$ ol $28 / L L / 20$

XoOdS lOXdS : $\exists 115$

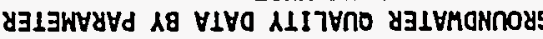

- 


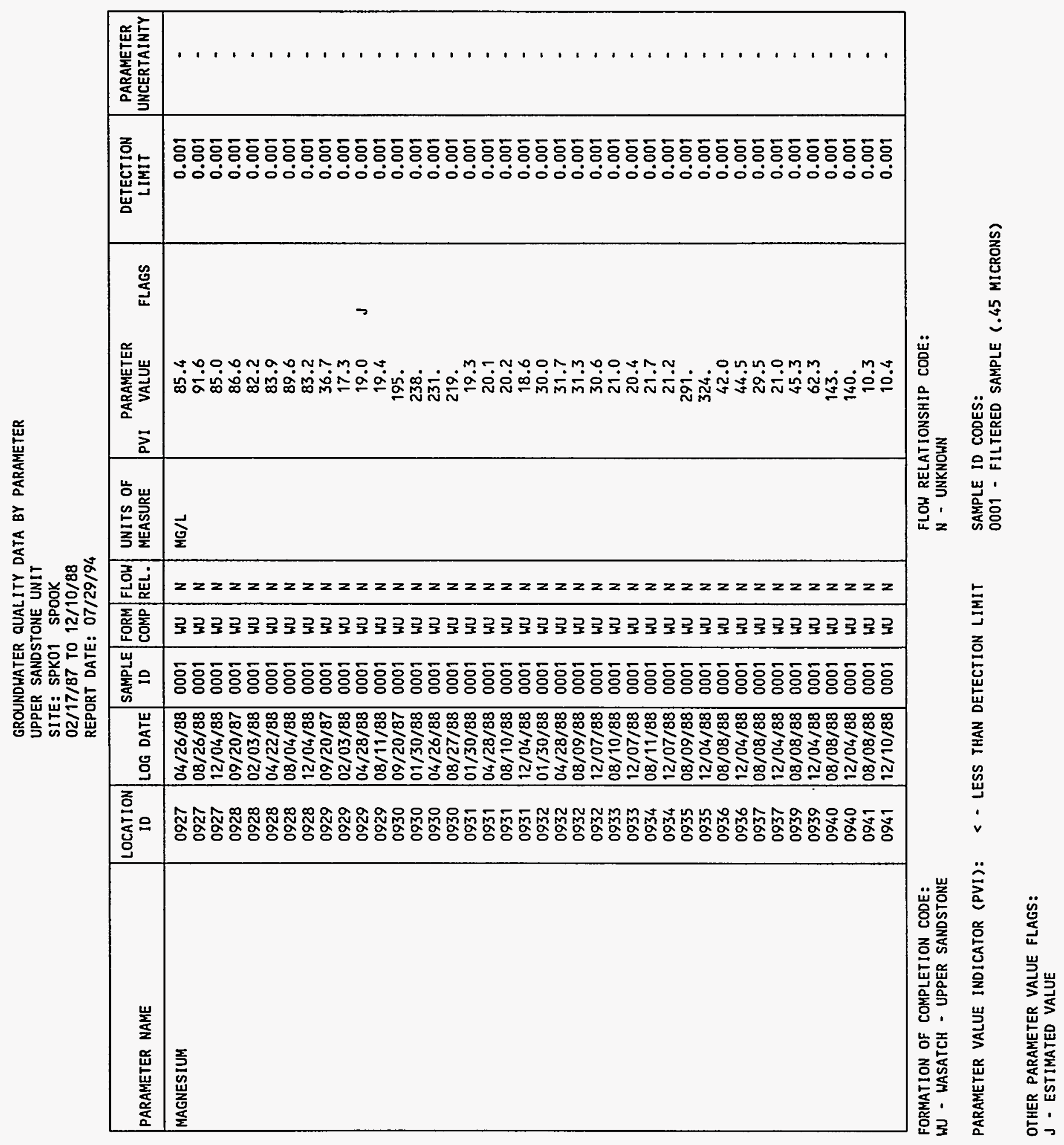


GROUNDWATER QUALITY DATA BY PARAMETER

UPPER SANDSTONE UNIT

SITE: SPKO1 SPOOK

02/17/87 TO $12 / 10 / 88$

REPORT DATE: $07 / 29 / 94$

\begin{tabular}{|c|c|c|c|c|c|c|c|c|c|c|}
\hline PARAMETER NAME & $\underset{\text { ID }}{\text { LOCATION }}$ & LOG DATE & $\underset{\text { ID }}{\text { SAMPLE }}$ & $\begin{array}{l}\text { FORM } \\
\text { COMP }\end{array}$ & $\begin{array}{l}\text { FLOH } \\
\text { REL. }\end{array}$ & $\begin{array}{l}\text { UNITS OF } \\
\text { MEASURE }\end{array}$ & PVI & $\begin{array}{l}\text { PARAMETER } \\
\text { VALUE }\end{array}$ & $\begin{array}{l}\text { DETECTION } \\
\text { LIMIT }\end{array}$ & $\begin{array}{l}\text { PARAMETER } \\
\text { UNCERTAINTY }\end{array}$ \\
\hline MAGNESIUM & $\begin{array}{l}0942 \\
0942 \\
0943 \\
0943 \\
0944 \\
0944\end{array}$ & $\begin{array}{l}08 / 08 / 88 \\
12 / 04 / 88 \\
08 / 09 / 88 \\
12 / 07 / 88 \\
08 / 11 / 88 \\
12 / 07 / 88\end{array}$ & $\begin{array}{l}0001 \\
0001 \\
0001 \\
0001 \\
0001 \\
0001\end{array}$ & $\begin{array}{l}W U \\
W U \\
W U \\
W U \\
W U \\
W U\end{array}$ & $\begin{array}{l}N \\
N \\
N \\
N \\
N \\
N\end{array}$ & MG/L & & $\begin{array}{l}23.3 \\
20.5 \\
15.7 \\
15.3 \\
36.3 \\
35.0\end{array}$ & $\begin{array}{l}0.001 \\
0.001 \\
0.001 \\
0.001 \\
0.001 \\
0.001\end{array}$ & $\begin{array}{l}- \\
: \\
-\end{array}$ \\
\hline MANGANESE & $\begin{array}{l}0911 \\
0911 \\
0911 \\
0911 \\
0911 \\
0911 \\
0912 \\
0912 \\
0912 \\
0912 \\
0912 \\
0913 \\
0913 \\
0913 \\
0913 \\
0913 \\
0913 \\
0916 \\
0916 \\
0916 \\
0916 \\
0916 \\
0916 \\
0917 \\
0917 \\
0917 \\
0917 \\
0917 \\
0917 \\
0918 \\
0918 \\
0918 \\
0918 \\
0918\end{array}$ & $\begin{array}{l}02 / 17 / 87 \\
09 / 18 / 87 \\
01 / 30 / 88 \\
04 / 22 / 88 \\
08 / 11 / 88 \\
12 / 10 / 88 \\
02 / 18 / 87 \\
02 / 03 / 88 \\
04 / 22 / 88 \\
08 / 27 / 88 \\
12 / 04 / 88 \\
02 / 18 / 87 \\
09 / 18 / 87 \\
02 / 03 / 88 \\
04 / 22 / 88 \\
08 / 04 / 88 \\
12 / 10 / 88 \\
02 / 20 / 87 \\
09 / 20 / 87 \\
02 / 03 / 88 \\
04 / 22 / 88 \\
08 / 04 / 88 \\
12 / 07 / 88 \\
02 / 19 / 87 \\
09 / 20 / 87 \\
01 / 30 / 88 \\
04 / 22 / 88 \\
08 / 27 / 88 \\
12 / 07 / 88 \\
02 / 20 / 87 \\
09 / 20 / 87 \\
02 / 03 / 88 \\
04 / 22 / 88 \\
08 / 26 / 88\end{array}$ & $\begin{array}{l}0001 \\
0001 \\
0001 \\
0001 \\
0001 \\
0001 \\
0001 \\
0001 \\
0001 \\
0001 \\
0001 \\
0001 \\
0001 \\
0001 \\
0001 \\
0001 \\
0001 \\
0001 \\
0001 \\
0001 \\
0001 \\
0001 \\
0001 \\
0001 \\
0001 \\
0001 \\
0001 \\
0001 \\
0001 \\
0001 \\
0001 \\
0001 \\
0001 \\
0001\end{array}$ & $\begin{array}{l}W U \\
W U \\
W U \\
W U \\
W U \\
W U \\
W U \\
W U \\
W U \\
W U \\
W U \\
W U \\
W U \\
W U \\
W U \\
W U \\
W U \\
W U \\
W U \\
W U \\
W U \\
W U \\
W U \\
W U \\
W U \\
W U \\
W U \\
W U \\
W U \\
W U \\
W U \\
W U \\
W U \\
W U\end{array}$ & $\begin{array}{l}N \\
N \\
N \\
N \\
N \\
N \\
N \\
N \\
N \\
N \\
N \\
N \\
N \\
N \\
N \\
N \\
N \\
N \\
N \\
N \\
N \\
N \\
N \\
N \\
N \\
N \\
N \\
N \\
N \\
N \\
N \\
N \\
N \\
N\end{array}$ & $M G / L$ & $<$ & $\begin{array}{l}0.40 \\
0.10 \\
0.10 \\
0.09 \\
0.10 \\
0.09 \\
0.02 \\
0.01 \\
0.02 \\
0.08 \\
0.06 \\
0.03 \\
0.03 \\
0.02 \\
0.02 \\
0.03 \\
0.02 \\
0.03 \\
0.04 \\
0.02 \\
0.03 \\
0.03 \\
0.03 \\
0\end{array}$ & $\begin{array}{l}0.01 \\
0.01 \\
0.01 \\
0.01 \\
0.01 \\
0.01 \\
0.01 \\
0.01 \\
0.01 \\
0.01 \\
0.01 \\
0.01 \\
0.01 \\
0.01 \\
0.01 \\
0.01 \\
0.01 \\
0.01 \\
0.01 \\
0.01 \\
0.01 \\
0.01 \\
0.01 \\
0.01 \\
0.01 \\
0.01 \\
0.01 \\
0.01 \\
0.01 \\
0.01 \\
0.01 \\
0.01 \\
0.01 \\
0.01\end{array}$ & 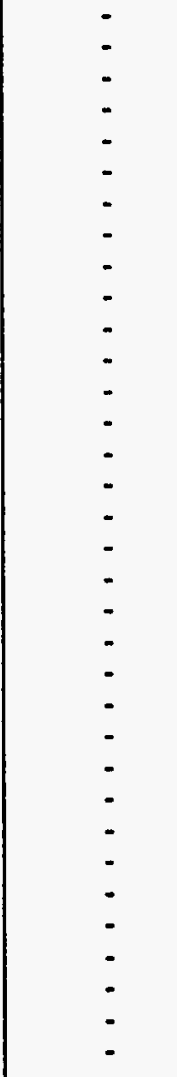 \\
\hline
\end{tabular}

FORMATION OF COMPLETION COOE:

WU - WASATCH - UPPER SANDSTONE

PARAMETER VALUE INDICATOR (PVI): < - LESS THAN DETECTION LIMIT
FLOW RELATIONSHIP CODE:

N - UNKNOWN 
GROUNDWATER QUALITY DATA BY PARAMETER

UPPER SANDSTONE UNIT

SITE: SPKO1 SPOOK

$02 / 17 / 87$ TO $12 / 10 / 88$

REPORT DATE : 07/29/94

\begin{tabular}{|c|c|c|c|c|c|c|c|c|c|c|c|}
\hline PARAMETER NAME & $\begin{array}{c}\text { LOCATION } \\
\text { ID }\end{array}$ & LOG DATE & $\begin{array}{c}\text { SAMPLE } \\
\text { ID }\end{array}$ & $\begin{array}{l}\text { FORM } \\
\text { COMP }\end{array}$ & $\begin{array}{l}\text { FLOW } \\
\text { REL. }\end{array}$ & $\begin{array}{l}\text { UNITS OF } \\
\text { MEASURE }\end{array}$ & PVI & $\begin{array}{l}\text { PARAMETER } \\
\text { VALUE }\end{array}$ & FLAGS & $\begin{array}{l}\text { DETECTION } \\
\text { LIMIT }\end{array}$ & $\begin{array}{l}\text { PARAMETER } \\
\text { UNCERTAINTY }\end{array}$ \\
\hline MANGANESE & $\begin{array}{l}0918 \\
0920 \\
0920 \\
0920 \\
0920 \\
0920 \\
0922 \\
0922 \\
0922 \\
0922 \\
0922 \\
0924 \\
0924 \\
0924 \\
0924 \\
0924 \\
0925 \\
0925 \\
0925 \\
0925 \\
0925 \\
0927 \\
0927 \\
0927 \\
0927 \\
0927 \\
0928 \\
0928 \\
0928 \\
0928 \\
0928 \\
0929 \\
0929 \\
0929 \\
0929 \\
0930 \\
0930 \\
0930 \\
0930 \\
0931\end{array}$ & $\begin{array}{l}12 / 04 / 88 \\
09 / 20 / 87 \\
01 / 30 / 88 \\
04 / 26 / 88 \\
08 / 27 / 88 \\
12 / 10 / 88 \\
09 / 20 / 87 \\
02 / 03 / 88 \\
04 / 26 / 88 \\
08 / 08 / 88 \\
12 / 10 / 88 \\
09 / 20 / 87 \\
02 / 04 / 88 \\
04 / 26 / 88 \\
08 / 26 / 88 \\
12 / 07 / 88 \\
09 / 20 / 87 \\
02 / 03 / 88 \\
04 / 26 / 88 \\
08 / 26 / 88 \\
12 / 07 / 88 \\
09 / 20 / 87 \\
02 / 04 / 88 \\
04 / 26 / 88 \\
08 / 26 / 88 \\
12 / 04 / 88 \\
09 / 20 / 87 \\
02 / 03 / 88 \\
04 / 22 / 88 \\
08 / 04 / 88 \\
12 / 04 / 88 \\
09 / 20 / 87 \\
02 / 03 / 88 \\
04 / 28 / 88 \\
08 / 11 / 88 \\
09 / 20 / 87 \\
01 / 30 / 88 \\
04 / 26 / 88 \\
08 / 27 / 88 \\
01 / 30 / 88\end{array}$ & $\begin{array}{l}0001 \\
0001 \\
0001 \\
0001 \\
0001 \\
0001 \\
0001 \\
0001 \\
0001 \\
0001 \\
0001 \\
0001 \\
0001 \\
0001 \\
0001 \\
0001 \\
0001 \\
0001 \\
0001 \\
0001 \\
0001 \\
0001 \\
0001 \\
0001 \\
0001 \\
0001 \\
0001 \\
0001 \\
0001 \\
0001 \\
0001 \\
0001 \\
0001 \\
0001 \\
0001 \\
0001 \\
0001 \\
0001 \\
0001 \\
0001\end{array}$ & $\begin{array}{l}W U \\
W U \\
W U \\
W U \\
W U \\
W U \\
W U \\
W U \\
W U \\
W U \\
W U \\
W U \\
W U \\
W U \\
W U \\
W U \\
W U \\
W U \\
W U \\
W U \\
W U \\
W U \\
W U \\
W U \\
W U \\
W U \\
W U \\
W U \\
W U \\
W U \\
W U \\
W U \\
W U \\
W U \\
W U \\
W U \\
W U \\
W U \\
W U\end{array}$ & $\begin{array}{l}N \\
N \\
N \\
N \\
N \\
N \\
N \\
N \\
N \\
N \\
N \\
N \\
N \\
N \\
N \\
N \\
N \\
N \\
N \\
N \\
N \\
N \\
N \\
N \\
N \\
N \\
N \\
N \\
N \\
N \\
N \\
N \\
N \\
N \\
N \\
N \\
N \\
N \\
N \\
N\end{array}$ & $M G / L$ & $<$ & $\begin{array}{c}0.01 \\
15.0 \\
14.2 \\
15.1 \\
15.6 \\
14.7 \\
0.39 \\
0.25 \\
0.26 \\
0.18 \\
0.22 \\
0.13 \\
0.14 \\
0.14 \\
0.13 \\
0.12 \\
0.04 \\
0.02 \\
0.01 \\
0.01 \\
0.01 \\
0.16 \\
0.15 \\
0.15 \\
0.14 \\
0.15 \\
0.02 \\
0.15 \\
0.02 \\
0.02 \\
0.02 \\
0.04 \\
0.044 \\
0.05 \\
0.07 \\
0.82 \\
19.0 \\
17.5 \\
12.7 \\
0.023\end{array}$ & $J$ & $\begin{array}{l}0.01 \\
0.01 \\
0.01 \\
0.01 \\
0.01 \\
0.01 \\
0.01 \\
0.01 \\
0.01 \\
0.01 \\
0.01 \\
0.01 \\
0.01 \\
0.01 \\
0.01 \\
0.01 \\
0.01 \\
0.01 \\
0.01 \\
0.01 \\
0.01 \\
0.01 \\
0.01 \\
0.01 \\
0.01 \\
0.01 \\
0.01 \\
0.01 \\
0.01 \\
0.01 \\
0.01 \\
0.01 \\
0.01 \\
0.01 \\
0.01 \\
0.01 \\
0.01 \\
0.01 \\
0.01 \\
0.01\end{array}$ & $\begin{array}{l}- \\
- \\
- \\
- \\
- \\
- \\
- \\
- \\
- \\
- \\
- \\
- \\
- \\
- \\
- \\
- \\
- \\
- \\
- \\
- \\
- \\
- \\
- \\
- \\
- \\
- \\
- \\
- \\
- \\
- \\
- \\
- \\
- \\
-\end{array}$ \\
\hline
\end{tabular}

FORMATION OF COMPLETION CODE:

WU - WASATCH - UPPER SANDSTONE

PARAMETER VALUE INDICATOR (PVI): < - LESS THAN DETECTION LIMIT
FLOW RELATIONSHIP CODE

N - UNKNOWN

SAMPLE ID CODES:

0001 - FILTERED SAMPLE (.45 MICRONS)

OTHER PARAMETER VALUE FLAGS:

$J$ - ESTIMATED VALUE 


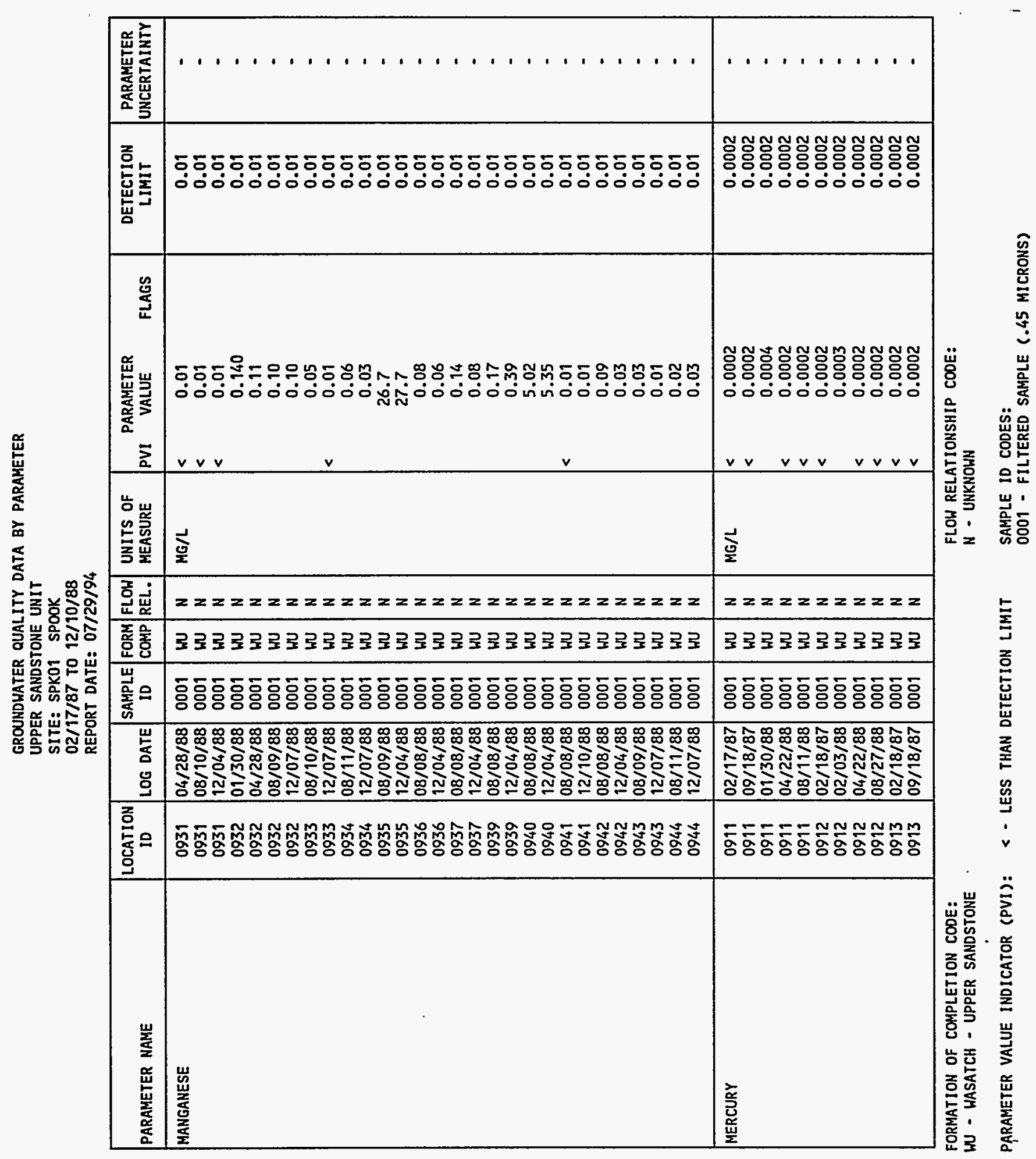


(SNOYJIW 5\%०) 37dWYS 03731714 - 1000

: $\$ 3003$ ol $\exists$ J7WHS

MMONXN - N

: $\exists 003$ dIHSNOIIV79У M07

\begin{tabular}{|c|c|c|c|c|c|c|c|c|c|c|c|}
\hline 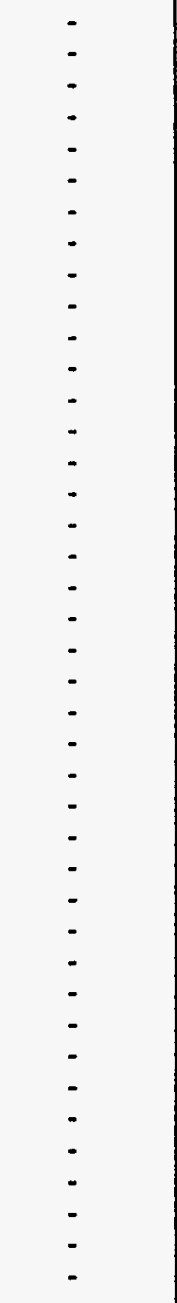 & $\begin{array}{l}2000^{\circ} 0 \\
2000^{\circ} 0 \\
2000^{\circ} 0 \\
2000^{\circ} 0 \\
2000^{\circ} 0 \\
2000^{\circ} 0 \\
2000^{\circ} 0 \\
2000^{\circ} 0 \\
2000^{\circ} 0 \\
2000^{\circ} 0 \\
2000^{\circ} 0 \\
2000^{\circ} 0 \\
2000^{\circ} 0 \\
2000^{\circ} 0 \\
2000^{\circ} 0 \\
2000^{\circ} 0 \\
2000^{\circ} 0 \\
2000^{\circ} 0 \\
2000^{\circ} 0 \\
2000^{\circ} 0 \\
2000^{\circ} 0 \\
2000^{\circ} 0 \\
2000^{\circ} 0 \\
2000^{\circ} 0 \\
2000^{\circ} 0 \\
2000^{\circ} 0 \\
2000^{\circ} 0 \\
2000^{\circ} 0 \\
2000^{\circ} 0 \\
2000^{\circ} 0 \\
2000^{\circ} 0 \\
2000^{\circ} 0 \\
2000^{\circ} 0 \\
2000^{\circ} 0 \\
2000^{\circ} 0 \\
2000^{\circ} 0 \\
2000^{\circ} 0 \\
2000^{\circ} 0 \\
2000^{\circ} 0 \\
2000^{\circ} 0 \\
2000^{\circ} 0\end{array}$ & & 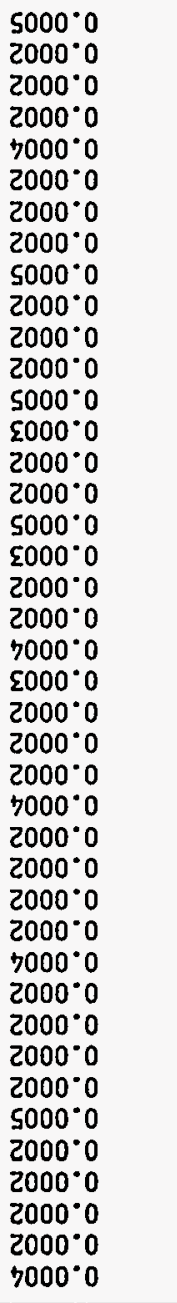 & $\begin{array}{l}> \\
> \\
> \\
> \\
> \\
> \\
> \\
> \\
> \\
> \\
> \\
> \\
> \\
> \\
> \\
> \\
> \\
> \\
> \\
> \\
>\end{array}$ & 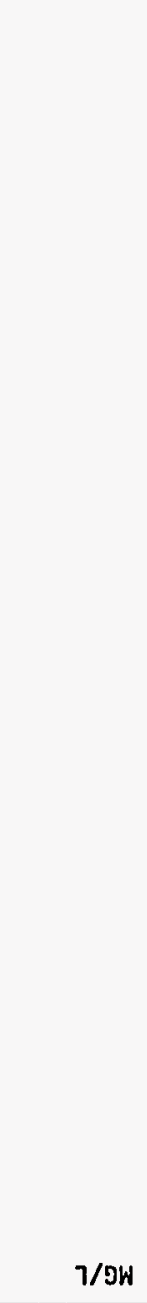 & $\begin{array}{l}N \\
N \\
N \\
N \\
N \\
N \\
N \\
N \\
N \\
N \\
N \\
N \\
N \\
N \\
N \\
N \\
N \\
N \\
N \\
N \\
N \\
N \\
N \\
N \\
N \\
N \\
N \\
N \\
N \\
N \\
N \\
N \\
N \\
N \\
N \\
N \\
N \\
N \\
N \\
N \\
N\end{array}$ & $\begin{array}{l}n M \\
n M \\
n M \\
n M \\
n M \\
n M \\
n M \\
n M \\
n M \\
n M \\
n M \\
n M \\
n M \\
n M \\
n M \\
n M \\
n M \\
n M \\
n M \\
n M \\
n M \\
n M \\
n M \\
n M \\
n M \\
n M \\
n M \\
n M \\
n M \\
n M \\
n M \\
n M \\
n M \\
n M \\
n M \\
n M \\
n M \\
n M \\
\cap M \\
n M \\
n M\end{array}$ & $\begin{array}{l}1000 \\
1000 \\
1000 \\
1000 \\
1000 \\
1000 \\
1000 \\
1000 \\
1000 \\
1000 \\
1000 \\
1000 \\
1000 \\
1000 \\
1000 \\
1000 \\
1000 \\
1000 \\
1000 \\
1000 \\
1000 \\
1000 \\
1000 \\
1000 \\
1000 \\
1000 \\
1000 \\
1000 \\
1000 \\
1000 \\
1000 \\
1000 \\
1000 \\
1000 \\
1000 \\
1000 \\
1000 \\
1000 \\
1000 \\
1000 \\
1000\end{array}$ & 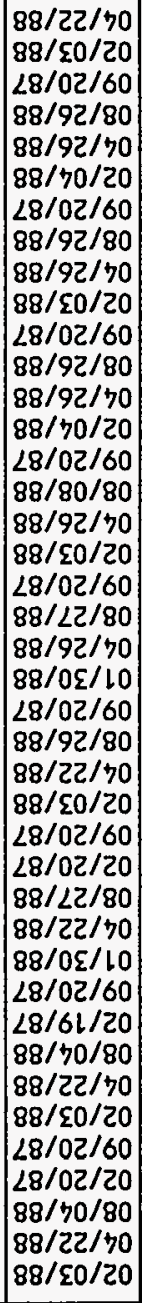 & $\begin{array}{l}8260 \\
8260 \\
8260 \\
L 260 \\
2260 \\
L 260 \\
L 260 \\
5260 \\
5260 \\
5260 \\
5260 \\
7260 \\
7260 \\
7260 \\
7260 \\
2260 \\
2260 \\
2260 \\
2260 \\
0260 \\
0260 \\
0260 \\
0260 \\
8160 \\
8160 \\
8160 \\
8160 \\
8160 \\
2160 \\
2160 \\
L 160 \\
L 160 \\
L 160 \\
9160 \\
9160 \\
9160 \\
9160 \\
9160 \\
\sum 160 \\
\varepsilon 160 \\
\Sigma 160 \\
\end{array}$ & มูกวมฺพ \\
\hline 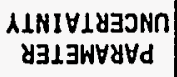 & $\begin{array}{c}11617 \\
\text { No1103130 }\end{array}$ & Sפชา & $\begin{array}{c}\exists \cap 7 \forall \wedge \\
y \Xi \perp \exists W \forall \forall \forall d\end{array}$ & Ind & $\begin{array}{l}\exists \forall \cap S \forall \exists W \\
\pm 0 \text { SIINn }\end{array}$ & $\begin{array}{l}-73 y \\
\text { moרy }\end{array}$ & dWOJ & $\begin{array}{c}\text { OI } \\
\text { כาdW }\end{array}$ & 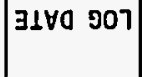 & $\begin{array}{c}\text { OI } \\
\text { NO11 }\end{array}$ & $\exists W \forall N$ УヨL $\exists W \forall Y \forall d$ \\
\hline
\end{tabular}

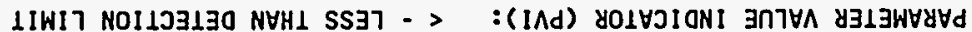

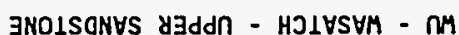
: $\exists 003$ NOILI7dWOJ to NOIIVWYOJ 


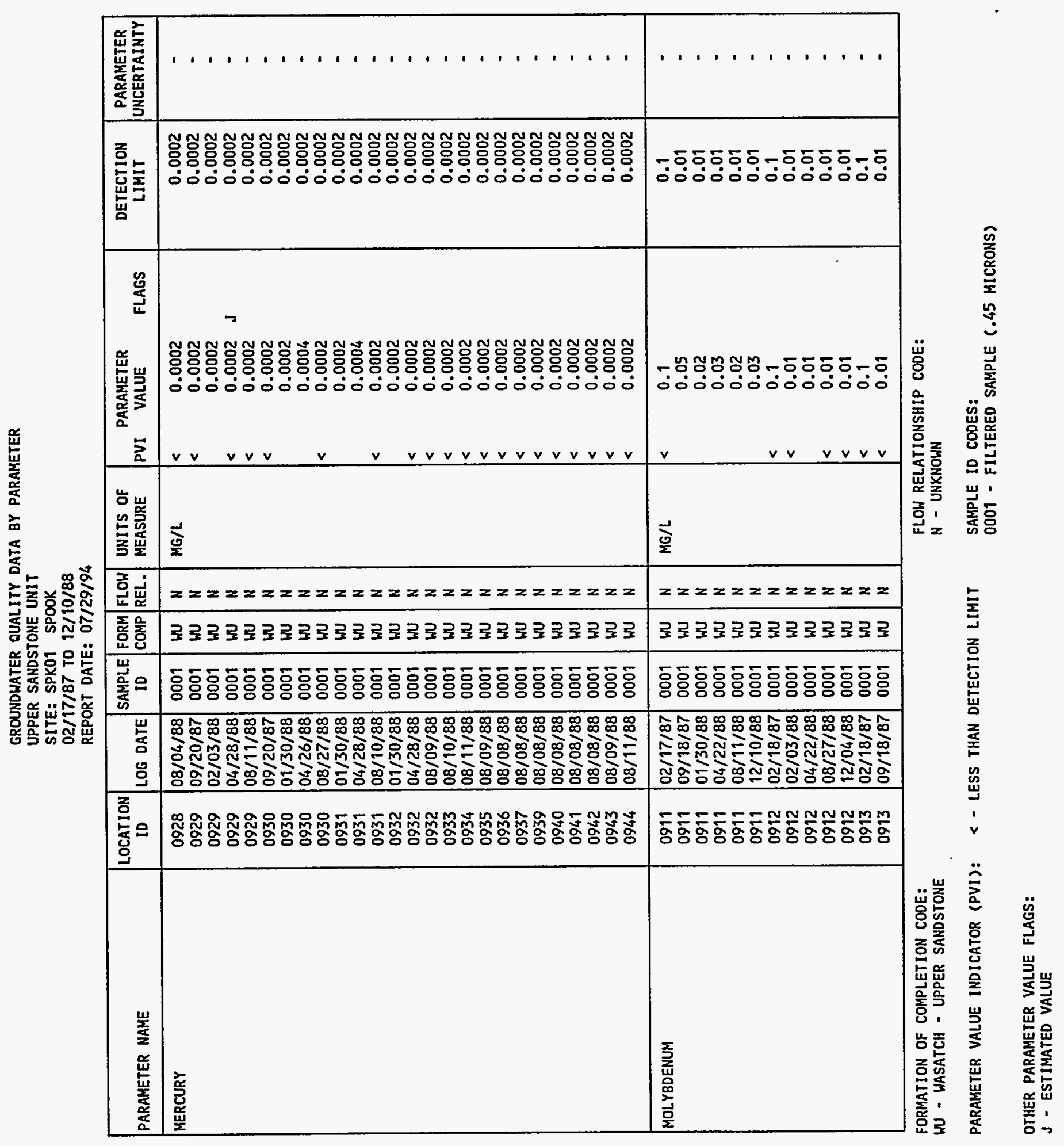




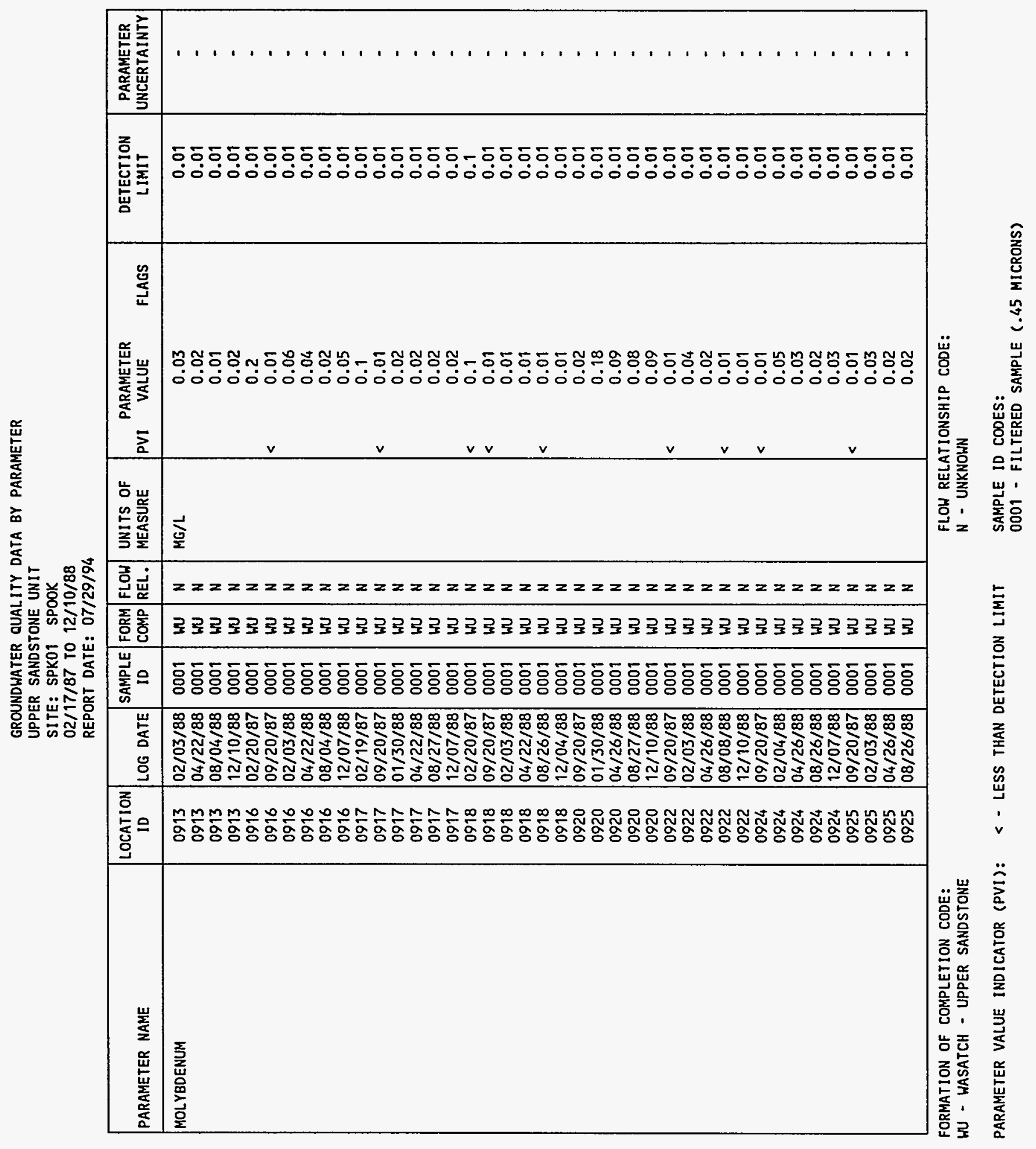




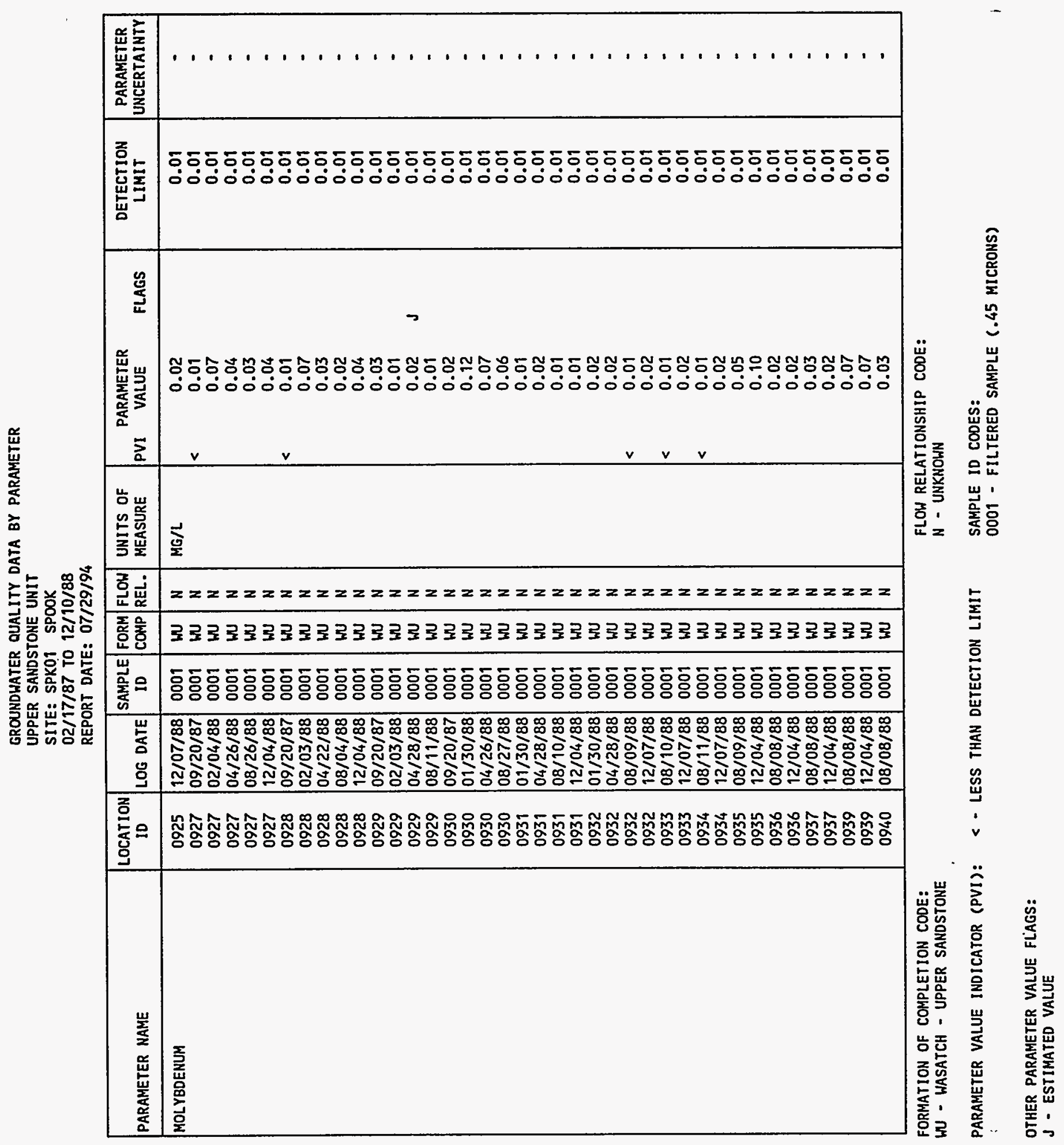


GROUNDWATER QUALITY DATA BY PARAMETER

UPPER SANDSTONE UNIT

SITE: SPKO1 SPOOK

REPORT DATE : $07 / 29 / 94$

\begin{tabular}{|c|c|c|c|c|c|c|c|c|c|c|}
\hline PARAMETER MAME & $\underset{\text { ID }}{\text { LOCATION }}$ & LOG DATE & $\begin{array}{c}\text { SAMPLE } \\
\text { ID }\end{array}$ & $\begin{array}{l}\text { FORM } \\
\text { COMP }\end{array}$ & $\begin{array}{l}\text { FLOW } \\
\text { REL. }\end{array}$ & $\begin{array}{l}\text { UNITS OF } \\
\text { MEASURE }\end{array}$ & PVI & $\begin{array}{l}\text { PARAMETER } \\
\text { VALUE }\end{array}$ & $\begin{array}{l}\text { DETECTION } \\
\text { LIMIT }\end{array}$ & $\begin{array}{l}\text { PARAMETER } \\
\text { UNCERTAINTY }\end{array}$ \\
\hline MOLYBDENUM & $\begin{array}{l}0940 \\
0941 \\
0941 \\
0942 \\
0942 \\
0943 \\
0943 \\
0944 \\
0944\end{array}$ & $\begin{array}{l}12 / 04 / 88 \\
08 / 08 / 88 \\
12 / 10 / 88 \\
08 / 08 / 88 \\
12 / 04 / 88 \\
08 / 09 / 88 \\
12 / 07 / 88 \\
08 / 11 / 88 \\
12 / 07 / 88\end{array}$ & $\begin{array}{l}0001 \\
0001 \\
0001 \\
0001 \\
0001 \\
0001 \\
0001 \\
0001 \\
0001\end{array}$ & $\begin{array}{l}\text { WU } \\
W U \\
W U \\
W U \\
W U \\
W U \\
W U \\
W U \\
W U\end{array}$ & $\begin{array}{l}N \\
N \\
N \\
N \\
N \\
N \\
N \\
N \\
N\end{array}$ & $M G / L$ & $\begin{array}{l}< \\
< \\
< \\
<\end{array}$ & $\begin{array}{l}0.06 \\
0.01 \\
0.01 \\
0.02 \\
0.02 \\
0.01 \\
0.02 \\
0.01 \\
0.02\end{array}$ & $\begin{array}{l}0.01 \\
0.01 \\
0.01 \\
0.01 \\
0.01 \\
0.01 \\
0.01 \\
0.01 \\
0.01\end{array}$ & $\begin{array}{l}- \\
- \\
- \\
- \\
- \\
- \\
- \\
-\end{array}$ \\
\hline NET GROSS ALPHA * & $\begin{array}{l}0911 \\
0911 \\
0911 \\
0911 \\
0911 \\
0912 \\
0912 \\
0912 \\
0912 \\
0913 \\
0913 \\
0913 \\
0913 \\
0913 \\
0916 \\
0916 \\
0916 \\
0916 \\
0916 \\
0917 \\
0917 \\
0917 \\
0917 \\
0917 \\
0918 \\
0918 \\
0918 \\
0918 \\
0918\end{array}$ & $\begin{array}{l}09 / 18 / 87 \\
01 / 30 / 88 \\
04 / 22 / 88 \\
08 / 11 / 88 \\
12 / 10 / 88 \\
02 / 03 / 88 \\
04 / 22 / 88 \\
08 / 27 / 88 \\
12 / 04 / 88 \\
09 / 18 / 87 \\
02 / 03 / 88 \\
04 / 22 / 88 \\
08 / 04 / 88 \\
12 / 10 / 88 \\
09 / 20 / 87 \\
02 / 03 / 88 \\
04 / 22 / 88 \\
08 / 04 / 88 \\
12 / 07 / 88 \\
09 / 20 / 87 \\
01 / 30 / 88 \\
04 / 22 / 88 \\
08 / 27 / 88 \\
12 / 07 / 88 \\
09 / 20 / 87 \\
02 / 03 / 88 \\
04 / 22 / 88 \\
08 / 26 / 88 \\
12 / 04 / 88\end{array}$ & $\begin{array}{l}0001 \\
0001 \\
0001 \\
0001 \\
0001 \\
0001 \\
0001 \\
0001 \\
0001 \\
0001 \\
0001 \\
0001 \\
0001 \\
0001 \\
0001 \\
0001 \\
0001 \\
0001 \\
0001 \\
0001 \\
0001 \\
0001 \\
0001 \\
0001 \\
0001 \\
0001 \\
0001 \\
0001 \\
0001\end{array}$ & $\begin{array}{l}W U \\
W U \\
W U \\
W U \\
W U \\
W U \\
W U \\
W U \\
W U \\
W U \\
W U \\
W U \\
W U \\
W U \\
W U \\
W U \\
W U \\
W U \\
W U \\
W U \\
W U \\
W U \\
W U \\
W U \\
W U \\
W U \\
W U \\
W U \\
W U\end{array}$ & $\begin{array}{l}N \\
N \\
N \\
N \\
N \\
N \\
N \\
N \\
N \\
N \\
N \\
N \\
N \\
N \\
N \\
N \\
N \\
N \\
N \\
N \\
N \\
N \\
N \\
N \\
N \\
N \\
N \\
N \\
N\end{array}$ & $\mathrm{PCl} / \mathrm{L}$ & & $\begin{array}{r}-254.80 \\
-203.06 \\
6.60 \\
106.46 \\
44.38 \\
-36.40 \\
119.16 \\
-106.22 \\
38.19 \\
-64.24 \\
-1099.60 \\
-290.62 \\
3515.96 \\
1327.84 \\
-677.00 \\
-2400.20 \\
-2675.60 \\
4597.40 \\
-3392.00 \\
-91.74 \\
-174.06 \\
184.00 \\
-1160.34 \\
-741.60 \\
-34.46 \\
-63.30 \\
31.14 \\
-189.34 \\
-320.26\end{array}$ & $\begin{array}{l}: \\
: \\
: \\
: \\
- \\
- \\
- \\
- \\
- \\
- \\
- \\
- \\
- \\
- \\
- \\
- \\
- \\
- \\
- \\
- \\
-\end{array}$ & $\begin{array}{l}- \\
\\
- \\
- \\
- \\
- \\
- \\
- \\
- \\
- \\
- \\
- \\
- \\
- \\
- \\
- \\
- \\
- \\
- \\
- \\
- \\
-\end{array}$ \\
\hline
\end{tabular}

* NET GROSS ALPHA (GROSS ALPHA - URANIUM) HITH 1 MG URANIUM a 686 PCI

FORMATION OF COMPLETION CODE:

WU - WASATCH - UPPER SANDSTONE

PARAMETER VALUE INDICATOR (PVI): < - LESS THAN DETECTION LIMIT
FLOW RELATIONSHIP CODE:

N - UNKNOWN

SAMPLE ID CODES:

0001 - FILTERED SAMPLE (.45 MICRONS) 
GROUNDWATER QUALITY DATA BY PARAMETER

UPPER SAHDSTONE UNIT

SITE: SPK01 SPOOK

O2/17/87 TO 12/10/88

REPORT DATE: $07 / 29 / 94$

\begin{tabular}{|c|c|c|c|c|c|c|c|c|c|c|}
\hline PARAMETER NAME & $\mid \begin{array}{c}\text { LOCATION } \\
\text { ID }\end{array}$ & LOG DATE & $\begin{array}{c}\text { SAMPLE } \\
\text { ID }\end{array}$ & $\begin{array}{l}\text { FORM } \\
\text { COMP }\end{array}$ & $\begin{array}{l}\text { FLOW } \\
\text { REL. }\end{array}$ & $\begin{array}{l}\text { UNITS OF } \\
\text { MEASURE }\end{array}$ & PVI & $\begin{array}{l}\text { PARAMETER } \\
\text { VALUE }\end{array}$ & $\begin{array}{l}\text { DETECTION } \\
\text { LIMIT }\end{array}$ & \begin{tabular}{|l} 
PARAMETER \\
UNCERTAINTY
\end{tabular} \\
\hline NET GROSS ALPHA * & $\begin{array}{l}0920 \\
0920 \\
0920 \\
0920 \\
0920 \\
0922 \\
0922 \\
0922 \\
0922 \\
0922 \\
0924 \\
0924 \\
0924 \\
0924 \\
0924 \\
0925 \\
0925 \\
0925 \\
0925 \\
0925 \\
0927 \\
0927 \\
0927 \\
0927 \\
0927 \\
0928 \\
0928 \\
0928 \\
0928 \\
0928 \\
0929 \\
0929 \\
0929 \\
0929 \\
0930 \\
0930 \\
0930 \\
0930 \\
0931\end{array}$ & $\begin{array}{l}09 / 20 / 87 \\
01 / 30 / 88 \\
04 / 26 / 88 \\
08 / 27 / 88 \\
12 / 10 / 88 \\
09 / 20 / 87 \\
02 / 03 / 88 \\
04 / 26 / 88 \\
08 / 08 / 88 \\
12 / 10 / 88 \\
09 / 20 / 87 \\
02 / 04 / 88 \\
04 / 26 / 88 \\
08 / 26 / 88 \\
12 / 07 / 88 \\
09 / 20 / 87 \\
02 / 03 / 88 \\
04 / 26 / 88 \\
08 / 26 / 88 \\
12 / 07 / 88 \\
09 / 20 / 87 \\
02 / 04 / 88 \\
04 / 26 / 88 \\
08 / 26 / 88 \\
12 / 04 / 88 \\
09 / 20 / 87 \\
02 / 03 / 88 \\
04 / 22 / 88 \\
08 / 04 / 88 \\
12 / 04 / 88 \\
09 / 20 / 87 \\
02 / 03 / 88 \\
04 / 28 / 88 \\
08 / 11 / 88 \\
09 / 20 / 87 \\
01 / 30 / 88 \\
04 / 26 / 88 \\
08 / 27 / 88 \\
01 / 30 / 88\end{array}$ & $\begin{array}{l}0001 \\
0001 \\
0001 \\
0001 \\
0001 \\
0001 \\
0001 \\
0001 \\
0001 \\
0001 \\
0001 \\
0001 \\
0001 \\
0001 \\
0001 \\
0001 \\
0001 \\
0001 \\
0001 \\
0001 \\
0001 \\
0001 \\
0001 \\
0001 \\
0001 \\
0001 \\
0001 \\
0001 \\
0001 \\
0001 \\
0001 \\
0001 \\
0001 \\
0001 \\
0001 \\
0001 \\
0001 \\
0001 \\
0001\end{array}$ & $\begin{array}{l}W U \\
W U \\
W U \\
W U \\
W U \\
W U \\
W U \\
W U \\
W U \\
W U \\
W U \\
W U \\
W U \\
W U \\
W U \\
W U \\
W U \\
W U \\
W U \\
W U \\
W U \\
W U \\
W U \\
W U \\
W U \\
W U \\
W U \\
W U \\
W U \\
W U \\
W U \\
W U \\
W U \\
W U \\
W U \\
W U \\
W U \\
W U\end{array}$ & $\begin{array}{l}N \\
N \\
N \\
N \\
N \\
N \\
N \\
N \\
N \\
N \\
N \\
N \\
N \\
N \\
N \\
N \\
N \\
N \\
N \\
N \\
N \\
N \\
N \\
N \\
N \\
N \\
N \\
N \\
N \\
N \\
N \\
N \\
N \\
N \\
N \\
N \\
N \\
N \\
N\end{array}$ & $\mathrm{PCI} / \mathrm{L}$ & & $\begin{array}{r}286.21 \\
294.63 \\
469.94 \\
176.56 \\
138.09 \\
6.63 \\
-12.50 \\
-18.38 \\
-0.72 \\
-9.81 \\
523.26 \\
127.22 \\
170.50 \\
-159.84 \\
296.10 \\
-268.62 \\
-113.74 \\
-72.68 \\
-207.22 \\
-408.38 \\
-1761.88 \\
140.44 \\
-260.34 \\
-518.40 \\
430.68 \\
208.20 \\
-137.70 \\
34.86 \\
-132.00 \\
-254.42 \\
23.49 \\
3.76 \\
-3.85 \\
4.27 \\
-53.32 \\
37.74 \\
40.02 \\
213.09 \\
-361.18\end{array}$ & 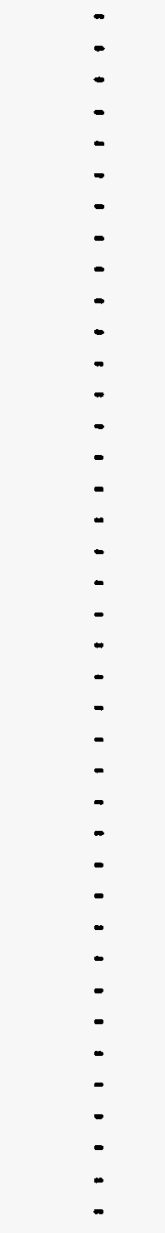 & $\begin{array}{l}- \\
- \\
- \\
- \\
- \\
- \\
- \\
- \\
- \\
- \\
- \\
- \\
- \\
- \\
- \\
- \\
- \\
- \\
- \\
- \\
- \\
- \\
- \\
- \\
- \\
- \\
- \\
- \\
- \\
- \\
- \\
- \\
-\end{array}$ \\
\hline
\end{tabular}

* NET GROSS ALPHA (GROSS ALPHA - URANIUM) WITH 1 MG URANIUM = 686 PCI

FORMATION OF COMPLETION CODE:

WU - HASATCH - UPPER SANDSTONE

PARAMETER VALUE INDICATOR (PVI): < - LESS THAN DETECTION LIMIT
FLOW RELATIONSHIP COOE:

H - UNKNOWN

SAMPLE ID CODES:

0001 - FILTERED SAMPLE (.45 MICRONS) 
GROUNDWATER QUALITY DATA BY PARAMETER

UPPER SANDSTONE UNIT

SITE: SPKO1 SPOOK

02/17/87 TO $12 / 10 / 88$

\begin{tabular}{|c|c|c|c|c|c|c|c|c|c|c|c|}
\hline PARAMETER NAME & $\underset{\text { LOCATION }}{\text { LD }}$ & LOG DATE & $\begin{array}{c}\text { SAMPLE } \\
10\end{array}$ & $\begin{array}{l}\text { FORM } \\
\text { COMP }\end{array}$ & $\begin{array}{l}\text { FLOW } \\
\text { REL. }\end{array}$ & $\begin{array}{l}\text { UNITS OF } \\
\text { MEASURE }\end{array}$ & PVI & $\begin{array}{l}\text { PARAMETER } \\
\text { VALUE }\end{array}$ & FLAGS & $\begin{array}{l}\text { DETECTION } \\
\text { LIMIT }\end{array}$ & $\begin{array}{l}\text { PARAMETER } \\
\text { UNCERTAINTY }\end{array}$ \\
\hline NET GROSS ALPHA * & $\begin{array}{l}0931 \\
0931 \\
0931 \\
0932 \\
0932 \\
0932 \\
0933 \\
0933 \\
0934 \\
0934 \\
0935 \\
0935 \\
0936 \\
0936 \\
0937 \\
0937 \\
0939 \\
0939 \\
0940 \\
0940 \\
0941 \\
0941 \\
0942 \\
0942 \\
0943 \\
0943 \\
0944 \\
0944\end{array}$ & $\begin{array}{l}04 / 28 / 88 \\
08 / 10 / 88 \\
12 / 04 / 88 \\
01 / 30 / 88 \\
04 / 28 / 88 \\
08 / 09 / 88 \\
08 / 10 / 88 \\
12 / 07 / 88 \\
08 / 11 / 88 \\
12 / 07 / 88 \\
08 / 09 / 88 \\
12 / 04 / 88 \\
08 / 08 / 88 \\
12 / 04 / 88 \\
08 / 08 / 88 \\
12 / 04 / 88 \\
08 / 08 / 88 \\
12 / 04 / 88 \\
08 / 08 / 88 \\
12 / 04 / 88 \\
08 / 08 / 88 \\
12 / 10 / 88 \\
08 / 08 / 88 \\
12 / 04 / 88 \\
08 / 09 / 88 \\
12 / 07 / 88 \\
08 / 11 / 88 \\
12 / 07 / 88\end{array}$ & $\begin{array}{l}0001 \\
0001 \\
0001 \\
0001 \\
0001 \\
0001 \\
0001 \\
0001 \\
0001 \\
0001 \\
0001 \\
0001 \\
0001 \\
0001 \\
0001 \\
0001 \\
0001 \\
0001 \\
0001 \\
0001 \\
0001 \\
0001 \\
0001 \\
0001 \\
0001 \\
0001 \\
0001 \\
0001\end{array}$ & $\begin{array}{l}W U \\
W U \\
W U \\
W U \\
W U \\
W U \\
W U \\
W U \\
W U \\
W U \\
W U \\
W U \\
W U \\
W U \\
W U \\
W U \\
W U \\
W U \\
W U \\
W U \\
W U \\
W U \\
W U \\
W U \\
W U \\
W U \\
W U \\
W U\end{array}$ & $\begin{array}{l}N \\
N \\
N \\
N \\
N \\
N \\
N \\
N \\
N \\
N \\
N \\
N \\
N \\
N \\
N \\
N \\
N \\
N \\
N \\
N \\
N \\
N \\
N \\
N \\
N \\
N \\
N \\
N\end{array}$ & $\mathrm{PCI/L}$ & & $\begin{array}{r}480.56 \\
-232.00 \\
-274.90 \\
45.68 \\
43.44 \\
0.11 \\
-444.56 \\
-458.86 \\
-139.72 \\
-185.18 \\
225.13 \\
866.07 \\
99.78 \\
-316.84 \\
760.52 \\
275.80 \\
61.80 \\
39.74 \\
1118.52 \\
-582.26 \\
-6.41 \\
4.28 \\
1.89 \\
2.30 \\
-349.40 \\
-255.68 \\
-71.99 \\
-50.54\end{array}$ & & $\begin{array}{l}- \\
: \\
: \\
: \\
: \\
: \\
: \\
: \\
- \\
- \\
: \\
- \\
- \\
- \\
- \\
- \\
- \\
- \\
-\end{array}$ & $\begin{array}{l}- \\
: \\
: \\
- \\
- \\
- \\
- \\
- \\
- \\
- \\
- \\
- \\
- \\
- \\
- \\
- \\
- \\
- \\
- \\
-\end{array}$ \\
\hline NICKEL & $\begin{array}{l}0911 \\
0911 \\
0911 \\
0911 \\
0912 \\
0912 \\
0912 \\
0913 \\
0913\end{array}$ & $\begin{array}{l}09 / 18 / 87 \\
01 / 30 / 88 \\
04 / 22 / 88 \\
08 / 11 / 88 \\
02 / 03 / 88 \\
04 / 22 / 88 \\
08 / 27 / 88 \\
09 / 18 / 87 \\
02 / 03 / 88\end{array}$ & \begin{tabular}{|l|}
0001 \\
0001 \\
0001 \\
0001 \\
0001 \\
0001 \\
0001 \\
0001 \\
0001
\end{tabular} & $\begin{array}{l}\text { WU } \\
W U \\
W U \\
W U \\
W U \\
W U \\
W U \\
W U \\
W U\end{array}$ & $\begin{array}{l}N \\
N \\
N \\
N \\
N \\
N \\
N \\
N \\
N\end{array}$ & $M G / L$ & $\begin{array}{l}< \\
< \\
< \\
< \\
< \\
< \\
< \\
<\end{array}$ & $\begin{array}{l}0.01 \\
0.01 \\
0.02 \\
0.04 \\
0.01 \\
0.01 \\
0.04 \\
0.01 \\
0.01\end{array}$ & $\begin{array}{l}\text { J } \\
\text { J } \\
\text { J } \\
\text { J } \\
\text { J } \\
\text { J }\end{array}$ & $\begin{array}{l}0.04 \\
0.04 \\
0.04 \\
0.04 \\
0.04 \\
0.04 \\
0.04 \\
0.04 \\
0.04\end{array}$ & $\begin{array}{l}- \\
- \\
- \\
- \\
- \\
- \\
-\end{array}$ \\
\hline
\end{tabular}

* NeT GROSS ALPHA (GROSS ALPHA - URANIUM) WITH 1 MG URANIUM = 686 PCI

FORMATION OF COMPLETION CODE:

WU - WASATCH - UPPER SANDSTONE

PARAMETER VALUE INDICATOR (PVI): < - LESS THAN DETECTION LIMIT
FLOW RELATIONSHIP CODE:

N - UNKNOWN

SAMPLE ID CODES:

0001 - FILTERED SAMPLE (.45 MICRONS)

OTHER PARAMETER VALUE FLAGS:

$J$ - ESTIMATED VALUE 


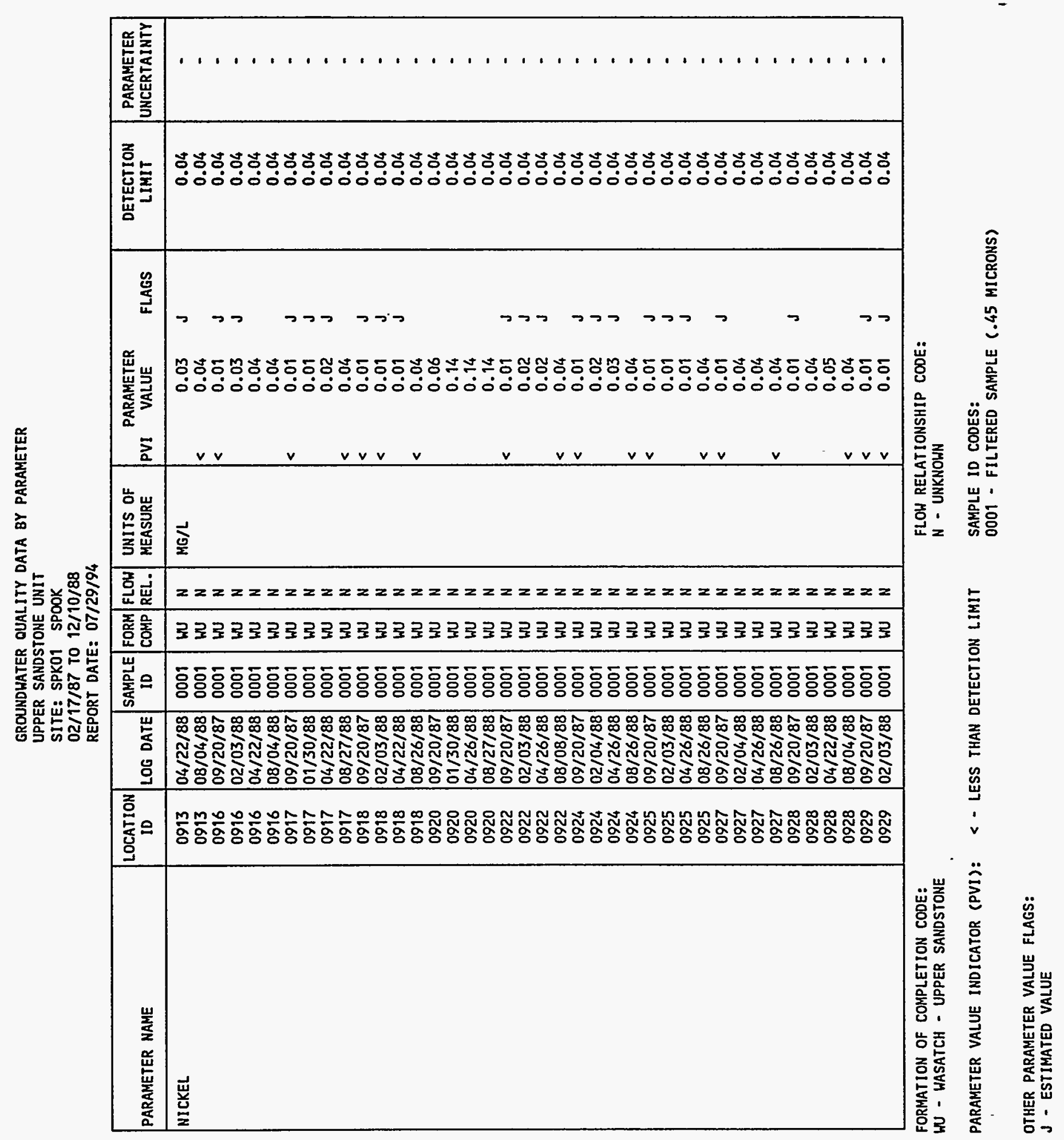




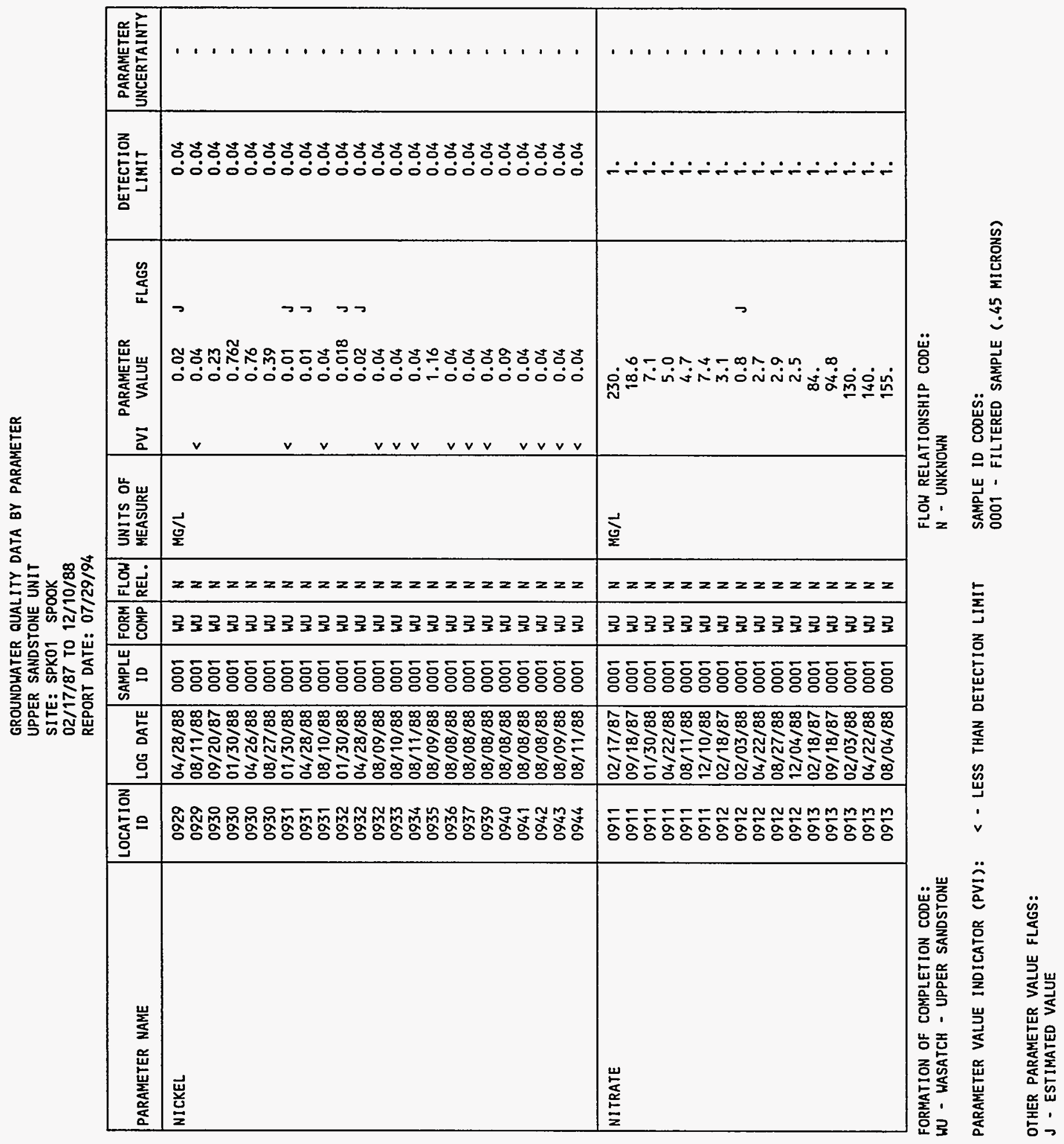




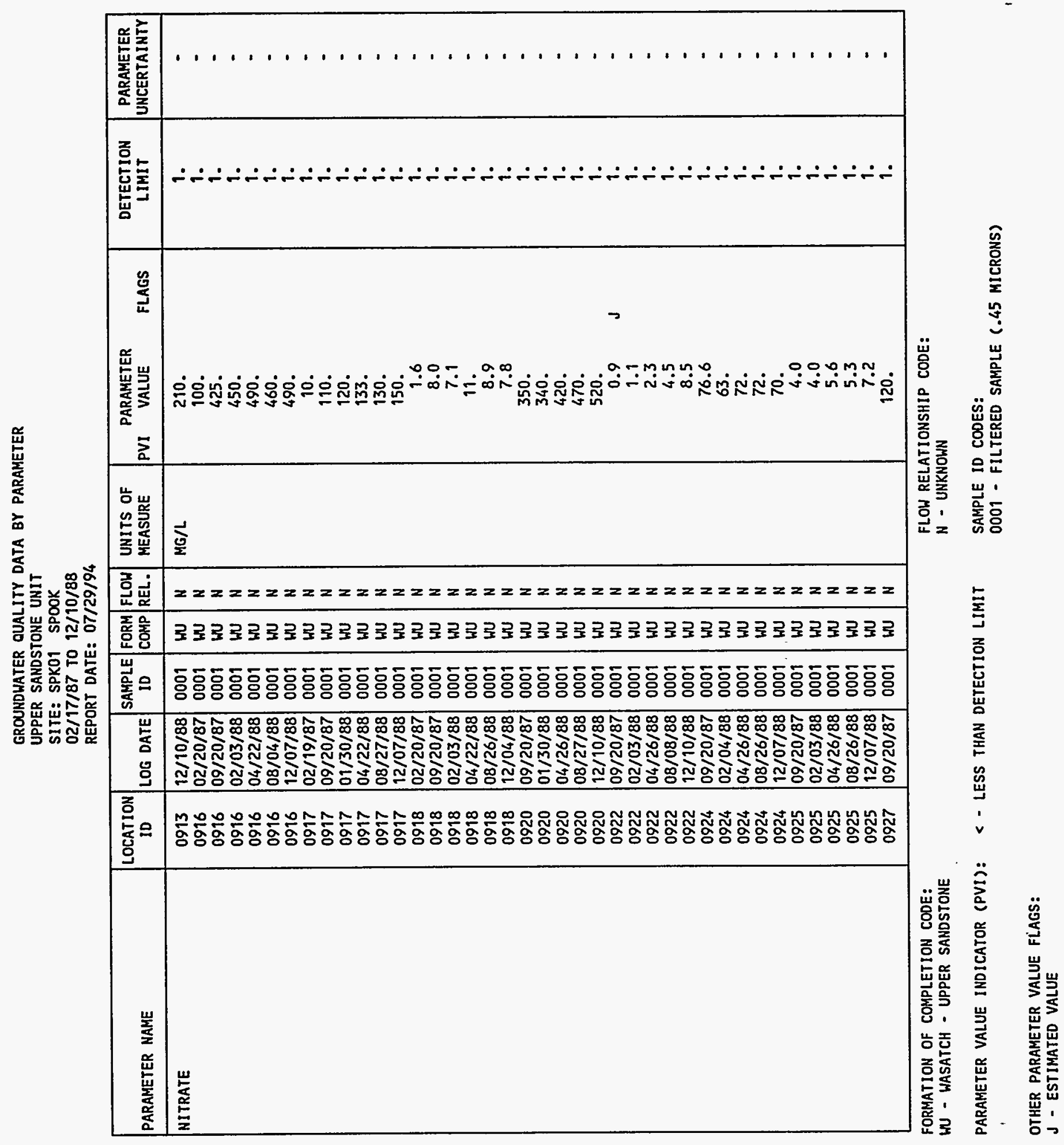




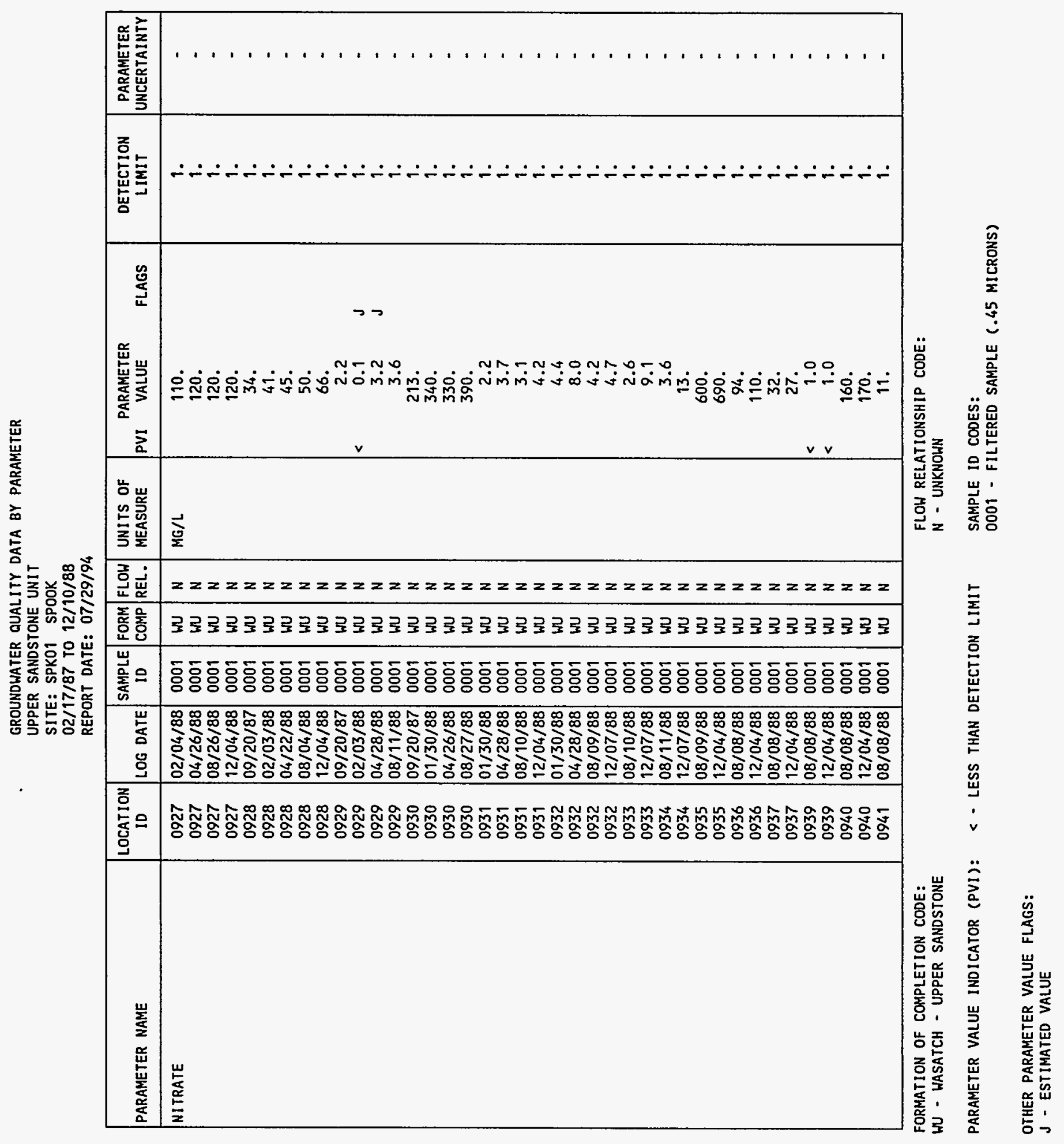


GROUNDWATER QUALITY DATA BY PARAMETER

UPPER SANDSTONE UNIT

SITE: SPKO1 SPOOK

$02 / 17 / 87$ TO $12 / 10 / 88$

REPORT DATE: $07 / 29 / 94$

\begin{tabular}{|c|c|c|c|c|c|c|c|c|c|c|}
\hline PARAMETER NAME & $\underset{\text { ID }}{\text { LOCATION }}$ & LOG DATE & $\underset{\text { ID }}{\text { SAMPLE }}$ & $\begin{array}{l}\text { FORM } \\
\text { COMP }\end{array}$ & $\begin{array}{l}\text { FLOW } \\
\text { REL. }\end{array}$ & $\begin{array}{l}\text { UNITS OF } \\
\text { MEASURE }\end{array}$ & $\begin{array}{c}\text { PARAMETER } \\
\text { PVI } \\
\text { VALUE }\end{array}$ & FLAGS & $\begin{array}{l}\text { DETECTION } \\
\text { LIMIT }\end{array}$ & $\begin{array}{l}\text { PARAMETER } \\
\text { UNCERTAINTY }\end{array}$ \\
\hline NITRATE & $\begin{array}{l}0941 \\
0942 \\
0942 \\
0943 \\
0943 \\
0944 \\
0944\end{array}$ & $\begin{array}{l}12 / 10 / 88 \\
08 / 08 / 88 \\
12 / 04 / 88 \\
08 / 09 / 88 \\
12 / 07 / 88 \\
08 / 11 / 88 \\
12 / 07 / 88\end{array}$ & $\begin{array}{l}0001 \\
0001 \\
0001 \\
0001 \\
0001 \\
0001 \\
0001\end{array}$ & $\begin{array}{l}W U \\
W U \\
W U \\
W U \\
W U \\
W U \\
W U\end{array}$ & $\begin{array}{l}N \\
N \\
N \\
N \\
N \\
N \\
N\end{array}$ & MG/L & $\begin{array}{l}15 . \\
40 . \\
47 . \\
5.3 \\
8.9 \\
3.2 \\
5.8\end{array}$ & & $\begin{array}{l}1 . \\
1 . \\
1 . \\
i . \\
1 .\end{array}$ & $\begin{array}{l}- \\
- \\
- \\
-\end{array}$ \\
\hline PH & $\begin{array}{l}0911 \\
0911 \\
0911 \\
0911 \\
0911 \\
0911 \\
0912 \\
0912 \\
0912 \\
0912 \\
0912 \\
0913 \\
0913 \\
0913 \\
0913 \\
0913 \\
0913 \\
0916 \\
0916 \\
0916 \\
0916 \\
0916 \\
0916 \\
0917 \\
0917 \\
0917 \\
0917 \\
0917 \\
0917 \\
0918 \\
0918 \\
0918 \\
0918\end{array}$ & $\begin{array}{l}02 / 17 / 87 \\
09 / 18 / 87 \\
01 / 30 / 88 \\
04 / 22 / 88 \\
08 / 11 / 88 \\
12 / 10 / 88 \\
02 / 18 / 87 \\
02 / 03 / 88 \\
04 / 22 / 88 \\
08 / 27 / 88 \\
12 / 04 / 88 \\
02 / 18 / 87 \\
09 / 18 / 87 \\
02 / 03 / 88 \\
04 / 22 / 88 \\
08 / 04 / 88 \\
12 / 10 / 88 \\
02 / 20 / 87 \\
09 / 20 / 87 \\
02 / 03 / 88 \\
04 / 22 / 88 \\
08 / 04 / 88 \\
12 / 07 / 88 \\
02 / 19 / 87 \\
09 / 20 / 87 \\
01 / 30 / 88 \\
04 / 22 / 88 \\
08 / 27 / 88 \\
12 / 07 / 88 \\
02 / 20 / 87 \\
09 / 20 / 87 \\
02 / 03 / 88 \\
04 / 22 / 88\end{array}$ & $\begin{array}{l}0001 \\
0001 \\
0001 \\
0001 \\
0001 \\
0001 \\
0001 \\
0001 \\
0001 \\
0001 \\
0001 \\
0001 \\
0001 \\
0001 \\
0001 \\
0001 \\
0001 \\
0001 \\
0001 \\
0001 \\
0001 \\
0001 \\
0001 \\
0001 \\
0001 \\
0001 \\
0001 \\
0001 \\
0001 \\
0001 \\
0001 \\
0001 \\
0001\end{array}$ & $\begin{array}{l}\text { WU } \\
W U \\
W U \\
W U \\
W U \\
W U \\
W U \\
W U \\
W U \\
W U \\
W U \\
W U \\
W U \\
W U \\
W U \\
W U \\
W U \\
W U \\
W U \\
W U \\
W U \\
W U \\
W U \\
W U \\
W U \\
W U \\
W U \\
W U \\
W U \\
W U \\
W U \\
W U \\
W U\end{array}$ & $\begin{array}{l}N \\
N \\
N \\
N \\
N \\
N \\
N \\
N \\
N \\
N \\
N \\
N \\
N \\
N \\
N \\
N \\
N \\
N \\
N \\
N \\
N \\
N \\
N \\
N \\
N \\
N \\
N \\
N \\
N \\
N \\
N \\
N \\
N\end{array}$ & SU & $\begin{array}{l}7.19 \\
7.43 \\
7.61 \\
7.36 \\
7.17 \\
7.74 \\
7.31 \\
7.68 \\
7.61 \\
7.29 \\
7.35 \\
6.98 \\
6.84 \\
6.93 \\
6.74 \\
6.67 \\
6.78 \\
6.97 \\
6.89 \\
6.79 \\
6.82 \\
6.68 \\
6.69 \\
7.34 \\
7.12 \\
7.01 \\
7.14 \\
7.01 \\
7.44 \\
7.28 \\
7.12 \\
7.47 \\
7.31\end{array}$ & & $\begin{array}{l}- \\
- \\
i \\
i \\
- \\
- \\
i \\
- \\
- \\
- \\
- \\
- \\
- \\
- \\
- \\
- \\
- \\
- \\
- \\
- \\
- \\
-\end{array}$ & $\begin{array}{l}- \\
- \\
- \\
- \\
- \\
- \\
- \\
- \\
- \\
- \\
- \\
- \\
- \\
- \\
- \\
- \\
- \\
- \\
- \\
- \\
- \\
- \\
- \\
-\end{array}$ \\
\hline
\end{tabular}

FORMATION OF COMPLETION CODE:

WU - HASATCH - UPPER SANDSTONE

FLOW RELATIONSHIP CODE:

PARAMETER VALUE INDICATOR (PVI): < - LESS THAN DETECTION LIMIT

SAMPLE ID CODES:

0001 - FILTERED SAMPLE (.45 MICRONS) 
SITE: SPKO1 SPOOK

$02 / 17 / 87$ TO $12 / 10 / 88$

REPORT DATE: $07 / 29 / 94$

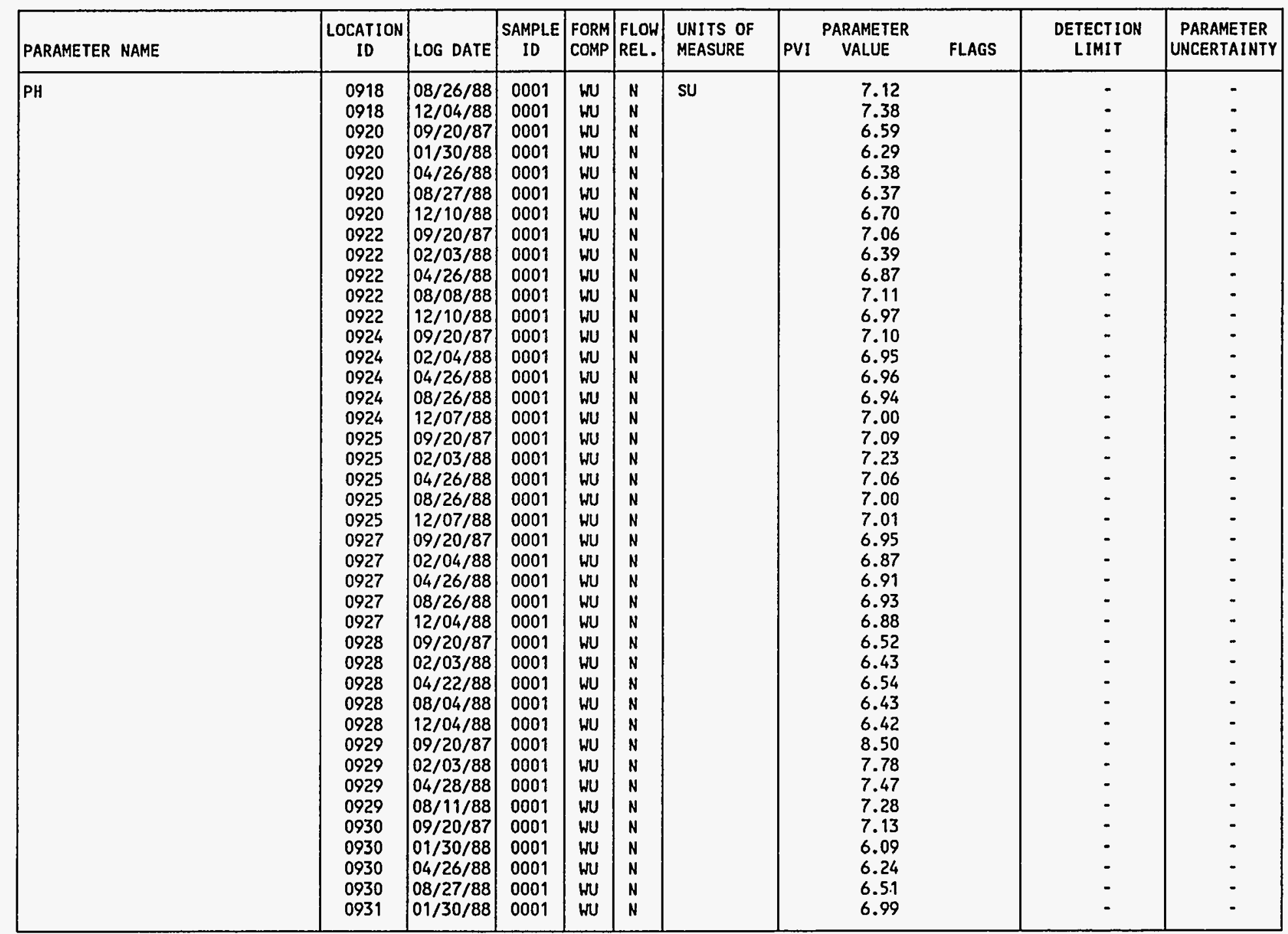

FORMATION OF COMPLETION CODE:

WU - WASATCH - UPPER SANDSTONE

FLOW RELATIONSHIP CODE:

PARAMETER VALUE INDICATOR (PVI): < - LESS THAN DETECTION LIMIT

SAMPLE ID CODES:

0001 - FILTERED SAMPLE (.45 MICRONS) 
GROUNDHATER QUALITY DATA BY PARAMETER

UPPER SANDSTONE UNIT

SITE: SPKOI SPOOK

$02 / 17 / 87$ TO $12 / 10 / 88$

REPORT DATE: 07/29/94

\begin{tabular}{|c|c|c|c|c|c|c|c|c|c|c|}
\hline |PARAMETER NAME & $\begin{array}{l}\text { LOCATION } \\
\text { ID }\end{array}$ & LOG DATE & $\underset{\text { ID }}{\text { SAMPLE }}$ & $\begin{array}{l}\text { FORM } \\
\text { COMP }\end{array}$ & $\begin{array}{l}\text { FLOW } \\
\text { REL. }\end{array}$ & $\begin{array}{l}\text { UNITS OF } \\
\text { MEASURE }\end{array}$ & PVI & $\begin{array}{l}\text { PARAMETER } \\
\text { VALUE }\end{array}$ & $\begin{array}{l}\text { DETECTION } \\
\text { LIMIT }\end{array}$ & $\begin{array}{l}\text { PARAMETER } \\
\text { UNCERTAINTY }\end{array}$ \\
\hline $\mathrm{PH}$ & $\begin{array}{l}0931 \\
0931 \\
0931 \\
0932 \\
0932 \\
0932 \\
0932 \\
0933 \\
0933 \\
0934 \\
0934 \\
0935 \\
0935 \\
0936 \\
0936 \\
0937 \\
0937 \\
0939 \\
0939 \\
0940 \\
0940 \\
0941 \\
0941 \\
0942 \\
0942 \\
0943 \\
0943 \\
0944 \\
0944\end{array}$ & $\begin{array}{l}04 / 28 / 88 \\
08 / 10 / 88 \\
12 / 04 / 88 \\
01 / 30 / 88 \\
04 / 28 / 88 \\
08 / 09 / 88 \\
12 / 07 / 88 \\
08 / 10 / 88 \\
12 / 07 / 88 \\
08 / 11 / 88 \\
12 / 07 / 88 \\
08 / 09 / 88 \\
12 / 04 / 88 \\
08 / 08 / 88 \\
12 / 04 / 88 \\
08 / 08 / 88 \\
12 / 04 / 88 \\
08 / 08 / 88 \\
12 / 04 / 88 \\
08 / 08 / 88 \\
12 / 04 / 88 \\
08 / 08 / 88 \\
12 / 10 / 88 \\
08 / 08 / 88 \\
12 / 04 / 88 \\
08 / 09 / 88 \\
12 / 07 / 88 \\
08 / 11 / 88 \\
12 / 07 / 88\end{array}$ & $\begin{array}{l}0001 \\
0001 \\
0001 \\
0001 \\
0001 \\
0001 \\
0001 \\
0001 \\
0001 \\
0001 \\
0001 \\
0001 \\
0001 \\
0001 \\
0001 \\
0001 \\
0001 \\
0001 \\
0001 \\
0001 \\
0001 \\
0001 \\
0001 \\
0001 \\
0001 \\
0001 \\
0001 \\
0001 \\
0001\end{array}$ & $\begin{array}{l}W U \\
W U \\
W U \\
W U \\
W U \\
W U \\
W U \\
W U \\
W U \\
W U \\
W U \\
W U \\
W U \\
W U \\
W U \\
W U \\
W U \\
W U \\
W U \\
W U \\
W U \\
W U \\
W U \\
W U \\
W U \\
W U \\
W U \\
W U \\
W U\end{array}$ & $\begin{array}{l}N \\
N \\
N \\
N \\
N \\
N \\
N \\
N \\
N \\
N \\
N \\
N \\
N \\
N \\
N \\
N \\
N \\
N \\
N \\
N \\
N \\
N \\
N \\
N \\
N \\
N \\
N \\
N \\
N\end{array}$ & su & & $\begin{array}{l}7.18 \\
7.21 \\
7.14 \\
6.87 \\
6.79 \\
6.75 \\
7.08 \\
7.27 \\
7.23 \\
7.12 \\
7.09 \\
6.06 \\
5.59 \\
7.46 \\
7.23 \\
7.30 \\
7.51 \\
7.02 \\
6.82 \\
6.48 \\
6.50 \\
7.40 \\
7.68 \\
7.39 \\
6.95 \\
7.27 \\
7.50 \\
6.99 \\
7.14\end{array}$ & 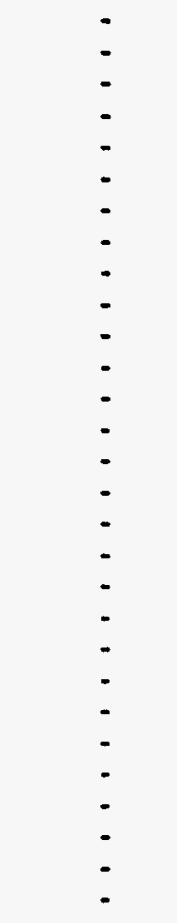 & $\begin{array}{l}- \\
- \\
- \\
- \\
- \\
- \\
- \\
- \\
- \\
- \\
- \\
- \\
- \\
- \\
- \\
- \\
- \\
- \\
- \\
- \\
- \\
-\end{array}$ \\
\hline PHOSPHATE & $\begin{array}{l}0911 \\
0911 \\
0911 \\
0911 \\
0911 \\
0912 \\
0912 \\
0912 \\
0912 \\
0913 \\
0913\end{array}$ & $\begin{array}{l}02 / 17 / 87 \\
09 / 18 / 87 \\
01 / 30 / 88 \\
04 / 22 / 88 \\
08 / 11 / 88 \\
02 / 18 / 87 \\
02 / 03 / 88 \\
04 / 22 / 88 \\
08 / 27 / 88 \\
02 / 18 / 87 \\
09 / 18 / 87\end{array}$ & $\begin{array}{l}0001 \\
0001 \\
0001 \\
0001 \\
0001 \\
0001 \\
0001 \\
0001 \\
0001 \\
0001 \\
0001\end{array}$ & $\begin{array}{l}\text { WU } \\
W U \\
W U \\
W U \\
W U \\
W U \\
W U \\
W U \\
W U \\
W U \\
W U\end{array}$ & $\begin{array}{l}N \\
N \\
N \\
N \\
N \\
N \\
N \\
N \\
N \\
N \\
N\end{array}$ & MG/L & $\begin{array}{l}< \\
< \\
< \\
< \\
< \\
< \\
< \\
<\end{array}$ & $\begin{array}{l}0.1 \\
0.2 \\
0.1 \\
0.3 \\
0.1 \\
0.1 \\
0.1 \\
0.3 \\
0.1 \\
0.1 \\
0.1\end{array}$ & $\begin{array}{l}0.1 \\
0.1 \\
0.1 \\
0.1 \\
0.1 \\
0.1 \\
0.1 \\
0.1 \\
0.1 \\
0.1 \\
0.1\end{array}$ & $\begin{array}{l}- \\
- \\
- \\
- \\
- \\
- \\
-\end{array}$ \\
\hline $\begin{array}{l}\text { FORMATION OF COMPLETION CODE: } \\
\text { WU - HASATCH - UPPER SANDSTONE }\end{array}$ & \multicolumn{10}{|c|}{$\begin{array}{l}\text { FLOW RELATIONSHIP CODE: } \\
N \text { - UNKNOWN }\end{array}$} \\
\hline
\end{tabular}




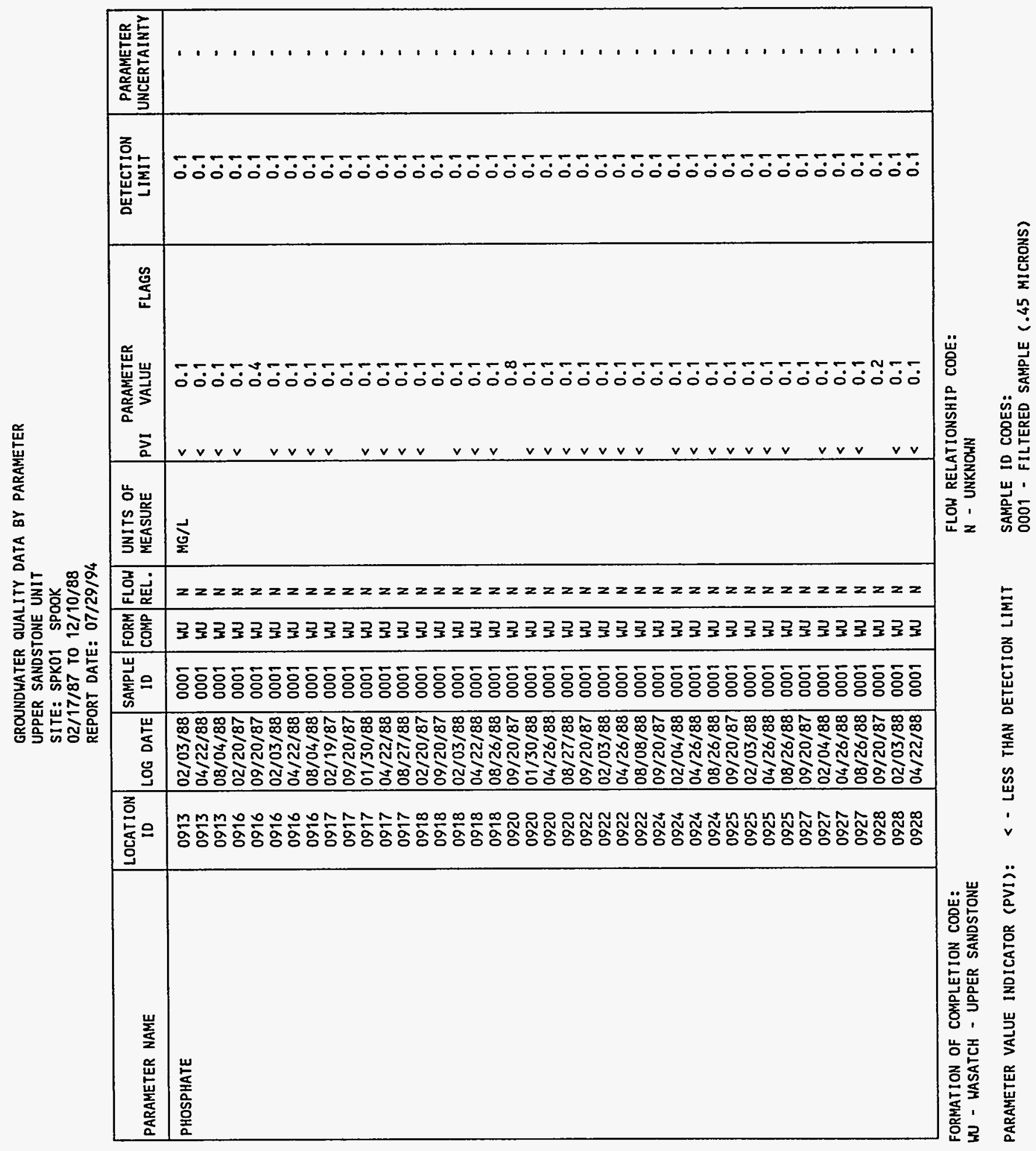




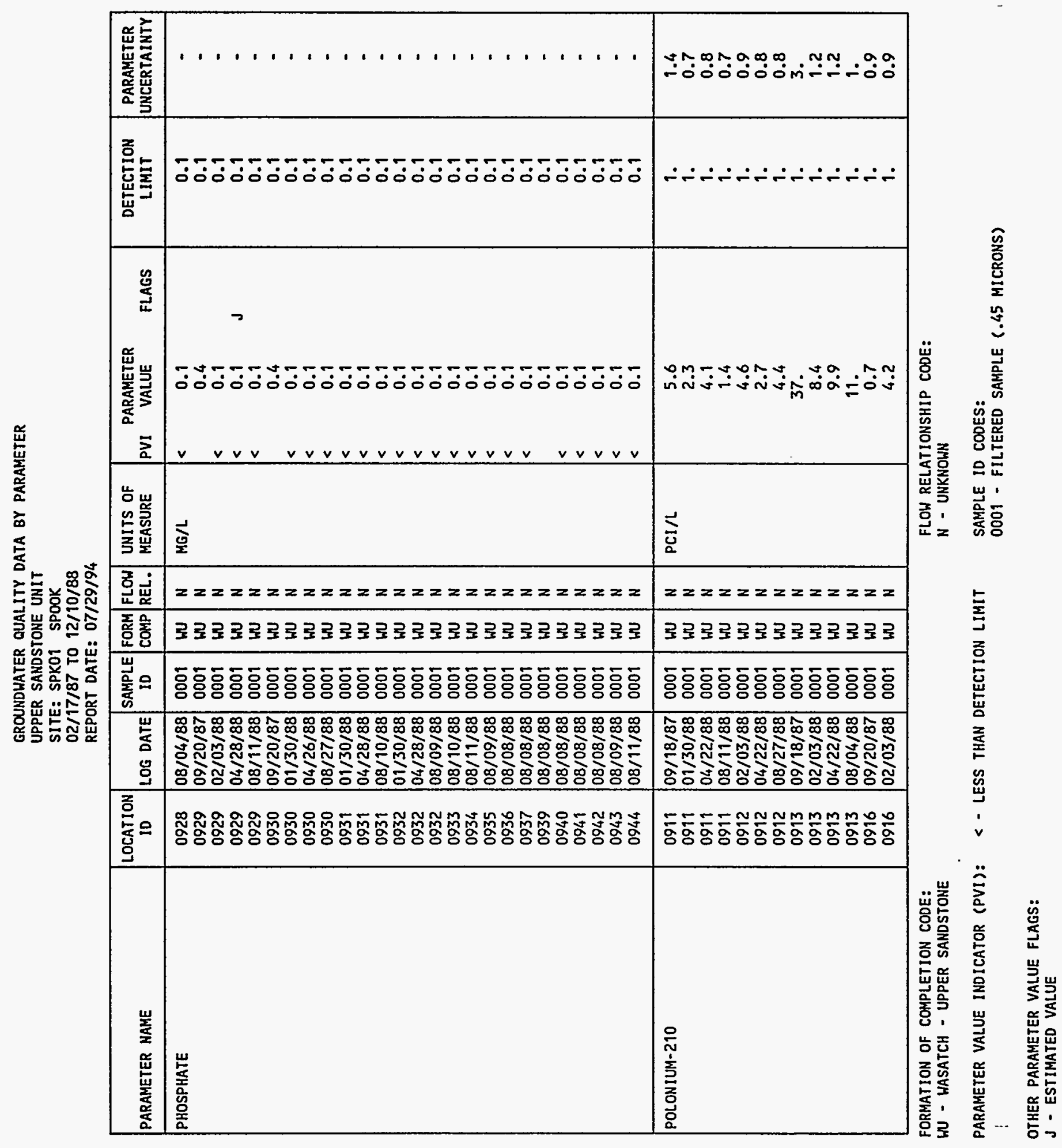


REPORT DATE: $07 / 29 / 9$

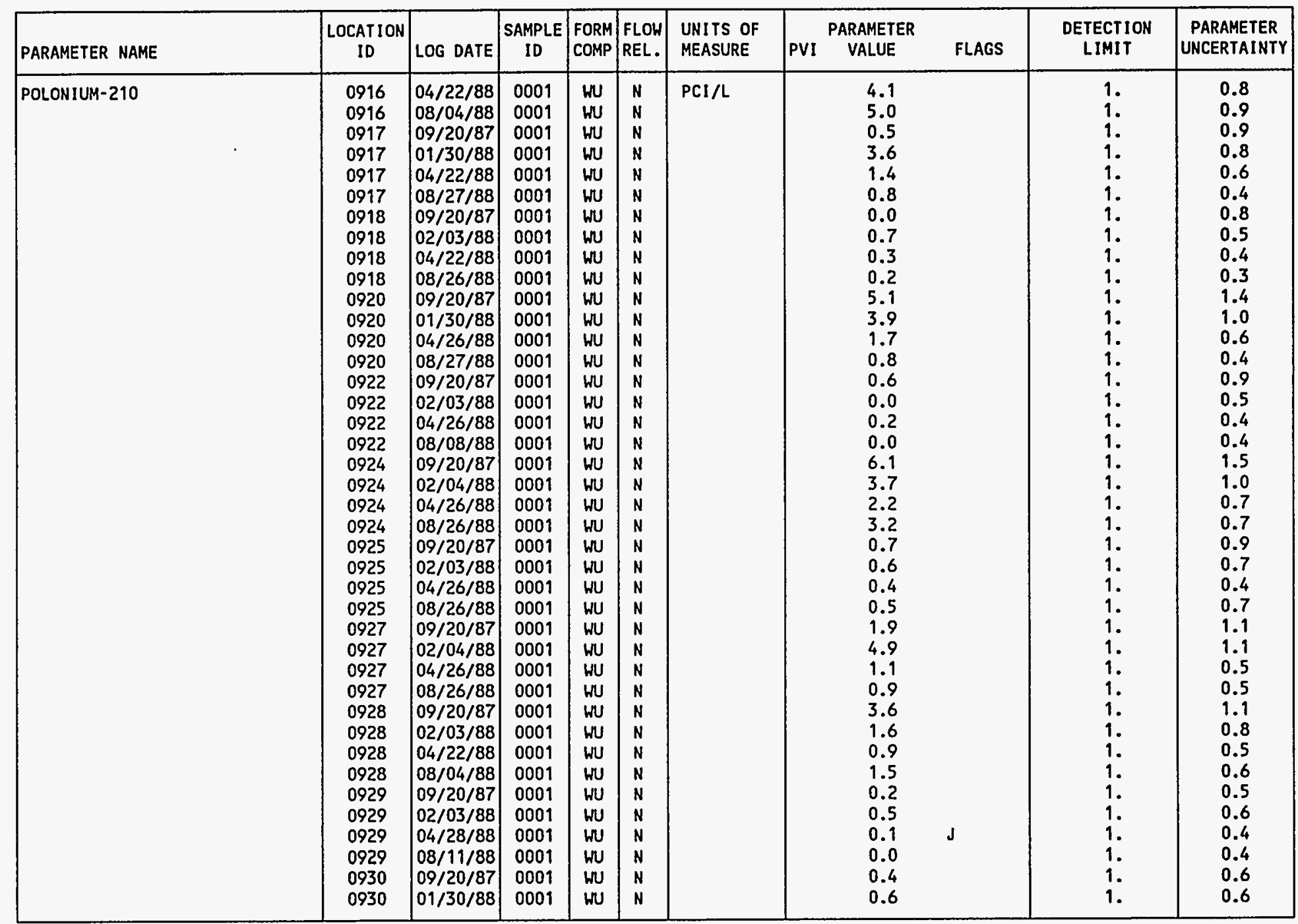

FORMATION OF COMPLETION CODE:

WU - HASATCH - UPPER SANDSTONE

PARAMETER VALUE INDICATOR (PVI): < - LESS THAN DETECTION LIMIT
FLOW RELATIONSHIP CODE:

SAMPLE ID CODES:

0001 - FILTERED SAMPLE (.45 MICRONS)

OTHER PARAMETER VALUE FLAGS:

$J$ - ESTIMATED VALUE 
GROUNDHATER QUALITY DATA BY PARAMETER

UPPER SANDSTONE UNIT

SITE: SPKOI SPOOK

$02 / 17 / 87$ TO $12 / 10 / 88$

REPORT DATE: $07 / 29 / 94$

\begin{tabular}{|c|c|c|c|c|c|c|c|c|c|c|}
\hline PARAMETER NAME & $\mid \begin{array}{c}\text { LOCATION } \\
\text { ID }\end{array}$ & LOG DATE & $\begin{array}{c}\text { SAMPLE } \\
\text { ID }\end{array}$ & $\begin{array}{l}\text { FORM } \\
\text { COMP }\end{array}$ & $\begin{array}{l}\text { FLOW } \\
\text { REL. }\end{array}$ & $\begin{array}{l}\text { UNITS OF } \\
\text { MEASURE }\end{array}$ & PVI & $\begin{array}{l}\text { PARAMETER } \\
\text { VALUE }\end{array}$ & $\begin{array}{l}\text { DETECTION } \\
\text { LIMIT }\end{array}$ & $\begin{array}{l}\text { PARAMETER } \\
\text { UNCERTAINTY }\end{array}$ \\
\hline POLONIUM-210 & $\begin{array}{l}0930 \\
0930 \\
0931 \\
0931 \\
0931 \\
0932 \\
0932 \\
0932 \\
0933 \\
0934 \\
0935 \\
0936 \\
0937 \\
0940 \\
0941 \\
0942 \\
0943 \\
0944\end{array}$ & $\begin{array}{l}04 / 26 / 88 \\
08 / 27 / 88 \\
01 / 30 / 88 \\
04 / 28 / 88 \\
08 / 10 / 88 \\
01 / 30 / 88 \\
04 / 28 / 88 \\
08 / 09 / 88 \\
08 / 10 / 88 \\
08 / 11 / 88 \\
08 / 09 / 88 \\
08 / 08 / 88 \\
08 / 08 / 88 \\
08 / 08 / 88 \\
08 / 08 / 88 \\
08 / 08 / 88 \\
08 / 09 / 88 \\
08 / 11 / 88\end{array}$ & $\begin{array}{l}0001 \\
0001 \\
0001 \\
0001 \\
0001 \\
0001 \\
0001 \\
0001 \\
0001 \\
0001 \\
0001 \\
0001 \\
0001 \\
0001 \\
0001 \\
0001 \\
0001 \\
0001\end{array}$ & $\begin{array}{l}W U \\
W U \\
W U \\
W U \\
W U \\
W U \\
W U \\
W U \\
W U \\
W U \\
W U \\
W U \\
W U \\
W U \\
W U \\
W U \\
W U \\
W U\end{array}$ & $\begin{array}{l}N \\
N \\
N \\
N \\
N \\
N \\
N \\
N \\
N \\
N \\
N \\
N \\
N \\
N \\
N \\
N \\
N \\
N\end{array}$ & $\mathrm{PCI} / \mathrm{L}$ & & $\begin{array}{l}0.2 \\
1.2 \\
0.8 \\
0.3 \\
0.7 \\
3.5 \\
1.8 \\
2.5 \\
0.0 \\
0.7 \\
0.2 \\
1.0 \\
4.5 \\
2.5 \\
0.1 \\
1.2 \\
0.0 \\
0.0\end{array}$ & $\begin{array}{l}1 . \\
1 . \\
1 . \\
1 . \\
1 . \\
1 . \\
1 . \\
1 . \\
1 . \\
1 . \\
1 . \\
1 . \\
1 . \\
1 . \\
1 .\end{array}$ & $\begin{array}{l}0.4 \\
0.5 \\
0.6 \\
0.4 \\
0.5 \\
1.0 \\
0.6 \\
0.7 \\
0.4 \\
0.5 \\
0.5 \\
0.6 \\
0.9 \\
0.7 \\
0.5 \\
0.6 \\
0.4 \\
0.5\end{array}$ \\
\hline POTASSIUM & $\begin{array}{l}0911 \\
0911 \\
0911 \\
0911 \\
0911 \\
0911 \\
0912 \\
0912 \\
0912 \\
0912 \\
0912 \\
0913 \\
0913 \\
0913 \\
0913 \\
0913 \\
0913 \\
0916 \\
0916 \\
0916 \\
0916 \\
0916\end{array}$ & $\begin{array}{l}02 / 17 / 87 \\
09 / 18 / 87 \\
01 / 30 / 88 \\
04 / 22 / 88 \\
08 / 11 / 88 \\
12 / 10 / 88 \\
02 / 18 / 87 \\
02 / 03 / 88 \\
04 / 22 / 88 \\
08 / 27 / 88 \\
12 / 04 / 88 \\
02 / 18 / 87 \\
09 / 18 / 87 \\
02 / 03 / 88 \\
04 / 22 / 88 \\
08 / 04 / 88 \\
12 / 10 / 88 \\
02 / 20 / 87 \\
09 / 20 / 87 \\
02 / 03 / 88 \\
04 / 22 / 88 \\
08 / 04 / 88\end{array}$ & $\begin{array}{l}0001 \\
0001 \\
0001 \\
0001 \\
0001 \\
0001 \\
0001 \\
0001 \\
0001 \\
0001 \\
0001 \\
0001 \\
0001 \\
0001 \\
0001 \\
0001 \\
0001 \\
0001 \\
0001 \\
0001 \\
0001 \\
0001\end{array}$ & $\begin{array}{l}W U \\
W U \\
W U \\
W U \\
W U \\
W U \\
W U \\
W U \\
W U \\
W U \\
W U \\
W U \\
W U \\
W U \\
W U \\
W U \\
W U \\
W U \\
W U \\
W U \\
W U \\
W U\end{array}$ & $\begin{array}{l}N \\
N \\
N \\
N \\
N \\
N \\
N \\
N \\
N \\
N \\
N \\
N \\
N \\
N \\
N \\
N \\
N \\
N \\
N \\
N \\
N \\
N\end{array}$ & $M G / L$ & & $\begin{array}{l}15.5 \\
8.67 \\
9.38 \\
8.66 \\
8.3 \\
8.5 \\
4.00 \\
6.25 \\
9.02 \\
5.7 \\
5.9 \\
9.42 \\
8.71 \\
9.72 \\
9.08 \\
9.5 \\
8.9 \\
10.5 \\
9.60 \\
11.6 \\
10.7 \\
11.8\end{array}$ & $\begin{array}{l}0.01 \\
0.01 \\
0.01 \\
0.01 \\
0.01 \\
0.01 \\
0.01 \\
0.01 \\
0.01 \\
0.01 \\
0.01 \\
0.01 \\
0.01 \\
0.01 \\
0.01 \\
0.01 \\
0.01 \\
0.01 \\
0.01 \\
0.01 \\
0.01 \\
0.01\end{array}$ & 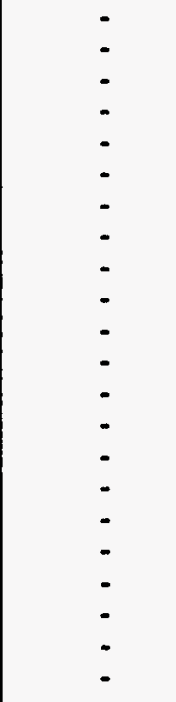 \\
\hline
\end{tabular}

FORMATION OF COMPLETION CODE:

WU - WASATCH - UPPER SANDSTONE

FLOH RELATIONSHIP COOE:

PARAMETER VALUE INDICATOR (PVI): < - LESS THAN DETECTION LIMIT

SAMPLE IO CODES:

0001 - FILTERED SAMPLE (.45 MICRONS) 


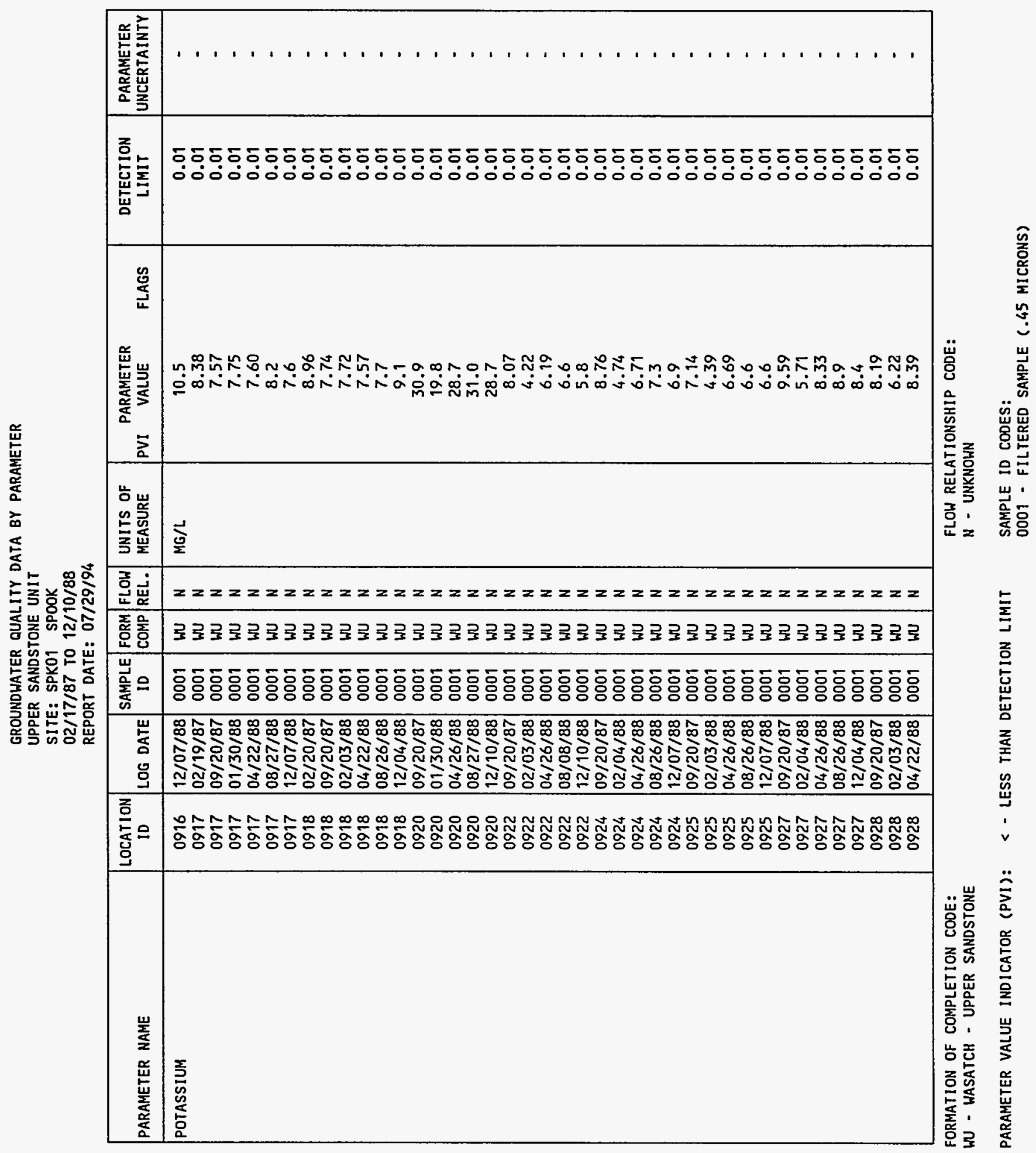




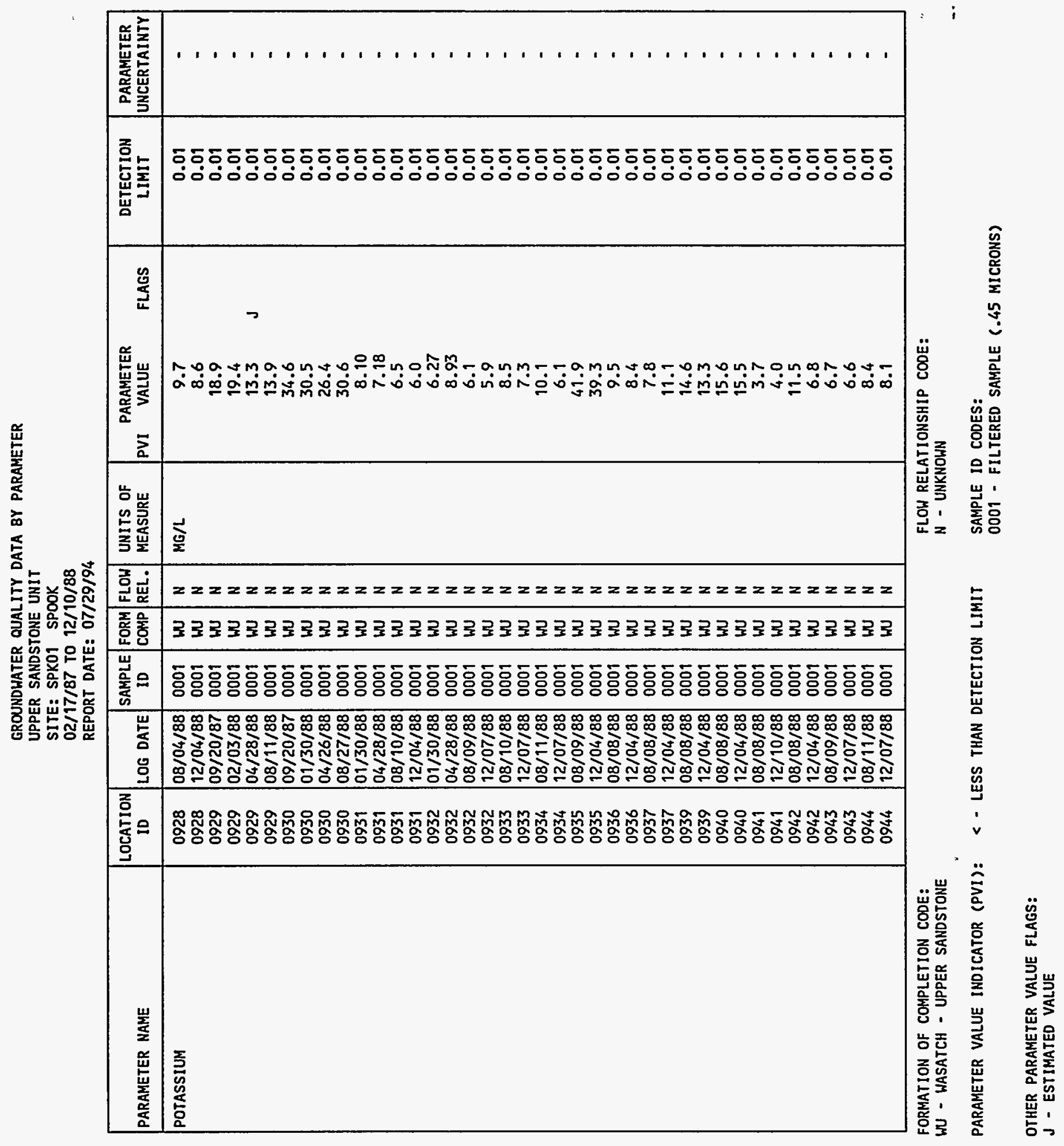


REPORT DATE: $07 / 29 / 94$

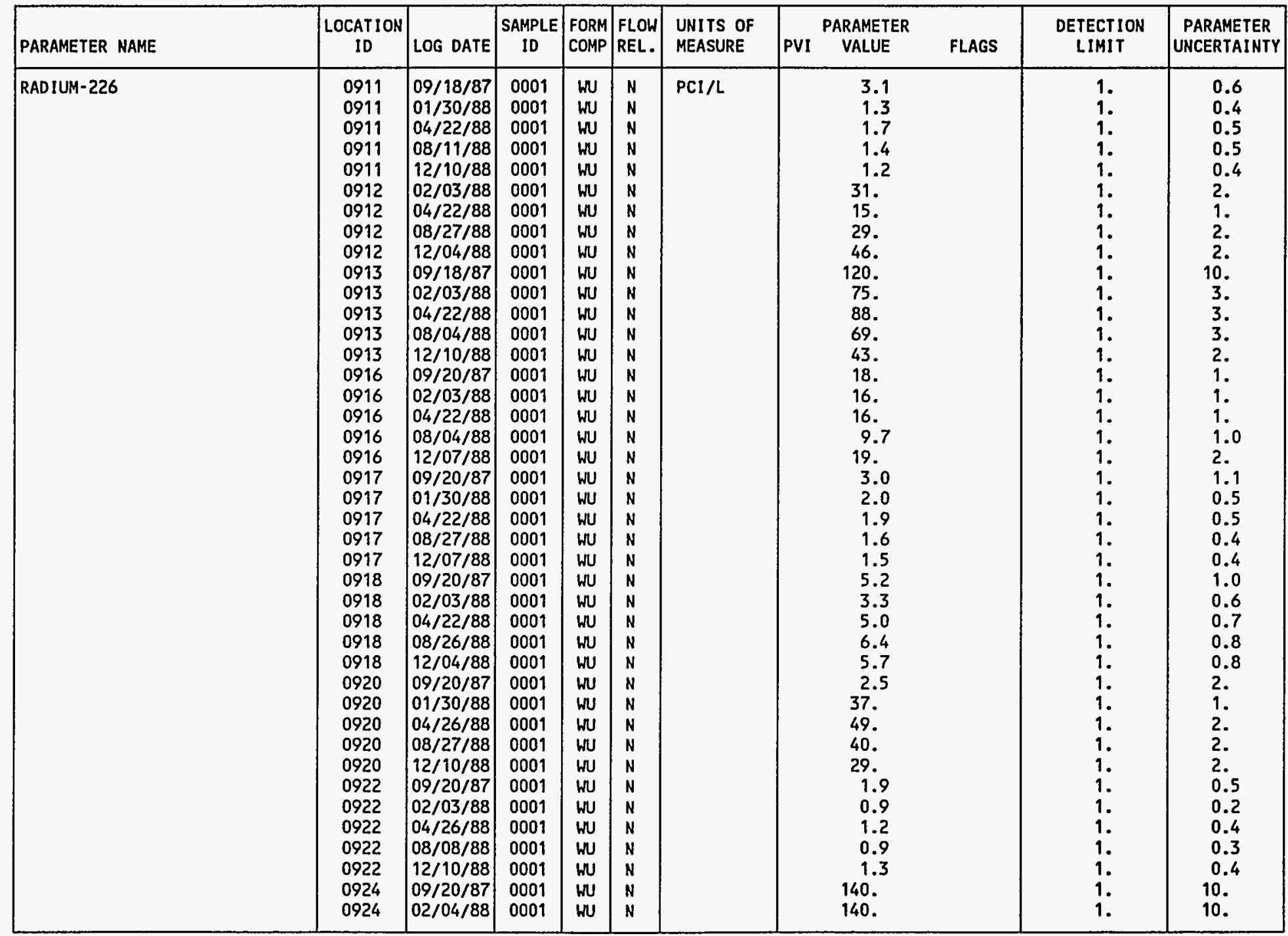

FORMATION OF COMPLETION CODE: WU - WASATCH - UPPER SANDSTONE

PARAMETER VALUE INDICATOR (PVI)
FLOW RELATIONSHIP CODE:

SAMPLE IO CODES:

0001 - FILTERED SAMPLE (.45 MICRONS) 
GROUNDHATER QUALITY DATA BY PARAMETER

UPPER SANDSTONE UNIT

SITE: SPKOI SPOOK

02/17/87 TO $12 / 10 / 88$

REPORT DATE: $07 / 29 / 94$

\begin{tabular}{|c|c|c|c|c|c|c|c|c|c|c|c|}
\hline PARAMETER NAME & $\underset{\text { ID }}{\text { LOCATION }}$ & LOG DATE & $\begin{array}{c}\text { SAMPLE } \\
\text { ID }\end{array}$ & $\begin{array}{l}\text { FORM } \\
\text { COMP }\end{array}$ & $\begin{array}{l}\text { FLOW } \\
\text { REL. }\end{array}$ & $\begin{array}{l}\text { UNITS OF } \\
\text { MEASURE }\end{array}$ & PVI & $\begin{array}{l}\text { PARAMETER } \\
\text { VALUE }\end{array}$ & FLAGS & $\begin{array}{l}\text { DETECTION } \\
\text { LIMIT }\end{array}$ & $\begin{array}{l}\text { PARAMETER } \\
\text { UNCERTAINTY }\end{array}$ \\
\hline RADIUM-226 & $\begin{array}{l}0924 \\
0924 \\
0924 \\
0925 \\
0925 \\
0925 \\
0925 \\
0925 \\
0927 \\
0927 \\
0927 \\
0927 \\
0927 \\
0928 \\
0928 \\
0928 \\
0928 \\
0928 \\
0929 \\
0929 \\
0929 \\
0929 \\
0930 \\
0930 \\
0930 \\
0930 \\
0931 \\
0931 \\
0931 \\
0931 \\
0932 \\
0932 \\
0932 \\
0932 \\
0933 \\
0933 \\
0934 \\
0934 \\
0935 \\
0935\end{array}$ & $\begin{array}{l}04 / 26 / 88 \\
08 / 26 / 88 \\
12 / 07 / 88 \\
09 / 20 / 87 \\
02 / 03 / 88 \\
04 / 26 / 88 \\
08 / 26 / 88 \\
12 / 07 / 88 \\
09 / 20 / 87 \\
02 / 04 / 88 \\
04 / 26 / 88 \\
08 / 26 / 88 \\
12 / 04 / 88 \\
09 / 20 / 87 \\
02 / 03 / 88 \\
04 / 22 / 88 \\
08 / 04 / 88 \\
12 / 04 / 88 \\
09 / 20 / 87 \\
02 / 03 / 88 \\
04 / 28 / 88 \\
08 / 11 / 88 \\
09 / 20 / 87 \\
01 / 30 / 88 \\
04 / 26 / 88 \\
08 / 27 / 88 \\
01 / 30 / 88 \\
04 / 28 / 88 \\
08 / 10 / 88 \\
12 / 04 / 88 \\
01 / 30 / 88 \\
04 / 28 / 88 \\
08 / 09 / 88 \\
12 / 07 / 88 \\
08 / 10 / 88 \\
12 / 07 / 88 \\
08 / 11 / 88 \\
12 / 07 / 88 \\
08 / 09 / 88 \\
12 / 04 / 88\end{array}$ & $\begin{array}{l}0001 \\
0001 \\
0001 \\
0001 \\
0001 \\
0001 \\
0001 \\
0001 \\
0001 \\
0001 \\
0001 \\
0001 \\
0001 \\
0001 \\
0001 \\
0001 \\
0001 \\
0001 \\
0001 \\
0001 \\
0001 \\
0001 \\
0001 \\
0001 \\
0001 \\
0001 \\
0001 \\
0001 \\
0001 \\
0001 \\
0001 \\
0001 \\
0001 \\
0001 \\
0001 \\
0001 \\
0001 \\
0001 \\
0001 \\
0001\end{array}$ & $\begin{array}{l}W U \\
W U \\
W U \\
W U \\
W U \\
W U \\
W U \\
W U \\
W U \\
W U \\
W U \\
W U \\
W U \\
W U \\
W U \\
W U \\
W U \\
W U \\
W U \\
W U \\
W U \\
W U \\
W U \\
W U \\
W U \\
W U \\
W U \\
W U \\
W U \\
W U \\
W U \\
W U \\
W U \\
W U \\
W U \\
W U \\
W U\end{array}$ & $\begin{array}{l}N \\
N \\
N \\
N \\
N \\
N \\
N \\
N \\
N \\
N \\
N \\
N \\
N \\
N \\
N \\
N \\
N \\
N \\
N \\
N \\
N \\
N \\
N \\
N \\
N \\
N \\
N \\
N \\
N \\
N \\
N \\
N \\
N \\
N \\
N \\
N \\
N \\
N \\
N \\
N\end{array}$ & $\mathrm{PCI} / \mathrm{L}$ & & $\begin{array}{c}123 . \\
135 . \\
190 . \\
2.5 \\
0.6 \\
0.2 \\
0.9 \\
0.7 \\
13 . \\
11 . \\
9.6 \\
13 . \\
10 . \\
6.8 \\
8.3 \\
7.3 \\
4.6 \\
7.6 \\
1.2 \\
1.9 \\
0.4 \\
1.7 \\
3.3 \\
2.1 \\
2.7 \\
13 . \\
0.7 \\
0.5 \\
1.0 \\
0.8 \\
14 . \\
13 . \\
47 . \\
46 . \\
0.7 \\
1.0 \\
3.8 \\
1.9 \\
46 . \\
74 .\end{array}$ & J & $\begin{array}{l}1 . \\
1 . \\
1 . \\
1 . \\
1 . \\
1 . \\
1 . \\
1 . \\
1 . \\
1 . \\
1 . \\
1 . \\
1 . \\
1 . \\
1 . \\
1 . \\
1 . \\
1 . \\
1 . \\
1 . \\
1 . \\
1 . \\
1 . \\
1 . \\
1 . \\
1 . \\
1 . \\
1 . \\
1 . \\
1 . \\
1 . \\
1 .\end{array}$ & $\begin{array}{c}4 . \\
4 . \\
10 . \\
0.6 \\
0.2 \\
0.5 \\
0.3 \\
0.3 \\
1 . \\
1 . \\
1.0 \\
1 . \\
1 . \\
0.9 \\
0.9 \\
0.9 \\
0.7 \\
1.0 \\
0.4 \\
0.5 \\
0.5 \\
0.5 \\
0.6 \\
0.5 \\
0.5 \\
1.5 \\
0.3 \\
0.3 \\
0.4 \\
0.3 \\
1 . \\
1 . \\
2 . \\
2 . \\
0.3 \\
0.4 \\
0.7 \\
0.5 \\
2 . \\
3 .\end{array}$ \\
\hline
\end{tabular}

FORMATION OF COMPLETION CODE:

FLOW RELATIONSHIP CODE:

WU - WASATCH - UPPER SANDSTONE

N - UNKNOHN

PARAMETER VALUE INDICATOR (PVI): < - LESS THAN DETECTION LIMIT

SAMLE 10 CODES:

0001 - FILTERED SAMPLE (.45 MICRONS)

OTHER PARAMETER VALUE FLAGS:

$J$ - EST IMATED VALUE 
GROUNDHATER QUALITY DATA BY PARAMETER

UPPER SAHDSTOHE UNIT

SITE: SPKO1 SPOOK

02/17/87 TO $12 / 10 / 88$

REPORT DATE: $07 / 29 / 94$

\begin{tabular}{|c|c|c|c|c|c|c|c|c|c|c|}
\hline PARAMETER NAME & $\begin{array}{l}\text { LOCATION } \\
\text { ID }\end{array}$ & LOG DATE & $\begin{array}{c}\text { SAMPLE } \\
\text { ID }\end{array}$ & $\begin{array}{l}\text { FORM } \\
\text { COMP }\end{array}$ & $\begin{array}{l}\text { FLOH } \\
\text { REL. }\end{array}$ & $\begin{array}{l}\text { UNITS OF } \\
\text { MEASURE }\end{array}$ & PVI & $\begin{array}{l}\text { PARAMETER } \\
\text { VALUE }\end{array}$ & $\begin{array}{l}\text { DETECTION } \\
\text { LIMIT }\end{array}$ & $\begin{array}{l}\text { PARAMETER } \\
\text { UNCERTAINTY }\end{array}$ \\
\hline RADIUM-226 & $\begin{array}{l}0936 \\
0936 \\
0937 \\
0937 \\
0939 \\
0939 \\
0940 \\
0940 \\
0941 \\
0941 \\
0942 \\
0942 \\
0943 \\
0943 \\
0944 \\
0944\end{array}$ & $\begin{array}{l}08 / 08 / 88 \\
12 / 04 / 88 \\
08 / 08 / 88 \\
12 / 04 / 88 \\
08 / 08 / 88 \\
12 / 04 / 88 \\
08 / 08 / 88 \\
12 / 04 / 88 \\
08 / 08 / 88 \\
12 / 10 / 88 \\
08 / 08 / 88 \\
12 / 04 / 88 \\
08 / 09 / 88 \\
12 / 07 / 88 \\
08 / 11 / 88 \\
12 / 07 / 88\end{array}$ & $\begin{array}{l}0001 \\
0001 \\
0001 \\
0001 \\
0001 \\
0001 \\
0001 \\
0001 \\
0001 \\
0001 \\
0001 \\
0001 \\
0001 \\
0001 \\
0001 \\
0001\end{array}$ & $\begin{array}{l}W U \\
W U \\
W U \\
W U \\
W U \\
W U \\
W U \\
W U \\
W U \\
W U \\
W U \\
W U \\
W U \\
W U \\
W U \\
W U\end{array}$ & $\begin{array}{l}N \\
N \\
N \\
N \\
N \\
N \\
N \\
N \\
N \\
N \\
N \\
N \\
N \\
N \\
N \\
N\end{array}$ & $\mathrm{PCI} / \mathrm{L}$ & & $\begin{array}{c}5.4 \\
4.2 \\
3.3 \\
4.2 \\
3.7 \\
3.7 \\
61 . \\
83 . \\
0.9 \\
1.3 \\
1.3 \\
0.9 \\
0.7 \\
0.6 \\
0.5 \\
0.9\end{array}$ & $\begin{array}{l}1 . \\
1 . \\
1 . \\
1 . \\
1 . \\
1 . \\
1 . \\
1 . \\
1 . \\
1 . \\
1 .\end{array}$ & $\begin{array}{l}0.8 \\
0.7 \\
0.6 \\
0.7 \\
0.8 \\
0.7 \\
3 . \\
3 . \\
0.3 \\
0.4 \\
0.4 \\
0.3 \\
0.3 \\
0.3 \\
0.2 \\
0.3\end{array}$ \\
\hline RADIUM-226 + RADIUM-228 & $\begin{array}{l}0911 \\
0911 \\
0911 \\
0911 \\
0911 \\
0912 \\
0912 \\
0912 \\
0912 \\
0913 \\
0913 \\
0913 \\
0913 \\
0913 \\
0916 \\
0916 \\
0916 \\
0916 \\
0916 \\
0917 \\
0917 \\
0917 \\
0917 \\
0917\end{array}$ & $\begin{array}{l}09 / 18 / 87 \\
01 / 30 / 88 \\
04 / 22 / 88 \\
08 / 11 / 88 \\
12 / 10 / 88 \\
02 / 03 / 88 \\
04 / 22 / 88 \\
08 / 27 / 88 \\
12 / 04 / 88 \\
09 / 18 / 87 \\
02 / 03 / 88 \\
04 / 22 / 88 \\
08 / 04 / 88 \\
12 / 10 / 88 \\
09 / 20 / 87 \\
02 / 03 / 88 \\
04 / 22 / 88 \\
08 / 04 / 88 \\
12 / 07 / 88 \\
09 / 20 / 87 \\
01 / 30 / 88 \\
04 / 22 / 88 \\
08 / 27 / 88 \\
12 / 07 / 88\end{array}$ & $\begin{array}{l}0001 \\
0001 \\
0001 \\
0001 \\
0001 \\
0001 \\
0001 \\
0001 \\
0001 \\
0001 \\
0001 \\
0001 \\
0001 \\
0001 \\
0001 \\
0001 \\
0001 \\
0001 \\
0001 \\
0001 \\
0001 \\
0001 \\
0001 \\
0001\end{array}$ & $\begin{array}{l}W U \\
W U \\
W U \\
W U \\
W U \\
W U \\
W U \\
W U \\
W U \\
W U \\
W U \\
W U \\
W U \\
W U \\
W U \\
W U \\
W U \\
W U \\
W U \\
W U \\
W U \\
W U \\
W U \\
W U\end{array}$ & $\begin{array}{l}N \\
N \\
N \\
N \\
N \\
N \\
N \\
N \\
N \\
N \\
N \\
N \\
N \\
N \\
N \\
N \\
N \\
N \\
N \\
N \\
N \\
N \\
N \\
N\end{array}$ & $\mathrm{PCI} / \mathrm{L}$ & & $\begin{array}{r}4.00 \\
1.40 \\
2.50 \\
2.60 \\
2.10 \\
31.30 \\
15.60 \\
30.20 \\
47.10 \\
122.70 \\
77.80 \\
90.10 \\
71.30 \\
45.30 \\
22.10 \\
21.10 \\
21.80 \\
15.10 \\
24.60 \\
5.50 \\
4.60 \\
6.00 \\
5.00 \\
5.90\end{array}$ & $\begin{array}{l}: \\
: \\
: \\
: \\
: \\
- \\
- \\
- \\
- \\
- \\
- \\
- \\
- \\
- \\
- \\
- \\
-\end{array}$ & $\begin{array}{l}- \\
- \\
- \\
- \\
- \\
- \\
- \\
- \\
- \\
- \\
- \\
- \\
- \\
- \\
- \\
-\end{array}$ \\
\hline
\end{tabular}

FORMATION OF COMPLETION CODE:

WU - WASATCH - UPPER SANDSTONE

FLOW RELATIONSHIP CODE:

PARAMETER VALUE INDICATOR (PVI): < - LESS THAN DETECTION LIMIT

SAMPLE ID CODES:

0001 - FILTERED SAMPLE (.45 MICRONS) 


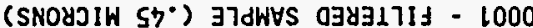

:S300J ol 37dwrs

MMONXNO - N

: 3003 dIHSNOII甘7ЭУ MOT

\begin{tabular}{|c|c|c|c|c|c|c|c|c|c|c|}
\hline 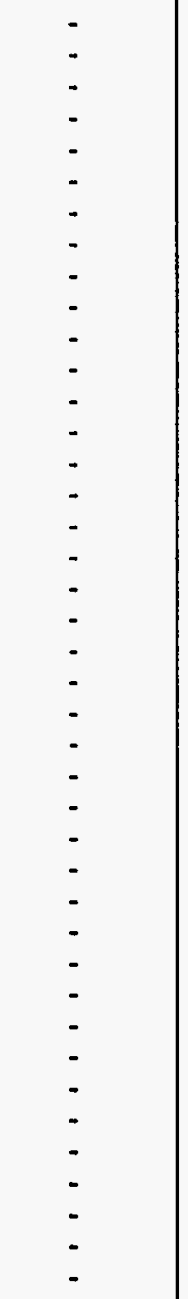 & 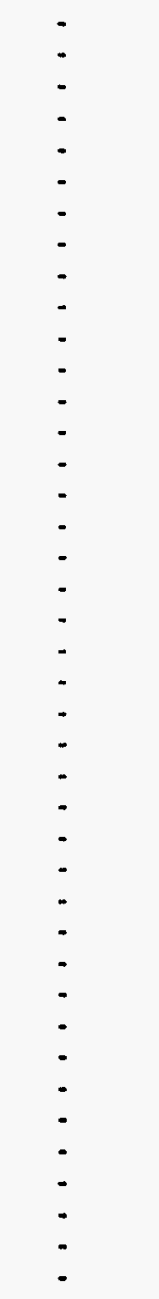 & & 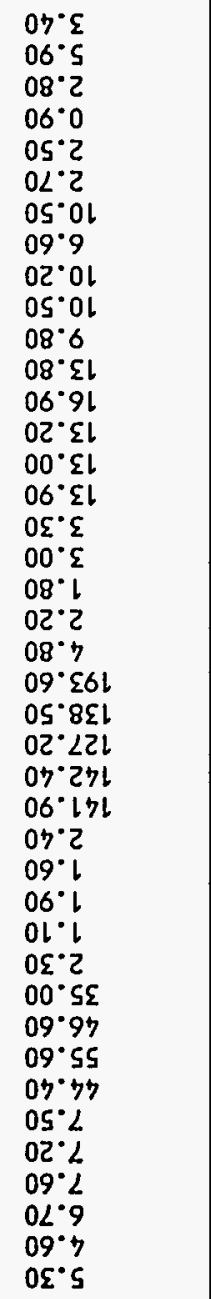 & (⿸丆口 & $\begin{array}{l}N \\
N \\
N \\
N \\
N \\
N \\
N \\
N \\
N \\
N \\
N \\
N \\
N \\
N \\
N \\
N \\
N \\
N \\
N \\
N \\
N \\
N \\
N \\
N \\
N \\
N \\
N \\
N \\
N \\
N \\
N \\
N \\
N \\
N \\
N \\
N \\
N \\
N \\
N \\
N \\
N\end{array}$ & $\begin{array}{l}n M \\
n M \\
n M \\
n M \\
n M \\
n M \\
n M \\
n M \\
n M \\
n M \\
n M \\
n M \\
n M \\
n M \\
n M \\
n M \\
n M \\
n M \\
n M \\
n M \\
n M \\
n M \\
n M \\
n M \\
n M \\
n M \\
n M \\
n M \\
n M \\
n M \\
n M \\
n M \\
N M \\
n M \\
n M \\
n M \\
n M \\
N M \\
n M \\
n M \\
n M\end{array}$ & $\begin{array}{l}1000 \\
1000 \\
1000 \\
1000 \\
1000 \\
1000 \\
1000 \\
1000 \\
1000 \\
1000 \\
1000 \\
1000 \\
1000 \\
1000 \\
1000 \\
1000 \\
1000 \\
1000 \\
1000 \\
1000 \\
1000 \\
1000 \\
1000 \\
1000 \\
1000 \\
1000 \\
1000 \\
1000 \\
1000 \\
1000 \\
1000 \\
1000 \\
1000 \\
1000 \\
1000 \\
1000 \\
1000 \\
1000 \\
1000 \\
1000 \\
1000\end{array}$ & 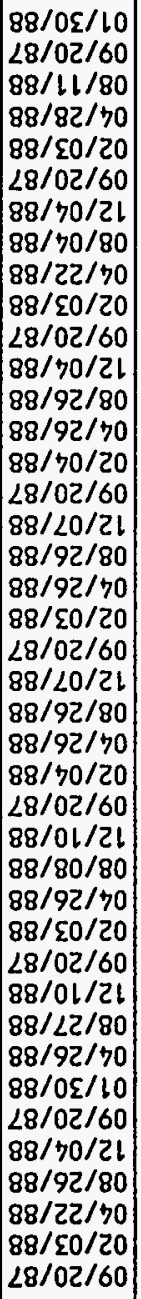 & $\begin{array}{l}0 乏 60 \\
0 £ 60 \\
6260 \\
6260 \\
6260 \\
6260 \\
8260 \\
8260 \\
8260 \\
8260 \\
8260 \\
2260 \\
2260 \\
2260 \\
2260 \\
2260 \\
5260 \\
5260 \\
5260 \\
5260 \\
5260 \\
\$ 260 \\
7260 \\
\$ 260 \\
\$ 260 \\
\$ 260 \\
2260 \\
2260 \\
2260 \\
2260 \\
2260 \\
0260 \\
0260 \\
0260 \\
0260 \\
0260 \\
8160 \\
8160 \\
8160 \\
8160 \\
8160\end{array}$ & (1) \\
\hline 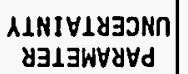 & 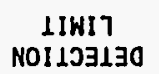 & SפชาA & 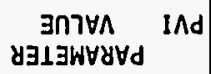 & $\begin{array}{l}\text { Jyns } \\
\text { jo S1INn }\end{array}$ & $\left|\begin{array}{c}798 \\
\text { M075 }\end{array}\right|$ & $\begin{array}{l}\text { dWOJ } \\
\text { WYOS }\end{array}$ & $\begin{array}{c}01 \\
\text { 3าdW }\end{array}$ & 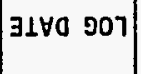 & aI & $\exists W \forall N$ \& $\exists \perp \exists W \forall Y \forall d$ \\
\hline
\end{tabular}

$76 / 62 / \angle 0$ : $\exists 1 \forall 0$ 180d $\exists y$

$88 / 0 l / 2 l$ ol $\angle 8 / L L / 20$

XOOdS LOXdS : $\exists 1 I S$

IIIn JNOLSOMYS y

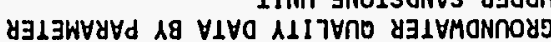

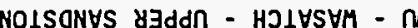
- 
GROUNDHATER QUALITY DATA BY PARAMETER

UPPER SANDSTONE UNIT

SITE: SPKO1 SPOOK

02/17/87 TO $12 / 10 / 88$

REPORT DATE: $07 / 29 / 94$

\begin{tabular}{|c|c|c|c|c|c|c|c|c|c|c|}
\hline PARAMETER NAME & $\underset{\text { ID }}{\text { LOCATION }}$ & LOG DATE & $\begin{array}{c}\text { SAMPLE } \\
10\end{array}$ & $\begin{array}{l}\text { FORM } \\
\text { COMP }\end{array}$ & $\begin{array}{l}\text { FL.OW } \\
\text { REL. }\end{array}$ & $\begin{array}{l}\text { UNITS OF } \\
\text { MEASURE }\end{array}$ & PVI & $\begin{array}{l}\text { PARAMETER } \\
\text { VALUE }\end{array}$ & $\begin{array}{l}\text { DETECTION } \\
\text { LIMIT }\end{array}$ & $\begin{array}{l}\text { PARAMETER } \\
\text { UNCERTAINTY }\end{array}$ \\
\hline RADIUM-226 + RADIUM-228 & $\begin{array}{l}0930 \\
0930 \\
0931 \\
0931 \\
0931 \\
0931 \\
0932 \\
0932 \\
0932 \\
0932 \\
0933 \\
0933 \\
0934 \\
0934 \\
0935 \\
0935 \\
0936 \\
0936 \\
0937 \\
0937 \\
0939 \\
0939 \\
0940 \\
0940 \\
0941 \\
0941 \\
0942 \\
0942 \\
0943 \\
0943 \\
0944 \\
0944\end{array}$ & $\begin{array}{l}04 / 26 / 88 \\
08 / 27 / 88 \\
01 / 30 / 88 \\
04 / 28 / 88 \\
08 / 10 / 88 \\
12 / 04 / 88 \\
01 / 30 / 88 \\
04 / 28 / 88 \\
08 / 09 / 88 \\
12 / 07 / 88 \\
08 / 10 / 88 \\
12 / 07 / 88 \\
08 / 11 / 88 \\
12 / 07 / 88 \\
08 / 09 / 88 \\
12 / 04 / 88 \\
08 / 08 / 88 \\
12 / 04 / 88 \\
08 / 08 / 88 \\
12 / 04 / 88 \\
08 / 08 / 88 \\
12 / 04 / 88 \\
08 / 08 / 88 \\
12 / 04 / 88 \\
08 / 08 / 88 \\
12 / 10 / 88 \\
08 / 08 / 88 \\
12 / 04 / 88 \\
08 / 09 / 88 \\
12 / 07 / 88 \\
08 / 11 / 88 \\
12 / 07 / 88\end{array}$ & $\begin{array}{l}0001 \\
0001 \\
0001 \\
0001 \\
0001 \\
0001 \\
0001 \\
0001 \\
0001 \\
0001 \\
0001 \\
0001 \\
0001 \\
0001 \\
0001 \\
0001 \\
0001 \\
0001 \\
0001 \\
0001 \\
0001 \\
0001 \\
0001 \\
0001 \\
0001 \\
0001 \\
0001 \\
0001 \\
0001 \\
0001 \\
0001 \\
0001\end{array}$ & $\begin{array}{l}W U \\
W U \\
W U \\
W U \\
W U \\
W U \\
W U \\
W U \\
W U \\
W U \\
W U \\
W U \\
W U \\
W U \\
W U \\
W U \\
W U \\
W U \\
W U \\
W U \\
W U \\
W U \\
W U \\
W U \\
W U \\
W U \\
W U \\
W U \\
W U \\
W U \\
W U \\
W U\end{array}$ & $\begin{array}{l}N \\
N \\
N \\
N \\
N \\
N \\
N \\
N \\
N \\
N \\
N \\
N \\
N \\
N \\
N \\
N \\
N \\
N \\
N \\
N \\
N \\
N \\
N \\
N \\
N \\
N \\
N \\
N \\
N \\
N \\
N \\
N\end{array}$ & PCI/L & & $\begin{array}{r}4.90 \\
16.80 \\
1.60 \\
2.20 \\
2.80 \\
2.60 \\
15.40 \\
14.00 \\
48.40 \\
47.80 \\
2.10 \\
2.90 \\
5.10 \\
3.50 \\
54.80 \\
89.00 \\
7.10 \\
6.40 \\
4.10 \\
5.80 \\
4.20 \\
4.80 \\
68.70 \\
94.00 \\
2.30 \\
3.20 \\
1.30 \\
1.00 \\
1.30 \\
1.80 \\
1.90 \\
3.40\end{array}$ & 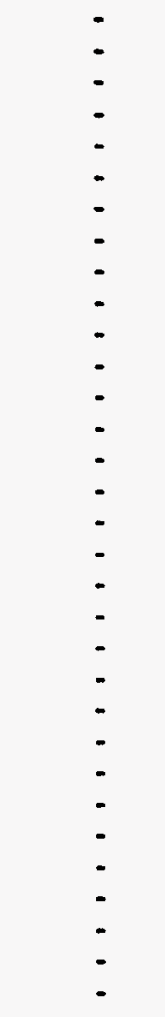 & $\begin{array}{l}- \\
- \\
- \\
- \\
- \\
- \\
- \\
- \\
- \\
- \\
- \\
- \\
- \\
- \\
- \\
- \\
- \\
- \\
- \\
- \\
- \\
- \\
- \\
-\end{array}$ \\
\hline RADIUM-228 & $\begin{array}{l}0911 \\
0911 \\
0911 \\
0911 \\
0911 \\
0912 \\
0912 \\
0912\end{array}$ & $\begin{array}{l}09 / 18 / 87 \\
01 / 30 / 88 \\
04 / 22 / 88 \\
08 / 11 / 88 \\
12 / 10 / 88 \\
02 / 03 / 88 \\
04 / 22 / 88 \\
08 / 27 / 88\end{array}$ & $\begin{array}{l}0001 \\
0001 \\
0001 \\
0001 \\
0001 \\
0001 \\
0001 \\
0001\end{array}$ & $\begin{array}{l}\text { WU } \\
W U \\
W U \\
W U \\
W U \\
W U \\
W U \\
W U\end{array}$ & $\begin{array}{l}N \\
N \\
N \\
N \\
N \\
N \\
N \\
N\end{array}$ & $\mathrm{PCI} / \mathrm{L}$ & & $\begin{array}{l}0.9 \\
0.1 \\
0.8 \\
1.2 \\
0.9 \\
0.3 \\
0.6 \\
1.2\end{array}$ & $\begin{array}{l}1 . \\
1 . \\
1 . \\
1 . \\
1 . \\
1 .\end{array}$ & $\begin{array}{l}1.2 \\
1.0 \\
0.7 \\
0.7 \\
0.9 \\
0.9 \\
0.8 \\
0.9\end{array}$ \\
\hline
\end{tabular}

FORMATION OF COMPLETION CODE:

FLOW RELATIONSHIP CODE:

WU - WASATCH - UPPER SANDSTONE

N - UNKNOWN

PARAMETER VALUE INDICATOR (PVI): < - LESS THAN DETECTION LIMIT

10 CODES:

0001 - FILTERED SAMPLE (.45 MICRONS) 


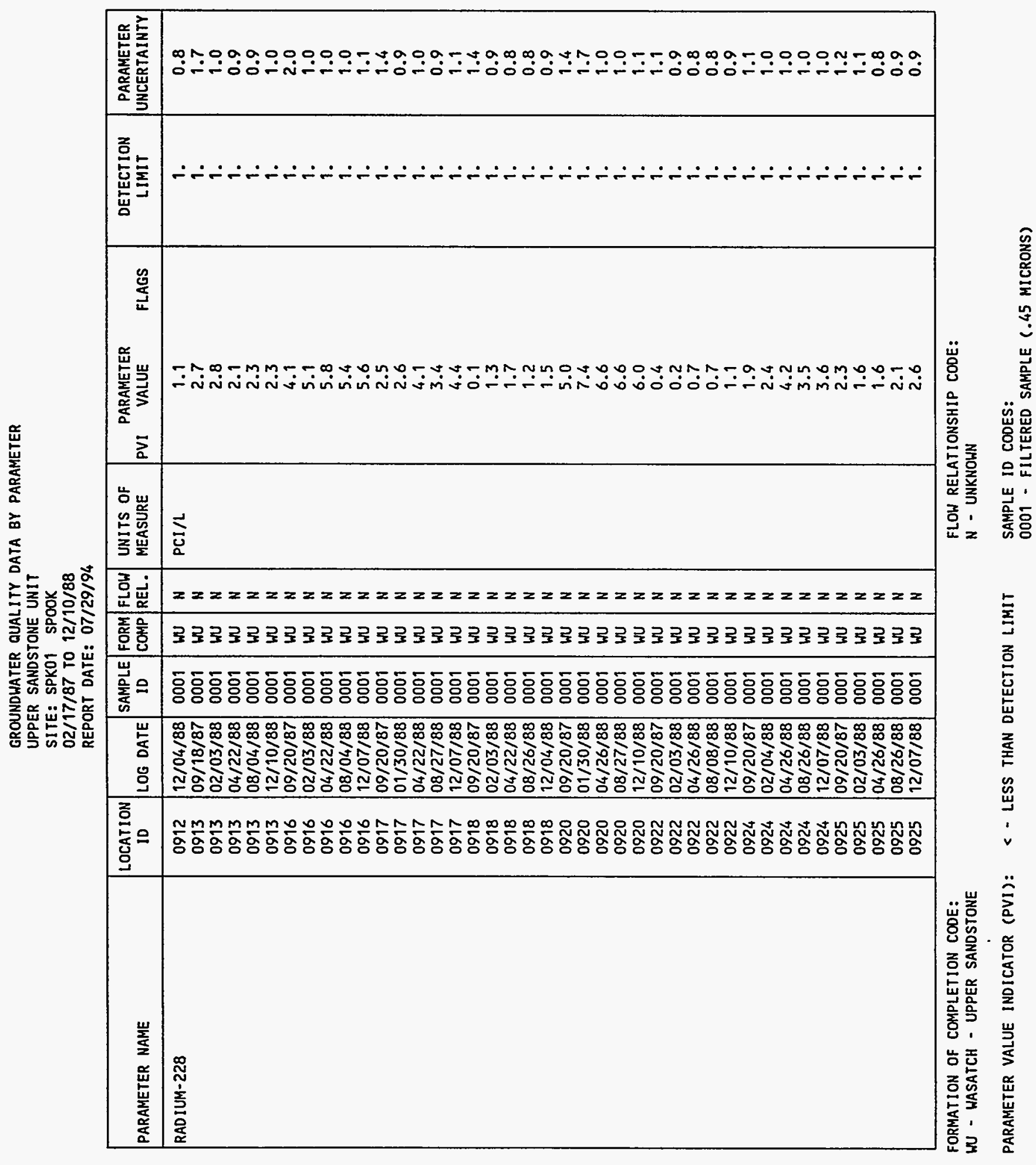




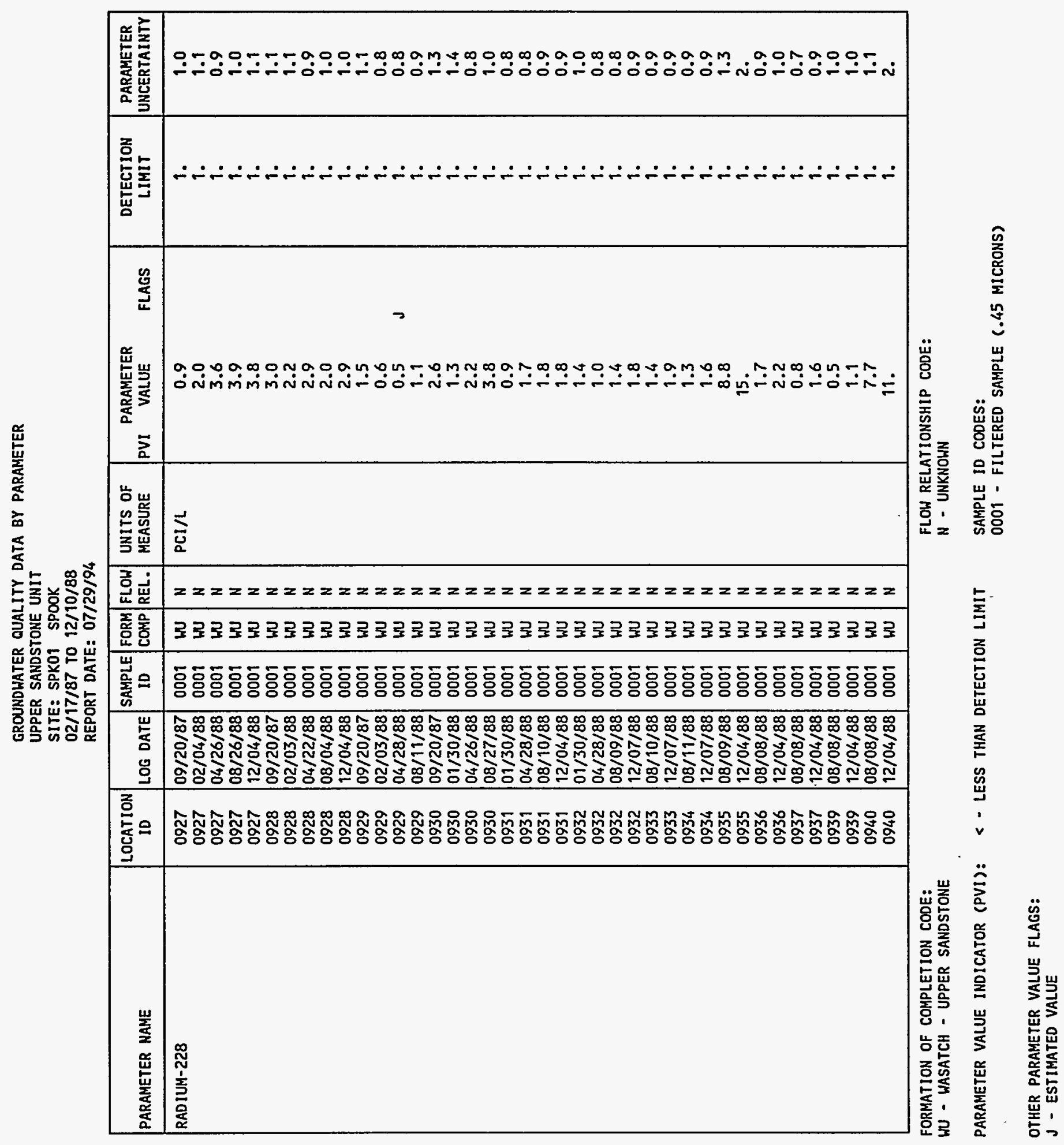




\begin{tabular}{|c|c|c|c|c|c|c|}
\hline & 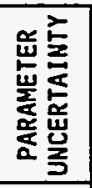 & 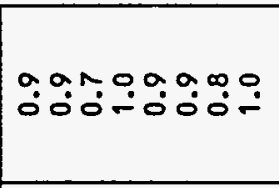 & 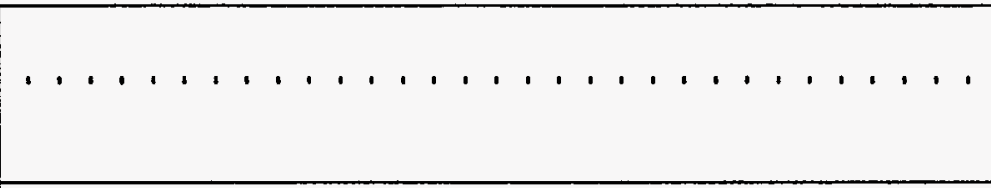 & \multirow{10}{*}{\multicolumn{2}{|c|}{ 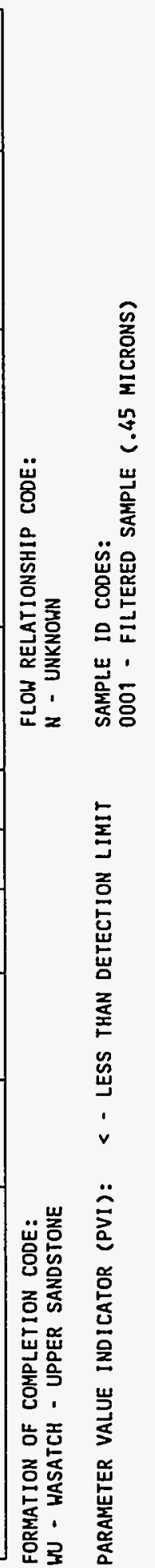 }} & \\
\hline \multirow{9}{*}{ 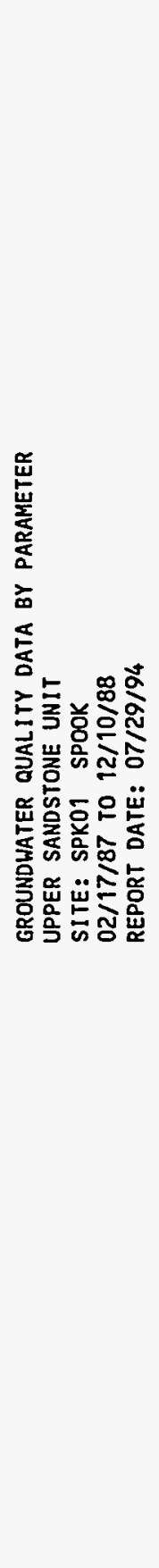 } & 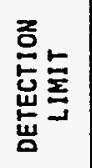 & 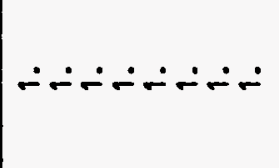 & 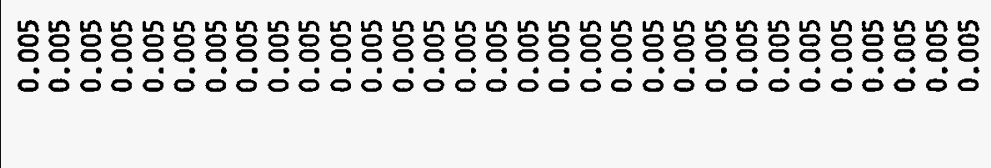 & & & \\
\hline & 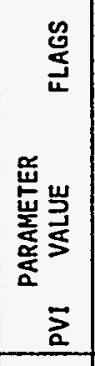 & 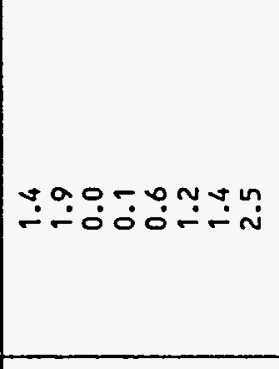 & 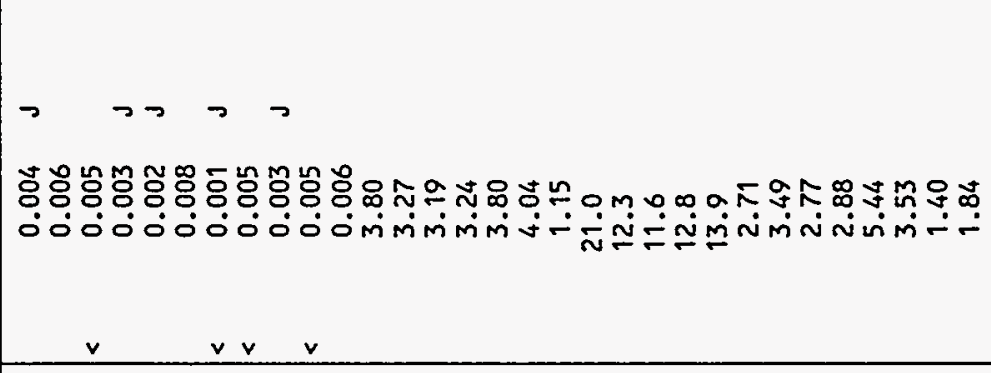 & & & \\
\hline & 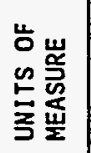 & $\mid \vec{\Xi}$ & ड़े & & & \\
\hline & 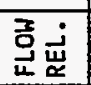 & $2 z 2 z 2 z 2 z$ & 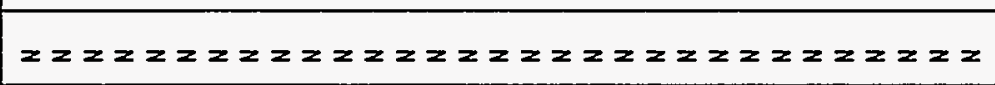 & & & \\
\hline & 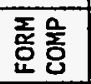 & 코로로코코코코 & 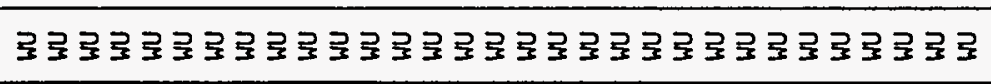 & & & \\
\hline & 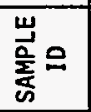 & 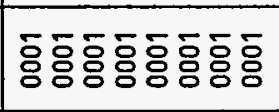 & 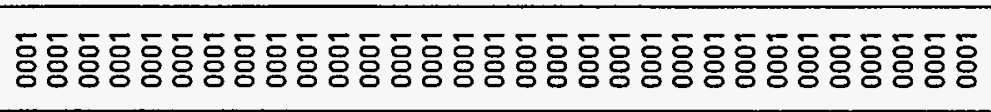 & & & \\
\hline & $\begin{array}{l}\text { 岁 } \\
\text { 号 } \\
\Xi \\
\end{array}$ & 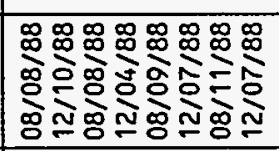 & 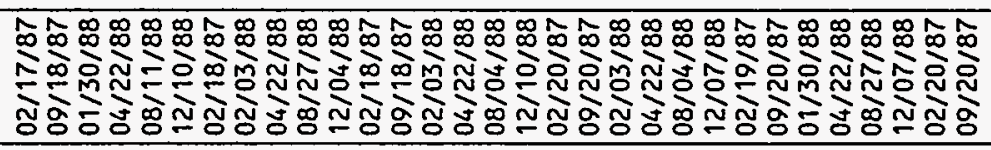 & & & \\
\hline & 悹 & 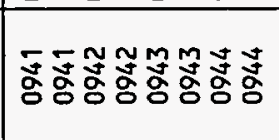 & 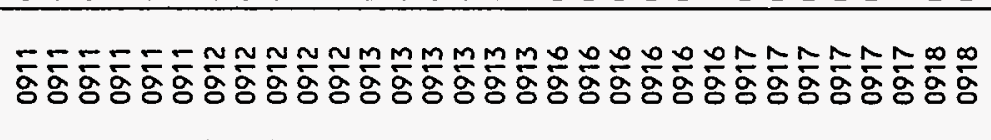 & & & \\
\hline & 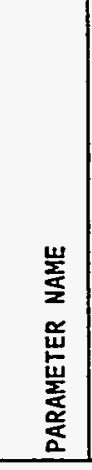 & 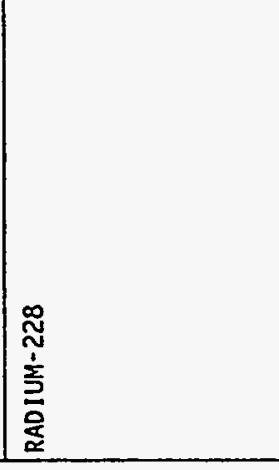 & 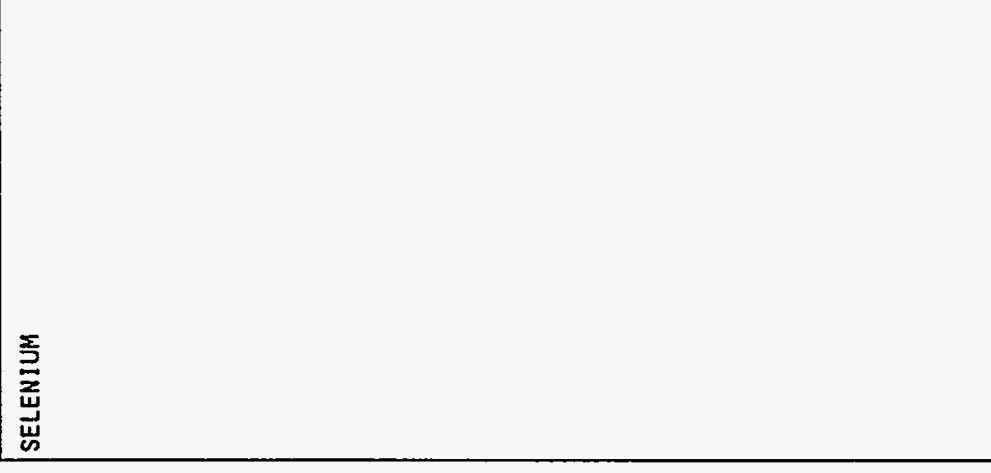 & & & 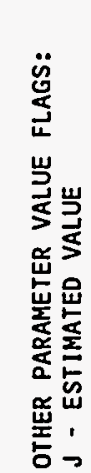 \\
\hline
\end{tabular}


GROUNDHATER QUALITY DATA BY PARAMETER

UPPER SANDSTONE UNIT

SITE: SPKO1 SPOOK

02/17/87 TO 12/10/88

REPORT DATE: $07 / 29 / 94$

\begin{tabular}{|c|c|c|c|c|c|c|c|c|c|c|c|}
\hline PARAMETER NAME & $\begin{array}{l}\text { LOCATION } \\
\text { ID }\end{array}$ & LOG DATE & $\begin{array}{c}\text { SAMPLE } \\
\text { ID }\end{array}$ & $\begin{array}{l}\text { FORM } \\
\text { COMP }\end{array}$ & $\begin{array}{l}\text { FLOW } \\
\text { REL. }\end{array}$ & $\begin{array}{l}\text { UNITS OF } \\
\text { MEASURE }\end{array}$ & PVI & $\begin{array}{l}\text { PARAMETER } \\
\text { VALUE }\end{array}$ & FLAGS & $\begin{array}{l}\text { DETECTION } \\
\text { LIMIT }\end{array}$ & $\begin{array}{l}\text { PARAMETER } \\
\text { UNCERTAINTY }\end{array}$ \\
\hline SELENIUM & $\begin{array}{l}0918 \\
0918 \\
0918 \\
0918 \\
0920 \\
0920 \\
0920 \\
0920 \\
0920 \\
0922 \\
0922 \\
0922 \\
0922 \\
0922 \\
0924 \\
0924 \\
0924 \\
0924 \\
0924 \\
0925 \\
0925 \\
0925 \\
0925 \\
0925 \\
0927 \\
0927 \\
0927 \\
0927 \\
0927 \\
0928 \\
0928 \\
0928 \\
0928 \\
0928 \\
0929 \\
0929 \\
0929 \\
0929 \\
0930 \\
0930\end{array}$ & $\begin{array}{l}02 / 03 / 88 \\
04 / 22 / 88 \\
08 / 26 / 88 \\
12 / 04 / 88 \\
09 / 20 / 87 \\
01 / 30 / 88 \\
04 / 26 / 88 \\
08 / 27 / 88 \\
12 / 10 / 88 \\
09 / 20 / 87 \\
02 / 03 / 88 \\
04 / 26 / 88 \\
08 / 08 / 88 \\
12 / 10 / 88 \\
09 / 20 / 87 \\
02 / 04 / 88 \\
04 / 26 / 88 \\
08 / 26 / 88 \\
12 / 07 / 88 \\
09 / 20 / 87 \\
02 / 03 / 88 \\
04 / 26 / 88 \\
08 / 26 / 88 \\
12 / 07 / 88 \\
09 / 20 / 87 \\
02 / 04 / 88 \\
04 / 26 / 88 \\
08 / 26 / 88 \\
12 / 04 / 88 \\
09 / 20 / 87 \\
02 / 03 / 88 \\
04 / 22 / 88 \\
08 / 04 / 88 \\
12 / 04 / 88 \\
09 / 20 / 87 \\
02 / 03 / 88 \\
04 / 28 / 88 \\
08 / 11 / 88 \\
09 / 20 / 87 \\
01 / 30 / 88\end{array}$ & $\begin{array}{l}0001 \\
0001 \\
0001 \\
0001 \\
0001 \\
0001 \\
0001 \\
0001 \\
0001 \\
0001 \\
0001 \\
0001 \\
0001 \\
0001 \\
0001 \\
0001 \\
0001 \\
0001 \\
0001 \\
0001 \\
0001 \\
0001 \\
0001 \\
0001 \\
0001 \\
0001 \\
0001 \\
0001 \\
0001 \\
0001 \\
0001 \\
0001 \\
0001 \\
0001 \\
0001 \\
0001 \\
0001 \\
0001 \\
0001 \\
0001\end{array}$ & $\begin{array}{l}W U \\
W U \\
W U \\
W U \\
W U \\
W U \\
W U \\
W U \\
W U \\
W U \\
W U \\
W U \\
W U \\
W U \\
W U \\
W U \\
W U \\
W U \\
W U \\
W U \\
W U \\
W U \\
W U \\
W U \\
W U \\
W U \\
W U \\
W U \\
W U \\
W U \\
W U \\
W U \\
W U \\
W U \\
W U \\
W U \\
W U \\
W U \\
W U\end{array}$ & $\begin{array}{l}N \\
N \\
N \\
N \\
N \\
N \\
N \\
N \\
N \\
N \\
N \\
N \\
N \\
N \\
N \\
N \\
N \\
N \\
N \\
N \\
N \\
N \\
N \\
N \\
N \\
N \\
N \\
N \\
N \\
N \\
N \\
N \\
N \\
N \\
N \\
N \\
N \\
N \\
N\end{array}$ & MG/L & $\mid \begin{array}{l}< \\
<\end{array}$ & $\begin{array}{l}1.49 \\
1.38 \\
2.66 \\
0.739 \\
1.41 \\
1.12 \\
0.953 \\
2.09 \\
0.865 \\
0.043 \\
0.153 \\
0.105 \\
0.177 \\
0.152 \\
2.14 \\
1.83 \\
1.80 \\
2.74 \\
1.85 \\
1.79 \\
1.43 \\
1\end{array}$ & $J$ & $\begin{array}{l}0.005 \\
0.005 \\
0.005 \\
0.005 \\
0.005 \\
0.005 \\
0.005 \\
0.005 \\
0.005 \\
0.005 \\
0.005 \\
0.005 \\
0.005 \\
0.005 \\
0.005 \\
0.005 \\
0.005 \\
0.005 \\
0.005 \\
0.005 \\
0.005 \\
0.005 \\
0.005 \\
0.005 \\
0.005 \\
0.005 \\
0.005 \\
0.005 \\
0.005 \\
0.005 \\
0.005 \\
0.005 \\
0.005 \\
0.005 \\
0.005 \\
0.005 \\
0.005 \\
0.005 \\
0.005 \\
0.005\end{array}$ & $\begin{array}{l}- \\
- \\
- \\
- \\
- \\
- \\
- \\
- \\
- \\
- \\
- \\
- \\
- \\
- \\
- \\
- \\
- \\
- \\
- \\
- \\
- \\
- \\
- \\
- \\
- \\
- \\
- \\
-\end{array}$ \\
\hline
\end{tabular}

FORMATION OF COMPLETION CODE: WU - WASATCH - UPPER SANDSTONE

PARAMETER VALUE INDICATOR (PVI):
FLOW RELATIONSHIP CODE:

N - UNKNOWN

SAMPLE ID CODES

0001 - FILTERED SAMPLE (.45 MICRONS)

OTHER PARAMETER VALUE FLAGS:

$J$ - ESTIMATED VALUE 
GROUNDWATER QUALITY DATA BY PARAMETER

UPPER SANDSTONE UNIT

SITE: SPKO1 SPOOK

REPORT DATE: $07 / 29 / 94$

\begin{tabular}{|c|c|c|c|c|c|c|c|c|c|c|}
\hline PARAMETER NAME & $\begin{array}{c}\text { LOCATION } \\
\text { ID }\end{array}$ & LOG DATE & $\begin{array}{c}\text { SAMPLE } \\
\text { ID }\end{array}$ & $\begin{array}{l}\text { FORM } \\
\text { COMP }\end{array}$ & $\begin{array}{l}\text { FLOW } \\
\text { REL. }\end{array}$ & $\begin{array}{l}\text { UNITS OF } \\
\text { MEASURE }\end{array}$ & PVI & $\begin{array}{l}\text { PARAMETER } \\
\text { VALUE }\end{array}$ & $\begin{array}{l}\text { DETECTION } \\
\text { LIMIT }\end{array}$ & $\begin{array}{l}\text { PARAMETER } \\
\text { UNCERTAINTY }\end{array}$ \\
\hline SELENIUM & $\begin{array}{l}0930 \\
0930 \\
0931 \\
0931 \\
0931 \\
0931 \\
0932 \\
0932 \\
0932 \\
0932 \\
0933 \\
0933 \\
0934 \\
0934 \\
0935 \\
0935 \\
0936 \\
0936 \\
0937 \\
0937 \\
0939 \\
0939 \\
0940 \\
0940 \\
0941 \\
0941 \\
0942 \\
0942 \\
0943 \\
0943 \\
0944 \\
0944\end{array}$ & $\begin{array}{l}04 / 26 / 88 \\
08 / 27 / 88 \\
01 / 30 / 88 \\
04 / 28 / 88 \\
08 / 10 / 88 \\
12 / 04 / 88 \\
01 / 30 / 88 \\
04 / 28 / 88 \\
08 / 09 / 88 \\
12 / 07 / 88 \\
08 / 10 / 88 \\
12 / 07 / 88 \\
08 / 11 / 88 \\
12 / 07 / 88 \\
08 / 09 / 88 \\
12 / 04 / 88 \\
08 / 08 / 88 \\
12 / 04 / 88 \\
08 / 08 / 88 \\
12 / 04 / 88 \\
08 / 08 / 88 \\
12 / 04 / 88 \\
08 / 08 / 88 \\
12 / 04 / 88 \\
08 / 08 / 88 \\
12 / 10 / 88 \\
08 / 08 / 88 \\
12 / 04 / 88 \\
08 / 09 / 88 \\
12 / 07 / 88 \\
08 / 11 / 88 \\
12 / 07 / 88\end{array}$ & $\begin{array}{l}0001 \\
0001 \\
0001 \\
0001 \\
0001 \\
0001 \\
0001 \\
0001 \\
0001 \\
0001 \\
0001 \\
0001 \\
0001 \\
0001 \\
0001 \\
0001 \\
0001 \\
0001 \\
0001 \\
0001 \\
0001 \\
0001 \\
0001 \\
0001 \\
0001 \\
0001 \\
0001 \\
0001 \\
0001 \\
0001 \\
0001 \\
0001\end{array}$ & $\begin{array}{l}W U \\
W U \\
W U \\
W U \\
W U \\
W U \\
W U \\
W U \\
W U \\
W U \\
W U \\
W U \\
W U \\
W U \\
W U \\
W U \\
W U \\
W U \\
W U \\
W U \\
W U \\
W U \\
W U \\
W U \\
W U \\
W U \\
W U \\
W U \\
W U \\
W U \\
W U\end{array}$ & $\begin{array}{l}N \\
N \\
N \\
N \\
N \\
N \\
N \\
N \\
N \\
N \\
N \\
N \\
N \\
N \\
N \\
N \\
N \\
N \\
N \\
N \\
N \\
N \\
N \\
N \\
N \\
N \\
N \\
N \\
N \\
N \\
N \\
N\end{array}$ & $M G / L$ & & $\begin{array}{l}0.749 \\
0.959 \\
0.909 \\
0.730 \\
0.826 \\
1.08 \\
0.072 \\
0.065 \\
0.043 \\
0.063 \\
0.275 \\
0.302 \\
0.277 \\
0.352 \\
0.262 \\
1.05 \\
5.25 \\
5.08 \\
0.305 \\
0.166 \\
0.025 \\
0.014 \\
3.83 \\
6.59 \\
0.101 \\
0.074 \\
0.272 \\
0.148 \\
0.351 \\
0.424 \\
0.052 \\
0.036\end{array}$ & $\begin{array}{l}0.005 \\
0.005 \\
0.005 \\
0.005 \\
0.005 \\
0.005 \\
0.005 \\
0.005 \\
0.005 \\
0.005 \\
0.005 \\
0.005 \\
0.005 \\
0.005 \\
0.005 \\
0.005 \\
0.005 \\
0.005 \\
0.005 \\
0.005 \\
0.005 \\
0.005 \\
0.005 \\
0.005 \\
0.005 \\
0.005 \\
0.005 \\
0.005 \\
0.005 \\
0.005 \\
0.005 \\
0.005\end{array}$ & $\begin{array}{l}: \\
: \\
- \\
: \\
: \\
- \\
- \\
- \\
- \\
- \\
- \\
- \\
- \\
- \\
- \\
- \\
- \\
- \\
- \\
- \\
- \\
- \\
-\end{array}$ \\
\hline SILICA - SIOL & $\begin{array}{l}0911 \\
0911 \\
0911 \\
0911 \\
0911 \\
0912 \\
0912 \\
0912\end{array}$ & $\begin{array}{l}02 / 17 / 87 \\
09 / 18 / 87 \\
01 / 30 / 88 \\
04 / 22 / 88 \\
08 / 11 / 88 \\
02 / 18 / 87 \\
02 / 03 / 88 \\
04 / 22 / 88\end{array}$ & $\begin{array}{l}0001 \\
0001 \\
0001 \\
0001 \\
0001 \\
0001 \\
0001 \\
0001\end{array}$ & $\begin{array}{l}W U \\
W U \\
W U \\
W U \\
W U \\
W U \\
W U \\
W U\end{array}$ & $\begin{array}{l}N \\
N \\
N \\
N \\
N \\
N \\
N \\
N\end{array}$ & $M G / L$ & & $\begin{array}{c}14 . \\
8.00 \\
10.6 \\
9.60 \\
11 . \\
20 . \\
14.3 \\
13.4\end{array}$ & $\begin{array}{l}2 . \\
2 . \\
2 . \\
2 . \\
2 . \\
2 . \\
2 . \\
2 .\end{array}$ & $\begin{array}{l}- \\
- \\
- \\
- \\
-\end{array}$ \\
\hline
\end{tabular}

FORMATION OF COMPLETION CODE:

WU - WASATCH - UPPER SANDSTONE

PARAMETER VALUE INDICATOR (PVI):
< - LESS THAN DETECTION LIMIT
FLOW RELATIONSHIP CODE:

- UNKNOWN

SAMPLE ID CODES

0001 - FILTERED SAMPLE (.45 MICRONS) 


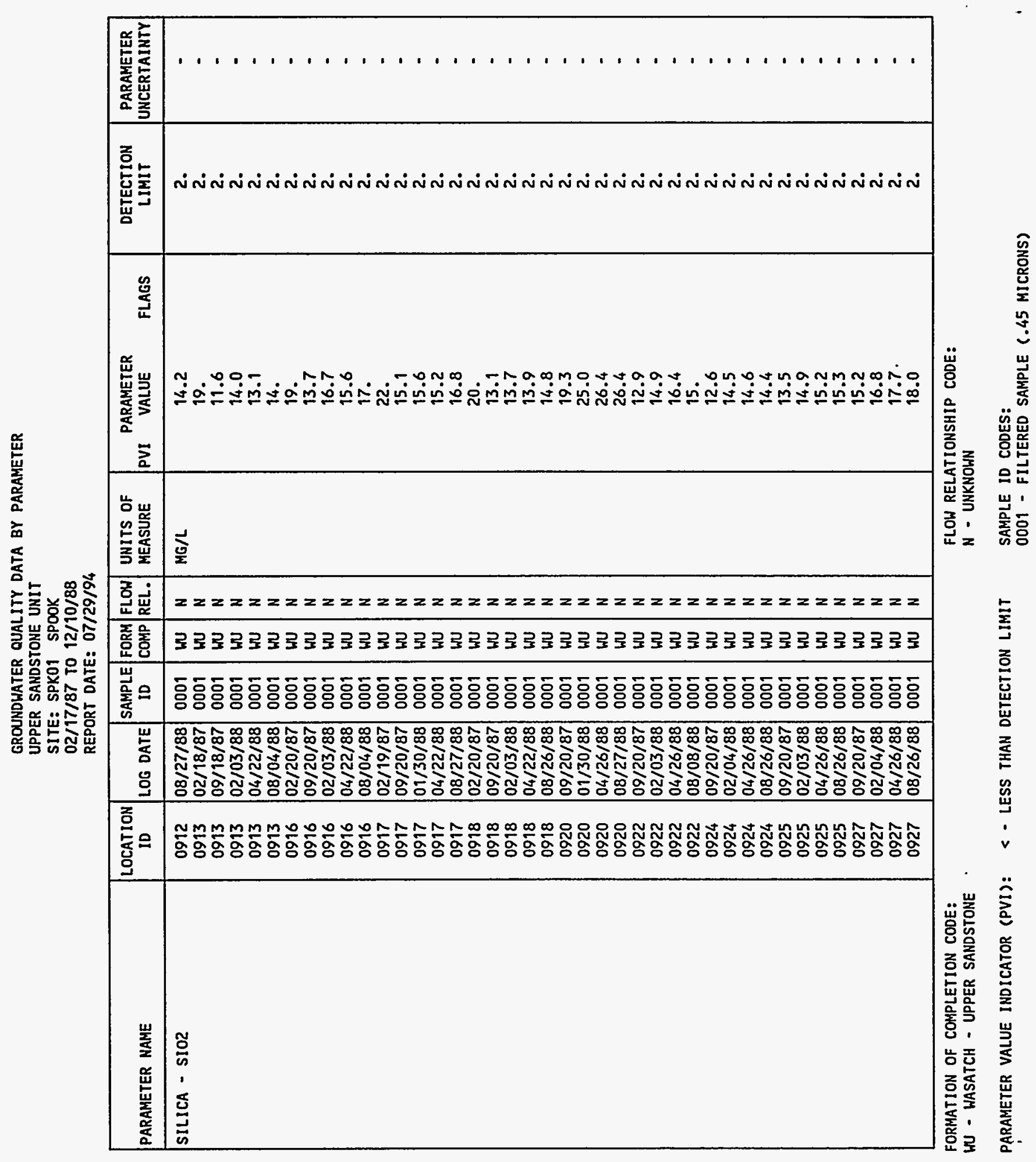


GROUNDWATER QUALITY DATA BY PARAMETER

UPPER SANDSTONE UNIT

SITE: SPKO1 SPOOK

$02 / 17 / 87$ TO $12 / 10 / 88$

REPORT DATE: $07 / 29 / 94$

\begin{tabular}{|c|c|c|c|c|c|c|c|c|c|c|c|}
\hline PARAMETER NAME & $\underset{\text { IOCATION }}{\text { ID }}$ & LOG DATE & $\begin{array}{c}\text { SAMPLE } \\
\text { ID }\end{array}$ & $\begin{array}{l}\text { FORM } \\
\text { COMP }\end{array}$ & $\begin{array}{l}\text { FLOW } \\
\text { REL. }\end{array}$ & $\begin{array}{l}\text { UNITS OF } \\
\text { MEASURE }\end{array}$ & PVI & $\begin{array}{l}\text { PARAMETER } \\
\text { VALUE }\end{array}$ & FLAGS & $\begin{array}{l}\text { DETECTION } \\
\text { LIMIT }\end{array}$ & $\begin{array}{l}\text { PARAMETER } \\
\text { UNCERTAINTY }\end{array}$ \\
\hline SILICA - SI02 & $\begin{array}{l}0928 \\
0928 \\
0928 \\
0928 \\
0929 \\
0929 \\
0929 \\
0929 \\
0930 \\
0930 \\
0930 \\
0930 \\
0931 \\
0931 \\
0931 \\
0932 \\
0932 \\
0932 \\
0933 \\
0934 \\
0935 \\
0936 \\
0937 \\
0939 \\
0940 \\
0941 \\
0942 \\
0943 \\
0944\end{array}$ & $\begin{array}{l}09 / 20 / 87 \\
02 / 03 / 88 \\
04 / 22 / 88 \\
08 / 04 / 88 \\
09 / 20 / 87 \\
02 / 03 / 88 \\
04 / 28 / 88 \\
08 / 11 / 88 \\
09 / 20 / 87 \\
01 / 30 / 88 \\
04 / 26 / 88 \\
08 / 27 / 88 \\
01 / 30 / 88 \\
04 / 28 / 88 \\
08 / 10 / 88 \\
01 / 30 / 88 \\
04 / 28 / 88 \\
08 / 09 / 88 \\
08 / 10 / 88 \\
08 / 11 / 88 \\
08 / 09 / 88 \\
08 / 08 / 88 \\
08 / 08 / 88 \\
08 / 08 / 88 \\
08 / 08 / 88 \\
08 / 08 / 88 \\
08 / 08 / 88 \\
08 / 09 / 88 \\
08 / 11 / 88\end{array}$ & $\begin{array}{l}0001 \\
0001 \\
0001 \\
0001 \\
0001 \\
0001 \\
0001 \\
0001 \\
0001 \\
0001 \\
0001 \\
0001 \\
0001 \\
0001 \\
0001 \\
0001 \\
0001 \\
0001 \\
0001 \\
0001 \\
0001 \\
0001 \\
0001 \\
0001 \\
0001 \\
0001 \\
0001 \\
0001 \\
0001\end{array}$ & $\begin{array}{l}W U \\
W U \\
W U \\
W U \\
W U \\
W U \\
W U \\
W U \\
W U \\
W U \\
W U \\
W U \\
W U \\
W U \\
W U \\
W U \\
W U \\
W U \\
W U \\
W U \\
W U \\
W U \\
W U \\
W U \\
W U \\
W U \\
W U \\
W U \\
W U\end{array}$ & $\begin{array}{l}N \\
N \\
N \\
N \\
N \\
N \\
N \\
N \\
N \\
N \\
N \\
N \\
N \\
N \\
N \\
N \\
N \\
N \\
N \\
N \\
N \\
N \\
N \\
N \\
N \\
N \\
N \\
N \\
N\end{array}$ & MG/L & & $\begin{array}{l}16.8 \\
16.8 \\
18.9 \\
18 . \\
4.75 \\
8.0 \\
7.60 \\
9.0 \\
15.0 \\
30.7 \\
28.9 \\
23.7 \\
15.7 \\
15.7 \\
16 . \\
16.4 \\
17.4 \\
17 . \\
15 . \\
15 . \\
39 . \\
14 . \\
9.0 \\
7 . \\
20 . \\
15 . \\
13 . \\
16 . \\
14 .\end{array}$ & J & $\begin{array}{l}2 . \\
2 . \\
2 . \\
2 . \\
2 . \\
2 . \\
2 . \\
2 . \\
2 . \\
2 . \\
2 . \\
2 . \\
2 . \\
2 . \\
2 . \\
2 . \\
2 . \\
2 . \\
2 . \\
2 . \\
2 . \\
2 . \\
2 . \\
2 . \\
2 . \\
2 . \\
2 . \\
2 . \\
2 .\end{array}$ & $\begin{array}{l}- \\
- \\
- \\
: \\
: \\
: \\
: \\
- \\
- \\
- \\
- \\
- \\
- \\
- \\
- \\
- \\
- \\
- \\
- \\
- \\
-\end{array}$ \\
\hline SILVER & $\begin{array}{l}0911 \\
0911 \\
0911 \\
0911 \\
0911 \\
0911 \\
0912 \\
0912 \\
0912 \\
0912\end{array}$ & $\begin{array}{l}02 / 17 / 87 \\
09 / 18 / 87 \\
01 / 30 / 88 \\
04 / 22 / 88 \\
08 / 11 / 88 \\
12 / 10 / 88 \\
02 / 18 / 87 \\
02 / 03 / 88 \\
04 / 22 / 88 \\
08 / 27 / 88\end{array}$ & $\begin{array}{l}0001 \\
0001 \\
0001 \\
0001 \\
0001 \\
0001 \\
0001 \\
0001 \\
0001 \\
0001\end{array}$ & $\begin{array}{l}\text { WU } \\
W U \\
W U \\
W U \\
W U \\
W U \\
W U \\
W U \\
W U \\
W U\end{array}$ & $\begin{array}{l}N \\
N \\
N \\
N \\
N \\
N \\
N \\
N \\
N \\
N\end{array}$ & $M G / L$ & $\begin{array}{l}< \\
< \\
< \\
< \\
< \\
< \\
< \\
< \\
< \\
<\end{array}$ & $\begin{array}{l}0.01 \\
0.01 \\
0.01 \\
0.01 \\
0.01 \\
0.01 \\
0.01 \\
0.01 \\
0.01 \\
0.01\end{array}$ & & $\begin{array}{l}0.01 \\
0.01 \\
0.01 \\
0.01 \\
0.01 \\
0.01 \\
0.01 \\
0.01 \\
0.01 \\
0.01\end{array}$ & $\begin{array}{l}- \\
- \\
- \\
- \\
\dot{-} \\
\dot{-} \\
-\end{array}$ \\
\hline
\end{tabular}

FORMATION OF COMPLETION CODE:

WU - WASATCH - UPPER SANDSTONE

PARAMETER VALUE INDICATOR (PVI): < - LESS THAN DETECTION LIMIT
FLOW RELATIONSHIP CODE:

N - UNKHOWN

SAMPLE ID CODES:

0001 - FILTERED SAMPLE (.45 MICRONS)

OTHER PARAMETER VALUE FLAGS:

J - ESTIMATED VALUE 
GROUNDHATER QUALITY DATA BY PARAMETER

UPPER SANDSTONE UNIT

SITE: SPKO1 SPOOK

REPORT DATE: $07 / 29 / 94$

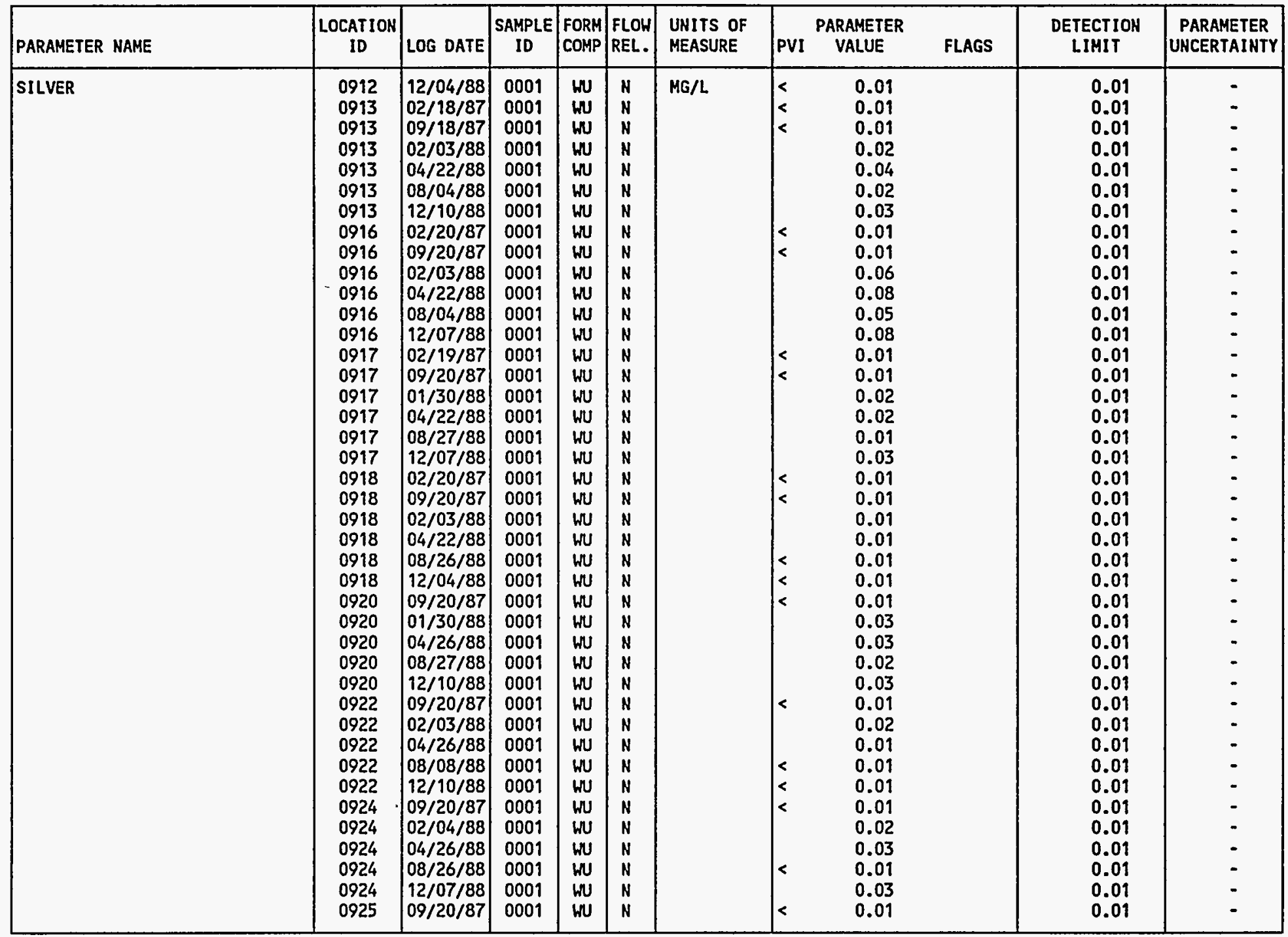

FORMATION OF COMPLETION CODE:

FLOW RELATIONSHIP CODE:

WU - HASATCH - UPPER SANDSTONE

N - UNKNOHN

PARAMETER VALUE INDICATOR (PVI): < - LESS THAN DETECTION LIMIT SAMPLE ID CODES:

0001 - FILTERED SAMPLE (.45 MICRONS) 
GROUNDWATER QUALITY DATA BY PARAMETER

UPPER SANDSTONE UNIT

SITE: SPKO1 SPOOK

12/10/88

REPORT DATE: 07/29/9

\begin{tabular}{|c|c|c|c|c|c|c|c|c|c|c|c|}
\hline PARAMETER NAME & $\begin{array}{c}\text { LOCATION } \\
\text { ID }\end{array}$ & LOG DATE & $\begin{array}{c}\text { SAMPLE } \\
\text { ID }\end{array}$ & $\begin{array}{l}\text { FORH } \\
\text { COMP }\end{array}$ & $\begin{array}{l}\text { FLOH } \\
\text { REL. }\end{array}$ & $\begin{array}{l}\text { UNITS OF } \\
\text { MEASURE }\end{array}$ & PVI & $\begin{array}{l}\text { PARAMETER } \\
\text { VALUE }\end{array}$ & FLAGS & $\begin{array}{l}\text { DETECTION } \\
\text { LIMIT }\end{array}$ & $\begin{array}{l}\text { PARAMETER } \\
\text { UNCERTAINTY }\end{array}$ \\
\hline SILVER & $\begin{array}{l}0925 \\
0925 \\
0925 \\
0925 \\
0927 \\
0927 \\
0927 \\
0927 \\
0927 \\
0928 \\
0928 \\
0928 \\
0928 \\
0928 \\
0929 \\
0929 \\
0929 \\
0929 \\
0930 \\
0930 \\
0930 \\
0930 \\
0931 \\
0931 \\
0931 \\
0931 \\
0932 \\
0932 \\
0932 \\
0932 \\
0933 \\
0933 \\
0934 \\
0934 \\
0935 \\
0935 \\
0936 \\
0936 \\
0937 \\
0937\end{array}$ & $\begin{array}{l}02 / 03 / 88 \\
04 / 26 / 88 \\
08 / 26 / 88 \\
12 / 07 / 88 \\
09 / 20 / 87 \\
02 / 04 / 88 \\
04 / 26 / 88 \\
08 / 26 / 88 \\
12 / 04 / 88 \\
09 / 20 / 87 \\
02 / 03 / 88 \\
04 / 22 / 88 \\
08 / 04 / 88 \\
12 / 04 / 88 \\
09 / 20 / 87 \\
02 / 03 / 88 \\
04 / 28 / 88 \\
08 / 11 / 88 \\
09 / 20 / 87 \\
01 / 30 / 88 \\
04 / 26 / 88 \\
08 / 27 / 88 \\
01 / 30 / 88 \\
04 / 28 / 88 \\
08 / 10 / 88 \\
12 / 04 / 88 \\
01 / 30 / 88 \\
04 / 28 / 88 \\
08 / 09 / 88 \\
12 / 07 / 88 \\
08 / 10 / 88 \\
12 / 07 / 88 \\
08 / 11 / 88 \\
12 / 07 / 88 \\
08 / 09 / 88 \\
12 / 04 / 88 \\
08 / 08 / 88 \\
12 / 04 / 88 \\
08 / 08 / 88 \\
12 / 04 / 88\end{array}$ & $\begin{array}{l}0001 \\
0001 \\
0001 \\
0001 \\
0001 \\
0001 \\
0001 \\
0001 \\
0001 \\
0001 \\
0001 \\
0001 \\
0001 \\
0001 \\
0001 \\
0001 \\
0001 \\
0001 \\
0001 \\
0001 \\
0001 \\
0001 \\
0001 \\
0001 \\
0001 \\
0001 \\
0001 \\
0001 \\
0001 \\
0001 \\
0001 \\
0001 \\
0001 \\
0001 \\
0001 \\
0001 \\
0001 \\
0001 \\
0001 \\
0001\end{array}$ & $\begin{array}{l}W U \\
W U \\
W U \\
W U \\
W U \\
W U \\
W U \\
W U \\
W U \\
W U \\
W U \\
W U \\
W U \\
W U \\
W U \\
W U \\
W U \\
W U \\
W U \\
W U \\
W U \\
W U \\
W U \\
W U \\
W U \\
W U \\
W U \\
W U \\
W U \\
W U \\
W U \\
W U \\
W U \\
W U \\
W U \\
W U \\
W U \\
W U \\
W U\end{array}$ & $\begin{array}{l}N \\
N \\
N \\
N \\
N \\
N \\
N \\
N \\
N \\
N \\
N \\
N \\
N \\
N \\
N \\
N \\
N \\
N \\
N \\
N \\
N \\
N \\
N \\
N \\
N \\
N \\
N \\
N \\
N \\
N \\
N \\
N \\
N \\
N \\
N \\
N \\
N \\
N \\
N \\
N\end{array}$ & $M G / L$ & $\begin{array}{l}< \\
< \\
< \\
< \\
< \\
< \\
< \\
< \\
< \\
< \\
< \\
< \\
< \\
< \\
< \\
< \\
< \\
<\end{array}$ & $\begin{array}{l}0.01 \\
0.02 \\
0.01 \\
0.01 \\
0.01 \\
0.03 \\
0.03 \\
0.02 \\
0.03 \\
0.01 \\
0.03 \\
0.03 \\
0.01 \\
0.03 \\
0.01 \\
0.01 \\
0.01 \\
0.01 \\
0.01 \\
0.018 \\
0.04 \\
0.02 \\
0.01 \\
0.02 \\
0.01 \\
0.01 \\
0.01 \\
0.01 \\
0.01 \\
0.01 \\
0.01 \\
0.01 \\
0.01 \\
0.01 \\
0.01 \\
0.03 \\
0.01 \\
0.03 \\
0.03 \\
0.02\end{array}$ & $J$ & $\begin{array}{l}0.01 \\
0.01 \\
0.01 \\
0.01 \\
0.01 \\
0.01 \\
0.01 \\
0.01 \\
0.01 \\
0.01 \\
0.01 \\
0.01 \\
0.01 \\
0.01 \\
0.01 \\
0.01 \\
0.01 \\
0.01 \\
0.01 \\
0.01 \\
0.01 \\
0.01 \\
0.01 \\
0.01 \\
0.01 \\
0.01 \\
0.01 \\
0.01 \\
0.01 \\
0.01 \\
0.01 \\
0.01 \\
0.01 \\
0.01 \\
0.01 \\
0.01 \\
0.01 \\
0.01 \\
0.01 \\
0.01\end{array}$ & $\begin{array}{l}- \\
- \\
- \\
- \\
- \\
- \\
- \\
- \\
- \\
- \\
- \\
- \\
- \\
- \\
- \\
- \\
- \\
- \\
- \\
- \\
- \\
- \\
- \\
- \\
- \\
- \\
- \\
- \\
- \\
- \\
- \\
- \\
- \\
- \\
- \\
- \\
-\end{array}$ \\
\hline
\end{tabular}

FORMATION OF COMPLETION CODE:

WU - WASATCH - UPPER SANDSTONE

PARAMETER VALUE INDICATOR (PVI): < - LESS THAN DETECTION LIMIT
FLOW RELATIONSHIP COOE:

N - UNKNOWN

SAMPLE ID CODES

0001 - FILTERED SAMPLE (.45 MICRONS)

OTHER PARAMETER VALUE FLAGS:

$J$ - ESTIMATED VALUE 


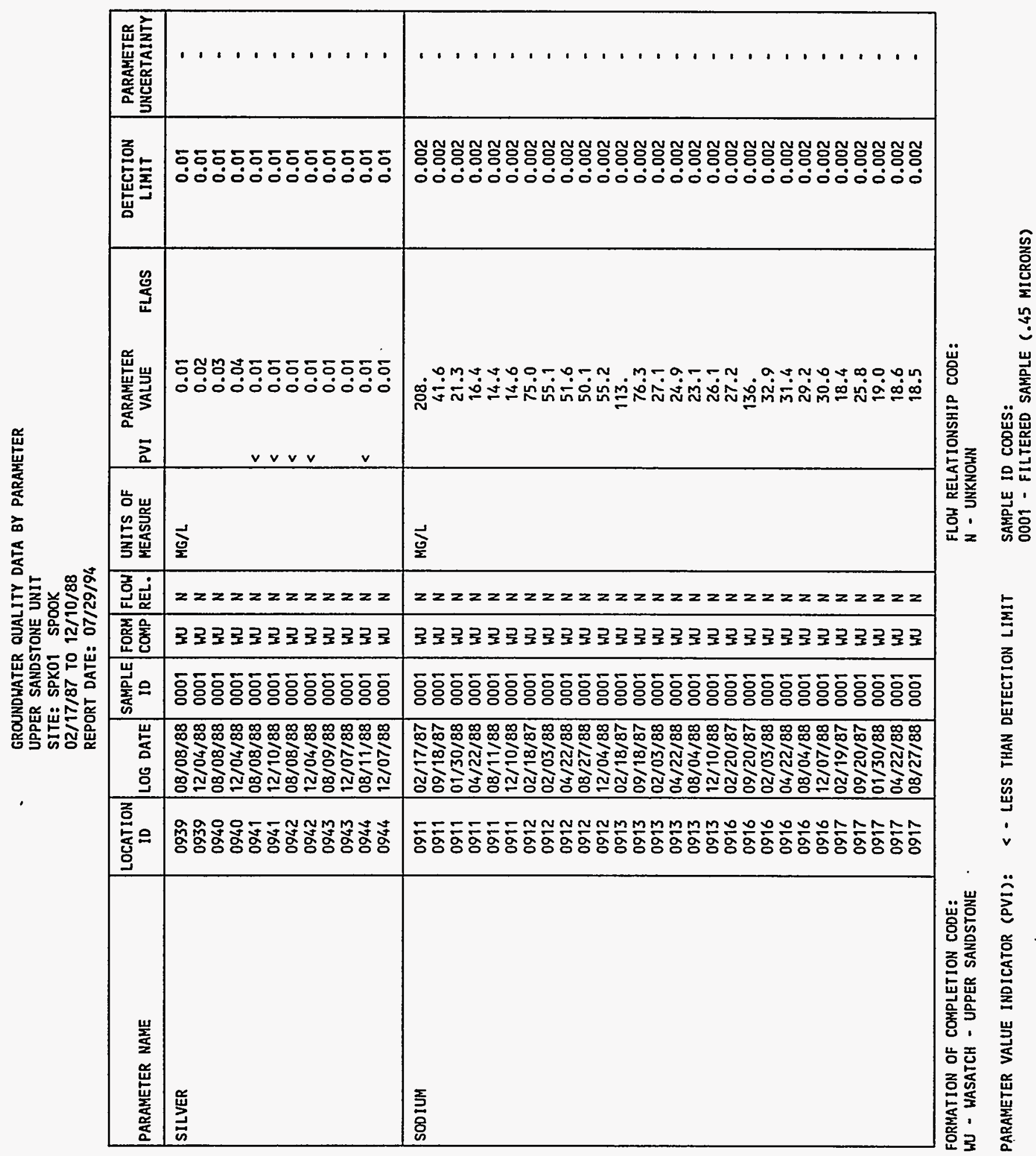


SITE: SPKO1 SPOOK

$02 / 17 / 87$ TO $12 / 10 / 88$

REPORT DATE : $07 / 29 / 94$

\begin{tabular}{|c|c|c|c|c|c|c|c|c|c|c|c|}
\hline PARAMETER NAME & $\underset{\text { ID }}{\text { LOCATION }}$ & LOG DATE & $\begin{array}{l}\text { SAMPLE } \\
\text { ID }\end{array}$ & $\begin{array}{l}\text { FORM } \\
\text { COMP }\end{array}$ & $\begin{array}{l}\text { FLOW } \\
\text { REL. }\end{array}$ & $\begin{array}{l}\text { UNITS OF } \\
\text { MEASURE }\end{array}$ & PVI & $\begin{array}{l}\text { PARAMETER } \\
\text { VALUE }\end{array}$ & FLAGS & $\begin{array}{l}\text { DETECTION } \\
\text { LIMIT }\end{array}$ & $\begin{array}{l}\text { PARAMETER } \\
\text { UNCERTAINTY }\end{array}$ \\
\hline SOD IUM & $\begin{array}{l}0917 \\
0918 \\
0918 \\
0918 \\
0918 \\
0918 \\
0918 \\
0920 \\
0920 \\
0920 \\
0920 \\
0920 \\
0922 \\
0922 \\
0922 \\
0922 \\
0922 \\
0924 \\
0924 \\
0924 \\
0924 \\
0924 \\
0925 \\
0925 \\
0925 \\
0925 \\
0925 \\
0927 \\
0927 \\
0927 \\
0927 \\
0927 \\
0928 \\
0928 \\
0928 \\
0928 \\
0928 \\
0929 \\
0929 \\
0929\end{array}$ & $\begin{array}{l}12 / 07 / 88 \\
02 / 20 / 87 \\
09 / 20 / 87 \\
02 / 03 / 88 \\
04 / 22 / 88 \\
08 / 26 / 88 \\
12 / 04 / 88 \\
09 / 20 / 87 \\
01 / 30 / 88 \\
04 / 26 / 88 \\
08 / 27 / 88 \\
12 / 10 / 88 \\
09 / 20 / 87 \\
02 / 03 / 88 \\
04 / 26 / 88 \\
08 / 08 / 88 \\
12 / 10 / 88 \\
09 / 20 / 87 \\
02 / 04 / 88 \\
04 / 26 / 88 \\
08 / 26 / 88 \\
12 / 07 / 88 \\
09 / 20 / 87 \\
02 / 03 / 88 \\
04 / 26 / 88 \\
08 / 26 / 88 \\
12 / 07 / 88 \\
09 / 20 / 87 \\
02 / 04 / 88 \\
04 / 26 / 88 \\
08 / 26 / 88 \\
12 / 04 / 88 \\
09 / 20 / 87 \\
02 / 03 / 88 \\
04 / 22 / 88 \\
08 / 04 / 88 \\
12 / 04 / 88 \\
09 / 20 / 87 \\
02 / 03 / 88 \\
04 / 28 / 88\end{array}$ & $\begin{array}{l}0001 \\
0001 \\
0001 \\
0001 \\
0001 \\
0001 \\
0001 \\
0001 \\
0001 \\
0001 \\
0001 \\
0001 \\
0001 \\
0001 \\
0001 \\
0001 \\
0001 \\
0001 \\
0001 \\
0001 \\
0001 \\
0001 \\
0001 \\
0001 \\
0001 \\
0001 \\
0001 \\
0001 \\
0001 \\
0001 \\
0001 \\
0001 \\
0001 \\
0001 \\
0001 \\
0001 \\
0001 \\
0001 \\
0001 \\
0001\end{array}$ & $\begin{array}{l}W U \\
W U \\
W U \\
W U \\
W U \\
W U \\
W U \\
W U \\
W U \\
W U \\
W U \\
W U \\
W U \\
W U \\
W U \\
W U \\
W U \\
W U \\
W U \\
W U \\
W U \\
W U \\
W U \\
W U \\
W U \\
W U \\
W U \\
W U \\
W U \\
W U \\
W U \\
W U \\
W U \\
W U \\
W U \\
W U \\
W U \\
W U \\
W U \\
W U\end{array}$ & $\begin{array}{l}N \\
N \\
N \\
N \\
N \\
N \\
N \\
N \\
N \\
N \\
N \\
N \\
N \\
N \\
N \\
N \\
N \\
N \\
N \\
N \\
N \\
N \\
N \\
N \\
N \\
N \\
N \\
N \\
N \\
N \\
N \\
N \\
N \\
N \\
N \\
N \\
N \\
N \\
N\end{array}$ & MG/L & & $\begin{array}{c}19.1 \\
18.6 \\
42.6 \\
19.6 \\
20.1 \\
19.0 \\
22.6 \\
421 . \\
395 . \\
411 . \\
400 . \\
432 . \\
23.6 \\
15.7 \\
15.4 \\
18.3 \\
16.6 \\
20.7 \\
19.0 \\
18.1 \\
18.2 \\
19.0 \\
16.2 \\
14.3 \\
13.8 \\
13.4 \\
13.9 \\
56.3 \\
51.9 \\
52.5 \\
53.8 \\
53.0 \\
28.1 \\
22.1 \\
22.7 \\
21.9 \\
22.2 \\
45.1 \\
24.8 \\
20.4\end{array}$ & 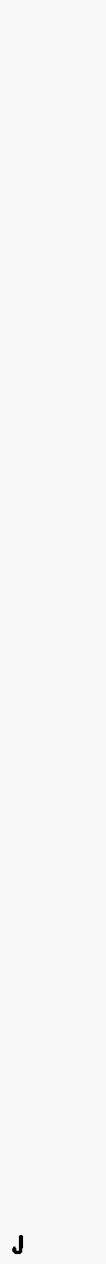 & $\begin{array}{l}0.002 \\
0.002 \\
0.002 \\
0.002 \\
0.002 \\
0.002 \\
0.002 \\
0.002 \\
0.002 \\
0.002 \\
0.002 \\
0.002 \\
0.002 \\
0.002 \\
0.002 \\
0.002 \\
0.002 \\
0.002 \\
0.002 \\
0.002 \\
0.002 \\
0.002 \\
0.002 \\
0.002 \\
0.002 \\
0.002 \\
0.002 \\
0.002 \\
0.002 \\
0.002 \\
0.002 \\
0.002 \\
0.002 \\
0.002 \\
0.002 \\
0.002 \\
0.002 \\
0.002 \\
0.002 \\
0.002\end{array}$ & $\begin{array}{l}- \\
- \\
- \\
- \\
- \\
- \\
- \\
- \\
- \\
- \\
- \\
- \\
- \\
- \\
- \\
- \\
- \\
- \\
- \\
- \\
- \\
- \\
- \\
- \\
- \\
- \\
- \\
- \\
-\end{array}$ \\
\hline
\end{tabular}

FORMATION OF COMPLETION CODE:

WU - HASATCH - UPPER SANDSTONE

FLOW RELATIONSHIP CODE:

PARAMETER VALUE INDICATOR (PVI): < - LESS THAN DETECTION LIMIT

SAMPLE ID CODES:

0001 - FILTERED SAMPLE (.45 MICRONS)

OTHER PARAMETER VALUE FLAGS:

$J$ - ESTIMATED VALUE 


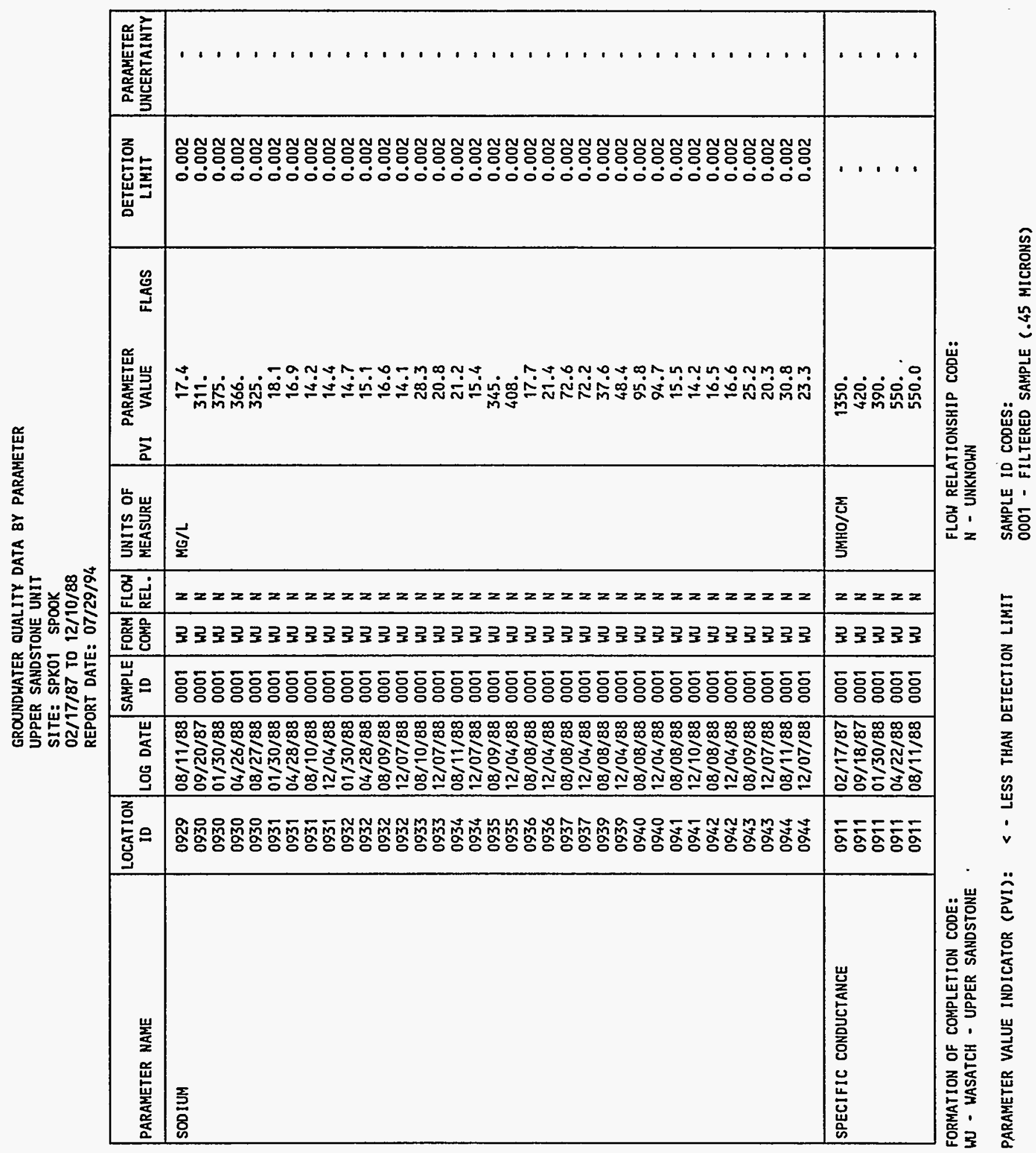


SITE: SPKO1 SPOOK

REPORT DATE: $07 / 29 / 94$

\begin{tabular}{|c|c|c|c|c|c|c|c|c|c|c|c|}
\hline PARAMETER NAME & $\underset{\text { LOCATION }}{\text { LO }}$ & LOG DATE & $\underset{\text { ID }}{\text { SAMPLE }}$ & $\begin{array}{l}\text { FORM } \\
\text { COMP }\end{array}$ & $\begin{array}{l}\text { FLOW } \\
\text { REL. }\end{array}$ & $\begin{array}{l}\text { UNITS OF } \\
\text { MEASURE }\end{array}$ & PVI & $\begin{array}{l}\text { PARAMETER } \\
\text { VALUE }\end{array}$ & FLAGS & $\begin{array}{l}\text { DETECTION } \\
\text { LIMIT }\end{array}$ & $\begin{array}{l}\text { PARAMETER } \\
\text { UNCERTAINTY }\end{array}$ \\
\hline SPECIFIC CONDUCTANCE & $\begin{array}{l}0911 \\
0912 \\
0912 \\
0912 \\
0912 \\
0912 \\
0913 \\
0913 \\
0913 \\
0913 \\
0913 \\
0913 \\
0916 \\
0916 \\
0916 \\
0916 \\
0916 \\
0916 \\
0917 \\
0917 \\
0917 \\
0917 \\
0917 \\
0917 \\
0918 \\
0918 \\
0918 \\
0918 \\
0918 \\
0918 \\
0920 \\
0920 \\
0920 \\
0920 \\
0920 \\
0922 \\
0922 \\
0922 \\
0922 \\
0922 \\
0924\end{array}$ & $\begin{array}{l}12 / 10 / 88 \\
02 / 18 / 87 \\
02 / 03 / 88 \\
04 / 22 / 88 \\
08 / 27 / 88 \\
12 / 04 / 88 \\
02 / 18 / 87 \\
09 / 18 / 87 \\
02 / 03 / 88 \\
04 / 22 / 88 \\
08 / 04 / 88 \\
12 / 10 / 88 \\
02 / 20 / 87 \\
09 / 20 / 87 \\
02 / 03 / 88 \\
04 / 22 / 88 \\
08 / 04 / 88 \\
12 / 07 / 88 \\
02 / 19 / 87 \\
09 / 20 / 87 \\
01 / 30 / 88 \\
04 / 22 / 88 \\
08 / 27 / 88 \\
12 / 07 / 88 \\
02 / 20 / 87 \\
09 / 20 / 87 \\
02 / 03 / 88 \\
04 / 22 / 88 \\
08 / 26 / 88 \\
12 / 04 / 88 \\
09 / 20 / 87 \\
01 / 30 / 88 \\
04 / 26 / 88 \\
08 / 27 / 88 \\
12 / 10 / 88 \\
09 / 20 / 87 \\
02 / 03 / 88 \\
04 / 26 / 88 \\
08 / 08 / 88 \\
12 / 10 / 88 \\
09 / 20 / 87\end{array}$ & $\begin{array}{l}0001 \\
0001 \\
0001 \\
0001 \\
0001 \\
0001 \\
0001 \\
0001 \\
0001 \\
0001 \\
0001 \\
0001 \\
0001 \\
0001 \\
0001 \\
0001 \\
0001 \\
0001 \\
0001 \\
0001 \\
0001 \\
0001 \\
0001 \\
0001 \\
0001 \\
0001 \\
0001 \\
0001 \\
0001 \\
0001 \\
0001 \\
0001 \\
0001 \\
0001 \\
0001 \\
0001 \\
0001 \\
0001 \\
0001 \\
0001 \\
0001\end{array}$ & $\begin{array}{l}W U \\
W U \\
W U \\
W U \\
W U \\
W U \\
W U \\
W U \\
W U \\
W U \\
W U \\
W U \\
W U \\
W U \\
W U \\
W U \\
W U \\
W U \\
W U \\
W U \\
W U \\
W U \\
W U \\
W U \\
W U \\
W U \\
W U \\
W U \\
W U \\
W U \\
W U \\
W U \\
W U \\
W U \\
W U \\
W U \\
W U\end{array}$ & $\begin{array}{l}N \\
N \\
N \\
N \\
N \\
N \\
N \\
N \\
N \\
N \\
N \\
N \\
N \\
N \\
N \\
N \\
N \\
N \\
N \\
N \\
N \\
N \\
N \\
N \\
N \\
N \\
N \\
N \\
N \\
N \\
N \\
N \\
N \\
N \\
N \\
N \\
N \\
N \\
N \\
N \\
N\end{array}$ & UMHO/CM & & $\begin{array}{c}\text { 500. } \\
375 . \\
300 . \\
400 . \\
380.0 \\
370 . \\
700 . \\
460 . \\
485 . \\
975 . \\
1050.0 \\
950 . \\
1150 . \\
950 . \\
1100 . \\
1750 . \\
1750.0 \\
1550 . \\
700 . \\
360 . \\
460 . \\
650 . \\
770.0 \\
700 . \\
450 . \\
280 . \\
380 . \\
625 . \\
600.0 \\
600 . \\
2100 . \\
1830 . \\
3600 . \\
3500.0 \\
3200 . \\
410 . \\
430 . \\
600 . \\
650.0 \\
600 . \\
700 .\end{array}$ & & 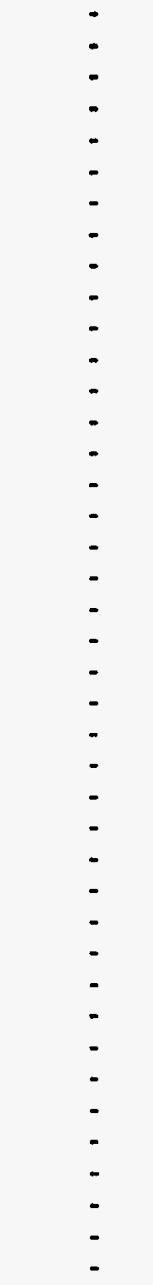 & 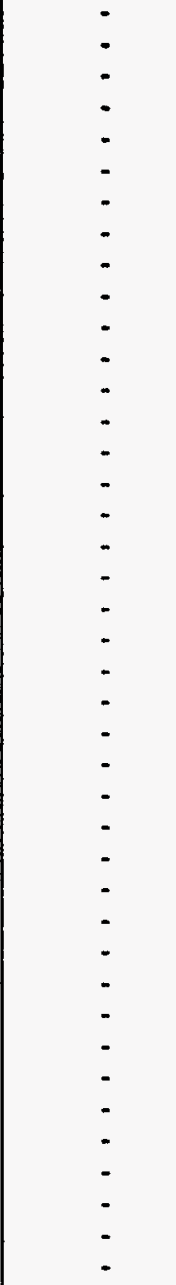 \\
\hline
\end{tabular}

FORMATION OF COMPLETION CODE:

WU - HASATCH - UPPER SANDSTONE

PARAMETER VALUE INDICATOR (PVI): < - LESS THAN DETECTION LIMIT
FLOW RELATIONSHIP CODE: N - UNKNOWN

SAMPLE ID CODES:

0001 - FILTERED SAMPLE (.45 MICRONS) 


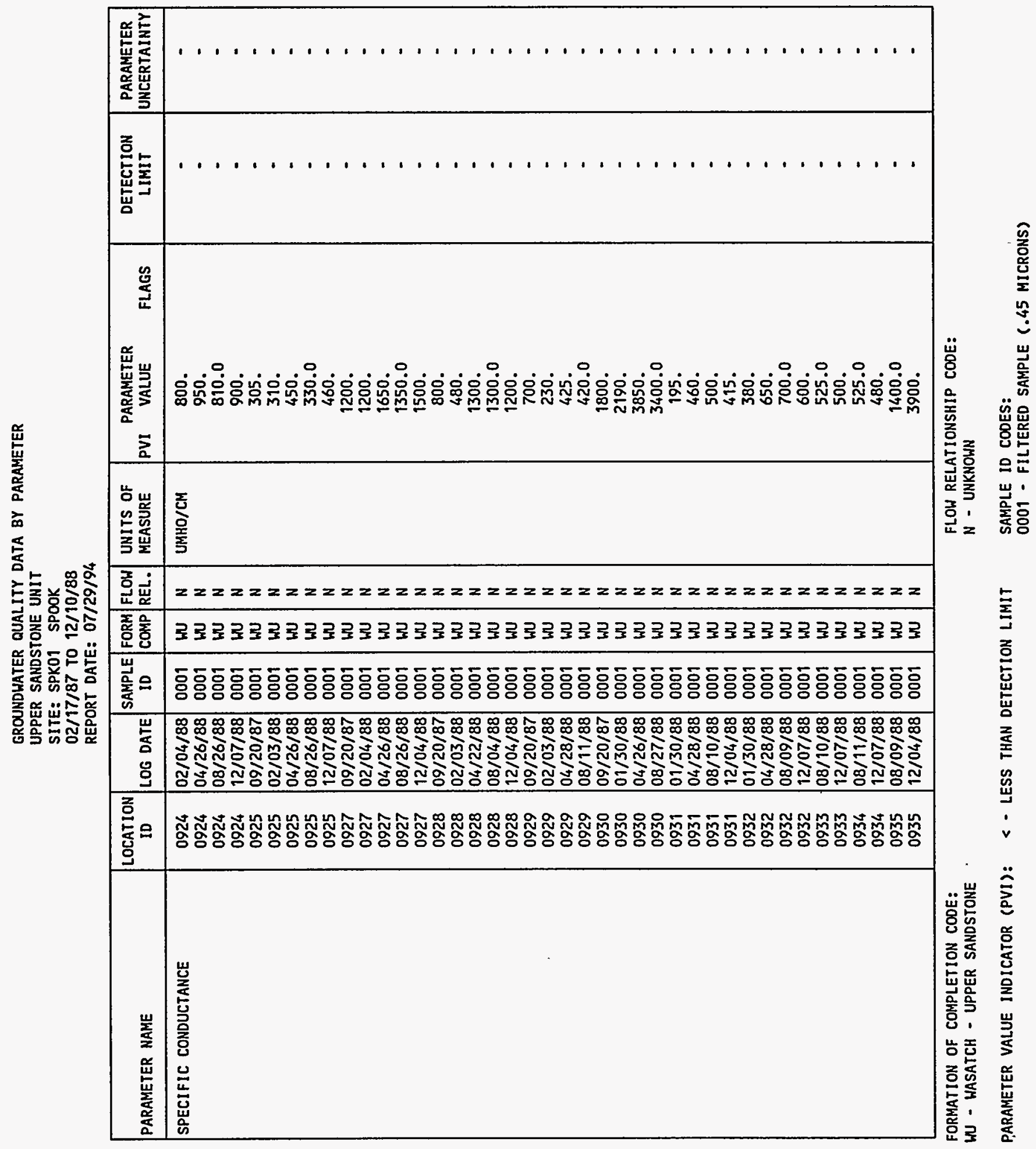


REPORT DATE: 07/29/94

\begin{tabular}{|c|c|c|c|c|c|c|c|c|c|c|}
\hline PARAMETER NAME & $\underset{\text { LOCATION }}{\text { ID }}$ & LOG DATE & $\begin{array}{l}\text { SAMPLE } \\
\text { ID }\end{array}$ & $\begin{array}{l}\text { FORM } \\
\text { COMP }\end{array}$ & $\begin{array}{l}\text { FLOW } \\
\text { REL. }\end{array}$ & $\begin{array}{l}\text { UNITS OF } \\
\text { MEASURE }\end{array}$ & PVI & $\begin{array}{l}\text { PARAMETER } \\
\text { VALUE }\end{array}$ & $\begin{array}{l}\text { DETECTION } \\
\text { LIMIT }\end{array}$ & $\begin{array}{l}\text { PARAMETER } \\
\text { UNCERTAINTY }\end{array}$ \\
\hline SPECIFIC CONDUCTANCE & $\begin{array}{l}0936 \\
0936 \\
0937 \\
0937 \\
0939 \\
0939 \\
0940 \\
0940 \\
0941 \\
0941 \\
0942 \\
0942 \\
0943 \\
0943 \\
0944 \\
0944\end{array}$ & $\begin{array}{l}08 / 08 / 88 \\
12 / 04 / 88 \\
08 / 08 / 88 \\
12 / 04 / 88 \\
08 / 08 / 88 \\
12 / 04 / 88 \\
08 / 08 / 88 \\
12 / 04 / 88 \\
08 / 08 / 88 \\
12 / 10 / 88 \\
08 / 08 / 88 \\
12 / 04 / 88 \\
08 / 09 / 88 \\
12 / 07 / 88 \\
08 / 11 / 88 \\
12 / 07 / 88\end{array}$ & $\begin{array}{l}0001 \\
0001 \\
0001 \\
0001 \\
0001 \\
0001 \\
0001 \\
0001 \\
0001 \\
0001 \\
0001 \\
0001 \\
0001 \\
0001 \\
0001 \\
0001\end{array}$ & $\begin{array}{l}W U \\
W U \\
W U \\
W U \\
W U \\
W U \\
W U \\
W U \\
W U \\
W U \\
W U \\
W U \\
W U \\
W U \\
W U \\
W U\end{array}$ & $\begin{array}{l}N \\
N \\
N \\
N \\
N \\
N \\
N \\
N \\
N \\
N \\
N \\
N \\
N \\
N \\
N \\
N\end{array}$ & UMHO/CH & & $\begin{array}{c}1300.0 \\
1000 . \\
800.0 \\
600 . \\
1100.0 \\
1150 . \\
3300.0 \\
2900 . \\
310.0 \\
310 . \\
700.0 \\
400 . \\
450.0 \\
10 . \\
800.0 \\
800 .\end{array}$ & $\begin{array}{l}- \\
: \\
: \\
: \\
- \\
- \\
- \\
- \\
- \\
- \\
-\end{array}$ & $\begin{array}{l}- \\
- \\
- \\
- \\
- \\
- \\
- \\
- \\
- \\
- \\
- \\
-\end{array}$ \\
\hline STRONT IUM & $\begin{array}{l}0911 \\
0911 \\
0911 \\
0911 \\
0912 \\
0912 \\
0912 \\
0913 \\
0913 \\
0913 \\
0913 \\
0916 \\
0916 \\
0916 \\
0916 \\
0917 \\
0917 \\
0917 \\
0917 \\
0918 \\
0918 \\
0918 \\
0918 \\
0920\end{array}$ & $\begin{array}{l}09 / 18 / 87 \\
01 / 30 / 88 \\
04 / 22 / 88 \\
08 / 11 / 88 \\
02 / 03 / 88 \\
04 / 22 / 88 \\
08 / 27 / 88 \\
09 / 18 / 87 \\
02 / 03 / 88 \\
04 / 22 / 88 \\
08 / 04 / 88 \\
09 / 20 / 87 \\
02 / 03 / 88 \\
04 / 22 / 88 \\
08 / 04 / 88 \\
09 / 20 / 87 \\
01 / 30 / 88 \\
04 / 22 / 88 \\
08 / 27 / 88 \\
09 / 20 / 87 \\
02 / 03 / 88 \\
04 / 22 / 88 \\
08 / 26 / 88 \\
09 / 20 / 87\end{array}$ & $\begin{array}{l}0001 \\
0001 \\
0001 \\
0001 \\
0001 \\
0001 \\
0001 \\
0001 \\
0001 \\
0001 \\
0001 \\
0001 \\
0001 \\
0001 \\
0001 \\
0001 \\
0001 \\
0001 \\
0001 \\
0001 \\
0001 \\
0001 \\
0001 \\
0001\end{array}$ & $\begin{array}{l}W U \\
W U \\
W U \\
W U \\
W U \\
W U \\
W U \\
W U \\
W U \\
W U \\
W U \\
W U \\
W U \\
W U \\
W U \\
W U \\
W U \\
W U \\
W U \\
W U \\
W U \\
W U \\
W U \\
W U\end{array}$ & $\begin{array}{l}N \\
N \\
N \\
N \\
N \\
N \\
N \\
N \\
N \\
N \\
N \\
N \\
N \\
N \\
N \\
N \\
N \\
N \\
N \\
N \\
N \\
N \\
N \\
N\end{array}$ & $M G / L$ & & $\begin{array}{l}1.17 \\
1.03 \\
0.87 \\
1.1 \\
0.49 \\
0.38 \\
0.5 \\
2.11 \\
1.84 \\
1.54 \\
1.8 \\
3.56 \\
3.37 \\
2.78 \\
3.3 \\
1.92 \\
1.70 \\
1.39 \\
1.9 \\
1.65 \\
1.42 \\
1.14 \\
1.6 \\
5.33\end{array}$ & $\begin{array}{l}0.1 \\
0.1 \\
0.1 \\
0.1 \\
0.1 \\
0.1 \\
0.1 \\
0.1 \\
0.1 \\
0.1 \\
0.1 \\
0.1 \\
0.1 \\
0.1 \\
0.1 \\
0.1 \\
0.1 \\
0.1 \\
0.1 \\
0.1 \\
0.1 \\
0.1 \\
0.1 \\
0.1\end{array}$ & $\begin{array}{l}- \\
- \\
- \\
- \\
- \\
- \\
- \\
- \\
- \\
- \\
- \\
- \\
- \\
- \\
- \\
- \\
- \\
- \\
-\end{array}$ \\
\hline
\end{tabular}

FORMATION OF COMPLETION CODE: WU - WASATCH - UPPER SANDSTONE

PARAMETER VALUE INDICATOR (PVI): < - LESS THAN DETECTION LIMIT
FLOW RELATIONSHIP CODE:

H - UNKNOWN

SAMPLE ID CODES:

0001 - FILTERED SAMPLE (.45 MICRONS) 
GROUNDHATER QUALITY DATA BY PARAMETER

UPPER SANDSTONE UNIT

SITE: SPKO1 SPOOK

$02 / 17 / 87$ TO $12 / 10 / 88$

REPORT DATE: $07 / 29 / 94$

\begin{tabular}{|c|c|c|c|c|c|c|c|c|c|c|c|}
\hline PARAMETER NAME & $\begin{array}{c}\text { LOCATION } \\
\text { ID }\end{array}$ & LOG DATE & $\begin{array}{c}\text { SAMPLE } \\
\text { ID }\end{array}$ & $\begin{array}{l}\text { FORM } \\
\text { COMP }\end{array}$ & $\begin{array}{l}\text { FLOW } \\
\text { REL. }\end{array}$ & $\begin{array}{l}\text { UNITS OF } \\
\text { MEASURE }\end{array}$ & PVI & $\begin{array}{l}\text { PARAMETER } \\
\text { VALUE }\end{array}$ & FLAGS & $\begin{array}{l}\text { DETECTION } \\
\text { LIMIT }\end{array}$ & $\begin{array}{l}\text { PARAMETER } \\
\text { UNCERTAINTY }\end{array}$ \\
\hline STRONTIUM & $\begin{array}{l}0920 \\
0920 \\
0920 \\
0922 \\
0922 \\
0922 \\
0922 \\
0924 \\
0924 \\
0924 \\
0924 \\
0925 \\
0925 \\
0925 \\
0925 \\
0927 \\
0927 \\
0927 \\
0927 \\
0928 \\
0928 \\
0928 \\
0928 \\
0929 \\
0929 \\
0929 \\
0929 \\
0930 \\
0930 \\
0930 \\
0930 \\
0931 \\
0931 \\
0931 \\
0932 \\
0932 \\
0932 \\
0933 \\
0934 \\
0935\end{array}$ & $\begin{array}{l}01 / 30 / 88 \\
04 / 26 / 88 \\
08 / 27 / 88 \\
09 / 20 / 87 \\
02 / 03 / 88 \\
04 / 26 / 88 \\
08 / 08 / 88 \\
09 / 20 / 87 \\
02 / 04 / 88 \\
04 / 26 / 88 \\
08 / 26 / 88 \\
09 / 20 / 87 \\
02 / 03 / 88 \\
04 / 26 / 88 \\
08 / 26 / 88 \\
09 / 20 / 87 \\
02 / 04 / 88 \\
04 / 26 / 88 \\
08 / 26 / 88 \\
09 / 20 / 87 \\
02 / 03 / 88 \\
04 / 22 / 88 \\
08 / 04 / 88 \\
09 / 20 / 87 \\
02 / 03 / 88 \\
04 / 28 / 88 \\
08 / 11 / 88 \\
09 / 20 / 87 \\
01 / 30 / 88 \\
04 / 26 / 88 \\
08 / 27 / 88 \\
01 / 30 / 88 \\
04 / 28 / 88 \\
08 / 10 / 88 \\
01 / 30 / 88 \\
04 / 28 / 88 \\
08 / 09 / 88 \\
08 / 10 / 88 \\
08 / 11 / 88 \\
08 / 09 / 88\end{array}$ & $\begin{array}{l}0001 \\
0001 \\
0001 \\
0001 \\
0001 \\
0001 \\
0001 \\
0001 \\
0001 \\
0001 \\
0001 \\
0001 \\
0001 \\
0001 \\
0001 \\
0001 \\
0001 \\
0001 \\
0001 \\
0001 \\
0001 \\
0001 \\
0001 \\
0001 \\
0001 \\
0001 \\
0001 \\
0001 \\
0001 \\
0001 \\
0001 \\
0001 \\
0001 \\
0001 \\
0001 \\
0001 \\
0001 \\
0001 \\
0001 \\
0001\end{array}$ & $\begin{array}{l}W U \\
W U \\
W U \\
W U \\
W U \\
W U \\
W U \\
W U \\
W U \\
W U \\
W U \\
W U \\
W U \\
W U \\
W U \\
W U \\
W U \\
W U \\
W U \\
W U \\
W U \\
W U \\
W U \\
W U \\
W U \\
W U \\
W U \\
W U \\
W U \\
W U \\
W U \\
W U \\
W U \\
W U \\
W U \\
W U \\
W U \\
W U \\
W U \\
W U\end{array}$ & $\begin{array}{l}N \\
N \\
N \\
N \\
N \\
N \\
N \\
N \\
N \\
N \\
N \\
N \\
N \\
N \\
N \\
N \\
N \\
N \\
N \\
N \\
N \\
N \\
N \\
N \\
N \\
N \\
N \\
N \\
N \\
N \\
N \\
N \\
N \\
N \\
N \\
N \\
N \\
N \\
N \\
N\end{array}$ & $M G / L$ & & $\begin{array}{l}5.95 \\
4.98 \\
6.5 \\
1.04 \\
0.81 \\
0.58 \\
0.9 \\
1.53 \\
1.51 \\
1.20 \\
1.6 \\
1.12 \\
1.01 \\
0.80 \\
1.1 \\
2.19 \\
2.33 \\
1.85 \\
2.3 \\
2.18 \\
2.33 \\
1.81 \\
2.0 \\
2.07 \\
0.94 \\
0.91 \\
1.0 \\
4.72 \\
5.50 \\
5.28 \\
6.0 \\
1.20 \\
1.12 \\
1.0 \\
0.98 \\
0.93 \\
1.0 \\
1.4 \\
1.0 \\
5.5\end{array}$ & $J$ & $\begin{array}{l}0.1 \\
0.1 \\
0.1 \\
0.1 \\
0.1 \\
0.1 \\
0.1 \\
0.1 \\
0.1 \\
0.1 \\
0.1 \\
0.1 \\
0.1 \\
0.1 \\
0.1 \\
0.1 \\
0.1 \\
0.1 \\
0.1 \\
0.1 \\
0.1 \\
0.1 \\
0.1 \\
0.1 \\
0.1 \\
0.1 \\
0.1 \\
0.1 \\
0.1 \\
0.1 \\
0.1 \\
0.1 \\
0.1 \\
0.1 \\
0.1 \\
0.1 \\
0.1 \\
0.1 \\
0.1 \\
0.1\end{array}$ & $\begin{array}{l}- \\
- \\
- \\
- \\
- \\
- \\
- \\
- \\
- \\
- \\
- \\
- \\
- \\
- \\
- \\
- \\
- \\
- \\
- \\
- \\
-\end{array}$ \\
\hline
\end{tabular}

FORMATION OF COMPLETION CODE:

FLOW RELATIONSHIP CODE:

WU - WASATCH - UPPER SANDSTONE

N - UNKNOWN

PARAMETER VALUE INDICATOR (PVI): < - LESS THAN DETECTION LIMIT

SAMPLE IO COOES:

0001 - FILTERED SAMPLE (.45 MICRONS)

OTHER PARAMETER VALUE FLAGS:

$J$ - ESTIMATED VALUE 


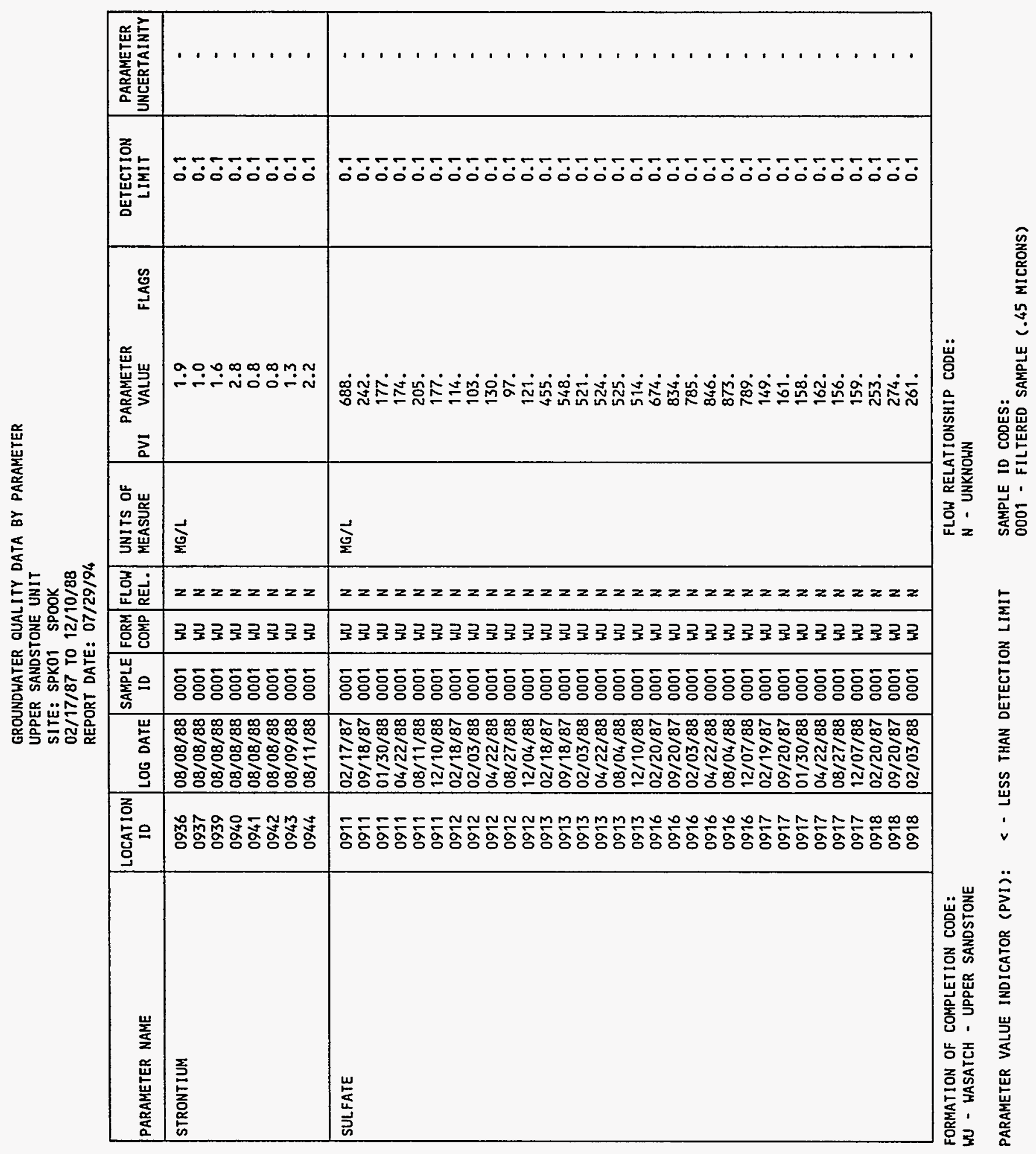


GROUNDWATER QUALITY DATA BY PARAMETER

UPPER SANDSTONE UNIT

SITE: SPKO1 SPOOK

02/17/87 TO 12/10/88

REPORT DATE: $07 / 29 / 94$

\begin{tabular}{|c|c|c|c|c|c|c|c|c|c|c|c|}
\hline PARAMETER NAME & $\mid \begin{array}{c}\text { LOCATION } \\
\text { ID }\end{array}$ & LOG DATE & $\begin{array}{c}\text { SAMPLE } \\
\text { ID }\end{array}$ & $\begin{array}{l}\text { FORM } \\
\text { COMP }\end{array}$ & $\begin{array}{l}\text { FLOWH } \\
\text { REL. }\end{array}$ & $\begin{array}{l}\text { UNITS OF } \\
\text { MEASURE }\end{array}$ & PVI & $\begin{array}{l}\text { PARAMETER } \\
\text { VALUE }\end{array}$ & FLAGS & $\begin{array}{l}\text { DETECTION } \\
\text { LIMIT }\end{array}$ & $\begin{array}{l}\text { PARAMETER } \\
\text { UNCERTAINTY }\end{array}$ \\
\hline SULFATE & $\begin{array}{l}0918 \\
0918 \\
0918 \\
0920 \\
0920 \\
0920 \\
0920 \\
0920 \\
0922 \\
0922 \\
0922 \\
0922 \\
0922 \\
0924 \\
0924 \\
0924 \\
0924 \\
0924 \\
0925 \\
0925 \\
0925 \\
0925 \\
0925 \\
0927 \\
0927 \\
0927 \\
0927 \\
0927 \\
0928 \\
0928 \\
0928 \\
0928 \\
0928 \\
0929 \\
0929 \\
0929 \\
0929 \\
0930 \\
0930 \\
0930\end{array}$ & $\begin{array}{l}04 / 22 / 88 \\
08 / 26 / 88 \\
12 / 04 / 88 \\
09 / 20 / 87 \\
01 / 30 / 88 \\
04 / 26 / 88 \\
08 / 27 / 88 \\
12 / 10 / 88 \\
09 / 20 / 87 \\
02 / 03 / 88 \\
04 / 26 / 88 \\
08 / 08 / 88 \\
12 / 10 / 88 \\
09 / 20 / 87 \\
02 / 04 / 88 \\
04 / 26 / 88 \\
08 / 26 / 88 \\
12 / 07 / 88 \\
09 / 20 / 87 \\
02 / 03 / 88 \\
04 / 26 / 88 \\
08 / 26 / 88 \\
12 / 07 / 88 \\
09 / 20 / 87 \\
02 / 04 / 88 \\
04 / 26 / 88 \\
08 / 26 / 88 \\
12 / 04 / 88 \\
09 / 20 / 87 \\
02 / 03 / 88 \\
04 / 22 / 88 \\
08 / 04 / 88 \\
12 / 04 / 88 \\
09 / 20 / 87 \\
02 / 03 / 88 \\
04 / 28 / 88 \\
08 / 11 / 88 \\
09 / 20 / 87 \\
01 / 30 / 88 \\
04 / 26 / 88\end{array}$ & $\begin{array}{l}0001 \\
0001 \\
0001 \\
0001 \\
0001 \\
0001 \\
0001 \\
0001 \\
0001 \\
0001 \\
0001 \\
0001 \\
0001 \\
0001 \\
0001 \\
0001 \\
0001 \\
0001 \\
0001 \\
0001 \\
0001 \\
0001 \\
0001 \\
0001 \\
0001 \\
0001 \\
0001 \\
0001 \\
0001 \\
0001 \\
0001 \\
0001 \\
0001 \\
0001 \\
0001 \\
0001 \\
0001 \\
0001 \\
0001 \\
0001\end{array}$ & $\begin{array}{l}W U \\
W U \\
W U \\
W U \\
W U \\
W U \\
W U \\
W U \\
W U \\
W U \\
W U \\
W U \\
W U \\
W U \\
W U \\
W U \\
W U \\
W U \\
W U \\
W U \\
W U \\
W U \\
W U \\
W U \\
W U \\
W U \\
W U \\
W U \\
W U \\
W U \\
W U \\
W U \\
W U \\
W U \\
W U \\
W U \\
W U \\
W U \\
W U\end{array}$ & $\begin{array}{l}N \\
N \\
N \\
N \\
N \\
N \\
N \\
N \\
N \\
N \\
N \\
N \\
N \\
N \\
N \\
N \\
N \\
N \\
N \\
N \\
N \\
N \\
N \\
N \\
N \\
N \\
N \\
N \\
N \\
N \\
N \\
N \\
N \\
N \\
N \\
N \\
N \\
N \\
N\end{array}$ & MG/L & & $\begin{array}{c}264 . \\
265 . \\
263 . \\
3030 . \\
3240 . \\
3240 . \\
2870 . \\
2760 . \\
330 . \\
274 . \\
275 . \\
304 . \\
293 . \\
556 . \\
579 . \\
606 . \\
617 . \\
634 . \\
152 . \\
151 . \\
150 . \\
156 . \\
154 . \\
1210 . \\
1220 . \\
1380 . \\
1360 . \\
1350 . \\
1180 . \\
1050 . \\
1190 . \\
1180 . \\
1150 . \\
576 . \\
109 . \\
100 . \\
98 . \\
2800 . \\
2940 . \\
3180 .\end{array}$ & $J$ & $\begin{array}{l}0.1 \\
0.1 \\
0.1 \\
0.1 \\
0.1 \\
0.1 \\
0.1 \\
0.1 \\
0.1 \\
0.1 \\
0.1 \\
0.1 \\
0.1 \\
0.1 \\
0.1 \\
0.1 \\
0.1 \\
0.1 \\
0.1 \\
0.1 \\
0.1 \\
0.1 \\
0.1 \\
0.1 \\
0.1 \\
0.1 \\
0.1 \\
0.1 \\
0.1 \\
0.1 \\
0.1 \\
0.1 \\
0.1 \\
0.1 \\
0.1 \\
0.1 \\
0.1 \\
0.1 \\
0.1 \\
0.1\end{array}$ & $\begin{array}{l}- \\
- \\
- \\
- \\
- \\
- \\
- \\
- \\
- \\
- \\
- \\
- \\
- \\
- \\
- \\
- \\
- \\
- \\
- \\
- \\
E \\
- \\
- \\
- \\
- \\
- \\
- \\
- \\
- \\
-\end{array}$ \\
\hline
\end{tabular}

FORMATION OF COMPLETION CODE:

FLOH RELATIONSHIP CODE:

WU - HASATCH - UPPER SANDSTONE

N - UNKNOWN

PARAMETER VALUE INDICATOR (PVI): < - LESS THAN DETECTION LIMIT

SAMPLE ID CODES:

0001 - FILTERED SAMPLE (.45 MICRONS)

OTHER PARAMETER VALUE FLAGS:

$J$ - ESTIMATED VALUE 


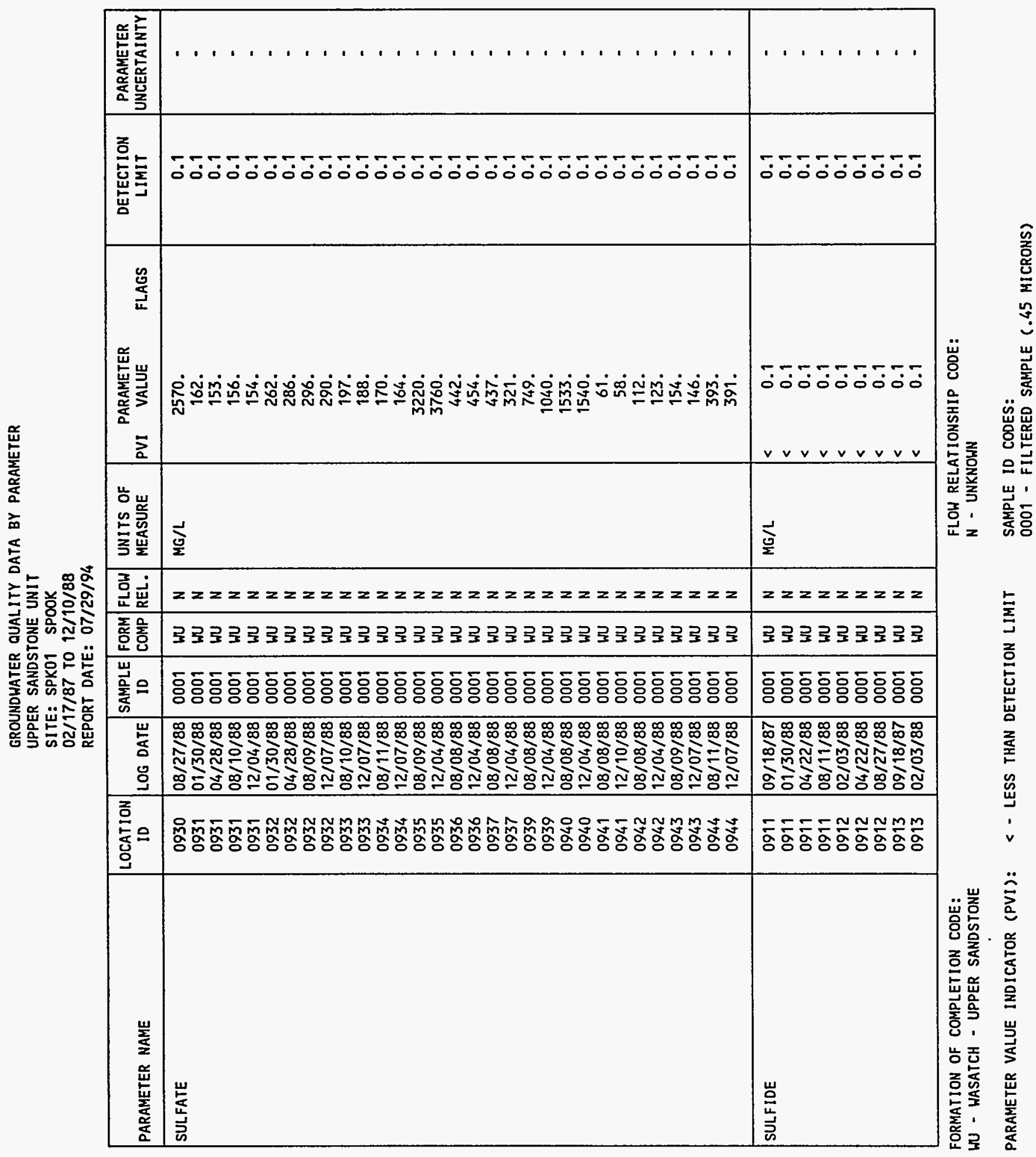




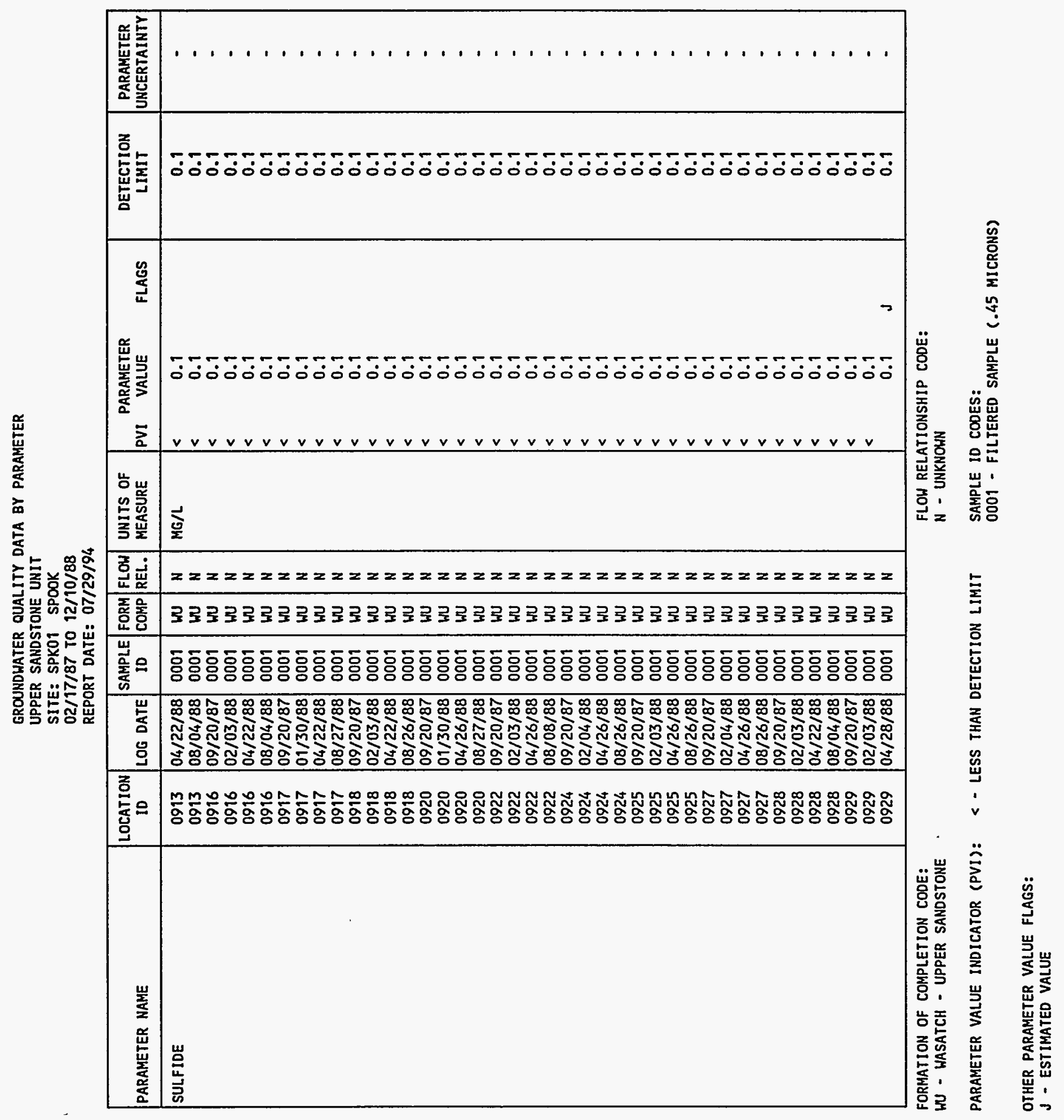




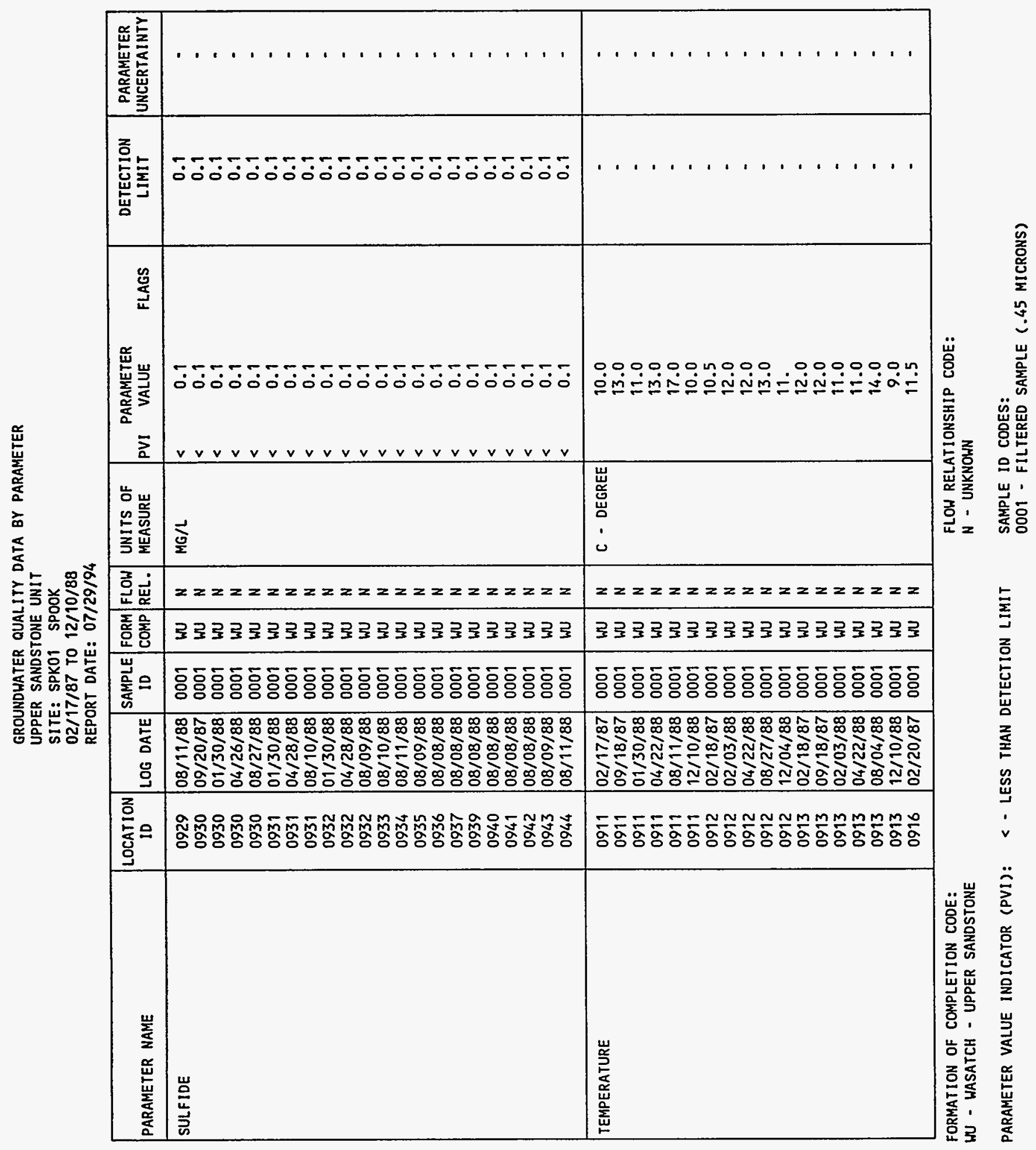




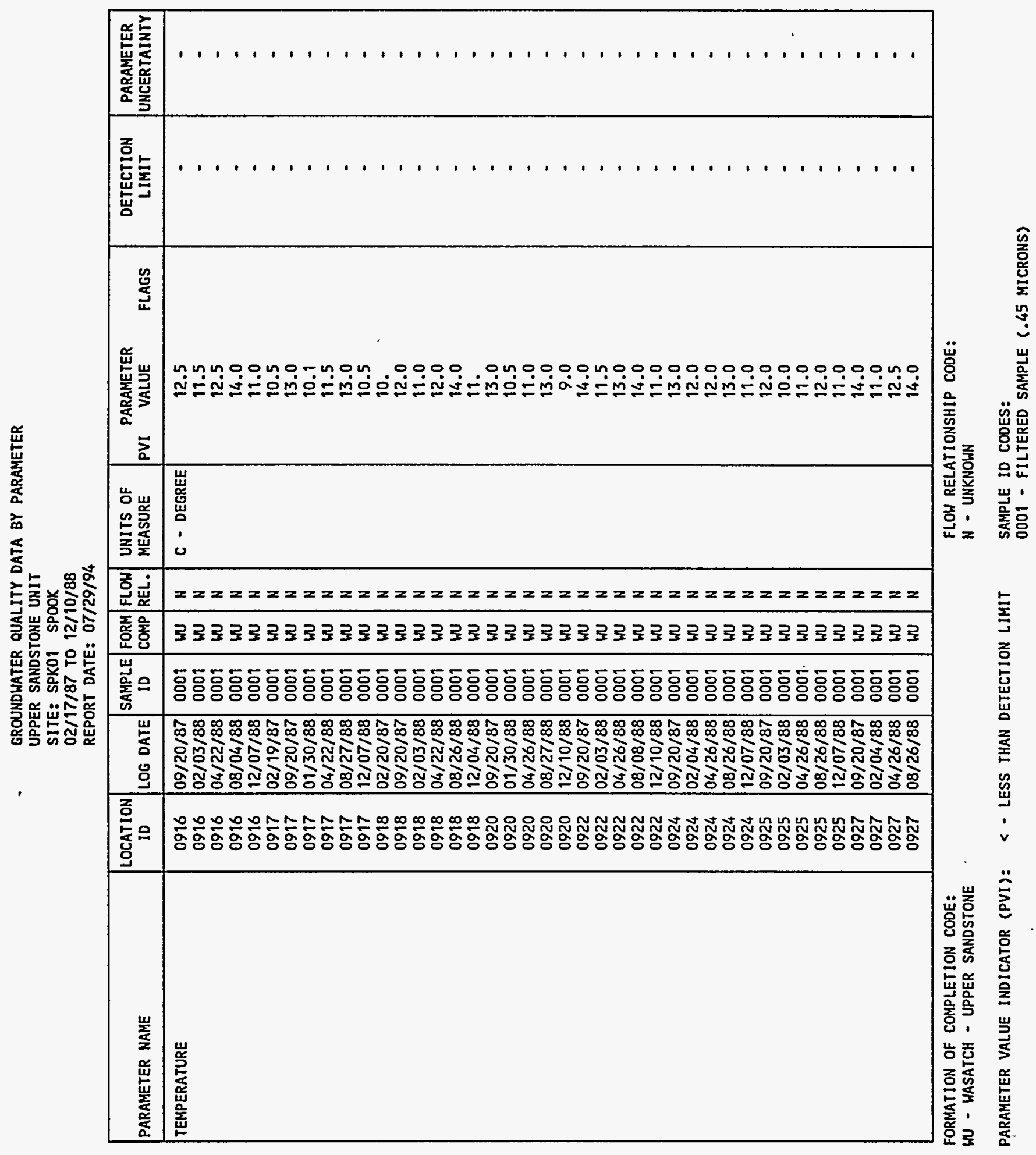


GROUNOWATER QUALITY DATA BY PARAMETER

UPPER SANDSTONE UNIT

SITE: SPKO1 SPOOK

$02 / 17 / 87$ TO $12 / 10 / 88$

REPORT DATE: $07 / 29 / 94$

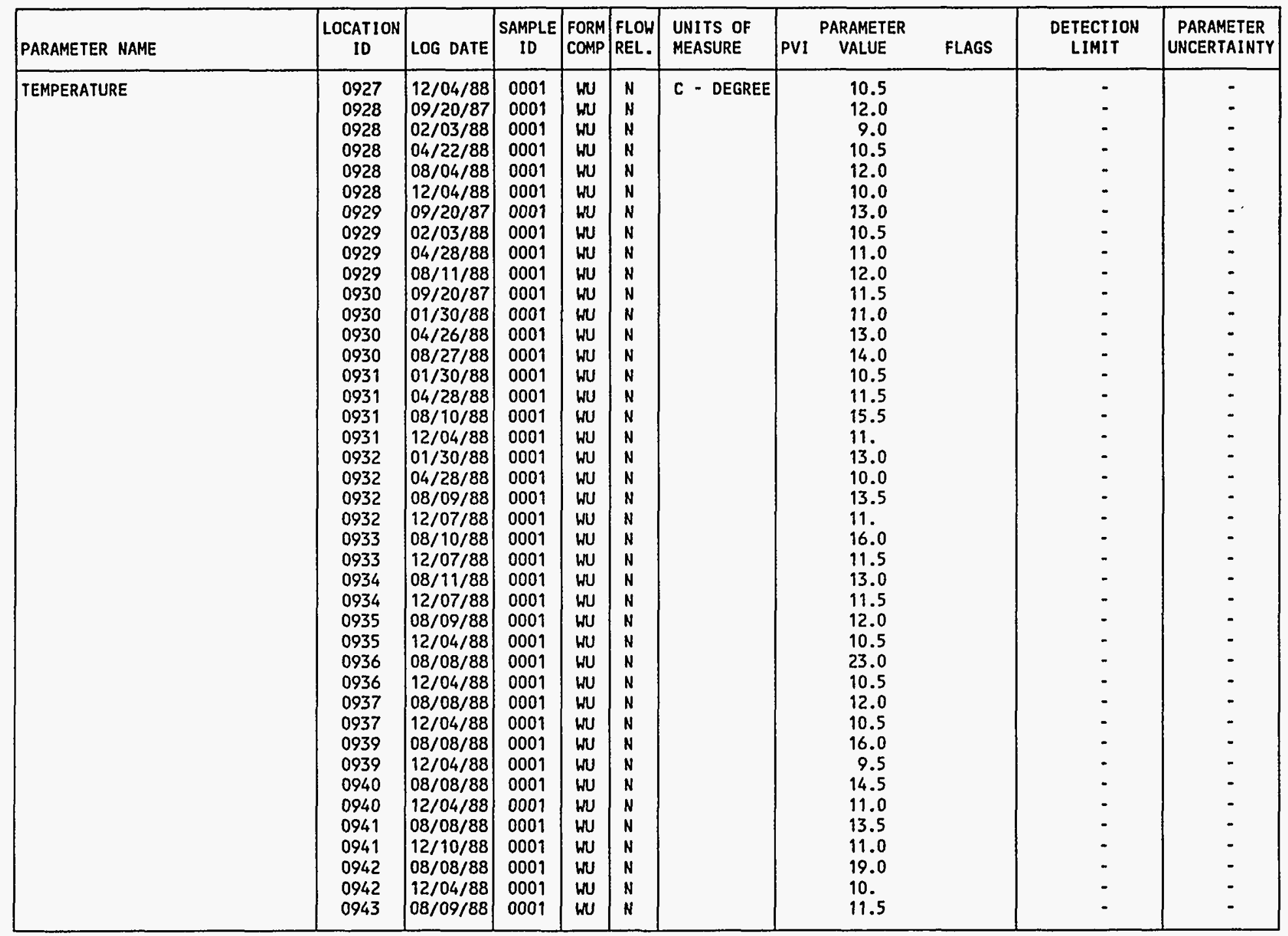

FOPMATION OF COMPLETION CODE:

WU - WASATCH - UPPER SANDSTONE

PARAMETER VALUE INDICATOR (PVI):
FLOW RELATIONSHIP CODE:

N - UNKNOWN

SAMPLE ID CODES

0001 - FILTERED SAMPLE (.45 MICRONS) 


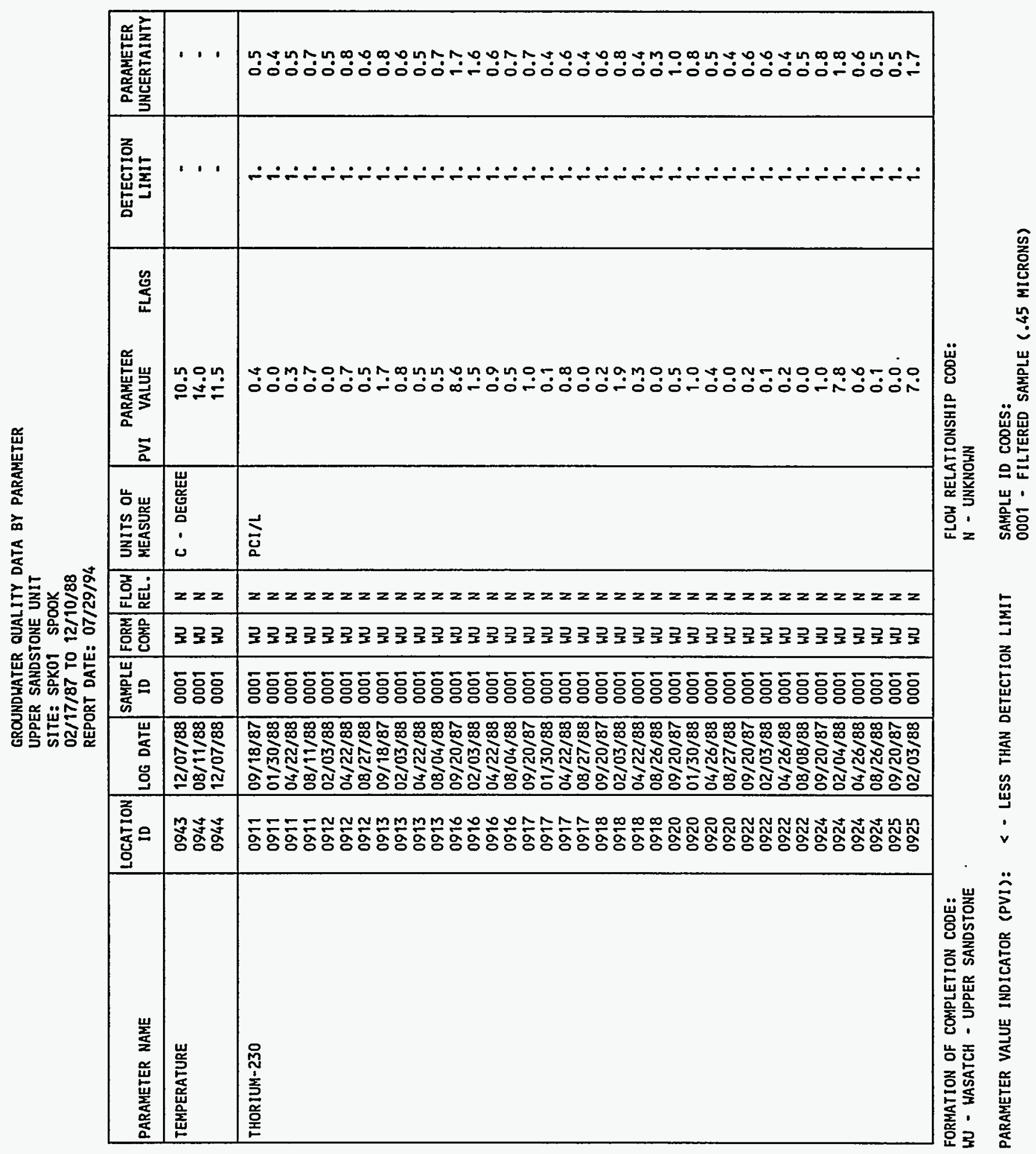


GROUNDWATER QUALITY DATA BY PARAMETER

UPPER SANDSTONE UNIT

SITE: SPKO1 SPOOK

02/17/87 TO $12 / 10 / 88$

REPORT DATE: 07/29/94

\begin{tabular}{|c|c|c|c|c|c|c|c|c|c|c|c|}
\hline PARAMETER NAME & $\begin{array}{c}\text { LOCATION } \\
10\end{array}$ & LOG DATE & $\begin{array}{c}\text { SAMPLE } \\
\text { ID }\end{array}$ & $\begin{array}{l}\text { FORM } \\
\text { COMP }\end{array}$ & $\begin{array}{l}\text { FLOW } \\
\text { REL. }\end{array}$ & $\begin{array}{l}\text { UNITS OF } \\
\text { MEASURE }\end{array}$ & PVI & $\begin{array}{l}\text { PARAMETER } \\
\text { VALUE }\end{array}$ & FLAGS & $\begin{array}{l}\text { DETECTION } \\
\text { LIMIT }\end{array}$ & $\begin{array}{l}\text { PARAMETER } \\
\text { UNCERTAINTY }\end{array}$ \\
\hline THORIUM-230 & $\begin{array}{l}0925 \\
0925 \\
0927 \\
0927 \\
0927 \\
0927 \\
0928 \\
0928 \\
0928 \\
0928 \\
0929 \\
0929 \\
0929 \\
0929 \\
0930 \\
0930 \\
0930 \\
0930 \\
0931 \\
0931 \\
0931 \\
0932 \\
0932 \\
0932 \\
0933 \\
0934 \\
0935 \\
0936 \\
0937 \\
0940 \\
0941 \\
0942 \\
0943 \\
0944\end{array}$ & $\begin{array}{l}04 / 26 / 88 \\
08 / 26 / 88 \\
09 / 20 / 87 \\
02 / 04 / 88 \\
04 / 26 / 88 \\
08 / 26 / 88 \\
09 / 20 / 87 \\
02 / 03 / 88 \\
04 / 22 / 88 \\
08 / 04 / 88 \\
09 / 20 / 87 \\
02 / 03 / 88 \\
04 / 28 / 88 \\
08 / 11 / 88 \\
09 / 20 / 87 \\
01 / 30 / 88 \\
04 / 26 / 88 \\
08 / 27 / 88 \\
01 / 30 / 88 \\
04 / 28 / 88 \\
08 / 10 / 88 \\
01 / 30 / 88 \\
04 / 28 / 88 \\
08 / 09 / 88 \\
08 / 10 / 88 \\
08 / 11 / 88 \\
08 / 09 / 88 \\
08 / 08 / 88 \\
08 / 08 / 88 \\
08 / 08 / 88 \\
08 / 08 / 88 \\
08 / 08 / 88 \\
08 / 09 / 88 \\
08 / 11 / 88\end{array}$ & $\begin{array}{l}0001 \\
0001 \\
0001 \\
0001 \\
0001 \\
0001 \\
0001 \\
0001 \\
0001 \\
0001 \\
0001 \\
0001 \\
0001 \\
0001 \\
0001 \\
0001 \\
0001 \\
0001 \\
0001 \\
0001 \\
0001 \\
0001 \\
0001 \\
0001 \\
0001 \\
0001 \\
0001 \\
0001 \\
0001 \\
0001 \\
0001 \\
0001 \\
0001 \\
0001\end{array}$ & $\begin{array}{l}W U \\
W U \\
W U \\
W U \\
W U \\
W U \\
W U \\
W U \\
W U \\
W U \\
W U \\
W U \\
W U \\
W U \\
W U \\
W U \\
W U \\
W U \\
W U \\
W U \\
W U \\
W U \\
W U \\
W U \\
W U \\
W U \\
W U \\
W U \\
W U \\
W U \\
W U \\
W U \\
W U \\
W U\end{array}$ & $\begin{array}{l}N \\
N \\
N \\
N \\
N \\
N \\
N \\
N \\
N \\
N \\
N \\
N \\
N \\
N \\
N \\
N \\
N \\
N \\
N \\
N \\
N \\
N \\
N \\
N \\
N \\
N \\
N \\
N \\
N \\
N \\
N \\
N \\
N \\
N\end{array}$ & $\mathrm{PCI} / \mathrm{L}$ & & $\begin{array}{l}0.0 \\
0.1 \\
1.1 \\
2.5 \\
0.2 \\
0.7 \\
0.3 \\
0.8 \\
3.8 \\
0.0 \\
0.0 \\
0.0 \\
0.8 \\
0.0 \\
0.5 \\
0.0 \\
0.3 \\
0.1 \\
8.1 \\
0.0 \\
0.0 \\
0.4 \\
0.3 \\
0.0 \\
0.1 \\
0.0 \\
0.0 \\
0.0 \\
0.0 \\
0.0 \\
0.0 \\
0.0 \\
0.0 \\
0.0\end{array}$ & . & $\begin{array}{l}1 . \\
1 . \\
1 . \\
1 . \\
1 . \\
1 . \\
1 . \\
1 . \\
1 . \\
1 . \\
1 . \\
1 . \\
1 . \\
1 . \\
1 . \\
1 . \\
1 . \\
1 . \\
1 . \\
1 . \\
1 . \\
1 . \\
1 . \\
1 . \\
1 . \\
1 . \\
1 . \\
1 . \\
1 . \\
1 . \\
1 . \\
1 .\end{array}$ & $\begin{array}{l}0.4 \\
0.5 \\
0.8 \\
1.1 \\
0.4 \\
0.6 \\
0.4 \\
0.8 \\
1.1 \\
0.5 \\
0.3 \\
0.4 \\
0.6 \\
0.4 \\
0.8 \\
0.4 \\
0.5 \\
0.5 \\
1.8 \\
0.3 \\
0.5 \\
0.6 \\
0.5 \\
0.5 \\
0.6 \\
0.4 \\
0.5 \\
0.5 \\
0.5 \\
0.5 \\
0.5 \\
0.4 \\
0.5 \\
0.5\end{array}$ \\
\hline TIN & $\begin{array}{l}0911 \\
0911 \\
0919 \\
0911 \\
0912\end{array}$ & $\begin{array}{l}09 / 18 / 87 \\
01 / 30 / 88 \\
04 / 22 / 88 \\
08 / 11 / 88 \\
02 / 03 / 88\end{array}$ & $\begin{array}{l}0001 \\
0001 \\
0001 \\
0001 \\
0001\end{array}$ & $\begin{array}{l}W U \\
W U \\
W U \\
W U \\
W U\end{array}$ & $\begin{array}{l}N \\
N \\
N \\
N \\
N\end{array}$ & $M G / L$ & $\mid \begin{array}{l}< \\
< \\
<\end{array}$ & $\begin{array}{l}0.018 \\
0.005 \\
0.009 \\
0.005 \\
0.005\end{array}$ & & $\begin{array}{l}0.005 \\
0.005 \\
0.005 \\
0.005 \\
0.005\end{array}$ & $\begin{array}{l}: \\
: \\
-\end{array}$ \\
\hline
\end{tabular}

FORMATION OF COMPLETION CODE:

WU - WASATCH - UPPER SANDSTONE

FLOW RELATIONSHIP CODE:

PARAMETER VALUE INDICATOR (PVI): < - LESS THAN DETECTION LIMIT

SAMPLE ID CODES:

0001 - FILTERED SAMPLE (.45 MICRONS)

OTHER PARAMETER VALUE FLAGS:

$J$ - ESTIMATEO VALUE 


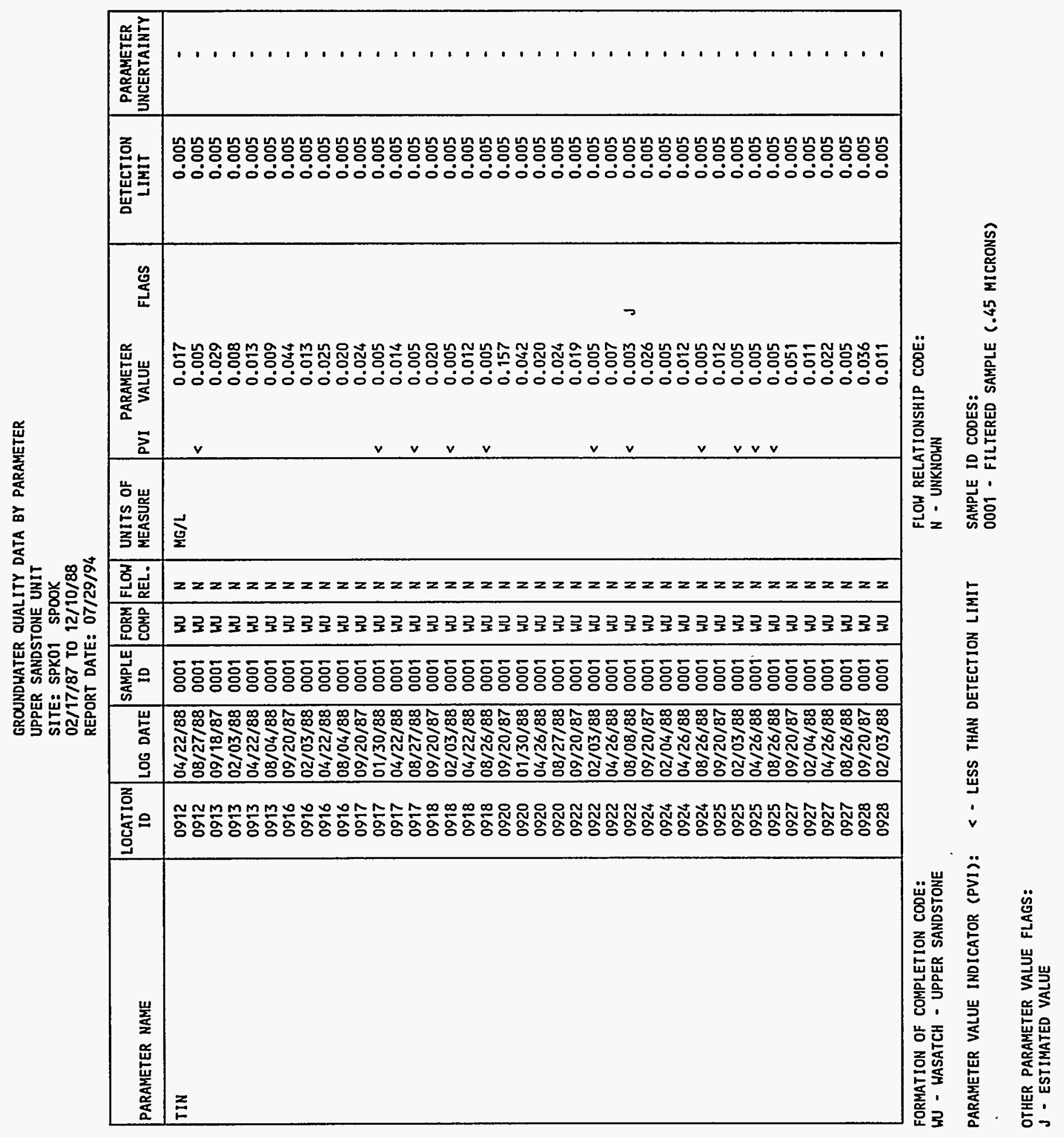




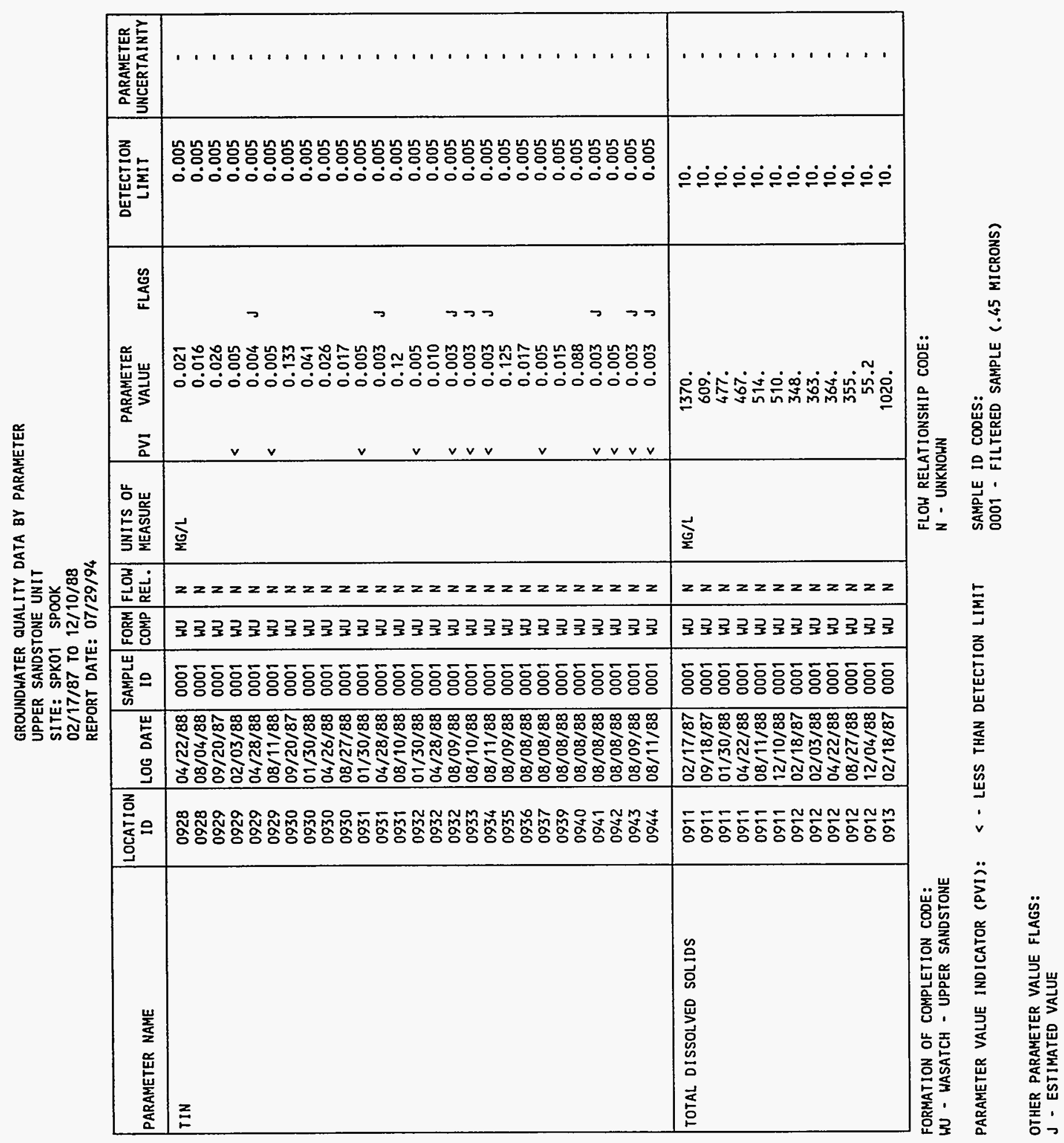




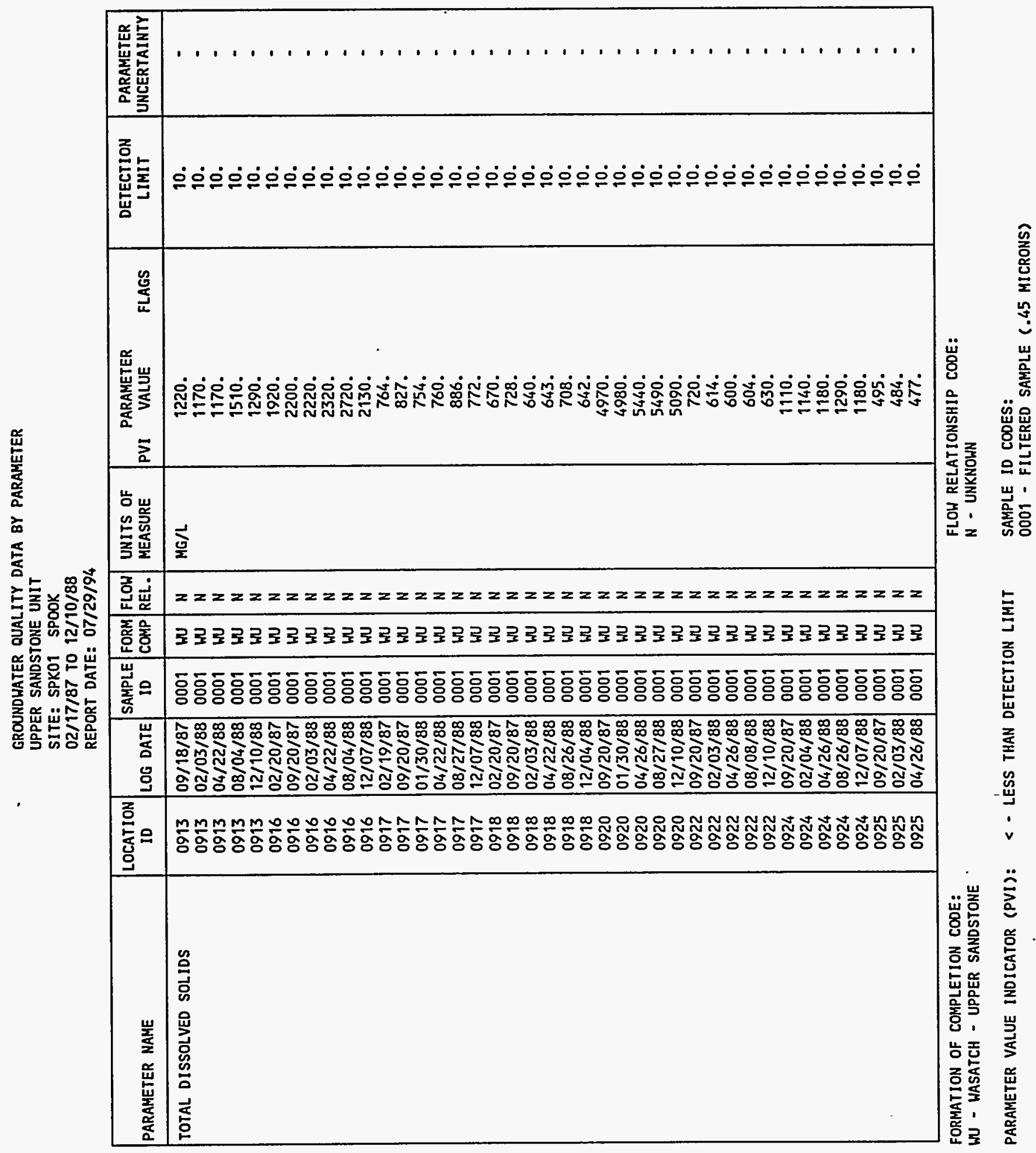


SITE: SPKO1 SPOOK

02/17/87 TO $12 / 10 / 88$

REPORT DATE: 07/29/94

\begin{tabular}{|c|c|c|c|c|c|c|c|c|c|c|c|}
\hline PARAMETER NAME & $\mid \begin{array}{c}\text { LOCATION } \\
\text { ID }\end{array}$ & LOG DATE & $\underset{\text { SAMPLE }}{\text { ID }}$ & $\begin{array}{l}\text { FORM } \\
\text { COMP }\end{array}$ & $\begin{array}{l}\text { FLOW } \\
\text { REL. }\end{array}$ & $\begin{array}{l}\text { UNITS OF } \\
\text { MEASURE }\end{array}$ & PVI & $\begin{array}{l}\text { PARAMETER } \\
\text { VALUE }\end{array}$ & FLAGS & $\begin{array}{l}\text { DETECTION } \\
\text { LIMIT }\end{array}$ & $\begin{array}{l}\text { PARAMETER } \\
\text { UNCERTAINTY }\end{array}$ \\
\hline TOTAL DISSOLVED SOLIDS & $\begin{array}{l}0925 \\
0925 \\
0927 \\
0927 \\
0927 \\
0927 \\
0927 \\
0928 \\
0928 \\
0928 \\
0928 \\
0928 \\
0929 \\
0929 \\
0929 \\
0929 \\
0930 \\
0930 \\
0930 \\
0930 \\
0931 \\
0931 \\
0931 \\
0931 \\
0932 \\
0932 \\
0932 \\
0932 \\
0933 \\
0933 \\
0934 \\
0934 \\
0935 \\
0935 \\
0936 \\
0936 \\
0937 \\
0937 \\
0939 \\
0939\end{array}$ & $\begin{array}{l}08 / 26 / 88 \\
12 / 07 / 88 \\
09 / 20 / 87 \\
02 / 04 / 88 \\
04 / 26 / 88 \\
08 / 26 / 88 \\
12 / 04 / 88 \\
09 / 20 / 87 \\
02 / 03 / 88 \\
04 / 22 / 88 \\
08 / 04 / 88 \\
12 / 04 / 88 \\
09 / 20 / 87 \\
02 / 03 / 88 \\
04 / 28 / 88 \\
08 / 11 / 88 \\
09 / 20 / 87 \\
01 / 30 / 88 \\
04 / 26 / 88 \\
08 / 27 / 88 \\
01 / 30 / 88 \\
04 / 28 / 88 \\
08 / 10 / 88 \\
12 / 04 / 88 \\
01 / 30 / 88 \\
04 / 28 / 88 \\
08 / 09 / 88 \\
12 / 07 / 88 \\
08 / 10 / 88 \\
12 / 07 / 88 \\
08 / 11 / 88 \\
12 / 07 / 88 \\
08 / 09 / 88 \\
12 / 04 / 88 \\
08 / 08 / 88 \\
12 / 04 / 88 \\
08 / 08 / 88 \\
12 / 04 / 88 \\
08 / 08 / 88 \\
12 / 04 / 88\end{array}$ & $\begin{array}{l}0001 \\
0001 \\
0001 \\
0001 \\
0001 \\
0001 \\
0001 \\
0001 \\
0001 \\
0001 \\
0001 \\
0001 \\
0001 \\
0001 \\
0001 \\
0001 \\
0001 \\
0001 \\
0001 \\
0001 \\
0001 \\
0001 \\
0001 \\
0001 \\
0001 \\
0001 \\
0001 \\
0001 \\
0001 \\
0001 \\
0001 \\
0001 \\
0001 \\
0001 \\
0001 \\
0001 \\
0001 \\
0001 \\
0001 \\
0001\end{array}$ & $\begin{array}{l}W U \\
W U \\
W U \\
W U \\
W U \\
W U \\
W U \\
W U \\
W U \\
W U \\
W U \\
W U \\
W U \\
W U \\
W U \\
W U \\
W U \\
W U \\
W U \\
W U \\
W U \\
W U \\
W U \\
W U \\
W U \\
W U \\
W U \\
W U \\
W U \\
W U \\
W U \\
W U \\
W U \\
W U \\
W U \\
W U \\
W U \\
W U \\
W U \\
W U\end{array}$ & $\begin{array}{l}N \\
N \\
N \\
N \\
N \\
N \\
N \\
N \\
N \\
N \\
N \\
N \\
N \\
N \\
N \\
N \\
N \\
N \\
N \\
N \\
N \\
N \\
N \\
N \\
N \\
N \\
N \\
N \\
N \\
N \\
N \\
N \\
N \\
N \\
N \\
N \\
N \\
N \\
N \\
N\end{array}$ & $M G / L$ & & $\begin{array}{c}474 . \\
477 . \\
2310 . \\
2230 . \\
2340 . \\
2360 . \\
2330 . \\
1900 . \\
1860 . \\
1860 . \\
1880 . \\
1790 . \\
1070 . \\
374 . \\
401 . \\
402 . \\
4360 . \\
4840 . \\
4670 . \\
4890 . \\
444 . \\
474 . \\
707 . \\
472 . \\
648 . \\
702 . \\
707 . \\
698 . \\
540 . \\
528 . \\
510 . \\
495 . \\
5450 . \\
5450 . \\
1200 . \\
1190 . \\
872 . \\
686 . \\
1250 . \\
1650 .\end{array}$ & $\mathbf{J}$ & $\begin{array}{l}10 . \\
10 . \\
10 . \\
10 . \\
10 . \\
10 . \\
10 . \\
10 . \\
10 . \\
10 . \\
10 . \\
10 . \\
10 . \\
10 . \\
10 . \\
10 . \\
10 . \\
10 . \\
10 . \\
10 . \\
10 . \\
10 . \\
10 . \\
10 . \\
10 . \\
10 . \\
10 . \\
10 . \\
10 . \\
10 . \\
10 . \\
10 . \\
10 . \\
10 . \\
10 . \\
10 . \\
10 . \\
10 . \\
10 . \\
10 .\end{array}$ & $\begin{array}{l}- \\
- \\
- \\
- \\
- \\
- \\
- \\
- \\
- \\
- \\
- \\
- \\
- \\
- \\
- \\
- \\
- \\
- \\
- \\
- \\
- \\
- \\
- \\
- \\
- \\
- \\
- \\
- \\
- \\
- \\
- \\
- \\
- \\
- \\
- \\
- \\
- \\
-\end{array}$ \\
\hline
\end{tabular}

FORMATION OF COMPLETION CODE:

WU - WASATCH - UPPER SANDSTONE

PARAMETER VALUE INDICATOR (PVI): < - LESS than DETECTION LIMIT
FLOW RELATIONSHIP COOE: $N$ - UNKNOWN

SAMPLE ID CODES:

0001 - FILTERED SAMPLE (.45 MICRONS)

OTHER PARAMETER VALUE FLAGS:

$J$ - ESTIMATED VALUE 
GROUNDWATER QUALITY DATA BY PARAMETER

UPPER SANDSTONE UNIT

SITE: SPKOT SPOOK

$02 / 17 / 87$ TO $12 / 10 / 88$

REPORT DATE: $07 / 29 / 94$

\begin{tabular}{|c|c|c|c|c|c|c|c|c|c|c|}
\hline PARAMETER NAME & $\underset{\text { ID }}{\text { LOCATION }}$ & LOG DATE & $\underset{\text { SAMPLE }}{\text { ID }}$ & $\begin{array}{l}\text { FORM } \\
\text { COMP }\end{array}$ & $\begin{array}{l}\text { FLOW } \\
\text { REL. }\end{array}$ & $\begin{array}{l}\text { UNITS OF } \\
\text { MEASURE }\end{array}$ & PVI & $\begin{array}{l}\text { PARAMETER } \\
\text { VALUE }\end{array}$ & $\begin{array}{l}\text { DETECTION } \\
\text { LIMIT }\end{array}$ & $\begin{array}{l}\text { PARAMETER } \\
\text { UNCERTAINTY }\end{array}$ \\
\hline TOTAL DISSOLVED SOLIDS & $\begin{array}{l}0940 \\
0940 \\
0941 \\
0941 \\
0942 \\
0942 \\
0943 \\
0943 \\
0944 \\
0944\end{array}$ & $\begin{array}{l}08 / 08 / 88 \\
12 / 04 / 88 \\
08 / 08 / 88 \\
12 / 10 / 88 \\
08 / 08 / 88 \\
12 / 04 / 88 \\
08 / 09 / 88 \\
12 / 07 / 88 \\
08 / 11 / 88 \\
12 / 07 / 88\end{array}$ & $\begin{array}{l}0001 \\
0001 \\
0001 \\
0001 \\
0001 \\
0001 \\
0001 \\
0001 \\
0001 \\
0001\end{array}$ & $\begin{array}{l}W U \\
W U \\
W U \\
W U \\
W U \\
W U \\
W U \\
W U \\
W U \\
W U\end{array}$ & $\begin{array}{l}N \\
N \\
N \\
N \\
N \\
N \\
N \\
N \\
N \\
N\end{array}$ & $M G / L$ & & $\begin{array}{c}4480 . \\
3670 . \\
292 . \\
304 . \\
433 . \\
449 . \\
475 . \\
464 . \\
859 . \\
823 .\end{array}$ & $\begin{array}{l}10 . \\
10 . \\
10 . \\
10 . \\
10 . \\
10 . \\
10 . \\
10 . \\
10 . \\
10 .\end{array}$ & $\begin{array}{l}- \\
- \\
- \\
- \\
- \\
- \\
-\end{array}$ \\
\hline URANIUM & $\begin{array}{l}0911 \\
0911 \\
0911 \\
0911 \\
0911 \\
0911 \\
0912 \\
0912 \\
0912 \\
0912 \\
0912 \\
0913 \\
0913 \\
0913 \\
0913 \\
0913 \\
0913 \\
0916 \\
0916 \\
0916 \\
0916 \\
0916 \\
0916 \\
0917 \\
0917 \\
0917 \\
0917 \\
0917 \\
0917 \\
0918\end{array}$ & $\begin{array}{l}02 / 17 / 87 \\
09 / 18 / 87 \\
01 / 30 / 88 \\
04 / 22 / 88 \\
08 / 11 / 88 \\
12 / 10 / 88 \\
02 / 18 / 87 \\
02 / 03 / 88 \\
04 / 22 / 88 \\
08 / 27 / 88 \\
12 / 04 / 88 \\
02 / 18 / 87 \\
09 / 18 / 87 \\
02 / 03 / 88 \\
04 / 22 / 88 \\
08 / 04 / 88 \\
12 / 10 / 88 \\
02 / 20 / 87 \\
09 / 20 / 87 \\
02 / 03 / 88 \\
04 / 22 / 88 \\
08 / 04 / 88 \\
12 / 07 / 88 \\
02 / 19 / 87 \\
09 / 20 / 87 \\
01 / 30 / 88 \\
04 / 22 / 88 \\
08 / 27 / 88 \\
12 / 07 / 88 \\
02 / 20 / 87\end{array}$ & $\begin{array}{l}0001 \\
0001 \\
0001 \\
0001 \\
0001 \\
0001 \\
0001 \\
0001 \\
0001 \\
0001 \\
0001 \\
0001 \\
0001 \\
0001 \\
0001 \\
0001 \\
0001 \\
0001 \\
0001 \\
0001 \\
0001 \\
0001 \\
0001 \\
0001 \\
0001 \\
0001 \\
0001 \\
0001 \\
0001 \\
0001\end{array}$ & $\begin{array}{l}W U \\
W U \\
W U \\
W U \\
W U \\
W U \\
W U \\
W U \\
W U \\
W U \\
W U \\
W U \\
W U \\
W U \\
W U \\
W U \\
W U \\
W U \\
W U \\
W U \\
W U \\
W U \\
W U \\
W U \\
W U \\
W U \\
W U \\
W U \\
W U \\
W U\end{array}$ & $\begin{array}{l}N \\
N \\
N \\
N \\
N \\
N \\
N \\
N \\
N \\
N \\
N \\
N \\
N \\
N \\
N \\
N \\
N \\
N \\
N \\
N \\
N \\
N \\
N \\
N \\
N \\
N \\
N \\
N \\
N \\
N\end{array}$ & $M G / L$ & & $\begin{array}{l}1.77 \\
1.80 \\
1.71 \\
1.90 \\
1.39 \\
1.67 \\
0.632 \\
0.607 \\
0.526 \\
0.563 \\
0.542 \\
8.23 \\
\quad 8.84 \\
8.60 \\
9.17 \\
8.14 \\
8.56 \\
15.4 \\
19.5 \\
20.7 \\
24.6 \\
19.1 \\
22.0 \\
5.43 \\
5\end{array}$ & $\begin{array}{l}0.003 \\
0.003 \\
0.003 \\
0.003 \\
0.003 \\
0.003 \\
0.003 \\
0.003 \\
0.003 \\
0.003 \\
0.003 \\
0.003 \\
0.003 \\
0.003 \\
0.003 \\
0.003 \\
0.003 \\
0.003 \\
0.003 \\
0.003 \\
0.003 \\
0.003 \\
0.003 \\
0.003 \\
0.003 \\
0.003 \\
0.003 \\
0.003 \\
0.003 \\
0.003\end{array}$ & 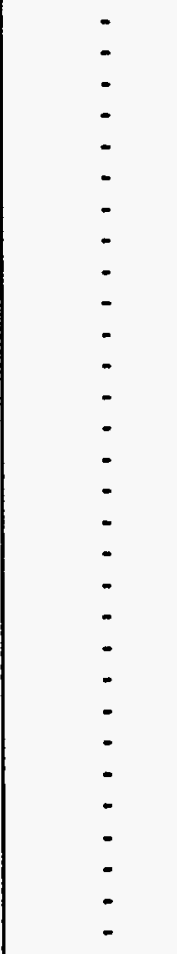 \\
\hline
\end{tabular}

FORMATION OF COMPLETION CODE:

WU - HASATCH - UPPER SANDSTONE

FLOW RELATIONSHIP CODE:

PARAMETER VALUE INDICATOR (PVI): < - LESS THAN DETECTION LIMIT SAMPLE ID CODES:

0001 - FILTERED SAMPLE (.45 MICRONS) 
REPORT DATE: $07 / 29 / 94$

\begin{tabular}{|c|c|c|c|c|c|c|c|c|c|c|c|}
\hline PARAMETER NAME & $\begin{array}{c}\text { LOCATION } \\
\text { ID }\end{array}$ & LOG DATE & $\begin{array}{l}\text { SAMPLE } \\
\text { ID }\end{array}$ & $\begin{array}{l}\text { FORM } \\
\text { COMP }\end{array}$ & $\begin{array}{l}\text { FLOW } \\
\text { REL. }\end{array}$ & $\begin{array}{l}\text { UNITS OF } \\
\text { MEASURE }\end{array}$ & PVI & $\begin{array}{l}\text { PARAMETER } \\
\text { VALUE }\end{array}$ & FLAGS & $\begin{array}{l}\text { DETECTION } \\
\text { LIMIT }\end{array}$ & $\begin{array}{l}\text { PARAMETER } \\
\text { UNCERTAINTY }\end{array}$ \\
\hline URANIUM & $\begin{array}{l}0918 \\
0918 \\
0918 \\
0918 \\
0918 \\
0920 \\
0920 \\
0920 \\
0920 \\
0920 \\
0922 \\
0922 \\
0922 \\
0922 \\
0922 \\
0924 \\
0924 \\
0924 \\
0924 \\
0924 \\
0925 \\
0925 \\
0925 \\
0925 \\
0925 \\
0927 \\
0927 \\
0927 \\
0927 \\
0927 \\
0928 \\
0928 \\
0928 \\
0928 \\
0928 \\
0929 \\
0929 \\
0929 \\
0929 \\
0930\end{array}$ & $\begin{array}{l}09 / 20 / 87 \\
02 / 03 / 88 \\
04 / 22 / 88 \\
08 / 26 / 88 \\
12 / 04 / 88 \\
09 / 20 / 87 \\
01 / 30 / 88 \\
04 / 26 / 88 \\
08 / 27 / 88 \\
12 / 10 / 88 \\
09 / 20 / 87 \\
02 / 03 / 88 \\
04 / 26 / 88 \\
08 / 08 / 88 \\
12 / 10 / 88 \\
09 / 20 / 87 \\
02 / 04 / 88 \\
04 / 26 / 88 \\
08 / 26 / 88 \\
12 / 07 / 88 \\
09 / 20 / 87 \\
02 / 03 / 88 \\
04 / 26 / 88 \\
08 / 26 / 88 \\
12 / 07 / 88 \\
09 / 20 / 87 \\
02 / 04 / 88 \\
04 / 26 / 88 \\
08 / 26 / 88 \\
12 / 04 / 88 \\
09 / 20 / 87 \\
02 / 03 / 88 \\
04 / 22 / 88 \\
08 / 04 / 88 \\
12 / 04 / 88 \\
09 / 20 / 87 \\
02 / 03 / 88 \\
04 / 28 / 88 \\
08 / 11 / 88 \\
09 / 20 / 87\end{array}$ & $\begin{array}{l}0001 \\
0001 \\
0001 \\
0001 \\
0001 \\
0001 \\
0001 \\
0001 \\
0001 \\
0001 \\
0001 \\
0001 \\
0001 \\
0001 \\
0001 \\
0001 \\
0001 \\
0001 \\
0001 \\
0001 \\
0001 \\
0001 \\
0001 \\
0001 \\
0001 \\
0001 \\
0001 \\
0001 \\
0001 \\
0001 \\
0001 \\
0001 \\
0001 \\
0001 \\
0001 \\
0001 \\
0001 \\
0001 \\
0001 \\
0001\end{array}$ & $\begin{array}{l}W U \\
W U \\
W U \\
W U \\
W U \\
W U \\
W U \\
W U \\
W U \\
W U \\
W U \\
W U \\
W U \\
W U \\
W U \\
W U \\
W U \\
W U \\
W U \\
W U \\
W U \\
W U \\
W U \\
W U \\
W U \\
W U \\
W U \\
W U \\
W U \\
W U \\
W U \\
W U \\
W U \\
W U \\
W U \\
W U \\
W U \\
W U \\
W U \\
W U\end{array}$ & $\begin{array}{l}N \\
N \\
N \\
N \\
N \\
N \\
N \\
N \\
N \\
N \\
N \\
N \\
N \\
N \\
N \\
N \\
N \\
N \\
N \\
N \\
N \\
N \\
N \\
N \\
N \\
N \\
N \\
N \\
N \\
N \\
N \\
N \\
N \\
N \\
N \\
N \\
N \\
N \\
N \\
N\end{array}$ & $M G / L$ & & 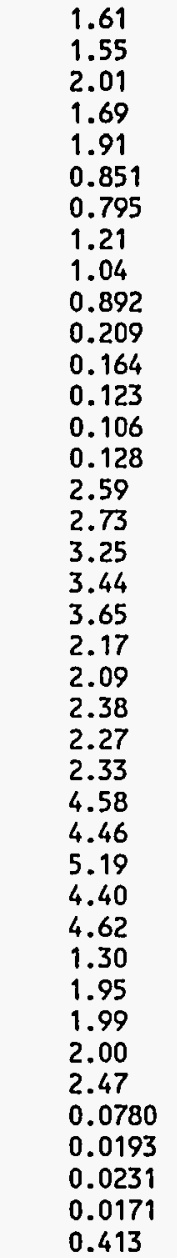 & $J$ & $\begin{array}{l}0.003 \\
0.003 \\
0.003 \\
0.003 \\
0.003 \\
0.003 \\
0.003 \\
0.003 \\
0.003 \\
0.003 \\
0.003 \\
0.003 \\
0.003 \\
0.003 \\
0.003 \\
0.003 \\
0.003 \\
0.003 \\
0.003 \\
0.003 \\
0.003 \\
0.003 \\
0.003 \\
0.003 \\
0.003 \\
0.003 \\
0.003 \\
0.003 \\
0.003 \\
0.003 \\
0.003 \\
0.003 \\
0.003 \\
0.003 \\
0.003 \\
0.003 \\
0.003 \\
0.003 \\
0.003 \\
0.003\end{array}$ & $\begin{array}{l}- \\
- \\
- \\
- \\
- \\
- \\
- \\
- \\
- \\
- \\
- \\
- \\
- \\
- \\
- \\
- \\
- \\
- \\
- \\
- \\
- \\
- \\
- \\
- \\
- \\
- \\
- \\
- \\
- \\
- \\
- \\
- \\
- \\
- \\
- \\
-\end{array}$ \\
\hline
\end{tabular}

FORMATION OF COMPLETION CODE:

WU - WASATCK - UPPER SANDSTONE

PARAMETER VALUE INDICATOR (PVI): < - LESS THAN DETECTION LIMIT
FLOW RELATIONSHIP CODE:

N - UNKNOWN

SAMPLE ID CODES

0001 - FILTERED SAMPLE (.45 MICRONS)

OTHER PARAMETER VALUE FLAGS:

$J$ - ESTIMATED VALUE 
GROUNDWATER QUALITY DATA BY PARAMETER

UPPER SANDSTONE UNIT

SITE: SPKOI SPOOK

$02 / 17 / 87$ TO $12 / 10 / 88$

REPORT DATE: 07/29/94

\begin{tabular}{|c|c|c|c|c|c|c|c|c|c|c|}
\hline PARAMETER NAME & $\begin{array}{c}\text { LOCATION } \\
\text { ID }\end{array}$ & LOG DATE & $\underset{\text { SAMPLE }}{\text { ID }}$ & $\begin{array}{l}\text { FORM } \\
\text { COMP }\end{array}$ & $\begin{array}{l}\text { FLOH } \\
\text { REL. }\end{array}$ & $\begin{array}{l}\text { UNITS OF } \\
\text { MEASURE }\end{array}$ & PVI & $\begin{array}{l}\text { PARAMETER } \\
\text { VALUE }\end{array}$ & $\begin{array}{l}\text { DETECTION } \\
\text { LIMIT }\end{array}$ & $\begin{array}{l}\text { PARAMETER } \\
\text { UNCERTAINTY }\end{array}$ \\
\hline URANIUM & $\begin{array}{l}0930 \\
0930 \\
0930 \\
0931 \\
0931 \\
0931 \\
0931 \\
0932 \\
0932 \\
0932 \\
0932 \\
0933 \\
0933 \\
0934 \\
0934 \\
0935 \\
0935 \\
0936 \\
0936 \\
0937 \\
0937 \\
0939 \\
0939 \\
0940 \\
0940 \\
0941 \\
0941 \\
0942 \\
0942 \\
0943 \\
0943 \\
0944 \\
0944\end{array}$ & $\begin{array}{l}01 / 30 / 88 \\
04 / 26 / 88 \\
08 / 27 / 88 \\
01 / 30 / 88 \\
04 / 28 / 88 \\
08 / 10 / 88 \\
12 / 04 / 88 \\
01 / 30 / 88 \\
04 / 28 / 88 \\
08 / 09 / 88 \\
12 / 07 / 88 \\
08 / 10 / 88 \\
12 / 07 / 88 \\
08 / 11 / 88 \\
12 / 07 / 88 \\
08 / 09 / 88 \\
12 / 04 / 88 \\
08 / 08 / 88 \\
12 / 04 / 88 \\
08 / 08 / 88 \\
12 / 04 / 88 \\
08 / 08 / 88 \\
12 / 04 / 88 \\
08 / 08 / 88 \\
12 / 04 / 88 \\
08 / 08 / 88 \\
12 / 10 / 88 \\
08 / 08 / 88 \\
12 / 04 / 88 \\
08 / 09 / 88 \\
12 / 07 / 88 \\
08 / 11 / 88 \\
12 / 07 / 88\end{array}$ & $\begin{array}{l}0001 \\
0001 \\
0001 \\
0001 \\
0001 \\
0001 \\
0001 \\
0001 \\
0001 \\
0001 \\
0001 \\
0001 \\
0001 \\
0001 \\
0001 \\
0001 \\
0001 \\
0001 \\
0001 \\
0001 \\
0001 \\
0001 \\
0001 \\
0001 \\
0001 \\
0001 \\
0001 \\
0001 \\
0001 \\
0001 \\
0001 \\
0001 \\
0001\end{array}$ & $\begin{array}{l}W U \\
W U \\
W U \\
W U \\
W U \\
W U \\
W U \\
W U \\
W U \\
W U \\
W U \\
W U \\
W U \\
W U \\
W U \\
W U \\
W U \\
W U \\
W U \\
W U \\
W U \\
W U \\
W U \\
W U \\
W U \\
W U \\
W U \\
W U \\
W U \\
W U \\
W U \\
W U \\
W U\end{array}$ & $\begin{array}{l}N \\
N \\
N \\
N \\
N \\
N \\
N \\
N \\
N \\
N \\
N \\
N \\
N \\
N \\
N \\
N \\
N \\
N \\
N \\
N \\
N \\
N \\
N \\
N \\
N \\
N \\
N \\
N \\
N \\
N \\
N \\
N \\
N\end{array}$ & MG/L & & $\begin{array}{l}0.0514 \\
0.0437 \\
0.185 \\
2 \\
2.13 \\
2.04 \\
2.00 \\
2.15 \\
0\end{array}$ & $\begin{array}{l}0.003 \\
0.003 \\
0.003 \\
0.003 \\
0.003 \\
0.003 \\
0.003 \\
0.003 \\
0.003 \\
0.003 \\
0.003 \\
0.003 \\
0.003 \\
0.003 \\
0.003 \\
0.003 \\
0.003 \\
0.003 \\
0.003 \\
0.003 \\
0.003 \\
0.003 \\
0.003 \\
0.003 \\
0.003 \\
0.003 \\
0.003 \\
0.003 \\
0.003 \\
0.003 \\
0.003 \\
0.003 \\
0.003\end{array}$ & 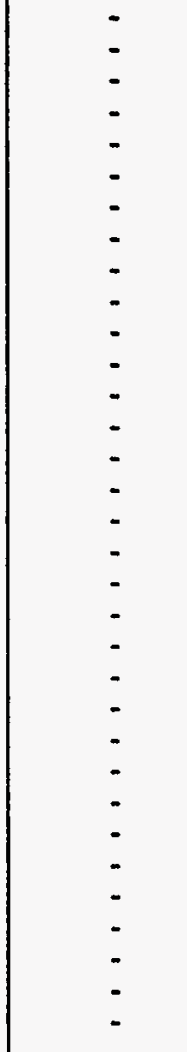 \\
\hline VANADIUM & $\begin{array}{l}0911 \\
0911 \\
0911 \\
0911 \\
0911 \\
0912 \\
0912\end{array}$ & $\begin{array}{l}09 / 18 / 87 \\
01 / 30 / 88 \\
04 / 22 / 88 \\
08 / 11 / 88 \\
12 / 10 / 88 \\
02 / 03 / 88 \\
04 / 22 / 88\end{array}$ & $\begin{array}{l}0001 \\
0001 \\
0001 \\
0001 \\
0001 \\
0001 \\
0001\end{array}$ & $\begin{array}{l}W U \\
W U \\
W U \\
W U \\
W U \\
W U \\
W U\end{array}$ & $\begin{array}{l}N \\
N \\
N \\
N \\
N \\
N \\
N\end{array}$ & $M G / L$ & $<$ & $\begin{array}{l}0.05 \\
0.01 \\
0.02 \\
0.01 \\
0.02 \\
0.01 \\
0.03\end{array}$ & $\begin{array}{l}0.01 \\
0.01 \\
0.01 \\
0.01 \\
0.01 \\
0.01 \\
0.01\end{array}$ & $\begin{array}{l}: \\
: \\
- \\
-\end{array}$ \\
\hline
\end{tabular}

FORMATION OF COMPLETION CODE:

FLOW RELATIONSHIP CODE:

WU - WASATCH - UPPER SANDSTONE

N - UNKNOWN

PARAMETER VALUE INDICATOR (PVI): < - LESS THAN DETECTION LIMIT SAMPLE ID CODES:

0001 - FILTERED SAMPLE (.45 MICRONS) 


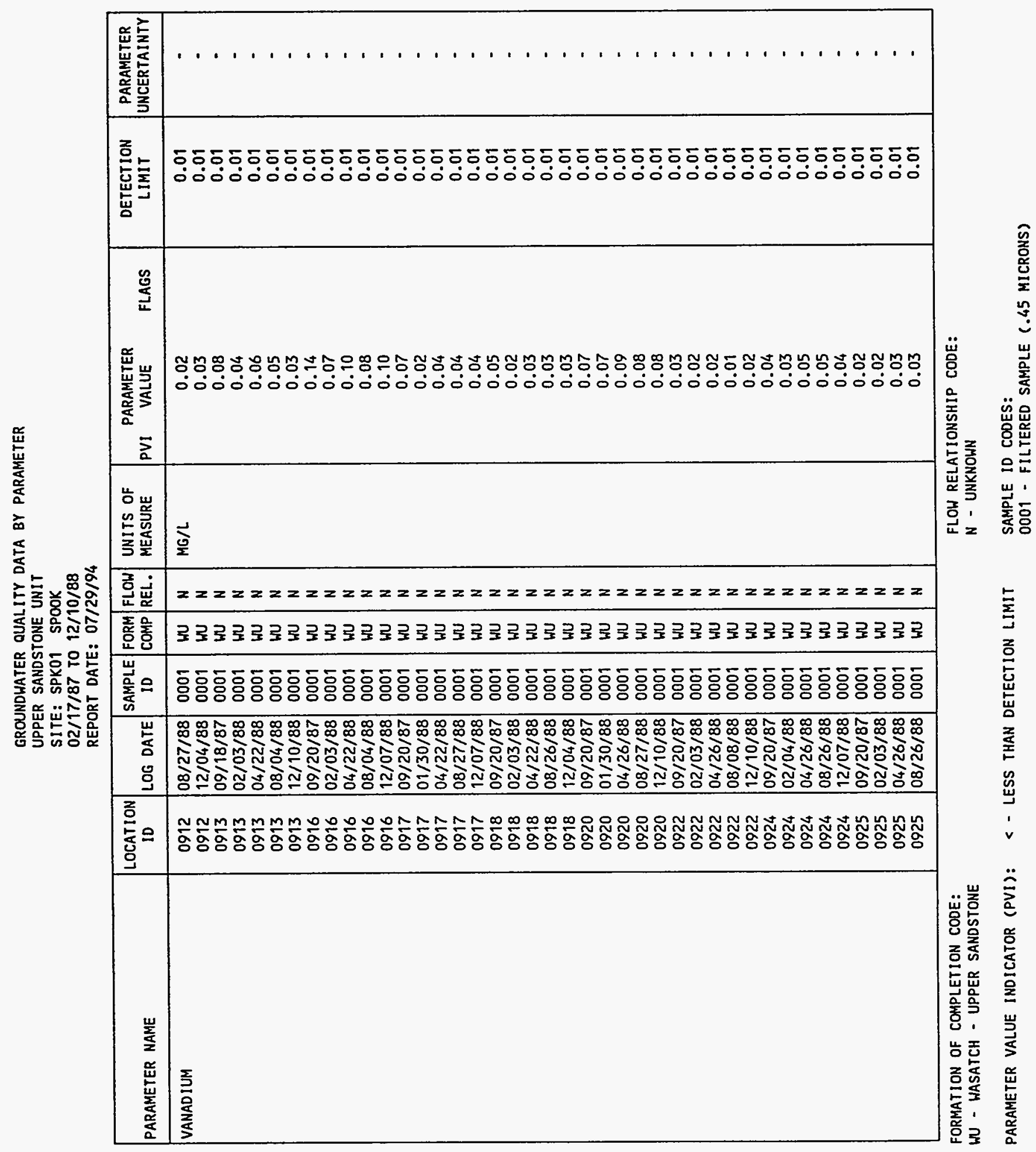




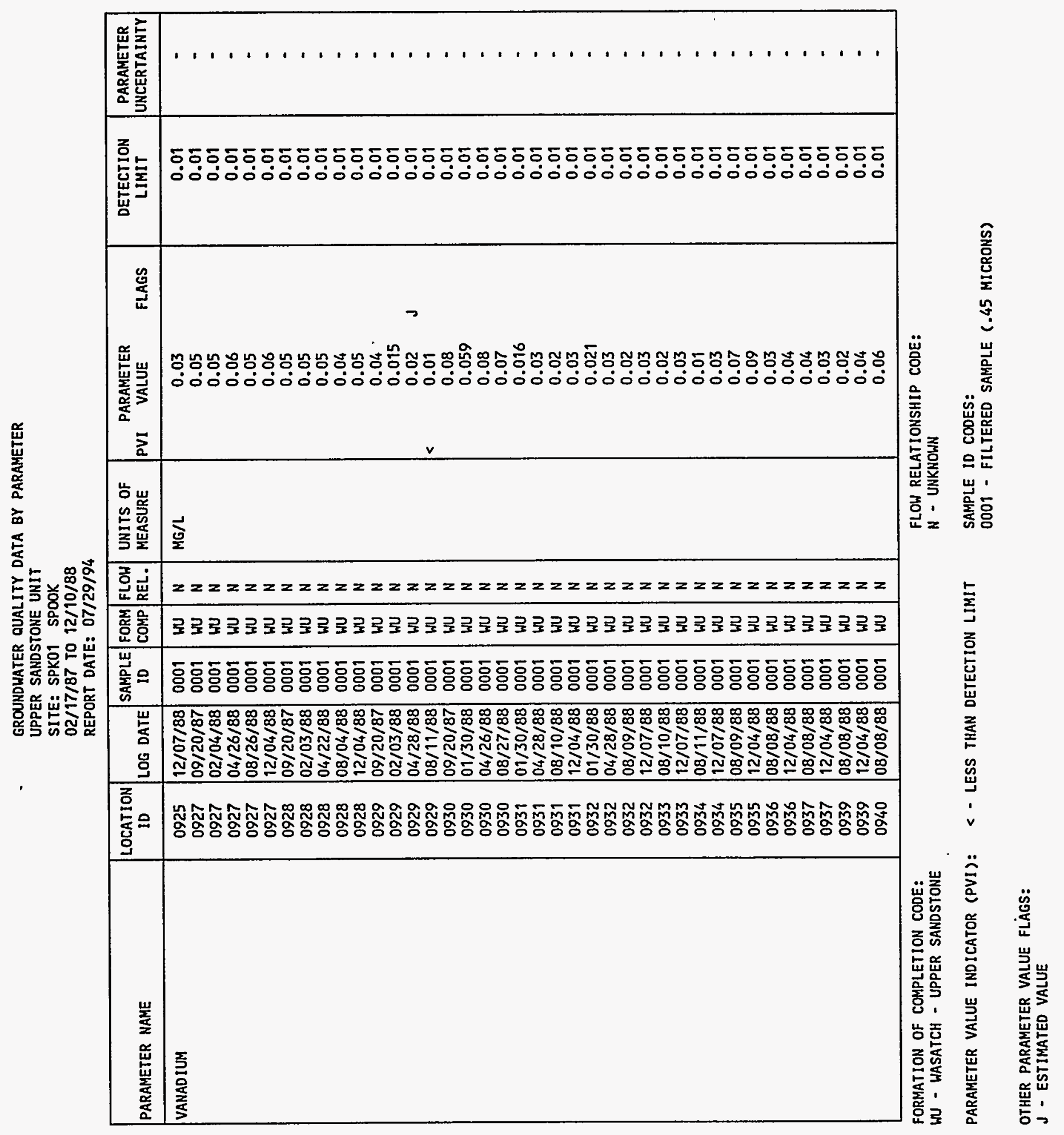




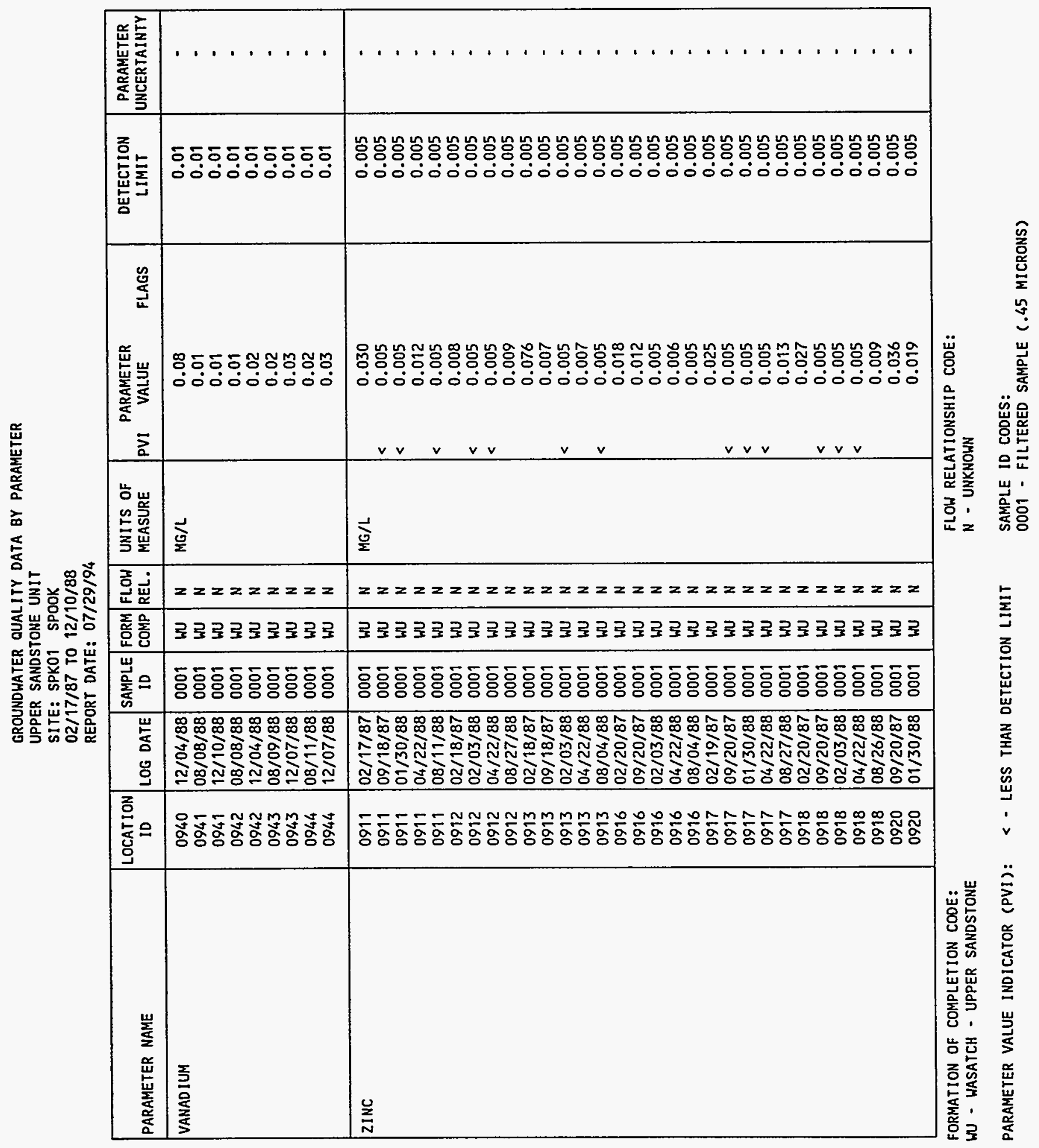




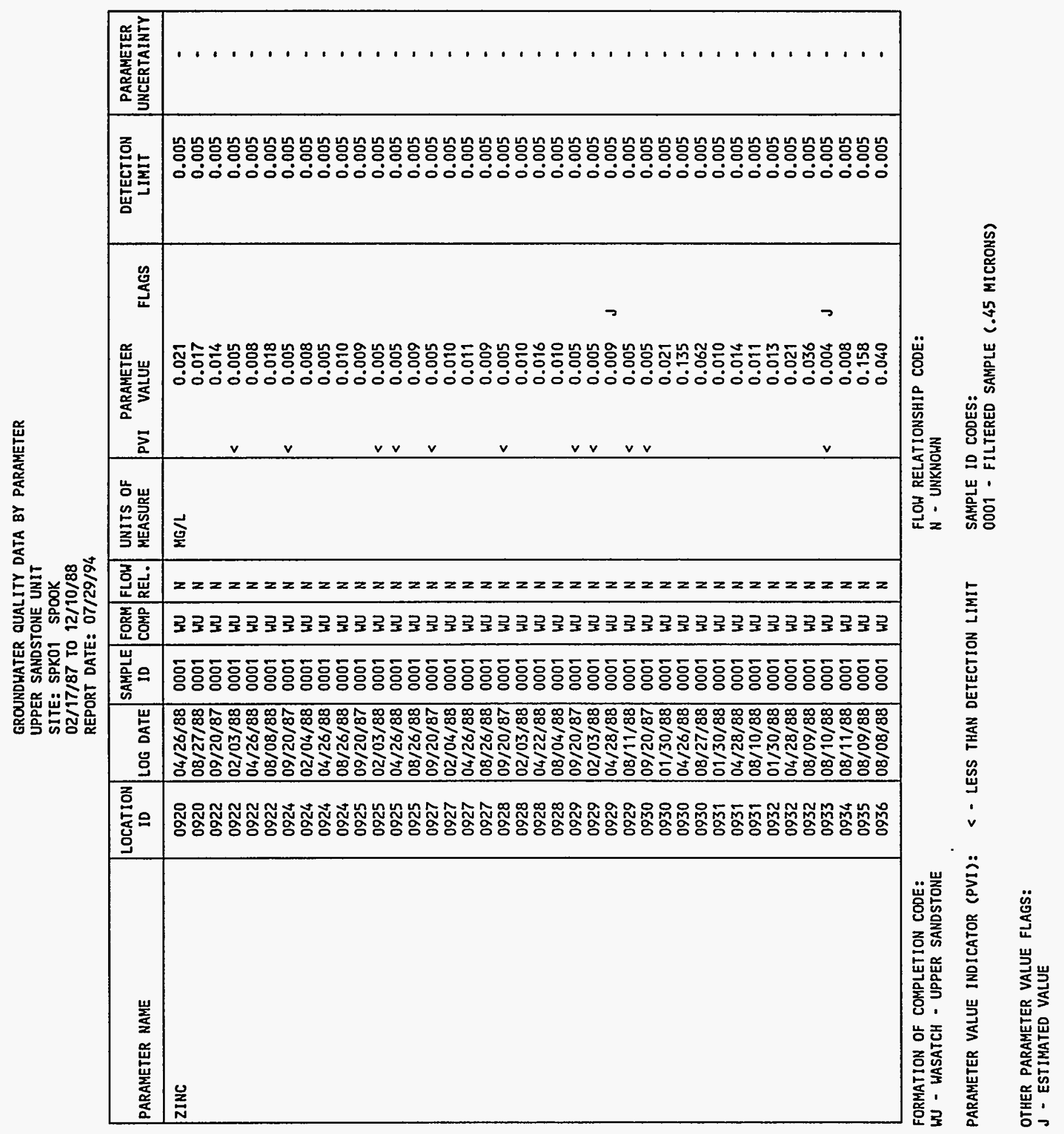


GROUNDWATER QUALITY DATA BY PARAMETER

UPPER SANDSTONE UNIT

SITE: SPKO1 SPOOK

02/17/87 TO $12 / 10 / 88$

REPORT DATE: 07/29/94

\begin{tabular}{|c|c|c|c|c|c|c|c|c|c|c|c|}
\hline PARAMETER NAME & $\underset{\text { LOCATION }}{\text { ID }}$ & LOG DATE & $\begin{array}{c}\text { SAMPLE } \\
\text { ID }\end{array}$ & $\begin{array}{l}\text { FORM } \\
\text { COMP }\end{array}$ & $\begin{array}{l}\text { FLOH } \\
\text { REL. }\end{array}$ & $\begin{array}{l}\text { UNITS OF } \\
\text { MEASURE }\end{array}$ & PVI & $\begin{array}{l}\text { PARAMETER } \\
\text { VALUE }\end{array}$ & FLAGS & $\begin{array}{l}\text { DETECTION } \\
\text { LIMIT }\end{array}$ & $\begin{array}{l}\text { PARAMETER } \\
\text { UNCERTAINTY }\end{array}$ \\
\hline ZINC & $\begin{array}{l}0937 \\
0939 \\
0940 \\
0941 \\
0942 \\
0943 \\
0944\end{array}$ & \begin{tabular}{|c|}
$08 / 08 / 88$ \\
$08 / 08 / 88$ \\
$08 / 08 / 88$ \\
$08 / 08 / 88$ \\
$08 / 08 / 88$ \\
$08 / 09 / 88$ \\
$08 / 11 / 88$
\end{tabular} & $\begin{array}{l}0001 \\
0001 \\
0001 \\
0001 \\
0001 \\
0001 \\
0001\end{array}$ & $\begin{array}{l}\text { WU } \\
W U \\
W U \\
W U \\
W U \\
W U \\
W U\end{array}$ & $\begin{array}{l}N \\
N \\
N \\
N \\
N \\
N \\
N\end{array}$ & $M G / L$ & < & $\begin{array}{l}0.007 \\
0.012 \\
0.006 \\
0.009 \\
0.005 \\
0.005 \\
0.005\end{array}$ & & $\begin{array}{l}0.005 \\
0.005 \\
0.005 \\
0.005 \\
0.005 \\
0.005 \\
0.005\end{array}$ & $\begin{array}{l}- \\
- \\
- \\
- \\
-\end{array}$ \\
\hline
\end{tabular}

FORMATION OF COMPLETION CODE:

WU - WASATCH - UPPER SANDSTONE

FLOW RELATIONSHIP CODE:

PARAMETER VALUE INDICATOR (PVI): < - LESS THAN DETECTION LIMIT

SAMPLE ID CODES:

0001 - FILTERED SAMPLE (.45 MICRONS)

DATA FILE NAME: M: \DART \SPK01\GWQ10001.DAT 
REPORT DATE: $07 / 29 / 9$

\begin{tabular}{|c|c|c|c|c|c|c|c|c|c|c|c|}
\hline PARAMETER HAME & $\begin{array}{l}\text { LOCATION } \\
\text { ID }\end{array}$ & LOG DATE & $\begin{array}{c}\text { SAMPLE } \\
\text { ID }\end{array}$ & $\begin{array}{l}\text { FORM } \\
\text { COMP }\end{array}$ & $\begin{array}{l}\text { FLOH } \\
\text { REL. }\end{array}$ & $\begin{array}{l}\text { UNITS OF } \\
\text { MEASURE }\end{array}$ & PVI & $\begin{array}{l}\text { PARAMETER } \\
\text { VALUE }\end{array}$ & FLAGS & $\begin{array}{l}\text { DETECTION } \\
\text { LIMIT }\end{array}$ & $\begin{array}{l}\text { PARAMETER } \\
\text { UNCERTAINTY }\end{array}$ \\
\hline ALKALINITY & $\begin{array}{l}0910 \\
0910 \\
0910 \\
0910 \\
0910 \\
0910 \\
0914 \\
0914 \\
0914 \\
0914 \\
0914 \\
0914 \\
0915 \\
0915 \\
0915 \\
0915 \\
0915 \\
0915 \\
0919 \\
0919 \\
0919 \\
0919 \\
0919 \\
0919 \\
0921 \\
0921 \\
0921 \\
0921 \\
0921 \\
0923 \\
0923 \\
0923 \\
0923 \\
0923 \\
0938 \\
0938\end{array}$ & $\begin{array}{l}02 / 17 / 87 \\
09 / 18 / 87 \\
01 / 30 / 88 \\
04 / 22 / 88 \\
08 / 11 / 88 \\
12 / 10 / 88 \\
02 / 18 / 87 \\
09 / 18 / 87 \\
02 / 03 / 88 \\
04 / 22 / 88 \\
08 / 08 / 88 \\
12 / 10 / 88 \\
02 / 20 / 87 \\
09 / 20 / 87 \\
02 / 03 / 88 \\
04 / 22 / 88 \\
08 / 10 / 88 \\
12 / 10 / 88 \\
02 / 19 / 87 \\
09 / 20 / 87 \\
01 / 30 / 88 \\
04 / 26 / 88 \\
08 / 27 / 88 \\
12 / 10 / 88 \\
09 / 20 / 87 \\
02 / 04 / 88 \\
04 / 26 / 88 \\
08 / 10 / 88 \\
12 / 10 / 88 \\
09 / 20 / 87 \\
01 / 30 / 88 \\
04 / 26 / 88 \\
08 / 09 / 88 \\
12 / 10 / 88 \\
08 / 09 / 88 \\
12 / 10 / 88\end{array}$ & $\begin{array}{l}0001 \\
0001 \\
0001 \\
0001 \\
0001 \\
0001 \\
0001 \\
0001 \\
0001 \\
0001 \\
0001 \\
0001 \\
0001 \\
0001 \\
0001 \\
0001 \\
0001 \\
0001 \\
0001 \\
0001 \\
0001 \\
0001 \\
0001 \\
0001 \\
0001 \\
0001 \\
0001 \\
0001 \\
0001 \\
0001 \\
0001 \\
0001 \\
0001 \\
0001 \\
0001 \\
0001\end{array}$ & $\begin{array}{l}W L \\
W L \\
H L \\
W L \\
W L \\
H L \\
W L \\
W L \\
H L \\
W L \\
W L \\
H L \\
W M \\
W M \\
W M \\
W M \\
W M \\
W M \\
W L \\
W L \\
H L \\
W L \\
W L \\
W L \\
W L \\
H L \\
H L \\
H L \\
H L \\
W L \\
W L \\
W L \\
H L \\
H L \\
W L \\
W L\end{array}$ & $\begin{array}{l}N \\
N \\
N \\
N \\
N \\
N \\
N \\
N \\
N \\
N \\
N \\
N \\
N \\
N \\
N \\
N \\
N \\
N \\
N \\
N \\
N \\
N \\
N \\
N \\
N \\
N \\
N \\
N \\
N \\
N \\
N \\
N \\
N \\
N \\
N\end{array}$ & $\mathrm{MG} / \mathrm{L} \mathrm{CACO} 3$ & & $\begin{array}{l}114 . \\
123 . \\
126 . \\
131.0 \\
125.0 \\
135 . \\
190 . \\
116 . \\
115 . \\
112 . \\
114.0 \\
111 . \\
127 . \\
134 . \\
117 . \\
121 . \\
99.0 \\
109 . \\
150 . \\
133 . \\
143 . \\
135 . \\
139.0 \\
165 . \\
208 . \\
260 . \\
194 . \\
215.0 \\
214 . \\
142 . \\
144 . \\
140 . \\
130.0 \\
145 . \\
204.0 \\
213 .\end{array}$ & $\mathbf{J}$ & $\begin{array}{l}- \\
- \\
- \\
- \\
- \\
- \\
- \\
- \\
- \\
- \\
- \\
- \\
- \\
- \\
- \\
- \\
- \\
- \\
- \\
- \\
- \\
- \\
- \\
- \\
- \\
-\end{array}$ & $\begin{array}{l}- \\
- \\
- \\
- \\
- \\
- \\
- \\
- \\
- \\
- \\
- \\
- \\
- \\
- \\
- \\
- \\
- \\
- \\
- \\
- \\
- \\
- \\
- \\
- \\
- \\
-\end{array}$ \\
\hline ALUMINUM & $\begin{array}{l}0910 \\
0910\end{array}$ & $\left|\begin{array}{l}02 / 17 / 87 \\
09 / 18 / 87\end{array}\right|$ & $\begin{array}{l}0001 \\
0001\end{array}$ & $\begin{array}{l}\text { HL } \\
\text { WL }\end{array}$ & $\begin{array}{l}N \\
N\end{array}$ & MG/L & $<$ & $\begin{array}{l}0.1 \\
0.13\end{array}$ & & $\begin{array}{l}0.1 \\
0.1\end{array}$ & - \\
\hline
\end{tabular}

FORMATION OF COMPLETION CODE:

WL - WASATCH - LOWER SANDSTONE

WM - WASATCH - MIDDLE SHALE

PARAMETER VALUE INDICATOR (PVI): < - LESS THAN DETECTION LIMIT

OTHER PARAMETER VALUE FLAGS:

$J$ - ESTIMATED VALUE
FLOW RELATIONSHIP CODE:

N - UNKNOWN

SAMPLE ID CODES

0001 - FILTERED SAMPLE (.45 MICRONS) 
GROUNDWATER QUALITY DATA BY PARAMETER

LOWER SANDSTONE AND MIDDLE SHALE UNITS

SITE: SPKO1 SPOOK

02/17/87 TO $12 / 10 / 88$

REPORT DATE: $07 / 29 / 94$

\begin{tabular}{|c|c|c|c|c|c|c|c|c|c|c|c|}
\hline PARAMETER NAME & $\underset{\text { ID }}{\text { LOCATION }}$ & LOG DATE & $\begin{array}{c}\text { SAMPLE } \\
\text { ID }\end{array}$ & $\begin{array}{l}\text { FORM } \\
\text { COMP }\end{array}$ & $\begin{array}{l}\text { FLOW } \\
\text { REL. }\end{array}$ & $\begin{array}{l}\text { UNITS OF } \\
\text { MEASURE }\end{array}$ & PVI & $\begin{array}{l}\text { PARAMETER } \\
\text { VALUE }\end{array}$ & FLAGS & $\begin{array}{l}\text { DETECTION } \\
\text { LIMIT }\end{array}$ & $\begin{array}{l}\text { PARAMETER } \\
\text { UNCERTAINTY }\end{array}$ \\
\hline ALUMINUM & $\begin{array}{l}0910 \\
0910 \\
0910 \\
0914 \\
0914 \\
0914 \\
0914 \\
0914 \\
0915 \\
0915 \\
0915 \\
0915 \\
0915 \\
0919 \\
0919 \\
0919 \\
0919 \\
0919 \\
0921 \\
0921 \\
0921 \\
0921 \\
0923 \\
0923 \\
0923 \\
0923 \\
0938\end{array}$ & $\begin{array}{l}01 / 30 / 88 \\
04 / 22 / 88 \\
08 / 11 / 88 \\
02 / 18 / 87 \\
09 / 18 / 87 \\
02 / 03 / 88 \\
04 / 22 / 88 \\
08 / 08 / 88 \\
02 / 20 / 87 \\
09 / 20 / 87 \\
02 / 03 / 88 \\
04 / 22 / 88 \\
08 / 10 / 88 \\
02 / 19 / 87 \\
09 / 20 / 87 \\
01 / 30 / 88 \\
04 / 26 / 88 \\
08 / 27 / 88 \\
09 / 20 / 87 \\
02 / 04 / 88 \\
04 / 26 / 88 \\
08 / 10 / 88 \\
09 / 20 / 87 \\
01 / 30 / 88 \\
04 / 26 / 88 \\
08 / 09 / 88 \\
08 / 09 / 88\end{array}$ & $\begin{array}{l}0001 \\
0001 \\
0001 \\
0001 \\
0001 \\
0001 \\
0001 \\
0001 \\
0001 \\
0001 \\
0001 \\
0001 \\
0001 \\
0001 \\
0001 \\
0001 \\
0001 \\
0001 \\
0001 \\
0001 \\
0001 \\
0001 \\
0001 \\
0001 \\
0001 \\
0001 \\
0001\end{array}$ & $\begin{array}{l}W L \\
W L \\
W L \\
W L \\
W L \\
W L \\
W L \\
W L \\
W H \\
W M \\
W M \\
W M \\
W M \\
W L \\
W L \\
W L \\
W L \\
W L \\
W L \\
W L \\
W L \\
W L \\
W L \\
W L \\
W L \\
W L \\
W L\end{array}$ & $\begin{array}{l}N \\
N \\
N \\
N \\
N \\
N \\
N \\
N \\
N \\
N \\
N \\
N \\
N \\
N \\
N \\
N \\
N \\
N \\
N \\
N \\
N \\
N \\
N \\
N \\
N \\
N \\
N\end{array}$ & $M G / L$ & $\begin{array}{l}< \\
< \\
< \\
< \\
< \\
< \\
<\end{array}$ & $\begin{array}{l}0.01 \\
0.03 \\
0.1 \\
0.1 \\
0.14 \\
0.02 \\
0.03 \\
0.1 \\
0.1 \\
0.17 \\
0.02 \\
0.05 \\
0.1 \\
0.1 \\
0.05 \\
0.08 \\
0.03 \\
0.1 \\
0.12 \\
0.14 \\
0.06 \\
0.1 \\
0.07 \\
0.09 \\
0.02 \\
0.1 \\
0.1\end{array}$ & $\begin{array}{l}J \\
J \\
J \\
J \\
J \\
J \\
J \\
J \\
J \\
J \\
J \\
J \\
J \\
J \\
J\end{array}$ & $\begin{array}{l}0.1 \\
0.1 \\
0.1 \\
0.1 \\
0.1 \\
0.1 \\
0.1 \\
0.1 \\
0.1 \\
0.1 \\
0.1 \\
0.1 \\
0.1 \\
0.1 \\
0.1 \\
0.1 \\
0.1 \\
0.1 \\
0.1 \\
0.1 \\
0.1 \\
0.1 \\
0.1 \\
0.1 \\
0.1 \\
0.1 \\
0.1\end{array}$ & $\begin{array}{l}- \\
- \\
- \\
- \\
- \\
- \\
- \\
- \\
- \\
- \\
- \\
- \\
- \\
- \\
- \\
- \\
- \\
-\end{array}$ \\
\hline AMMONIUM & $\begin{array}{l}0910 \\
0910 \\
0910 \\
0910 \\
0910 \\
0910 \\
0914 \\
0914 \\
0914 \\
0914 \\
0914\end{array}$ & $\begin{array}{l}02 / 17 / 87 \\
09 / 18 / 87 \\
01 / 30 / 88 \\
04 / 22 / 88 \\
08 / 11 / 88 \\
12 / 10 / 88 \\
02 / 18 / 87 \\
09 / 18 / 87 \\
02 / 03 / 88 \\
04 / 22 / 88 \\
08 / 08 / 88\end{array}$ & $\begin{array}{l}0001 \\
0001 \\
0001 \\
0001 \\
0001 \\
0001 \\
0001 \\
0001 \\
0001 \\
0001 \\
0001\end{array}$ & $\begin{array}{l}W L \\
W L \\
W L \\
W L \\
W L \\
W L \\
H L \\
W L \\
W L \\
W L \\
W L\end{array}$ & $\begin{array}{l}N \\
N \\
N \\
N \\
N \\
N \\
N \\
N \\
N \\
N \\
N\end{array}$ & $\mathrm{MG} / \mathrm{L}$ & $\mid<$ & $\begin{array}{l}0.1 \\
0.4 \\
1.7 \\
1.3 \\
1.3 \\
0.5 \\
0.1 \\
2.3 \\
0.1 \\
0.1 \\
0.1\end{array}$ & & $\begin{array}{l}0.1 \\
0.1 \\
0.1 \\
0.1 \\
0.1 \\
0.1 \\
0.1 \\
0.1 \\
0.1 \\
0.1 \\
0.1\end{array}$ & $\begin{array}{l}- \\
- \\
- \\
- \\
- \\
- \\
- \\
-\end{array}$ \\
\hline
\end{tabular}

FORMATION OF COMPLETION CODE:

WL - HASATCH - LOWER SAMDSTOHE

FLOW RELATIONSHIP CODE:

WM - WASATCH - MIDDLE SHALE

N - UNKNOWN

PARAMETER VALUE INDICATOR (PVI): < - LESS THAN DETECTION LIMIT

SAMPLE ID CODES:

0001 - FILTERED SAMPLE (.45 MICRONS)

OTHER PARAMETER VALUE FLAGS:

$J$ - ESTIMATED VALUE 
REPORT DATE: $07 / 29 / 94$

\begin{tabular}{|c|c|c|c|c|c|c|c|c|c|c|c|}
\hline PARAMETER NAME & $\begin{array}{c}\text { LOCATION } \\
\text { ID }\end{array}$ & LOG DATE & $\begin{array}{c}\text { SAMPLE } \\
\text { ID }\end{array}$ & $\begin{array}{l}\text { FORM } \\
\text { COMP }\end{array}$ & $\begin{array}{l}\text { FLOW } \\
\text { REL. }\end{array}$ & $\begin{array}{l}\text { UNITS OF } \\
\text { MEASURE }\end{array}$ & PVI & $\begin{array}{l}\text { PARAMETER } \\
\text { VALUE }\end{array}$ & FLAGS & $\begin{array}{l}\text { DETECTION } \\
\text { LIMIT }\end{array}$ & $\begin{array}{l}\text { PARAMETER } \\
\text { UNCERTAINTY }\end{array}$ \\
\hline AMMONIUM & $\begin{array}{l}0914 \\
0915 \\
0915 \\
0915 \\
0915 \\
0915 \\
0915 \\
0919 \\
0919 \\
0919 \\
0919 \\
0919 \\
0919 \\
0921 \\
0921 \\
0921 \\
0921 \\
0921 \\
0923 \\
0923 \\
0923 \\
0923 \\
0923 \\
0938 \\
0938\end{array}$ & $\begin{array}{l}12 / 10 / 88 \\
02 / 20 / 87 \\
09 / 20 / 87 \\
02 / 03 / 88 \\
04 / 22 / 88 \\
08 / 10 / 88 \\
12 / 10 / 88 \\
02 / 19 / 87 \\
09 / 20 / 87 \\
01 / 30 / 88 \\
04 / 26 / 88 \\
08 / 27 / 88 \\
12 / 10 / 88 \\
09 / 20 / 87 \\
02 / 04 / 88 \\
04 / 26 / 88 \\
08 / 10 / 88 \\
12 / 10 / 88 \\
09 / 20 / 87 \\
01 / 30 / 88 \\
04 / 26 / 88 \\
08 / 09 / 88 \\
12 / 10 / 88 \\
08 / 09 / 88 \\
12 / 10 / 88\end{array}$ & $\begin{array}{l}0001 \\
0001 \\
0001 \\
0001 \\
0001 \\
0001 \\
0001 \\
0001 \\
0001 \\
0001 \\
0001 \\
0001 \\
0001 \\
0001 \\
0001 \\
0001 \\
0001 \\
0001 \\
0001 \\
0001 \\
0001 \\
0001 \\
0001 \\
0001 \\
0001\end{array}$ & $\begin{array}{l}H L \\
W M \\
W M \\
W M \\
W M \\
W M \\
W M \\
W L \\
H L \\
H L \\
W L \\
W L \\
W L \\
W L \\
W L \\
W L \\
W L \\
W L \\
H L \\
W L \\
W L \\
W L \\
H L \\
W L \\
W L\end{array}$ & $\begin{array}{l}N \\
N \\
N \\
N \\
N \\
N \\
N \\
N \\
N \\
N \\
N \\
N \\
N \\
N \\
N \\
N \\
N \\
N \\
N \\
N \\
N \\
N \\
N \\
N \\
N\end{array}$ & $M G / L$ & $\begin{array}{l}< \\
< \\
< \\
< \\
< \\
< \\
< \\
< \\
< \\
< \\
< \\
< \\
< \\
< \\
< \\
< \\
< \\
< \\
< \\
< \\
< \\
< \\
< \\
< \\
< \\
< \\
<\end{array}$ & $\begin{array}{l}0.1 \\
0.1 \\
0.1 \\
0.1 \\
0.1 \\
0.1 \\
0.1 \\
0.5 \\
0.1 \\
0.1 \\
0.1 \\
0.1 \\
0.1 \\
0.1 \\
0.1 \\
0.1 \\
0.1 \\
0.1 \\
0.1 \\
0.1 \\
0.1 \\
0.1 \\
0.1 \\
0.1 \\
0.1\end{array}$ & J & $\begin{array}{l}0.1 \\
0.1 \\
0.1 \\
0.1 \\
0.1 \\
0.1 \\
0.1 \\
0.1 \\
0.1 \\
0.1 \\
0.1 \\
0.1 \\
0.1 \\
0.1 \\
0.1 \\
0.1 \\
0.1 \\
0.1 \\
0.1 \\
0.1 \\
0.1 \\
0.1 \\
0.1 \\
0.1 \\
0.1\end{array}$ & $\begin{array}{l}- \\
- \\
- \\
- \\
- \\
- \\
- \\
- \\
- \\
- \\
- \\
- \\
- \\
- \\
- \\
- \\
- \\
-\end{array}$ \\
\hline ANTIMONY & $\begin{array}{l}0910 \\
0910 \\
0910 \\
0910 \\
0910 \\
0914 \\
0914 \\
0914 \\
0914 \\
0914 \\
0915 \\
0915 \\
0915\end{array}$ & $\begin{array}{l}02 / 17 / 87 \\
09 / 18 / 87 \\
01 / 30 / 88 \\
04 / 22 / 88 \\
08 / 11 / 88 \\
02 / 18 / 87 \\
09 / 18 / 87 \\
02 / 03 / 88 \\
04 / 22 / 88 \\
08 / 08 / 88 \\
02 / 20 / 87 \\
09 / 20 / 87 \\
02 / 03 / 88\end{array}$ & $\begin{array}{l}0001 \\
0001 \\
0001 \\
0001 \\
0001 \\
0001 \\
0001 \\
0001 \\
0001 \\
0001 \\
0001 \\
0001 \\
0001\end{array}$ & $\begin{array}{l}\text { WL } \\
W L \\
W L \\
W L \\
W L \\
W L \\
W L \\
W L \\
W L \\
W L \\
\text { WM } \\
\text { WM } \\
\text { WM }\end{array}$ & $\begin{array}{l}N \\
N \\
N \\
N \\
N \\
N \\
N \\
N \\
N \\
N \\
N \\
N \\
N\end{array}$ & MG/L & $\mid \begin{array}{l}< \\
< \\
< \\
< \\
< \\
< \\
< \\
< \\
< \\
<\end{array}$ & $\begin{array}{l}0.003 \\
0.004 \\
0.003 \\
0.004 \\
0.005 \\
0.003 \\
0.003 \\
0.003 \\
0.003 \\
0.003 \\
0.003 \\
0.003 \\
0.004\end{array}$ & $J$ & $\begin{array}{l}0.003 \\
0.003 \\
0.003 \\
0.003 \\
0.003 \\
0.003 \\
0.003 \\
0.003 \\
0.003 \\
0.003 \\
0.003 \\
0.003 \\
0.003\end{array}$ & $\begin{array}{l}- \\
: \\
: \\
: \\
: \\
- \\
- \\
-\end{array}$ \\
\hline
\end{tabular}

FORMATION OF COMPLETION CODE:

HL - HASATCH - LOWER SANDSTONE

HM - HASATCH - MIDDLE SHALE

PARAMETER VALUE INDICATOR (PVI): < - LESS THAN DETECTION LIMIT
FLOW RELATIONSHIP CODE:

N - UNKNOWN

SAMPLE ID CODES:

0001 - FILTERED SAMPLE (.45 MICRONS)

OTHER PARAMETER VALUE FLAGS:

$J$ - ESTIMATED VALUE 
GROUNDWATER QUALITY DATA BY PARAMETER

LOHER SANDSTONE AND MIDDLE SHALE UNITS

SITE: SPKO1 SPOOK

REPORT DATE: $07 / 29 / 94$

\begin{tabular}{|c|c|c|c|c|c|c|c|c|c|c|c|}
\hline PARAMETER NAME & $\underset{\text { ID }}{\text { LOCATION }}$ & LOG DATE & SAMPLE & $\begin{array}{l}\text { FORM } \\
\text { COMP }\end{array}$ & $\begin{array}{l}\text { FLOH } \\
\text { REL. }\end{array}$ & $\begin{array}{l}\text { UNITS OF } \\
\text { MEASURE }\end{array}$ & PVI & $\begin{array}{l}\text { PARAMETER } \\
\text { VALUE }\end{array}$ & FLAGS & $\begin{array}{c}\text { DETECTION } \\
\text { LIMIT }\end{array}$ & $\begin{array}{l}\text { PARAMETER } \\
\text { UNCERTAINTY }\end{array}$ \\
\hline ANTIMONY & $\begin{array}{l}0915 \\
0915 \\
0919 \\
0919 \\
0919 \\
0919 \\
0919 \\
0921 \\
0921 \\
0921 \\
0921 \\
0923 \\
0923 \\
0923 \\
0923 \\
0938\end{array}$ & $\begin{array}{l}04 / 22 / 88 \\
08 / 10 / 88 \\
02 / 19 / 87 \\
09 / 20 / 87 \\
01 / 30 / 88 \\
04 / 26 / 88 \\
08 / 27 / 88 \\
09 / 20 / 87 \\
02 / 04 / 88 \\
04 / 26 / 88 \\
08 / 10 / 88 \\
09 / 20 / 87 \\
01 / 30 / 88 \\
04 / 26 / 88 \\
08 / 09 / 88 \\
08 / 09 / 88\end{array}$ & $\begin{array}{l}0001 \\
0001 \\
0001 \\
0001 \\
0001 \\
0001 \\
0001 \\
0001 \\
0001 \\
0001 \\
0001 \\
0001 \\
0001 \\
0001 \\
0001 \\
0001\end{array}$ & $\begin{array}{l}W M \\
W M \\
W L \\
W L \\
W L \\
W L \\
W L \\
W L \\
W L \\
W L \\
W L \\
W L \\
W L \\
W L \\
W L \\
W L\end{array}$ & $\begin{array}{l}N \\
N \\
N \\
N \\
N \\
N \\
N \\
N \\
N \\
N \\
N \\
N \\
N \\
N \\
N \\
N\end{array}$ & MG/L & $\mid \begin{array}{l}< \\
< \\
< \\
< \\
< \\
< \\
< \\
< \\
< \\
< \\
< \\
< \\
< \\
< \\
<\end{array}$ & $\begin{array}{l}0.003 \\
0.003 \\
0.003 \\
0.003 \\
0.004 \\
0.003 \\
0.003 \\
0.003 \\
0.004 \\
0.003 \\
0.003 \\
0.003 \\
0.003 \\
0.003 \\
0.003 \\
0.003\end{array}$ & $\mathbf{J}$ & $\begin{array}{l}0.003 \\
0.003 \\
0.003 \\
0.003 \\
0.003 \\
0.003 \\
0.003 \\
0.003 \\
0.003 \\
0.003 \\
0.003 \\
0.003 \\
0.003 \\
0.003 \\
0.003 \\
0.003\end{array}$ & $\begin{array}{l}- \\
- \\
- \\
- \\
- \\
- \\
- \\
- \\
- \\
- \\
-\end{array}$ \\
\hline ARSENIC & $\begin{array}{l}0910 \\
0910 \\
0910 \\
0910 \\
0910 \\
0910 \\
0914 \\
0914 \\
0914 \\
0914 \\
0914 \\
0914 \\
0915 \\
0915 \\
0915 \\
0915 \\
0915 \\
0915 \\
0919 \\
0919 \\
0919 \\
0919\end{array}$ & $\begin{array}{l}02 / 17 / 87 \\
09 / 18 / 87 \\
01 / 30 / 88 \\
04 / 22 / 88 \\
08 / 11 / 88 \\
12 / 10 / 88 \\
02 / 18 / 87 \\
09 / 18 / 87 \\
02 / 03 / 88 \\
04 / 22 / 88 \\
08 / 08 / 88 \\
12 / 10 / 88 \\
02 / 20 / 87 \\
09 / 20 / 87 \\
02 / 03 / 88 \\
04 / 22 / 88 \\
08 / 10 / 88 \\
12 / 10 / 88 \\
02 / 19 / 87 \\
09 / 20 / 87 \\
01 / 30 / 88 \\
04 / 26 / 88\end{array}$ & $\begin{array}{l}0001 \\
0001 \\
0001 \\
0001 \\
0001 \\
0001 \\
0001 \\
0001 \\
0001 \\
0001 \\
0001 \\
0001 \\
0001 \\
0001 \\
0001 \\
0001 \\
0001 \\
0001 \\
0001 \\
0001 \\
0001 \\
0001\end{array}$ & $\begin{array}{l}W L \\
W L \\
W L \\
W L \\
W L \\
W L \\
W L \\
W L \\
W L \\
W L \\
W L \\
W L \\
W M \\
W M \\
W M \\
W M \\
W M \\
W M \\
W L \\
W L \\
H L \\
H L\end{array}$ & $\begin{array}{l}N \\
N \\
N \\
N \\
N \\
N \\
N \\
N \\
N \\
N \\
N \\
N \\
N \\
N \\
N \\
N \\
N \\
N \\
N \\
N \\
N \\
N\end{array}$ & $M G / L$ & $\begin{array}{l}< \\
< \\
< \\
< \\
< \\
< \\
< \\
< \\
< \\
< \\
< \\
< \\
< \\
< \\
< \\
< \\
< \\
< \\
< \\
< \\
<\end{array}$ & $\begin{array}{l}0.001 \\
0.002 \\
0.001 \\
0.001 \\
0.01 \\
0.01 \\
0.001 \\
0.001 \\
0.001 \\
0.001 \\
0.01 \\
0.01 \\
0.001 \\
0.001 \\
0.001 \\
0.001 \\
0.01 \\
0.01 \\
0.001 \\
0.001 \\
0.01 \\
0.001\end{array}$ & $\begin{array}{l}J \\
J \\
J \\
J \\
J \\
J \\
J \\
J \\
J \\
J \\
J \\
J \\
J \\
J \\
J\end{array}$ & $\begin{array}{l}0.01 \\
0.01 \\
0.01 \\
0.01 \\
0.01 \\
0.01 \\
0.01 \\
0.01 \\
0.01 \\
0.01 \\
0.01 \\
0.01 \\
0.01 \\
0.01 \\
0.01 \\
0.01 \\
0.01 \\
0.01 \\
0.01 \\
0.01 \\
0.01 \\
0.01\end{array}$ & $\begin{array}{l}- \\
- \\
- \\
- \\
- \\
- \\
- \\
- \\
- \\
- \\
- \\
- \\
- \\
- \\
- \\
-\end{array}$ \\
\hline
\end{tabular}

FORMATION OF COMPLETION CODE:

WM - WASATCH - MIDDLE SHALE

WL - HASATCH - LOWER SANDSTONE

PARAMETER VALUE INDICATOR (PVI): < - LESS THAN DETECTION LIMIT
FLOW RELATIONSHIP CODE

N - UNKNOWN

SAMPLE ID COOES

0001 - FILTERED SAMPLE (.45 MICRONS)

OTHER PARAMETER VALUE FLAGS:

$J$ - ESTIMATED VALUE 
SITE: SPKO1 SPOOK

02/17/87 TO $12 / 10 / 88$

REPORT DATE : $07 / 29 / 94$

\begin{tabular}{|c|c|c|c|c|c|c|c|c|c|c|c|}
\hline PARAMETER NAME & $\underset{\text { LOCATION }}{\text { LD }}$ & LOG DATE & $\begin{array}{c}\text { SAMPLE } \\
10\end{array}$ & $\begin{array}{l}\text { FORM } \\
\text { COMP }\end{array}$ & $\begin{array}{l}\text { FLOH } \\
\text { REL. }\end{array}$ & $\begin{array}{l}\text { UNITS OF } \\
\text { MEASURE }\end{array}$ & PVI & $\begin{array}{l}\text { PARAMETER } \\
\text { VALUE }\end{array}$ & FLAGS & $\begin{array}{l}\text { DETECTION } \\
\text { LIMIT }\end{array}$ & $\begin{array}{l}\text { PARAMETER } \\
\text { UNCERTAINTY }\end{array}$ \\
\hline ARSENIC & $\begin{array}{l}0919 \\
0919 \\
0921 \\
0921 \\
0921 \\
0921 \\
0921 \\
0923 \\
0923 \\
0923 \\
0923 \\
0923 \\
0938 \\
0938\end{array}$ & $\begin{array}{l}08 / 27 / 88 \\
12 / 10 / 88 \\
09 / 20 / 87 \\
02 / 04 / 88 \\
04 / 26 / 88 \\
08 / 10 / 88 \\
12 / 10 / 88 \\
09 / 20 / 87 \\
01 / 30 / 88 \\
04 / 26 / 88 \\
08 / 09 / 88 \\
12 / 10 / 88 \\
08 / 09 / 88 \\
12 / 10 / 88\end{array}$ & $\begin{array}{l}0001 \\
0001 \\
0001 \\
0001 \\
0001 \\
0001 \\
0001 \\
0001 \\
0001 \\
0001 \\
0001 \\
0001 \\
0001 \\
0001\end{array}$ & $\begin{array}{l}\text { WL } \\
W L \\
W L \\
W L \\
W L \\
W L \\
W L \\
W L \\
W L \\
W L \\
W L \\
W L \\
W L \\
W L\end{array}$ & $\begin{array}{l}N \\
N \\
N \\
N \\
N \\
N \\
N \\
N \\
N \\
N \\
N \\
N \\
N \\
N\end{array}$ & $M G / L$ & $\begin{array}{l}< \\
< \\
< \\
< \\
< \\
< \\
< \\
< \\
< \\
< \\
< \\
< \\
<\end{array}$ & $\begin{array}{l}0.01 \\
0.01 \\
0.004 \\
0.01 \\
0.001 \\
0.01 \\
0.01 \\
0.001 \\
0.01 \\
0.001 \\
0.01 \\
0.01 \\
0.01 \\
0.01\end{array}$ & $\begin{array}{l}\text { J } \\
\text { J } \\
\text { J } \\
\text { J } \\
\text { J }\end{array}$ & $\begin{array}{l}0.01 \\
0.01 \\
0.01 \\
0.01 \\
0.01 \\
0.01 \\
0.01 \\
0.01 \\
0.01 \\
0.01 \\
0.01 \\
0.01 \\
0.01 \\
0.01\end{array}$ & $\begin{array}{l}- \\
- \\
- \\
- \\
- \\
- \\
- \\
- \\
- \\
-\end{array}$ \\
\hline BARIUM & $\begin{array}{l}0910 \\
0910 \\
0910 \\
0910 \\
0910 \\
0914 \\
0914 \\
0914 \\
0914 \\
0914 \\
0915 \\
0915 \\
0915 \\
0915 \\
0915 \\
0919 \\
0919 \\
0919 \\
0919 \\
0919 \\
0921 \\
0921 \\
0921 \\
0921\end{array}$ & $\begin{array}{l}02 / 17 / 87 \\
09 / 18 / 87 \\
01 / 30 / 88 \\
04 / 22 / 88 \\
08 / 11 / 88 \\
02 / 18 / 87 \\
09 / 18 / 87 \\
02 / 03 / 88 \\
04 / 22 / 88 \\
08 / 08 / 88 \\
02 / 20 / 87 \\
09 / 20 / 87 \\
02 / 03 / 88 \\
04 / 22 / 88 \\
08 / 10 / 88 \\
02 / 19 / 87 \\
09 / 20 / 87 \\
01 / 30 / 88 \\
04 / 26 / 88 \\
08 / 27 / 88 \\
09 / 20 / 87 \\
02 / 04 / 88 \\
04 / 26 / 88 \\
08 / 10 / 88\end{array}$ & $\begin{array}{l}0001 \\
0001 \\
0001 \\
0001 \\
0001 \\
0001 \\
0001 \\
0001 \\
0001 \\
0001 \\
0001 \\
0001 \\
0001 \\
0001 \\
0001 \\
0001 \\
0001 \\
0001 \\
0001 \\
0001 \\
0001 \\
0001 \\
0001 \\
0001\end{array}$ & $\begin{array}{l}W L \\
W L \\
W L \\
W L \\
W L \\
W L \\
W L \\
W L \\
W L \\
W L \\
W M \\
W M \\
W M \\
W M \\
W M \\
W L \\
W L \\
W L \\
W L \\
W L \\
W L \\
W L \\
W L \\
W L\end{array}$ & $\begin{array}{l}N \\
N \\
N \\
N \\
N \\
N \\
N \\
N \\
N \\
N \\
N \\
N \\
N \\
N \\
N \\
N \\
N \\
N \\
N \\
N \\
N \\
N \\
N \\
N\end{array}$ & $M G / L$ & $\begin{array}{l}< \\
< \\
< \\
< \\
< \\
< \\
< \\
< \\
< \\
< \\
< \\
< \\
< \\
< \\
<\end{array}$ & $\begin{array}{l}0.1 \\
0.02 \\
0.1 \\
0.02 \\
0.1 \\
0.1 \\
0.03 \\
0.1 \\
0.02 \\
0.1 \\
0.1 \\
0.03 \\
0.1 \\
0.02 \\
0.1 \\
0.1 \\
0.01 \\
0.01 \\
0.02 \\
0.1 \\
0.01 \\
0.01 \\
0.02 \\
0.1\end{array}$ & $\begin{array}{l}\text { J } \\
\text { J } \\
\text { J } \\
J \\
J \\
J \\
J \\
J \\
J \\
J \\
J \\
J\end{array}$ & $\begin{array}{l}0.1 \\
0.1 \\
0.1 \\
0.1 \\
0.1 \\
0.1 \\
0.1 \\
0.1 \\
0.1 \\
0.1 \\
0.1 \\
0.1 \\
0.1 \\
0.1 \\
0.1 \\
0.1 \\
0.1 \\
0.1 \\
0.1 \\
0.1 \\
0.1 \\
0.1 \\
0.1 \\
0.1\end{array}$ & $\begin{array}{l}- \\
- \\
- \\
- \\
- \\
: \\
- \\
- \\
- \\
- \\
- \\
- \\
- \\
- \\
- \\
- \\
- \\
- \\
-\end{array}$ \\
\hline
\end{tabular}

FORMATION OF COMPLETION CODE:

WL - WASATCH - LOWER SANDSTONE

WM - WASATCH - MIDDLE SHALE

PARAMETER VALUE INDICATOR (PVI): < - LESS THAN DETECTION LIMIT
FLOW RELATIONSHIP COOE:

N - UNKNOHN 


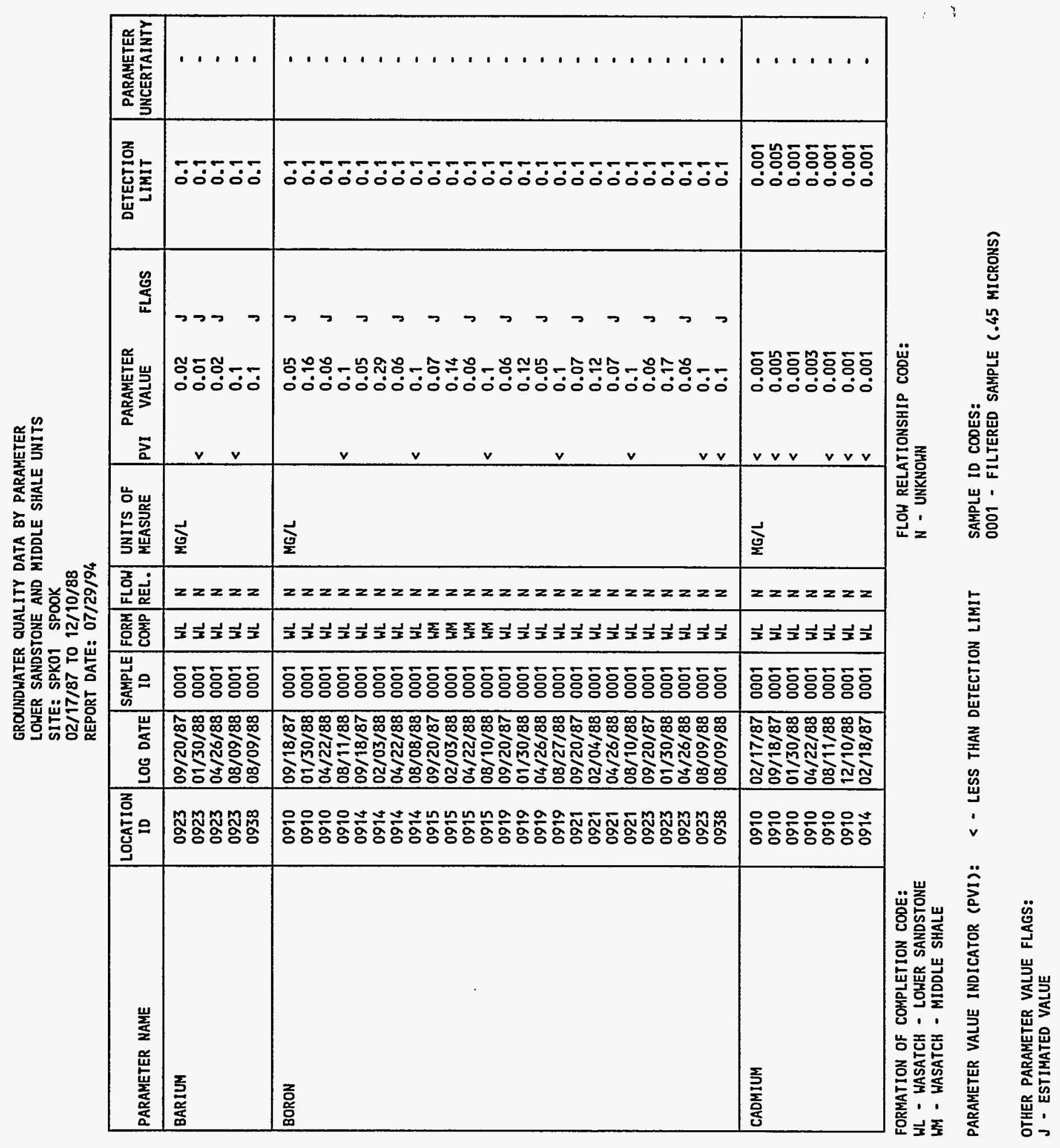


SITE: SPKO1 SPOOK

$02 / 17 / 87$ TO $12 / 10 / 88$

REPORT DATE: $07 / 29 / 94$

\begin{tabular}{|c|c|c|c|c|c|c|c|c|c|c|c|}
\hline PARAMETER NAME & $\begin{array}{c}\text { LOCATION } \\
\text { ID }\end{array}$ & LOG DATE & $\begin{array}{c}\text { SAMPLE } \\
\text { ID }\end{array}$ & $\begin{array}{l}\text { FORM } \\
\text { COMP }\end{array}$ & $\begin{array}{l}\text { FLOW } \\
\text { REL. }\end{array}$ & $\begin{array}{l}\text { UNITS OF } \\
\text { MEASURE }\end{array}$ & PVI & $\begin{array}{l}\text { PARAMETER } \\
\text { VALUE }\end{array}$ & FLAGS & $\begin{array}{l}\text { DETECTION } \\
\text { LIMIT }\end{array}$ & $\begin{array}{l}\text { PARAMETER } \\
\text { UNCERTAINTY }\end{array}$ \\
\hline CADMIUM & $\begin{array}{l}0914 \\
0914 \\
0914 \\
0914 \\
0914 \\
0915 \\
0915 \\
0915 \\
0915 \\
0915 \\
0915 \\
0919 \\
0919 \\
0919 \\
0919 \\
0919 \\
0919 \\
0921 \\
0921 \\
0921 \\
0921 \\
0921 \\
0923 \\
0923 \\
0923 \\
0923 \\
0923 \\
0938 \\
0938\end{array}$ & $\begin{array}{l}09 / 18 / 87 \\
02 / 03 / 88 \\
04 / 22 / 88 \\
08 / 08 / 88 \\
12 / 10 / 88 \\
02 / 20 / 87 \\
09 / 20 / 87 \\
02 / 03 / 88 \\
04 / 22 / 88 \\
08 / 10 / 88 \\
12 / 10 / 88 \\
02 / 19 / 87 \\
09 / 20 / 87 \\
01 / 30 / 88 \\
04 / 26 / 88 \\
08 / 27 / 88 \\
12 / 10 / 88 \\
09 / 20 / 87 \\
02 / 04 / 88 \\
04 / 26 / 88 \\
08 / 10 / 88 \\
12 / 10 / 88 \\
09 / 20 / 87 \\
01 / 30 / 88 \\
04 / 26 / 88 \\
08 / 09 / 88 \\
12 / 10 / 88 \\
08 / 09 / 88 \\
12 / 10 / 88\end{array}$ & $\begin{array}{l}0001 \\
0001 \\
0001 \\
0001 \\
0001 \\
0001 \\
0001 \\
0001 \\
0001 \\
0001 \\
0001 \\
0001 \\
0001 \\
0001 \\
0001 \\
0001 \\
0001 \\
0001 \\
0001 \\
0001 \\
0001 \\
0001 \\
0001 \\
0001 \\
0001 \\
0001 \\
0001 \\
0001 \\
0001\end{array}$ & $\begin{array}{l}W L \\
W L \\
W L \\
W L \\
W L \\
W M \\
W M \\
W M \\
W M \\
W M \\
W M \\
W L \\
W L \\
W L \\
W L \\
W L \\
W L \\
W L \\
W L \\
W L \\
W L \\
W L \\
W L \\
W L \\
W L \\
W L \\
W L \\
W L \\
W L\end{array}$ & $\begin{array}{l}N \\
N \\
N \\
N \\
N \\
N \\
N \\
N \\
N \\
N \\
N \\
N \\
N \\
N \\
N \\
N \\
N \\
N \\
N \\
N \\
N \\
N \\
N \\
N \\
N \\
N \\
N \\
N \\
N\end{array}$ & $M G / L$ & $\begin{array}{l}< \\
< \\
< \\
< \\
< \\
< \\
< \\
< \\
< \\
< \\
< \\
< \\
< \\
< \\
< \\
< \\
< \\
< \\
< \\
< \\
< \\
< \\
< \\
< \\
< \\
<\end{array}$ & $\begin{array}{l}0.005 \\
0.001 \\
0.002 \\
0.002 \\
0.001 \\
0.001 \\
0.005 \\
0.001 \\
0.001 \\
0.005 \\
0.001 \\
0.002 \\
0.005 \\
0.001 \\
0.001 \\
0.001 \\
0.001 \\
0.005 \\
0.001 \\
0.001 \\
0.001 \\
0.001 \\
0.005 \\
0.001 \\
0.001 \\
0.001 \\
0.001 \\
0.001 \\
0.001\end{array}$ & J & $\begin{array}{l}0.005 \\
0.001 \\
0.001 \\
0.001 \\
0.001 \\
0.001 \\
0.005 \\
0.001 \\
0.001 \\
0.001 \\
0.001 \\
0.001 \\
0.005 \\
0.001 \\
0.001 \\
0.001 \\
0.001 \\
0.005 \\
0.001 \\
0.001 \\
0.001 \\
0.001 \\
0.005 \\
0.001 \\
0.001 \\
0.001 \\
0.001 \\
0.001 \\
0.001\end{array}$ & $\begin{array}{l}- \\
: \\
- \\
: \\
: \\
: \\
- \\
- \\
- \\
- \\
- \\
- \\
- \\
- \\
- \\
- \\
- \\
- \\
- \\
-\end{array}$ \\
\hline CALCIUM & $\begin{array}{l}0910 \\
0910 \\
0910 \\
0910 \\
0910 \\
0910 \\
0914 \\
0914 \\
0914\end{array}$ & $\begin{array}{l}02 / 17 / 87 \\
09 / 18 / 87 \\
01 / 30 / 88 \\
04 / 22 / 88 \\
08 / 11 / 88 \\
12 / 10 / 88 \\
02 / 18 / 87 \\
09 / 18 / 87 \\
02 / 03 / 88\end{array}$ & $\begin{array}{l}0001 \\
0001 \\
0001 \\
0001 \\
0001 \\
0001 \\
0001 \\
0001 \\
0001\end{array}$ & $\begin{array}{l}\text { WL } \\
\text { WL } \\
\text { WL. } \\
W L \\
W L \\
W L \\
W L \\
W L \\
W L\end{array}$ & $\begin{array}{l}\mathbf{N} \\
\mathbf{N} \\
\mathbf{N} \\
\mathbf{N} \\
\mathbf{N} \\
\mathbf{N} \\
\mathbf{N} \\
\mathbf{N} \\
\mathbf{N}\end{array}$ & $M G / L$ & & $\begin{array}{l}55.9 \\
48.2 \\
51.2 \\
51.7 \\
51.4 \\
49.7 \\
41.1 \\
33.3 \\
36.1\end{array}$ & & $\begin{array}{l}0.01 \\
0.01 \\
0.01 \\
0.01 \\
0.01 \\
0.01 \\
0.01 \\
0.01 \\
0.01\end{array}$ & $\begin{array}{l}- \\
: \\
- \\
- \\
- \\
-\end{array}$ \\
\hline
\end{tabular}

FORMATION OF COMPLETION CODE:

HL - HASATCH - LOWER SANDSTONE

WM - HASATCH - MIDDLE SHALE

PARAMETER VALUE INDICATOR (PVI): < - LESS THAN DETECTION LIMIT
FLOH RELATIONSHIP CODE:

N - UNKNOWN

SAMPLE ID COOES:

0001 - FILTERED SAMPLE (.45 MICRONS)

OTHER PARAMETER VALUE FLAGS:

$J$ - ESTIMATEO VALUE 
SITE: SPKO1 SPOOK

REPORT DATE: $07 / 29 / 94$

\begin{tabular}{|c|c|c|c|c|c|c|c|c|c|c|c|}
\hline PARAMETER NAME & $\underset{\text { ID }}{\text { LOCATION }}$ & LOG DATE & $\begin{array}{c}\text { SAMPLE } \\
\text { ID }\end{array}$ & $\begin{array}{l}\text { FORM } \\
\text { COMP }\end{array}$ & $\begin{array}{l}\text { FLOW } \\
\text { REL. }\end{array}$ & $\begin{array}{l}\text { UNITS OF } \\
\text { MEASURE }\end{array}$ & PVI & $\begin{array}{l}\text { PARAMETER } \\
\text { VALUE }\end{array}$ & FLAGS & $\begin{array}{l}\text { DETECTION } \\
\text { LIMIT }\end{array}$ & $\begin{array}{l}\text { PARAMETER } \\
\text { UNCERTAINTY }\end{array}$ \\
\hline CALCIUM & $\begin{array}{l}0914 \\
0914 \\
0914 \\
0915 \\
0915 \\
0915 \\
0915 \\
0915 \\
0915 \\
0919 \\
0919 \\
0919 \\
0919 \\
0919 \\
0919 \\
0921 \\
0921 \\
0921 \\
0921 \\
0921 \\
0923 \\
0923 \\
0923 \\
0923 \\
0923 \\
0938 \\
0938\end{array}$ & \begin{tabular}{|l|}
$04 / 22 / 88$ \\
$08 / 08 / 88$ \\
$12 / 10 / 88$ \\
$02 / 20 / 87$ \\
$09 / 20 / 87$ \\
$02 / 03 / 88$ \\
$04 / 22 / 88$ \\
$08 / 10 / 88$ \\
$12 / 10 / 88$ \\
$02 / 19 / 87$ \\
$09 / 20 / 87$ \\
$01 / 30 / 88$ \\
$04 / 26 / 88$ \\
$08 / 27 / 88$ \\
$12 / 10 / 88$ \\
$09 / 20 / 87$ \\
$02 / 04 / 88$ \\
$04 / 26 / 88$ \\
$08 / 10 / 88$ \\
$12 / 10 / 88$ \\
$09 / 20 / 87$ \\
$01 / 30 / 88$ \\
$04 / 26 / 88$ \\
$08 / 09 / 88$ \\
$12 / 10 / 88$ \\
$08 / 09 / 88$ \\
$12 / 10 / 88$
\end{tabular} & $\begin{array}{l}0001 \\
0001 \\
0001 \\
0001 \\
0001 \\
0001 \\
0001 \\
0001 \\
0001 \\
0001 \\
0001 \\
0001 \\
0001 \\
0001 \\
0001 \\
0001 \\
0001 \\
0001 \\
0001 \\
0001 \\
0001 \\
0001 \\
0001 \\
0001 \\
0001 \\
0001 \\
0001\end{array}$ & $\begin{array}{l}W L \\
W L \\
W L \\
W M \\
W M \\
W M \\
W M \\
W M \\
W M \\
W L \\
W L \\
W L \\
W L \\
W L \\
W L \\
W L \\
W L \\
W L \\
W L \\
W L \\
W L \\
W L \\
W L \\
W L \\
W L \\
W L \\
H L\end{array}$ & $\begin{array}{l}N \\
N \\
N \\
N \\
N \\
N \\
N \\
N \\
N \\
N \\
N \\
N \\
N \\
N \\
N \\
N \\
N \\
N \\
N \\
N \\
N \\
N \\
N \\
N \\
N \\
N \\
N\end{array}$ & $M G / L$ & & $\begin{array}{c}36.5 \\
36.6 \\
33.7 \\
46.0 \\
40.2 \\
45.3 \\
44.0 \\
43.6 \\
41.9 \\
55.8 \\
61.1 \\
57.5 \\
59.9 \\
60.7 \\
57.2 \\
131 . \\
101 . \\
119 . \\
108 . \\
104 . \\
58.1 \\
51.1 \\
53.9 \\
55.0 \\
49.5 \\
14.3 \\
9.50\end{array}$ & J & $\begin{array}{l}0.01 \\
0.01 \\
0.01 \\
0.01 \\
0.01 \\
0.01 \\
0.01 \\
0.01 \\
0.01 \\
0.01 \\
0.01 \\
0.01 \\
0.01 \\
0.01 \\
0.01 \\
0.01 \\
0.01 \\
0.01 \\
0.01 \\
0.01 \\
0.01 \\
0.01 \\
0.01 \\
0.01 \\
0.01 \\
0.01 \\
0.01\end{array}$ & 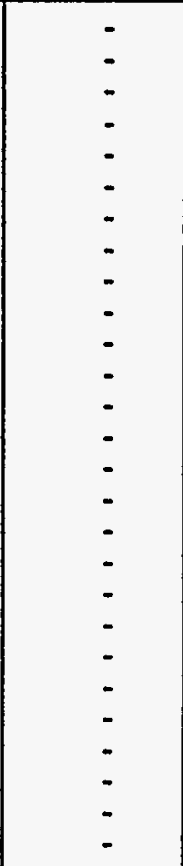 \\
\hline CHLORIDE & $\begin{array}{l}0910 \\
0910 \\
0910 \\
0910 \\
0910 \\
0910 \\
0914 \\
0914 \\
0914 \\
0914 \\
0914\end{array}$ & $\begin{array}{l}02 / 17 / 87 \\
09 / 18 / 87 \\
01 / 30 / 88 \\
04 / 22 / 88 \\
08 / 11 / 88 \\
12 / 10 / 88 \\
02 / 18 / 87 \\
09 / 18 / 87 \\
02 / 03 / 88 \\
04 / 22 / 88 \\
08 / 08 / 88\end{array}$ & $\begin{array}{l}0001 \\
0001 \\
0001 \\
0001 \\
0001 \\
0001 \\
0001 \\
0001 \\
0001 \\
0001 \\
0001\end{array}$ & $\begin{array}{l}W L \\
W L \\
W L \\
W L \\
W L \\
W L \\
W L \\
W L \\
W L \\
W L \\
W L\end{array}$ & $\begin{array}{l}N \\
N \\
N \\
N \\
N \\
N \\
N \\
N \\
N \\
N \\
N\end{array}$ & $M G / L$ & $<$ & $\begin{array}{l}2.3 \\
2.9 \\
3.8 \\
1.7 \\
1.0 \\
1.0 \\
2.3 \\
3.4 \\
3.8 \\
3.0 \\
1.5\end{array}$ & & $\begin{array}{l}1 . \\
1 . \\
1 . \\
1 . \\
i . \\
1 . \\
1 . \\
1 .\end{array}$ & $\begin{array}{l}- \\
- \\
- \\
- \\
- \\
- \\
-\end{array}$ \\
\hline
\end{tabular}

FORMATION OF COMPLETION CODE:

WL - WASATCH - LOWER SANDSTONE

WM - WASATCH - MIDDLE SHALE

PARAMETER VALUE INDICATOR (PVI): < - LESS THAN DETECTION LIMIT
FLOW RELATIONSHIP CODE:

N - UNKNOWN

OTHER PARAMETER VALUE FLAGS:

$J$ - ESTIMATED VALUE 
GROUNDWATER QUALITY DATA BY PARAMETER

LOWER SANDSTONE AND MIDDLE SHALE UNITS

SITE: SPKO1 SPOOK

02/17/87 TO $12 / 10 / 88$

REPORT DATE: 07/29/94

\begin{tabular}{|c|c|c|c|c|c|c|c|c|c|c|c|}
\hline PARAMETER NAME & $\underset{\text { ID }}{\text { LOCATION }}$ & LOG DATE & $\underset{\text { ID }}{\text { SAMPLE }}$ & $\begin{array}{l}\text { FORM } \\
\text { COMP }\end{array}$ & $\begin{array}{l}\text { FLOW } \\
\text { REL. }\end{array}$ & $\begin{array}{l}\text { UNITS of } \\
\text { MEASURE }\end{array}$ & PVI & $\begin{array}{l}\text { PARAMETER } \\
\text { VALUE }\end{array}$ & FLAGS & $\begin{array}{l}\text { DETECTION } \\
\text { LIMIT }\end{array}$ & $\begin{array}{c}\text { PARAMETER } \\
\text { UNCERTAINTY }\end{array}$ \\
\hline CHLORIDE & $\begin{array}{l}0914 \\
0915 \\
0915 \\
0915 \\
0915 \\
0915 \\
0915 \\
0919 \\
0919 \\
0919 \\
0919 \\
0919 \\
0919 \\
0921 \\
0921 \\
0921 \\
0921 \\
0921 \\
0923 \\
0923 \\
0923 \\
0923 \\
0923 \\
0938 \\
0938\end{array}$ & $\begin{array}{l}12 / 10 / 88 \\
02 / 20 / 87 \\
09 / 20 / 87 \\
02 / 03 / 88 \\
04 / 22 / 88 \\
08 / 10 / 88 \\
12 / 10 / 88 \\
02 / 19 / 87 \\
09 / 20 / 87 \\
01 / 30 / 88 \\
04 / 26 / 88 \\
08 / 27 / 88 \\
12 / 10 / 88 \\
09 / 20 / 87 \\
02 / 04 / 88 \\
04 / 26 / 88 \\
08 / 10 / 88 \\
12 / 10 / 88 \\
09 / 20 / 87 \\
01 / 30 / 88 \\
04 / 26 / 88 \\
08 / 09 / 88 \\
12 / 10 / 88 \\
08 / 09 / 88 \\
12 / 10 / 88\end{array}$ & $\begin{array}{l}0001 \\
0001 \\
0001 \\
0001 \\
0001 \\
0001 \\
0001 \\
0001 \\
0001 \\
0001 \\
0001 \\
0001 \\
0001 \\
0001 \\
0001 \\
0001 \\
0001 \\
0001 \\
0001 \\
0001 \\
0001 \\
0001 \\
0001 \\
0001 \\
0001\end{array}$ & $\begin{array}{l}W L \\
W M \\
W M \\
W M \\
W M \\
W M \\
W M \\
W L \\
W L \\
W L \\
W L \\
W L \\
W L \\
W L \\
W L \\
W L \\
W L \\
W L \\
W L \\
W L \\
W L \\
W L \\
W L \\
W L \\
W L\end{array}$ & $\begin{array}{l}N \\
N \\
N \\
N \\
N \\
N \\
N \\
N \\
N \\
N \\
N \\
N \\
N \\
N \\
N \\
N \\
N \\
N \\
N \\
N \\
N \\
N \\
N \\
N \\
N\end{array}$ & $M G / L$ & $<$ & $\begin{array}{l}1.0 \\
3.5 \\
4.5 \\
5.1 \\
3.0 \\
1.5 \\
1.4 \\
2.3 \\
2.8 \\
0.7 \\
0.5 \\
2.1 \\
1.0 \\
3.9 \\
1.3 \\
1.8 \\
1.5 \\
2.1 \\
2.8 \\
2.5 \\
0.5 \\
1.0 \\
1.0 \\
1.5 \\
1.0\end{array}$ & $J$ & $\begin{array}{l}1 . \\
1 . \\
1 . \\
1 . \\
1 . \\
1 . \\
1 . \\
1 . \\
1 . \\
1 . \\
1 . \\
1 . \\
1 . \\
1 . \\
1 . \\
1 . \\
1 . \\
1 . \\
1 .\end{array}$ & $\begin{array}{l}- \\
- \\
- \\
- \\
- \\
- \\
- \\
- \\
- \\
- \\
- \\
- \\
- \\
- \\
- \\
- \\
- \\
-\end{array}$ \\
\hline CHROMIUM & $\begin{array}{l}0910 \\
0910 \\
0910 \\
0910 \\
0910 \\
0914 \\
0914 \\
0914 \\
0914 \\
0914 \\
0915 \\
0915 \\
0915\end{array}$ & $\begin{array}{l}09 / 18 / 87 \\
01 / 30 / 88 \\
04 / 22 / 88 \\
08 / 11 / 88 \\
12 / 10 / 88 \\
09 / 18 / 87 \\
02 / 03 / 88 \\
04 / 22 / 88 \\
08 / 08 / 88 \\
12 / 10 / 88 \\
09 / 20 / 87 \\
02 / 03 / 88 \\
04 / 22 / 88\end{array}$ & $\begin{array}{l}0001 \\
0001 \\
0001 \\
0001 \\
0001 \\
0001 \\
0001 \\
0001 \\
0001 \\
0001 \\
0001 \\
0001 \\
0001\end{array}$ & $\begin{array}{l}W L \\
W L \\
W L \\
W L \\
W L \\
W L \\
W L \\
W L \\
W L \\
W L \\
W M \\
W M \\
W M\end{array}$ & $\begin{array}{l}N \\
N \\
N \\
N \\
N \\
N \\
N \\
N \\
N \\
N \\
N \\
N \\
N\end{array}$ & $M G / L$ & $<<$ & $\begin{array}{l}0.01 \\
0.01 \\
0.03 \\
0.02 \\
0.05 \\
0.01 \\
0.01 \\
0.02 \\
0.02 \\
0.01 \\
0.01 \\
0.01 \\
0.02\end{array}$ & $\mathbf{J}$ & $\begin{array}{l}0.01 \\
0.01 \\
0.01 \\
0.01 \\
0.01 \\
0.01 \\
0.01 \\
0.01 \\
0.01 \\
0.01 \\
0.01 \\
0.01 \\
0.01\end{array}$ & $\begin{array}{l}- \\
- \\
- \\
- \\
- \\
- \\
- \\
- \\
-\end{array}$ \\
\hline
\end{tabular}

FORMATION OF COMPLETION CODE:

WL - WASATCH -

WM - WASATCH - MIDDLE SHALE

PARAMETER VALUE INDICATOR (PVI): < - LESS THAN DETECTION LIMIT

OTHER PARAMETER VALUE FLAGS:

$J$ - ESTIMATED VALUE
FLOW RELATIONSHIP CODE:

N - UNKNOHN

SAMPLE ID CODES

0001 - FILTERED SAMPLE (.45 MICRONS) 
GROUNDHATER QUALITY DATA BY PARAMETER

LOWER SANDSTONE AND MIDDLE SHALE UNITS

SITE: SPKO1 SPOOK

$02 / 17 / 87$ To $12 / 10 / 88$

REPORT DATE: $07 / 29 / 94$

\begin{tabular}{|c|c|c|c|c|c|c|c|c|c|c|c|}
\hline PARAMETER NAME & $\underset{\text { ID }}{\text { LOCATION }}$ & LOG DATE & $\begin{array}{c}\text { SAMPLE } \\
\text { ID }\end{array}$ & $\begin{array}{l}\text { FORH } \\
\text { COMP }\end{array}$ & $\begin{array}{l}\text { FLOW } \\
\text { REL. }\end{array}$ & $\begin{array}{l}\text { UNITS OF } \\
\text { MEASURE }\end{array}$ & PVI & $\begin{array}{l}\text { PARAMETER } \\
\text { VALUE }\end{array}$ & FLAGS & $\begin{array}{l}\text { DETECTION } \\
\text { LIMIT }\end{array}$ & \begin{tabular}{|} 
PARAMETER \\
UNCERTAINTY
\end{tabular} \\
\hline CHROMIUM & $\begin{array}{l}0915 \\
0915 \\
0919 \\
0919 \\
0919 \\
0919 \\
0919 \\
0921 \\
0921 \\
0921 \\
0921 \\
0921 \\
0923 \\
0923 \\
0923 \\
0923 \\
0923 \\
0938 \\
0938\end{array}$ & $\begin{array}{l}08 / 10 / 88 \\
12 / 10 / 88 \\
09 / 20 / 87 \\
01 / 30 / 88 \\
04 / 26 / 88 \\
08 / 27 / 88 \\
12 / 10 / 88 \\
09 / 20 / 87 \\
02 / 04 / 88 \\
04 / 26 / 88 \\
08 / 10 / 88 \\
12 / 10 / 88 \\
09 / 20 / 87 \\
01 / 30 / 88 \\
04 / 26 / 88 \\
08 / 09 / 88 \\
12 / 10 / 88 \\
08 / 09 / 88 \\
12 / 10 / 88\end{array}$ & $\begin{array}{l}0001 \\
0001 \\
0001 \\
0001 \\
0001 \\
0001 \\
0001 \\
0001 \\
0001 \\
0001 \\
0001 \\
0001 \\
0001 \\
0001 \\
0001 \\
0001 \\
0001 \\
0001 \\
0001\end{array}$ & $\begin{array}{l}W M \\
W H \\
W L \\
W L \\
W L \\
W L \\
W L \\
W L \\
W L \\
W L \\
W L \\
W L \\
W L \\
W L \\
W L \\
W L \\
W L \\
W L \\
W L\end{array}$ & $\begin{array}{l}N \\
N \\
N \\
N \\
N \\
N \\
N \\
N \\
N \\
N \\
N \\
N \\
N \\
N \\
N \\
N \\
N \\
N \\
N\end{array}$ & MG/L & $\mid \begin{array}{l}< \\
< \\
< \\
<\end{array}$ & $\begin{array}{l}0.02 \\
0.01 \\
0.01 \\
0.02 \\
0.04 \\
0.02 \\
0.02 \\
0.01 \\
0.04 \\
0.05 \\
0.03 \\
0.04 \\
0.01 \\
0.03 \\
0.04 \\
0.02 \\
0.02 \\
0.02 \\
0.01\end{array}$ & $J$ & $\begin{array}{l}0.01 \\
0.01 \\
0.01 \\
0.01 \\
0.01 \\
0.01 \\
0.01 \\
0.01 \\
0.01 \\
0.01 \\
0.03 \\
0.01 \\
0.01 \\
0.01 \\
0.01 \\
0.01 \\
0.01 \\
0.01 \\
0.01\end{array}$ & $\begin{array}{l}- \\
: \\
- \\
: \\
- \\
- \\
- \\
- \\
- \\
- \\
- \\
-\end{array}$ \\
\hline COPPER & $\begin{array}{l}0910 \\
0910 \\
0910 \\
0910 \\
0910 \\
0914 \\
0914 \\
0914 \\
0914 \\
0914 \\
0915 \\
0915 \\
0915 \\
0915 \\
0915 \\
0919 \\
0919 \\
0919 \\
0919\end{array}$ & $\begin{array}{l}02 / 17 / 87 \\
09 / 18 / 87 \\
01 / 30 / 88 \\
04 / 22 / 88 \\
08 / 11 / 88 \\
02 / 18 / 87 \\
09 / 18 / 87 \\
02 / 03 / 88 \\
04 / 22 / 88 \\
08 / 08 / 88 \\
02 / 20 / 87 \\
09 / 20 / 87 \\
02 / 03 / 88 \\
04 / 22 / 88 \\
08 / 10 / 88 \\
02 / 19 / 87 \\
09 / 20 / 87 \\
01 / 30 / 88 \\
04 / 26 / 88\end{array}$ & $\begin{array}{l}0001 \\
0001 \\
0001 \\
0001 \\
0001 \\
0001 \\
0001 \\
0001 \\
0001 \\
0001 \\
0001 \\
0001 \\
0001 \\
0001 \\
0001 \\
0001 \\
0001 \\
0001 \\
0001\end{array}$ & $\begin{array}{l}W L \\
W L \\
W L \\
W L \\
W L \\
W L \\
W L \\
W L \\
W L \\
W L \\
W H \\
W M \\
W M \\
W M \\
W M \\
W L \\
W L \\
W L \\
H L\end{array}$ & $\begin{array}{l}N \\
N \\
N \\
N \\
N \\
N \\
N \\
N \\
N \\
N \\
N \\
N \\
N \\
N \\
N \\
N \\
N \\
N \\
N\end{array}$ & $M G / L$ & $\begin{array}{l}< \\
< \\
< \\
< \\
< \\
< \\
< \\
< \\
< \\
< \\
< \\
< \\
< \\
< \\
< \\
< \\
< \\
<\end{array}$ & $\begin{array}{l}0.01 \\
0.01 \\
0.01 \\
0.01 \\
0.02 \\
0.01 \\
0.01 \\
0.01 \\
0.01 \\
0.02 \\
0.01 \\
0.01 \\
0.01 \\
0.01 \\
0.02 \\
0.01 \\
0.01 \\
0.01 \\
0.01\end{array}$ & $\begin{array}{l}J \\
J \\
J \\
J \\
J \\
J \\
J \\
J \\
J \\
J \\
J \\
J \\
J \\
J \\
J \\
J\end{array}$ & $\begin{array}{l}0.02 \\
0.02 \\
0.02 \\
0.02 \\
0.02 \\
0.02 \\
0.02 \\
0.02 \\
0.02 \\
0.02 \\
0.02 \\
0.02 \\
0.02 \\
0.02 \\
0.02 \\
0.02 \\
0.02 \\
0.02 \\
0.02\end{array}$ & $\begin{array}{l}- \\
- \\
- \\
- \\
- \\
- \\
- \\
- \\
- \\
- \\
- \\
- \\
-\end{array}$ \\
\hline
\end{tabular}

FORMATION OF COMPLETION CODE:

WM - WASATCH - MIDDLE SHALE

WL - WASATCH - LOWER SANDSTONE

PARAMETER VALUE INDICATOR (PVI): < - LESS THAN DETECTION LIMIT
FLOH RELATIONSHIP COOE:

- UNKNOWN

SAMPLE ID CODES

0001 - FILTERED SAMPLE (.45 MICRONS)

OTHER PARAMETER VALUE FLAGS:

$J$ - ESTIMATED VALUE 
GROUNDWATER QUALITY DATA BY PARAMETER

LOWER SANDSTONE AND MIDDLE SHALE UNITS

SITE: SPKO1 SPOOK

$02 / 17 / 87$ TO $12 / 10 / 88$

REPORT DATE: $07 / 29 / 94$

\begin{tabular}{|c|c|c|c|c|c|c|c|c|c|c|c|}
\hline PARAMETER NAME & ${ }_{10}^{\text {LOCATION }}$ & LOG DATE & $\begin{array}{c}\text { SAMPLE } \\
\text { ID }\end{array}$ & $\begin{array}{l}\text { FORM } \\
\text { COMP }\end{array}$ & $\begin{array}{l}\text { FLOW } \\
\text { REL. }\end{array}$ & $\begin{array}{l}\text { UNITS OF } \\
\text { MEASURE }\end{array}$ & PVI & $\begin{array}{l}\text { ARAMETER } \\
\text { VALUE }\end{array}$ & FLAGS & $\begin{array}{l}\text { DETECTION } \\
\text { LIMIT }\end{array}$ & $\begin{array}{l}\text { PARAMETER } \\
\text { UNCERTAINTY }\end{array}$ \\
\hline COPPER & $\begin{array}{l}0919 \\
0921 \\
0921 \\
0921 \\
0921 \\
0923 \\
0923 \\
0923 \\
0923 \\
0938\end{array}$ & $\begin{array}{l}08 / 27 / 88 \\
09 / 20 / 87 \\
02 / 04 / 88 \\
04 / 26 / 88 \\
08 / 10 / 88 \\
09 / 20 / 87 \\
01 / 30 / 88 \\
04 / 26 / 88 \\
08 / 09 / 88 \\
08 / 09 / 88\end{array}$ & $\begin{array}{l}0001 \\
0001 \\
0001 \\
0001 \\
0001 \\
0001 \\
0001 \\
0001 \\
0001 \\
0001\end{array}$ & $\begin{array}{l}\text { WL } \\
\text { WL } \\
\text { HL } \\
\text { HL } \\
\text { WL } \\
\text { WL } \\
\text { WL } \\
\text { WL } \\
\text { WL } \\
\text { HL }\end{array}$ & $\begin{array}{l}N \\
N \\
N \\
N \\
N \\
N \\
N \\
N \\
N \\
N\end{array}$ & $M G / L$ & $\begin{array}{l}< \\
< \\
< \\
< \\
<\end{array}$ & $\begin{array}{l}0.02 \\
0.02 \\
0.01 \\
0.01 \\
0.02 \\
0.01 \\
0.01 \\
0.01 \\
0.02 \\
0.02\end{array}$ & $\begin{array}{l}\text { J } \\
J \\
J \\
J \\
J \\
J\end{array}$ & $\begin{array}{l}0.02 \\
0.02 \\
0.02 \\
0.02 \\
0.02 \\
0.02 \\
0.02 \\
0.02 \\
0.02 \\
0.02\end{array}$ & $\begin{array}{l}- \\
- \\
- \\
- \\
- \\
- \\
-\end{array}$ \\
\hline FLUORIDE & $\begin{array}{l}0910 \\
0910 \\
0910 \\
0910 \\
0910 \\
0914 \\
0914 \\
0914 \\
0914 \\
0914 \\
0915 \\
0915 \\
0915 \\
0915 \\
0915 \\
0919 \\
0919 \\
0919 \\
0919 \\
0919 \\
0921 \\
0921 \\
0921 \\
0921 \\
0923 \\
0923 \\
0923 \\
0923\end{array}$ & $\begin{array}{l}02 / 17 / 87 \\
09 / 18 / 87 \\
01 / 30 / 88 \\
04 / 22 / 88 \\
08 / 11 / 88 \\
02 / 18 / 87 \\
09 / 18 / 87 \\
02 / 03 / 88 \\
04 / 22 / 88 \\
08 / 08 / 88 \\
02 / 20 / 87 \\
09 / 20 / 87 \\
02 / 03 / 88 \\
04 / 22 / 88 \\
08 / 10 / 88 \\
02 / 19 / 87 \\
09 / 20 / 87 \\
01 / 30 / 88 \\
04 / 26 / 88 \\
08 / 27 / 88 \\
09 / 20 / 87 \\
02 / 04 / 88 \\
04 / 26 / 88 \\
08 / 10 / 88 \\
09 / 20 / 87 \\
01 / 30 / 88 \\
04 / 26 / 88 \\
08 / 09 / 88\end{array}$ & $\begin{array}{l}0001 \\
0001 \\
0001 \\
0001 \\
0001 \\
0001 \\
0001 \\
0001 \\
0001 \\
0001 \\
0001 \\
0001 \\
0001 \\
0001 \\
0001 \\
0001 \\
0001 \\
0001 \\
0001 \\
0001 \\
0001 \\
0001 \\
0001 \\
0001 \\
0001 \\
0001 \\
0001 \\
0001\end{array}$ & $\begin{array}{l}W L \\
W L \\
W L \\
W L \\
W L \\
W L \\
W L \\
W L \\
W L \\
W L \\
W M \\
W M \\
W M \\
W M \\
W M \\
W L \\
W L \\
W L \\
W L \\
W L \\
W L \\
W L \\
W L \\
W L \\
W L \\
W L \\
W L \\
W L\end{array}$ & $\begin{array}{l}N \\
N \\
N \\
N \\
N \\
N \\
N \\
N \\
N \\
N \\
N \\
N \\
N \\
N \\
N \\
N \\
N \\
N \\
N \\
N \\
N \\
N \\
N \\
N \\
N \\
N \\
N \\
N\end{array}$ & $\mathrm{MG} / \mathrm{L}$ & & $\begin{array}{l}0.3 \\
0.30 \\
0.23 \\
0.27 \\
0.2 \\
0.3 \\
0.25 \\
0.23 \\
0.25 \\
0.2 \\
0.3 \\
0.32 \\
0.31 \\
0.32 \\
0.3 \\
0.3 \\
0.21 \\
0.20 \\
0.21 \\
0.2 \\
0.13 \\
0.12 \\
0.13 \\
0.1 \\
0.23 \\
0.22 \\
0.22 \\
0.2\end{array}$ & $J$ & $\begin{array}{l}0.1 \\
0.1 \\
0.1 \\
0.1 \\
0.1 \\
0.1 \\
0.1 \\
0.1 \\
0.1 \\
0.1 \\
0.1 \\
0.1 \\
0.1 \\
0.1 \\
0.1 \\
0.1 \\
0.1 \\
0.1 \\
0.1 \\
0.1 \\
0.1 \\
0.1 \\
0.1 \\
0.1 \\
0.1 \\
0.1 \\
0.1 \\
0.1\end{array}$ & $\begin{array}{l}- \\
- \\
- \\
- \\
- \\
- \\
- \\
- \\
- \\
- \\
- \\
- \\
- \\
- \\
- \\
- \\
- \\
- \\
- \\
- \\
-\end{array}$ \\
\hline
\end{tabular}

FORMATION OF COMPLETION CODE: WL - WASATCH - LOWER SANDSTONE WM - HASATCH - MIDDLE SHALE

PARAMETER VALUE INDICATOR (PVI): < - LESS THAN DETECTION LIMIT
FLOW RELATIONSHIP CODE:

N - UNKNOHN

AMPLE ID CODES:

0001 - FILTERED SAMPLE (.45 MICRONS)

OTHER PARAMETER VALUE FLAGS:

$J$ - ESTIMATED VALUE 
GROUNDHATER QUALITY DATA BY PARAMETER

LOWER SANDSTONE AND MIDDLE SHALE UNITS

SITE: SPKO1 SPOOK

REPORT DATE: $07 / 29 / 94$

\begin{tabular}{|c|c|c|c|c|c|c|c|c|c|c|c|}
\hline PARAMETER NAME & $\underset{\text { ID }}{\text { LOCATION }}$ & LOG DATE & $\begin{array}{c}\text { SAMPLE } \\
\text { ID }\end{array}$ & $\mid \begin{array}{l}\text { FORM } \\
\text { COMP }\end{array}$ & FLOW & $\begin{array}{l}\text { UNITS OF } \\
\text { MEASURE }\end{array}$ & PVI & $\begin{array}{l}\text { PARAMETER } \\
\text { VALUE }\end{array}$ & FLAGS & $\begin{array}{l}\text { DETECTION } \\
\text { LIMIT }\end{array}$ & $\begin{array}{l}\text { PARAMETER } \\
\text { UNCERTAINTY }\end{array}$ \\
\hline FLUORIDE & 0938 & $08 / 09 / 88$ & 0001 & HL & N & MG/L & & 0.5 & $\mathrm{~J}$ & 0.1 & - \\
\hline GROSS ALPHA & $\begin{array}{l}0910 \\
0910 \\
0910 \\
0910 \\
0910 \\
0914 \\
0914 \\
0914 \\
0914 \\
0914 \\
0915 \\
0915 \\
0915 \\
0915 \\
0915 \\
0919 \\
0919 \\
0919 \\
0919 \\
0919 \\
0921 \\
0921 \\
0921 \\
0921 \\
0921 \\
0923 \\
0923 \\
0923 \\
0923 \\
0923 \\
0938 \\
0938\end{array}$ & $\begin{array}{l}09 / 18 / 87 \\
01 / 30 / 88 \\
04 / 22 / 88 \\
08 / 11 / 88 \\
12 / 10 / 88 \\
09 / 18 / 87 \\
02 / 03 / 88 \\
04 / 22 / 88 \\
08 / 08 / 88 \\
12 / 10 / 88 \\
09 / 20 / 87 \\
02 / 03 / 88 \\
04 / 22 / 88 \\
08 / 10 / 88 \\
12 / 10 / 88 \\
09 / 20 / 87 \\
01 / 30 / 88 \\
04 / 26 / 88 \\
08 / 27 / 88 \\
12 / 10 / 88 \\
09 / 20 / 87 \\
02 / 04 / 88 \\
04 / 26 / 88 \\
08 / 10 / 88 \\
12 / 10 / 88 \\
09 / 20 / 87 \\
01 / 30 / 88 \\
04 / 26 / 88 \\
08 / 09 / 88 \\
12 / 10 / 88 \\
08 / 09 / 88 \\
12 / 10 / 88\end{array}$ & $\begin{array}{l}0001 \\
0001 \\
0001 \\
0001 \\
0001 \\
0001 \\
0001 \\
0001 \\
0001 \\
0001 \\
0001 \\
0001 \\
0001 \\
0001 \\
0001 \\
0001 \\
0001 \\
0001 \\
0001 \\
0001 \\
0001 \\
0001 \\
0001 \\
0001 \\
0001 \\
0001 \\
0001 \\
0001 \\
0001 \\
0001 \\
0001 \\
0001\end{array}$ & $\begin{array}{l}H L \\
H L \\
W L \\
W L \\
W L \\
W L \\
W L \\
W L \\
W L \\
H L \\
W M \\
W M \\
W M \\
W M \\
W M \\
W L \\
W L \\
W L \\
H L \\
W L \\
W L \\
H L \\
W L \\
W L \\
W L \\
H L \\
W L \\
W L \\
W L \\
W L \\
W L \\
W L\end{array}$ & $\begin{array}{l}N \\
N \\
N \\
N \\
N \\
N \\
N \\
N \\
N \\
N \\
N \\
N \\
N \\
N \\
N \\
N \\
N \\
N \\
N \\
N \\
N \\
N \\
N \\
N \\
N \\
N \\
N \\
N \\
N \\
N \\
N\end{array}$ & $\mathrm{PCI} / \mathrm{L}$ & & $\begin{array}{c}14 . \\
27 . \\
17 . \\
13 . \\
12 . \\
5.3 \\
4.6 \\
4.1 \\
1.8 \\
11 . \\
27 . \\
13 . \\
13 . \\
5.9 \\
8.4 \\
7.5 \\
12 . \\
6.9 \\
6.4 \\
4.9 \\
6.9 \\
11 . \\
9.7 \\
3.0 \\
0.0 \\
8.9 \\
6.2 \\
6.1 \\
2.5 \\
4.1 \\
0 . \\
1.5\end{array}$ & $\jmath$ & $\begin{array}{l}0.2 \\
0.2 \\
0.2 \\
0.2 \\
0.2 \\
0.2 \\
0.2 \\
0.2 \\
0.2 \\
0.2 \\
0.2 \\
0.2 \\
0.2 \\
0.2 \\
0.2 \\
0.2 \\
0.2 \\
0.2 \\
0.2 \\
0.2 \\
0.2 \\
0.2 \\
0.2 \\
0.2 \\
0.2 \\
0.2 \\
0.2 \\
0.2 \\
0.2 \\
0.2 \\
0.2 \\
0.2\end{array}$ & $\begin{array}{l}4 . \\
6 . \\
5 . \\
3 . \\
4 . \\
2.6 \\
2.7 \\
2.8 \\
2.2 \\
3 . \\
5 . \\
4 . \\
4 . \\
2.7 \\
2.8 \\
3.2 \\
4 . \\
3.5 \\
3.0 \\
3.0 \\
4.0 \\
5 . \\
5.4 \\
3.8 \\
3.7 \\
3.2 \\
4.0 \\
4.0 \\
2.9 \\
3.3 \\
100 . \\
2.2\end{array}$ \\
\hline GROSS BETA & $\begin{array}{l}0910 \\
0910 \\
0910 \\
0910\end{array}$ & $\begin{array}{l}09 / 18 / 87 \\
01 / 30 / 88 \\
04 / 22 / 88 \\
08 / 11 / 88\end{array}$ & $\begin{array}{l}0001 \\
0001 \\
0001 \\
0001\end{array}$ & $\begin{array}{l}W L \\
W L \\
W L \\
W L\end{array}$ & $\begin{array}{l}N \\
N \\
N \\
N\end{array}$ & $\begin{array}{c}\mathrm{PCI} / \mathrm{L} \\
.\end{array}$ & & $\begin{array}{l}13 . \\
14 . \\
12 . \\
9.6\end{array}$ & & $\begin{array}{l}1 . \\
1 . \\
1 .\end{array}$ & $\begin{array}{l}3 . \\
3 . \\
2 . \\
1.5\end{array}$ \\
\hline
\end{tabular}

FORMATION OF COMPLETION CODE:

WL - WASATCH - LOWER SANDSTONE
WM - WASATCH - MIDDLE SHALE

FLOW RELATIONSHIP CODE:

N - UNKNOWN

PARAMETER VALUE INDICATOR (PVI): < - LESS THAN DETECTION LIMIT

SAMPLE ID CODES:

0001 - FILTERED SAMPLE (.45 MICRONS)

OTHER PARAMETER VALUE FLAGS:

$J$ - ESTIMATED VALLUE 
SITE: SPK01 SPOOK

02/17/87 TO $12 / 10 / 88$

REPORT DATE: $07 / 29 / 94$

\begin{tabular}{|c|c|c|c|c|c|c|c|c|c|c|c|}
\hline PARAMETER NAME & $\underset{\text { ID }}{\text { LOCATION }}$ & LOG DATE & $\begin{array}{c}\text { SAMPLE } \\
\text { ID }\end{array}$ & $\begin{array}{l}\text { FORM } \\
\text { COMP }\end{array}$ & $\begin{array}{l}\text { FLOW } \\
\text { REL. }\end{array}$ & $\begin{array}{l}\text { UNITS OF } \\
\text { MEASURE }\end{array}$ & |PVI & $\begin{array}{l}\text { PARAMETER } \\
\text { VALUE }\end{array}$ & FLAGS & $\begin{array}{l}\text { DETECTION } \\
\text { LIMIT }\end{array}$ & $\begin{array}{l}\text { PARAMETER } \\
\text { UNCERTAINTY }\end{array}$ \\
\hline GROSS BETA & $\begin{array}{l}0910 \\
0914 \\
0914 \\
0914 \\
0914 \\
0914 \\
0915 \\
0915 \\
0915 \\
0915 \\
0915 \\
0919 \\
0919 \\
0919 \\
0919 \\
0919 \\
0921 \\
0921 \\
0921 \\
0921 \\
0921 \\
0923 \\
0923 \\
0923 \\
0923 \\
0923 \\
0938 \\
0938\end{array}$ & $\begin{array}{l}12 / 10 / 88 \\
09 / 18 / 87 \\
02 / 03 / 88 \\
04 / 22 / 88 \\
08 / 08 / 88 \\
12 / 10 / 88 \\
09 / 20 / 87 \\
02 / 03 / 88 \\
04 / 22 / 88 \\
08 / 10 / 88 \\
12 / 10 / 88 \\
09 / 20 / 87 \\
01 / 30 / 88 \\
04 / 26 / 88 \\
08 / 27 / 88 \\
12 / 10 / 88 \\
09 / 20 / 87 \\
02 / 04 / 88 \\
04 / 26 / 88 \\
08 / 10 / 88 \\
12 / 10 / 88 \\
09 / 20 / 87 \\
01 / 30 / 88 \\
04 / 26 / 88 \\
08 / 09 / 88 \\
12 / 10 / 88 \\
08 / 09 / 88 \\
12 / 10 / 88\end{array}$ & $\begin{array}{l}0001 \\
0001 \\
0001 \\
0001 \\
0001 \\
0001 \\
0001 \\
0001 \\
0001 \\
0001 \\
0001 \\
0001 \\
0001 \\
0001 \\
0001 \\
0001 \\
0001 \\
0001 \\
0001 \\
0001 \\
0001 \\
0001 \\
0001 \\
0001 \\
0001 \\
0001 \\
0001 \\
0001\end{array}$ & $\begin{array}{l}W L \\
W L \\
W L \\
W L \\
W L \\
W L \\
W M \\
W M \\
W M \\
W M \\
W M \\
W L \\
H L \\
W L \\
W L \\
H L \\
H L \\
W L \\
W L \\
W L \\
H L \\
W L \\
W L \\
W L \\
W L \\
W L \\
W L \\
W L\end{array}$ & $\begin{array}{l}N \\
N \\
N \\
N \\
N \\
N \\
N \\
N \\
N \\
N \\
N \\
N \\
N \\
N \\
N \\
N \\
N \\
N \\
N \\
N \\
N \\
N \\
N \\
N \\
N \\
N \\
N \\
N\end{array}$ & $\mathrm{PCI} / \mathrm{L}$ & & $\begin{array}{c}7.4 \\
7.3 \\
6.4 \\
6.4 \\
6.1 \\
4.1 \\
19 . \\
7.6 \\
12 . \\
6.6 \\
5.7 \\
10 . \\
10 . \\
9.4 \\
8.6 \\
6.9 \\
15 . \\
11 . \\
11 . \\
11 . \\
17 . \\
22 . \\
7.7 \\
9.1 \\
7.1 \\
6.4 \\
93 . \\
4.3\end{array}$ & J & $\begin{array}{l}1 . \\
1 . \\
1 . \\
1 . \\
1 . \\
1 . \\
1 . \\
1 . \\
1 . \\
1 . \\
1 . \\
1 . \\
1 . \\
1 . \\
1 . \\
1 . \\
1 . \\
1 . \\
1 . \\
1 . \\
1 . \\
1 . \\
1 . \\
1 . \\
1 . \\
1 .\end{array}$ & $\begin{array}{l}1.8 \\
2.2 \\
2.0 \\
1.6 \\
1.3 \\
1.4 \\
2 . \\
2.2 \\
2 . \\
1.3 \\
1.4 \\
2 . \\
2 . \\
1.7 \\
1.6 \\
1.7 \\
3 . \\
3 . \\
3 . \\
3 . \\
3 . \\
3 . \\
2.8 \\
2.0 \\
2.1 \\
3.9 \\
51 . \\
1.4\end{array}$ \\
\hline IRON & $\begin{array}{l}0910 \\
0910 \\
0910 \\
0910 \\
0910 \\
0910 \\
0914 \\
0914 \\
0914 \\
0914\end{array}$ & $\begin{array}{l}02 / 17 / 87 \\
09 / 18 / 87 \\
01 / 30 / 88 \\
04 / 22 / 88 \\
08 / 11 / 88 \\
12 / 10 / 88 \\
02 / 18 / 87 \\
09 / 18 / 87 \\
02 / 03 / 88 \\
04 / 22 / 88\end{array}$ & $\begin{array}{l}0001 \\
0001 \\
0001 \\
0001 \\
0001 \\
0001 \\
0001 \\
0001 \\
0001 \\
0001\end{array}$ & $\begin{array}{l}W L \\
W L \\
W L \\
W L \\
W L \\
W L \\
W L \\
W L \\
W L \\
W L\end{array}$ & $\begin{array}{l}N \\
N \\
N \\
N \\
N \\
N \\
N \\
N \\
N \\
N\end{array}$ & $M G / L$ & $\begin{array}{l}< \\
< \\
< \\
<\end{array}$ & $\begin{array}{l}0.03 \\
0.02 \\
0.11 \\
0.04 \\
0.03 \\
0.04 \\
0.03 \\
0.01 \\
0.12 \\
0.02\end{array}$ & $\begin{array}{l}\mathbf{J} \\
\mathbf{J}\end{array}$ & $\begin{array}{l}0.03 \\
0.03 \\
0.03 \\
0.03 \\
0.03 \\
0.03 \\
0.03 \\
0.03 \\
0.03 \\
0.03\end{array}$ & $\begin{array}{l}- \\
- \\
- \\
- \\
- \\
-\end{array}$ \\
\hline
\end{tabular}

FORMATION OF COMPLETION CODE:

WL - WASATCH - LOWER SANDSTONE

HM - HASATCH - MIDDLE SHALE

PARAMETER VALUE INDICATOR (PVI): < - LESS THAN DETECTION LIMIT
FLOW RELATIONSHIP CODE:

N - UNKNOWN

SAMPLE ID CODES:

0001 - FILTERED SAMPLE (.45 MICRONS)

OTHER PARAMETER VALUE FLAGS:

$\checkmark$ - ESTIMATED VALUE 
SITE: SPKO1 SPOOK

02/17/87 TO $12 / 10 / 88$

\begin{tabular}{|c|c|c|c|c|c|c|c|c|c|c|c|}
\hline PARAMETER NAME & $\underset{\text { ID }}{\text { LOCATION }}$ & LOG DATE & $\begin{array}{c}\text { SAMPLE } \\
\text { ID }\end{array}$ & $\begin{array}{l}\text { FORM } \\
\text { COMP }\end{array}$ & $\begin{array}{l}\text { FLOH } \\
\text { REL. }\end{array}$ & $\begin{array}{l}\text { UNITS OF } \\
\text { MEASURE }\end{array}$ & PVI & $\begin{array}{l}\text { PARAMETER } \\
\text { VALUE }\end{array}$ & FLAGS & $\begin{array}{l}\text { DETECTION } \\
\text { LIMIT }\end{array}$ & $\begin{array}{l}\text { PARAMETER } \\
\text { UNCERTAINTY }\end{array}$ \\
\hline IRON & $\begin{array}{l}0914 \\
0914 \\
0915 \\
0915 \\
0915 \\
0915 \\
0915 \\
0915 \\
0919 \\
0919 \\
0919 \\
0919 \\
0919 \\
0919 \\
0921 \\
0921 \\
0921 \\
0921 \\
0921 \\
0923 \\
0923 \\
0923 \\
0923 \\
0923 \\
0938 \\
0938\end{array}$ & $\begin{array}{l}08 / 08 / 88 \\
12 / 10 / 88 \\
02 / 20 / 87 \\
09 / 20 / 87 \\
02 / 03 / 88 \\
04 / 22 / 88 \\
08 / 10 / 88 \\
12 / 10 / 88 \\
02 / 19 / 87 \\
09 / 20 / 87 \\
01 / 30 / 88 \\
04 / 26 / 88 \\
08 / 27 / 88 \\
12 / 10 / 88 \\
09 / 20 / 87 \\
02 / 04 / 88 \\
04 / 26 / 88 \\
08 / 10 / 88 \\
12 / 10 / 88 \\
09 / 20 / 87 \\
01 / 30 / 88 \\
04 / 26 / 88 \\
08 / 09 / 88 \\
12 / 10 / 88 \\
08 / 09 / 88 \\
12 / 10 / 88\end{array}$ & $\begin{array}{l}0001 \\
0001 \\
0001 \\
0001 \\
0001 \\
0001 \\
0001 \\
0001 \\
0001 \\
0001 \\
0001 \\
0001 \\
0001 \\
0001 \\
0001 \\
0001 \\
0001 \\
0001 \\
0001 \\
0001 \\
0001 \\
0001 \\
0001 \\
0001 \\
0001 \\
0001\end{array}$ & $\begin{array}{l}W L \\
W L \\
W M \\
W M \\
W M \\
W M \\
W M \\
W M \\
W L \\
W L \\
W L \\
W L \\
W L \\
W L \\
W L \\
W L \\
W L \\
W L \\
W L \\
W L \\
W L \\
W L \\
W L \\
W L \\
W L \\
W L\end{array}$ & $\begin{array}{l}N \\
N \\
N \\
N \\
N \\
N \\
N \\
N \\
N \\
N \\
N \\
N \\
N \\
N \\
N \\
N \\
N \\
N \\
N \\
N \\
N \\
N \\
N \\
N \\
N \\
N\end{array}$ & MG/L & $\mid<$ & $\begin{array}{l}0.08 \\
0.03 \\
0.03 \\
0.01 \\
0.17 \\
0.04 \\
0.07 \\
0.03 \\
0.11 \\
0.07 \\
0.07 \\
0.10 \\
0.10 \\
0.05 \\
0.61 \\
0.63 \\
0.70 \\
0.67 \\
0.68 \\
0.06 \\
0.07 \\
0.08 \\
0.06 \\
0.07 \\
0.03 \\
0.03\end{array}$ & $J$ & $\begin{array}{l}0.03 \\
0.03 \\
0.03 \\
0.03 \\
0.03 \\
0.03 \\
0.03 \\
0.03 \\
0.03 \\
0.03 \\
0.03 \\
0.03 \\
0.03 \\
0.03 \\
0.03 \\
0.03 \\
0.03 \\
0.03 \\
0.03 \\
0.03 \\
0.03 \\
0.03 \\
0.03 \\
0.03 \\
0.03 \\
0.03\end{array}$ & $\begin{array}{l}- \\
- \\
- \\
- \\
- \\
- \\
- \\
- \\
- \\
- \\
- \\
- \\
- \\
- \\
- \\
- \\
- \\
-\end{array}$ \\
\hline LEAD & $\begin{array}{l}0910 \\
0910 \\
0910 \\
0910 \\
0910 \\
0910 \\
0914 \\
0914 \\
0914 \\
0914 \\
0914 \\
0914\end{array}$ & $\begin{array}{l}02 / 17 / 87 \\
09 / 18 / 87 \\
01 / 30 / 88 \\
04 / 22 / 88 \\
08 / 11 / 88 \\
12 / 10 / 88 \\
02 / 18 / 87 \\
09 / 18 / 87 \\
02 / 03 / 88 \\
04 / 22 / 88 \\
08 / 08 / 88 \\
12 / 10 / 88\end{array}$ & $\begin{array}{l}0001 \\
0001 \\
0001 \\
0001 \\
0001 \\
0001 \\
0001 \\
0001 \\
0001 \\
0001 \\
0001 \\
0001\end{array}$ & $\begin{array}{l}\text { WL } \\
\text { WL } \\
W L \\
W L \\
W L \\
W L \\
\text { HL } \\
\text { WL } \\
W L \\
W L \\
W L \\
W L\end{array}$ & $\begin{array}{l}N \\
N \\
N \\
N \\
N \\
N \\
N \\
N \\
N \\
N \\
N \\
N\end{array}$ & $M G / L$ & $\begin{array}{l}< \\
< \\
< \\
< \\
< \\
< \\
< \\
< \\
< \\
< \\
< \\
<\end{array}$ & $\begin{array}{l}0.001 \\
0.02 \\
0.01 \\
0.01 \\
0.01 \\
0.01 \\
0.001 \\
0.02 \\
0.01 \\
0.01 \\
0.01 \\
0.01\end{array}$ & J & $\begin{array}{l}0.01 \\
0.02 \\
0.01 \\
0.01 \\
0.01 \\
0.01 \\
0.01 \\
0.02 \\
0.01 \\
0.01 \\
0.01 \\
0.01\end{array}$ & $\begin{array}{l}- \\
- \\
- \\
- \\
- \\
- \\
- \\
-\end{array}$ \\
\hline
\end{tabular}

FORMATION OF COMPLETION CODE:

HL - WASATCH - LOWER SANDSTONE

WM - WASATCH - MIDDLE SHALE

PARAMETER VALUE INDICATOR (PVI): < - LESS THAN DETECTION LIMIT
FLOH RELATIONSHIP CODE:

N - UNKNOWN

SAMPLE ID CODES

0001 - FILTERED SAMPLE (.45 MICRONS)

OTHER PARAMETER VALUE FLAGS

$J$ - ESTIMATED VALUE 


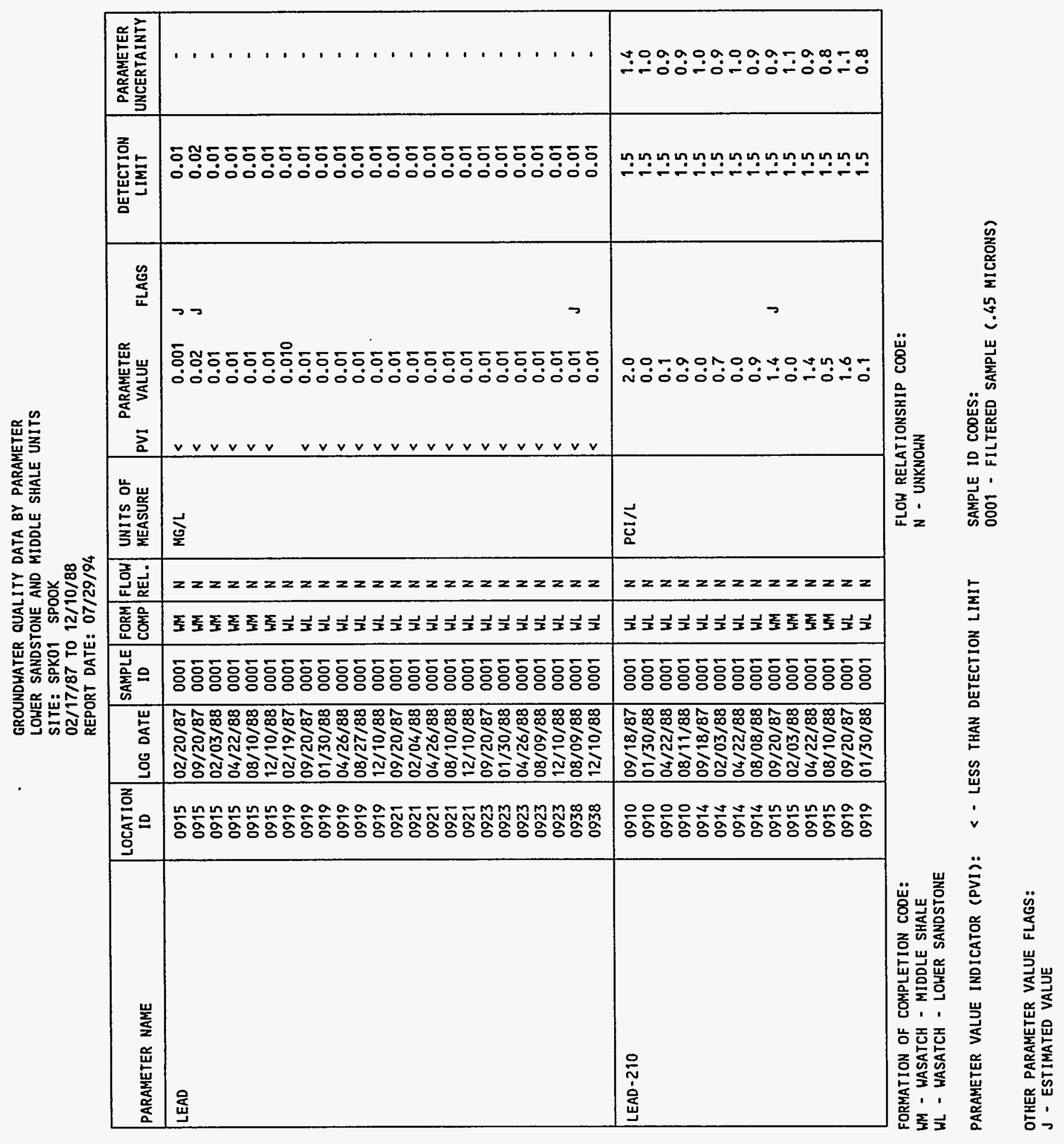


GROUNDHATER QUALITY DATA BY PARAMETER

LOWER SANDSTONE AND MIDDLE SHALE UNITS

SITE: SPKOI SPOOK

REPORT DATE: $07 / 29 / 94$

\begin{tabular}{|c|c|c|c|c|c|c|c|c|c|c|c|}
\hline PARAMETER NAME & $\begin{array}{c}\text { LOCATION } \\
\text { ID }\end{array}$ & LOG DATE & $\begin{array}{c}\text { SAMPLE } \\
\text { ID }\end{array}$ & $\begin{array}{l}\text { FORM } \\
\text { COMP }\end{array}$ & $\begin{array}{l}\text { FLOW } \\
\text { REL. }\end{array}$ & $\begin{array}{l}\text { UNITS OF } \\
\text { MEASURE }\end{array}$ & PVI & $\begin{array}{l}\text { PARAMETER } \\
\text { VALUE }\end{array}$ & FLAGS & $\begin{array}{l}\text { DETECTION } \\
\text { LIMIT }\end{array}$ & $\begin{array}{l}\text { PARAMETER } \\
\text { UNCERTAINTY }\end{array}$ \\
\hline LEAD-210 & $\begin{array}{l}0919 \\
0919 \\
0921 \\
0921 \\
0921 \\
0921 \\
0923 \\
0923 \\
0923 \\
0923 \\
0938\end{array}$ & $\begin{array}{l}04 / 26 / 88 \\
08 / 27 / 88 \\
09 / 20 / 87 \\
02 / 04 / 88 \\
04 / 26 / 88 \\
08 / 10 / 88 \\
09 / 20 / 87 \\
01 / 30 / 88 \\
04 / 26 / 88 \\
08 / 09 / 88 \\
08 / 09 / 88\end{array}$ & $\begin{array}{l}0001 \\
0001 \\
0001 \\
0009 \\
0001 \\
0001 \\
0001 \\
0001 \\
0001 \\
0001 \\
0001\end{array}$ & $\begin{array}{l}W L \\
W L \\
W L \\
W L \\
W L \\
H L \\
W L \\
W L \\
W L \\
H L \\
W L\end{array}$ & $\begin{array}{l}\text { N } \\
\text { N } \\
\text { N } \\
\text { N } \\
\text { N } \\
N \\
N \\
N \\
N \\
N \\
N\end{array}$ & PCI/L & & $\begin{array}{l}0.0 \\
0.1 \\
1.6 \\
0.0 \\
0.0 \\
0.6 \\
0.6 \\
0.1 \\
0.0 \\
0.6 \\
0.9\end{array}$ & $J$ & $\begin{array}{l}1.5 \\
1.5 \\
1.5 \\
1.5 \\
1.5 \\
1.5 \\
1.5 \\
1.5 \\
1.5 \\
1.5 \\
1.5\end{array}$ & $\begin{array}{l}0.8 \\
0.7 \\
1.0 \\
0.8 \\
0.6 \\
1.2 \\
0.9 \\
0.8 \\
0.8 \\
1.2 \\
1.0\end{array}$ \\
\hline MAGNESIUM & $\begin{array}{l}0910 \\
0910 \\
0910 \\
0910 \\
0910 \\
0910 \\
0914 \\
0914 \\
0914 \\
0914 \\
0914 \\
0914 \\
0915 \\
0915 \\
0915 \\
0915 \\
0915 \\
0915 \\
0919 \\
0919 \\
0919 \\
0919 \\
0919 \\
0919 \\
0921 \\
0921 \\
0921\end{array}$ & $\begin{array}{l}02 / 17 / 87 \\
09 / 18 / 87 \\
01 / 30 / 88 \\
04 / 22 / 88 \\
08 / 11 / 88 \\
12 / 10 / 88 \\
02 / 18 / 87 \\
09 / 18 / 87 \\
02 / 03 / 88 \\
04 / 22 / 88 \\
08 / 08 / 88 \\
12 / 10 / 88 \\
02 / 20 / 87 \\
09 / 20 / 87 \\
02 / 03 / 88 \\
04 / 22 / 88 \\
08 / 10 / 88 \\
12 / 10 / 88 \\
02 / 19 / 87 \\
09 / 20 / 87 \\
01 / 30 / 88 \\
04 / 26 / 88 \\
08 / 27 / 88 \\
12 / 10 / 88 \\
09 / 20 / 87 \\
02 / 04 / 88 \\
04 / 26 / 88\end{array}$ & $\begin{array}{l}0001 \\
0001 \\
0001 \\
0001 \\
0001 \\
0001 \\
0001 \\
0001 \\
0001 \\
0001 \\
0001 \\
0001 \\
0001 \\
0001 \\
0001 \\
0001 \\
0001 \\
0001 \\
0001 \\
0001 \\
0001 \\
0001 \\
0001 \\
0001 \\
0001 \\
0001 \\
0001\end{array}$ & $\begin{array}{l}W L \\
W L \\
W L \\
W L \\
H L \\
W L \\
W L \\
W L \\
W L \\
W L \\
W L \\
W L \\
W M \\
W M \\
W M \\
W M \\
W M \\
W M \\
W L \\
W L \\
W L \\
W L \\
W L \\
W L \\
W L \\
W L \\
W L\end{array}$ & $\begin{array}{l}N \\
N \\
N \\
N \\
N \\
N \\
N \\
N \\
N \\
N \\
N \\
N \\
N \\
N \\
N \\
N \\
N \\
N \\
N \\
N \\
N \\
N \\
N \\
N \\
N \\
N \\
N\end{array}$ & $M G / L$ & & $\begin{array}{l}12.6 \\
9.36 \\
9.70 \\
9.18 \\
9.37 \\
8.93 \\
5.44 \\
5.29 \\
5.58 \\
5.36 \\
5.54 \\
5.19 \\
7.37 \\
6.29 \\
6.88 \\
6.57 \\
6.27 \\
6.35 \\
10.4 \\
10.1 \\
10.3 \\
10.2 \\
10.7 \\
10.2 \\
20.9 \\
21.3 \\
22.2\end{array}$ & J & $\begin{array}{l}0.001 \\
0.001 \\
0.001 \\
0.001 \\
0.001 \\
0.001 \\
0.001 \\
0.001 \\
0.001 \\
0.001 \\
0.001 \\
0.001 \\
0.001 \\
0.001 \\
0.001 \\
0.001 \\
0.001 \\
0.001 \\
0.001 \\
0.001 \\
0.001 \\
0.001 \\
0.001 \\
0.001 \\
0.001 \\
0.001 \\
0.001\end{array}$ & $\begin{array}{l}- \\
- \\
: \\
- \\
- \\
- \\
- \\
- \\
- \\
- \\
- \\
- \\
- \\
- \\
- \\
- \\
- \\
-\end{array}$ \\
\hline
\end{tabular}

FORMATION OF COMPLETION CODE:

WL - HASATCH - LOWER SANDSTONE

WM - WASATCH - MIDDLE SHALE

PARAMETER VALUE INDICATOR (PVI): < - LESS THAN DETECTION LIMIT
FLOW RELATIONSHIP CODE:

N - UNKNOWN

SAMPLE ID CODES:

0001 - FILTERED SAMPLE (.45 MICRONS)

OTHER PARAMETER VALUE FLAGS:

$J$ - ESTIMATED VALUE 


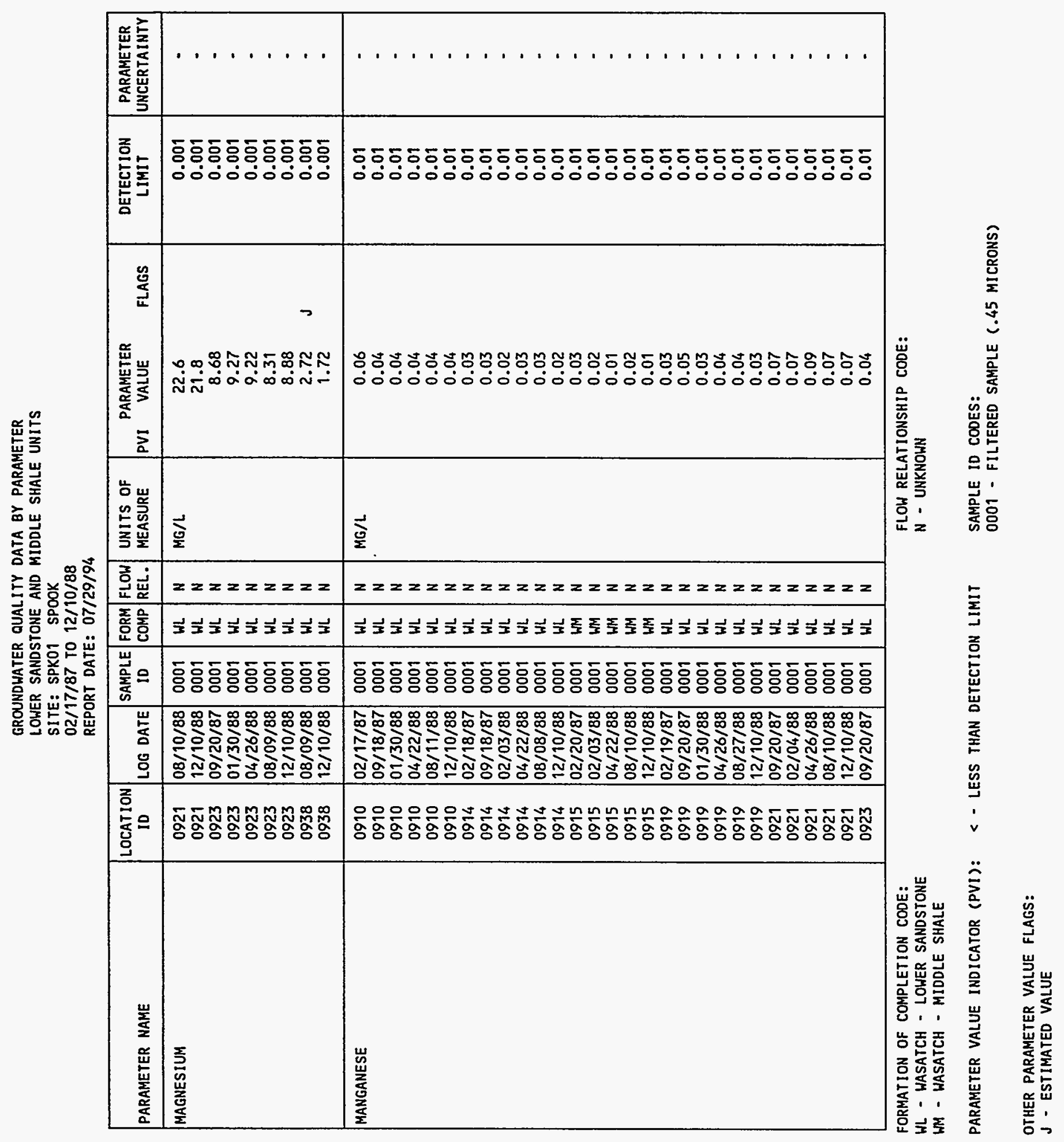


SITE: SPKO1 SPOOK

REPORT DATE: $07 / 29 / 94$

\begin{tabular}{|c|c|c|c|c|c|c|c|c|c|c|c|}
\hline PARAMETER NAME & $\begin{array}{c}\text { LOCATION } \\
\text { ID }\end{array}$ & LOG DATE & $\underset{\text { ID }}{\text { SAMPLE }}$ & $\begin{array}{l}\text { FORM } \\
\text { COMP }\end{array}$ & $\begin{array}{l}\text { FL.OW } \\
\text { REL. }\end{array}$ & $\begin{array}{l}\text { UNITS OF } \\
\text { MEASURE }\end{array}$ & PVI & $\begin{array}{l}\text { PARAMETER } \\
\text { VALUE }\end{array}$ & FLAGS & $\begin{array}{l}\text { DETECTION } \\
\text { LIMIT }\end{array}$ & \begin{tabular}{|} 
PARAMETER \\
UNCERTAINTY
\end{tabular} \\
\hline MANGANESE & $\begin{array}{l}0923 \\
0923 \\
0923 \\
0923 \\
0938 \\
0938\end{array}$ & $\begin{array}{l}01 / 30 / 88 \\
04 / 26 / 88 \\
08 / 09 / 88 \\
12 / 10 / 88 \\
08 / 09 / 88 \\
12 / 10 / 88\end{array}$ & $\begin{array}{l}0001 \\
0001 \\
0001 \\
0001 \\
0001 \\
0001\end{array}$ & $\begin{array}{l}W L \\
W L \\
W L \\
H L \\
W L \\
W L\end{array}$ & $\begin{array}{l}N \\
N \\
N \\
N \\
N \\
N\end{array}$ & $M G / L$ & $<$ & $\begin{array}{l}0.03 \\
0.04 \\
0.03 \\
0.04 \\
0.01 \\
0.01\end{array}$ & $\mathbf{J}$ & $\begin{array}{l}0.01 \\
0.01 \\
0.01 \\
0.01 \\
0.01 \\
0.01\end{array}$ & $\begin{array}{l}- \\
- \\
- \\
-\end{array}$ \\
\hline MERCURY & $\begin{array}{l}0910 \\
0910 \\
0910 \\
0910 \\
0910 \\
0914 \\
0914 \\
0914 \\
0914 \\
0914 \\
0915 \\
0915 \\
0915 \\
0915 \\
0915 \\
0919 \\
0919 \\
0919 \\
0919 \\
0919 \\
0921 \\
0921 \\
0921 \\
0921 \\
0923 \\
0923 \\
0923 \\
0923 \\
0938\end{array}$ & $\begin{array}{l}02 / 17 / 87 \\
09 / 18 / 87 \\
01 / 30 / 88 \\
04 / 22 / 88 \\
08 / 11 / 88 \\
02 / 18 / 87 \\
09 / 18 / 87 \\
02 / 03 / 88 \\
04 / 22 / 88 \\
08 / 08 / 88 \\
02 / 20 / 87 \\
09 / 20 / 87 \\
02 / 03 / 88 \\
04 / 22 / 88 \\
08 / 10 / 88 \\
02 / 19 / 87 \\
09 / 20 / 87 \\
01 / 30 / 88 \\
04 / 26 / 88 \\
08 / 27 / 88 \\
09 / 20 / 87 \\
02 / 04 / 88 \\
04 / 26 / 88 \\
08 / 10 / 88 \\
09 / 20 / 87 \\
01 / 30 / 88 \\
04 / 26 / 88 \\
08 / 09 / 88 \\
08 / 09 / 88\end{array}$ & $\begin{array}{l}0001 \\
0001 \\
0001 \\
0001 \\
0001 \\
0001 \\
0001 \\
0001 \\
0001 \\
0001 \\
0001 \\
0001 \\
0001 \\
0001 \\
0001 \\
0001 \\
0001 \\
0001 \\
0001 \\
0001 \\
0001 \\
0001 \\
0001 \\
0001 \\
0001 \\
0001 \\
0001 \\
0001 \\
0001\end{array}$ & $\begin{array}{l}W L \\
W L \\
W L \\
W L \\
W L \\
W L \\
W L \\
W L \\
W L \\
W L \\
W M \\
W M \\
W M \\
W M \\
W M \\
W L \\
W L \\
W L \\
W L \\
W L \\
W L \\
W L \\
W L \\
W L \\
W L \\
W L \\
W L \\
W L \\
W L\end{array}$ & $\begin{array}{l}N \\
N \\
N \\
N \\
N \\
N \\
N \\
N \\
N \\
N \\
N \\
N \\
N \\
N \\
N \\
N \\
N \\
N \\
N \\
N \\
N \\
N \\
N \\
N \\
N \\
N \\
N \\
N \\
N\end{array}$ & $M G / L$ & $\begin{array}{l}< \\
< \\
< \\
< \\
< \\
< \\
< \\
< \\
< \\
< \\
< \\
< \\
< \\
< \\
< \\
< \\
< \\
< \\
<\end{array}$ & $\begin{array}{l}0.0002 \\
0.0002 \\
0.0004 \\
0.0002 \\
0.0002 \\
0.0002 \\
0.0002 \\
0.0004 \\
0.0002 \\
0.0002 \\
0.0002 \\
0.0002 \\
0.0004 \\
0.0002 \\
0.0002 \\
0.0003 \\
0.0002 \\
0.0003 \\
0.0004 \\
0.0002 \\
0.0002 \\
0.0002 \\
0.0005 \\
0.0002 \\
0.0002 \\
0.0002 \\
0.0007 \\
0.0002 \\
0.0002\end{array}$ & $J$ & $\begin{array}{l}0.0002 \\
0.0002 \\
0.0002 \\
0.0002 \\
0.0002 \\
0.0002 \\
0.0002 \\
0.0002 \\
0.0002 \\
0.0002 \\
0.0002 \\
0.0002 \\
0.0002 \\
0.0002 \\
0.0002 \\
0.0002 \\
0.0002 \\
0.0002 \\
0.0002 \\
0.0002 \\
0.0002 \\
0.0002 \\
0.0002 \\
0.0002 \\
0.0002 \\
0.0002 \\
0.0002 \\
0.0002 \\
0.0002\end{array}$ & 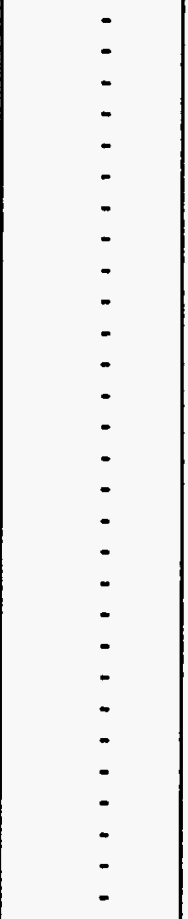 \\
\hline MOLYBBDENUM & $\begin{array}{l}0910 \\
0910\end{array}$ & $\begin{array}{l}02 / 17 / 87 \\
09 / 18 / 87\end{array}$ & $\begin{array}{l}0001 \\
0001\end{array}$ & $\begin{array}{l}\text { WL } \\
\text { WL }\end{array}$ & $\stackrel{N}{N}$ & $M G / L$ & $<<$ & $\begin{array}{l}0.1 \\
0.01\end{array}$ & & $\begin{array}{l}0.1 \\
0.01\end{array}$ & $\dot{-}$ \\
\hline
\end{tabular}

FORMATION OF COMPLETION CODE:

WL - WASATCH - LOWER SANDSTONE

HM - HASATCH - MIDDLE SHALE

PARAMETER VALUE INDICATOR (PVI): < - LESS THAN DETECTION LIMIT
FLOW RELATIONSHIP CODE:

N - UNKNOWN

OTHER PARAMETER VALUE FLAGS:

$\checkmark$ - ESTIMATED VALUE 
REPORT DATE: $07 / 29 / 9$

\begin{tabular}{|c|c|c|c|c|c|c|c|c|c|c|c|}
\hline PARAMETER NAME & $\begin{array}{c}\text { LOCATION } \\
\text { ID }\end{array}$ & LOG DATE & $\begin{array}{c}\text { SAMPLE } \\
\text { ID }\end{array}$ & $\begin{array}{l}\text { FORM } \\
\text { COMP }\end{array}$ & $\begin{array}{l}\text { FLOW } \\
\text { REL. }\end{array}$ & $\begin{array}{l}\text { UNITS OF } \\
\text { MEASURE }\end{array}$ & PVI & $\begin{array}{l}\text { PARAMETER } \\
\text { VALUE }\end{array}$ & FLAGS & $\begin{array}{l}\text { DETECTION } \\
\text { LIMIT }\end{array}$ & $\begin{array}{l}\text { PARAMETER } \\
\text { UNCERTAINTY }\end{array}$ \\
\hline MOLYBDENUM & $\begin{array}{l}0910 \\
0910 \\
0910 \\
0910 \\
0914 \\
0914 \\
0914 \\
0914 \\
0914 \\
0914 \\
0915 \\
0915 \\
0915 \\
0915 \\
0915 \\
0915 \\
0919 \\
0919 \\
0919 \\
0919 \\
0919 \\
0919 \\
0921 \\
0921 \\
0921 \\
0921 \\
0923 \\
0923 \\
0923 \\
0923 \\
0923 \\
0938 \\
0938\end{array}$ & $\begin{array}{l}01 / 30 / 88 \\
04 / 22 / 88 \\
08 / 11 / 88 \\
12 / 10 / 88 \\
02 / 18 / 87 \\
09 / 18 / 87 \\
02 / 03 / 88 \\
04 / 22 / 88 \\
08 / 08 / 88 \\
12 / 10 / 88 \\
02 / 20 / 87 \\
09 / 20 / 87 \\
02 / 03 / 88 \\
04 / 22 / 88 \\
08 / 10 / 88 \\
12 / 10 / 88 \\
02 / 19 / 87 \\
09 / 20 / 87 \\
01 / 30 / 88 \\
04 / 26 / 88 \\
08 / 27 / 88 \\
12 / 10 / 88 \\
09 / 20 / 87 \\
02 / 04 / 88 \\
08 / 10 / 88 \\
12 / 10 / 88 \\
09 / 20 / 87 \\
01 / 30 / 88 \\
04 / 26 / 88 \\
08 / 09 / 88 \\
12 / 10 / 88 \\
08 / 09 / 88 \\
12 / 10 / 88\end{array}$ & $\begin{array}{l}0001 \\
0001 \\
0001 \\
0001 \\
0001 \\
0001 \\
0001 \\
0001 \\
0001 \\
0001 \\
0001 \\
0001 \\
0001 \\
0001 \\
0001 \\
0001 \\
0001 \\
0001 \\
0001 \\
0001 \\
0001 \\
0001 \\
0001 \\
0001 \\
0001 \\
0001 \\
0001 \\
0001 \\
0001 \\
0001 \\
0001 \\
0001 \\
0001\end{array}$ & $\begin{array}{l}W L \\
W L \\
W L \\
W L \\
W L \\
W L \\
W L \\
W L \\
W L \\
W L \\
W M \\
W M \\
W M \\
W M \\
W M \\
W M \\
W L \\
W L \\
W L \\
W L \\
W L \\
W L \\
W L \\
W L \\
W L \\
W L \\
W L \\
W L \\
W L \\
W L \\
W L \\
W L \\
W L\end{array}$ & $\begin{array}{l}N \\
N \\
N \\
N \\
N \\
N \\
N \\
N \\
N \\
N \\
N \\
N \\
N \\
N \\
N \\
N \\
N \\
N \\
N \\
N \\
N \\
N \\
N \\
N \\
N \\
N \\
N \\
N \\
N \\
N \\
N \\
N \\
N\end{array}$ & MG/L & $\begin{array}{l}< \\
< \\
< \\
< \\
< \\
< \\
< \\
< \\
< \\
< \\
< \\
< \\
< \\
< \\
< \\
< \\
< \\
< \\
< \\
< \\
<\end{array}$ & $\begin{array}{l}0.01 \\
0.01 \\
0.01 \\
0.01 \\
0.1 \\
0.01 \\
0.01 \\
0.01 \\
0.01 \\
0.01 \\
0.2 \\
0.01 \\
0.01 \\
0.01 \\
0.01 \\
0.01 \\
0.1 \\
0.01 \\
0.02 \\
0.01 \\
0.01 \\
0.01 \\
0.01 \\
0.03 \\
0.01 \\
0.01 \\
0.01 \\
0.02 \\
0.01 \\
0.01 \\
0.01 \\
0.01 \\
0.01\end{array}$ & $\mathbf{J}$ & $\begin{array}{l}0.01 \\
0.01 \\
0.01 \\
0.01 \\
0.1 \\
0.01 \\
0.01 \\
0.01 \\
0.01 \\
0.01 \\
0.01 \\
0.01 \\
0.01 \\
0.01 \\
0.01 \\
0.01 \\
0.1 \\
0.01 \\
0.01 \\
0.01 \\
0.01 \\
0.01 \\
0.01 \\
0.01 \\
0.01 \\
0.01 \\
0.01 \\
0.01 \\
0.01 \\
0.01 \\
0.01 \\
0.01 \\
0.01\end{array}$ & 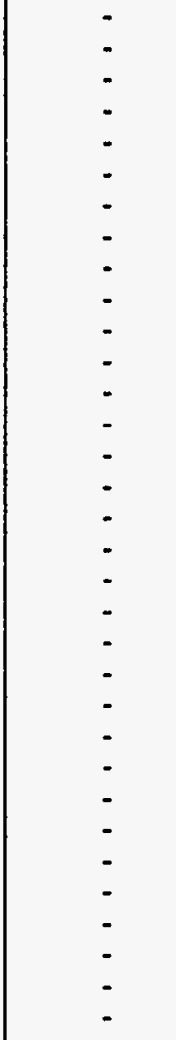 \\
\hline NET GROSS ALPHA * & $\begin{array}{l}0910 \\
0910 \\
0910\end{array}$ & $\begin{array}{l}09 / 18 / 87 \\
01 / 30 / 88 \\
04 / 22 / 88\end{array}$ & $\begin{array}{l}0001 \\
0001 \\
0001\end{array}$ & $\begin{array}{l}W L \\
W L \\
W L\end{array}$ & $\begin{array}{l}N \\
N \\
N\end{array}$ & $\mathrm{PCI} / \mathrm{L}$ & & $\begin{array}{r}4.46 \\
14.38 \\
4.79\end{array}$ & & - & - \\
\hline
\end{tabular}

* Net gross alPha (GROSS ALPHA - URANIUM) WITH 1 MG URANIUM = 686 PCI

FORMATION OF COMPLETION CODE:

WL - HASATCH - LOWER SANDSTONE

WM - WASATCH - MIDDLE SHALE

PARAMETER VALUE INDICATOR (PVI): < - LESS THAN DETECTION LIMIT
FLOH RELATIONSHIP CODE:

N - UNKNOWN

SAMPLE ID CODES

0001 - FILTERED SAMPLE (.45 MICRONS)

OTHER PARAMETER VALUE FLAGS

$J$ - ESTIMATED VALUE 
OLPORT DATE: $07 / 29 / 94$

\begin{tabular}{|c|c|c|c|c|c|c|c|c|c|c|c|}
\hline PARAMETER NAME & $\underset{\text { ID }}{\text { LOCATION }}$ & LOG DATE & $\begin{array}{c}\text { SAMPLE } \\
\text { ID }\end{array}$ & $\left|\begin{array}{l}\text { FORM } \\
\text { COMP }\end{array}\right|$ & $\begin{array}{l}\text { FLOW } \\
\text { REL. }\end{array}$ & $\begin{array}{l}\text { UNITS OF } \\
\text { MEASURE }\end{array}$ & PVI & $\begin{array}{l}\text { PARAMETER } \\
\text { VALUE }\end{array}$ & FLAGS & $\begin{array}{l}\text { DETECTION } \\
\text { LIMIT }\end{array}$ & $\begin{array}{l}\text { PARAMETER } \\
\text { UNCERTAINTY }\end{array}$ \\
\hline NET GROSS ALPHA * & $\begin{array}{l}0910 \\
0910 \\
0914 \\
0914 \\
0914 \\
0914 \\
0914 \\
0915 \\
0915 \\
0915 \\
0915 \\
0915 \\
0919 \\
0919 \\
0919 \\
0919 \\
0919 \\
0921 \\
0921 \\
0921 \\
0921 \\
0921 \\
0923 \\
0923 \\
0923 \\
0923 \\
0923 \\
0938 \\
0938\end{array}$ & $\begin{array}{l}08 / 11 / 88 \\
12 / 10 / 88 \\
09 / 18 / 87 \\
02 / 03 / 88 \\
04 / 22 / 88 \\
08 / 08 / 88 \\
12 / 10 / 88 \\
09 / 20 / 87 \\
02 / 03 / 88 \\
04 / 22 / 88 \\
08 / 10 / 88 \\
12 / 10 / 88 \\
09 / 20 / 87 \\
01 / 30 / 88 \\
04 / 26 / 88 \\
08 / 27 / 88 \\
12 / 10 / 88 \\
09 / 20 / 87 \\
02 / 04 / 88 \\
04 / 26 / 88 \\
08 / 10 / 88 \\
12 / 10 / 88 \\
09 / 20 / 87 \\
01 / 30 / 88 \\
04 / 26 / 88 \\
08 / 09 / 88 \\
12 / 10 / 88 \\
08 / 09 / 88 \\
12 / 10 / 88\end{array}$ & $\begin{array}{l}0001 \\
0001 \\
0001 \\
0001 \\
0001 \\
0001 \\
0001 \\
0001 \\
0001 \\
0001 \\
0001 \\
0001 \\
0001 \\
0001 \\
0001 \\
0001 \\
0001 \\
0001 \\
0001 \\
0001 \\
0001 \\
0001 \\
0001 \\
0001 \\
0001 \\
0001 \\
0001 \\
0001 \\
0001\end{array}$ & 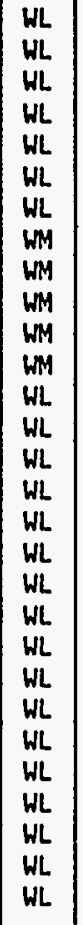 & $\begin{array}{l}N \\
N \\
N \\
N \\
N \\
N \\
N \\
N \\
N \\
N \\
N \\
N \\
N \\
N \\
N \\
N \\
N \\
N \\
N \\
N \\
N \\
N \\
N \\
N \\
N \\
N \\
N \\
N \\
N\end{array}$ & $\mathrm{PCI} / \mathrm{L}$ & & $\begin{array}{r}4.97 \\
5.07 \\
0.84 \\
3.09 \\
2.45 \\
0.63 \\
10.18 \\
9.92 \\
4.49 \\
-3.74 \\
1.17 \\
4.08 \\
6.54 \\
4.04 \\
5.32 \\
6.06 \\
3.87 \\
6.21 \\
10.38 \\
9.01 \\
2.66 \\
-0.41 \\
7.46 \\
5.03 \\
5.07 \\
1.47 \\
3.35 \\
-1.03 \\
1.23\end{array}$ & & $\begin{array}{l}- \\
- \\
- \\
i \\
i \\
- \\
- \\
- \\
- \\
- \\
- \\
- \\
- \\
- \\
- \\
- \\
- \\
- \\
- \\
-\end{array}$ & $\begin{array}{l}- \\
- \\
- \\
- \\
- \\
- \\
- \\
- \\
- \\
- \\
- \\
- \\
- \\
- \\
- \\
- \\
- \\
- \\
- \\
- \\
- \\
- \\
- \\
- \\
-\end{array}$ \\
\hline NICKEL & $\begin{array}{l}0910 \\
0910 \\
0910 \\
0910 \\
0914 \\
0914 \\
0914\end{array}$ & $\begin{array}{l}09 / 18 / 87 \\
01 / 30 / 88 \\
04 / 22 / 88 \\
08 / 11 / 88 \\
09 / 18 / 87 \\
02 / 03 / 88 \\
04 / 22 / 88\end{array}$ & $\begin{array}{l}0001 \\
0001 \\
0001 \\
0001 \\
0001 \\
0001 \\
0001\end{array}$ & $\begin{array}{l}\text { WL } \\
W L \\
W L \\
W L \\
W L \\
W L \\
W L\end{array}$ & $\begin{array}{l}N \\
N \\
N \\
N \\
N \\
N \\
N\end{array}$ & $M G / L$ & $\begin{array}{l}< \\
< \\
< \\
< \\
< \\
<\end{array}$ & $\begin{array}{l}0.01 \\
0.01 \\
0.01 \\
0.04 \\
0.01 \\
0.01 \\
0.01\end{array}$ & $\begin{array}{l}J \\
J \\
j \\
j \\
j \\
J\end{array}$ & $\begin{array}{l}0.04 \\
0.04 \\
0.04 \\
0.04 \\
0.04 \\
0.04 \\
0.04\end{array}$ & $\begin{array}{l}- \\
- \\
- \\
- \\
-\end{array}$ \\
\hline
\end{tabular}

* NET GROSS ALPHA (GROSS ALPHA - URANIUM) HITH 1 MG URANIUM $=686$ PCI

FORMATION OF COMPLETION CODE:

WL - WASATCH - LOWER SANDSTONE

WM - WASATCH - MIDDLE SHALE

PARAMETER VALUE INDICATOR (PVI): < - LESS THAN DETECTION LIMIT

OTHER PARAMETER VALUE FLAGS:

$J$ - ESTIMATED VALUE
FLOW RELATIONSHIP CODE: N - UNKNOWN

SAMPLE ID CODES:

0001 - FILTERED SAMPLE (.45 MICRONS) 
SITE: SPKO1 SPOOK

02/17/87 TO $12 / 10 / 88$

REPORT DATE: $07 / 29 / 94$

\begin{tabular}{|c|c|c|c|c|c|c|c|c|c|c|c|}
\hline PARAMETER NAME & $\underset{10}{\text { LOCATION }}$ & LOG DATE & $\begin{array}{c}\text { SAMPLE } \\
\text { ID }\end{array}$ & $\begin{array}{l}\text { FORM } \\
\text { COMP }\end{array}$ & $\begin{array}{l}\text { FLOW } \\
\text { REL. }\end{array}$ & $\begin{array}{l}\text { UNITS OF } \\
\text { MEASURE }\end{array}$ & PVI & $\begin{array}{l}\text { PARAMETER } \\
\text { VALUE }\end{array}$ & FLAGS & $\begin{array}{l}\text { DETECTION } \\
\text { LIMIT }\end{array}$ & $\begin{array}{l}\text { PARAMETER } \\
\text { UNCERTAINTY }\end{array}$ \\
\hline NICKEL & $\begin{array}{l}0914 \\
0915 \\
0915 \\
0915 \\
0915 \\
0919 \\
0919 \\
0919 \\
0919 \\
0921 \\
0921 \\
0921 \\
0921 \\
0923 \\
0923 \\
0923 \\
0923 \\
0938\end{array}$ & $\begin{array}{l}08 / 08 / 88 \\
09 / 20 / 87 \\
02 / 03 / 88 \\
04 / 22 / 88 \\
08 / 10 / 88 \\
09 / 20 / 87 \\
01 / 30 / 88 \\
04 / 26 / 88 \\
08 / 27 / 88 \\
09 / 20 / 87 \\
02 / 04 / 88 \\
04 / 26 / 88 \\
08 / 10 / 88 \\
09 / 20 / 87 \\
01 / 30 / 88 \\
04 / 26 / 88 \\
08 / 09 / 88 \\
08 / 09 / 88\end{array}$ & $\begin{array}{l}0001 \\
0001 \\
0001 \\
0001 \\
0001 \\
0001 \\
0001 \\
0001 \\
0001 \\
0001 \\
0001 \\
0001 \\
0001 \\
0001 \\
0001 \\
0001 \\
0001 \\
0001\end{array}$ & $\begin{array}{l}W L \\
W M \\
W M \\
W M \\
W M \\
W L \\
W L \\
W L \\
W L \\
W L \\
W L \\
W L \\
W L \\
W L \\
W L \\
W L \\
W L \\
W L\end{array}$ & $\begin{array}{l}N \\
N \\
N \\
N \\
N \\
N \\
N \\
N \\
N \\
N \\
N \\
N \\
N \\
N \\
N \\
N \\
N \\
N\end{array}$ & $M G / L$ & $\begin{array}{l}< \\
< \\
< \\
< \\
< \\
< \\
< \\
< \\
< \\
< \\
< \\
< \\
<\end{array}$ & $\begin{array}{l}0.04 \\
0.01 \\
0.01 \\
0.01 \\
0.04 \\
0.01 \\
0.01 \\
0.01 \\
0.04 \\
0.01 \\
0.01 \\
0.02 \\
0.04 \\
0.01 \\
0.01 \\
0.01 \\
0.04 \\
0.04\end{array}$ & $\begin{array}{l}J \\
J \\
J \\
J \\
J \\
J \\
J \\
J \\
J \\
J \\
J \\
J \\
J\end{array}$ & $\begin{array}{l}0.04 \\
0.04 \\
0.04 \\
0.04 \\
0.04 \\
0.04 \\
0.04 \\
0.04 \\
0.04 \\
0.04 \\
0.04 \\
0.04 \\
0.04 \\
0.04 \\
0.04 \\
0.04 \\
0.04 \\
0.04\end{array}$ & $\begin{array}{l}- \\
- \\
- \\
- \\
- \\
- \\
- \\
- \\
- \\
- \\
- \\
- \\
- \\
-\end{array}$ \\
\hline NI TRATE & $\begin{array}{l}0910 \\
0910 \\
0910 \\
0910 \\
0910 \\
0910 \\
0914 \\
0914 \\
0914 \\
0914 \\
0914 \\
0914 \\
0915 \\
0915 \\
0915 \\
0915 \\
0915 \\
0915 \\
0919 \\
0919\end{array}$ & $\begin{array}{l}02 / 17 / 87 \\
09 / 18 / 87 \\
01 / 30 / 88 \\
04 / 22 / 88 \\
08 / 11 / 88 \\
12 / 10 / 88 \\
02 / 18 / 87 \\
09 / 18 / 87 \\
02 / 03 / 88 \\
04 / 22 / 88 \\
08 / 08 / 88 \\
12 / 10 / 88 \\
02 / 20 / 87 \\
09 / 20 / 87 \\
02 / 03 / 88 \\
04 / 22 / 88 \\
08 / 10 / 88 \\
12 / 10 / 88 \\
02 / 19 / 87 \\
09 / 20 / 87\end{array}$ & $\begin{array}{l}0001 \\
0001 \\
0001 \\
0001 \\
0001 \\
0001 \\
0001 \\
0001 \\
0001 \\
0001 \\
0001 \\
0001 \\
0001 \\
0001 \\
0001 \\
0001 \\
0001 \\
0001 \\
0001 \\
0001\end{array}$ & $\begin{array}{l}W L \\
W L \\
W L \\
W L \\
W L \\
W L \\
W L \\
W L \\
W L \\
W L \\
W L \\
W L \\
W M \\
W M \\
W M \\
W M \\
W M \\
W M \\
W L \\
W L\end{array}$ & $\begin{array}{l}N \\
N \\
N \\
N \\
N \\
N \\
N \\
N \\
N \\
N \\
N \\
N \\
N \\
N \\
N \\
N \\
N \\
N \\
N \\
N\end{array}$ & $M G / L$ & $<$ & $\begin{array}{r}60.2 \\
2.7 \\
0.1 \\
2.2 \\
1.0 \\
7.6 \\
0.4 \\
1.8 \\
0.1 \\
3.8 \\
1.6 \\
7.0 \\
0.4 \\
0.9 \\
0.7 \\
3.9 \\
3.8 \\
1.0 \\
0.8 \\
0.1\end{array}$ & $\begin{array}{l}J \\
J \\
J \\
J \\
J \\
J\end{array}$ & $\begin{array}{l}1 . \\
1 . \\
1 . \\
i . \\
1 . \\
1 . \\
1 . \\
1 . \\
1 . \\
1 . \\
1 . \\
1 . \\
1 . \\
1 . \\
1 .\end{array}$ & $\begin{array}{l}- \\
- \\
- \\
- \\
- \\
: \\
- \\
- \\
- \\
- \\
- \\
- \\
- \\
- \\
- \\
-\end{array}$ \\
\hline
\end{tabular}

FORMATION OF COMPLETION CODE:

WL - WASATCH - LOWER SANDSTONE

WM - WASATCH - MIDDLE SHALE

PARAMETER VALUE INDICATOR (PVI): < - LESS THAN DETECTION LIMIT
FLOW RELATIONSHIP CODE:

N - UNKNOWN

\section{SAMPLE ID CODES:}

0001 - FILTERED SAMPLE (.45 MICRONS)

OTHER PARAMETER VALUE FLAGS:

$J$ - ESTIMATED VALUE 
GROUMDHATER QUALITY DATA BY PARAMETER

LOWER SANDSTONE AND MIDDLE SHALE UNITS

SITE: SPKO1 SPOOK

$02 / 17 / 87$ TO $12 / 10 / 88$

REPORT DATE: $07 / 29 / 94$

\begin{tabular}{|c|c|c|c|c|c|c|c|c|c|c|c|}
\hline PARAMETER NAME & $\underset{\text { LOCATION }}{\text { LO }}$ & LOG DATE & $\begin{array}{c}\text { SAMPLE } \\
\text { ID }\end{array}$ & $\begin{array}{l}\text { FORM } \\
\text { COMP }\end{array}$ & $\begin{array}{l}\text { FLOW } \\
\text { REL. }\end{array}$ & $\begin{array}{l}\text { UNITS OF } \\
\text { MEASURE }\end{array}$ & PVI & $\begin{array}{l}\text { PARAMETER } \\
\text { VALUE }\end{array}$ & FLAGS & $\begin{array}{l}\text { DETECTION } \\
\text { LIMIT }\end{array}$ & $\begin{array}{l}\text { PARAMETER } \\
\text { UNCERTAINTY }\end{array}$ \\
\hline NITRATE & $\begin{array}{l}0919 \\
0919 \\
0919 \\
0919 \\
0921 \\
0921 \\
0921 \\
0921 \\
0921 \\
0923 \\
0923 \\
0923 \\
0923 \\
0923 \\
0938 \\
0938\end{array}$ & $\begin{array}{l}01 / 30 / 88 \\
04 / 26 / 88 \\
08 / 27 / 88 \\
12 / 10 / 88 \\
09 / 20 / 87 \\
02 / 04 / 88 \\
04 / 26 / 88 \\
08 / 10 / 88 \\
12 / 10 / 88 \\
09 / 20 / 87 \\
01 / 30 / 88 \\
04 / 26 / 88 \\
08 / 09 / 88 \\
12 / 10 / 88 \\
08 / 09 / 88 \\
12 / 10 / 88\end{array}$ & $\begin{array}{l}0001 \\
0001 \\
0001 \\
0001 \\
0001 \\
0001 \\
0001 \\
0001 \\
0001 \\
0001 \\
0001 \\
0001 \\
0001 \\
0001 \\
0001 \\
0001\end{array}$ & $\begin{array}{l}W L \\
W L \\
W L \\
H L \\
W L \\
W L \\
H L \\
W L \\
W L \\
H L \\
W L \\
W L \\
H L \\
H L \\
H L \\
W L\end{array}$ & $\begin{array}{l}N \\
N \\
N \\
N \\
N \\
N \\
N \\
N \\
N \\
N \\
N \\
N \\
N \\
N \\
N \\
N\end{array}$ & $M G / L$ & $\begin{array}{l}< \\
< \\
< \\
< \\
<\end{array}$ & $\begin{array}{l}0.1 \\
2.3 \\
1.9 \\
1.0 \\
0.1 \\
1.8 \\
0.8 \\
1.0 \\
5.2 \\
0.4 \\
0.4 \\
1.6 \\
1.0 \\
5.5 \\
1.0 \\
5.9\end{array}$ & $\begin{array}{l}J \\
J \\
J \\
J\end{array}$ & $\begin{array}{l}1 . \\
1 . \\
1 . \\
1 . \\
1 . \\
1 . \\
1 . \\
1 . \\
1 . \\
1 . \\
1 . \\
1 . \\
1 . \\
1 .\end{array}$ & $\begin{array}{l}- \\
- \\
- \\
- \\
- \\
- \\
- \\
- \\
- \\
- \\
-\end{array}$ \\
\hline PH & $\begin{array}{l}0910 \\
0910 \\
0910 \\
0910 \\
0910 \\
0910 \\
0914 \\
0914 \\
0914 \\
0914 \\
0914 \\
0914 \\
0915 \\
0915 \\
0915 \\
0915 \\
0915 \\
0915 \\
0919 \\
0919 \\
0919 \\
0919\end{array}$ & $\begin{array}{l}02 / 17 / 87 \\
09 / 18 / 87 \\
01 / 30 / 88 \\
04 / 22 / 88 \\
08 / 11 / 88 \\
12 / 10 / 88 \\
02 / 18 / 87 \\
09 / 18 / 87 \\
02 / 03 / 88 \\
04 / 22 / 88 \\
08 / 08 / 88 \\
12 / 10 / 88 \\
02 / 20 / 87 \\
09 / 20 / 87 \\
02 / 03 / 88 \\
04 / 22 / 88 \\
08 / 10 / 88 \\
12 / 10 / 88 \\
02 / 19 / 87 \\
09 / 20 / 87 \\
01 / 30 / 88 \\
04 / 26 / 88\end{array}$ & $\begin{array}{l}0001 \\
0001 \\
0001 \\
0001 \\
0001 \\
0001 \\
0001 \\
0001 \\
0001 \\
0001 \\
0001 \\
0001 \\
0001 \\
0001 \\
0001 \\
0001 \\
0001 \\
0001 \\
0001 \\
0001 \\
0001 \\
0001\end{array}$ & $\begin{array}{l}H L \\
W L \\
W L \\
W L \\
W L \\
W L \\
W L \\
W L \\
H L \\
W L \\
W L \\
W L \\
W M \\
W M \\
W M \\
W M \\
W M \\
W M \\
H L \\
W L \\
W L \\
W L\end{array}$ & $\begin{array}{l}N \\
N \\
N \\
N \\
N \\
N \\
N \\
N \\
N \\
N \\
N \\
N \\
N \\
N \\
N \\
N \\
N \\
N \\
N \\
N \\
N \\
N\end{array}$ & su & & $\begin{array}{l}8.54 \\
7.77 \\
8.00 \\
7.81 \\
7.67 \\
7.96 \\
8.18 \\
8.09 \\
8.12 \\
8.03 \\
7.85 \\
8.15 \\
7.68 \\
7.70 \\
7.43 \\
7.78 \\
7.62 \\
7.67 \\
7.87 \\
7.44 \\
7.75 \\
7.47\end{array}$ & & $\begin{array}{l}- \\
: \\
- \\
- \\
- \\
- \\
- \\
- \\
- \\
- \\
- \\
- \\
- \\
- \\
- \\
- \\
- \\
-\end{array}$ & $\begin{array}{l}- \\
- \\
- \\
- \\
: \\
: \\
- \\
- \\
- \\
- \\
- \\
- \\
- \\
- \\
-\end{array}$ \\
\hline
\end{tabular}

FORMATION OF COMPLETION CODE:

FLOW RELATIONSHIP CODE:

WL - HASATCH - LOHER SANDSTONE

N - UNKNOWN

WASATCH - MIODLE SHALE

SAMPLE ID CODES:

0001 - FILTERED SAMPLE (.45 MICRONS)

OTHER PARAMETER VALUE FLAGS

$J$ - EST IMATED VALUE 
GROUNDWATER QUALITY DATA BY PARAMETER

LOWER SANDSTONE AND MIDDLE SHALE UNITS

SITE: SPKO1 SPOOK

$02 / 17 / 87$ TO $12 / 10 / 88$

REPORT DATE: 07/29/94

\begin{tabular}{|c|c|c|c|c|c|c|c|c|c|c|c|}
\hline PARAMETER NAME & $\mid \begin{array}{c}\text { LOCATION } \\
\text { ID }\end{array}$ & LOG DATE & $\begin{array}{c}\text { SAMPLE } \\
10\end{array}$ & $\begin{array}{l}\text { FORM } \\
\text { COMP }\end{array}$ & $\begin{array}{l}\text { FLOH } \\
\text { REL. }\end{array}$ & $\begin{array}{l}\text { UNITS OF } \\
\text { MEASURE }\end{array}$ & PVI & $\begin{array}{l}\text { PARAMETER } \\
\text { VALUE }\end{array}$ & FLAGS & $\begin{array}{l}\text { DETECTION } \\
\text { LIMIT }\end{array}$ & $\begin{array}{l}\text { PARAMETER } \\
\text { UNCERTAINTY }\end{array}$ \\
\hline PH & $\begin{array}{l}0919 \\
0919 \\
0921 \\
0921 \\
0921 \\
0921 \\
0921 \\
0923 \\
0923 \\
0923 \\
0923 \\
0923 \\
0938 \\
0938\end{array}$ & $\begin{array}{l}08 / 27 / 88 \\
12 / 10 / 88 \\
09 / 20 / 87 \\
02 / 04 / 88 \\
04 / 26 / 88 \\
08 / 10 / 88 \\
12 / 10 / 88 \\
09 / 20 / 87 \\
01 / 30 / 88 \\
04 / 26 / 88 \\
08 / 09 / 88 \\
12 / 10 / 88 \\
08 / 09 / 88 \\
12 / 10 / 88\end{array}$ & $\begin{array}{l}0001 \\
0001 \\
0001 \\
0001 \\
0001 \\
0001 \\
0001 \\
0001 \\
0001 \\
0001 \\
0001 \\
0001 \\
0001 \\
0001\end{array}$ & $\begin{array}{l}W L \\
W L \\
W L \\
W L \\
W L \\
W L \\
W L \\
W L \\
W L \\
W L \\
W L \\
W L \\
W L \\
W L\end{array}$ & $\begin{array}{l}N \\
N \\
N \\
N \\
N \\
N \\
N \\
N \\
N \\
N \\
N \\
N \\
N \\
N\end{array}$ & su & & $\begin{array}{l}7.43 \\
7.55 \\
7.25 \\
7.13 \\
7.15 \\
7.13 \\
7.28 \\
7.57 \\
7.85 \\
7.53 \\
7.48 \\
7.71 \\
7.94 \\
8.55\end{array}$ & & $\begin{array}{l}: \\
: \\
: \\
: \\
: \\
: \\
: \\
- \\
-\end{array}$ & $\begin{array}{l}- \\
- \\
- \\
- \\
- \\
- \\
- \\
- \\
- \\
- \\
-\end{array}$ \\
\hline PHOSPHATE & $\begin{array}{l}0910 \\
0910 \\
0910 \\
0910 \\
0910 \\
0914 \\
0914 \\
0914 \\
0914 \\
0914 \\
0915 \\
0915 \\
0915 \\
0915 \\
0919 \\
0919 \\
0919 \\
0919 \\
0919 \\
0921 \\
0921 \\
0921 \\
0921 \\
0923\end{array}$ & $\begin{array}{l}02 / 17 / 87 \\
09 / 18 / 87 \\
01 / 30 / 88 \\
04 / 22 / 88 \\
08 / 11 / 88 \\
02 / 18 / 87 \\
09 / 18 / 87 \\
02 / 03 / 88 \\
04 / 22 / 88 \\
08 / 08 / 88 \\
02 / 20 / 87 \\
09 / 20 / 87 \\
02 / 03 / 88 \\
04 / 22 / 88 \\
02 / 19 / 87 \\
09 / 20 / 87 \\
01 / 30 / 88 \\
04 / 26 / 88 \\
08 / 27 / 88 \\
09 / 20 / 87 \\
02 / 04 / 88 \\
04 / 26 / 88 \\
08 / 10 / 88 \\
09 / 20 / 87\end{array}$ & $\begin{array}{l}0001 \\
0001 \\
0001 \\
0001 \\
0001 \\
0001 \\
0001 \\
0001 \\
0001 \\
0001 \\
0001 \\
0001 \\
0001 \\
0001 \\
0001 \\
0001 \\
0001 \\
0001 \\
0001 \\
0001 \\
0001 \\
0001 \\
0001 \\
0001\end{array}$ & $\begin{array}{l}W L \\
W L \\
W L \\
W L \\
W L \\
W L \\
W L \\
W L \\
W L \\
W L \\
W M \\
W M \\
W M \\
W M \\
W L \\
W L \\
W L \\
W L \\
W L \\
H L \\
W L \\
W L \\
W L \\
W L\end{array}$ & $\begin{array}{l}N \\
N \\
N \\
N \\
N \\
N \\
N \\
N \\
N \\
N \\
N \\
N \\
N \\
N \\
N \\
N \\
N \\
N \\
N \\
N \\
N \\
N \\
N \\
N\end{array}$ & $M G / L$ & $\begin{array}{l}< \\
< \\
< \\
< \\
< \\
< \\
< \\
< \\
\\
< \\
< \\
< \\
< \\
< \\
< \\
< \\
< \\
< \\
< \\
< \\
< \\
< \\
< \\
<\end{array}$ & $\begin{array}{l}0.1 \\
0.2 \\
0.1 \\
0.3 \\
0.1 \\
0.1 \\
0.1 \\
0.1 \\
0.1 \\
0.1 \\
0.1 \\
0.1 \\
0.1 \\
0.1 \\
0.1 \\
0.1 \\
0.1 \\
0.1 \\
0.1 \\
0.1 \\
0.1 \\
0.1 \\
0.1 \\
0.1\end{array}$ & $J$ & $\begin{array}{l}0.1 \\
0.1 \\
0.1 \\
0.1 \\
0.1 \\
0.1 \\
0.1 \\
0.1 \\
0.1 \\
0.1 \\
0.1 \\
0.1 \\
0.1 \\
0.1 \\
0.1 \\
0.1 \\
0.1 \\
0.1 \\
0.1 \\
0.1 \\
0.1 \\
0.1 \\
0.1 \\
0.1\end{array}$ & $\begin{array}{l}- \\
- \\
- \\
: \\
: \\
- \\
- \\
- \\
- \\
- \\
- \\
- \\
- \\
- \\
- \\
- \\
-\end{array}$ \\
\hline
\end{tabular}

FORMATION OF COMPLETION CODE:

FLOW RELATIONSHIP CODE:

WL - WASATCH - LOWER SANDSTO
WM - WASATCH - MIDOLE SHALE

N - UNKNOWN

PARAMETER VALUE INDICATOR (PVI): < - LESS THAN DETECTION LIMIT

SAMPLE ID CODES:

0001 - FILTERED SAMPLE (.45 MICRONS)

OTHER PARAMETER VALUE FLAGS:

J. ESTIMATED VALUE 
GROUNDHATER QUALITY DATA BY PARAMETER

LOHER SANDSTONE AND MIDDLE SHALE UNITS

SITE: SPKOT SPOOK

OLFT7/87 TO

REPORT DATE: $07 / 29 / 94$

\begin{tabular}{|c|c|c|c|c|c|c|c|c|c|c|c|}
\hline PARAMETER NAME & $\begin{array}{c}\text { LOCATION } \\
\text { ID }\end{array}$ & LOG DATE & $\begin{array}{c}\text { SAMPLE } \\
\text { ID }\end{array}$ & $\begin{array}{l}\text { FORM } \\
\text { COMP }\end{array}$ & $\begin{array}{l}\text { FLOW } \\
\text { REL. }\end{array}$ & $\begin{array}{l}\text { UNITS OF } \\
\text { MEASURE }\end{array}$ & PVI & $\begin{array}{l}\text { ARAMETER } \\
\text { VALUE }\end{array}$ & FLAGS & $\begin{array}{l}\text { DETECTION } \\
\text { LIMIT }\end{array}$ & $\begin{array}{l}\text { PARAMETER } \\
\text { UNCERTAINTY }\end{array}$ \\
\hline PHOSPHATE & $\begin{array}{l}0923 \\
0923 \\
0923 \\
0938\end{array}$ & $\begin{array}{l}01 / 30 / 88 \\
04 / 26 / 88 \\
08 / 09 / 88 \\
08 / 09 / 88\end{array}$ & $\begin{array}{l}0001 \\
0001 \\
0001 \\
0001\end{array}$ & $\begin{array}{l}\text { WL } \\
\text { WL } \\
\text { WL } \\
\text { WL }\end{array}$ & $\begin{array}{l}N \\
N \\
N \\
N\end{array}$ & $M G / L$ & $\begin{array}{l}< \\
< \\
< \\
<\end{array}$ & $\begin{array}{l}0.1 \\
0.1 \\
0.1 \\
0.1\end{array}$ & J & $\begin{array}{l}0.1 \\
0.1 \\
0.1 \\
0.1\end{array}$ & $\begin{array}{l}- \\
-\end{array}$ \\
\hline POLONIUM-210 & $\begin{array}{l}0910 \\
0910 \\
0910 \\
0910 \\
0914 \\
0914 \\
0914 \\
0914 \\
0915 \\
0915 \\
0915 \\
0915 \\
0919 \\
0919 \\
0919 \\
0919 \\
0921 \\
0921 \\
0921 \\
0921 \\
0923 \\
0923 \\
0923 \\
0923 \\
0938\end{array}$ & $\begin{array}{l}09 / 18 / 87 \\
01 / 30 / 88 \\
04 / 22 / 88 \\
08 / 11 / 88 \\
09 / 18 / 87 \\
02 / 03 / 88 \\
04 / 22 / 88 \\
08 / 08 / 88 \\
09 / 20 / 87 \\
02 / 03 / 88 \\
04 / 22 / 88 \\
08 / 10 / 88 \\
09 / 20 / 87 \\
01 / 30 / 88 \\
04 / 26 / 88 \\
08 / 27 / 88 \\
09 / 20 / 87 \\
02 / 04 / 88 \\
04 / 26 / 88 \\
08 / 10 / 88 \\
09 / 20 / 87 \\
01 / 30 / 88 \\
04 / 26 / 88 \\
08 / 09 / 88 \\
08 / 09 / 88\end{array}$ & $\begin{array}{l}0001 \\
0001 \\
0001 \\
0001 \\
0001 \\
0001 \\
0001 \\
0001 \\
0001 \\
0001 \\
0001 \\
0001 \\
0001 \\
0001 \\
0001 \\
0001 \\
0001 \\
0001 \\
0001 \\
0001 \\
0001 \\
0001 \\
0001 \\
0001 \\
0001\end{array}$ & $\begin{array}{l}W L \\
W L \\
W L \\
W L \\
W L \\
W L \\
W L \\
W L \\
W M \\
W M \\
W M \\
W M \\
W L \\
W L \\
W L \\
W L \\
W L \\
W L \\
W L \\
W L \\
W L \\
W L \\
W L \\
W L \\
W L\end{array}$ & $\begin{array}{l}N \\
N \\
N \\
N \\
N \\
N \\
N \\
N \\
N \\
N \\
N \\
N \\
N \\
N \\
N \\
N \\
N \\
N \\
N \\
N \\
N \\
N \\
N \\
N \\
N\end{array}$ & $\mathrm{PCI} / \mathrm{L}$ & & $\begin{array}{l}2.2 \\
0.0 \\
0.0 \\
0.0 \\
0.3 \\
0.2 \\
0.0 \\
0.8 \\
1.2 \\
0.1 \\
0.0 \\
2.5 \\
0.0 \\
0.3 \\
0.1 \\
0.0 \\
0.3 \\
0.0 \\
0.0 \\
0.0 \\
0.1 \\
0.0 \\
0.7 \\
0.0 \\
0.0\end{array}$ & J & $\begin{array}{l}1 . \\
1 . \\
1 . \\
1 . \\
1 . \\
1 . \\
1 . \\
1 . \\
1 . \\
1 . \\
1 . \\
1 . \\
1 . \\
1 . \\
1 . \\
1 . \\
1 . \\
1 . \\
1 . \\
1 .\end{array}$ & $\begin{array}{l}1.1 \\
0.3 \\
0.3 \\
0.4 \\
0.9 \\
0.4 \\
0.3 \\
0.5 \\
1.0 \\
0.3 \\
0.4 \\
0.7 \\
0.8 \\
0.6 \\
0.4 \\
0.3 \\
0.9 \\
0.6 \\
0.4 \\
0.4 \\
0.8 \\
0.5 \\
0.5 \\
0.4 \\
0.4\end{array}$ \\
\hline POTASSIUM & $\begin{array}{l}0910 \\
0910 \\
0910 \\
0910 \\
0910 \\
0910 \\
0914 \\
0914\end{array}$ & $\begin{array}{l}02 / 17 / 87 \\
09 / 18 / 87 \\
01 / 30 / 88 \\
04 / 22 / 88 \\
08 / 11 / 88 \\
12 / 10 / 88 \\
02 / 18 / 87 \\
09 / 18 / 87\end{array}$ & $\begin{array}{l}0001 \\
0001 \\
0001 \\
0001 \\
0001 \\
0001 \\
0001 \\
0001\end{array}$ & $\begin{array}{l}W L \\
W L \\
W L \\
W L \\
W L \\
W L \\
W L \\
W L\end{array}$ & $\begin{array}{l}N \\
N \\
N \\
N \\
N \\
N \\
N \\
N\end{array}$ & $M G / L$ & & $\begin{array}{l}8.82 \\
4.74 \\
5.25 \\
4.88 \\
5.2 \\
4.8 \\
4.21 \\
3.56\end{array}$ & & $\begin{array}{l}0.01 \\
0.01 \\
0.01 \\
0.01 \\
0.01 \\
0.01 \\
0.01 \\
0.01\end{array}$ & $\begin{array}{l}: \\
: \\
: \\
:\end{array}$ \\
\hline
\end{tabular}

FORMATIOH OF COMPLETION CODE:

WL - WASATCH - LOWER SANDSTONE

WM - HASATCH - MIDDLE SHALE

PARAMETER VALUE INDICATOR (PVI): < - LESS thaN DETECTION LIMIT
FLOH RELATIONSHIP CODE:

N - UNKNOWN

SAMPLE ID CODES

0001 - FILTERED SAMPLE (.45 MICRONS)

OTHER PARAMETER VALUE FLAGS

$J$ - ESTIMATED VALUE 


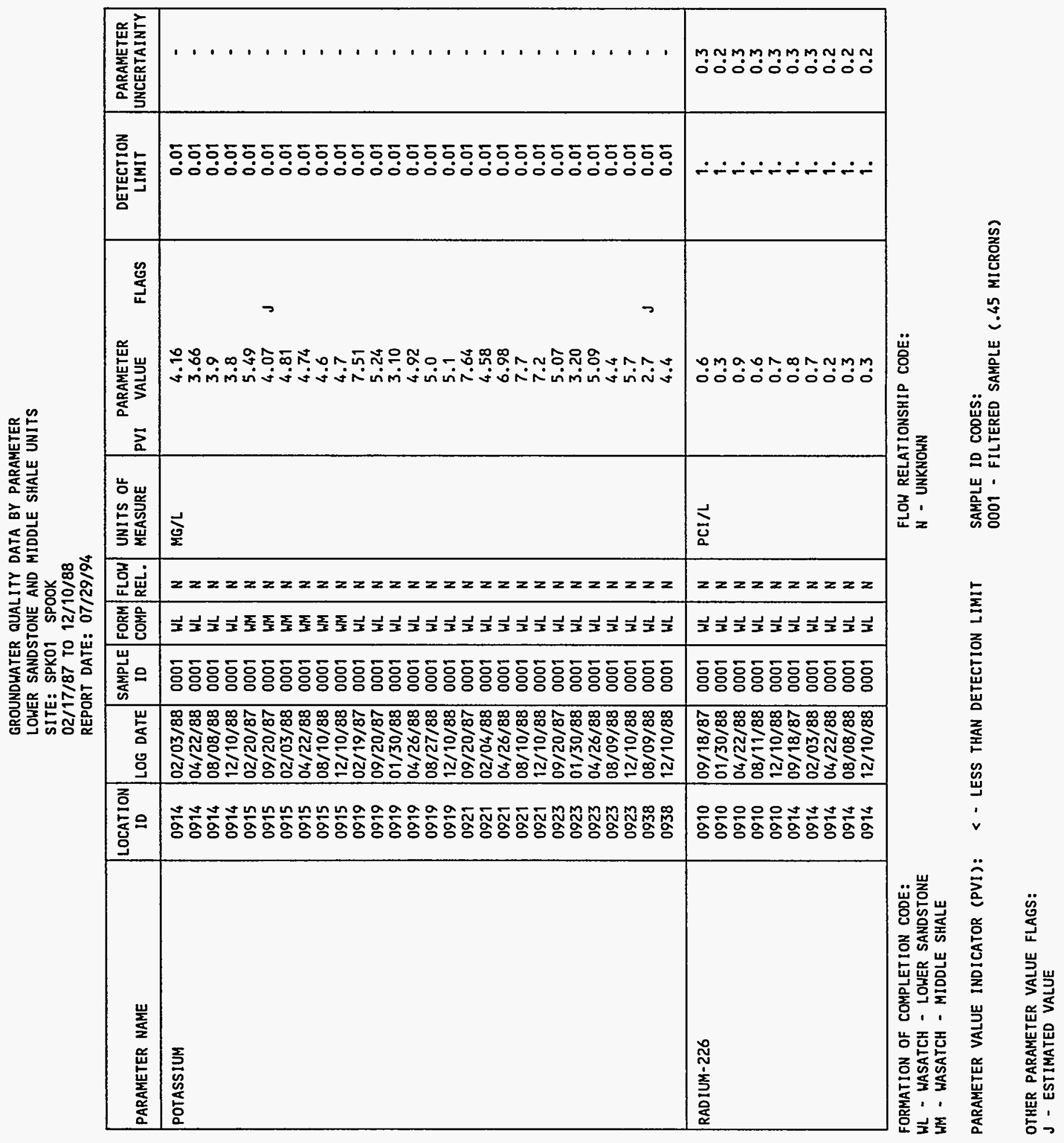


LOHER SANDSTONE AND MIDDLE SHALE UNITS

SITE: SPKO1 SPDOK

02/17/87 TO $12 / 10 / 88$

REPORT DATE: $07 / 29 / 9$

\begin{tabular}{|c|c|c|c|c|c|c|c|c|c|c|c|}
\hline PARAMETER NAME & $\underset{\text { ID }}{\text { LOCATION }}$ & LOG DATE & $\begin{array}{l}\text { SAMPLE } \\
\text { ID }\end{array}$ & $\begin{array}{l}\text { FORH } \\
\text { COMP }\end{array}$ & $\begin{array}{l}\text { FLOW } \\
\text { REL. }\end{array}$ & $\begin{array}{l}\text { UNITS OF } \\
\text { MEASURE }\end{array}$ & PVI & $\begin{array}{l}\text { PARAMETER } \\
\text { VALUE }\end{array}$ & FLAGS & $\begin{array}{l}\text { DETECTIOH } \\
\text { LIMIT }\end{array}$ & $\begin{array}{l}\text { PARAMETER } \\
\text { UNCERTAINTY }\end{array}$ \\
\hline RADIUM-226 & $\begin{array}{l}0915 \\
0915 \\
0915 \\
0915 \\
0915 \\
0919 \\
0919 \\
0919 \\
0919 \\
0919 \\
0921 \\
0921 \\
0921 \\
0921 \\
0921 \\
0923 \\
0923 \\
0923 \\
0923 \\
0923 \\
0938 \\
0938\end{array}$ & $\begin{array}{l}09 / 20 / 87 \\
02 / 03 / 88 \\
04 / 22 / 88 \\
08 / 10 / 88 \\
12 / 10 / 88 \\
09 / 20 / 87 \\
01 / 30 / 88 \\
04 / 26 / 88 \\
08 / 27 / 88 \\
12 / 10 / 88 \\
09 / 20 / 87 \\
02 / 04 / 88 \\
04 / 26 / 88 \\
08 / 10 / 88 \\
12 / 10 / 88 \\
09 / 20 / 87 \\
01 / 30 / 88 \\
04 / 26 / 88 \\
08 / 09 / 88 \\
12 / 10 / 88 \\
08 / 09 / 88 \\
12 / 10 / 88\end{array}$ & $\begin{array}{l}0001 \\
0001 \\
0001 \\
0001 \\
0001 \\
0001 \\
0001 \\
0001 \\
0001 \\
0001 \\
0001 \\
0001 \\
0001 \\
0001 \\
0001 \\
0001 \\
0001 \\
0001 \\
0001 \\
0001 \\
0001 \\
0001\end{array}$ & $\begin{array}{l}W M \\
W H \\
W M \\
W H \\
W H \\
H L \\
W L \\
W L \\
W L \\
H L \\
H L \\
W L \\
W L \\
W L \\
W L \\
W L \\
W L \\
W L \\
H L \\
W L \\
W L \\
W L\end{array}$ & $\begin{array}{l}N \\
N \\
N \\
N \\
N \\
N \\
N \\
N \\
N \\
N \\
N \\
N \\
N \\
N \\
N \\
N \\
N \\
N \\
N \\
N \\
N \\
N\end{array}$ & $\mathrm{PCl} / \mathrm{L}$ & & $\begin{array}{l}2.1 \\
0.3 \\
0.8 \\
0.3 \\
0.5 \\
1.3 \\
1.2 \\
0.9 \\
1.1 \\
1.3 \\
0.5 \\
0.6 \\
0.4 \\
0.3 \\
0.6 \\
0.5 \\
0.8 \\
0.7 \\
0.3 \\
0.8 \\
0.3 \\
0.3\end{array}$ & J & $\begin{array}{l}1 . \\
1 . \\
1 . \\
1 . \\
1 . \\
1 . \\
1 . \\
1 . \\
1 . \\
1 . \\
1 . \\
1 . \\
1 . \\
1 . \\
1 . \\
1 . \\
1 .\end{array}$ & $\begin{array}{l}0.5 \\
0.2 \\
0.3 \\
0.2 \\
0.3 \\
0.4 \\
0.3 \\
0.3 \\
0.3 \\
0.4 \\
0.3 \\
0.2 \\
0.3 \\
0.2 \\
0.3 \\
0.3 \\
0.2 \\
0.3 \\
0.2 \\
0.3 \\
0.2 \\
0.2\end{array}$ \\
\hline RADIUM-226 + RADIUM-228 & $\begin{array}{l}0910 \\
0910 \\
0910 \\
0910 \\
0910 \\
0914 \\
0914 \\
0914 \\
0914 \\
0914 \\
0915 \\
0915 \\
0915 \\
0915 \\
0915 \\
0919\end{array}$ & $\begin{array}{l}09 / 18 / 87 \\
01 / 30 / 88 \\
04 / 22 / 88 \\
08 / 11 / 88 \\
12 / 10 / 88 \\
09 / 18 / 87 \\
02 / 03 / 88 \\
04 / 22 / 88 \\
08 / 08 / 88 \\
12 / 10 / 88 \\
09 / 20 / 87 \\
02 / 03 / 88 \\
04 / 22 / 88 \\
08 / 10 / 88 \\
12 / 10 / 88 \\
09 / 20 / 87\end{array}$ & $\begin{array}{l}0001 \\
0001 \\
0001 \\
0001 \\
0001 \\
0001 \\
0001 \\
0001 \\
0001 \\
0001 \\
0001 \\
0001 \\
0001 \\
0001 \\
0001 \\
0001\end{array}$ & $\begin{array}{l}W L \\
W L \\
H L \\
H L \\
W L \\
W L \\
W L \\
H L \\
W L \\
H L \\
H M \\
H M \\
W H \\
W H \\
W M \\
H L\end{array}$ & $\begin{array}{l}N \\
N \\
N \\
N \\
N \\
N \\
N \\
N \\
N \\
N \\
N \\
N \\
N \\
N \\
N \\
N\end{array}$ & $\mathrm{PCI} / \mathrm{L}$ & & $\begin{array}{l}1.00 \\
0.90 \\
1.20 \\
1.10 \\
1.60 \\
0.80 \\
0.70 \\
0.50 \\
0.50 \\
1.10 \\
2.10 \\
0.30 \\
1.70 \\
0.70 \\
0.90 \\
3.30\end{array}$ & & $\begin{array}{l}. \\
\dot{-} \\
. \\
- \\
- \\
- \\
- \\
- \\
- \\
- \\
-\end{array}$ & $\begin{array}{l}- \\
: \\
: \\
: \\
- \\
- \\
- \\
- \\
- \\
-\end{array}$ \\
\hline
\end{tabular}

FORMATION OF COMPLETION CODE:

WM - HASATCH - MIDDLE SHALE

FLOH RELATIONSHIP CODE

WL - HASATCH - LOWER SANDSTONE

N - UNKNOWN

PARAMETER VALUE INDICATOR (PVI): < - LESS THAN DETECTION LIMIT

SAMPLE ID CODES:

0001 - FILTERED SAMPLE (.45 MICRONS)

OTHER PARAMETER VALUE FLAGS:

J - ESTIMATED VALUE 


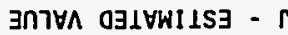

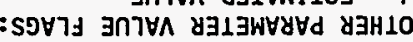

(SNOYJIK 5\%) 37dWHS 03831714 - 1000

:S3000 of $37 \mathrm{dWHS}$

LIWIT NOILJコ130 NYHL SS37 - >

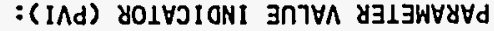

MMONXN - $N$

: $\exists 003$ dIHSNOII

\begin{tabular}{|c|c|c|c|c|c|c|c|c|c|c|}
\hline $\begin{array}{l}6 \cdot 0 \\
1 \cdot 1 \\
8 \cdot 0 \\
2 \cdot 0 \\
8 \cdot 0 \\
6 \cdot 0 \\
0 \cdot 1 \\
8 \cdot 0 \\
2 \cdot 0 \\
8 \cdot 0 \\
2 \cdot 0 \\
5 \cdot 1 \\
8 \cdot 0 \\
2 \cdot 0 \\
8 \cdot 0 \\
L \cdot 0 \\
2 \cdot 1 \\
6 \cdot 0 \\
2 \cdot 0 \\
L \cdot 0 \\
6 \cdot 0 \\
7 \cdot 1\end{array}$ & 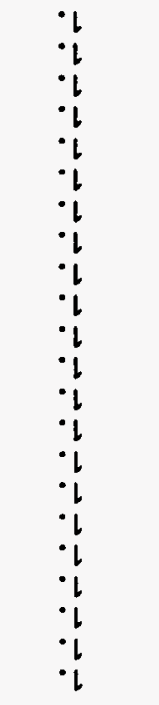 & 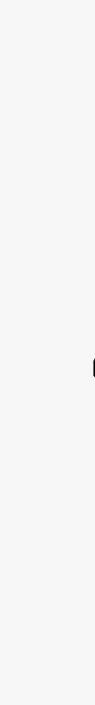 & $\begin{array}{l}5 \cdot 0 \\
6 \cdot 0 \\
L \cdot 1 \\
0 \cdot 1 \\
2 \cdot 1 \\
8 \cdot 0 \\
0 \cdot 2 \\
7 \cdot 0 \\
7 \cdot 0 \\
6 \cdot 0 \\
0 \cdot 0 \\
0 \cdot 0 \\
8 \cdot 0 \\
2 \cdot 0 \\
5 \cdot 0 \\
0 \cdot 0 \\
0 \cdot 0 \\
6 \cdot 0 \\
5 \cdot 0 \\
5 \cdot 0 \\
9 \cdot 0 \\
7 \cdot 0\end{array}$ & . & $\begin{array}{l}N \\
N \\
N \\
N \\
N \\
N \\
N \\
N \\
N \\
N \\
N \\
N \\
N \\
N \\
N \\
N \\
N \\
N \\
N \\
N \\
N \\
N\end{array}$ & $\begin{array}{l}7 M \\
7 M \\
7 M \\
7 M \\
7 M \\
7 M \\
7 M \\
W M \\
W M \\
W M \\
W M \\
W M \\
7 M \\
7 M \\
7 M \\
7 M \\
7 M \\
7 M \\
7 M \\
7 M \\
7 M \\
7 M\end{array}$ & $\begin{array}{l}1000 \\
1000 \\
1000 \\
1000 \\
1000 \\
1000 \\
1000 \\
1000 \\
1000 \\
1000 \\
1000 \\
1000 \\
1000 \\
1000 \\
1000 \\
1000 \\
1000 \\
1000 \\
1000 \\
1000 \\
1000 \\
1000\end{array}$ & 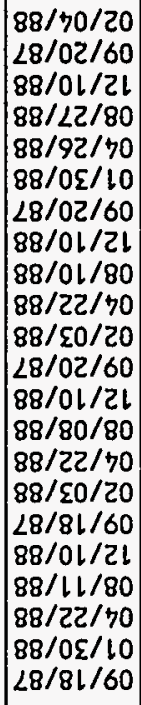 & $\begin{array}{l}1260 \\
1260 \\
6160 \\
6160 \\
6160 \\
6160 \\
6160 \\
\text { sl60 } \\
\text { sl60 } \\
\text { sl60 } \\
\text { sl60 } \\
\text { sl60 } \\
7160 \\
7160 \\
7160 \\
7160 \\
7160 \\
0160 \\
0160 \\
0160 \\
0160 \\
0160\end{array}$ & 8Z2-wกI Q \\
\hline $\begin{array}{l}- \\
: \\
: \\
: \\
- \\
- \\
- \\
- \\
- \\
- \\
-\end{array}$ & $\begin{array}{l}: \\
: \\
: \\
: \\
: \\
- \\
- \\
- \\
- \\
-\end{array}$ & & 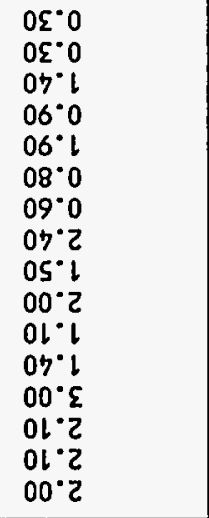 & 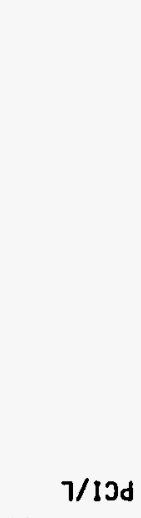 & $\begin{array}{l}N \\
N \\
N \\
N \\
N \\
N \\
N \\
N \\
N \\
N \\
N \\
N \\
N \\
N \\
N \\
N\end{array}$ & $\begin{array}{l}7 M \\
7 M \\
7 M \\
7 M \\
7 M \\
7 M \\
7 M \\
7 M \\
7 M \\
7 M \\
7 M \\
7 M \\
7 M \\
7 M \\
7 M \\
7 M\end{array}$ & $\begin{array}{l}1000 \\
1000 \\
1000 \\
1000 \\
1000 \\
1000 \\
1000 \\
1000 \\
1000 \\
1000 \\
1000 \\
1000 \\
1000 \\
1000 \\
1000 \\
1000\end{array}$ & $\begin{array}{l}88 / 01 / 2 L \\
88 / 60 / 80 \\
88 / 01 / 21 \\
88 / 60 / 80 \\
88 / 92 / 70 \\
88 / 0 \Sigma / 10 \\
28 / 02 / 60 \\
88 / 01 / 2 L \\
88 / 01 / 80 \\
88 / 92 / 70 \\
88 / 70 / 20 \\
28 / 02 / 60 \\
88 / 01 / 21 \\
88 / L 2 / 80 \\
88 / 92 / 70 \\
88 / 0 \Sigma / 10\end{array}$ & $\begin{array}{l}8 \sum 60 \\
8 \varepsilon 60 \\
\varepsilon 260 \\
\varepsilon 260 \\
\varepsilon 260 \\
\varepsilon 260 \\
\varepsilon 260 \\
1260 \\
1260 \\
1260 \\
1260 \\
1260 \\
6160 \\
6160 \\
6160 \\
6160\end{array}$ & 更 \\
\hline 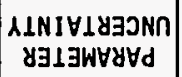 & $\begin{array}{l}\text { IIWIT } \\
\text { NoI1J3130 }\end{array}$ & Sפชาง & $\underset{\substack{\exists \cap 7 \forall \wedge \\
d \exists \perp \exists W \forall y \forall d}}{ } I \Lambda d$ & $\begin{array}{l}\text { JUnSHבW } \\
\text { JO SIINก }\end{array}$ & $\begin{array}{l}\cdot 7 \exists y \\
\text { Mold }\end{array}$ & $\begin{array}{l}\text { dWOJ } \\
\text { WYOS }\end{array}$ & OI & $\exists \perp \forall 0$ э0า & $\begin{array}{c}01 \\
\text { NOI1 }\end{array}$ & 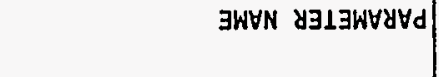 \\
\hline
\end{tabular}

$76 / 62 / 20$ : 3170 180d38

$88 / 0 \mathrm{~L} / 2 \mathrm{~L} 01 \mathrm{~L} 2 \mathrm{~L} / \mathrm{LL} / 20$

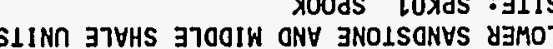

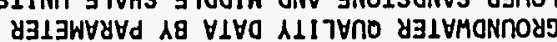

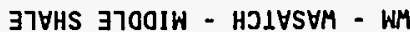

בNOISONYS YZMOT - HJIYSYM - IM

: 


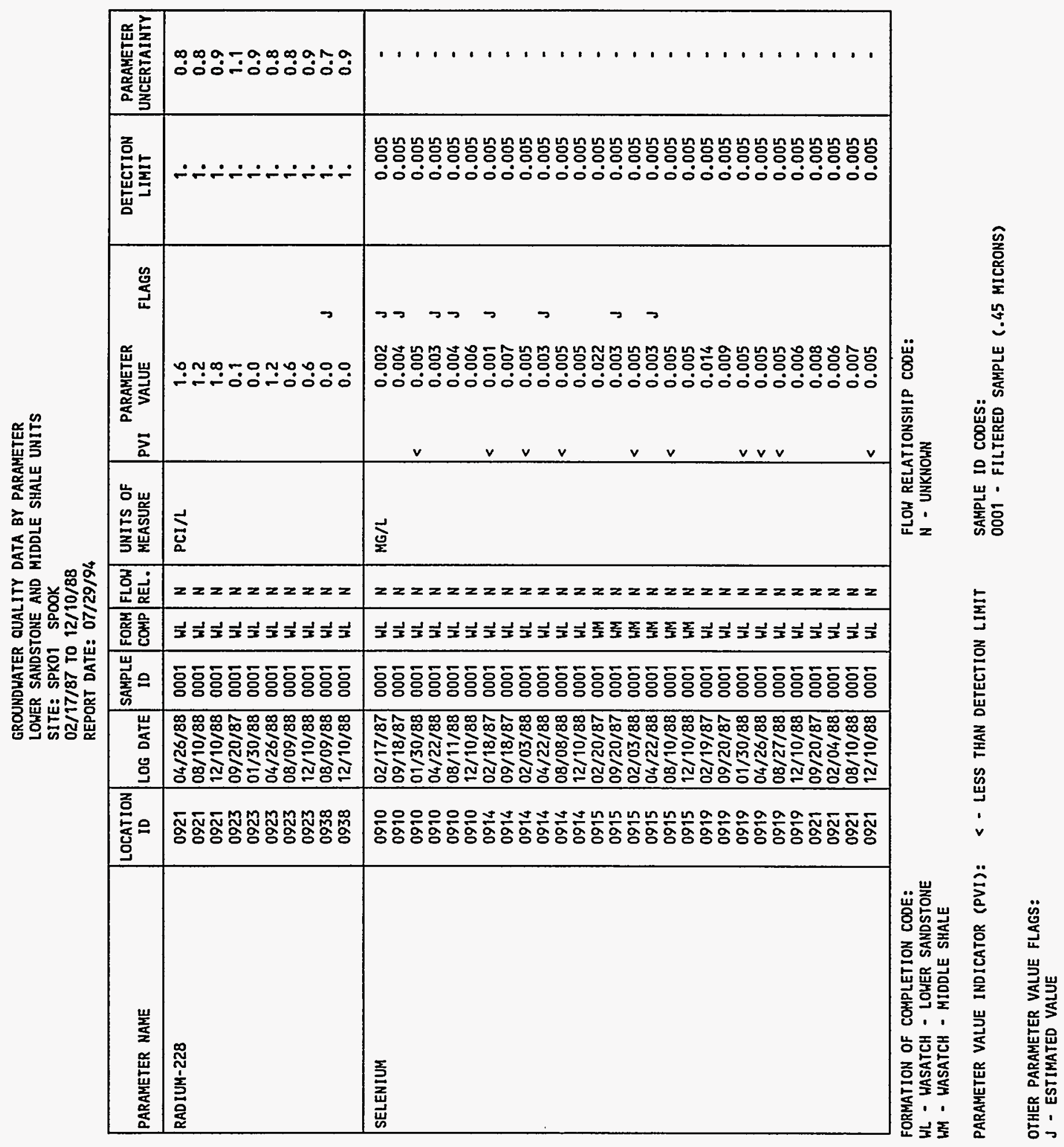


SITE: SPKO1 SPOOK

REPORT DATE. $07 / 29 / 94$

\begin{tabular}{|c|c|c|c|c|c|c|c|c|c|c|c|}
\hline PARAMETER NAME & $\underset{10}{\text { LOCATION }}$ & LOG DATE & $\begin{array}{c}\text { SAMPLE } \\
\text { I0 }\end{array}$ & $\begin{array}{l}\text { FORM } \\
\text { COMP }\end{array}$ & $\begin{array}{l}\text { FLOW } \\
\text { REL. }\end{array}$ & $\begin{array}{l}\text { UNITS OF } \\
\text { MEASURE }\end{array}$ & PVI & $\begin{array}{l}\text { PARAMETER } \\
\text { VALUE }\end{array}$ & FLAGS & $\begin{array}{l}\text { DETECTION } \\
\text { LIMIT }\end{array}$ & $\begin{array}{l}\text { PARAMETER } \\
\text { UNCERTAINTY }\end{array}$ \\
\hline SELENIUM & $\begin{array}{l}0923 \\
0923 \\
0923 \\
0923 \\
0923 \\
0938 \\
0938\end{array}$ & $\begin{array}{l}09 / 20 / 87 \\
01 / 30 / 88 \\
04 / 26 / 88 \\
08 / 09 / 88 \\
12 / 10 / 88 \\
08 / 09 / 88 \\
12 / 10 / 88\end{array}$ & $\begin{array}{l}0001 \\
0001 \\
0001 \\
0001 \\
0001 \\
0001 \\
0001\end{array}$ & $\begin{array}{l}W L \\
W L \\
W L \\
W L \\
W L \\
W L \\
W L\end{array}$ & $\begin{array}{l}N \\
N \\
N \\
N \\
N \\
N \\
N\end{array}$ & $M G / L$ & $\mid \begin{array}{l}< \\
< \\
< \\
<\end{array}$ & $\begin{array}{l}0.007 \\
0.005 \\
0.005 \\
0.006 \\
0.005 \\
0.005 \\
0.005\end{array}$ & J & $\begin{array}{l}0.005 \\
0.005 \\
0.005 \\
0.005 \\
0.005 \\
0.005 \\
0.005\end{array}$ & $\begin{array}{l}- \\
- \\
- \\
- \\
- \\
-\end{array}$ \\
\hline SILICA - SIO2 & $\begin{array}{l}0910 \\
0910 \\
0910 \\
0910 \\
0910 \\
0914 \\
0914 \\
0914 \\
0914 \\
0914 \\
0915 \\
0915 \\
0915 \\
0915 \\
0915 \\
0919 \\
0919 \\
0919 \\
0919 \\
0919 \\
0921 \\
0921 \\
0921 \\
0921 \\
0923 \\
0923 \\
0923 \\
0923 \\
0938\end{array}$ & $\begin{array}{l}02 / 17 / 87 \\
09 / 18 / 87 \\
01 / 30 / 88 \\
04 / 22 / 88 \\
08 / 11 / 88 \\
02 / 18 / 87 \\
09 / 18 / 87 \\
02 / 03 / 88 \\
04 / 22 / 88 \\
08 / 08 / 88 \\
02 / 20 / 87 \\
09 / 20 / 87 \\
02 / 03 / 88 \\
04 / 22 / 88 \\
08 / 10 / 88 \\
02 / 19 / 87 \\
09 / 20 / 87 \\
01 / 30 / 88 \\
04 / 26 / 88 \\
08 / 27 / 88 \\
09 / 20 / 87 \\
02 / 04 / 88 \\
04 / 26 / 88 \\
08 / 10 / 88 \\
09 / 20 / 87 \\
01 / 30 / 88 \\
04 / 26 / 88 \\
08 / 09 / 88 \\
08 / 09 / 88\end{array}$ & $\begin{array}{l}0001 \\
0001 \\
0001 \\
0001 \\
0001 \\
0001 \\
0001 \\
0001 \\
0001 \\
0001 \\
0001 \\
0001 \\
0001 \\
0001 \\
0001 \\
0001 \\
0001 \\
0001 \\
0001 \\
0001 \\
0001 \\
0001 \\
0001 \\
0001 \\
0001 \\
0001 \\
0001 \\
0001 \\
0001\end{array}$ & $\begin{array}{l}W L \\
W L \\
W L \\
W L \\
W L \\
W L \\
W L \\
W L \\
W L \\
W L \\
W M \\
W M \\
W M \\
W M \\
W M \\
W L \\
W L \\
W L \\
W L \\
W L \\
W L \\
W L \\
W L \\
W L \\
W L \\
W L \\
W L \\
W L \\
W L\end{array}$ & $\begin{array}{l}N \\
N \\
N \\
N \\
N \\
N \\
N \\
N \\
N \\
N \\
N \\
N \\
N \\
N \\
N \\
N \\
N \\
N \\
N \\
N \\
N \\
N \\
N \\
N \\
N \\
N \\
N \\
N \\
N\end{array}$ & MG/L & & $\begin{array}{l}13 . \\
9.84 \\
11.5 \\
11.4 \\
12 . \\
14 . \\
9.16 \\
10.6 \\
10.0 \\
10 . \\
16 . \\
10.2 \\
11.1 \\
10.3 \\
11 . \\
20 . \\
13.8 \\
14.2 \\
14.8 \\
15.0 \\
14.8 \\
15.7 \\
17.3 \\
17 . \\
11.8 \\
12.5 \\
13.4 \\
12 . \\
10 .\end{array}$ & $\mathbf{J}$ & $\begin{array}{l}2 . \\
2 . \\
2 . \\
2 . \\
2 . \\
2 . \\
2 . \\
2 . \\
2 . \\
2 . \\
2 . \\
2 . \\
2 . \\
2 . \\
2 . \\
2 . \\
2 . \\
2 . \\
2 . \\
2 . \\
2 . \\
2 . \\
2 . \\
2 . \\
2 . \\
2 . \\
2 . \\
2 . \\
2 .\end{array}$ & 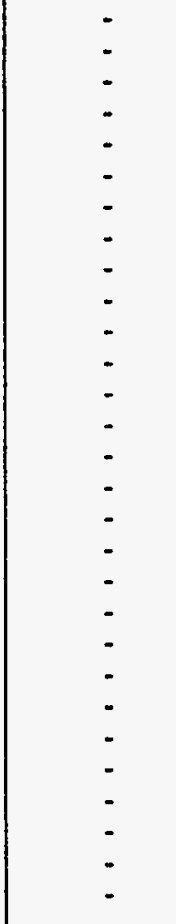 \\
\hline SILVER & 0910 & $02 / 17 / 87$ & 0001 & WL & $\mathbf{N}$ & MG/L & $<$ & 0.01 & & 0.01 & - \\
\hline
\end{tabular}

FORMATION OF COMPLETION CODE:

WL - WASATCH - LOWER SANDSTONE

FLOW RELATIONSHIP CODE:

WM - WASATCH - MIDDLE SHALE

N - UNKNOWN

PARAMETER VALUE INDICATOR (PVI): < - LESS THAN DETECTION LIMIT

SAMPLE ID CODES:

0001 - FILTERED SAMPLE (.45 MICRONS)

OTHER PARAMETER VALUE FLAGS:

$\mathrm{J}$ - ESTIMATED VALUE 
GROUNDHATER QUALITY DATA BY PARAMETER

LOWER SANDSTONE AND MIDDLE SHALE UNITS

SITE: SPKO1 SPOOK

REPORT DATE: $07 / 29 / 94$

\begin{tabular}{|c|c|c|c|c|c|c|c|c|c|c|c|}
\hline PARAMETER NAME & $\mid \begin{array}{c}\text { LOCATION } \\
\text { ID }\end{array}$ & LOG DATE & $\begin{array}{c}\text { SAMPLE } \\
\text { ID }\end{array}$ & $\begin{array}{l}\text { FORM } \\
\text { COMP }\end{array}$ & $\begin{array}{l}\text { FLOH } \\
\text { REL. }\end{array}$ & $\begin{array}{l}\text { UNITS OF } \\
\text { MEASURE }\end{array}$ & PVI & $\begin{array}{l}\text { PARAMETER } \\
\text { VALUE }\end{array}$ & FLAGS & $\begin{array}{l}\text { DETECTION } \\
\text { LIMIT }\end{array}$ & $\begin{array}{l}\text { PARAMETER } \\
\text { UNCERTAINTY }\end{array}$ \\
\hline SILVER & $\begin{array}{l}0910 \\
0910 \\
0910 \\
0910 \\
0910 \\
0914 \\
0914 \\
0914 \\
0914 \\
0914 \\
0914 \\
0915 \\
0915 \\
0915 \\
0915 \\
0915 \\
0915 \\
0919 \\
0919 \\
0919 \\
0919 \\
0919 \\
0919 \\
0921 \\
0921 \\
0921 \\
0921 \\
0921 \\
0923 \\
0923 \\
0923 \\
0923 \\
0923 \\
0938 \\
0938\end{array}$ & $\begin{array}{l}09 / 18 / 87 \\
01 / 30 / 88 \\
04 / 22 / 88 \\
08 / 11 / 88 \\
12 / 10 / 88 \\
02 / 18 / 87 \\
09 / 18 / 87 \\
02 / 03 / 88 \\
04 / 22 / 88 \\
08 / 08 / 88 \\
12 / 10 / 88 \\
02 / 20 / 87 \\
09 / 20 / 87 \\
02 / 03 / 88 \\
04 / 22 / 88 \\
08 / 10 / 88 \\
12 / 10 / 88 \\
02 / 19 / 87 \\
09 / 20 / 87 \\
01 / 30 / 88 \\
04 / 26 / 88 \\
08 / 27 / 88 \\
12 / 10 / 88 \\
09 / 20 / 87 \\
02 / 04 / 88 \\
04 / 26 / 88 \\
08 / 10 / 88 \\
12 / 10 / 88 \\
09 / 20 / 87 \\
01 / 30 / 88 \\
04 / 26 / 88 \\
08 / 09 / 88 \\
12 / 10 / 88 \\
08 / 09 / 88 \\
12 / 10 / 88\end{array}$ & $\begin{array}{l}0001 \\
0001 \\
0001 \\
0001 \\
0001 \\
0001 \\
0001 \\
0001 \\
0001 \\
0001 \\
0001 \\
0001 \\
0001 \\
0001 \\
0001 \\
0001 \\
0001 \\
0001 \\
0001 \\
0001 \\
0001 \\
0001 \\
0001 \\
0001 \\
0001 \\
0001 \\
0001 \\
0001 \\
0001 \\
0001 \\
0001 \\
0001 \\
0001 \\
0001 \\
0001\end{array}$ & $\begin{array}{l}W L \\
W L \\
W L \\
W L \\
W L \\
W L \\
H L \\
W L \\
W L \\
W L \\
W L \\
W M \\
W M \\
W M \\
W M \\
W M \\
W M \\
W L \\
W L \\
W L \\
W L \\
W L \\
W L \\
W L \\
W L \\
W L \\
W L \\
W L \\
W L \\
W L \\
W L \\
W L \\
W L \\
W L \\
W L\end{array}$ & $\begin{array}{l}N \\
N \\
N \\
N \\
N \\
N \\
N \\
N \\
N \\
N \\
N \\
N \\
N \\
N \\
N \\
N \\
N \\
N \\
N \\
N \\
N \\
N \\
N \\
N \\
N \\
N \\
N \\
N \\
N \\
N \\
N \\
N \\
N \\
N \\
N\end{array}$ & MG/L & $\begin{array}{l}< \\
< \\
< \\
< \\
< \\
< \\
< \\
< \\
< \\
< \\
< \\
< \\
< \\
< \\
< \\
< \\
< \\
< \\
< \\
< \\
< \\
< \\
< \\
< \\
< \\
< \\
< \\
< \\
< \\
< \\
< \\
< \\
< \\
<\end{array}$ & $\begin{array}{l}0.01 \\
0.01 \\
0.01 \\
0.01 \\
0.01 \\
0.01 \\
0.01 \\
0.01 \\
0.01 \\
0.01 \\
0.01 \\
0.01 \\
0.01 \\
0.01 \\
0.01 \\
0.01 \\
0.01 \\
0.01 \\
0.01 \\
0.01 \\
0.01 \\
0.01 \\
0.01 \\
0.01 \\
0.02 \\
0.01 \\
0.01 \\
0.01 \\
0.01 \\
0.01 \\
0.01 \\
0.01 \\
0.01 \\
0.01 \\
0.01\end{array}$ & . & $\begin{array}{l}0.01 \\
0.01 \\
0.01 \\
0.01 \\
0.01 \\
0.01 \\
0.01 \\
0.01 \\
0.01 \\
0.01 \\
0.01 \\
0.01 \\
0.01 \\
0.01 \\
0.01 \\
0.01 \\
0.01 \\
0.01 \\
0.01 \\
0.01 \\
0.01 \\
0.01 \\
0.01 \\
0.01 \\
0.01 \\
0.01 \\
0.01 \\
0.01 \\
0.01 \\
0.01 \\
0.01 \\
0.01 \\
0.01 \\
0.01 \\
0.01\end{array}$ & 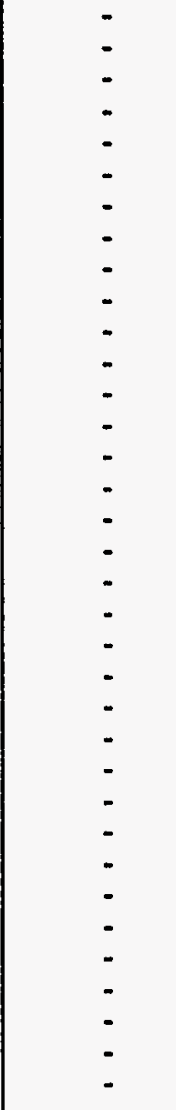 \\
\hline SOOIUM & $\begin{array}{l}0910 \\
0910 \\
0910\end{array}$ & $\begin{array}{l}02 / 17 / 87 \\
09 / 18 / 87 \\
01 / 30 / 88\end{array}$ & $\begin{array}{l}0001 \\
0001 \\
0001\end{array}$ & $\begin{array}{l}W L \\
W L \\
W L\end{array}$ & $\begin{array}{l}N \\
N \\
N\end{array}$ & MG/L & & $\begin{array}{l}102 . \\
85.9 \\
82.6\end{array}$ & & $\begin{array}{l}0.002 \\
0.002 \\
0.002\end{array}$ & $\begin{array}{l}- \\
-\end{array}$ \\
\hline
\end{tabular}

FORMATION OF COMPLETION CODE:

WL - WASATCH - LOWER SANDSTONE

WM - HASATCH - MIDDLE SHALE

PARAMETER VALUE INDICATOR (PVI): < - LESS THAN DETECTION LIMIT
FLOW RELATIONSHIP CODE:

N - UNKNOWN

SAMPLE IO CODES

0001 - FILTERED SAMPLE (.45 MICRONS)

OTHER PARAMETER VALUE FLAGS:

$\checkmark$ - ESTIMATED VALUE 
GROUNDWATER QUALITY DATA BY PARAMETER

LOWER SANDSTONE AND MIDDLE SHALE UNITS

SITE: SPK01 SPOOK

$02 / 17 / 87$ TO $12 / 10 / 88$

REPORT DATE: $07 / 29 / 94$

\begin{tabular}{|c|c|c|c|c|c|c|c|c|c|c|c|}
\hline PARAMETER NAME & $\underset{\text { ID }}{\text { LOCATION }}$ & LOG DATE & $\underset{\text { ID }}{\text { SAMPLE }}$ & $\begin{array}{l}\text { FORM } \\
\text { COMP }\end{array}$ & $\begin{array}{l}\text { FLOW } \\
\text { REL. }\end{array}$ & $\begin{array}{l}\text { UNITS OF } \\
\text { MEASURE }\end{array}$ & PVI & $\begin{array}{l}\text { PARAMETER } \\
\text { VALUE }\end{array}$ & FLAGS & $\begin{array}{l}\text { DETECTION } \\
\text { LIMIT }\end{array}$ & $\begin{array}{l}\text { PARAMETER } \\
\text { UNCERTAINTY }\end{array}$ \\
\hline SOOIUM & $\begin{array}{l}0910 \\
0910 \\
0910 \\
0914 \\
0914 \\
0914 \\
0914 \\
0914 \\
0914 \\
0915 \\
0915 \\
0915 \\
0915 \\
0915 \\
0915 \\
0919 \\
0919 \\
0919 \\
0919 \\
0919 \\
0919 \\
0921 \\
0921 \\
0921 \\
0921 \\
0921 \\
0923 \\
0923 \\
0923 \\
0923 \\
0923 \\
0938\end{array}$ & $\begin{array}{l}04 / 22 / 88 \\
08 / 11 / 88 \\
12 / 10 / 88 \\
02 / 18 / 87 \\
09 / 18 / 87 \\
02 / 03 / 88 \\
04 / 22 / 88 \\
08 / 08 / 88 \\
12 / 10 / 88 \\
02 / 20 / 87 \\
09 / 20 / 87 \\
02 / 03 / 88 \\
04 / 22 / 88 \\
08 / 10 / 88 \\
12 / 10 / 88 \\
02 / 19 / 87 \\
09 / 20 / 87 \\
01 / 30 / 88 \\
04 / 26 / 88 \\
08 / 27 / 88 \\
12 / 10 / 88 \\
09 / 20 / 87 \\
02 / 04 / 88 \\
04 / 26 / 88 \\
08 / 10 / 88 \\
12 / 10 / 88 \\
09 / 20 / 87 \\
01 / 30 / 88 \\
04 / 26 / 88 \\
08 / 09 / 88 \\
12 / 10 / 88 \\
12 / 10 / 88\end{array}$ & $\begin{array}{l}0001 \\
0001 \\
0001 \\
0001 \\
0001 \\
0001 \\
0001 \\
0001 \\
0001 \\
0001 \\
0001 \\
0001 \\
0001 \\
0001 \\
0001 \\
0001 \\
0001 \\
0001 \\
0001 \\
0001 \\
0001 \\
0001 \\
0001 \\
0001 \\
0001 \\
0001 \\
0001 \\
0001 \\
0001 \\
0001 \\
0001 \\
0001\end{array}$ & $\begin{array}{l}W L \\
W L \\
W L \\
W L \\
W L \\
W L \\
W L \\
W L \\
W L \\
W M \\
W M \\
W M \\
W M \\
W M \\
W M \\
W L \\
W L \\
W L \\
W L \\
W L \\
W L \\
W L \\
W L \\
W L \\
W L \\
H L \\
W L \\
W L \\
W L \\
W L \\
W L \\
W L\end{array}$ & $\begin{array}{l}N \\
N \\
N \\
N \\
N \\
N \\
N \\
N \\
N \\
N \\
N \\
N \\
N \\
N \\
N \\
N \\
N \\
N \\
N \\
N \\
N \\
N \\
N \\
N \\
N \\
N \\
N \\
N \\
N \\
N \\
N \\
N\end{array}$ & $M G / L$ & & $\begin{array}{r}79.1 \\
75.0 \\
77.2 \\
84.8 \\
61.3 \\
59.8 \\
56.7 \\
51.5 \\
56.4 \\
39.3 \\
44.0 \\
51.9 \\
70.5 \\
37.3 \\
43.6 \\
55.1 \\
54.2 \\
54.5 \\
55.6 \\
54.7 \\
57.6 \\
41.7 \\
32.9 \\
35.3 \\
39.3 \\
36.0 \\
101 . \\
86.3 \\
92.5 \\
103 . \\
97.6 \\
80.3\end{array}$ & $\mathrm{~J}$ & $\begin{array}{l}0.002 \\
0.002 \\
0.002 \\
0.002 \\
0.002 \\
0.002 \\
0.002 \\
0.002 \\
0.002 \\
0.002 \\
0.002 \\
0.002 \\
0.002 \\
0.002 \\
0.002 \\
0.002 \\
0.002 \\
0.002 \\
0.002 \\
0.002 \\
0.002 \\
0.002 \\
0.002 \\
0.002 \\
0.002 \\
0.002 \\
0.002 \\
0.002 \\
0.002 \\
0.002 \\
0.002 \\
0.002\end{array}$ & $\begin{array}{l}- \\
- \\
- \\
- \\
- \\
- \\
- \\
- \\
- \\
- \\
- \\
- \\
- \\
- \\
- \\
- \\
- \\
- \\
- \\
- \\
- \\
- \\
- \\
- \\
-\end{array}$ \\
\hline SPECIFIC CONDUCTANCE & $\begin{array}{l}0910 \\
0910 \\
0910 \\
0910 \\
0910 \\
0910\end{array}$ & $\begin{array}{l}02 / 17 / 87 \\
09 / 18 / 87 \\
01 / 30 / 88 \\
04 / 22 / 88 \\
08 / 11 / 88 \\
12 / 10 / 88\end{array}$ & $\begin{array}{l}0001 \\
0001 \\
0001 \\
0001 \\
0001 \\
0001\end{array}$ & $\begin{array}{l}W L \\
W L \\
W L \\
W L \\
W L \\
W L\end{array}$ & $\begin{array}{l}N \\
N \\
N \\
N \\
N \\
N\end{array}$ & UMHO/CM & & $\begin{array}{l}525 . \\
275 . \\
330 . \\
470 . \\
460.0 \\
475 .\end{array}$ & & $\begin{array}{l}- \\
- \\
- \\
-\end{array}$ & $\begin{array}{l}- \\
- \\
- \\
-\end{array}$ \\
\hline
\end{tabular}

FORMATION OF COMPLETION CODE:

WL - HASATCH - LOWER SANDSTONE

FLOW RELATIONSHIP CODE:

WM - HASATCH - MIDDLE SHALE

N - UNKNOWN

PARAMETER VALUE INDICATOR (PVI): < - LESS THAN DETECTION LIMIT

SAMPLE ID CODES:

0001 - FILTERED SAMPLE (.45 MICRONS)

OTHER PARAMETER VALUE FLAGS

$J$ - ESTIMATED VALUE 
GROUNDUATER QUALITY DATA BY PARAMETER

LONER SAMDSTONE AMD MIDOLE SHALE UMITS

SITE: SPK01 SPOOK

$02 / 17 / 87$ TO $12 / 10 / 88$

REPORT DATE: $07 / 29 / 94$

\begin{tabular}{|c|c|c|c|c|c|c|c|c|c|c|c|}
\hline PARAMETER NAME & $\begin{array}{c}\text { LOCAT ION } \\
\text { ID }\end{array}$ & LOG DATE & $\begin{array}{c}\text { SAMPLE } \\
10\end{array}$ & $\begin{array}{l}\text { FORM } \\
\text { COMP }\end{array}$ & $\begin{array}{l}\text { FLOW } \\
\text { REL. }\end{array}$ & $\begin{array}{l}\text { UNITS OF } \\
\text { MEASURE }\end{array}$ & PVI & $\begin{array}{l}\text { PARAMETER } \\
\text { VALUE }\end{array}$ & FLAGS & $\begin{array}{l}\text { DETECTION } \\
\text { LIMIT }\end{array}$ & $\begin{array}{l}\text { PARAMETER } \\
\text { UNCERTAINTY }\end{array}$ \\
\hline STRONTIUM & $\begin{array}{l}0915 \\
0915 \\
0915 \\
0919 \\
0919 \\
0919 \\
0919 \\
0921 \\
0921 \\
0921 \\
0921 \\
0923 \\
0923 \\
0923 \\
0923 \\
0938\end{array}$ & $\begin{array}{l}02 / 03 / 88 \\
04 / 22 / 88 \\
08 / 10 / 88 \\
09 / 20 / 87 \\
01 / 30 / 88 \\
04 / 26 / 88 \\
08 / 27 / 88 \\
09 / 20 / 87 \\
02 / 04 / 88 \\
04 / 26 / 88 \\
08 / 10 / 88 \\
09 / 20 / 87 \\
01 / 30 / 88 \\
04 / 26 / 88 \\
08 / 09 / 88 \\
08 / 09 / 88\end{array}$ & $\begin{array}{l}0001 \\
0001 \\
0001 \\
0001 \\
0001 \\
0001 \\
0001 \\
0001 \\
0001 \\
0001 \\
0001 \\
0001 \\
0001 \\
0001 \\
0001 \\
0001\end{array}$ & $\begin{array}{l}\text { UM } \\
W M \\
W L \\
W L \\
W L \\
W L \\
W L \\
W L \\
W L \\
W L \\
W L \\
W L \\
W L \\
W L \\
W L \\
W L\end{array}$ & $\begin{array}{l}N \\
N \\
N \\
N \\
N \\
N \\
N \\
N \\
N \\
N \\
N \\
N \\
N \\
N \\
N \\
N\end{array}$ & $M G / L$ & < & $\begin{array}{l}0.53 \\
0.43 \\
0.3 \\
0.744 \\
0.67 \\
0.52 \\
0.8 \\
1.35 \\
1.23 \\
0.98 \\
1.4 \\
0.655 \\
0.62 \\
0.47 \\
0.8 \\
0.1\end{array}$ & $\mathrm{~J}$ & $\begin{array}{l}0.1 \\
0.1 \\
0.1 \\
0.1 \\
0.1 \\
0.1 \\
0.1 \\
0.1 \\
0.1 \\
0.1 \\
0.1 \\
0.1 \\
0.1 \\
0.1 \\
0.1 \\
0.1\end{array}$ & $\begin{array}{l}- \\
- \\
- \\
- \\
- \\
- \\
- \\
- \\
- \\
- \\
- \\
-\end{array}$ \\
\hline SULFATE & $\begin{array}{l}0910 \\
0910 \\
0910 \\
0910 \\
0910 \\
0910 \\
0914 \\
0914 \\
0914 \\
0914 \\
0914 \\
0914 \\
0915 \\
0915 \\
0915 \\
0915 \\
0915 \\
0915 \\
0919 \\
0919 \\
0919 \\
0919\end{array}$ & $\begin{array}{l}02 / 17 / 87 \\
09 / 18 / 87 \\
01 / 30 / 88 \\
04 / 22 / 88 \\
08 / 11 / 88 \\
12 / 10 / 88 \\
02 / 18 / 87 \\
09 / 18 / 87 \\
02 / 03 / 88 \\
04 / 22 / 88 \\
08 / 08 / 88 \\
12 / 10 / 88 \\
02 / 20 / 87 \\
09 / 20 / 87 \\
02 / 03 / 88 \\
04 / 22 / 88 \\
08 / 10 / 88 \\
12 / 10 / 88 \\
02 / 19 / 87 \\
09 / 20 / 87 \\
01 / 30 / 88 \\
04 / 26 / 88\end{array}$ & $\begin{array}{l}0001 \\
0001 \\
0001 \\
0001 \\
0001 \\
0001 \\
0001 \\
0001 \\
0001 \\
0001 \\
0001 \\
0001 \\
0001 \\
0001 \\
0001 \\
0001 \\
0001 \\
0001 \\
0001 \\
0001 \\
0001 \\
0001\end{array}$ & $\begin{array}{l}W L \\
W L \\
W L \\
W L \\
W L \\
W L \\
H L \\
W L \\
W L \\
W L \\
W L \\
W L \\
W M \\
W M \\
W H \\
W M \\
W M \\
W M \\
H L \\
W L \\
H L \\
H L\end{array}$ & $\begin{array}{l}N \\
N \\
N \\
N \\
N \\
N \\
N \\
N \\
N \\
N \\
N \\
N \\
N \\
N \\
N \\
N \\
N \\
N \\
N \\
N \\
N \\
N\end{array}$ & $M G / L$ & & $\begin{array}{l}290 . \\
217 . \\
212 . \\
214 . \\
216 . \\
198 . \\
124 . \\
124 . \\
119 . \\
126 . \\
133 . \\
110 . \\
116 . \\
125 . \\
135 . \\
135 . \\
114 . \\
96 . \\
171 . \\
174 . \\
155 . \\
173 .\end{array}$ & $J$ & $\begin{array}{l}0.1 \\
0.1 \\
0.1 \\
0.1 \\
0.1 \\
0.1 \\
0.1 \\
0.1 \\
0.1 \\
0.1 \\
0.1 \\
0.1 \\
0.1 \\
0.1 \\
0.1 \\
0.1 \\
0.1 \\
0.1 \\
0.1 \\
0.1 \\
0.1 \\
0.1\end{array}$ & $\begin{array}{l}- \\
- \\
- \\
- \\
- \\
- \\
- \\
- \\
- \\
- \\
- \\
- \\
- \\
- \\
- \\
- \\
- \\
-\end{array}$ \\
\hline
\end{tabular}

FORMATION OF COMPLETION COOE:

WM - HASATCH - MIDDLE SHALE

FLOW RELATIONSHIP COOE:

WL - MASATCH - LONER SAMDSTONE

N - UMKNONI

PARAMETER VALUE INDICATOR (PVI): < - LESS THAN DETECTION LIMIT SAMPLE ID CODES:

0001 - FILTERED SAMPLE (.45 MICROMS)

OTHER PARAMETER VALUE FLAGS:

J - ESTIMATED VALUE 


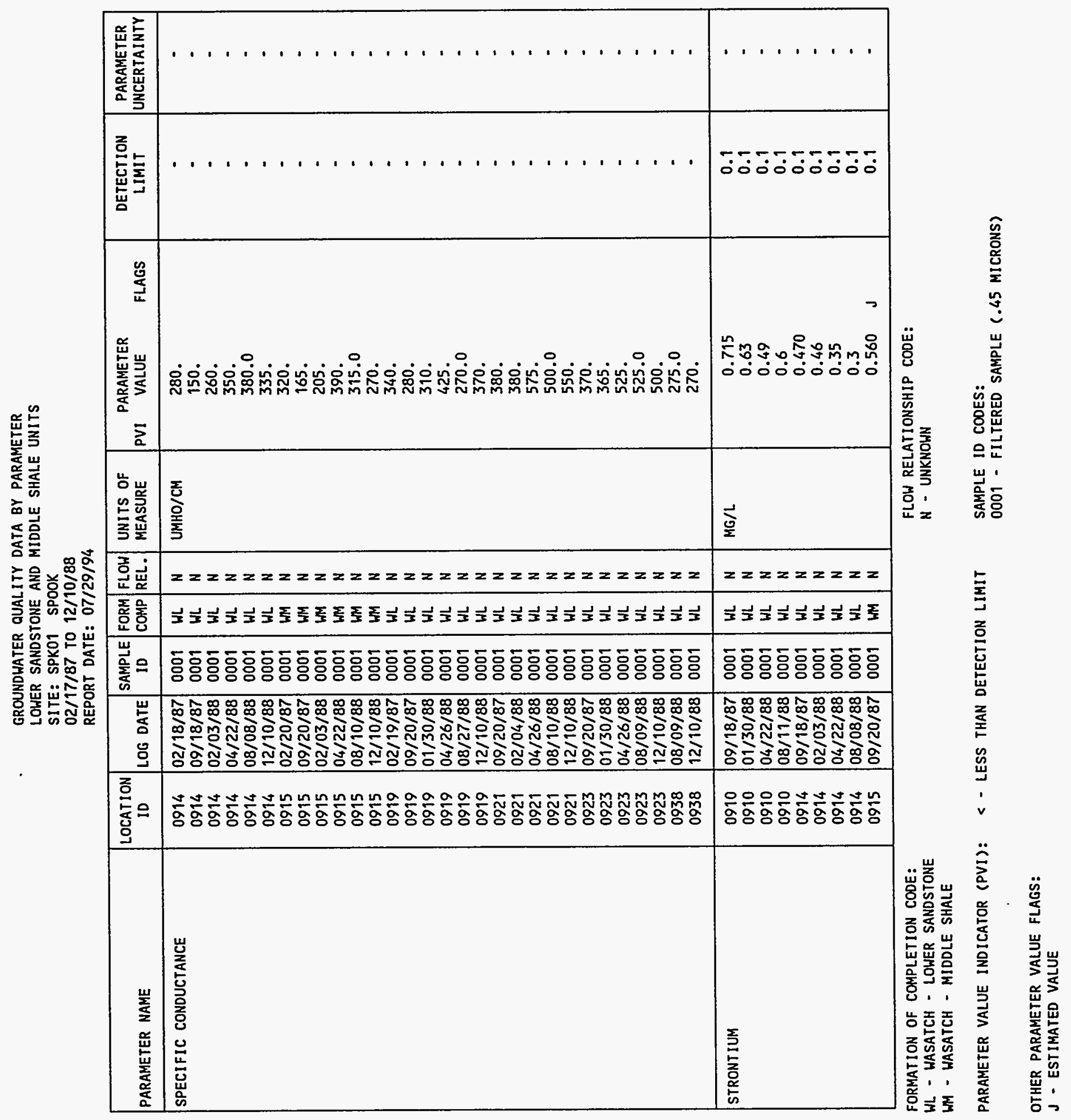




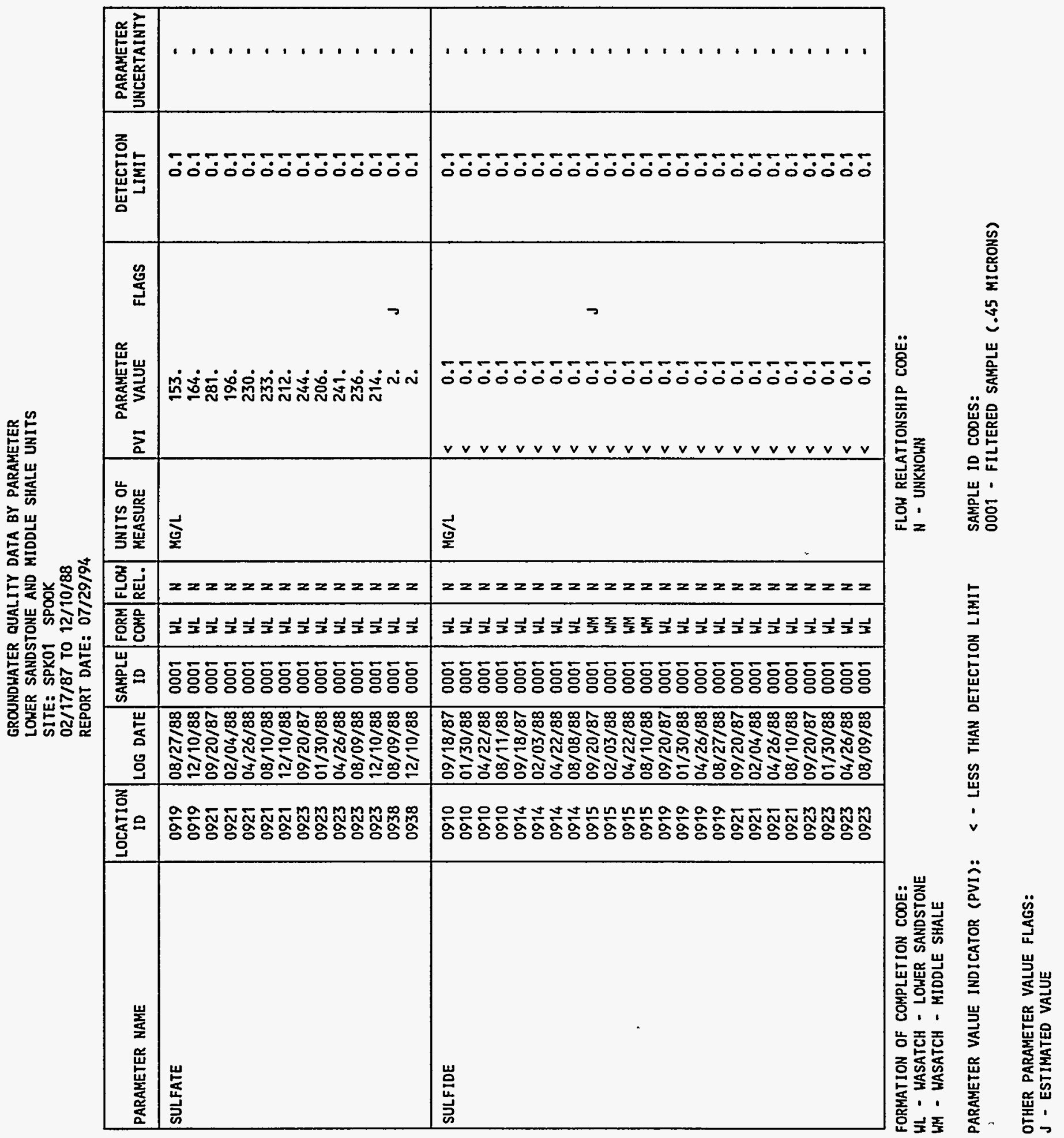


GROUNDWATER QUALITY DATA BY PARAMETER

LOWER SANDSTONE AND MIDDLE SHALE UNITS

SITE: SPKO1 SPOOK

REPORT TO $12 / 10 / 88$

REPORT DATE: $07 / 29194$

\begin{tabular}{|c|c|c|c|c|c|c|c|c|c|c|}
\hline PARAMETER NAME & $\underset{\text { LOCATION }}{\text { ID }}$ & LOG DATE & $\begin{array}{c}\text { SAMPLE } \\
\text { ID }\end{array}$ & $\begin{array}{l}\text { FORM } \\
\text { COMP }\end{array}$ & $\begin{array}{l}\text { FLOW } \\
\text { REL. }\end{array}$ & $\begin{array}{l}\text { UNITS OF } \\
\text { MEASURE }\end{array}$ & $\begin{array}{l}\text { PARAMETER } \\
\text { PVI VALUE }\end{array}$ & FLAGS & $\begin{array}{l}\text { DETECTION } \\
\text { LIMIT }\end{array}$ & $\begin{array}{l}\text { PARAMETER } \\
\text { UNCERTAINTY }\end{array}$ \\
\hline SULFIDE & 0938 & $08 / 09 / 88$ & 0001 & $W L$ & H & $M G / L$ & 0.1 & J & 0.1 & - \\
\hline TEMPERATURE & $\begin{array}{l}0910 \\
0910 \\
0910 \\
0910 \\
0910 \\
0910 \\
0914 \\
0914 \\
0914 \\
0914 \\
0914 \\
0914 \\
0915 \\
0915 \\
0915 \\
0915 \\
0915 \\
0915 \\
0919 \\
0919 \\
0919 \\
0919 \\
0919 \\
0919 \\
0921 \\
0921 \\
0921 \\
0921 \\
0921 \\
0923 \\
0923 \\
0923 \\
0923 \\
0923 \\
0938 \\
0938\end{array}$ & $\begin{array}{l}02 / 17 / 87 \\
09 / 18 / 87 \\
01 / 30 / 88 \\
04 / 22 / 88 \\
08 / 11 / 88 \\
12 / 10 / 88 \\
02 / 18 / 87 \\
09 / 18 / 87 \\
02 / 03 / 88 \\
04 / 22 / 88 \\
08 / 08 / 88 \\
12 / 10 / 88 \\
02 / 20 / 87 \\
09 / 20 / 87 \\
02 / 03 / 88 \\
04 / 22 / 88 \\
08 / 10 / 88 \\
12 / 10 / 88 \\
02 / 19 / 87 \\
09 / 20 / 87 \\
01 / 30 / 88 \\
04 / 26 / 88 \\
08 / 27 / 88 \\
12 / 10 / 88 \\
09 / 20 / 87 \\
02 / 04 / 88 \\
04 / 26 / 88 \\
08 / 10 / 88 \\
12 / 10 / 88 \\
09 / 20 / 87 \\
01 / 30 / 88 \\
04 / 26 / 88 \\
08 / 09 / 88 \\
12 / 10 / 88 \\
08 / 09 / 88 \\
12 / 10 / 88\end{array}$ & $\begin{array}{l}0001 \\
0001 \\
0001 \\
0001 \\
0001 \\
0001 \\
0001 \\
0001 \\
0001 \\
0001 \\
0001 \\
0001 \\
0001 \\
0001 \\
0001 \\
0001 \\
0001 \\
0001 \\
0001 \\
0001 \\
0001 \\
0001 \\
0001 \\
0001 \\
0001 \\
0001 \\
0001 \\
0001 \\
0001 \\
0001 \\
0001 \\
0001 \\
0001 \\
0001 \\
0001 \\
0001\end{array}$ & $\begin{array}{l}W L \\
W L \\
W L \\
W L \\
W L \\
W L \\
W L \\
W L \\
W L \\
W L \\
W L \\
W L \\
W M \\
W M \\
W M \\
W M \\
W M \\
W M \\
W L \\
W L \\
W L \\
W L \\
W L \\
W L \\
W L \\
W L \\
W L \\
W L \\
W L \\
W L \\
W L \\
W L \\
W L \\
W L \\
W L \\
W L\end{array}$ & $\begin{array}{l}N \\
N \\
N \\
N \\
N \\
N \\
N \\
N \\
N \\
N \\
N \\
N \\
N \\
N \\
N \\
N \\
N \\
N \\
N \\
N \\
N \\
N \\
N \\
N \\
N \\
N \\
N \\
N \\
N \\
N \\
N \\
N \\
N \\
N \\
N \\
N\end{array}$ & C - DEGREE & $\begin{array}{l}12.0 \\
13.0 \\
12.0 \\
13.0 \\
14.0 \\
11.5 \\
11.0 \\
12.5 \\
12.0 \\
13.0 \\
18.5 \\
11.5 \\
10 . \\
12.0 \\
11.5 \\
14.5 \\
14.0 \\
10.5 \\
11 . \\
13.0 \\
11.5 \\
12.0 \\
13.0 \\
10.0 \\
13.0 \\
11.5 \\
12.5 \\
14.0 \\
11.0 \\
13.0 \\
12.5 \\
13.0 \\
13.75 \\
12.0 \\
13.75 \\
12.0\end{array}$ & & $\begin{array}{l}- \\
: \\
: \\
- \\
- \\
- \\
- \\
- \\
- \\
- \\
- \\
- \\
- \\
- \\
- \\
- \\
- \\
- \\
- \\
- \\
- \\
- \\
- \\
-\end{array}$ & $\begin{array}{l}- \\
: \\
: \\
: \\
- \\
- \\
- \\
- \\
- \\
- \\
- \\
- \\
- \\
: \\
- \\
- \\
- \\
- \\
- \\
- \\
- \\
- \\
- \\
- \\
- \\
- \\
-\end{array}$ \\
\hline THORIUM-230 & 0910 & $09 / 18 / 87$ & 0001 & WL & N & $\mathrm{PCI} / \mathrm{L}$ & 0.0 . & & 1. & 0.5 \\
\hline
\end{tabular}

FORMATION OF COMPLETION CODE:

FLOW RELATIONSHIP CODE:

HL - WASATCH - LOWER SAMDSTONE

N - UNKNOWN

IM - WASATCH - MIDDLE SHALE

SAMPLE ID CODES:

0001 - FILTERED SAMPLE (.45 MICRONS)

OTHER PARAMETER VALUE FLAGS:

$J$ - ESTIMATED VALUE 
GROUNDHATER QUALITY DATA BY PARAMETER

LOWER SANDSTONE AND MIDDLE SHALE UNITS

SITE: SPKO1 SPOOK

REPORT DATE: $07 / 29 / 94$

\begin{tabular}{|c|c|c|c|c|c|c|c|c|c|c|c|}
\hline PARAMETER NAME & $\begin{array}{l}\text { LOCATION } \\
\text { ID }\end{array}$ & LOG DATE & $\begin{array}{c}\text { SAMPLE } \\
\text { ID }\end{array}$ & $\begin{array}{l}\text { FORM } \\
\text { COMP }\end{array}$ & $\begin{array}{l}\text { FLOW } \\
\text { REL. }\end{array}$ & $\begin{array}{l}\text { UNITS OF } \\
\text { MEASURE }\end{array}$ & PVI & $\begin{array}{l}\text { PARAMETER } \\
\text { VALUE }\end{array}$ & FLAGS & $\begin{array}{l}\text { DETECTION } \\
\text { LIMIT }\end{array}$ & $\begin{array}{l}\text { PARAMETER } \\
\text { UNCERTAINTY }\end{array}$ \\
\hline THORIUH-230 & $\begin{array}{l}0910 \\
0910 \\
0910 \\
0914 \\
0914 \\
0914 \\
0914 \\
0915 \\
0915 \\
0915 \\
0915 \\
0919 \\
0919 \\
0919 \\
0919 \\
0921 \\
0921 \\
0921 \\
0921 \\
0923 \\
0923 \\
0923 \\
0923 \\
0938\end{array}$ & $\begin{array}{l}01 / 30 / 88 \\
04 / 22 / 88 \\
08 / 11 / 88 \\
09 / 18 / 87 \\
02 / 03 / 88 \\
04 / 22 / 88 \\
08 / 08 / 88 \\
09 / 20 / 87 \\
02 / 03 / 88 \\
04 / 22 / 88 \\
08 / 10 / 88 \\
09 / 20 / 87 \\
01 / 30 / 88 \\
04 / 26 / 88 \\
08 / 27 / 88 \\
09 / 20 / 87 \\
02 / 04 / 88 \\
04 / 26 / 88 \\
08 / 10 / 88 \\
09 / 20 / 87 \\
01 / 30 / 88 \\
04 / 26 / 88 \\
08 / 09 / 88 \\
08 / 09 / 88\end{array}$ & $\begin{array}{l}0001 \\
0001 \\
0001 \\
0001 \\
0001 \\
0001 \\
0001 \\
0001 \\
0001 \\
0001 \\
0001 \\
0001 \\
0001 \\
0001 \\
0001 \\
0001 \\
0001 \\
0001 \\
0001 \\
0001 \\
0001 \\
0001 \\
0001 \\
0001\end{array}$ & $\begin{array}{l}W L \\
W L \\
W L \\
W L \\
W L \\
W L \\
W L \\
W N \\
W N \\
W M \\
W N \\
W L \\
W L \\
W L \\
W L \\
W L \\
W L \\
W L \\
W L \\
W L \\
W L \\
W L \\
W L \\
W L\end{array}$ & $\begin{array}{l}N \\
N \\
N \\
N \\
N \\
N \\
N \\
N \\
N \\
N \\
N \\
N \\
N \\
N \\
N \\
N \\
N \\
N \\
N \\
N \\
N \\
N \\
N \\
N\end{array}$ & $\mathrm{PCI} / \mathrm{L}$ & & $\begin{array}{l}0.0 \\
0.0 \\
0.1 \\
0.0 \\
0.1 \\
0.0 \\
0.1 \\
0.0 \\
0.0 \\
0.0 \\
0.0 \\
0.0 \\
0.0 \\
0.1 \\
0.2 \\
0.0 \\
0.4 \\
0.0 \\
0.1 \\
0.2 \\
0.2 \\
0.0 \\
0.0 \\
0.2\end{array}$ & $\mathbf{J}$ & $\begin{array}{l}1 . \\
1 . \\
1 . \\
1 . \\
1 . \\
1 . \\
1 . \\
1 . \\
1 . \\
1 . \\
1 . \\
1 . \\
1 . \\
1 . \\
1 . \\
1 . \\
1 . \\
1 . \\
1 . \\
1 . \\
1 .\end{array}$ & $\begin{array}{l}0.4 \\
0.3 \\
0.5 \\
0.5 \\
0.6 \\
0.4 \\
0.6 \\
0.4 \\
0.5 \\
0.4 \\
0.5 \\
0.5 \\
0.4 \\
0.4 \\
0.5 \\
0.4 \\
0.6 \\
0.2 \\
0.6 \\
0.6 \\
0.6 \\
0.3 \\
0.5 \\
0.6\end{array}$ \\
\hline TIN & $\begin{array}{l}0910 \\
0910 \\
0910 \\
0910 \\
0914 \\
0914 \\
0914 \\
0914 \\
0915 \\
0915 \\
0915 \\
0915 \\
0919 \\
0919\end{array}$ & $\begin{array}{l}09 / 18 / 87 \\
01 / 30 / 88 \\
04 / 22 / 88 \\
08 / 11 / 88 \\
09 / 18 / 87 \\
02 / 03 / 88 \\
04 / 22 / 88 \\
08 / 08 / 88 \\
09 / 20 / 87 \\
02 / 03 / 88 \\
04 / 22 / 88 \\
08 / 10 / 88 \\
09 / 20 / 87 \\
01 / 30 / 88\end{array}$ & $\begin{array}{l}0001 \\
0001 \\
0001 \\
0001 \\
0001 \\
0001 \\
0001 \\
0001 \\
0001 \\
0001 \\
0001 \\
0001 \\
0001 \\
0001\end{array}$ & $\begin{array}{l}W L \\
W L \\
W L \\
W L \\
W L \\
W L \\
W L \\
W L \\
W N \\
W M \\
W N \\
W N \\
W L \\
W L\end{array}$ & $\begin{array}{l}N \\
N \\
N \\
N \\
N \\
N \\
N \\
N \\
N \\
N \\
N \\
N \\
N \\
N\end{array}$ & MG/L & $\begin{array}{l}< \\
< \\
< \\
< \\
<\end{array}$ & $\begin{array}{l}0.026 \\
0.005 \\
0.010 \\
0.005 \\
0.021 \\
0.005 \\
0.010 \\
0.005 \\
0.018 \\
0.005 \\
0.010 \\
0.005 \\
0.020 \\
0.005\end{array}$ & J & $\begin{array}{l}0.005 \\
0.005 \\
0.005 \\
0.005 \\
0.005 \\
0.005 \\
0.005 \\
0.005 \\
0.005 \\
0.005 \\
0.005 \\
0.005 \\
0.005 \\
0.005\end{array}$ & $\begin{array}{l}- \\
- \\
- \\
- \\
- \\
- \\
- \\
- \\
-\end{array}$ \\
\hline
\end{tabular}

FORMATION OF COMPLETION CODE: HL - HASATCH - LOWER SANDSTONE WM - HASATCH - MIDDLE SHALE

PARAMETER VALUE INDICATOR (PVI): < - LESS THAN DETECTION LIMIT
FLOW RELATIONSHIP CODE:

N - UNKNOHN

AMPLE IO CODES

0001 - FILTERED SAMPLE (.45 MICRONS)

OTHER PARAMETER VALUE FLAGS:

$J$ - ESTIMATED VALUE 
REPORT DATE: $07 / 29 / 9$

\begin{tabular}{|c|c|c|c|c|c|c|c|c|c|c|c|}
\hline PARAMETER NAME & $\begin{array}{c}\text { LOCATION } \\
\text { ID }\end{array}$ & LOG DATE & $\begin{array}{c}\text { SAMPLE } \\
\text { ID }\end{array}$ & $\begin{array}{l}\text { FORM } \\
\text { COMP }\end{array}$ & $\begin{array}{l}\text { FLOH } \\
\text { REL. }\end{array}$ & $\begin{array}{l}\text { UNITS OF } \\
\text { MEASURE }\end{array}$ & PVI & $\begin{array}{l}\text { PARAMETER } \\
\text { VALUE }\end{array}$ & FLAGS & $\begin{array}{l}\text { DETECTION } \\
\text { LIMIT }\end{array}$ & $\begin{array}{l}\text { PARAMETER } \\
\text { UNCERTAINTY }\end{array}$ \\
\hline TIN & $\begin{array}{l}0919 \\
0919 \\
0921 \\
0921 \\
0921 \\
0921 \\
0923 \\
0923 \\
0923 \\
0923 \\
0938\end{array}$ & $\begin{array}{l}04 / 26 / 88 \\
08 / 27 / 88 \\
09 / 20 / 87 \\
02 / 04 / 88 \\
04 / 26 / 88 \\
08 / 10 / 88 \\
09 / 20 / 87 \\
01 / 30 / 88 \\
04 / 26 / 88 \\
08 / 09 / 88 \\
08 / 09 / 88\end{array}$ & $\begin{array}{l}0001 \\
0001 \\
0001 \\
0001 \\
0001 \\
0001 \\
0001 \\
0001 \\
0001 \\
0001 \\
0001\end{array}$ & $\begin{array}{l}W L \\
W L \\
W L \\
W L \\
W L \\
W L \\
W L \\
W L \\
W L \\
W L \\
W L\end{array}$ & $\begin{array}{l}N \\
N \\
N \\
N \\
N \\
N \\
N \\
N \\
N \\
N \\
N\end{array}$ & $M G / L$ & $\mid \begin{array}{l}< \\
< \\
< \\
< \\
< \\
<\end{array}$ & $\begin{array}{l}0.005 \\
0.005 \\
0.016 \\
0.005 \\
0.007 \\
0.003 \\
0.026 \\
0.005 \\
0.007 \\
0.003 \\
0.005\end{array}$ & $\begin{array}{l}\text { J } \\
\text { J } \\
\text { J }\end{array}$ & $\begin{array}{l}0.005 \\
0.005 \\
0.005 \\
0.005 \\
0.005 \\
0.005 \\
0.005 \\
0.005 \\
0.005 \\
0.005 \\
0.005\end{array}$ & $\begin{array}{l}: \\
: \\
: \\
: \\
: \\
: \\
:\end{array}$ \\
\hline TOTAL DISSOLVED SOLIDS & $\begin{array}{l}0910 \\
0910 \\
0910 \\
0910 \\
0910 \\
0910 \\
0914 \\
0914 \\
0914 \\
0914 \\
0914 \\
0914 \\
0915 \\
0915 \\
0915 \\
0915 \\
0915 \\
0915 \\
0919 \\
0919 \\
0919 \\
0919 \\
0919 \\
0919 \\
0921 \\
0921 \\
0921\end{array}$ & $\begin{array}{l}02 / 17 / 87 \\
09 / 18 / 87 \\
01 / 30 / 88 \\
04 / 22 / 88 \\
08 / 11 / 88 \\
12 / 10 / 88 \\
02 / 18 / 87 \\
09 / 18 / 87 \\
02 / 03 / 88 \\
04 / 22 / 88 \\
08 / 08 / 88 \\
12 / 10 / 88 \\
02 / 20 / 87 \\
09 / 20 / 87 \\
02 / 03 / 88 \\
04 / 22 / 88 \\
08 / 10 / 88 \\
12 / 10 / 88 \\
02 / 19 / 87 \\
09 / 20 / 87 \\
01 / 30 / 88 \\
04 / 26 / 88 \\
08 / 27 / 88 \\
12 / 10 / 88 \\
09 / 20 / 87 \\
02 / 04 / 88 \\
04 / 26 / 88\end{array}$ & $\begin{array}{l}0001 \\
0001 \\
0001 \\
0001 \\
0001 \\
0001 \\
0001 \\
0001 \\
0001 \\
0001 \\
0001 \\
0001 \\
0001 \\
0001 \\
0001 \\
0001 \\
0001 \\
0001 \\
0001 \\
0001 \\
0001 \\
0001 \\
0001 \\
0001 \\
0001 \\
0001 \\
0001\end{array}$ & $\begin{array}{l}W L \\
W L \\
W L \\
W L \\
W L \\
W L \\
W L \\
W L \\
W L \\
W L \\
W L \\
W L \\
W M \\
W M \\
W M \\
W M \\
W M \\
W M \\
W L \\
W L \\
W L \\
W L \\
W L \\
W L \\
W L \\
W L \\
W L\end{array}$ & $\begin{array}{l}N \\
N \\
N \\
N \\
N \\
N \\
N \\
N \\
N \\
N \\
N \\
N \\
N \\
N \\
N \\
N \\
N \\
N \\
N \\
N \\
N \\
N \\
N \\
N \\
N \\
N \\
N\end{array}$ & $M G / L$ & & $\begin{array}{l}545 . \\
510 . \\
443 . \\
447 . \\
459 . \\
472 . \\
346 . \\
371 . \\
311 . \\
309 . \\
403 . \\
344 . \\
340 . \\
350 . \\
330 . \\
332 . \\
306 . \\
265 . \\
436 . \\
413 . \\
454 . \\
416 . \\
405 . \\
57.6 \\
600 . \\
611 . \\
585 .\end{array}$ & $J$ & $\begin{array}{l}10 . \\
10 . \\
10 . \\
10 . \\
10 . \\
10 . \\
10 . \\
10 . \\
10 . \\
10 . \\
10 . \\
10 . \\
10 . \\
10 . \\
10 . \\
10 . \\
10 . \\
10 . \\
10 . \\
10 . \\
10 . \\
10 . \\
10 . \\
10 . \\
10 . \\
10 . \\
10 .\end{array}$ & $\begin{array}{l}: \\
- \\
- \\
- \\
: \\
- \\
- \\
- \\
- \\
- \\
- \\
- \\
- \\
- \\
- \\
- \\
- \\
- \\
- \\
-\end{array}$ \\
\hline
\end{tabular}

FORMATION OF COMPLETION CODE: WL - WASATCH - LOWER SANDSTONE WM - WASATCH - MIDDLE SHALE

PARAMETER VALUE INDICATOR (PVI): < - LESS THAN DETECTION LIMIT

OTHER PARAMETER VALUE FLAGS:

$J$ - ESTIMATED VALUE
FLOH RELATIONSHIP CODE:

N - UNKNOWN

SAMPLE ID CODES:

0001 - FILTERED SAMPLE (.45 MICRONS) 
GROUNDWATER QUALITY DATA BY PARAMETER

LOWER SANDSTONE AND MIDDLE SHALE UNITS

SITE: SPKO1 SPOOK

$02 / 17 / 87$ To $12 / 10 / 88$

REPORT DATE: 07/29/94

\begin{tabular}{|c|c|c|c|c|c|c|c|c|c|c|c|}
\hline PARAMETER NAME & $\begin{array}{l}\text { LOCATION } \\
\text { ID }\end{array}$ & LOG DATE & $\begin{array}{c}\text { SAMPLE } \\
\text { ID }\end{array}$ & $\begin{array}{l}\text { FORM } \\
\text { COMP }\end{array}$ & $\begin{array}{l}\text { FLOW } \\
\text { REL. }\end{array}$ & $\begin{array}{l}\text { UNITS OF } \\
\text { MEASURE }\end{array}$ & PVI & $\begin{array}{l}\text { ARAMETER } \\
\text { VALUE }\end{array}$ & FLAGS & $\begin{array}{l}\text { DETECTION } \\
\text { LIMIT }\end{array}$ & $\begin{array}{l}\text { PARAMETER } \\
\text { UNCERTAINTY }\end{array}$ \\
\hline TOTAL DISSOLVED SOLIDS & $\begin{array}{l}0921 \\
0921 \\
0923 \\
0923 \\
0923 \\
0923 \\
0923 \\
0938 \\
0938\end{array}$ & $\begin{array}{l}08 / 10 / 88 \\
12 / 10 / 88 \\
09 / 20 / 87 \\
01 / 30 / 88 \\
04 / 26 / 88 \\
08 / 09 / 88 \\
12 / 10 / 88 \\
08 / 09 / 88 \\
12 / 10 / 88\end{array}$ & $\begin{array}{l}0001 \\
0001 \\
0001 \\
0001 \\
0001 \\
0001 \\
0001 \\
0001 \\
0001\end{array}$ & $\begin{array}{l}\text { WL } \\
W L \\
W L \\
W L \\
H L \\
W L \\
W L \\
H L \\
W L\end{array}$ & $\begin{array}{l}N \\
N \\
N \\
N \\
N \\
N \\
N \\
N \\
N\end{array}$ & MG/L & & $\begin{array}{l}564 . \\
555 . \\
523 . \\
549 . \\
509 . \\
503 . \\
510 . \\
265 . \\
264 .\end{array}$ & J & $\begin{array}{l}10 . \\
10 . \\
10 . \\
10 . \\
10 . \\
10 . \\
10 . \\
10 . \\
10 .\end{array}$ & $\begin{array}{l}- \\
- \\
- \\
- \\
- \\
-\end{array}$ \\
\hline URANIUM & $\begin{array}{l}0910 \\
0910 \\
0910 \\
0910 \\
0910 \\
0910 \\
0914 \\
0914 \\
0914 \\
0914 \\
0914 \\
0914 \\
0915 \\
0915 \\
0915 \\
0915 \\
0915 \\
0915 \\
0919 \\
0919 \\
0919 \\
0919 \\
0919 \\
0919 \\
0921 \\
0921 \\
0921 \\
0921 \\
0921\end{array}$ & $\begin{array}{l}02 / 17 / 87 \\
09 / 18 / 87 \\
01 / 30 / 88 \\
04 / 22 / 88 \\
08 / 11 / 88 \\
12 / 10 / 88 \\
02 / 18 / 87 \\
09 / 18 / 87 \\
02 / 03 / 88 \\
04 / 22 / 88 \\
08 / 08 / 88 \\
12 / 10 / 88 \\
02 / 20 / 87 \\
09 / 20 / 87 \\
02 / 03 / 88 \\
04 / 22 / 88 \\
08 / 10 / 88 \\
12 / 10 / 88 \\
02 / 19 / 87 \\
09 / 20 / 87 \\
01 / 30 / 88 \\
04 / 26 / 88 \\
08 / 27 / 88 \\
12 / 10 / 88 \\
09 / 20 / 87 \\
02 / 04 / 88 \\
04 / 26 / 88 \\
08 / 10 / 88 \\
12 / 10 / 88\end{array}$ & $\begin{array}{l}0001 \\
0001 \\
0001 \\
0001 \\
0001 \\
0001 \\
0001 \\
0001 \\
0001 \\
0001 \\
0001 \\
0001 \\
0001 \\
0001 \\
0001 \\
0001 \\
0001 \\
0001 \\
0001 \\
0001 \\
0001 \\
0001 \\
0001 \\
0001 \\
0001 \\
0001 \\
0001 \\
0001 \\
0001\end{array}$ & $\begin{array}{l}H L \\
W L \\
W L \\
W L \\
H L \\
W L \\
W L \\
W L \\
W L \\
W L \\
W L \\
H L \\
W M \\
W M \\
W M \\
W M \\
W M \\
W M \\
W L \\
W L \\
W L \\
W L \\
W L \\
W L \\
H L \\
W L \\
W L \\
H L \\
H L\end{array}$ & $\begin{array}{l}N \\
N \\
N \\
N \\
N \\
N \\
N \\
N \\
N \\
N \\
N \\
N \\
N \\
N \\
N \\
N \\
N \\
N \\
N \\
N \\
N \\
N \\
N \\
N \\
N \\
N \\
N \\
N \\
N\end{array}$ & $M G / L$ & & $\begin{array}{l}0.0822 \\
0.0139 \\
0.0184 \\
0.0178 \\
0.0117 \\
0.0101 \\
0.0072 \\
0.0065 \\
0.0022 \\
0.0024 \\
0.0017 \\
0.0012 \\
0.0579 \\
0.0249 \\
0.0124 \\
0.0244 \\
0.0069 \\
0.0063 \\
0.0078 \\
0.0014 \\
0.0116 \\
0.0023 \\
0.0005 \\
0.0015 \\
0.0010 \\
0.0009 \\
0.0010 \\
0.0005 \\
0.0006\end{array}$ & $\begin{array}{l}J \\
J \\
J \\
J \\
J \\
J \\
J \\
J \\
J \\
J\end{array}$ & $\begin{array}{l}0.003 \\
0.003 \\
0.003 \\
0.003 \\
0.003 \\
0.003 \\
0.003 \\
0.003 \\
0.003 \\
0.003 \\
0.003 \\
0.003 \\
0.003 \\
0.003 \\
0.003 \\
0.003 \\
0.003 \\
0.003 \\
0.003 \\
0.003 \\
0.003 \\
0.003 \\
0.003 \\
0.003 \\
0.003 \\
0.003 \\
0.003 \\
0.003 \\
0.003\end{array}$ & $\begin{array}{l}- \\
- \\
- \\
- \\
- \\
- \\
- \\
- \\
- \\
- \\
- \\
- \\
- \\
- \\
- \\
- \\
- \\
- \\
- \\
- \\
- \\
-\end{array}$ \\
\hline
\end{tabular}

FORMATION OF COMPLETION CODE:

WL - HASATCH - LOWER SANDSTONE

WM - HASATCH - MIDDLE SHALE

PARAMETER VALUE INDICATOR (PVI): < - LESS THAN DETECTION LIMIT
FLOH RELATIONSHIP CODE:

N - UNKNOWN

SAMPLE ID CODES:

0001 - FILTERED SAMPLE (.45 MICRONS)

OTHER PARAMETER VALUE FLAGS:

$J$ - ESTIMATED VALUE 


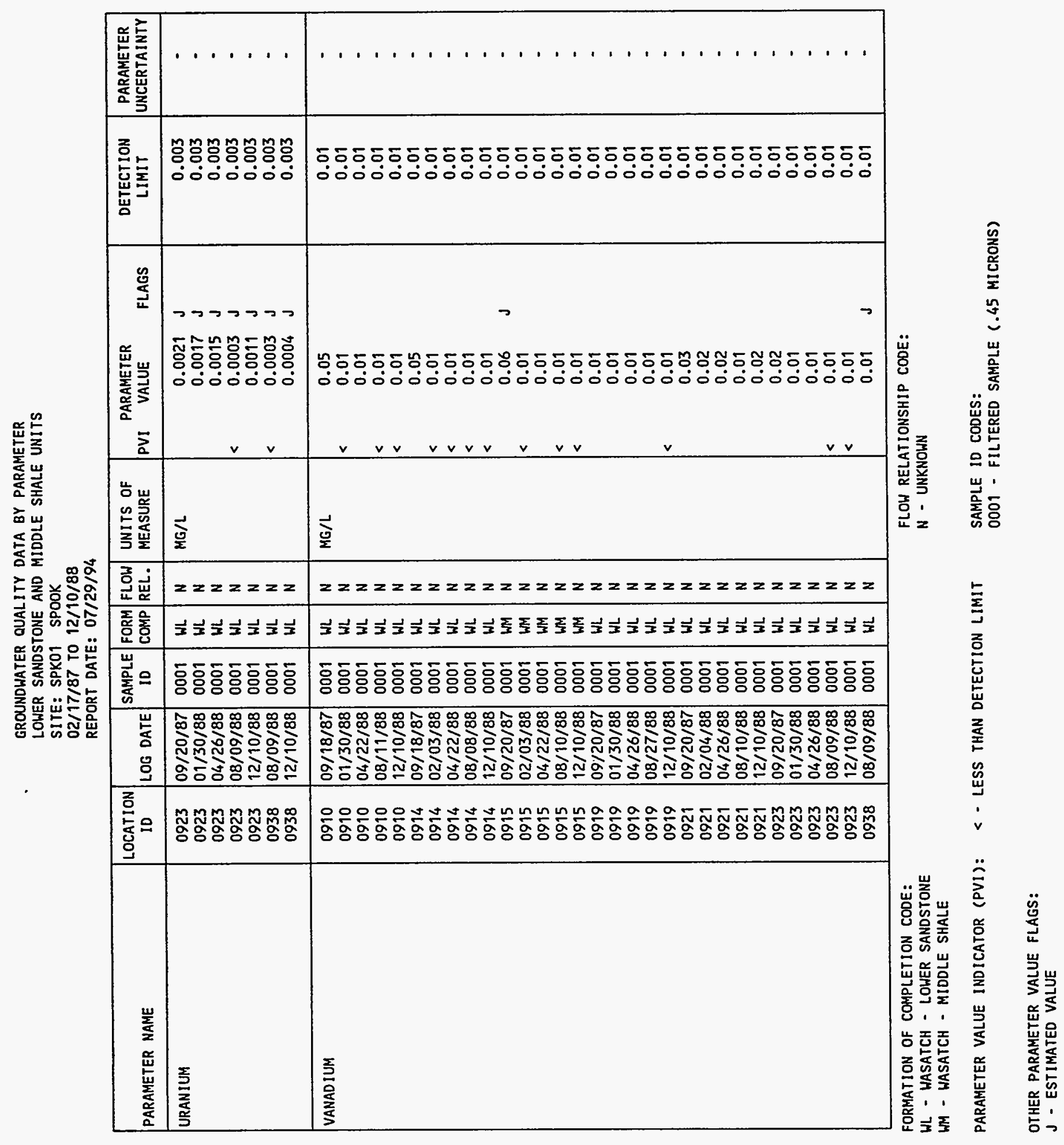




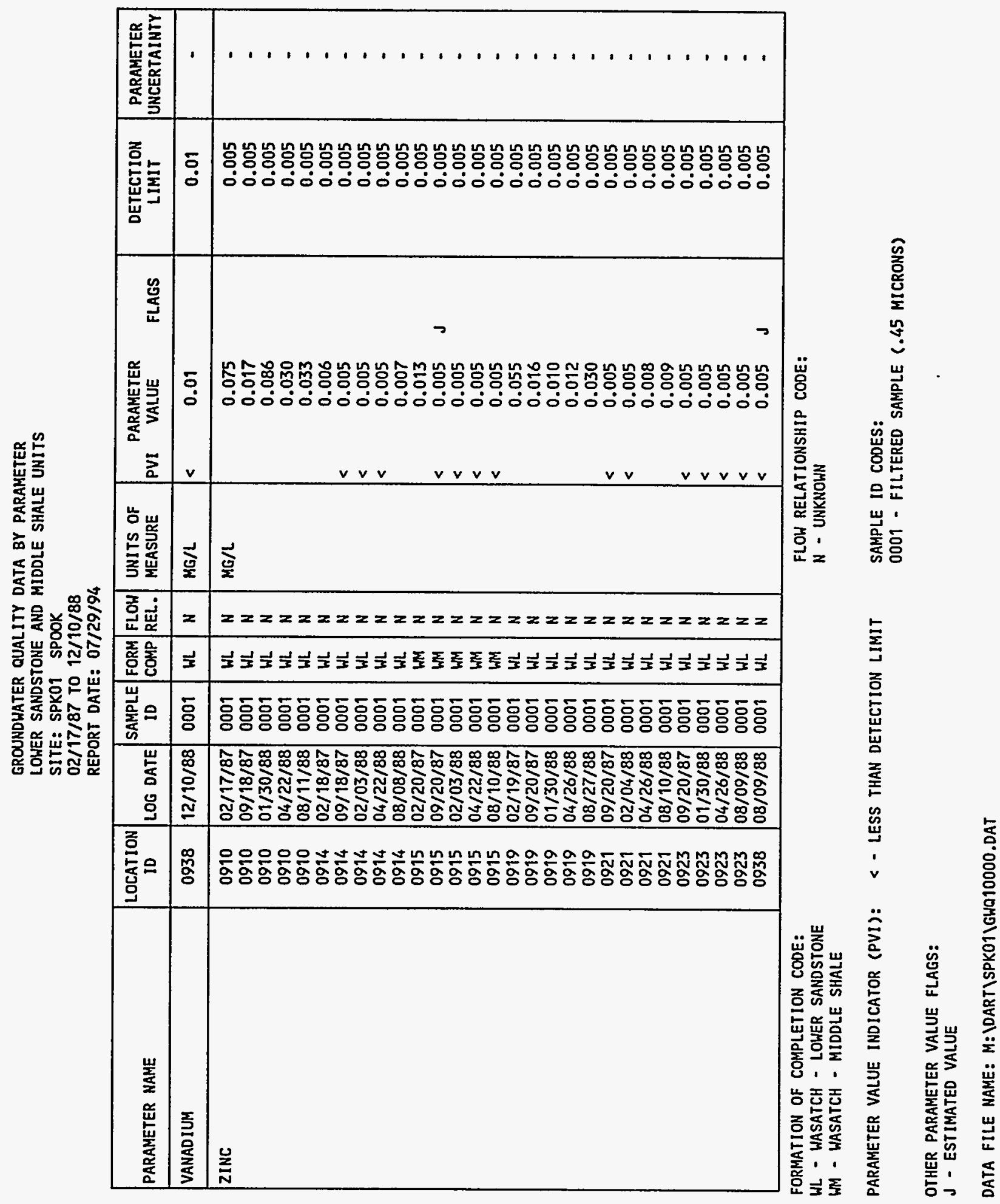


GROUNDWATER QUALITY DATA BY PARAMETER

Domestic Hells

SITE: SPKO1 SPOOK

$11 / 18 / 85$ TO $08 / 27 / 88$

REPORT DATE: $07 / 29 / 94$

\begin{tabular}{|c|c|c|c|c|c|c|c|c|c|c|c|}
\hline PARAMETER NAME & $\underset{\text { LOCATION }}{\text { ID }}$ & LOG DATE & $\begin{array}{c}\text { SAMPLE } \\
\text { ID }\end{array}$ & $\begin{array}{l}\text { FORM } \\
\text { COMP }\end{array}$ & $\begin{array}{l}\text { FLOW } \\
\text { REL. }\end{array}$ & $\begin{array}{l}\text { UNITS OF } \\
\text { MEASURE }\end{array}$ & PVI & $\begin{array}{l}\text { PARAMETER } \\
\text { VALUE }\end{array}$ & FLAGS & $\begin{array}{l}\text { DETECTION } \\
\text { LIMIT }\end{array}$ & $\begin{array}{l}\text { PARAMETER } \\
\text { UNCERTAINTY }\end{array}$ \\
\hline ALKALINITY & $\begin{array}{l}0800 \\
0803 \\
0903 \\
0903 \\
0903\end{array}$ & $\begin{array}{l}08 / 11 / 88 \\
08 / 27 / 88 \\
11 / 18 / 85 \\
02 / 19 / 87 \\
08 / 11 / 88\end{array}$ & $\begin{array}{l}0001 \\
0001 \\
0001 \\
0001 \\
0001\end{array}$ & $\begin{array}{l}\text { NR } \\
\text { NR } \\
\text { NR } \\
\text { NR } \\
\text { NR }\end{array}$ & $\begin{array}{l}N \\
N \\
N \\
N \\
N\end{array}$ & $\mathrm{MG} / \mathrm{L} \quad \mathrm{CACO} 3$ & & $\begin{array}{l}160.0 \\
165.0 \\
232 . \\
214 . \\
174.0\end{array}$ & & $\begin{array}{l}\ddot{-} \\
\dot{-} \\
\dot{-}\end{array}$ & :- \\
\hline ALUMINUM & $\begin{array}{l}0800 \\
0803 \\
0903 \\
0903 \\
0903\end{array}$ & $\begin{array}{l}08 / 11 / 88 \\
08 / 27 / 88 \\
11 / 18 / 85 \\
02 / 19 / 87 \\
08 / 11 / 88\end{array}$ & $\begin{array}{l}0001 \\
0001 \\
0001 \\
0001 \\
0001\end{array}$ & $\begin{array}{l}\text { NR } \\
\text { NR } \\
\text { NR } \\
\text { NR } \\
\text { NR }\end{array}$ & $\begin{array}{l}\mathrm{N} \\
\mathrm{N} \\
\mathrm{N} \\
\mathrm{N} \\
\mathrm{N}\end{array}$ & $M G / L$ & $\begin{array}{l}< \\
< \\
<\end{array}$ & $\begin{array}{l}0.1 \\
0.1 \\
0.4 \\
0.1 \\
0.1\end{array}$ & & $\begin{array}{l}0.1 \\
0.1 \\
0.1 \\
0.1 \\
0.1\end{array}$ & $:$ \\
\hline AMMONIUM & $\begin{array}{l}0800 \\
0803 \\
0903 \\
0903 \\
0903\end{array}$ & $\begin{array}{l}08 / 11 / 88 \\
08 / 27 / 88 \\
11 / 18 / 85 \\
02 / 19 / 87 \\
08 / 11 / 88\end{array}$ & $\begin{array}{l}0001 \\
0001 \\
0001 \\
0001 \\
0001\end{array}$ & $\begin{array}{l}\text { NR } \\
\text { NR } \\
\text { NR } \\
\text { NR } \\
\text { NR }\end{array}$ & $\begin{array}{l}\mathrm{N} \\
\mathrm{N} \\
\mathrm{N} \\
\mathrm{N} \\
\mathrm{N}\end{array}$ & $M G / L$ & $<$ & $\begin{array}{l}0.1 \\
0.1 \\
0.1 \\
0.5 \\
0.1\end{array}$ & & $\begin{array}{l}0.1 \\
0.1 \\
0.1 \\
0.1 \\
0.1\end{array}$ & : \\
\hline ANTIMONY & $\begin{array}{l}0800 \\
0803 \\
0903 \\
0903 \\
0903\end{array}$ & $\begin{array}{l}08 / 11 / 88 \\
08 / 27 / 88 \\
11 / 18 / 85 \\
02 / 19 / 87 \\
08 / 11 / 88\end{array}$ & $\begin{array}{l}0001 \\
0001 \\
0001 \\
0001 \\
0001\end{array}$ & $\begin{array}{l}\text { NR } \\
\text { NR } \\
\text { NR } \\
\text { NR } \\
\text { NR }\end{array}$ & $\begin{array}{l}N \\
N \\
N \\
N \\
N\end{array}$ & $M G / L$ & $\mid \begin{array}{l}< \\
< \\
< \\
< \\
<\end{array}$ & $\begin{array}{l}0.003 \\
0.003 \\
0.003 \\
0.003 \\
0.003\end{array}$ & $\mathrm{~J}$ & $\begin{array}{l}0.003 \\
0.003 \\
0.003 \\
0.003 \\
0.003\end{array}$ & $\begin{array}{l}- \\
- \\
-\end{array}$ \\
\hline ARSENIC & $\begin{array}{l}0800 \\
0803 \\
0903 \\
0903 \\
0903\end{array}$ & $\begin{array}{l}08 / 19 / 88 \\
08 / 27 / 88 \\
11 / 18 / 85 \\
02 / 19 / 87 \\
08 / 11 / 88\end{array}$ & $\begin{array}{l}0001 \\
0001 \\
0001 \\
0001 \\
0001\end{array}$ & $\begin{array}{l}\text { NR } \\
\text { NR } \\
\text { NR } \\
\text { NR } \\
\text { NR }\end{array}$ & $\begin{array}{l}N \\
N \\
N \\
N \\
N\end{array}$ & MG/L & $\mid \begin{array}{l}< \\
< \\
< \\
< \\
<\end{array}$ & $\begin{array}{l}0.01 \\
0.01 \\
0.01 \\
0.001 \\
0.01\end{array}$ & J & $\begin{array}{l}0.01 \\
0.01 \\
0.01 \\
0.01 \\
0.01\end{array}$ & $:$ \\
\hline BARIUM & $\begin{array}{l}0800 \\
0803 \\
0903 \\
0903 \\
0903\end{array}$ & $\begin{array}{l}08 / 11 / 88 \\
08 / 27 / 88 \\
11 / 18 / 85 \\
02 / 19 / 87 \\
08 / 11 / 88\end{array}$ & $\begin{array}{l}0001 \\
0001 \\
0001 \\
0001 \\
0001\end{array}$ & $\begin{array}{l}\text { NR } \\
\text { NR } \\
\text { NR } \\
\text { NR } \\
\text { NR }\end{array}$ & $\begin{array}{l}N \\
N \\
N \\
N \\
N\end{array}$ & $M G / L$ & $<$ & $\begin{array}{l}0.1 \\
0.1 \\
0.2 \\
0.2 \\
0.1\end{array}$ & & $\begin{array}{l}0.1 \\
0.1 \\
0.1 \\
0.1 \\
0.1\end{array}$ & $\begin{array}{l}- \\
- \\
-\end{array}$ \\
\hline BORON & $\begin{array}{l}0800 \\
0803 \\
0903 \\
0903\end{array}$ & $\begin{array}{l}08 / 11 / 88 \\
08 / 27 / 88 \\
11 / 18 / 85 \\
08 / 11 / 88\end{array}$ & $\begin{array}{l}0001 \\
0001 \\
0001 \\
0001\end{array}$ & $\begin{array}{l}\text { NR } \\
\text { NR } \\
\text { NR } \\
\text { NR }\end{array}$ & $\begin{array}{l}N \\
N \\
N \\
N\end{array}$ & $M G / L$ & $<$ & $\begin{array}{l}0.1 \\
0.1 \\
0.3 \\
0.1\end{array}$ & & $\begin{array}{l}0.1 \\
0.1 \\
0.1 \\
0.1\end{array}$ & :- \\
\hline
\end{tabular}

FORMATION OF COMPLETION CODE:

NR - NO RECOVERY OF DATA FOR CLASSIFYING

PARAMETER VALUE INDICATOR (PVI):

< - LESS THAN DETECTION LIMIT
FLOW RELATIONSHIP CODE

N - UNKNOHN

SAMPLE ID CODES:

0001 - FILTERED SAMPLE (.45 MICRONS)

OTHER PARAMETER VALUE FLAGS

$J$ - ESTIMATED VALUE 
SITE: SPKO1 SPOOK

$11 / 18 / 85$ TO $08 / 27 / 88$

REPORT DATE: $07 / 29 / 94$

\begin{tabular}{|c|c|c|c|c|c|c|c|c|c|c|c|}
\hline PARAMETER NAME & $\underset{\text { ID }}{\text { LOCATION }}$ & LOG DATE & $\begin{array}{c}\text { SAMPLE } \\
\text { ID }\end{array}$ & $\begin{array}{l}\text { FORM } \\
\text { COMP }\end{array}$ & $\begin{array}{l}\text { FLOH } \\
\text { REL. }\end{array}$ & $\begin{array}{l}\text { UNITS OF } \\
\text { MEASURE }\end{array}$ & PVI & $\begin{array}{l}\text { ARAMETER } \\
\text { VALUE }\end{array}$ & FLAGS & $\begin{array}{l}\text { DETECTION } \\
\text { LIMIT }\end{array}$ & $\begin{array}{l}\text { PARAMETER } \\
\text { UNCERTAINTY }\end{array}$ \\
\hline CADMIUM & $\begin{array}{l}0800 \\
0803 \\
0903 \\
0903 \\
0903\end{array}$ & $\begin{array}{l}08 / 11 / 88 \\
08 / 27 / 88 \\
11 / 18 / 85 \\
02 / 19 / 87 \\
08 / 11 / 88\end{array}$ & $\begin{array}{l}0001 \\
0001 \\
0001 \\
0001 \\
0001\end{array}$ & $\begin{array}{l}\text { NR } \\
\text { NR } \\
\text { NR } \\
\text { NR } \\
\text { NR }\end{array}$ & $\begin{array}{l}N \\
N \\
N \\
N \\
N\end{array}$ & $M G / L$ & $\begin{array}{l}< \\
< \\
< \\
< \\
<\end{array}$ & $\begin{array}{l}0.001 \\
0.001 \\
0.009 \\
0.001 \\
0.001\end{array}$ & & $\begin{array}{l}0.001 \\
0.001 \\
0.001 \\
0.001 \\
0.001\end{array}$ & $\begin{array}{l}- \\
- \\
-\end{array}$ \\
\hline CALCIUM & $\begin{array}{l}0800 \\
0803 \\
0903 \\
0903 \\
0903\end{array}$ & $\begin{array}{l}08 / 11 / 88 \\
08 / 27 / 88 \\
11 / 18 / 85 \\
02 / 19 / 87 \\
08 / 11 / 88\end{array}$ & $\begin{array}{l}0001 \\
0001 \\
0001 \\
0001 \\
0001\end{array}$ & $\begin{array}{l}\text { NR } \\
\text { NR } \\
\text { NR } \\
\text { NR } \\
\text { NR }\end{array}$ & $\begin{array}{l}N \\
N \\
N \\
N \\
N\end{array}$ & $M G / L$ & & $\begin{array}{l}13.7 \\
10.8 \\
18.5 \\
24.1 \\
41.0\end{array}$ & & $\begin{array}{l}0.01 \\
0.01 \\
0.01 \\
0.01 \\
0.01\end{array}$ & $\begin{array}{l}- \\
- \\
-\end{array}$ \\
\hline CHLORIDE & $\begin{array}{l}0800 \\
0803 \\
0903 \\
0903 \\
0903\end{array}$ & $\begin{array}{l}08 / 11 / 88 \\
08 / 27 / 88 \\
11 / 18 / 85 \\
02 / 19 / 87 \\
08 / 11 / 88\end{array}$ & $\begin{array}{l}0001 \\
0001 \\
0001 \\
0001 \\
0001\end{array}$ & $\begin{array}{l}\text { NR } \\
\text { NR } \\
\text { NR } \\
\text { NR } \\
\text { NR }\end{array}$ & $\begin{array}{l}N \\
N \\
N \\
N \\
N\end{array}$ & MG/L & $<$ & $\begin{array}{l}1.0 \\
3.4 \\
7 . \\
4.7 \\
1.0\end{array}$ & & $\begin{array}{l}1 . \\
1 . \\
1 . \\
1 .\end{array}$ & $\begin{array}{l}- \\
- \\
-\end{array}$ \\
\hline CHROMIUM & $\begin{array}{l}0800 \\
0803 \\
0903 \\
0903\end{array}$ & $\begin{array}{l}08 / 11 / 88 \\
08 / 27 / 88 \\
11 / 18 / 85 \\
08 / 11 / 88\end{array}$ & $\begin{array}{l}0001 \\
0001 \\
0001 \\
0001\end{array}$ & $\begin{array}{l}\text { NR } \\
\text { NR } \\
\text { NR } \\
\text { NR }\end{array}$ & $\begin{array}{l}N \\
N \\
N \\
N\end{array}$ & $M G / L$ & $<$ & $\begin{array}{l}0.01 \\
0.01 \\
0.01 \\
0.02\end{array}$ & & $\begin{array}{l}0.01 \\
0.01 \\
0.01 \\
0.01\end{array}$ & - \\
\hline COBALT & 0903 & $11 / 18 / 85$ & 0001 & NR & N & $M G / L$ & $<$ & 0.05 & & 0.05 & - \\
\hline COPPER & $\begin{array}{l}0800 \\
0803 \\
0903 \\
0903 \\
0903\end{array}$ & $\begin{array}{l}08 / 11 / 88 \\
08 / 27 / 88 \\
11 / 18 / 85 \\
02 / 19 / 87 \\
08 / 11 / 88\end{array}$ & $\begin{array}{l}0001 \\
0001 \\
0001 \\
0001 \\
0001\end{array}$ & $\begin{array}{l}\text { NR } \\
\text { NR } \\
\text { NR } \\
\text { NR } \\
\text { NR }\end{array}$ & $\begin{array}{l}\mathbf{N} \\
\mathbf{N} \\
\mathbf{N} \\
\mathbf{N} \\
\mathbf{N}\end{array}$ & $M G / L$ & $\mid \begin{array}{l}< \\
< \\
<\end{array}$ & $\begin{array}{l}0.02 \\
0.02 \\
0.04 \\
0.01 \\
0.02\end{array}$ & J & $\begin{array}{l}0.02 \\
0.02 \\
0.02 \\
0.02 \\
0.02\end{array}$ & $\begin{array}{l}- \\
- \\
- \\
-\end{array}$ \\
\hline CYANIDE & 0903 & $11 / 18 / 85$ & 0001 & NR & N & $M G / L$ & $<$ & 0.01 & & 0.01 & - \\
\hline FLUORIDE & $\begin{array}{l}0800 \\
0803 \\
0903 \\
0903 \\
0903\end{array}$ & $\begin{array}{l}08 / 11 / 88 \\
08 / 27 / 88 \\
11 / 18 / 85 \\
02 / 19 / 87 \\
08 / 11 / 88\end{array}$ & $\begin{array}{l}0001 \\
0001 \\
0001 \\
0001 \\
0001\end{array}$ & \begin{tabular}{l|} 
NR \\
NR \\
NR \\
NR \\
NR
\end{tabular} & $\begin{array}{l}N \\
N \\
N \\
N \\
N\end{array}$ & $M G / L$ & & $\begin{array}{l}0.2 \\
0.3 \\
1.2 \\
0.5 \\
0.3\end{array}$ & & $\begin{array}{l}0.1 \\
0.1 \\
0.1 \\
0.1 \\
0.1\end{array}$ & $\begin{array}{l}: \\
-\end{array}$ \\
\hline GROSS ALPHA & 0800 & $08 / 11 / 88$ & 0001 & NR & N & $\mathrm{PCI} / \mathrm{L}$ & & 1.7 & & 0.2 & 2.0 \\
\hline
\end{tabular}

FORMATION OF COHPLETION CODE:

NR - NO RECOVERY OF DATA FOR CLASSIFYING

PARAMETER VALUE INDICATOR (PVI): < - LESS THAN DETECTION LIMIT
FLOH RELATIONSHIP CODE:

N - UNKNOHN

SAMPLE ID CODES:

0001 - FILTERED SAMPLE (.45 MICRONS)

OTHER PARAMETER VALUE FLAGS:

$J$ - ESTIMATED VALUE 
GROUNDWATER QUALITY DATA BY PARAMETER

Domestic wells

SITE: SPK01 SPOOK

$11 / 18 / 85$ TO $08 / 27 / 88$

REPORT DATE: $07 / 29 / 94$

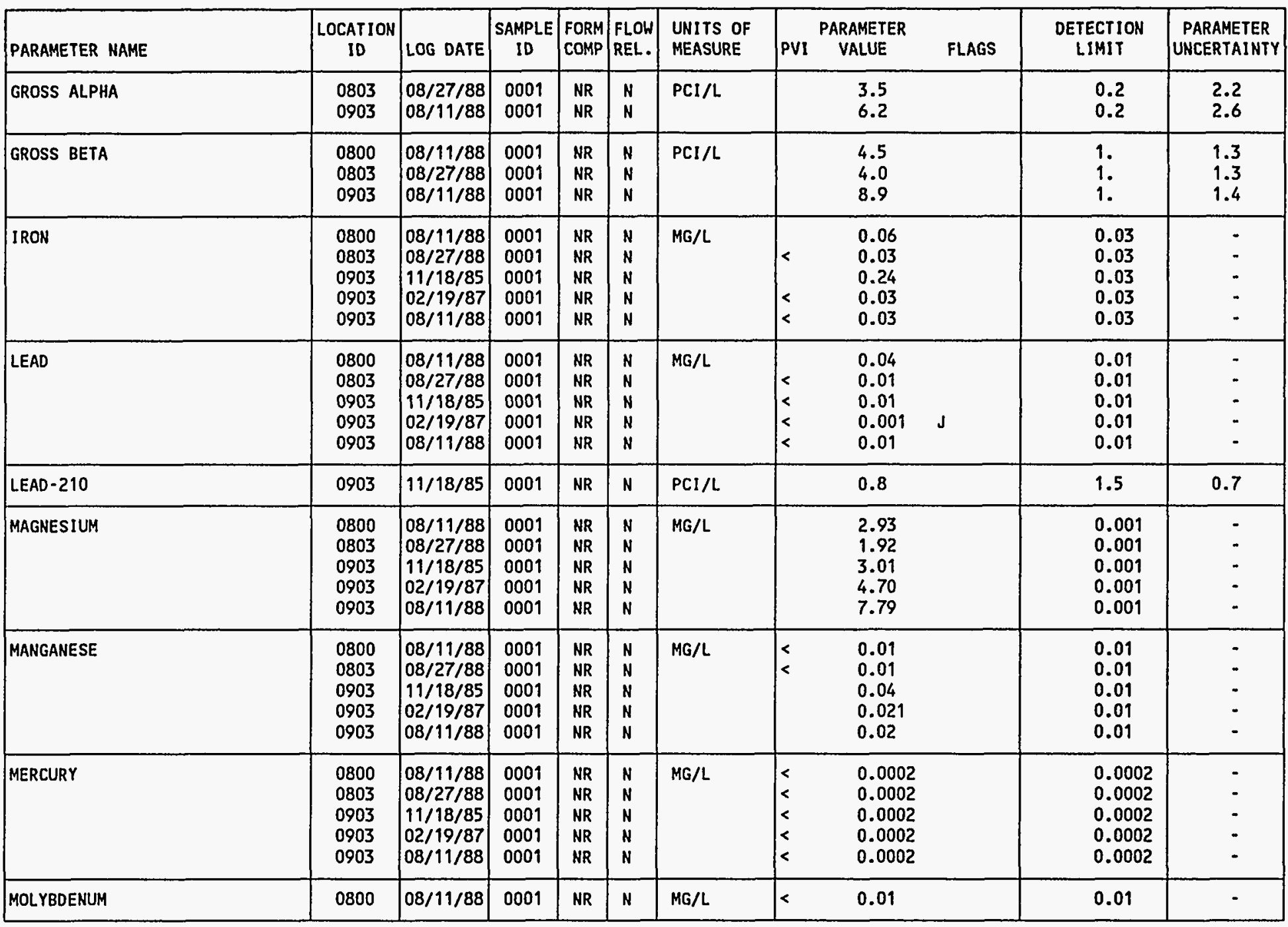

FORMATION OF COMPLETION CODE:

HR - NO RECOVERY OF DATA FOR CLASSIFYING

PARAMETER VALUE INDICATOR (PVI): < - LESS THAN DETECTION LIMIT
FLOW RELATIONSHIP CODE:

N - UNKNOWN

SAMPLE ID CODES:

0001 - FILTERED SAMPLE (.45 MICRONS)

OTHER PARAMETER VALUE FLAGS:

$J$ - ESTIMATED VALUE 
GROUNDWATER QUALITY DATA BY PARAMETER

Domestic Hells

SITE: SPKO1 SPOOK

$11 / 18 / 85$ TO $08 / 27 / 88$
REPORT DATE: $07 / 29 / 94$

\begin{tabular}{|c|c|c|c|c|c|c|c|c|c|c|c|}
\hline PARAMETER NAME & $\underset{\text { ID }}{\text { LOCATION }}$ & LOG DATE & $\begin{array}{c}\text { SAMPLE } \\
\text { ID }\end{array}$ & $\begin{array}{l}\text { FORM } \\
\text { COMP }\end{array}$ & $\begin{array}{l}\text { FLOH } \\
\text { REL. }\end{array}$ & $\begin{array}{l}\text { UNITS OF } \\
\text { MEASURE }\end{array}$ & PVI & $\begin{array}{l}\text { ARAMETER } \\
\text { VALUE }\end{array}$ & FLAGS & $\begin{array}{l}\text { DETECTION } \\
\text { LIMIT }\end{array}$ & $\begin{array}{l}\text { PARAMETER } \\
\text { UNCERTAINTY }\end{array}$ \\
\hline MOLYBDENUM & $\begin{array}{l}0803 \\
0903 \\
0903 \\
0903\end{array}$ & $\begin{array}{l}08 / 27 / 88 \\
11 / 18 / 85 \\
02 / 19 / 87 \\
08 / 11 / 88\end{array}$ & $\begin{array}{l}0001 \\
0001 \\
0001 \\
0001\end{array}$ & $\begin{array}{l}\text { AR } \\
\text { NR } \\
\text { NR } \\
\text { NR }\end{array}$ & $\begin{array}{l}N \\
N \\
N \\
N\end{array}$ & $M G / L$ & $\mid \begin{array}{l}< \\
<\end{array}$ & $\begin{array}{l}0.01 \\
0.06 \\
0.1 \\
0.01\end{array}$ & & $\begin{array}{l}0.01 \\
0.01 \\
0.1 \\
0.01\end{array}$ & $\begin{array}{l}- \\
- \\
.\end{array}$ \\
\hline NET GROSS ALPHA * & $\begin{array}{l}0800 \\
0803 \\
0903\end{array}$ & $\begin{array}{l}08 / 11 / 88 \\
08 / 27 / 88 \\
08 / 11 / 88\end{array}$ & $\begin{array}{l}0001 \\
0001 \\
0001\end{array}$ & $\begin{array}{l}\text { NR } \\
\text { NR } \\
\text { NR }\end{array}$ & $\begin{array}{l}\mathbf{N} \\
N \\
N\end{array}$ & $\mathrm{PCI} / \mathrm{L}$ & & $\begin{array}{l}0.19 \\
2.47 \\
5.03\end{array}$ & & - & : \\
\hline NICKEL & $\begin{array}{l}0800 \\
0803 \\
0903 \\
0903\end{array}$ & $\begin{array}{l}08 / 11 / 88 \\
08 / 27 / 88 \\
11 / 18 / 85 \\
08 / 11 / 88\end{array}$ & $\begin{array}{l}0001 \\
0001 \\
0001 \\
0001\end{array}$ & $\begin{array}{l}\text { NR } \\
\text { NR } \\
\text { NR } \\
\text { NR }\end{array}$ & $\begin{array}{l}N \\
N \\
N \\
N\end{array}$ & MG/L & $\begin{array}{l}< \\
<\end{array}$ & $\begin{array}{l}0.04 \\
0.04 \\
0.04 \\
0.04\end{array}$ & & $\begin{array}{l}0.04 \\
0.04 \\
0.04 \\
0.04\end{array}$ & $:$ \\
\hline NITRATE & $\begin{array}{l}0800 \\
0803 \\
0903 \\
0903 \\
0903\end{array}$ & $\begin{array}{l}08 / 11 / 88 \\
08 / 27 / 88 \\
11 / 18 / 85 \\
02 / 19 / 87 \\
08 / 11 / 88\end{array}$ & $\begin{array}{l}0001 \\
0001 \\
0001 \\
0001 \\
0001\end{array}$ & $\begin{array}{l}\text { NR } \\
\text { NR } \\
\text { NR } \\
\text { NR } \\
\text { NR }\end{array}$ & $\begin{array}{l}\mathbf{N} \\
\mathbf{N} \\
\mathbf{N} \\
\mathrm{N} \\
\mathrm{N}\end{array}$ & $M G / L$ & $<$ & $\begin{array}{l}1.0 \\
3.7 \\
1 . \\
0.4 \\
1.3\end{array}$ & J & $\begin{array}{l}1 . \\
1 . \\
1 . \\
1 .\end{array}$ & $\begin{array}{l}- \\
- \\
-\end{array}$ \\
\hline NI TRI TE & 0903 & $11 / 18 / 85$ & 0001 & NR & N & $M G / L$ & $<$ & 0.1 & & 0.1 & - \\
\hline PH & $\begin{array}{l}0800 \\
0803 \\
0903 \\
0903 \\
0903\end{array}$ & $\begin{array}{l}08 / 11 / 88 \\
08 / 27 / 88 \\
11 / 18 / 85 \\
02 / 19 / 87 \\
08 / 11 / 88\end{array}$ & $\begin{array}{l}0001 \\
0001 \\
0001 \\
0001 \\
0001\end{array}$ & $\begin{array}{l}\text { NR } \\
\text { NR } \\
\text { NR } \\
\text { NR } \\
\text { NR }\end{array}$ & $\begin{array}{l}N \\
N \\
N \\
N \\
N\end{array}$ & SU & & $\begin{array}{l}8.10 \\
8.39 \\
7.85 \\
7.80 \\
7.58\end{array}$ & & : & $\begin{array}{l}- \\
- \\
-\end{array}$ \\
\hline PHOSPHATE & $\begin{array}{l}0800 \\
0803 \\
0903 \\
0903 \\
0903\end{array}$ & $\begin{array}{l}08 / 11 / 88 \\
08 / 27 / 88 \\
11 / 18 / 85 \\
02 / 19 / 87 \\
08 / 11 / 88\end{array}$ & $\begin{array}{l}0001 \\
0001 \\
0001 \\
0001 \\
0001\end{array}$ & $\begin{array}{l}\text { NR } \\
\text { NR } \\
\text { NR } \\
\text { NR } \\
\text { NR }\end{array}$ & $\begin{array}{l}N \\
N \\
N \\
N \\
N\end{array}$ & $M G / L$ & $\begin{array}{l}< \\
< \\
< \\
<\end{array}$ & $\begin{array}{l}0.1 \\
0.1 \\
0.1 \\
0.1 \\
0.1\end{array}$ & & $\begin{array}{l}0.1 \\
0.1 \\
0.1 \\
0.1 \\
0.1\end{array}$ & :- \\
\hline POLONIUM-210 & 0903 & $11 / 18 / 85$ & 0001 & NR & N & $\mathrm{PCI} / \mathrm{L}$ & & 0.6 & & 1. & 0.6 \\
\hline POTASSIUM & $\begin{array}{l}0800 \\
0803\end{array}$ & $\begin{array}{l}08 / 11 / 88 \\
08 / 27 / 88\end{array}$ & $\begin{array}{l}0001 \\
0001\end{array}$ & $\begin{array}{l}\text { NR } \\
\text { NR }\end{array}$ & $\begin{array}{l}\mathrm{N} \\
\mathrm{N}\end{array}$ & $M G / L$ & & $\begin{array}{l}2.0 \\
1.6\end{array}$ & & $\begin{array}{l}0.01 \\
0.01\end{array}$ & - \\
\hline
\end{tabular}

* Net gRoss alPHa (GROSS ALPHA - URANIUM) WITH 1 MG URANIUM = 686 PCI

FORMATION OF COMPLETION CODE:

NR - NO RECOVERY OF DATA FOR CLASSIFYING

PARAMETER VALUE INDICATOR (PVI): < - LESS THAN DETECTION LIMIT
FLOH RELATIONSHIP CODE:

N - UNKNOWN

SAMPLE ID CODES:

0001 - FILTERED SAMPLE (.45 MICRONS)

OTHER PARAMETER VALUE FLAGS:

J - ESTIMATED VALUE 
GROUNDHATER QUALITY DATA BY PARAMETER

Domestic Wells

SITE: SPKO1 SPOOK

$11 / 18 / 85$ TO $08 / 27 / 88$

REPORT DATE: $07 / 29 / 94$

\begin{tabular}{|c|c|c|c|c|c|c|c|c|c|c|c|}
\hline PARAMETER NAME & $\underset{\text { ID }}{\text { LOCATION }}$ & LOG DATE & $\begin{array}{c}\text { SAMPLE } \\
10\end{array}$ & $\begin{array}{l}\text { FORM } \\
\text { COMP }\end{array}$ & $\begin{array}{l}\text { FLOW } \\
\text { REL. }\end{array}$ & $\begin{array}{l}\text { UNITS OF } \\
\text { MEASURE }\end{array}$ & PVI & $\begin{array}{l}\text { PARAMETER } \\
\text { VALUE }\end{array}$ & FLAGS & $\begin{array}{l}\text { DETECTION } \\
\text { LIMIT }\end{array}$ & $\begin{array}{l}\text { PARAMETER } \\
\text { UNCERTAINTY }\end{array}$ \\
\hline POTASSIUM & $\begin{array}{l}0903 \\
0903 \\
0903\end{array}$ & $\begin{array}{l}11 / 18 / 85 \\
02 / 19 / 87 \\
08 / 11 / 88\end{array}$ & $\begin{array}{l}0001 \\
0001 \\
0001\end{array}$ & $\begin{array}{l}\text { NR } \\
\text { NR } \\
\text { NR }\end{array}$ & $\begin{array}{l}N \\
N \\
N\end{array}$ & $M G / L$ & & $\begin{array}{l}3.78 \\
3.34 \\
4.4\end{array}$ & & $\begin{array}{l}0.01 \\
0.01 \\
0.01\end{array}$ & $\dot{-}$ \\
\hline RADIUM-226 & 0903 & $11 / 18 / 85$ & 0001 & NR & N & $\mathrm{PCI} / \mathrm{L}$ & & 1. & & 1. & 0.4 \\
\hline RADIUM-226 + RADIUM-228 & 0903 & $11 / 18 / 85$ & 0001 & NR & $N$ & PCI/L & & 1.30 & & - & - \\
\hline RADIUM-228 & 0903 & $11 / 18 / 85$ & 0001 & NR & $\mathbf{N}$ & PCI/L & & 0.3 & & 1. & 0.9 \\
\hline SELENIUM & $\begin{array}{l}0800 \\
0803 \\
0903 \\
0903 \\
0903\end{array}$ & $\begin{array}{l}08 / 11 / 88 \\
08 / 27 / 88 \\
11 / 18 / 85 \\
02 / 19 / 87 \\
08 / 11 / 88\end{array}$ & $\begin{array}{l}0001 \\
0001 \\
0001 \\
0001 \\
0001\end{array}$ & $\begin{array}{l}\text { NR } \\
\text { NR } \\
\text { NR } \\
\text { NR } \\
\text { NR }\end{array}$ & $\begin{array}{l}N \\
N \\
N \\
N \\
N\end{array}$ & $M G / L$ & $\begin{array}{l}< \\
< \\
< \\
<\end{array}$ & $\begin{array}{l}0.004 \\
0.005 \\
0.005 \\
0.001 \\
0.001\end{array}$ & $\begin{array}{l}J \\
J \\
J\end{array}$ & $\begin{array}{l}0.005 \\
0.005 \\
0.005 \\
0.005 \\
0.005\end{array}$ & $\begin{array}{l}- \\
- \\
-\end{array}$ \\
\hline SILICA - SIOZ & $\begin{array}{l}0800 \\
0803 \\
0903 \\
0903 \\
0903\end{array}$ & $\begin{array}{l}08 / 11 / 88 \\
08 / 27 / 88 \\
11 / 18 / 85 \\
02 / 19 / 87 \\
08 / 11 / 88\end{array}$ & $\begin{array}{l}0001 \\
0001 \\
0001 \\
0001 \\
0001\end{array}$ & $\begin{array}{l}\text { NR } \\
\text { NR } \\
\text { NR } \\
\text { NR } \\
\text { NR }\end{array}$ & $\begin{array}{l}N \\
N \\
N \\
N \\
N\end{array}$ & $M G / L$ & & $\begin{array}{l}15 . \\
12.2 \\
4 . \\
17 . \\
16 .\end{array}$ & & $\begin{array}{l}2 . \\
2 . \\
2 . \\
2 . \\
2 .\end{array}$ & $\begin{array}{l}\dot{-} \\
\dot{-} \\
-\end{array}$ \\
\hline SILVER & $\begin{array}{l}0800 \\
0803 \\
0903 \\
0903 \\
0903\end{array}$ & $\begin{array}{l}08 / 11 / 88 \\
08 / 27 / 88 \\
11 / 18 / 85 \\
02 / 19 / 87 \\
08 / 11 / 88\end{array}$ & $\begin{array}{l}0001 \\
0001 \\
0001 \\
0001 \\
0001\end{array}$ & $\begin{array}{l}\text { NR } \\
\text { NR } \\
\text { NR } \\
\text { NR } \\
\text { NR }\end{array}$ & $\begin{array}{l}N \\
N \\
N \\
N \\
N\end{array}$ & $M G / L$ & $\begin{array}{l}< \\
< \\
< \\
<\end{array}$ & $\begin{array}{l}0.01 \\
0.01 \\
0.01 \\
0.01 \\
0.01\end{array}$ & & $\begin{array}{l}0.01 \\
0.01 \\
0.01 \\
0.01 \\
0.01\end{array}$ & $\begin{array}{l}- \\
- \\
-\end{array}$ \\
\hline SODIUM & $\begin{array}{l}0800 \\
0803 \\
0903 \\
0903 \\
0903\end{array}$ & $\begin{array}{l}08 / 11 / 88 \\
08 / 27 / 88 \\
11 / 18 / 85 \\
02 / 19 / 87 \\
08 / 11 / 88\end{array}$ & $\begin{array}{l}0001 \\
0001 \\
0001 \\
0001 \\
0001\end{array}$ & $\begin{array}{l}\text { NR } \\
\text { NR } \\
\text { NR } \\
\text { NR } \\
\text { NR }\end{array}$ & $\begin{array}{l}N \\
N \\
N \\
N \\
N\end{array}$ & $\mathrm{MG} / \mathrm{L}$ & & $\begin{array}{l}86.3 \\
99.6 \\
72.8 \\
76.6 \\
56.6\end{array}$ & & $\begin{array}{l}0.002 \\
0.002 \\
0.002 \\
0.002 \\
0.002\end{array}$ & $\begin{array}{l}- \\
- \\
- \\
-\end{array}$ \\
\hline SPECIFIC CONDUCTANCE & $\begin{array}{l}0800 \\
0803 \\
0903 \\
0903 \\
0903\end{array}$ & $\begin{array}{l}08 / 11 / 88 \\
08 / 27 / 88 \\
11 / 18 / 85 \\
02 / 19 / 87 \\
08 / 11 / 88\end{array}$ & $\begin{array}{l}0001 \\
0001 \\
0001 \\
0001 \\
0001\end{array}$ & $\begin{array}{l}\text { NR } \\
\text { NR } \\
\text { NR } \\
\text { NR } \\
\text { NR }\end{array}$ & $\begin{array}{l}\mathrm{N} \\
\mathrm{N} \\
\mathrm{N} \\
\mathrm{N} \\
\mathrm{N}\end{array}$ & UMHO/CM & & $\begin{array}{l}370.0 \\
335.0 \\
220 . \\
295 . \\
295.0\end{array}$ & & : & $\begin{array}{l}- \\
- \\
-\end{array}$ \\
\hline STRONTIUM & 0800 & $08 / 11 / 88$ & 0001 & NR & $\mathbf{N}$ & $M G / L$ & $<$ & 0.1 & & 0.1 & - \\
\hline
\end{tabular}

FORMATION OF COMPLETION CODE:

UR - NO RECOVERY OF DATA FOR CLASSIFYING

FLOH RELATIONSHIP CODE:

PARAMETER VALUE INDICATOR (PVI): < - LESS THAM DETECTION LIMIT

SAMPLE ID CODES:

0001 - FILTERED SAMPLE (.45 MICRONS)

OTHER PARAMETER VALUE FLAGS:

$J$ - estimated Value 
REPORT DATE: $07 / 29 / 94$

\begin{tabular}{|c|c|c|c|c|c|c|c|c|c|c|c|}
\hline PARAMETER NAME & $\underset{\text { ID }}{\text { LOCATION }}$ & LOG DATE & $\underset{\text { SAMPLE }}{\text { ID }}$ & $\begin{array}{l}\text { FORM } \\
\text { COMP }\end{array}$ & $\begin{array}{l}\text { FLOW } \\
\text { REL. }\end{array}$ & $\begin{array}{l}\text { UNITS OF } \\
\text { MEASURE }\end{array}$ & PVI & $\begin{array}{l}\text { PARAMETER } \\
\text { VALUE }\end{array}$ & FLAGS & $\begin{array}{l}\text { DETECTION } \\
\text { LIMIT }\end{array}$ & \begin{tabular}{|c|} 
PARAMETER \\
UNCERTAINTY
\end{tabular} \\
\hline STRONTIUM & $\begin{array}{l}0803 \\
0903 \\
0903\end{array}$ & $\mid \begin{array}{l}08 / 27 / 88 \\
11 / 18 / 85 \\
08 / 11 / 88\end{array}$ & $\begin{array}{l}0001 \\
0001 \\
0001\end{array}$ & $\begin{array}{l}\text { NR } \\
\text { NR } \\
\text { NR }\end{array}$ & $\begin{array}{l}N \\
N \\
N\end{array}$ & $M G / L$ & $<$ & $\begin{array}{l}0.1 \\
0.1 \\
0.5\end{array}$ & & $\begin{array}{l}0.1 \\
0.1 \\
0.1\end{array}$ & : \\
\hline SULFATE & $\begin{array}{l}0800 \\
0803 \\
0903 \\
0903 \\
0903\end{array}$ & $\begin{array}{l}08 / 11 / 88 \\
08 / 27 / 88 \\
11 / 18 / 85 \\
02 / 19 / 87 \\
08 / 11 / 88\end{array}$ & $\begin{array}{l}0001 \\
0001 \\
0001 \\
0001 \\
0001\end{array}$ & $\begin{array}{l}\text { NR } \\
\text { NR } \\
\text { NR } \\
\text { NR } \\
\text { NR }\end{array}$ & $\begin{array}{l}N \\
N \\
N \\
N \\
N\end{array}$ & MG/L & & $\begin{array}{l}81 . \\
80 . \\
5.4 \\
46.1 \\
81 .\end{array}$ & & $\begin{array}{l}0.1 \\
0.1 \\
0.1 \\
0.1 \\
0.1\end{array}$ & $\begin{array}{l}- \\
- \\
-\end{array}$ \\
\hline SULFIDE & $\begin{array}{l}0800 \\
0803 \\
0903 \\
0903\end{array}$ & $\begin{array}{l}08 / 11 / 88 \\
08 / 27 / 88 \\
11 / 18 / 85 \\
08 / 11 / 88\end{array}$ & $\begin{array}{l}0001 \\
0001 \\
0001 \\
0001\end{array}$ & $\begin{array}{l}\text { NR } \\
\text { NR } \\
\text { NR } \\
\text { NR }\end{array}$ & $\begin{array}{l}N \\
N \\
N \\
N\end{array}$ & $M G / L$ & $\begin{array}{l}< \\
< \\
< \\
<\end{array}$ & $\begin{array}{l}0.1 \\
0.1 \\
0.1 \\
0.1\end{array}$ & & $\begin{array}{l}0.1 \\
0.1 \\
0.1 \\
0.1\end{array}$ & $\begin{array}{l}- \\
- \\
-\end{array}$ \\
\hline TEMPERATURE & $\begin{array}{l}0800 \\
0803 \\
0903 \\
0903 \\
0903\end{array}$ & $\begin{array}{l}08 / 11 / 88 \\
08 / 27 / 88 \\
11 / 18 / 85 \\
02 / 19 / 87 \\
08 / 11 / 88\end{array}$ & $\begin{array}{l}0001 \\
0001 \\
0001 \\
0001 \\
0001\end{array}$ & $\begin{array}{l}\text { NR } \\
\text { NR } \\
\text { NR } \\
\text { NR } \\
\text { NR }\end{array}$ & $\begin{array}{l}\mathrm{N} \\
\mathrm{N} \\
\mathrm{N} \\
\mathrm{N} \\
\mathrm{N}\end{array}$ & C - DEGREE & & $\begin{array}{c}16.0 \\
17.25 \\
3 . \\
8.0 \\
12.0\end{array}$ & & $\begin{array}{l}- \\
- \\
-\end{array}$ & $\begin{array}{l}- \\
- \\
-\end{array}$ \\
\hline THORIUM-230 & 0903 & $11 / 18 / 85$ & 0001 & NR & N & $\mathrm{PCI} / \mathrm{L}$ & & 0.5 & & 1. & 0.4 \\
\hline TIN & $\begin{array}{l}0800 \\
0803 \\
0903 \\
0903\end{array}$ & \begin{tabular}{|l|}
$08 / 11 / 88$ \\
$08 / 27 / 88$ \\
$11 / 18 / 85$ \\
$08 / 11 / 88$
\end{tabular} & $\begin{array}{l}0001 \\
0001 \\
0001 \\
0001\end{array}$ & $\begin{array}{l}\text { NR } \\
\text { NR } \\
\text { NR } \\
\text { NR }\end{array}$ & $\begin{array}{l}\mathbf{N} \\
\mathbf{N} \\
\mathrm{N} \\
\mathrm{N}\end{array}$ & $M G / L$ & $\begin{array}{l}< \\
< \\
< \\
<\end{array}$ & $\begin{array}{l}0.005 \\
0.005 \\
0.005 \\
0.005\end{array}$ & & $\begin{array}{l}0.005 \\
0.005 \\
0.005 \\
0.005\end{array}$ & - \\
\hline TOTAL DISSOLVED SOLIDS & $\begin{array}{l}0800 \\
0803 \\
0903 \\
0903 \\
0903\end{array}$ & $\begin{array}{l}08 / 11 / 88 \\
08 / 27 / 88 \\
11 / 18 / 85 \\
02 / 19 / 87 \\
08 / 11 / 88\end{array}$ & $\begin{array}{l}0001 \\
0001 \\
0001 \\
0001 \\
0001\end{array}$ & $\begin{array}{l}\text { NR } \\
\text { NR } \\
\text { NR } \\
\text { NR } \\
\text { NR }\end{array}$ & $\begin{array}{l}N \\
N \\
N \\
N \\
N\end{array}$ & $M G / L$ & & $\begin{array}{l}302 . \\
299 . \\
320 . \\
280 . \\
310 .\end{array}$ & & $\begin{array}{l}10 . \\
10 . \\
10 . \\
10 . \\
10 .\end{array}$ & $\begin{array}{l}- \\
- \\
-\end{array}$ \\
\hline TOTAL ORGANIC CARBON & 0903 & $11 / 18 / 85$ & 0001 & NR & $N$ & $M G / L$ & & 51. & & 1. & - \\
\hline URANIUM & $\begin{array}{l}0800 \\
0803 \\
0903 \\
0903\end{array}$ & $\begin{array}{l}08 / 11 / 88 \\
08 / 27 / 88 \\
11 / 18 / 85 \\
02 / 19 / 87\end{array}$ & $\begin{array}{l}0001 \\
0001 \\
0001 \\
0001\end{array}$ & $\begin{array}{l}\text { NR } \\
\text { NR } \\
\text { NR } \\
\text { NR }\end{array}$ & $\begin{array}{l}\mathbf{N} \\
\mathbf{N} \\
\mathbf{N} \\
\mathbf{N}\end{array}$ & $M G / L$ & $\begin{array}{l}< \\
< \\
<\end{array}$ & $\begin{array}{l}0.0022 \\
0.0003 \\
0.0003 \\
0.0003\end{array}$ & $\begin{array}{l}J \\
J \\
J \\
J\end{array}$ & $\begin{array}{l}0.003 \\
0.003 \\
0.003 \\
0.003\end{array}$ & $\begin{array}{l}- \\
-\end{array}$ \\
\hline
\end{tabular}

FORMATION OF COMPLETION CODE:

NR - NO RECOVERY OF DATA FOR CLASSIFYING

FLOW RELATIONSHIP CODE:

PARAMETER VALUE INDICATOR (PVI): < - LESS THAN DETECTION LIMIT 
GROUNDWATER QUALITY DATA BY PARAMETER

Domestic Hells

SITE: SPKO1 SPOOK

$11 / 18 / 85$ TO $08 / 27 / 88$

REPORT DATE: $07 / 29 / 94$

\begin{tabular}{|c|c|c|c|c|c|c|c|c|c|c|}
\hline PARAMETER NAME & $\underset{\text { ID }}{\text { LOCATION }}$ & LOG DATE & $\begin{array}{c}\text { SAMPLE } \\
\text { I0 }\end{array}$ & $\begin{array}{l}\text { FORM } \\
\text { COMP }\end{array}$ & $\begin{array}{l}\text { FLOW } \\
\text { REL. }\end{array}$ & $\begin{array}{l}\text { UNITS OF } \\
\text { MEASURE }\end{array}$ & PVI & $\begin{array}{l}\text { PARAMETER } \\
\text { VALUE }\end{array}$ & $\begin{array}{l}\text { DETECT ION } \\
\text { LIMIT }\end{array}$ & $\begin{array}{l}\text { PARAMETER } \\
\text { UNCERTAINTY }\end{array}$ \\
\hline URANIUM & 0903 & $08 / 11 / 88$ & 0001 & NR & $N$ & $M G / L$ & & $0.0017 \mathrm{~J}$ & 0.003 & - \\
\hline VANADIUM & $\begin{array}{l}0800 \\
0803 \\
0903 \\
0903\end{array}$ & \begin{tabular}{|l|}
$08 / 11 / 88$ \\
$08 / 27 / 88$ \\
$11 / 18 / 85$ \\
$08 / 11 / 88$
\end{tabular} & $\begin{array}{l}0001 \\
0001 \\
0001 \\
0001\end{array}$ & $\begin{array}{l}\text { NR } \\
\text { NR } \\
\text { NR } \\
\text { NR }\end{array}$ & $\begin{array}{l}N \\
N \\
N \\
N\end{array}$ & MG/L & $\mid<$ & $\begin{array}{l}0.01 \\
0.01 \\
0.19 \\
0.01\end{array}$ & $\begin{array}{l}0.01 \\
0.01 \\
0.01 \\
0.01\end{array}$ & . \\
\hline ZINC & $\begin{array}{l}0800 \\
0803 \\
0903 \\
0903 \\
0903\end{array}$ & $\begin{array}{l}08 / 11 / 88 \\
08 / 27 / 88 \\
11 / 18 / 85 \\
02 / 19 / 87 \\
08 / 11 / 88\end{array}$ & $\begin{array}{l}0001 \\
0001 \\
0001 \\
0001 \\
0001\end{array}$ & $\begin{array}{l}\text { NR } \\
\text { NR } \\
\text { NR } \\
\text { NR } \\
\text { NR }\end{array}$ & $\begin{array}{l}N \\
N \\
N \\
N \\
N\end{array}$ & $M G / L$ & $<$ & $\begin{array}{l}0.005 \\
0.011 \\
0.082 \\
0.005 \\
0.005\end{array}$ & $\begin{array}{l}0.005 \\
0.005 \\
0.005 \\
0.005 \\
0.005\end{array}$ & $\begin{array}{l}- \\
- \\
-\end{array}$ \\
\hline
\end{tabular}

FORMATION OF COMPLETION CODE

NR - NO RECOVERY OF DATA FOR CLASSIFYING

FLOW RELATIONSHIP CODE:

N - UNKNOWN

PARAMETER VALUE INDICATOR (PVI): < - LESS THAN DETECTION LIMIT

SAMPLE ID CODES

0001 - FILTERED SAMPLE (.45 MICRONS)

OTHER PARAMETER VALUE FLAGS:

J - ESTIMATED VALUE

DATA FILE NAME: M: IDARTISPK01\GWQ10010.DAT 


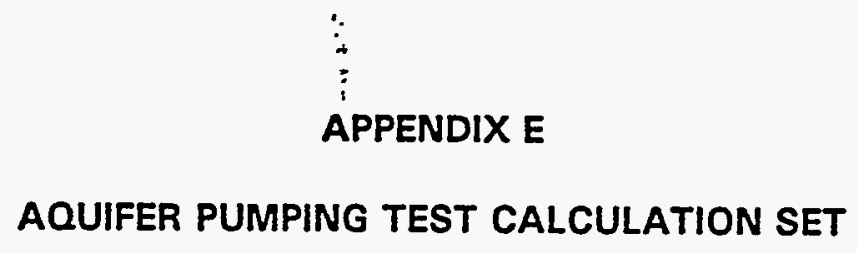




\section{CALCULATION COVER SHEET}

CALC 'NO. 07-756-01-00 DISCIPLINE Hydrology NO. OF SHEETS 24

PROJECT: Site Choracteiziztion

SITE:

$$
\text { spook, wyoming }
$$

Feature: Hydroulic Conductivity

SOURCES OF DATA:

$$
\begin{aligned}
& \text { Aguifer pumping tests } \\
& \text { mow } 913 \text { s } 928
\end{aligned}
$$

SOURCES OF FORMULAE \& REFERENCES:

Krusemon, G.P., and De Ridder, N.A., 1970. Anohysis ant Evalustor of Pumping'Test Data luteinotionsl instite for lond Reclomator and Tmpovenent/ ILRI, wo jeningen, The NeThertonds.

lohmor, S. W, 1972, Grounbusth Hydionlies, USES Professional fopen 700 .

Depatment of Interion, surpar of Reclomoxion, 1977. Grownd woth Monvit, A woth Peoorser Technicd Putelication.

PRELIMINaAy Calc. $\square$ final Calc. $\$$ Supersedes Calc. no.

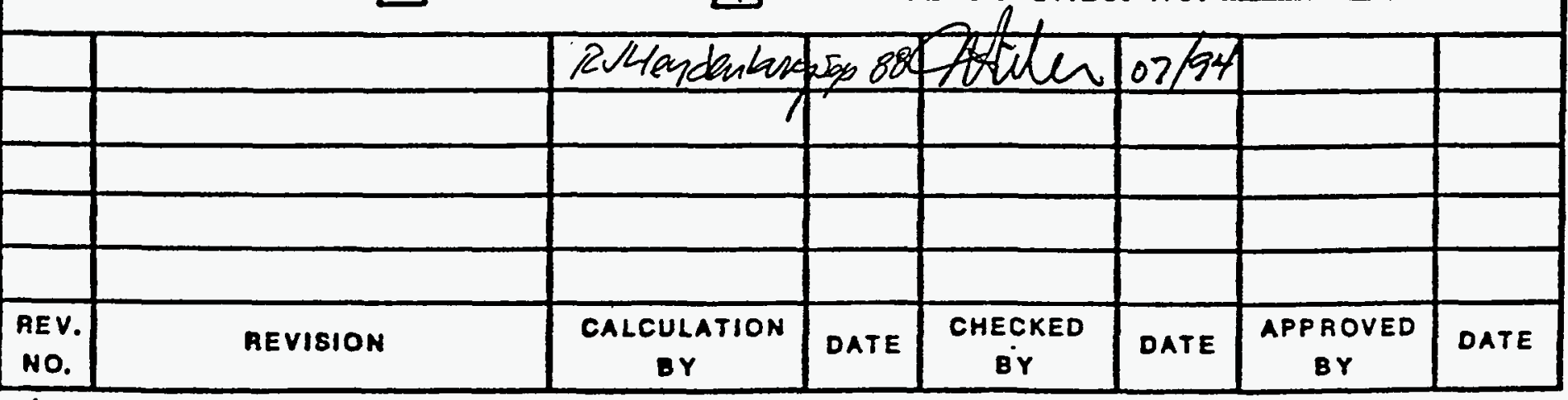


SPOOR UMTRA PROJECT SITE, PYOMING

AQUIFER PUMPING TESTS

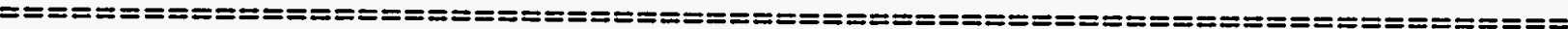

A. Purpose of calculation:

To calculate transmissivity (T), storativity (S), and hydraulic conductivity $(K)$ in sediments of the upper sandstone unit (uppermost aquifer) of the Tertiary Wasatch Formation beneath the Spook processing site.

\section{B. Methods and procedures:}

Aquifer pumping tests were performed in two monitor wells (SPKO1-0913 and -0928) at the spook processing site during August 1988 (see attached figure for location). The monitor wells were pumped at a relatively constant rate for various periods of time. Water from the pumped wells was discharged into the spook open pit mine. Water level measurements were recorded using an electric sounder during pumping and recovery phases of the tests.

C. Assumptions :

$\rightarrow \quad$ Aquifer has seemingly infinite areal extent.

$\rightarrow \quad$ Aquifer homogeneous, isotropic, and of uniform thickness over area influenced by pumping test.

$\rightarrow \quad$ Potentiometric surface nearly horizontal.

$\rightarrow \quad$ Aquifer pumped at a constant discharge rate.

$\rightarrow \quad$ Pumped well penetrates entire aquifer and thus received

- water from entire thickness by horizontal flow.

\section{Material properties:}

The upper sandstone unit of the Tertiary Wasatch Formation beneath the Spook site consists of moderately cemented, fineto coarse-grained sandstone containing silty shale lenses. It ranges in thickness from 70 to 140 feet. Ground water occurs under unconfined conditions at depths ranging from 20 to 120 feet below ground surface. The observed saturated thickness of the upper sandstone unit is approximately 20 feet in the vicinity of the site. This unit is underlain by an aquitard consisting of well consolidated silty shale.

\section{E. Data sources:}

Aquifer pumping test data were collected at the spook 
processing site during August 1988 (see attached sheets).

F. Calculations and analyses:

Drawdown and recovery versus time graphs, equations, and calculations for $T, K$, and $S$ are shown on the attached sheets for each monitor well tested.

G. Results:

Results of the calculations are summarized at the end of each monitor well section and on the attached table. 


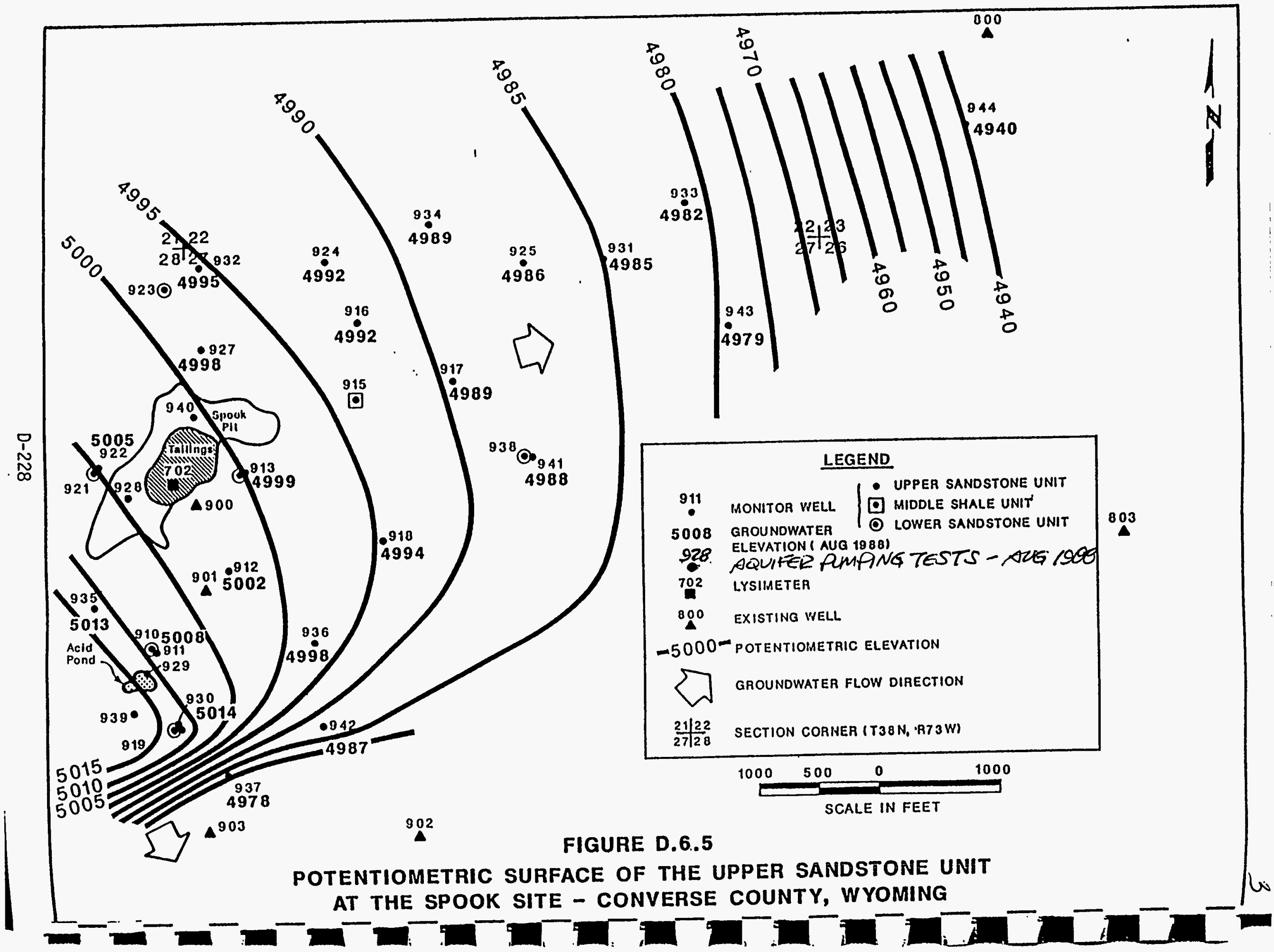




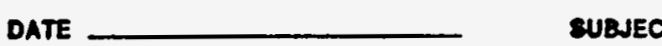

SUBNECT

$$
\text { DY }
$$

CHKD.

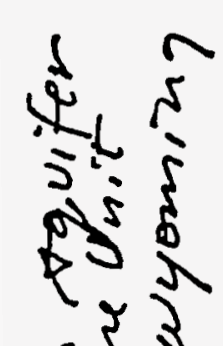

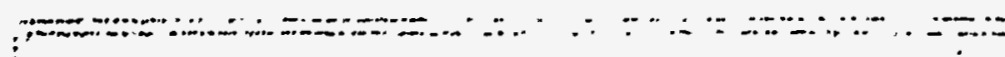

…

$$
\text { ! }
$$

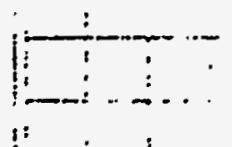

य

$\therefore 5^{6} 8$

专

定

(
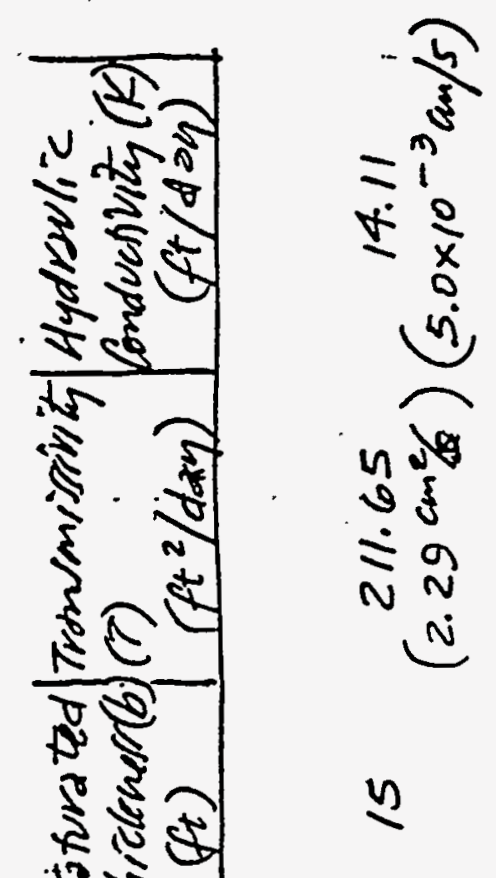

है

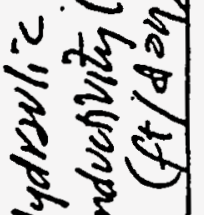

$=i$

$\pm \frac{2}{x}$

तू

\. 15

$9<1$

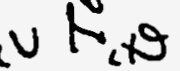

$=25$

f

$-25$ 205

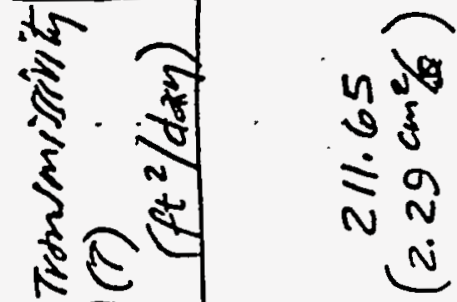

से है

$m+$

Ni

M.

है

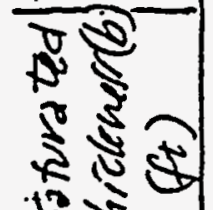

n

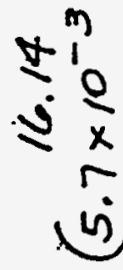

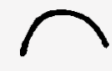

0

$\frac{1}{1}$

$\theta$

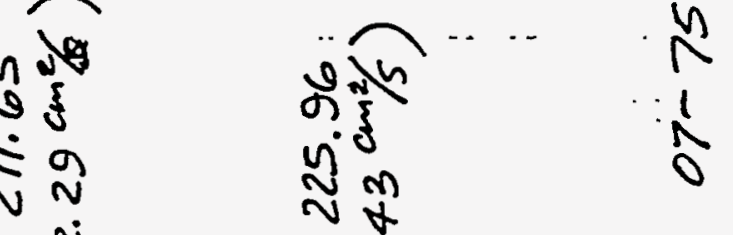

4 .

$y$
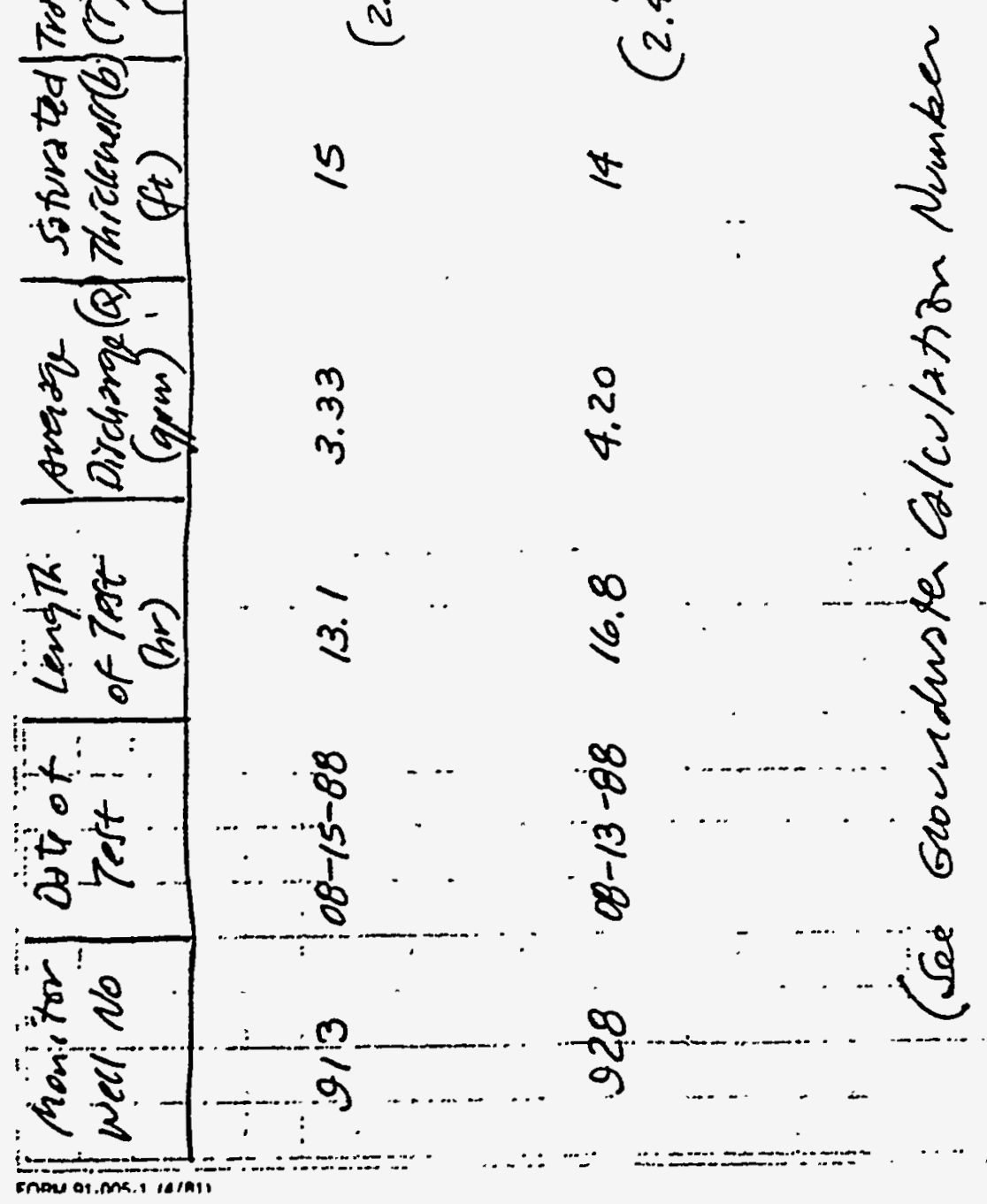
UNITED STATES DEPARTMENT OF THE INTERIOR GEOLOGICAL SURVEY

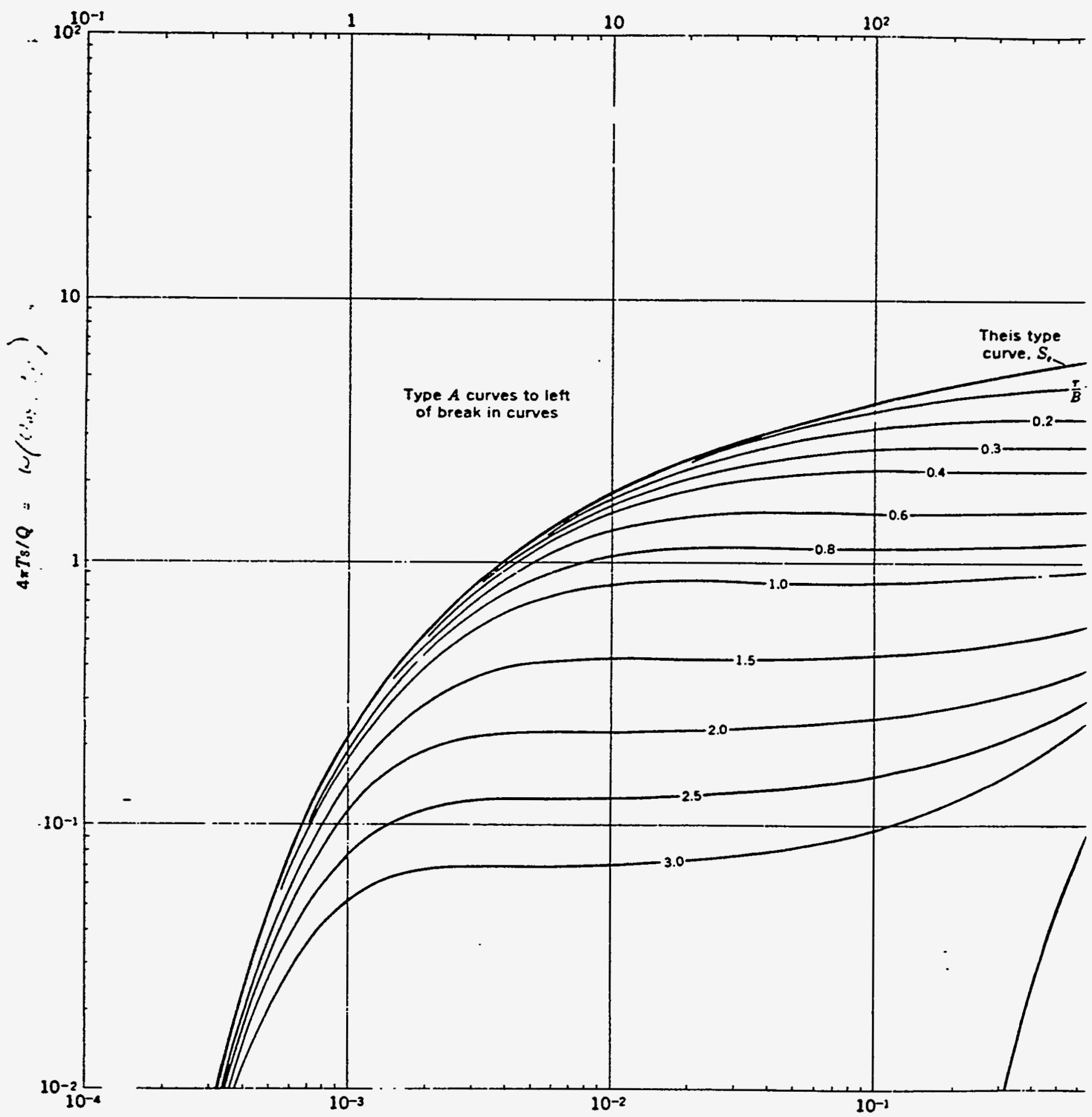

DELAYED-YIF 
PROFESSIONAL PAPER 708 PLATE 8

$\Gamma t / \tau^{2} S_{\text {c }}: i$.

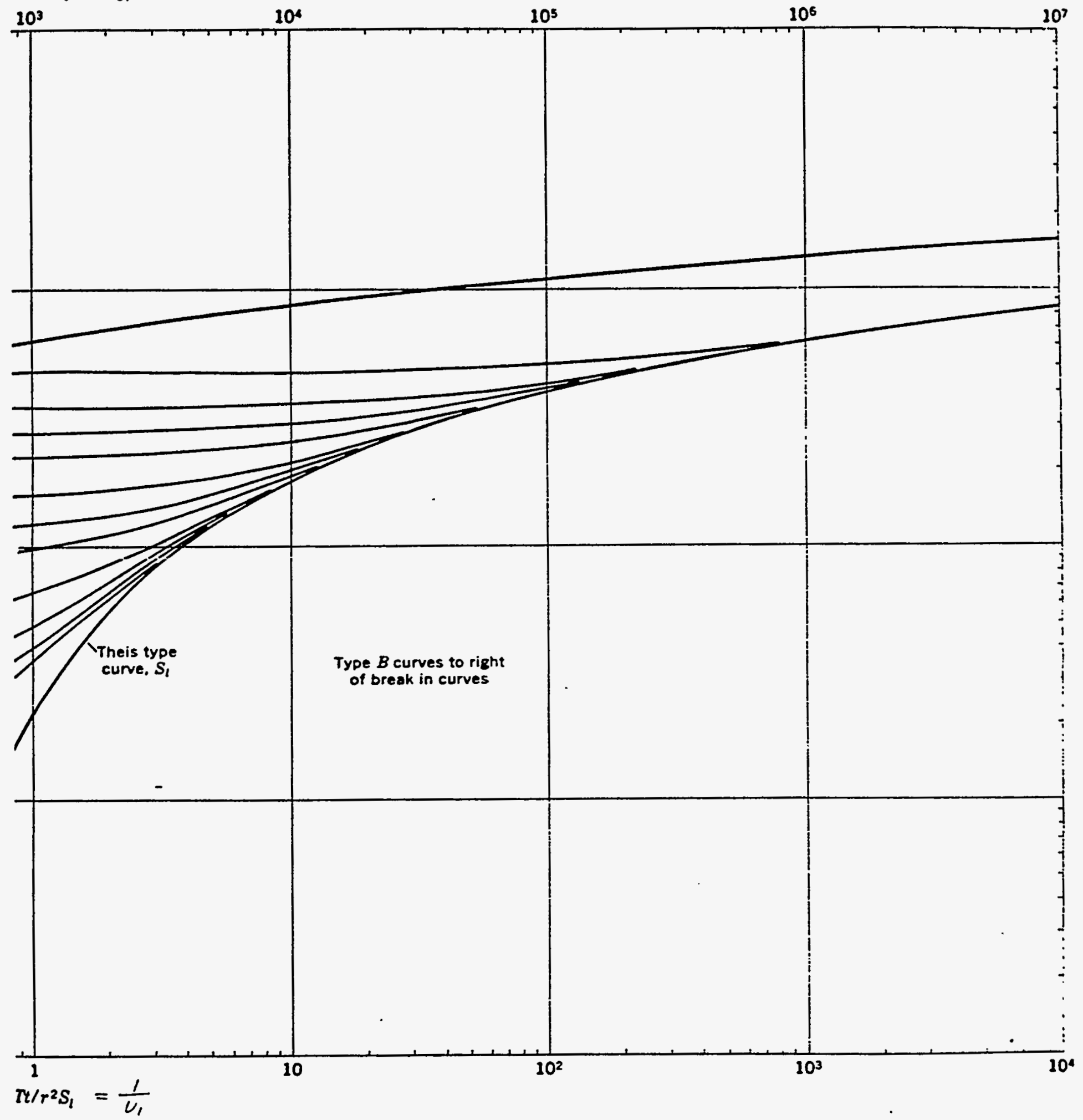

\section{DD TYPE CURVES}

$\operatorname{ton}(1963$, tiz. 1) 
JACOBS ENGINEERING SPOOK, wT

7

DATE

DY एU/ CHKO.
SUBNECT

AQUIFER PUMPING TEST
SHEET NO.

JOB NO.

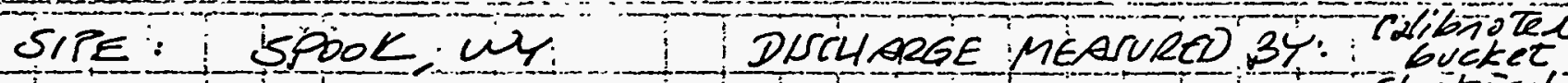

LOCDTION: MN-913 DROUSOWN MEAUVED BY: Electiced
soumder Topof sounder

$$
\because
$$

:PUMP-TEST: WEL REREENGE POINT: cosfing

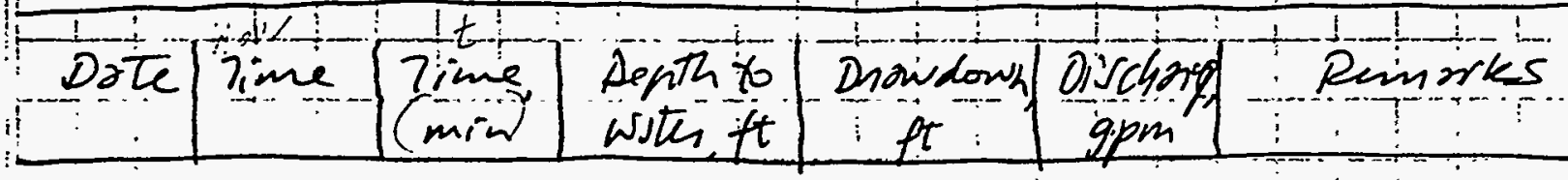

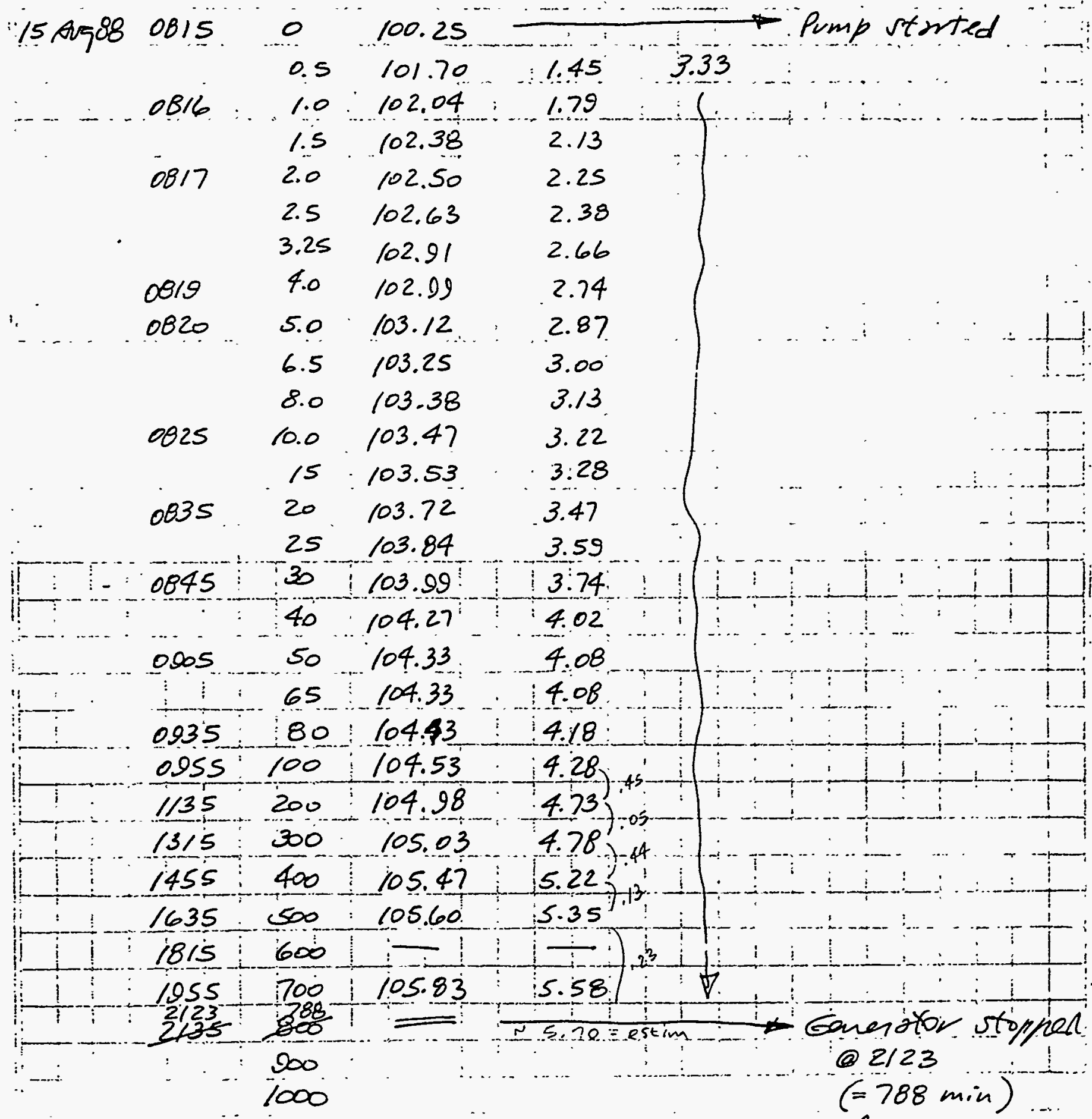

$\rightarrow$ Pump of

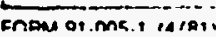




\section{J] JACOBS ENGINEERING GPOK, wy}

DATE

BY R4 chro.
SUBJECT

AQUIFER AMPING TEST
SHEETNO.

JOB NO.

SITE!: SPOOK WUY

LOCSTION: MW-913

PECOVER:-TEST WEIC

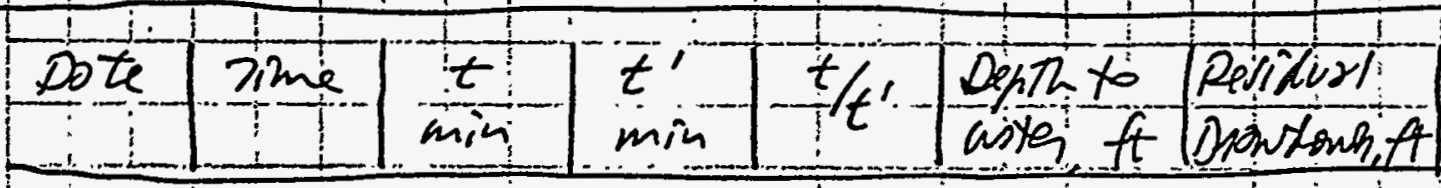

$\left[\begin{array}{llllll}15 A 8 B 8 & 2123 & 788 & 0 & 10.0 & \end{array}\right.$

(126 $2126 \quad 39263.7 \quad 102.40: 2.153 .55$

$1: 2127 \quad 722 \quad 4 \quad 1198.0 \quad 10217 \quad 1.023 .79$

$2128 \quad 793 \quad 5 \quad 158.6 \quad 102.00 \quad 1 \% 35.95$

$\begin{array}{llllllll}2129 & 794 & 6 & 132.3 & 10 / .88 & 1.63 & 4.07\end{array}$

$\begin{array}{llllllll}2130 & 795 & 7 & 113.6 & 101.80 & 1.55 & 4.15\end{array}$

$2131.796 \quad 8 \quad 29.5 \ldots 101.75 \ldots 1.50$ 4.6.

$\begin{array}{rlllll}2133 & 798 \ldots & 10 & 70.8 & 101.68 & 1.434 .27 \\ 2138 & 803 & 15 & 53.5 & 101.60 & 1.35\end{array}$

$2143 \cdot 808$ 20 $40.4 \cdot 101.53 \quad 1.28 \% 42$

$\begin{array}{lllllll}2148 & 813 & 25 & 32.5 & 101.47 & 1.22 .4 .43\end{array}$

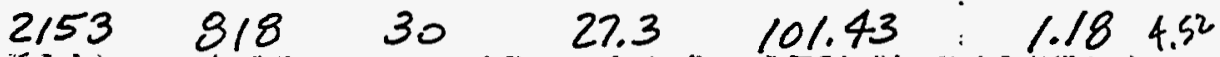

$2203 \quad 828 \quad 40: 20.7 \div 10137: 12415$

$2213 \quad 838 \quad 50: 16.8 \quad 101.32 \quad 1.07 \quad 463$

$2228 \quad 853-65 \quad 13.1 \% 101.24: 0.99$

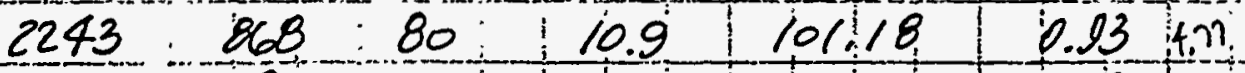

$2303.888100 .8 .9 \quad 101.13 \quad 0.884 .84$

$2330.915 \quad 127 \quad 7.2 \quad 101.08 \quad 0.834 .97$ 
IR JACOBS ENGINEERING SPDOK, WY

$\subseteq$

DATE

PUMP MW-913- OBRERVIITON WCU 1 , $r=18.15^{\prime}$ IS30w

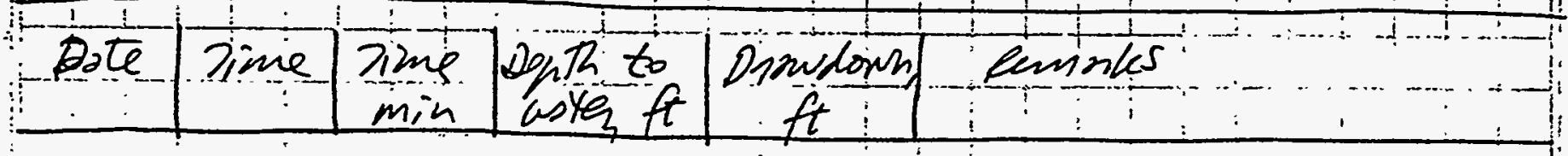

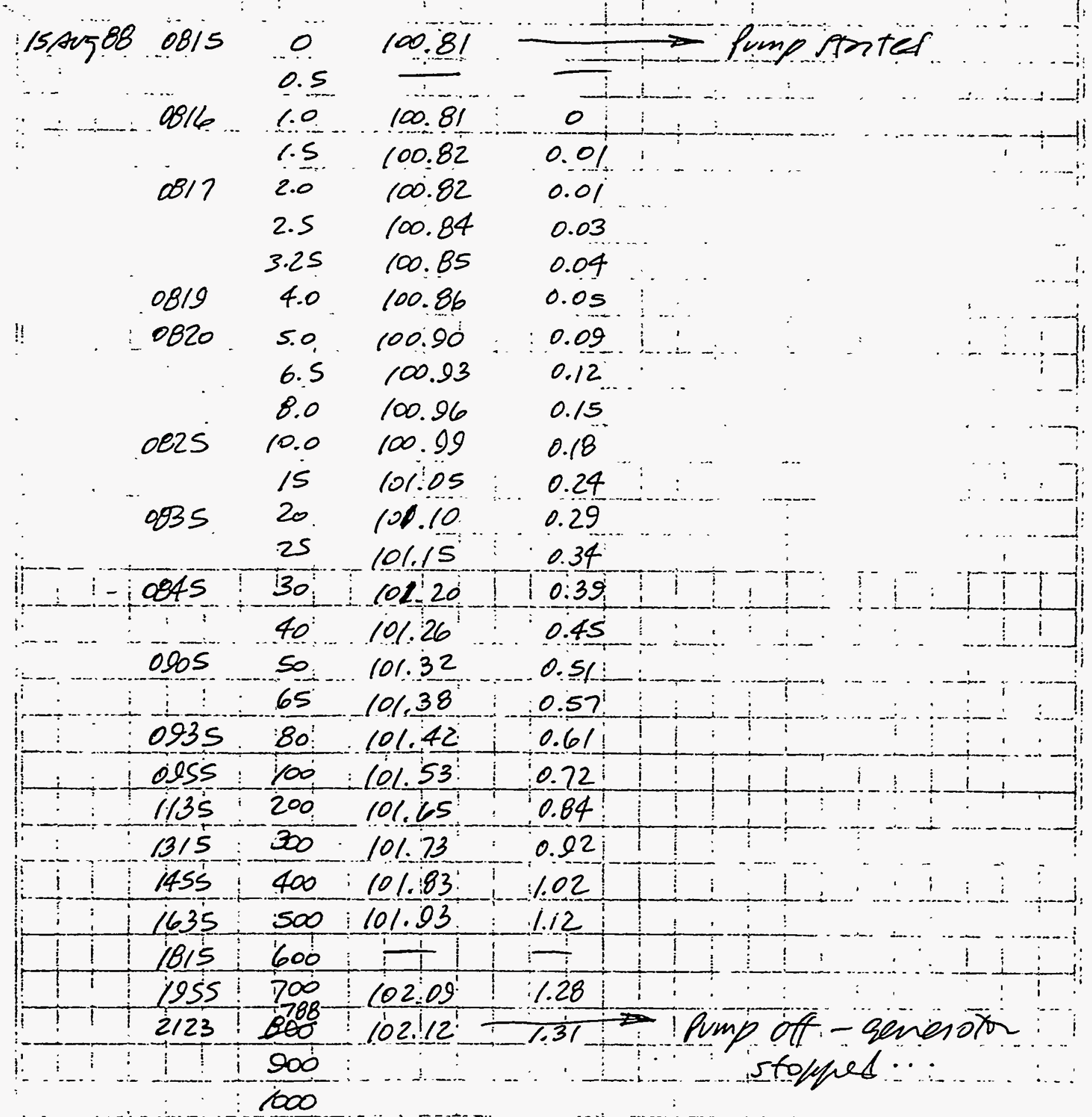




\section{JE JACOBS ENGINEERING viDOK, wY}

$y / 3-4$

DATE

SUBNECT

Br $2 / 4$ CHKD

PQUIFER PUMPING TEST

SHEET NO.

SITE SPOOK U4

LoCATroN: ow-9/3-1

RELOVER - OS ERVOTION

WeZ

JOBNO.

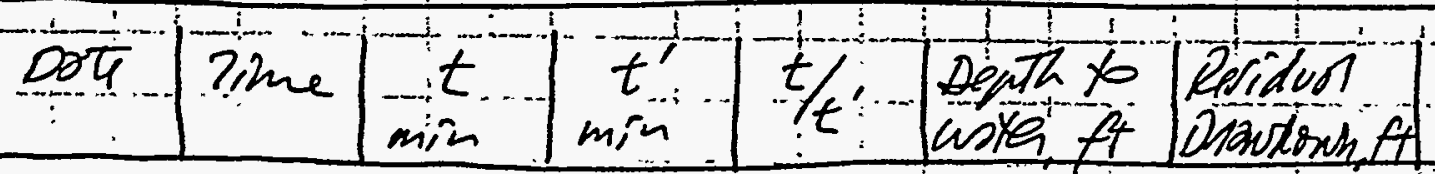

15 prepse

...

$2123, ? 88$

$2124 \quad 789$

790.5

$2128: 793$

794.5

2131 796

$2133 \ldots 798$

0 .

$0.0 \quad 19212$

$1 / 31$

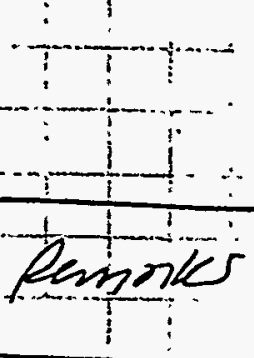

$2138 \quad 803 \quad 15$

1. $\quad 789.0,102.10$

1.290 .02

316.2 i 102.70

1.290 .02

$159.6 \quad 102.03$

1.220 .09

$122.2,102.00$

$1.19 \quad 0.12$

$99.5 \quad 101.96$

$1.15 \quad 0.16$

$79.8 \quad 101.93$

$1.12 \quad 0.19$

$2143 \quad 808$

$2148 \cdot B / 3$

$2153 \quad B 1 \theta$

2203

828

$2213 \quad 838$

2228

$B 53$

2243

868

2303

888

915

$53.5 \quad 101.88$

1.070 .24

40.4 101.83 1.020.29

2330

$32.5-101.79$

$\begin{array}{lll}0.98 & 0.33^{\circ}\end{array}$

30. $27.3 \quad 101.76$

0.950 .36

$40: 20.7 \quad 101.70$

50 $16.8 \div 101.67$

0.890 .42

65

$13=1$

101.63

$0.860 \mathrm{ks}^{5}$

80.10.9

col. 59

0.820 .49

\begin{tabular}{|l|l|l|l|l|l|l|l|}
\hline & 1 & 1. & 230 \\
\hline & & & 233
\end{tabular}

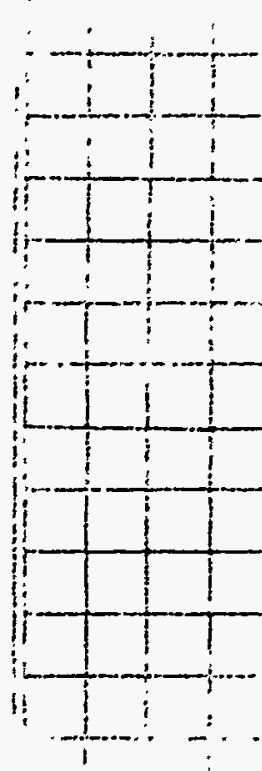

100

8.9

127 1.2 10149

0.780 .33 
$0.1 \quad 2^{2} \quad 3^{4} \quad 567891.0 \quad 2 \quad 3 \quad 4 \quad 5678910$ 2

$3 \quad 456789100$ 2 34567891000

(1)

7 ?

:

\section{7 車}

17ri

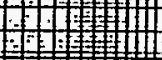

$+$.

4 -

Q

H.

3 3

-1. 2. 1.

>

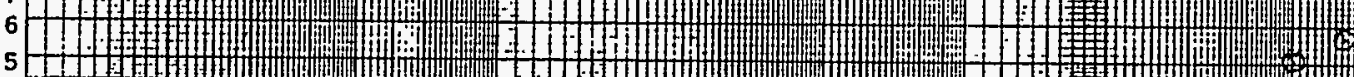

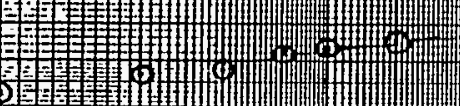

(स)

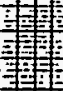

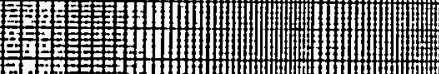

4.

$\infty$

(4)

3 -

Yั)

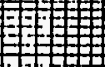

\section{(9)}

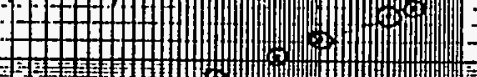

(t)

(1)

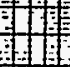

Y)

Y)

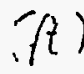

2 2

$\mathrm{r}$
$\mathrm{r}$

$$
\begin{aligned}
& t\left(i_{\text {min }}\right) \\
& \text { time sffa pumping storted } \\
& \text { SPOK, WY: } \\
& \text { nw-9/3 } \\
& \text { Perping : }
\end{aligned}
$$




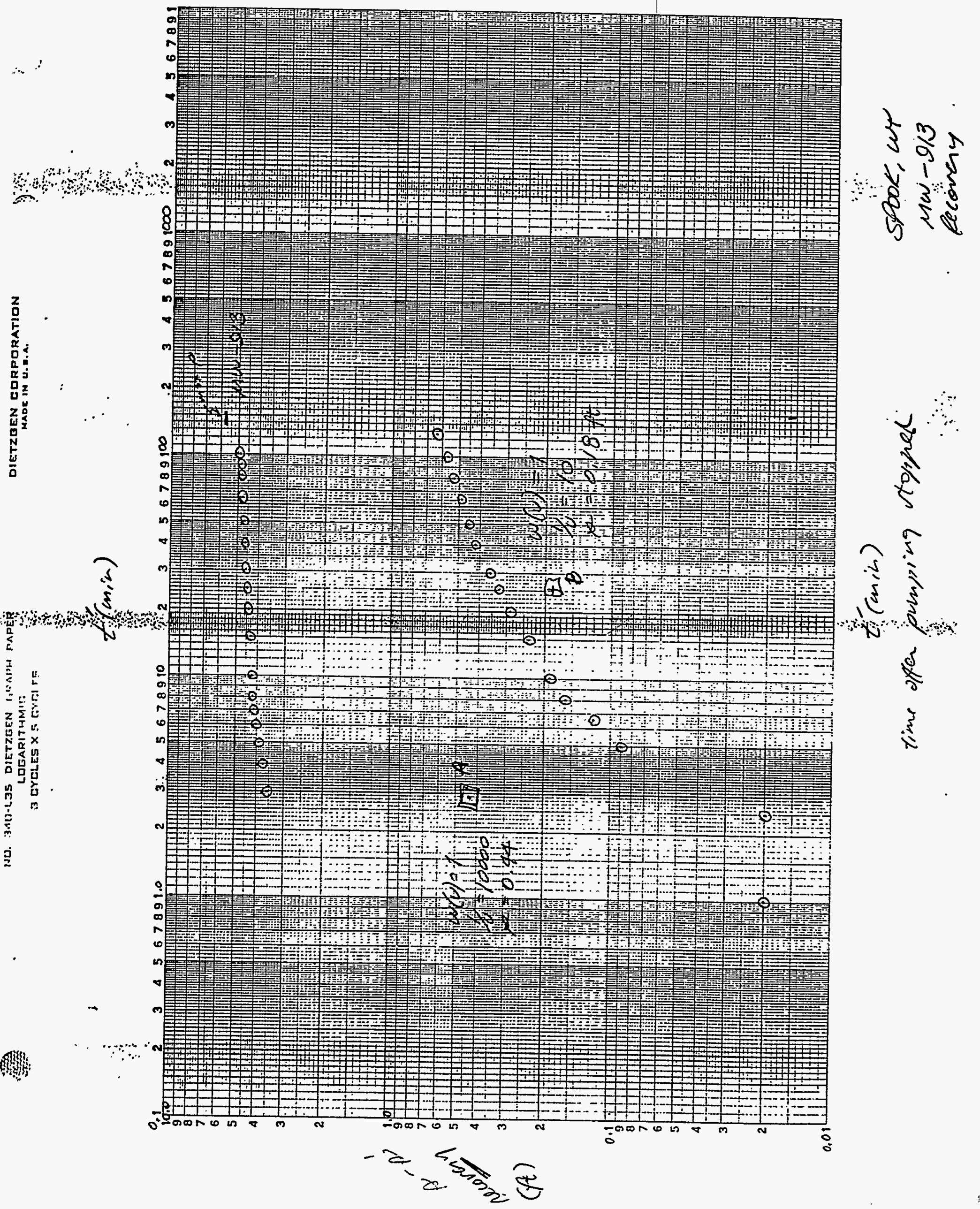


JE JACOBS ENGINEERING

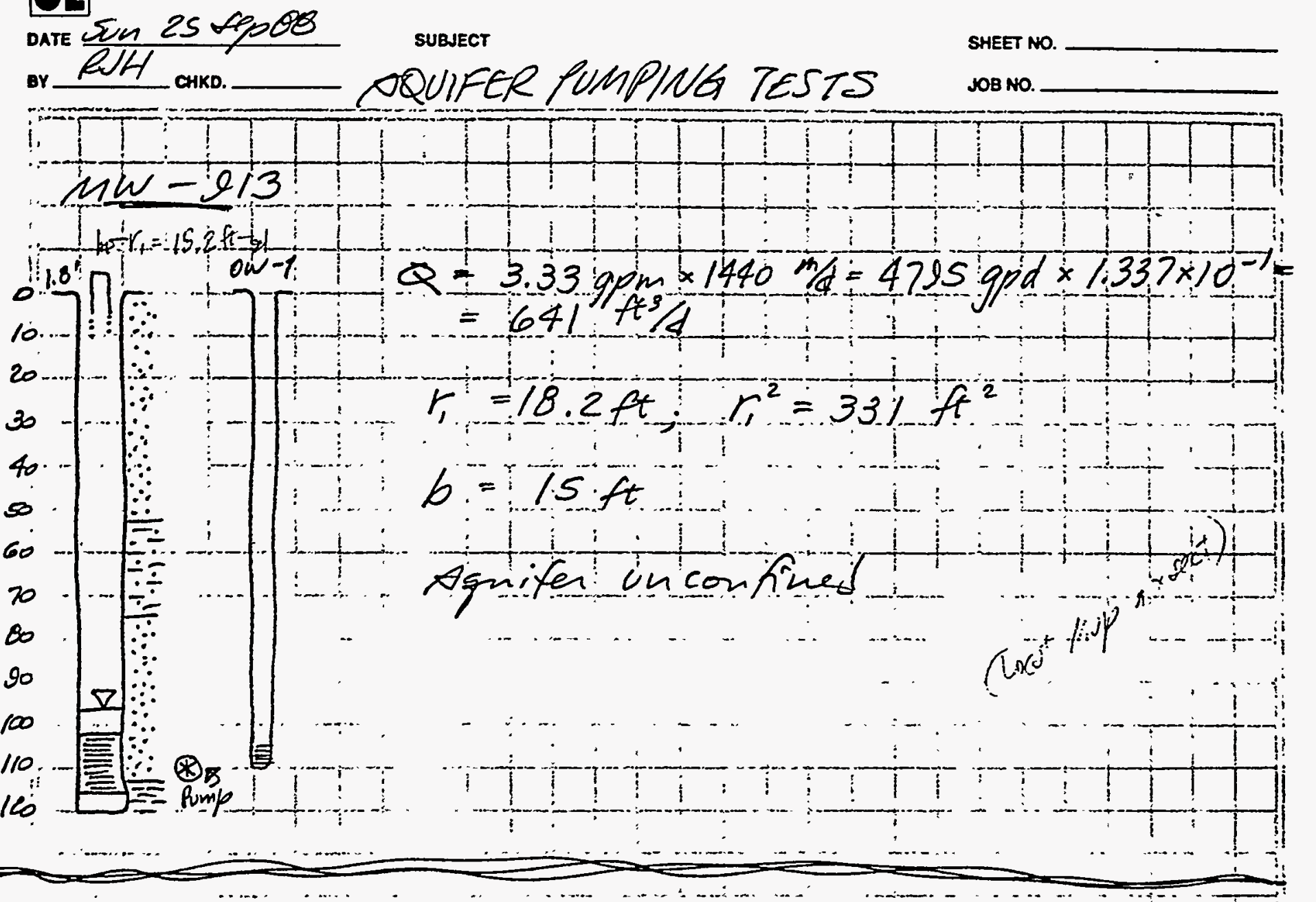

1) Mw-913 Pmping (The,is)

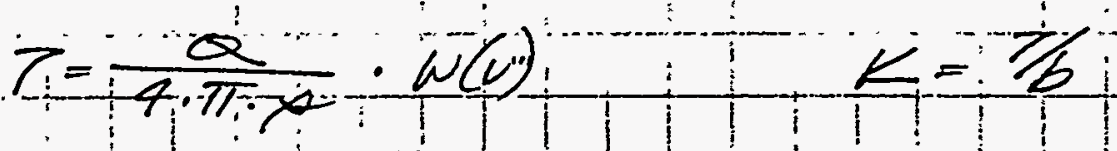

$$
\begin{aligned}
& \text { A. } 7(\text { endi })=\frac{641}{4 \cdot \pi \cdot 2 \cdot 0 \cdot 1=25.50 A^{2}}
\end{aligned}
$$

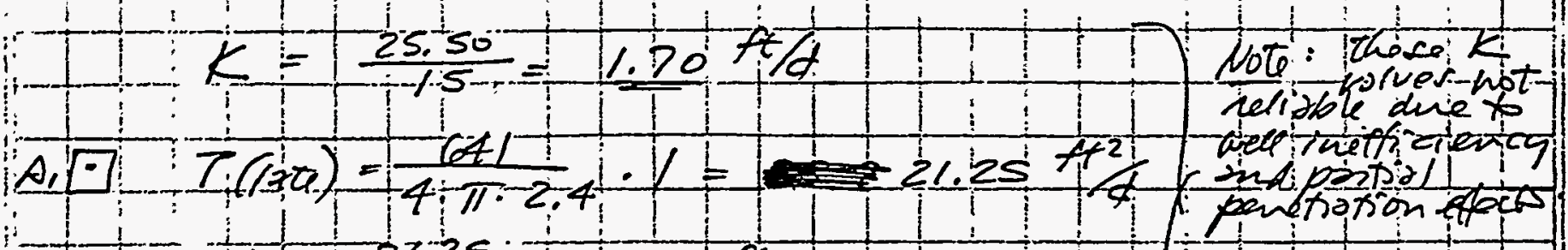

$$
\begin{aligned}
& \quad K=\frac{27.25}{1-5}=1.42{ }^{A t} / 1 \quad 1
\end{aligned}
$$

(2) Mw-D13 Recoirny (Pheiv)

$$
\begin{aligned}
& A-T \frac{641}{4-\pi 10044} 1=1-5.93 A^{2} / d \\
& K=\frac{175.93 \mathrm{ft}^{2} / \mathrm{A}}{15 \mathrm{ft}}=23 \mathrm{ft} / \mathrm{A}
\end{aligned}
$$


JI. JACOBS ENGINEERING

SPook w w

$5 / 3-9$

DATE vUn 25 eape8

в $\mathrm{e} 4 \mathrm{C}$ сако.

SUBJECT

SHEET NO.

AQUIFER RMPING TESTS



3) $M w-913$ Reconeny (Ataightline)

$1 K=10.18$ Ald (ae colculatous on groph

4) ow-e13-1 bumping (Theiv)

$B B \quad T=\frac{641}{4 \cdot \pi \cdot 0.24} \cdot 1=212.54 \mathrm{At}^{2} / \mathrm{d}$
$K=\frac{212.54}{15}=14.17 \mathrm{~A} / \mathrm{d}$

5) ow $=9 / 3-1$

$$
\begin{aligned}
& v^{\prime}=\frac{4 T t}{r^{2}} \\
& v^{\prime}
\end{aligned}=\frac{4 \cdot 212.54 \cdot 0.025 \cdot 0.1}{331}=6.4 \times 10^{-3} \quad(\mathrm{e} 2 \mathrm{r} / \mathrm{g})
$$

(as indicatoon of Beloned yiclit phenomenon Aि $x_{1}^{2}(10 t)$.

6). $0 w-913-1$ pecover (Theis)

$$
\begin{aligned}
& 7=\frac{1641}{4 . \pi+0.18}=283.38 A^{2} / d \\
& K=\frac{203.38}{15}=\frac{10.89}{14}
\end{aligned}
$$

i) ow $213-1$ fecovan (Borghtiline)

$$
K=19.59 \text { fta (ae galculoron on pronh...) }
$$


JI JACOBS ENGINEERING

SPOOK, WY

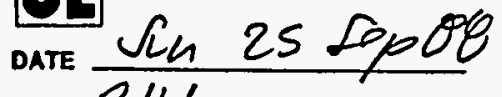

or CH chik.

SUBJECT

SQUIFER PUMPINGTESTS

SHEET NO.

$M w-9 / 3$ (cout)

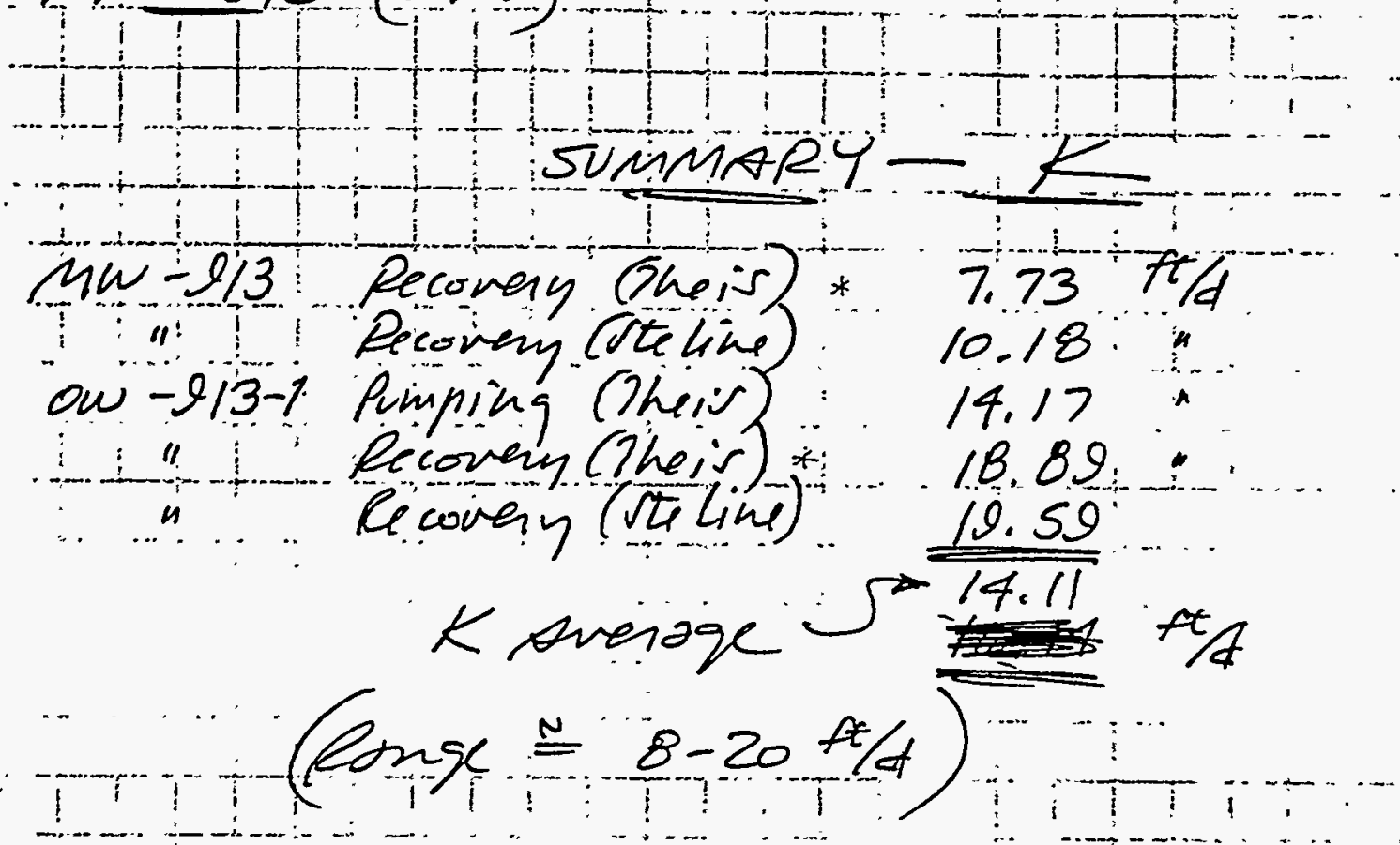

L

NOBNO.

$+1$

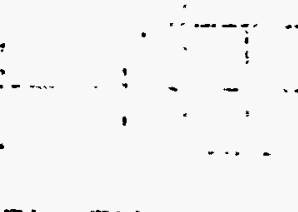

$$
\begin{aligned}
& \text { Mu-li3 Peconery (Tais) * }
\end{aligned}
$$

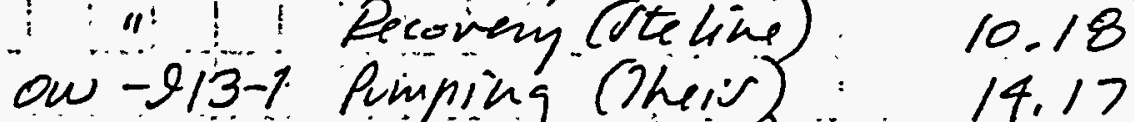

$$
\begin{aligned}
& \text { Ricoven (Theiv . 18.89 } \\
& K \text { Average } 5=14.11
\end{aligned}
$$

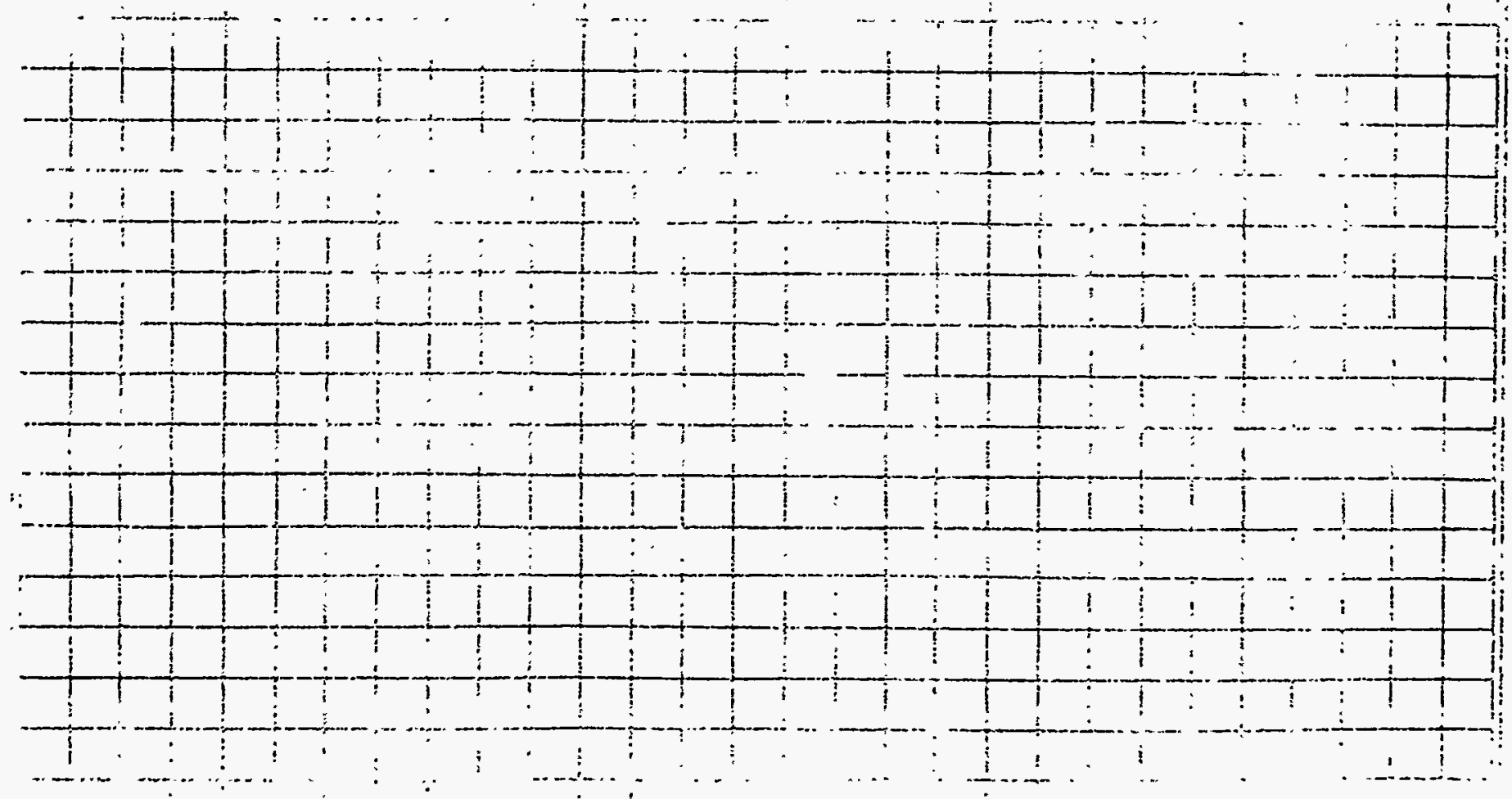


JACOBS ENGINEERING

se00k, wy

DATE

or PAT Cho.

SUBNECT

SHEET NO.

OQUIFEE PMPPNGTEST

JOB NO

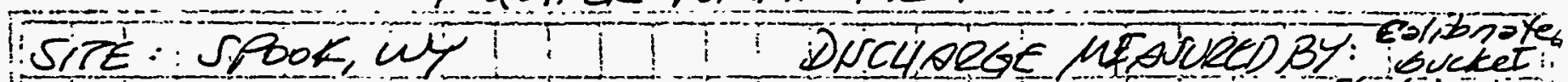

LOCDTION: $110 \mathrm{~N}-98$

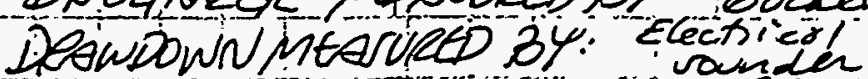

PUMP:TEST WELL PEFEDENCE BINT: "Top of piv.

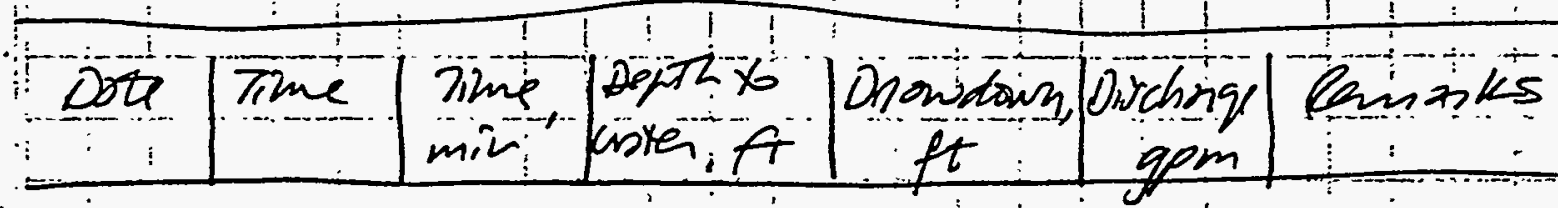

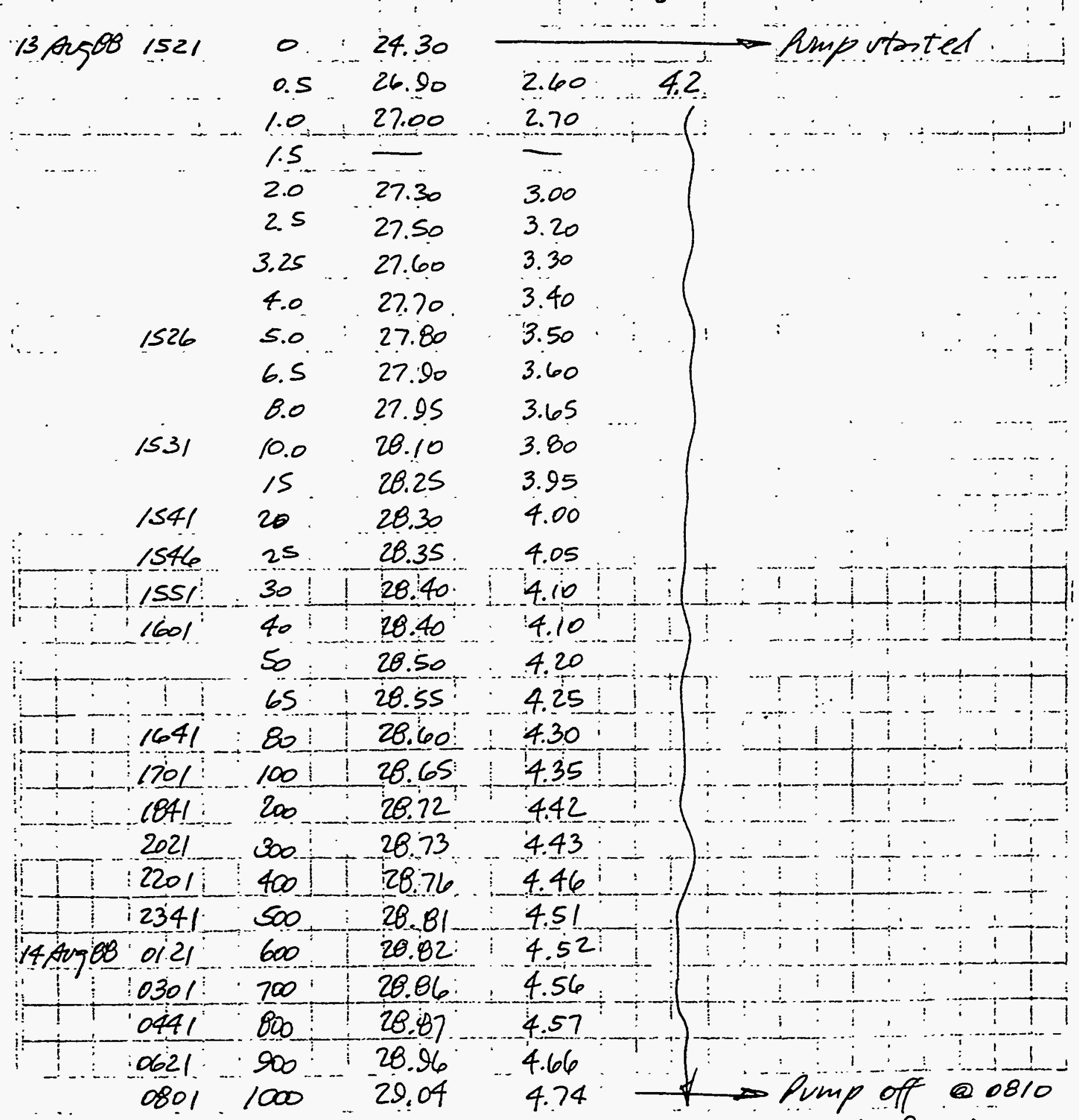

$=1009 \mathrm{~min}$ 
JACOBS ENGINEERING

spook, wy

18

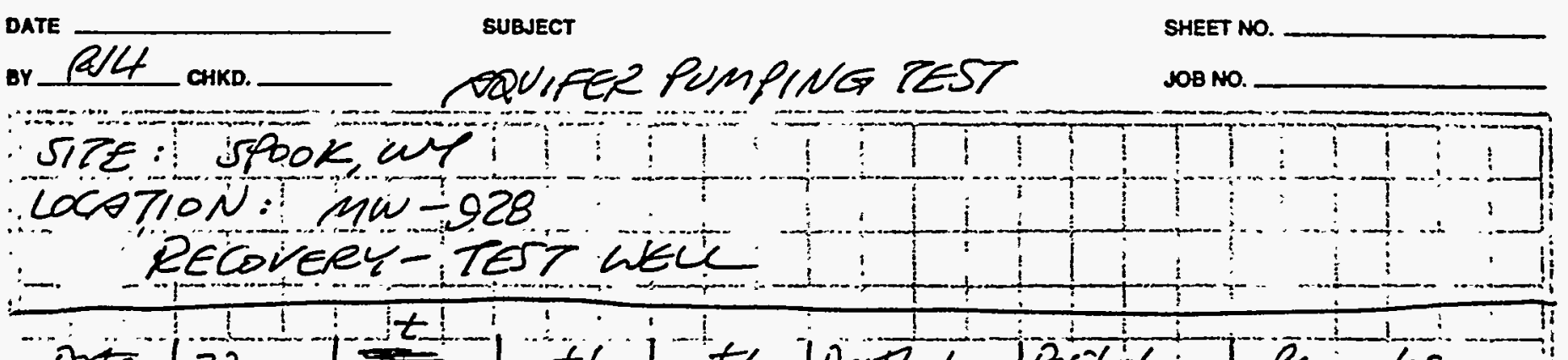

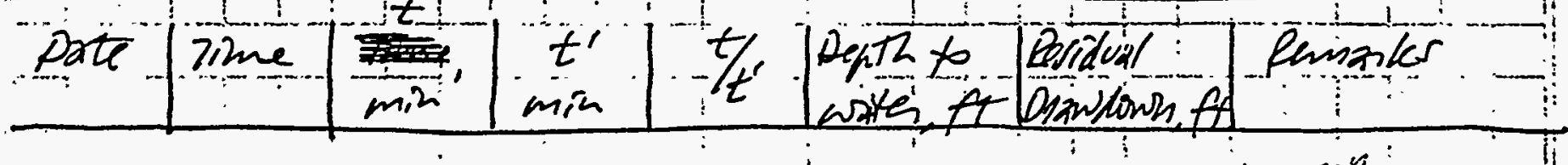

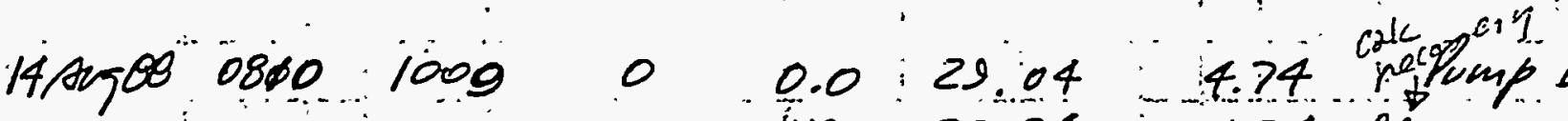

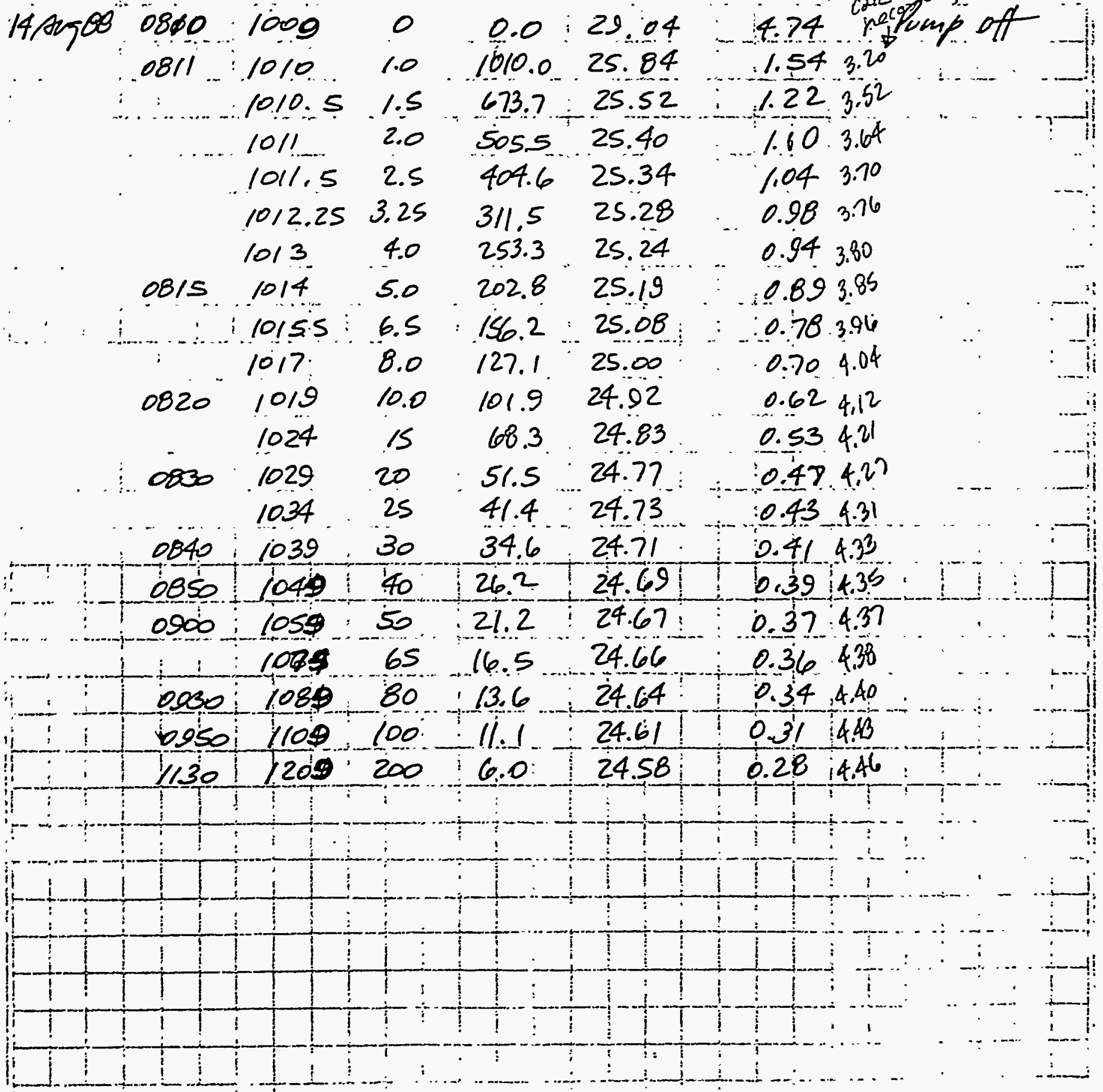


JF JACOBS ENGINEERING SPOOK, aY

$\begin{array}{ll}000 & 1\end{array}$

DATE

SUBUECT

BY 241 chKo.

PQVIFER PUMPING TEST

SHEET NO

SITE $5900 \mathrm{~K}$ W Refeerne folnt: zop of pro LOCA7I0N: ow-928-1

OUMP MN-928 - OBSERUATION WECI $Y=20.0^{\prime}$ 'S30 W

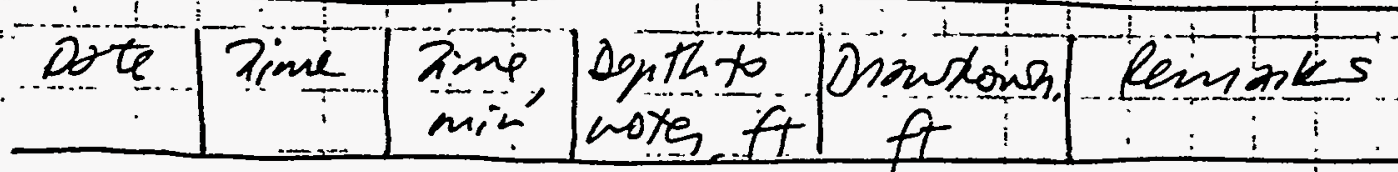

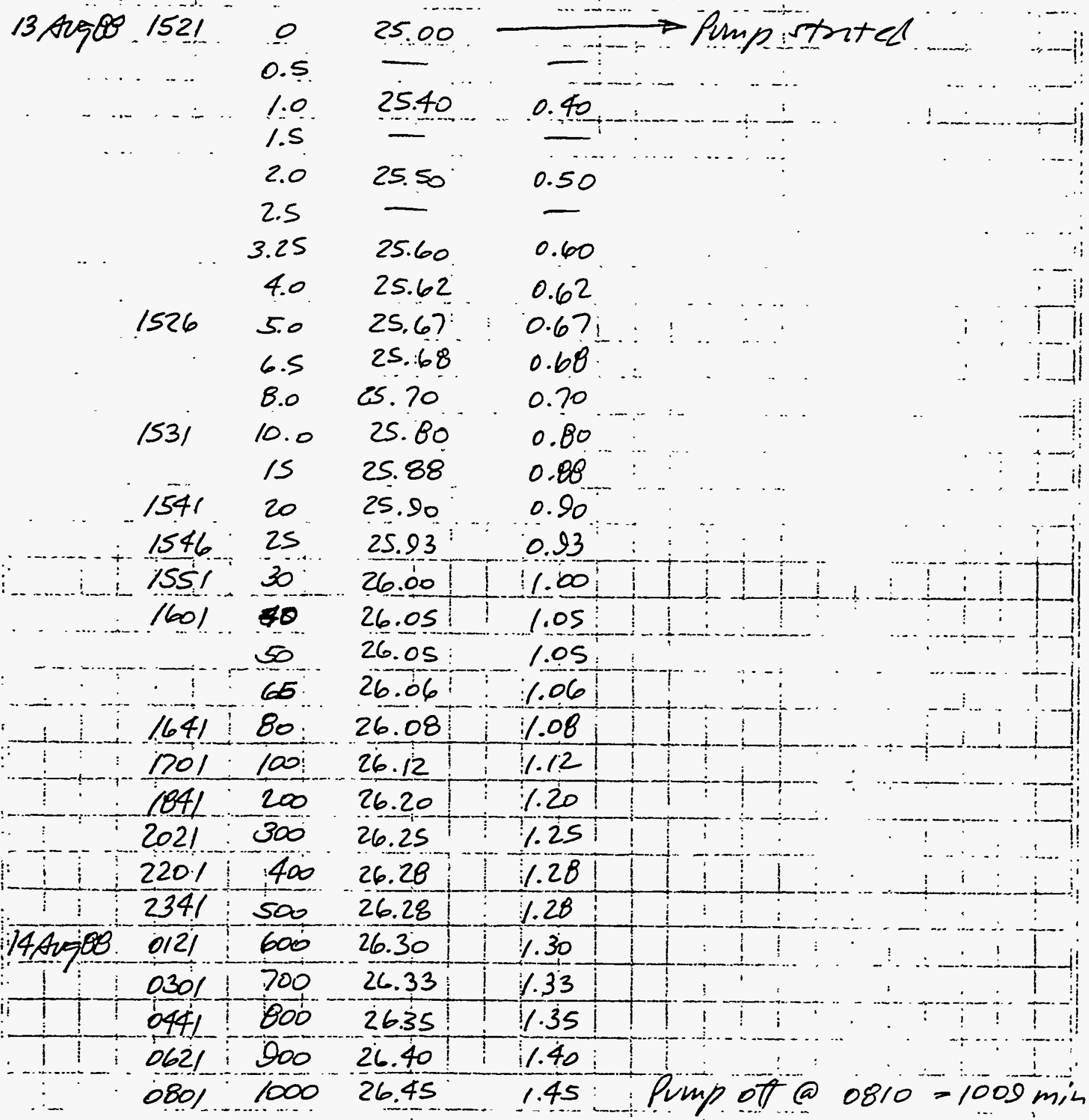


JACOBS ENGINEERING SPOOK, WY

$y<6-4$

20

DATE

SUBNECT

SHEET NO.

BY $2 / 4$ chKo.

OQUIFE MMPING TEST

NOBNO.

STE: spook wY

GOCNTON: OW -928-1

RECOVERY OBSERVATIOU UEUI

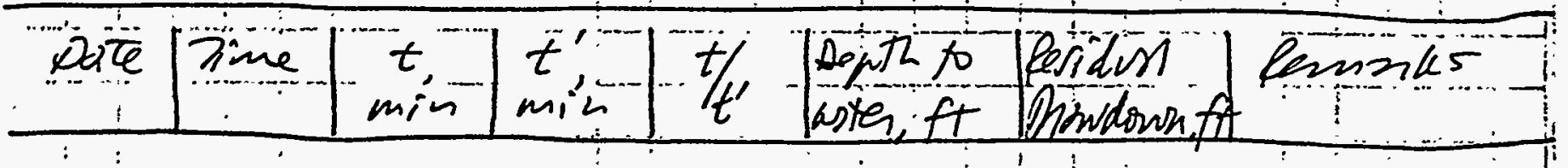

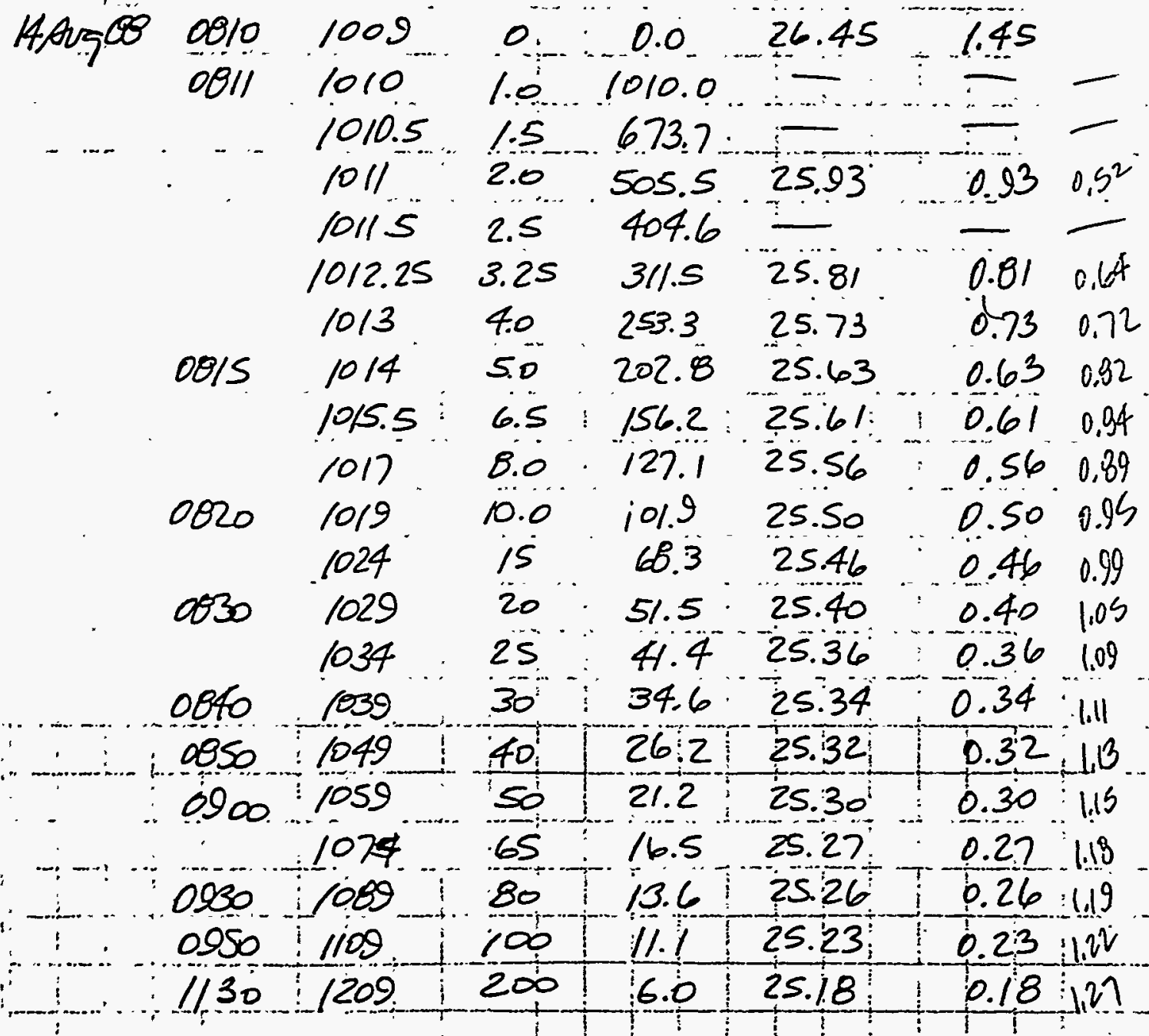


TIF JACOBS ENGINEERING

spook, wh

21

DATE

SUBNECT

ORUIFER PUMPINATEST
SHEET NO.

108 No.

ropotipic=

SITE: SPOOK WY

LOCATION: OW-928-2

PUNP MW-928 - OBVERVATION WELL $2, r=81.0^{\prime}$ NaO W.

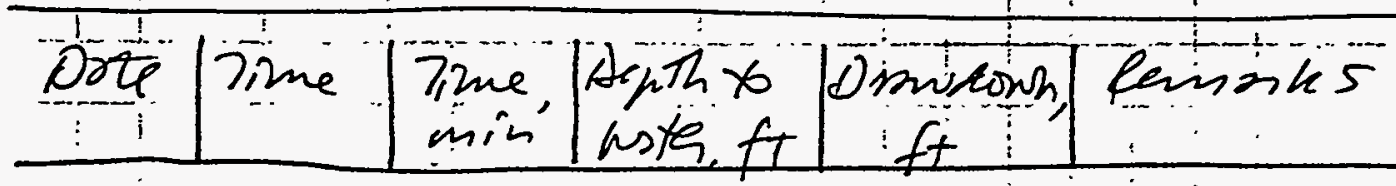

$\begin{array}{llllll}\text { Bongeg } 1521 & 0 & 25.00 & 0.5 & \ldots\end{array}$

$\begin{array}{llll}1.0 & & & \\ 1.5 & 25.00 & 0.00 & \cdots \\ 2.0 & - & - & \cdots \\ 2.5 & 25.00 & 0.00 & \end{array}$

$\therefore \quad 1526 \begin{array}{ccc}4.0 & = & \\ 5.0 & = & 1 \\ 6.5 & -1 & \\ 0.0 & 25.20 & 0.20\end{array}$

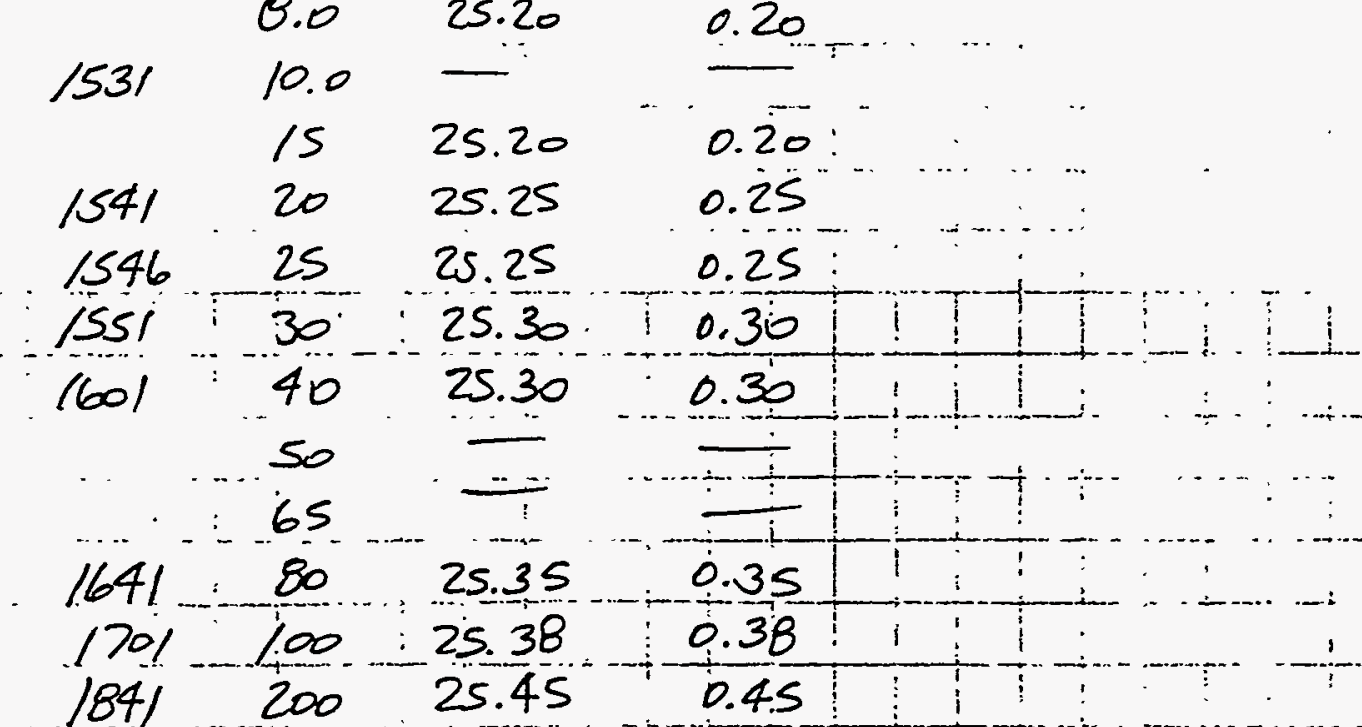

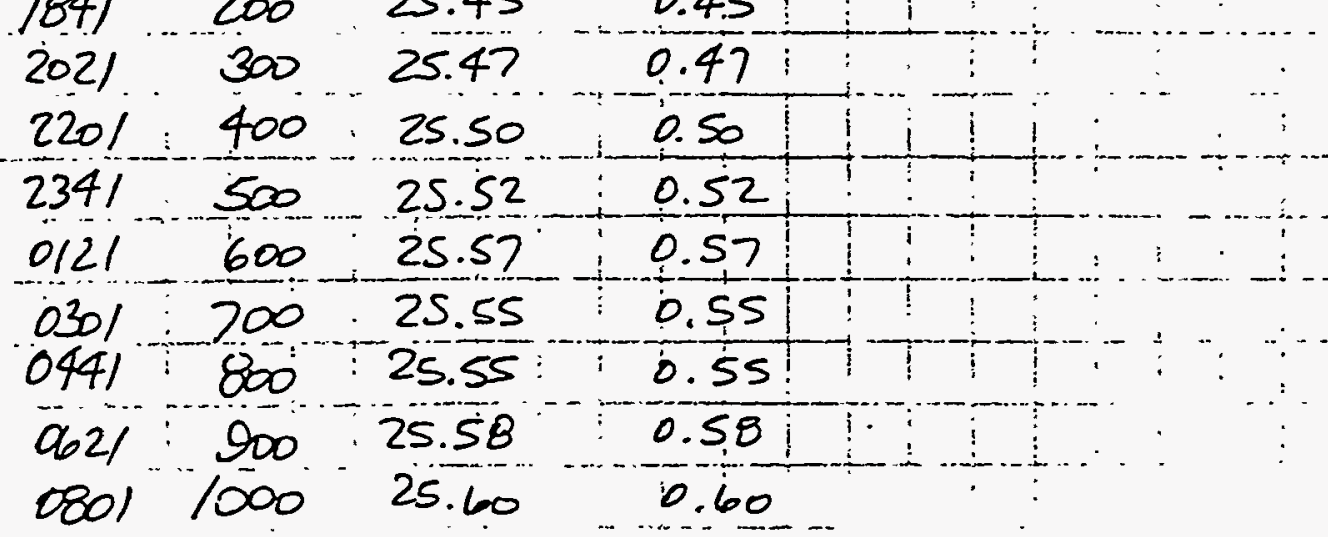


JE JACOBS ENGINEERING SPOOE, wy

ont

DV RU4 CHKD. NUIFER AMPING TEST

STTE: POOK WIT

LOCATION: on $-228-2$

- RECOVERY TOBSGRATION WEL 2

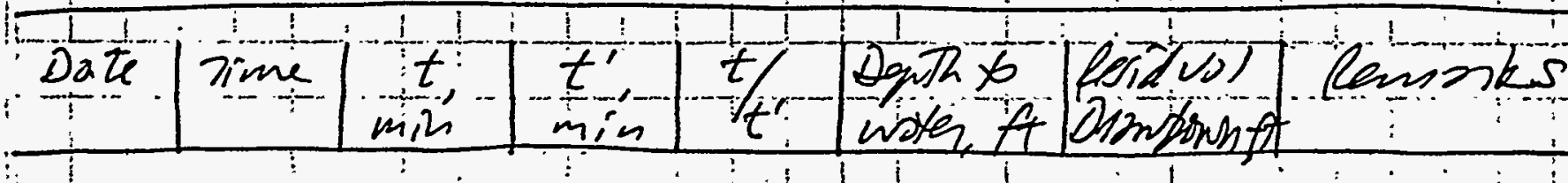

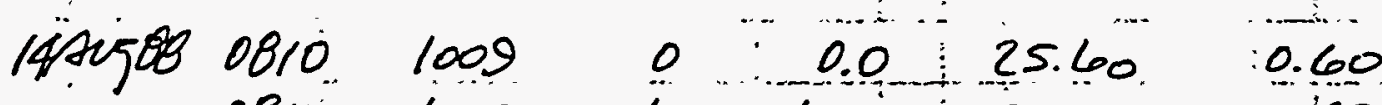

0811.1010 1.0 1010.0 25.5?

$1010.5-1.5 \quad 673.7$

$1011 \quad 2.0 \cdot 5055$

$1011.5 \quad 2.5 \quad 404.6$

$1012.25 \quad 3.25 \quad 311.5$

$\begin{array}{lllllll}1013 & 4.0 & 253.3 & 25.50 & 0.50 & 0.10\end{array}$

$0 B 15 \quad 1014 \quad 5.0 \quad 202.8$

$1015.5 \quad 6.5156 .2$

$1017 \quad$ B.0 $127.1=25.45$

o820. $1019 \quad 10.0 \quad 101.9 \quad 25.40$

1024 is 68.3 . 25.36

0830.1029 20 $51.5 \quad 25.33$

1034 25 $41.4 \quad 2531$

$0840.1039 \quad 30 \quad 34.6 \quad 2530$

OBSO $1049 \quad 40 \quad 26.2 \quad 25.29$

0900.1059 so 121.2525

$1074-65-16.5-25.25$

0930: 1089 . 80 13.6 25.24

0950.1109 .100

11.125 .22

$1130 \quad 1209 \quad 200 \quad 6.0125 .19$
SHEET NO.

NOB N. 

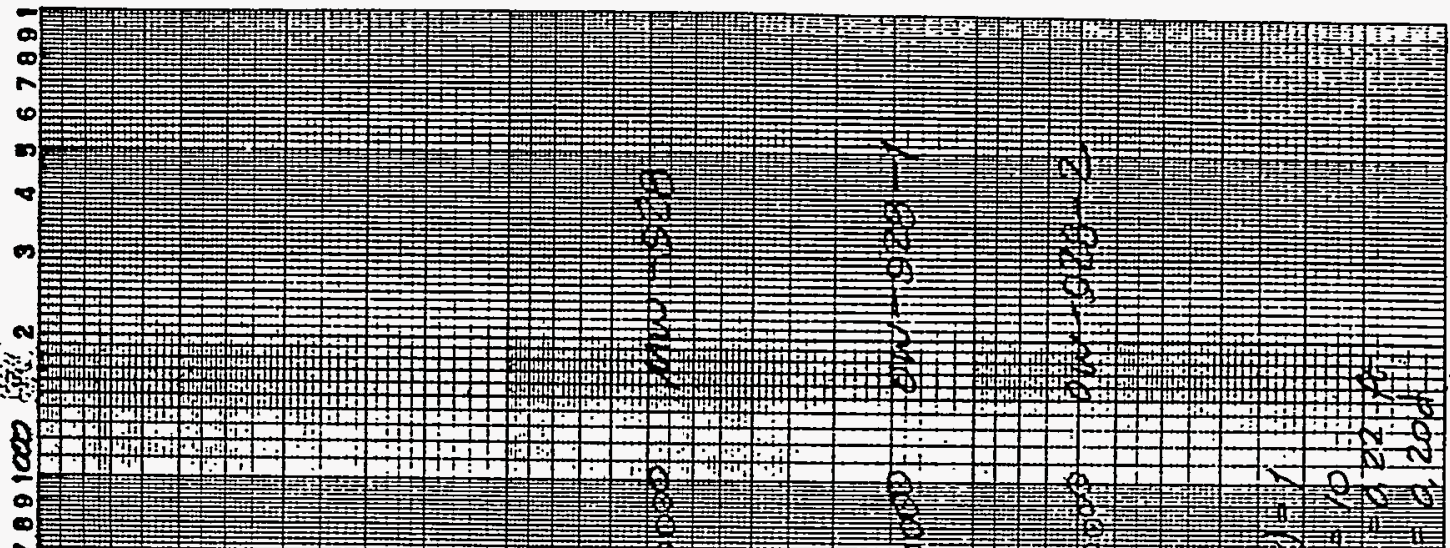
11 1

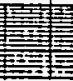
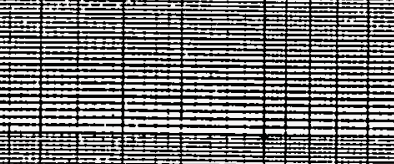

1

+2

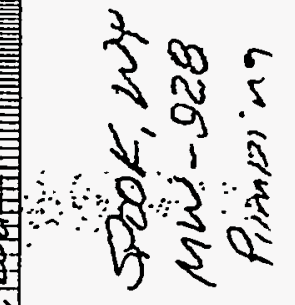

(n)

$\infty$

ए।

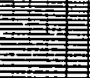

1

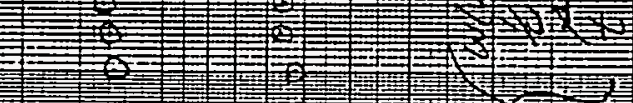

1

L

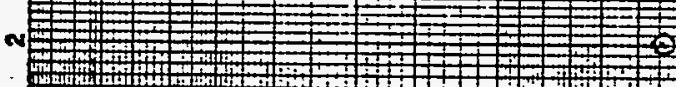

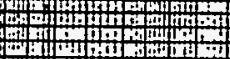

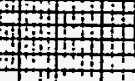

+

$8,+1+11$

$+1+1$

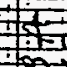

$\sin$

W

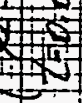

$1+$

急

is

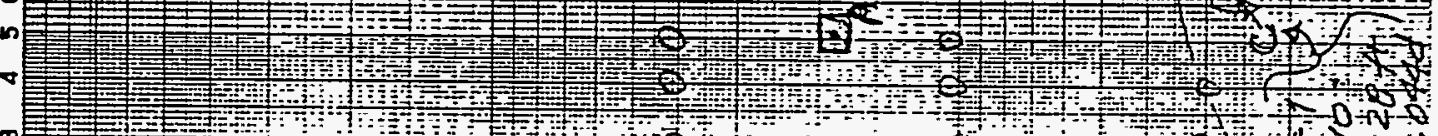

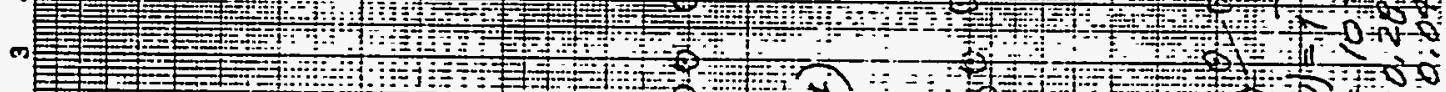

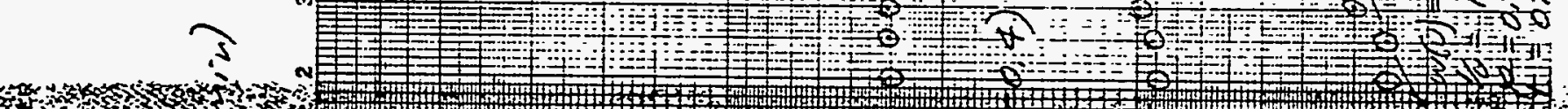

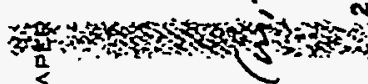

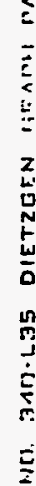

$=N$

$\frac{1}{2}$

$=$

$z \sum$

足

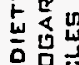

络

$\dot{\dot{s}}$

(4)
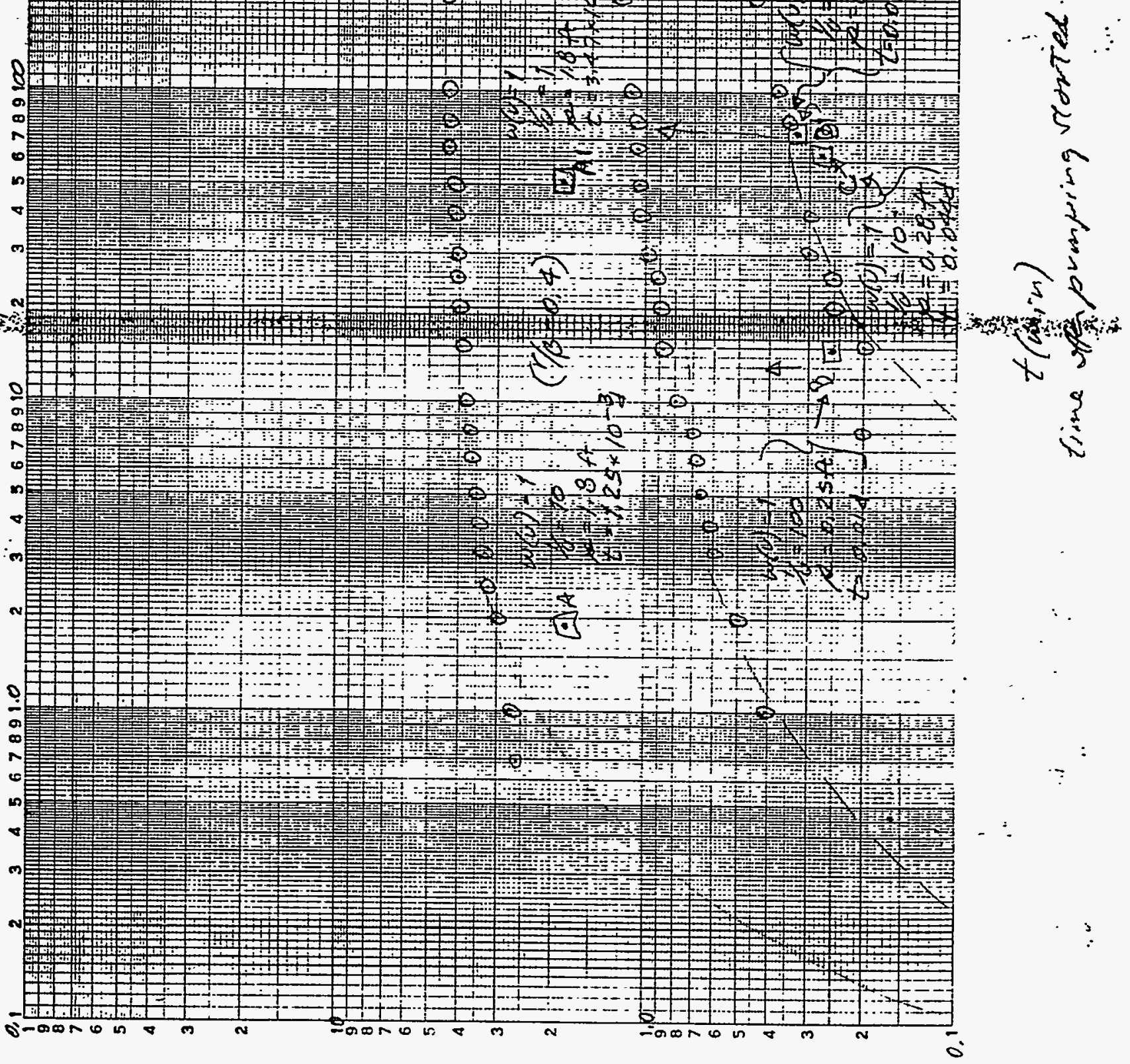


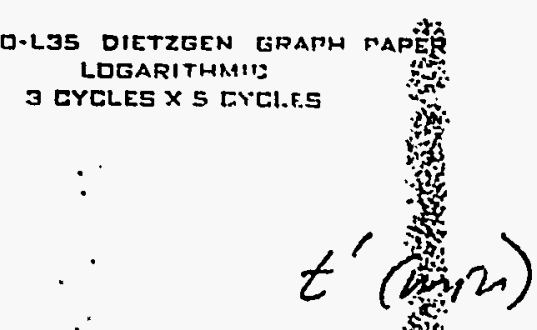

DIETZIEN CORPORATION

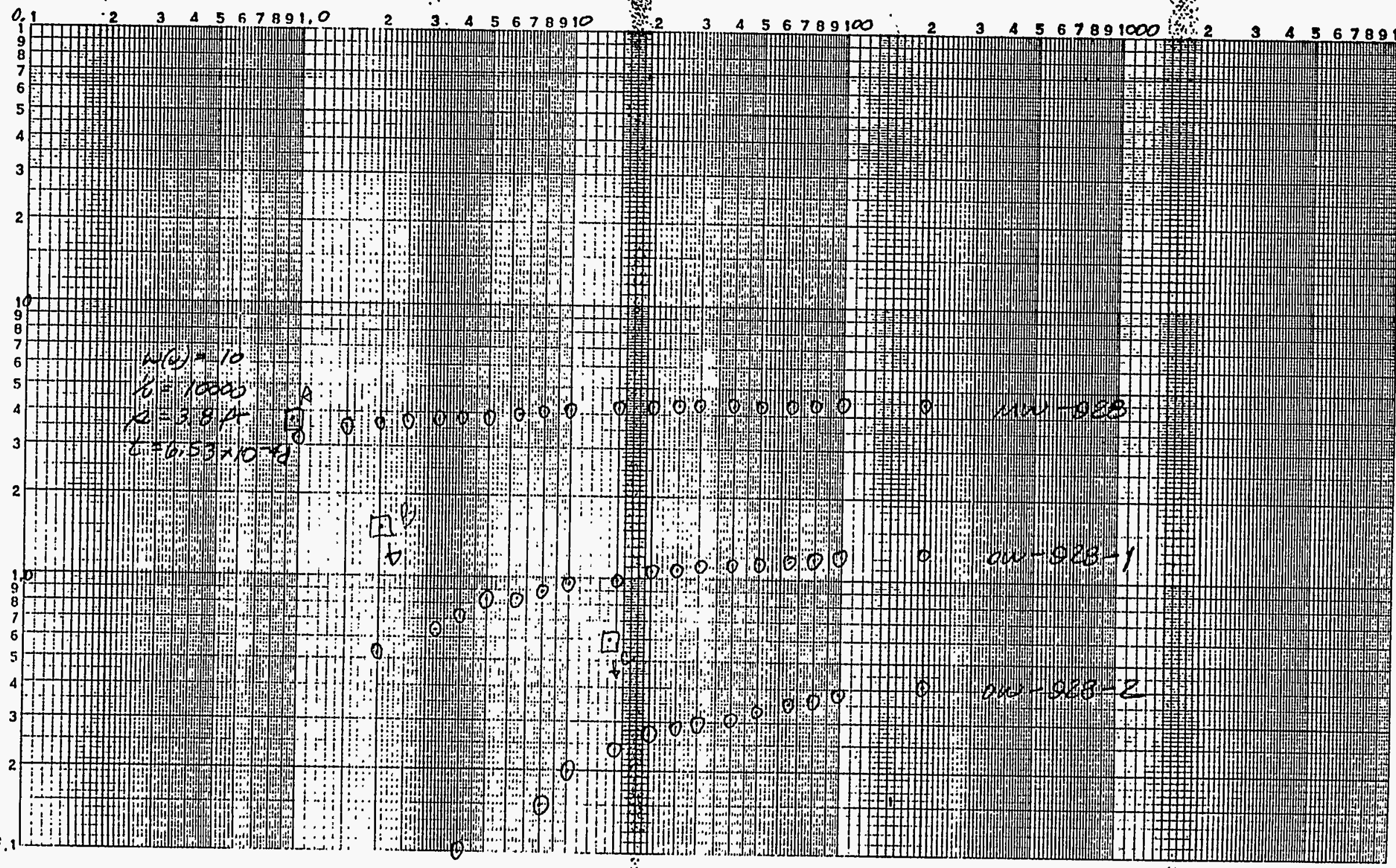
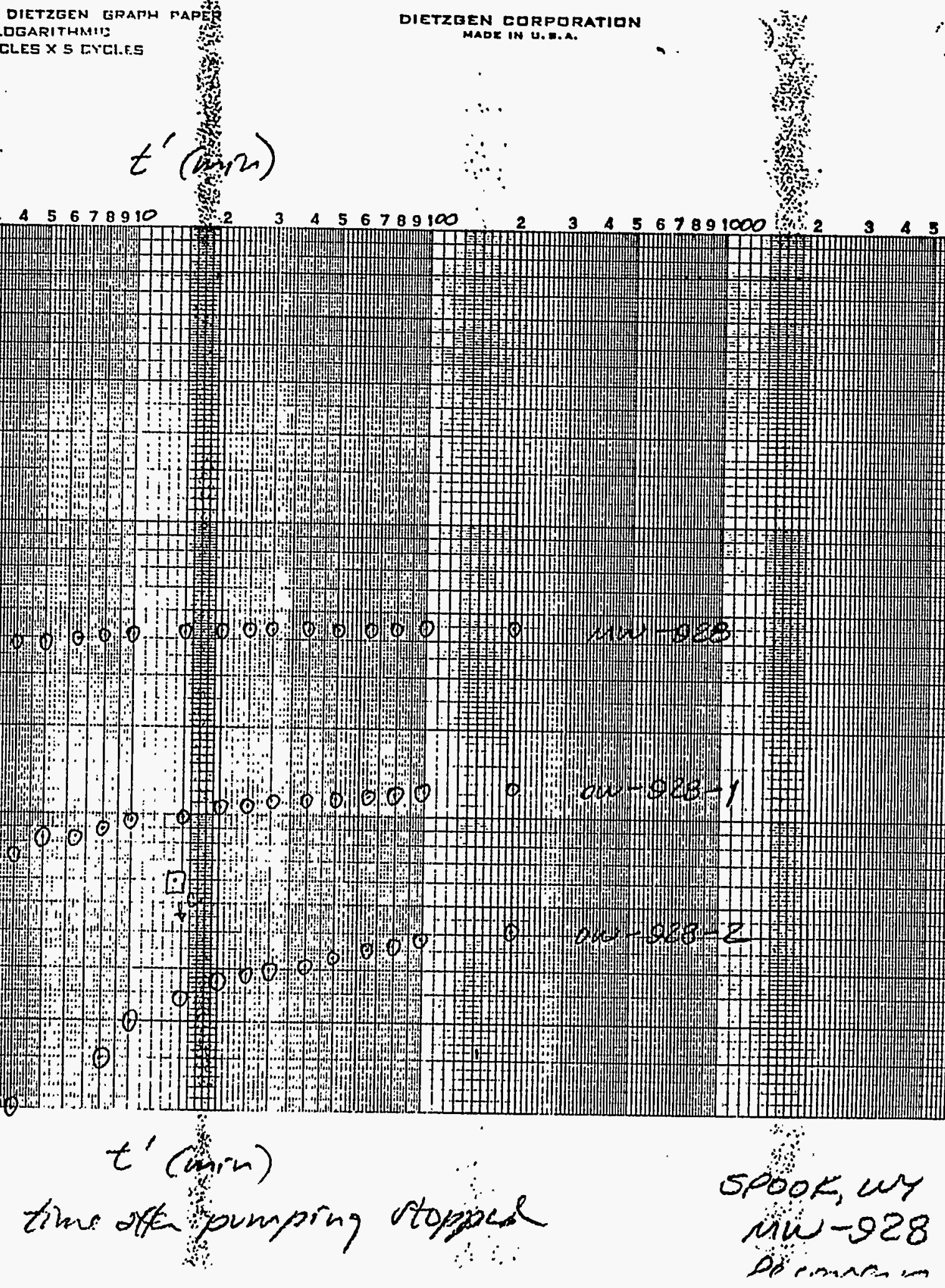

2

DATE Sun $25 \operatorname{sep} 88$

SUBJECT

SHEET NO.

or Pull chic.

AQUIFER PIPING TESTS

SOB NO.

Mw- 228

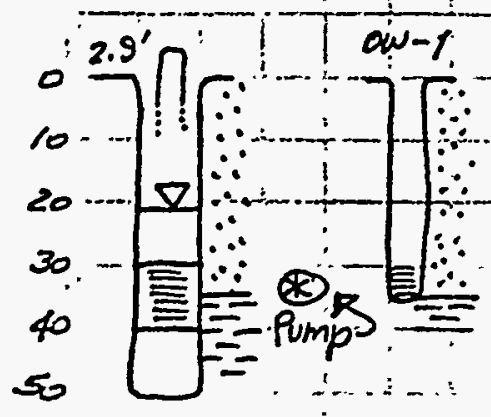

$$
\begin{aligned}
& Q=4.29 p m \times 1440 \mathrm{~m} / \mathrm{d}=6048 \mathrm{gpd} \times 1.337 \times 10^{-7}=1 \\
& =808.6 \quad \mathrm{ft}^{3} / \mathrm{s} \\
& r_{1}=20 \mathrm{ft} ; r_{1}^{2}=400 \mathrm{ft}^{2} ; r_{2}=81 \mathrm{ft} ; r_{2}^{2}=656 / \mathrm{ft}^{2} \\
& b=14 f t
\end{aligned}
$$

Ognife - unconfined

1) inw-e28 Pimping (This)

$$
\frac{1}{T}=\frac{1}{4 \pi p} \cdot a(0 \ldots) \quad K=: T / 6
$$

$A \square T($ err th $)=\frac{808.6 A^{3 / 4}}{4 \cdot \pi \cdot 1.8 \mathrm{At}} \cdot 1=35.75 \mathrm{ft}^{2} / \mathrm{d}$

$$
K=\frac{35.75}{14}=2.5 \mathrm{ft} / \mathrm{d}
$$

$A, 0 T($ est $)=$ sine 20 dove $=2.55 A / d=K$

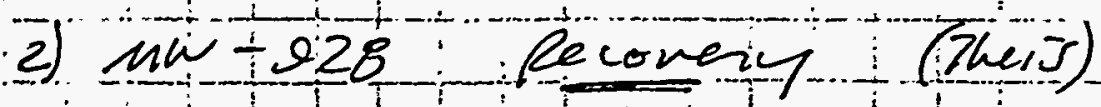

AD $7=\frac{1808.6}{4 \cdot \pi \cdot 0} \cdot 10=16933 \mathrm{ft}^{2}$

$$
K=\frac{169.33}{14}=12.10 \mathrm{ft} / \mathrm{s}
$$

3) $1 w-928$ Recovery (Aright line)

$$
K=1 / 513 \text { At /d (ae colculzsoris on graph }
$$


TIE JACOBS ENGINEERING SPOOL, WY

27

DATE Jin 25 EAp 08

SUBJECT

OQUIFE PUMPINGTESTS

SHEET NO

CHKD

JOB NO.

$M w-228$ (cont)

4) ow-228-1 fumping. (Theis)

$$
\begin{gathered}
\text { BU(som) }=\frac{808.6}{4 \cdot \pi \cdot 0.25}+1=257.39 A^{2} / 4 \\
K=\frac{257.39}{14}=18.38 \mathrm{~A} / 4
\end{gathered}
$$

$$
\begin{gathered}
B_{1} \text { I (r.t) }=\frac{80 B .6}{4 \cdot \pi \cdot 0.33}=194.99 \mathrm{~A}^{2} / \mathrm{d} \\
K=\frac{104.99}{14}=13.92 \mathrm{~A} / \mathrm{d}
\end{gathered}
$$

$$
\begin{aligned}
& \text { 5) } 0 w-228-1 \\
& W^{\prime}=\frac{4 T t u}{r^{2}}
\end{aligned}
$$

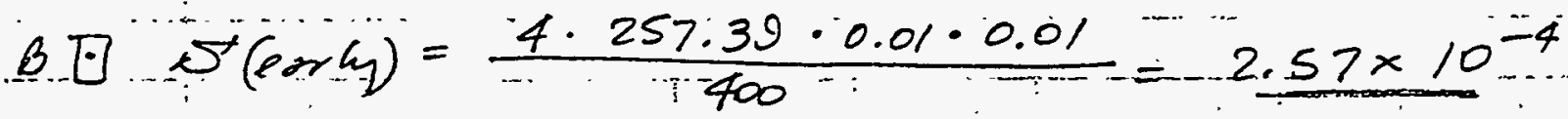

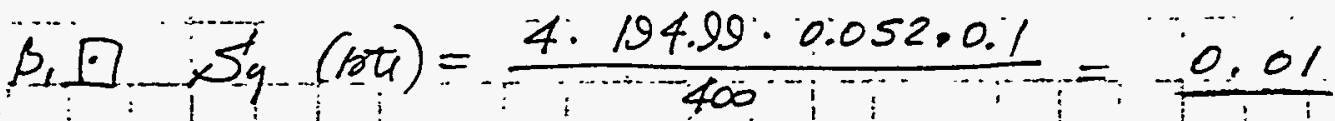

$$
\begin{aligned}
& 7=\frac{8+S_{4}}{S^{5}}>6.5=\frac{2.57 \times 10-4 \cdot 0.05}{2.57 \times 10^{4}}=39.9
\end{aligned}
$$

6) ow- $0,28-1$ Recoivery (Noinhtline)

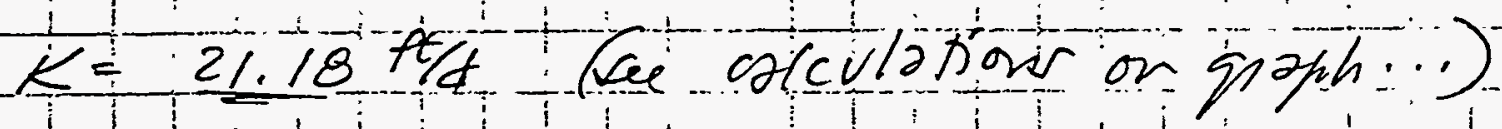


JACOBS ENGINEERING

$2 B$

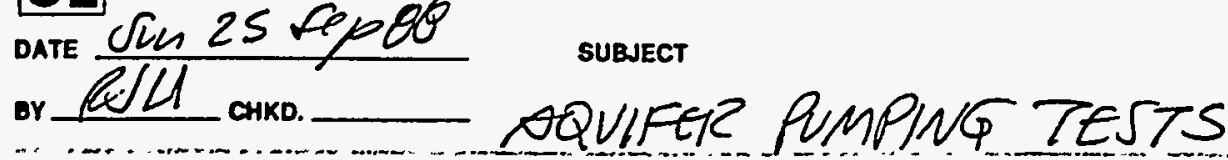

Sun 25 AgpoO

SHEET NO.

$M w-22 \theta$ (cont)

Los No.

त). $0 w-928+2$ founping (Oheis)

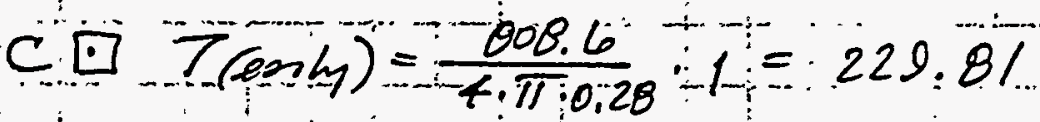

$$
\begin{aligned}
& K=\frac{229 \cdot 81-16}{14 \cdot 16.41} \mathrm{~A} / \mathrm{d} \\
& c_{1} \boxminus T \cdot(120)=\frac{808: 6}{4: \pi 70.2^{2}} \cdot 1=292.48 \mathrm{At}^{2} / 6
\end{aligned}
$$

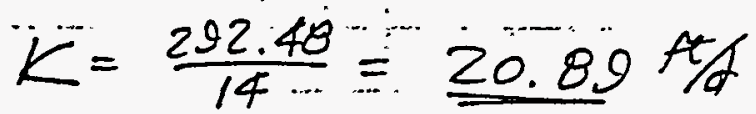

8) . ow $-92 B-2$

$$
\begin{aligned}
& \text { C四 S(6xim) }=\frac{4-229.81 \cdot 0.044 \cdot 0.1}{0.6561}=6.16 \times 10^{-4} L \\
& c_{1} \square \sigma_{y}^{\prime}(10.6)=\frac{4 \cdot 292.49 \cdot 0.20 \cdot 0.1}{6561}=0.004 \\
& \eta=\frac{5+5 y}{5.5} \geq 6.5=7.49
\end{aligned}
$$

SUNMAR $=\underline{K}$

Mu-928 Reconen (ruein) $12.10 \mathrm{Ft} / \mathrm{A}$

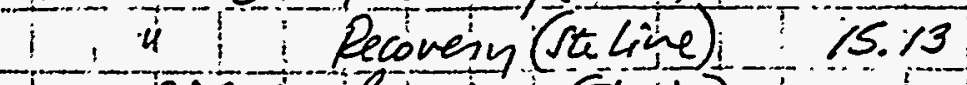

low-928-1 Rimping (Geniv) 16.15

$$
\text { Reconeng (retiane) } 21.18
$$

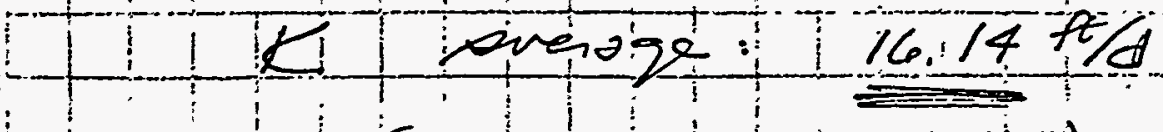

(eonge $=12-21$ ftdd) 


\section{IF LACOBS ENGINEERING GROUP INC. \\ EE' ADVANCED SYSTEMS DIVISION, ALBUEUERQUE OPERATIONS}

\section{CALCULATION COVER SHEET}

CALC Nọ. 07-756-01-00 DISCIPLINE Hydrology NO. OF SHEETS 24 PROJECT: Sit Choracteizization

SITE: spook, wyoming

FEATURE: Hydroulic Conductivity

SOURCES OF DATA:

Oguifen pumping tests

SOURCES OF FORMULAE \& REFERENCES:

Krusemon, G.P., and De Ridder, N.A., 1970. Onohyis ant Evalustion

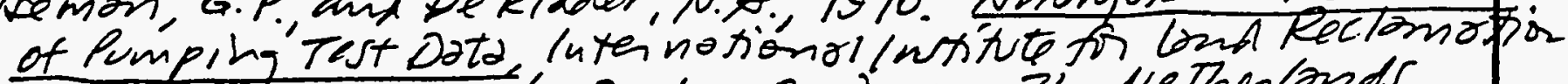
and Tmporement/ILRI, wa genihgen, the Netherlonds.

lohmon, S. $w_{-1}$ 1972, Grountuste Hydinlics, USG5

Profestiond fixen 700 .

Depntment of Intenion, surpor of Reclomoxion, 1977. Ground wath Monvil, A wath Peorras Technicol Publicstion.

PRELIMINARY CALC. $\square$ FINAL CALC. $\$$ SUPERSEDES CALC. NO.

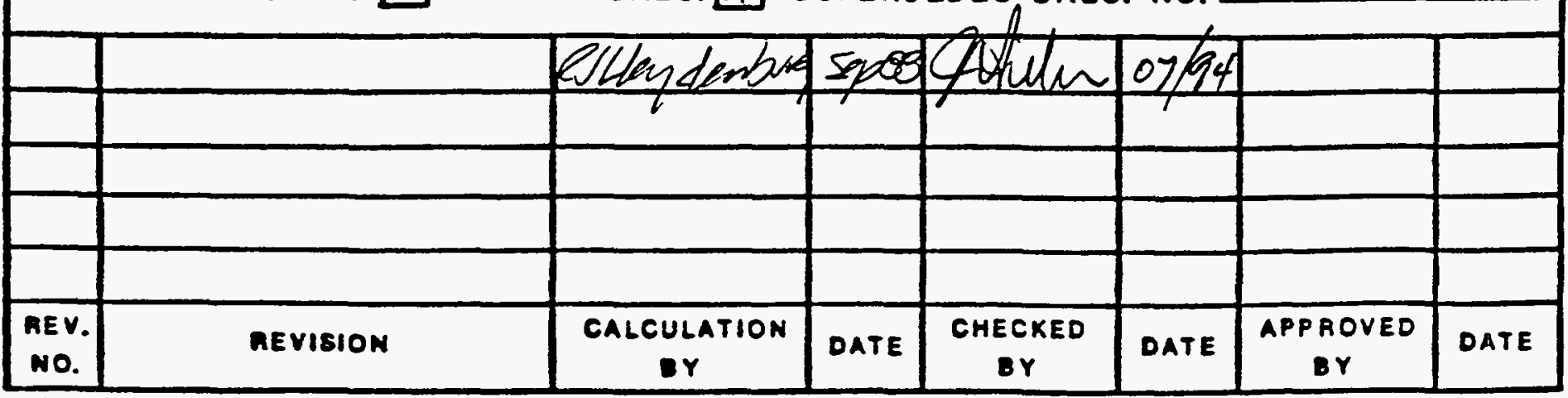


STK- AQUIFER RUMPING TESTS

A vimminy cheet will be incented utsing:

A. Purpace of calculation:

B. Methoots a fhocedures:

c. Assumptions:

D. nathispropaties:

E. Das Gurcer:

F. Calculations a sonahoder:

G. Rerutts: 


\section{UNITED STATES DEPARTMENT OF THE INTERIOR}

OEOLOGICAL SURVEY

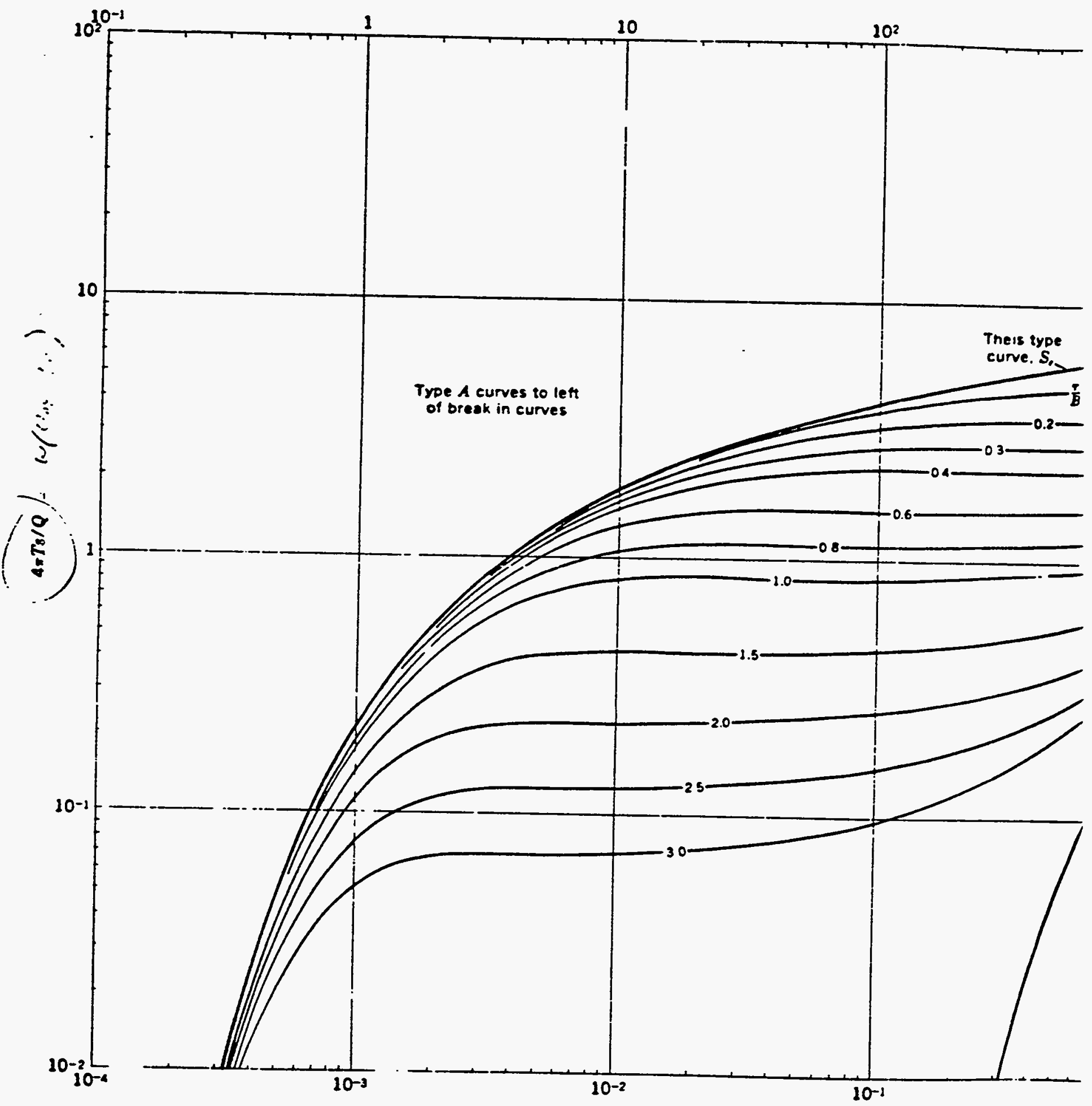


PROFESSIONAL PAPER 708

$n t / \tau^{2} S$ : $\frac{1}{\vdots}$

PLATE 8

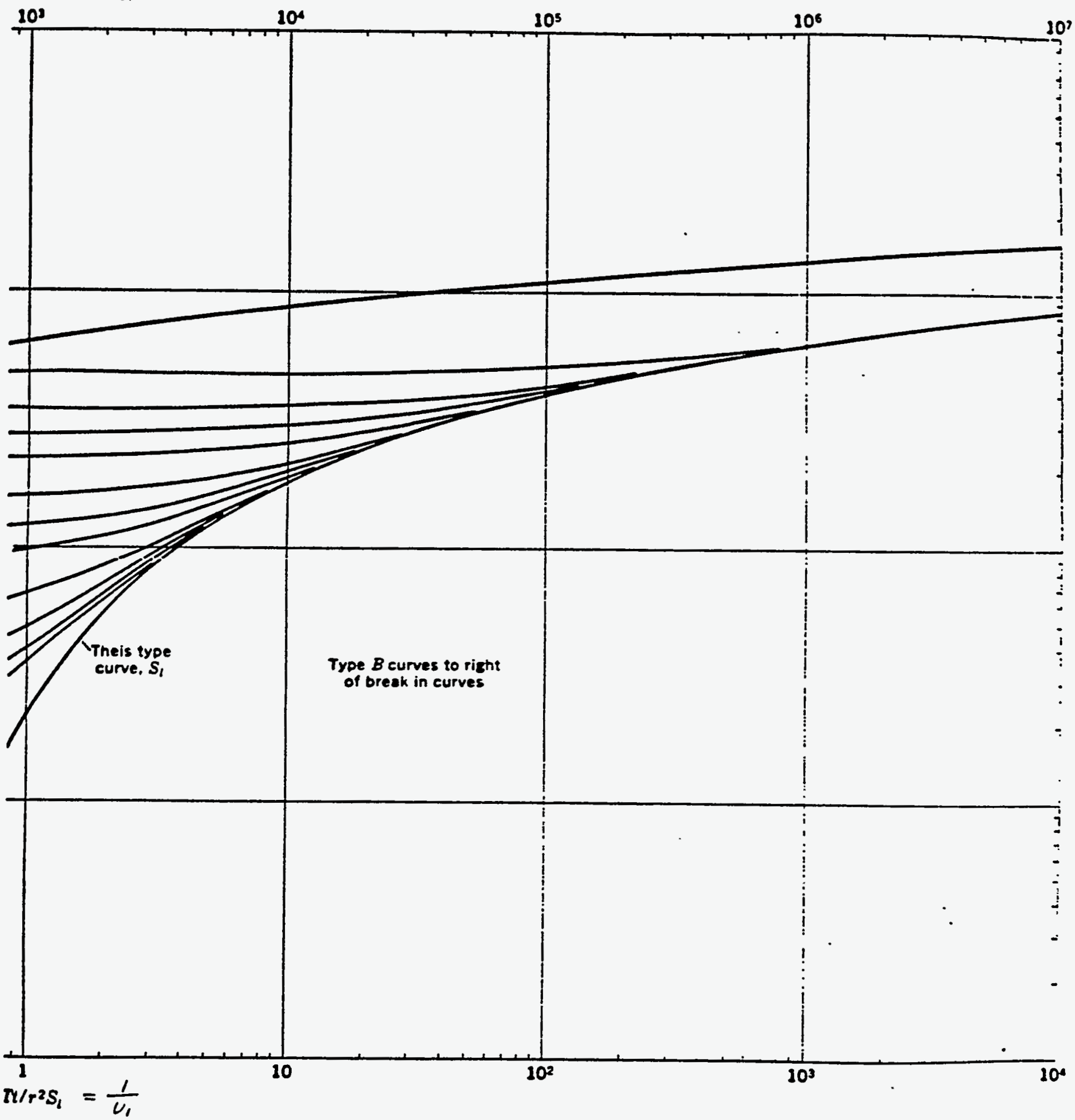

\section{TYPE CURVES}

$\operatorname{con}(2953$, fis. 1$)$ 
TE. JACOBS ENGINEERING BOOOK, w4

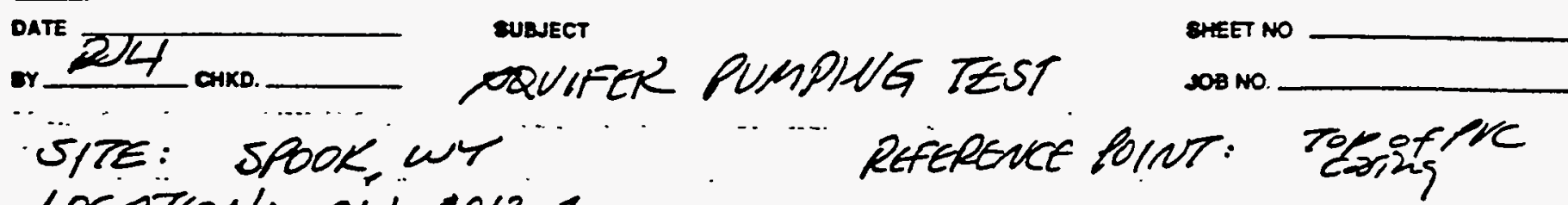
LOCOTION: OW"-1013-1

REFERENE POINT: Topofle
weZl $1: r=18.15^{\circ}$ s30w

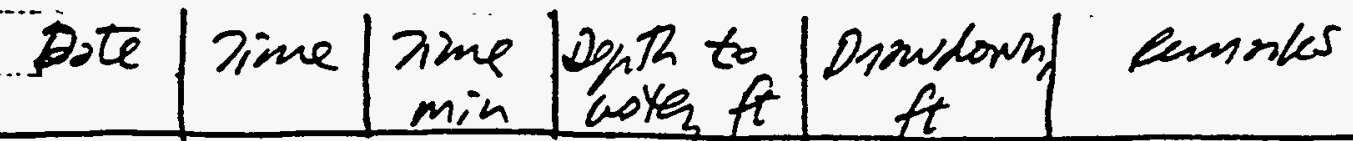

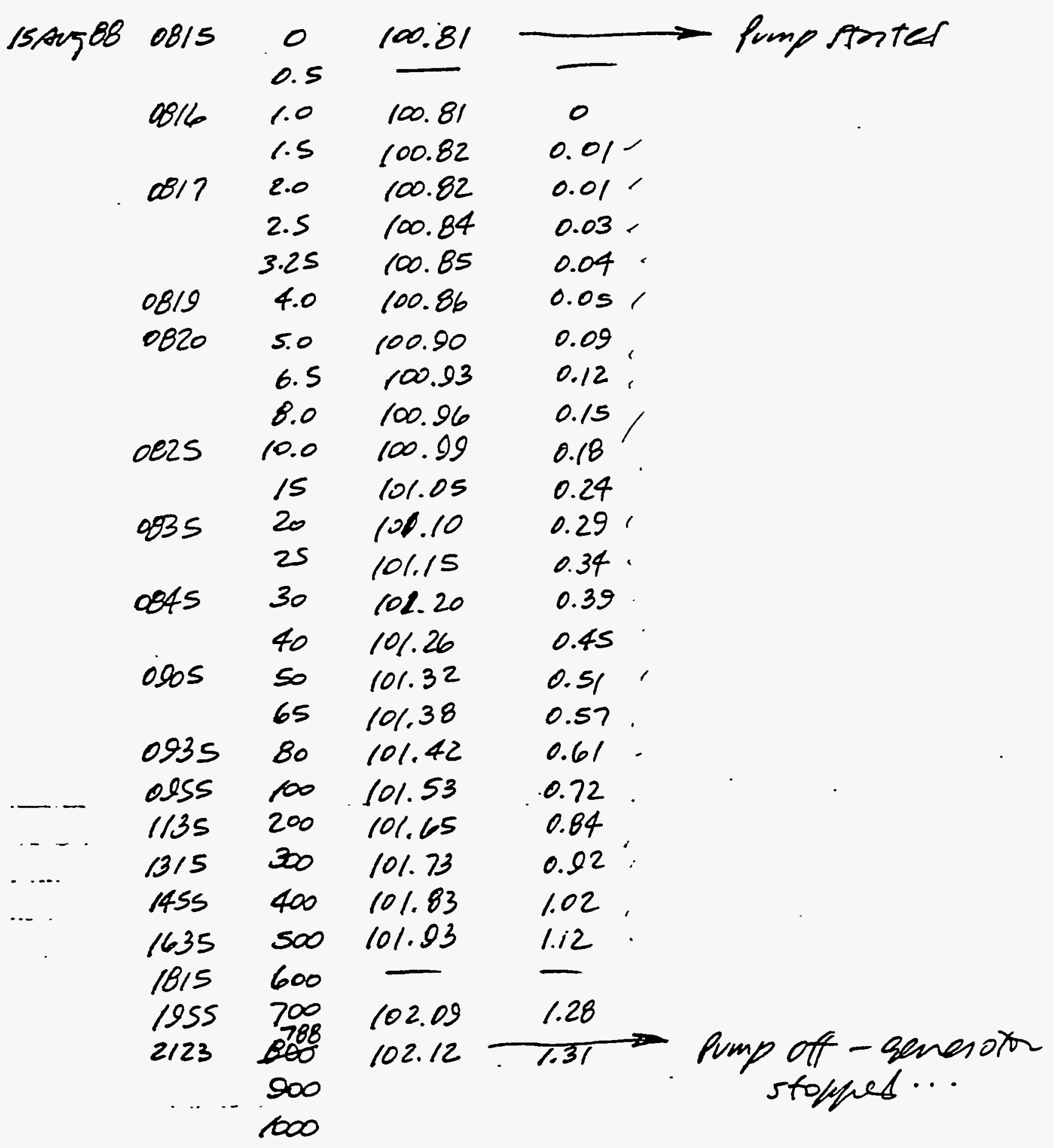

ropun 91.005 .1 14:81) 
IF JACOBS ENGINEERING SPOOK, wY

DATE

NUNECT

AQUIFER PUMPING TEST
SHEET NO.

$\cos 10$

SITE: SPDOK wi

DISCUARGE MEANURED BY: CXIbIOTES

LOCATION: MNU-9/3

DROUTH MEATRED BH: Elucket

PUMP-TEST WEL REFERNEE BOINT: resticiler roposfing

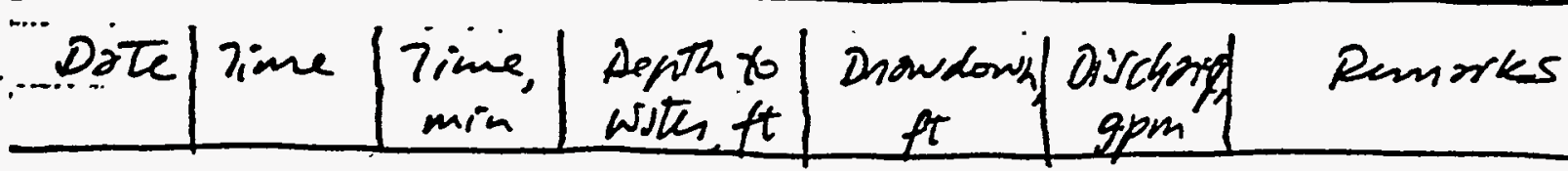

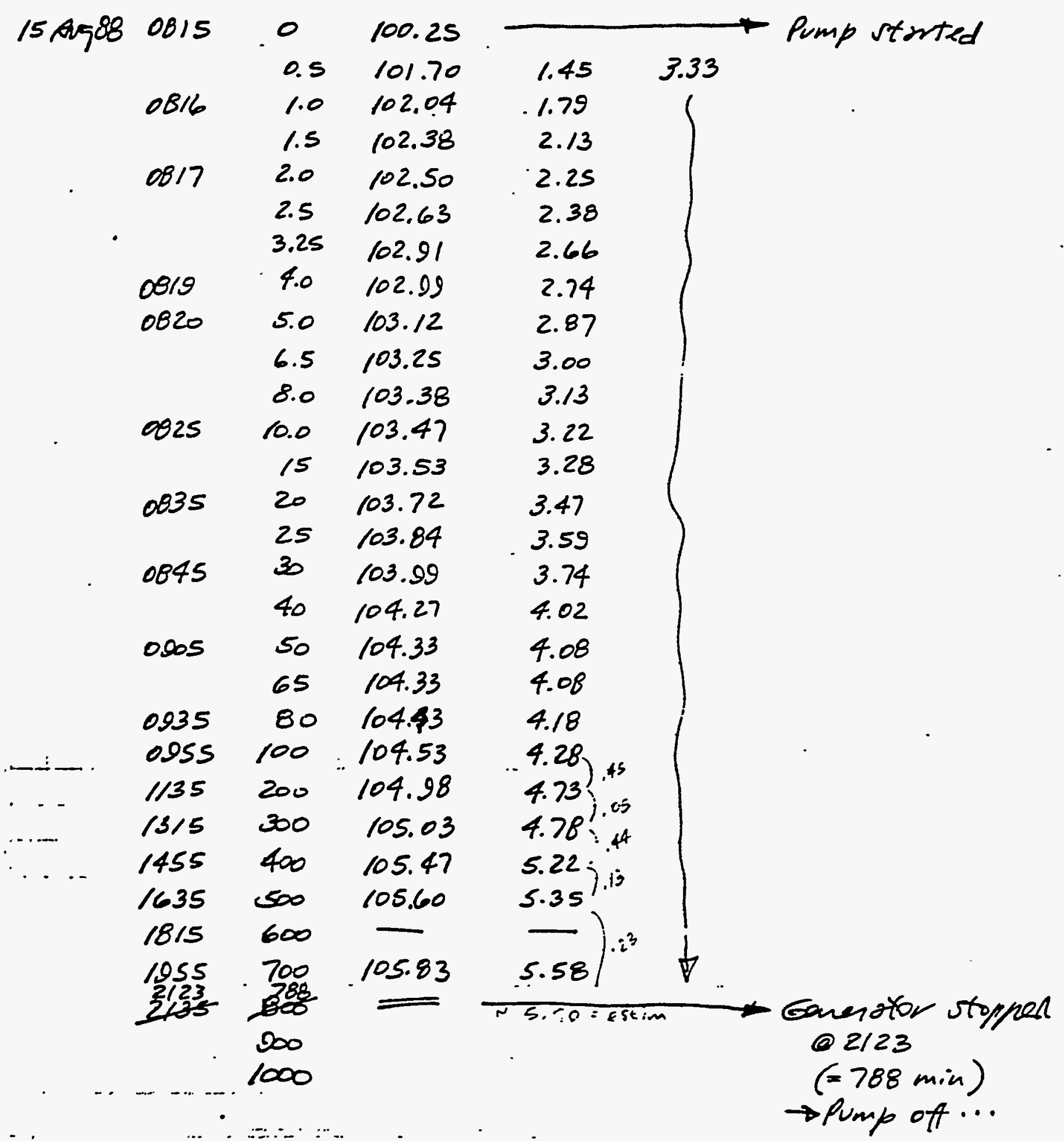




\section{JE JACOBS ENGINEERING UPBOK, w4}

DATE

BHEET NO.

Los No.

SITE: SPOOK, WY

LOCATION: MW-913

I PECOVER -TEST WEZ

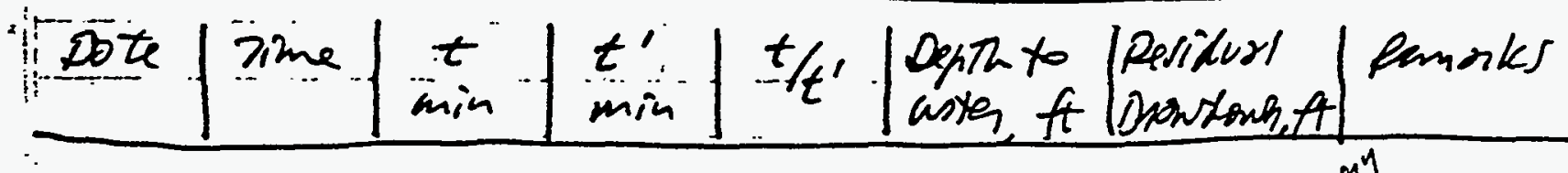

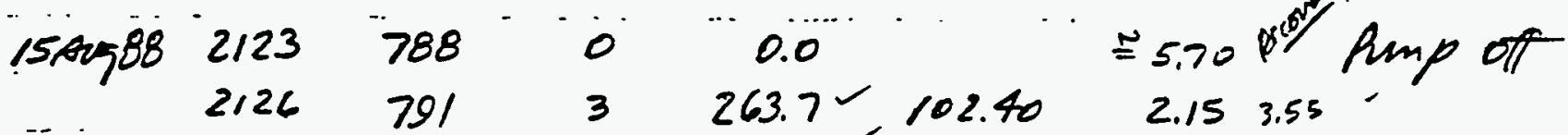

$2127 \quad 792 \quad 4$

$\begin{array}{lll}2128 & 793 & 5\end{array}$

$\begin{array}{llll}2129 & 794 \quad 6 & 132.3-101.88\end{array}$

$2130795 ? 7.113 .6 \div 101.80$

$2131 \quad 796 \quad 8 \quad 99.5^{-101.75}$

$2133 \cdot 798 \cdot 10$

$2138 \quad 803 \quad 15$

$2143 \quad 808 \quad 20$

$2148 \quad 813 \quad 25$

$2153 \quad 818 \quad 30$

$79.8=101.68$

1.023 .78 -

$198.0 \div 102.17$

1.753 .55

$2203 \quad 828 \quad 40$

$53.5^{\prime} 101.60$

$40.4 \sqrt{3} 101.53$

$32.5 \times 101.47$

1.63407,

$1.55 \quad 4.15$

$1.50 \quad 4.20$

$2213 \quad 838 \quad 50$

$27.3^{\circ} 101.43$

$1.43 \quad 4.27$

$2228 \quad 853 \quad 65$

$20.7-101.37$

$16.8^{\prime} \quad 101.32$

$2243 \quad 868 \quad 80$

13.1

10.9

$2303 \quad 888 \quad 100$

8.9

$\begin{array}{lll}2330 & 915 \quad 127\end{array}$

$7.2 \sim 101.08$

$1.35 \quad 4.35$

1.28 a d

$1.22 \quad 44.3$

$1.18 \quad 4.52$

$1.12+15$

$1.07 \quad 463$

$\begin{array}{lll}101.24 & 0.99 & 4.11 ; \\ 101.18 & 0.93 & 4.1\end{array}$

0.864 .32$,

0.834 .25 


\section{JE JACOBS ENGINEERING SPOOK, wY}

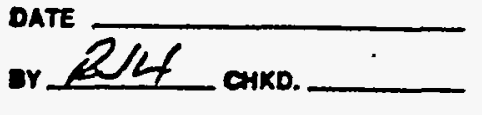

SUDECT

SITE: SPOK Ü

AQUIFCR PUMPINE TEST

LOCATION: OW-9/3-1

RECONERY - OSNERVITION NEL

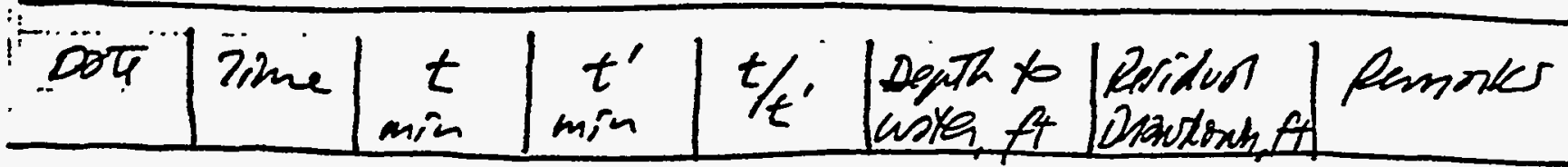

$\begin{array}{ccccccc}15,02988 & 2123 & 788 & 0 & 0.07 & 102.12 & 1.31 \\ 2124 & 789 & 1 & 789.0 & 102.10 & 1.29 & 0.02-\end{array}$

Pump of $790.5^{-} \quad 2.5 \quad 316.2^{\prime}, 102.70$

$2128793 \ldots .5 \quad . .159 .6$ \% 102.03

$\begin{array}{lllll}794.5 & 6.5 & 122.2^{\prime} & 102.00\end{array}$

$2131796 \quad B \quad 99.5-101.96$

$2133 \quad 798 \quad 10 \quad 79.8-101.93$

$2 / 38 \quad 803 \quad 15$

$53.5-101.88$

1.29 c.02r

$2143 \quad B 08 \quad 20$

40.4 10/.83

$1.220 .09 ?$

$2148 \quad B / 3 \quad 25$

$32.5,10 \% .79$

$1.190 .12^{2}$

$1.150 .16-$

$1.128 .19^{2}$

$B 18 \quad 30$

27.3 .101 .76

1.030 .24

$2203 \quad 828$

40

20.7

$1.020 .2^{2}$

$2213 \quad 838 \quad 50$

$16.8^{\prime} 101.67$

$2228 \quad B 53 \quad 65$

$13.1 \quad 101.63$

$0.981 .33^{\circ}$

2243

868

80

10.9

$2303 \quad 888 \quad 100$

101.59

0.950 .36

2330

$9 / 5$

127

$8.9^{\circ} \quad 101.56$

$0.890 .4^{2}$

$0.86 \mathrm{o.t}^{\mathrm{b}}$

0.820 .48

0.780 .53

$7.2^{\prime} \quad 101.49$

$0.750 .5 \%$

0.68 u. 63 
JACOBS ENGINEERING

uroot, wh

DATE $\sqrt{\text { Un }} 25$ appos

ऽUENECT

SHEET NO

or ell aro.

oqUIFER fUMPING TESTS

Nos no.

3) Mü-213 Tecovery (thaightline)

$K=10.18$ Ald (Coe colculations on graph...)

4) ow $-913-1$. Lumping (Sheis)

$$
\begin{aligned}
& \text { B日 } T=\frac{641}{7 \cdot \pi \cdot 0.24} \cdot i=212.54 \mathrm{At} / \mathrm{d} \\
& K=\frac{212.54}{15}=14.11^{5} \mathrm{~A} / \mathrm{d}
\end{aligned}
$$

5) ow $-9 / 3-1$

$$
\begin{aligned}
\sigma^{\prime} & =\frac{4 T t u}{r^{2}} \\
\sigma^{2} & =\frac{4 \cdot 212.54 \cdot 0.025 \cdot 0.1}{331}=6.4 \times 10^{-3}{ }^{-} \text {(ear/y) }
\end{aligned}
$$

(as indicdtion of deloned yield phenomenon An $\tilde{x}_{1}^{\phi_{1}}(10 t)$.

6) ow-9/3-1 fecovern (Their)

$$
\begin{aligned}
& 7=\frac{.64}{4 \cdot \pi \cdot 0.10} \cdot 1=203.3-36 \mathrm{At}^{2} / 4 \\
& K=\frac{203.38}{15}=10.89 \mathrm{~A} / \mathrm{d} \mathrm{V}
\end{aligned}
$$

2) ow-913-1 fecovan (thorghtline)

$K=19.59^{\circ}$ A/d (bee calculotions on ppoph...) 


\section{尺}

:

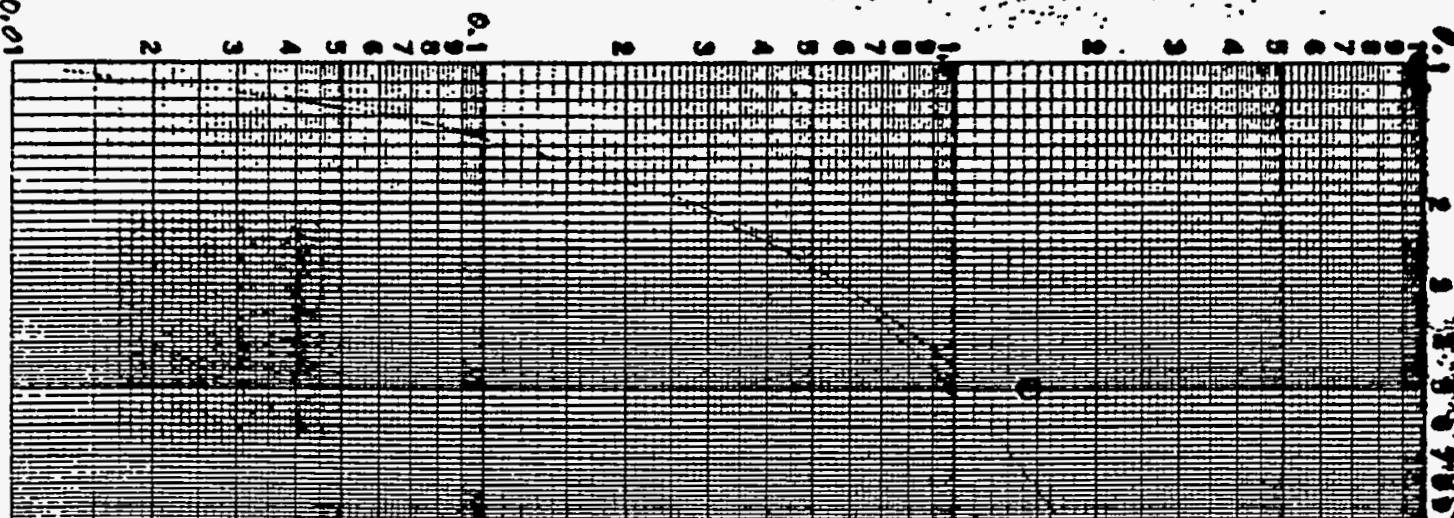

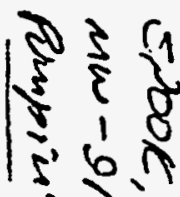

$\rightarrow$ L

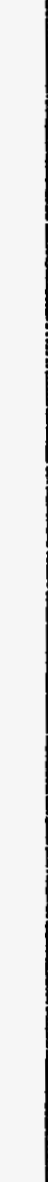

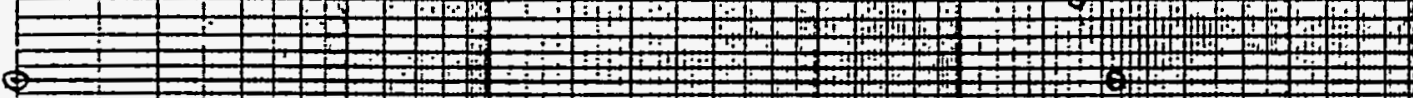

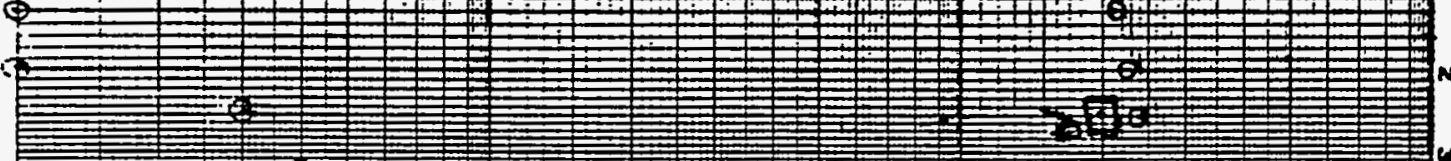

है

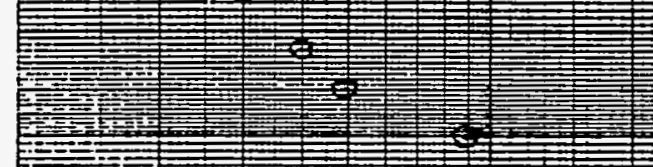

$\stackrel{3}{3}$

2
5
5
5
5
5

ฉู

i

\section{展}




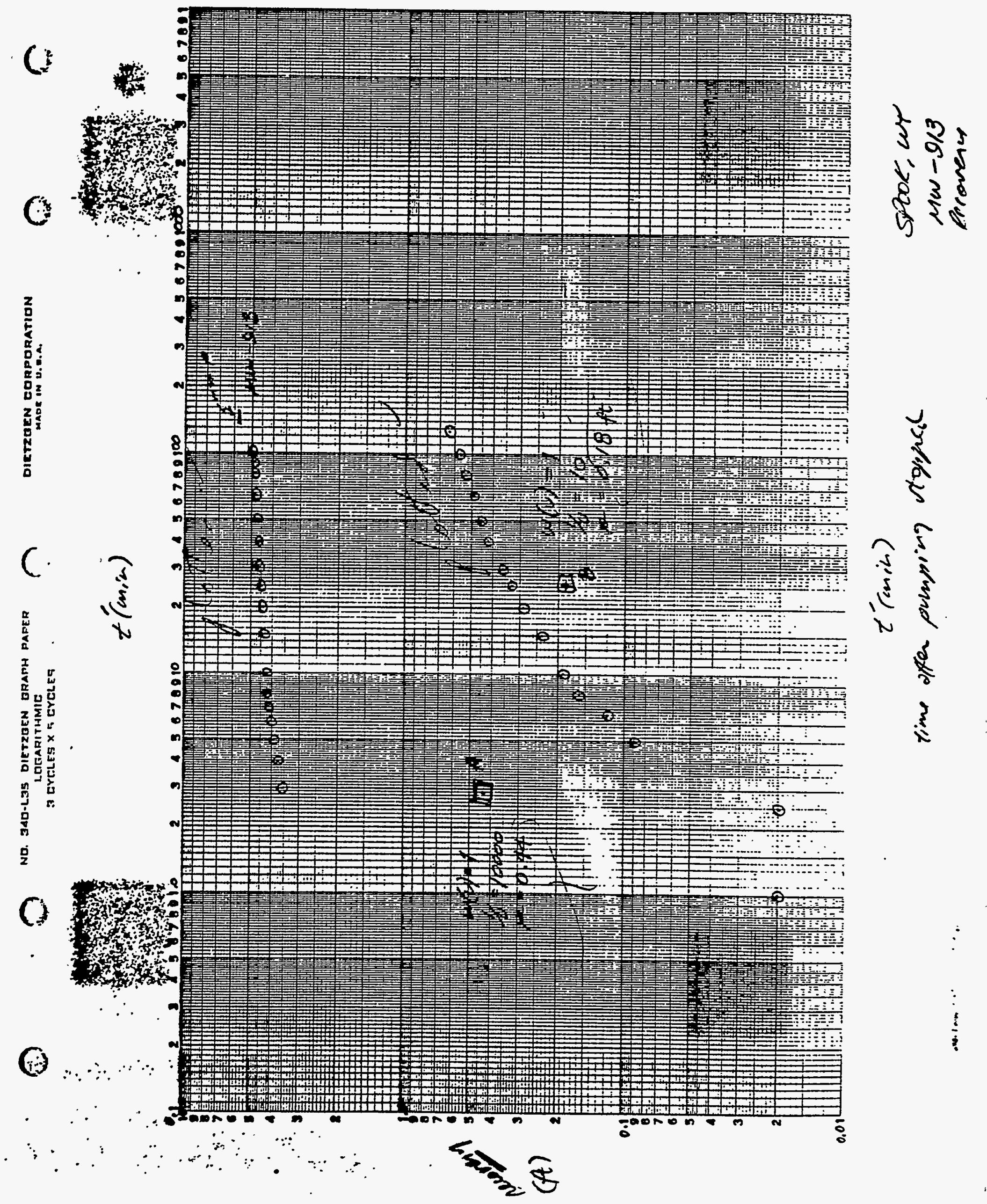


JE JACOBS ENGINEERING

SPOOE, WY

$5 / 3-8$

Date $\sin 25$ tepos

or EHA caxo.

OQUIFER PUMPING TESTS

SHEET NO.

SOB NO.

MN-913

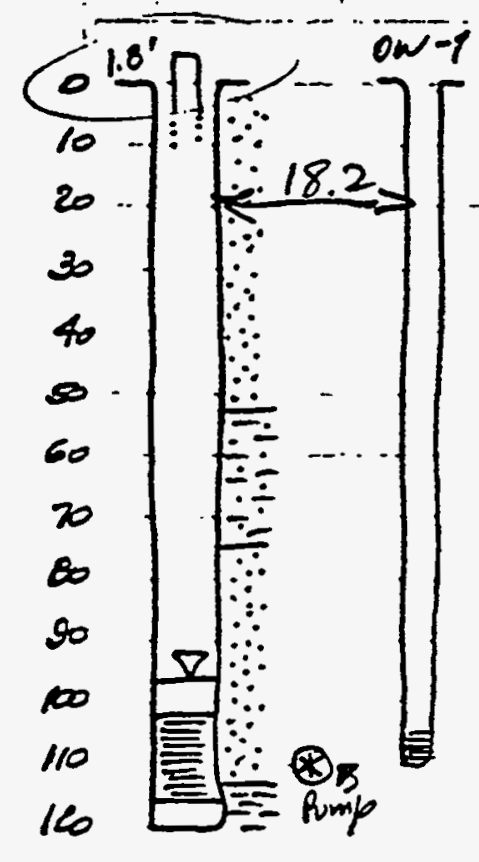

$$
\begin{aligned}
Q & =3.33 \mathrm{gpm} \times 1440 \mathrm{~m} / \mathrm{d}=4795 \mathrm{gpd} \times 1.337 \times 10^{-1}= \\
& =641 \mathrm{ft} / \mathrm{s} \\
r_{1} & =18.2 \mathrm{ft} ; \quad r_{1}^{2}=331 \mathrm{ft}^{2} \\
b & =15 \mathrm{ft}
\end{aligned}
$$

Agnifer un confined

1) Mw-913 Pmping (The,15)

$$
T=\frac{Q}{4 \cdot \pi \cdot p} \cdot w(v) \quad K=\geqslant
$$

$A Q \cdot T($ enty $)=\frac{641}{4 \cdot \pi \cdot 2 \cdot 0} \cdot 1=25.50 A^{2} / d$

$$
K=\frac{25.50}{15}=1.70 \mathrm{ft} / \mathrm{d}
$$

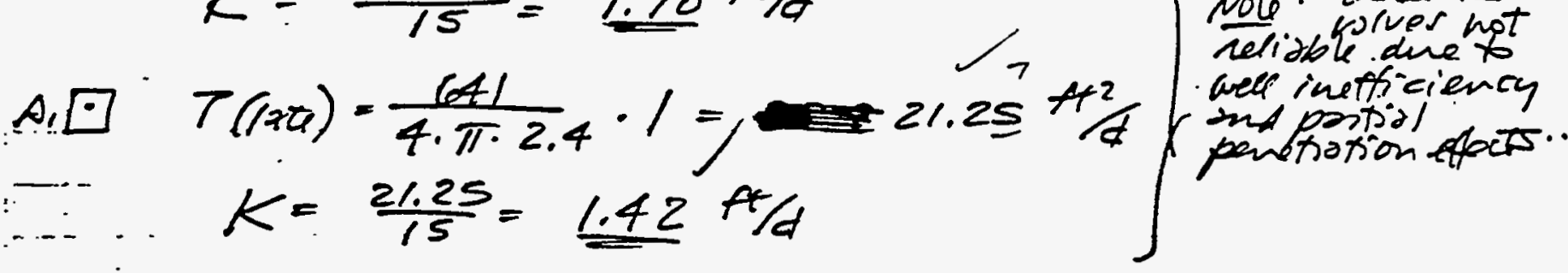

2) Mw-913 Recovery (Theis)

$\therefore 5=6$

A $\sigma^{\prime} T=\frac{641}{4 \cdot \pi \cdot 0.44} \cdot 1=115.93 \mathrm{At}^{2} / \mathrm{d}$

$$
K=\frac{115.93 \mathrm{~A}^{3 / 4}}{75 \pi}=7.73^{2} \mathrm{ft} / \mathrm{A}
$$

corm 91.005 .1 (a/ 181$)=$ 
WE JACOBS ENGINEERING

DATE $\frac{\Omega_{4} 25 \text { SOp O8 }}{\text { RU }}$

SUET

"PUL Ono. SQUIFER PUMPING TESTS.

SHEET NO

sos no.

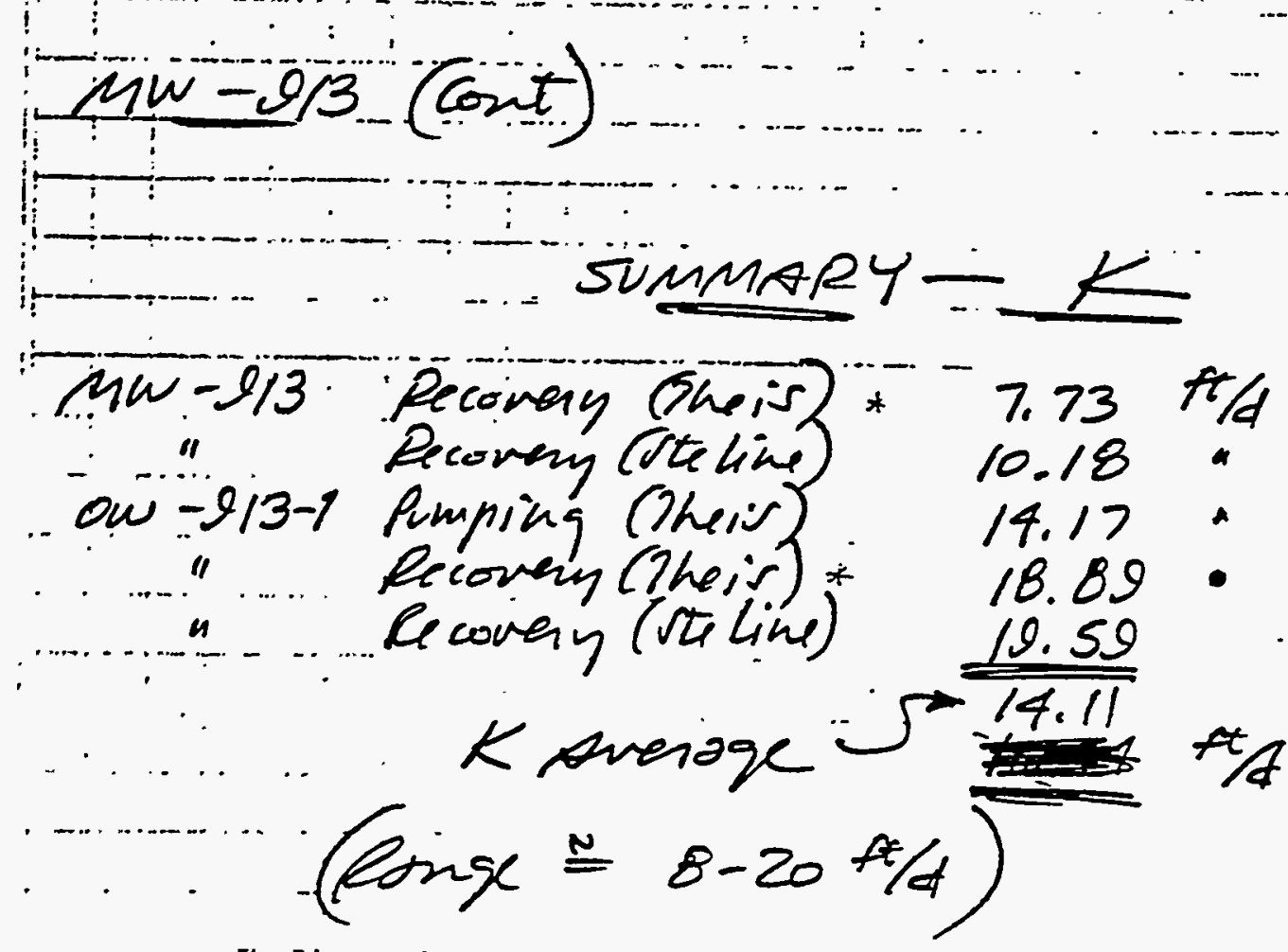

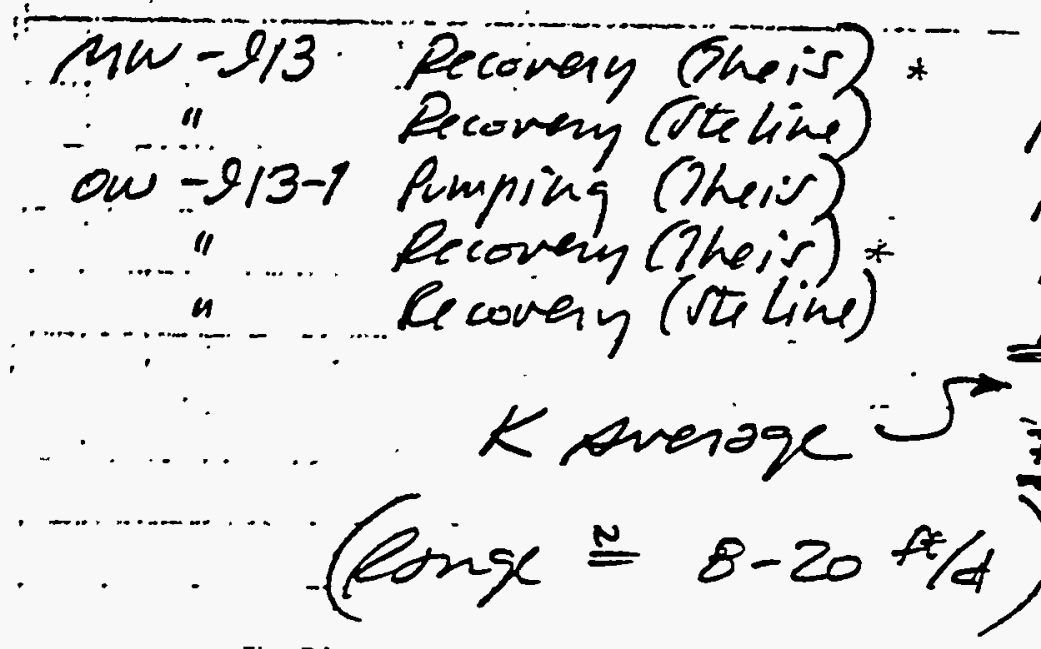




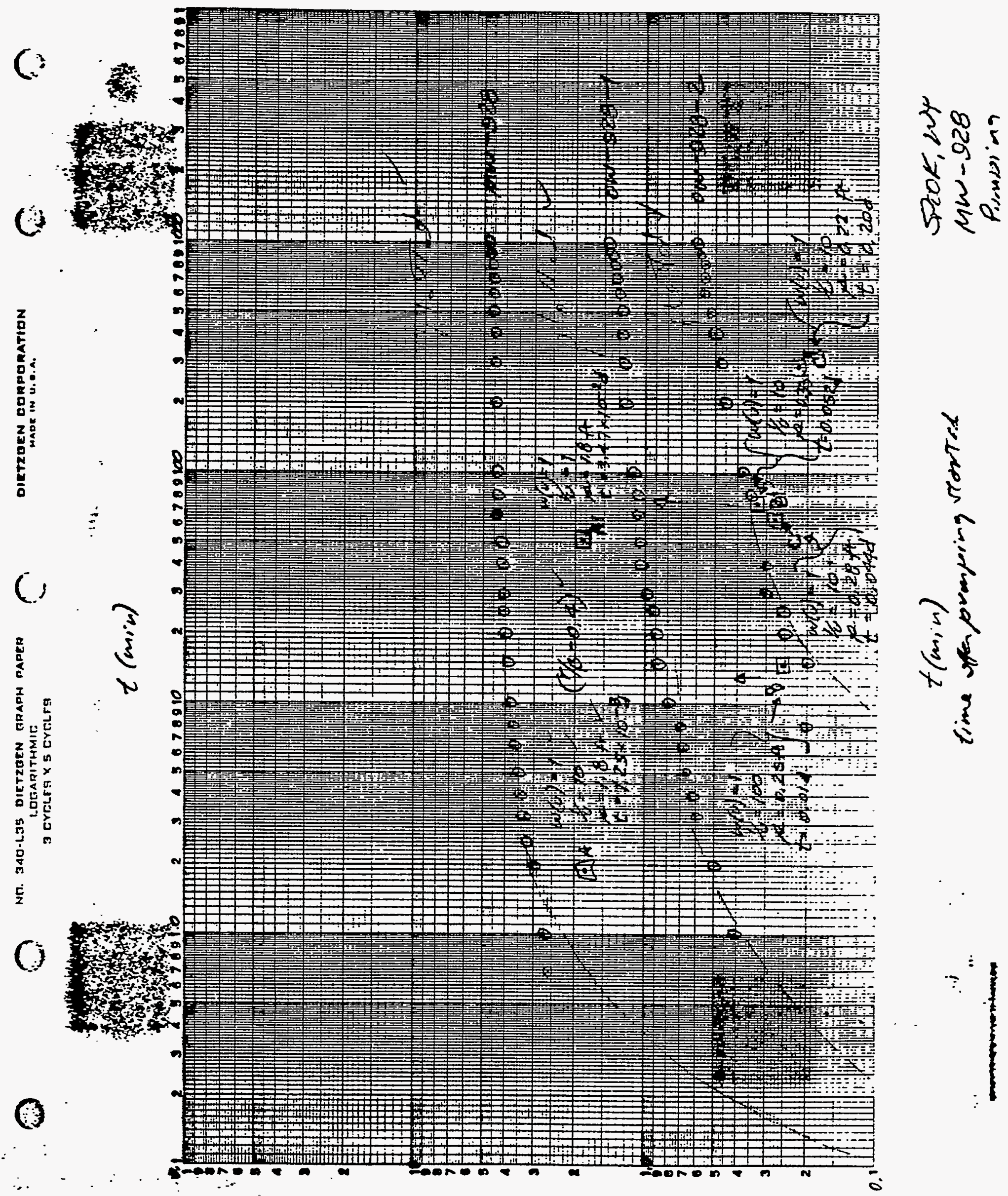




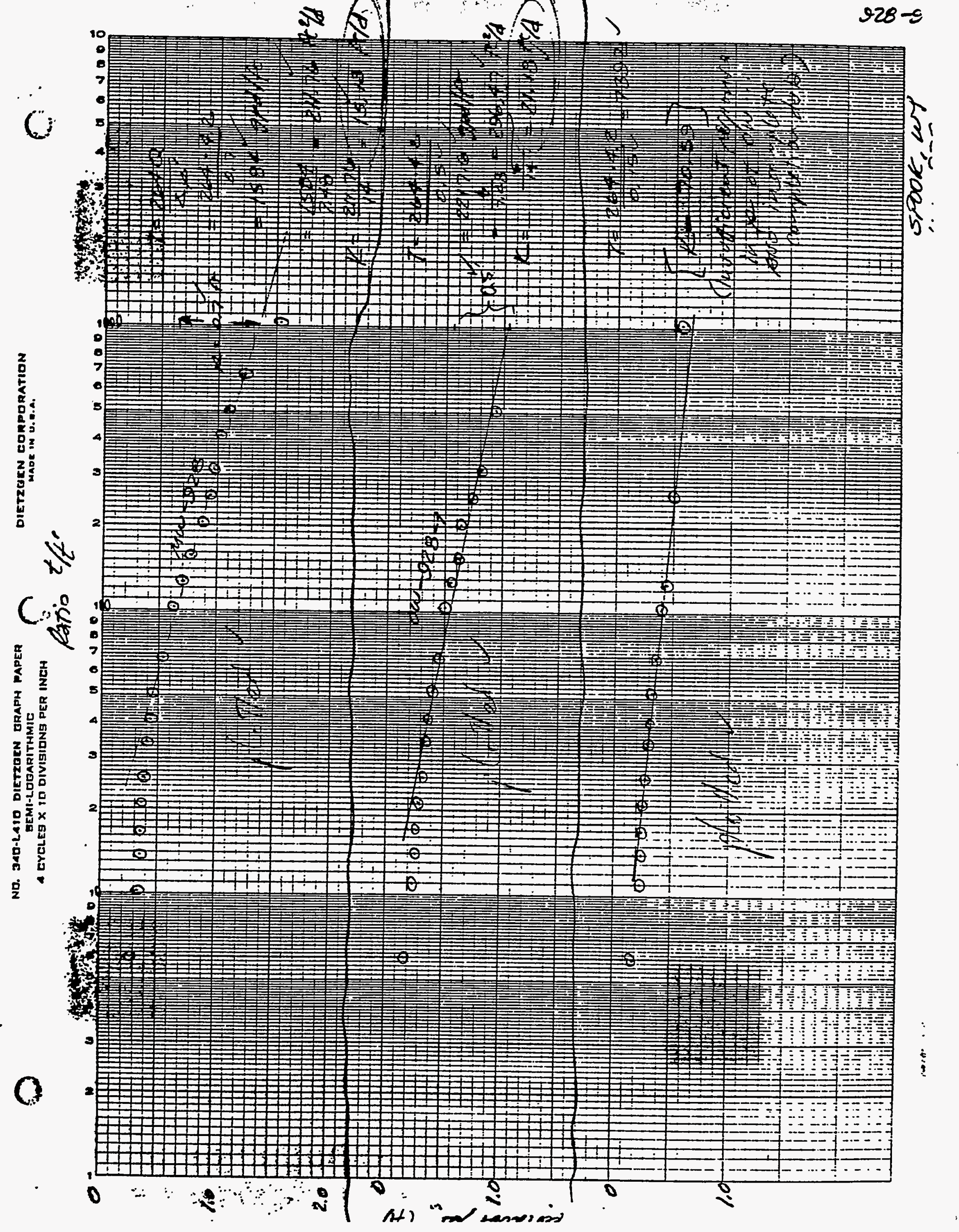


DATE

JACOBS ENGINEERING

se00k, wy

or $2 / 4$ ark.

SUsPECT

SHEET NO.

$\cos n$

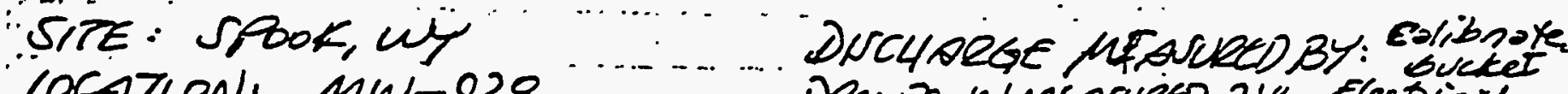

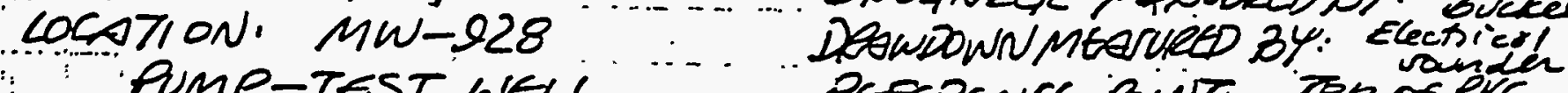

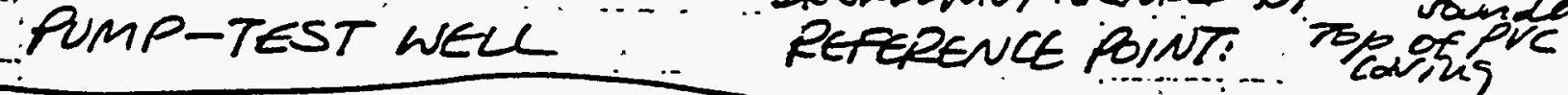

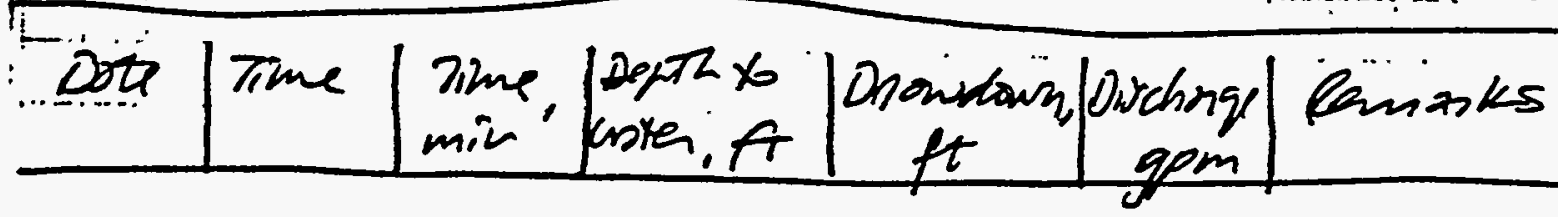

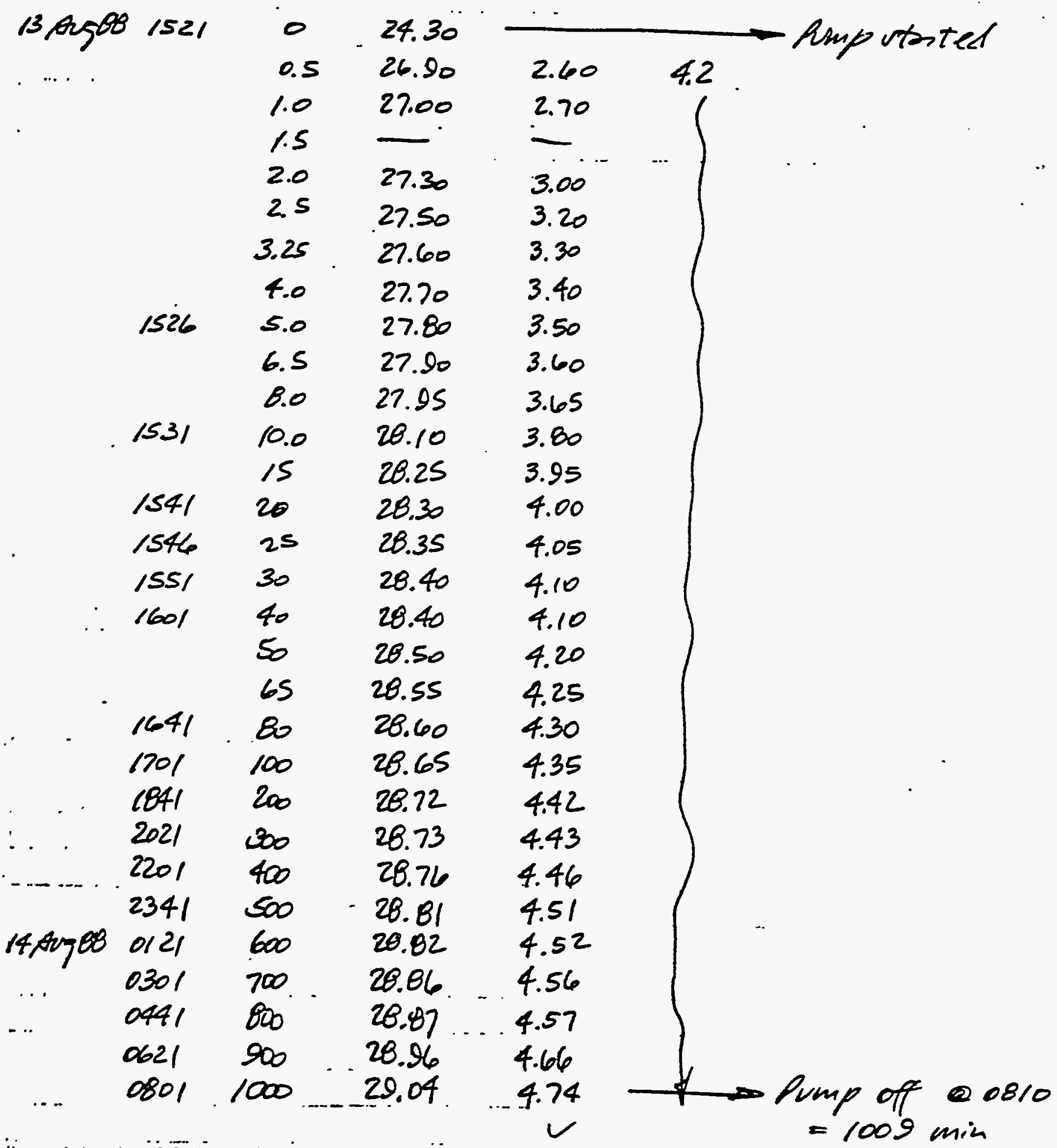


JE JACOBS ENGINEERING SPOOK, WY

$y<0-<$

DATE

PUDECT

SHEET NO.

or Q/4 CHKo.

OQUIFER PUMPING TEST

sor no.

Site: SPook, wil

LOCATION: MW-928

RECOVERY-TEST ÜLU

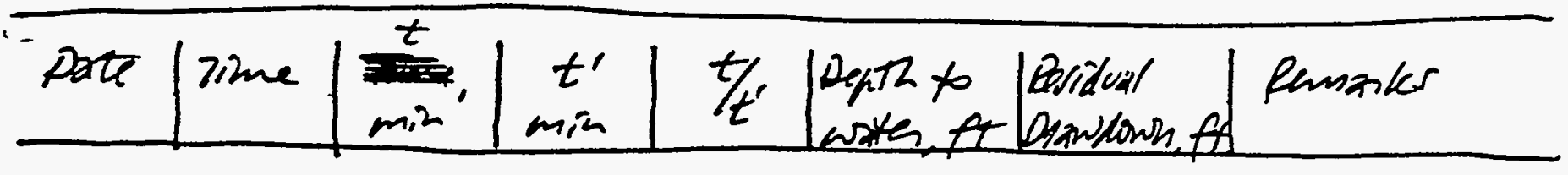

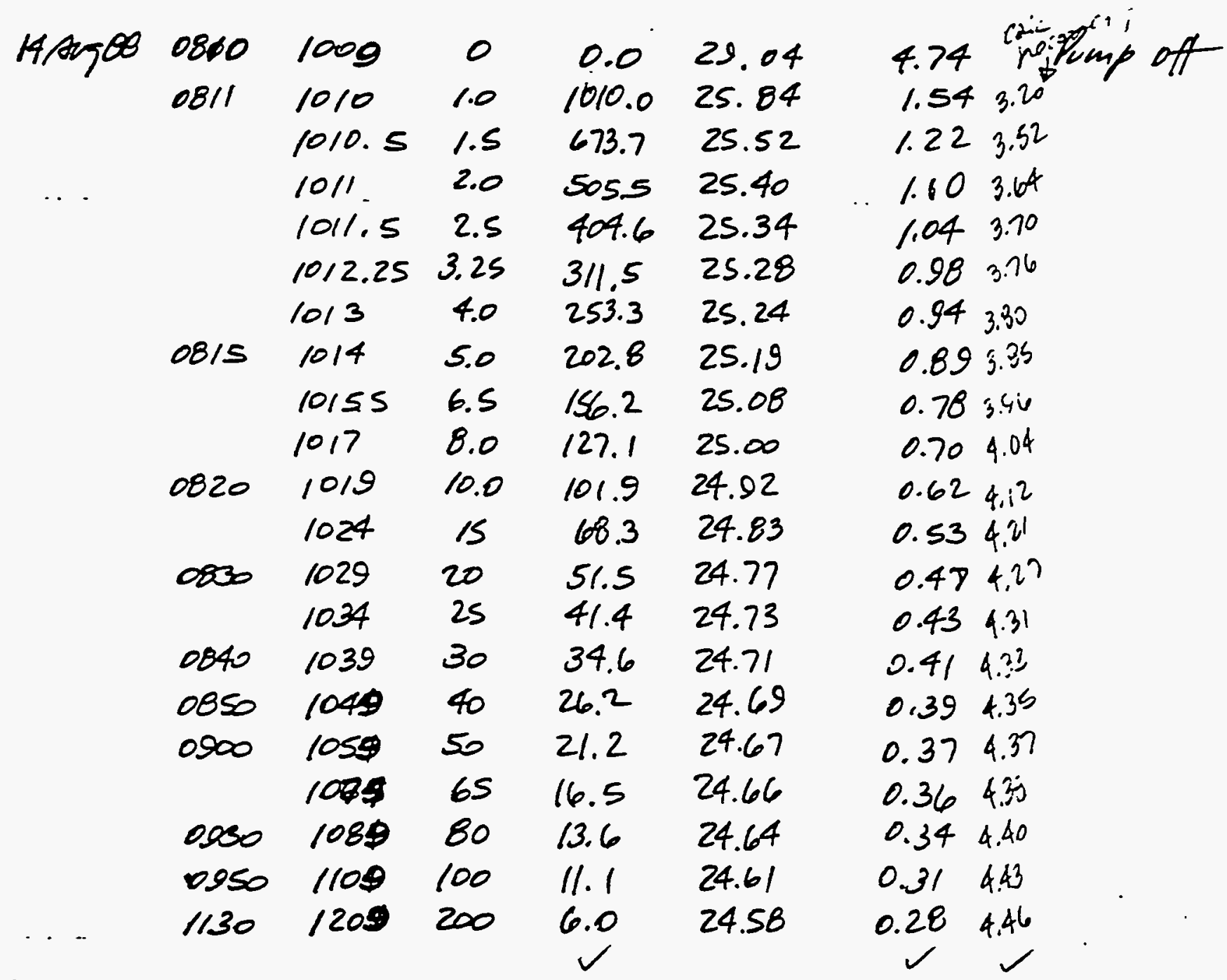

$\cos 91.005 .1$ (4/81) 
JE JACOBS ENGINEERING SPOOK, aY

DATE

SULEET

or $2 L 1$ arko.

SITE: seook ay

PRVIFER PUMPINGTEST

208 no.

LOCOTION: ow -928-1

REFERENCE foint: Topof pro

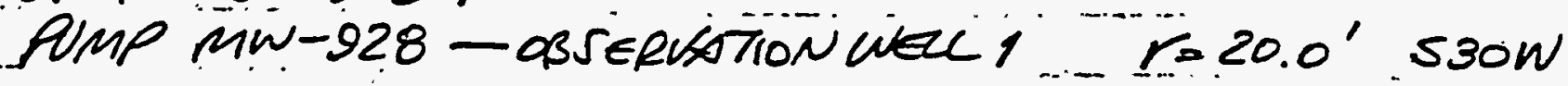

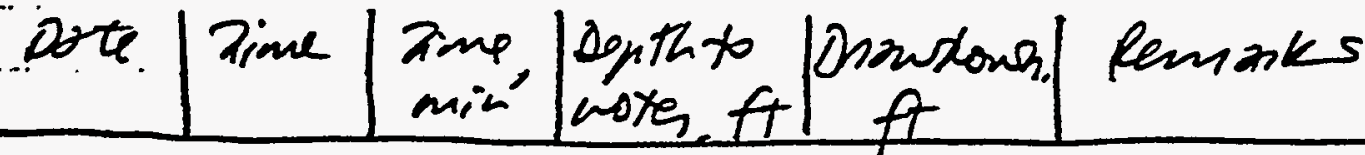

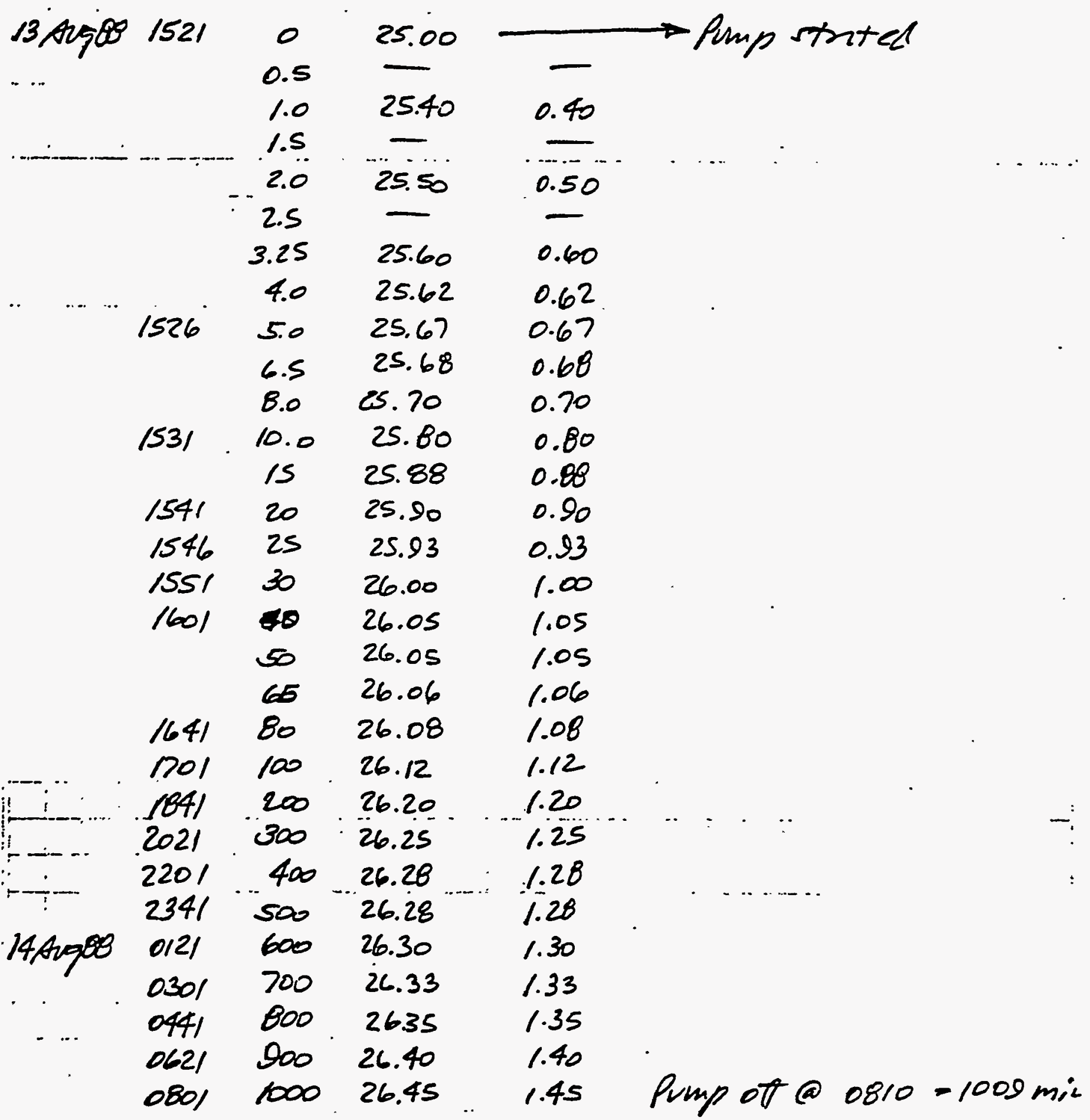

$\operatorname{ronu} 81 \cdot 00 \cos 1(4 / 8)$ 


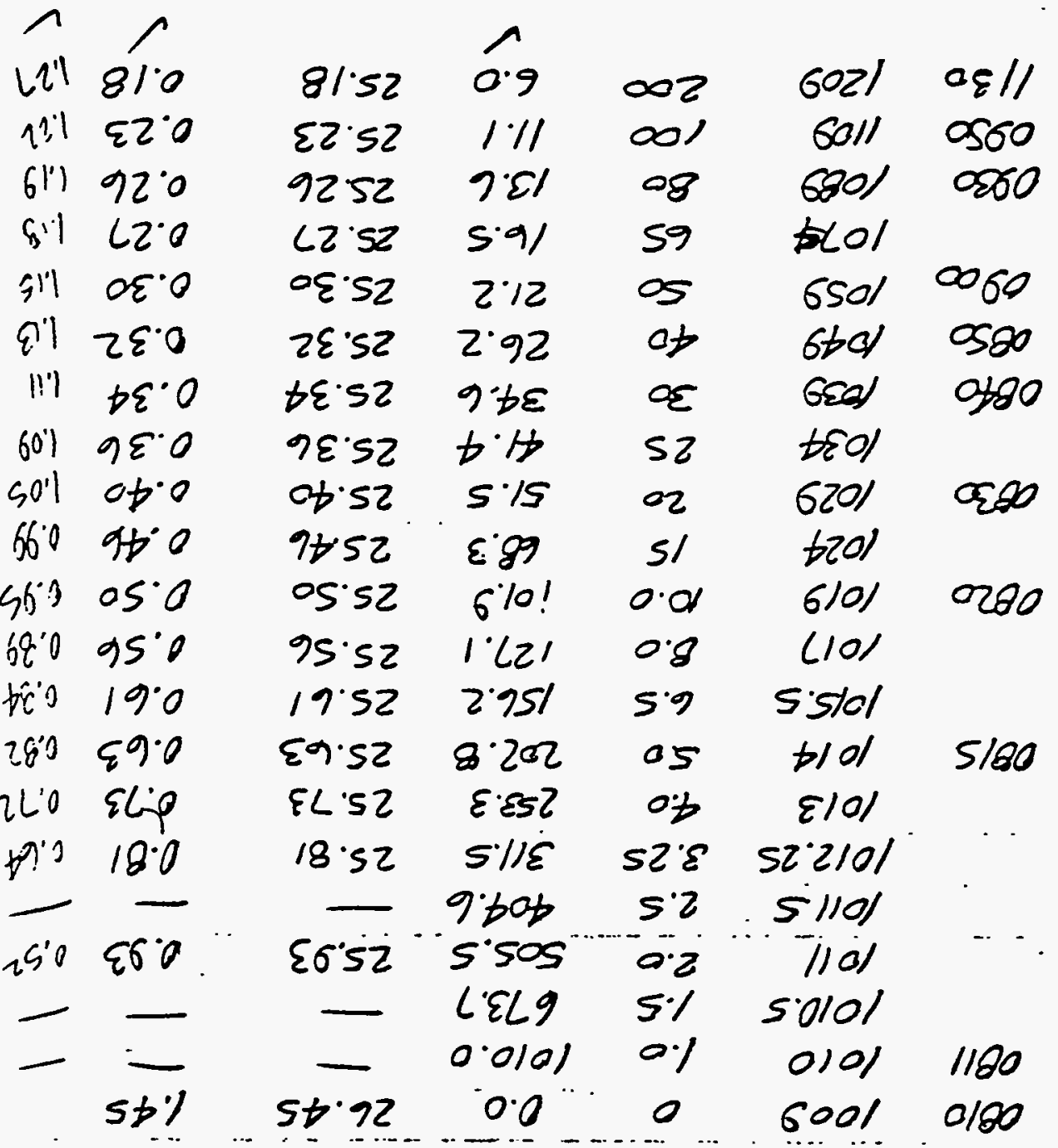

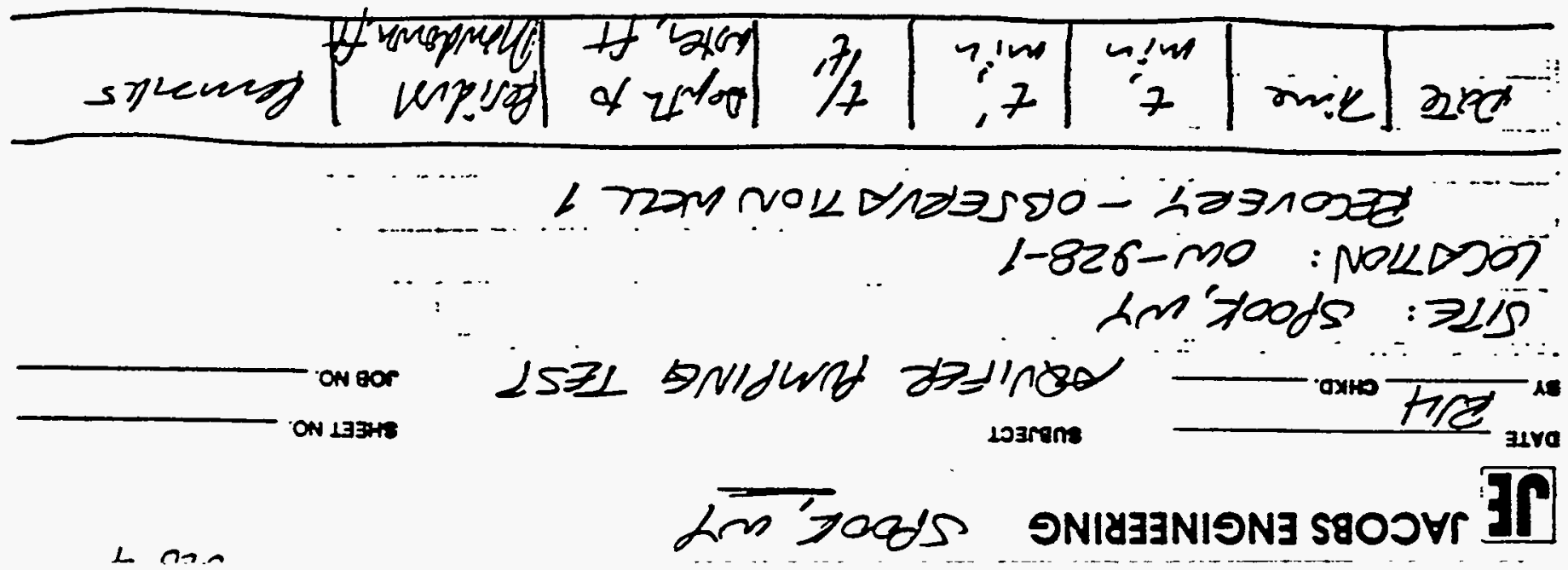


oATE

JACOBS ENGINEERING

NUBJECT

QHEET NO.

SITE: SPOOK, WT

$\infty 03$ n.

Location: ow-928-2

PUMP. MW-928 = 06UECVATION wELL $2 \quad r=81.0^{\prime}$ NGOW

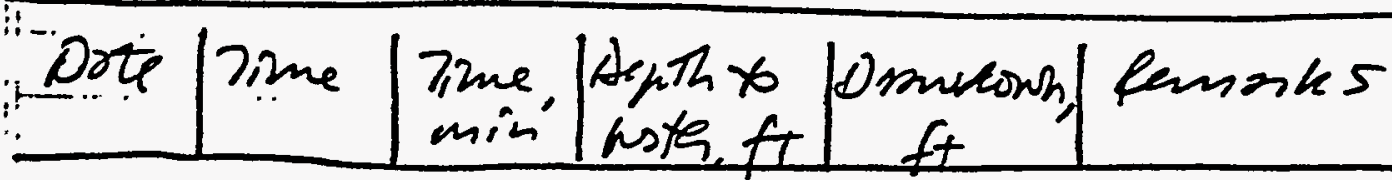

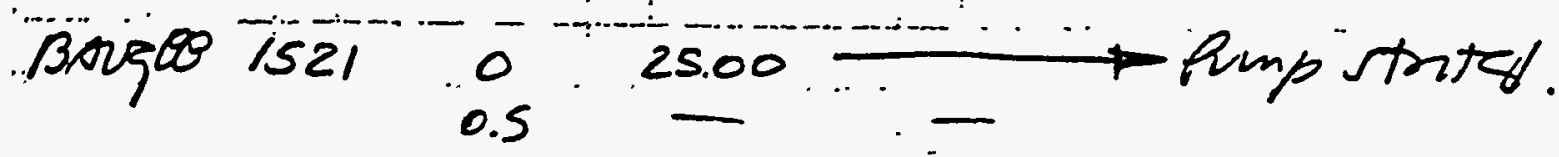

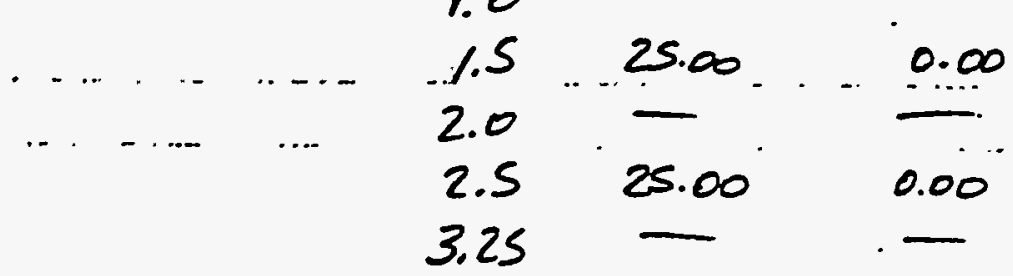

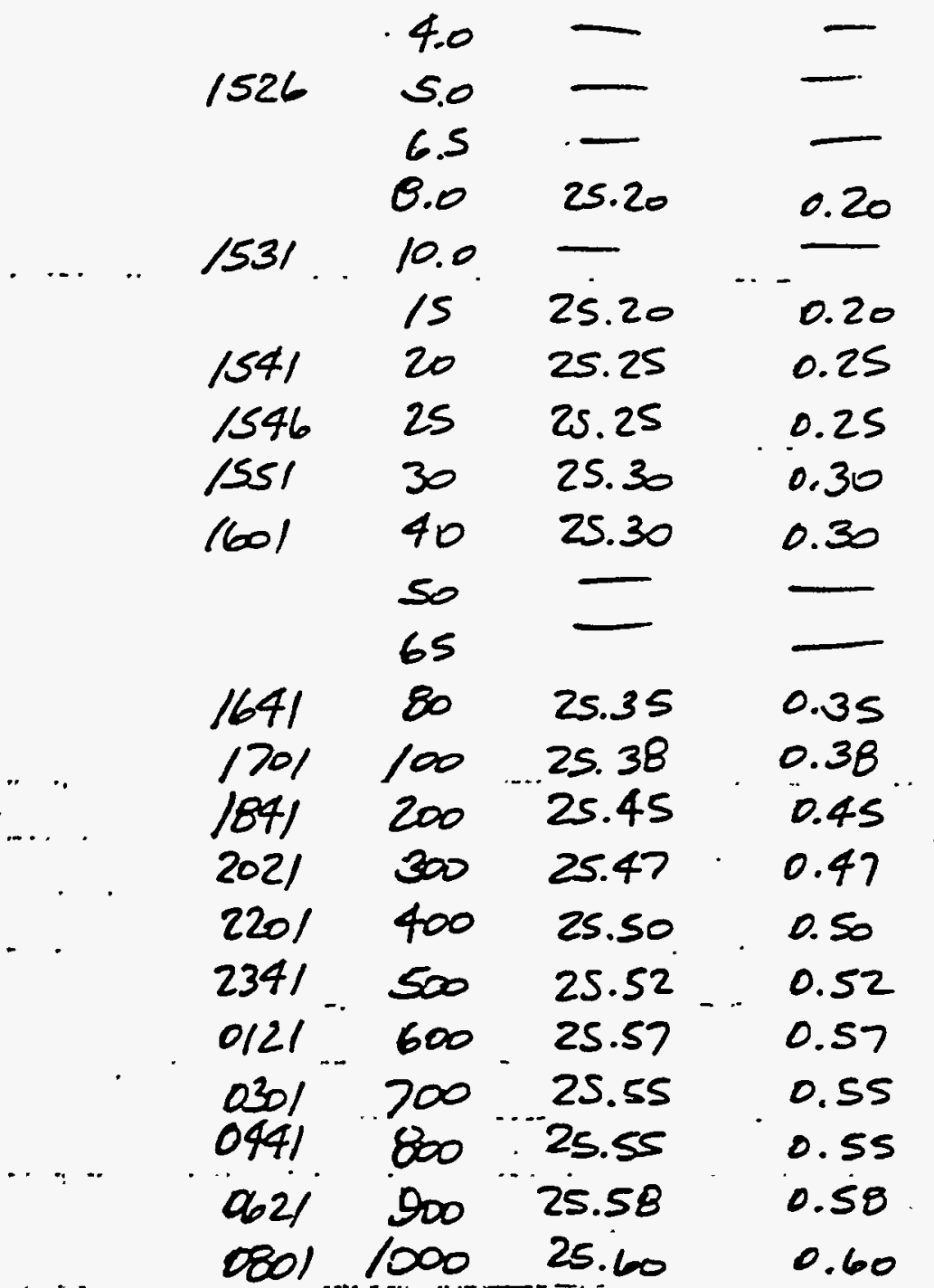

romen $91.005 .1(4 / 81)$ 
1.0 1.0

16.061 .0

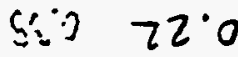

$61.52 \quad 0.9$

orio tzo

22.52

$1 \%$

$\infty$

$\infty$

का 52 9.

08

sio s2:

52.52

$5 \cdot 7$

59

:०० $12 \cdot 0$

$42 \cdot 52$

$2 \cdot \pi$

9

$62.52 \quad 2 \pi$

of

clis os.0

०ह52

9te

\&

62.0 $1 \varepsilon^{\circ} 0$

I $\varepsilon 52$

Dit

$\varepsilon \varepsilon \cdot \Omega$

$5 \% 15$

वह's2

$\varepsilon 89$

s?

L१ ह ह०

ot:S2

$6 \% 10$

stisz

$1 \cdot(2)$

$2 \cdot 751$

820

$019 \infty .0$

20

$\varepsilon \cdot 252$

or

52

0.01

o'g

5.9

0.5

oss

$s \cdot 1 / \varepsilon$

0.6

$52 \%$

52

5505.2

LIQ

5\%

$0 \%$

45.52

0.010

0.0

0

0101

6001 0180

1180

og. 0

0952

$$
0.0
$$

$28 \Omega 0$ - hdت 0232

Z-826-ma : Noll0so7

in $\log 05: 325$ 
JE JACOBS ENGINEERING

DATE $\sin 25$ sp 88 sUaEct

bUل ano.

OQUIFER PUMPING TESTS

in-928

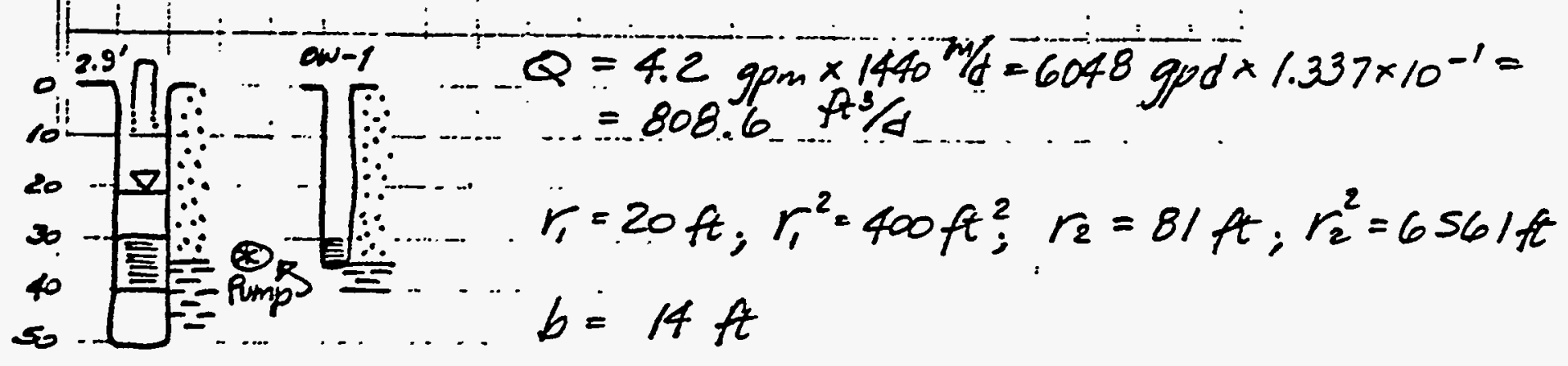

Aguifar incourined

1) Mw-928 Pumping (Theis)

$$
\dot{T}=\frac{a}{4 \pi p} \cdot \omega(u \ldots) \quad K=7 / 6
$$

A回 $\left.J_{\text {(err }}\right)=\frac{808.6 \mathrm{~A}^{2} / 4}{4 \cdot \pi \cdot 1.8 \mathrm{~A}} \cdot 1=35.75 \mathrm{At}^{2} / \mathrm{d}$

$$
K=\frac{35.75}{14}=2.55 \mathrm{At} / \mathrm{d}
$$

$A, \square T($ fott $)=$ sme as above $=2.55 \mathrm{f} / \mathrm{d}=K$ )

2) nw-928 fecovery (Theis)

$A 7=\frac{808.6}{4 \cdot \pi \cdot 3 \cdot 0} \div 10=169.33 \mathrm{ft} / \mathrm{d}$

$$
K=\frac{169.33}{14}=12.10 \mathrm{Ft} / \mathrm{s}
$$

3) Mw-928 fecoiry (stioightline)

$\dot{K}=15.13 \mathrm{~A} / \mathrm{d}$ (ae colculations on graph...) 
JACOBS ENGINEERING GPOL, wy

Date $\operatorname{tin} 25$ gpe8

or ento

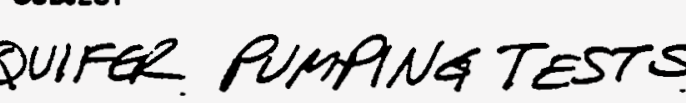

HEET NO

$108 \mathrm{NO}$.

Mw-028 (cont)

4)

$$
\begin{aligned}
& \text { B.] } T(\operatorname{sos} 4)=\frac{808.6}{4 \cdot \pi \cdot 0.25} \cdot 1=257.39 \mathrm{~A} / 4 \\
& K=\frac{257.30}{14}=18.38 \pi 4 \\
& B, D T(10.6)=\frac{808.6}{4 \cdot \pi \cdot 0.33} \cdot 1=194.99 \mathrm{At} / \mathrm{d} \\
& K=\frac{104.99}{14}=13.02 \mathrm{~A} / \mathrm{A}
\end{aligned}
$$

$$
\begin{aligned}
& \text { 5) } 0 w-928-1 \\
& W^{\prime}=\frac{4 T t u}{r^{2}} \\
& \text { BD } \sigma(\text { errly })=\frac{4 \cdot 257.39 \cdot 0.01 \cdot 0.01}{400}=2.57 \times 10^{-4} \\
& \text { B.D s. } \sigma_{y}(\text { tot })=\frac{4 \cdot 194.99 \cdot 0.052 \cdot 0.1}{400}=0.01 \\
& \eta=\frac{s+S_{y}}{s}>6.5=\frac{2.57 \times 10^{-1} \cdot 0.01}{2.57 \times 10^{-4}}=39.9
\end{aligned}
$$

b) ow-928-1 Recovery (Stroightline)

$K=21.18 \mathrm{Ald}$ (Fee olculation on giah...) 
UE JALUSS ENGINEERING

DATE Sun 25 tepes

ofdU Ono.

wiLEeT

HeET no.

cos no.

Mw-e2e(cont)

7) ow-928-2.'Pumping (Jhei)

$$
\begin{aligned}
& \text { CD T(terih) }=\frac{008.6}{4 \cdot \pi \cdot 0.28} \cdots 1=229.81 \\
& K=\frac{229.81}{14}=16.41 \mathrm{~A} / \mathrm{d} \\
& c_{1} \square T(100)=\frac{B O B .2}{4 \cdot \pi \cdot 0.2^{2}} \cdot 1=292.48 \mathrm{~A}^{2} / \mathrm{g} \\
& K=\frac{292.48}{14}=20.89 \mathrm{AJ}
\end{aligned}
$$

8) ow $-928-2$.

$$
\begin{aligned}
& C \boxminus \quad S^{\prime}(\mathrm{benh})=\frac{4 \cdot 229.81 \cdot 0.044 \cdot 0.1}{6561}=6.16 \times 10^{-4} \text {. } \\
& \text { c... } \sigma_{4}^{\prime}(126)=\frac{4 \cdot 222.48 \cdot 0.20 \cdot 0.1}{6561}=0.004 \\
& \eta=\frac{\sigma+5 y}{s}>6.5=7.49
\end{aligned}
$$

SUMMART - K

$$
\begin{aligned}
& \text { Mw-928. Reconang(reis) } 12.10 \mathrm{f} / \mathrm{A} \\
& \text { "ow-928-1. Pecovery (suline) . 15.13" " } \\
& \text { ow-928-1 Rumping (Then) ... 16.15" } \\
& \text { E. preijo : . 16.14 f/d } \\
& \text { (fongs: } \div 12-21 \mathrm{ft} / \mathrm{d})
\end{aligned}
$$


JACOBS ENGINEERING

Foof, WY

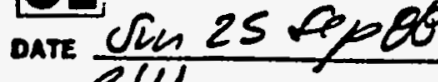

WUECT

HEET NO.

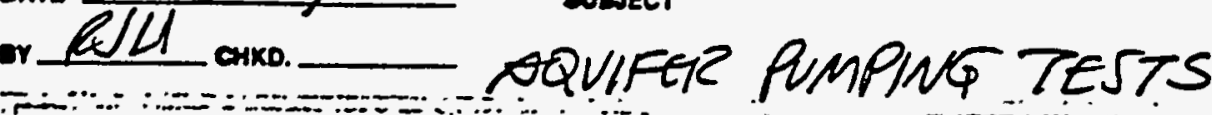

sos no.

nw-220 (cont)

7) ow-928-2 +. Puming (Jhoi)

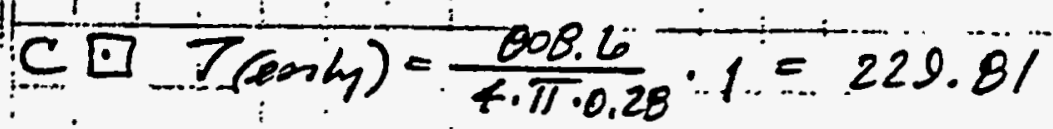

$$
\begin{aligned}
& K=-\frac{229 \cdot 81}{14}=16.41 \mathrm{~A} / \mathrm{d} \\
& c_{1} \square T T(120)=\frac{800.6}{4 \cdot \pi \cdot 0.22} \cdot 1=292.48 A^{2} / 6 \\
& K=\frac{292.48}{14} \equiv 20.89 \mathrm{At}
\end{aligned}
$$

8) ow-928-2

$$
\begin{aligned}
& \text { C曰 } S^{\prime}(\mathrm{erm})=\frac{4 \cdot 229.81 \cdot 0.044 \cdot 0.1}{6561}=6.16 \times 10^{-4} \\
& \text { c..]. } \sigma_{4}^{\prime}(126)=\frac{4 \cdot 292.48 \cdot 0.20 .0 .1}{6561}=0.004 \\
& \ddot{\eta}=\frac{v+s_{4}}{5}>6.5=7.49
\end{aligned}
$$

SUMMARY - K

Mw-928. Recovang(Feis) $12.10 \mathrm{ft} / \mathrm{A}$

...." Recovery (Juline)

ow-928-7 Rumping (Theiv) 15.13

$\because$..: Recontiy (stiline) 21.18

K... prajoge: $16.14 \mathrm{ft} / \mathrm{d}$

(fongs $\div 12-21 \mathrm{ft} / \mathrm{d})$ 
APPENDIX F

RISK ANALYSIS 


\section{RISK ANALYSIS}

To demonstrate the naturally poor water quality and substantiate the lack of probable use, a risk analysis of background ground water quality from the uppermost aquifer was performed. Potential risks due to adult resident ingestion of background ground water from the uppermost aquifer were derived based on the average detected contaminant concentrations in the background monitor wells. The risk calculations were based on standard EPA risk assessment guidance (EPA, 1989), which assumes a 154-pound 70-kilogram) adult drinking a half-gallon (2 liters) of affected water per day, 350 days per year, for a period of 44 years (representing a farm residence). Potential risk for noncancer effects is evaluated by comparing the calculated intake of each constituent of concern (based on the presumed exposure scenario and intake parameters) with the EPA reference dose, which is the conservatively derived dose level considered by EPA to be protective of human health. Potential risk for cancer effects is given by the excess lifetime cancer risk. The National Contingency Plan (40 CFR Part 300 (1994)) states that exposures to noncarcinogenic chemicals should present no appreciable risk of significant adverse effects and that the cumulative excess lifetime cancer risk should not exceed $10^{-4}$ (40 CFR $\S 300.430(e)(2)(i)(2))$.

The doses based on the average background concentrations of selenium and soluble uranium salts $(0.4$ and $2 \mathrm{mg} / \mathrm{L}$, respectively) exceeded the respective EPA reference doses for those chemicals (by 2.2 and 18 times the reference dose, respectively). The calculated excess lifetime cancer risk for the average detected uranium-234 and -238 concentration in the background ground water $(1350 \mathrm{pCi} / \mathrm{L})$ was $6.7 \times 10^{-4}$, which exceeds the $10^{-4}$ limit for acceptable cancer risk. Thus, the background concentrations of selenium and uranium are associated with unacceptable cancer and noncancer risks, which supports the conclusion that background ground water from the uppermost aquifer is not an acceptable source of drinking water for humans.

Potential risk to agricultural crops irrigated with background ground water from the uppermost aquifer was evaluated by comparing current monitoring data to maximum recommended safe concentrations for irrigation water (EPA, 1972). The results of that comparison are presented in Table F.1. The EPA comparison values reflect average rainfall conditions. Crops in arid regions, such as at the Spook site, are more susceptible to damage from elevated contaminant concentrations in irrigation water (relative to those in more humid regions) because of the increased requirement for irrigation water and the extended duration of the required irrigation period (i.e., crops in arid regions may require irrigation throughout the growing season rather than only during limited periods of the growing season) (EPA, 1972). Thus, it is possible that crop damage may occur at contaminant concentrations that are somewhat lower than those shown in Table F.1 because of the arid conditions at the site.

Two of the background contaminants (molybdenum and selenium) have maximum 10.03 and $2.17 \mathrm{mg} / \mathrm{L}$, respectively) and average (0.018 and $0.4 \mathrm{mg} / \mathrm{L}$, respectively) concentrations exceeding the maximum recommended safe concentrations in irrigation water $(0.010$ and $0.020 \mathrm{mg} / \mathrm{h}$, respectively). These results indicate that naturallyoccurring concentrations of contaminants in ground water from the uppermost aquifer are above the recommended concentrations for irrigation. 


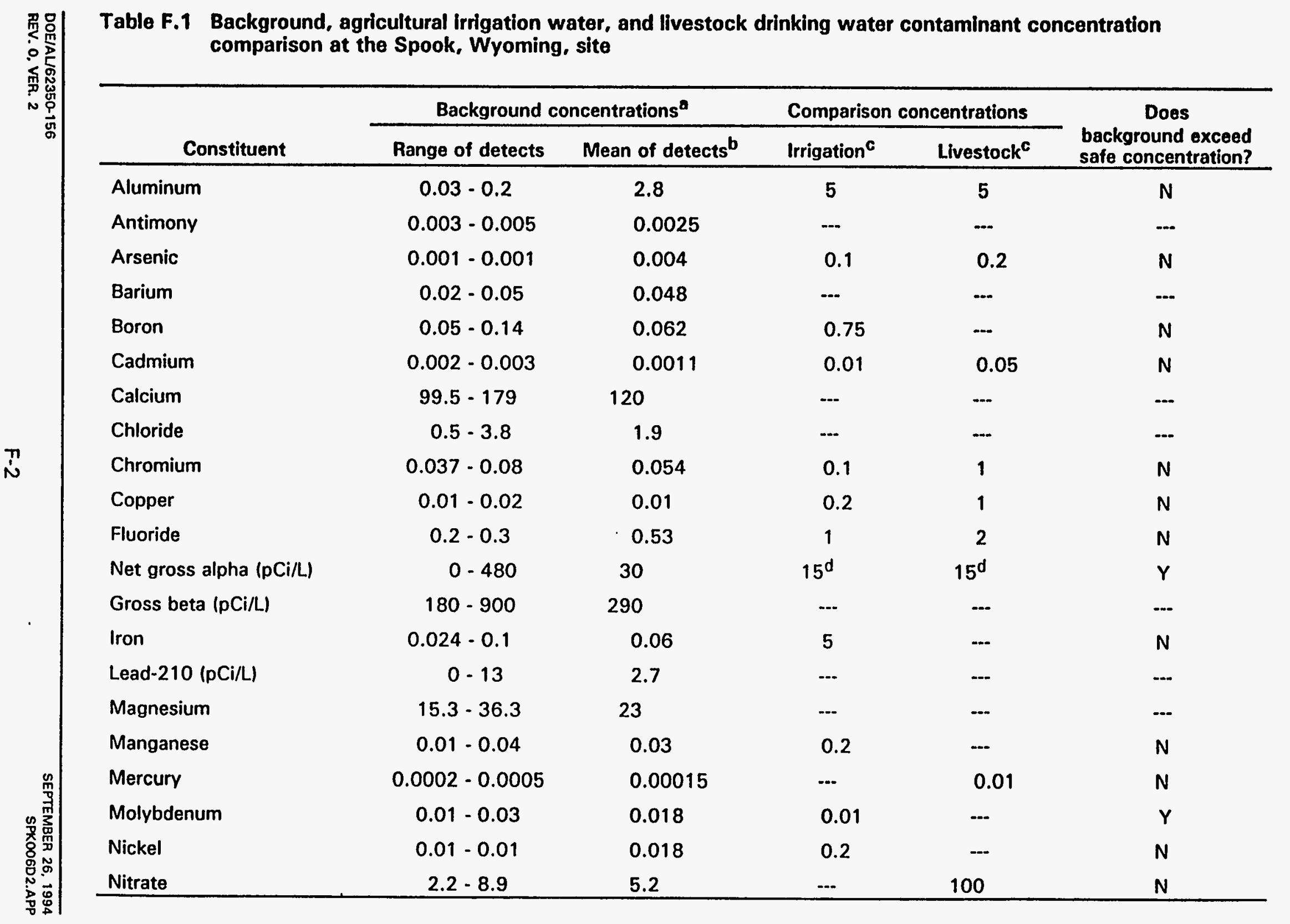


Table F.1 Background, agricultural irrigation water, and livestock drinking water contaminant concentration comparison at the Spook, Wyoming, site (concluded)

\begin{tabular}{|c|c|c|c|c|c|}
\hline \multirow[b]{2}{*}{ Constituent } & \multicolumn{2}{|c|}{ Background concentrations ${ }^{a}$} & \multicolumn{2}{|c|}{ Comparison concentrations } & \multirow{2}{*}{$\begin{array}{l}\text { Does } \\
\text { background exceed } \\
\text { safe concentration? }\end{array}$} \\
\hline & Range of detects & Mean of detects ${ }^{b}$ & Irrigationc & Livestock ${ }^{c}$ & \\
\hline Polonium-210 (pCi/L) & $0-0.8$ & 0.15 & $\cdots$ & $\cdots$ & $-\cdots$ \\
\hline Potassium & $4.39-8.4$ & 7.4 & -- & -- & $\cdots$ \\
\hline Radium-226 (pCi/L) & $0.2-2.5$ & 0.74 & 20 & 20 & $\mathbf{N}$ \\
\hline Radium-226/-228 (pCi/L) & $1.3-4.8$ & 2.3 & 5 & 5 & $\mathbf{N}$ \\
\hline Selenium & $0.036-2.17$ & 0.4 & 0.02 & 0.05 & Y \\
\hline Silica $-\mathrm{SiO}_{2}$ & $13.5-16$ & 15 & $\cdots$ & $\cdots$ & $\cdots$ \\
\hline Silver & $0.01-0.02$ & 0.011 & $\cdots$ & -- & -- \\
\hline Sodium & $13.4-30.8$ & 23 & $-\cdots$ & $\cdots$ & -- \\
\hline Strontium & $0.8-2.2$ & 1.5 & --- & -- & $--\cdot$ \\
\hline Sulfate & $150-393$ & 220 & $-\cdots$ & -- & $\ldots$ \\
\hline Thorium-230 (pCi/L) & $0-8.1$ & 0.7 & $\cdots$ & --- & --- \\
\hline Tin & $0.003-0.12$ & 0.017 & -- & $\cdots$ & $\cdots$ \\
\hline Uranium & $0.89-2.9$ & 2 & $-\cdots$ & -- & $\cdots$ \\
\hline Uranium-234/238 (pCi/L) & $610-1990$ & 1350 & $30^{d}$ & $30^{d}$ & $\gamma$ \\
\hline Vanadium & $0.016-0.03$ & 0.025 & 0.1 & 0.1 & $N$ \\
\hline Zinc & $0.005-0.014$ & 0.0054 & 2 & 25 & $N$ \\
\hline
\end{tabular}

${ }^{a}$ Concentrations are reported in milligrams per liter $(\mathrm{mg} / \mathrm{L})$ unless noted otherwise in first column.

b Mean of the average of detects for each background well. If a well had no detects, one-half the detection limit was used.

CMaximum safe concentrations, EPA, 1972.

'EPA (1972) states that the maximum recommended safe concentration for radionuclides in water for irrigation and livestock should be taken as the Federal Drinking Water Standard. 
Livestock can be exposed to contaminants in ground water directly by drinking water or indirectly through ingestion of fodder irrigated with the affected ground water. Because ground water from the uppermost aquifer is not recommended for use as a source of irrigation water, the indirect exposure pathway is not relevant.

Potential risks related to direct exposure of livestock to background ground water concentrations were evaluated to demonstrate that naturally-occurring contaminant concentrations exceed recommended safe levels for ingestion of the water. Analysis of the potential toxicity of background ground water to livestock consisted of comparing background ground water concentrations to approximate drinking-water concentrations considered to be protective of livestock (EPA, 1972).

The average and maximum detected concentrations for selenium in the background ground water $(0.4$ and $2.17 \mathrm{mg} / \mathrm{L}$, respectively) exceed the $0.05 \mathrm{mg} / \mathrm{L}$ level considered to be protective of livestock (EPA, 1972). These results indicate that the naturally elevated selenium concentration causes the ground water from the uppermost aquifer to be unusable for ingestion by livestock.

The indirect exposure pathways associated with ingestion of crops irrigated with background ground water or with ingestion of meat or milk from livestock that drink the ground water were not evaluated because the screening level risk analysis demonstrated that background ground water from the uppermost aquifer is not usable as a source of crop irrigation water or as a source of drinking water for cattle.

\section{Conclusions of the risk analysis}

The screening level risk analysis of background ground water from the uppermost aquifer demonstrates that use of naturally-occurring ground water from the uppermost aquifer (represented by monitor wells SPK-01-931, -933-943, and -944) (Figure F.1) would constitute unacceptable risks to humans, livestock, and irrigated crops. The background risk analysis indicated that there is no beneficial use of the naturally-occurring ground water from the uppermost aquifer; therefore, a baseline risk assessment was not performed for the Spook site.

The results of this risk analysis support the DOE's proposed compliance strategy of no further remediation based on the application for supplemental standards. The affected ground water from the uppermost aquifer at the site poses no risk to human health and the environment because of the absence of relevant or realistic exposure pathways. The screening level risk analysis for background ground water from the uppermost aquifer demonstrates that naturally-occurring concentrations of uranium, selenium, and nitrate in that ground water cause unacceptable risks to humans, and the ground water is not recommended for use for livestock or irrigation. 


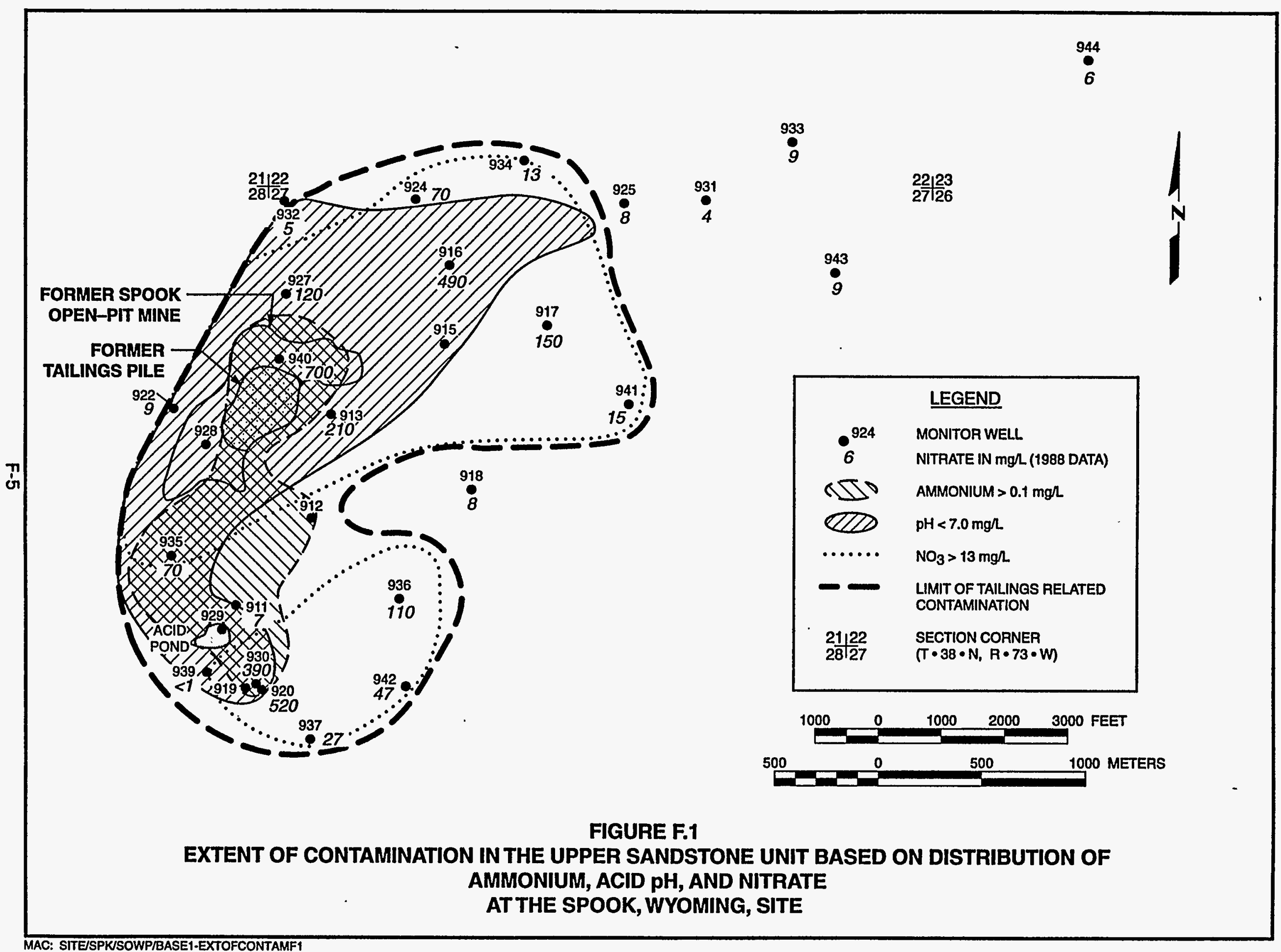




\section{REFERENCES}

EPA (U.S. Environmental Protection Agency), 1989. Risk Assessment Guidance for Superfund, Volume I, Human Health Evaluation Manual, Part A, Interim Final, EPA/540/1-89/002, Office of Solid Waste, Waste Management Division, Washington, D.C.

EPA (U.S. Environmental Protection Agency), 1972. Quality Criteria for Water 1972, A Report of the Committee on Water Quality Criteria, prepared by the National Academy of Sciences-National Academy of Engineering, Environmental Studies Board, Washington.

\section{CODE OF FEDERAL REGULATIONS}

40 CFR Part 300, "National Oil and Hazardous Substance Pollution Contingency Plan," U.S. Environmental Protection Agency (1994). 